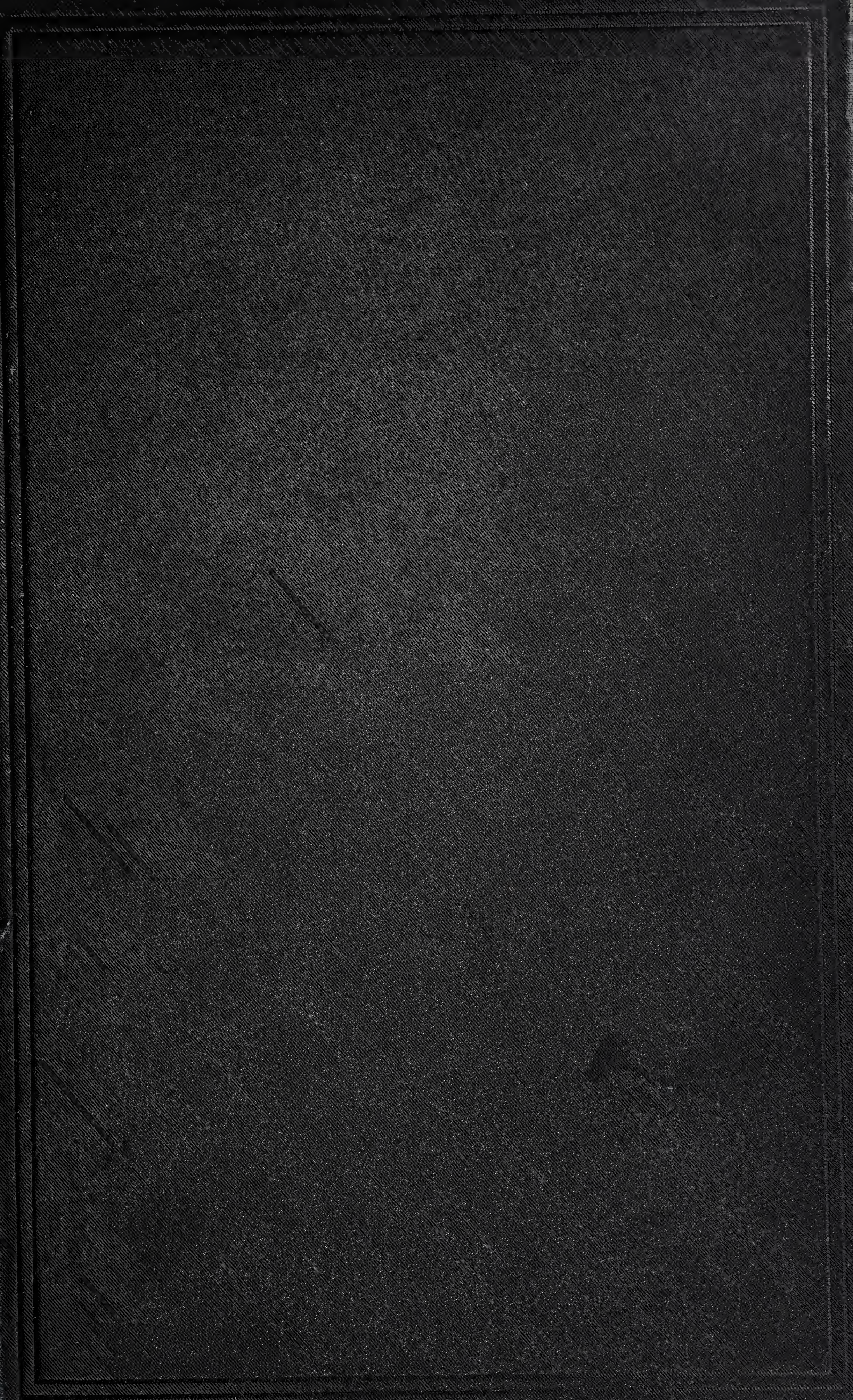




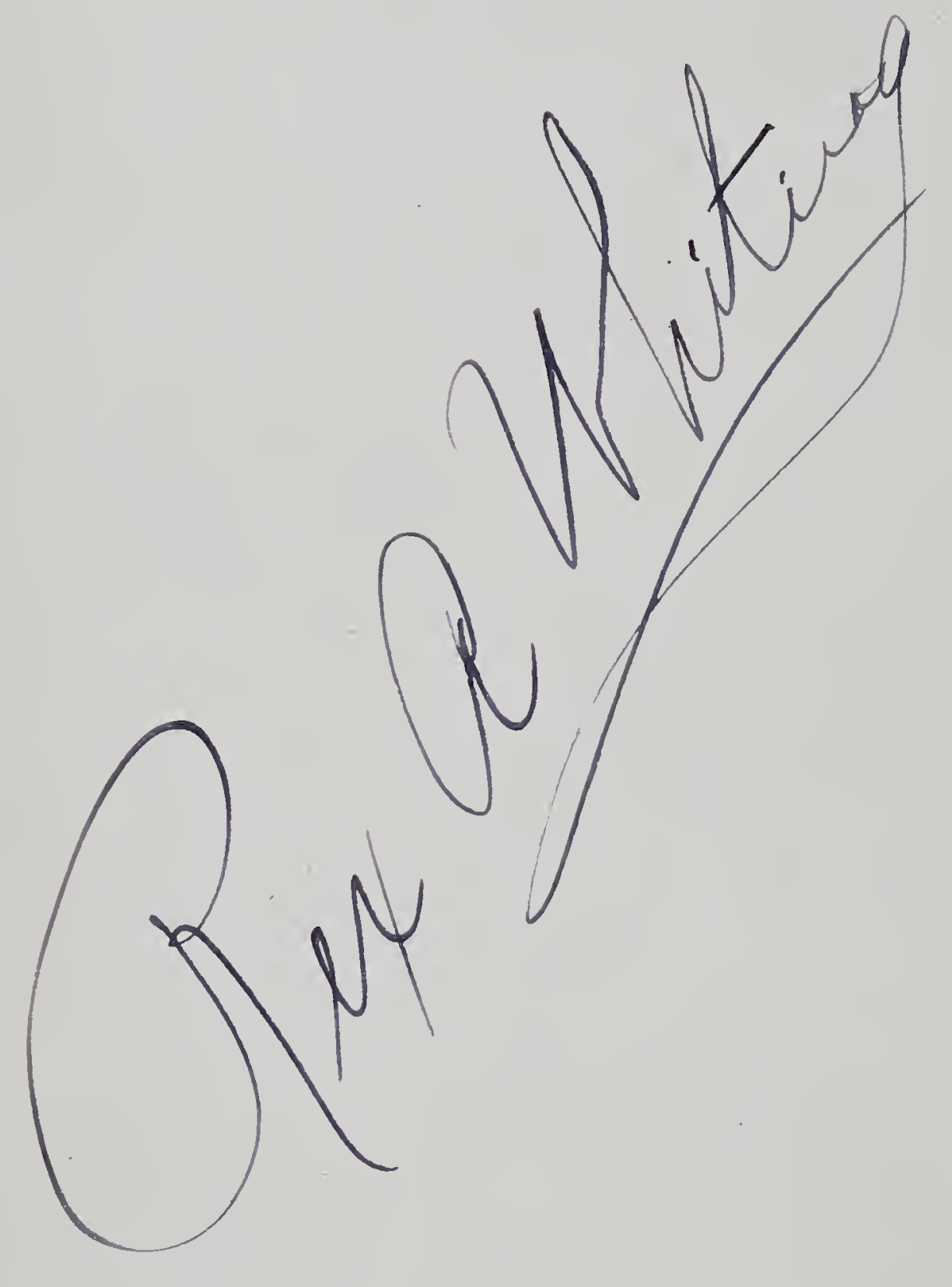


Pex. A. Whiting 





\section{THE PRACTICE}

$\mathrm{OF}$

\section{VETERINARY SURGERY.}

VOL. III.-REGIONAL SURGERY. 
BI THE SAME AUTHOR.

Royal sro, 438 pages, 406 illustrations.

A Handbook of Horse-shoeing.

With Introductory Chapters on the Anatomy and Physiology of the Horse's Foot. The section on the "Practice of Shoeing" contains $5 t$ full-page plates and descriptive notes supplied by ALBERT WHEATLEY, F.R.C.V.S.

60 large pages $\left(14 \frac{1}{2} \times 10\right.$ inches $), 315$ illustrations.

An Atlas of Yeterinary Surgical Operations.

Demy sto, 42 pages, 16 full-page plates.

A Surgical Operating Table for the Horse.

Royal Sro, 618 pages, 95 illustrations.

Studies in Clinical Veterinary Medicine and Surgery.

By P. J. Cadiot and Jno. A. W. Doluar.

Royal $8 r_{0}, 269$ pages, 27 i illustrations.

The Practice of Veterinary Surgery.

Vol. I.-OPERATIVE TEChNIQUE. 


\title{
REGIONAL
}

\section{VETERINARY SURGERY}

BY

\author{
Dr. H. MÖLLER
}

FORMERLY PROFESSOR OF SURGERY AT THE VETERINARY HIGH SCHOOL OF BERLIN

AND

JNO. A. W. DOLLAR, M.R.C.V.S., F.R.S.E. CORRESPONDING MEMBER OF THE CENTRAL SOCIETY OF VETERINARY MEDICINE OF PARIS, ASSOCIATE OF THE SOCIETY OF VETERINARY MEDICINE OF BRABANT (BELGIUM), LIFE MEMBER OF THE ROYAL ITALIAN SOCIETY OF HYGIENE, ETC., ETC.

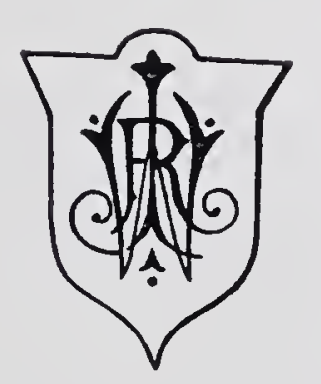

New YorK

WILLIAM R. JENKINS

VETERINARY PUBLISHER AND BOOKSELLER

851 AND 853 STXTh AVENue

1904

[All rights reserved] 
Digitized by the Internet Archive in 2017 with funding from IMLS LG-70-15-0138-15 


\section{PREFACE.}

The rapidity with which a large edition of "Möller's Veterinary Surgery" has been taken up by the veterinary profession in this and other English-speaking countries rendered it necessary to prepare a new volume to take its place. This I now have the pleasure of placing before the public. The experience gained since the issue of the first volume, the valuable criticisms of fellow-practitioners, and the continued advances in the science of veterinary surgery have made indispensable a thorough reconstruction of the material, so that whilst I have deviated as little as possible from the original plan of arrangement, I have excised much that was of doubtful or secondary importance, and have added freely from my own experience and from the teachings of the British and European veterinary Press. Despite my best efforts to keep it within reasonable compass, the book has grown by more than one hundred pages of letter-press, and the illustrations have been much more than doubled.

To Professor McQueen, my faithful coadjutor in former works, I am indebted for many valuable suggestions, and for performing the indispensable but onerous task of reading the proof sheets of the book as it passed through the press.

Herr Wilhelm Braumüller, of Vienna, Herren Schönefeld, of Dresden, and Messrs. Asselin and Houzeau, of Paris, very lindly consented to supply me with some of the new illustrations, whilst others were generously placed at my disposal by Messrs. Arnold \& Son, of London. The greater number, however, have been specially drawn for me by $\mathrm{Mr}$. Beecroft, of London, and engraved by John Swaine \& Sons.

As on a previous occasion, I have to plead for lenient consideration of work performed in all the storm and stress of a busy practitioner's life; a life, moreover, complicated by many undertakings other than those strictly appertaining to the profession of veterinary surgery, and 
enlivened, if not sweetened, by the necessity for conducting frequent and strenuous political controversies. From my fellow practitioners, however, of whose number I am proud to regard myself as one, I have always received generous treatment, and to their judgment I now, without further words, respectfully submit the present volume.

56, New Bond Street,

JNO. A. W. DOLLAR.

LONDON, W.

July, 1903. 


\section{CONTENTS.}

\section{DISEASES OF THE HEAD.}

I. Diseases of the Lips and Cheers

(1.) Wounds and Bruises . • .

(2.) Acute Inflammation of the Lips and Cheeks . 3

(3.) Tumours of the Lips and Cheeks . . . . . . 4

(4.) Paralysis of the Lips. Facial Paralysis . . . . 6

II. Diseases of the Mouth . . . . . . . . 10

(1.) Foreign Bodies in the Mouth _. 10

(2.) Diseases of the Tongue . . . . . . . . 13

(A) Mechanical Injuries . . . . . . 13

(B) Acute Inflammation of the Tongue (Glossitis Acuta) . • 15

(C) Chronic Inflammation and New Growths in the Tongue (Makroglossia) • $\quad . \quad \cdot \quad \cdot \quad 16$

(a) Glossitis Chronica Interstitialis Fibrosa . . 16

(b) Actinomycosis of the Tongue. Glossitis Actinomycotica 17

(D) Paralysis of the Tongue (Glossoplegia) . . . 19

(E) Ranula . . . . 20

(F) Fracture of the Hyoid Bone . . . . . 21

(G) New Growths in the Tongue : $\quad . \quad 5 \quad 22$

(3.) Fracture of the Premaxillary Bone . . . . . 22

(4.) Injuries of the Interdental Space . . . . . . . 23

(5.) Diseases of the Teeth . . . . . . 25

(A) Irregularities in the Development of the Teeth . . 26

(a) Extra or Adventitions Teeth (Polyodontia, Hyper-
(lentition)

(b) Trregularities in Replacement of the Teeth : $: 27$

(c) Displacement of the Teeth . . . 28

(B) Irregularities of Wear in the Teeth . . . $\quad 29$

(a) The Angular or Sharp Mouth, the so-called Prog-
nathous Jaw.

(b) The Shear-like Mouth : $\quad 33$

(c) The Wave-formed Mouth : : $: 34$

(d) The Step-formed Mouth . . . . 35

(e) Premature Wear of the Teeth . . : : $\quad 37$

(f) The Smooth Mouth . . . . . . 38

(C) Diseases of the Tooth Proper . . . . . . 38

(a) Dental Caries (Caries Dentium) . . . 38

(b) Fissures and Cavities in the Teeth . . . 40

(c) Dental Tartar (Cremor Dentium) : . : 41

(D) Diseases of the Alveoli _ : • • • 42

(a) Alveolar Periostitis (Periostitis Alveolaris) : : 42

(a) Inflammation of the Alveolar Periosteum in Herbivora

(b) Inflammation of the Alveolar Periosteum in
Carnivora

(b) Dental Fistula . $\quad . \quad \cdot \quad \cdot \quad \cdot 054$

(c) Neoplasms of the Gums and Alveoli (Epulis) : : $\quad 57$

(d) Dental Tumours . . . . . . 57 
III. Diseases of the Nose, Nostrils, and the Spaces communicating WITH THEMI

(1.) Wounds of the Alæo of the Nostrils

(2.) Fracture of the Nasal Bones

(3.) Foreign Bodies and Tumours in the Nostrils

(4.) Diseases of the Frontal and Superior Maxillary Sinuses Trephining the Superior Maxillary and Frontal Sinuses

(5.) Necrosis of the Turbinated Bones

(6.) Epistaxis (Bleeding from the Nose)

PAGE

IV. Diseases of tile Salivary Glands 。 . . . . 76

(1.) Wounds and Injuries of the Salivary Glands . . . 76

(2.) Inflammation of the Salivary Glands (Parotitis) • . 76

(3.) Abscess Formation in the Sub-Parotid Lrmph Glands . . 80

(t.) Salivary Calculi and Concretions . . . 81

(5.) Salivary Fistulæe . . . . . 82

(6.) Tumours of the Salivary Glands . . . 86

(7.) Actinomycosis in the Parotideal Region . . . . 87

V. Diseases of the Face and Iower Jaw . . . . 90

(1.) Fractures of Bones of the Face . . . . . . . 90

(2.) Fracture of the Lower Jaw . . . . . . 94

(3.) Dislocation (Luxation) of the Lower Jaw . . . 99

(4.) Inflammation of the Articulation of the Jaw . . . 98

(5.) Paralysis of the Muscles of Mastication. Paralysis of the Lower Jaw (so-called) . . . . . . . Pos

(6.) Periostitis and Exostosis on the Posterior Border of the Lower Jaw . . . . . . . 101

(7.) Tumours on the Lower Jaw . . . . . . 101

(8.) Actinomycosis of the Maxilla, Spinarentosa . . . 103

(9.) Inflammation and New Growths in the Submaxillary Lymph Glands .

VI. Diseases of the Ear and Guttural Pouches . . . . 107

(1.) Injury of the External Ear . . . . . . 107

(2.) Inflammation of the External Ear . 108

(3.) Edema of the Free Border of the Ear-External Canker (Othrematoma)

(4.) Inflammation of the External Auditory Meatus (Otitis Externa). Otorrhoea.

(5.) Fistula of the Ear. Dentigerous Cysts

(6.) Chronic Catarrh of the Guttural Pouches : $\quad \cdot \quad \cdot \quad 114$

(7.) Tympanites of the Guttural Pouch . . . . . . 121

VII. Diseases of the Sirull . . . . . . . . 123

(1.) Fractures and Injuries to the Cranial Bones . . . . 123

(2.) Concussion' of the Brain (Commotio cerebri) . . . 126

(3.) Fractures of the Frontal Bone . . . . . . . 126

(4.) Loosening of the Horns . $\quad$. $\quad$. $\quad . \quad 129$

(5.) Wounds and Injuries to the Skull $\quad . \quad$. $\quad$. 129

(6.) Yoke-injuries in working oxen : • • . 130

(7.) Trephining the Cranial Cavity in Sheep or Cattle, or Opening by the Trochar 


\section{DISEASES OF THE NECK.}

I Wounds and Bruises of THe Tissues of THe Neor

II. FraCtures of the Cervicat Vertebre

III. Distortion of the Neck (Torticollis, Capu't Obstipum) . . 142

IV. Goltre, Struma . . . . . . . . . 149

V. Ixflamiation of the Bursa of the Ligamentum Nucha $\cdot 154$

VI. Phlebi'sis. Inflamination of the Jugular Vein. (Pmlebisis Vexæ Jugularis) . . . . . . . . . 157

VII. Diseases of the Pharynx and Cesophagus . . . . 160

(1.) Foreign Bodies in the Pharnynx and Esophagus . . 160

(A) Foreign Bodies in the Pharynx and Esophagus of the Horse 162

(B) Foreign Bodies in the Esophagus of Cattle . . . 163

(C) Foreign Bodies in the (Esophagus of Swine . . . 169

(D) Foreign bodies in the Pharynx and Esophagus of Carnivora 170

(E) Impaction of the Crop in Birds . . 170

(2.) Injuries to the Pharynx and CEsophagus (CEsophageal Fistula) . 171

(3.) Ectasiae, Diverticula, and Ruptures of the Cisophagus . . 173

(4.) Stenosis and Compression of the CEsophagus . . . 176

(5.) Paralysis of the Pharynx and Cisophagus. . . . . 176

(6.) Tumours in the Pharynx and (Esophagus . . . 178

VIII. Diseases of the Larynx and Trachea . . . . 182

(1.) Injuries, Inflammations, and Tumours in the Larynx (Laryngitis. Perilaryngitis). . . . . . . 182

(2.) Neuroses of the Larynx-Spasm of the Rima GlottidisSpasmus Glottidis - Laryngismus Stridulus - LaryngoSpasmus-Hemiplegia and Liplegia Laryngis . . 187

(3.) Fracture, Deformity, and Stenosis of the Trachea..$\quad 189$

(4.) Foreign Bodies, Tumours, and Parasites in the 'Trachea . . 192

(5.) Tracheotomy . . . . . . . 194

(6.) Laryngotomy . . . . . . . . . . 200

\section{DISEASES OF THE THORAX.}

I. Fractures of the Ribs-Fracturze Costarum . . . 217

II. Costal Sinus . . . . . . . . . . 219

III. Sternal Sinus and Fradture of the Sternum . • . 220

IV. Wouxds and Bruises of the Sof't Parts of the Chest-Wall . 222

(1.) Wounds of the Chest-Wall without Perforation . . . 222

(2.) Wounds of the Pleura and Thoracic Cavity . . . 224

V. Shoulder Abscess . . . . . . . . 228

VI. Saddle and Collar Galls-Injuries from Pressure . . 233

(1.) Injuries Produced by the Saddle-Saddle-Galls . . . 233

(2.) Bruises Produced by the Collar or other Portions of the Harness 242

VII. Tumours of the Breast . . . . . . 243 
ViII. Tapping the Cinest (Paracentesis Thoracis, Thoracocentesis) .

(1.) Puncture of the Thorax for Removal of Serous or Pleuritic Exudate.

(2.) Puncture of the Thorax for Removal of Purulent Fluid .

(3.) Tapping the Pericardium in the Dog

\section{SURGICAL DISEASES OF THE ABDOMEN.}

I. Bruises of the Abdoninal Parietes . . . . . 251

II. Wounds of the Abdomen . . . . . . 253

III. Bowel Fistura (Anus Preternaturalis) · . . 259

IV. Paracentesis Abdominis (Puncture of time Abdonex) . . 261

V. Hernia . . . . . . . . . . 263

VI. Umbilical Heria (Omphalocele, Exomphalos) · • . 273

VII. Inguinal Hernia, Scrotal Hervia (Hernia Inguinalis, Heria SCRO'TALIS) . $\quad . \quad . \quad \cdot \quad \cdot \quad \cdot \quad \cdot 280$

(1.) Inguinal Hernia in the Horse . . . . . . 281

(A) Treatment of Non-Strangulated Inguinal Hernia . 285

(B) Treatment of Incarcerated Inguinal Hernia 289

(2.) False Inguinal Hernia (Hernia Inguinalis Interstitialis) • 292

(3.) Inguinal Hernia in Ruminants . . . . . 294

(4.) Perineal Hernia in Swine . . . . . . 295

(5.) Inguinal Hernia in Dogs . . . . . . . 297

VIII. Crural Hernia (Hernia Cruralis) . . . . . . 298

iX. Perineal Hernia . . . . . . . . 299

X. Ventral Hersia (Hernia Ventralis) . . . . . 301

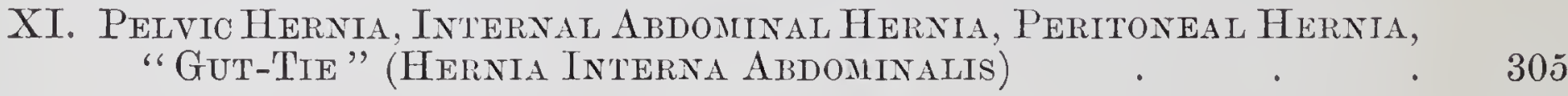

XII. Diaphragmatic Hermia (Hernia Diaphragitatica) • • 308

\section{SURGICAL DISEASES OF' THE STOMACH AND BOTELS.}

I. Foreign Bodies in the Digestive Tract . . . . 310

II. Puncture of the Bowel in Horses (Punctio Intestini) · · 315

III. Puncture of the Runien and Rumenotomy . . . . . 321

IV. Intussusception or Intagination of the Bowel . . . 328

V. Twist or Rotation of the Colon in Horses . . . . 330

SURGICAL DISEASES OF THE POSTERIOR PORTIONS OF THE RECTUM AND OF THE ANUS.

I. Congenital Mathormations. Absence of the Anus and Formation of Cloacd (Atresia Ani et Recti, Fistula Recto-Vaginalis) · 335

II. Injuries to the Rectudi and Anal Region . . . . 338 
III. Inflanimation of the Mucous Membrane of the Rectuni and Anus

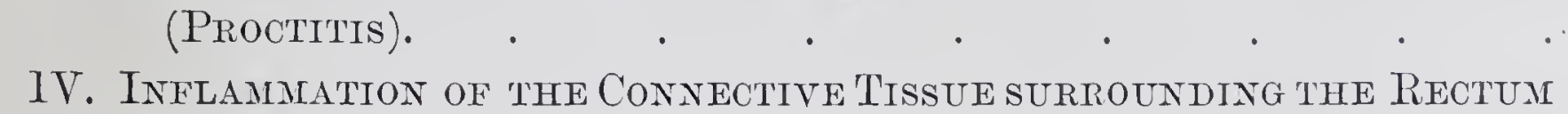
(Periproctitis or Paraproctitis Apostematosa) . . .

V. Prolapse of the Rectum and Anus (Prolapsus Recti ét Ani) VI. Dilatation, Stentosis, and Paralysis of the Rectum and Anus .

TII. Anal Fistula and Recto-Vaginal Fistula.

\section{DISEASES OF THE URINARY ORGANS.}

I. Congenital Malformations

(1.) Fissuring of the Male [Meatus Urinarius (Hypospadia and Epispadia)

(2.) Pervious Urachus

II. Urinary Calculi

(1.) Urinary Calculi in the Horse

(2.) Urethral Calculi in Ruminants .

(3.) Urinary Calculi in Swine

(4.) Urinary Calculi in the Dog'

III. Puncture of the Bladder (Punctio Vesicte) .

IV. Injuries, Inflamation, and Strictures of the Urethra . $37 j$

V. Paralysis of the Urinary Bladder (Cystoplegia). Catheterisis

VI. Inflamation of the Urinary Bladder (Cystitis) . •

VII. Prolapse and Inversion of the Urinary Bradder in Female Animals (Prolapsus ét Inversio Vesice)

\section{DISEASES OF THE MALE ORGANS OF GENERATION.}

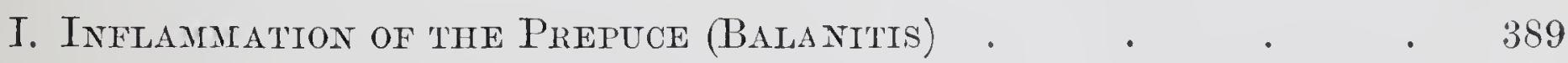

(1.) Inflammation of the Prepuce in the Hor:se . . . . $\quad 389$

(2.) Inflammation of the Prepuce in Oxen . . . . . . 391

(3.) Inflammation of the Prepuce in Swine . $\quad . \quad$. 892

(4.) Inflammation of the Prepuce in Carnivora . . . . 393

II. Phimosis, Parapimiosis Paralysis of the Muscles of the Penis, INJURIES to THE PENIS . . . . . . . 394

III. Tumours of the Sheatil and Penis. Amputation of the Penis . 398

Amputation of the Penis . . . . . . . . 399

TV. Diseases of the Prostate . . . . . . . . . . 403

V. Diseases of the Scrottul . . . . . . . . . . 405

VI. Diseases of the Testicle. Castration of Cryptorchids • • 407

(1.) Congenital Defects and Retention of the Testicles . . 407

(2.) Inflammation of the Testicle and Epididymis. Orchitis, Periorchitis, Epididymitis . . . . . . 413

(3.) Tumours of the Testicle (Sarcocele) and of the Epididymis . 414

VII. Hydrocele, Henatocele, and Varicocele . . . . 415

VIIT. Inflamation of the Spermatic Cord. Scirrhous Cord. FunicuLitis ChronicA . 


\section{DISEASES OF THE FEMALE ORGANS OF GENERATION.}

I. Injuries and Inflamation of the Vulva, Vagina, and Uterus .

II. Prolapse (Inversion) of the Vagina. Protaapsus (Irversio) VAGIN ex

III. Prolapse (Ixversion) of ahe U'ierus. Prolapsus (Inversio) Ut'eri IV. Tumours of the Vagina and Uterus .

V. Diseases of the Mamalary Gland

(1.) Wounds and Bruises of the Udder. Lacteal Calculi .

(2.) Acute Inflammation of the Udder (Mastitis Acuta). Its pathology and Varieties

(A) Acute Inflammations of the Udder :

(a) Phlegmonous Mastitis. Mastitis Phlegmonosid

(b) Catar'h of the Udder. M. Catarrhalis .

(c) Purulent Intiammation of the Udder. Nastitis Apostematosa

(d) Gangrenous Inflammation of the Uider. Mastitis Giangrenosa

(B) Chronic Inflammations of the Udder

(a) Infectious Catarrhal Mastitis

(b) Tuberculosis of the Udder. Mastitis T'uberculosil

(c) Actinomycosis of the Udder

(d) Bothryomycosis of the Udder

(4.) Tumours of the Udder

DISEASES OF THE SPINAL COLUMN AND PELVIS.

I. Fractures, Luxations, and Sub-Iuxations of the Dorsal and Lumbar Verítebize. Comalotio Spixalis.

II. Fraciures of the Sacral and Caudal Veríterra . . . 468

iit. Curvature of the Spine . . . . . . 470

IV. Fracture of the Peltis. . . . . . . 472

T. Diastasis or Luxation of the Sacro-Iliac Articulation . • 480.

VI. Luxation of the Syaphysis Ossium Pubis . . . . 482

VII. Hygrona of the Subcutaneous Bursa of the luber Ischit in Cattrle .

IT Paralysis of the Hind Extremities. Paraplegia • • • 483

IX. Diseases of the Tail . . . . . . . . . 491

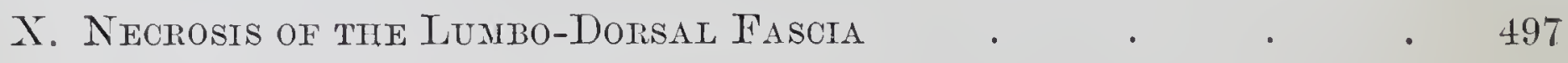

\section{DISEASES OF THE FORE LIMB.}

\section{A. DISEASES OF THE SHOULDER REGION.}

I. Fractures of the Scapula

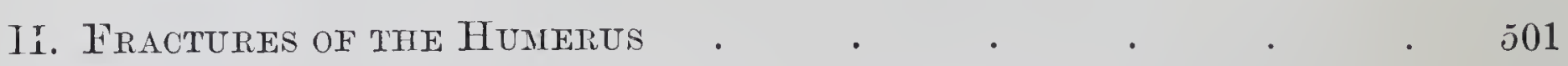

III. Diseases of The Shoulder-Joln't 
IV. Inflamimation of the Bursa of tire Biceps, Flexor Brachil or Coraco Radialis Muscle (Bursitis Intertubercularis).

T. Ixflamation of the Tendon of the Postea-Spinatus Muscle AND OF ITS BURSA

VI. Paralysis of the Suprascapular Nerve . . . . . 510

VII. Paralysis of the Radial Nerve (Paralysis Nerit Radialis) • 513

VIII. Paralysis of the Brachial Plexus . . . . . . J19

IX. Shoumer Liameness . . . . . . . . . 521

B. DISEASES OF THE ELBOW AND FORE ARM.

I. Fracture of the Ulia . . . . . . . . 526

II. Fracture of the Radius. $\quad$. . . . . 528

III. Luxation of trie Elbow-Jont . . . . . . . . 529

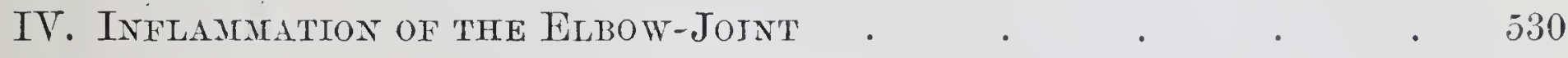

V. Capped Elbow tn the Horse . . . . . . . . . 531

VI. Wounds and Bruises of the Fore A ril • . • • . 536

\section{DISEASES OF THE KNEE.}

I. Fractures of the Bones of the Kree · . . . . . 539

II. Injuries to the Anterior Surface of the Kree . . . 540

(1.) Injuries to the Knee in the Horse . . . . . 540

(2.) Tumour's of the Knee in Cattle . . . . . . . . . 546

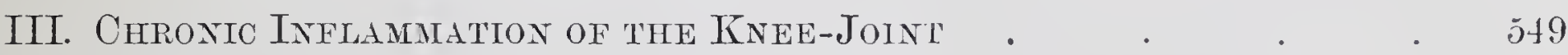

IV. Inflamation of tue Bursa of the Flexor Pedis . . . . 500

T. Exlargement of Articular and Tendinous Synortal Cavities in THE FORE LintB

\section{DISEASES OF THE METACARPUS.}

I. Fractures of the Metacarpus . . . . . . . 561

II. Wounds of the Metachlipus . . . . . . . 562

III. Rupture of the Flexor Tendons and Suspensory Liganent $56 j$

IV. Inflamination of the Flexor Tendons (Texdinitis) and of the Suspensory and Check Liganents

V. Contracted Tendons ("Knuchling Over") in Foals . . öS"

VI. Splints . . . . . . . . . . . 586

E. DISEASES OF THE PHALANGES.

I. Luxation of the Phalanges . . . . . . . . 594

II. Spratn of the Coronary Jolnt . . . . . . . . . 596

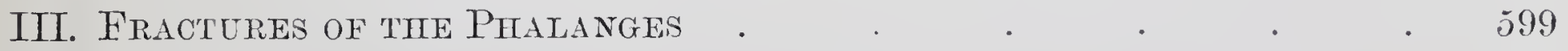

IV. RING-Bone . . . . . . . . . . . 605

Plantar Neurectomy . . . . . . . 614

Median Neurectomy .

Ulnar Neurectomy

T. Inflannation of the Posterior Liganients of the Coronet-Join'i 622

VI. Fractures of the Sesamoid Bones . . . . 624 


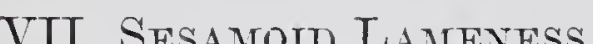

ViII. Ossification of the Lateral Cartilage.- Side-bone $\quad$. 628

IX. Fracture of the Navicular Bone . . . . . 632

X. Navicular Disease . . . . . . 633

XI. Deraratiris in the Fetrock Region. Mud-Fever-Cracked Heels -Grease

\section{DISEASES OF THE HIND LIMB.}

\section{A. DISEASES OF THE QUARTER.}

I. Injuries to the Muscles and Fascize of the Quarter . . 655

II. Fracture of the Fendur . . . . . . . $60 ̄ 7$

III. Luxation of the Fendur a . . . . . 661

IV. Inflamation of the Hip-Join't (Coxitis) . . . . . . $66 \tilde{0}$

V. Inflamination of the Tendon and Tendon Bursa of the Gluteus Medius Musche. Bursitis Glúxæl Medir. S. TroCHANTERICA .

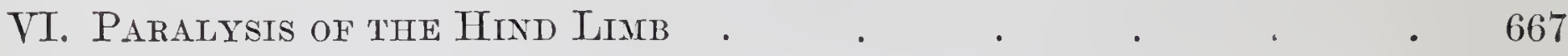

A. Paralysis in the region supplied by the gluteal nerves . . 668

B. Paralysis in the region supplied by the great sciatic nerve . 668

C. Paralysis of the anterior crural nerve and quadriceps femoris muscle. Rupture of rectus femoris and vasti muscles, and of the straight ligaments of the patella . .673

D. Paralysis of the obturator nerve . . . . . . . 676

VII. Thrombosis of the Posterior Aorta and of its Branches . 677

VIII. Displacement of the Biceps Femoris Muscle . • . . 680

IX. Hip LameNess . . . . . . . . . 682

\section{B. DISEASES OF THE STIFLE-JONNT.}

I. Luxation and Sprain of the Stifle-Joint ‘ . . . . 687

II. Displacenent of the Pátella. Luxatio Patelile . . . 688

III. Rupture of the Straigite Ligaments of the Patelia . . 693

IV. Fracture of the Patella . . . . . . . 694

V. Injuries and Acute Inflamalation of the Stifle-Joint. Gonitis 695

VI. Chronic Ixflamimation of the Stifle-Joint. (Gonitis Chronica
Sicca).

VIL. Bursitis Prepatellaris . . . . . . . 698

\section{DISEASES OF TIIE LOWER THIGH OR LEG.}

I. Wounds and Injukies . . . . . . . . . 699

II. Fracture of the Tibla . . . . . . . . . 700

IIT. Rupture of the Tibio-Fibular Tnterosseous Liganent • . 704

IV. Rupture of the Flexor Metatarsi Musche . . . . . 704

V. Rupture of the Tendo Achillis and Gastrocnemin Muscles • 708 


\section{DISEASES OF THE HOCK.}

I. Fracture of the Bones of the Hock .

711

II. Luxation ANd Sprain of The Hock-Jorn'T . . . . . 713

III. Wounds and ACute Inflammation of The Hock-Joint • • 714

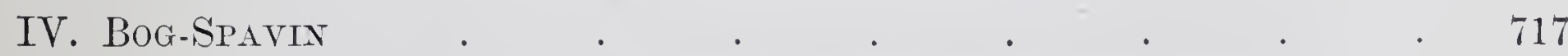

V. Chronic Inflametion of the Hock. Spavin. Arthritis Chronica

VI. Enlargeirents on the Outer Surface of the Hock . . . . 741

VII. CURB . . . . . . . . . . . . . 742

VIII. CAPPED HoCK . . . . . . . . . . . . 744

IX. Luxation of the Flexor Pedis Perforatus Texdon . . 748

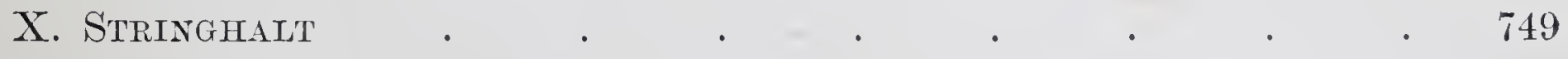

E. DISEASES OF THE METATARSTS.

I. Diseases of the Tendons and Tendon Sheaths in the Tarsal and Metatarsal Regions

II. Infectious Inflanimition of the Lower Synovial Sheath of the PERFORANS TENDON . . . . . . .

III. IxJuries produced BY Striking (INTERfering) AND their ComplCATIONS

\section{F. DISEASES OF THE FOOT.}

(A) Acute Inflamination of the Podophyllous Membrane. PododerMATITIS ACUTA

I. Pricks or Stabs in Shoeing

II. Picked-up Nails. Purulext Cellulitis of the Fibro-Fatty Frog. Resection of the Flexor Pedis Perforats

III. Treads and Injurifs to the Coronet. Purulent Inflamiation of the Subcoronary Connective Tissue . Inflammation of the Subcoronary Connective Tissue . . . 787

IV. CoRns.

V. QUITTOR

VI. Laninitis. Inflamination of the Sensitive Lamine

(B) Chronic Inflamiation of the Sensitive Iandine. Pododernatitis Chronica

I. Keratona.

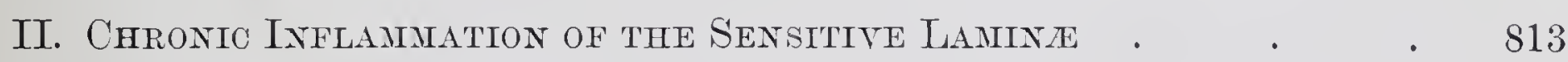

III. Canker of the Sole and Frog (Pododermatitis Chronica VerRUCOSA VEL Migrans)

\section{G. DISEASES OF THE CIAWS IN CATTLE AND SHEEP.}

I. Bruises of the Sensitive Sole and Bulbs of the Foot in Oxen .

II. Wounds of the Soft Tissues of the Clat . . . . 825

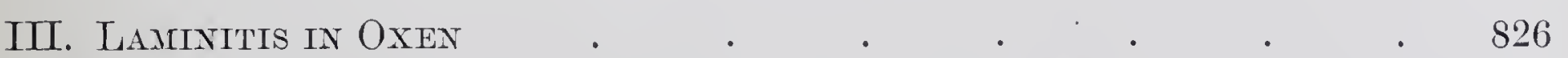

IV. Whithow of the Claw, Joint Felon, Panaritiul . . . 826 
V. A BNorymatites of THE Horvy CLAW — 831

VI. Amputation of the Claws in Oxen . . . . . . . . 831

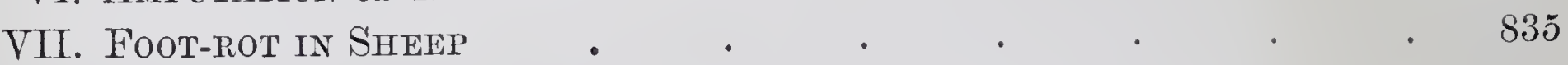

H. DISEASES OF THE CLAWS IN CARNIVORA.

I. Growing-in of the Claws (Incarnatio Unguis). Dislocation of the Claws. Chronid Ixflammation of the Clath Matrix. LOSS OF THE Claws. . . . . . . . 837

II. Bruises axd Wounds of the Pads of the Sole and Toe . $\quad 839$

INDEX. 


\section{LIST OF. ILLUSTRATIONS.}

FIG.

1. Atheroma of the false nostril

2. Right-sided facial paralysis (central)

3. Double-sided facial paralysis (peripheral) .

4. Position of nostrils and nose in double-sided (peripheral) facial paralysis

5. Supernumerary tooth (horse) .

6. Normal position of the molars in the horse 30

7. Tooth rasp .

8, 9. Tooth chisels

10. Tooth cutter

11. Left-sided shear-mouth

12. Grinding surfaces in shear-mouth and in normal mouth . . . . 33

13, 14. Tooth shears . . . . . . . . . . . . 36

15. Tooth screw . . . . . . . . . . 37

16. Metznik's tooth shears . . . . . . . . . . 37

17. Central dry caries of a horse's upper molar _ . . . . . 39

18. Teeth scalers for dogs . . . . . . . . . . . . 41

19. Necrosis of a dental alveolus, with dislocation of the last molar and extensive periostitis of the lower jaw (horse) . . . . . . 4 j

20. Güther's forceps for upper molars _ . . . . . . . 48

21. Günther's forceps for lower molar's . . . . . . . 48

22. Giunther's exporteur . . . . . . . . . . . 48

23. Guinther's beaked forceps . . . . . . . . . . . 48

24. Günther's forceps for first lower molars . _ . . . . . . 49

25. Günther's forceps for first upper molars . $\quad$. $\quad$. $\quad$. $\quad$. 49

26. Frick and Hauptner's "Universal " forceps . $\quad$ • $\quad$. . . 49

27. Showing the incisor and molar teeth of a six year old horse in position . . . . . . . . . . . . . . . . 50

28. Guinther's tooth pickers . . . . . . . . . . . . 50

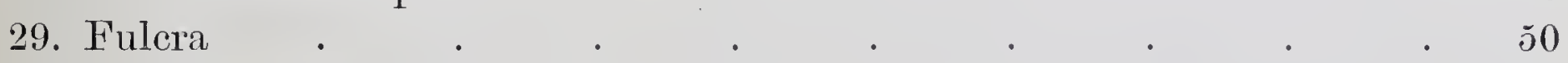

30. Günther's forceps for the incisors . . . . . . . . . . . 52

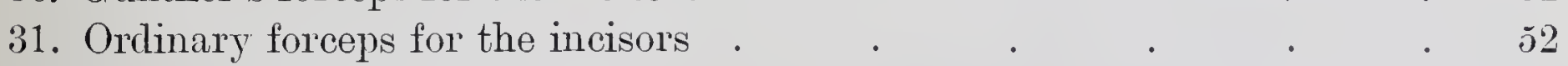

32, 33, 34. Dental forceps for dogs . . . . . . . . . ot

35. Periostitis alveolaris ossificans of the third molar (horse). . . . 5う

36. Epulis in a mastiff; showing deformity of face and upper jaw . . . js

37. The same; interior of the mouth . . . . . . . . . 5s

38. Facial oedema after injury . . . . . . . . . . 63

39. Osteomata from skull of a three jear old bullock _ . . . . 65

40. Stock trephine . . . . . . . . . . . . 68

41. Hand trephine , . , , . . . . . 68

V.S. 
FIG.

69

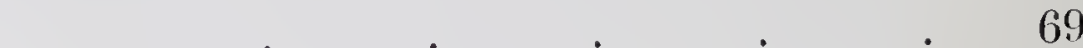

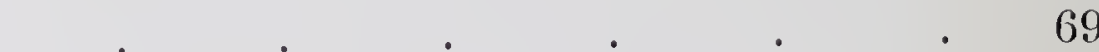

46. Exfoliative trephine

47. Perforating trephine . . . . . . . . 69

48. Head of a horse (B). .

49. Lateral view of horse's skull; the facial sinuses exposed _ • • 71

50. Necrosis of the turbinated bones . • . . . . . . 73

j1. Infero-lateral view of the laryngeal region $(\mathrm{B}) \quad$. $\quad . \quad$. $\quad 83$

52. Parotid gland and Stenson's duct. (Semi-schematic.) (C) . . 84

53. Actinomycosis of the parotid gland in an ox _ . . . . $\quad 88$

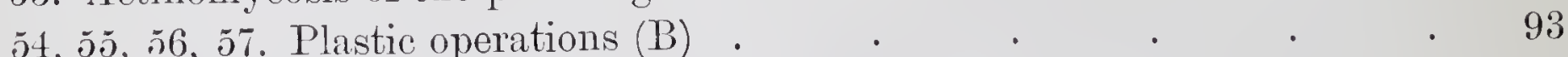

58 . Incomplete union of fracture of the body of the lower jaw . . 95

59. Fracture of the lower jaw united by wiring $(\mathrm{C})$ • . . . . . 96

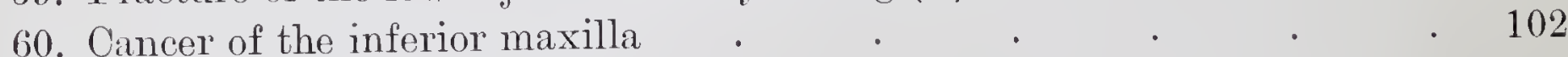

61. Bothryomycosis of the lower jaw . . . . . . . 103

62. Osteoporosis of the upper jaw . . . . . . . 104

63. Pre-auricular fistula due to presence of a dentigerous cyst $\quad$. 114

64. Giinther's catheter • • • • • • • • 117

65. Deep tissues in the parotid region; opening the guttural pouches. (Semi-schematic.) (C) . . . . . . . . . 118

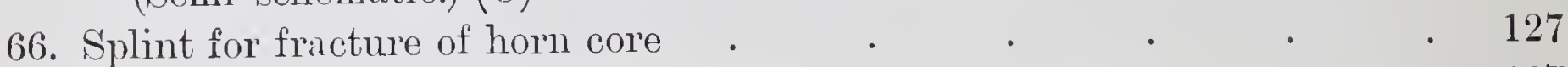

67. Form of splint for fracture of horn core . $\quad . \quad$ • . . . 127

68. Zehden's syringe

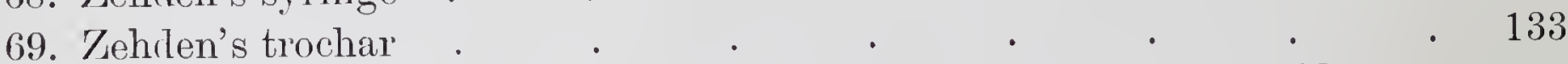

70. Sub-luxation of the 3rd cervical vertebra, as seen from the left side $\quad 144$

71. Sub-luxation of the 3rd cervical vertebra, as seen from the right side . . . . . . . . . . . 144

72. Iistortion of the neck (torticollis) in consequence of the calkin of a hind shoe becoming caught in the head-collar . . . . . 14 ó

73. Knudsen's iron splint for distortion of the neck . . . . . 148

74. Probang and mouth gag for cattle . . . . . . . . 164

75. Fisophageal screw . . . . . . . . . . . . 165

76. Esophageal screw . . . . . . . . . . . . 16 . . . .

77. Delvos and Hertwig's oesophageal forceps _ • • . . . 165

78. To illustrate the operation of oesophagotomy. (Semi-schematic.) (C) $\quad 167$

79. Section at right angles to the long axis of the neck through the upper third of the sixth cervical vertebra $(\mathrm{E})$. • . . . . $16 \mathrm{~s}$

80. A papilloma from the oesophagus of an ox $\quad . \quad$. $\quad . \quad 180$

81. Tampon-canula, or Trendelenberg-canula _ . . . . . . . 186

82. Hayne's tracheal trochar . . . . . . . . . . . . 195

83, 84, 85. Thompson's tracheotome . . . . . . . . 196

86. Making the incision in tracheotomy (B) . . . . . . . . 197

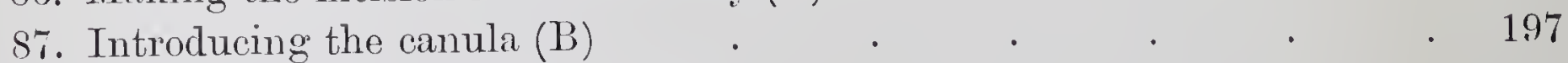

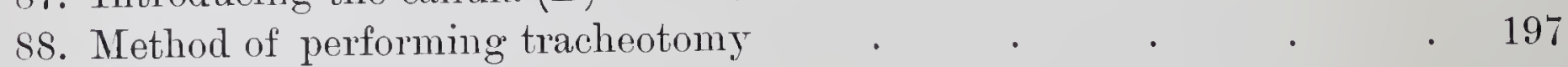

89. Barthélemy's canula . . . . . . . . . . . . 198

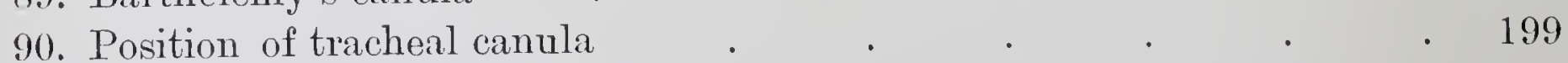

91. Leblanc's jointed canula . . . . . . . . . . 200

92. Leblanc's jointed canula . . . . . . . . . . . 200 
93. Peuch's canula

94. Field's tracheotomy tube • • . 201

• •

95. Coleman's tracheotomy tube . . . . . . . . 202

96. Double-tube canula . . . . . . . 202

97. Superior orifice of the larynx and glottis of a horse affected with chronic roaring .

9s. Möller's operation.

99. Scalperion of the larynx 206

• • • • 207

$\cdot . \quad \cdot 207$

101. Curved scissors • • • • . . . . . . 208

102. Forceps for grasping the arytenoid . . . . . . . . 208

103. Curved needle for snturing laryngeal mucous membrane . . . 208

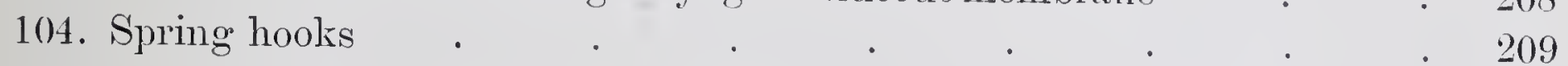

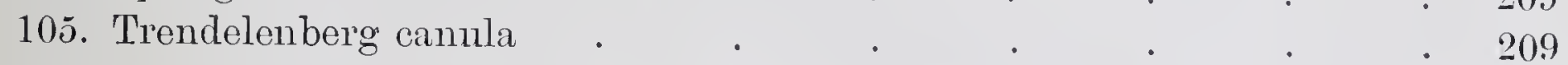

106. Arytenotomy. Second phase of the operation . $\quad$ • . . . 210

107. Third phase $(a)$. Incision of mucous membrane $\quad$. . . 210

108. Third phase $(b)$. Section of vocal cord . . . . . . 211

109. Third phase (b). Dissection of arytenoid _ . . . . . 211

110. Third phase (c). Division of arytenoid . . . . . . . 212

111. Third phase $(d)$. Excision of arytenoid . . . . . . 212

112. Fourth phase. Suture . . . . . . . . . . . 213

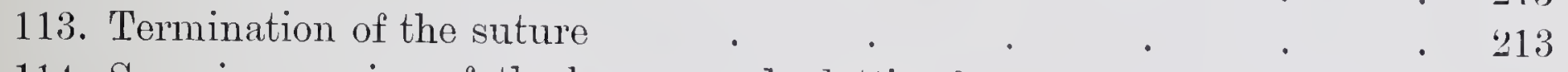

114. Superior opening of the larynx and glottis of a "roarer" that died of pneumonia eighteen days after the operation . . . . . 214

115. Pulveriser for disinfecting the.laryngeal wound . . . . 21

116. Antero-posterior section of the preceding larynx . . . . . 21

117. Multiple abscess formation in the shoulder-region due to Dis'rmyces equi $(\mathrm{C})$.

118. Transverse section through horse's thorax. (Semi-diagrammatic, after Franck.).

• .0 .246

121, 122. Showing mechanism of incarceration of intestine $\quad \cdot \quad 247$

123. Kühn's needle for ligation of the sac in umbilical hernia . $\quad 277$

124. Bordonnat's clam . . . . . . . . . . . 278

125. Combe's clam . . . . . . . . . . . . . $\quad$. 278

126. Incomplete inguinal hernia $\quad . \quad$. $\quad$. $\quad$. $\quad$. 282

127. Inguinal hernia of the horse . . . . . . . . . . 282

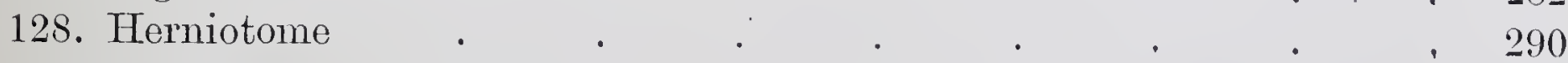

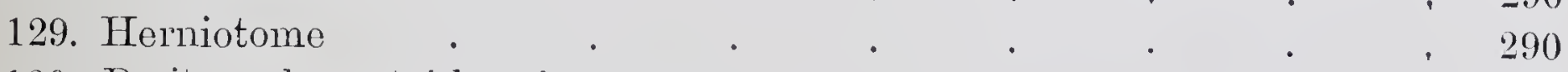

130. Peritoneal-scrotal hernia . . . . . . . . . 293

131. Suture for rupture of perinaeum . . . . . . . . $\quad 300$

132. Ventral hernia in a mare : . . . . . . . . 302

133. Schema illustrating Degive's operation for umbilical and ventral herniae 304

134. Anker's guarded knife for pelvic hernia . . . . . 307

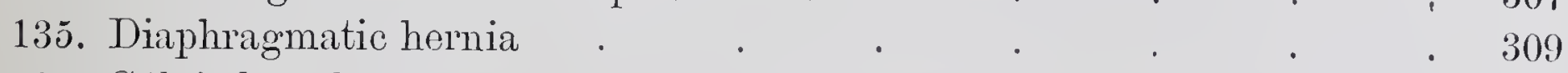

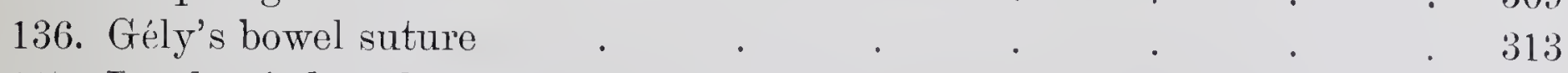

137. Lembert's bowel suture . . . . . . . . . . . . . . 313

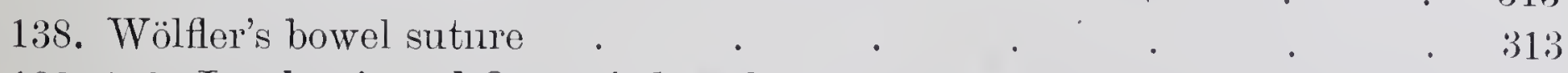

139, 140. Tembert's and Czerny's bowel sutures , , , 313 
FIG.

141. Vertical section of the horse's body between the last rib and external angle of the ilium . . . . . . . , 316

142. Friedberger's intestinal trochar for horses . • • • . 317

143. Showing the relative positions of the thoracic and abdominal organs. Left side .

144. Showing the relative positions of the thoracic and abdominal organs. Right side

145. Abdominal organs seen from below (L) , . . . . . . . . . . .

146. Mouth gag for oxen . . . . . . . . . . . 322

147. 'Trochar and canula for puncture af the rumen . _ . . . 323

148. Fertical section of the ox's body, showing relative position of rumen and other organs. . , , , , , . 324

149. Bräuer's trochar . . . . . . . . . . . 327

150. Forceps for use with Bräuer's trochar . . . . . . . 327

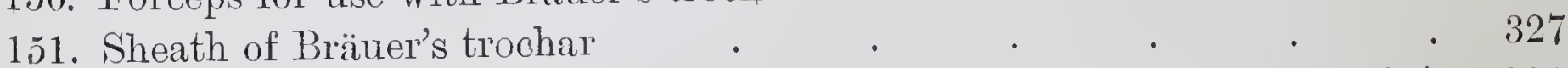

152. Congenital malformation-atresia ani in the bitch. (Semi-diagrammatic.) 336

1á3. Congenital malformation-atresia ani et recti in the bitch. (Semidiagrammatic.) , . . . . . . . . . 336

154. Prolapsus recti with invagination in the bitch. (Semi-diagrammatic.) . 344

15う. Prolapsus recti with invagination in the horse . . . . . 34 . 34

156. Stockfleth's wooden ring , . . . . . . . . . . 348

157, 158. Method of ligation in prolapsus recti _ . . . . . . 348

1.59. Caloulus passed spontaneously. Natural size . . . . 359

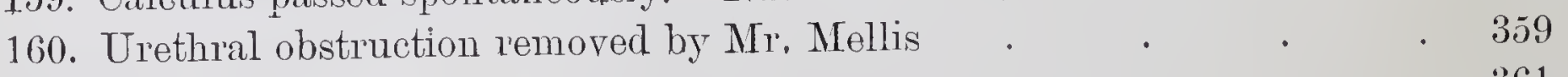

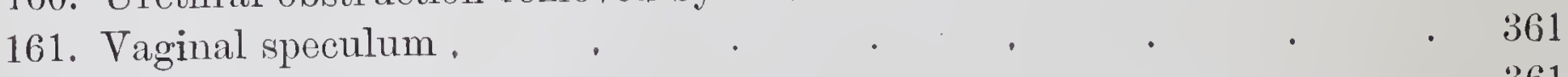

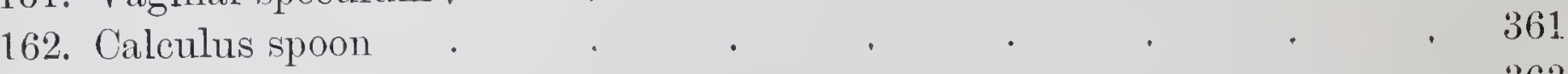

163. Calculus forceps . . . . . . . . . . . . . 363

164. Bouley's calculus forceps . . . . - . . . . . . . 364

165. Vesical calculus composed of phosphate and oxalate of lime . . . 365

166. Cystic calculus removed by V.-Tient. Walker . • . . . 365

167. Section of calculus removed by V.-Lieut. Walker _ . . . . 365

168. Course of the urethra in the ox . . . . . . . . . . . 366

169. Flourant's trochar . . . . . . . . . 373

170. Median section of the bladder, urethra, vagina, and vulva of a cow (B) 378

171. Inversion of the bladder in a mare $\quad . \quad$. . . . . . . 383

172. Amputation of the penis by the elastic ligature . . . . 401

173. Amputation of the penis showing stump and urethral orifice . $\quad 402$

174. Carcinoma of the testiole in a horse . . . . . . . . . 415

175. "Champignon" formation in the horse . . . . . . . . . 419

176. Prolapse (inversion) of the vagina . . . . . . . . 430

177. Simple vaginal speculum . . . . . . . . . . . 437

178. Expanding vaginal speculum (closed) . . . . . . . . . 437

179. Expanding vaginal speculum (open) . • . . . . . 438

180. Fracture (by crushing) of the body of a vertebra (C) . . . . . 464

181. Bernardot and Butel's apparatus for prevention of broken back when casting . . . . . . . . . 467

182. Schema illustrating fractures of the pelvis in the horse . . . . $\quad 473$

183. Docking knife . . . . . . . . . . . 494

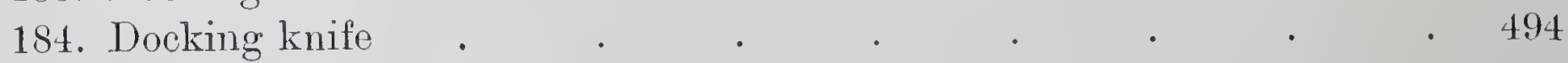

185. Ampntation of tail by flap operation $(\mathrm{B}) \cdot$. . . . . . $\quad$. 49 o 
1S6. Amputation of tail by flap operation, second stage (B) . . . 495

1S7. Amputation of tail by flap operation, third stage (B) . 496

1SS. Amputation of tail by flap operation, fourth stage (B) 496

189. Total necrosis of the scapula . . . . . . . 500

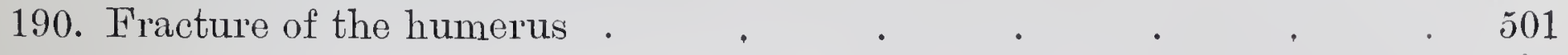

191. Lameness from bursitis intertubercularis . . . . . 50s

192. Fractured first rib . . . . . . . . . 51う

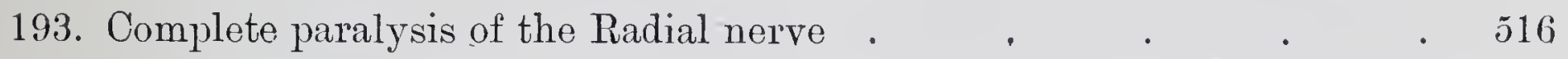

194. First portion of the stride in a case of paralysis of the radial nerve $(\mathrm{C})$. $\quad$ o17

195. Fracture of the ulna in a horse . . . . . . . . . . . . 527

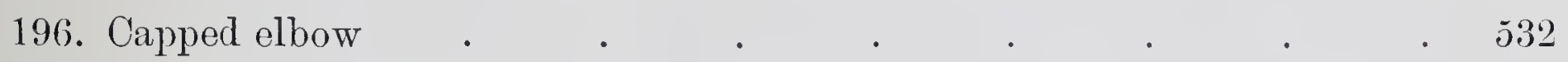

197. Iygroma of the knee resulting from bruising . . . . . . 541

198. Plastic operation for removal of scars from the knee (B) • • $\quad 543$

199. Plastic operation for removal of scars from the knee (operation completed) (B) .

200. Plastic operation for the removal of scars from the knee (the knee two months after operation) (B)

201. Knee tumour (cutaneous form) .

202. Distension of the flex or pedis bursa (most marked on the outer side of the $\lim b$ ).

203. Schema of the more important tendon sheaths of bursa of the fore limb, seen from in front and without.

204. The same, as seen from the front . . . . . . . joj4

205. Onter surface of the fore limb; to show synovial sheaths (L) . 556

206. Inner surface of the fore limb; to show synovial sheaths (L) • 556

207. Showing relative positions of tendons, \&c., in the metacarpal region (C) 565

208. Rupture of the flexor pedis perforans and perforatus tendons (C) 566

209. Rupture of the flexor pedis perforans behind the fetlock in consequence of suppuration in the tendon sheath . . . . . . . . . 568

210. Rupture of the superior sessamoidal or suspensory ligament 569

211. Excessive "knuckling over" in a horse . . . . . . . . 574

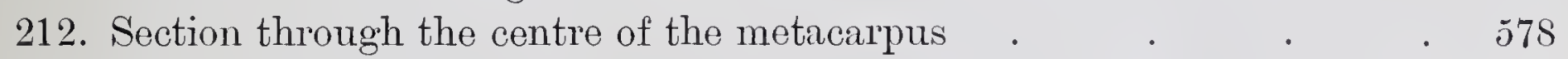

213. Tenotomy-first stage (B) . . . . . . . . . . 579

214. Tenotomy-second stage (B) . . . . . . . . 579

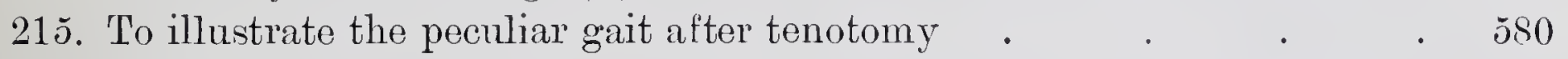

216. Excessive dorsal flexion after section of the perforans and perforatus tendons

217. Special shoe for "knuckling" . . . . . . . . . . . . . . . . . . . .

218. Double-sided knuckling in a two year old colt .

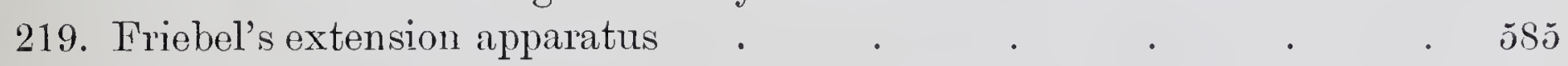

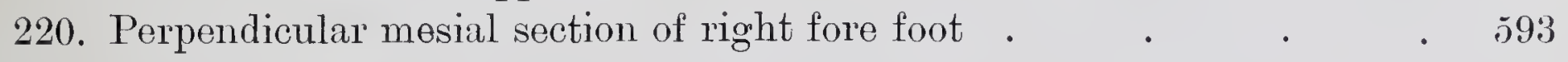

221, 222. Superficial and deep ligaments of the fetlock, pastern, and navicular

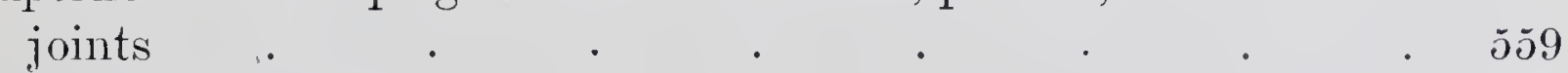

223. Fracture of os suffraginis . . . . . . . . . . . . $\quad 601$

224. Fracture of os suffraginis with formation of exostosis . . . . 601

225. Transverse fracture of os suffraginis . . . . . . . . . 601

226. Double fracture of upper end of os suffraginis . . . . . . . 601

227. Tongitudinal fracture of os suffraginis . . . . . . . . 601

22S. Articular ring-bone . . . . . . . . . . . 605

229. Periarticular ring-bone , . , , : , , 60 a 
FIG.

230. The outside of the right coronet shows ring-bone formation ; the left is normal

231. Showing course of internal plantar nerve

232. Section through the metacarpus about 3 to 4 inches above the fetlockjoint (E) .

233. Section through the fetlock-joint .

234. Neurectomy below the fetlock

235. Neurectomy above the fetlock .

236. Section through the fore arm, near the bottom of the upper fourth of the radius (E)

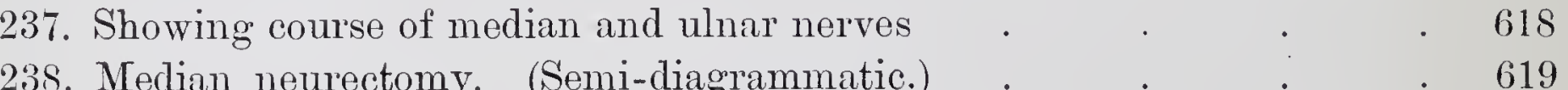

239. Operation of median neurectomy. The nerve exposed . . . 620

240. Section of neuroma from the divided end of the median nerve, three months after operation .

241. Longitudinal section of the median nerve, 6 inches below the point at which neurectomy was performed

242, 243. Neurectomy of the ulnar nerve

244. The flexor pedis perforans and perforatus tendons in a case of sesamoid lameness .

24j. Showing lateral cartilages and their attachments

247. Pedal bone, with almost complete ossification of the lateral cartilages .

248. Preparation illustrating navicular disease

249. Navicular bone in chronic navicular disease

250. Flexor pedis perforans tendon in chronic navicular disease . .

251. Straight seton needle

252. Curved seton needle

253. Paralysis of the external popliteal nerve (C)

254. Paralysis of internal popliteal nerve

25j. Paralysis of the anterior crural nerve

256. Incomplete crural paralysis

630

634

635

636

643

643

669

671

672

675

25\%. Left-sided chronic inflammation of the stifle-joint (Gonitis chronica) .

258. Bilateral chronic inflammation of the stifle joint (Gonitis chronica bilateralis)

267. Operation for division of the inner terminal tendon of the flexor metatarsi muscle $(\mathrm{C})$. $\quad . \quad$. . . . . . 733

268. Periosteotomy knives . . . . . . . . 735

269. Horizontal section through the hind limb about the lower quarter of the tibia (E) .

270. Rubber cord applied to thigh to check bleeding . 
273. Showing relations of the posterior tibial nerve (B) . . . . $\quad 739$

274. Neurectomy of the posterior tibial nerve (B) . . . . . 740

275. Curb . . . . . . . . . . . . 743

276. Capped hock (dropsy of the subcutaneous bursa of the hock) . . . 745

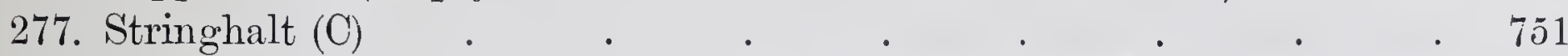

278. Tendon sheaths and bursæ of the hind limb of the horse, seen from without. (Semi-diagrammatic.)

279. Tendon sheaths and bursa of the hock-joint, seen from within. (Semidiagrammatic.) .

280. External surface of the horse's hock, to show synovial sheaths (L)

281. Internal surface of the horse's hock to show synovial sheaths (L)

282. Distension of bursa of flexor tendons in right hock . . . . Ts

283. Distension of sheath of flexor pedis perforans . . . . . . 75s

284. Distension of the metatarso-phalangeal (sesamoid) sheath (C) . . 759

285. Enlargement of the bursa of the extensor pedis tendon $(\mathrm{C}) \quad . \quad$. $\quad$ i61

286. Position of the limb, in infectious inflammation of the flexor pedis perforatus sheath in the fetlock region

287. Rupture of the flexor pedis perforans tendon $\cdot$.
288. To illustrate the superficial structures involved in surgical injuries of the foot.

289. To illustrate the deeper-seated structures involved in surgical injuries of the foot.

290. To illustrate the joints and deep-seated arteries, veins, and nerves involved in surgical injuries of the foot.

291. Vertical cross section of foot

292. Vertical cross section of foot . . . . . . . .

293. Cross section of a sound and well-shod hoof, showing the proper position of the nails

294. Operation for partial resection of the aponeurosis of the flexor pedis perforans tendon $(\mathrm{C})$

295. Operation for complete resection of the aponeurosis of the flexor pedis perforans tendon $(\mathrm{C})$

296. Lateral view of fore foot, with nerves and injected vessels, showing some of the structures involved in inflammation of the sub-coronary connective tissue, and its complications

297. Three-quarter bar shoe

298. Ordinary three-quarter shoe

299. Showing the lateral cartilage and its relations (L) . . . . 792

300. Hoof knives and knives for excision of lateral cartilage . 798

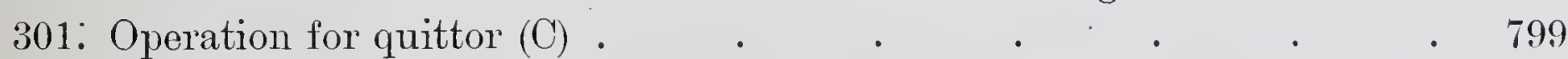

302. Celluloid injection preparation of the veins and arteries of the foot prepared by maceration (B)

310. Hoof after laminitis (shod) , . , . . . 810 
311. Portion of the inner surface of the wall showing changes after oldstanding corr . . . . . . . . 812

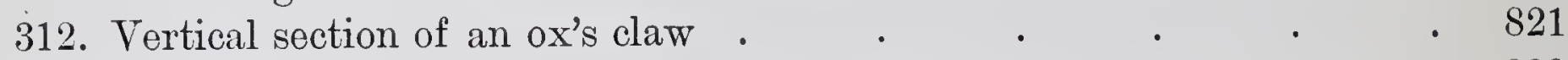

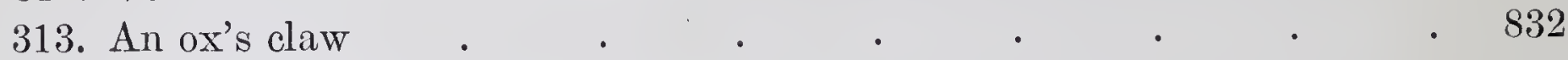

314. Disarticulation of the ox's claw. Primary incision (C) . . . 833

315. Disarticulation of the ox's claw (C) . $\quad . \quad$. $\quad . \quad$. 834

In the above list the added letters have the following signification: (B), from Bayer's "Operationslehre"; (C), from Cadiot and Almy's "Traité de Thérapeutique Chirurgicale"; (E), from Ellenberger and Baum's "Anatomie"; and (L), from Leisering's "Atlas." Most of these figures have been redrawn. 


\section{REGIONAL VETERINARY SURGERY.}

\section{DISEASES OF THE HEAD.}

\section{I.-DISEASES OF THE LIPS AND CHEEKS.}

\section{(1.) WOUNDS AND BRUISES.}

IN horses, wounds of the lips frequently result from bites or kicks, from collisions and falls on hard ground, or from entanglement with sharp portions of the harness, or with hooks, etc., fixed in stable racks and mangers. Bruises are produced in a similar manner, and by the severe and repeated application of the twitch. The corners of the mouth may be injured by thin or badly-fitting bits, especially in young and hard-mouthed horses, or in those suffering from brain disease, whilst the cheeks are sometimes lacerated by kicks, horn thrusts, and, on their inner surfaces, by the sharp edges of the molars. Cattle at grass may suffer from torn wounds produced by the oxherd's dogs, while sporting dogs may be bitten by the quarry.

The great mobility of the injured parts, and the constant soiling which occurs during mastication, almost always interfere with the healing of bruises and contused wounds. On the other hand, reparative processes are assisted by the richness of this region in blood-vessels and loose connective tissue, so that healing sometimes takes place by primary intention, even when wounds are extensively bruised, their edges already dry, and their flaps dissected from underlying tissues over considerable areas. Deep wounds in the corner of the mouth, where the mucous membrane is divided and the opening of the mouth lengthened, give most trouble. Perforating wounds of the cheek also heal with difficulty, and sometimes produce fistulæ. Injuries to the great venous plexus in the cheek occasion considerable bleeding, which, however, is seldom dangerous. The division of Stenson's duct often results in salivary fistula. Emphysema of the facial region frequently accompanies wounds of the cheek, but calls for no special treatment, and usually disappears spontaneously. Necrosis of the margins of wounds may 
produce defects in the lips and cheeks, which both injure the animal's appearance and interfere with feeding and drinking. Such defects frequently follow wounds in the corner of the mouth, where the buccal opening becomes lengthened (Malkrostomy).

Treatment. In surface injuries, small flaps of skin can be removed with scissors, when healing usually follows. To prevent deformity following extensive injury, attempts should be made to bring about healing by first intention. After removing loose shreds, the wound, which is usually dry, must be freshened, i.e., the surface removed with the scalpel to furnish the moist or bleeding flaps necessary for immediate union. The wound and its surroundings are then cleansed, the neighbouring long hair removed, the parts flooded with some fluid disinfectant (corrosive sublimate, creolin or carbolic solution), and the edges brought together as evenly and completely as possible. On account of the great mobility of the lips and their neighbourhood, pin sutures are here preferable. The pins should be inserted deeply and at distances of about $\frac{1}{2}$ to 1 inch, being secured by a continuous thickish thread applied in a figure of 8 . Ordinary sutures sometimes suffice. For further security, and to fix the edges, the wound may be smeared with collodion or wound gelatine, ${ }^{1}$ over which may be placed tow or jute or strips of gauze.

Deep wounds at the corners of the mouth or on the cheeks require particular precautions. Button sutures (see Dollar's "Operative Technique," p. 140, etc.) are most useful, and the ligature, which must be strong, should be passed right through the cheek; lead or brass wire is also suitable. Injury to the wound can be avoided by putting the horse on the pillar reins. Where healing by primary intention is desired, water alone must be given for the first 24 hours, and during the following few days only gruel or bran mash. After six to eight days the stitches can be removed from the lips, but those in the corners of the mouth or in the cheeks should be left a couple of days longer. In fistulæ about the cheeks the hardened walls are removed by caustics or the actual cautery, and a purse-string suture. inserted. The inner opening of the fistula may sometimes be closed by passing a suture through the mucous membrane.

\footnotetext{
I Wound gelatine was first described by Frick as "sublimate glycerine gelatine" (Arch. für pract. u. wiss. Thierheithunde, 1886, p. 397). To prepare it, ordinary sheet gelatine is soaked in sufficient 1 per cent. sublimate solution to cover it. After it has become quite solt, it is melted by gentle heat, and a quantity of glycerine equal to one-tenth of the weight of the dry gelatine added. This gives the material an elastic character. To use it, it is melted on a slow fire and painted over the wound. It sets in a few seconds, acheres equally well to moist or dry surfaces, and is much preferable to collodion. The surface can be strengthened by laying on it, while moist, shreds of tow or strips of gauze.
} 


\section{(2.) ACUTE INFLAMMATION OF THE LIPS AND CHEEKS.}

Acute inflammation of the lips in the domesticated animals is frequently caused by licking blistering ointments, by partaking of irritating materials, by infectious disorders, like aphtha or stomatitis pustulosa, or by such injuries as have previously been referred to.

Specific inflammations are treated of in works on internal disorders. Dogs, being much exposed to infection; sometimes show cellular inflammation of the upper and lower lips after slight injuries. Diseases of the skin, like eczema and mange, are not infrequently transmitted to the lips as a result of licking the diseased spots. Thence they generally spread to the bridge of the nose, producing a dermatitis chronica apostematosa, which gives great trouble, especially if the area involved is too great to permit of all diseased skin being removed with knife and scissors. The follicular inflammation of the lips in young dogs, associated with lymphangitis and suppuration in the submaxillary lymphatic glands, which is described by Fröhner, is probably due to extensive outbreaks of acne pustules. A similar affection in an old dachshund was described in the Berliner Thierärztliche Wochenschrift. Death resulted from general infection.

In dogs and cattle, spontaneous gangrene of the cheek occurs, resembling noma of children. In dogs the disease begins with formation of an almost invisible eschar at the corner of the mouth; the great swelling which simultaneously appears indicating the character of the malady. Gangrene makes rapid progress, often attaining the size of a florin in a few days. The skin is soft, greyish-brown, and easily removable; the submaxillary lymph glands are swollen, and fever and severe salivation exist. In dogs the process seldom terminates before destroying a large portion of the cheek. The appearance of granulations and of pus formation on the boundary indicate the commencement of healing. After the gangrenous portion sloughs, the molars may become visible, and great difficulty exist in taking fluids. In spite of this, complete recovery usually occurs; difficulty in feeding disappears, and even the animal's outward appearance does not permanently suffer. The disease is rare in dogs, and its cause is as little understood as that of noma in the human subject, though it probably consists in infection by a specific micro-organism.

Treatment. The parts should at once be disinfected, preferably with the cautery. Where this is impossible, lotions of permanganate of potassium or carbolic acid, sublimate or creolin can be applied. Thin fluid nourishment is indicated, and may be given from a bottle. As soon as the defect in the cheek interferes with feeding, fluid or semisolid nutriment becomes indispensable. If required, animal broths may be given. 
Stockfleth has described a similar disease in cattle, resulting from injuries, but this does not extend as in dogs. Most commonly an abscess forms in the cheek, and recovery occurs in a few days. The treatment of other inflammatory processes should be adapted to their special features. The cause must be removed and care taken that the affected part is not irritated more than necessary, either by rubbing or by mastication. Attention is accordingly required in selecting and preparing the food, and in the management of the animal. Complications are treated on general surgical principles. In suppurative forms of skin inflammation, diseased parts should be promptly removed with the knife.

Chronic inflammation of the lips and cheeks in oxen is sometimes due to actinomycosis. Klepzow, who examined 2,000 slaughtered cattle, found actinomycosis of the lips in 5.6 per cent. Several hard, painless swellings appear in a line with the molars. The skin covering them is at first mobile, but later becomes adherent and finally breaks, a mucopurulent fluid being discharged from the wound, which is surrounded by flabby granulations which readily bleed.

The cause is infection with actinomyces from the cavity of the mouth.

Treatment consists in early opening of the swellings, drastic curetting of the parts and dressing or plugging with tow saturated in tinct. iodi.

Like actinomycosis in cattle, bothryomycosis of the lips and cheeks in horses is not uncommon. Small fibrous swellings form, most frequently at the points where skin and mucous membrane are coterminous.

Treatment should be undertaken early; it consists in removal of the swellings, careful disinfection and suturing of the wound.

\section{(3.) TUMOURS OF THE LIPS AND CHEEKS.}

(A.) Warts. In dogs and horses the lips are not infrequently the seat of great numbers of small pedunculated warts. These new growths occur both on the mucous membrane and on the skin, and vary from the size of a pin's head to that of a grain of linseed. Their covering is often wanting in pigment, in consequence of which they appear red on the surface and are often thought by laymen to be parasites. They are almost always found in young animals, cause no particular inconvenience, and may disappear after a short time without apparent cause. Attempts to inoculate other animals have proved ineffective (Garcia). If mastication be interfered with, they can be removed with scissors; but if they cause no inconvenience it is better to await their spontaneous disappearance.

(B.) Encysted tumours also occur frequently on the lips of horses; 
they lie close under the mucous membrane, sometimes attain the size of a pigeon's egg, and contain a thick, fluid, honey-like material. 'They are really retention cysts, produced by obstruction of the ducts of mucous glands. So long as they attain no considerable size, nor become the seat of inflammatory changes, they are seldom observed. To detect them, the thumb is laid on the external skin, the fingers on the mucous membrane of the lip, which is allowed to glide slowly through the fingers. When such tumours become inflamed, they cause enlargement of the submaxillary lymph glands, displace the lips, and impart to the face somewhat of the appearance of facial paralysis. If they become inflamed, or interfere with feeding, treatment will be required. It is generally. sufficient to lay open the parts and dress the interior with nitrate of silver, sulphate of copper, 2 per cent. corrosive sublimate solution, or $10-20$ per cent. solution of chloride of zinc.

A typical tumour develops above the base of the false nostril in horses, usually resulting from occlusion of a sebaceous follicle. It is filled with a granular material. It may become as large as a hen's egg, is round, painless, and freely movable, but seldom causes any difficulty in breathing. By passing the finger into the false nostril, it may be readily felt, and is some-

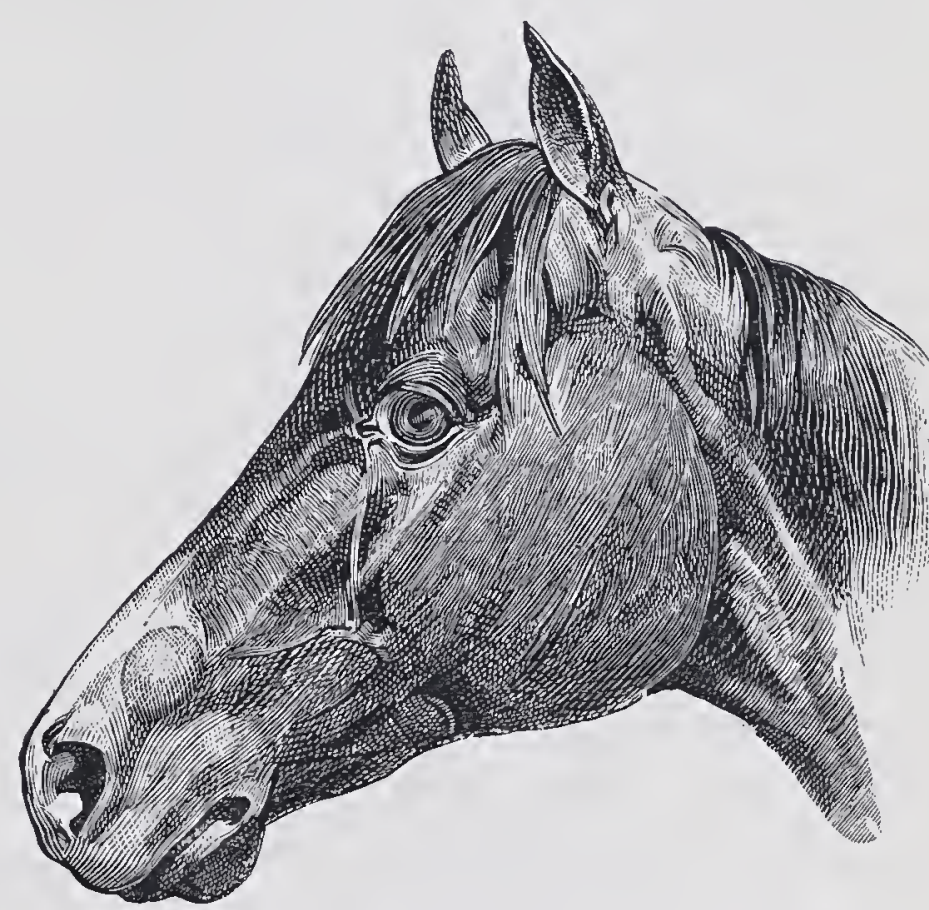

FIG. 1.-Atheroma of the false nostril.

times visible from without. (Fig. 1.) Such growths are seen oftenest in young foals. They are easily removed. The animal is cast-though in very quiet subjects this is scarcely necessary-and an incision made through the skin in the long direction of the head. The tumour is then grasped with forceps and freed from its surroundings, care being taken not to incise it, as its removal is thus rendered more difficult. Should such an accident happen, a dark-grey, granular material is discharged, and the inner wall of the tumour comes in view. The nasal mucous membrane being firmly adherent to the swelling may be injured, when froth from the nostril will appear in the wound. But even where the mucous membrane has been cut, healing by primary intention occurs. The wound is at once sutured, and finally covered with iodoform collodion or wound gelatine. Tempel removed a dentigerous cyst from a horse's upper lip. With the exception of those named, new growths on the cheeks and lips are comparatively rare in domesticated animals. Fibromata, sarcomata, carcinomata and melanomata have 
been seen and operated on with varying success, depending on the kind and age of the tumour.

Treatment consists in careful removal of the new growths, which is seldom very difficult if the knife is used early and boldly.

\section{(4.) PARALYSIS OF THE LIPS. FACIAL PARALYSIS.}

The facial is the motor nerve of the muscles of the ears, eyelids, nose, lips, and cheeks. Arising from the pons, it enters the inner ear with the $\mathrm{N}$. acousticus, passes through the Fallopian canal, and outwards through the stylomastoid foramen of the petrous temporal bone, penetrates the parotid gland; and then passes over the posterior border of the lower jaw, on the external surface of which it divides. According to their points of origin, the following three portions may be differentiated.

I. In the Fallopian canal arise :

(1) A nerve for the stapedius muscle.

(2) A nerve for the chorda tympani.

II. At the stylomastoid foramen :

(1) The posterior auricular nerve giving twigs to the cervico auriculares, and the parieto auricularis externus and internus.

(2) The middle auricular, distributed to the skin lining the interior of the ear.

III. Thence to the point of termination :

(1) Nerve to the occipito-styloid, stylo-hyoid; and digastricus muscles.

(2) The zygomatico temporalis nerve gives off-

(a) The anterior auricular nerves.

(b) Twigs to the temporalis muscle.

(c) Twigs to the orbicularis palpebrarum and the external levator palpebrarum.

(3) The cervical branch, which gives off motor twigs to the depressor of the ear and the superficial cervical muscles, afterwards passes over the external surface of the lower jaw, and, as a motor nerve, supplies the muscles of the nose, lips, and cheeks.

Paralysis of the facial nerve occurs rather frequently in horses, but is rarer in oxen and dogs; it is commonly confined to one side, often to the nerve supply of the upper lip; the deformity consequently is slight, and the mischief may be overlooked. But double-sided paralysis interferes very noticeably with feeding, and therefore with nutrition; while at a later stage the respiration may become impaired owing to paralysis of the nostrils.

The cause is most frequently external injury impairing the conductivity of the nerve. 'The malady is therefore common in horses suffering from such illness as colic or injuries to the feet, and which in consequence, lie a great deal. Siedamgrotzky first noted that this nerve often becomes bruised at the point where it winds round the lower jaw. The cheekstraps of the head-collar, and especially the buckle at the left side, may 
easily injure the nerve when the animal is lying. The double-sided paralysis described by Grebe, and erroneously regarded as reflex paralysis, is similarly produced. In earlier times, facial paralysis was often caused by inserting hair setons in the cheeks as a remedy in diseases of the eye. It occurs during the progress of serious infectious disorders, such as influenza, petechial fever, \&c., and may also be produced, as in man, by severe chills. Utz saw the condition after an animal had been struck by lightning.

The paralysis is mostly confined to the facial muscles. The lips are distorted, the nostrils narrowed, the upper lip, and sometimes the under, are drawn towards the sound side. The condition is best recognised by looking at the lips from in front. So long as one side alone is affected, feeding is little interfered with; but in double-sided paralysis the lips hang flaccid, a condition best seen in the under lip, and feeding now becomes extremely difficult. The food can only be grasped with the teeth, and, in drinking, the corners of the mouth must be immersed in order to prevent the water flowing back. Even when carefully tended, the animals rapidly loose condition, for they have difficulty not only in grasping food, but, owing to the paralysis of the cheeks, also in masticating it. The food cannot be brought between the opposing rows of teeth and so accumulates between the cheek and molars. These are the appearances when injury has occurred at the point where the nerve passes round the under jaw; but where in addition the subzygomatic nerve is involved, sensation is lost in the skin of the affected side.

Facial paralysis due to central injury is different both in its symptoms and consequences. Should the lesion be sufficiently near the brain to involve the zygomatico-temporalis nerve, then, in addition to the above appearances, paralysis affects the levator palpebrarum, as well as various muscles of the ear. The upper eyelid will droop (ptosis) whilst the orbicularis palpebrarum will be unable to properly close the eye, and the ear on the affected side will hang limply. (Fig. 2.) Zahn found the bulbus of the affected side smaller, and the cornea cloudy. Disturbance of hearing could also be detected. Not infrequently paralysis of other portions of the body accompanies paralysis of the facial; thus Jewtichilew saw hemiplegia, Tempel paralysis of the trigeminal nerve, and Fuchs of the hypoglossus and glossopharyngeus.

The causes are various. Melanotic tumours, developing in the parotid, as well as swellings in the Fallopian canal, may press on the nerve. Occasionally the origin is central. Thus the post-mortem of a horse suffering from double-sided paralysis, disclosed sarcoma of the pons. Götze noted paralysis of the lips and ears in a horse after influenza, and found on section an abscess in the cerebellum. In another case, Lydtin saw sarcoma on the petrous temporal bone, the growth extending from 
the posterior convolutions of the cerebrum to the point of origin of the twelfth nerve. Thomassen and Hamburger found hrmorrhage in the facial nerve centre. In a dog that suffered from unilateral paralysis Monfallet found tuberculosis of the meninges.

It is difficult to say if rheumatic paralysis of the facial nerve occurs in animals as well as in men. The greater number of cases, at any rate, are of traumatic origin. Disease of the middle ear being rare in the horse, it cannot often be a determining agent, and Möller has never seen a case of facial paralysis in dogs, though in them middle ear disease is comparatively common. But Trofimow relates that a bitch showed onesided paralysis in consequence of catching cold; the upper eyelid was

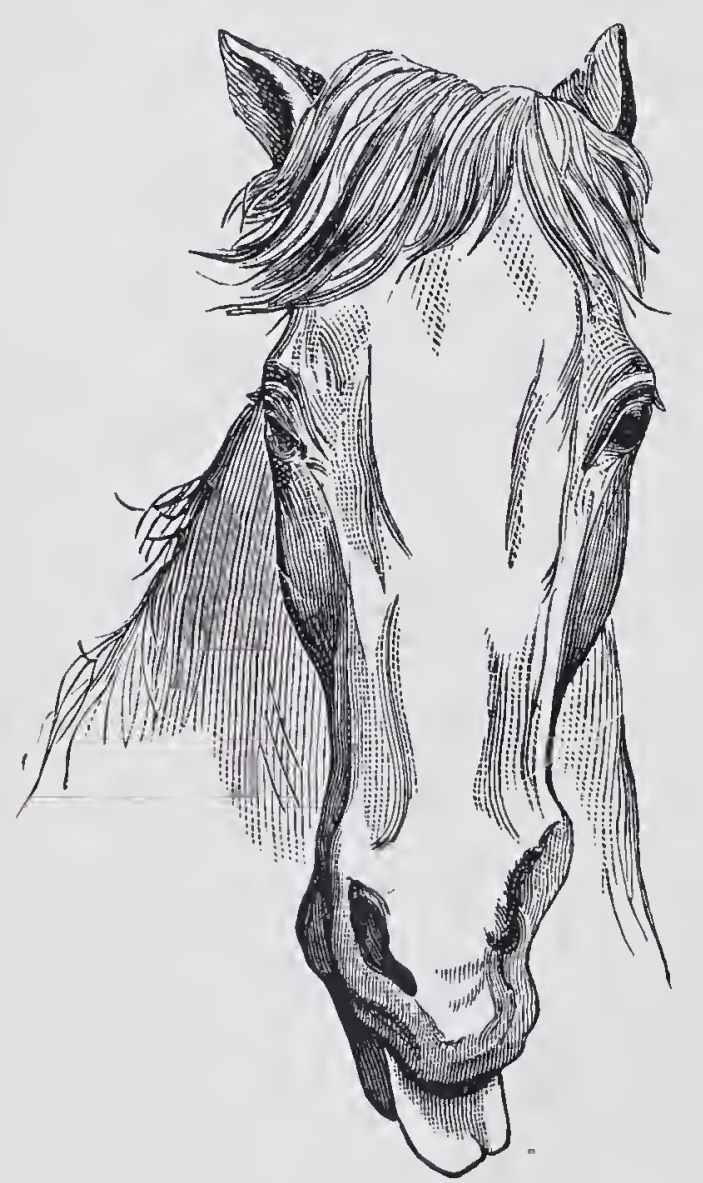

FIG. 2.-Right-sided facial paralysis (central). The protrusion of the tongue is accidental-it does not necessarily occur in facial paralysis.

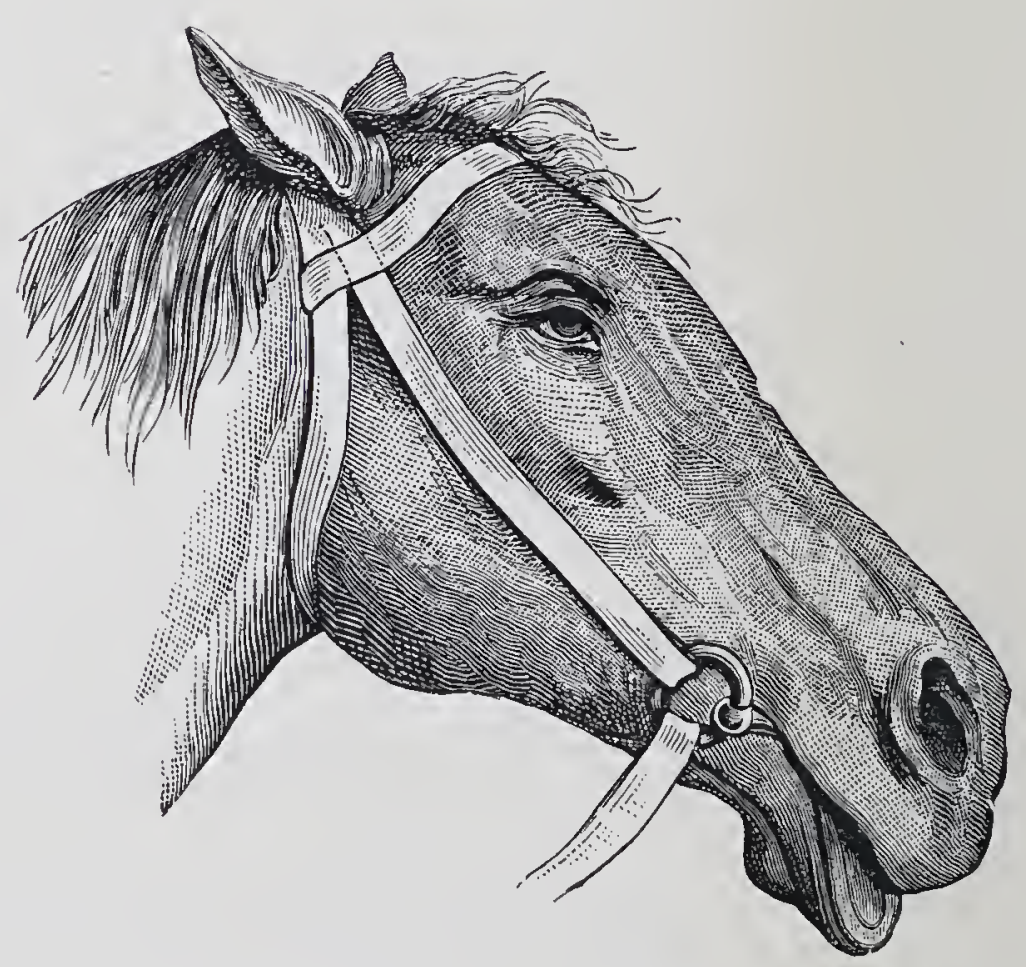

FIg. 3.-Double-sided facial paralysis (peripheral).

involved; cure was effected in two months, but a relapse is said to have occurred later. Cattle seldom suffer from facial paralysis, probably because the nerve is protected against mechanical injury by the horns. In double-sided paralysis both nostrils fall in, but a sufficient opening remains for ordinary quiet respiration. Immediately, however, that breathing is hurried, from such causes as excitement or rapid movement, a snoring sound becomes audible during inspiration. As the horse breathes only through the nose, the narrowed opening becomes insufficient, the edges of the nostrils are pressed inwards, and inspiratory dyspnoea ensues. That asphyxia is always caused, as Claude Bernard states, has not been borne out by the experiments of Günther and Ellenberger. Möller's observations support those of Ellenberger, but it 
must be allowed, as shown in a case related by Schöneberger, that an animal affected with this form of paralysis, if excited and forcibly driven, may not only suffer from severe dyspnœa, but may even die outright. In one case of double-sided peripheral paralysis the breathing was of a snoring character, even when the patient was at rest, whilst during inspiration the nostrils became contracted and the false nostrils collapsed. (Fig. 4.)

Diagnosis of central paralysis is not difficult, though determination of the cause and its exact position require much care. Should the paralysis be confined to one or both lips, it is peripheral; where the muscles both of the eyes and ears are affected at the same time, the injury is above the point where the nerve winds round the jaw. Central paralysis may sometimes be recognised by the continued existence of reflex irritability in the affected parts, showing that conductivity of the nerve is not destroyed.

Prognosis. Most cases of peripheral paralysis recover in from four to six weeks. Return of irritability on faradisation points towards recovery, and this usually occurs little by little. When the animal is in a state of rest, it may be noted that the lip is returning to its normal position; should it be excited, however, the distortion again becomes visible. A prognosis is, therefore, best arrived at while the patient stands quietly in the stable. Cases of incomplete paralysis generally recover; those where the eyes and ears are affected are not hopeful, and where tumours are the cause a cure is not

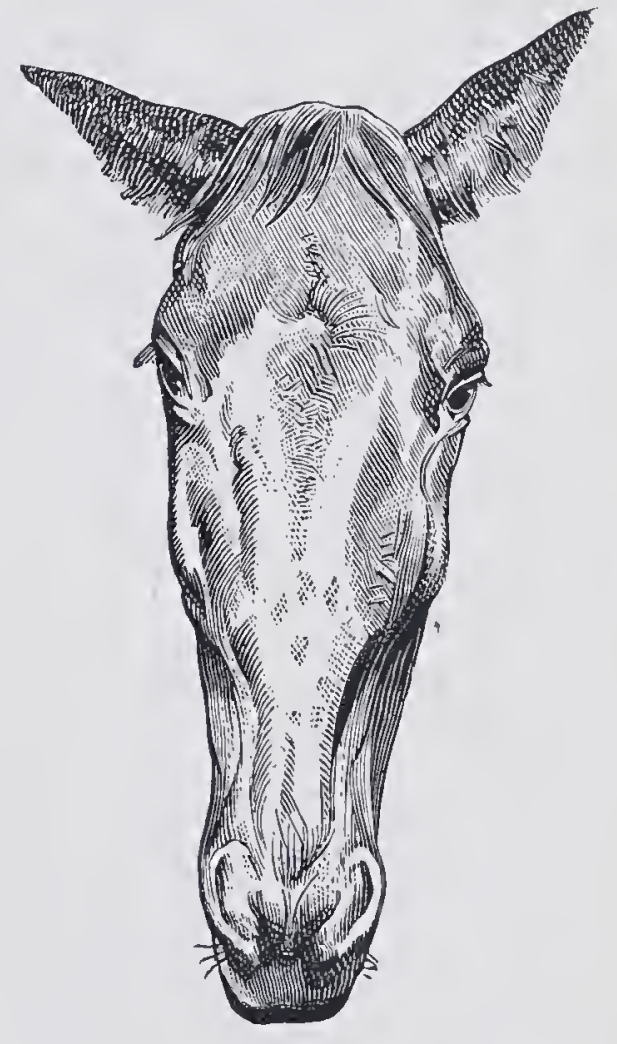

FIG. 4.-Position of nostrils and nose in double-sided (peripheral) facial paralysis (from a photograph). to be expected. Further, when the paralysis has been in existence for more than two or three months, the prognosis is always unfavourable. Double-sided is generally less hopeful than single-sided paralysis, while, if the appearances point to some central cause, little hope of cure can be entertained.

Treatment. In one-sided incomplete paralysis the food which has accumulated in the cheek must be removed after each meal. Nothing further is necessary. In complete paralysis of one side, soft, easily masticated food should be given, and the surface on the upper margin of the lower jaw, where the nerve emerges, rubbed with some resolvent material (Ugt.hydrarg). Such measures serve to satisfy the owner, and the rubbing certainly appears to alleviate inflammatory action. The frequently recommended injection of veratrine into the cheeks has proved of little value. Electricity is inapplicable, owing to the excessive 
sensibility of the horse to this agent. In double-sided paralysis the principal point is attention to the food, which should consist of mashes, gruel, and green stuffs. Corn should be given crushed and in a deep receptacle, so that the animal can more easily seize it with the teeth. To avoid the tendency to dyspnœa, Schöneberger advised that wire sutures be passed through each nostril, and brought together over the nose-a suggestion he first made in 1874. Tracheotomy is sometimes useful.

Voigtländer observed periodical attacks of cramp in the region supplied by the N. facialis in a horse; "on the right side the upper eyelid began to twitch, a slight contraction like a shadow ran across the masseter as far as the lip, and then began powerful contractions, which drew the right half of the upper lip upwards and outwards, and set in motion the entire half of the head behind the eye." These attacks came on every five minutes, and occurred even during feeding. The owner stated that the disease had been in existence for several years. Degive noticed paralysis of the tongue and lips in horses, a disorder which is said to be frequent in Belgium, and which has a certain resemblance to bulbar paralysis in man. He found general progressive paralysis of the bulbar nerves, especially the hypoglossus and facialis, which was attended with salivation, paralysis of the masticatory muscles and of those of the tongue and lips, with consequent difficulty in eating, portions of the food falling out of the mouth. Paralysis of the soft palate and pharyngeal muscles was sometimes present and interfered with swallowing. The malady always ended in death, generally in five to six months, sometimes later. Post-mortem showed gangrenous pneumonia (mechanical pneumonia), atrophy of the roots of the bulbar nerves (hypoglossus, vagus and facialis), and degeneration of the muscles of the tongue and cheeks.

The cause is clearly a morbid process in the medulla, leading to the degeneration and atrophy of the motor roots of the hypoglossus, facialis and vagus, which arise here. The disease does not yet appear to have been observed in countries other than Belgium.

\section{II.-DISEASES OF THE MOUTH.}

\section{(1.) FOREIGN BODIES IN THE MOUTH.}

In domesticated animals, and especially in dogs and cattle, foreign bodies taken into the mouth along with the food, or picked up in play, are apt to become fixed in position. In dogs, bones and needles are the most common objects; in cattle, pieces of wood, which lie between the teeth or the teeth and cheeks; while needles usually penetrate the tongue. The animals slobber, and chew when the mouth is apparently empty; some shake the head or make cautious attempts to swallow; and, if the foreign body is not removed, they become thin from insufficient nourishment. Where such symptoms are met with, the mouth should always be carefully examined. 'The objects being often very small, like needles or splinters of wood, it is necessary to search closely. Where hard bodies 
become fixed between the upper and lower teeth, the mouth can sometimes neither be closed by the animal itself nor by external force. In dogs and cats, sewing needles are often fixed in the base of the tongue.

Apart from difficulty in feeding and loss of condition, other symptoms may supervene. - A horse has been seen to die of bleeding from the palatine artery, primarily brought about by a needle penetrating the tongue. Most veterinary surgeons have removed pieces of bone from between the molars in dogs, which were unable to close their mouths, and in consequence had been suspected of rabies. Lindenberg saw epileptiform attacks in a cow result from a sharp molar injuring the tongue; attempts to eat immediately produced an attack. To remove a foreign body the mouth should be forced wide open, and the object seized either with the hand or with forceps. Needles are always found penetrating the tongue from behind forwards, assuming this position in consequence of the struggles of the animal when choking, or the attempts it makes to displace the needle with the tongue. For this reason, when extracting a foreign body, the tongue should be powerfully drawn forward, and the object thrust upwards and backwards. From neglect of this principle, attempts to pull out the needle by means of the thread which it often carries are always unsuccessful.

Injuries to the hard palate are sometimes complicated with severe bleeding, and require most careful attention. When the palatine artery is wounded, animals may bleed to death, as the movements of the tongue interfere with the formation of thrombi. In venous bleeding the following treatment is generally sufficient: after covering the tongue with flour to the thickness of an inch, the mouth is firmly bound and the horse left at rest for $6-8$ hours. In severe arterial bleeding, which often follows penetration of the vessel, the horse should be cast, the mouth kept open with a gag, and the artery ligatured. Under some circumstances pressure may be employed, as, for instance, in injuries of the anterior parts of the palate. After placing on the bleeding spot a hard pad of tow or jute, preferably soaked with perchloride of iron solution, a bandage or handlkerchief is passed round it, and firmly tied around the jaw, beneath the upper lip. This should remain in place for 12 hours. A piece of thick leather or board will further ensure its not shifting. Stoppage of the bleeding may at times be effected by burning the part with a round-headed iron, but this treatment is not reliable; the bleeding usually stops for a few seconds and then recommences, and it is better to occupy this interval in applying a dressing. Under any circumstances, hard food must not be given during the ensuing 24 hours. In spite of the unpleasant smell, the above described wounds usually heal rapidly after removal of the foreign body. Where deep cuts exist, the mouth should be cleansed after each meal and the 
animal prevented, either by muzzling or tying up short, from again fouling the parts.

The tongue is sometimes strangulated by a cord or rubber band; this has repeatedly been seen, both in horses and dogs. Either from ill-will and a desire to injure the owner, or with the view of keeping the horse quiet during cleaning, or preventing its protruding the tongue, a piece of string is tied round it. Kirchner relates a remarkable case of the kind in a cow, where the tongue became snared in consequence of chewing some string. In dogs and cats, sections of blood-vessels or elastic bands sometimes slip on to the tongue and remain fast. In one of the two cases described by Barrier, a section of aorta was found encircling the tongue, which was necrotic. Carrucci relates a similar case, where the tongue was swollen to four times its normal size. Kitt, when making a post-mortem examination of a goat, found an iron ring, $\frac{3}{4}$ inch broad and $2 \frac{3}{4}$ inches in circumference, firmly fixed round the tongue. The animal had shown difficulty in eating and was finally killed.

Such a condition is recognised at the first glance by the great swelling and bluish-red colour of the tongue. The affected portion is sharply defined posteriorly and the adjoining part is still perfectly normal : closer examination discovers the encircling object, which may at first be covered by the swelling. The condition is one of strangulation, and, if not relieved, necrosis of the tongue results from interference with circulation and nutrition. But this necrosis does not always set in rapidly, and healing sometimes occurs, even where the tongue is already insensible and cold, and of a bluish-black colour. In these cases, however, the surface of the mucous membrane is destroyed and sloughs away later.

Treatment. The first thing is to remove the foreign body. Sometimes this is sufficient, but not always so, for the mucous membrane, being relatively thick and strong, is unable to yield to the extent required by the excessive swelling, and the tongue runs a risk of becoming necrotic. In such cases, scarification is advisable, longitudinal incisions being made with the bistoury over the whole swelling, and to the extent of half its thickness. Deeper incisions might wound the hypoglossal artery, and give rise to profuse and dangerous bleeding. The swelling generally subsides rapidly after this operation, but at times may continue for several days. Bathing with alum solution assists healing and checks putrefactive changes. As long as much swelling remains, fluid nourishment must be given, and in cats and dogs this may appropriately be administered as a drench. 


\section{(2.) DISEASES OF THE TONGUE.}

\section{(A) MECHANICAL INJURIES.}

With the exception of those wounds previously described produced by foreign bodies, injuries to the tongue occur most frequently in horses. The use of the bit sufficiently accounts for this. Moreover, stablemen, in order to control unruly or sensitive horses during grooming, not infrequently pass a cord around the tongue. If this be sharply pulled, the tongue may easily be cut through, and the thinner the cord the more easily does the accident occur. Snaffle bits, especially if worn, produce the same effect. In horses and ruminants the tongue may also be injured by sharp or displaced teeth.

Rupture of the frænum linguæ sometimes occurs in horses, resulting in suppuration, abscess formation, and the production of fistulæ. In oxen the dorsum of the tongue is abraded by rough fodder. Steffen saw the point of a foal's tongue become gangrenous and slough, after having been violently handled during some dental operation. His report of the case points to a blood-vessel having been ruptured. The injuries so frequently found near the base of the tongue in oxen are not always of a traumatic nature; more frequently they are due to actinomycotic infection, and will be described later. Dogs often bite their own, or one another's tongues in falling or playing.

Diagnosis presents no difficulty. The irritation in the mouth, salivation, want of appetite, or slow, cautious mastication readily indicate the nature of the injury and its extent. Healing is usually rapid and certain, though transverse wounds of the tongue may leave a deep depression. But even this is no great drawback, and is only worth notice inasmuch as the animal wastes food in eating, and the tongue may be lacerated if forcibly handled during examination. But a portion of the tongue may be torn away in the first instance or later, and if the frænum linguæ be involved, mastication will be rendered difficult.

The attempts to cure protrusion of the tongue have shown that in horses the removal of 3-4 inches causes no inconvenience. But where more is lost the animals are unable to bring the food between the back teeth. At times they seek to effect this by holding the head in the air like chickens when drinking, but at best some food must be wasted, and mastication takes longer.

Graf records that a horse, which had lost the point of the tongue, had severe swelling of the remainder, accompanied by salivation and inability to eat solid food: only fluids and mashes could be taken. When the wound had eicatrised, the stump only extended about $\frac{3}{4}$ of an 
inch beyond the first molar. In three weeks the horse could again eat ordinary food, but took three times as long as formerly to do so. Lüdecke described a similar case, in which the tongue was lost as far as the commencement of the frænum, but nevertheless the horse could eat as usual. Cadiot and Dollar describe two cases, in one of which the right side of the tongue was lost from a point just in front of the first molars; recovery occurred in about three weeks, and there was no subsequent difficulty in prehension or mastication. In the other the tongue was divided transversely, the stump only extending two inches below the first molars. Despite the mutilation there was no difficulty in grasping or masticating food ("Clinical Veterinary Medicine and Surgery," p. 333).

In dogs defects in the tongue interfere especially with drinking, as some of the water flows back. But in time both dogs and horses learn to eat and drink in the usual manner. The superficial vessels sometimes are torn, but the arteria lingux profunda may not be divided, and in this rare contingency the nutrition of the anterior part of the tongue is not interfered with. When, however, the profunda is torn, necrosis of the point of the tongue may easily follow. Severe bleeding after the injury is, therefore, an unfavourable symptom. Cagny shows that even severe injuries to the tongue may heal. The end of a horse's tongue had almost been cut off about four inches from the tip by a rubber ring; nevertheless the wound entirely healed, without suturing and without loss by necrosis.

Treatment consists in carefully cleansing the cavity of the mouth after each meal. The horse is then either muzzled or tied up, to prevent it soiling the wound by eating. Clean water should always be kept in the stall. Deep wounds, i.e., such as reach nearly half through the tongue, must be carefully sutured. Very strong silk is the best material, and the stitches are inserted deeply and close together, otherwise they are liable to tear out. Sometimes it is even possible in this manner to bring about healing by first intention. If in transverse wounds the point of the tongue is already gangrenous, it will be needful to amputate, but there must be no haste, and the operation should be deferred until unmistakable signs of necrosis have appeared, for not infrequently the tip may be preserved, even where the cut extends three quarters through the thickness of the tongue. Profuse bleeding results on division of the hypoglossal artery, but if the horse has been cast for operation the vessel can easily be taken up with forceps and tied. Amputation by the elastic ligature obviates this complication. Injury to the frænum often leads to formation of abscesses, which may require to be opened. Under any circumstances, the parts must be kept scrupulously clean. 


\section{(B) ACUTE INFLAMMATION OF THE TONGUE (GLOSSITIS ACUTA).}

Compared with injuries produced by external agencies, acute inflammatory processes in the tongue are seldom seen; but specific inflammations, the result of infection, occasionally occur, especially in cattle and horses. The condition termed gloss-anthrax, formerly regarded as a form of anthrax, occurs also in "wildseuche," a peculiar epizootic showing many points of resemblance to anthrax.

Infection is particularly favoured by hard prickly fodder. Cattle and horses usually suffer in consequence from acute glossitis, which often becomes enzootic, and is clearly due to the entrance of pathological micro-organisms. That septic processes and cellular inflammation may extend from the pharynx to the tongue is shown by Fürstenberg's observations on sheep.

The tongue swells at some particular spot and becomes hard and painful. The swelling increases, feeding becomes difficult, and salivation soon sets in. In cattle, excessive œdema about the pharynx occurs at an early stage, and the lymph glands become swollen. Should an abscess form in a superficial position the pus is usually discharged into the mouth, but deep-seated abscesses of the tongue often break in the submaxillary space, and in cattle discharge a peculiarly offensive pus.

Singard observed a similar disease in young cattle leading to necrosis of the tongue ; and reports finding bacilli in the necrotic parts, which, even after many cultivations, reproduced the disease. Gresswell described an enzootic glossitis terminating in necrosis, and stated having found bacilli which were identical in appearance with those of malignant œdema. Stockfleth also gives an account of gangrenous glossitis in cattle and horses. Even in 1787 the municipality of Detmold gave directions concerning the measures to be taken to check a disease of the tongue in horned cattle and horses, which, from the description given, appears to have been an exceedingly acute and rapid gangrenous process. The animals were said frequently to die in 24 hours. Scarifications were recommended. Kolb noticed in cattle an inflammation of the tongue characterised by severe salivation and cedema of the pharynx, with painful swellings on the frænum linguæ varying in size from a pigeon's to a hen's egg. The swellings where scarified soon healed, otherwise they formed abscesses with foetid contents. Rehrs reports a similar condition in horses.

Diagnosis and treatment must be founded on the character and extent of the disease. In general, scarification is indicated, to allow disease products to escape and prevent necrosis. Should fluctuation appear, recourse must at once be had to the knife. After treatment consists in thorough local disinfection, and feeding with nutritious fluids or semisolids. 


\section{(C) CHRONIC INFLAMMATION AND NEW GROWTHS IN THE TONGUE (MAKROGLOSSIA).}

Under the names of tuberculosis of the tongue, degeneration of the tongue, wooden tongue, and chronic inflammation of the tongue, many different conditions have been described, which have a certain similarity, inasmuch as in all the tongue gradually becomes larger and thicker, and eating and breathing are rendered difficult.

The nature of these processes until lately was doubtful. Numerous foci being found in the lungs, they were described as tubercular. In 1875 Siedamgrotzliy, in view of their pathological and anatomical appearances, suggested that they were due to the entrance of some irritant. More recently they have been carefully investigated and it is clear that several diseases have been confounded. Micro-organisms such as actinomyces, bothryomyces, and tubercle bacilli have frequently been found, and less often psorospermia. The latter, however, cannot always be viewed as pathological, inasmuch as they are sometimes found in perfectly healthy parts of the tongue. At times no cause whatever can be assigned for the chronic inflammatory process by which the fibrous tissue of the tongue increases at the expense of the muscles. Probably malignant new growths occur, but owing to the want of proper investigations nothing certain can be said on this head. Tumours, at any rate, are much less common in animals than in men, in whom carcinoma and syphilis furnish a large number of tongue diseases. The ox is the most frequent sufferer, and in it disease is generally due to actinomyces or bothryomyces. Pflug has urged, however, that chronic indurating glossitis does not always result from actinomycotic infection, but may be due to hyperplasia of the muscular connective tissue. Imminger has found only 4 to 8 per cent. of these tongue diseases to be due to actinomycosis. Probably bothryomyces and staphylococci are sometimes the cause. Truelsen and many others have detected actinomyces in the hor'se's tongue. Compared with actinomycosis, tuberculosis of the tongue in oxen is comparatively rare.

\section{(A) GLOSSITIS CHRONICA INTERSTITIALIS FIBROSA.}

This disease, first described by Truelsen as occurring in the ox, consists essentially in simple hyperplasia of the interstitial connective tissue of the tongue. The tongue feels hard and craunches under the

knife. The cut surface is smooth and greyish white. The muscular substance has almost disappeared. Imminger describes two forms, viz.: (1) Disease of the tip of the tongue in young, and (2) Disease of the base of the tongue in old animals.

The cause is hitherto unknown. 
The affected oxen can chew but little or not at all. They certainly attempt to take food but chew slowly and let fall most of what is grasped; on the other hand they can swallow fluids. Salivation soon sets in and the manger is often covered with foamy saliva. The tongue feels hard and rigid, but is smooth and not painful on manipulation.

In well-developed cases and in full-grown cattle the prognosis is unfavourable. Such animals had best be slaughtered. Under opposite circumstances something can be done. Early treatment in young animals is usually completely successful. Older animals are usually incurable. As in actinomycosis, iodide of potassium is given in full doses and the tongue is painted with tincture of iodine, if necessary after scarification.

(B) ACtinomycosis of the tongue. GLOSSITIS ACtinonycotica.

This disease is very common, and in horses the number of recorded cases increases. Certain localities seem particularly affected. France appears nearly exempt, but Russia, Denmark, America and North Africa furnish numerous cases. The disease is fairly common in Germany, $9 \cdot 1$ per cent. of the total number of oxen slaughtered in Berlin showing it.

The changes in the tongue consist in chronic proliferation of the connective tissue, which exhibits numerous granulating centres. In these the specific fungi are embedded. Sometimes the centres suppurate. The swellings and abscesses usually rise above the general surface of the tongue and are readily visible. Sometimes the appearances consist in ulceration of the base of the tongue just in front of the swelling. Though such lesions are often due to actinomycosis their true nature is not usually recognised.

Appearances and Progress. The tongue gradually becomes thicker and larger, especially at its base, and, on account of its stiffness, feeding is rendered difficult, whilst in swallowing, the head and neck are abnormally extended. Salivation frequently exists, and difficulty in breathing may appear, particularly during mastication and swallowing. At the same time respiration becomes audible, whistling or rattling. These symptoms slowly becoming aggravated, the mouth is examined, and the tongue found thickened, its base being unusually stiff and hard. The veins are abnormally large, and the surface has a dark blue colour, as in strangulation, but acute inflammatory symptoms (pain and odema) are absent. Hard swellings, which vary from the size of a pea to that of a pigeon's egg, are occasionally visible on the surface, and may show signs of ulceration. They are best felt when the tongue is allowed to glide through the hand. Zschokke states that these swellings lie immediately V.S. 
beneath the mucous membrane, and can thus be readily detected. Not infrequently they are of a distinctly yellow colour. In other cases the tongue is simply increased in size. After a time the submaxillary lymphatic glands swell, and at a later stage cedema is present. The difficulty in swallowing prevents the animals taking anything but fluid nourishment, and slow wasting ensues.

Henschel and Falk at the Berlin slaughter-houses saw actinomycosis of the tongue appearing as white or yellow hard swellings, varying in size from a pin's head to a bean, sometimes lying in the mucous membrane, sometimes in the deeper tissues. The disease always started from the lower (anterior) border of the dorsum. They think that a certain comnection exists between the localisation of the disease and the manner in which oxen gather their food. In grazing, the tongue is rotated and passed sideways round the blades of grass. Injuries are thus inflicted which afterwards allow the entrance into the tissues of actinomyces or of particles of food. Of the total animals slaughtered, $9 \cdot 1$ per cent. showed such excoriations, and $7 \cdot 2$ per cent. were affected with actinomycosis.

Treatment. Prognosis is unfavourable, and animals fit for the butcher had best be slaughtered. Fluid food is indicated. Although hitherto looked upon as incurable, some cases are said to have recovered after local scarification and the use of iodine. Bassi, Thomassen, Ostertag, and others recommend iodide of potassium internally, 1 to $2 \frac{1}{2}$ drachms in a quart of water for six succeeding days. This is said, however, to have occasionally produced a kind of poisoning. Actinomycosis may not only be checked but absolutely cured by deep scarification and painting with iodine solution (Thomassen, Ostertag, and others). Of 100 cases Strebel claims to have completely cured one-third and to have so improved others that they could be successfully fattened; only 50 per cent. he regards as incurable. To these belong the advanced cases, and those in which the root of the tongue is principally involved. Where the point and middle alone are invaded, the prognosis is much more favourable, for in these parts deep incisions may be made without danger.

Dressing with tincture of iodine may take place two or three times a day after eating. Ostertag, however, assigns most importance to careful application of the drug; after laying open all swellings, he applies the tincture personally, using a stiff brush. The application is renewed once a week. Bass noted a relapse after iodine treatment. The later observations of Thomassen, Nocard, Ostertag, and others give a high value to the administration of iodide of potassium internally, and the local use of tinct. iodi. Under any circumstances it is better, when dealing with an infectious disease, not to place too much reliance on complete or lasting recovery, and as soon as sufficient improvement is 
declared to prepare the animal for the butcher. Such animals fatten most readily on distillers' and brewers' grains, which make only slight demands on their masticating powers.

\section{(D) PARALYSIS OF THE TONGUE (GLOSSOPLEGIA).}

Inflammatory processes may interfere with the movements of the tongue; but its paralysis depends on injury to the hypoglossal nerve, which supplies with motor filaments the collective muscles of the tongue, and most of those of the hyoid bone.

Wounds, abscesses, or inflammatory processes may affect the nerve at some point of its course, or at its origin on the inferior surface of the medulla, and thus produce glossoplegia. Kater saw one-sided paralysis occur in a foal which three months before had been wounded in the throat with a knife. On the left side the muscles of the tongue had so completely disappeared that at that point the upper and lower coverings of mucous membrane were in contact. In the case of a horse which had first suffered from left-sided, and afterwards from general paralysis of the tongue, Hallander discovered on the left side of the medulla a sarcoma which had originated in the guttural pouch. This paralysis is also seen during severe infections, like contagious pleuro-pneumonia of the horse (see Cadiot and Dollar's "Clinical Vet. Med. and Surgery," p. 185). In central paralysis both nerves usually suffer, and, of course, both sides of the tongue, for the two hypoglossal nerves arise very close together. In the horse paralysis of the tongue sometimes accompanies acute meningitis or hydrocephalus. But every case of double-sided paralysis is not necessarily central. Diplegia occurs in horses whose tongues have been roughly handled, and where both nerves have been injured. In dogs double-sided paralysis is regularly observed during rabies, but it also appears without any preceding illness, and is sometimes accompanied by masticatory facial paralysis. Here the cause is probably central. A somewhat similar paralysis of the tongue in a dog, accompanied by rather extensive disease of the brain, is thus described by Frick :

The dog, which was about five to six years old, usually stood with its back arched, and with the limbs drawn under the body; there was general quivering of the entire surface. The eyes were cloudy and watery; tears ran over the cheeks and fluid from the nostrils. Long threads of tenacious saliva hung from the mouth. There was a weak, rather frequent, cough. The rectal temperature was $1.03 \cdot 2^{\circ} \mathrm{F}$. Examination of the cavity of the mouth showed that about 1 to $1 \frac{1}{2}$ inches of the free end of the tongue was relaxed and incapable of voluntary movement. When the affected part was thrust backwards the animal could not return it to its normal position. The soft palate hung flaccid and was not under control. Food was fairly well taken and swallowed. The animal eagerly took water, which, however, at once ran out of its mouth again. To overcome this difficulty a rubber tube was passed into the cesophagus, and water administered by pouring it into a funnel 
attached to the free uplifted end. After a short time, however, the water was returned, part being lost through the mouth and part passing down the trachea, causing the animal to cough.

Diagnosis. Paralysis of the soft palate, point of the tongue, pharynx and osophagus.

The dog was removed by the owner without undergoing treatment.

Jürgens thinks that numbers of the observations published in veterinary literature as glossoplegia really relate to inflammatory affections; but paralysis of the tongue may certainly result from acute inflammation of that organ.

The symptoms of one-sided paralysis are displacement of the tongue and difficulty in mastication and deglutition. In double-sided paralysis both acts become nearly impossible, particularly the latter. The tongue generally hangs from the mouth. In protracted cases the muscles atrophy, though, of course, in single-sided paralysis only those of the paralysed side suffer.

The disease must not be confounded with the so-called "protrusion," where the tongue is voluntarily lolled out of the mouth. Paralysis is shown by distortion and inability to retract the tongue.

Prognosis is generally unfavourable in double-sided paralysis. The animals cannot be fattened, and therefore, if the case is persistent, it is better to slaughter. Monoplegia is of little consequence, as the animals can still feed well.

Treatment can do little. Should the disease be caused by external injuries, these must be dealt with on general principles, otherwise one can only await developments or slaughter. In fat animals the latter course is preferable, as condition is rapidly lost.

\section{(E) RANULA.}

Ranula is commonest in dogs and cattle. Under the point of the tongue on the frænum linguæ, a long roundish swelling develops, which may attain the size of a pigeon's, or even of a hen's egg. It is greyish-yellow, soft, and not inflamed. When opened, a yellow, thick fluid escapes, and the walls collapse. The swelling, if of any considerable size, interferes with feeding, and produces salivation. In man the voice at times assumes a harsh, croaking tone, hence the German name "Froschgeschwulst" (Frog swelling). The pathological cause has not yet been determined even in man. It was thought to be due to occlusion of Wharton's duct, but this is generally found to be clear. More recently it has been regarded as stoppage of a mucous duct (retention tumour). The condition in the dog is no better understood. One should beware of regarding every swelling or tumour on or near the frænum linguæ as ranula, only the true cyst deserving 
this title. Marked swelling of the loose sublingual connective tissue is often mistaken for the condition in question. Such swelling frequently accompanies cellulitis at the base of the tongue or in the pharynx. A flaccid swelling containing blood-stained or yellowish fluid is then found under the tongue.

Prognosis is favourable, though simply laying the cyst open is ineffective, as it always fills again. But this can easily be prevented by removing the thin wall with scissors and forceps. Ellinger has employed injections of pilocarpine with success. Stockfleth has described as ranula in cattle an entirely different condition, which takes a much more troublesome course, and must be regarded as a malignant inflammation of the submaxillary lymph glands (compare with affections of lymph glands). Hohenleitner states having seen two cases of ranula caused by actinomyces. The ranula disappeared after painting with iodine, though stiffness of the tongue persisted.

\section{(F) FRACTURE OF THE HYOID BONE.}

On account of its sheltered position, fractures of the hyoid bone are rare, but have nevertheless been observed. In horses and cattle they are produced by thrusts with the horns, and blows with the feet, or even by violent traction on the tongue. In dogs they result from the animal being roughly seized by the throat, as is sometimes done by the police in securing stray animals.

The Symptoms comprise salivation, prolapse of the tongue, difficulty in eating and especially in swallowing, accumulation of food in the mouth and swelling in the throat. In complicated fractures there may also be bleeding from the mouth, possibly of a severe character. Crepitation on moving the tongue can seldom be detected.

Union of subcutaneous fractures is usually complete in four weeks. But it not infrequently happens that fragments of bone perforate the mucous membrane. Intense inflammatory swelling then develops, which may quickly prove fatal (Herraud); or mastication and swallowing may be interfered with, and death occur from inanition, or the patients may require to be slaughtered. Fatal bleeding sometimes results from splinters of bone injuring neighbouring blood-vessels. Rupprecht relates that the broken hyoid of a horse perforated the guttural pouch and caused death by lacerating a large vessel. Asphyxia caused by such bleeding occurring: into the larynx and trachea is spoken of by Bolle. But even cases complicated by exfoliation of large pieces of the hyoid may recover in from six to eight weeks, as Schade's experience shows.

Treatment in simple fracture is confined to supplying suitable food which must be easily digestible and require little mastication. At the 
commencement, water may be given per rectum, especially if deglutition is difficult, and soluble nourishment may be administered in the same way. In complicated cases the wound must be frequently cleaned. Should the skin be wounded by perforating fragments (an exceptional occurrence), ordinary antiseptic treatment must be adopted, and loose pieces of bone removed.

\section{(G) NEW GROWTHS IN THE TONGUE.}

Whilst carcinoma of the tongue is common in man it is comparatively rare in domesticated animals, but the following growths have been observed :

In young dogs, calves, and horses, numerous papillomafa are not uncommon. They vary in size from a pin's head to a walnut, and are usually partially macerated in the saliva. The significance of these growths in young animals has not yet been explained. Frick saw a young sporting dog whose tongue was thickly coated with such growths. The condition is seldom of importance, does not interfere with eating and disappears spontaneously. Treatment consists in snipping off the growths with scissors or enucleating them. Fowler's solution of arsenic internally is useful.

Fibromata, sarcomata and carcinomata are seen in oxen and horses. They only receive surgical treatment when in or near the tip of the tongue, whence they can be removed by amputation. When seated further back treatment is useless, and the patient had better be slaughtered.

Mucoid cysts have frequently been seen at the base of the tongue in horses. In many cases they have caused no complication, and have only been discovered after death apparently from an epileptiform fit. In other cases they have caused difficulty in swallowing, breathing and mastication. Pedunculated cysts may be removed with the écraseur; the flatter kinds are laid open and their interior destroyed with the cautery or by dressing with tincture of iodine.

\section{(3.) FRACTURE OF THE PREMAXILLARY BONE.}

Such fractures are caused by falling or running against obstacles; in horses by kicks, in dogs by blows. Sometimes the nasal process alone breaks; sometimes the alveolar portion with one or more incisor teeth is involved; sometimes the bodies of both bones are broken through.

Diagnosis is based on the painful character of the swelling, and on the result of examination of the bone, which lies almost immediately under the skin. Transverse fractures of the body of this bone produce results similar to those of the body of the under jaw; the upper incisor 
teeth and the alveolar margin of the bone appear movable, or are more or less displaced backwards. The upper lip often hangs down obliquely, so that on casual examination this injury might be mistaken for facial paralysis. So long as the fracture is confined to a single alveolus or to the nasal process, it heals rapidly and completely. Transverse fractures of the body give greater trouble, especially when the alveolar margin and the incisors are movable, or when a complicated fracture exists. Treatment of such cases is only advisable in valuable or favourite animals. Where the free edge is firm, recovery is much more certain.

Treatment aims at fixing the fragments in position with wire in the same way as in the lower jaw. In complicated fractures strict antiseptic precautions must be observed. In some cases the incisor teeth, which have been dislocated backwards, can only be replaced in their normal position after the lapse of some time, and by exercising considerable force. But as soon as they are brought into position, so that the upper and lower teeth are in contact, the movements of mastication usually suffice to complete replacement. In horses transverse fractures of the inter-maxillary bone just behind the alveolar process have often been seen: in one case the upper incisors had all been thrust downwards and backwards in consequence of the animal falling on the mouth. Reduction can often be effected, even several days after the accident, by using as a lever the thin handle of a hammer. Sometimes strong pressure with the thumbs is sufficient.

\section{(4.) INJURIES OF THE INTERDENTAL SPACE.}

The interdental space is that portion of the jaw which intervenes between the corner incisor and the first molar tooth. The bone here presents a more or less sharp border, and is covered with periosteum and strong, thick mucous membrane. On this part the pressure of the bit falls. The bit usually rests on the tongue and edges of the lips, but the action of the reins presses it against the jaw, and thus, especially in riding-horses, produces wounds. The more severe the bit, and the lower it is fixed, the more easily this happens. Such injuries are also more frequent with a high and sharp conformation of the bone, a thin tongue and relaxed lips, and with riders whose hands are heavy.

The diseased processes so produced are of various kinds, and may be distinguished as follows :-

(1) The simplest injury consists in erosion of the mucous membrane covering the interdental space. The epithelium being removed by the rubbing of the bit occasionally makes sensitive riding-horses troublesome, but yieldis to treatment in a few days. 
(2) Should the force be greater the mucous membrane itself may be bruised or wounded. Simple bruises without penetration are seldom very troublesome, but actual wounds may easily lead to periostitis and its results (necrosis).

(3) The severe use of sharp bits ends in disease of the periosteum or of the bone. Sometimes this takes the form of periostitis ossificans, which goes on to the formation of exostoses, often seen in the skeletons of old riding-horses. Purulent periostitis, always met with where the wound has extended to the bone, produces necrosis, which may lead to purulent affection of the medulla (osteomyelitis suppurativa). The disease products later find exit on the lower maroin of the jaw, and necrotic portions of bone, as large as a finger, may be discharged. Though in such cases recovery is naturally slow, it is always complete, and no permanent injury is left.

Symptoms. Attention is often first directed to an erosion of the mucous membrane by the excitability of the horse when reined in. Bruises are recognised by the local swelling, redness and pain; wounds are directly visible. The parts are best examined by introducing the forefinger into the mouth; and should pain, swelling, or tears in the membrane be detected, the diagnosis can be confirmed by visual inspection. Periostitis ossificans can only be discovered by touch, otherwise it often remains unnoticed, the horse's pulling being assigned to bad temper, either on its part or on that of its rider. Moderate pressure on the inflamed spot produces marked pain. Wounds in the mucous membrane or necrosis of the bone are easily detected with the finger or a metallic probe. Osteomyelitis is recognised by swelling of the bone, usually extending from above downwards towards the lower edge of the jaw. When the enlargement breaks a sanguineo-purulent discharge mixed with necrotic bone fragments escapes, and emits the peculiarly unpleasant smell of bone pus. The probe often passes from the lower edge of the jaw right into the mouth. Salivation and painful mastication, though they sometimes occur, are not essential characteristics.

Treatment must be based on the anatomical changes. If the parts are merely abraded, it will be sufficient to rest the horse, or use a simple nose-band or smooth snaffle, in place of a more severe bit, until the epithelium has again grown, or the bit may be fixed higher in the mouth. In wounds of the mucous membrane, rest or absolute avoidance of bar bits is necessary. The parts must be cleansed after each meal, and the horse muzzled or tied up. The wound should subsequently be treated according to its character. Immediately the bone or periosteum begins to suffer, the bit must certainly be laid on one side. In periostitis ossificans recovery usually follows this treatment in a few weeks; but in 
purulent periostitis and myelitis improvement will only occur after removal of the necrotic bone. This can sometimes be effected through the mouth by using dressing forceps, otherwise the swelling should be allowed to rupture, and the bone taken away from without. Both the external wound and the opening in the bone must be kept as clean as possible. Syringing with solutions of carbolic acid, creolin, or diluted hydrochloric or-sulphuric acid is here indicated. A plug of iodoform gauze may afterwards be introduced, in order to prevent food particles entering. In caries of the lower jaw, Greiner injects the fistula with concentrated solution of lactic acid, and then introduces tampons soaked in the same fluid. The wound requires cleansing after each meal, and treating as above indicated. In some cases good results follow the use of a pointed cautery.

Unfortunately, wounds are often discovered only when the jaws are considerably swollen. Injuries to the interdental space are very common in the army after general manœuvres, and when cavalry ride great distances without veterinary superintendence. Under such circumstances the mouths require to be examined as regularly as the backs and saddles. In tender-mouthed horses leather or rubber covered bits prevent this injury, and should be used for some time after healing; but as long as wounds exist they are of little service, and in erosion of the mucous membrane are positively hurtful.

\section{(5.) DISEASES OF THE TEETH.}

This chapter will take cognisance of all pathological conditions of the teeth, and of all irregularities in the conformation of the mouth, which interfere with mastication. Without this wider knowledge abnormalities of the teeth cannot be understood, nor can a reliable diagnosis be made. Diseases of the molars are most important in herbivora, because the molar teeth perform the whole duty of grinding the herbage, while the incisors only cut it. The dental disorders of vegetable feeders will, therefore, first receive attention.

The clinical appearances, although varying in the different conditions, generally show marked agreement. In horses (of which we are now more particularly speaking), the following symptoms are usually present:-

(1) Deliberate cautious mastication, subject to sudden interruptions and rolling of the tongue, the head being held on one side.

(2) Dropping of food from the mouth; balls of hay are found in the manger ; in popular phrase the animal "quids" its food.

(3) An important symptom is the presence of food in the mouth, generally in the cheek, a considerable time after eating. Grinding the teeth when no food is in the mouth is also significant. 
(4) At a later stage wasting and loss of strength. In cattle epileptiform convulsions have been seen as a result of dental disease. In dogs salivation should always draw attention to the state of the mouth, and particularly of the teeth.

Alveolar periostitis is the most common dental disease in animals, and always gives the mouth an extremely offensive smell.

Immediately any such symptoms are present, the mouth, and especially the teeth, should be thoroughly examined. In some horses, irregularities of the teeth can be detected through the thickness of the cheeks by palpation from without. Sometimes the bone swells and fistulx form. The incisor's are easily inspected, but examination of the molar's requires certain precautions varying in the different classes of animals. Deviation of the teeth from their absolute or relative positions likewise points to disease.

Diseases of the teeth may be clinically divided into the following four groups :-
A. Irregularities in development.
B. Irregularities in wear.
C. Diseases of the tooth proper.
D. Diseases of the alveolar periosteum.

\section{(A) IRREGULARITIES IN THE DEVELOPMENT OF THE TEETH.}

(A) EXTRA OR AdVENTITIOUS TEETH (POLYOdONTIA, HYPERDENTITION).

Kollmann states that in man during fotal life more enamel germ is sometimes formed than is required for the normal number of teeth, and that this determines the production of supernumerary teeth. Kitt looks on hyperdentition as a result of atavism, and points to the fact that in former ages foals had more teeth than at present. The same theory explains the appearance of the pre-molars in the horse, whose ancestors (Hipparion, \&c.) always had four pre-molars. Kitt describes as a typical hyperdentition the apparently purposeless excess of teeth, an example of which is cited by Goubaux, where a certain horse had double the normal number of incisors. Günther, Stockfleth, and others have noted supernumerary molars. Their commonest situation seems to be behind the third molar, but they may lie alongside the normal teeth, being either in contact with the tongue or cheek. At times milk teeth may be retained by becoming fixed between their permanent successors.

Supernumerary teeth are seen in most of the domestic animals, supernumerary incisors, canines, and molars all being represented, the latter most frequently. A regular series of such observations in horses and oxen has been compiled by Morot, and in the dog by Sussdorf.

Excess teeth seldom cause trouble until, by continued unopposed growth, 
they come in contact with and wound soft tissues. Röll and Dieckerhoff have seen cases where a tooth in the lower jaw has become so long as finally to penetrate the opposite bone. The nasal cavity may even be pierced, and a suspicious discharge produced. Möller records two such cases. Supernumerary molars, when on the inside of the row, are apt to wound the tongue, and, when on the outside, the cheek. Walther speaks of two accessory molars in the horse which appeared, one on the inner side of each of the third upper molars. The horse had difficulty in chewing. Möller saw two cases where a supernumerary tooth was present on the inner side of the fourth upper molar. (Fig. 5.) Both teeth suffered from alveolar periostitis, penetrated the maxillary sinus, and produced chronic nasal discharge. In another case the extra tooth was on the inside of the third premolar, and interfered with eating.

Treatment consists in shortening or removing the offending molar. Extraction is difficult where the tooth stands close to another, and therefore camnot be grasped. In these cases it may either be shortened or punched out. Such teeth may be removed by using forceps with sufficiently thin jaws to pass between the diseased tooth and its neighboul. In the front of the mouth the two teeth may sometimes be thrust asunder with a strong chisel.

(B) IRREgULARITIES IN REPLACEMENT OF THE 'TEETH.

Occasionally the milk teeth remain fixed in position, and cause the permanent teeth to grow

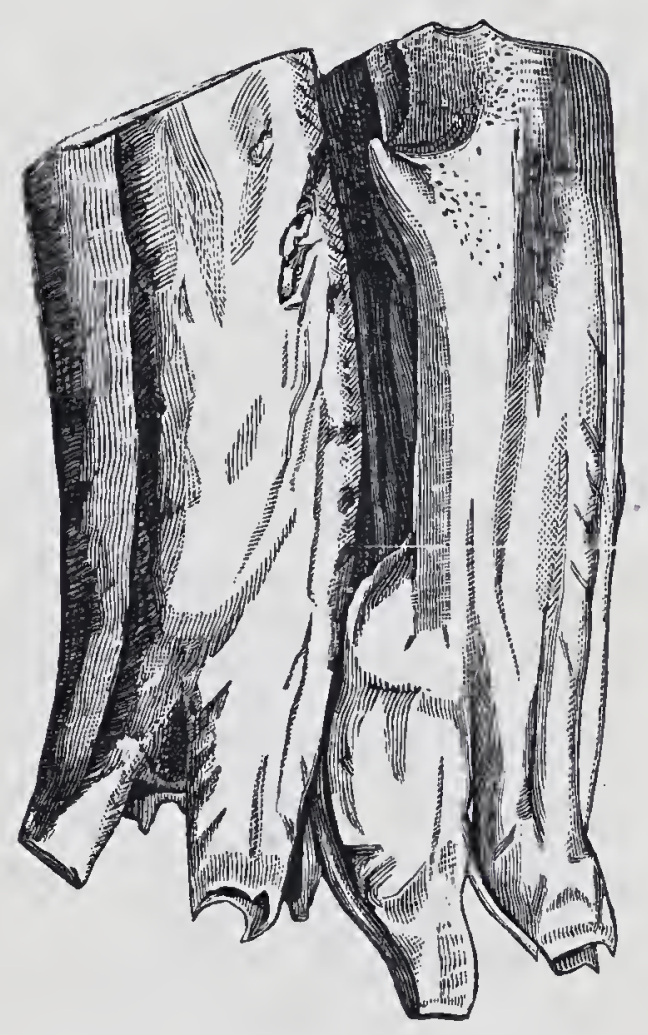

Fig. 5.--Supernumerary tooth (horse). irregularly. Such milk teeth may be removed with ordinary forceps, though Günther's are preferable. (Fig. 30.) It should be noted that the milk tooth is always in front, the permanent behind. In extracting the first tooth, the projection in front of the jaws of the forceps should lie on the permanent tooth. The incisor teeth are sometimes absent in dogs, especially in such as have suffered from severe attacks of some infectious disease during very early life. It seems possible that under such circumstances the germ of the permanent teeth may have undergone atrophy. The eruption of the molars seldom gives rise to trouble, though at times severe pain accompanies the process, and soft food may be required. Occasionally, however, the animal loses condition to such a degree as to necessitate operative interference. Frick removed six temporary molars from a foal's mouth; they had remained fixed on the erupting teeth like caps, and during 
mastication continually injured the gums and cheelss. The animal's appetite and condition immediately improved after the operation.

\section{(c) DISPLACEMENT OF THE TEETH.}

Molars may become displaced in consequence of disease of the alveolar periosteum and loosening of the teeth. This condition will be noticed later. Abnormalities occur in development; one or other of the permanent incisors, instead of appearing in the site of the deciduous tooth, sometimes makes its appearance at a point further back, and wounds the tongue. Stockfleth mentions a case of this kind.

In horses an incisor is sometimes rotated on its axis, the convex surface being turned inwards, or it lies horizontally, and grows beyond the lips, injuring the animal's appearance. The molars may be similarly displaced. Kitt describes a mouth in which the first molar lay with its crown alongside the outer aspect of the second and fourth, whilst its root projected from the jaw on the inner side at a point about an inch and a half below the alveolar ridge.

Kitt ascribes such deviations from normal position either to temporary or permanent want of space at the time the tooth appears, or to abnormal conformation of the mouth or incidence of pressure. A milk tooth may thus give an abnormal direction to a permanent tooth just developing, or this abnormal direction may exist from the first.

False position, due to irregular development of the jaw, may be considered under this heading. Either jaw may be affected. If the upper is too long (Prognathia superior), or the lower too short (Brachygnathia inferior), the so-called "overshot jaw" is the result; whilst an opposite conformation produces "undershot jaw." These variations result from faulty development of the bones, and are often accompanied by corresponding changes in the molars. In undershot jaw the lower rows of molars are displaced anteriorly so that the first lower molar projects beyond that of the upper jaw, whilst the last upper molar does not come in contact with its fellow of the lower jaw. In overshot jaw the case is reversed. The teeth consequently either partially or entirely escape wear, and thus become too long and impede mastication. The nose, or even the entire head, may be distorted, producing displacement of the teeth. This is termed by Gurlt campylorhinus. (Fig. 11.) Leisering saw such a case during life. Dose noted a peculiar abnormality in a cow. The incisors stood in pairs, one behind the other. Although the animal was three years old, no teeth had been shed. The under jaw was deformed, and Gurlt considered this to be the real and primary cause of the peculiarity.

When slight, these changes are of little importance, but immediately they become pronounced they produce various undesirable results. The 
animal has difficulty in grazing, can no longer tear off the short grass, and where the incisors are much affected mastication even of cut food may prove difficult and painful. If the molars fail to correspond, the grinding surfaces wear away unevenly, and the overlapping part grows until it either wounds the opposite gum or even the palate, and thus produces pain in chewing. Deviation of the axis of the tooth in either a backward or forward direction, is continually aggravated by mastication, which tends still further to thrust the tooth out of position and is apt finally to produce alveolar periostitis.

Schrader saw a horse in which the first left upper molar was immediately behind the tush, while the second lay at the inner side of the third, so that the first molar was separated from the others by a space of 2 inches. The two first lower molars had grown into this space and perforated the palate, and in drinking, the water passed through this aperture and was discharged by the nostrils. The horse, being much wasted, was slaughtered.

Too great a space between the teeth is at once abnormal and injurious, especially in the case of the molars. The crowns of the teeth should stand close together, so as to afford mutual support, and prevent food entering the interspaces. Where intervals occur food is driven into them, penetrates even the alveoli, and may produce inflammation there. Such alveolar periostitis is not uncommon in old animals.

Treatment consists in shortening or removing the offending tooth. In young animals too much of the tooth must not be removed at once, as the pulp cavity may be exposed, and alveolar periostitis set up. Where intervals occur between the teeth the evil is palliated by giving soft food, so as to lessen or remove the need for mastication.

\section{(B) IRREGULARITIES OF WEAR IN THE TEETH.}

In the horse's under jaw the rows of molars form two almost straight lines, which posteriorly diverge slightly. In animals of average size the first pair of teeth lie about 2 inches, the last about 4 inches, from each other. In the upper jaw the space between the two rows (which are bowed outwards) is considerably greater, the two first molars on either side standing about 3 inches, the last molars about 4 to $4 \frac{1}{4}$ inches from each other. At the boundary between the pre-molars and molars the cross measurement in the upper jaw is about $4 \frac{1}{4}$ to $4 \frac{3}{4}$ inches, in the under jaw about $3 \frac{1}{8}$ inches. It will thus be obvious that during rest the grinding surfaces of the upper and lower rows do not cover one another; the upper row overhangs the outer edge of the lower; while, on the other hand, the inner edge of the lower row projects farther inwards than that of the upper. (Fig. 6.) Moreover, the grinding surfaces, when 
viewed from in front, are inclined to the horizontal, their outer borders being $\frac{3}{16}$ to $\frac{3}{8}$ of an inch lower than their inner. If the lines of the grinding surfaces are prolonged, they meet in an obtuse angle below the palate. (Fig. 6.) Baume places the teeth of the horse in a class between those which grow continuously, and have an open pulp cavity, and those like the teeth of men and carnivora, the pulp cavity of which is closed, and which grow from the root.

The ox's molars differ from those of the horse in having a much rougher grinding surface. The edges of the folds of enamel project prominently, forming, even in normal teeth, quite sharp points. The lower rows of molars lie almost parallel with each other, or, at most, are

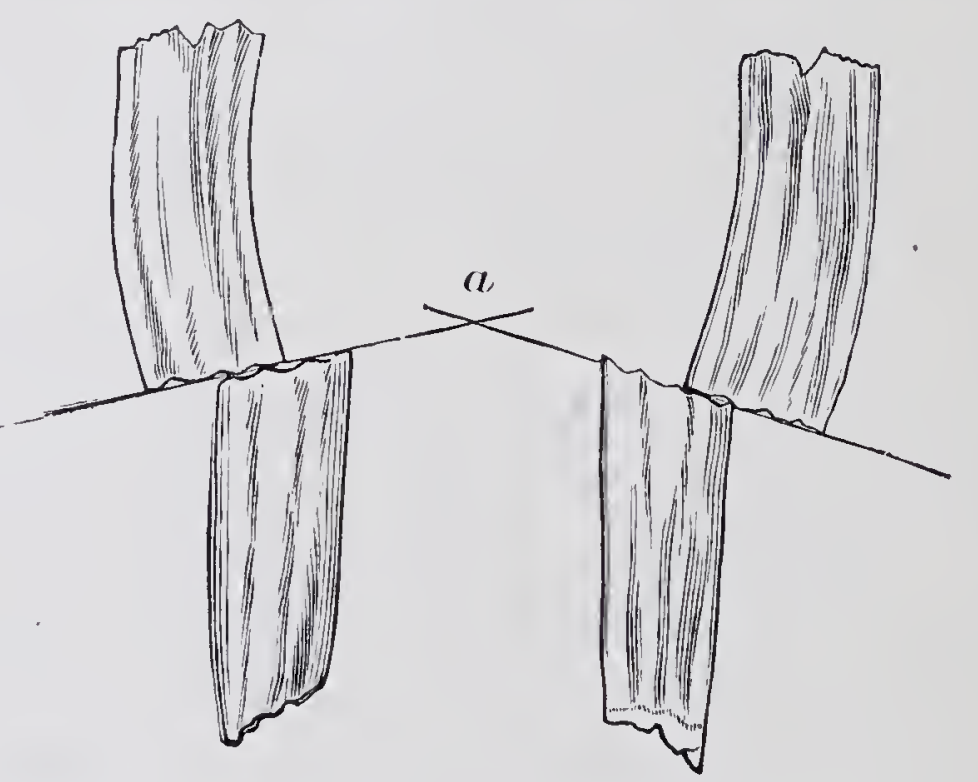

FIG. 6.-Normal position of the molars in the horse.

slightly bowed outwards. Sometimes the upper and lower rows of molars altogether fail to coincide or to cover. In many cases, when seen from in front, the outer edges of the lower molars are at the same height as the inner edges of the upper. The grinding surfaces are inclined as in the horse. Günther states that the normal rate of wear, which in herbivora is very considerable, amounts to about $2 \mathrm{~mm}$. (nearly one line) per year. In consequence of this continued wear, the grinding and cutting surfaces should eventually attain perfect contact. But where they do not, certain areas are insufficiently worn, and grow abnormally fast (Exsuperantia dentis, Kitt), while certain others wear too quickly. Hence arise the following irregularities :-

(a) The angular or sharp mouth.

(b) The shear-like mouth.

(c) The wave-formed mouth, where the row of teeth, seen from the side, appears undulatory.

(d) The step-formed mouth, where, from similar irregular wear, the row is composed of alternate high and low teeth.

(e) Premature wear of the teeth.

(f) The smooth mouth. 


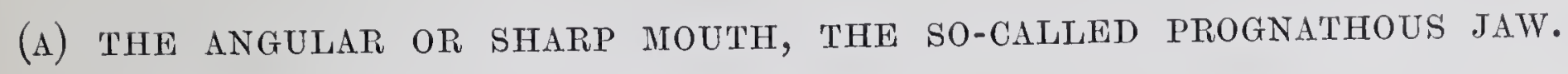

Under ordinary circumstances mastication causes general wear of the entire grinding surface of the molars, but if any part fails to be worn, that portion will become too long. Owing to the peculiar relations of the two jaws, the points which generally escape wear are on the inner side of the lower row and on the outer side of the upper. Either single teeth, an entire row, or even several rows, may be involved. If the two rows do not terminate at the same point, the upper molar being too far forward, or the first lower molar too far back, the unopposed points will continue to grow until their sharp elongations may injure the opposite gum.

Causes. This irregularity may be caused by a narrow formation of the lower jaw, as was shown by Defay, and later by Günther and others, though such formation is not the only cause; otherwise the ox, which very rarely exhibits the condition, should be a frequent sufferer. It is assisted by limitation of the movements of mastication, which in its turn is said by Lorge to be dependent on faulty development of the muscles of the jaw. In chewing, the ox moves the jaw laterally through a wide angle; hence, although its upper and lower rows of molars do not coincide, it very seldom suffers from sharp edges. As soon as sharp edges form on the teeth, the inside of the cheeks may be wounded. The further limitation thus placed on movements of the jaw aggravates the production of sharp edges, and the mischief increases. It is easy to operate on the sharp edges and points of the teeth, but this does not remove the cause, and the malady is liable to recur. The sharp edges of the lower teeth lacerate the tongue, while those of the upper injure the cheek. These injuries may eventually induce general wasting and loss of power. Such a condition is seen especially in old horses, seldom in other animals.

Symptoms. The animal feeds badly, chews slowly and cautiously, and holds the head to one side. Food is often dropped from the mouth into the manger ; portions also remain between the teeth and cheeks; while movements of the jaws or tongue are as much as possible avoided. Wounds of the mucous membrane produce copious salivation. Jessen remarked that this condition was formerly often overlooked; but that its importance is apt nowadays to be over-estimated, and the diagnosis of "sharp teeth" is sometimes used as a cloak for ignorance. Irregularities, however, frequently produce no mischief, and can only be regarded as causes of imperfect mastication when they occasion wounds of the tongue or cheeks. Unless where very marked, they are unimportant in young animals.

Treatment. Sharp points or edges can either be removed with the rasp (fig. 7) or one of the several forms of chisel. (Figs. 8, 9, and 10.) If 
necessary, perfectly efficient instruments can be made from an ordinary foot-rasp. Large projections require the use of the chisel and mallet or

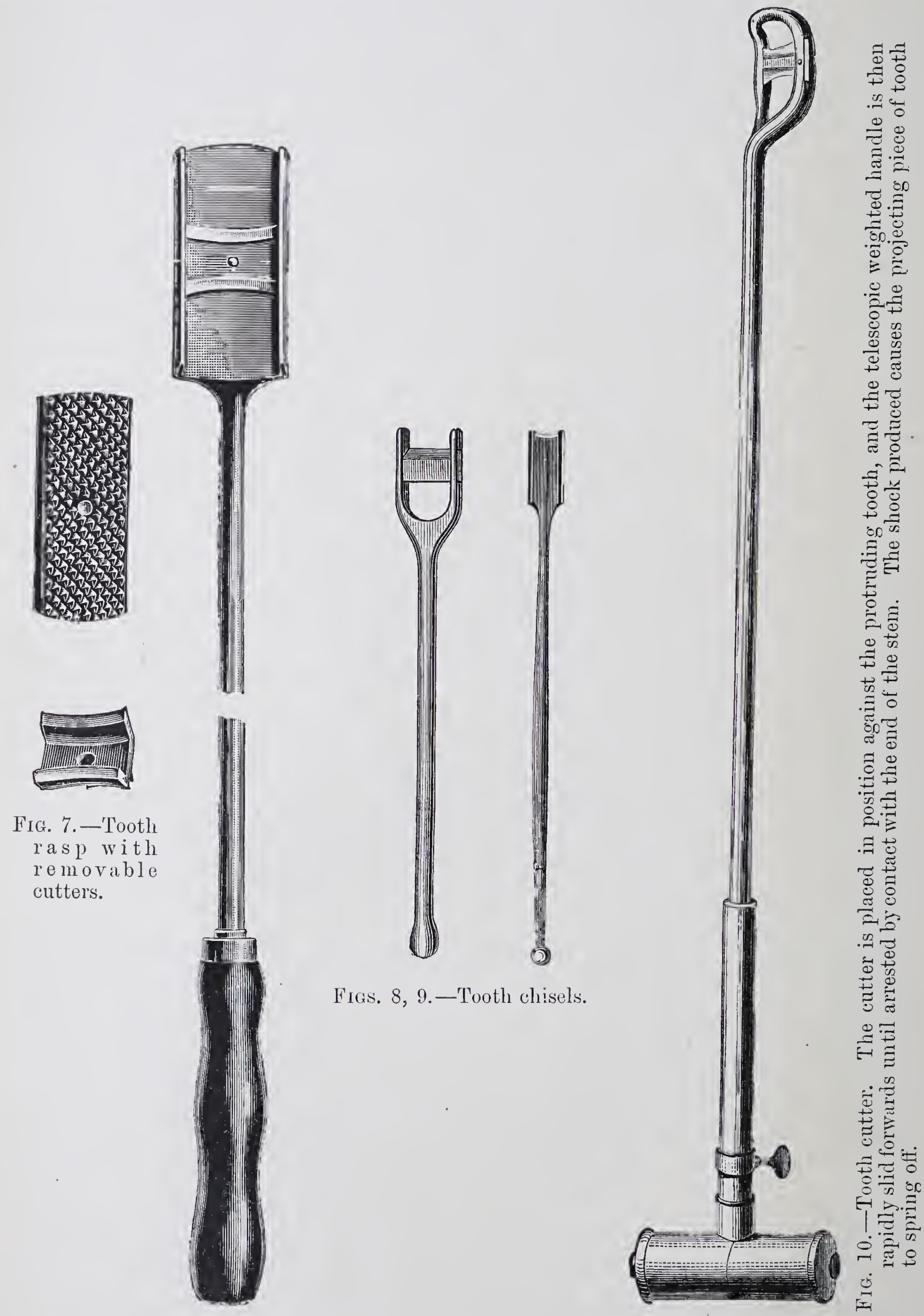

hammer. In the case of the back molars the chisel must be used with caution, so as to avoid injuring soft structures. The blow must be sharp but short; while, to prevent the chisel travelling too far forward, the 
left hand, in which it is held, can be rested against the incisor teeth. The skilled practitioner can dispense with instruments having rounded guards near the cutting edge, and also with Brogniez's "odontriteur," a chisel in which the blow is produced by an iron bolt sliding on the handle. The rasping and chiselling of the teeth sometimes produce their good results indirectly, by making one or more teeth sensitive, and thus throwing the patient off its feed, time is given for recovery from gastric affections.

In old horses, chewing on one side of the mouth sometimes shortens the incisors of that side. This condition, described by Günther as "oblique mouth," seldom causes trouble, but is interesting because often associated with irregular wear of the molars:

\section{(B) SHEAR-LIKE MIOUTH.}

Shear-like mouth consists in a considerable increase in the obliquity of the wearing surfaces of the molars. Their outer edges in both jaws are too low, the inner too high, so that the wearing surfaces, if prolonged,

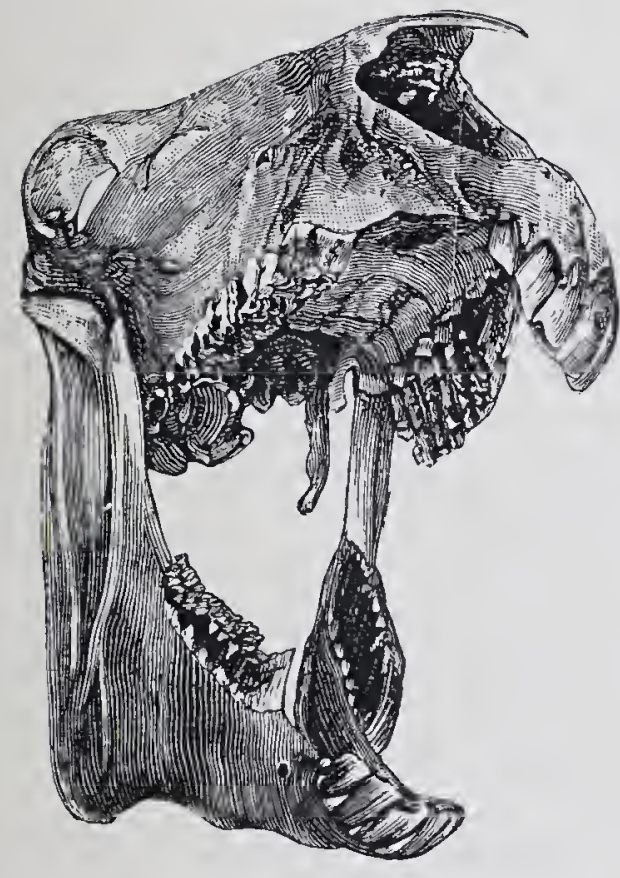

FIG. 11.-Left-sided shear-mouth (from a photograph).

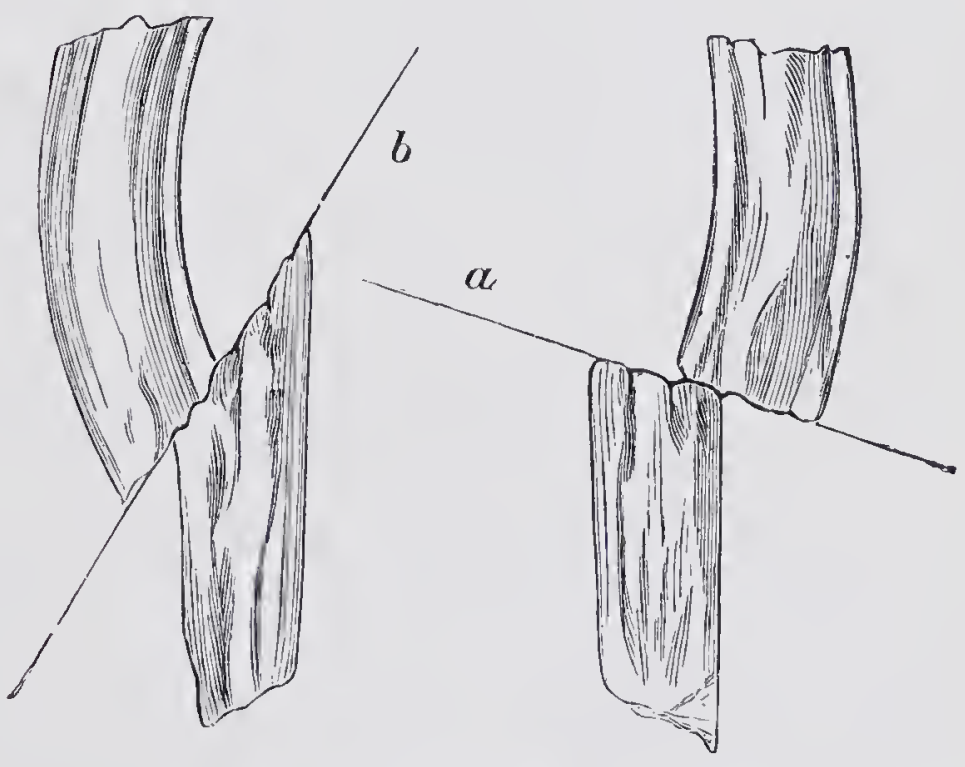

FIG. 12.-Grinding surfaces

would meet in an acute angle above the palate. In other words, the crowns lie not over, but alongside, one another, so that the mouth resembles that of a flesh feeder. (Figs. 11 and 12.) The rows of teeth, therefore, do not grind, but cut, meeting one another like the blades of a pair of great shears, in which the inner blade is formed by the lower molars, the outer blade by the upper. When confined to single teeth, this change most frequently affects the fourth molars, because they are more liable to lateral displacements; but, as a rule, one whole row is affected, constituting simple shear mouth; occasionally both sides suffer (double shear mouth).

The condition is brought about thus: The inner edge of the lower v.s. 
molars and the outer edge of the upper are not worn away; both continue to grow until the former meets the hard palate, while the latter injures the gums of the lower jaw. Coupled with impaired mastication, such wounds prevent the horse either taking or chewing food. The overgrown teeth sometimes penetrate the bone above, and the hard palate may even be completely perforated. The side thrust on the teeth loosens them, while wounds of the gum are apt to produce inflammatory changes in the alveolar periosteum. Hence "shear mouth," especially in old horses, generally produces alveolar periostitis.

Its causes are the same as those of angular or sharp mouth. A narrow jaw and limited movement are notable predisponents. It is unnecessary to discuss the correctness of Lorge's supposition that the muscles of the jaw are imperfectly developed; or Günther's, that the affection is due to variations in hardness of the molars. Esser saw shear mouth result from the articulation of the jaw being wounded, lateral movement in one direction being shortened, and irregular wear thus induced.

Prognosis depends on the degree of development, condition of the teeth, and existing complications. The earlier stages escape notice; it is only when chewing becomes difficult that its presence is suspected. Provided the teeth are still sound and firm, temporary relief may at all events be given; but where they are loose, or alveolar periostitis has already set in, the chances of recovery are slight, and in long-standing cases cure is impossible.

Treatment was formerly confined to removing the sharp edges with the rasp or chisel, and lightening the work of mastication by giving soft food. Even nowadays nothing more can be done if the teeth are loose or shear mouth is moderately developed. But where the patients are young and valuable, and the teeth still firm, relief may be assured, at least for some time, by removing the projections with tooth shears. Möller operated on several cases in this way, and the animals immediately afterwards were able to take their food and masticate quite satisfactorily. If several teeth have to be shortened, the horse must be cast, but the operation is not difficult, and Möller more than once operated on double shear mouth in a quarter to half an hour, and had most excellent results. As to the use of the shears, see section (D) hereafter.

\section{(c) THE WAVE-FORMED MOUTH.}

The wearing surfaces of the several molars on each side above and below are normally of similar height, and form a plane surface; but variation of this plane produces the wave-formed mouth. It is generally bilateral, the fourth lower molar being the shortest, the corresponding upper tooth the longest in their particular rows. The 
molars in front and behind these become respectively longer or shorter. In the lower jaw they are usually too long, in the upper jaw too short. Sometimes the state of things is reversed.

This form of mouth usually depends on unequal durability of the individual teeth, and sometimes on disease of the alveoli. Slight inequalities cause little discomfort; but when well developed, and especially if the alveoli are involved, mastication is greatly impaired, and the sharp points and edges wound the opposing soft parts. Certain teeth may be worn down level with the gum, and mastication made exceedingly painful. The disease occurring in early life is especially serious, as it becomes aggravated with age.

Treatment. Sharp points and edges must be removed. Excessively long teeth which injure soft structures should be shortened or extracted; and the diet should consist mainly of crushed food and slops.

(D) THE STEP-FORIED MOUTH.

This irregularity is closely allied to the foregoing. The only difference between them is that the neighbouring molars vary in height, not gradually but suddenly, a short one being followed by a much longer one, or vice versî. The same cause, viz., unequal hardness, seems at work here. The condition may be due to some unknown constitutional peculiarity, as evidenced by its attacking both sides of the mouth. The spaces resulting from loss of teeth are often responsible for its production.

The prognosis depends on the degree and extent of the irregularity. Mastication is usually more impeded by this than by the wave-formed mouth, because lateral movement of the jaws is here more difficult. The disease is gravest where the animals are young, the soft tissues wounded, or where several teeth have been lost.

Treatment is merely palliative. Soft food will assist mastication. The longest teeth must be reduced or removed. The crowns can be shortened with Möller's tooth shears. This is preferable to extraction, which often presents great difficulties on account of want of room, whilst the use of file and chisel demands too much time and care.

Möller's shears (fig. 13) have been frequently varied, but not much improved. The central screw lying between the limbs of the instrument ensures great power without disturbing the position of the instrument. Every part must be fashioned very strongly and carefully of the best steel, for, owing to the immense power of the screw, it might otherwise break or bend. With quiet horses one or more front molars may be cut without previous casting. But it is better to lay the patient down when it is highcouraged or troublesome, or when several teeth or any of the back molars are
to be dealt with. After inserting a rather large mouth gag, and drawing the
D 2 
tongue away from the part to be operated on, the shears are adjusted on the tooth to be shortened, and the screw turned, while the instrument is held by one or two assistants. After a few turns, made as quickly as possible, the desired portion of the tooth will spring off with a loud noise. At this moment the horse usually makes a movement with its head, but, as the shears are already free, this is of no importance. Möller has cut many strong upper molars without ever having an accident. The tooth breaks smoothly off. In old horses, it may happen that the movement of the head loosens the tooth, and it comes away with the shears, affording, however, the desired relief. Most difficulty is met with in back molars, and where the

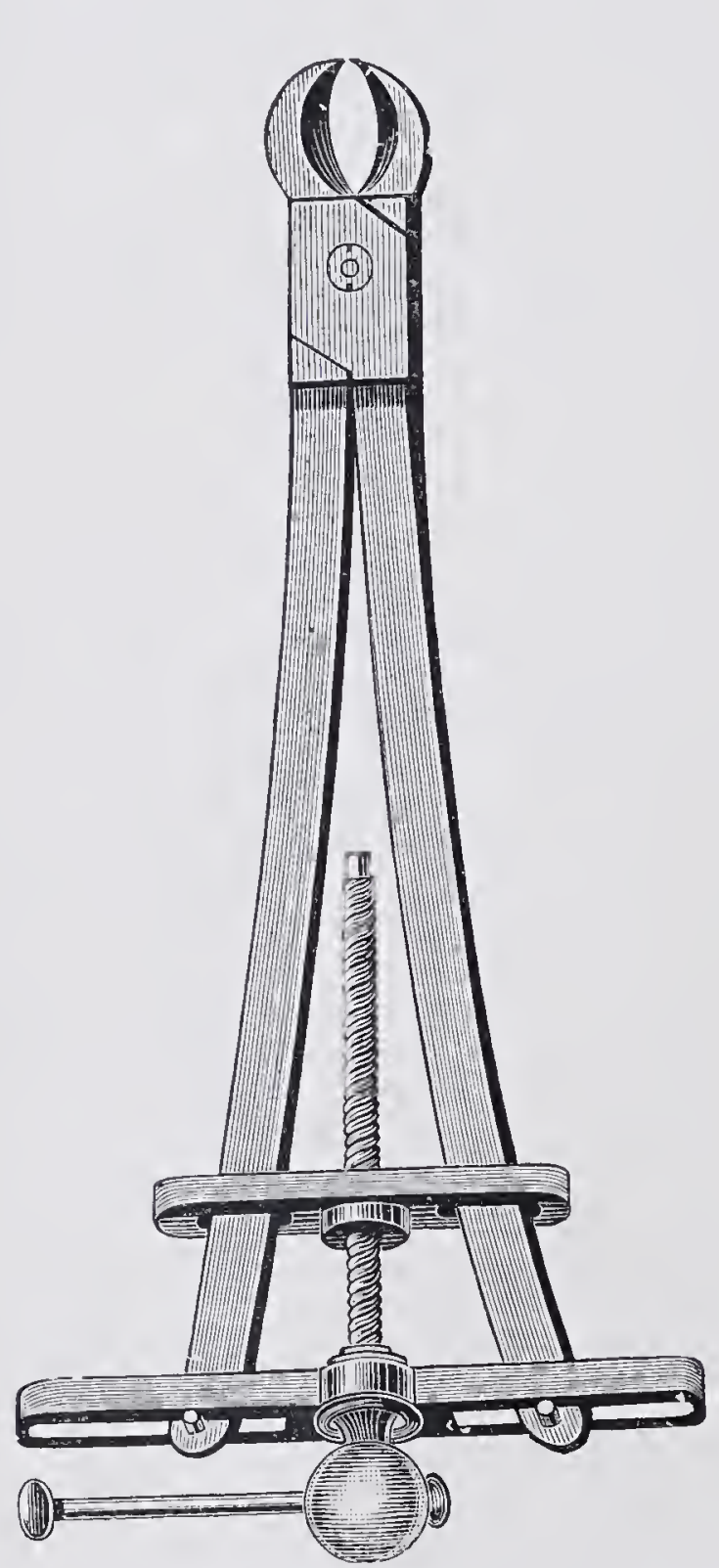

Frg. 13. -Tooth shears.

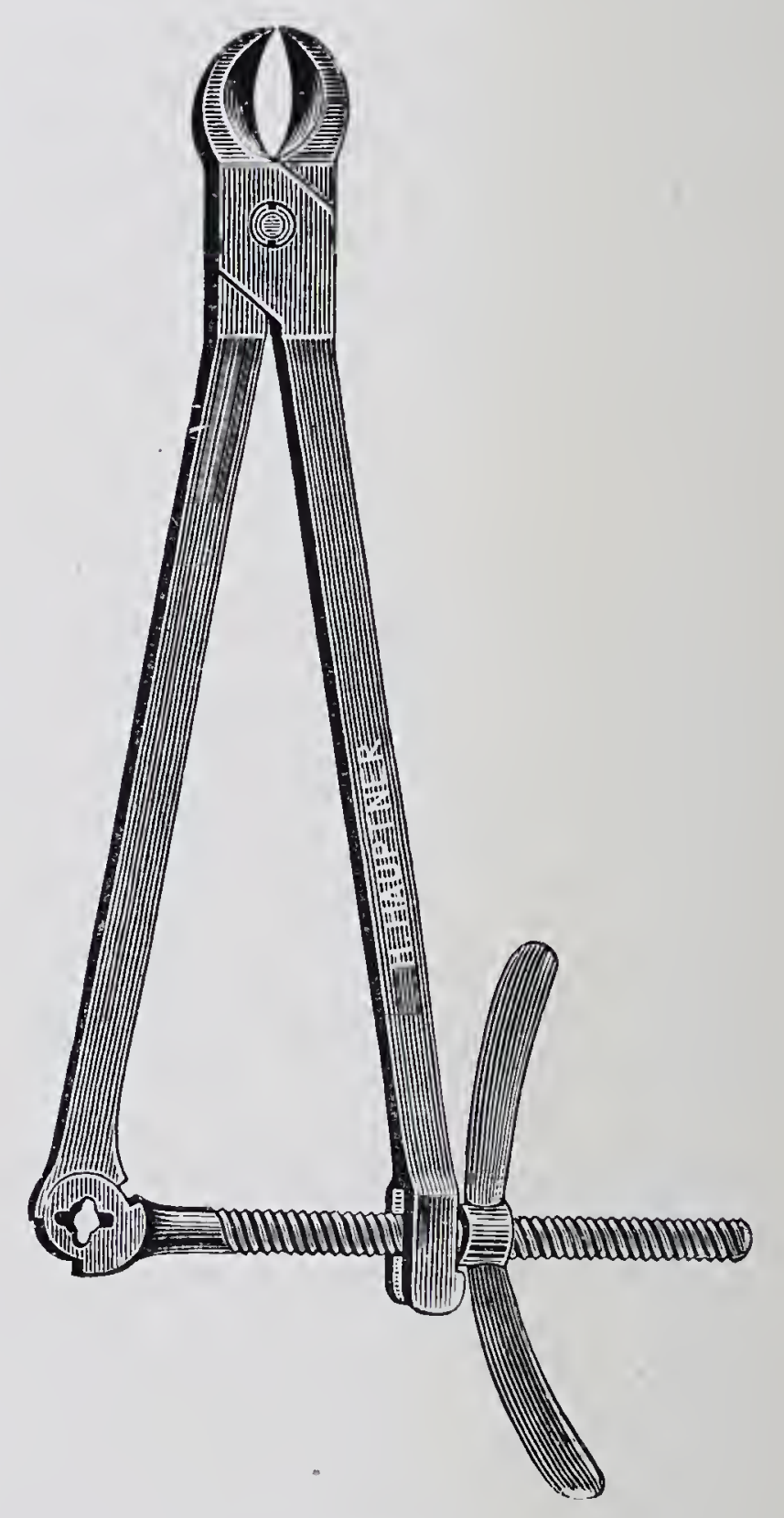

Fig. 14. - Tooth shears.

sides of the teeth have been worn away obliquely. The gag must be wide enough to allow the open shears to be easily introduced. When the shears slide off the sides of the teeth, Möller's tooth screw (fig. 15) may preferably be employed. In this instrument the power of the screw acts directly on the cutter. The upper arms are for holding the instrument in position. It is used in the same way as the shears, and will be found very useful.

Metznik has invented a pair of shears in which the strength of the hands, acting through a series of levers, is found sufficient to cut teeth without having recourse to a screw (fig. 16). It is of service, but would be better if the limbs were narrower and more easily movable. Even when using interchangeable jaws, it is occasionally found too narrow for broad teeth, and too broad for narrow ones. 
(E) PREMATURE WEAR OF THE TEETH.

Defective resisting power in the teeth and the consumption of hard food sometimes produce premature wear, so that the animal becomes

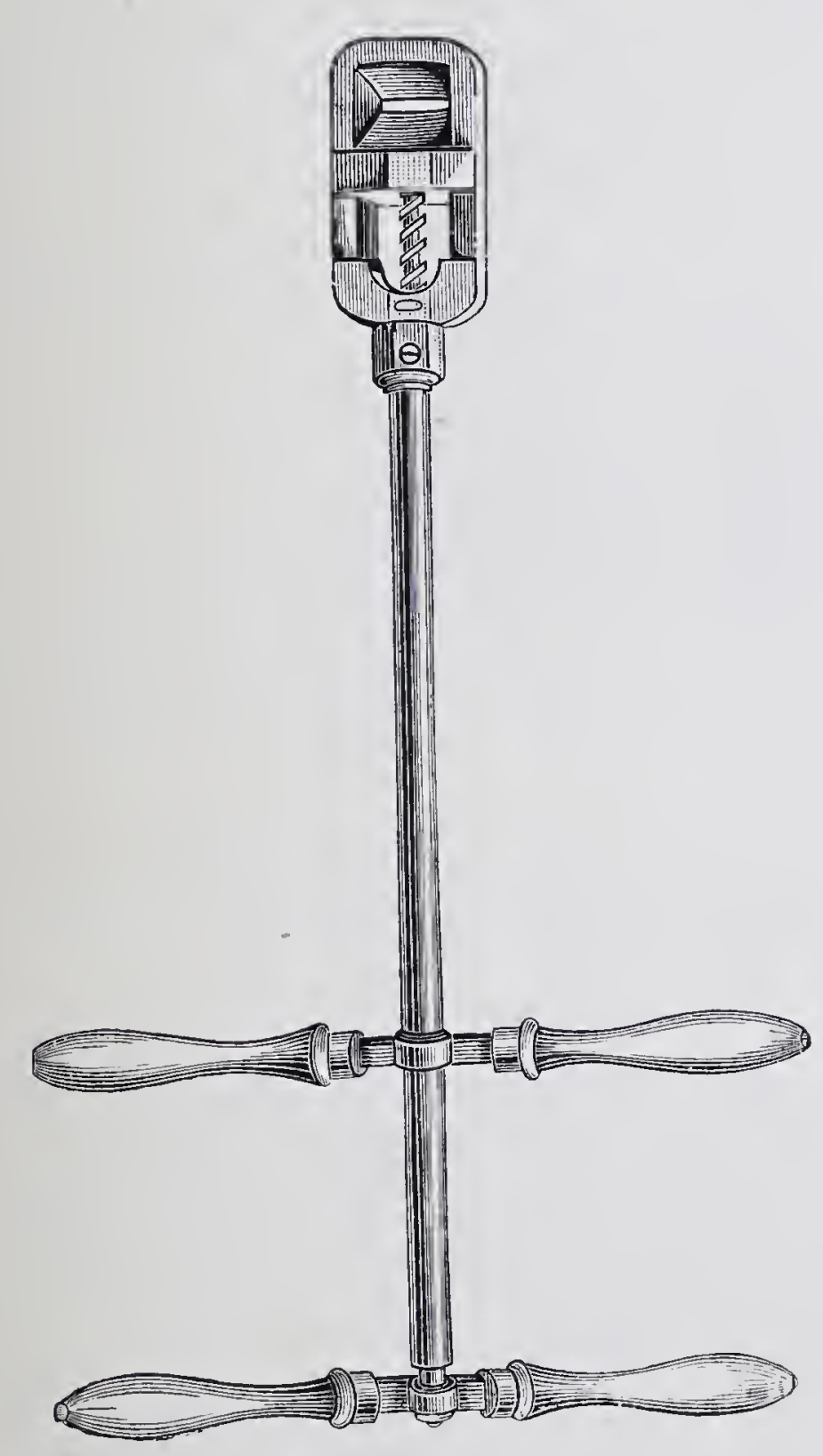

Fig. 15.-Tooth screw.

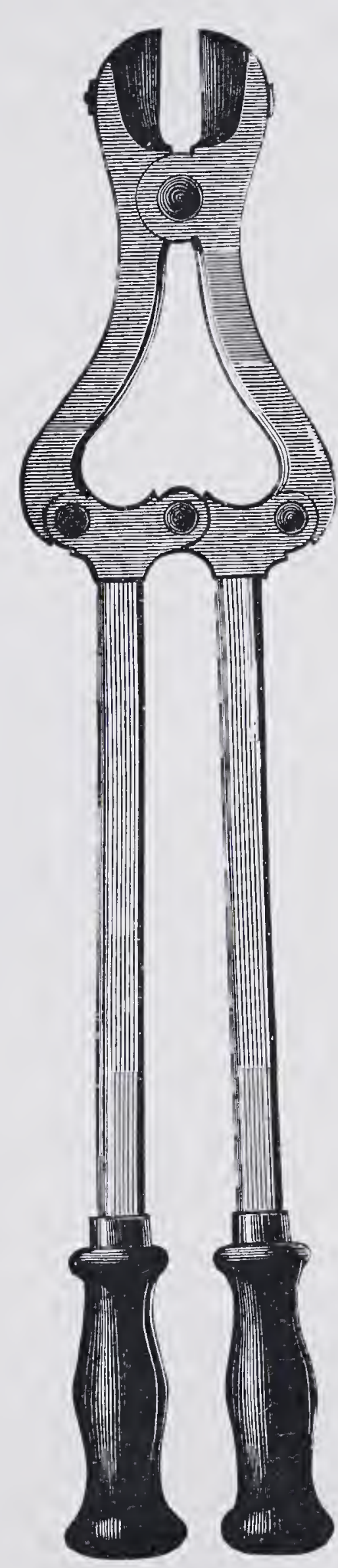

Fig. 16.-Metznik's improved Tooth Shears with interchangeable jaws.

unable to grind its food properly. This condition is met with in herbivora as well as in carnivora. Dralle found all the molars in a twelve-year old horse so much worn and so loose, that they could be partially withdrawn from their sockets with the fingers. Pallin wrongly 
described this condition in a thirty-year old horse as periodontitis. Only exceptionally in middle-aged horses are the molars worn down to the root and mastication impeded. Cases occurring in early life are usually serious. They clearly result from individual idiosyncrasy, consisting in defective hardness of the enamel and dentine. The only useful treatment consists in giving soft and crushed food to assist mastication and prolong the worliable period.

\section{(F) THE SHOOTH IOUTH.}

Enamel and dentine being of unequal hardness, the latter wears away more rapidly and produces the roughened fold-like appearance on the grinding surface of the molar, requisite for effectively triturating the food. But when enamel and dentine wear at the same rate the surface becomes even and polished. In advanced age this normally occurs, because in the deeper portions of the tooth the enamel ceases. It also occasionally occurs in young animals on account of insufficient hardness and durability of the enamel, and is more serious than in older horses. The smooth mouth during mastication resembles an uncut millstone during grinding. Mastication is of course less impeded when single teeth are affected than when the condition is general.

Treatment is confined to palliatives, giving crushed or ground grain, gruel, or bran mashes, and allowing longer time for feeding.

The opposite condition in horses is termed "ruminant's mouth." The grinding faces become exceedingly uneven. This is normal, however, so long as the molar's have not come into wear ; but it rarely persists, and is seldom troublesome.

\section{(C) DISEASES OF THE TOOTH PROPER.}

\section{(a) Dental Caries (CARIES Dentium).}

The term "Caries" is used to describe the process which results in the gradual destruction of the cement and dentine of the teeth, the enamel remaining comparatively intact. In veterinary surgery this term is often erroneously used to describe alveolar periostitis. At one time Möller was doubtful whether caries occurred in animals, but Kitt's observations and his own later experience show that this opinion was not strictly correct, and he has lately reported some cases of undoubted caries in the molars of horses. Kitt has pointed out that in animals it takes the form of dry chronic caries, and Baume has noted the same fact in connection with ruminants and dogs. He states that the process starts either in the cement of the enamel folds, or in the centre of an "island" of dentine. Stockfleth and. Kitt believe that caries starts in the remains of the 
osteo-cement pulp, that is, in the tissue, which in the embryo forms the cement. This material, or at least the space left by its contraction, is seen when a tooth is cut through with the shears. It begins just below the grinding surface, and reaches downwards as far as the involution of the enamel. Sometimes it is even visible on the grinding surface, and food then penetrates, filling the cavity with a black powder. This decomposes, micro-organisms develop in it, and the surrounding cement and dentine become carious; a hole thus gradually burrows in the tooth, and increases in size (fig. 17). The process, therefore, usually starts at the wearing surface, and, having destroyed the crown, extends to the base of the enamel cavity, and even to the pulp. The pulp cavity may

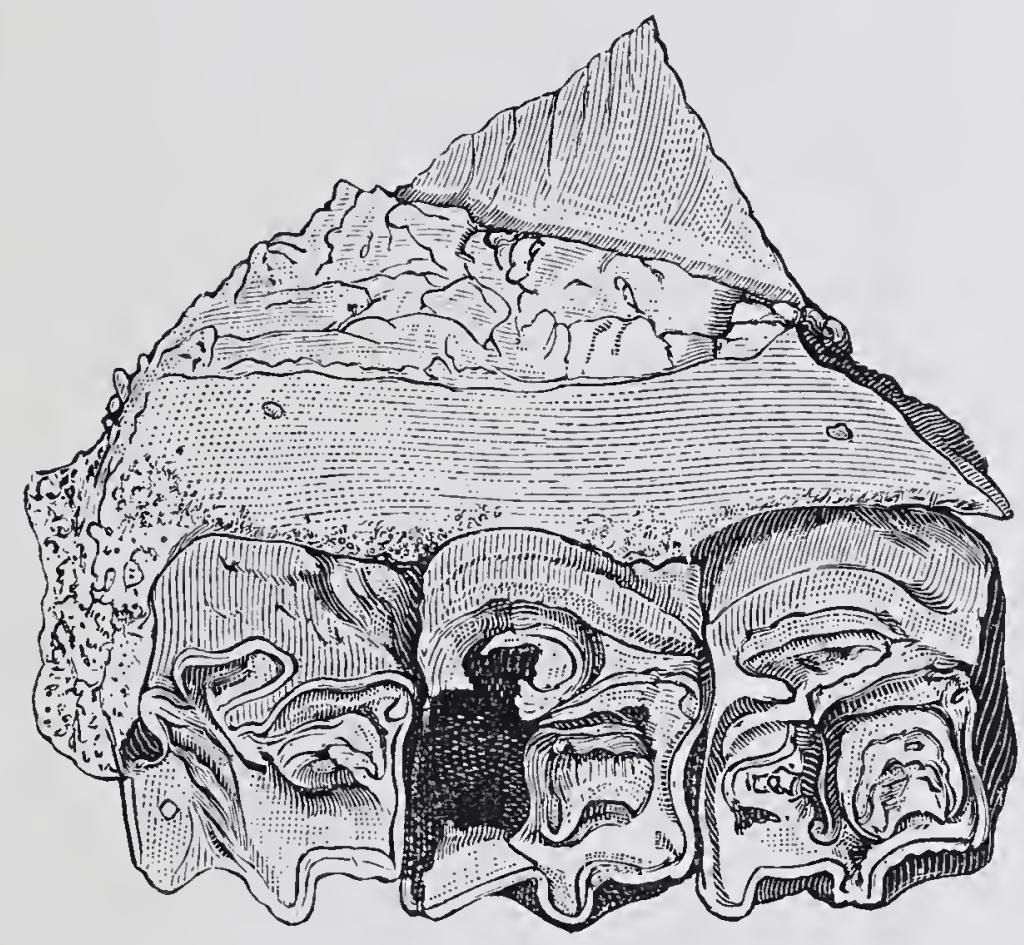

FIG. 17.-Central dry caries of a horse's upper molar (Kitt).

then be crammed with particles of food, and purulent alveolar periostitis result, with its attendant symptoms. The tooth itself takes a darker colour, and becomes loose. It may then split lengthways during mastication (spontaneous fracture), or pieces may break off.

Symptoms. The disease is seldom recognised at first, the only sign being a scarcely appreciable darkening, generally on the wearing surface. At this point a cavity forms which gradually increases in size (fig. 17), its walls, formed of the remains of the dentine, being of a black-brown colour. This stage is rarely noticed, and the disease only receives attention when the tooth has split and mastication become difficult, or when alveolar periostitis has set in. On attempting to extract the tooth it is found to be soft and fragile.

The process may continue for long periods, and finally lead to more or less complete destruction of the affected tooth, though the production of alveolar periostitis or splintering usually necessitates extraction. When removed, one or more deep cavities are found in the dentine. Stockfleth says the disease occurs even at six or seven years of age, 
though it is commoner at nine or ten; but in older horses it is less frequent.

Causes. In man, caries is caused by decomposition of the fluids of the mouth, or of food remaining between the teeth. This process, without doubt, is assisted by micro-organisms, perhaps by the Leptothrix buccalis, and may extend to neighbouring teeth. In animals the disease probably depends on similar causes, but it is exceedingly rare. It is not known whether this rarity is due to the more rapid wear of the teeth in animals, or to some peculiar property of the saliva. Stockfleth considers that a predisposition to caries is often inherited.

Prognosis depends entirely on the extent of the caries. In man its extension can be checked by "stopping" the tooth. With this object the cavity is drilled, cleared of all carious matter, thoroughly disinfected, and filled with some material which will protect the still healthy parts from further attack. The "filling" consists either of metal, like gold or amalgam, or of some other hard substance, such as cement. Very rarely, however, can this be done in animals, least of all in horses. It is, therefore, impossible to save a tooth when once attacked, the more so as the condition is usually only noted when disease is extensive. It then becomes a question whether the animal is able to masticate sufficiently with the teeth that will remain after those which are diseased have been extracted. In the horse, caries seldom affects a number of teeth, hence this question is usually determined in the affirmative. Varnell states having used a stopping of gutta-percha, and Wulff a resin composition. The details of both cases are, however, so meagre that it is impossible to say whether the condition was really one of caries. Possibly the stoppings served to replace lost teeth. Stoppings can certainly be used for this purpose.

Treatment consists in early extraction. Care, however, is required in using the forceps, as the softened crown is liable to break off. The "Universal" forceps (fig. 26) must not be screwed up too tightly, and if the tooth be loose particular care is necessary. Extraction is easiest where purulent alveolar periostitis exists, or where the tooth is splintered. In such case Günther's pointed forceps are sometimes sufficient. Where the crown is broken but the tooth remains firm it can only be removed by punching.

\section{(B) Fissures aNd CAVITIES IN THE TEETH.}

Fissuring is commonest in the horse's molars. It is rarer in oxen, not because these animals less frequently pick up stones, nails or similar hard bodies - the contrary is the case-but because they swallow them directly, without attempts at mastication, and because such objects are then retained in one or another of the stomachs. The fourth molar, which stands in the centre of the curved row, and suffers most 
under the severe friction of one grinding surface on the other, is most frequently affected. Without doubt want of hardness, which is often produced by caries, greatly favours splitting, while other causes may lower the resistance of the tooth. Such conditions may possibly originate during foetal life, a view which receives confirmation from corresponding teeth on either side being often splintered. Hard substances accidentally present in the food also produce splitting, especially in animals which masticate energetically. Sometimes only a piece of the crown breaks off, but very frequently the crack extends as far as the root. The tooth may be broken into several pieces, which separate, injure the tongue or cheek, and make chewing painful. While fissuring is confined to the crown no serious consequences ensue; but should the fracture extend to the alveolus, particles of food and fluids may enter and inflame the alveolar periosteum.

Treatment consists in removing the splin tered tooth, and is seldom difficult. Sometimes the pieces are too small to be grasped by ordinary forceps, and in such cases Günther's pointed forceps are useful. (Fig. 23.)

(c) Dental tartar (CREMor Dentium).

Alike in animals and men the fluids of the mouth deposit on the teeth a grey-brown coating called tartar. It is commonest in dogs and vegetable feeders, but very rare in cats. Pet dogs suffer oftener than others. According to Fürstenberg's investigations tartar in dogs and horses consists principally of phosphate of calcium, carbonate of calcium, carbonate of magnesium, and organic substances. Iron and
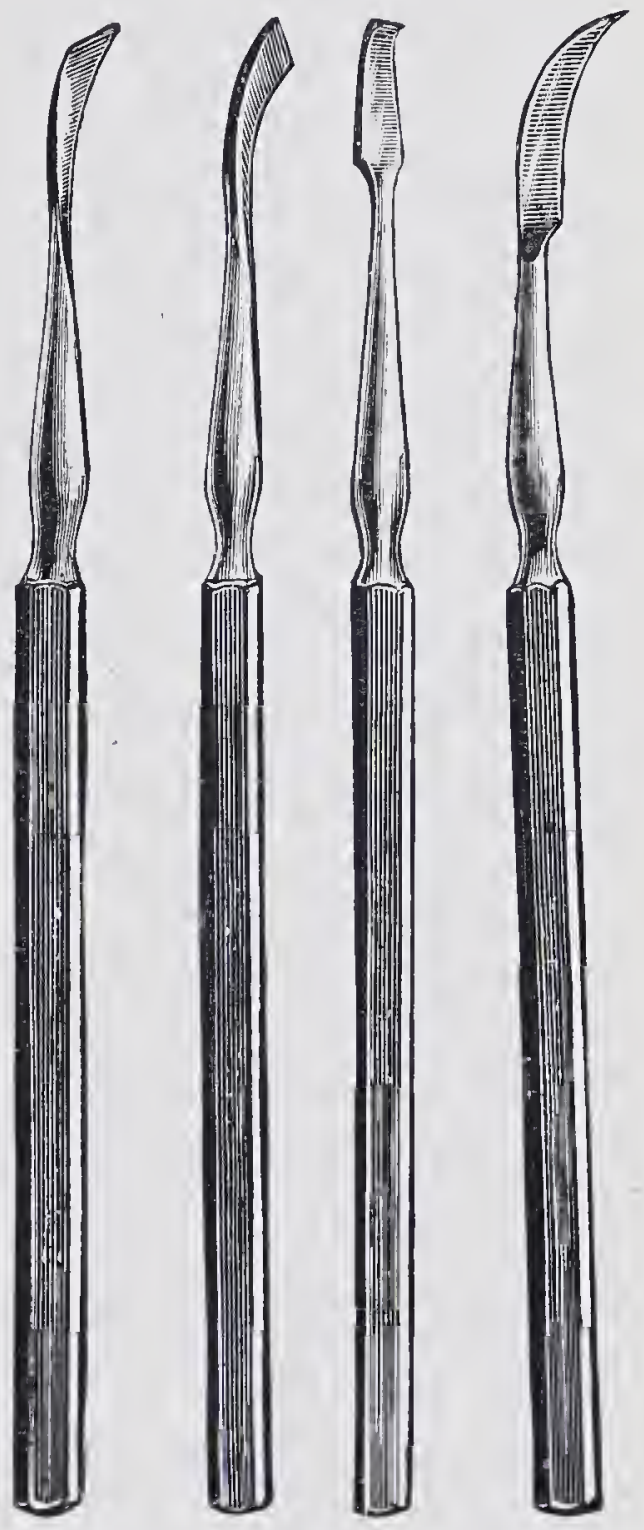

Fig. 18. - Teeth scalers for dogs. manganese have also been detected. Microscopical examination shows that the material is formed chiefly of vegetable particles, various bacteria, epithelium from the cavity of the mouth, and several salts of calcium held together by salivary mucus. The deposit is oftenest seen on the outer surface of the crown, turned towards the cheek. It commences in the neighbourhood of the gums, and gradually spreads upwards and downwards. Incisors and molars are alike affected, the latter more frequently, but in horses the tushes are the commonest seat.

A pale yellow or greenish brown chalk-like substance, rather rough on the surface, covers that portion of the crown lying nearest the gum. In 
the dog it stands out clearly on the shining white surface of the tooth. Hertwig states that in goats tartar sometimes appears black and metallic from admixture of oxide of iron.

The deposit spreading may completely encapsule the crown above; while, as it increases below, it produces atrophy and necrosis of the gum. Here lies the chief danger. As soon as the alveolus is laid bare, it becomes inflamed by the fluids of the mouth. This is by far the commonest cause of alveolar periostitis in dogs, and is especially frequent in house dogs.

The food decomposes in the alveolus, producing a grey slimy material which can be partly squeezed out by pressure, and has a peculiarly penetrating odour. The gum is bluish-red and swollen, bleeds easily, and is sometimes studded with little abscesses. 'The disease has hence been named "scurvy," whilst, on account of the offensive smell, it is also known as "mouth rot."

The black coloration, produced by feeding on husks and brewers' grains, must not be confounded with tartar. Its exact nature is not yet known, but it affects only the surface of the crown. The significance of tartar lies in the possibility of its exciting alveolar disease. Provided the alveolus is healthy, recovery follows removal of the deposit; but teeth already much loosened can only be extracted. The smell is often so offensive that the dog cannot be tolerated in the house.

Treatment. The deposit is removed with teeth scalers or similar instruments (fig. 18), avoiding injury of the gum or alveolus. To get rid of the incrustation, the teeth may be regularly cleansed for some time with pumice. Sporting dogs being the usual patients, these measures can be carried out thoroughly. The gum is protected by placing the instrument close to it, and working towards the top of the tooth. Immediately the tooth becomes loose, extraction is the only resource.

\section{(D) DISEASES OF THE ALVEOLI.}

\section{(A) ALVEolar PERIOSTITIS (PERIOSTITIS ALVEOLARIS).}

This is by far the commonest dental disease of herbivora and carnivora, and especially of dogs. As its development, appearance, and causes vary considerably in different classes of animals, it is advisable to study it separately in each class.

\section{(a) Inflammation of the Alveolar Periosteum in Herbivora.}

The roots of the teeth are fixed in the alveoli by the alveolar periosteum or alveolar dental membrane. 'The outer layer of the periosteum 
lines the alveolus, the inner layer the root of the teeth. At the point where the periosteum becomes continuous with the outer surface of the jaw-bone, the gum is attached, and its function is to prevent foreign bodies like food entering the alveolus.

Depending on their character, two forms of alveolar periostitis may be distinguished. (1) Chronic ossifying, and (2) purulent alveolar periostitis. Those forms of alveolar disease resulting from local invasions of actinomyces or from tumour formation are described elsewhere. The chronic ossifying form is characterised by the formation of exostoses at the root of the tooth, which appears either roughened, or covered with flat, bony deposits, or with knobby masses of new bone; the latter rendering extraction of the tooth impossible. In the purulent form the periosteum is usually thickened and extremely vascular; at points it is covered with granulations, and separated from the wall of the alveolus or root of the tooth by a quantity of grey-coloured, offensive bone pus. Opposite these points the bone or root of the tooth is often eroded to the extent of one-sixteenth of an inch or more. In occasional cases, one sees extensive bone necrosis followed by suppurative osteomyelitis of the lower jaw. The periosteum usually becomes diseased in spots or on one side of the tooth, but in very old-standing cases disease may be so extensive that the tooth lies loosely in the alveolus and can be removed with the fingers. Frick has seen some cases where only a few platelike fragments, the remains of the enamel, filled the alveolus. In all old-standing cases the smell is very offensive.

The above two conditions are often associated, so that at one point the tooth shows discrete exostoses and at another limited areas of purulent alveolar periostitis.

Causes. Alveolar periostitis almost always results from injury to the gum, or its separation from the tooth. Fissures of the tooth reaching to the root, and laying open the root-canal, occasionally produce it. Portions of hard food, especially hard chaff, may easily become insinuated between the tooth and the gum, and remain fast, movements of the tongue and cheeks not sufficing to remove them. Purulent periostitis, with separation of the periosteum from the tooth, supervenes. Masses of food penetrate into this enlarged space, widening the division between the alveolar wall and the tooth, and increasing inflammatory action. Separated from the periosteum, the tooth loses its firm seat in the alveolus, and, yielding to the pressure of its neighbour, is diverted from the common line, and may be so displaced that it can be removed with the fingers. Its root appears completely denuded of periosteum, and bathed in pus. Similar results are produced by the entrance of food between the teeth, as happens especially when the crowns are not in close contact.

In dogs and horses, alveolar periostitis often results from epulis, the 
tumour, either a sarcoma or carcinoma, having loosened the tooth and allowed food to enter.

The disease develops more rapidly when the tooth has been fissured, allowing food or fluid to enter the alveolus, and when compound fractures of the jaw extend to the alveolus. Less frequently the malady arises from the root canal. During wear of the tooth the canal is progressively closed with cement substance. But should wear outstrip this protective process, the root canal and pulp are laid bare on the grinding surface; food or decomposing buccal secretions enter, and occasion purulent inflammation of the pulp (pulpitis purulenta). This may extend downwards to the base of the alveolus, reach the alveolar periosteum, and finally produce purulent periostitis. In the lower molar of a five-year-old horse Frick found the pulp cavity occupied throughout its length of 4 inches by a fragment of straw, which had set up alveolar periostitis, and eventually dental fistula.

Kitt states that in the molars of old horses the central invaginated portion of enamel often becomes worn away, and the pulp chamber exposed. Food then enters and produces pulpitis, which extends to the alveolar periosteum.

Complicated fractures, or even external injuries, of the jaw may occasionally cause alveolar periostitis.

This is commonest in young horses, in which the roots of both the upper and lower molars are at points only covered by periosteum and skin. In such cases injuries may directly affect the root itself or the alveolar periosteum, but the inflammation usually remains localised. A predisposition to alveolar periostitis is observed in various forms of faulty mouth, especially in the shear mouth, and where the teeth are far separated. In consequence of their slighter make and lesser strength, the lower molars are oftener diseased than the upper. The third and fourth molars are most commonly affected, which Günther ascribes to their central position in the fan-shaped arrangement (fig. 27), and to their consequent exposure to powerful compression, both from in front and from behind.

Alveolar periostitis of the incisor's is very rare in horses and ruminants, is generally of a secondary nature, and is due to injury of the interdental space or compound fracture of the alveolar process.

Though single teeth are often affected, it is common for several to become diseased together, frequently corresponding teeth, like the two third lower molars. The cause of this symmetrical occurrence is not quite clear, though it may be due to abnormalities during development. Where several neighbouring alveoli are diseased, and especially where the teeth are very loose, a suspicion of some new growth is always justified. 
Many cases remain confined to the alveolus. When an upper molar is completely loosened it may fall out; lower molars are not so readily displaced, though they may be equally loose; for this reason, the loose molars which can be extracted with the hand are usually found in the lower jaw. Sometimes inflammation seizes on the thin plate of bone which forms the alveolus and extends to the medulla. Purulent osteomyelitis thus results. This irritates the periosteum of the outer surface of the bone, and produces periostitis ossificans, which causes the bone to swell. Finally the pus breaks through the bone, and a dental fistula results. After discharge of the pus, granulations form, and the point of perforation heals up, until only a narrow channel remains communicating with the root of the tooth. This persists (compare "dental

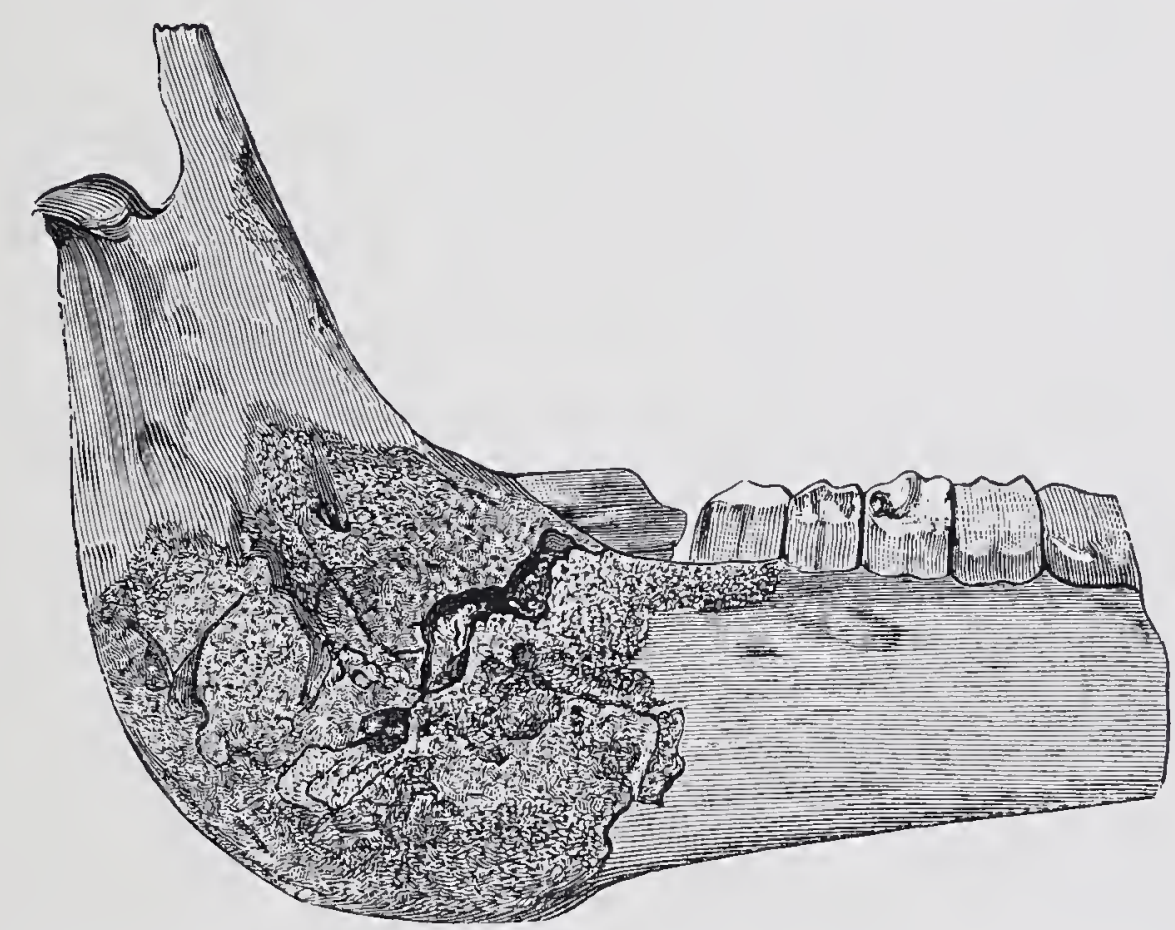

FIG. 19. - Necrosis of a dental alveolus, with dislocation of the last molar and extensive periostitis of the lower jaw (horse).

fistula"). In disease of the upper molars, the pus sometimes breaks into the superior maxillary sinus. Perforation into the nasal cavity may follow disease of the pre-molars and produce a chronic nasal discharge. Occasionally the alveolar wall becomes necrotic, and extensive periostitis ensues. Fig. 19 shows how much a tooth may be displaced. The last molar lay horizontally, and under it a piece of bone the size of a hen's egg had become necrotic.

The symptoms consist in slow, interrupted, one-sided mastication, rolling of the tongue, slobbering, and dropping food from the mouth. Even from the outside it may be discovered that one or other of the teeth is displaced; while the buccal pouches are distended with masses of food. On passing the hand into the mouth, the exact displacement is ascertained. The peculiar, repulsive so-called "carious" smell, especially noticeable in the retained masses of food, is quite characteristic of alveolar periostitis. In dogs the smell, whilst very offensive, is somewhat different to 
that in horses. Closer examination shows that the gum has receded from the affected tooth, which is so loose as sometimes to be movable by the fingers. It is usually pushed out of line or lies deeper than the others. As might be expected from the direction of their wearing surfaces, the upper molars are generally displaced outwards, the lower inwards.

In cattle, epileptiform seizures occur. In chronic cases the animal wastes. In the lower jaw the bone sometimes swells and fistulæ form, indicating the position of the diseased tooth. If the interior of the mouth be illuminated by a mirror, changes in the teeth as well as in the gums may usually be very plainly seen.

Treatment. Extraction is the only certain method of dealing with alveolar periostitis. This is comparatively easy in old subjects, whether horses or oxen, but may be more difficult in younger animals, especially when the crown of the tooth is broken, as not seldom happens. Several teeth may be simultaneously diseased, or one soon after another, and under such conditions the prognosis is less favourable. It may then be a question whether the animal, with its still remaining teeth, can chew sufficient food, and vegetable feeders frequently cannot do so.

The only available cure for alveolar periostitis in the larger animals lies in extraction of the faulty tooth. Operating on man and small animals requires considerable strength, and a vastly greater degree is needed in herbivora, and especially in horses. The two Günthers, Robertson, and Gowing have all rendered their profession valuable service in constructing necessary instruments for operations on the teeth of horses, and laying down general principles for their use. Punching out the diseased tooth, as adopted in former times, is now employed only where the forceps cannot be used, owing to the loss of the crown. Where the anterior molars of the upper jaw are diseased, it is still employed. The operator commences by trephining the jawbone as nearly opposite the root of the affected tooth as possible. The success of the operation depends on the choice of the proper spot. In the upper jaw the landmark is the lower end of the zygomatic ridge of the superior maxillary bone, which lies at the division between the third and fourth molars. Alterations in the diseased tooth or its gum may usually be detected by introducing the hand into the mouth. Should the tooth be displaced and loose, there need be no doubt as to its condition. A round punch about one half to three quarters of an inch thick and with a flattened end is now placed on the root of the diseased tooth, care being taken that the punch points in the general direction of the tooth itself. A few strong blows on the punch with a rather heavy hammer drive the tooth from its socket and it falls into the mouth. The pain of the operation makes the use of 
an anæsthetic necessary, and even in deep narcosis some animals react to the blows of the hammer.

Only the pre-molars and the two first molars of the upper jaw can be removed by punching. The last molar having its root in the hindmost portion of the superior maxillary sinus cannot be so reached, nor can the back molars of the lower jaw, lying as they do too far from the lower edge of the bone. Care should be taken in using the punch only to strike the affected tooth, otherwise neighbouring teeth, or even the maxillary bone may be split.

During extraction a mouth gag must be used to allow of the hand being introduced to catch the tooth and prevent its being swallowed. A preferable method consists in only driving the tooth so far downwards as to enable it to be reached from the mouth with forceps, with which extraction is completed. This gives greater security against such an accident. Bouley and Stockfleth, by performing an osophageal operation, succeeded in removing teeth which had been swallowed; but in another case the tooth produced perforation of the cæcum and death. In the upper jaw it is necessary to avoid injuring the seventh nerve, in the lower jaw the fifth. After removing the tooth the socket is plugged with a piece of antiseptic tow or jute, which, if possible, should be renewed daily. In some cases this is indispensable to enable the animal to drink. The opening usually closes in three to six weeks, but sometimes not till later; occasionally a fistula remains. Bad results seldom follow skilful removal, but where the tooth or jaw bone are splintered serious bleeding or pus formation may ensue.

The use of the forceps is much preferable to the above described process. It is simpler and less painful, although requiring some practice, strength, and judgment. Drawing a tooth is much less difficult in old than in young animals, in which the molars, having been little worn, are still long.

In such cases attention must be paid to the following points:-

1. Choice of Forceps.-Those designed by F. and K. Günther, Robertson and Gowing are amongst the best. They must be very strong, so as not to spring much, but not very thick in the jaws, as this makes adjustment difficult. As the horse's molars do not lie parallel to one another, attention must be given to the direction of the pull, and Günther therefore constructed especial forceps for each tooth.

Forceps acting as levers of the first class, and those as levers of the second class, must be distinguished. In the former the jaws are at the end (figs. 20 and 21), in the latter at some distance from it (figs. 24 and 25). As the molars are arranged in a fan shape, the crowns of the pre-molars look backwards, those of the molars forwards; and as it is of the greatest importance that the pull should come in the direction of the axis of the tooth, it is clear that the second class lever forceps are suitable for the pre-molars, the first class lever for the molars. For the upper pre-molars the forceps must be somewhat bent just in front of the jaws, so that in pulling, their legs may not strike against the 
incisors of the lower jaw (fig. 25). Forceps for the upper molars require wider jaws or removable joints. The limbs of the molar forceps must be very long. In using them force has to be exerted in two ways-firstly, to grasp the tooth, and secondly, to remove it. Attempts have therefore been frequently made to assist the operator by special arrangements in the forceps. The simplest addition is an S-shaped hook, but as this must be provided of many different sizes, it is not very practical. Wendenberg fixed to one leg of the forceps an iron chain, with links perforated with holes, so that after placing one leg of the forceps in position these could be pushed over the other limb.

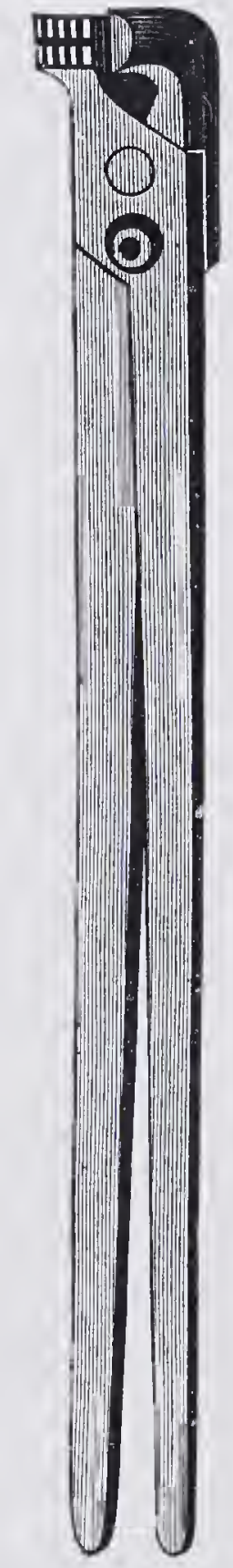

FIG. 20.-Giunther's forceps for upper molars.

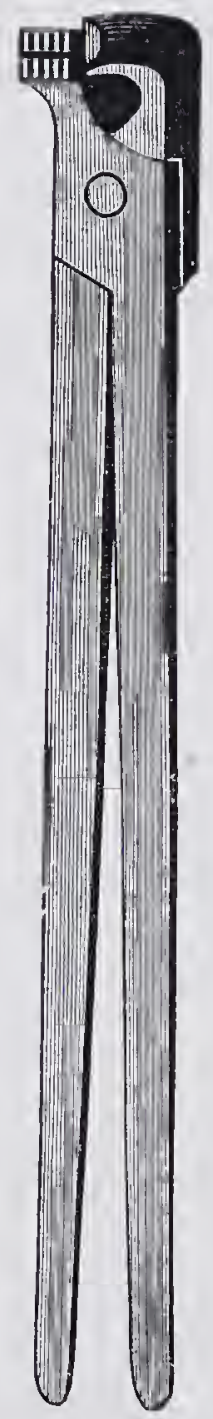

Fig. 21.-Günther's forceps for lower molars.

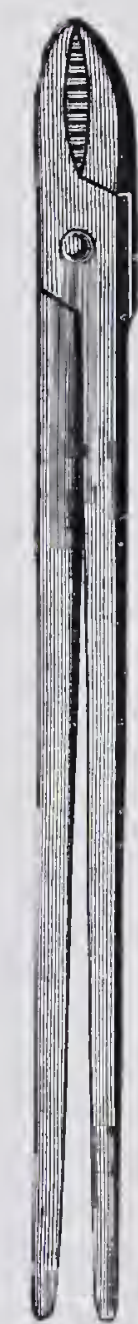

FIG. 22. - Günther's exporteur.

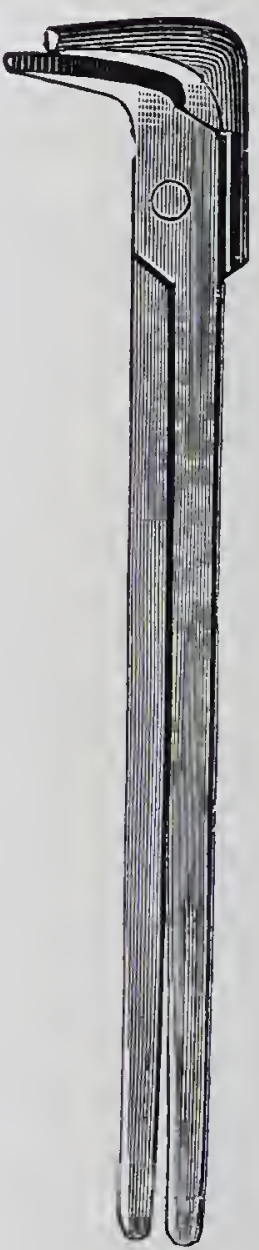

FIG. 23.-Günther's beaked forceps.

Gowing constructed forceps operated by a screw, like Plasse's. These arrangements are, however, too complicated and difficult to handle.

It is to be hoped that some device will yet be discovered to replace or assist the excessive exertion of power required. Up to the present, however, none of the discoveries tending to this object can be described as practical.

Frick and Hauptner have lately constructed forceps in which the power required for gripping the tooth is furnished by a screw. This instrument has the further advantage that it can be used either as a first or second class lever forceps. Fig. 26 shows its construction. The two limbs are turned on their long axis by the screw at the end, and the mouth of the forceps thus closed. 
The use of the forceps shown in figs. 20 and 21 necessitates fulcra or pivots of different thicknesses. In young horses the pre-molars and their crowns are directed backwards, the molars forwards (the second and third more than the first), and these facts must be kept in sight both in applying the forceps and in choosing fulcra. The direction of pull or traction line must follow the longitudinal axis of the tooth. As will be seen from fig. 27, which shows the teeth of a six-year-old horse, the roots of the pre-molars point more or less forwards, whilst those of the molars take an opposite direction. The pre-molars accordingly must be grasped deep down and a thick fulcrum selected. In drawing molars

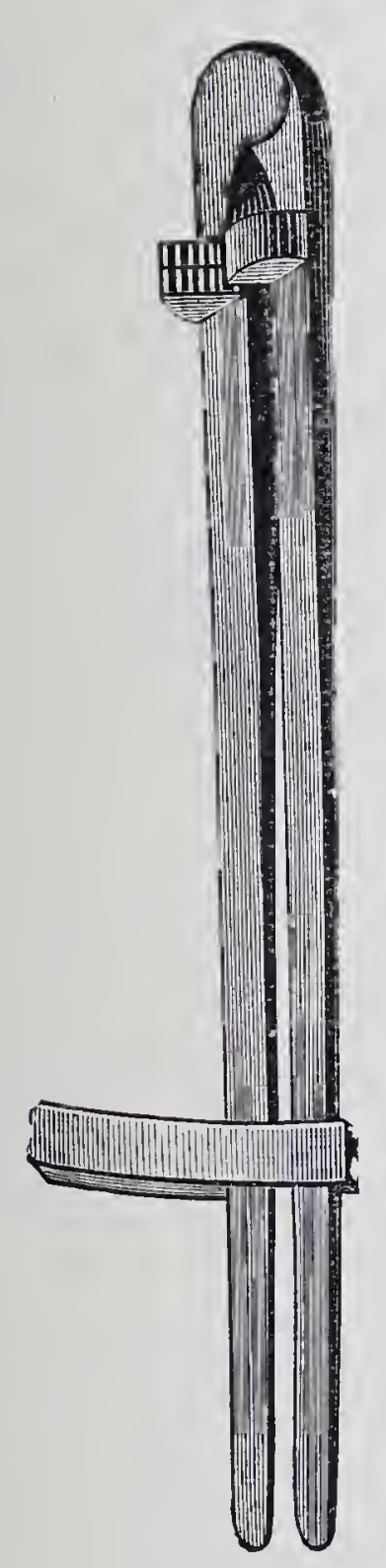

Fig. 24.-Giinther's forceps for first lower molars.

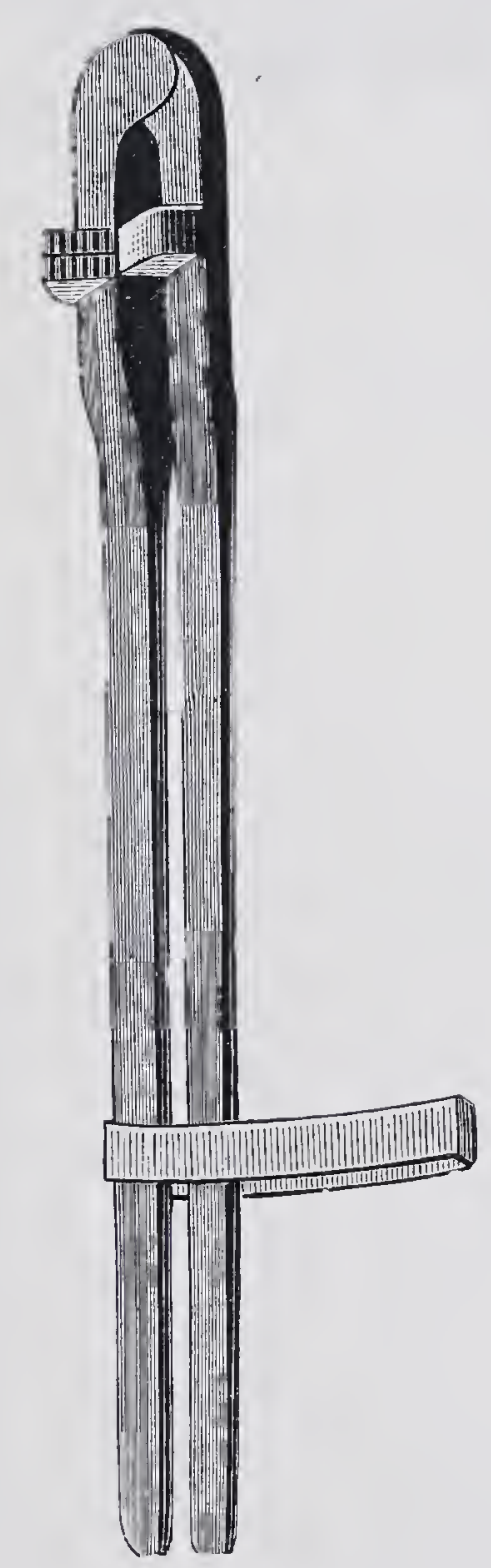

Fig. 25.-Giinther's forceps for first upper molars.
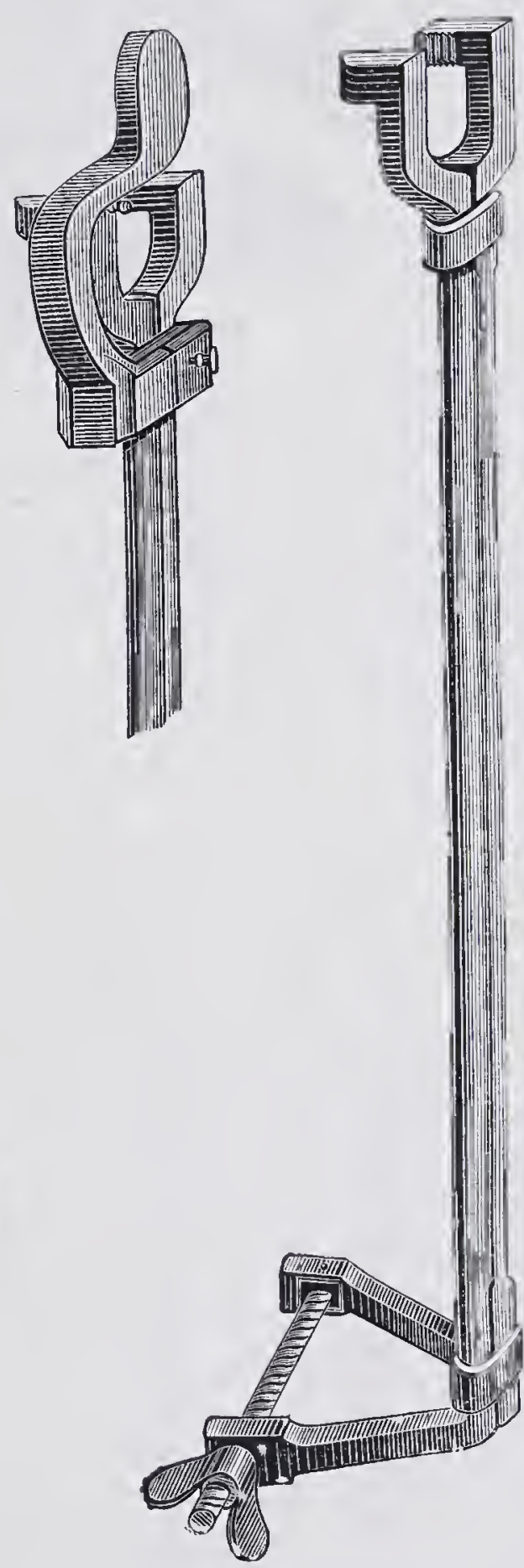

Fig. 26.--Frick and Hauptner's "Universal" forceps.

the practice is reversed; the tooth is gripped just below its upper surface; the pivot should be thin; better still, a pair of second-class lever forceps may be used.

In old and quiet horses the tooth may be drawn standing; younger and fidgety animals are better cast. A halter is put on and a mouth-gag inserted. An assistant sitting on the horse's neck directs its head towards the light, whilst another passes a cord across the upper interdental space, and draws the head upwards and backwards.

The operator having satisfied himself as to the condition of the mouth, and 
provided the proper forceps and fulcrum, performs extraction, which may be divided into the following three stages:-

(a) Adjustment of the forceps. - In carrying this out, the above facts must be

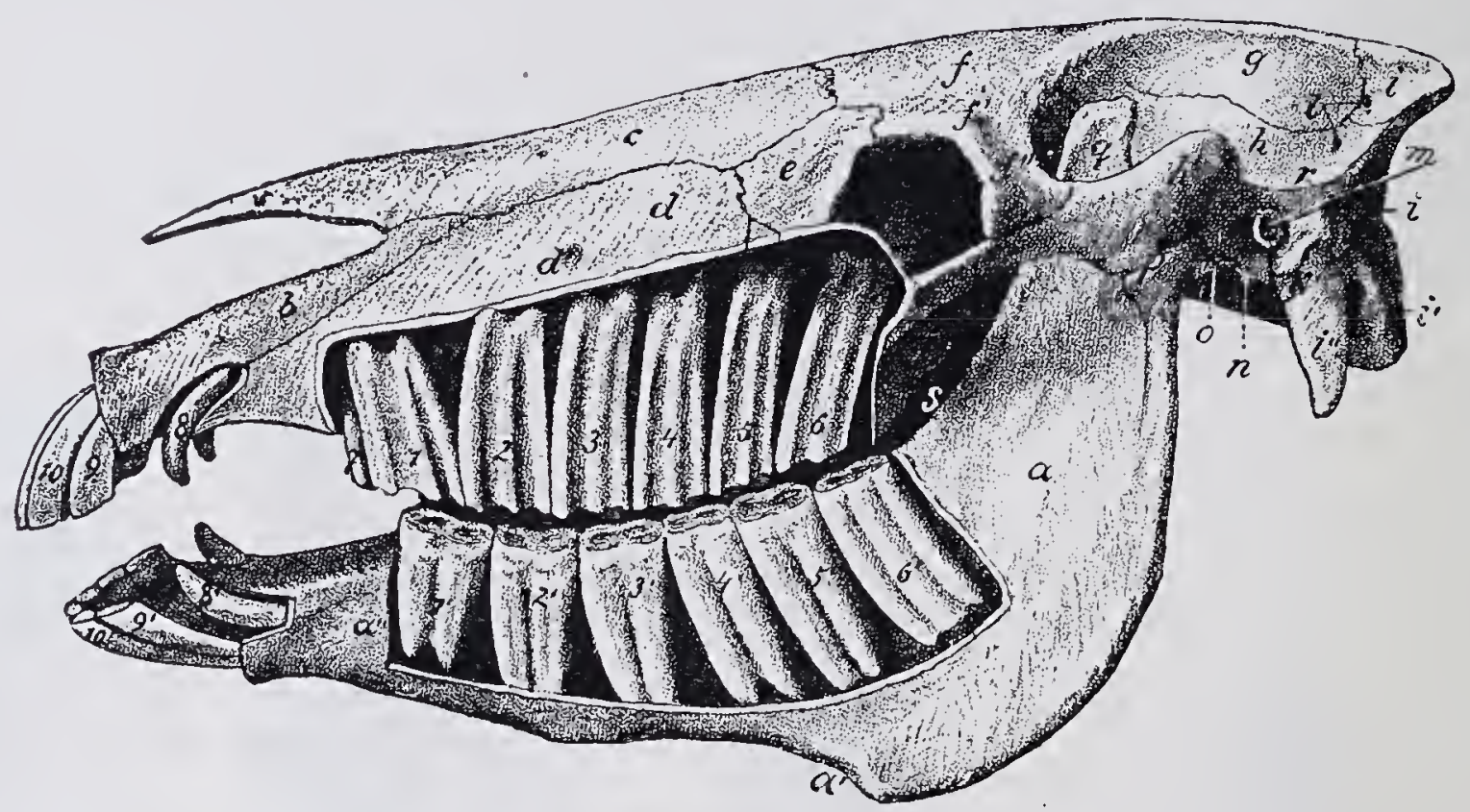

FIG. 27. - Showing the incisor and molar teeth of a six-year-oll horse in position.

(After Ellenberger and Baum.)

remembered. Difficulties often arise at once; sometimes room is wanting between tooth and cheek, either because the jaws of the instrument are too thick,

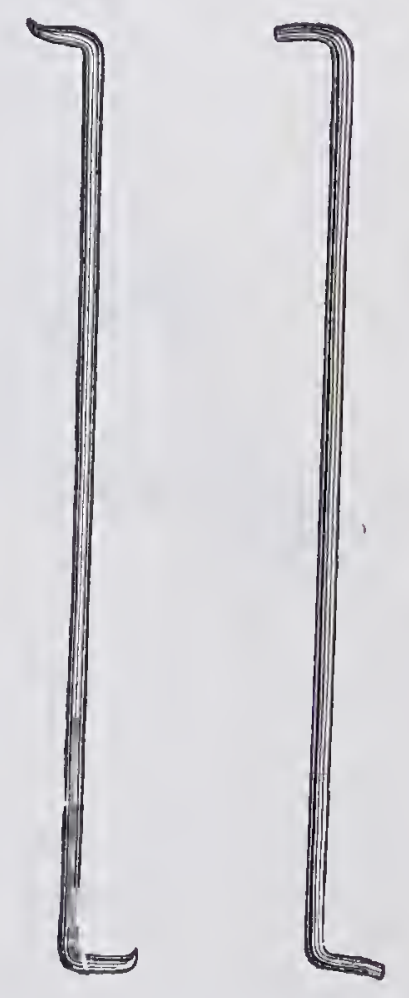

FI*. 28. --Tooth picker's (Giinther).

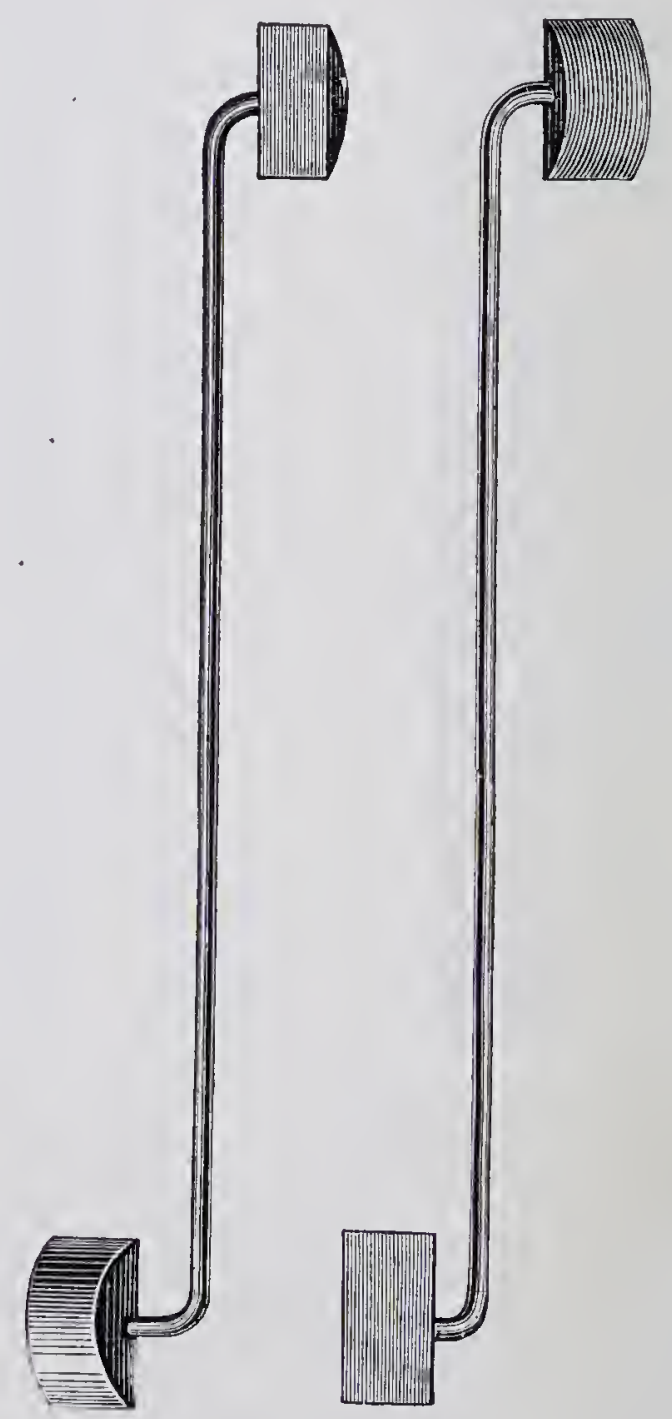

FIG. 29.-Fulcra.

or because food has accumulated; or, finally, because the crown of the tooth has been pushed against the cheek. In the first case another pair of forceps is selected in the second, the food is removed with a toothpick (fig. 28); in the 
last the tooth should be pushed back, if possible; or if this is impracticable, the opening of the gag must be diminished, and the jaw pushed sideways in the direction of the displaced tooth.

Sometimes room is wanting between the upper and lower rows of teeth. Should the size of the forceps be to blame, they should be changed; where the opposite tooth is too long, it must be cut shorter or removed. In placing the forceps in position, care is required not to grasp more than the diseased tooth. In extracting pre-molars, one hand is introduced into the mouth to guide the instrument. The displacement of the tooth often simplifies the determination of its position and the adjustment of the forceps. In using the Frick-Hauptner forceps (fig. 26), the correct position is assured by counting the teeth in front of the jaws of the instrument.

(b) Teeth that are still firm can be loosened by light side movements of the instrument. But to avoid breaking the crown of the tooth or fracturing the jaw, it is needful to proceed slowly and cautiously.

(c) Adjustment and selection of the fulcrum.-When using a first-class lever forceps, as already stated, the selection of the fulcrum must be made with a knowledge of the general direction of the axis of the tooth, to which the fulcrum should be brought as near as possible, in order to give greater leverage.

(d) Now follows the extraction of the tooth. -The operator takes up a firm position, with his elbows on his sides, and giving the word to hold fast, exercises a steady but strong pull on the forceps, throwing on them the weight of the body. The power required to draw a tooth in the prone subject is developed by the muscles of the thigh, the power of the arms not being usually sufficient. The arms, or rather the hands, are employed in grasping the limbs of the forceps, so as to retain hold of the tooth.

Should the attempt be successful, the tooth is felt to yield, and a hissing noise may sometimes be heard, due to the air rushing into the alveolus. In such case the tooth is still firmly held, but the pull is relaxed for an instant. A deeper fulcrum is slipped under the forceps, and a light pull will now generally remove the tooth. As in young animals the teeth are longer, a fresh and deeper hold is often needful. Short teeth can be removed directly with the forceps, but longer ones may require the assistance of the hand or exporteur (fig. 22). The opposite teeth sometimes prevent removal of the loosened molar. In such cases more room must be made, either by opening the gag further, or by closing it and pushing the lower jaw to one side, in order that the two rows of teeth no longer oppose each other. The tongue, however, should not be loosened, for fear of the tooth falling into the mouth and being swallowed.

If, during extraction, the animal makes violent movements with the head, the operation is stopped, but the movements are followed by the forceps. This is especially necessary when operating on horses standing. If the forceps are held rigidly, the jaw may easily be fractured by the animal's own movements.

If the forceps are not firmly held they may slip off the tooth and require to be readjusted. Should this happen repeatedly, and, even after considerable efforts, no loosening of the tooth occur, endeavour must be made to discover the cause of failure, which most frequently results from an improper hold having been taken, the pull being in a wrong direction, or several teeth being grasped. A change of forceps or of pivot is often desirable. It may be repeated, that the direction of pull should correspond with the axis of the tooth, otherwise power is lost by the tooth grasped being forced against its neighbour. In certain cases, like dental fistula, growths, either in the alveolus or on the tooth, render extraction very difficult, or even impossible. 
For extracting molars, a tooth-key, resembling that formerly used in human dentistry, has been recommended. But, unless for removing the short teeth of old horses, this instrument is useless.

The extracted tooth generally shows evidence of pathological processes. Where purulent periostitis has existed, the root is roughened and denuded of periosteum. Other portions of the root exhibit shreds of periosteum, and sometimes exostoses.

Treatment after extraction is seldom necessary, but when cleansing the parts a plug of tow or jute soaked in a disinfectant may be introduced into the tooth socket. It is particularly useful where dental fistulæ have existed, but must be renewed daily.

In drawing several teeth at one time, it is well to first extract the lowest

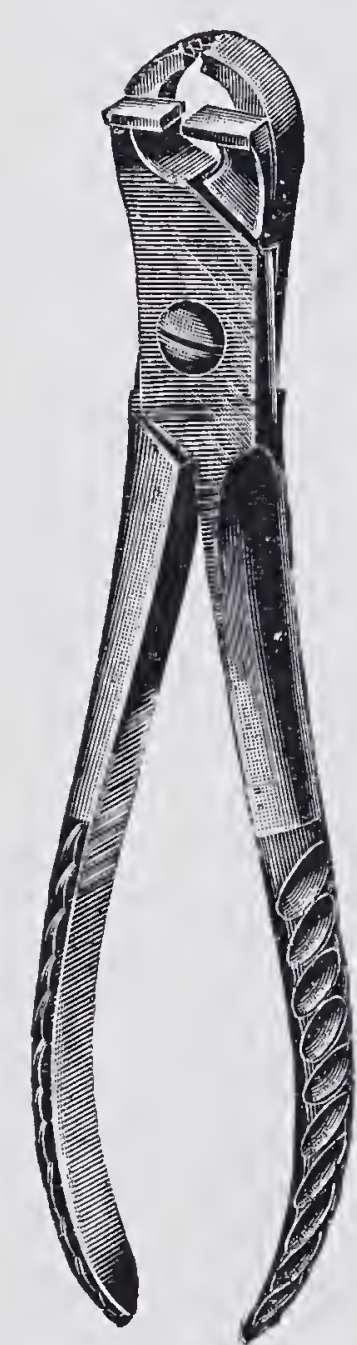

FIG. 30.-Giinther's forceps for the incisors.

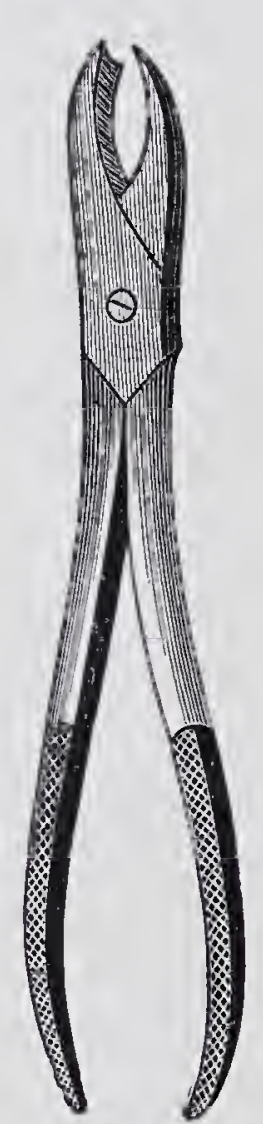

Fig. 31.-Ordinary furceps for the incisors. and most posterior. This minimises the difficulties caused by bleeding. Cases of unusually large teeth have been reported. Degive extracted a horse's tooth which weighed 15 ounces. Teeth are said to have been successfully transplanted, but such reports must be received with caution. South describes having, in 1886, replaced a carious third molar by a similar tooth removed from a dead horse. How long this remained in position is not stated.

In 1887, in the clinique of the Berlin college, the first upper molar of a carthorse was removed by punching, as the tooth crown was broken away. In 1890 the horse was brought back, the owner stating that it had for a long time suffered from an offensive nasal discharge. An examination showed that the left nostril was blocked with masses of food, which had entered by an opening in the now empty alveolus. The passage was large enough to admit the little finger. The upper maxillary sinus proved healthy on trephining. After carefully removing every particle of food from the nostril, and cutting down the overgrown first molar of the lower jaw, the alveolus was thoroughly cleansed and stopped with a mass of gutta-percha, softened in hot water. To give it a more secure hold, a few grooves had previously been filed on the teeth on either side. The nasal discharge ceased after this operation, and several weeks later the gutta-percha was found still in position, and quite fulfilling its object. Mastication appeared in no way disturbed, and the horse lived for several years in the same condition. Voigtländer describes a similar case, though the horse had finally to be killed. Some very interesting cases of this character will be found in Cadiot and Dollar's "Clinical Veterinary

A communication from Paris describes the extraction of an elephant's tusk measuring $1 \frac{1}{2}$ yards in length. This was first sawn off, and the root, which weighed $18 \mathrm{lbs}$. (?), then drawn with forceps. The elephant suffered a good deal, but is said to have completely recovered. (Her. Rep. vol. xiii. p. 178.)

It is seldom needful to extract incisors, though the operation is often done 
by horse-dealers to give horses the appearance of greater age. The operation is called for where milk teeth remain confined between the permanent incisors, or where the inter-maxillary or submaxillary bone is fractured and the alveoli exposed.

Günther's forceps are the best (fig. 30). The projection just in front of the jaws of the instrument serves as a fulcrum, and should be rested on one of the neighbouring teeth. Incisors can also be pulled with human forceps made rather longer and stronger than usual (fig. 31). The same instrument serves as an exporteur.

\section{(b) Inflammation of the Alveolar Periosteum in Carnirora.}

Dogs, especially those kept in the house, are the most frequent sufferers. Compared with that in herbivora, the disease differs both in its advent and progress. A large number of teeth are usually attacked, sometimes nearly all; both molars and incisors suffer, and old dogs may be reduced to an absolutely toothless condition.

Symptoms. The edges of the gums are swollen, more or less reddened, and bleed on the least touch. At points where the tongue cannot reach, as along the external borders, the gums are moist with a grey slimy fluid of a particularly penetrating odour. The crowns of the teeth are partly covered with grey chalky masses of tartar, which intrude under the gum in the direction of the alveolus, loosening the tooth, and causing it to fall out. Where the disease is extensive, the animals salivate freely, eat badly, and either avoid gnawing bones, or whine occasionally during the process, whilst the mouth emits a most offensive smell. Sometimes single teeth become loosened and partly displaced, preventing the animal closing the mouth, and giving rise to suspicion of rabies.

The condition was formerly regarded as systemic, and received such names as scurvy, mouth-rot, \&c. But no fever or other constitutional symptom is present, and recovery takes place as soon as all diseased teeth are removed.

The formation of tartar, or the extension of caries, as already stated, is the immediate cause of alveolar periostitis. The tartar is deposited on the teeth, and continually advances towards the gums, producing inflammation and necrosis. The alveolus is finally exposed, putrefactive organisms enter from the mouth and keep up the inflammatory action. The grey stinking material discharged when the tooth is pressed upon consists almost entirely of putrefactive bacteria. A predisposition to this formation of tartar exists in certain small races of dogs, possibly caused by inappropriate feeding.

Preventive treatment calls for the removal of tartar before alveolar disease has appeared. The incrustation can be scraped off in the manner already described. It is advised to paint the diseased spot with 
a 2 to 5 per cent. solution of alum or permanganate of potassium, which is further useful in removing smell. All loose teeth must be removed, otherwise success will never be attained.

In dogs, extraction presents no difficulty: an assistant holds the animal, and human forceps may be used. The tooth is seized with forceps of suitable size, and a rapid drawing movement made, usually towards the tongue. Operating on the right side, torsion is made towards the left, and vice versî. The crown is usually firm, but the root
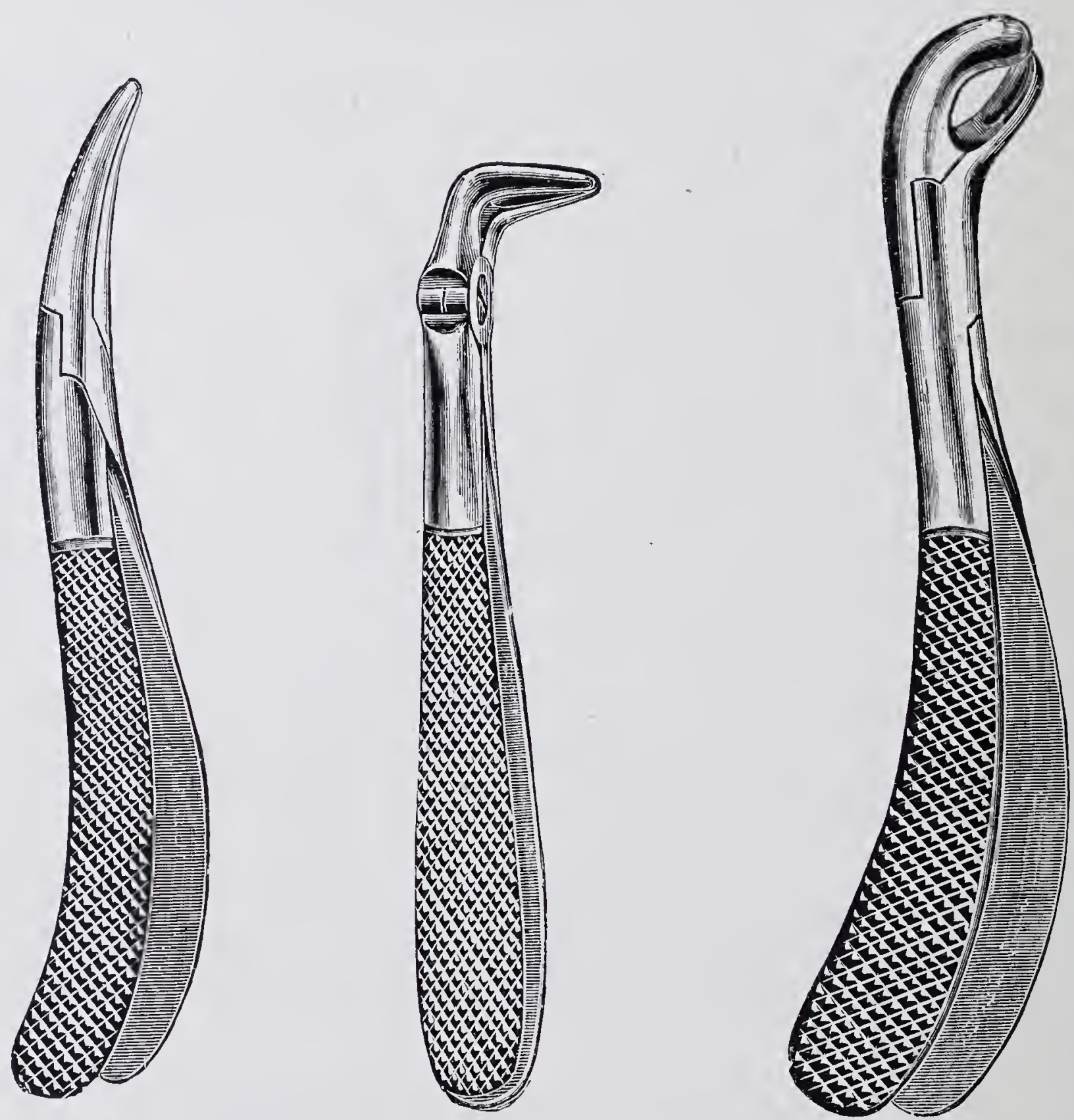

Figs. 32, 33, 34. -Dental forceps for dogs.

loose. The tooth key may be used if thought suitable. Artificial teeth have been used in dogs. Moseley reports having fitted a full set with such success that the dog could afterwards crush bones. A Schipperke wearing a gold plate was exhibited at the Central Veterinary Medical Society. But these achievements are in the nature of scientific amusements.

\section{(B) Dental Fistula.}

This may be defined as a tube-like canal communicating at one end with the external air and at the other with a tooth root or socket. It 
almost always results from alveolar periostitis, the inflarnmatory process extending and producing an osteomyelitis purulenta, which finally leads to perforation externally. Inflammation then abates, until the alveolus alone remains inflamed. The small quantities of pus produced find exit through the communicating sinus.

Dental fistula occurs oftenest in young horses. It affects the first and second pre-molars, seldom the third, and is commoner in the lower than in the upper jaw. Fistula is very rare in the molars, because their roots are covered by thick masses of muscle, and in the upper jaw project into the superior maxillary sinus. Despite these facts, such fistulæ do occur. They open into the superior maxillary sinus or into the nostril, producing empyema of the sinus and chronic nasal discharge, which gives the peculiarly offensive smell of alveolar periostitis.

In dogs, dental fistulæ originate most frequently from the upper molars. The outer opening is usually near the lower eyelid, and might be mistaken for that of a lachrymal fistula, were it not that probing shows it to communicate with a molar tooth. For a description of several cases of dental fistula in the horse, dog and cat, see p. 331 of Cadiot and Doliar's "Clinical Veterinary Medicine and Surgery."

Causes. Fistulæ, though generally preceded by alveolar periostitis, may be produced by complicated fractures or injuries of the maxilla. Hertwig believed pre-molar fistulæ in the lower jaw to be commonly caused by wounds inflicted by narrow and inclined mangers. We can support this view, having

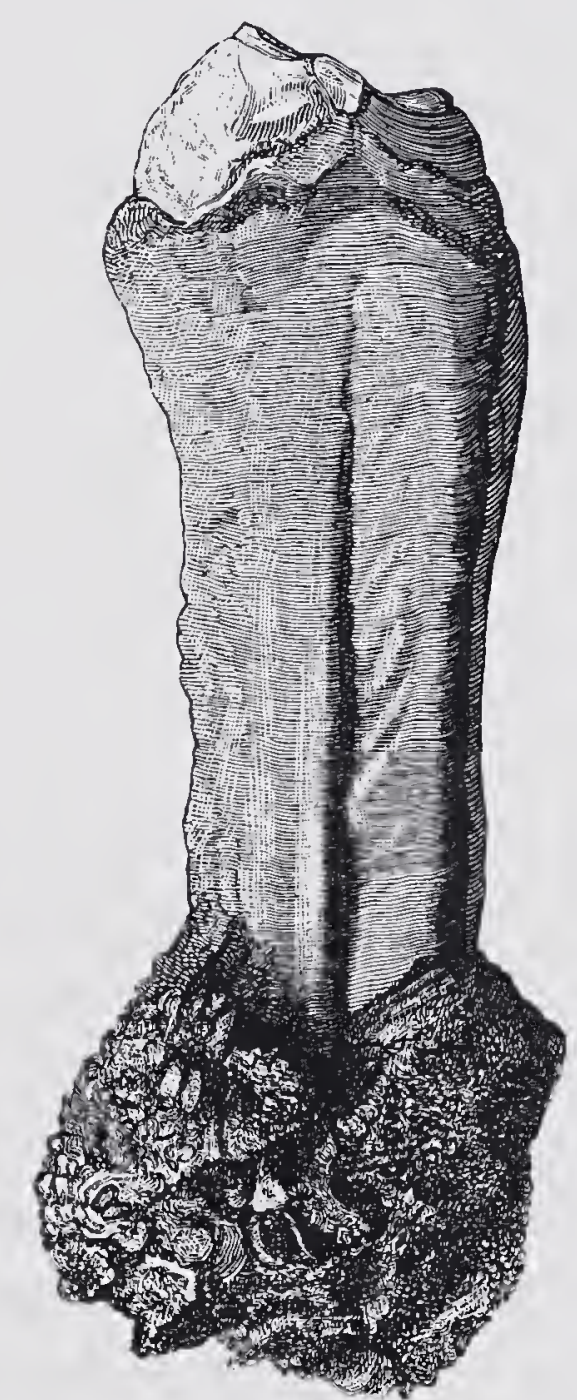

FIG. 35.-Periostitis alveolaris ossificans of the third molar (horse). seen many cases of pre-molar fistula in young animals exhibiting no disease of the alveolus, but traceable to external injury of the posterior border of the lower jaw. The root of the tooth is often thickened, surrounded by actively-growing granulation tissue, which becomes covered with fresh cement; a periostitis alveolaris ossificans results, with formation of hyperostosis radicis (fig. 35), and extraction is rendered difficult, or even impossible.

The symptoms consist in the appearance of a little funnel-shaped depression in the skin, either of the lower margin of the under jaw, or of the upper jaw at the height of the roots of the pre-molars. Sometimes the fistula opens within the mouth near the affected tooth. A fine 
sound, about half a line in thickness, being introduced, meets with a hard substance-tooth, bone, \&c. Round the external opening and over the course of the canal the bone is rarefied and swollen. Mastication is not always impeded. Examination usually betrays signs of alveolar periostitis in the affected tooth.

Prognosis. Removal of the diseased.tooth is essential. Hertwig describes a cure after application of the actual cautery and mopping with tincture of aloes, but such recoveries are exceptional. When the lower pre-molars have become diseased from external injury, recovery sometimes takes place without removal of the tooth. But usually the carious. root continues to promote pus formation, and regenerative processes are unequal to the task of separating the necrotic tissue-a condition obviously very unfavourable to healing. Moreover, in such cases there is generally a difficulty in extracting the tooth. In fistula affecting the upper jaw a radical cure is impossible without removal of the tooth. In prognosis account must be taken not only of the disturbances caused by the diseased tooth, but also of the dangers of extraction. Where inconvenience is slight, it may appear advisable to refrain from treatment. If, however, the fistula opens into the upper maxillary sinus or nostril, the tooth must be removed and the sinus trephined. Where perforation into the nostril is attended with necrosis of the turbinated bones, as not infrequently happens, the prognosis is unfavourable. The nasal discharge continues after extraction of the tooth, and betrays the peculiar smell of bone pus. After a time pieces of the turbinated bones become loose, and are discharged with the nasal fluids.

Where, however, perforation of the nostril and necrosis have not taken place, recovery usually occurs soon after removal of the tooth. But it is unwise to prophesy the termination-time alone can determine. To detect perforation into the nostril, Günther's catheter for the guttural pouch may be employed. On introducing it into the lower meatus, the point where the fistula opens will be felt as a raised and uneven patch.

Treatment may be attempted without sacrifice of the teeth if the fistula has resulted from an external injury, and produces no serious inconvenience, and if the teeth in question give no evidence of disease. Proceeding on general surgical principles, the canal is washed out, its walls scraped with the curette, and dressed with chloride of zinc $(1-10)$, or iodoform dissolved in ether. Solution of lactic acid has been recommended. If the alveolus is diseased the tooth is removed, and little further attention is required. Healing is hastened by inserting a plug of tow, saturated with a disinfectant. This may be renewed daily. In dogs the molar should always be removed, even though the crown appears sound. 


\section{(c) NeOplasis of THE GUMS AND ALVEOLi (EPUlis).}

Under the common title of epulis are grouped all tumours originating in the gum or alveolus. In former times growths due, in the horse to bothryomyces, and in the ox to actinomyces, were included under the general designation "Epulis." The special neoplasm is indicated thus: epulis sarcomatosa, carcinomatosa, \&c. Dammann saw in horses cancerous tumours of considerable size, which sprang from the necks of the incisors. Roloff has described an epulis sarcomatosa in horses and cattle, and we have repeatedly seen such cases both in horses and dogs. The case illustrated (figs. 36 and 37) was a myxo-sarcoma of very rapid growth, affecting a mastiff bitch. Beginning on the left side of the upper jaw, it extended from the first canine tooth for a distance of three inches backwards, involving all the pre-molars and molars, across the mouth to the left side, causing loosening of all the molars of that side; in an upward direction it affected the whole of the superior maxilla on both sides. The lower jaw, especially on the left side, was also involved. Sections revealed cystic spaces filled with blood-stained fluid; the interior of the superior maxilla resembled a piece of hepatised lung and the bones cut like those affected with osteoporosis. (A. E. Mettam, The Veterinarian, 1901, p. 129.)

Diagnosis is not difficult. The new growth may attain such dimensions as to hinder mastication; then the teeth at the affected spot become loose and fall out, while symptoms of alveolar periostitis are present. Where in herbivora several neighbouring teeth are loose, one should always look for epulis formation. Keiper observed after fracture of the lower jaw in a foal a rapidly growing neoplasm, which he regarded as sarcoma. In fourteen days it attained the size of a child's head, and, owing to its position near the incisors, interfered with feeding. As it recurred after removal, the foal was killed.

The prognosis is usually unfavourable. As soon as the new growth spreads beyond the alveolus its complete removal becomes very difficult. From any portions remaining the tumour is apt to grow again.

Treatment consists in complete extirpation, which, owing to the position of the tumour, is difficult. As the operation is very painful, an anæsthetic should be given. The growth may then be dissected out, the last traces being removed with the curette or actual cautery, Paquelin's form being the most useful.

\section{(D) DENTAL TUMOURS.}

True dental tumours, odontomata, have very rarely been described in domesticated animals. Imminger saw an odontosarcoma in a horse, succeeding to sarcoma of the jaw. Many of the "odontomata" recorded 

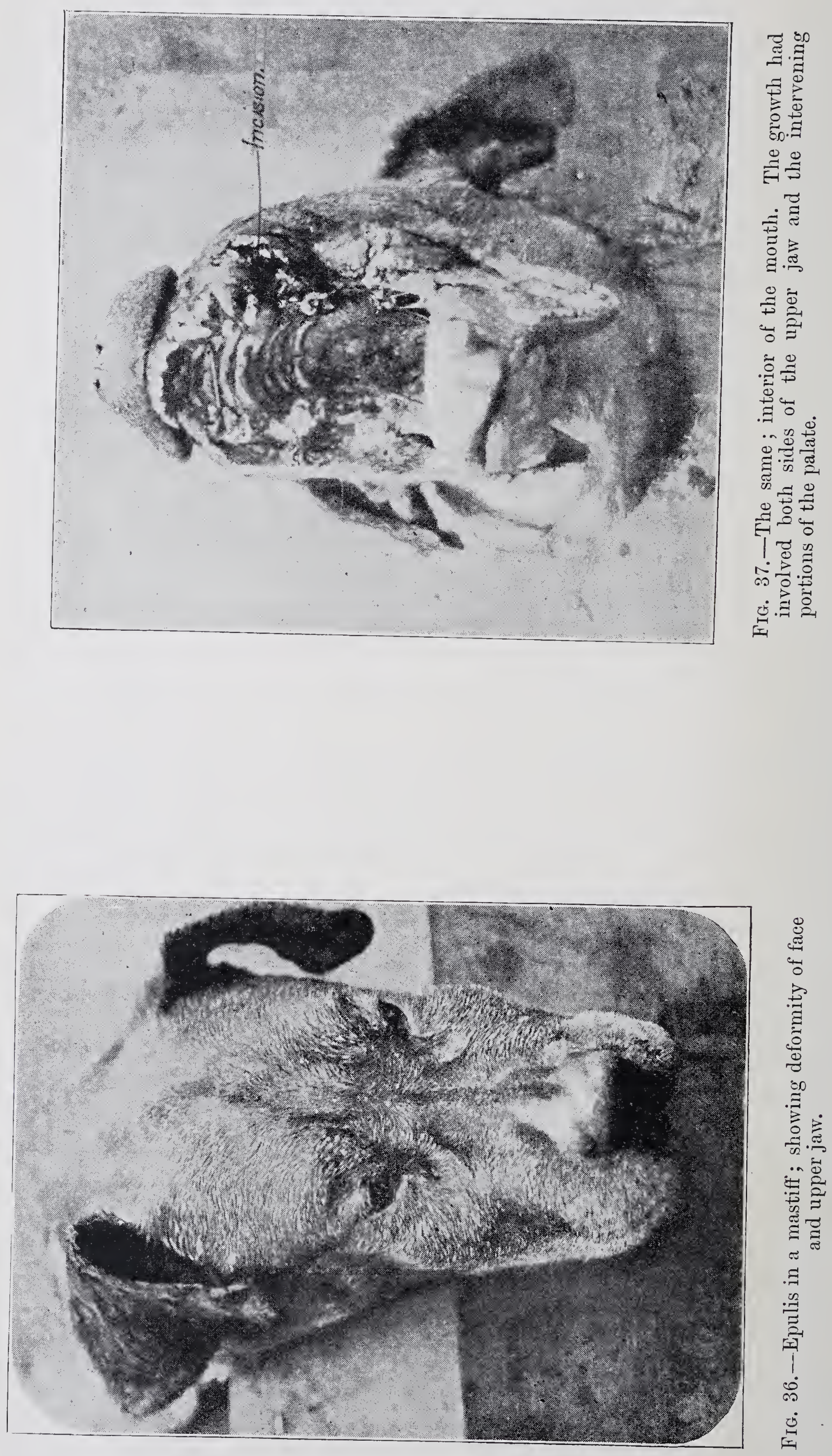
in current literature are only large exostoses developed on teeth in consequence of alveolar periostitis.

Dental cysts, found in the most varying positions in the animal body, are due to aberrant portions of the fotal paradental epithelium and are dealt with elsewhere. Williams, after trephining a horse's right superior maxillary sinus for an apparent tumour growth, found in its interior several hundred rudimentary teeth. The growth had extended into the mouth.

\section{III.-DISEASES OF THE NOSE, NOSTRILS, AND THE SPACES COMMUNICATING WITH THEM.}

\section{(1.) WOUNDS OF THE ALA OF THE NOSTRILS.}

These occur most frequently in horses, which, when rapidly moving the head, may chance to catch the nostrils on such objects as hooks or nails. Sometimes they result from bites from other horses. Some savage tribes slit the false nostril, thinking to assist breathing. Injuries to the nose are less frequent in other animals. Though they generally take the form of torn wounds, they heal steadily and quickly owing to the abundant connective tissue in their neighbourhood. But immediately the cartilaginous portions of the nose are injured, healing becomes difficult. Inflammation of the cartilage is apt to result, causing chronic inflammation, thickening, or even fistula formation. In this way stenosis of the nostrils may be produced and the breathing affected.

Treatment of fresh wounds, especially of the nasal cartilage, should aim at healing by first intention. The parts must be cleansed, the edges of the wounds freshened and disinfected, and brought together with pin sutures, so as to lie firmly in apposition. The wound should then be painted with collodion or wound gelatine, and the horse put on the pillar reins to prevent its rubbing out the stitches. In workinghorses small flaps of slin may simply be cut off. Healing is usually so perfect that neither the area of the nostril, and consequently the breathing, nor even the horse's appearance suffers. Necrosis of the nasal cartilage is serious and may continue for months. The fistulæ should be laid open and the parts frequently dressed with disinfectants, if necessary with chloride of zinc or nitrate of silver or even touched with the actual cautery.

\section{(2.) FRACTURE OF THE NASAL BONES.}

In horses these fractures generally result from the animal running away, and the nose being brought in collision with some hard object. Falls, 
kicks, or powerful blows also produce them. Horses suffering from brain disease occasionally fracture the nasal bones by striking the head against walls. In other animals the accident is rarer. In dogs it is sometimes due to a blow with a stick. The fracture may be single or double-sided, longitudinal or transverse, subcutaneous or compound. Fragments of bone may perforate either the skin or mucous membrane, and thus produce a compound fracture.

Diagnosis is not difficult. There is usually deformity of the nose and the neighbouring parts, bleeding from the nostrils, and sometimes difficulty in breathing, produced by narrowing of the nostril or accumulation of blood. Injuries to the lachrymal duct are indicated by the appearance of blood-stained tears and ecchymoses in the inner canthus of the eye. Tearing of the schneiderian membrane may be followed by emphysema about the face. Diagnosis only becomes difficult where much swelling exists.

Prognosis is on the whole not unfavourable. In rare cases death results from bleeding or suffocation, or at a later stage from septic poisoning. So long as the fracture is subcutaneous and no great displacement exists, complete recovery may be looked for. In severe displacements, and especially where both nostrils are fractured, an asthma nasale may remain. Caries of the turbinated bones sometimes follows compound fractures.

Treatment of subcutaneous fractures without much dislocation requires nothing more than a few days' rest. Compound fractures must, as far as possible, be antiseptically treated, loose splinters of bone removed, and dislocated bones replaced. Where portions of bone have been driven inwards, replacement is most difficult. In large animals it may be effected by introducing into the nostril a stick, with the rounded end wrapped in lint or tow, and therewith pressing the bone forcibly outwards, but this only succeeds in the lower part of the nostril. In the upper part the upper turbinated bone lies in contact with the nasal bone, and may easily be damaged by such manipulation, caries resulting. Care must also be taken not to convert a simple into a compound fracture. In some cases it is advisable to trephine, and remove pieces of bone which have been pushed into the nasal meatus.

It must not be forgotten that dyspnœa may be produced by general swelling of the nasal mucous membrane, and that during the first fortyeight hours tracheotomy may become necessary. The owner should be warned of this contingency, especially when the practitioner lives at a distance. In certain cases it may be advisable at once to perform the operation. 


\section{(3.) FOREIGN BODIES AND TUMOURS IN THE NOSTRILS.}

Foreign bodies occasionally obtain entrance to the nostrils of horses and cattle, and remain for long periods in situ. With the exception of pentastomum tænioides, foreign bodies are rarely found in the nostrils of the dog. Sponges or similar objects have even been inserted into the nostrils of horses in order to conceal a discharge, such as that of glanders. Hermann found a wisp of straw, Körner a piece of ribbon, in a horse's nostrils. In a horse Möller had under treatment the lower meatus was discovered to be full of food, which had entered through the alveolus of a molar tooth. Dusseau, in making the autopsy of a horse, found in the nostrils forty bean-like, hard white bodies, consisting of dried mucus and epithelium, and believed them to have been formed in the guttural pouch.

New growths, in the form of polypi, often occur in the nostrils. According to Ercolani, Cato and Absyrtus were acquainted with nasal polypi; and in 1784, Icart is said to have removed one weighing $1 \frac{3}{4} \mathrm{lbs}$. from a horse. The growths are most commonly fibromata and myxomata, though lipomata, osteomata, enchondromata, angiomata, sarcomata, and carcinomata have been found. They often originate from the cribriform plate of the ethmoid or the lateral wall of the nostril. Gurlt has seen them develop from the nasal septum, Rizot from the turbinated bones. Hamburger discovered an extensive myxofibroma in the septum nasi, whilst Lammers met with a case where the mucous membrane was thickened, and showed cavities containing numerous examples of strongylus armatus; Kitt observed a sarcoma in the nostril of a dog. Strerath found tuberculosis of the nasal mucous membrane in a calf. The animal had shown difficulty in breathing after taling food or water, and usually breathed through the mouth, making a snoring noise. The growths were of varying size, some as large as grains of corn, some like peas, others again were confluent and gave the membrane a fatty appearance. Strebel described a cyst containing a piece of bone, which he found in an ox. He considered it had originated in the ethmoid bone. New growths occasionally extend into the nostril from the brain cavity and maxillary sinuses. Cases of bothryomycosis and actinomycosis of the nasal mucous membrane have also been observed in the horse and ox.

Symptoms. Both tumours and foreign bodies in the nostrils produce a muco-purulent discharge, which is generally one-sided, and often accompanied by bleeding. The pharyngeal glands are usually swollen. The air-stream from the affected nostril is weaker, and not infrequently a loud breathing sound, resembling that in "roaring," is present, constituting asthma nasale. Compression of the lachrymal duct 
produces overflow of tears. When the condition becomes further developed, the nasal or superior maxillary bones or the hard palate appear swollen. Sometimes the growth even extends through the plate of bone. More exact information as to the nature of the case is obtained on examination with a speculum, with a sound, or merely with the finger. Polypi sometimes become so long as to protrude from the nostril. Where they start from high in the meatus, the earliest symptoms are the before-mentioned asthma nasale and swelling of the nasal bones, but if their origin is low down, they may be felt and even seen soon after dyspnœa begins to be apparent. When in the latter position they usually grow from the outer wall of the meatus, just over the base of the false nostril. This is a fact to be remembered both in diagnosis and treatment.

Dogs and cats with nasal growths sneeze and scratch, or rub the nose with the paw; sometimes they run along with the head pressed on the ground. In the former pentastomum tænioides produces attacks suggesting rabies. Several cases of tumour formation in the nasal cavities in dogs are described at length in Cadiot and Dollar"s "Clinical Veterinary Medicine and Surgery," pp. 319 et seq.

Treatment, to be successful, requires the removal of the foreign bodies or new growths. Necrosis of the turbinated bones and such complications as the growth of sarcomata are frequently irremediable. Foreign bodies can, as a rule, be seized and removed with a pair of dressing forceps. Sometimes it is sufficient to wash out the nasal cavity with a powerful jet of water. In men foreign bodies can be removed by blowing forcibly into the opposite nostril.

New growths, seated in the lower part of the nostril, may be extracted with the help of the so-called polypus forceps or Leverett's hooks (decapitating hooks). When somewhat above this, and especially if on the outer wall of the meatus, they may be removed thus:-An incision is made at the point of junction of the nasal and inter-maxillary bones, on the inner side of the false nostril parallel with the nasal bone, and the finger inserted to ascertain the size and position of the polypus. The growth may then be removed either with the fingers, with a wire snare, or with the écraseur. But if the polypi are higher placed, the nostril must be trephined. Difficulty is often experienced in diagnosing the seat of the growth; this may sometimes be effected with a long sound, or with Günther's catheter for the guttural pouch. Trephining, if adopted, is better performed too high than too low. The growth, if possible, is removed by blunt dissection or ligation. Where the base of the polypus is broad, a curette is often of considerable service, but whatever the means employed, the principal object is to completely remove the growth. If bleeding prove alarming, the head may be placed in a pendent position, or the superior meatus or the upper part of the posterior naris 
may be filled with tow or jute, in order to check the flow of blood into the larynx. ${ }^{1}$ But the tampons must be secured by strong tape, that they may not fall into the pharynx and produce danger of suffocation. Under such circumstances it is advisable to perform tracheotomy and insert a tampon canula. One of Möller's patients died from cerebritis, owing to the inflammation extending from the point of operation to the brain.

The term "rhinoscleroma" was formerly given to a disease of the nose in man, usually following nasal catarrh, and producing general swelling both of the nasal mucous membrane and the external skin. The swelling, as the name indicates, is distinguished by its hardness, and may be of such dimensions that the nostrils are completely occluded. The schneiderian membrane appears livid. Anatomically, the thickening is like that of elephantiasis, and, according to later investigations, is caused by a specific infection -a micro-organism, similar to Friedländer's pneumococcus, has been found in the growth. Whether this condition occurs in the lower animals has yet to be determined. The cases hitherto reported show peculiarities indicating other than microorganismal causes. This view seems to be supported by a reported case of Jacobi's, where recovery followed the injection of Lugol's solution of iodine into the subcutaneous tissue. A similar case was given in the Zeitschrift für Veterinürkunde. It cannot be denied that the nasal mucous membrane and the nostrils do become swollen as in elephantiasis, but whether such swellings should be looked upon as rhinoscleroma cannot be determined without a fuller knowledge of their nature.

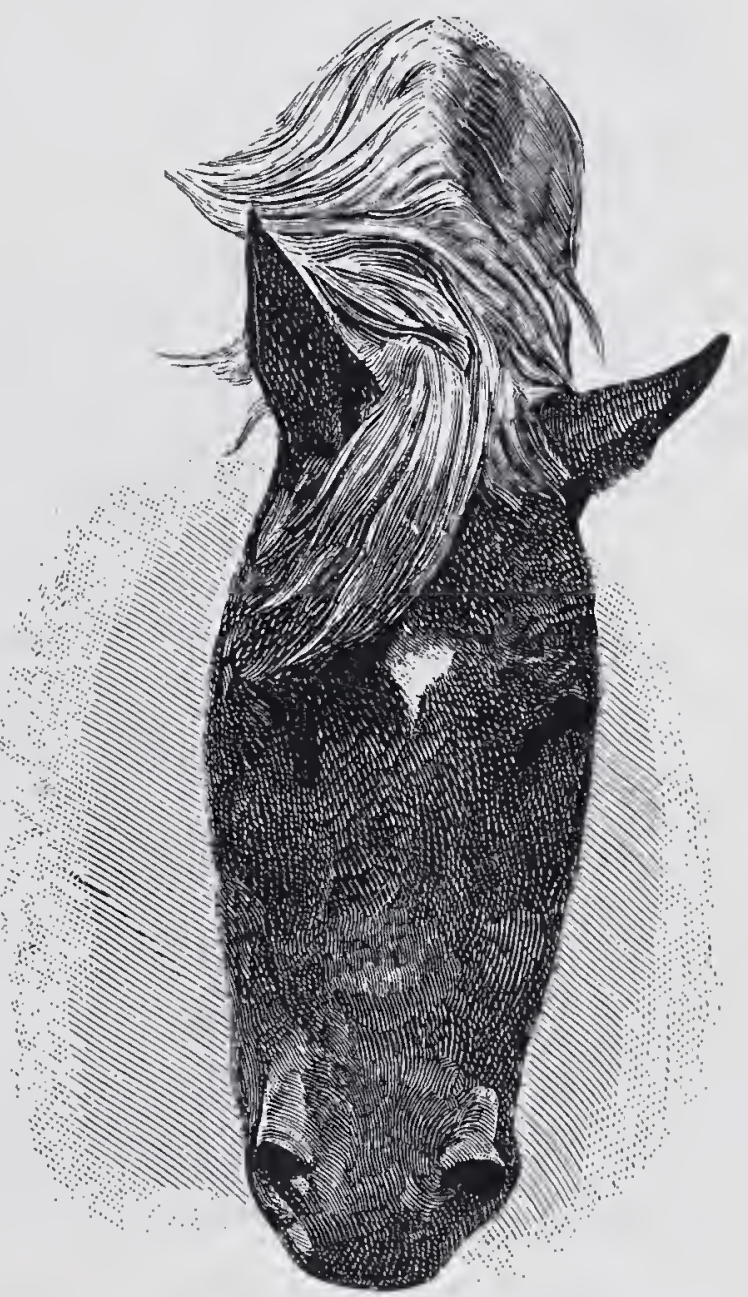

Fig. 38.-Facial œedema after injury. Thickening occurring about the nose in glanders might, however, be mistaken for rhinoscleroma.

In an omnibus horse the upper and lower lips and the nose became extremely thickened. Both nostrils and the lower lip felt hard. The swelling was smooth on the surface and the skin covering it was denuded of hair and closely adherent. Some spots on the upper lip were deprived of pigment and appeared dull red in colour. The swelling was not sharply marginated above, but gradually shaded off about 4 inches above the nostrils, extending somewhat higher, however, as it receded from the middle line of the face, and being continued in the form of a slender cordiform enlargement up to the masseter muscles.

The nostrils were $3 \frac{3}{4}$ inches in length. The anterior edge of the lips extended about 3 inches beyond the incisor teeth. The mucous membrane of the lips and nose was little changed, and there was neither nasal discharge nor difficulty in breathing.

The submaxillary and prepectoral lymphatic glands were swollen and hard.

\footnotetext{
${ }_{1}^{1}$ Adrenalin chloride ( 1 in 1,000) applied to the mucous membrane before incision produces powerful contraction of the arterioles, and permits of practically bloodless operation.
} 
A small firm swelling was noted above the right eyelid and a hard, ill-defined but somewhat extensive swelling below the chest. The latter was not adherent to the skin.

The condition had attained the above development in two months despite treatment.

Extensixe œdema of the head sometimes occurs in the horse in petechial fever. and influenza, and in cattle in malignant catarrhal fever; it affects both the head and neck as the result of wounds in these regions. Where the head is held low for considerable periods, as after bruising or sprain of the muscles of the neck or painful disease of the cervical vertebræ, marked oedema may develop, completely transforming the appearance of the animal, while swellings affecting the nasal mucous membrane and adjacent structures induce difficulty in breathing. A photograph of such a case is presented in fig. 38 .

This condition demands attention, as, being often accompanied by fever, it may, on superficial investigation, be mistaken for a symptom of certain infectious disorders. Such an error is, however, avoided by consideration of the history of the case and the condition of the neck muscles, or the cervical
vertebræ. Marked dyspnœa may necessitate tracheotomy. As soon as possible the head should be raised and so maintained. The result is often astonishing; a large swelling of this kind disappeared in a few hours. Massage is useful to promote resorption of the extravasated fluid.

\section{(4.) DISEASES OF THE TEMPORAL AND SUPERIOR MAXILLARY SINUSES.}

The mucous membrane of the facial and maxillary sinuses is in direct connection with that of the nostrils. In the sinuses it is closely attached to the bone, to which it acts as periosteum, and contains numerous small mucous glands. From its sheltered position this membrane is seldom diseased. But when catarrh or inflammation arises, the disease readily becomes chronic, for the secretion, finding no regular outlet, remains in the cavity and decomposes, irritating the mucous membrane. 'This tendency to chronicity is induced by various causes.

On clinical grounds, diseases of the frontal and superior maxillary sinuses must be considered together despite their varying characters and causes. The following forms are recognised :-

(1) Dropsy of the sinus. A serous or mucoid fluid fills one side or occasionally both sides of the cavity. There is no marked change of
the lining mucous membrane.

(2) Empyema of the sinus. The sinus contains pus; in acute cases and in oxen blood may also be present. The mucous membrane is thickened, vascular and covered with granulations.

(3) Tumour formation in the sinus. Carcinomata, sarcomata, osteomata, odontomata, \&c., may be present, accompanied by varying quantities
of a muco-purulent fluid.

1 For fuller treatment of "Tumours in the Facial Sinuses and Cancer of the Superior Maxilla," see Carliot and Dollar's "Clinical Veterinary Medicine and Surgery," pp. 22 et seq. 
(4) Specific inflammation due to glanders, bothryomycosis, or actinomycosis. The glanders, ulcers, or specific new growths are accompanied by a puruient fluid.

The causes are as varied as the processes themselves. In the horse the superior maxillary sinus is often involved in consequence of suppurative alveolar periostitis attacking the socket of a molar tooth and the discharge escaping into the cavity. It may also suffer owing to the development of new growths or the entrance of foreign bodies. Finally, the sinuses may become affected by extension of a disease like glanders or strangles from the nasal mucous membrane.

Stockfleth found in this cavity a piece of bone, the result of unskilful trephining. Stenersen found pieces of sand-sedge, which had obtained

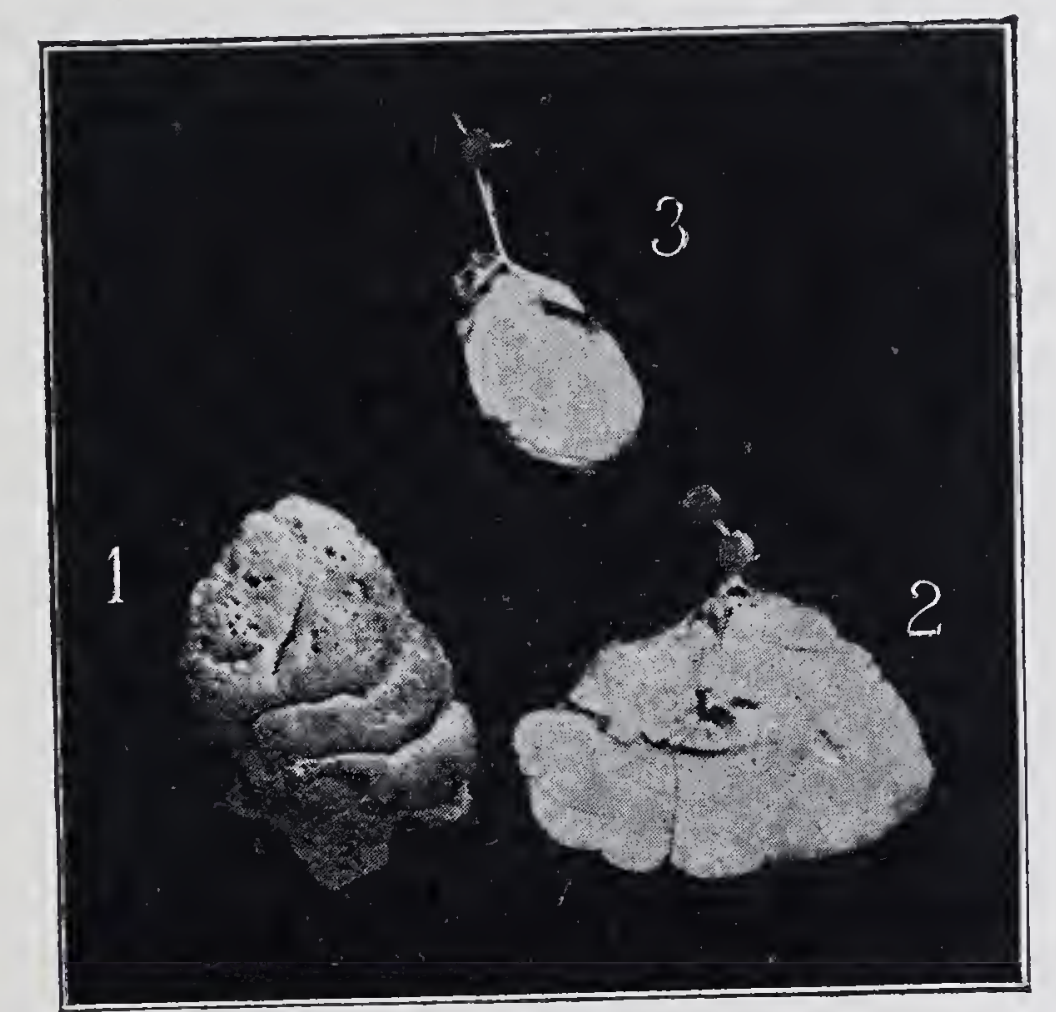

FIG. 39. - Osteomata from skull of a three year old bullock.

entrance through the socket of a diseased upper molar.' Decomposed food may enter in the same way. Fractures of the frontal and superior maxillary bones, and in cattle, injury of the horn-core, also act as causes.

Cadiot believes that chills due to cold external applications or cold irrigation of the sinuses may develop disease. Larvæ of Gastrophilus are occasionally found in the sinuses in horses.

In oxen the causes are usually fractures of the horn-core, or severe bruising and necrosis of bone due to the yoke. Tumours are rarer in oxen than in horses.

J. J. Vahey (Ballinrobe, Ireland) saw a three year old bullock with slight enlargement above the line of molar teeth on the left side, apparently involving the maxillary sinus. In four months the head increased to nearly double the normal size, and became much distorted. The left eye was almost closed, probably by a large osteoma within the. V.S. 
maxillary sinus. On slaughter bony tumours were found "everywhere, all over the head." Professor Mettam examined the skull. The largest tumour was situated in the left maxillary sinus, and had protruded into the orbital cavity. The smaller of the two tumours illustrated (fig. 39) was in the right maxillary sinus. Ten or twelve other smaller tumours were obtained from different parts of the skull, and one the size of the fist from the body of the inferior maxilla. 'This last was interesting because it had included within it at least one incisor tooth. A tumour was also removed from between the bodies of the premaxillæ, and one from the angle of the left ramus of the inferior maxilla. The three tumours last mentioned were attached or included within the bones, but there "were no distinct pedicles." The bones in which they were situated were reduced to mere shells. The large tumours photographed were not pedunculated at all. Sections resembled ivory. The larger of two was $16.5 \mathrm{~cm}$. in length, and $11 \mathrm{~cm}$. in greatest width. It had a circumference of $40.7 \mathrm{~cm}$. It weighed $4 \mathrm{lbs} .3 \mathrm{ozs}$, or nearly 2 kilos. The smaller tumour was $8 \mathrm{~cm}$. in length, nearly $6 \mathrm{~cm}$. in breadth, and weighed $12 \mathrm{ozs}$. (380 grammes). Part of the surface of the larger tumour resembled a growing coral. The remainder of the surface was bosselated. An irregular cavity in the interior was filled with decomposing animal matter. Although the osteoma was so hard and resistant to the saw, yet one half which was accidentally let fall broke into three pieces; the fractures were clean and conchoidal, giving one the impression that this large tumour had resulted from the fusion of several of small size. The smaller tumour included in its structure a molar tooth.-The Veterinarian, March, 1899, p. 174.

In sheep oestrus larvæ sonetimes cause disease of the frontal and superior maxillary sinuses. In dogs tumours are the commonest cause, though pentastomum trnioides plays an occasional part.

The diagnosis can often be made only by the method of elimination. The first symptom is a one-sided mucopurulent and sometimes illsmelling nasal discharge, which may only appear at intervals or when the head is depressed, as in disease of the guttural pouch. If the disease has been caused through alveolar periostitis, the peculiar odour of a decayed tooth will be notable. The submaxillary glands of the same side often swell, and the case may be suspected to be glanders. At a later stage the bones inclosing the simus become swollen, and on percussion over the diseased spot emit a duller, less resounding tone. In making this test the hol'se's mouth must be closed, and the sinus on each side struck in turn over exactly corresponding points, using the plain end of the percussion hammer or a strong key. 'Thinning of the bone, so that it yields to strong pressure, is sometimes seen in cases of tumour growth and in actinomycosis or bothryomycosis. Perforation of the bone 
and exuberant granulation is only seen in rare cases, and is due to tumour formation or one of the specific inflammations.

In oxen the nasal discharge is usually blood-streaked at first, and only becomes purulent at a later period. If the frontal sinus be affected, signs of brain disturbance, like dulness, lowering of the head, giddiness, falling or convulsions, may appear. In unilateral disease the animals usually hold the head obliquely towards the diseased side.

Similar sensory disturbance is seen in dogs and sheep when the frontal sinuses are affected. Dogs are then apt to be very snappish.

Recovery never occurs spontaneously, though death from extension of the process to the brain or from septicæmia is rare. A fatal termination is commoner in oxen. In most cases the disease becomes chronic; in oxen the pus occasionally breaks through at the base of the horn.

Treatment must be preceded by trephining. The cases said to have been cured by disinfectant inhalations are open to considerable doubt. The contents of the sinus are washed out, a disinfecting solution, such as carbolic, sublimate, or creolin, injected, and the opening closed with a plug of tow or jute. Irrigation must be repeated daily, until the nasal discharge ceases. The operation wound soon closes completely.

\section{TREPHINING THE SUPERIOR MAXILLARY AND FRONTAL SINUSES.}

Trephining the super-maxillary and frontal sinuses has been undertaken from very early times. It was frequently vainly employed for the cure of glanders, and has occasionally been of service in aiding: diagnosis in cases of suspected glanders, for which purpose it is commended by Haubner as affording evidence of the condition of the lining membrane of the sinuses, and explaining any dubious discharge. But trephining is still more useful in the treatment of such local affections of the facial sinuses as chronic catarrh, in removing tumours and foreign bodies, and in punching out certain molars.

Various instruments are used for removing portions of bone from the walls of the skull.

(1.) The trephine, a circular-shaped saw, which is either affixed to a stock similar to a carpenter's (stock trephine, fig. 40), or to a handle (hand trephine, fig. 41). Usually the latter simple form is used. The crown, a steel cylinder of varying size, which forms the saw, is smooth on its external surface, differing in this respect from those formerly in use, which were conical, and provided externally with sharp saw teeth to allow of their entering the bone. In the centre of the crown stands the centre pin, a piece of steel which can be fixed by means of a screw, and caused to project somewhat beyond the cutting edge of the crown

(2.) The elevator (fig. 42) carries at one end a ring, by which it is 
held. The other end is fashioned into a conical screw, which can be inserted in the piece of loose bone to remove it: under certain circumstances the elevator may be used to bring dislocated fragments of bone into their former position.

(3.) The lenticular knife (fig. 44). With this the sharp edges of the opening left by the trephine are removed, to prevent the finger being injured when introduced during examination.

(4.) The "periosteum knife" (fig. 45) is for scraping the periosteum from the point of operation, but may be replaced by a blunt knife,

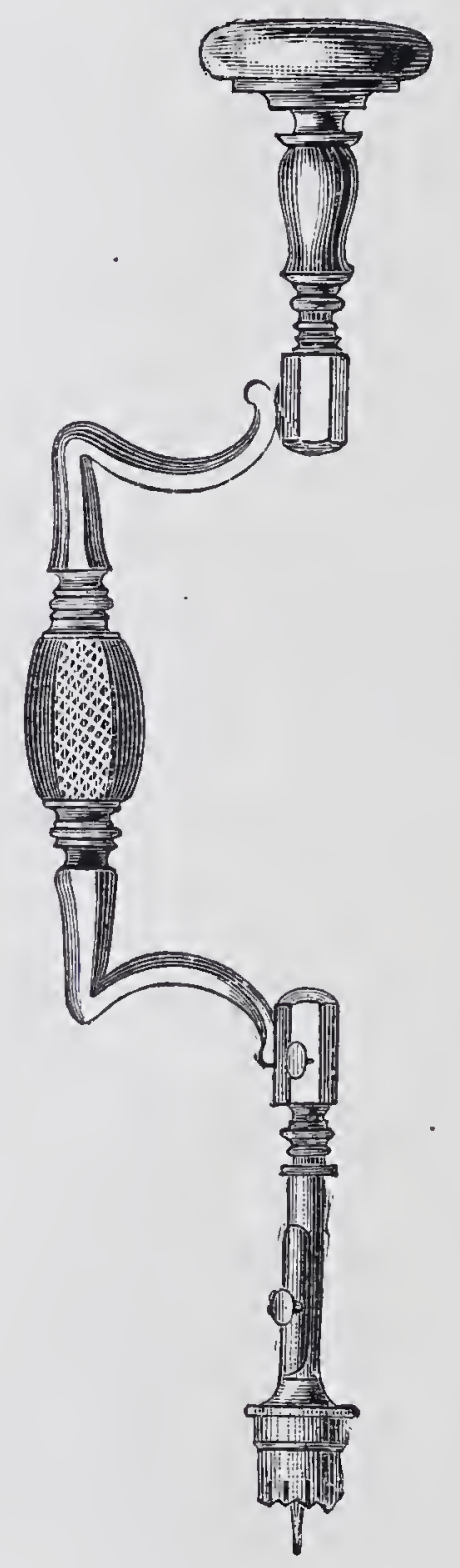

FIG. 40. - Stock trephine.

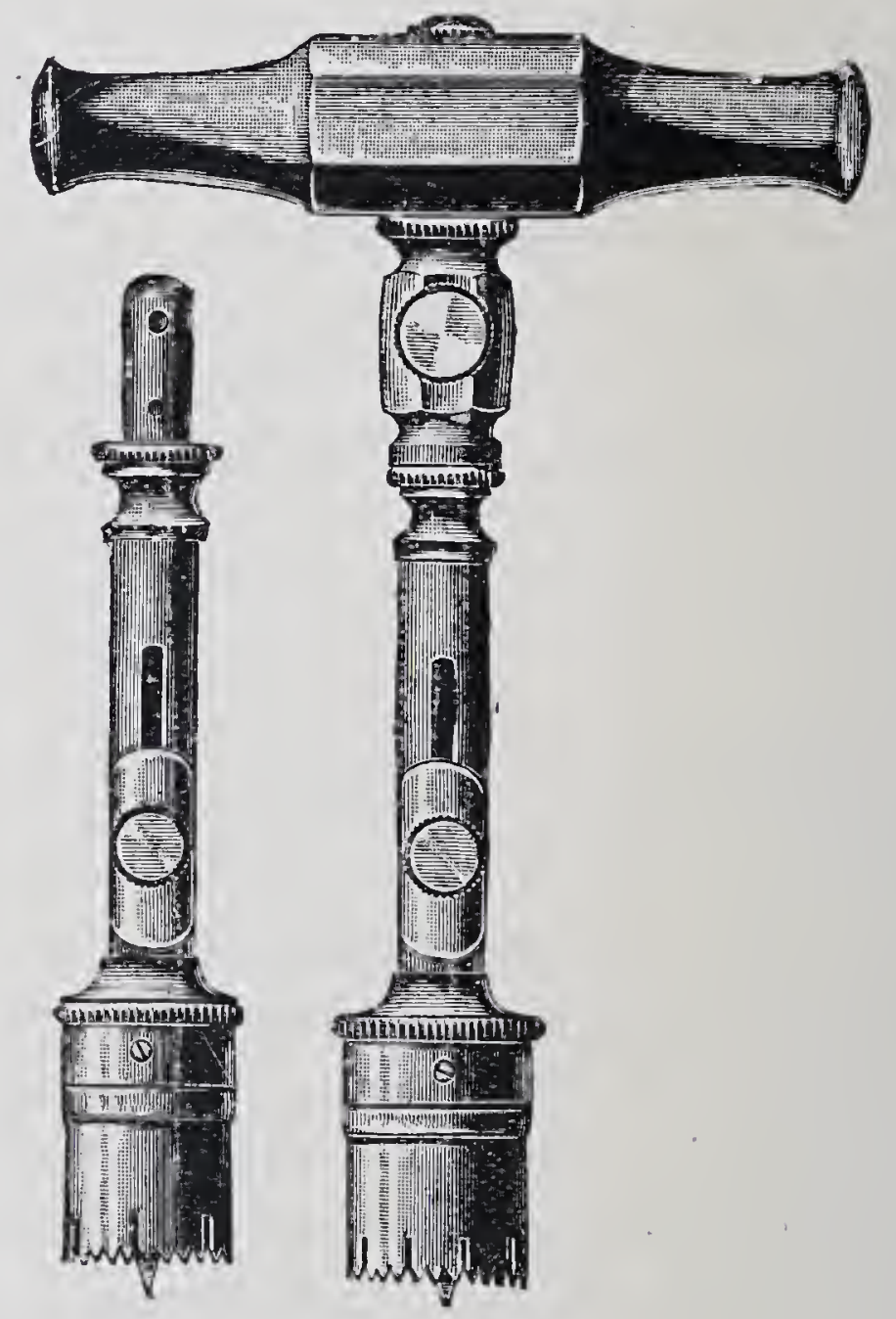

Fig. 41.-Hand trephine.

curette or gouge. The set of instruments usually comprises a chisel (fig. 43) to remove pieces of bone such as those between two trephine apertures, and is useful in making large openings. Where a trephine is not to land, the opening may be made with an ordinary borer (exfoliative trephine, fig. 46), but if it is merely necessary to make an aperture in the bone, the perforating trephine is used (fig. 47). The last-named instruments can, however, generally be dispensed with.

Trephining the facial and superior maxillary sinuses of the horse.

In opening the facial sinus, the frontal bone is trephined at a point on a level with the supra-orbital process of the frontal bone, and about 1 inch from the middle line. Here the skin lies almost immediately on 
the periosteum. The superior maxillary sinus in the horse is generally divided by a thin plate of bone into an upper larger and a lower smaller division. Lanzilotti describes this plate as having been present in 52 out of 74 cases operated on. It lies nearly in the centre of a rectangle, whose longer sides are formed by the zygomatic ridge, and a line drawn parallel with it, starting from the inner angle of the eye, and whose shorter sides are bounded by the rim of the orbit, and a line drawn perpendicularly to the lower end of the zygomatic process of the malar bone (see figs. 48 and 49). According to Lanzilotti, the septum is always wanting in the ass, and often in the mule. Trephining about $1 \frac{1}{4}$ inches above the lower end of the zygomatic ridge, and $\frac{3}{4}$ to $1 \frac{1}{4}$ inches away from

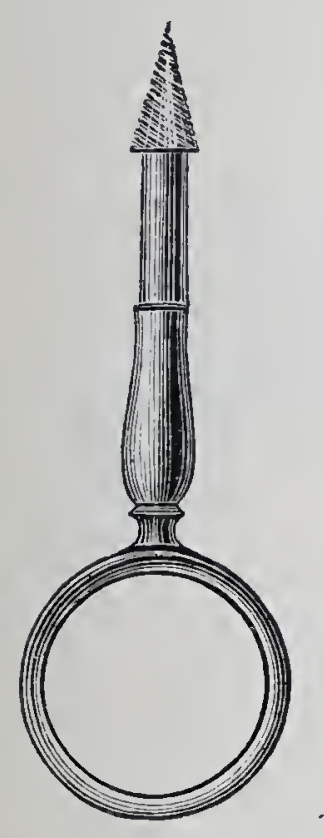

FIG. 42.Bone elevator or screw.

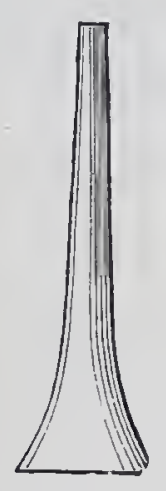

Frg. 43.Chisel.

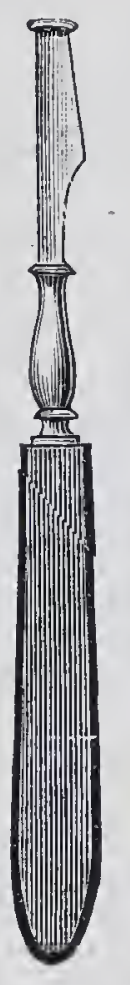

FrG. 44 Lenticular knife.

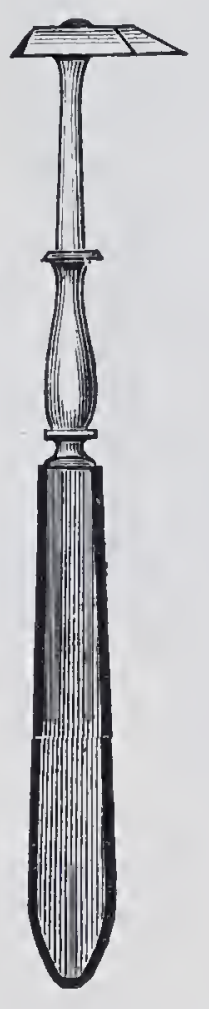

FIG. 45.-

Periosteum knife.

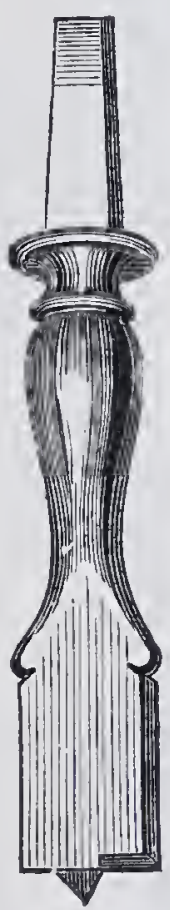

Fig. 46.Exfoliative trephine.

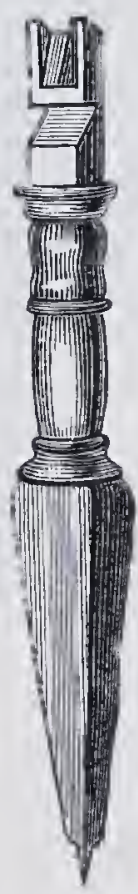

Fig. 47.Perforating trephine.

it, this division is cut into, and both portions of the sinus are opened. Where one sinus alone has been opened, the dividing wall can be broken down. In foals one operates somewhat further from the edge of the zygomatic ridge, in order not to injure the roots of the fourth and fifth molars which lie in the lower division of the superior maxillary sinus.

The horse had better be cast, though quiet animals may be operated on standing. After removing the hair from a space about 2 to $2 \frac{1}{2}$ inches in circumference, a longitudinal cut 2 to 3 inches in length is made through the skin. This should be parallel with the middle line, and $\frac{3}{4}$ to $1 \frac{1}{4}$ inches removed from it.

In trephining the superior maxillary sinus, the cut should be parallel with, and about 1 inch above, the zygomatic ridge. A " $\mathrm{T}$ " or " $\mathrm{V}$ " shaped incision was formerly recommended, but Gerlach has rightly condemned this procedure, because the loose flaps of skin retract and 
give rise to excessive granulation. The late Professor Robertson recommended removing a circular patch of skin. Cicatrisation is much more perfect after this procedure, and in one or two months no visible wound

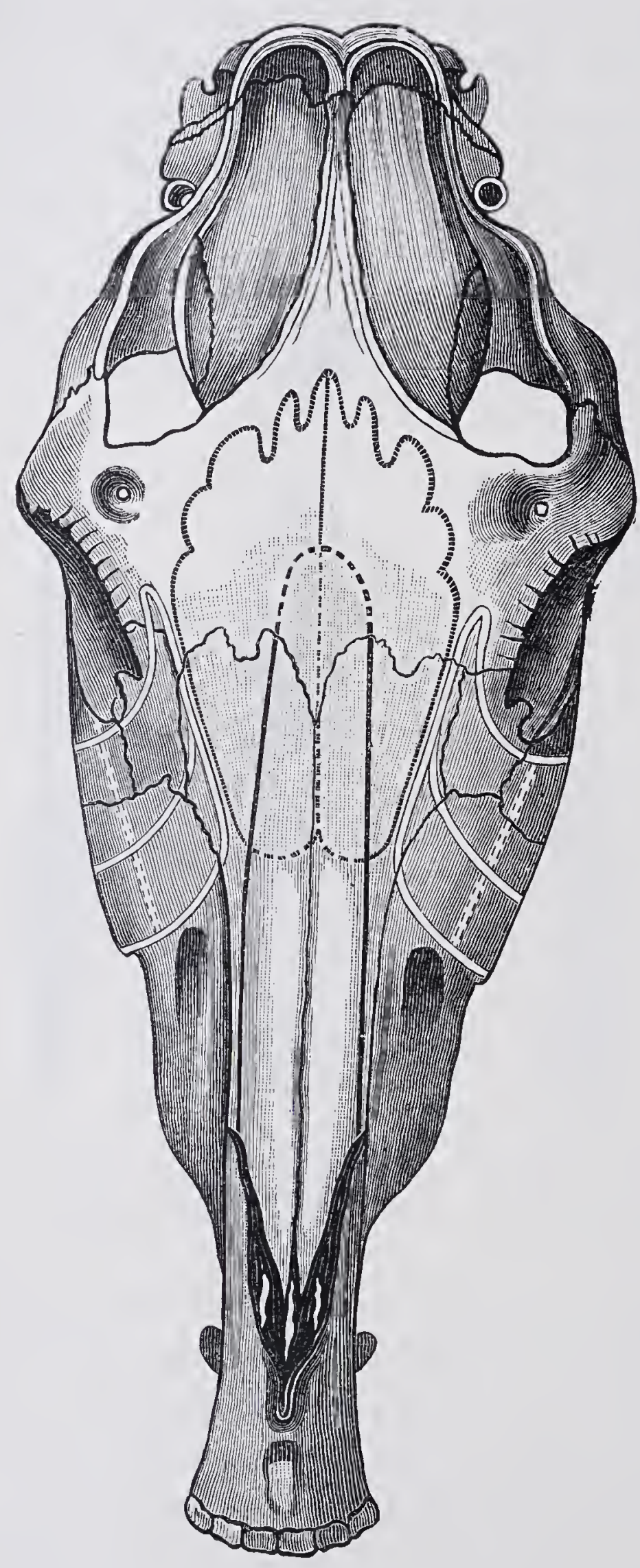

Fig. 48. - Head of a horse. |H||||||| boundaries of the frontal sinus. $\begin{array}{llll}- & , & , & \text { nasal meati. } \\ ---- & , & ,, & \text { superior maxillary sinus. }\end{array}$ Where the latter is very thick it will be neribed until the bone is perforated. Where the latter is very thick it will be necessary from time to time to clean the teeth of the trephine with a brush. An increased sense of yielding gradually becomes apparent, whereupon sawing is more cautiously proceeded with. As soon as the bone is thought to be cut through, the edge of the wound a stitch is inserted, by which the flaps are drawn asunder. The skin is separated from the sub-lying tissues with the knife in order to make room for the trephine crown between the two edges of the wound. Should the operation on the superior maxilla be performed rather higher than above described, the belly of the levator labii superioris is encountered, and must be pushed to one side. The trephine crown is now placed in position, and to facilitate removal of the pieces of periosteum a cut is made around it with a guarded bistoury, and the membrane separated with a scraper or blunt knife. A central hole having been made with a gimlet, the trephine, with its centre pin in advance, is replaced in position, and by light rotary movements caused to enter the bone. The instrument in case of need is supported by the thumb and index finger of the operator's left hand. The teeth of the saw soon begin to act, but, before the piece of bone to be excised is loosened, the elevator should be inserted. The trephine, being again placed in position, is used as before de-

remains. In the middle of each 
elevator is applied and the piece lifted out. But if this cannot be effected, recourse must again be had to the trephine. The lining mucous membrane generally remains in situ, or hanging in shreds to the sides of the opening.

After removing sharp edges with the lenticular knife, the finger may be introduced for examination. Bleeding is usually slight. If it renders examination difficult, the sinus is syringed out with water and dried with a small sponge, when the mucous membrane may be more closely examined. In empyema, pus usually flows from the opening spontaneously, and whatever remains can be removed by washing. Any new

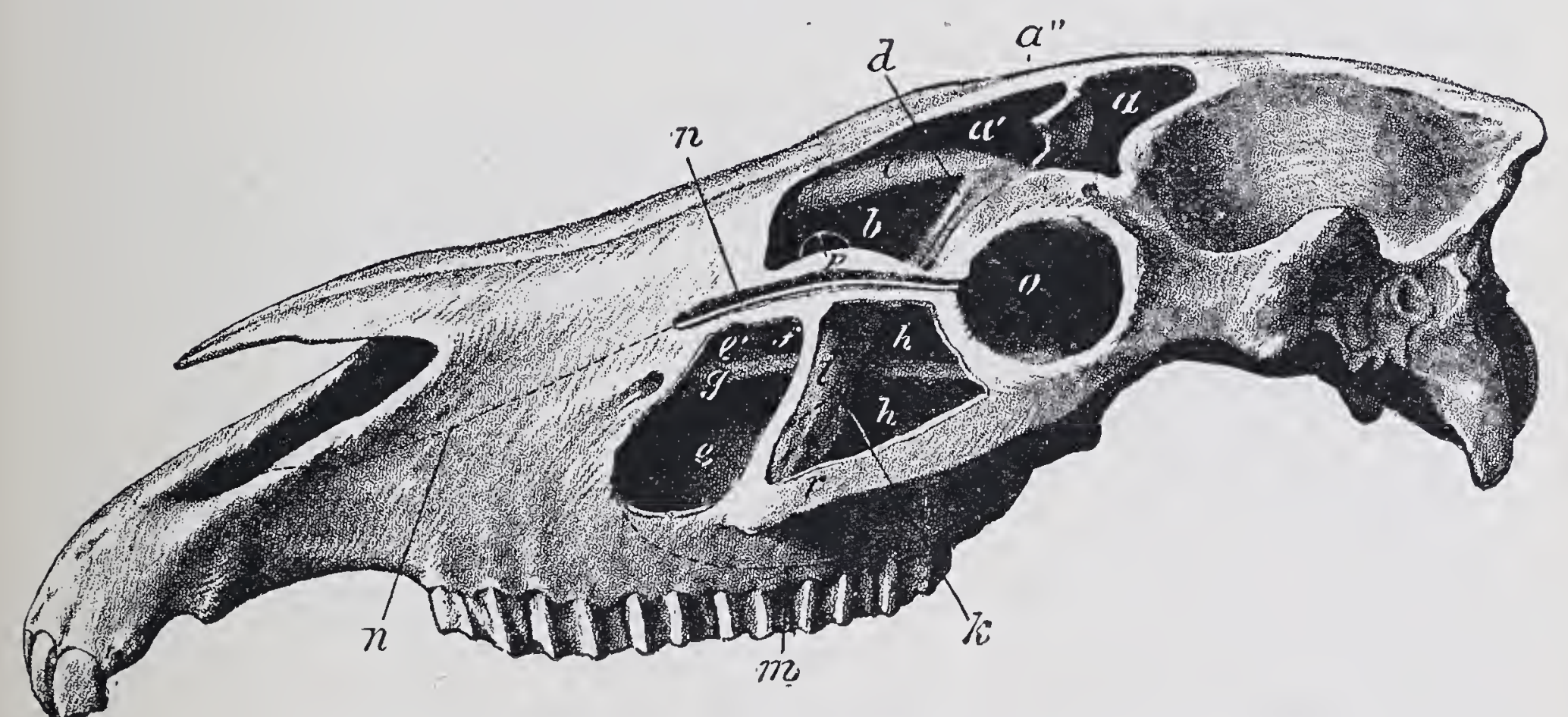

Fig. 49.-Lateral view of horse's skull; the facial sinuses exposed.

$\alpha$, Posterior portion of frontal sinus ; $a^{\prime}$, anterior portion of same; $\alpha^{\prime \prime}$, the septum dividing them ; $b$, posterior portion of the anterior turbinated bone ; $c$, cavity of nostril ; $d$, ethmoid bone; e, superior inaxillary sinus (lower part), which is divided from the upper part $h \hbar$ by the thin septum $i ; e^{\prime}$ bony plate forming the lateral boundary of the posterior turbinated bone $f$; $g$, infraorbital canal; $k$, line dividing the upper (thin) from the lower (thick) portion of the septum; $m$, lower margin of the maxillary sinus; $n$, ductus ad llasam; $o$, orbit; $p$, zygomatic ridge.

growths can also be taken away. The opening is closed with a plug of tow or jute.

To facilitate drainage Siedamgrotzky has recently suggested a modified method of operation. He operates about $\frac{1}{2}$ to $\frac{3}{4}$ of an inch in front of a horizontal line drawn through the two inner angles of the eyes, and $1 \frac{1}{4}$ to $1 \frac{1}{2}$ inches from the middle line of the face. After washing out the sinus cavity he effects a communication between it and that of the nose by thrusting a director downwards and invards at the deepest spot, breaking through the thin plate of bone and the mucous membrane covering it, and enlarging the opening so made by excising with a tenotome an oval fragment $\frac{3}{4}$ inch long and $\frac{3}{8}$ inch wide. To avoid injuring the septum nasi the knife is only introduced a short distance and the operation performed at the deepest spot. The resulting bleeding ceases in a fer minutes; if not, the cavity may be plugged. To prevent 
inspiration of blood the horse is at once allowed to rise; for a similar reason anæsthetics are withheld.

During the first three or four days after operation the artificial opening may remain closed with blood or pus, but at the end of that time discharge occurs freely and recovery is usually easy.

After-treatment must depend on the nature of the diseased processes. In chronic catarh, flushings of the affected sinus, with antiseptics like 2 per cent. carbolic solution, are indicated. Where putrefactive processes are actively going on, 3 per cent. permanganate of potash, or solutions of astringent materials-such as alum 2 per cent., tannin 2 per cent.-may be used. The opening is then closed with a plug, so as to guard the mucous membrane against the direct influence of the air. Treatment should be continued as long as any nasal discharge is present. The wound is afterwards encouraged to heal, only soft plugs being used in the trephine opening. In about three or four weeks it closes completely, and after the lapse of two or three months scarcely a trace of the operation can be observed. Where large tumours are to be removed from the frontal or superior maxillary sinuses a simple opening may not be sufficient; a second is bored in the neighbourhood of the first, and the intervening bone removed with the assistance of the chisel (fig. 43) and the hammer. Möller has frequently had occasion to trephine the nostril in this way in order to remove tumours or necrotic portions of the turbinated bones. A saw similar to a key-hole saw is useful here in order to enlarge the trephine opening.

Although diseases of the frontal sinuses occur in herbivora, they are less common than in horses. In sheep, on the other hand, the presence of oestrus ovis may necessitate the opening of the frontal and maxillary sinuses. This is usually done in the middle line, in order that both sides can be opened simultaneously, and washed out by injections. The frontal sinus, as in other ruminants, is connected with the horn-core, and may be opened by sawing through the horns; but this operation is not only more painful, but is also accompanied by greater loss of blood, which in cattle already weakened may be attended with undesirable consequences.

\section{(5) NECROSIS OF THE TURBINATED BONES.}

After Jessen's description of a peculiar disease of the turbinated bones of the horse, accompanied by chronic thickening, Stockfleth and others published descriptions of similar cases. The condition is not common in North Germany, and usually appears after strangles, while a few cases of Möller's have bəen caused by disease of the upper molars. Sand, on making the post-mortem examination of an old horse which 
had shown an offensive nasal discharge, found the mucous membrane was thickened, and the surface of the ethmoid cells necrotic. The bony plates had broken dorrn, forming a grey, grumous material. Möller has seen two cases caused by disease of the upper molars, and Fröhner published three of a similar kind. For a very full description of this condition see Cadiot and Dollar's "Clinical Veterinary Medicine and Surgery," pp. 15 et seq.

Sand describes a peculiar disease of the turbinated and neighbouring bones which he terms mucous degeneration (schleim-degeneration). The disease was seen oftenest in foals, and was distinguished by dyspnœea, swelling of the bones of the face, and a muco-serous discharge. The post-mortem showed

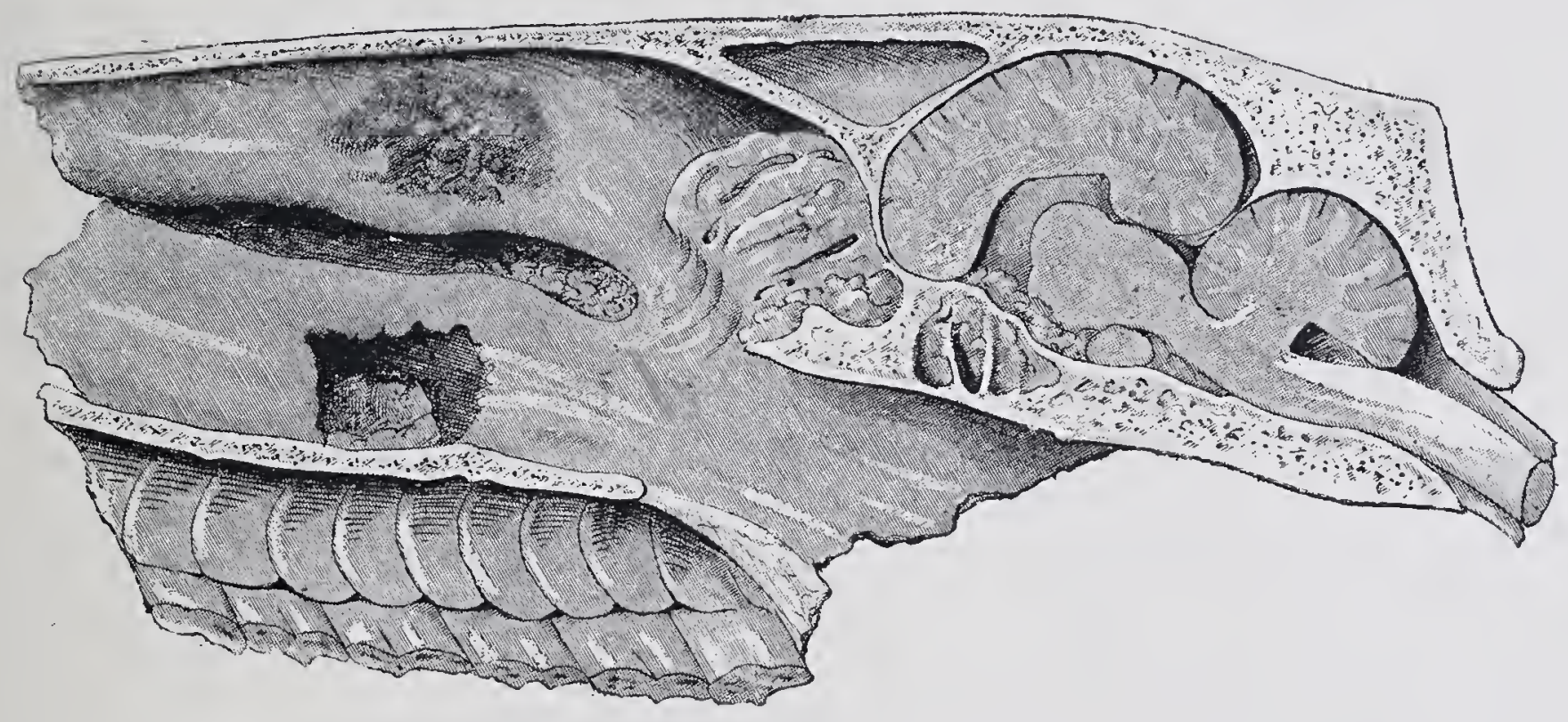

FI $x .50 .-N e c r o s i s$ of the turbinated bones.

the bones of the face to be much thinned, partly perforated, and the sinuses of the head greatly dilated. The remains of bone structure in the turbinated bones could only be recognised microscopically; the latter appearing to be almost transformed into a mucoid tissue. The walls of the superior maxillary and frontal sinuses showed the same changes, and the cavities themselves were greatly increased in size. Their contents were sometimes purulent, sometimes muco-serous.

The early symptoms consist of a stinking discharge from one nostril, snoring breathing, and diminution in the stream of air of the affected side. At a later stage the nasal bones become swollen, and not unfrequently the thickening in the turbinated bone can be directly seen, or felt by the finger. As swelling of the pharyngeal glands is usually present, the condition is sometimes difficult to distinguish from nasal polypus, and is occasionally only recognised exactly after trephining.

Treatment. Trephining the nasal cavity and removal of the diseased turbinated bone is the only method likely to prove successful:

Cadiot and Dollar describe removal of the posterior (maxillary) turbinated bone (p. 317 of "Clinical Veterinary Medicine and Surgery"). The left nasal cavity was trephined, an incision about $\frac{3}{4}$ of an inch long being 
made opposite the centre of the nasal bone; at each end two circular trephine openings were made and the intervening bone removed with scissors. The anterior part of the diseased bone was removed through an opening in the left false nostril and the remainder through the trephine incision. In four weeks recovery was almost complete. Höyer, in a case of necrosis of the turbinated bones, removed the diseased part with a muscle hook and secured healing, whilst the cases described by Jessen were also cured by resection of the diseased turbinated bone through a trephine opening. Hering and Jessen recommend making an incision through the skin close to the middle line, and extending the entire length of the nasal bone. At the upper end of this cut the trephine is inserted, and a piece of the nasal bone an inch broad and as long as the cut through the skin removed by a key-hole saw. Through such an incision the diseased turbinated bone could also be removed. But a case of caries treated in this way by Möller was still uncured after the lapse of eight weeks. Complete resection of the turbinated bones is attended with no slight difficulty; any fragments left behind are apt to renew the diseased processes. Schlegel described a case in a horse of osteosarcoma of the left lower turbinated bone, which eventually led to pulmonary gangrene and metastatic growths, endocarditis, gastritis ulcerosa, and tendovaginitis of the flexor tendons of both hind legs. The animal died. A horse treated by Cadiot for necrosis of the turbinated bones died of consecutive meningitis and pyæmia.

Sand recommends, in cases of mucoid degeneration of the turbinated bones, to trephine early, and having established free drainage, to wash the parts out regularly with antiseptic solutions. This is said to usually check or completely cure the disease. In August, 1888, a four year old mare was sent to hospital after suffering for some weeks from ill-smelling nasal discharge. She was well-nourished, and had no appearance of bodily illness, but an offensive muco-purulent discharge, smelling of bone pus, was discharging in moderate quantities from the left nostril ; the submaxillary glands of the left side were somewhat swollen. The use of Günther's catheter disclosed the existence, on the floor of the left meatus, of a slight unevenness and swelling at the height of the third molar. The resulting examination of the mouth disclosed the fact that the third upper molar on the left side had lost its crown, and that all the appearances of purulent periostitis of the alveolus existed. Scars left by previous trephinings were visible on the temporal and superior maxillary bones of the left side. After removing the diseased tooth by punching, the operation wound healed regularly, but the nasal discharge continued. Trephining the nostril was undertaken, when it was found that the turbinated bones were necrotic, and denuded of mucous membrane at several points. Attempts to remove these portions were not successful; and on account of the comparatively small value of the horse, the owner decided
to have it killed.

Necrosis of the cartilage of the nostril is described by Cadiot and Dollar ("Clinical Veterinary Medicine and Surgery," p. 315). The inner wing of the nostril was swollen and indurated over the region corresponding to the cartilaginous plate, with which a sinus, masked by granulations, communicated. This sinus was laid open and the parts swabbed with iodine tincture. Treatment was intermittent and a second operation became necessary, but
recovery occurred in four months. 


\section{(6) EPISTAXIS (BLEEDING FROM THE NOSE).}

Bleeding from the nose may occur either without injury to or disease of the mucous membrane or as a symptom of disease of the mucous membrane, as in glanders and tumour formation.

Extensive venous plexuses (centrum venosum) exist in the mucous membrane of the nostril of the horse, and especially in that investing the septum nasi. Injuries of these plexuses may give rise to considerable bleeding. Hence nasal bleeding is most frequently seen in this animal. It may be caused by external injuries, such as carelessly wiping out the nose with a rough cloth, by fracture of the nasal bones with dislocation of the fragments internally, by other accidental wounds, occasionally from foreign bodies and tumours in the nostril. Schindellia noted severe bleeding after fracture of the septum. In a fifteen year old horse, which for ten years had suffered periodically from epistaxis, especially after severe exertion, Deigendesch found an angioma of the septum which had become ulcerated. Great exertion, like racing, sometimes occasions bleeding, as do dusty, irritating fodders. Bigoteau observed nasal bleeding in a great number of horses which had been fed with dusty sainfoin. It is further noteworthy that ulcerative changes like those of glanders give rise to repeated and profuse bleeding, which is also liable to occur in mercurial poisoning. When nasal bleeding continually recurs in horses without visible cause, it must awaken very great suspicion, particularly in animals which have been exposed to glanders infection. The quantity of blood so lost, and the time of bleeding, are exceedingly varied. In glanders, and other ulcerative processes, bleeding is occasionally so severe as to threaten immediate death.

Treatment. Immediately bleeding becomes of a character to require therapeutic treatment, cold applications are made to the head, and the nostril of the affected side washed out by means of the irrigator, with cold water, to which tannin or vinegar may appropriately be added. In bleeding from the lower portions of the nostril, plugging may be necessary, but where this is resorted to the patient must be carefully watched, and the plugs removed immediately the breathing is difficult. Or tracheotomy can be performed, a tampon canula inserted, and both nostrils plugged. Even this, however, will not invariably stop the bleeding. Insufflation of finely powdered alum has been recommended, and is worth trying when the blood comes from the lower portions of the nostrils. Where fatal results threaten, secale cornutum (ergot) may ba tried. Sohngen saw recovery in the horse after subcutaneous injection of 12 grains extr. secal. cornut. Extractum hydrastis canadensis is useful, but adrenalin chloride is now the best hæmostatic for bleeding from mucous membranes. Astrachanez plugged the nostril with tampons 
of tow saturated with turpentine, and injected turpentine into the nostril with success. The cause must be removed.

Vapour of vinegar, recommended by some authorities, has been declared useless by others. Although fatal bleeding has been repeatedly observed in horses, the flow usually ceases when the animal is kept quiet, and when hæmorrhage has lowered blood pressure. The head should be kept elevated.

\section{IY.-DISEASES OF THE SALIVARY GLANDS.}

\section{(1.) WOUNDS AND INJURIES OF THE SALIVARY GLANDS.}

Owing to the position of the parotid it suffers from external injuries more frequently than the other salivary glands. As long as important blood-vessels are not injured, wounds of this gland are not particularly serious. If proper treatment be adopted at once, even the danger of salivary fistulæ is not great. The injuries most to be feared are those in which the ducts of the gland, and especially Stenson's duct, are injured. In sheep and dogs Stenson's duct passes obliquely across the cheek, while in horses and cattle it lies protected below the jaw, and hence is less exposed to external wounds. The duct is more frequently injured by sharp objects. The condition may be recognised by the position of the wound, and the outflow of clear saliva, the secretion of which is increased by feeding, and even by the sight of food. In many cases the wounds heal completely; in other's a small opening is left from which saliva discharges (salivary fistula). To avoid this result, healing by first intention must be sought for ; the flow of blood checked, the wound carefully cleansed and sutured : during twenty-four to forty-eight hours the animal should receive no food, and should be placed by itself in order that the secretion of saliva be not excited by the feeding of its fellows. Small duses of atropine may also be given, which check or altogether stop salivary secretion. Further treatment must be based on the general principles of surgery.

\section{(2.) INFLAMMATION OF THE SALIVARY GLANDS (PAROTITIS).}

The salivary glands at times become the seat of inflammation. The parotid is most frequently affected, the submaxillary and sublingual only occasionally. Foreign bodies obtaining access to the glands may produce inflammation. But many cases result from causes not clearly recognised, some of them apparently of an infectious nature. They 
produce their effects either by irritating the gland ducts directly, or through the blood stream. In men, as in animals, endemic parotitis sometimes shows itself. That in man is clearly of an infectious character, and is vulgarly known as mumps. Animals, and especially dogs, suffer from a similar disease. Not infrequently parotitis appears symptomatically during the course of equine strangles and influenza, and of distemper in dogs, and it has been seen repeatedly in cats.

The inflammatory condition arises in the parenchyma of the gland. The organ swells, its divisions appear dark red, and their epithelium turbid in small areas. The process soon spreads to the connective tissue, which, after a short time, is infiltrated with organisable lymph. Finally pus formation takes place both here and in the alveoli. By confluence of small abscesses greater ones are formed, extensive areas of the gland and its connective tissue become necrotic, and the abscess finally perforates, not infrequently destroying a large piece of skin. The contents of such an abscess contain necrotic shreds, and possess an exceedingly offensive smell. Not all abscesses in the parotid region are necessarily due to parotitis; many are only subparotideal, but perforate by way of the parotid. The abscesses in this region seen in swine and oxen mostly originate in the pharynx. In oxen the foreign body causing the abscess may often be detected by exploring the abscess cavity with the finger.

Symptoms and course. Acute parotitis, the most frequent disease of this kind, is accompanied by the early appearance of a diffuse swelling in the parotid region. The gland appears hard, hot, and painful; the head is extended, and inclined towards the sound side; salivation exists, and not infrequently fever. When the swelling is great, dyspnoea and difficulty in swallowing may be present. At times facial paralysis is produced owing to pressure on the nerve, or to extension to it of inflammatory processes. The swelling gradually becomes fluctuating at its most prominent point, and if not opened a large quantity of ill-smelling greyish-red fluid, mixed with flakes of dead connective tissue, will be spontaneously discharged. The swelling seldom becomes absorbed. Once necrosis has taken place, abscess formation usually results in eight to ten days; but in fourteen days to three weeks complete healing may be expected. Recovery is the most common termination; septicæmia and pyæmia with fatal results are rare. Owing to high fever and disturbance of appetite the animals fall off in condition, and mill secretion abates. Salivary fistulæ and facial paralysis are seldom observed as sequelæ of this affection. In a case of Möller's the optic nerve was involved and optic paralysis with amaurosis remained, having evidently resulted from retrobulbar neuritis, produced by extension of inflammation into the orbit. Extensive burrowing of pus may also be followed by fatal consequences. 
Differential diagnosis. Parotitis may easily be mistaken at the commencement for swelling of the sub-parotideal lymph gland, which does not, however, remain confined to the parotid region, but is more diffused. From distension of the guttural pouches parotitis is distinguished by the presence of inflammation, and by the absence of the purulent nasal discharge which is so diagnostic of disease of the guttural pouch. 'Tumours in the parotid region are unaccompanied by inflammation, and never develop so rapidly as the swelling of parotitis. The submaxillary and sublingual glands also become diseased, but less frequently. Parotitis usually originates in the mucous lining of the ducts, the inflammation extending from catarrhal conditions of the buccal membrane. Outbreaks of an enzootic type are recorded in horses. A long flattish swelling appears in the throat, which extends under the parotid, and salivation is present. The termination of Wharton's duct on the septum linguæ not infrequently appears stopped. Should pus formation result, the abscess commonly breaks into the mouth, less frequently externally, but in fourteen days convalescence is established. The abscesses so often seen in the laryngeal region in oxen are almost always the result of injury to the tongue or lower portion of the cavity of the mouth and local infection.

Stockfleth has described, under the name of "ranula," a disease in cows, which presents the appearances of virulent inflammation of the submaxillary gland. There is cedema of the throat, and an elastic swelling as large as a hen's egg is found on the frænum linguæ. If this be opened early, the symptoms soon disappear, but if not, the head and neck rapidly swell, and breathing and swallowing are so disturbed that the animals often die in a few days.

Inflammation and abscess formation occur in the ducts of the salivary glands, especially in Stenson's and Wharton's ducts. Meyer found in Stenson's duct in a horse a spikelet of Timothy grass. Concretions may also inflame the gland duct, producing pus formation and salivary fistula. Foreign bodies in the duct can often be removed by pressure in the direction of the buccal opening; if this fail the duct must be incised.

Cattle, especially in low-lying situations, are liable to chronic inflammation of the parotid glands ascribed by Stockfleth to bad pastures, but his description rather points to actinomycosis or some musculo-fibromatous condition. In such cases inflammatory appearances develop slowly; hard, indurated, slightly painful swellings first appear, scarcely increasing the size of the part, but later abscesses form and discharge a thick pus; the adjoining lymph glands swell, contributing to the serious difficulty in breathing and swallowing which finally sets in. The patients rapidly go back in condition, and are disfigured by the formation of salivary fistulæ. Even with liberal feeding complete recovery is uncertain, and it is generally desirable to prepare the animals for slaughter. Thiernesse described a disease in dogs similar to parotitis, but withstanding all ordinary curative methods, and for which he 
recommended complete extirpation of the gland. In similar disease occurring in horses, the same surgical treatment must be adopted. A case of chronic inflammation of the submaxillary gland in a horse occurred in Möller's clinique. The swelling appeared slowly in the region of the larynx, produced perforation and a permanent fistulous wound. A long, hard, painful swelling was found extending upwards in the course of the submaxillary gland, and a fistulous opening below in the throat, from which a canal ran throughout the greater part of the swelling. Healing was effected by passing a seton needle upwards through the swelling, making an opening, and inserting a drainagetube. Under chronic inflammation of the tongue must be included infection with bothryomyces (horse) and with actinomyces (ox). The former are rare, and may be surgically treated.

Treatment. Owing to the specific character of acute parotitis, cold applications are usually contra-indicated; while warmth, in the form of Preisnitz's poultices, inrubbing of camphorated oil, and warm applications, are to be recommended. Warmth either leads to dispersal or abscess formation, and, in any case, shortens the progress of the disease. When marked fluctuation appears the part had better be opened, but this may be needful earlier if dyspnoea threatens; and where breathing is much interfered with, tracheotomy becomes imperative. In ordinary circumstances distinct fluctuation is waited for, thus avoiding the danger of the incision wounding large blood-vessels or nerves, and of producing salivary fistulæ. The cut is made parallel with the large blood-vessels and nerves, and the proceeding conducted as in opening a sub-parotideal abscess. Caution is required in employing the often recommended trochar, for injuries to large blood-vessels and considerable bleeding are not altogether avoided, even by its careful use. As the contents of the abscess are often under high pressure, and spurt out in a stream, Stockfleth recommended maling the opening from the opposite side, and standing on a block of wood during the operation. The method described in the next section is preferable.

With chronic parotitis, little can be done. Cattle should be prepared for slaughter without delay, because difficulty in breathing and swallowing: often appear later, and make feeding absolutely impossible. Abscesses should be opened and their cavities washed out with 10 per cent. chloride of zinc, or similar disinfectant. In valuable animals, or such as have no slaughter value, total extirpation of the parotid may be adopted. In acute inflammation of the submaxillary gland and in chronic diseases, extirpation of the gland may be necessary, but is attended with great difficulty, especially in ruminants. In inflammatory diseases of the ducts of these glands endeavour should be made to render patent the buccal opening of the gland, give exit to its contents, and thus diminish the risk of abscess and fistula formation. 


\section{(3.) ABSCESS FORMATION IN THE SUBPAROTID LYMPH GLANDS.}

The lymph glands lying below the parotid often become diseased, especially during the course of strangles, sore-throat, and other complaints, a phlegmonous inflammation developing, and gradually leading to abscess formation. In cattle and dogs the condition is oftener due to injuries of the pharynx by foreign bodies; in swine to specific infections, like those of tuberculosis, swine-fever, and actinomycosis.

Symptoms. Swelling appears in the parotid region, sometimes close under the ear, more frequently, however, in the lower posterior border of the gland, and usually spreads over the upper portion of the neck. The swelling increases, producing difficulty in breathing and swallowing, while the head is extended and held towards the sound side. Slight fever exists. Where both sides are affected, dyspnœea often develops to such a degree, especially during inspiration, that suffocation threatens, and tracheotomy becomes necessary. The difficulty in swallowing is attended by the danger of mechanical pneumonia.

At first the swelling appears hard and firm, and painful on pressure. Gradually the centre bulges, and in eiglıt to fourteen days unmistakable fluctuation may be detected. Failing an artificial opening, perforation occurs spontaneously in a few days. This commonly takes place externally, but at times the abscess breaks into the pharynx, and danger ensues of pus flowing into the trachea and bronchi, and producing fatal pneumonia. In a few cases rupture takes place both inwardly and outwardly. From the external wound there then flows pus, mixed with necrotic material, saliva and particles of food and water, and the formation of a pharyngeal fistula becomes possible.

Diagnosis. The disease resembles parotitis and distension of the guttural pouch. From parotitis it is distinguished by the swelling: being now diffused, and not confined to the parotid region; from disease of the guttural pouch, by the special characters of such attacks, by the progress of the case and the presence of fever.

Treatment. Early opening of the abscess is desirable, but the use of sharp instruments for the purpose is contra-indicated, as, owing to the displacement of parts, the parotid may be wounded, producing salivary fistula, or the great blood-vessels injured, causing fatal bleeding.

Viborg describes the following excellent method, which is simple and without danger. A twitch having been applied, and the seat of operation cleansed and disinfected, an incision is made through the skin over the highest point of the swelling, and, if possible, below the edge of the parotid, the underlying fascia divided, and the forefinger inserted. The thick connective tissue or gland structure is now broken down, the free 
hand meanwhile pressing on the swelling and guiding the inserted finger towards the abscess. This attained, a strong thrust breaks it and allows the escape of a stream of thick, creamy pus, which often spurts to a distance of several yards. To avoid being struck, it is better to place oneself on the opposite side. It may be necessary to operate on both glands. Some operators prefer to make the incision at the lower border of the parotid, below the submaxillary vein. The finger is then passed upwards and inwards; sometimes the abscess can only be opened by using a closed pair of round-pointed scissors, as the tissue is very resistant. This method gives equal security against injuring the parotid or important vessels, and gives a depending orifice.

With drainage of the abscess, fever falls, and difficulties in breathing and swallowing disappear. Healing occurs in eight to fourteen days, but complete closure of the abscess sometimes requires from three to six weeks. Warmth and moist applications (Preisnitz's poultices) further the breaking down of the swelling; the pus is removed by pressure and washing out the cavity with carbolic or sublimate lotion; further treatment is not usually necessary. This procedure, as already stated, is preferable to the use of sharp instruments, and especially to the oft recommended trochar. Cadiot and Dollar describe a case of "cold abscess" in this region which had existed for three months. A little inodorous whitish mucous discharge ran from the nostrils. A swelling occupied the submaxillary and laryngeal regions and extended on either side of the parotis. Swallowing was difficult, and the animal ate and drank slowly. The swelling was opened as above described, a pint of whitish laudable pus evacuated, and a drainage-tube inserted. Recovery was rapid. ("Clinical Veterinary Medicine and Surgery," p. 336.)

\section{(4) SALIVARY CALCULI AND CONCRETIONS.}

Calculus deposits are sometimes encountered in the ducts of the salivary glands, especially of the parotid, in horses and cattle, and may attain the size of a goose's egg. They are commonest in the horse, ass, ox, and sheep. Schumann discovered one in a horse which weighed over 7 ounces, and Stockffeth a similar one of $12 \frac{3}{4}$ ounces, but calculi have been found weighing 75 ounces. Their form is oval, colour greyishyellow, surface usually smooth, though where several are together they show facets. In herbivora, according to Lasaigne, they consist of 80 to 90 per cent. carbonate of calcium, 3 per cent. phosphate of calcium, and 9 to 10 per cent. of organic substances, composed chiefly of salivary corpuscles and bacteria. In men and carnivora, phosphate of calcium is an important ingredient. Foreign bodies entering the salivary duct, or injuries of the mucous membrane, are the usual immediate causes. The v.s. 
cut surface of such concretions often presents a stratified appearance, and a central nucleus of awns, straw, \&c.

Symptoms. The concretion is only remarked after it has attained a certain size. It appears as a hard, sharply defined, slightly movable swelling, generally lying on the outer surface of the under jaw, close in front of the buccal opening of Stenson's duct, but sometimes on the posterior border of the under jaw. The salivary duct is usually distended behind the swelling, and when the flow of saliva is entirely shut off the gland is enlarged. Inflammation is seldom present, but may appear and lead to formation of abscesses.

Treatment consists in operative removal. Small concretions may perhaps be forced through the duct, which will necessarily be somewhat fissured; the larger require an incision to be made in the duct. In this case a transverse cut is preferable to a longitudinal one, on account of its healing more easily, and not so frequently leading to salivary fistula. Recovery is hastened by observing strict antiseptic precautions before and during operation, carefully suturing the wound, and withdrawing food for one to two days. Bayer removed a concretion from the duct through a longitudinal incision, and after closing the wound with Lembert's suture, effected healing by first intention. In some cases, where the calculus is lodged very near the opening of the duct, it may be removed by incision from the buccal cavity. Such cases are rare, but the fact is worth remembering, as this method avoids the occurrence of a salivary fistula.

\section{(5) SALIVARY FISTULA土.}

Wounds of the salivary glands and their ducts often fail to heal, because the continual flow of saliva pushes aside the granulations and hinders closure. The gland epithelium finally unites with that of the outer skin, and through the opening so formed saliva flows continuously (salivary fistula). A distinction must be made between fistulæ of the salivary gland and of the salivary duct. The latter occur most frequently in Stenson's duct. Although the general condition of the animal is only slightly affected, much saliva escapes during eating and mats the hair of the cheek, finally producing a blemish. Fistulæ of salivary glands heal more easily than those of salivary ducts, - sometimes, indeed, without treatment of any lind.

Treatment aims at closing the external opening. This may be done by cauterising with nitric acid, silver nitrate, concentrated carbolic acid, or the actual cautery. A scab is thus formed, which checks the outflow of saliva, allows the formation of granulations, and promotes cicatrisation. 'These methods sometimes fail at first, and require to be repeated. The draw-purse suture may prove useful. 
The treatment of fistule of the salivary duct is more difficult. Before recovery can take place, it is necessary to provide for the exit of saliva
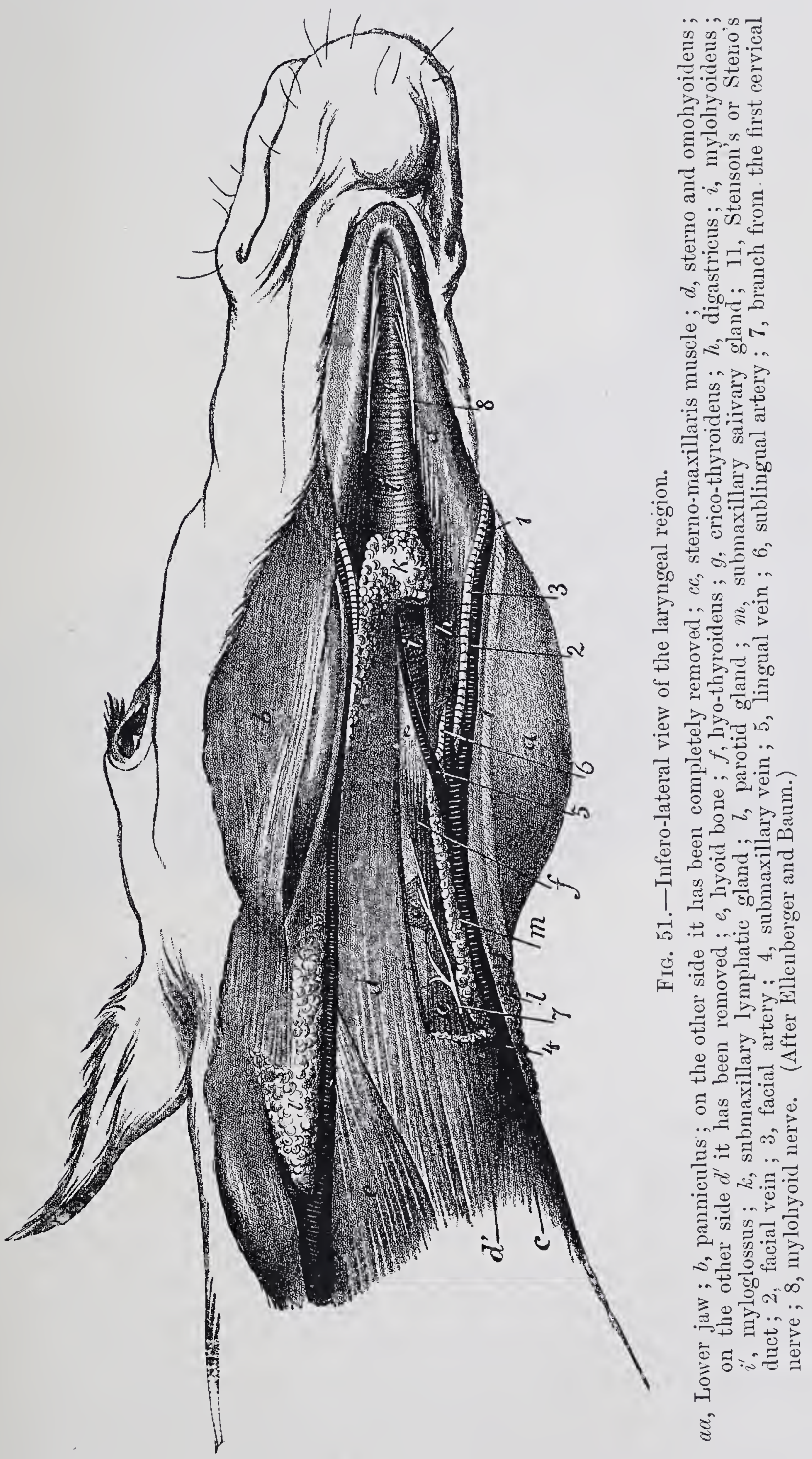
into the mouth. Where stricture of the duct occurs, as it often does, at a point between the fistula and the natural opening, this must be remedied. Lindenberg recommends passing a strong probe, and then attemping to reclose the walls of the fistula by caustic or the cautery. The resulting inflammatory swelling may close the wound, but healing will be more assured if a stitch be inserted. The purse-string stitch is the best. With a strongly curved needle a thread is passed through the

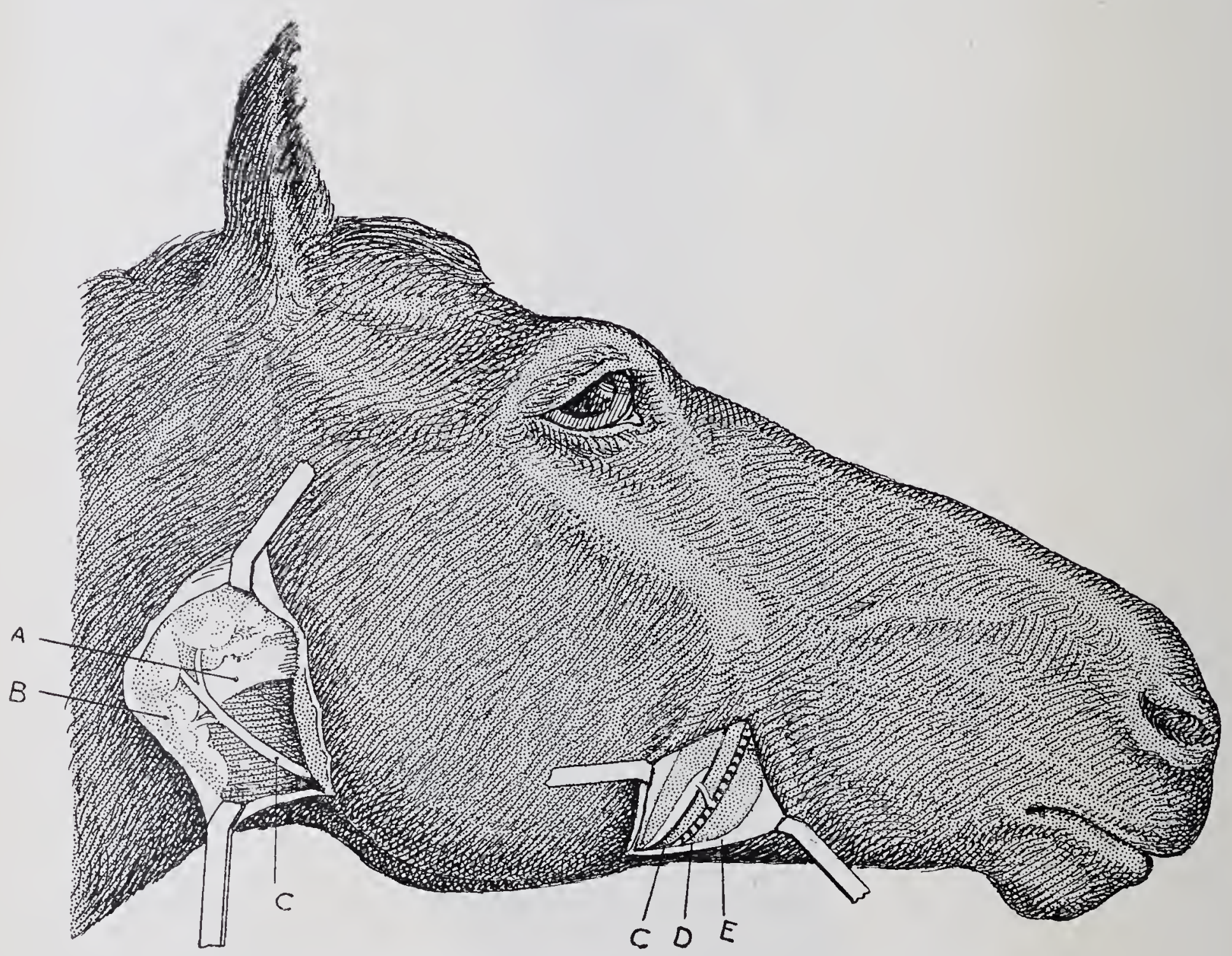

FiG. 52. - Parotid gland and Stenson's duct. (Semi-schematic.)

$\Lambda$, Tendon of the stylo-maxillaris ; B, parotid gland ; C, Stenson's duct. The lines D and E should be prolonged. They are intended to point to the facial vein and artery-the latter indicated by the strong cross-shading, the vein being next it towards the left.

skin around the opening of the fistula, and the margins thus brought together. Where the natural opening into the mouth cannot be renewed, an artificial conduit must be provided. Stockfleth recommends exposing the duct behind the fistula, and piercing the cheek in an oblique direction with a trochar. The free portion of the salivary duct is introduced into this opening, and made fast with a suture. Lafosse and Hering pass a thread through the cheek by means of a trochar, and fasten the ends together in the corner of the mouth. After the thread has remained in position for some weeks it is removed, and the opening closed with a suture. In man a thin drainage-tube or piece of lead-wire has been used in a similar way. Labat, in a horse, kept the artificial opening 
free by means of a simple plug of tow, and so produced healing. Should this procedure not be attended with success, destruction of the function of the gland alone remains. For this purpose one of the following methods may be adopted :---

(1) Tying the salivary duct above the fistula. The duct is isolated between the fistula and the gland, and tied with a stout thread. A strong ligature is essential. The animal should fast for the next twentyfour to forty-eight hours, and then receive only fluid nourishment. Soon afterwards swelling of the gland appears (tumour salivalis), and is followed by gradual atrophy. Not infrequently, however, the ligature cuts, to prevent which a stout ligature should be chosen. Bassi observed great dilatation of Stenson's duct after ligation; he applied a second ligature above the swelling, but a fistula of the salivary duct resulted. He then injected 6 drachms of ethyl alcohol into the gland on two occasions, at intervals of two days. The gland was destroyed and the fistula healed.

(2) Transverse section of the duct, after being tried by Reynal and F. Günther, was again recommended by Gerlach, while Siedamgrotzky and Harms have also tested it, though Harms has lately questioned its usefulness. They found that the end of the duct became closed through inflammatory processes, and that the duct itself afterwards atrophied. Even this method, however, sometimes fails.

Harms very appropriately points out that experiments, although successful on healthy horses, do not demonstrate the efficacy of similar treatment in diseased ones. Reynal found that it was not always possible to produce adhesion of the duct by section. The fact must not be lost sight of that in fistula of the salivary duct the opening of the duct into the mouth is generally occluded. Permanent closure of the duct gradually leads to atrophy of the parotid. Möller saw a horse whose right Stenson's duct was dilated to the thickness of a man's thumb, and, was without opening into the mouth. The gland had entirely disappeared, and the parotid region presented a marked depression.

(3) Injection of irritant fluids into the gland. Haubner recommends liquor amm. caust. 10 to 15 per cent. Tincture of iodine, creosote, nitrate of silver, \&c., have since been used. Bassi injected 30 per cent. of alcohol; Labat the following mixture,-20 to 40 per cent. of tincture of iodine, 1 per cent. iodide of potassium, and 60 per cent. of water; Delamotte, 50 per cent. tincture of iodine; Bergeron, a 20 per cent. solution of lactic acid. Concentrated tincture of iodine usually produces severe inflammation of the gland, sometimes ending in necrosis, and therefore diluted solution of iodine in iodide of potassium is to be preferred. The injection can be repeated if necessary. Abscesses often result, but in no way interfere with success. 


\section{(6) TUMOURS OF THE SALIVARY GLANDS.}

The nature of new growths occurring in the salivary glands of animals has not hitherto been fully or sufficiently investigated. In man, connective tissue tumours, such as sarcomata, fibromata, and enchondromata, are most frequent. The tumours so common in grey horses are usually melano-sarcomata, and in cattle actinomycosis. As little is known of the causes of these new growths as of other tumours. Cohnhein refers the development of enchondroma to cartilaginous fragments of the branchial arch. Actinomycosis depends on infection.

Their sharply defined appearance, slow development, and the absence of inflammatory symptoms, render the diagnosis of tumours comparatively easy. Their precise nature, however, can usually only be determined by microscopical examination. Actinomycosis will be referred to later.

Treatment must clearly be of an operative character. Simple, sharply defined tumours can easily be removed with knife or scissors. By careful suturing and appropriate treatment of the wound, it is easy to avoid producing salivary fistulæ. Large and extended tumours may necessitate removal of the entire gland. Total extirpation of the parotid is attended with danger, and therefore should only be resolved on in case of pressing necessity, as when dyspnœa is produced by the tumour, or when exceptionally valuable animals, which cannot be slaughtered, have to be dealt with. Leblanc is said to have first performed this operation, and to have employed it both for removal of tumours and for the cure of salivary fistulæ. Important vessels and nerves-the carotid and jugular, with their branches-and the great nerve stems (nervus facialis) must be avoided. Provision must, however, be made for ligaturing vessels. The operating place should be well lighted, and antiseptic precautions are, of course, necessary.

The skin is divided from the upper to the lower end of the parotid, and where adherent to the tumour, is removed. In large animals a second incision is usually carried in a backward direction perpendicular to the former, and starting from about its centre. The fascia underlying the skin is divided and separated as far as possible from the parotid. The gland, thus exposed, is now separated from its surroundings, beginning at the lower end, so that large bleeding vessels may be more easily dealt with. For the same purpose a thread may be passed around the carotid, which, in case of need, can be used as a ligature, though the precaution is almost unnecessary if two or three torsion forceps are at hand. As the recurrent nerve may easily be injured, it is better, in valuable horses, to avoid exposing the carotid. The gland should as far as possible be separated with blunt instruments, assisted by the fingers. Vessels 
are ligatured as exposed, the larger doubly ligatured and then cut through. In this way, and by free use of sponges or dossils of lint, the field of operation is kept clear, and the gland removed without dangerous bleeding. The wound is then cleansed with antiseptic fluids, dusted with iodoform, covered with jute or wood wool, and the edges brought together with sutures. In twenty-four to forty-eight hours the stitches are loosened, the dressings removed, the wound washed out, dusted with iodoform and tannin, and the whole treated as an open wound. Healing will be complete in three or four weeks. To allow the air free entrance to the wound surface, the flaps should be drawn back with a few stitches, and pledgets of wood-wool wadding inserted in the depths of the wound, so as to take up the secretions rapidly. The external skin should also be divided, to permit discharges to escape rapidly. The insertion of a drainage-tube may be required. If during the next few days portions of the wound appear damp, they should be dried with wadding, and powdered with a mixture of iodoform and tannin $(1-3)$. Where a dry scab has formed, the wound is better left undisturbed. Dry scabs, firmly adherent to the underlying tissues, should under no circumstances be removed.

\section{(7) ACTINOMYCOSIS IN THE PAROTIDEAL REGION.}

Although actinomycosis of the parotideal region is also occasionally seen in horses and swine, it is very much commoner in cattle. By proliferation of the connective tissue new growths are slowly formed in the subparotideal and retropharyngeal regions. These were formerly erroneously regarded and described as tumours. Their true cause is to be sought in local infection with the actinomyces fungus.

The parasites are probably taken along with the food, and find entrance into the mucous membrane and deeper-lying tissues through wounds caused by the rough character of the food. The circumstance that herbivora most frequently suffer from the disease, supports the theory that infection results in this way. Johne detected vegetable material invaded by actinomyces in the tonsils of swine; Grawitz found portions of a head of corn in the lower jaw of a calf, which were surrounded by granulation tissue. Bang found the disease more widely distributed where much barley straw was given as food. Fischer describes a wound of the human tongue produced whilst chewing some barley; ray fungi were found in the abscess which resulted, and the portions of grain discharged were also covered with them. According to Imminger, epizootics of aphtha favour infection, for he found that after these actinomycosis broke out over large areas. Frick considers that the fungus is often carried by the fodder, in proof of which he relates the 
following:-As a protection against anthrax certain farmers in his practice were in the habit of thoroughly steaming all cattle food before giving it to the animals. Although in other parts of the same district actinomycosis, especially of the parotid region, was extremely common, the farms on which this practice was observed remained entirely free of it.

The pharyngeal mucous membrane is probably the commonest point of entry of the actinomyces fungus, and the primary swellings almost always affect the pharyngeal walls, though infection may occasionally occur through the skin. One certainly sees cases of infection in that part of the neck which oftenest rubs against the manger. Actinomycotic

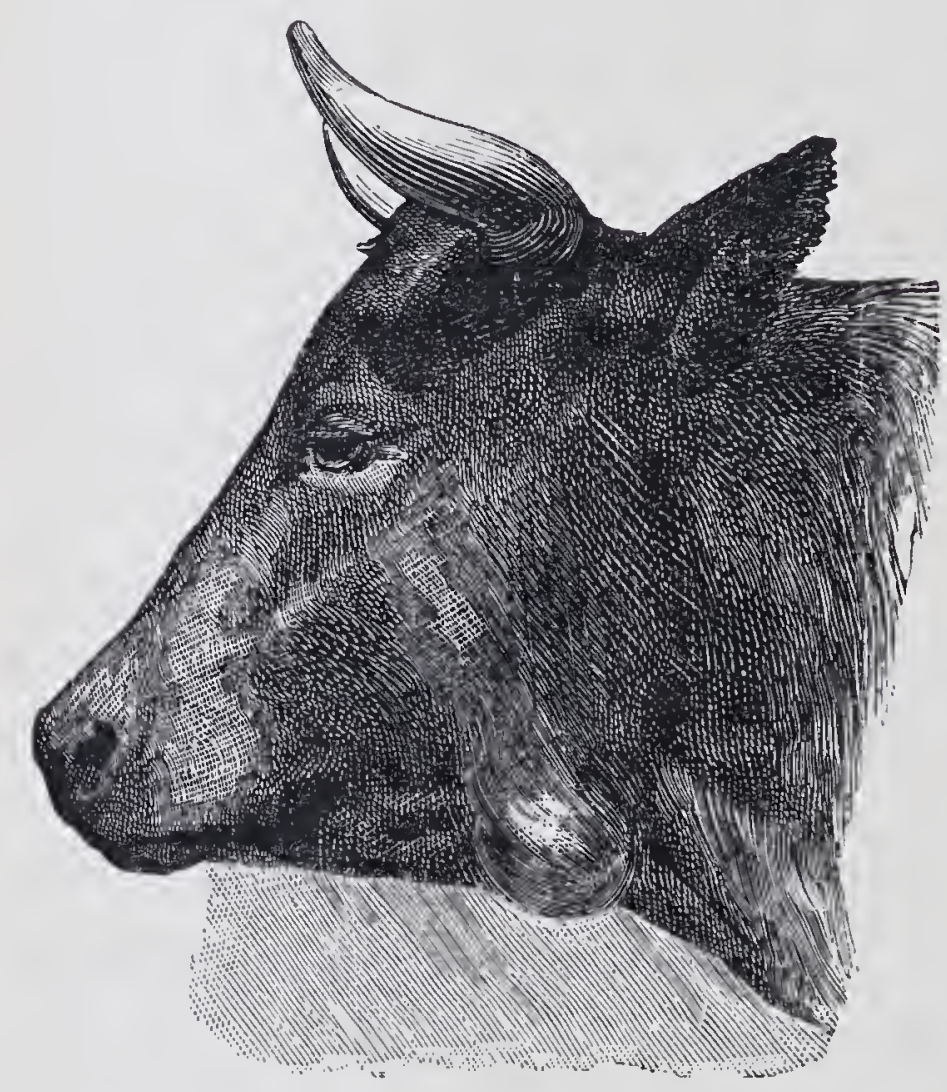

FIG. 53. - Actinomycosis of the parotid gland in all ox, from a photo by Esser. abscesses of the parotid gland discharge infective pus on the edge of the manger, and the tiny splinters of wood always present there penetrate the skin and implant the fungi in any fresh animal placed in the same stall. This mode of infection is, however, rare. The disease varies greatly in frequency in given districts and in different years. On one farm almost every animal may be affected, whilst on another in the immediate neighbourhood the disease may be practically unknown. In certain years the disease appears almost epizootic, in others it is strictly sporadic. The reasons remain obscure, but are possibly connected with the condition in which the fodder
is harvested.

The symptoms consist of a small, insensitive, sharply defined, and somewhat hard tumour, from which a cord-like thickening extends into the depths. The swelling gradually becomes larger, fluctuation appears, and if the slin is without pigment, the yellow-coloured contents may show through. Incision liberates a thick, muco-purulent fluid, in which careful examination discovers the above-mentioned yellow grains. If not opened, the tumour breaks spontaneously. Cicatricial contraction very seldom brings about healing. As a rule, the growth proceeds, sooner or later producing, according to its position, functional disturbance, difficulty in swallowing, dyspnœa, and finally, in consequence of advancing exhaustion, death. 'The swellings in the parotid region occasionally attain a great size, sometimes becoming as large as a child's head, but such 
growths require several months. From the wound made by opening the abscess, grows a soft, dark-red mass of granulation tissue, which bleeds readily, and contains large quantities of the specific parasites.

Prognosis must depend on whether the new formation can be completely removed, which is seldom the case. Lasting improvement can only be expected where treatment has been commenced early, that is, before infection has become general, and treatment is more successful in disease of the skin or subcutaneous tissues than where deeper-seated structures or the pharynx or tongue are involved. Disease of the bones of the upper or lower jaw, said by Esser and others to arise from the first molars, is always very intractable. Reference should be made to the sections dealing with these structures. Cases affecting the parotid region, unless recent, are generally regarded as hopeless. Whilst Esser supports this view, Preusse has observed recovery in forty-one out of forty-five animals. It is of prime importance to remove the tumour early, before it has attained, say, the size of the hand, and while it is still circumscribed. If left longer, the growth recurs, even after careful removal. The results of iodine treatment have lately proved so remarkably favourable, and been so well spoken of by different practitioners, that the prognosis of this disease must now be looked on as much more hopeful than formerly.

Treatment. Prophylaxis demands the avoidance of suspicious fodder ; but this is troublesome and scarcely practicable, on account of the difficulty of recognising whether such suspicious materials are infected with actinomyces. In dealing with cattle it may be possible to steam or boil most of the food, and special care must be taken that the abscesses and their contents do not contaminate any food. Although direct infection has not been clearly proved, actinomycosis occurs in man, and veterinarians and those handling infected subjects should hence exercise due caution.

A case is related by Meyer where a peasant's son who tended some cows affected with actinomycosis himself became the subject of actinomycosis of the jaw. Owners and attendants of diseased animals should therefore be warned.

Of the various methods of treatment, operation is certainly the oldest. It consists in total extirpation of the infected connective tissue.

In operating the animal is cast, the head placed on one side, and the nose forced downwards. The operator works round the tumours, always operating in the still healthy tissue, and removes the growth as far as possible with the fingers, assisted by knife and scissors. In this way severe bleeding is avoided, and small vessels can afterwards be ligatured. For checking parenchymatous bleeding, Esser and Preusse recommend the actual cautery, which has the additional advantage of destroying at 
the same time any infectious material that may have remained. The wound is washed out with carbolic solution, filled with some aseptic material (salicylic wadding, Esser), and the skin sewn up. When the stitches are removed next day, the parts are washed out with a disinfectant solution, and treated as an open wound. Should the condition return, repetition of the operation is seldom of any use.

Injections of iodine tincture and 1 per cent. sublimate solution have been recommended, but are tedious and unreliable. In some cases deeply incising the swelling and daily painting the surfaces with iodine tincture (not solution) is useful. Destruction of the fibrous tissue of the growth with arsenic has been highly spoken of. Arsenic, however, can only be used advantageously in the parotid region and in cases where the growth does not extend to the larynx or pharynx. Small solid fragments of arsenious acid weighing from 3 to 7 grains are thrust into the midst of the growth in the direction of the hard cord which can be felt extending into the depth. A passage can first be made with round-pointed scissors, and the fragment of arsenic inserted with slender forceps. Another method consists in incising the skin, passing a fairly wide trochar and canula to the hase of the growth, removing the trochar, inserting the fragment of arsenic into the canula, and thrusting it to the bottom by replacing the trochar. The entire instrument is then withdrawn. In from six to twelve weeks the diseased tissue sloughs a way, leaving a wound which heals by granulation. Worling oxen may be used throughout the treatment. Arsenic has also been used in the form of an ointment.

Within recent years the internal use of potassium iodide has been warmly recommended. One and a half to $2 \frac{1}{2}$ drachms of the salt, dissolved in water, are given daily. Under this treatment the swellings diminish, but it must often be continued for many weeks before recovery is complete. Moreover, success is not invariable, though the real value of the treatment cannot be called in question. The other methods can always be tried in case of failure.

Edgar used the red iodide of mercury dissolved in water by the addition of potassium iodide, in place of the latter salt alone.

\section{Y.-DISEASES OF THE FACE AND LOWER JAW.}

The face is here regarded as comprising that section of the head whose base is formed by the upper jaw and the malar and lachrymal bones.

\section{(1.) FRACTURES OF BONES OF THE FACE.}

On account of its sheltered position, the upper jaw is seldom fractured. In horses fracture is often due to dental operations, and in dogs to bites; less frequently to such external violence as kicks, or collision with fixed 
objects. During the progress of dental operations fractures result if the animal, especially when operated on standing, suddenly moves, and the operator does not follow the movement with his forceps. Incautious attempts to loosen the tooth with the forceps may also produce fracture of the submaxilla. In compound fractures the broken ends or splinters of the bone are found in the wound. When the facial plate of the superior maxilla is thus affected the corresponding sinus is opened, and air passes in and out during respiration.

The symptoms consist of swelling, salivation, defective and painful mastication; the molar's are found to be loose, and the gums wounded; crepitation may sometimes be detected.

Prognosis depends chiefly upon whether the fracture is subcutaneous or complicated. In the former instance, recovery generally occurs in three weeks; in the latter, a much longer time is required. Complications result both from injury to the skin, and to the mucous membrane of the mouth and gums. In compound fractures with exposure of the superior maxillary sinus or alveoli of the teeth the prognosis should be cautious.

Treatment of subcutaneous fractures only requires restriction to soft food. Removal of loosened teeth, which may easily convert a simple fracture into a compound one, should be deferred. When the gum remains attached, and the root is not exposed, loose teeth again become firm. Fractures of the superior maxilla, complicated with cutaneous wounds, must be treated by antiseptic methods. If pus formation has commenced, strict cleanliness must be enforced, and loose splinters of bone removed. Complicated fractures, involving wounds of the gum, require similar precautions. Even with abundance of soft food, animals rapidly loose condition, and economical considerations often suggest that cattle be killed rather than treated. In a thoroughbred mare Möller saw transverse fracture of the superior maxilla nearly above the roots of the incisors. The fracture was caused by falling on the mouth, and all the incisors were displaced downwards and backwards towards the tongue. As the fracture had already existed for several days, replacement could not be immediately effected, but was at length attained by the attendant, according to instructions, daily exercising pressure on the dislocated teeth. Being gradually brought into normal contact with the lower incisors, the act of mastication helped to maintain them in position, and complete recovery resulted.

The gravity of fractures of the malar bone depends on their position. When involving the orbital process, they resemble those of the orbital process of the frontal bone and the zygomatic process of the temporal bone. When affecting other portions, they may open the superior maxillary sinus, or loosen the attachment of the masseter muscle, causing difficulty in feeding, while the fragments may become 
separated, and muscular contraction prevent their being replaced in position.

Diagnosis of fractures of the malar, caused by external violence, is only difficult when much swelling exists. If not due to cellulitis, to the presence of new growths, or disease of the superior maxillary sinus, such swelling should arouse suspicion of a fracture, especially if there be any considerable difficulty in mastication. Where the superior maxillary sinus is laid open, or the zygomatic ridge injured, doubt can no longer exist. Damage to the alveoli of the upper molars, or extensive tearing of muscular insertions, give rise to difficulty in feeding. Subcutaneous fractures of the malar bone, which are of rare occurrence, unite easily, and compound fractures only cause difficulty when the aveoli of teeth are exposed, and purulent alveolar periostitis results.

Treatment of subcutaneous fractures merely requires regulation of diet in many cases. When involving both sides, fracture of the premaxilla may result in the incisors and the bone being displaced downwards and backwards or in the bone being split and the fragments displaced laterally. In the former case a stout baton of wood can be inserted into the mouth and used as a lever to replace the bone and teeth, which then usually remain in position on account of the opposing row of teeth supporting them; in the latter case a shallow groove is formed in the corner incisors of each side and a thick copper wire passed several times around the six incisor teeth, supporting them and holding the two bones together. Compound fractures heal most rapidly under antiseptic treatment. Should purulent alveolar periostitis supervene, the affected teeth must be removed. In endeavouring to replace the fragments dislocated by the pull of the muscles of mastication, it has been recommended first to perform myotomy. But this entails the danger of making a simple subcutaneous fracture into a compound one; while, if already compound, exfoliation of bone may ensue. Further treatment is regulated by general principles. Reference may be made to fractures of the frontal bone, and diseases of the superior maxillary sinus.

Figs. 54 and 55 illustrate a plastic operation performed by Bayer to remedy loss of skin in two carriage horses which had run away and damaged the bone to such an extent as to necessitate removal of considerable portions. The skin having become adherent to the mucous lining of the sinus, it was necessary to dissect considerable flaps in order to cover the defects. The parts were carefully shaved and disinfected, and a slender paring removed from the edges of the wound to ensure fresh surfaces for union. In the first case an incision was carried from $d$ successively to points $f, g$, and $h$, and the flap dissected free from subjacent tissues, leaving it connected with the rest of the skin only at $a h$. The prolongation to $f$ and the large size of the flap were necessitated by 
the knowledge that contraction would occur. The edges a $c d f$ were then brought in contact with $a b c$, and $f g$ were united to $c d$ with closely placed sutures. Healing of the flap was perfect, and the exposed surface $h d \cdot f^{\prime}$ ? soon granulated and was finally covered by skin.

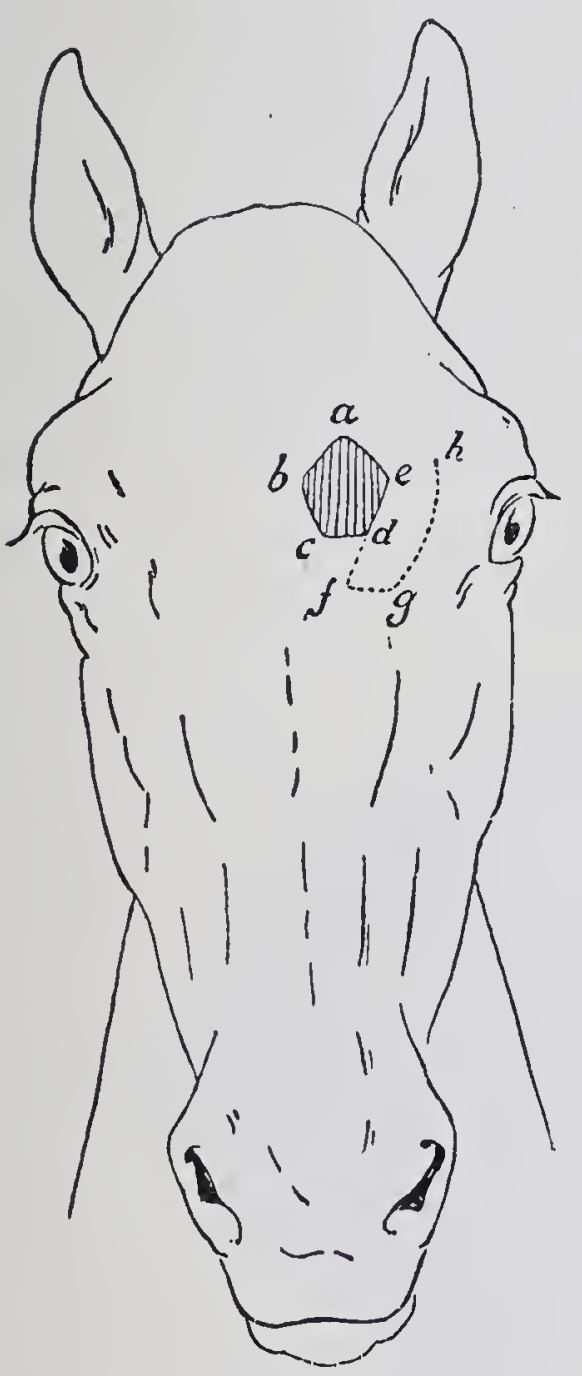

FI 54 .

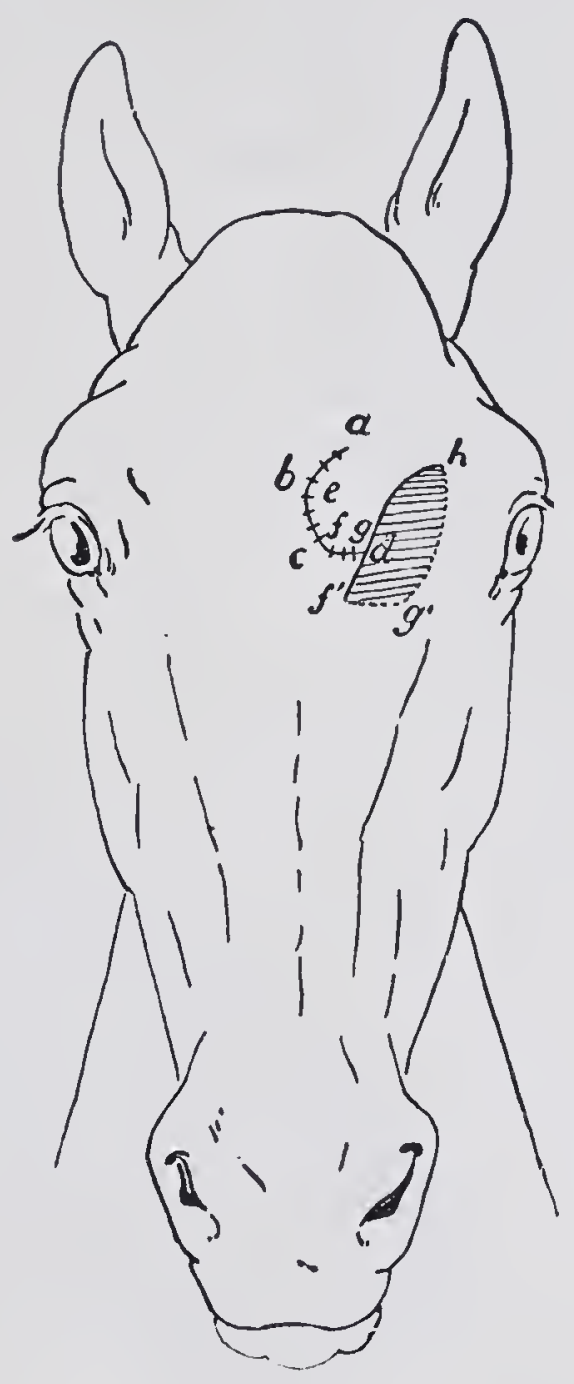

FIG. 55.

When, in consequence of injuries in the neighbourhood of the neck or withers, large indolent wounds are left, healing can often be effected

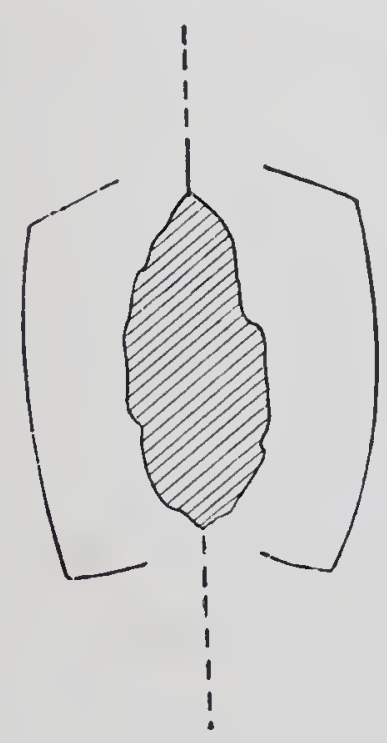

Fig. 56.

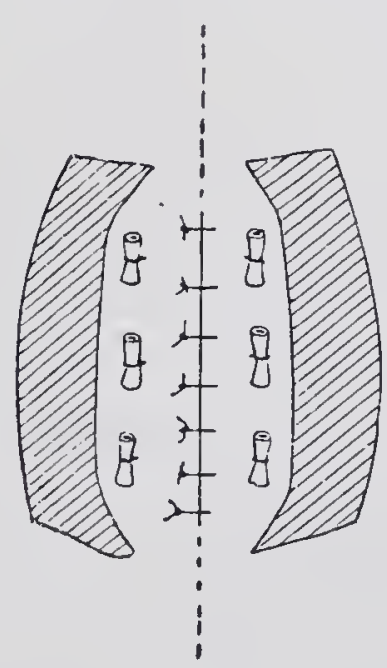

FIG. 57.

by incising the skin and subcutaneous tissues on either side of the wound, dissecting the slin free so as to form flaps, and uniting these in the middle line as indicated in figs. 56 and 57. 


\section{(2.) FRACTURE OF THE LOWER JAW.}

Of all the bones of the head the under jaw is the most frequent seat of fracture: this is true of all classes of animals, and notably of the horse. The causes are external violence, kicks and falls; occasionally the excessive action of the muscles of mastication, as when the mouth gag is applied to horses suffering from brain disease, or in the simultaneous application of the mouth gag and twitch. Hertwig considers that, under these circumstances, the animals are unable rightly to estimate the degree of contraction of the masseter muscles. Hering saw a case in the horse, caused by the clumsy use of the tooth chisel. In dental operations fractures may occur under the same circumstances as in the upper jaw. Transverse fracture of the body of the jaw in calves not infrequently results from violent manipulation during delivery. In a similar way fracture of the symphisis of the lower jaw may be caused by parturition hooks being inserted in the angle behind the body of the jaw. In staghounds they result from the prey striking out at the moment when the dog has fastened on to the hind-limb.

The fracture is sometimes subcutaneous, and remains confined to one branch of the lower jaw ; but not infrequently both branches break, and usually at the neck, where teeth are wanting. Vormeng noted brealkage of both branches in the middle line, an accident which occurs more frequently in foals than in older animals. Fractures of the border of the alveoli of the lower incisors are often seen, in consequence of the animal biting, being kicked, or falling and striking fixed objects. Fractures of the joint or coronoid process, although occasionally reported, occur less frequently.

Subcutaneous fractures of one branch of the lower jaw are not so readily diagnosed; suddenly developed difficulty in mastication is the chief symptom. In fractures in the region of the molars the teeth may continue to hold the fragments together in such a way that neither crepitation nor unusual mobility of the parts can be detected. In such cases one or more teeth may be found to be loose.

Symptoms. In transvere fractures of the body of the bone the chin and lower incisors hang limply and are abnormally mobile, while crepitation may be detected. Fractures of the coronoid process or joint, or in their neighbourhood, often interfere seriously with mastication, and may be mistaken for inflammation of the articulation. The broken fragments of the coronoid process are drawn upwards by the temporalis muscle, and can be detected on palpation.

Prognosis. Fractures in the median line and subcutaneous injuries of one branch unite regularly and completely in from three to five weeks. Double-sided fractures present the greatest difficulty, and often affect 
mastication so seriously that condition is lost in a marked degree. Transverse fractures of the body are difficult to set, and sometimes eventuate in formation of callus fibrosus, with sinking of the alveolar margin and protrusion of the tongue (fig. 58). Grün cured the above transverse fracture in a calf by applying a suitable splint and giving nourishment through an œesophageal canula. The case recovered rapidly. Fractures of the articulatory process, or in its neighbourhood, are the most serious. Those of the coronoid process unite, but sometimes by the formation of a callus fibrosus. Compound fractures of the lower jaw often prove very troublesome, but are not always incurable, as recorded instances show. Nor can fractures of the articulatory process be regarded

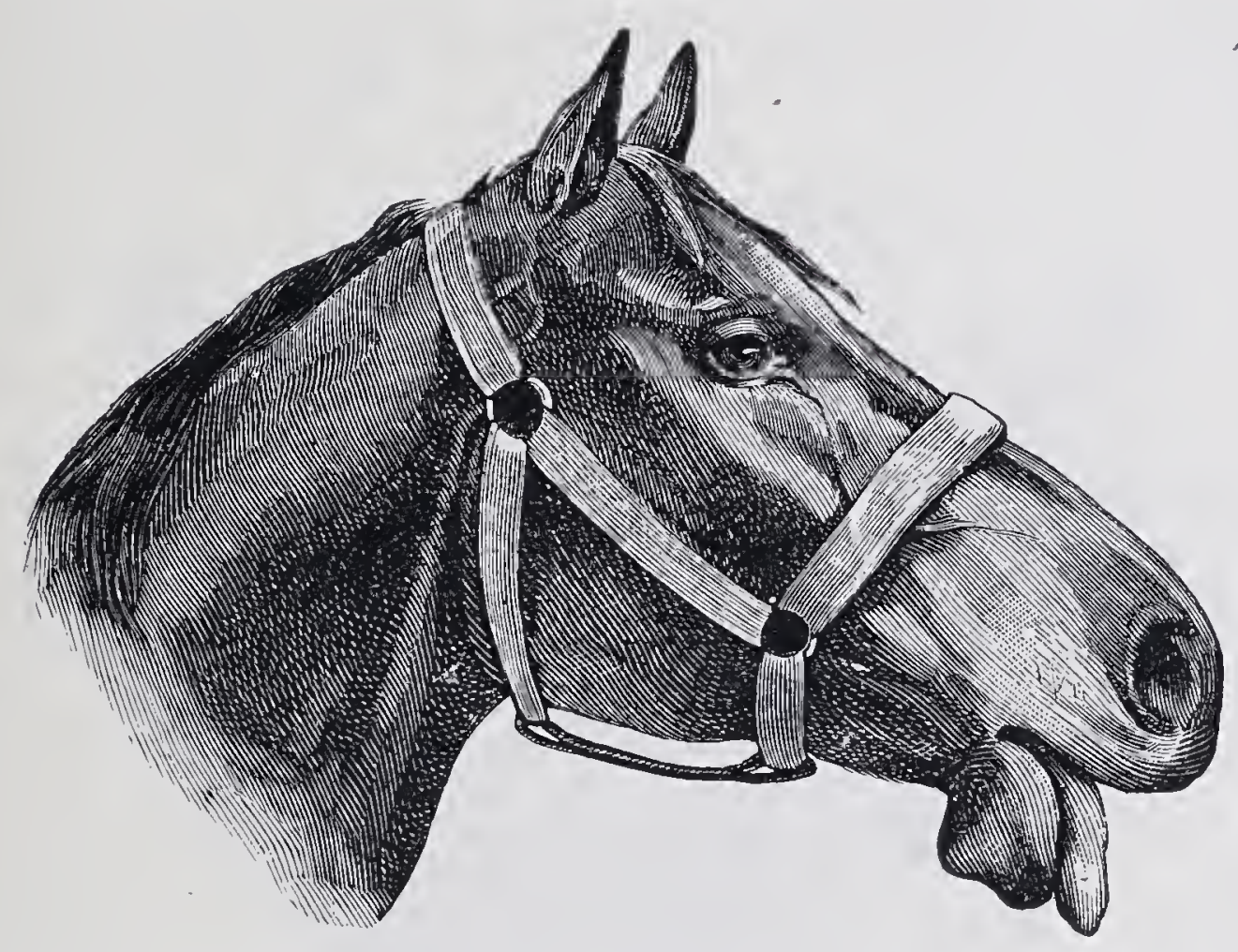

Fig. 58. - Incomplete union of fracture of the body of the lower jaw, from a photograph.

as hopeless, since Fröhner has shown that recovery may follow resection of the maxillary joint.

Treatment in simple one-sided fracture consists merely in the administration of soft food. Dislocated fragments must be brought into position. As long as the fracture remains confined to one branch no mechanical appliance is necessary; but when double-sided, considerable ingenuity is required. Prince recommends a wedge-shaped splint of wood, which fits into the submaxillary space, and is fastened with straps passed over the animal's neck and head. In small patients, and in valuable large animals, a similar splint formed of gutta-percha is pressed into the space whilst soft, so as to form an exact reproduction of it; its posterior broad end is supported against the animal's throat. Another arrangement is due to Mazza. Girard arranged tivo bars of iron, bound together at an angle, corresponding to the direction of the posterior borders of the lower jaw, and carrying at their point of union a spoonshaped extension for supporting the chin. 'The other ends were provided 
with rings for attachment to the halter or to a neck strap, while a strap passed over the animal's nose, about half-way up, secured the under portion of the splint in place. Instead of rods, Marrel used a gutter-like splint, formed of tinned iron, which fitted the posterior border of the lower jaw. On the lower part were four rings for fastening to the halter and to the animal's nose and head. Fractures in the inter-dental space of the lower jaw may sometimes be reduced and fixed, by using the teeth as points d'appui. Thus Delamotte bored a hole between the first and second molars, and carried a wire thence around the incisor teeth. The wire having been in position for three and a half months, the fracture was found to be united. More recently bone sutures have been successfully used. As a final resort, the body of the bone may be resected without destroying the power to masticate. This has been accomplished

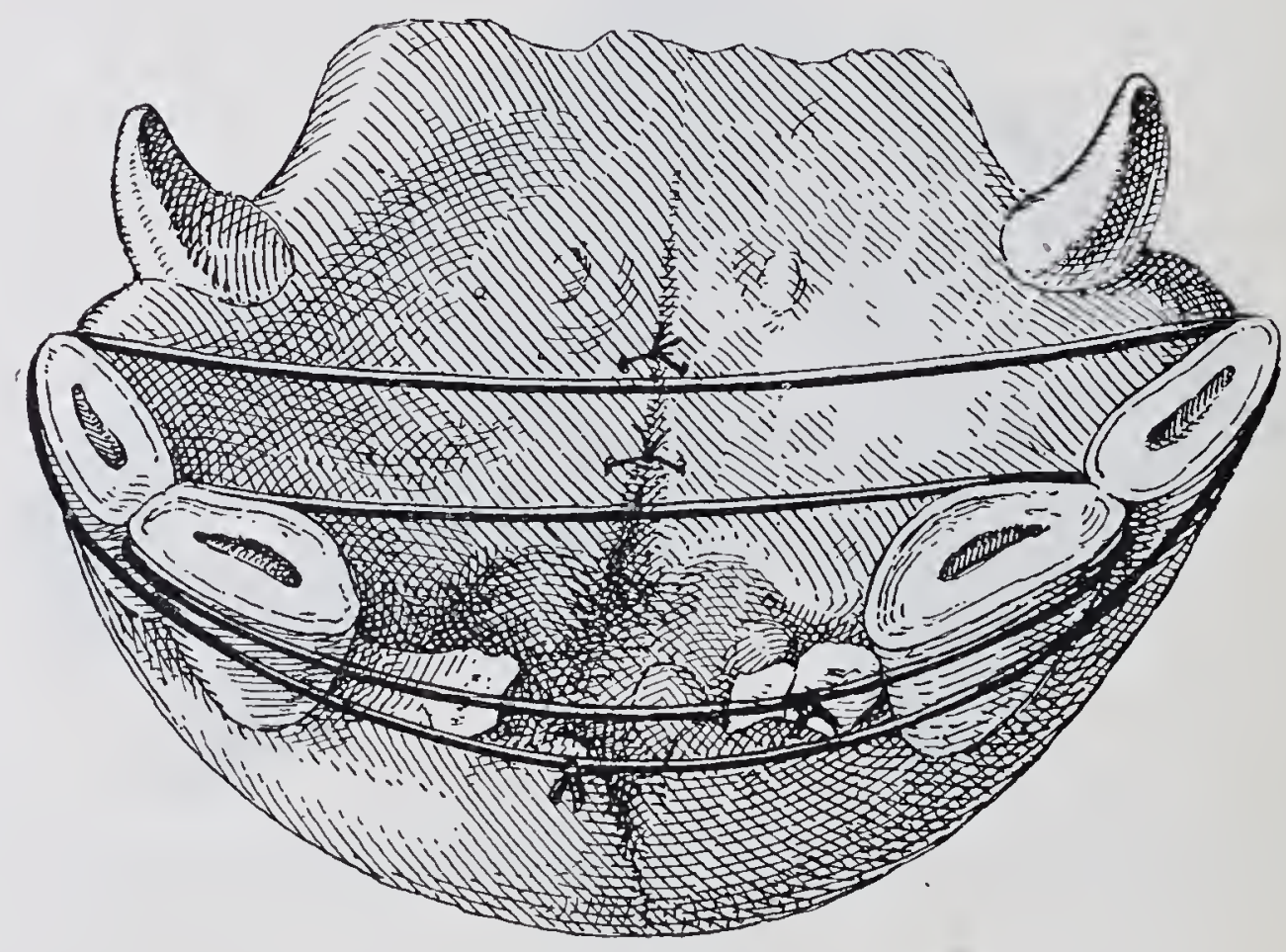

FIG. 59. - Fracture of the lower jaw united by wiring.

by more than one operator, both in the case of the horse and dog. In treating fractures at the height of the first molars, Ohlsen inserted an iron splint the shape of an ordinary magnet, which was pushed into the mouth legs-foremost, and embraced the back teeth, holding both splint and bone in position: unfortunately this method is liable to loosen the teeth. After attending to diet, treatment of fractures in the neighbourhood of the articulatory process becomes expectant. Hence it is generally advisable to slaughter cattle early, rather than await an uncertain healing process. It need scarcely be added that all compound fractures must be treated on antiseptic principles.

Littlewood saw double fracture of the lower jaw in a horse, the maxilla being broken near the symphysis, and between the second and third incisor teeth. The pieces of bone were replaced during anæsthesia, a hole drilled through them, and a metal wire so inserted as to fix them in position. The operation was completely successful. 
Cadiot and Dollar describe a case of double fracture of the lower jaw -viz., through the neck and through the branch under the masseter muscle-in an eight month old poodle, in consequence of a kick from a horse. A dressing formed of layers of linen smeared with pitch was applied, and further secured by bands of tarlatan passed over the head and round the neck. A muzzle was eventually applied. The patient was at first spoon-fed with liquid food and afterwards with chopped lean meat. Union was complete in one month, and the animal could take its ordinary food, though there was a slight deformity from callus formation.

\section{(3.) DISLOCATION (LUXATION) OF THE LOWER JAW.}

This occurs only in carnivora: the long coronoid process and limited mobility of the joint render it impossible in herbivora unless after fracture. Staghounds, and sporting and drovers' dogs most frequently suffer ; the first often, from being struck by the hind foot of the animal they are pursuing. Dislocation is frequently complicated with fracture. Sometimes it is confined to one side, but double-sided luxations also occur, thus resembling the condition usual in man.

Symptoms and course. The mouth hangs open, and can neither be closed voluntarily nor by external assistance. Salivation, protrusion of the tongue, and inability to eat are also present. Sometimes the ball of the eye is pushed forward by pressure of the dislocated coronoid process (exophthalmus). In one-sided dislocation the jaw hangs towards the sound side. The condition may be mistaken for paralysis of the lowe. jaw, and for the presence of foreign bodies in the mouth or between the teeth. But in paralysis, the lower jaw is passively movable, and the mouth can be closed by pressure, whilst the presence of foreign bodies is determined by local examination. As in rabies the mouth often hangs open very much as in luxation of the jaw, care should be observed in making the local examination. A favourable course is insured if professional assistance is sought early and no complications exist; but recurrences nevertheless occur, and animals should therefore not be used for some time after apparent recovery. Where much bleeding has taken place into the orbit, replacement of the prolapsed eye sometimes proves difficult.

Reduction is most easily effected by inserting a stick about $\frac{3}{4}$ of an inch in thickness transversely into the mouth, and pushing it well back. The operator then seizes the front of both jaws and presses them together, the stick acting as a fulcrum to the two-armed lever. The coronoid process is thus drawn downwards, and can be brought into normal position by pushing the dislocated jaw sideways towards the middle line.

v.s. 
In small animals both jaws are seized, and the dislocated coronoid process moved first downward and then backward; but care is required to avoid being bitten, and for this reason the first method deserves preference. Recurrence is prevented by giving soft food, interdicting bones, and applying a muzzle.

\section{(4.) INFLAMMATION OF THE ARTICULATION OF THE JAW.}

This articulation, on account of its exposed position, is often injured by blows or "treads," and purulent inflammation so caused. Cellulitis affecting neighbouring structures may extend to the joint, or it may become inflamed in horses which, in consequence of painful affections, lie continuously (decubitus). Gurlt found the joint immobile in a goat, on account of the formation of extensive exostoses. The condition had resulted from severe bruising. Dry chronic arthritis (arthritis chronica sicca) of this joint is also seen in horses.

Symptoms and course. Impaired mastication is the most frequent accompaniment. The mouth cannot be properly opened, whence the condition is sometimes mistaken for trismus, from which it may be distinguished by a swelling affecting one or other, but seldom both articulations. The lower jaw is generally displaced sideways, so that the grinding surfaces of the teeth do not exactly correspond, and patients, in consequence, quickly fall off in condition, and sometimes cannot take any food at all. They often develop the shear-like mouth. At the same time the animals rapidly fall away in condition, as might be expected. Sometimes the disease ends in anchylosis of the joint.

Bösenroth saw a case of this kind in a horse, and has described the post-mortem appearance. Several similar cases occurred in Möller's practice. In a dog, which suffered from inability to open the mouth, and showed extensive atrophy of the muscles of mastication, Möller found formation of new bony material in both articulations. This had been produced by an arthritis chronica. Siedamgrotzky has described a similar case.

The disease is of a very grave character, especially in animals which have to consume much food, such as working-horses and milch-cows. Cases like that described by Weiss, where a horse was able to masticate regularly after displacement of the inter-articular cartilage, are exceedingly rare. An error in diagnosis may possibly have occurred in the case described.

Treatment. The patient's strength must be conserved, suitable diet provided, wounds and inflammatory processes properly treated, and the ill effects of decubitus guarded against. So long as no purulent arthritis 
exists, recovery, as in Siedamgrotzky's case, may be expected. When suppuration has occurred, the parts must be washed out with sublimate solution, solution of iodine in iodide of potash, or with iodoform xther. Any fragments of bone must be removed. Re-section of the joint was successfully performed by Fröhner, but is only commendable as a last resort. Delamotte recommends injecting the cavity with carbolic or sublimate solution, and limiting the movement of the lower jaw as far as possible by means of a tightly applied nose strap. Fluid nourishment alone is indicated.

\section{(5.) PARALYSIS OF THE MUSCLES OF MASTICATION.}

\section{PARALYSIS OF THE LOWER JAW (SO-CALLED).}

A condition in animals has been described where, in consequence of diplegia of the masticatory muscles, the mouth cannot be closed. It may more correctly be termed paralysis of the muscles of mastication, or masticatory facial paralysis, as it is styled in man. According to present information, it seems almost entirely confined to dogs and cats, but Röll, Waltrup, and Lydtin have also seen it in the horse.

Both the masseters and the temporal muscle are supplied with motor twigs from the third branch of the fifth cranial nerve, which arises in the pons varolii. One-sided paralysis of this nerve is much less frequent in animals than double-sided paralysis, but double-sided paralysis in cats and dogs has been repeatedly described. The condition occurs almost invariably during the progress of rabies, in fact is seldom seen unassociated with it, and therefore its presence always awakens suspicion. Although Gerlach insisted on its acceptance as a sure sign of madness, dogs and cats thus affected often recover completely, the surest proof that Gerlach's statement is incorrect. Körber and Fröhner have noticed the same thing in dogs.

The anatomical changes causing the disease are at present unknown; they are probably due to some diseased condition in the pons varolii. As a great number of nerve centres exist in this portion of the brain, it is not remarkable that this disease is frequently accompanied by other nervous disorders. In the case described by Körber, paralysis of the pharynx and muscles of the throat and breast supervened. Tempel records a case where a fibro-sarcoma extending from the guttural pouch had pressed on the facial and trigeminal nerves, paralysing them and producing the above symptoms. But that isolated cases of paralysis of these motor nerves occur, and may recover, is shown by the following illustrations :-

A grey pointer was sent to hospital on the 1st February, 1883, with the report that it had received an excessive quantity of beer some days previously, 
and had afterwards slept for a long time. When it awoke next day, its mistress remarked that its tongue was protruding, and that it was unable to close its mouth. The animal appeared low-spirited; the under jaw hung flaccid; and when pressed into position again fell. The tongue was dry and hung out; food could not be taken, though pieces of meat placed in the mouth were swallowed with great relish. During the next few days the dog appeared quiet, somewhat apathetic, and lay continuously in a corner of his kennel. Five days later, the disease had already so far yielded to expectant treatment that the animal could again take nourishment regularly.

A similar case, which, however, appeared incurable, occurred in Möller's practice in 1884. A nine months old dog, used for drawing a barrow, showed complete paralysis of the nerve, could not withdraw the tongue, the mouth remained continually open, and in taking fluid nourishment or water, the head was thrust into the fluid as high as the eyes. The muscles of mastication, and particularly the temporal muscles, showed marked atrophy. The tongue and buccal membrane were insensitive to injuries; twitchings occurred in the muscles of the rump, like those often seen following distemper; no improvement appeared after lengthened observation and faradisation. As the owner objected to have the animal killed, a post-mortem unfortunately could not be made.

Röll and Lydtin saw cases of one-sided trigeminal paralysis in the horse. Röll states having found all three branches paralysed; the skin and mucous membrane of the affected half of the head and the cornea being insensitive. Salivation, difficulty in chewing, and accumulation of food between the cheeks and teeth existed. The mucous membrane of the nose, mouth, and conjunctiva was hyperæmic. Owing to inability to close the lids and protect the eye, corneal ulcers formed. Post-mortem discovered fatty degeneration of the roots of the nerve and meningitis at the base of the brain. Lydtin observed the disease in an old horse. Marked atrophy of the masseter and temporalis muscles of the right side had been developing for ten years, swallowing was difficult, salivation existed, the molars showed partial shear-mouth. The animal was regarded as incurable, and killed. Post-mortem showed the masseter, temporalis, and pterygoid muscles completely atrophied; their weight being only one-eighth of those of the healthy side. At the base of the petrous temporal bone, and just over Gasser's ganglion, lay a fibro-sarcoma as large as the cerebellum, which must be regarded as having caused the paralysis. Fröhner considered one case occurring in his experience to have been caused by catching cold.

Cadéac saw trigeminal paralysis in a dog. Recovery occurred in a week. Schmidt recorded a case where a sporting dog showed sudden paralysis of the lower jaw and marked convergence of the optical axes. The animal recovered in one week.

The symptoms consist of salivation, protrusion of the tongue (which is often dry on the surface), and inability to take nourishment, to masticate, or to close the mouth completely. These symptoms, and the fact that the mouth can easily be closed by pressing on the lower jaw, distinguish this condition from dislocation of the lower jaw, or from foreign bodies lodged between the molars. Masticatory paralysis, as stated, is a constant symptom of rabies in the dog.

Prognosis must be based on general principles. Where the paralysis has only existed a short time, and is incomplete, some hope may be 
given. Under other circumstances, and especially where extensive atrophy and degeneration have set in, treatment is of no value. In Waltrup's case in the horse, and Fröhner's in the dog, improvement occurred gradually. Frick records three cases in the dog; all appeared suddenly without visible cause, and all recovered completely.

Treatment calls for appropriate nourishment, food must be placed in the mouth, and the stomach tube is sometimes serviceable. The induced or constant electric current should be tried, the poles being applied to the masseter and temporalis muscles; Fröhner states that by its daily use recovery occurs in one to two months.

\section{(6.) PERIOSTITIS AND EXOSTOSIS ON THE POSTERIOR BORDER OF THE LOWER JAW.}

Circumscribed periostitis sometimes occurs in the horse on the posterior border of the lower jaw at the height of the first molar, and induces exostosis. The most frequent cause is external violence, especially striking against narrow mangers while feeding. The periostitis seldom produces disturbance; pain is sometimes indicated by careful mastication, but the presence of exostosis usually first attracts notice. On the posterior border of the lower jaw a hard, sharply defined, round, sometimes knobby, painless swelling appears, firmly attached to the bone. Sometimes the swelling is flatter, and may then be mistaken for that produced by alveolar periostitis, in which, however, the rarefaction of bone and the swelling are on the lateral surface of the lower jaw, and mount upwards. Periostitis caused by local injury, on the other hand, remains confined to the posterior edge.

Prognosis is favourable, the mature exostosis only producing an unimportant blemish.

Treatment must conform to general principles. Prominent exostoses may be removed: a circular cut is first made through the skin and periosteum, and the chisel or saw then applied. Flat swellings are better left alone.

\section{(7.) TUMOURS ON THE LOWER JAW.}

True tumours of the lower jaw are much less common in animals than in men. Only enchondromata, carcinomata, and epitheliomata have hitherto been described, and these sparingly. Cadiot and Dollar describe a case of lobulated pavement epithelioma of the lower jaw in a horse, of which an illustration is given overleaf. "The left branch of the lower maxilla opposite the first molars appeared 
destroyed throughout its entire depth. In two months the tumour destroyed the central portion of the right branch of the lower maxilla, produced great disturbance, general decline, and death." ("Clinical Veterinary Medicine and Surgery," p. 330.) Almost all the tumours in cattle described as sarcomata are in reality caused by actinomyces. As regards true tumours the prognosis is usually unfavourable, re-section of

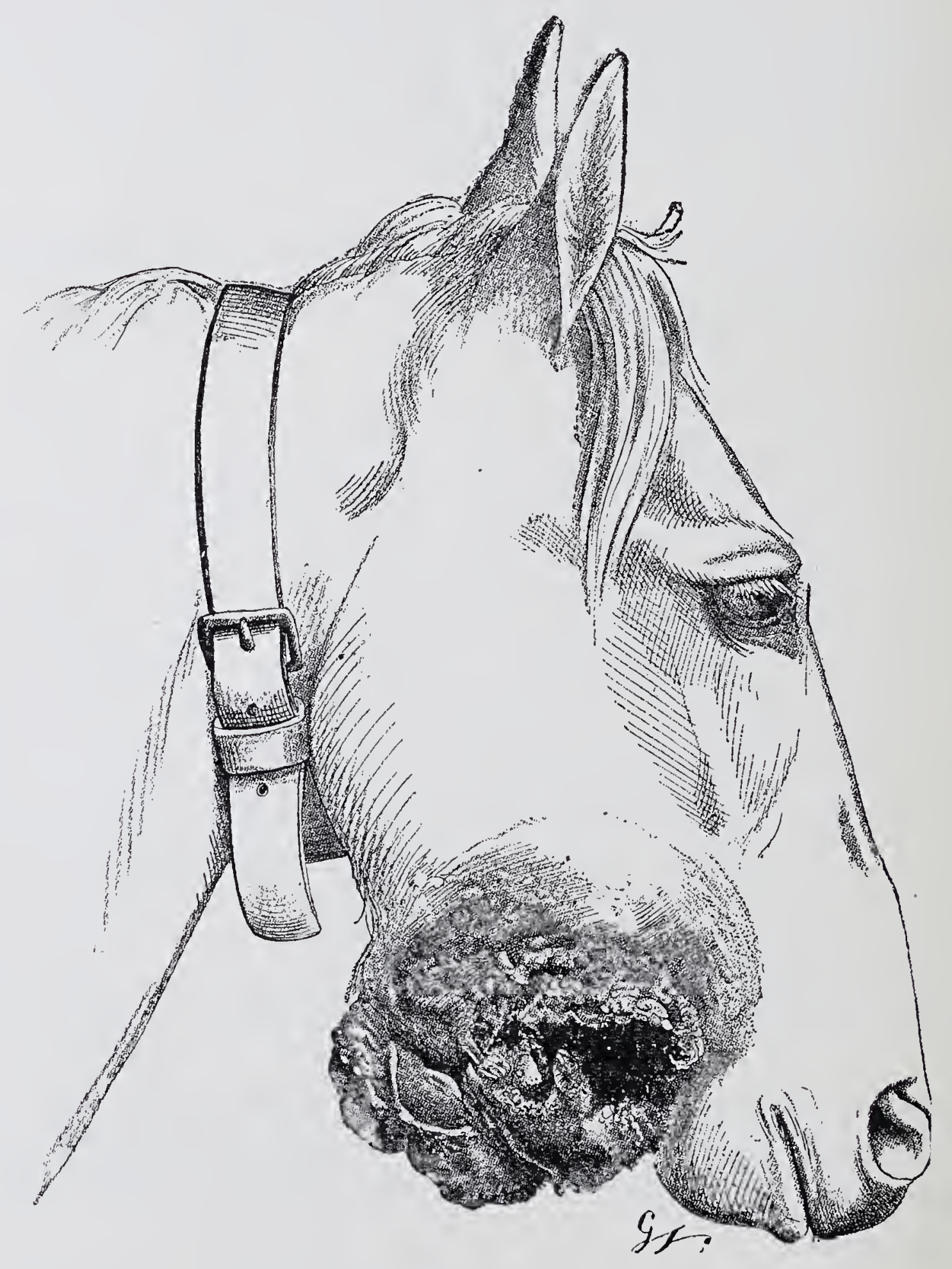

FIg. 60.-Cancer of the inferior maxilla.

the jaw being the only reliable treatment. It is therefore almost always best to slaughter the animal. Keiper observed an osteo-sarcoma (?) in the horse, springing from the point of fracture of the lower jaw, between the incisors and pre-molars. This rapidly recurred after extirpation, and in twenty-two days attained a height of six and a breadth of four inches. Möller has several times removed similar growths, which have proved to be mycofibromata. They usually appear on the buccal 
membrane, and possessing a narrow base are easily extirpated. They must not be mistaken for those new growths which arise from the alveolus of the tooth (epulis), and are much less easy to deal with. It is important to note from what point the tumour arises and whether

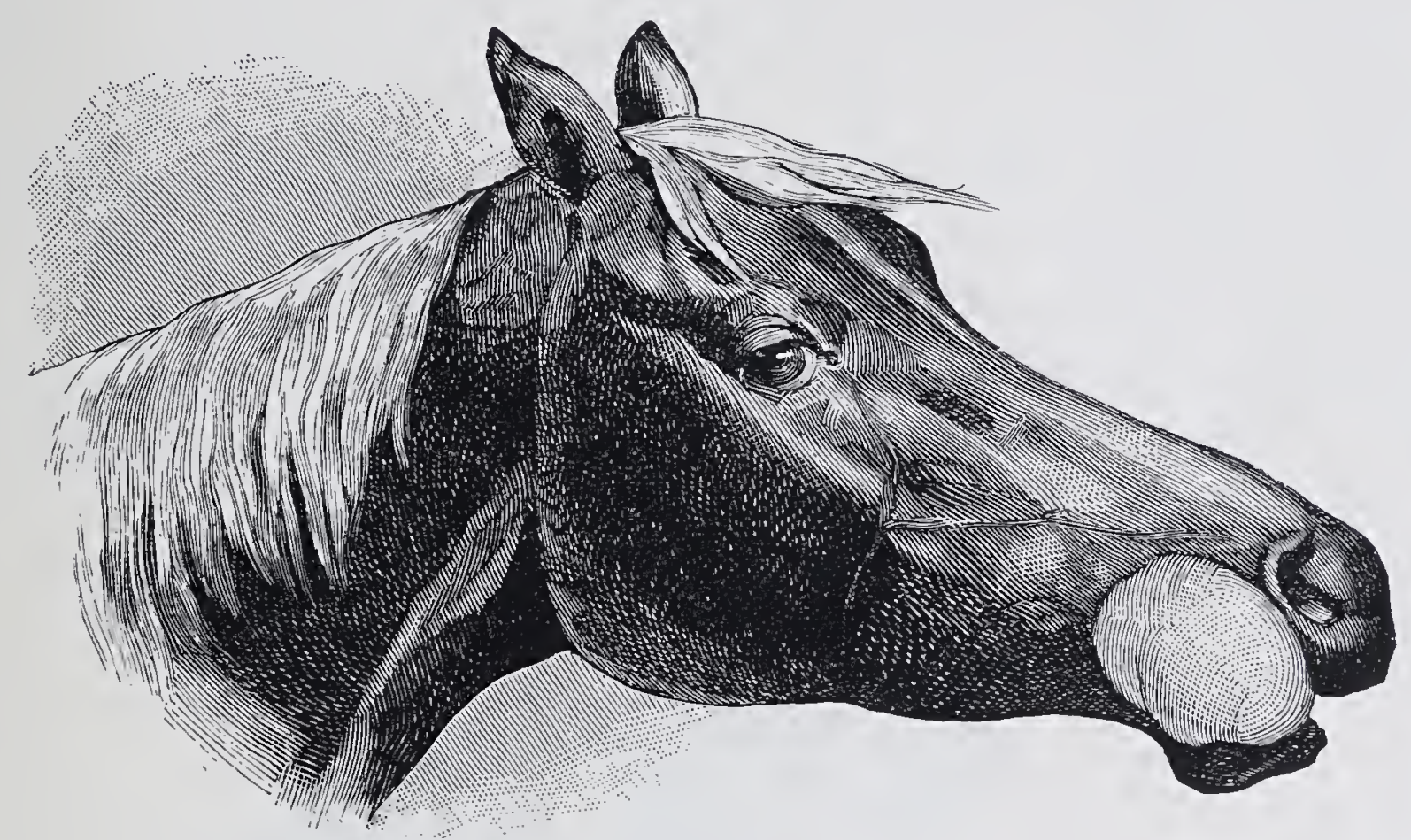

Fic. 61.-Bothryomyeosis of the lower jaw.

swelling of the submaxillary glands already exists. Tumours of the jaw of epithelial type are probably due in most instances to paradental epithelial débris, thus resembling the dentigerous and dermoid cysts.

\section{(8.) ACTINOMYCOSIS OF THE MAXILLA, SPINAVENTOSA.}

Actinomycosis of the maxillæ occurs in all domesticated animals, and has even been seen in elephants, though the ox is by far the most frequent sufferer. Imminger found the disease localised in the jaw in 14 out of 15 cases of actinomycosis. Marey records 541 cases; 117 of these affected the bones of the skull. The changes produced by the actinomyces resemble those due to tumour growth, and have frequently been mistaken for the latter. Vachetta describes the growths as osteosarcomata. All the older reported cases of "sarcoma" of the maxilla are probably attributable to actinomycosis.

Infection with actinomyces following injuries to the gums often causes osteomyelitis and rarefying ostitis of the jaw. This generally attacks the lower jaw, but sometimes the upper in addition, and is frequently seen in ruminants, particularly in cattle, but also in goats. The disease usually arises from one or other pre-molar. Pilz saw a tumour of this kind in a horse : it had developed in the neighbourhood of the first and 
second lower molars, and had four fistulous openings, from which a yellowish pus was discharged.

Symptoms. A hard, firm swelling develops on the lower jaw, which careful examination shows to be caused by an increase in the volume of the bone. The swelling spreads and increases in size; and although it is at first only slightly painful, the animal soon shows difficulty in chewing, masticating slowly, holding the head obliquely on one side, and even allowing food to fall from the mouth. Closer examination discloses the presence of purulent alveolar periostitis of one or several molars, those, namely, at the point where the swelling appears externally. The diseased teeth lie deeper in the jaw than usual, or are pressed out of line. The masticated food collected around them possesses a

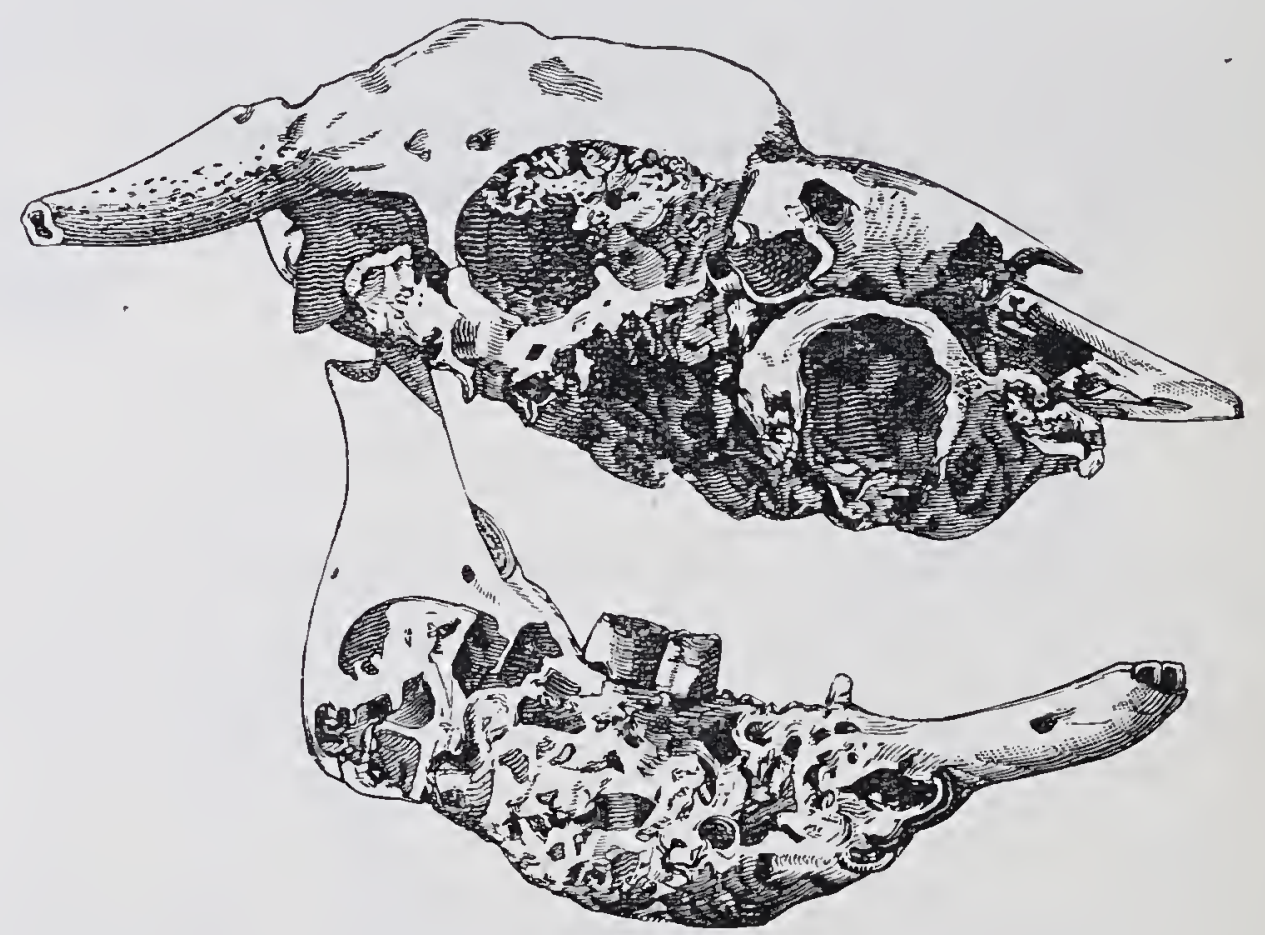

Fig. 62. - Osteoporosis of the upper jaw (Esser).

peculiarly penetrating and unpleasant smell, always noticed in alveolar periostitis of herbivora. The external skin increases in thickness, and gradually becomes adherent to the swelling. Later, perforation takes place, and a thick fluid yellow pus is discharged, in which actinomyces may be found. A probe penetrates deeply into the bone and easily breaks down its thin trabeculæ. Granulations form around the wounds, and bleed readily when touched. As a rule, feeding is disturbed, and loss of condition sets in.

Prognosis and course. Thenature and position of the diseased processes make it evident that little can be done therapeutically. Actinomyces possess considerable powers of resistance, and when within the bone, are exceedingly difficult to reach. The disease must almost always be incurable, though Pilz and others claim to have had recoveries after giving iodide of potassium internally, and applying tincture of iodine locally. Nevertheless many unsuccessful cases have been recorded. Moussu unhesitatingly states that potassium iodide alone is insufficient 
in actinomycosis of the jaw. Frick confirms this. The prognosis depends on the extent to which mastication is interfered with, and on whether the animal can still be used for any purpose. It is better to slaughter, immediately the general condition begins to suffer. Postmortem shows the bones to be perforated with granulation masses and pus cavities. The existing osteoporosis is most clearly seen after macerating the bone (fig. 62).

Treatment, even when early adopted, offers little hope. Loose teeth must be removed under any circumstances, the alveoli afterivards washed out with a disinfectant fluid, and plugged with tow saturated with tincture of iodine. Where abscesses have already perforated, antiseptic injections may be tried. Tincture of iodine seems to deserve preference. The internal administration of iodide of potassium has been extensively tried in this disease, but with very varied and often negative results. Moussu's experience leads him to prefer energetic curettage of the parts and subsequent internal use of potassium iodide. But more important than these therapeutic measures is the administration of such food as makes little call on mastication, as brewers' or distillers' grains.

Pilz gave an eighteen months old foal 4 to 5 drams of iodide of potassium, and injected the swelling with tincture of iodine daily. In five weeks symptoms of poisoning occurred, appetite and condition were lost, the coat was dry and staring, the limbs swollen, and pulse accelerated, but these symptoms disappeared after the material had been discontinued for a fortnight. Iodide of potassium was afterwards given for periods of fourteen days at a time, until two pounds of iodide of potassium and one pound of tincture of iodine had been used. At the same time the parts were regularly washed out with different materials. Great improvement was noted; but as the foal was soon afterwards sold, the final result of the treatment could not be determined.

\section{(9.) INFLAMMATION AND NEW GROWTHS IN THE SUBMAXILLARY LYMPH GLANDS.}

Infectious processes in the lips, nose, cheeks, or nostrils usually cause swelling of the submaxillary lymph glands. This is especially noted in glanders, strangles, certain forms of cellulitis, and also in some malignant new growths, such as carcinoma, mycofibroma, and actinomycosis. In the last-named disease chronic lymphadenitis, accompanied by marked swelling, pus formation, and ulceration, may also occur.

Horses, oxen, and dogs all suffer from primary tumours of the submaxillary lymphatic glands, in which lymphomata, atheromata, and carcinomata are occasionally found.

Symptoms. The acute inflammatory diseases of the submaxillary glands are described in works on pathology. The chronic are characterised 
by swelling, which is slightly painful and moderately firm, sometimes appears as a circumscribed new growth, sometimes as a diffuse enlargement of the whole gland, or of single sections of it. It develops slowly, finally breaking in several places. So soon as the ray-shaped fungi of actinomycosis or the well-known bothryomyces forms are discharged, the nature of the disease may be recognised. In the horse Möller, however, repeatedly found chronic disease of these lymph glands, with ulceration of the skin and multiple abscess formation in the glands, without the presence of the above-named parasites; but in such cases pyogenic cocci were present.

Treatment requires extirpation of the gland, or of those portions affected by the new growth. Resorbent or disinfecting materials are never satisfactory. In the horse Möller frequently removed the collective submaxillary lymph glands (see fig. 51), and even a portion of the sublingual; he recommends proceeding as follows:- The horse should be cast, chloroformed, and laid on its back. The skin is now cut through at the point where it has become adherent to the underlying structures. The connective tissue which surrounds the gland is thus exposed, and the tumour divided from the sound structures by the fingers, aided by scissors and knife. Great care must be taken not to injure the external facial artery and vein, or Stenson's duct. If this seems unavoidable, they should previously be ligatured. Wherever practicable, the operation should commence at the posterior part, in order that the injured vessels be more conveniently ligatured. If the sublingual gland is diseased, it must also be removed. The seat of operation is then washed out, its surface sprinkled with iodoform and tannin, and, in order to check bleeding, a mass of tow or jute firmly inserted before sewing up the wound. The tampon is removed after twenty-four hours, and the wound treated as an open one, when regular healing usually follows. The appearance of normal or blood-stained saliva is unimportant. The discharge ceases with the appearance of granulation.

Diseases of the laryngeal region, of which little is to be found in recognised text-books, are sometimes seen in the horse and dog. Thus Vachetta has seen fistulæ due to the non-closure of the branchial arches in the horse and dermoid cysts in horses and dogs. In the latter mucoid cysts, varying in size between a pigeon's egg and a man's fist, are seen in or near the submaxillary region; they usually extend downwards, sometimes behind the larynx and œsophagus. The cause of their formation is unknown; Fröhner regards them as retention cysts due to the persistence of isolated fragments of the embryonic prototype of the sublingual gland. They appear' as fluctuating swellings of slow growth, unaccompanied by inflammation. Surgical treatment is difficult, inasmuch as the entire growth can seldom be removed, and a fragment is 
usually left which leads to a return of the condition or to fistula formation.

The treatment of these cysts is purely surgical. Removal of the cyst is followed by recovery, but the operation is very troublesome on account of the richness of the surrounding tissues in vessels and nerves. Möller claims to have had many successes after operation. In one case, however, in a dog, the animal gradually wasted, became dull, and died without at any time showing fever.

On account of the difficulty of total extirpation Fröhner recommends injecting 2 to 5 per cent. iodine solution, which produces suppuration in the cyst. The contents are first withdrawn with a small syringe and the iodine solution injected, no antiseptic precautions being observed. Should suppuration not occur within three or four days, as can be ascertained by puncture, the iodine injection is repeated. As soon as pus is freely formed the cyst can be laid open and treated as a common abscess cavity.

Owing to failures with this method Frick now prefers to lay open the cyst fully and suture the flaps to the neighbouring skin. He then carefully mops out the cavity and thoroughly cauterises the interior with the Pacquelin cautery. The necrotic portions are shed and the cavity fills up by granulations.

\section{VI.-DISEASES OF THE EAR AND GUTTURAL POUCHES.}

\section{(1.) INJÜRY OF THE EXTERNAL EAR.}

Injuries of the external ear are commonest in dogs, though seen in other animals. They are produced in cropping the ears and from bites. In long-eared varieties, shaking the head persistently may lead to injuries. Larger animals meet with wounds of the ears by bringing the head forcibly against nails or hooks, and occasionally by being bitten by their companions. The practice of applying a twitch to the ear, more common formerly than now, was also a frequent cause.

Prognosis. The wound, when confined to the skin, is unimportant; but the cartilage being closely attached to the skin, often suffers, and may be more or less injured. In a horse Möller saw a torn wound extending from the base of the ear to its summit, and dividing it into two exactly equal portions. Transverse wounds occur, or pieces of the cartilage (recognised by its white colour) may be entirely lost. In long-eared dogs, the cartilage does not reach to the point of the ear, but is continued by a fascia-like membrane. Healing, easily effected if the parts are undisturbed, is often checked by the animal continually rubbing 
or shaking the ears. Longitudinal wounds heal better than transverse ones. Injuries to the point, produced by shaking the head, are difficult to heal, because the animal is prone to irritate them afresh. After a time thickening results, and ulceration of the edges of the wound occurs, laying the cartilage bare and producing exfoliation. The surfaces are usually invested with a brown crust, and bleed easily. This condition, termed "external canker," is indicative of chronic mischief, and is usually a sequel of otitis externa.

Treatment. Loose shreds, brought into apposition with sutures and treated antiseptically, will often adhere; but where appearance is not regarded, they may be removed with the scissors. After cleansing and freshening the edges with scissors, the bare and projecting margins of the cartilage are pushed back as far as possible, and pin sutures inserted on both the external and internal surfaces. It is usually sufficient to pass the pins through the skin, but it may be desirable to insert a few through the cartilage. After another thorough cleansing, the wound should be covered with gelatine and wadding, and in large animals a thin bandage passed round the ear. In dogs, the ears may be bound together on the sides of the head; whilst in horses movement can be prevented by placing the animals on the pillar reins. If the dressing remains dry, it should be left in position for about a week. In ulceration of the cartilage, it is of first importance to prevent the head being shaken. This can be effected by bandaging the ears to the head, but preferably by the treatment recommended in inflammation of the external auditory meatus. Sections of skin which have lost their blood supply are removed.

\section{(2.) INFLAMMATION OF THE EXTERNAL EAR.}

This condition is seldom seen in domestic animals other than the dog and pig. Pofeld saw a cow which lost a large portion of both ears from septic cellulitis. In dogs the condition usually results from excoriations of the external ear, due very frequently to otitis externa. Bites are a rare cause. On the other hand, cellulitis of the external ear is a common disease in herds of swine. Young pigs often bite each other's ear's, producing a disease which has been described as erysipelas, but is in reality an inflammation of the loose connective tissue of the outer ear and of its base.

The animals usually hold the head inclined towards the affected side. Dogs often begin to shake the head, but are soon deterred by pain and give up the attempt. The external ear is greatly swollen, in pigs of a fiery red, and shows numerous scratches and tears, from which serum discharges. Handling is very painful, and the parts feel intensely 
inflamed. In pigs the inflammation soon extends to the inner ear and brain, as shown by somnolence, loss of appetite, and burrowing in the straw, where the animal seeks to hide itself. Pigs not infrequently die of this disease, an event of very rare occurrence in dogs.

In dogs the inflammation subsides in a few days after the application of Goulard's extract or dilute solutions of lead acetate. Scarification and disinfectant applications are of assistance in the early stages in pigs, but, if somnolence has appeared or suppuration set in, destruction of the external or even of the middle ear often follows and treatment is hopeless. For this reason fat pigs should be slaughtered at an early stage.

(3.) (EDEMA OF THE FREE BORDER OF THE EAR -EXTERNAT CANKER (OTHÆMATOMA).

Rupture of blood-vessels, and extravasation of blood between the cartilage and skin of the external ear, is often brought about in longeared dogs by the ears being rubbed or bitten, but more frequently from their being vigorously shaken to allay irritation consequent on otitis. As a rule, the rupture occurs under the perichondrium, and the condition, therefore, in the dog consists of sub-perichondrial blood extravasation. This usually remains fluid for a lengthened period, appears oftenest on the inner surface, less frequently on the outer, at times on both. From the position of the lesion, absorption is slow and usually incomplete.

Hoffmann, misled by the above facts, erroneously regarded the extravasate as consisting of lymph.

Symptoms. The dog holds its head on one side, and examination reveals a slightly painful, fluctuating swelling, sharply defined, rounded or longish, and varying in size from a bean to an orange. An incision liberates fluid or clotted blood, and displays the yellow white surface of the cartilage, which later becomes covered with granulations, which appear as little discrete red flecks, and gradually, during perhaps several weeks, spread over the exposed surface. Healing is tardy, and the parts generally remain thickened. Where the swelling is not opened it may persist indefinitely, and produce marked distortion.

Treatment. In slight hæmatoma, absorption may be produced by massage, though operation is usually preferable. The inner surface of the ear is incised to the full extent of the swelling, the lining membrane completely removed, and the cavity disinfected. Catgut sutures are then passed completely through the external ear so as to bind together the walls of the cavity, the knots being tied on the outer surface of the ear. To keep the edges of the wound clean, and bring about rapid healing, 
the wound may be painted with tincture of iodine and dusted with iodoform. Healing is further assisted by binding the ears close to the head with a broad bandage, or by using a net. This disease must be distinguished from the next in series.

\section{(4.) INFLAMMATION OF THE EXTERNAL AUDITORY MEATUS (OTITIS EXTERNA). OTORRHCEA.}

This is termed in Germany "internal canker," in contradistinction to the ulcerative and chronic inflammatory processes of the cartilage of the ear, which have been described as "external canker." To avoid mistakes, it would be better to discontinue the use of the terms otitis externa and interna.

The meatus extending from the auditory opening to the tympanic membrane has externally a cartilaginous foundation, internally a bony one. It is lined by skin, rich in large spherical wax glands.

Causes. In dogs with long ears, occasionally in other animals, the waxy secretion decomposes in consequence of exclusion of air, high temperature, and restricted evaporation, and its products irritate the lining of the meatus. Inflammation is seldom caused by the entry of foreign bodies into the external auditory opening, though Hering has met with acari of sarcoptes cynotis, or more properly dermatophagus canis. Although examining many dogs suffering from otitis externa, Möller has never found acari in the external meatus, but has seen the disease caused by dermatocoptes cuniculi in rabbits, and Zürn states that dermatophagus cuniculi produces the same result. Cadiot ascribes parasitic otitis in the dog and cat to the presence of Symbiotes auricularum and in the goat and rabbit to Psoroptes communis. Ostertag found bird-lice (Gamasus auris) in the external meatus of a cow: attention was drawn to the animal by its violently shaking its head. In dogs facial eczema sometimes invades the external ear, and, on the other hand, otorrhoa may produce eczema on the ear.

Two kinds of otitis externa are distinguished-the acute and chronic. The acute develops in the dog as a sequel to eczema of neighbouring parts, or following the entrance into the meatus of discharges from neighbouring wounds. A yellowish-brown secretion, usually mixed with pus, flows from the ear, and produces eczema of the skin below. Proper treatment often induces recovery in eight to fourteen days.

In neglected cases chronic otitis develops. It may be divided into a superficial and deep form. The first is commonest in dogs, and owes its origin to irritation produced by decomposing secretions. Abnormal pruritus is present; the unpigmented skin of the meatus is reddened, and covered with a blackish-brown fatty material; light pressure at the 
base of the cartilage produces an agreeable sensation, the animal leaning towards the operator, and holding the head on one side. A bubbling sound may perhaps be heard, owing to motion of the fluid contents of the ear. After some time the secretions become yellowish-brown, and excoriated spots appear in the depths of the meatus. In the deep form one meets with a purulent discharge (otorrhcea); granulations appear on the surface of the diseased meatus, and caries of the petrous temporal bone may ensue. Sometimes pus formation preponderates; sometimes formation of granulations. The latter may obliterate the external opening. Perforation of the tympanum and otitis media are rare. Whilst irritation disappears early in the chronic superficial form, the deep form is usually accompanied by greater pain. Fröhner records vomiting in dogs, which he referred to irritation of the ramus auricularis of the vagus nerve. Implication of the middle ear or brain may produce epileptiform fits and other brain symptoms; but, considering the frequency of such ear diseases in dogs, these complications are seldom observed. Masch describes such a case in the horse, which showed a purulent discharge containing fragments of bone from the external ear. The horse was killed, and on post-mortem examination was found to be suffering from necrosis of the petrous temporal bone and an abscess under the dura mater.

In order to view the largest possible portion of the meatus, the head is placed downwards, the cartilage grasped with both hands, and held in such a position as to direct the passage, which lies sideways, somewhat towards the front. The aural speculum is of no great value in dogs, as the tympanum is not visible. The resistance of the animal, moreover, interferes both with the insertion of the instrument and the examination.

Prognosis. Acute otitis is readily curable, but chronic otitis externa is often extremely obstinate. In the superficial form care and perseverance will usually succeed, but the deep form is usually incurable; the more profuse the discharge of pus, the less the chance of recovery.

The treatment of acute otitis can usually be effected by repeated cleansing of the meatus and the use of mild astringents. A small soft sponge fastened to a stick is dipped into lukewarm water or solution of acetate of lead, gently squeezed and inserted into the meatus. Slow rotary movements remove the secretion, when the meatus may be washed out with a solution of acetate of lead, care being taken to thoroughly remove all traces of fluid with a sponge. Excoriations can be painted with a 2 per cent. solution of nitrate of silver. Where pain is excessive, lukewarm oil of henbane dropped into the ear often gives relief. The washing of the meatus must be repeated at least once a day, and continued until the discharge has completely disappeared.

In chronic superficial otitis the first requisite is careful cleaning of the meatus with lukewarm soap and water and an ear-sponge, followed by 
washing with a 5 per cent. lotion of equal parts of tannic and salicylic acids in spirit. Alcohol is of service, and 3 per cent. solution of resorcin in spirit can be strongly recommended. Butel recommends a 6 per cent. solution of boric acid in alcohol. This treatment must be repeated daily ; and where the disease has been neglected, and to prevent its return, must be continued for some weeks. No fluid must be left in the ears. If great pain be present, henbane oil is useful, Bayer, after cleansing, powders the ears with boric acid, while resorcin, iodoform, and other materials have been recommended; but less depends on the materials used than on their careful application. Nocard recommends using night and morning an ointment consisting of 10 parts of salicylic acid, 100 parts of vaselin, and 20 drops of tincture of benzoin. A piece the size of a hazel-nut is placed in the ear. Internally, preparations of arsenic and iodine (Donovan's solution) are useful.

Exuberant granulations are removed with a curette, and, on account of their often possessing a neck, this is easy.

Inflammation of the middle ear-that is, of the tympanum-is termed otitis media; that of the labyrinth, otitis interna; but neither is usually recognised during life. They occur from the spread of inflammation from the external meatus, or the entrance of foreign bodies. Attacks of delirium have been observed by Stadler and Schumacher in cattle, produced by acari in the middle ear (dermanyssus avium). Schütz and Siedamgrotzky detected tuberculosis of the middle ear in pigs. In rabbits, formation of pus in the middle ear has produced epileptiform attacks, and dermatocoptes have also been found in the meatus and middle ear, causing cerebral symptoms. The animals sometimes made rotary, sometimes rolling movements. Manege movements have been seen in tuberculosis of the middle ear in pigs.

In addition to those mentioned some rarer diseases of the outer ear may here be referred to.

Tumours are commonest in dogs, and are chiefly represented by papillomata and fibromata; the former not infrequently follow otitis externa. They are also seen in horses. Their treatment is purely surgical, and follows general principles. Several observers have seen horns on the skin of the ear in cattle.

Paralysis of the ear in horses is usually an accompaniment of central facial paralysis. Frick has seen primary paralysis of the levator muscles of the ear after the animal had been lifted by the ears. Rupture of the muscles can almost always be detected under such circumstances. Recovery is sometimes protracted.

Neuralgia at the basis of the external ear accompanied by epileptiform attacks has been noted in carriage horses in which the bridle straps were 
too short, causing the head piece to press on the base of the ear. The following case of Möller's belongs to the same category.

A horse showed powerful cramp-like movements immediately a finger was introduced into one or other ear. Passing it into the left ear, the horse at once endeavoured to bring the left hind-foot as near the ear as possible, and rapid movements were made with the muscles of the hind-foot and of the neck. Pressure on the base of the ear immediately produced these cramp. like movements. The same happened on the right side. Pressure of the bit caused similar symptoms, so that the animal was useless for work. Examination of the ears and auditory opening during life showed no change; hearing was undisturbed. Unfortunately Möller was unable to make a postmortem, but during life he was unable to detect the slightest change in the ears.

\section{(5.) FISTULA OF THE EAR. DENTIGEROUS CYSTS. ${ }^{1}$}

Fistulæ are sometimes seen in the horse at the base and near the anterior surface of the cartilage, extending downwards towards the malar bone for a distance of 1 to $1 \frac{1}{2}$ inches. A little serous fluid discharges on pressure. On introducing a probe, a hard body may be discovered at the bottom of the canal which, if the latter be laid open, will be recognised as of the nature of a tooth. Sometimes several small teeth are found in the swelling. Strictly speaking, the condition is not a fistula, but represents the remainder of the incompletely developed branchial arch. On this account the fistula sometimes communicates with the guttural pouch.

The phenomenon must be referred to the development of embryonic branchial arches and clefts. Sometimes the lateral plates of these visceral cavities are ruptured, and remain so, thus giving rise to fissures in the ear, pharynx, œsophagus, and neck (fistula auris et colli congenita), whilst partial persistence of the unruptured embryonic furrows produces blind sacs and dermoid cysts. The dental furrow which occurs in this portion of the embryonic apparatus results from a primitive fold of the buccal epithelium, which, as in the jaw, can produce enamel. The teeth are oftenest found in the squamous portion of the malar bone. Several-i.e., from 2 to 4 -may be present; they resemble molars. Sometimes a cyst alone is present, and the tooth wanting.

Such teeth may be present (abnormally) in other positions, as the gums, the superior maxillary sinus, the testicle, and the ovary. In the malar bone they often remain long unremarked, until either acute inflam. mation produces swelling and prominence, or until attention is accidentally directed to the fistulous opening. They are commonest in young animals, especially during the period of dentition, and may even be seen within a few weeks of birth. The swelling is hard, and firmly connected with the

1 See also Cadiot and Dollar's "Clinical Veterinary Medicine and Surgery," pp. 1 et seq. and also p. 298.

V.S. 
malar bone, over which the skin is freely movable. Sometimes cellular inflammation develops, followed by abscess formation, but producing no great disturbance. In horses of little value objection is accordingly raised to removal of the teeth.

Treatment. Where treatment is necessary the swelling is opened to the bottom, the tooth removed, the interior cauterised with chloride of zinc or chromic acid, 1 to 10, and recovery usually occurs in four to six weeks. Ligature of vessels is rarely necessary. When the tooth is deeply implanted in one of the cranial bones it may be removed with forceps, but in such case there is considerable danger of meningo-encephalitis, even though strict antisepsis be observed, and it is often preferable to

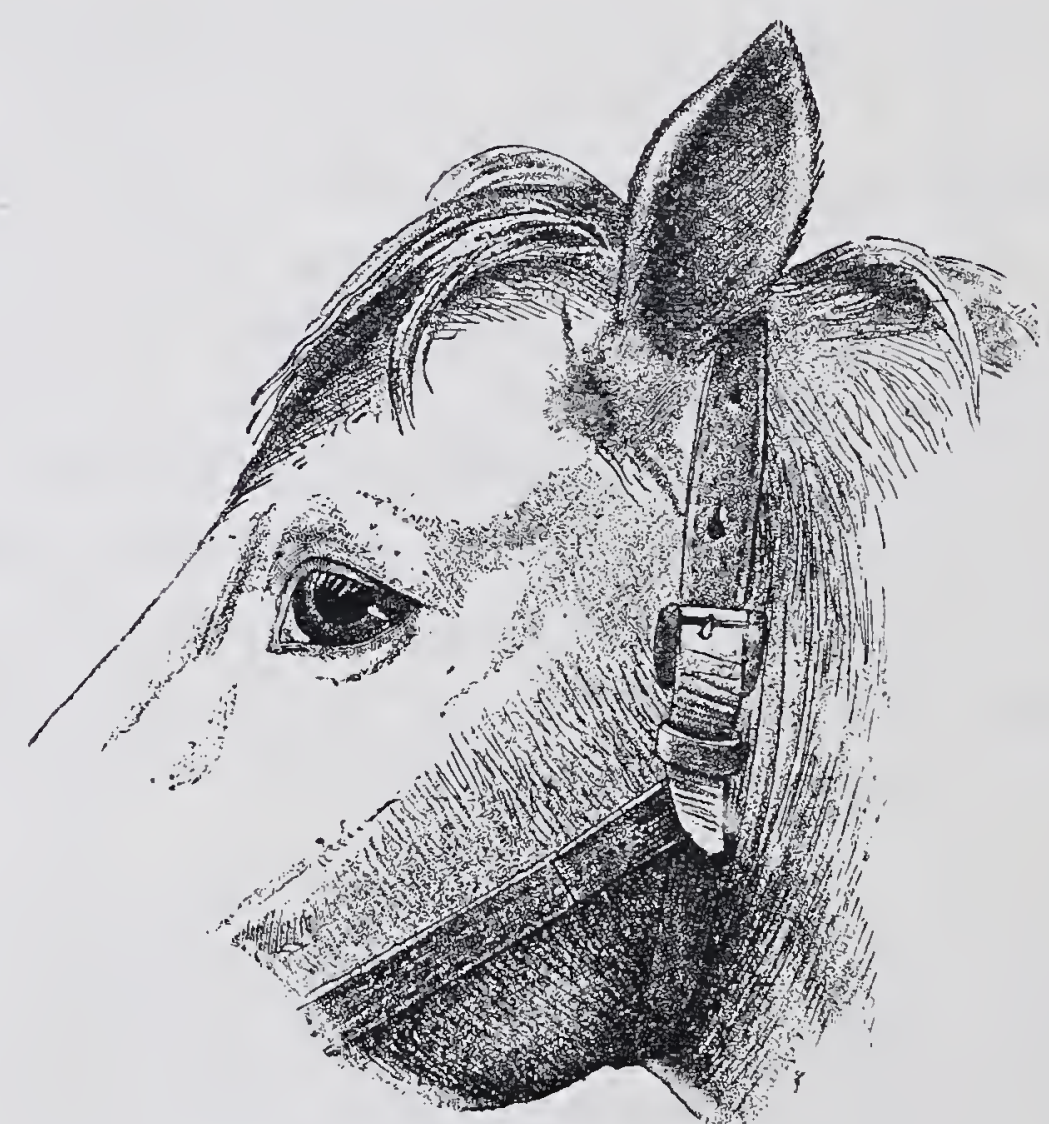

Fig. 63.--Pre-auricular fistula due to presence of a dentigerous cyst.

renounce operation. The disease occurs very seldom in animals other than the horse. Verwey found a dental cyst on the petrous temporal bone of a dog, which suffered in consequence from otitis externa.

Dermoid cysts closely resemble those above described, and occur in similar situations, but usually contain a mass of deeply pigmented tissue more or less covered with hair.

\section{(6.) CHRONIC CATARRH OF THE GUTTURAL POUCHES.}

In the horse the mucous membrane of the guttural pouch consists of an extension of that of the pharynx. The Eustachian tabe is about $\frac{1}{8}$ of an inch in thickness, rich in mucous glands, and lined with ciliated epithelium. The pouches possess a considerable area, and are attached 
to the neighbouring parts by connective tissue. From their protected position they seldom suffer from inflammatory disorders; but when these do occur, they are generally of a chronic character. Secretion is retained, becomes decomposed, and then irritates the membrane. 'The fluid part is mostly resorbed, while the solid, from movement within the pouches, becomes fashioned into chestnut-like bodies, which sometimes attain the size of a hen's egg. Their surface appear's yellowish-brown, their interior ellowish, and on account of their cartilaginous consistency they have been described as chondroids. They are in many instances of the nature of inspissated pus. Uhlich counted 317 small chondroids weighing collectively 17 ounces. Savarese removed 240 ; they weighed from 5 grains up to $1 \frac{3}{4}$ drams. In other cases a turbid, porridge-like fluid, containing great numbers of greyish-white grains, sometimes mixed with food materials, occupy the diseased sac. Thomassen describes dropsy of the guttural pouch in a two months old foal; Johow found the pouch filled with thick mucus; the entrance to the pharynx was displaced by the swelling. The distended pouch presses on the larynx and trachea, and causes dyspnoe; thickenings or polypoid growths are often seen on the surface of the mucous membrane. Generally only one pouch is diseased, seldom both.

The causes include inflammatory processes extending from the mucous membrane of the pharynx through the Eustachian tube during the course of sore throat and strangles, and foreign bodies and food passing into the pouch. Whether in such cases congenital defects exist in the Eustachian tube cannot be determined by the communications of observer's. Possibly changes in the valve at the entrance to the Eustachian tube may cause the entry of food into the guttural pouch. Schlampp found 27 ounces of food in the pouch of a horse. Ruprecht records injury to the sac from a piece of bone derived from a fracture of the hyoid. Hering and Hahn discovered in a glandered horse cicatrices, small abscesses and ulcers in the mucous membrane of the sac; the latter was distended with a mucoid secretion. Hallander found a sarcoma in the guttural pouch. It had broken into the spinal canal and caused hemiplegia. The pouch showed signs of catarrh.

Symptoms. (1) The nasal discharge is one-sided, muco-purulent, generally without unpleasant smell, occasionally appears for a time in considerable quantity, and may then entirely disappear. Pressure on the guttural pouch, placing the bit in position or lowering the head, increase the discharge.

(2) The swelling is sometimes slight, sometimes well marked. In one horse, whose right sac held more than 2 pounds of concretions, scarcely any swelling could be observed from the outside. In another, swelling occurred in a marked degree in the parotid region, especially 
when the head was lowered. Occasionally the swelling of one side is also appreciable on the other, and may give the impression that both pouches are diseased. The greater the swelling, the more marked its fluctuating character. Entrance of air or gases into the diseased pouch causes a churning sound when the horse moves its head, and percussion reveals pretty clearly the division between fluid and gases. Should the horse eat from the ground, or be ridden or driven, a portion of the contents of the sac is discharged, and the swelling visibly diminishes.

(3) Severe distension compresses the larynx, trachea, and pharynx, and produces difficulty in breathing and swallowing. Inspiration soon begins to produce a rough sound, and at a later stage expiration becomes loud. The seeming contradiction between clinical observations and the experiments of Günther, in which filling the sac with a mass of plaster of paris produced no difficulty in breathing, is explained by the fact that the dyspncea is not produced by the filling of the sac, but by its distension and pressing on the larynx and trachea. Filling the pouch with large quantities of fluid or gases is usually accompanied by dyspnoea, whilst filling with hard materials, such as chondroids, produces no such result. Experiment confirms this statement, which also agrees with most of the cases reported.

(4) In severe distension the animal holds the head towards the sound side - a condition particularly pronounced when the horse is ridden.

Günther's catheter for the guttural pouch gives absolute information as to the presence of the disease.

The disease is sometimes mistaken for swelling of the parotid or subparotideal lymph glands, in which the swelling is, however, less sharply defined. Ulcerative processes in the pharynx produce similar discharges.

Diagnosis may be confirmed by the use of Günther's catheter. After a little practice it can even be inserted whilst the animal is standing.

Prognosis is, as a rule, unfavourable. The condition is not usually recognised until the mucous membrane of the sac has undergone considerable anatomical changes, and its walls have become concentrically thickened, when cure is impossible.

Appropriate treatment is sometimes followed by recovery. Although Günther describes one case, spontaneous recovery seldom occurs.

Treatment. Vapour baths, so frequently recommended, are, as Haubner has pointed out, quite worthless. Their reputation is due to error in diagnosis. Recovery is only obtained by complete removal of the contents of the pouch, and by direct treatment of its lining membrane. Entrance to the pouch may either be obtained through the Eustachian tube or by an operative wound. The first method is rarely successful, for it does not remove such solids as chondroids, nor provide sufficient exit even for fluid contents. Günther's tube or catheter is therefore of 
little value in treatment though it is sometimes used for diagnostic purposes.

It consists of a brass tube about 20 inches in length; one end is closed, but is provided with two lateral openings, and is somewhat curved. The other end is also slightly bent, possesses a long opening to take the so-called index, and receives the screw of an iron handle. The index consists of a spring, about 8 inches long, and serves to fix the distance of the Eustachian tube from the entrance to the nostril. By marking with the spring the distance of the temporal canthus, which lies at an equal distance from the entrance to the nostril, one knows, on introducing the instrument, when the upper end has attained the Eustachian tube. To use the catheter the patient's head must be moderately extended. After applying the twitch and fixing the spring, the tube is passed, the bent end directed towards the palate, into the lower meatus of the nostril, until the index shows that the end of the instrument has attained the entrance of the Eustachian tube. A quarter turn is then made with the handle of the instrument, so that the point is directed to the side, and the handle of the catheter pressed towards the septum nasi, probing movements being made, until the sound passes into the guttural pouch. Unopposed progress of the instrument, without back pressure, shows that it has entered the pouch. The handle is now removed, and the contents of the sac allowed to flow through the tube. In a similar manner fluids may be injected into the sac through the tube. Where the catheter is passed in an animal which has been cast, the mouth should be placed rather higher, in order to bring the bent point of the catheter sideways into the Eustachian tube, by moving the handle towards the septum nasi.

For opening the GutTural pouch various operations have been recommended, all of which have their advantages and their drawbacks. Although very quiet horses have been operated on standing, most operators will prefer the prone position. Where excessive dyspnca exists, it may, however, be aggravated by casting, and Leblanc and others recommend that tracheotomy should first be performed; but this seems undesirable, and it suffices if the instruments for tracheotomy are at hand.

(1) Schabert, in 1779, propounded the oldest method, viz, hyovertobrotomy, where the pouch is penetrated through the stylo-maxillaris muscle. The hair in front of the wing of the atlas for an area of 2 to 4 inches is shaved; an incision is made through the skin about $\frac{3}{8}$ of an inch in front of the anterior border of the wing, and parallel with

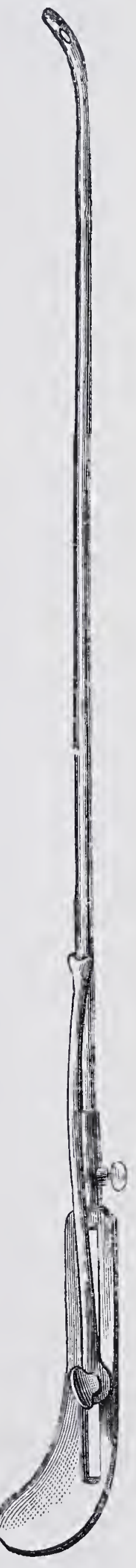

FIG. $64 .-$ Giinther's catheter. it. Just in front of the wing lies the auricular nerve, arising from the second cervical nerve, which must be avoided.

Separating the subcutis by a few light strokes, the parotid, recognised 
by its light-yellowish appearance, appear's, and is laid forward. The fascia of one of the muscles of the neck, now in view, is divided in the direction and to the extent of the skin incision. The stylo-maxillaris muscle

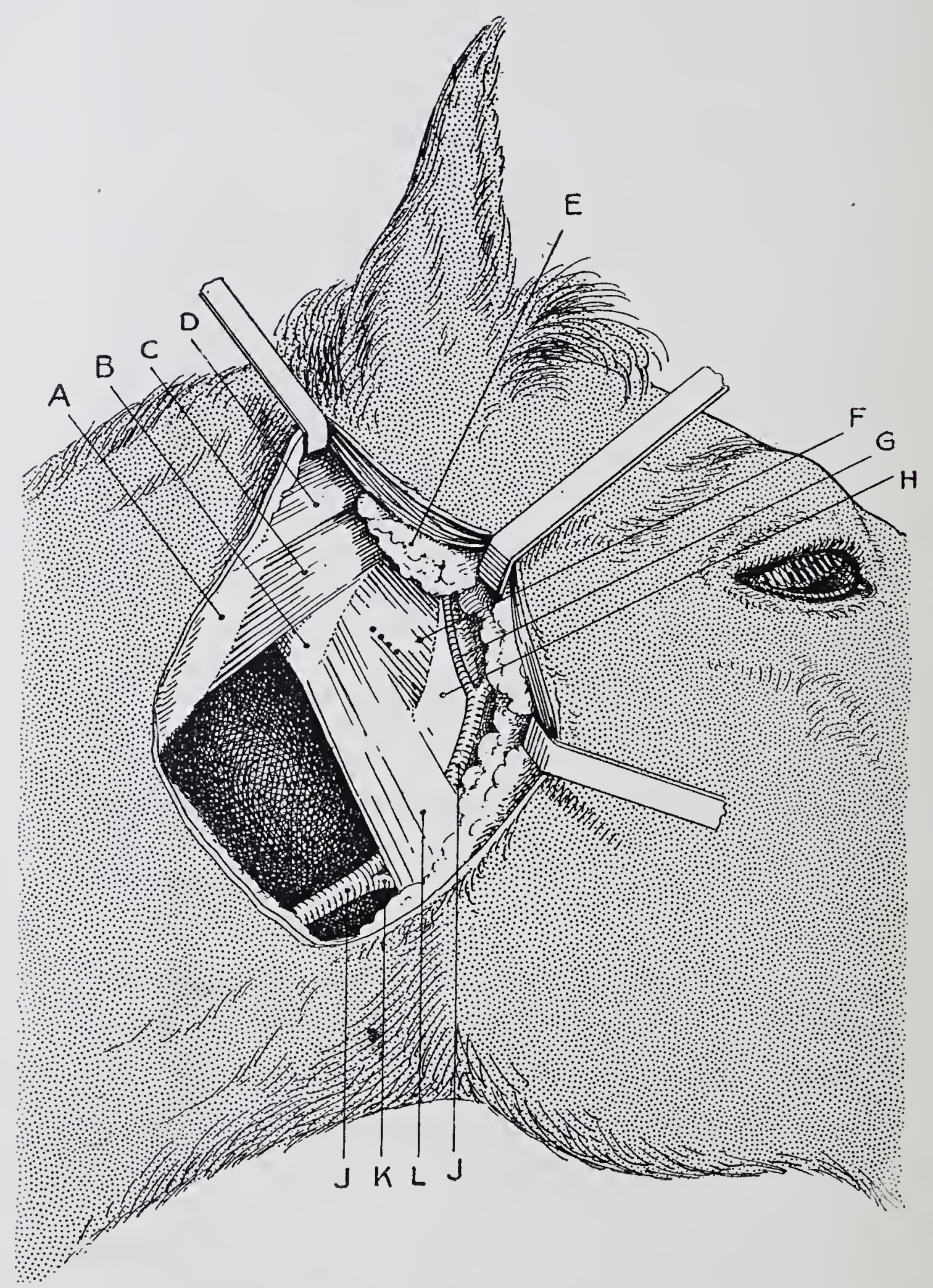

FIG. 65.-Deep tissues in the parotid region; opening the guttural pouches. (Semi-schematic.)

A, Atlas ; B, styloid process of the occipital; C, obliquus capitis superior ; D, tendon of the complexus and rectus capitis posticus major (Percivall's complexus minor); E, parotid gland ; F, occipito-styloid muscle; $G$, post auricular artery ; $H$, hyoid bone ; JJ, cxternal carotid artery; K, submaxillary artery ; I, digastricus; the stylo-maxillaris forms the posterior portion of this muscular mass. The dotted line indicates the point where the
occipito-styloid is usually punctured.

thus exposed is pierced with a pointed bistoury, the back of the knife being turned towards the wing of the atlas, and the handle a little inclined towards it. In this way the point of the knife enters the angle made by the 9 th and 10 th cerebral nerves with the inner carotid, and without injuring these. Through this opening the finger can be 
inserted into the guttural pouch. This operation has the disadvantage of endangering the nerves and vessels mentioned, nor is the opening into the sac sufficient for the removal of such solid contents as chondroids.

(2) Lecocq recommended that the sac should be entered through the stylo-hyoid muscle. The procedure is the same as Schabert's method, but the incision is made rather higher. This operation is even more objectionable than the last. Not only the external and internal carotids, but also the facial and lingual nerves are endangered. The risks of such injury are increased by the great swelling and consequent abnormal relation of the parts. Such operations, especially in dead animals, can, however, be easily carried out experimentally.

(3) Viborg recommended opening the sac below from the triangle which is formed by the tendon of the sterno-maxillaris muscle and the submaxillary vein, with the border of the lower jaw. In the middle of this triangle, and parallel with the muscle named, an incision is made in the skin, about 2 to 4 inches in length, and reaching to the border of the lower jaw. After separating the panniculus of the neck, and dividing the connective tissue, the guttural pouch is perforated with a trochar, and the opening can then be enlarged with the fingers. This method has the advantage of opening the pouch at its deepest point, thus more easily removing both fluids and solids. The pouch, when distended, is easily reached by this method, which, however, is not always free from difficulty. As has been shown by Hering; some cases of supposed hyovertobrotomy have only been the evacuation of a parotid abcess.

(4) The following method is probably the best. It is at first like Schabert's, but the cutaneous incision is carried a little further, extending over the lower edge of the wing of the atlas. The parotid is pushed to one side. The fascia of the throat muscles are cut through, bringing in view the stylo-maxillaris, digastricus and occipito-styloid muscles.

Without pushing aside the guttural pouch from the inner face of the latter muscle, the two forefingers are inserted, the connective tissue pushed on one side, and the space between the styloid process of the occipital bone and the posterior surface of the long horn of the hyoid is discovered. The forefinger of the left hand is introduced, with its volar surface turned towards the point of division, and a sharp pointed bistoury slid along the dorsal surface of the finger and passed through the occipito-styloid muscle into the guttural pouch. Without removing the left hand, the right forefinger is inserted in the sac, and the left immediately follows to enlarge the opening by tearing if found necessary.

To pass a seton or drainage-tube through the guttural pouch, an 
opening must be made in Viborg's triangle. For this purpose Dieterich employs a curved trochar. But it is more easily managed with a blunt seton needle passed outwards from the guttural pouch towards this triangle, the incision being made according to Viborg's directions. This method no doubt deserves preference; it lessens the danger of injuring large vessels or nerves, and gives a large opening, allowing easy exit of secretions, and insuring complete evacuation of the sac. Where, on account of much distension of the sac, there is difficulty in finding the blood-vessels, a blunt seton needle is inserted at the point of division of the vessels. Following Dieterich's directions, Möller succeeded in passing the entire hand into the guttural pouch, and examining from this point the Eustachian tube. After-treatment consists in flushing out the sac with disinfectants, astringents, and other therapeutic agents. Too much fluid must be avoided, as it may enter the pharynx, trachea, or lungs by way of the Eustachian tube. Degive lost a horse in this way from a solution of potash entering the lungs and producing pneumonia.

On 11th of March, 1886, an aged grey working-mare, showing no constitutional symptoms, was sent into hospital on account of swollen throat. The head was held extended, and side movements avoided. A thick yellowish-white frothy fluid ran from the nose, and was increased and became purulent on depression of the head. A snoring inspiratory sound could be heard when the horse was resting, which, on the slightest excitement or movement, became audible also in expiration. Deglutition visibly caused difficulty ; part of the water taken flowed back through the nostril. A swelling existed in the parotid region, most marked on the right side, where it was pear-shaped, the smaller end lying at the base of the ear, the lower border overpassing the anterior edge of the neck by about 2 inches, and extending over the trachea. The swelling was 14 inches long and $11 \frac{1}{2}$ inches broad at its greatest breadth. On the left side it presented a rounder form, was of less size and less sharply defined. Its length was $4 \frac{3}{4}$ inches and greatest breadth 6 inches. The skin on both sides of the neck showed traces of the application of irritants. Distinct fluctuation could be detected, percussion produced in the lower sections of the right side a hollow note, which was distinctly tympanitic in the upper part. The percussion sound on the left side was everywhere resonant. Movement of the head and rapid pressure on the swelling produced on both sides a distinct splashing sound. The cicatrix of a tracheotomy wound was visible in the middle of the neck. The larynx seemed to have retained its normal position, though the trachea was bent at a point below the swelling. No doubt could exist as to the diagnosis, and operation was decided on by Dieterich's method, with the modification that the incision was made with a seton needle. The great swelling and displacement of the organs rendered it impossible to discover the point of division of the arteries. Immediately on incision a quantity of unpleasantly smelling gases was discharged. After making an opening in Viborg's triangle, about five pints of turbid fluid, containing white lumps, flowed out. The cavity was washed, and a thick drainagetube inserted. When the horse got up, the swelling had disappeared on both sides, the breathing was regular, and food could be taken without difficulty. From March 13th the guttural pouch was washed out once daily, either with 3 per cent. solution of tannic acid or of permanganate of potash. The running from the nose decreased greatly, though a muco-purulent discharge continued 
to flow from the lower operation wound. The condition now remained at a standstill for a long time, and a lotion of acetate of aluminum was used for rinsing the guttural pouch. The wound closed after removal of the drainage-tube, but had to be re-opened on April 22nd, because the sac had again filled. Washings with permanganate, acetate of aluminum and 1 per cent. of corrosive sublimate were without success. The discharge continued, though in smaller quantities. On June 9th the patient was cast, and the operation wound, especially the upper part, so enlarged that the hand could be passed into the pouch and the fingers introduced into the Eustachian tube. The finger of the left hand, introduced from the mouth, could also be passed into the Eustachian tube, so that both hands met here. The tube appeared widely dilated; but it was further laid open with a guarded tenotome, in accordance with Bassi and Niebuhr's suggestions. The sac continued to be washed out, and from time to time painted throughout with a 2 per cent. solution of sublimate. But this treatment remained unsuccessful. The discharge continued. No marked contraction occurred in the mucous membrane, and the guttural pouch preserved its abnormal size. When it was seen on June 17th that the horse showed no dyspnœa, even on movement, treatment was discontinued, and the animal sent to grass. In December, 1886 , the horse was quite capable of work; the operative wound was not then closed, though it had become smoothed off. The secretion was slight, and the guttural pouch markedly smaller.

This case shows how obstinate the disease may be. Such difficulties are to be expected. Where the condition has existed for a long time, and the sac has been much dilated, it cannot, owing to its connection with neighbouring parts, readily resume its normal volume. Possibly dilatation of the opening of the Eustachian tube produced by the lasting discharge also forms an obstacle to healing.

Thomassen's case of hydrops of the pouch also showed swelling, slight respiratory dyspnœa, and want of appetite. An experimental opening. was made, and four pints of amber-coloured serum allowed to escape. A drainage-tube was inserted, and recovery occurred in three months.

Cadiot and Dollar describe a case of chronic pharyngitis and catarrh of the guttural pouches cured by double hyovertobrotomy. The animal was thin, had difficulty in swallowing, showed a muco-purulent discharge mixed with fragments of food from both nostrils, and occasionally suffered from violent attacks of coughing. Part of the drinking water returned by the nostrils. Both guttural pouches were opened and daily irrigated with antiseptic solutions for a period of three weeks. Recovery was almost complete in a month. ("Clinical Veterinary Medicine and Surgery," p. 335.)

\section{(7.) TYMPANITES OF THE GUTTURAL POUCH.}

The disease described by French authors, and especially by Vatel and Gohier, as guttural tympanites, has been observed repeatedly by others. Friebel and Kühnert found this condition in foals soon after birth. It consists in the accumulation of abnormal quantities of air or 
gas in the guttural pouches, which become so dilated as to cause severe dyspnoa. A swelling appears in the region of the parotid which is resonant on percussion, and on strong pressure sometimes produces a whistling sound in consequence of air escaping from the Eustachian tube. It usually occurs in young animals, foals, and affects both guttural pouches. If the sac is opened the gases escape, but reaccumulate as soon as the opening closes. Reported cases show that tympany may be due to either of two causes.

(1) Atmospheric air enters through the Eustachian tube and gradually accumulates in the guttural pouches. This probably occurs during deglutition, and is caused by deformities in the tube allowing its opening to act as a valve, which admits air but prevents its exit. Even in normal subjects, exit of air appears to be difficult. Degive injected air through a trochar into the guttural pouch of a dead animal, and noticed that it was retained for a long time. Gerlach referred the condition to paralysis of the elevator of the soft palate, which he considered should close the Eustachian tube, because in one of his cases these muscles seemed atrophied on the diseased side. K. Günther also considered that the pouch might become distended in this way. The air which has so entered, according to Gerlach, is unable to return, because the tube closes like a valve at its point of entry into the guttural pouch. Investigations in the horse, carried out with the pharyngeal speculum, do not, however, support this idea. The open end of the Eustachian tube can be directly observed with the instrument. Closure by the soft palate may certainly occur during swallowing, and entrance of air be caused by the soft palate not properly covering the opening of the Eustachian tube.

The significance of the guttural pouches has been variously interpreted. Franck looks on them as safety valves to control the air pressure in the middle ear, and prevent excessive tension of the tympanum by alteration of pressure. The suggestions that they are involved in voice production, or that the respiratory air is warmed during its stay in them, seem improbable.

Perosino discovered, by experiment, that during expiration the pouches were dilated, and that during inspiration they collapsed again. He introduced a tube containing alcohol into the pouch through a trochar, and noticed that the fluid rose during expiration and fell during inspiration. The phenomenon was exaggerated during violent expiration. Perosino therefore considered the guttural pouches were intended to moderate the stream of inspiratory air during violent exertion. Prince, on the other hand, supports the view first suggested by Prange, viz., that the guttural pouches serve to assist movements of the head by acting as elastic cushions. Perosino states that, in animals suffocated by stopping up the nostrils, the guttural pouches are enormously dilated.

(2) Other published observations ascribe the condition to the development of gas during catarrhal disease of the guttural pouch (Bassi, Degive, Mölier). Cases associated with disease of the mucous membrane and 
accumulation of fluid secretion must be viewed and treated according to the principles previously described.

Treatment. Where atmospheric air accumulates, Günther's catheter is used; and if the condition recurs, the guttural pouch is opened, and a seton or drainage-tube inserted. Stockfleth employed this method successfully. Friebel pierced the cavity with a trochar, and injected astringents like 2 per cent. solution of sulphate of zinc. Where such methods are unsuccessful, it is better to divide the opening of the Eustachian tube, as was done by Niebuhr with good results. Possibly the valve-like action of the tube is thus done away with. McFadyean failed to effect a cure even after several times operating. On post-mortem examination of the case he found the opening of the Eustachian tube contracted and indurated. Thomassen recommends opening the sac at the posterior border of the under jaw, dilating the opening with the forefinger, and washing out with some antiseptic fluid. On account of the dyspnœa often present, the same principles obtain as in treatment of catarrhal affections of the guttural pouch.

\section{VII.-DISEASES OF THE SKULL.}

In animals the brain and portion of the head described as the skull have a much less extensive development than in man. One portion, especially in ruminants, is covered by the frontal sinuses. Injuries to this portion are of less importance than in the human subject. The fact, however, that most fractures of the skull are only discovered on post-mortem examination suggests the need for particular care in forecasting the probable results in cases of injury.

\section{(1.) FRACTURES AND INJURIES TO THE CRANIAL BONES.}

The cranial cavity is formed by the parietal, interparietal, temporal, occipital, and sphenoid bones, in front by the ethmoid and the upper section of the frontal. Fractures of the cranial bones are caused by falls (either forwards or backwards), collisions, kicks, horn thrusts, and in carnivora by bites from other animals, kicks, and similar injuries. The sphenoid is sometimes broken by the contrecoup resulting from collisions. Subcutaneous fractures of the above-named bones occur, but complicated fractures are most common. In some cases concussion exists simultaneously with injury of the brain or spinal cord produced by dislocated fragments of bone.

König has given a résumé of the frequency with which the cranial bones are respectively fractured, based on the official statistics of the Prussian 
army. Of 2,984 cases of cranial fracture, 55 affected the occipital bone, 40 the sphenoid, 33 the frontal, 10 the parietal, 4 the temporal, and 2 the ethmoid.

The symptoms of cranial fracture are, in the absence of displacement, often very ill-defined. Local swelling and pain are often absent, even slight depressions in the skull often escape observation, and do not always produce cerebral disturbance. Should the dislocation be considerable, marked interference with consciousness, equilibrium, respiration, and circulation may result. The animal shows spasmodic seizures, and is unable to stand.

Prognosis is uncertain. As long as only the external plate is injured, the fracture goes on well, especially if no dangerous complications exist. But where the inner plate is divided the condition becomes exceedingly grave, doubly so where the fracture is complicated. Recovery occurs, however, even in such cases. Uebelen saw a dog and Meyer a cow recover, in both of which the brain was exposed and injured. Whitlamsmith saw a dog in which the brain protruded in consequence of injury with a knife. A piece as large as a bean was removed. For two weeks the dog showed paralysis of the right side and manege movements; then gradual recovery began and finally became complete. Möller has often seen injuries in horses and dogs successfully treated where both skull and brain were injured. In a dog deafness remained, and its behaviour suggested that sensation was also defective. Some years later death resulted, and post-mortem showed a well-marked injury to the temporal bone and to the subjacent portions of the hemisphere of the cerebrum. Where the wounds become infected the animals usually die of purulent meningitis. In horses Möller has repeatedly treated injuries of the parietal bone caused by animals rumning against sharp objects. In one the dura mater was exposed. As the patients came for treatment soon after injury, and antisepsis was carefully carried out, healing was effected by primary intention in from three to four weeks, without either constitutional disturbance or formation of pus. It is often impossible to discover the extent of the injuries produced by the fracture. Prognosis must accordingly be guarded, notably when brain symptoms make their appearance, and especially when these have existed for several days, or have appeared after the lapse of some time. A favourable termination is to be expected when no brain symptoms like dulness, irritability, spasms, \&c., appear during the first eight days after injury. Until this lapse of time prognosis must always remain doubtful.

Gröning observed fracture of the left parietal in a horse through falling over backwards. Some days later slight improvement set in, but, though feeding was not interrupted, periodical excitement and fright were present. On the sixth day epileptiform attacks occurred, and death followed on the seventh, 
Post-mortem showed a small extravasation of blood in the cranial cavity, 3 drams of a clear light-red fluid in the ventricle, and a linear fracture of $2 \frac{3}{8}$ inches in length in the left parietal bone. Conti saw fracture of the skull produced by casting. The animal remained unconscious for a short time, and then struck out violently with the feet. It died on the fourth day, and a postmortem showed three lines of fracture starting from the occipital bone. One ran from the left condyle to the foramen lacerum basis cranii; the second reached to the base of the right condyle; whilst the third divided the occipital from the temporal bone. The bodies of the occipital and sphenoid bones were further fractured in several places. Pflug saw fracture of the skull in the horse caused by falling over backwards. The animal died on the spot. Postmortem showed the cranium to be completely divided in a transverse direction into two parts. The medulla was torn away from the brain.

Franco Gonelli describes a case of fracture of the base of the skull in a horse brought about by falling into a trench; the horse's mouth struck on the edge. The animal was able to travel more than two miles after the accident, but then showed signs of coma, the temperature fell to $97 \cdot 6^{\circ} \mathrm{F}$., and the heart's action to twenty-four beats per minute. The respiration was irregular. After some hours the horse died. Post-mortem examination showed the entire base of the skull to be fractured. Extra meningeal bleeding had occurred and extended as far as the commencement of the vertebral canal.

Fractures of the sphenoid and of the occipital, and even of the other bones of the skull, usually produce death in a short time, often after a few seconds. Fractures of other cranial bones may prove fatal if attended with much bleeding into the brain cavity. Mariot saw a horse, after falling, die with loss of consciousness and advancing dyspnoea. Becker records that a horse, after having struck its head against a wall, immediately died. Post-mortem showed a comminuted fracture of the occipital, with severe extravasation of blood on the medulla oblongata.

Treatment. In subcutaneous fractures, without much dislocation, rest alone is required. Cold applications, laxatives, and spare diet ward off brain symptoms, and suffice in small fractures where dislocation of the fragments is only slight, and the brain functions are not disturbed. Replacement should be attempted where it can be effected without making a wound, and thus endangering aseptic healing. Strict antisepsis must be adopted in compound or complicated fractures where the injury is still recent, i.e., has not existed for more than twenty-four hours. The hair is cut or shaved, the wound examined with a disinfected finger, splinters of bone and foreign bodies removed as far as possible, and the entire surface carefully washed out. Plenty of disinfecting fluid must be used, preferably in the form of a strong stream. No harm is done if the fluid penerrates the connective tissue and produces odema. Loose shreds of tissue are removed with the scissors, the wound sutured with sterilised material (catgut or silk), and a dressing applied, kept in place in the horse with the help of the halter, to which the turns of the bandage are fastened. The horse should then be placed on the pillar reins, so that it cannot rub off the bandage, as it frequently attempts to 
do. During the first forty-eight hours the patient must be watched, and the usual measures taken to ward off brain symptoms. Rest, restricted diet, and laxatives are also to be recommended.

\section{(2.) CONCUSSION OF THE BRAIN (COMMOTIO CEREBRI).}

Concussion is induced in horses by falls or collisions, and less frequently by kicks from a shod foot. Wilhelm diagnosed the condition in a cow which had fallen a distance of 14 feet off a wall. Dogs and cats suffer from falls from windows as well as from blows on the skiull.

The symptoms consist of loss of consciousness, inability to stand and walk, sometimes pallor of the mucous membrane of the head, and a small, infrequent pulse. In carnivora vomiting may be present. Respiration is sometimes irregular. Various views are held concerning the alterations produced in the brain : molecular displacement is scarcely sufficient explanation, nor do the experiments of Koch and Filehne demonstrate the precise pathological conditions.

Course. Where concussion is not associated with fracture of the skull, bleeding into the cranial cavity, or further injuries, the symptoms usually disappear in a short time, often after a few hours, at latest after some days. A so-called reaction sometimes follows, the mucous membranes become red, the pulse more frequent and wiry-conditions indicating febrile mischief.

Medical interference is usually contra-indicated, and if adopted must be confined to treating symptoms. The drugs oftenest employed are heart stimulants.

Absolute rest and easily digestible food in moderate quantity are desirable. In a case reported by Wilhelm, complete recovery occurred after two days.

\section{(3.) FRACTURES OF THE FRONTAL BONE.}

When occurring on the upper portion (cerebral surface), prognosis and treatment are the same as in fractures of the skull. It is otherwise where the wall of the frontal sinus or the external plate of the bone are alone involved. Fractures here have a different signification according to their position. In most cases the prognosis is favourable and treatment successful, as experience in trephining the frontal sinus would indicate. In ruminants, fracture of the frontal bone only becomes serious when the inner plate of bone is affected; then treatment is similar to that in fractures of the skull. Fracture of the horn core will be considered later. In those of the orbital process of the temporal bone the eye may 
be endangered, as in fracture of the orbital process of the malar bone, either on account of dislocated fragments or extravasated blood pressing on the bulbus and producing exophthalmos. Swelling of the orbit may, moreover, extend to the optic nerve, and through its atrophy produce blindness. Sometimes the lower jaw is also endangered.

The guides to diagnosis are the displacement of the frastured process,

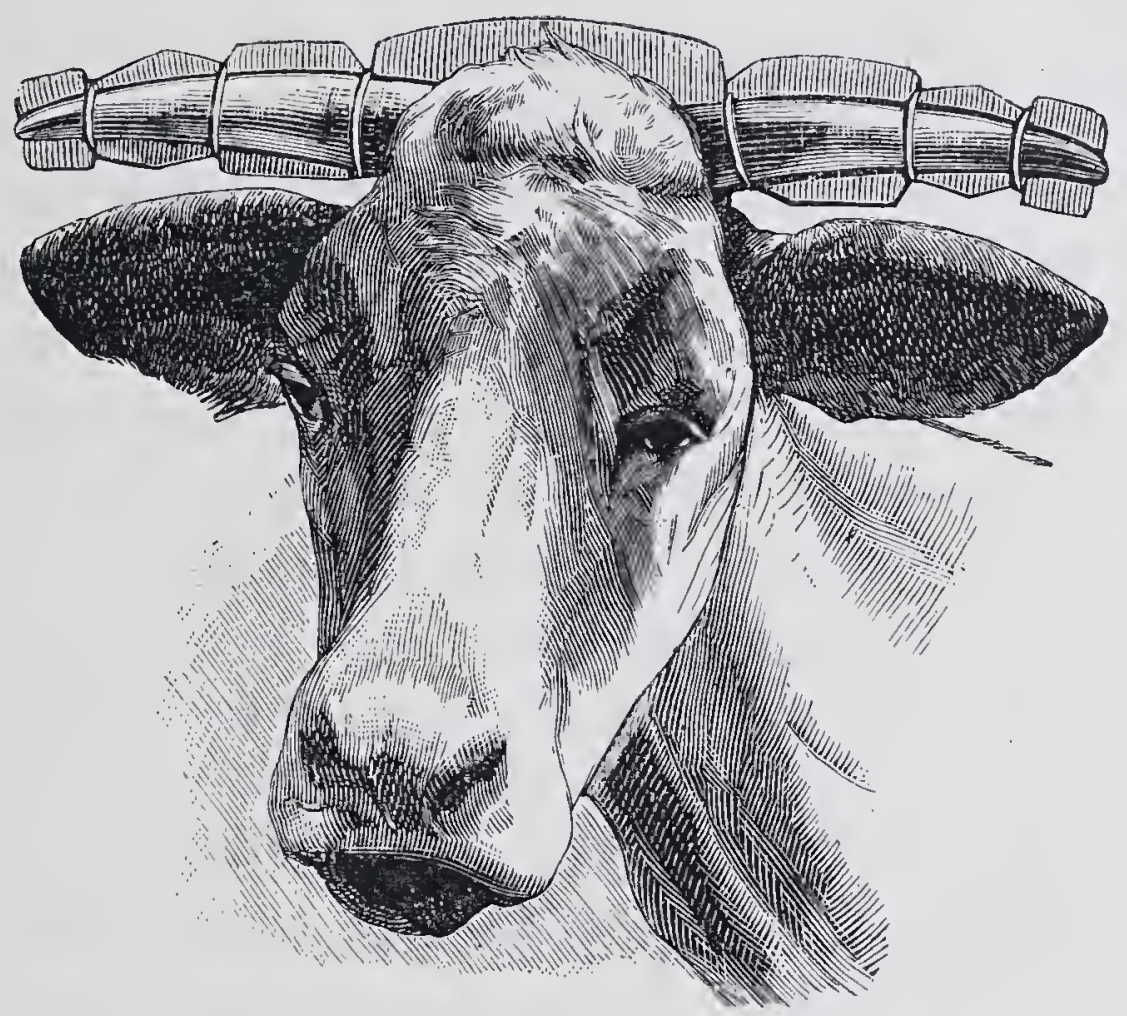

FIa. 66. - Splint for fracture of horn core.

the position and extent of the swelling, and the appearance of the connective tissue of the eye, but where there is much swelling diagnosis may be doubtful.

Treatment. Subcutaneous fractures of the orbital process, without marked dislocation, only require cold applications to minimise bleeding, and consequent danger to the eye. The animal should receive no food

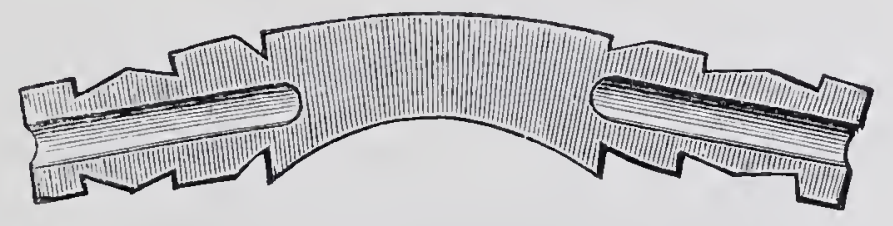

F1G. 67.-Form of above splint.

during the first twenty-four hours, for masticatory movements are not only painful but produce bleeding. Complete resection of the orbital process is not difficult, and in cases of compound fracture may become necessary. Any considerable displacement should be rectified, if needful, with the help of the bone elevator. Hendrickx recommends trephining. Antiseptic measures must be adopted from the outset, non-irritant fluids being selected. A bandage is then applied.

Fractures of the horn core are not uncommon in ruminants. They are recognised by the animal's resistance to manipulation of the parts, 
by abnormal mobility of the horn, and swelling, or even crepitation. Where blood has entered the frontal sinus there is often blood-stained or (in cases of longer standing) purulent discharge from the nose; in empyema of the frontal sinus the head is often held lower on the affected side.

The course is generally favourable; union goes on regularly in subcutaneous and often in complicated cases, especially if antiseptic treatment is early applied. In neglected and bad cases it is often advisable to remove the horn core entirely. In cows grave consequences are sometimes observed. Textor describes the occurrence of epileptiform seizures, which, however, disappeared when the sanguineo-purulent contents of the frontal sinus had been removed by trephining. After such fracture, a bull was unable to cover cows; erections occurred, but the animal, when attempting to spring, fell towards the diseased side, and was useless for stud purposes. In compound fractures pus formation may involve the brain, and bring about death from meningitis.

Treatment in subcutaneous fractures requires fixation of the horn core, which may be effected by a suitable splint passed over both horns, and secured by bandages (fig. 67). Skin injuries must be previously disinfected. The animal is tied up so that the horn cannot be displaced. When the horn is but slightly loosened it is often sufficierit to pass a bandage in a figure of eight tightly round both horns. Oblique fractures are often sufficiently kept in place by the horn itself, and the application of a tar bandage is quite sufficient. Where the core is so completely separated that union is not probable, it should be removed with the saw, and a dressing applied.

Fractures of those portions of the temporal bone which cover the brain are often attended with dislocation of bone and injury to the mucous membrane of the frontal sinus, and are indicated by swelling, depression on the forehead, a nasal discharge, at first blood-stained, and later purulent. These fractures, although usually healing without disturbance, are sometimes followed by chronic purulent disease of the frontal sinus. When the fracture remains subcutaneous, and no blood is discharged from the nose, recovery occurs without trouble.

Compound fractures are treated antiseptically; any cutaneous injury requires complete asepsis; where the mucous membrane of the frontal sinus is injured, as indicated by blood-stained discharge, complete asepsis is more difficult. But even here healing may be effected, and often before this is complete the animals may be returned to work. Severe dislocation inwardly may be remedied by the use of the bone elevator, or by trephining; the latter used, however, only when nasal discharge continues after union of the fracture. Other symptoms are treated according to general principles. 


\section{(4.) LOOSENING OF THE HORNS.}

Loss of one or both horns occurs under similar circumstances to fracture of the horn core. Sometimes the connection between horn and horn core is only loosened. Sometimes the horn, although completely divided from the core, can be replaced, but the new horn never attains the natural form, and a blemish results. Otherwise, loss of the horn scarcely exercises any other visibly injurious effect. Where the horn is loosened, its exterior is thoroughly cleansed, and a bandage smeared with tar applied. If completely loosened, the horn is removed, and the same treatment applied.

\section{(5.) WOUNDS AND INJURTES TO THE SKULL.}

Wounds and injuries, unaccompanied by fracture, require no particular treatment. Concussion may be produced by severe shocks, and is recognised by deafness, uncontrollable movements, and staggers. During phrenitis, horses sometimes have their heads injured, and it may be difficult to determine whether the brain affection or the injury stood first in order. In the horse, the skin carrying the forelock is sometimes extensively torn by sudden awkward movements of the head against fixed objects, and union is often difficult. Where primary union cannot be effected, the divided flap contracts, causing a blemish and depreciation of value. The injury is very common in pit ponies which work in low seams and without some special protective cap. In such cases the flap can be entirely removed, as appearances are of little importance, and such animals are not troubled by flies. Where a fragment of the occipital crest has been detached it should be removed, whether the wound be recent or of old standing. Healing is usually easy.

In the dog pusformation in the temporal muscles is often kept up by foreign bodies. From a hunting-dog Möller removed a splinter of wood $3 \frac{1}{4}$ inches long, said to have been in position for nine months. It had caused continually recurring abscess formation. The wounds in cattle resulting from pressure of the yoke will be described under the head of pressure injuries.

Prognosis and treatment are simple. Attention must be given to concussion of the brain, which is sometimes present. Rest and antiphlogistic laxative treatment are indicated. After careful disinfection, the edges of the wound are brought together with button sutures, or by deep sutures placed at some distance from the margins, and a bandage applied. When pus formation has set in, retraction of the lappets may sometimes be prevented by inserting deep stitches with a thickish thread. Where pus formation is seen in the temporal muscles of dogs, examination 
of the face should be made, to insure the removal of such foreign bodies as shots or particles of wood.

\section{(6.) YOKE-INJURTES IN WORKING OXEN.}

In working oxen the yolke often injures the head, necessitating skilled treatment. The causes of such injuries are very varied. Sometimes the slin alone, especially at the base of the horns, is bruised. Extravasation of blood under the slin is rare, though excoriations and even superficial necrosis of the skin occur frequently. Periostitis in the yoke bed is probably more common than is thought, but escapes observation. So long as the injury remains aseptic no great harm results, but when the parts become infected serious symptoms may follow. Extensive bruising of the skin with excoriation and free suppuration is rare, but furunculosis on the other hand is fairly common. Deep-seated suppuration, with necrosis of the periosteum, suppurative periostitis, necrosis of bone, and disease of the frontal sinuses, occurs in exceptional cases. Suppuration near the base of the horns is of particular importance, as the horns may thus be lost, seriously impairing the animal's usefulness.

The causes of these yoke-injuries are partly of a constitutional character. A narrow forehead concentrates the pressure on one spot, a bulging one causes the yoke to rock. Failure to keep clean the parts on which the yoke rests predisposes to injury, especially in wet weather. Badly-fitting yokes are a fruitful cause of bruises and excoriations; but want of skill in padding the yolie is less serious than unskilful padding in the case of horse collars. On the other hand, unequal yoking and bad driving often leads to injury, one ox doing an unfairly large share of work and his companion correspondingly less.

Symptoms. So long as the injuries are of an aseptic character the animal only shows a certain unwillingness to pull. This is often attributed to temper, and the veterinary surgeon is therefore rarely called in, his services being first invoked when there is some visible wound. Injuries are most liable to occur when the horns grow in a forward and downward direction.

The prognosis is usually favourable; even extensive necrosis of the frontal bone heals under suitable treatment. Injuries at the base of the horns must always be regarded with suspicion, as they may lead to loss or displacement of the horn so that the yoke can no longer be applied.

Treatment is chiefly of a prophylactic character. The yoke should fit well and the yoke bed be inspected and cleaned frequently. The oxen working together should be of similar speed and temperament, and the driver should see that each does an equal share of work. The local 
injuries are treated on general principles. In cases of necrosis, separation of the periosteum or horns from underlying tissues or secretion of fluid products, free use of the knife is called for. Every effort must be made to preserve the horns and to ensure the resulting cicatrix being as small as possible. If it is imperative that the animals continue at work, circumscribed injuries may be shielded by surrounding them with pads and so relieving them of pressure. Extensive disease of the yolie bed necessitates either entire rest or the use of a collar.

\section{(7.) TREPHINING THE CRANTAL CAVITY IN SHEEP OR CATTLE, OR OPENING BY THE TROCHAR.}

The cysticercus form of the tape-worm of dogs (Tænia cœnurus) often develops in the brain of the sheep, and occasionally in that of cattle and other animals. It usually attains the size of a duck's egg, and animals may die from the resulting disturbance. So-called gid or sturdy generally appear's in the first or second year of the sheep's life, producing significant phenomena. No treatment short of operation is effectual. Picric acid and other chemicals, cauterisation, and refrigeration of the skull with ice, recently recommended in England, and usually requiring to be persisted with for three weeks, are of no avail. Trephining or opening the skull with a trochar is the only satisfactory treatment. Even in the previous century this was attempted by laymen in a primitive way with the pocket-knife, but success could only be satisfactorily attained when proper instruments had been constructed. These consist of a small trephine about $\frac{3}{8}$ of an inch in breadth, or of a special set of trochars, which have been perfected by Zehden.

It is of primary importance to fix the position of the cysticercus. This is usually in the neighbourhood of the surface of one hemisphere of the brain. Sometimes it can be recognised by the softness and yielding of the bone on strong pressure. Percussion gives a dull, less loud sound over the affected spot, a symptom to which Villborg directed attention last century. But these appearances are often entirely wanting, and the position of the parasite can only be conjectured from noting the peculiarities of the patient's movements.

(1) Where the patient shows rotary movements, the cyst generally lies on the surface of one hemisphere, and on that side towards which the animal turns. Though this symptom, according to Möller's experience, is by far the most reliable, exceptions occur, especially where the cyst is large, and presses on the deeper-lying portions of the brain.

(2) I'wisting of the head towards the hind-quarter's points to the cyst lying in the depth of the hemisphere of the same side, or in the base of the opposite ventricle. 
(3) A depressed position of the head, with a desire to make trotting movements, and abnormal raising of the fore-limbs, suggests that the site is towards the front of the hemisphere, or in the depths of the hemisphere towards the corpus striatum.

(4) Staggering, with general uncertainty of movement, points to the cerebellum or posterior portions of the cerebrum as the position of the cyst.

(5) The base of the cerebellum, or the pons varolii or medulla oblongata, is affected where the animals fall down and make rolling movements around the long axis of the body.

(6) Stumbling, and frequent falling, and holding the head high (Segler), suggest the growth to be between the cerebrum and cerebellum.

These conclusions are evidently not very reliable. Experience, however, testifies that in most cases the skull over the hemispheres must be selected as the site of the operation. The bladder being of considerable size, it is not necessary to discover its central point. Accordingly, when the skull gives no direct evidence of the precise site, most operators trephine about $\frac{3}{8}$ of an inch behind the inner border of the horn in rams, and in ewes about $\frac{3}{8}$ to $\frac{3}{4}$ of an inch behind the horn core. The centre of the posterior lobe will be met by operating about $\frac{3}{4}$ of an inch from the middle line. To avoid injuring the sinus longitudinalis, the point of operation must not approach nearer than $\frac{3}{16}$ of an inch to the middle line. The best possible antiseptic precautions must be adopted. After opening the cranial cavity the thinned and protruding dura mater must be divided, and the bladder, which will generally be found lying below it, removed with forceps. The skin is then closed with catgut or silk sutures, powdered with iodoform, and covered with turpentine or tar. Wound gelatine is also suitable.

Möller's experience with trephining has not been favourable, and he prefers the trochar. The following is the method of procedure:-The animal is laid on its side on a table, the surface of the skull shorn, the wool removed, and the skin disinfected. In rams having large horns, where the skin inclines to form folds, this is rather difficult. The trochar is provided with a canula suitable to the thickness of the skull, that is, one in which the round shield is not much further from the end of the canula than the thickness of the skull, so that the instrument shall not enter the brain too deeply (fig. 69). With some care and practice, however, this appliance is not required. It must be remembered that the thickness of the skull varies considerably; in ewe lambs it may only be a few lines; in rams with large horns it is often more than $\frac{3}{8}$ of an inch. The point of the trochar (previously disinfected by boiling), being directed rather towards the middle line and backwards, is driven through the roof of the skull by a few light blows from a hammer.

The stilette is then removed, and if the bladdor has been struck its 
clear serous contents are discharged. After spontaneous discharge has ceased, the empty syringe (fig. 68) is inserted through the canula, and any remaining fluid removed.

The canula is now withdrawn, but the skin in the neighbourhood of the wound fixed by an assistant, so that the opening in the bone may be more easily found if the syringe has to be subsequently inserted. Where this precaution is not observed, the search for the trochar opening often takes much longer than would be expected. The syringe is held in the right hand with the thumb in the ring of the piston, and the point towards the little finger. Inserting it about as far as the canula penetrates, the piston is slowly drawn up by the thumb. Any water in the sac enter's the syringe and is removed, and the operation is repeated as often as required. A portion of the sac

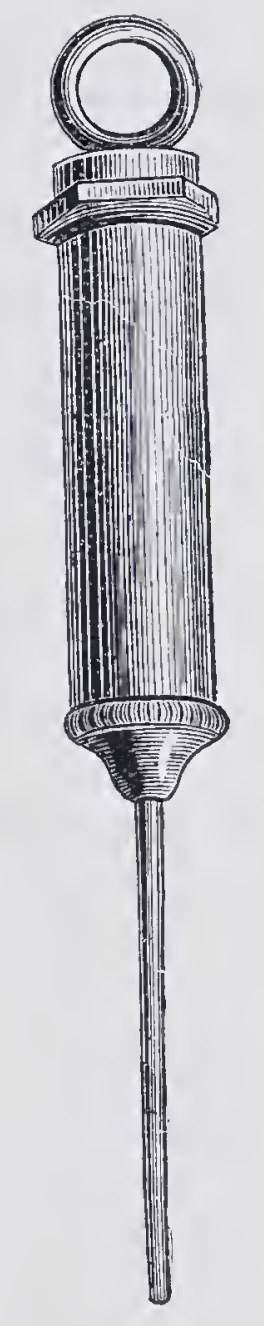

Fig. 68.Zehden's syringe. wall may enter the syringe, when resistance will be felt to the withdrawal of the piston. In such case the pull is maintained, and the syringe, drawing with it the sac and contents, are cautiously removed, until the sac wall comes in sight and can be grasped with blunt forceps and completely taken away. If the bladder remain full of serum, and prove difficult to abstract, it may be pierced with a thin steel probe, but bladder and parasite must both be completely removed. The skin wound is disinfected, covered with turpentine, collodion, or tar, and usually heals satisfactorily.

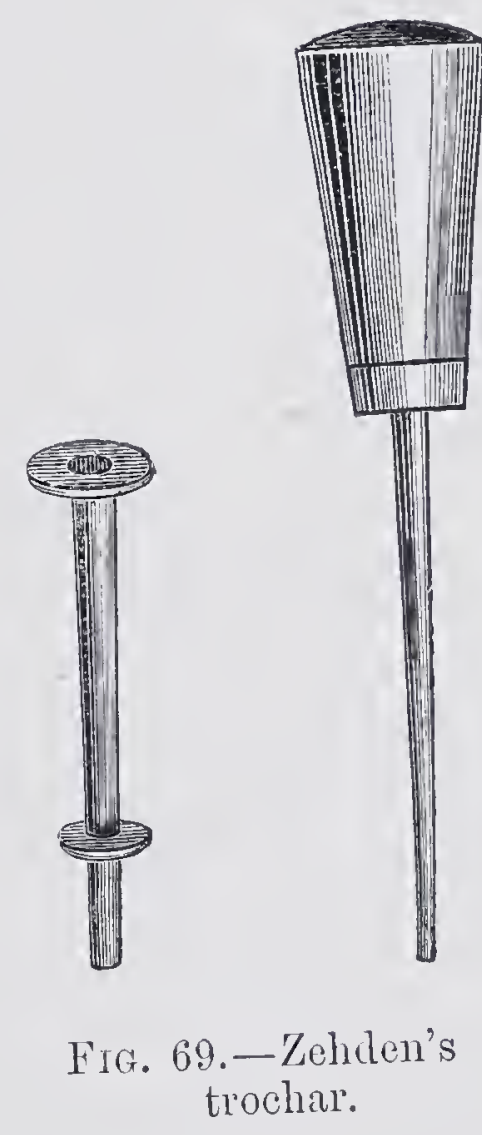

Difficulties, however, are sometimes met with. Animals apparently successfully operated on die frequently after a few hours, and post. mortem shows bleeding from some of the larger meningeal vessels. Where careful antisepsis is not carried out, inflammatory processes may also result. Injury to a lateral ventricle is especially dangerous, being apt to be followed by fatal hydrocephalus purulentus, running its course in a few days. Frequently the coenurus is in the cerebellum, when its removal is almost impossible. When several parasites exist in different positions in the brain the case presents serious difficulties. Sometimes the bladder is not met with when the trochar is introduced, no fluid discharges after removal of the stilette, and it becomes a question 
whether to introduce the trochar more deeply or to operate at another point. When antiseptically treated from the first, the wound seldom gives much trouble, and no objection exists to renewing the attempt in another place. Sometimes the sac lies so deep as to be impossible of removal, and little good can be done, even with the explorer first designed by Stürig, and improved by Lehmann. The injuries inseparable from the use of this instrument do not, however, occasion any
particular danger.

Rams with large well-developed horns give most trouble. In them the frontal sinuses are large, and cover so much of the skull that only the posterior folds of the cerebrum can be directly reached from the exterior: Möller endeavours, in such cases, firstly, to trephine the frontal sinuses, and thence to open the roof of the cranium with a trochar. It is difficult, however, to make the frontal sinus aseptic, and, in rinsing it out, large quantities of fluid pass into the nostrils, producing difficulty in breathing. Hence, during operation, the head should be pendulous, though this is attended with considerable discomfort to the operator. The thin mucous membrane can hardly be kept aseptic, and from it inflammatory processes are easily propagated to the cranial cavity. The easiest subjects are ewes, in which both skin and bone are
alike thin.

At best the losses are heavy. Generally only 10 per cent. are saved, and of these some die later of the sequelæ of the disease. The operation is usually confined to rams and specially valuable animals. The percentage of cures claimed varies greatly. Englehart records 6 per cent.; Stöhr, 15 per cent.; Scholz, 25 per cent.; Dammann, 33 per cent.; Reboul, who operates in primitive style with a cobbler's awl and feather quill, states that he has saved 50 per cent.; and Sütner, even 70 per cent. Kuhlmann claims to have saved from one-half to one-fourth of those operated on, exclusive of the cases in which no bladder could be the skilfulness of the operators.

The failures, in recent years, depend in part on the formation of skull and many operators do not remove the bladder, and the in part on the fact that relief. The prevention of such parasitism is secured by close observation of dogs and their treatment with vermicides, is secured by close observation of young sheep. Preferable to operation is the prompt stall feeding of lambs and of all subjects so soon as they manifest the prompt feeding and early slaughter

In cattle, sturdy is rare in North Germany, but occurs of the disease.

South. Cysticerci were found in the Salzburg slaughter-houses in in the numbers of cattle, and sturdy is said to be rather common in cattle througe out Steiermark, Kärnten, the Tyrol, Bukowina, and Dalmatia. Differing from of existence, cattle suffer at more advanced ages 
the same in both classes of animals, and consist of the peculiar position of the head, dilatation of one or both pupils, and abnormal movements, similar to those in sheep.

In operating, the marked differences in the skull formation of the two classes of animals must, however, be borne in mind. In cattle the frontal sinuses extend over the greater part of the cranium, and only through them can the cerebral hemispheres be reached. Hence diagnosis and treatment meet with the same difficulties as in large-horned rams. Softening of the external cranial plates evidently cannot occur here; but the inner temporal plates are sometimes so bulged that they come in contact with the external plates. This may be discovered by percussion, and the position of the parasite localised by sounding the frontal and superior maxillary sinuses, using the metallic end of the percussion hammer.

If an operation be deemed desirable, the frontal sinus must first be trephined, a soft point in the bony covering of the cranium may be discovered, and trephining or the trochar resorted to according to the rules previously given.

In cattle the disease often takes an acute course, rendering operation useless. Nor if performed does it insure success, the result often depending on accidental circumstances. Although observations of successful operations have been published (Cooper, Bertholet, and others), reports of unfavourable results are not wanting.

As the result is always uncertain, it is better to slaughter affected animals early, though treatment may be attempted in particularly valuable breeding animals.

Treatment is strictly surgical. Albrecht, Merkt, and others claim to have had a fair degree of success. Münch cured 8 cases out of 11 operated on ; Merkt had 50 per cent. of recoveries. Only those animals should be selected for operation which (1) eat well and show no marked interference with sensation, and (2) which always turn towards the same side.

The animal is cast and the operation performed with strict antiseptic precautions. Merkt, who is supported by Albrecht, describes the mode of operation as follows:-

An incision about $1 \frac{1}{2}$.inches long is made through the skin parallel with and distant $\frac{3}{8}$ of an inch from the middle line. The incision should not be commenced too high, as in that direction the two plates of the temporal bone lie wider apart. At right angles to this incision a second, about 1 inch in length, is made and then the second longitudinal cut. An assistant, kneeling behind the animal's head, holds back the flap with a hook, the exposed periosteum is carefully removed and a hole about $\frac{1}{2}$ inch in diameter is made with a trephine. This exposes the inner or cranial plate of the temporal bone. Very often, however, this cranial plate is in contact with the outer or has become absorbed; if not it must be removed with the gouge or bone forceps and bone screw, as it can seldom be reached with the trephine. In any case the trephine must be used cautiously, and the cut be made slowly, to prevent the instrument suddenly breaking through when near the end of the cut and so injuring the brain. Where the second plate exists it is necessary to remove with bone forceps all the thin, softened portions of bone covering the cyst so as to facilitate removal of the bladder, which is often of large size.

Having at last produced a hole about $\frac{1}{2}$ an inch in diameter, the brain will be seen, in favourable cases, to be pressing against the edges, and on incising the meninges the distended bladder will at once protrude. As soon as this occurs, the straw bedding should be removed from below the affected side of the head so as to allow it to descend and thus render removal of the bladder easier. The cyst usually bursts spontaneously; if not it can be ruptured 
with a director, after which the wall is cautiously grasped with forceps
and rvithdrawn.

The point of operation is then cleansed and the skin flap simply laid in place. The wound always heals readily. After-treatment consists in keeping the animal quiet and the wound undisturbed. Before allowing the animal to rise all assistants except two withdraw. The entire body, including the head, faction has passed. Where allowed to lie until the temporary period of stupethe head, attempts to sit upright, thetic struggles begin and the patient, lifting hours elapse before this oecight, the hobbles are removed. Sometimestwelve better than when the patient gets up quicklyses the results are always much

rhe animal is supported gets up quickly.

strong sack or length of cloth is sewn toghich it is placed backwards. A envelop the lower part of theth is sewn together so as to form a collar to are fastened respectively to two posts driven fis are attached two ropes which tance apart of one yard. This posts driven firmly into the ground at a disthe head. If it goes forward it haps the animal in position without touching the wall. The eyes should be bandaged in the sacking collar; if back it meets Animals sometimes recover very rapialy and the stable be kept perfectly quiet. 


\section{DISEASES OF THE NECK.}

Anatomy. In the domestic animals the cervical vertebræ, which are comparatively long, are surrounded by well-developed muscular tissues, and thus protected from injury. Anteriorly, i.s, below the cervical portion of the vertebral column, lie the trachea and osophagus, the jugular and carotid, with the large nerve trunks of the vagus and sympathetic ; behind the vertebræ, the ligamentum nuchæ, which gives attachment to many of the muscles of the neck. The latter consists of a round portion forming the upper border of the neck, and a flat portion which extends in the middle line from the vertebrx to the cordiform portion. The collective muscles of the neck are surrounded by two fasciæ or aponeuroses, which extend to the withers and back, and become attached to the cordiform portion of the ligamentum nuchæ. In cattle the skin is folded at the lower end of the neck to form the so-called dewlap.

\section{I.-WOUNDS AND BRUISES OF THE TISSUES OF THE NECK.}

Wounds confined to the skin are of no great consequence. Deeper reaching injuries may involve important organs. To this class belong-

(1) Bruises and wounds of the fascia of the neck and of the ligamentum nuchæ. When healing results by primary intention, these cases are also unimportant, but should pus formation occur, the tendinous structures (fascia, ligamentum nuchæ) usually become necrotic. Separation of dead particles requires a long time, and during the process fresh tracts of tissue are often destroyed. Healing is thus retarded and rendered difficult. This is especially frequent in injuries to the poll, withers, ligamentum nuchæ, or adjacent fasciæ, which lead to chronic pus formation and fistulie. The process often starts at the withers and extends thence to the ligamentum nuchæ and muscles of the neck; these conditions are treated of under "Diseases of the Back." Ii is here only necessary to remark that all such cases are treated by thorough asepsis, and preventing as far. as possible the burrowing of wound discharges.

(2) Injuries to the large blood-vessels of the neck, carotid and jugular. Injuries to the carotid by sabre cuts, thrusts from lances or other sharp objects, as well as those inflicted during venesection may endanger life, by producing fatal bleeding or suffocation. Injuries to the jugular vein are less grave, though its complete section may lead to fatal hæmorrhage; while there is also a danger of air entering the blood stream, an accident 
which is favoured by the negative blood pressure in this vessel, and by its liability to gape in consequence of attachment to neighbouring tissues. The escape of dark-coloured blood in a strong steady stream points to injury of the jugular vein, and calls for its complete section, or secure ligation, which latter must be performed on both sides of the wound; on the peripheral side to stop bleeding, on the central side to prevent entrance of air into the blood stream.

Free bleeding in jerks indicates injury of the carotid or of some other of the larger arteries, and necessitates ligation of the injured vessel. The closure of small skin wounds in no way removes the danger, because the blood accumulating under the skin and loose connective tissue of the throat presses on the trachea, and may cause suffocation. There should, therefore, be no hesitation in ligaturing the carotid.' At the point of bleeding, a cut must be made parallel with and a little behind the jugular, dividing the skin and subscapulo-hyoideus muscle. The loose connective tissue is pressed to one side with the index finger, and the vessel, easily recognised by its distinct pulsation, sought, drawn forward, separated from the vagus, sympathetic and recurrent, and after being twice ligatured, cut through between the two threads. The peripheral ligature is necessary on account of the collateral communication with arteries of the other side of the neck (occipital and innominate arteries). Section of the vessel is intended to prevent tearing away at the point of ligation, which is favoured by the natural tension of the carotid.

It is necessary to remark that the blood may flow in a regular stream, and not in jerks, even in injuries of the carotid. This is the case where the blood does not find exit directly, but flows under the skin or into the cavities of the connestive tissue. A red colour and powerful stream
always suggest injury to the carotid.

(3) The larger nerve trunks, like the vagus, sympathetic and recurrent, may be injured. One-sided division of the vagus, or even of the sympathetic and recurrent, do not endanger life, but cause unilateral paralysis of the larynx. From such injuries roaring has repeatedly been seen to result in the horse. Where injury to the nerve exists, or is suspected, the strictest antiseptic precautions must be observed to prevent extension of inflammatory processes to the nerves named, and to bring about as regular an union of the nerve endings as possible.

(4) Wounds in the neck may be complicated with perforation of the trachea; and though such a condition is seldom directly fatal, it occasionally leads to death, from blood entering the trachea and lungs and producing suffocation. The emphysema often seen about the neck after injuries to the trachea is an unpleasant, if not an actually dangerous, complication. Tracheal injuries are recognised by the bloodstained discharge from the nose, the frothy condition of the wound 
secretions, or of the blood discharge from the wound, as well as by emphysema in the neighbourhood of the wound. Bloody discharge from the nose, coughing, rattling in the throat, and dyspnoea point to the entrance of a large quantity of blood into the trachea. Where these symptoms occur, the bleeding vessels must be immediately tied and patients with injuries to the trachea, or in its neighbourhood, should not be left unattended. Extravasations in the neck sometimes lead to compression of the trachea and death from suffocation, and, where this threatens, tracheotomy becomes necessary. It is also called for where much blood has entered the lungs. A tampon canula is inserted, or the trachea plugged above the canula to prevent the blood passing downwards. In such cases the tracheal tube must be fixed with especial care to prevent the tampons falling into the trachea. For this purpose, and in order to be able to remove the tampon more easily, it is well to encircle it with a piese of tape, which can be fastened round the neck. For further information, refer to the article on tracheotomy.

(5) Injuries to the œsophagus are not so frequent. They usually occur in the lower third, on the left side of the throat. Cisophageal mischief is shown by the presence of chewed food or saliva in the wound. Though such wounds are not dangerous to life, they require a long time to heal.

(6) Wounds lower down on the neck, usually produced by the carriage pole, or by running against obstacles, may extend 8 to 12 inches deep between the shoulder blade and thorax, and cause extensive destruction of the muscular tissues of the shoulder and breast. They are still more serious when they extend to the first ribs, or the space between them. Complicated fractures of the ribs are often produced in this manner.

The wound, if confined to the muscular tissue, requires no particular attention, although fatal results occasionally ensue from extensive burrowing of pus, or from septicamia. The extent of such injuries is sometimes difficult to ascertain at first, and for a few days diagnosis should be guarded. Where one or other of the ribs is injured, purulent pleuritis and death can seldom be averted. Early and continuous irrigation should be adopted, and the wound in the skin and muscles laid open to allow free exit of discharge. This is even more requisite where the injury extends to the muscular tissue of the breast, and under the shoulder. With free opening and the use of drainagetubes such wounds, however, usually heal satisfactorily. Injuries involving the sternum are dangerous, because this bone is liable to become necrotic, producing so-called sternal fistula, which seldom or never heals. 'To prevent such complications the wound must be carefully cleansed, continuously irrigated, or frequently rinsed out, and drainage-tubes or setons used. 


\section{II.-FRACTURES OF THE CERYICAL VERTEBRÆ.}

The above fractures are not uncommon in horses. Fractures of the atlas, of the condyles of the occipital bone, and of the other vertebræ of the neck occur, sometimes singly and sometimes together. The usual cause is falling on the racecourse-or in steeplechasing-or being driven over, or, in the case of refractory jibbers, from a rope or chain being passed around its neck and another horse harnessed to it. Ridinghorses in violently bucking or falling over backwards, or even in arching the neck excessively, not infrequently fracture a cervical vertebra, usually the third or fourth. In bucking or leaping, if the fore-limbs are not rapidly extended, the animal comes to the ground on its mouth, and thus falling, even on soft ground, may fracture the third or fourth vertebra. Hertwig states having seen fracture of the odontoid process of the second cervical under these conditions. But such fractures are rare. Vertebral fractures are seldom produced by liicks or thrusts with the carriage pole. Cattle cause them by violently tilting at each other. In small animals they result from blows with heavy sticks or attempts to crawl through narrow openings in which they become fixed and struggle to get free.

Symptoms. In horses, death usually occurs instantly from arrest of respiratory and cardiac action, a result of the implication of the phrenic and other nerves of respiration. But in fractures of the first or of the last two cervical vertebræ, the animals may live for some time. Fractures of the last two are often followed by paralysis of the muscles of the shoulder, while fractures of the oblique processes produce
torticollis.

A riding-horse, injured by falling, was ridden for some miles, but died four days later, and post-mortem revealed fracture of the sixth cervical. O. R. Scharfenberg described the following case:-A horse, after running against a door, fell, rose again, and worked for three hours. Next day paralysis set in, second cervical broken completely across. A horse after falline body of the course was ridden some four or five miles further after falling on the raceappeared, and two hours after the fall the further. Signs of paralysis then a transverse fracture of the body of the fourthe died. The autopsy showed examined after death by Rabe, had fractured cervical vertebra. A horse, by falling, carried its head awry, showed swelling in the neichbougust 21st the atlas, and threatened to fall immediately any attempt was made to raise
the head. After standing quietly in the stable until Septo was moved, rapidly became worse, and in consequil September 8th, it ox thrown down whilst struggling with an consequence was killed. An placed in a stall, however, it soon become another could still run; on being vertebra was found, oner, it soon became paralysed, and the second cervical

Köhne records fon post-mortem, to be broken into four pieces.

fallen. On the following of both wings of the atlas in a horse which had and marked stiffness of the theck med difficulty in breathing, rolling gait, 
moving the latter. These symptoms continued; and having fallen on the twenty-second day, the horse was unable to rise again, and died three days later. Post-mortem showed formation of callus on the broken wings, and inflammatory processes in the meninges of the medulla oblongata. A cow whose horms had become fast in an iron rack was found unable to stand, to move the head, or to eat. Post-mortem discovered fracture of the second cervical vertebra, and bleeding into the vertebral canal.

During the year 1891 the following vertebral fractures were noted amongst the Prussian army horses:--Thirty cervical fractures, six dorsal, and seven lumbar.

Injuries to the spinal cord in man are generally accompanied by abnormal rise in temperature, reaching 43 to 44 centigrade; but in animals no observations are available on this point.

The course of the disease is, then, similar to that in man, although in him death occurs somewhat later, because the medulla oblongata or the spinal cord is not so often injured. Displacement of the pieces of bone, bleeding into the spinal canal, or inflammatory processes generally disturb the function of the spinal cord later, and bring about death. In fractures of the $3 r^{r}$ to 6 th cervical vertebræ, death usually occurs immediately, with appearances of suffocation. As the diaphragmatic nerve is formed by the 5th, 6th, and 7 th cervical nerves, its action is soon destroyed in fractures of the last cervical vertebræ, and asphyxia must necessarily result. If the fracture is confined to one or other of the vertebral processes, the animal may live for a time, or even recover.

Treatment of fracture of the body of a vertebra is usually impossible, because death is almost immediate. Otherwise, especially in animals worthless for slaughter, it is of the first importance to prevent displacement of the broken fragments. Where such a fracture, or one affecting any of the processes, is believed to exist, the animal is placed in a condition of absolute rest, and measures adopted to prevent, as far as possible, all movements of the head and neck. If soft parts are injured, antiseptic methods must be employed to prevent pus formation, necrosis and spread of inflammatory processes.

Complete displacement of the cervical vertebræ, said to occur in the horse, may here be mentioned. As it usually causes immediate death, there is seldom opportunity for a precise diagnosis. Conditions described as luxations of the vertebræ have doubtless often been merely fractures. This is true of a case described by Vives. A mule, which shortly before had been actively galloping about, was observed standing immovable, with head sunk, extended, and turned to the left. Attempts to lift the head produced much pain, the neck was swollen, and the occipital region distorted. The right wing of the atlas extended further forward than usual; the left could not be felt. Next day severe swelling of the head had set in. On the thirty-fifth day the condition remained essentially the same. 


\section{III.-DISTORTION OF THE NECK (TORTICOLLIS, CAPUT OBSTIPUM).}

The collective term torticollis, or caput obstipum, has been used in human medicine since olden times to describe many different conditions, which, on careful examination, have nothing in common with that now under consideration. In animals, distortion of the neck sometimes occurs, but the precise anatomical changes on which the abnormality depends are not recognised. On this account, while adopting the above designation, we shall endeavour to point out the nature of the more frequent of these curvings or distortions.

(1) Cramp or contraction of the muscles of the neck occurs both in men. and animals. In men the most frequently affected muscle is the sterno-cleido-mastoideus; its shortening is often congenital, or develops through cicatrisation after birth. Such conditions have not been observed in animals. But Uebele records that an eight days foal showed at short intervals attacks of cramp in the left cervical muscles, drawing the head to the left; when not supported, it fell; the condition disappeared in fourteen days. Möller has repeatedly seen temporary displacement of the head and neck in horses, consequent on rheumatic affections of the above-named and other muscles. These abnormalities were accompanied by a lameness, most marked when the limb was being lifted. In dogs similar rheumatic contractions in the muscles of the neck also occur, usually on both sides; can be recognised by local pain and swelling, and may exist only for a few hours. In pugs, in which it is common, such attacks often recur.

(2) Paralysis of the muscles of the neck. Whilst the diseased conditions causing the above-named distortions are to be sought on the concave side of the curvature of the neck, the cause of paralytic torticollis exists on the convex side, as may be seen in dogs and rabbits during the course of diseases of the middle ear. In the German Army Reports a horse is mentioned in which paralysis of the muscles and production of torticollis resulted from a carcinomatous growth on the petrous temporal bone. Torticollis appears in horses as an accompaniment of the general paralysis of meningitis cerebralis, and in diseases of the medulla oblongata and medulla spinalis in the neck. Wilden speaks of a horse which showed torticollis during an acute brain attack, but recovered as the cerebral symptoms disappeared. Leisering saw a dog which suffered from torticollis, and simultaneously from hemiplegia and paralysis of the ear and eye, with softening of the pons varolii, medulla oblongata, and cerebellum. In birds-hens and ducks-Möller has often seen the head bent in a semicircle (so that the beak was turned backwards) in 
consequence of brain disease, or following intoxication produced by coal gas. The same is noticed in canaries.

(3) Inflammation of the soft parts, especially of the muscles, consequent on severe strains and lacerations, is produced in horses by falling, and is often described as subluxation of the cervical vertebræ. Fambach had under observation a horse which, by hanging back in the halter, produced rupture of the round portion of the ligamentum nuchæ just behind the occiput; pus formation and necrosis occurred, and were followed by death. Flessa describes a case of torticollis in a horse produced by rupture of the levator humeri muscle at the border between the upper and middle thirds; recovery occurred in two months.

(4) Subluxations and fractures of the cervical vertebræ. Complete luxations of the body of the vertebræ, as above stated, are almost always fatal. On the other hand, subluxations, accompanied by distortion of the neck, may occur without injury to the spinal cord. In France the condition is described as "entorse vertebro-cervicale," and consists in subluxation of one or other of the oblique processes of the bone. As the bodies of the vertebræ are attached to one another by cartilage, their division is more appropriately described as diastasis, but this is a condition not infrequently complicated with fracture of the oblique processes. According to Schrader, Hippocrates declared that the conditions described as displacement of the cervical vertebræ were often only muscular diseases, and that Absyrtos, in his contemporary History, expressed himself in the same sense. ${ }^{1}$ Lebel, Hurtrel d'Arboval, and others combated the possibility of displacement of the cervical vertebræ, and supported their contention by citing cases of spontaneous recovery.

Hertwig has shown, however, that such displacement may occur in consequence of rupture of the ligaments of the oblique processes. Williams found degenerative processes in the inter-articular ligaments. In other cases the oblique processes are fractured. Guitaud and others could even detect distinct crepitation. Busse states having directly felt the broken oblique process of the vertebra. Möller detected this fracture on post-mortem examination. In 1892 he treated a horse which had been found one morning cast in its stall and unable to rise even with assistance. In spite of all efforts it was only got up next day, and then showed unmistakable symptoms of torticollis. The neck was bent at its middle point, at a rather sharp angle, and directed downwards and towards the right side. The head could certainly be pushed towards the left, producing crepitation, but immediately fell back into the abnormal position when released. Post-mortem showed the oblique processes of the 4 th and 5 th cervical vertebræ to

1 Sed te nolo latere, non luxationem esse sed perversionem (Schrader). 
be fractured, and their surroundings infiltrated with blood. There was no injury either to the bodies of the vertebræ, the spinal cord, or its covering, nor had bleeding occurred into the vertebral canal. The

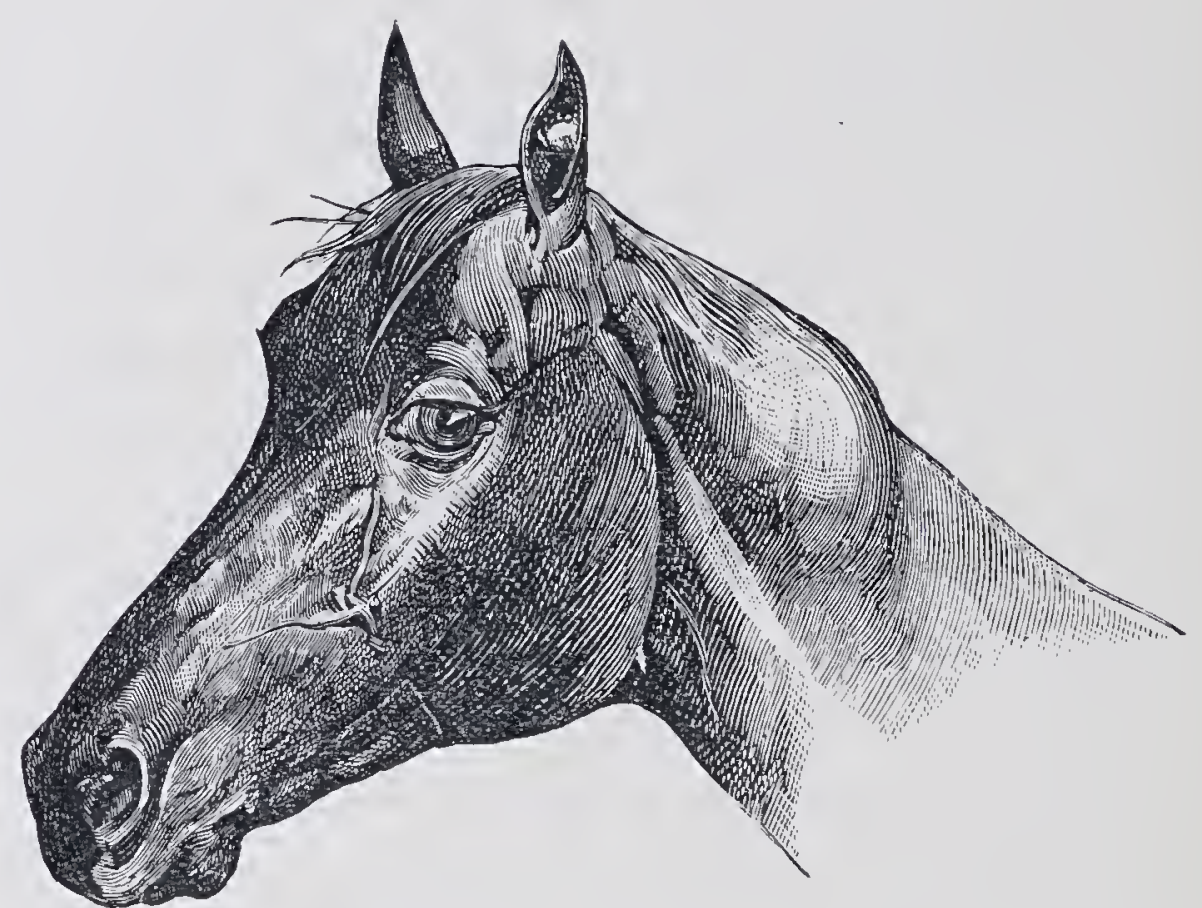

FIG. 70.--Subluxation of the third cervical vertebra, as seen from the left side. torticollis was clearly not the mere result of fracture, but of hæmorrhage, producing paralysis of the cervical nerves. Labat saw a similar case.

In the same year a thoroughbred was sent to Möller, which had fallen and produced distortion of the neck in the region of the 2 nd and $3 \mathrm{rd}$

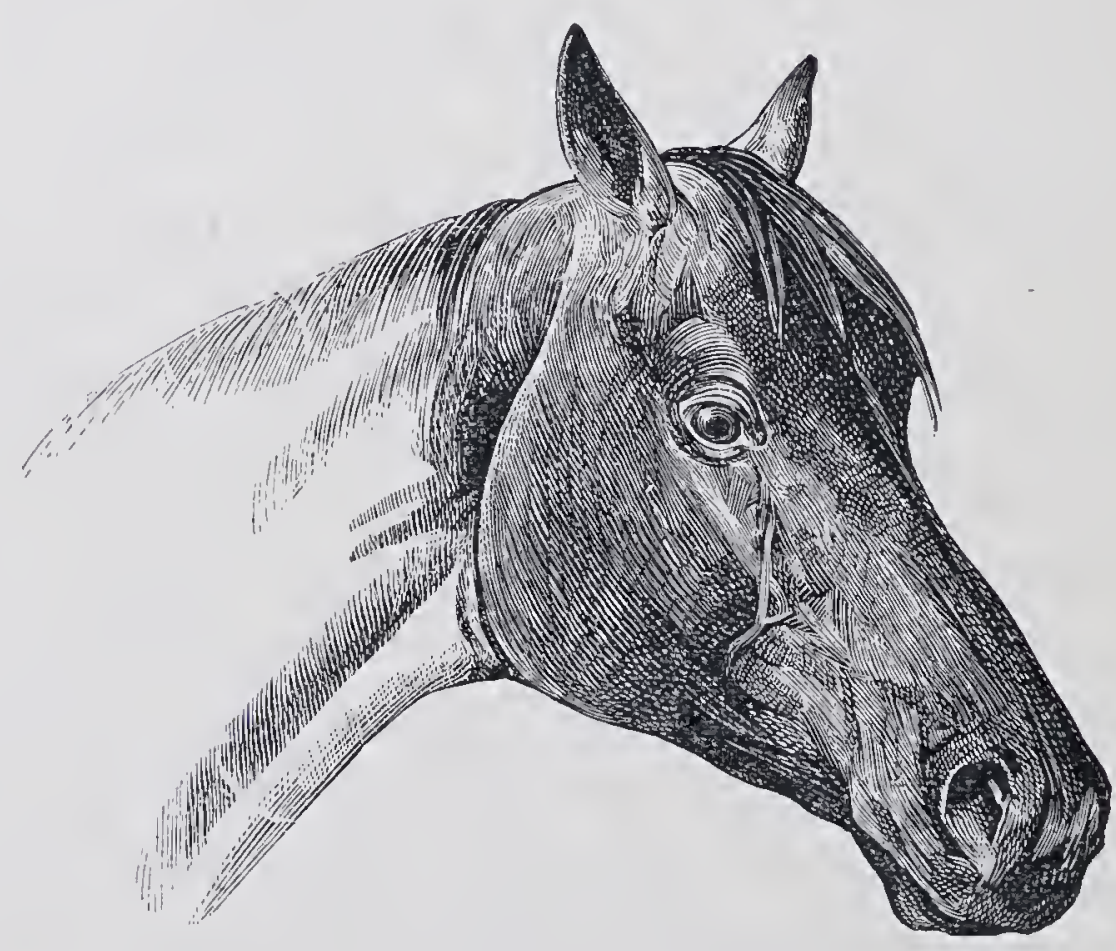

FıG. 71.- Subluxation of the third cervical vertebra, as seen from the right side.

vertebrx. The swelling, which had at first been considerable, had mostly disappeared. On the left side, between the 2nd and $3 r d$ cervical vertebræ, a distinct prominence was seen (fig. 70); whilst at the corresponding point on the right, a depression existed sufficiently large to accommodate the two hands when extended (fig. 71). The condition was doubtless due to subluxation between the 2 nd and $3 r d$ cervical vertebræ. 
Werner saw a horse which after running into and striking a carriage with its head lay senseless for three minutes, but on getting up was able to resume work. Ten hours later the head was bent towards the right; on the left side of the neck over the second cervical vertebra was a marked swelling. The gait was unsteady. The neck could not be brought into a straight line. Post-mortem examination showed a

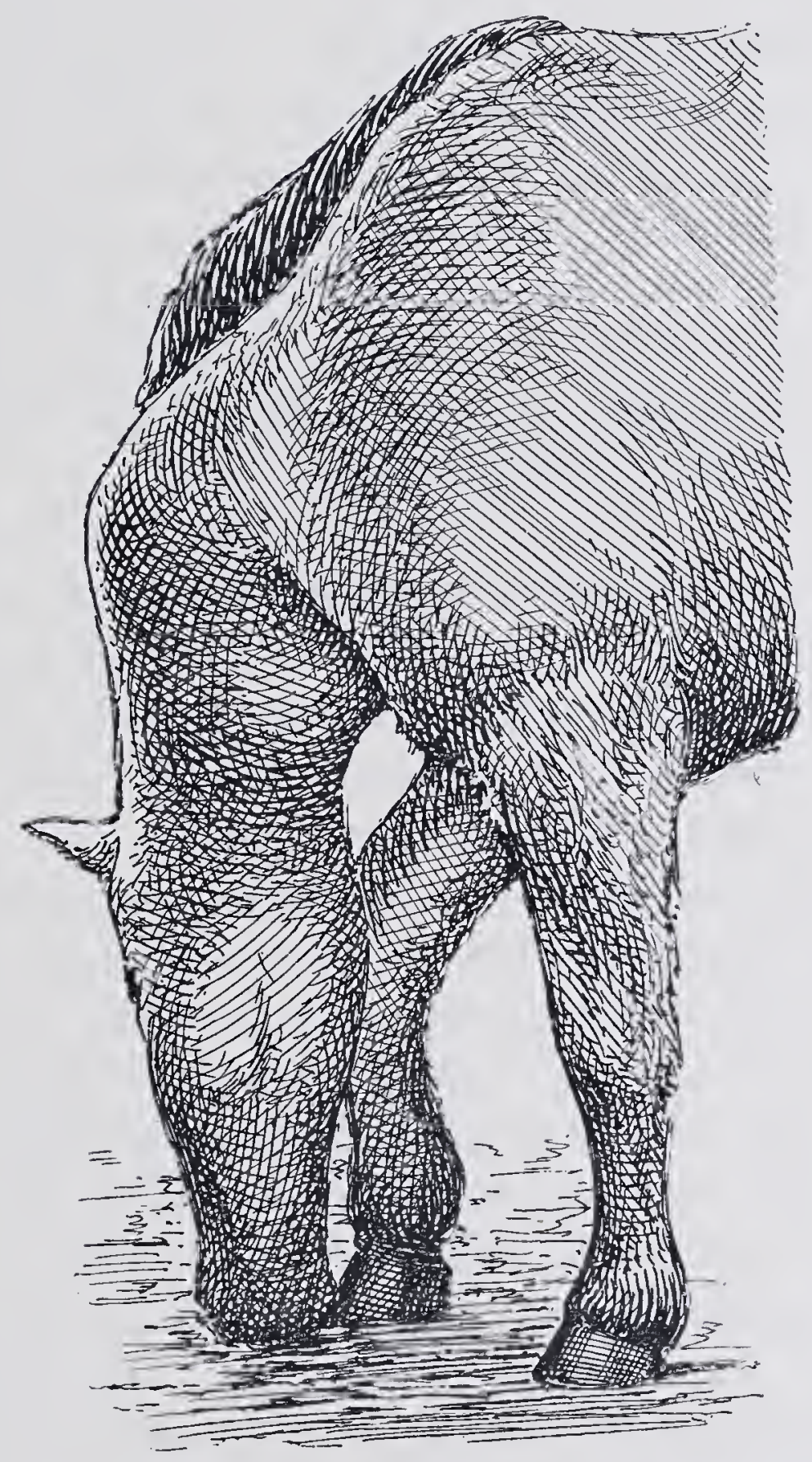

FIG. 72.-Distortion of the neck (torticollis) in consequence of the calkin of a hind shoe becoming caught in the head-collar. (Redrawn from a photograph.)

transverse fracture of the second cervical vertebra and submeningeal hæmorrhage.

Mongiardino had the opportunity of making autopsies on two horses showing torticollis. In one the intervertebral dises between the $3 \mathrm{rd}$, 4th, and 5th cervical vertebræ were partly torn through. The head of the fourth vertebra slipped partly out of the cup of the third when the neck was bent. In the other case the capsular ligaments and the intervertebral ligaments on the convex side of the neck were strained so that the articular surfaces no longer corresponded. The cervical muscles on the convex side were paralysed and had undergone degeneration. 
A horse mentioned by Grams had hung back on a halter; when its head was turned towards the right the middle of the neck sprang outwards, when towards the left the dislocation was reduced. The animal recovered after the application of a special splint.

The case described by Mr. Martin (The Veterinarian, 1896, p. 39) as dislocation of the cervical vertebre may have been a subluxation or' only a muiscular injury. The pony had been "cast" with its off hind shoe fixed in the head collar, probably for a considerable time. When released and got up it presented the appearance shown. Attempts at replacing the parts produced staggering, strabismus, convulsive picking up of the hind legs, and spasms of the body muscles. Splints were applied to the neck and the animal was tied up, but fell during the night and pulled down a beam. On the sixth day after the accident improvement was noticeable and the splints were readjusted. In less than three weeks recovery was complete.

Hering's failure to produce the condition artificially in the horse does not disprove the occurrence of such subluxations and fractures, but only shows that simple injuries to the muscles produce changes in the manner of carrying the neck. Gerlach observed the disease in cows and horses, and described it as distortion of the cervical vertebræ. With incomplete displacement of the latter, the head is directed sometimes to one side (abduction-luxation); but sometimes the neck thdergoes more or less pronounced rotation (rotation-luxation) which, strictly speaking, alone corresponds with torticollis. The condition is seen most frequently in horses; which, when scratching the head, get the hind-foot fixed in the halter and are thrown, or in.jibbers, around whose neck is passed a rope or chain, to which another horse is harnessed.

Diagnosis. The conditions described cannot always be differentiated, even on careful examination. Cognisance must be taken not only of the symptoms, but of the history of the case. Distortion produced by muiscular diseases is usually distinguished by pain, swelling, and other inflammatory changes, while the neck, although it may be brought into its normal position, reverts to its distorted state when force or pressure is removed. Cicatrices are a rare cause in the lower animals. When inflammatory appearances are absent, paralysis of the muscles on the convex aspect of the neck may be suspected, especially if cicatricial contraction can be excluded as a cause.

Distortions arising from fractures and subluxations may be distinguished from those connected with muscular lesions by crepitation, and still more notably by the fact that the parts, even if difficult to bring into position, retain that position when once reduced. Many observers state that in subluxation the neck is excessively mobile; but although it may be so in certain directions, general freedom of movement is 
diminished. Egelling saw what he considered to be persistent huxation of the cervical vertebræ. The horse could even be ridden.

Slight curvatures usually produce little inconvenience, but considerable displacement of the head leads to difficulty in movement, and the animal is sometimes unable to get up or to stand, though it can do both immediately the head is supported in its normal position. Others make "circus movements" towards the side to which the neck is curved. Where curvature becomes excessive, as is frequently the case in dogs and rabbits, the animals attempt to rise, but at once fall back, and make rolling movements around the long axis of the body. This is occasionally seen in dogs with disease of the middle ear, and in rabbits affected with mange of the ear. Sometimes, in horses which have suffered from severe strain of the collective muscles of the neck, the head is carried very low, producing considerable cedematous swelling, and causing the animal's head to resemble that of a hippopotamus.

Ruptures of the funicular portion of the ligamentum nuchæ can be directly felt and recognised by the head being carried very low. The same position is assumed in disease of the upper vertebræ, and of their joints. Möller had a horse with purulent arthritis between the first and second cervical vertebræ, produced by the breaking through of a retropharyngeal abscess. The horse held the head and neck stiffly, and threatened to fall when attempts were made to lift the former. In purulent arthritis fever usually exists, but is altogether wanting in diseases of the muscles produced in a mechanical way.

Course and prognosis. Distortion of the neck due to rheumatic disease of the muscles usually disappears in a few days. It often continues only a few hours, although in certain individuals it may recur, and is signalised by great pain, which causes whimpering or crying. Though caput obstipum caused by paralysis may disappear in a short time, it sometimes remains for long periods, or even proves incurable. In forming a sound prognosis, it is important to discover the cause of the paralysis. In Möller's practice, cases connected with ear disease have all proved incurable. Paralysis which has occurred suddenly, or existed only a short time, and is due to rheumatic or traumatic causes, may generally be expected to disappear earlier than that which has developed more slowly, and depends on disease of the central nervous system. In the latter case, prognosis is always doubtful, and is still graver when the condition is complicated with convulsions. On the other hand, distortion of the neck produced by muscular strain is cured in eight to fourteen days, or even earlier. Hertwig emphasises the fact that diagnosis must be cautious during the first few days; a safe judgment cannot be formed from early appearances; severe disturbances 
often disappear quickly, whilst apparently slight cases may take an unfavourable course.

Conditions caused by muscular disease seldom leave any lasting ill results, though extensive ruptures may later be recognised by depressions. Schrader found, on the neck of a three year old colt, a furrow in which the fist could be laid. After luxations or fractures, cicatricial thickenings are often observed. Harvermann noted these in horses, on the concave side of the diseased neck. An army horse with subluxation of the $3 \mathrm{rd}$ and 4th cervical vertebræ again became useful after some months. It is important to note whether the animals can stand and take food; if not they may die of decubitus, ${ }^{1}$ or lose condition and value. As a rule, in such cases slaughter is to be recommended, especially where no considerable improvement has resulted within several days after the attack. Still more unfavourable is the prognosis where purulent disease

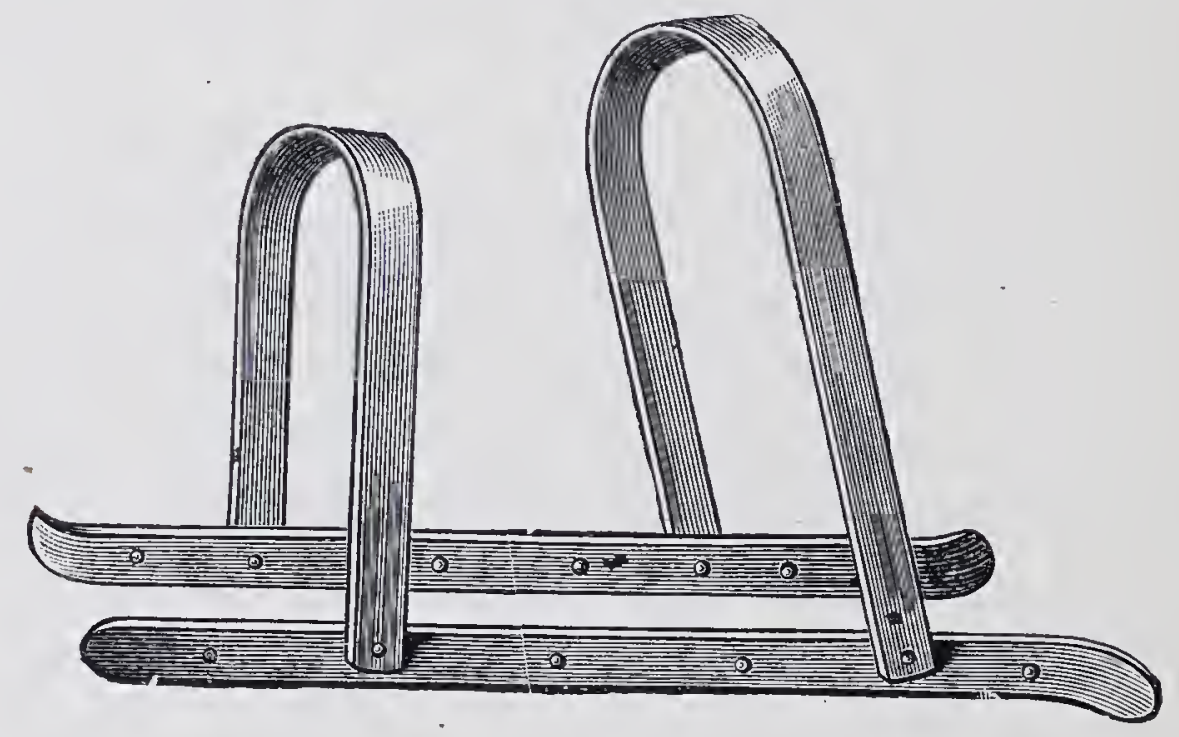

FIG. 73.- Knudsen's iron splint for distortion of the neck.

invades joints like that between the occiput and the first cervical vertebra.

Treatment of rheumatic torticollis comprises warm coverings, friction with volatile stimulants, and administration of salicylic acid, potassium iodide, antipyrine, and similar drugs. It is especially desirable to keep the animal standing where distortion depends on sprains of the muscular tissues or disease of the spinal column. The neck may usually be kept straight by a girth, cradle, or narrow piece of wood, fastened with the roller and a strong halter to the concave side of the neck; sometimes by simply fastening up the head.

Where luxation is present, replacement must be attempted. In heavy horses counter-extension may be effected by the weight of the body. Where this is impracticable, the animal may be fixed with the help of a horse collar, while, with a strong, well-fitting halter, extension may be

\footnotetext{
${ }^{1}$ Decubitus.--This is a convenient medical term, signifying the continued assumption of the recumbent position which often accompanies debilitating or painful diseases like laminitis.-
[JNO.A.W . . . ]
} 
effected by several strong assistants, or by the help of a compound pulley. Godine states having thus produced an extension of $3 \frac{1}{4}$ inches in the neck of a horse. Immelmann passed a strong hop sack, doubled, round the head of the horse, and allowed fifteen persons to pull on it. During extension, the operator endeavours to effect reduction by pressing or turning the neck at the middle of the convexity. This attempt generally succeeds, the distortion immediately disappearing. Busse laid his patient on the ground, with the concavity of the neck downwards, placed a board on the convex side, and exerted strong pressure on it. 'The displacement, even when reduced, may recur on the horse rising. In such cases fracture of the oblique process usually exists, and the application of a bandage is necessary. Hertwig recommended two splints of wood or tin, which fitted exactly the side of the neck and reached from the shoulder to the posterior border of the lower jaw, and were fastened together at the upper and lower ends of the neck by bands. Stockfleth recommends an iron splint (fig. 73) constructed by Knudsen. Owing to its yielding nature, it is easily fitted. The splint, when applied to the convex side of the neck, must be padded with tow or wadding to prevent bruising. The horse is supported on rising, and afterwards fastened up short to the wall. The use of slings may be desirable. Cattle can be treated in the same way. The apparatus should remain in position eight to fourteen days, sometimes a little longer, care being taken that the animal does not lie down, and that the splints are kept carefully padded.

Inflammatory processes sometimes require the use of cold applications. Massage may be used later to bring about absorption. Where the head is sunk and oedematous swellings result, producing dyspncea by narrowing the nostrils, tracheotomy may be performed if necessary. Swelling usually disappears on raising the head and supporting it with a girth.

\section{IV.-GOITRE. STRUIMA.}

The thyroid gland, though not so frequently the seat of disease in domesticated animals as in man, does become affected, especially in carnivora,-less frequently in horses and cattle. Acute inflammation (thyroiditis is unknown in animals, though transitory swelling of the glands, without other inflammatory appearances, is seen in horses and doge during the progress of catarrhal affections of the pharynx, but disappears during convalescence. More frequently new growths, whose nature is not precisely understood, invade the glands. Hyperplastic processes, since olden times named Struma, also occur. New growths resembling: these develop in the gland, but being difficult to precisely identify, are included in the clinical term Goitre. The following varieties can be distinguished:-(1) Real goitre, dependent on hyperplasia of the 
follicles of the thyroid gland, with colloid change of their contents, which are chiefly albuminous. The swelling is mainly due to enlargement of the follicles, and is termed struma follicularis. It may attack the entire organ or only one-half, less frequently it is confined to certain sections. (2) In fibrous goitre the connective tissue is specially increased, the follicles, on the other hand, being little affected. This form is generally confined to single portions of the gland, and seems more often to be of an inflammatory character. (3) The gland is exceedingly rich in blood-vessels, and these, especially the veins, become greatly dilated, causing varicose goitre (struma varicosa). (4) At tirnes single follicles undergo distension, producing the so-called cystic goitre (struma cystica).

Specific new growths, like carcinoma, have been seen in dogs by Bruckmüller, Zschokke, and others. Zschokke says that at least 30 to 40 per cent. of old dogs suffer from goitre, and whilst in young animals the condition consists simply of hyperplasia of the gland, the goitre of old dogs is almost always of a cancerous nature, and is prone to degeneration and bleeding. Gerlach and Honert found sarcoma of the gland in horses. Tubereulous swellings have been met with by Bruckmüller and others in cattle. Johne has described carcinoma of the thyroid, accompanied by secondary carcinoma in the lungs of a horse. But the older reports of these growths are not absolutely reliable, as the classification of tumours was not then so precise as now.

The causes of goitre are just as little lnown as those of other tumours. Endemic outbrealss are stated to have occurred in animals as in men (Baillanger in mules, Wörz in cattle). French veterinarians have further insisted that in regions where goitre frequently occurs in men, it is also common in animals. 'That locality has some predisposing effect seems undeniable, but complete uncertainty exists as to the immediate cause of the disease. Whether the existence of magnesia or the absence of iodine and bromine in the soil exercises any influence, as has been asserted, is at present undecided. Little is lnown of the connection of goitre with cretinism, no veterinary statisties being available.

In animals, and especially in carnivora, goitre appears most frequently during the earlier years of life. Without doubt it may, under certain circumstances, be inherited, and Möller has repeatedly seen almost all the puppies of one litter affected. Lydtin related the case of a stallion which transmitted goitre to five of his offspring. Johne saw a case of inherited goitre in the dromedary. According to Haubner, the lambs of one flock were attacked enzootically in certain years. In one season six, and in the following, ten new-born lambs were affected, and the disease, it was believed, was transmitted by the ram. Similar observations have been made by Gurlt in goats. It should, however, be noted that the size 
of the thyroid varies considerably in certain animals, especially in horses. Not infrequently its lobes, normally the size of a chestnut in the horse and ox, become as large as a duck's egg, whilst the commissure remains little affected. This liability to variation is widely observed in particular: families, and appears, to a certain degree, to be inherited. Such conditions cannot consistently be described as goitre, for the gland, although abnormal, does not continue to grow larger. Many references to inherit: ance and enzootic outbreaks of goitre in animals are clearly to be referred to this condition.

Symptoms. The most striking feature is the swelling close under the larynx, and towards the side of the trachea. In horses the gland on either side, usually not larger than a chestnut, attains the dimensions of a man's fist. It sometimes becomes as large in dogs. As the gland grows in size and weight it sinks, and becomes more prominent. Either one or both sides may be affected. In dogs it is easily displaced, and may descend as far as the lower portion of the neck, and lie immediately in front of the chest; but it seldom exhibits inflammation, pain, or increased temperature.

As long as the size of the thyroid is not much increased, goitre in animals is of little significance. In the horse, however, Massot and Truelsen have observed dyspnce from the swelling pressing on the trachea. This occurs more often in dogs, which may be unable to lie down, on account of the swelling compressing the trachea and interfering with breathing. The animals live for some time continuously supported on the extended front legs, until finally it becomes necessary to kill them. The trachea may be narrowed until it closely resembles a scabbard. Zschokke found carcinomata in dogs invading the trachea and cesophagus, and producing difficulty both in breathing and swallowing. Johne reports the case of a dromedary calf which died at birth from suffocation produced by a goitre swelling weighing nearly 13 pounds. In a horse, sarcoma of the thyroid spread entirely over the larynx during the space of a year and a half, and finally caused death by suffocation.

Prognosis. In animals, and especially in young dogs, goitre is not simply a blemish, although some varieties occurring in horses cause no inconvenience. The significance of the case depends upon the size of the swelling and its rate of increase, but our knowledge of the condition does not justify very sanguine expectations. Extirpation entails not only the danger of excessive bleeding, but also the development of peculiar symptoms described as cachexia strumipriva, which are frequently seen in young dogs. Some weeks after removal of the thyroid in its entirety, marked weakness appears, accompanied by anæmia and wasting, from which the animals finally die. These symptoms, however, can be prevented by giving a daily dose of fresh thyroid gland. Goitre, in man, 
is associated with œdema in different portions of the body (myxœdema) and with sensorial disturbances (cretinismus). But the exact relation these have to disease or removal of the thyroid is not yet explained.

Von Eiselsberg observed the disease in lambs. Two months after extirpation of the swelling, the animals' growth became checked. They remained much smaller, weaker, and of less weight than the control animals. The anterior portion of the head was shorter, the posterior portion enlarged, the abdomen distended, the testicles atrophied, the wool badly developed. One of the sheep suffered from catarrhal attack of the lungs, but von Eiselsberg regarded this as an accidental symptom. The temperature was 1.5 to 2 degrees Cent. below normal. Von Eiselsberg noted similar symptoms in a goat whose thyroid had been removed at the age of six weeks.

It was formerly believed that this gland was a secretory organ, which could be dispensed with without danger to life. But Grützner demonstrated that, although one-twentieth the size of the brain, the thyroid has as large arterial vessels. Horsley testifies to its important secretory functions, and points out that myxcedema does not appear, if half or a part of the gland be left. Others (Munk) believe that the disturbances caused by extirpation result from unfavourable wound healing, or from injury to the vagus or sympathetic, and moreover are aggravated by feeding the patients on flesh. Breisacher tested this question by a series of experiments on dogs, and found that the strumous symptoms, especially the clonic and tonic muscular contractions, and respiratory cramp receded, when the animals were restricted to milk diet; but reappeared when they were again fed on meat and soup; boiled meat, however, appeared not to be injurious.

Other dangers of extirpation are the risk of injuring the recurrent nerve or vagus, and of causing dangerous bleeding. But operations are sometimes successful. Massotremoved the thyroid in two horses without bad results, and in one of them the dyspnœa consequent on pressure disappeared after operation. Möller once performed the operation in a horse without the results described, but operating on dog's he repeatedly observed cachexia strumipriva. Removal of a portion of the gland is, however, not attended with serious consequences. Haubner stated that lambs affected with goitre were sometimes born dead, sometimes died soon after birth. A lamb operated on by Haubner remained healthy, though for how long is not said. Carcinomatous goitre can be recognised by the uneven, knotty character of the surface of the swelling. It is very dangerous.

Treatment. Medicines administered internally, or applied by infriction, are usually without success; but iodide of potassium may be prescribed or used as ointment. Painting with tincture of iodine has been recommended, but I have only seen this serviceable when the thyroid was acutely swollen in consequence of disease of the pharyngeal mucous membrane. Siedamgrotzky suggests infriction with iodoform and unguentum hydrarg. 'Truelsen recommends rubbing in three times a day an ointment of 1 part of pure iodine, 5 parts iodide of potassium, 30 parts sapo virid, and gives inteŕnaliy iodide of potassium and nitrate of 
potassium in doses of 30 grains of each. A marked diminution of the swelling and removal of the dyspnoea is said to occur in three weeks. In true goitre more active results are obtained by intraparenchymatous injections made with a Pravaz syringe, the needle being inserted into the tissues of the gland, and from 5 to 15 drops of tincture of iodine passed in. Inflammation usually develops, but disappears in eight to fourteen days, when the injection may again be used and repeated at intervals. Good results have been reported from injection of a 15 per cent. solution of iodoform in æther or glycerine. Möller obtained favourable results in a dog from intraparenchymatous injection of a watery solution of papain ( 1 to 10$)$. The swelling in forty-eight hours was uniformly soft; on incision the digested parenchyma of the gland was discharged as a milky fluid, and although it did not completely disappear, the swelling markedly decreased and the dyspnœa became less. In dogs the gland sometimes undergoes cystic changes, producing a swelling outwardly resembling an abscess. Should it be laid open, however, death generally results (Zschokke).

More recently very good results have been obtained, both in animals and men, from the administration of thyroid juice or extract. Even after a few doses of $1 \frac{1}{2}$ to 7 grains, dogs have shown marked improvement and diminution in the swelling. On the other hand, Stabel found that, in man, neither thyroiodin nor thyroden could be relied on to control the effects subsequent to the removal of the thyroid gland.

Rydygier, in human patients, recently tried ligature of the arteries of supply but their simultaneous ligation was only favourable in parenchymatous goitre. In struma fibrosa et cystica the operation was useless. Schmidt used the "écraseur" for extirpation in the horse. Massot exposed the gland, drew it forward with the fingers, and resorted to torsion before ligaturing. After" dissecting back the skin, Möller lays free the gland with the fingers, and ligatures the visible vessels, finally applying a still stronger ligature and removing the gland above it. Care should be taken not to divide the gland too closely, as the ligature may slip off. It is better to leave some gland tissue, so as to give the ligature a better hold. After-treatment must be regulated according to general principles. Lanzilotti removed a goitre swelling as large as a child's head from a horse. It had developed in six months. The right section of the gland was as large as a potato, and was retained. The animal returned to work after a month's rest. Examination of the tumour showed it to be a simple adenoma. Munk's and Breisacher's experiments seem to indicate milk diet as a valuable means of treatment. Where only one side of the gland is diseased, removal of this portion can be undertaken without serious consequences.

In man goitre is often accompanied by so-called Basedow's disease (exophthalmic goitre), which Jewsejenko and Cadiot state having observed also in the dog and horse. A three year old female pug had a sudden epileptic fit, showed marked frequency of the pulse and disturbance of digestion, with swelling of the thyroid, exophthalmus and ulceration of the cornea. The eyes were treated with sublimate lotion, the introduction of eserin, and the application of compresses moistened with 2 per cent. boric acid; 8 drops 
of tincture of iodine were injected into the thyroid, and iodide of potassium given internally; improvement was noted, and recovery occurred in six weeks.

Jewsejenko reported the case of a four year old English thoroughbred mare, which lost appetite after a race and showed great thirst, weakness, palpitation of the heart, and frequency of pulse, with swelling of the thyroid and eyelids and reddening of the conjunctiva. Fourteen days later exophthalmus occurred, the thyroid gland showed pulsation, and death occurred in four weeks, with symptoms of anæmia and exhaustion. Whether paralysis of the sympathetic, thought to be the cause of Basedow's disease in man, was also present here cannot be decided. A horse in Cadiot's practice was found to be much wasted, low in condition, and to have one fore-foot painfully swollen. Edematous swellings were present at different points on the body, and the left half of the thyroid was much enlarged. The arteries lying near the surface, amongst others the carotid, the posterior auricular, and the metatarsal arteries, showed powerful rhythmical movements ; the frequency of the pulse was from 70 to 80 per minute. In the cardiac region the labouring action of the heart was distinctly noticeable, the thoracic wall visibly moving. Examination of the blood showed no tendency to leukæmia. The patient died on the third day without having shown exophthalmus. The heart weighed $14 \mathrm{lbs}$, and the great blood-vessels were twice the normal diameter.

Brisot observed marked enlargement of the thymus gland in a two year old cow. The swelling extended from the 3rd ring of the trachea to the sternum, and transversely from one jugular to the other. It was hard, painless, and weighed at the time of death $13 \frac{1}{2} \mathrm{lbs}$.

\section{Y.-INFLAMMATION OF THE BURSA OF THE LIGAMENTUM NUCHÆ.}

In the horse the funicular portion of the ligamentum nuchæ is provided on the summit of the second cervical vertebra with a mucous bursa, which attains the size of an apple, and is covered on both sides of the ligament by the complex muscles. Loose connective tissue attaches the inner surfaces of these muscles to the bursa. Poll-evil is due, then, to a bursitis, produced by bruising, less frequently by metastatic inflammation like that of strangles, and, though usually acute at first, tends to become chronic. The first injury may be caused by the animal striking its poll against a rack or low door; by a fall, or blow with a heavy whip-handle; less commonly from pressure of the halter, or, as Hertwig believed, from violently bending the neck when being reined up. Among animals at grass, this bursitis is more generally caused by external injuries than by straining the muscles of the neck during grazing.

Symptoms. A characteristic longish round swelling, about 4 to 6 inches long, appears over the first two cervical vertebræ close to the middle line, is accompanied by inflammatory symptoms, and sometimes affects one side, sometimes both. At first fluctuating and sharply defined, it soon extends to the surrounding soft parts, becomes diffuse and less yielding. 
The condition primarily consists in the outpouring of inflammatory exudate or blood into the bursa. Later parabursitis sets in, and the swelling loses its sharply-defined form. The accompanying pain generally causes the animal to hold the head extended, low and fixed. Sometimes brain symptoms, like those of staggers, appear. Slight fever either accompanies the condition from the outset, or develops subsequently.

If by appropriate treatment asepsis can be maintained, the material poured into the bursa may be reabsorbed, and recovery occur in four to six weeks. But more frequently infection, produced through skin abrasions caused when the bursa was injured, extends. The bursa takes on chronic inflammation, its walls and surroundings become thickened; small spherical growths, proceeding from the inner surface of the bursa, separate after a time, so that the cavity may become filled with a great number of rice-like grains. There are frequently large masses of these corpora oryzoidea. Vincent erroneously considered them to be entozoa. When chronic inflammation has occurred, recovery is slow; but the working usefulness of the horse may not greatly suffer. The swelling in such cases becomes smaller, harder, and less movable with the lapse of time. In most cases, however, infectious inflammation of the bursa sets in; the inflammatory appearances increase greatly, spread to the neighbouring connective tissue and muscles (parabursitis), and finally, after acute pain and fever, end in abscess formation and perforation of the skin. The finger, introduced into the abscess cavity or bursa, detects necrosis of the ligamentum nuchæ and purulent inflammation of the bursa, which may continue for months and baffle the best of treatment. Sometimes inflammatory symptoms, and especially pus formation, abate, although purulent fluid, mixed with synovia, continues to flow from the small fistulous opening. Necrosis may extend to the deeper lying portions of the ligamentum nuchæ and cause inflammatory intermuscular swelling in the throat, and sometimes necrosis of the ridge of the second cervical vertebra. Recovery is most tedious, especially if proper treatment is not adopted, and unless in valuable subjects, slaughter may be advisable. The thickening left produces no difficulty in moving the neck; but permanent stiffness occasionally results from adhesion between two vertebræ (Lafosse); and fatal cases occur from embolism, from the continuation of the fever, or finally, from pus breaking into the vertebral canal. Death then occurs suddenly, with general paralysis and epileptiform seizures,

The course depends principally on whether the tissues remain aseptic. Should this be the case recovery follows in four to six weeks; otherwise the above described complications are very apt to occur and the disease to become chronic. 'The prognosis is then unfavourable. Special care must be exercised in giving an opinion where there is much suppuration, 
swelling and pain. Unless in. valuable animals treatment is seldom justified when suppuration has extended to deep-seated structures and the lower portions of the ligamentum nuchæ have become necrotic.

Treatment. In recent aseptic conditions of the bursa, inflammation should be combated by cold antiseptic applications, made with a towel folded several times and fastened to the mane. Slight steady pressure also favours reabsorption of extravasated material. The halter must be removed, and the animal placed loose in a box, and liept from work. Where a box cannot be obtained, a neck strap may be applied instead of a head collar. As soon as acute inflammatory symptoms have somewhat receded, gentle massage with mildly stimulating applications is indicated. Blistering with sublimate or iodide of mercury ointment has been recommended, but should only be practised with great caution.

As soon as abscess formation reveals infection, it is advisable to provide for discharge of pus at the earliest possible moment, but care must be taken not to injure the occipital artery. Should this or one of its branches be cut, bleeding may generally be stopped by ligature or compress. The incision must be wide and deep enough to allow free exit to the matter, and, if necessary, counter openings may be made and setons or drainage-tubes inserted. When necrosis of the ligamentum nuchæ has taken place, section of the funicular portion may become necessary; it is easily effected even in the standing position with a tenotome. The necrotic material is removed, and the pus washed from the abscess and bursa once or twice daily. The fear that difficulty in lifting the head may follow is said by Hertwig to be unfounded. He declares that even after resection of portions of the ligamentum nuchæ the patient soon lifts its head as high and moves it as freely as before. Subcutaneous section of the ligamentum nuchr, recommended by Lafosse, can only be advised where there is some prospect of maintaining asepsis. But section of the ligamentum nuchæ is not to be lightly adventured on. Under any circumstances, division of the shin across the top of the neck is to be avoided, as it gives rise to wounds, which heal with difficulty. In disease at the side of the neck, the knife must be employed in a free manner, and, where possible, the fistula laid open to its termination. The same courageous use of the linife is called for in providing exit for pus burrowing amongst the muscles of the neck.

Infection may extend to the occipital bone and occipito-atloid ligament, causing arthritis and septic meningitis, with compression of the spinal cord. As a rule, treatment is not justified in cases of this kind, though, where the bone and ligament are alone involved, free removal of diseased tissue with the linife and curette, followed by continuous irrigation with an antiseptic solution might be tried. 


\section{VI.-PHLEBITIS. INFLAMIMATION OF THE JUGULAR VEIN (PHLEBITIS VEN JE JUGLARIS).}

In former times, when bleeding was much more general, horses and cattle frequently exhibited this sequel of the operation. It was described as venous fistula, but its nature remained long unrecognised. Lafosse and Hunter had drawn attention to thrombus formation occurring in the vein; but until Virchow's experiments, the relation of this coagulation to phlebitis was not understood. The ordinary wound in the vein, caused by lancet or fleam, closes by the edges coming together and uniting. Apposition is favoured by the negative blood pressure in the jugular, and no visible change is usually left in the vessel. The skin wound, if closed by pin sutures, commonly heals by first intention.

Causes of venous fistulæ are,-1st, blood coagulating in the vein; and 2nd, purulent breaking down or septic softening of the thrombus. The following circumstances favour thrombus formation :-

The venous wound, if inflicted by a blunt fleam or lancet, and not smoothly cut, but torn through, may be followed by coagulation of blood on the bruised surface and complete thrombosis of the vessel. The same occurs if the wound is not made exactly in the longitudinal course of the vein, but somewhat obliquely, or when, owing to repeated use of the instrument, a double wound has been formed. Injury to a valve in the vein can also induce coagulation, though Hertwig has declared that the danger so produced is not great. Venous fistulæ, further, arise from any circumstance which prevents proper approximation of the edges of the venous wound, and most notably from considerable subcutaneous bleeding. The extravasation displaces the edges of the vessel, and the subcutaneous clot extends into the vein. Extensive extravasations at the point of bleeding are produced by pressure of the collar on the unhealed wound, by animals, suffering from staggers, leaning against the manger, by those with disease of the feet continuously lying; or by openings being made in the vein, as was formerly done to introduce drugs into the blood stream. Viworg, who practised intravenous administration of medicines by means of a funnel, had extensive opportunities of observing this disease. Unclean (by this we do not mean septic) instruments, do not cause phlebitis so frequently as has been supposed.

The thrombus, howsoever caused, usually undergoes purulent disintegration or septic softening, and induces inflammation in the intima of the vessel, which soon spreads to the media and adventitia, and leads to perforation of the walls at different points, producing discharge of the offensive purulent contents. Clotting and subsequent organisation seldom occur; the thrombus usually breaks down. In this case the vein is also permanently occluded. Organisation only takes a short time, 
but is very seldom seen. The disease scarcely ever occurs without a wound of the vessel, though it must be allowed that inflammatory processes may extend from without to the wall of the vein. Such a case has been described by Peter's.

Symptoms. On removing the pin a day or two after blood-letting, the skin wound, instead of being closed and dry, is swollen, and discharges a somewhat dark fluid blood. Within the next fer days the swelling enlarges, and the vein is now found to consist of a round, firm fixed cord, which extends from the wound in the skin to a varying distance above and below. The lattel symptom proves the existence of a thrombus. The discharge of blood may be produced by extravasation under the skin, and, therefore, it is important to examine the vein. Filling of the vein on pressure at the base of the neck and collapse on removal of the pressure prove the non-existence of a thrombus. The use of a probe for diagnosis is not only superfluous but dangerous, because clotting and embolism in the lungs may thus be produced.

During the next few days the wound discharges decomposed blood, which is later mixed with clots and pus. The swelling increases towards the head, and more or less severe bleeding may take place, especially during feeding. If thombus formation is rapid, cerebral disturbance resembling stagger's may be produced, because the blood is unable to escape freely from the brain.

Endophlebitis septica rapidly involves the several venous coats and surlounding connective tissues, producing multiple abscesses. Septic decomposition of the thrombus may produce pyæmia, but this seldom occurs, for the blood usually coagulates quickly within the vessel, completely closing it before septic decomposition can set in, or emboli be carried to the heart or lungs. Embolic pneumonia is, however, occasionally produced, and is invariably fatal. Where this complication is averted the disease usually takes a chronic course. The thrombus undergoes purulent or septic degeneration, the thickening of the venous wall finally leads to complete occlusion; obturation or obliteration usually occurring in three to four weeks. Inflammatory processes, the repeated abscess formation, and circulatory disturbances gradually disappear, circulation being re-established by dilatation of collateral vessels. Skin veins sometimes become as large as a man's finger, thus completing: the communication between the still patent portions of the vessel.

Prognosis. So far as the animal's life is concerned, the disease usually takes a favourable cour'se, unless where pulmonary embolism supervenes, producing dyspnoea, high fever, \&c. Although obliteration of the vessel produces no lasting bad results, the animal cannot be worked during the active stages of the disease, nor can a horse with obliterated 
jugular usually be grazed, as from its depressed position the head swells owing to interference with the returning blood stream.

Treatment. From prophylactic considerations it is desirable after blood-letting to rest the animal, and avoid any pressure on the vein, especially below the wound, hence for twenty-four hours the collar must not be worn. Moreover, everything likely to produce extravasation must be avoided, and the fleam or lancet never applied twice at the same spot. Where the wound has not closed after removal of the pin, it should be washed with sublimate or carbolic solution, to prevent decomposition of the extravasated blood. Once the vein is thrombosed, it cannot again be rendered patent, nor can septic change often be prevented. Further mischief may sometimes be checked by repeated disinfection of the skin wound and of the extravasation with the abovenamed or similar antiseptics. Should inflammatory reaction or swelling become very marked, moist warmth will remove tension and pain, and infriction with unguentum hydrargyri may be found useful. In applying the ointment severe pressure and rubbing must be avoided, lest the thrombus be broken and emboli set free. Abscesses should be opened, and free exit given to secretions by increasing the size of the skin wound. In France a seton is passed through the vein. Under certain circumstances, as when severe bleeding occurs repeatedly, it may be advisable to ligature the vessel below, and if the thrombus does not extend too far upwards, it may also be ligatured above. The operation is performed according to general principles, the vessel divided between the points of ligation and removed. If it has been ligatured above the diseased point, the isolated section of the vein can be completely laid open. French surgeons often remove the degenerated portion of the vein, but in cases of suppuration Cadiot prefers free drainage. For some days after ligation the animal must be kept perfectly quiet, while, to avoid bleeding, only fluid nourishment should be given. Schley recommends washing the vein out with sublimate solution.

In removing the diseased portion of vein a ligature must first be applied above and below. The vein is then divided transversely at the upper end, and completely separated from neighbouring structures. No danger results if the wound be treated as an open one, but care is required to avoid injuring the carotid, and, therefore, the knife should be sparingly used, and the fingers or blunt end of the scissors used in preference. Sometimes the vein tears cleanly away from surrounding tissues if moderate traction be exercised.

Several cases were successfully treated by laying open the sinuous wound and dressing with iodine solution, succeeded on subsequent days by carbolic or creolin lotions. (Cadiot and Dollar, "Clinical Veterinary Medicine and Surgery," p. 341.) 


\section{VII.-DISEASES OF THE PHARYNX AND ESOPHAGUS.}

\section{(1.) FOREIGN BODIES IN THE PHARYNX AND ESOPHAGUS.}

Foreign bodies in the pharynx are most frequently found in carnivora. Bones, fish spines, needles, pieces of wood, taken with the food, or picked up in play, sometimes stick in the pharynx. (See Cadiot and Dollar's "Clinical Veterinary Medicine and Surgery," p. 346, for reported cases.) In dogs and cats, sewing needles are often found at the base of the tongue close in front of the epiglottis. Pieces of potato or of other roots get lodged in the pharyngeal pouch of swine. In herbivora, pieces of wood, bones, hair-pins, and the like may become fixed in the mucous membrane of the pharynx, but more frequently lodge in the œsophagus. In ruminants, especially in cattle, the offending substances are generally shoes, fœtal membranes, cloth, pieces of potato, fruit, or turnip ; in hor'ses, carrots, chaff, linseed cake or hay; whilst occasionally the obstacle is a tooth, a hen's egg, a bolus, a portion of a prickly plant, or a piece of wood or bone.

Grimm found a piece of a lamp chimney in the œsophagus of a cow. Möbius found a thorn about 5 inches in length. Möller removed a whip handle about 3 feet long from a horse's œesophagus. Dandrieux extracted a snake 10 inches in length from the gullet of a cow. Iwersen found a hair ball in the œsophagus of an ox, eructated from the stomach.

Foreign bodies remain fixed either because they are sharp and penetrate the mucous membrane, or are too large to pass the narrow portion of the osophagus, or because the csophagus has undergone contraction, which interferes with their passage. In horses, stoppage of the csophagus with hay results from swelling of the bronchial glands, from the presence of tumours in it (melanosarcomata), and from external compression. Cadiot and Dollar report a case of cesophagus obstruction in a horse from swallowing a piece of carrot. A hypodermic injection of pilocarpine and eserine resulted in the foreign body passing onwards into the stomach in about three-quarter's of an hour. "Clinical Veterinary Medicine and Surgery," p. 345.) In moribund animals, the food taken sometimes remains in the wesophagus, and occludes long sections of it. Whether paralysis of the tube ever occurs is questionable. The portions of the cesophagus where bodies are usually arrested are-

(1) The commencement of the tube immediately behind the pharynx.

(2) The lower portion in the neck.

(3) The point where it perforates the diaphragm.

Diseased conditions which produce obstruction will be dealt with elsewhere. At this point only the two first of the above conditions will be considered; and as obstruction produces different results in different 
kinds of animals, it will be considered separately in each. The obstructive bodies are divisible into two classes:-(a) Wound-producing foreign bodies which pierce the mucous membrane, and remain in position in consequence of their rough surface; and $(b)$ mere mechanical obstructions which, on account of their size, are unable to pass through the tube. The first are most frequently found in the pharynx, the latter in the osophagus, though the first variety are also met with here.

According to Rubeli's experiments, the striped muscular fibres, which in man are confined to the first two-thirds of the osophagus, are in animals continued almost to the stomach. In ruminants the oesophagus possesses no unstriped muscular tissue whatever. This first appears in the rumen. In all animals the mucous membrane is invested with a thick stratified epithelium, covered with numerous papillæ, and the mucosa possesses lymph follicles together with mucous glands. The area of the cosophageal tube stands in inverse proportion to the strength of its muscular coat. In the region of the cardiac opening a thickening and narrowing occur in all domesticated animals, with the exception of cattle and dogs.

The osophagus of the horse is absolutely and relatively the longest, but also the narrowest. According to Franck, its length is nearly 49 inches. Rubeli found it to be from 50 to 52 inches, measured in position, and in larger animals even 56 to 60 inches. Its walls are strong, and become so much thicker between the diaphragm and the cardiac opening that the lumen of the tube almost entirely disappears, explaining the frequent occurrence in this region of diverticula, stenoses, and obstructions.

The œsophagus of ruminants and carnivora is more cylindrical. In oxen a contraction occurs at the lower end of the upper third, and from this point onwards the diameter increases, while the wall is comparatively thin, and decreases in strength from above downwards.

In sheep the thickness increases from below upwards, but the muscular wall is very thin in comparison with the surface of the mucous membrane. The narrowing at the middle of the cesophagus, remarked in the goat, is wanting in the sheep.

Swine have also a contraction at the middle of the tube, which from this point enlarges both above and below. In dogs the narrowest point lies at the height of the lower portion of the cricoid cartilage, the width being about $1 \frac{3}{4}$ inches. The tube increases from here to a circumference of $2 \frac{3}{4}$ inches, then narrows to 2 inches, dilates for a second time, and, about 6 inches behind the pharynx, attains a circumference of 3 inches. The last contraction, some $2 \frac{3}{8}$ inches in front of the cardia, measures $2 \frac{1}{4}$ inches. From here the œsophagus widens like a funnel, until it enters the stomach (the figures refer to the width of the mucous membrane).

In cats two contractions exist: the upper lies $\frac{3}{4}$ inch behind the pharynx, the lower at the point where the osophagus pierces the diaphragm. The latter is the more distinct, the circumference only measuring about 2 lines:

The views with regard to the mechanism of swallowing are very varied. It was formerly believed that the masticated food was advanced by peristaltic movements of the csophagus, but Kronecker and Meltzer have ascribed this duty to the muscles of the pharynx, especially the broad hyoid by which the soft fluid nourishment is injected into the stomach. Hard substances, as well as any materials remaining in the pharynx, are passed forward by the constrictors. The process is a reflex act produced by stimuli from the glossopharyngeal nerve and transmitted by the superior laryngeal (Wasilieff).

v.s. 


\section{(A) FOREIGN BODIES IN THE PHARYNX AND CEOPHAGUS OF THE HORSE.}

In horses foreign bodies very seldom become fixed in the pharynx, but aremore frequent in the osophagus. As stated, they generally consist of pieces of oil-cake, potatoes, hay, boluses, eggs, or grass. Greedy feeders supplied with soft hay are common sufferers. A considerable section of the csophagus is sometimes blocked, and the condition may recur at short intervals. Mollereau observed several recurrences within fourteen days. Graf found the esophagus filled with hay throughout. The thoracic portion, or lower cervical portion, is usually the seat of the stoppage. Walther saw a horse whose cosophagus became occluded in consequence of its swallowing a safety pin which had perforated the asophagus transversely.

Symptoms. Animals cease feeding, slobber at the mouth, make choking movements, and stand with extended neck, and strained, anxious countenance. Any food or water taken is discharged from the nose. Not even the saliva can be swallowed, and cough is often distressing. Considerable quantities of fodder accumulated in the thoracic portion may press on the trachea and produce dyspnoea. Foreign bodies in the neck portion may sometimes be recognised by swelling, or can be detected by palpation. ' In the hor'se the condition is seldom dangerous, as the material generally softens, and passes downwards, and boluses and pieces of oil-cake, as well as hay and grass, may thus be removed without external assistance. The condition becomes graver, and horses may die, when such hard substances as eggs, boluses, \&c., remain fixed in the thoracic portion, producing pressure on the trachea, and consequent suffocation, or mechanical pneumonia. Food or drink attempted to be taken are returned into the pharynx, and thence may enter the trachea.

Treatment must take cognisance of this danger. All food should be lemoved, but, after a time, a little water may be given to discover if the animal can swallow. Foreign bodies, fixed in the upper portion of the osophagus, can be directly removed by drawing them back into the pharynx with the fingers. Immelmann administered pilocarpine, which produced excessive salivation, lubricating both the obstruction and its surroundings, thus facilitating the former being swallowed. This remedy increases, however, the danger of mechanical pneumonia, and caution is required in employing it. The probang or oesophageal tube can be used in quiet horses without casting them, but with somewhat greater difficulty than in oxen. Walther removed a potato with it, but hay often presents considerable resistance, and injury may be done if force be used. It is, therefore, best to wait quietly for twenty-four to 
forty-eight hours, when the material generally passes downwards. Further treatment in horses is much the same as in cattle, and under that heading fuller details are given.

\section{(B) FOREIGN BODIES IN THE ESOPHAGUS OF CATTLE.}

From the nature of their food, and the narrowing of the gullet from the pharynx to the lower part of the neck, choking is more common in the ox than in the horse. Obstructions, as a rule, all occur close behind the pharynx, but are also tolerably frequent in the neck or thoracic portion. Death from suffocation sometimes results from cows attempting to swallow the after-birth, a portion of the membranes sticking in the œsophagus and pharynx, occluding the opening of the larynx, and so causing rapid suffocation.

Symptoms, broadly the same as in the horse, comprise salivation, inability to swallow, attempts at eructation, difficult breathing, and, in ruminants, the addition of a grave symptom--tympanites. A portion of the gases formed in the stomach are normally discharged through the œsophagus; but immediately the œsophagus is obstructed, these gases accumulate in the stomach and bowels, causing dangerous inflation. Increasing distension interferes with breathing, and may cause suffocation. These symptoms can be completely developed in a few hours, and hence it is necessary to at once carry out appropriate treatment. It is worthy of note, however, that Favereau, who has treated many cases, states that in the majority the obstruction will pass into the rumen and recovery will occur if the rumen be punctured and an expectant attitude be assumed thereafter. The canula may even be left in position for several days.

Treatment may be by one of the following methods-

(1) Return of the foreign body into the pharnyx, usually successful where roots have remained fixed close behind the pharynx. The operator places himself on the left side of the animal, passes his right arm over the neck, and with both hands (preferably with the thumbs) endeavours to push the obstructing body upwards by pressing in the osophageal furrow. This usually succeeds if the animal's head be left free. But, if firmly held, the animal becomes disturbed, and stretches out its head, and even if the foreign body is forced into the pharynx its further outward passage is arrested. Where the first attempt fails, it can, however, be repeated, and, with a little dexterity, usually succeeds. In cattle, foreign bodies may be reached and removed from the pharynx or osophagus by introducing the hand through the mouth. The head must be carefully fixed, and a mouth gag applied. The hand, protected with a glove, unprovided with fingers, is introduced rapidly and energetically, but it must not be kept too long in the pharynx, as pressure on 
the rima glottidis may produce dyspnœa and struggling. In the horse, the narrowness of the space between the rows of molars and the soft palate render this procedure difficult, and it

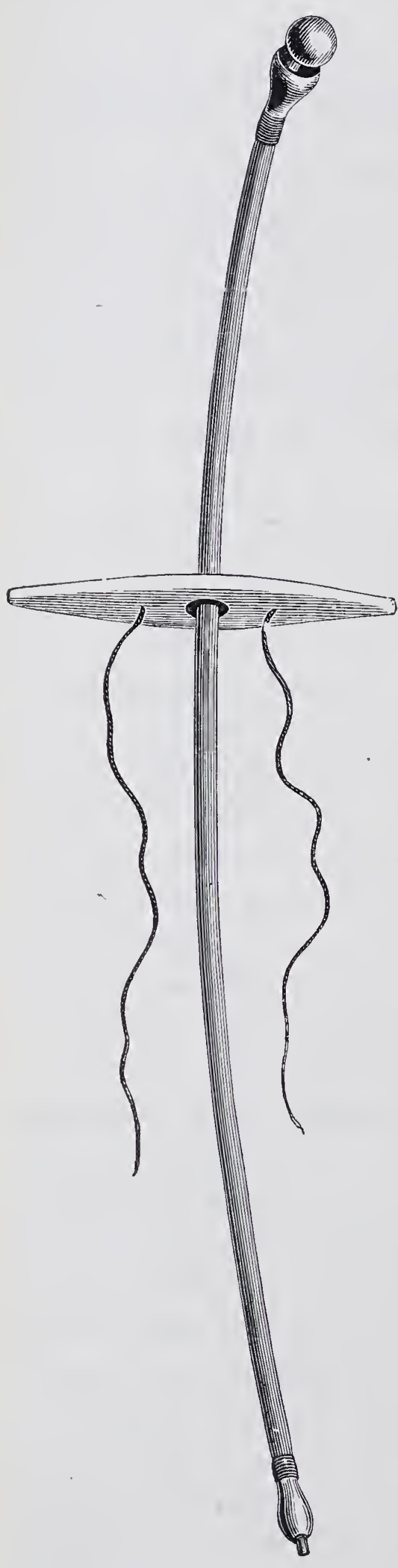

F1G. 74.-Probang and mouth gag for cattle. should not be attempted unless the patient is cast, when the hand may be introduced as far as the pharynx.

(2) Reduction in size of the foreign body in situ may be effected in the case of potatoes and other boiled roots when they lie in the neck portion of the cesophagus by powerful pressure with both hands. Pieces of oilcake, masses of hay, and boluses can sometimes be moved in this way, and caused to gravitate towards the stomach. It has been recommended to place a piece of wood against one side of the neck, and to break down the foreign body from the other side with a hammer, taking care not to injure the œsophagus or produce solutions of continuity in the trachea. This, however, is a very rough-and-ready procedure, deprecated by all British teachers.

(3) Forcing the foreign body onwards into the stomach. Where the above-described methods are unsuccessful, efforts should be made to pass the foreign body on into the stomach. This must not be attempted with sharp substances, such as bones, but may be successful with bodies having smooth surfaces or of soft consistence, as, for instance, roots, oilcake, and hay. For this purpose Munro's cesophageal sound is used in horses; failing this, a flexible cane may be selected, and the thin end, to avoid injury, surrounded with several folds of cloth firmly secured with whipcord, or a strong, carefully oiled rope may be used. Although more easy and successful in oxen, Möller states that this procedure may also be used in horses even in the standing position. A mouth gag is applied; in cattle, a simple piece of wood with a hole in the centre may be used (fig. 74). In the absence of a gag, the tongue is held by an assistant (best with the help of a cloth), and drawn well towards one side. A cord is passed round the 
horns, and the head drawn up to a cross-beam, and extended as far as possible. In horses a twitch should be applied. The œsophageal sound is passed over the base of the tongue into the pharynx, and thence into the oesophagus. To avoid injury it must be introduced with caution. The only danger is that the instrument may pass into the larynx and trachea, but this accident is immediately evidenced by coughing and difficulty in breathing. Should it occur, the probang must be drawn back and the attempt repeated, until the instrument passes freely forward and can be felt at the left side of the neck in the csophageal furrow - a proof that it has entered the asophagus, down which it is slowly pushed until it reaches the foreign body, where further progress is resisted. Cautious attempts are made to push the obstacle onwards. The entry of the sound into the stomach is attested by the cessation of resistance, as well as by the length of the instrument passed. In horses, foreign bodies like hay are sometimes so firmly impacted, that even this instrument cannot move them.

(4) Extraction of foreign bodies by means of instruments. Amongst these is Meier's wire snare. It consists of a piece of brass or copper wire, about $\frac{1}{8}$ th of an inch in thickness, doubled and fastened by the ends to a handle, and works somewhat like the string snare, used for removing corks from the interior of bottles. It is introduced like the probang, the loop thrust behind the foreign body, which may sometimes be extracted by turning and pulling on the instrument. But

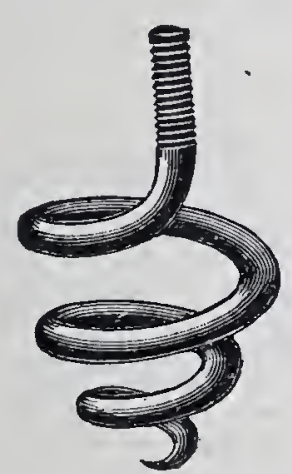

FIG. 75.Esophageal screw.

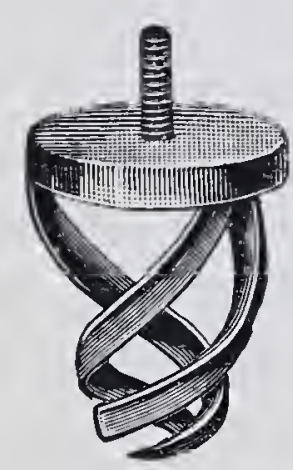

FIG. 76. (Esophageal screw. this device succeeds only where the obstruction lies in the upper portion of the tube, and is of such a character that it can thus be snared and held. The methods already described are, however, usually more successful, and the snare is therefore little used.

The so-called œsophageal screw, for transfixing pieces of potato or turnip, is not of much service. The oesophageal forceps constructed by Delvos and Hertwig are more useful (fig. 77). They are introduced closed, but when the foreign body is felt, the jaws are opened by a screw, the instrument advanced, and the substance grasped and removed. These appliances have, however, only a narrow field of usefulness. Although their working is often interfered with by the 
curvature of the esophagus at the entrance of the chest, notable especially in horses, one or other may be tried where the foreign body is low down and cannot otherwise be forced on. Dinter deprecates the forcible use of the œsophageal sound, and when it is unsuccessful, performs rumenotomy, and awaits the passage of the foreign body, which almost invariably occurs in six to eight hours. Strebel confirms this, but in one case had to wait forty-eight hours for the passage of the obstruction. He also suggests giving small doses of oil. Willach also recommends this expectant treatment.

(5) Incision into the æsophagus. Essophagotomy. This operation can only be performed in the neck portion of the oesophagus, and is only indicated where the measures above described fail. It is the last possible resource, where one has to deal with sharp substances, like bones, \&c. Its difficulties and dangers are usually exaggerated; it is not dangerous to life, and is only occasionally followed by bad results, though some degree of stricture must be expected. Quiet animals, such as cattle, may be operated on in the erect position. After clipping the hair from the left side of the neck, over the foreign body, and rather below than above this, an incision is made through the skin, about 4 inches in length, on the anterior or under border of the jugular vein, and parallel with it. After dividing the muscles of the neck or their fascix, the loose connective tissue encountered must be torn through by means of the two forefingers. The carotid is first sought, and will be immediately recognised by its pulsation. The finger is then passed forward in the direction of the posterior aspect of the trachea, where the œsophagus can easily be found, especially if a foreign body is fixed in it. It is drawn forward, and its two coats divided as far as seems necessary for removal of the foreign body.

As Dette has correctly remarked, it is desirable to make the incision in the skin under the jugular vein, and not over it, as is often described, in order that wound discharges and any portions of food may flow away as easily as possible. Further, the operation wound should not be larger than is absolutely necessary; whilst the cesophagus should be separated as little as possible from its surroundings. Under certain circumstances the potatoes or roots may be broken down within the osophagus and the pieces removed through a smaller wound. The parts must be sutured, as far as possible, with chromic catgut, first bringing the mucous membrane together, and then the muscular coat. The skin wound is better left unsutured, as healing by primary intention cannot be expected. The catgut threads need not be removed, as they become absorbed. The wound is treated on general principles. For the first twenty-four hours food and drink must be completely withdrawn, though, if absolutely necessary, pure water may be given. 
During the next few days soft food or hay may be supplied. Tainturier gives nothing but hay and pure water to cattle, and in one case obtained healing in thirty-five, and in another in twenty-five days. Malzew performed cesophagotomy five times in oxen and six in dogs;

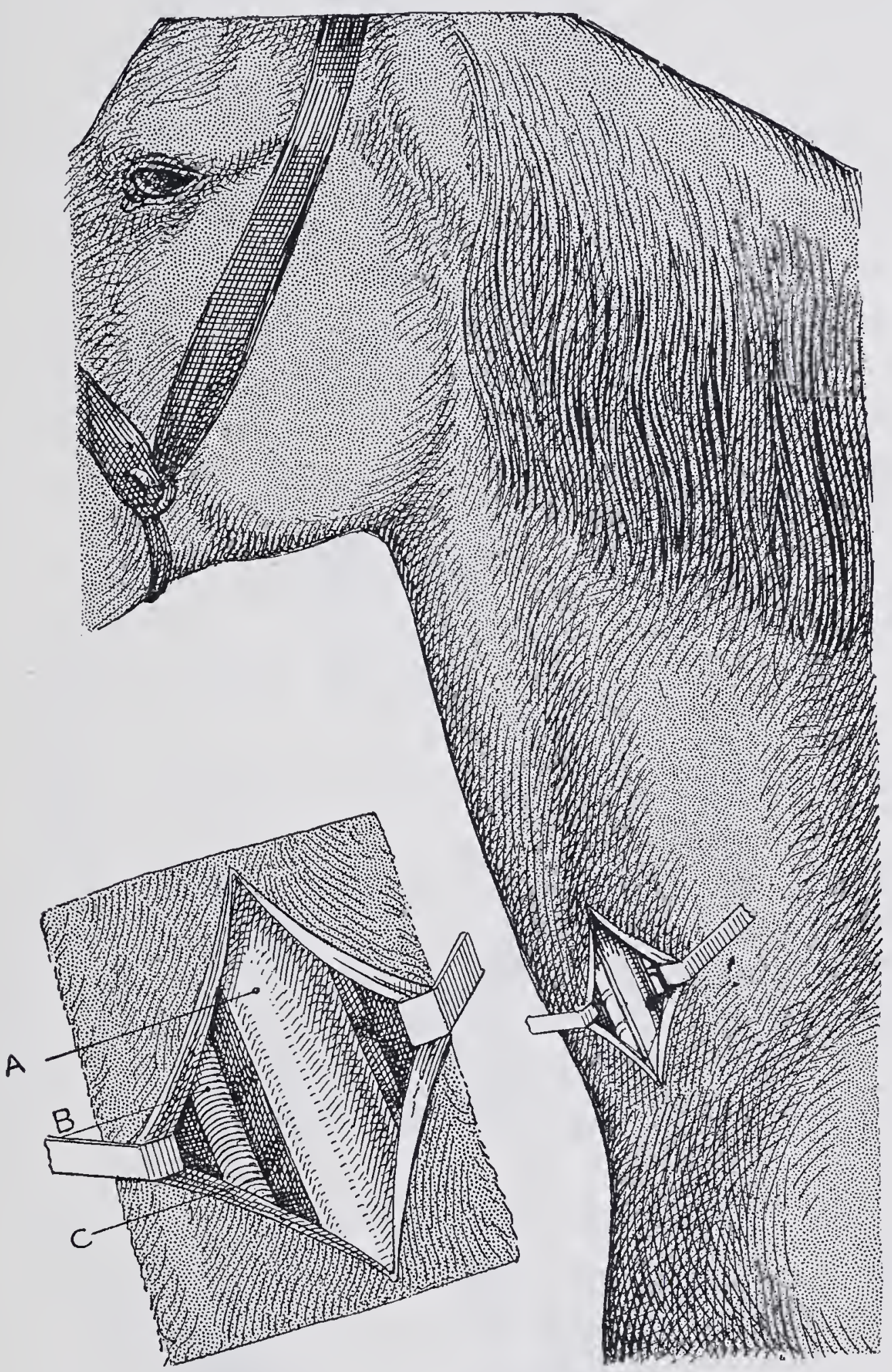

FiG. 78. - To illustrate the operation of oesophagotomy.

A, csophagus; $\mathrm{B}$, carotid artery; C, trachea. (Semi-schematic.)

healing occurred within eighteen to twenty-three days in the oxen, and in twelve to eighteen in the dogs.

Although the animals fall away in condition, bad consequences are rarely observed. The subcutaneous breaking down of the foreign body, suggested by Lafosse, is liable to injure the jugular, and is usually followed by pus formation, necessitating enlargement of the wound. Mauri has, nevertheless, recommended this treatment recently, Should difficulties or delays occur in removing the foreign body, and considerable 


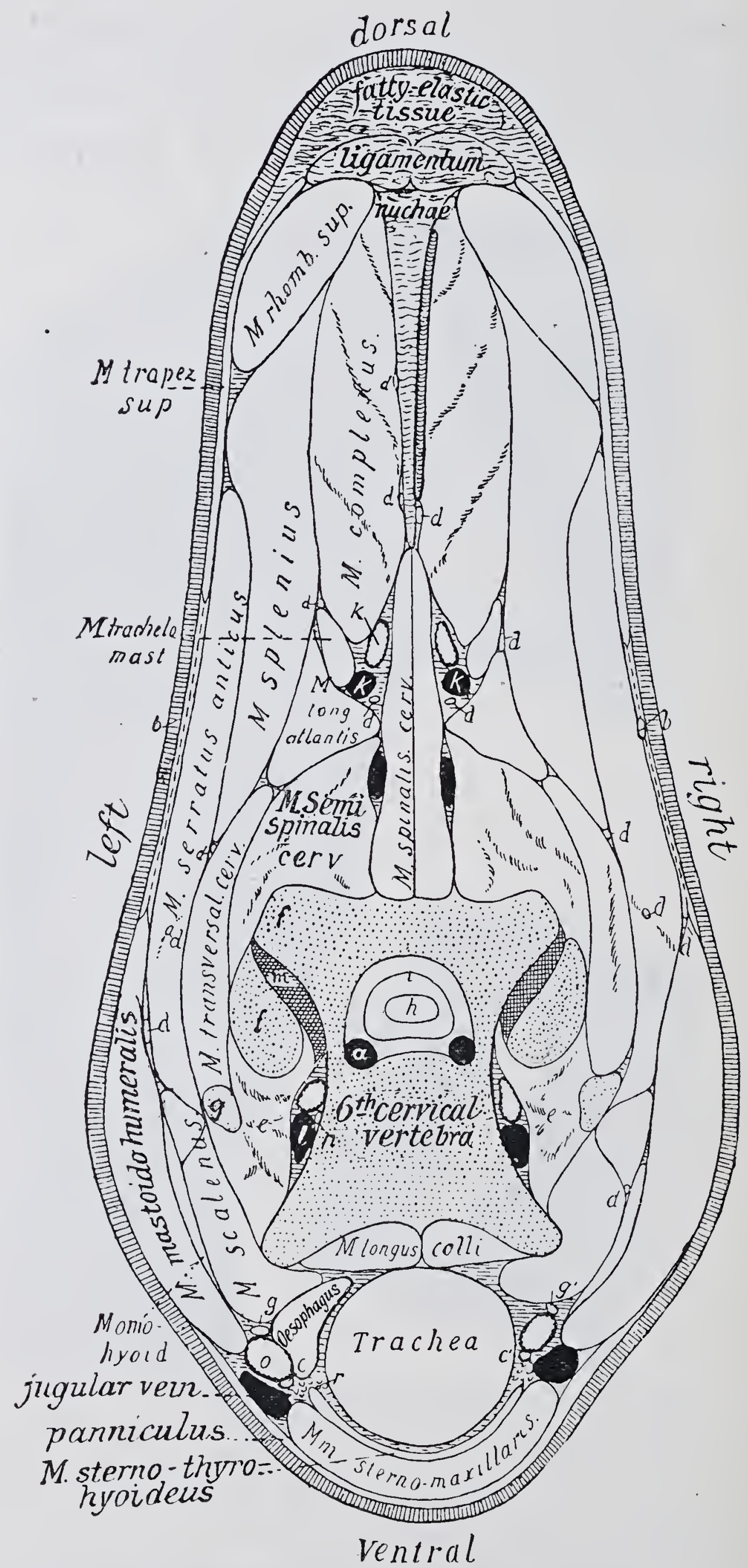

Fig. 79. - Section at right angles to the long axis of the neck through the upper third of the 6 th cervical vertebra.

$a$, Veius of spinal cord ; $b$, branch of spinal accessory nerve ; $c$, recurrent nerve $d$, inconstant nerve twigs; $e$, intertransversales muscles; $f f q$, portions of vertebra; $h$, spinal cord ; $i$, its membranes; $k k^{\prime}$, superior cervical artery and vein; $l l^{\prime}$, vertebral artery and vein $n$, brancl of the sympathetic; o, carotid artery; $p$, jugular vein ; $q q$, vagus and sympathetic nerves; $r$, tracheal lymphatic gland. 
tympanites result, as is frequent in cattle, the rumen must be punctured to ward off suffocation.

If the removal of the offending substance by the described methods fails, as is usually the case when it lies in the thoracic,portion, drenching with thick oily materials may be tried, if no difficulty in breathing exists. Caution, however, is necessary to prevent the fluid passing into the trachea and producing mechanical pneumonia. Small quantities must be given at a time and the animal watched, so that, if coughing occurs, the administration may at once be stopped. After puncture of the rumen, it is desirable to wait for twelve to twenty-four hours for softening and dispersal of the foreign body. Michalski gave a bull $1 \frac{1}{2}$ grains of veratrine dissolved in $2 \frac{1}{2}$ drams of spirit subcutaneously, producing violent oesophageal spasms in fifteen minutes and the disappearance of the obstruction. Immelmann's statements point to the possible usefulness of subcutaneous injections of pilocarpine, which provoke profuse salivation. Apomorphine favours cesophageal peristalsis and eructation, although this latter act is only produced exceptionally. Arecolin and eserin given hypodermically and chloride of barium intravenously have also been recommended.

\section{(C) FOREIGN BODIES IN THE CESOPHAGUS OF SWINE.}

When housed, swine are usually fed on boiled roots, carefully cut potatoes, or semi-fluid gruels, so that food rarely causes obstructions. But when roaming in great.herds, and fed on uncut potatoes or other roots, pieces may stick in the pharyngeal pouch or in the osophagus, and provoke symptoms similar to those in other animals. According to Lothes, foreign bodies in the pharyngeal pouch of swine produce difficulty in swallowing, salivation, and a peculiar change in the voice; instead of grunting, a shrill shrieking sound is produced, and if the subjects are in poor condition, the obstruction may be detected by palpation. Foreign bodies fixed in the œsophagus sometimes induce vomiting, and are thus ejected; but where long retained, tympanites develops, and death ensues from suffocation.

Early assistance is necessary, but in pigs little can be done manually. If the obstruction is in the pharyngeal pouch, pressure must be exercised simultaneously on both sides directly over the region of the larynx, and an effort made to push the contents of the pouch upwards into the pharynx. Where the foreign body is fixed in the top of the pharynx or just beyond it, Stockfleth advises that the pig be laid on a table, when removal may sometimes be made with a blunt hook, but if the object is deeper seated it may be pushed downwards with a flexible stick. Teetz removed a piece of broomstick $12 \frac{1}{2}$ inches long from the upper part of a pig's cesophagus. Apomorphine can be tried as an emetic; if this does 
not at once succeed fat pigs had better be slaughtered. CEsophagotomy may be performed in those in poorer condition; it is scarcely more difficult or dangerous than in other animals. Hering reports two successful cases; the wound healed without being sutured.

\section{(D) FOREIGN BODIES IN THE PHARYNX AND ESOPHAGUS OF CARNIVORA.}

Attention has already been given to the occurrence and treatment of foreign bodies in the pharynx of dogs and cats. They occur usually at the base of the tongue, often close under the epiglottis, though sometimes behind the soft palate in the posterior wall or in the sides of the pharynx. Removal must be effected according to the directions before given. Bones, particularly vertebræ of birds and game, fish-bones, needles, and other sharp objects, are liable to get fixed immediately behind the pharynx and occasionally just before the cardiac opening. Smooth rounded objects are seldom found obstructing the oesophagus in these animals; they generally pass onwards to the bowel. Reichenbach removed from a dog's œsophagus by incision a piece of wood $4 \frac{1}{2}$ inches long, $\frac{5}{8}$ of an inch broad, and $\frac{1}{2}$ an inch thick. Restlessness, anxiety, choking and vomiting, avoidance of food and drink, and most notably discharge of ropy saliva, suggest the presence of a foreign body. Pressure on the cosophagus sometimes gives pain.

Sharp substances perforating the cesophagus in the neck portion cause swelling and inflammation, in the thoracic portion, rapidly fatal pleurisy. They can be successfully extracted only by way of the pharynx, as described in the chapter "Foreign Bodies in the Mouth." The foreign body, when in the lower portion of the asophagus, may be pushed onwards with a flexible catheter. This failing, emetics, such as apomorphine subcutaneously, may be tried, and, if unsuccessful, œsophagotomy, which in dogs as in other animals is without danger, must be performed. Möller often operated successfully. In one case the cosophagus was perforated at two points, but the piece of bone was removed, and healing followed without stricture or other bad result.

Coleman removed a rabbit's vertebra from the osophagus of a dog by incision. Complete healing in a fortnight. Porcher and Morey state having discovered by Röntgen photography the presence of a coffee spoon in the last portion of a dog's œsophagus and having removed it by gastrotomy. Healing by primary intention.

\section{(E) IMPACTION OF THE CROP IN BIRDS.}

The crop is a diverticulum of the osophagus, in which the food is prepared for digestion as in the first compartments of the ruminant's 
stomach. Impaction results from excessive distension with dry foods, or with indigestible foreign bodies, and from compression of the osophagus in diseases of the lungs.

Symptoms comprise excessive fulness and distension of the crop, which feels hard and firm, want of appetite, and sometimes discharge of offensive fluid from the beak, which is usually held open. If the condition persists for long or frequently recurs, the crop becomes greatly dilated, the animals gradually waste, and may die. The condition is seen in fowls, ducks, geese, and pigeons and by recurrence becomes chronic.

Treatment consists in massage. Attempts should be made to empty the crop or to break down the hardened contents by pressure and kneading. Zürn recommends hydrochloric acid. If this proves useless, as in distention with foreign bodies like sand and stones, the crop must be emptied by operation, which is well sustained by most birds. After removing the feathers, the crop is divided in the long direction of the neck far enough to allow of the finger entering and the contents being withdrawn. The wound is cleansed and sutured, and generally heals by first intention. According to Zürn pigeons are not good subjects for operation, especially during breeding, the mucous membrane of the crop being swollen and very rich in blood-vessels.

\section{(2.) INJURIES TO THE PHARYNX AND CESOPHAGUS (GSOPHAGEAL FISTULA).}

Injuries of the walls of the pharynx occur in all large animals, but most commonly in horses. When rasping the teeth the chisel or rasp, if carelessly handled, may severely injure the pharyngeal wall, and even produce death from bleeding or acute inflammatory processes. Like injuries result from the use of sticks in giving balls; from awkward employment of pharyngeal sounds, forceps, and other instruments; and in all animals, and notably in carnivora, from sharp foreign bodies. Merkl found a hairpin in a horse's pharynx. Injuries to the cesophagus are almost invariably caused by sharp foreign bodies, or by osophageal instruments used for their removal. Injuries from without are rare, though Graf and Braun have both seen injuries to the œsophagus in horses caused by kicks from other animals.

The course of such injuries depends entirely upon their character. As already stated, fatal bleeding may occur, or suffocation from passage of blood into the trachea. Where the inflammatory processes are superficial, and foreign bodies have been promptly removed, healing often occurs quickly; but deep-seated inflammation of the mucous membrane of the pharynx and adjacent parts may supervene (angina phlegmonosa), 
threatening suffocation. Foreign bodies injuring the mucous membrane of these regions sometimes produce extensive inflammation and the formation of abscesses, which may break externally and cause oesophageal fistula, or into the thorax or abdomen, inducing fatal septic pleuritis or peritonitis, and rapidly leading to death. Laser records, in a remount horse, the rare case of double rupture of the osophagus-one in the neck portion, the second in the thorax; death ensued. External perforation is most frequently met with at the upper end of the oesophagus, close behind the pharynx; Möller has several times seen this in horses. The abscess breaks and discharges an exceedingly offensive pus, often mixed with food, after which saliva, food, and water escape during swallowing, proving beyond question that the disease has originated in the osophagus or pharynx. The wound closes gradually, but a small fistulous canal remains, discharging water and saliva, and healing often with the greatest difficulty. This constitutes œesophageal or pharyngeal fistula. Butters recently described such a case. The inflammatory processes and consequent swelling produce difficulty in swallowing, and not infrequently feverish symptoms (angina). A swelling develops on the left side, gradually increases, becomes soft, and finally breaks, when the difficulty in swallowing and the fever disappear. Guilmot saw a case of abscess formation in the lumen of the osophagus of a calf, causing difficulty in swallowing, vomiting, and tympanites; on introducing the osophageal sound, about two pints of pus were discharged, the symptoms disappearing some days later. Haubold saw an osophageal fistula produced in the horse by abscess formation in the retro-pharyngeal lymph glands; the animal recovered in three weeks. Stamm had a similar case following strangles. Injuries of the walls of the pharynx by balling-guns or tooth instruments, indicated by salivation and disturbance in swallowing, may heal, provided they do not take a fatal course from bleeding or inflammation. In small animals their position and extent can be determined by direct inspection.

Treatment. As already indicated, care must be exercised in the use of cesophageal and tooth instruments, and sharp balling-guns or sticks avoided. The course of abscess formation in the throat may be shortened by early and cautious opening, by washing out the abscess, and making provision for regular discharge of the contents, inflammatory products, and saliva. Esophageal fistulæ sometimes give much trouble. Definite closure may often be produced by passing a thread steeped in sublimate, or by applying the actual cautery, but is not always successful. In a horse under Möller's treatment these and other measures were used without result. Widening the fistula and suturing the opening in the osophagus generally produces healing, though the operation is by no means easy, and does not in every case produce the desired effect. In 
Butter's case injections of nitrate of silver proved useless, but the fistula closed spontaneously in four months.

\section{(3.) ECTASTAE, DIVERTICULA, AND RUPTURES OF THE GSOPHAGUS.}

The term ectasia is applied where the skin, muscular and mucous tissues have undergone dilatation. Ectasiæ sometimes result from stricture of the oesophagus, and, like it, usually develop slowly. They occur both in large animals and in carnivora.

Langrehr saw a cow which for three months had always vomited after taking food, and especially when drink was given soon after feeding. The cow was very thin, and when slaughtered exhibited a dilatation of the œsophagus close under the diaphragm, produced by distension of all its coats, and capable of holding nearly three quarts of fluid. Wagner examined a horse which had a dilatation of the osophagus in front of the 4 th cervical vertebra, and also just before its passage through the diaphragm. The condition had led to an attack of mechanical pneumonia and death.

Sometimes dilatations take a spindle form, sometimes they are sharply defined. Where greatly developed, difficulty in swallowing occurs, and wasting afterwards sets in.

Diverticula are here taken to mean ruptures of the muscular coat of the oesophagus, and passage of the elastic buti unbroken mucosa through the opening. This has been described as cesophagus ventriculosus, or cesophagocele, and is oftenest met with in horses and cattle. It sometimes results from greedy consumption of rough and prickly clover. Such ruptures occur in the neck portion of the tube, but more frequently close to its entrance into the stomach.

Both the muscular and mucous coats may become ruptured at the same time, or the mucous coat may yield somewhat later than the muscular.

Fuchs discovered a dilatation of the œesophagus after death, where the neck portion had a circumference of $9 \frac{1}{2}$ inches, the thoracic portion of $12 \frac{3}{4}$ inches, and the portion in front of the diaphragm was distended to $20 \frac{1}{2}$ inches. Dr. S. describes a diverticulum in the horse formed in consequence of the muscular coat being ruptured for a distance of 11 inches in the thorax. The sac-like dilatation held five pints of water, and the horse suffered from symptoms of broken wind. Schellenberg saw a similar diverticulum in the dog, produced by a bite and subsequent abscess formation. On post-mortem, six months later, a diverticulum was discovered as large as a man's fist. It had resulted from cicatricial contraction.

Of twenty-six cases collected by Rubeli, the diverticulum was, in eleven, close before the diaphragm, in seven in the thoracic portion, and in eight in the neck portion, usually in the lower part of it. The anatomical disposition of the oesophagus in horses explains this distribution. The mucous membrane may subsequently be ruptured. Illustrations of these several classes of cases are recorded. 
Ruland relates that a horse which had suffered from colic, with severe attacks of vomiting, was found to have the œsophagus ruptured close in front of its passage through the diaphragm. Leisering describes a diverticulum in a horse employed in transport duty, and which died from suffocation : 3 feet from the pharynx the distended oesophagus had a circumference of about 16 inches, 8 inches lower the circumference was 12 inches, $13 \frac{3}{4}$ inches higher it measured $12 \frac{1}{2}$ inches; close in front of the cardiac opening was a second diverticulum. Grünwald saw a horse which died a day after suffering from a shivering and "blowing" attack. The post-mortem discovered a quantity of turbid fluid mixed with food in the thorax. The œsophagus was ruptured for $4 \frac{1}{4}$ inches close in front of the diaphragm, and in front of this again was dilated for a distance of 16 inches, its walls being more than $\frac{1}{2}$ inch thick, and its circumference 6 inches. At the point of rupture the mucous membrane formed a cavity as large as a hen's egg. This communicated with the rupture. Grünwald considered that an abscess had formed in the osophageal wall and caused the rupture. But it also seems possible that the condition was a simple diverticulum. Harms, Hartenstein, and Roloff describe cases of œsophageal diverticula in cows; one detailed by Harms situated $2 \frac{3}{4}$ inches in front of the rumen.

The symptoms of these conditions bear a general resemblance to one another. Ectasiæ and diverticula are recognised by choking and a tendency to vomiting, usually appearing soon after taking food. Where the lesion is in the neck portion of the cesophagus, a swelling appears on the left side of the neck during feeding, which is not painful, or only slightly so, shows no inflammatory symptoms, but may be accompanied by salivation, choking, or attempts at vomiting, and usually disappears after one to two hours. Pressure on it produces symptoms of suffocation and attempts at vomiting.; It is commonly more prominent after consumption of dry chaff than after hay or water. It may continue for a long time without impairing general health, but when large, especially if situated in the thoracic portion, or when distended with food, such swellings excite choking and vomiting or compress the trachea and produce dyspnoa. The horse described by Leisering, if smartly exercised, especially after feeding with hay, exhibited dyspnoea and coughing, which disappeared later. The animal eventually died from suffocation. Other cases have been described. The symptoms may continue for a long time without causing more than loss of condition and slowness in feeding, though the dyspnœea sometimes produces death, as shown by the cases reported by Leisering, Fuchs, and others. Finally, obstructions may lead to mechanical pneumonia and death in consequence of interference with swallowing, as in Wagner's case.

In complete rupture of the œsophagus symptoms are produced similar to those of severe injury to the œsophagus. If in the cervical portion, they comprise emphysema in the neck and formation of abscesses, which by-and-by perforate and discharge pus mixed with food. These may heal like asophageal wounds. Spontaneous healing in a cow is related by Schleg; but death may ensue from excessive burrowing of pus and 
sepsis, as Laurent's and Graf'ś observations show. Graf's case was caused by a kick from another horse. Schleg's case in a cow shows that spontaneous recovery is nevertheless possible. Krebs records dilatation and rupture of the esophagus in a horse in consequence of fatty degeneration of the muscular coat.

Should the osophagus become ruptured in the thorax or abdomen, death soon follows from septic pleuritis or peritonitis. Kehm and Grünwald report such cases in the horse. The condition is, however, seldom recognised before death. Accumulation of food may cause inflammation and destruction of the mucous membrane, especially during the earlier stages and before it becomes gradually thickened and more resistant.

Prognosis in these cases is usually unfavourable. If ectasia does not endanger life, it seriously interferes with nutrition and the use of the animal, and is usually incurable. By regulating the diet it may be possible to keep the animals at work ; fat cattle should be prepared for slaughter.

Treatment. In ectasia, which sometimes extends over large areas, therapeutic treatment scarcely promises any good result, but in diverticula in the cervical portion of the œsophagus something may be done, both in horses and cattle, by operation, as is shown by the cases related by Reinemann, Schwerdtfeger, Moisant, Reichel, and others. The dilated portion of the mucosa may be replaced or removed altogether, and the rupture in the muscles brought together by sutures. With antiseptic precautions, the operation is not attended with much danger. This is the most promising treatment in animals, but in chronic cases is more easily described than carried out, especially if the rupture in the muscular coat is already cicatrised and fixed to the neighbouring parts by much cicatricial tissue. The operation can, however, be tried.

Procedure is similar to that of incising the osophagus. After returning the mucosa, the muscular coat is, where practicable, carefully sutured with catgut or silk ligatures inserted close together; bad results naturally follow if the stitches tear out. Where this course is impossible, the diverticulum must be opened, a piece of the dilated mucous membrane excised, and the parts brought together in the above fashion. Moisant applied a sort of clamp during eating and drinking, and attained good results in a horse, and afterwards in two cows. Careful stitching of the muscular and mucous coats is, however, not only simpler, but equally successful. Reinemann effected a cure, although the stitches tore out in two days, owing to inappropriate feeding. Granulations gradually close the opening. Reichel operated on oxen in the standing position. T'o prevent the stitches tearing out, nothing more than water should be given during the ensuing twenty-four hours, and during the next few days only fluid nourishment. In complete rupture of the cervical portion of the 
œsophagus, the same treatment is recommended as in injuries from sharp substances. Nothing can be donein ruptures of the thoracic and abdominal portions.

\section{(4.) STENOSIS AND COMPRESSION OF THE AESOPHAGUS.}

Stenosis of the oesophagus may result from chronic inflammatory processes in its walls. Andersen relates that a horse had for four months repeatedly suffered from impaction of the osophagus, and on post-mortem showed a ring-like contraction, about 8 inches below the pharynx; the portion above this point was widened (ectastic). Köhne describes as stricture what appears to have been a diverticulum.

A similar case is described by Friedenreich; a horse, after suffering for a long time from difficulty in swallowing, finally diéd from hunger, and on post-mortem a duplication of the mucous membrane was discovered arising from the upper pharyngeal wall, reducing to one-third the dimensions of the pharynx. The mucous membrane did not appear diseased.

Compression of the cesophagus is caused by tumours in the thorax, or by swelling of the bronchial lymphatic glands (compression stenosis). Johne and others report such cases in oxen, in which tuberculous bronchial glands compressed the osophagus, and caused difficulty in swallowing and disturbance of nutrition. Animals are not so frequently affected as men, either by contraction of the mucous membrane, from the swallowing of irritants, or by narrowing of the lumen of the tube by tumours or parasites like spiroptera sanguinolenta, constituting obturation stenosis. These conditions can seldom be diagnosed with certainty. Their chronic course distinguishes them from the disturbances produced by foreign bodies or by diverticula of the asophagus. Cattle often show chronic tympanites, and where this is accompanied by coughing and wasting; suspicion of tuberculosis must occur.

There is seldom opportunity for treatment, the condition in most cases being only definitely recognised on post-mortem examination.

\section{(5.) PARALYSIS OF THE PHARYNX AND GSOPHAGUS.}

The muscles of the pharynx and of the upper two-thirds of the asophagus receive their motor nerves from the 9 th pair, the last third of the osophagus is supplied by the 10th pair. (For the innervation of deglutition, see "Foreign Bodies in the Pharynx," \&c.) Paralysis of these nerves may induce functional disturbance. Records are numerous of socalled paralysis of the osophagus, but the descriptions are not always reliable or complete, and some of the cases depend not so much on 
paralysis as on structural changes or the presence of foreign bodies. No strict division or sufficient points of distinction can be made between paralysis of the pharynx and osophagus. Conditions viewed as paralysis of the œsophagus must sometimes be referred to the pharynx. Whether paralysis of the muscles of the pharnyx, especially of the constrictors, can produce difficulty in swallowing, and of what particular form, is not at present known. In serious diseases, especially in brain disorders and rabies, as well as towards the end of life, innervation of deglutition often suffers, and food consequently remains fixed in the osophagus. Hence post-mortem examination of horses often discovers accumulation of food in the osophagus, without any apparent obstruction in the lumen of the tube; but these cases have no further clinical interest.

Möller treated a horse which showed difficulty in swallowing for a long time; no cause could be discovered, no other morbid systems were presenteven on post-mortem nothing abnormal could be detected. In another horse, recovery from a laryngeal injury was followed by difficulty in swallowing, and though food was chewed as usual, no attempt was made to swallow it, but the horse allowed it to fall from the mouth again. The tongue appeared normal, both in appearance and function. It, therefore, seems probable that paralysis of the pharynx existed, though the strict proof was wanting. We have seen similar difficulty in deglutition several times after resection of the arytenoid cartilage; in one case caused by great increase in the connective tissue about the larynx and pharynx; in another case the symptoms completely disappeared after some weeks. Possibly this case depended on inflammatory swelling in the pharynx, though the animal showed no other disturbance. Dieckerhoff saw a similar case in a horse. That inability to swallow may be caused by impaction in the œsophagus appears evident from Puschmann's record of a horse which had the tube packed tightly with hay 2 inches from the cardiac orifice. The cases described by Cadéac and others as osophageal cramp may probably be referred either to impaction of the osophagus or other hindrance to the passage of food (diverticulum, stenosis). Complete blocking of the osophagus naturally prevents the possibility of swallowing. This is easily explained if one adopts Kronecker and Meltzer's view, that food is injected immediately into the stomach from the pharynx.

Köhne saw six otherwise healthy horses which appeared unable to swallow, and in consequence very rapidly fell away in condition; they showed some œdema under the chest. The autopsy revealed nothing of a positive character.

Stietenroth saw some similar cases; the pupils of the eyes were markedly dilated, and at a later stage there was loss of sensation over almost the whole surface of the body, a condition which Stietenroth interpreted as pointing to belladonna poisoning. This view seemed to be supported by the fact that the disease attacked two horses in the same stable within a short interval.

Schmidt has indicated the possible occurrence of one-sided paralysis of the pharynx and osophagus. An old mare had a left-sided nasal discharge ; the left nostril was filled with food, and on drinking, a great part of the water flowed back through the nose. She soon died, and examination showed the left superior maxillary sinus filled with food, mechanical pneumonia existed, and the left half of the soft palate was atrophied v.s. 
("only rudiments existed"), while the wall of the pharynx on the left side was dilated in a pouch-like form. Pharyngeal paralysis was also seen in an army horse, but disappeared after eight days treatment with strychnine.

Reports as to inability to swallow appear enigmatical. The horses can take food, chew, and swallow in the usual way, but are unable to make drinking movements. They go greedily towards the water, make snapping movements with the lips, and move the tongue without being able to take a single swallow of water. Friedberger and Fröhner believed that a congenital defect existed in the nervous centre (sucking centre). But it is remarkable that the animals under these circumstances had lived so long, the one mentioned by Friedberger being eight years old. Leitenstern reports cases of slowly developing cramp of the cardia in man. A person who had regularly vomited the food swallowed without its being mixed with gastric juice was, after death, found to have dilatation of the entire oesophagus, but no mechanical obstruction could be found.

Symptoms. Inability to swallow, especially if associated with stoppage of the œsophagus with food, reveals the nature of the case. It is often noted in rabid dogs that the animals attempt to drink, but that little or none is swallowed. It is difficult to determine whether the inability to swallow depends alone on the masticatory facial muscle paralysis or in part on paralysis of the pharynx.

Treatment. It is of primary importance to attempt the removal of the frequently existing obstruction in the cesophagus, and for this end the probang is most useful. Appropriate diet must also be given. Drugs are of little use, though strychnine might be tried. It is more hopeful to treat the general conditions to which the disease is sometimes due.

\section{(6.) TUMOURS IN THE PHARYNX AND ESOPHAGUS.}

Such tumours are more common in cattle than in other animals. New growths in this region have long been recognised; their gravity depends upon their nature. Roloff considered them to be retention tumours, arising from the mucous glands of the pharynx. Harms declared them to be lymphomata, but recently the conviction has gained ground that they are usually cases of actinomycosis. They generally develop singly on the posterior upper wall of the pharynx, under the sphenoid bone, or close above and behind the larynx. Harms named the former, which usually attain the size of an apple, "anterior," the latter, which are commonly much larger, "posterior pharyngeal actinomycomata." Considering their frequency in cattle, infection is doubtless often produced by food containing spores of actinomyces. The comparatively thin, tender mucous membrane injured by the passage of hard sharp forage is easily infected. As the growths enlarge, the following symptoms appear :-

(1) Dyspnoa. 'The respiration is snoring and rattling, attended with 
cough, and the dyspnoea occurs particularly during feeding, and sometimes at every effort to swallow. When the head is bent, this disturbance is more marked, a circumstance valuable in diagnosis. (2) Difficulty in swallowing. The tumour, as it becomes larger, interferes with deglutition. The head is extended, and food entering the pharynx excites coughing. (3) Palpation from without sometimes discovers swelling in the region of the pharynx. In making this examination, the head must be extended as much as possible, and the two hands pushed upwards above the larynx. Harms once found dislocation of the larynx. On introducing the hand into the pharynx, the condition, size, character, and position of the new growth can be fully determined.

This examination is necessary to differentiate the condition in question from other diseases of the tongue like makroglossia, or from swelling of the retropharyngeal lymph glands, though the latter is not very frequent in oxen. Nevertheless, it has been several times observed, and Prietsch describes a case. A well-nourished cow gradually developed snoring respiration four to five months after the first calving. It was most marked when eating hay, and sometimes became so severe that chewing was interrupted, the head extended, and the tongue thrust out of the mouth. The larynx was swollen and enlarged. Post-mortem showed tuberculous changes in the lungs and retropharyngeal lymph glands.

According to Harms, the anterior pharyngeal swellings contain a greyishred material, sometimes calcified, in the interior of which actinomyces can be detected. The posterior have a smooth fibrous form, and contain a greenish decomposed material. They develop slowly. Harms states that the swelling may exist for five months or more, without producing danger of suffocation, but the symptoms, and especially the respiratory disturbance, generally increase. He further remarks that in the posterior pharyngeal swellings arrest of growth may occur, so that clinical symptoms remain stationary.

Prognosis is unfavourable; complete recovery improbable, although improvement may be secured for a long time by operative treatment.

Treatment. Harms casts the animal, raises the neck, and makes a longitudinal incision in the middle line under the larynx, through which the hand is introduced, the connective tissue at the side of the larynx is then divided, the tumour reached and removed by breaking down the tissues around it. Meier casts the animal on the right side, inserts a mouth gag, raises the head, introduces the right hand into the mouth and pharynx with its volar surface towards the palate, and grasps and tears off the anterior actinomyces swellings. The posterior growths, on account of their firm coating of connective tissue, cannot thus be dealt with, and, therefore, Meier pierces these with the finger, allowing the contents to escape. Harms prefers the former of these methods, and of the latter remarks that it is difficult to carry out, and does not always remove the dyspnœa. The "écraseur" is often very useful. With it the entire tumour, under certain conditions, may doubtless be removed. Esser, after extensive experience, has come to the conclusion that the 
operation seldom produces lasting good results. Todide of potassium may be tried.

Tumours in the pharynx of horses are rare, and little is at present known as to their nature. The symptoms resemble those in oxen, and the methods for removal are similar ; but it is not always possible to introduce the hand into

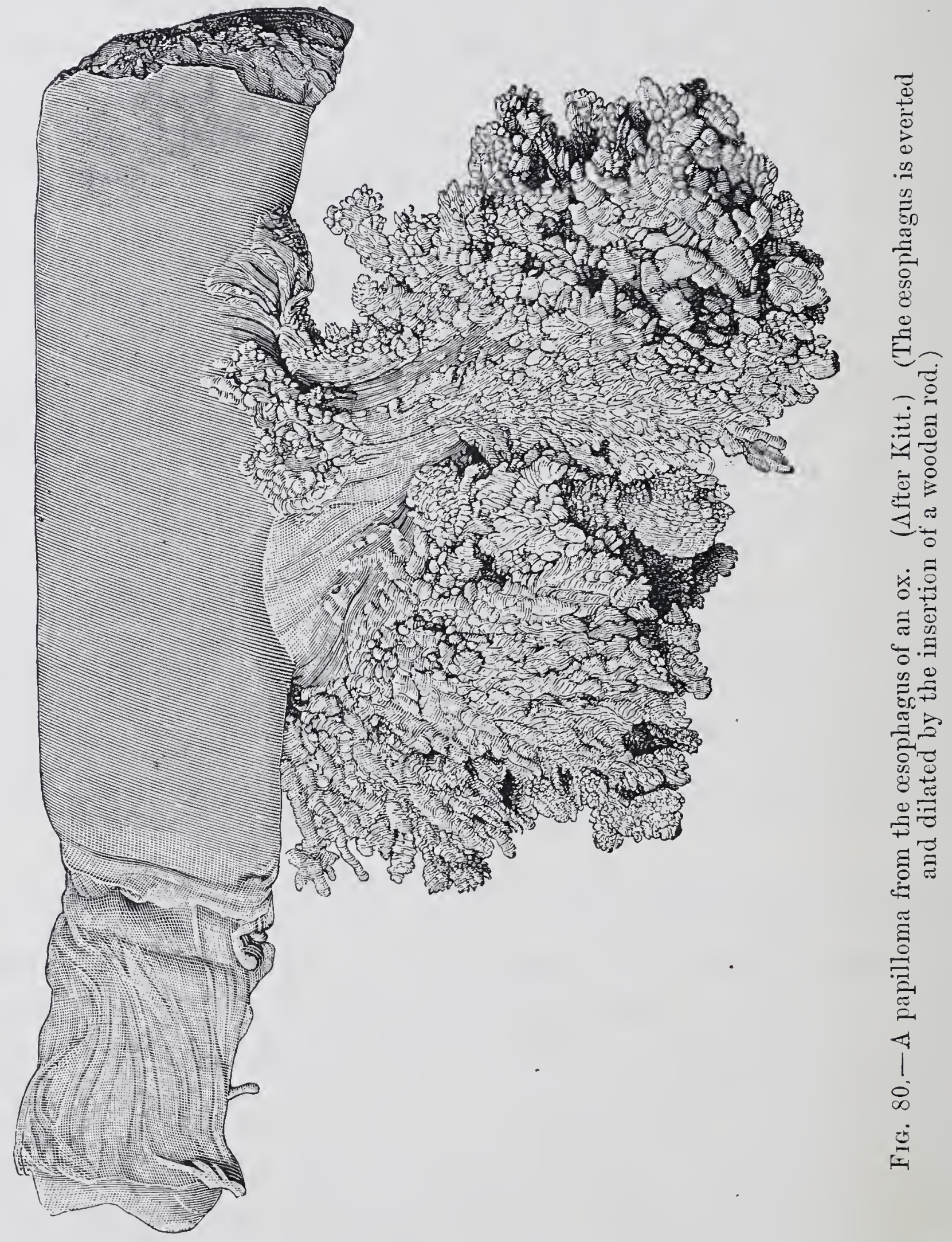

the pharynx. Degive, on making the post-mortem of a horse which had repeatedly suffered from sudden attacks of dyspnœa, threatening death from suffocation, observed a cystoid tumour as large as a hen's egg on the floor of the pharynx close in front of the epiglottis Labat operated on a horse which had suffered from nasal bleeding and difficulty in breathing and swallowing. By opening the larynx, and dividing the soft palate, he succeeded in removing a tumour from above the larynx by the écraseur. The growth proved to be an epithelioma (compare with next chapter as to occurrence of laryngeal tumours).

Such tumours are rare in carnivora. Diericx removed from the pharynx of 
a dog, with the help of a snare, a double polypus 2 inches long, which on microscopical examination proved to be a fibroma. In spite of extended practice, Möller has not yet seen similar disease in dogs.

Tumours of the pharynx are most frequent in ruminants, especially in cattle, and may be due either to actinomyces (Siedamgrotzky, De Jong) or be simple papillomata (Schütz). The former are usually single, the latter multiple. Fessler describes a case of multiple papilloma in the osophagus of an ox. The entire surface of the osophageal mucous membrane was covered with warty brush-like outgrowths, which were only the size of a grain of barley in the neighbourhood of the pharynx, but became as large as a bean in the middle of the œsophagus, and formed bristle-like tufts. Lower down they were not so numerous. These papillomata resembled bunches of bristles, numbering thirty or more, which grew from a conical base in the form of a brush.

Microscopical examination showed the following appearances :-The propria mucosa, below the papilloma, contained spherical masses of blood-vessels, in which the veins formed large cavernous spaces. The papilloma was provided with a solid root containing loops of blood-vessels from which processes of connective tissue extended into the threads and bristles. The connective tissue was covered with epithelium. Beel noticed a case of multiple papilloma in the œsophagus of an ox, marked by difficulty in deglutition, swelling on the left side of the neck, difficulty in introducing the probang, chronic tympanites, and reduced condition. Post-mortem showed "intra-cesophageal stenosis," with dilatation in front of the contracted spot. The latter, which was of uniform width, began below the pharynx, was $6 \frac{3}{4}$ inches in length, and ended at the height of the second rib; its greatest circumference was $9 \frac{1}{2}$ inches, the thickness of the muscular coat $\frac{1}{8}$ inch. The mucous membrane was covered with a great number of wart-like papillæ of varying size, some not exceeding that of a pin's head, others as large as a hazel nut; forty-five of them were from 1 inch to $1 \frac{1}{4}$ inches long; it was contracted at the level of the second rib, but still allowed the probang to pass. Beel believed that the periodicity of the symptoms was caused by masses of food inducing occasional occlusion at this spot. Microscopical examination gave similar results to those described by Fessler. The growth illustrated (fig. 80) is of an analogous character-viz., papilloma coralliforme.

Psorospermia have repeatedly been met with in the oesophageal walls in goats and sheep, but seem to produce no difficulty in swallowing. Possibly some of the cases described as paralysis of the pharynx are due to such parasites. Koch found tuberculous abscesses in the œsophageal walls in cows. In tropical countries (Java) cysts have been discovered in the oesophageal walls of dogs, which were filled with spiroptera sanguinolenta, and had completely occluded the osophagus. Harms, during the autumn, found female filarix below the osophageal epithelium in sheep. These have been stated by Zürn to be identical with spiroptera scutata œesophagea bovis. In dogs spiroptera sanguinolenta often occurs in the œesophagus.

These new growths, varying in size and form, produce greater or less difficulty in deglutition; often only fluids can be swallowed, and that with great effort. Sometimes suffocation and vomiting occur; wasting is a regular result. Curability depends on the condition and number of the growths, and on whether they can be reached. Polypi with narrow pedicles may be torn off and discharged during violent vomiting. In carnivora, as in man, these usually have their seat in the pharynx or close behind it, and during -vomiting may be thrown forward into the pharynx, and can then be removed, as shown by a case reported by Diericx. Where the swelling in the neck portion of the oesophagus can be detected externally, which, however, is seldom the case, it may be removed 
by performing œsophagotomy. The uncertainty of diagnosis during life generally prevents rational treatment. Lorenz saw a horse die from pleurisy, and found a carcinoma which had led to rupture of the œsophagus. Molni detected a tumour in the left oesophageal furrow in a cow, which, after each feeding time, showed tympanites. He regarded the case as one of goitre.

\section{VIII.-DISEASES OF THE LARYNX AND TRACHEA.}

\section{(1.) INJURIES, INFLAMMATIONS, AND TUMOURS IN THE LARYNX. (LARYNGITIS. PERILARYNGITIS.)}

Only in carnivora is the larynx accessible to direct inspection. This is effected for clinical purposes by drawing out the tongue and depressing it with some blunt instrument (scissors). The laryngeal mirror used by Nawratil and Schmidt is of little value, and is seldom used on account of the animal's resistance. Until recently, inspection of the larynx in the larger animals appeared impossible, but Polansky and Schindelka, with the assistance of the Vienna optician, Leiter, have constructed instruments for examining the larynx and pharynx of the horse, and amongst others the rhino-laryngoscope, which affords not only clear but extensive views of this region. Its construction is complicated, its use requires the electric light, and its application in veterinary practice has accordingly as yet been limited; but it is very serviceable for clinical observation and for teaching.

On account of its protected position between the branches of the lower jaw, the larynx seldom suffers from injuries from without, though sharp foreign bodies swallowed with the food may pierce and inflame the mucous membrane. Rowland removed from the pharynx of a horse a fish-hook, which had penetrated the larynx. Injuries of the larynx occur oftener than is supposed, but not so frequently as those of the pharynx, and produce either acute or chronic inflammatory processes. The acute are clinically comprised under acute laryngitis, and are considered in the text-boolis on special pathology and therapeutics. The chronic lead to proliferation of connective tissue in the neighbourhood of the laryngeal cartilages (perilaryngitis chronica fibrosa) or pus and abscess formation.

Kühnert saw a case of perichondritis suppurativa laryngea in a pig. An abscess had formed in the submucous tissue of the right arytenoid cartilage, and markedly narrowing the glottis, caused dyspnœa; the animal was slaughtered. Leisering detected swelling of the vocal cords and of the mucous membrane of the laryngeal pouches in a dog which had suffered from severe cough; and Hutchinson makes a similar report of a pig, killed on account of dyspnoa. Necrosis of the cricoid cartilage had occurred.

Perilaryngitis chronica fibrosa is commonest in horses. Progressive increase of connective tissue occurs, which sometimes undergoes ossification; its retraction fixes the arytenoid cartilages, narrowing the glottis, and producing more or less severe dyspnca. Gurlt reports this condition 
in a horse which had suffered from broken wind. The larynx was ossified. Werner speaks of an ox which, after suffering for over a year from gradually increasing difficulty in breathing, was found to have a laryngeal growth which extended into the trachea. Möller has often seen chronic perilaryngitis in horses. In four cases no cause could be discovered; another happened after resection of the arytenoid cartilage, causing failure of the operation. Chronic inflammatory diseases of the mucous membrane of the larynx are not very rare. In cows tuberculous processes occur. In horses and dogs chronic catarrh of the larynx is occasionally seen, though it seldom requires surgical treatment. Chronic irritation in the deep layers of the mucous membrane, causing thickening and proliferation, occurs oftenest on the vocal cords, which are often locally thickened. Lee found both vocal cords in a horse covered with cartilaginous growths.

Symptoms. Chronic laryngitis and perilaryngitis are recognised primarily by dyspnoea, which ensues more rapidly in cases where pus formation is proceeding than in those where connective tissue is being formed. At first inspiratory dyspnoea is alone recognisable, but difficulty in expiration may also be noted later. So long as the difficulty is confined to inspiration, the disease cannot easily be distinguished from paralysis of the recurrent nerve, and is clinically included under the term "whistling" or "roaring." External examination seldom discovers any structural changes, but if the process has extended to the outer surface of the cricoid and thyroid cartilages, the larynx appears of unusual size, although, its outlines seem less sharply defined. Ossification is recognised by an unusual hardness and resistance to pressure, usually first remarked on attempting to make the horse cough by squeezing the larynx. Laryngitis chronica, with thickening in the mucous membrane, gradually produces dyspnca, whistling sets in, sometimes in aggravated form, and may be noted both in inspiration and expiration, or may not differ from that produced by paralysis of the recurrent nerve. A further symptom of disease of the vocal cords is marked hoarseness or loss of voice (aphonia).

Tumours are most frequent in oxen, though also found in horses and other animals. Their nature has not yet been sufficiently investigated; a large number have been described as atheromatous. Their general position at the base of the epiglottis makes it probable that many are actinomycotic. The new growths described as cystic tumours of the larynx are possibly of this character. Gurlt describes the majority of the tumours in the larynx as polypi. Some may be mycotic, especially those associated with pus formation. A laryngeal tumour of the ox, discovered by Peschel, was stated by Johne to be adenoma of the mucous glands. 
Lee removed a cartilaginous growth from the vocal cord of a horse which had suffered from broken wind and was unable to neigh; recovery was complete in six weeks. Besnard lost a six year old mare by suffocation produced by a pedunculated cystic polypus, as large as a walnut, which hadits seat at the base of the epiglottis, and caused difficulty in swallowing, together with severe dyspnoea and attacks of coughing. Degive believed that apnoea arose from the epiglottis being pushed into the larynx by the swelling during deglutition, and not by the latter being swallowed, as Besnard thought. Lehnhard found a retention cyst, as large as a hen's egg and full of clear fluid, on the anterior surface of the epiglottis in a horse which had suddenly died from suffocation.

Tuberculosis of the larynx is common in oxen. Confusion with actinomycosis tumours often occurs, but a case of tuberculous new growth in the larynx of an ox was described by Johne, who had already drawn attention to the similarity of the two diseases. Prietsch and others noted tuberculosis of the larynx in cows on post-mortem.

Cadiot and Dollar (p. 100 of "Clinical Veterinary Medicine and Surgery") describe a number of cases of tuberculous ulcer in the neck in the dog and cat. The pathogeny of the condition is as follows:--In animals with tuberculous lesions of the lung coughing is common, and virulent tuberculous material is continually being passed into the pharynx, the mucous membrane of which becomes inoculated (auto-inoculation). The disease soon extends to the neighbouring lymphatic glands, which, under the pressure of the collar, become inflamed. Suppuration of the surrounding tissues and ulceration of the skin follow. The wound thus produced rarely heals, and in most of the cases death occurs within a few weeks or months from generalised tuberculosis.

Symptoms and progress. The first stages in the development of new growths are naturally beyond the field of clinical observation. Dyspnœea and, under certain circumstances, disturbance in swallowing are only produced after they have reached a certain size. On the epiglottis, however, they may attain considerable dimensions before marked symptoms appear ; but difficulty both of breathing and swallowing are then noted. As in diseases of the tongue, and in the so-called pharyngeal lymphoma, dyspnoea may occur, particularly during feeding. The disturbed respiration and deglutition are not uniform in their appearance, as Fricker and Dieck have noted in horses. Pedunculated tumours on the base of the tongue, dropping on to the larynx, suddenly produce symptoms of severe inspiratory dyspnœa, from which the animals sometimes fall and occasionally die of asphyxia. The sudden appearance of dyspnoea, without febrile or other constitutional disturbance, denotes the presence of such tumours. 'I he condition may be easily mistaken for cramp or odema of the glottis.

The exterior of the larynx is seldom visibly altered. Only where the new growth has spread to the exterior, or has produced marked changes in the wall of the trachea, does the region of the larynx appear fuller 
and swelling show itself. Prietsch also observed this in cows which suffered from laryngeal tuberculosis.

Examination throngh the mouth often gives more definite information. In carnivora, by drawing forward the tongue with a cloth, and pressing down its base with a pair of scissors or the handle of a spoon, it is possible to view the interior of the larynx and determine the presence of tumours on its upper borders. In horses this may be effected with the laryngoscope constructed by Polansky and Schindelka. When this instrument is not available, the pharynx may sometimes be examined when the horse is cast, provided the position of the molars and the soft palate allow the passage of the hand. Such examination is more easy in oxen, and, if the head is fixed, may be made while the animal is standing.

In doubtful cases the larynx can be opened and a view of its interior obtained, an operation entirely without danger, and to be made use of wherever it is important to remove doubts.

Tumours in the trachea are first remarked when they narrow the lumen of the tube considerably, and produce dyspncea. This is generally accompanied by loud breathing sounds, which occur both during inspiration and expiration, though occasionally only during one portion of the act. It is characteristic of tumours in the trachea that the noise appears especially or exclusively during expiration. The position and nature of the new growth explain this peculiarity. Dyspnoea increases with the growth of the swelling, and may terminate in suffocation. Only where malignant tumours perforate the wall of the trachea and extend outwards can their presence be directly recognised. Diagnosis is rarely aided by palpation of the trachea; but pressure on the diseased spot may, perhaps, increase or decrease the dyspnoa.

Prognosis is in general unfavourable. Pus formation and the discharge of abscesses into the trachea may occasion pneumonia. In chronic laryngitis with thickening of the mucous membrane, resection of the vocal cords may be serviceable. Prognosis of perilaryngitis fibrosa, and other diseases, depends in great part on the degree of dyspnoca. Tracheotomy is certainly a palliative. The prognosis of most laryngeal tumours is unfavourable; but pedunculated growths, situated even in the larynx, may sometimes be removed without recurrence, section being made of the cricoid cartilage and first rings of the trachea. Where the operation is unsuccessful, tracheotomy will still render the animals workable for some time.

Treatment. Tumours on the epiglottis can sometimes be removed in the same way as pharyngeal tumours (p. 179) in cattle, by tearing them away with the liand; in dogs, with the assistance of instruments. Dieck removed a swelling as large as a hen's egg from the epiglottis of a hor'se 
with a specially-constructed instrument in the form of a hooked knife. Thickenings and tumours on the vocal cords can be excised. 'The horse should be cast, and, if restless, anæsthetised. The hair over the larynx is shaved, and a cut made through the skin in the middle line, from the thyroid to below the 1st, $2 \mathrm{nd}$, or $3 \mathrm{rd}$ tracheal rings. After ligaturing any bleeding skin vessels, the second cut is made, dividing the subscapulo-hyoideus and the sterno-thyro-hyoideus exactly in the

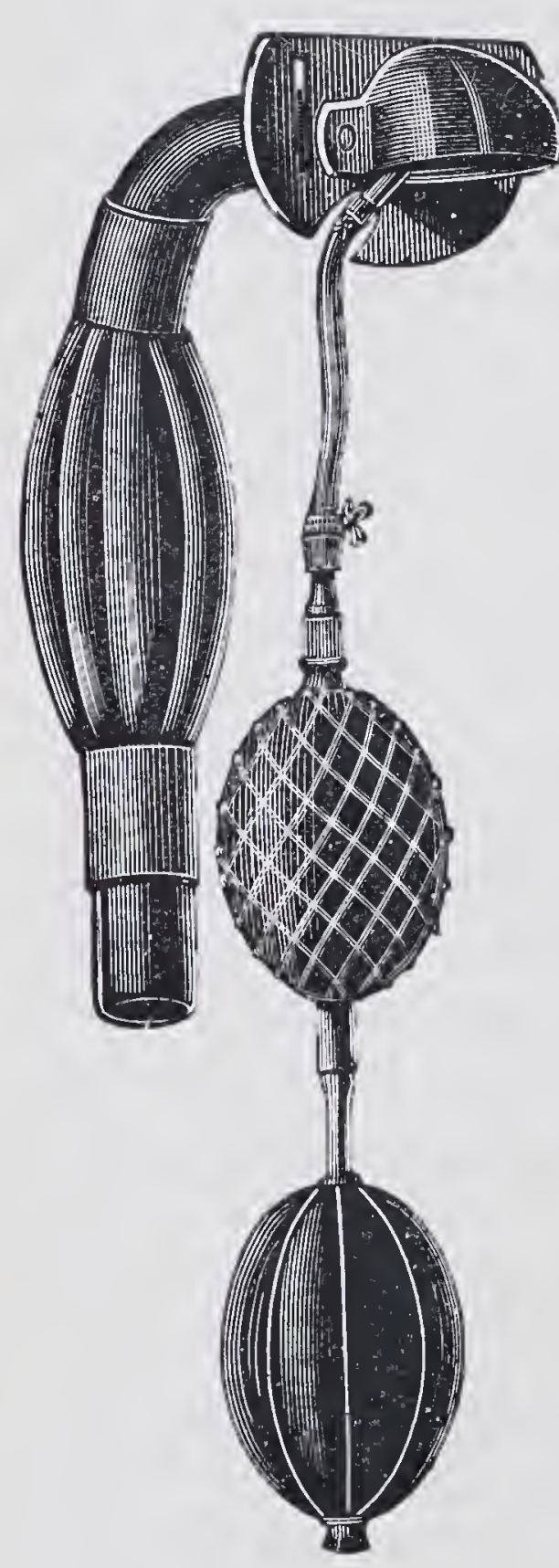

Fig. 81. - Tampon-canula or Trendelenberg-canula.

middle line. This exposes the cricoid cartilage and trachea, which are then incised. The edges of the wound are held apart with broad hooks or retractors. Where a tampon-canula (fig. 81) is available, it should be inserted in the trachea to prevent danger of suffocation from entrance of blood. The finger is introduced into the larynx, the vocal cords examined, and either of them exhibiting the above-described changes is excised with scissors. The cord is previously fixed by grasping it with a torsion or clamp forceps; or, in their absence, it is made tense by a ligature passed with a curved needle over its lower anterior end. Bleeding is slight, and is of no importance if a tampon-canula is inserted. Should it occur, it can be checked by plugging the larynx. The tampon-canula and tampons can be removed next day, but, for security, the canula is better left in position for some days, especially when the animal is not under the eye of the operator. Serious swelling of the mucous membrane and dyspnœa occasionally occur, but are rare.

When tumours are within the laryngeal cavity, their size and character are ascertained by opening the larynx, and their removal, especially if pedunculated, is effected with the scissors and the "écraseur." In dealing with new growths removal must be complete; Paquelin's cautery, in certain circumstances, does good service, and also checks bleeding. Where marked dyspnœa exists, or occurs periodically, tracheotomy must either be performed before casting the horse, and a canula inserted; or the instruments must be in readiness, so that, in case of need, a tube can be inserted. In the treatment of perilaryngitis chronica fibrosa, or of new growths with broad bases in the larynx, tracheotomy below the growth is the only means of removing dyspnœa. 


\section{(2.) NEUROSES OF THE LARYNX-SPASM OF THE RIMA GLOTTIDIS-SPASMUS GLOTTIDIS-LARYN- GISMUS STRIDULUS - LARYNGO-SPASMUS - HEMIPLEGIA AND DIPLEGTA LARYNGIS.}

These te:ms are applied to attacks of difficult breathing, occurring periodically, and apparently caused by spasmodic closure of the rimaglottidis. As a rule, the dyspnoea is inspiratory, and may occasionally be so severe as to constitute apnœa,- - that is, temporary cessation of respiration.

The causes consist either in direct irpitation of the mucous membrane of the larynx, or in reflex irritation of the vagus and recurrent nerves supplying the constrictors of the larynx. Records of many such cases have been published, but are seldom sufficient to determine the exact cause of the attack. Acute inflammatory changes, catarrh, cdema of the glottis, food materials, or foreign bodies in the upper air passages, have all been assigned as causes. Post-mortem examination has discovered tumours in the pharynx, larynx, or trachea, but true neuroses of the larynx are occasionally met with.

Ebinger describes a horse suddenly attacked by severe dyspnœa, threatening suffocation, and accompanied by roaring. When at rest the breathing was normal and the health undisturbed, but the slightest work or pressure on the larynx, or indeed on any point of the neck above the jugular furrow, caused immediately severe dyspnœa and marked roaring, which continued during the next 8-10 respirations. Light pressure sufficed again to produce the attack. The symptoms disappeared after eight days' treatment with subcutaneous injections of acetate of morphine. Ebinger, not without justification, believes this to have been a neurosis of the recurrent nerve. The cases described by Holzendorf and Neumann as spasm of the larynx, or cramp of the muscles of the glottis, may have been due to other causes. In that recorded by Holzendorf, inflammatory disease of the larynx was clearly present; while the obstinate dyspnoea treated by Neumann does not appear to have been neuropathic.

Möller saw similar cases, apparently of neuropathic origin, in otherwise healthy horses; Seffner during recovery from acute bronchitis.

King and Robertson referred similar attacks to ingestion of certain leguminous seeds, which they consider had a specific action on the recurrent nerve.

Penberthy regards the condition as bronchial asthma, in which inhalations of chloroform and amyl nitrite might be useful.

Trumbower describes six cases of spasm of the larynx in horses which worked together in the same field. Death occurred in a few hours. Postmortem examination showed the existence of acute laryngitis.

When danger of suffocation is associated with these conditions, tracheotomy must be resorted to at once. It is further noteworthy that the inspiratory dyspnoea is increased by the anxiety and unrest associated with it. All excitement must, therefore, be avoided, the horse placed in 
a quiet stall, and care taken that the air be pure and the temperature suitable. Caution must be observed in the use of morphine; doses over 8 grains often cause excitement. Clysters of chloral hydrate deserve preference. Preisnitz's poultices, prepared by dipping poroplastic or thick felt in hot water, may be applied to the throat and neck, but must not be heavy, and may sometimes be preferably replaced by dry coverings.

Myopathic paralysis of single muscles, or of groups of laryngeal muscles, is not known in animals, but may possibly occur. Neuropathic paralysis of the muscles of one side of the larynx (hemiplegia laryngis) is, however, very frequent in horses. The greater number, if not all the muscles of the larynx, which move the vocal cord are supplied by the recurrent nerve. Of late a second branch of the vagus has been recognised by Exner as a motor nerve to the larynx, but it has not yet been determined what influence it exerts 'on the function of its muscles. From the construction of the larynx, as a valve opening outwards, it is clear that simultaneous paralysis of the constrictors and dilators must cause disturbed breathing during inspiration. Cramp of the constrictors alone might produce expiratory dyspnœa, but this very rarely occurs, for the expiratory muscles, assisted by abdominal pressure, are almost always sufficiently powerful to open the larynx, even against the constrictors. It is otherwise in inspiration. Should the dilators of the larynx become paralysed, the arytenoid cartilage is forced towards the opening of the glottis by the instreaming air, and roaring results. With a few exceptions it is caused by paralysis of the recurrent nerve, and is so described in the text-books, as well as in another section of the present work. This paralysis is usually incurable, but the associated dyspnoea may be relieved either by removing the arytenoid cartilage, which has fallen below its position in the larynx, or by tracheotomy, thus providing a direct entrance for the air below the larynx. Both operations are fully described in sections 5 and 6 immediately hereafter.

Roaring is also occasionally seen in other animals besides the horse; Utz and Ollmann found it in cows. Esser has described paralysis of the recurrent in a dog, and Möller noted the disease in two dogs. Möller also records several cases of double-sided paralysis of the larynx (diplegia. laryngis) in horses and dogs producing severe inspiratory dyspnœe. After being walked for a short time, whistling or roaring was produced. The condition generally pursues a chronic course, and may be taken for chronic inflammation or tumour formation in the larynx.

Diagnosis of roaring belongs to the province of special pathology, but the detection of double-sided paralysis of the larynx and its distinction from chronic inflammation or tumour formation may call for operative interference, or for the use of the rhino-laryngoscope. By using the latter instrument one sees clearly at the moment of inspiration that the 
two arytenoids sink downwards and approach each other, and in paralysis may even come in contact, completely closing the larynx. If not provided with the instrument, one may divide the cricoid cartilage and crico-thyroid ligament, and by introducing the forefinger through the opening, directly examine the arytenoid cartilages. Whilst in the normal larynx they make distinct to-and-fro movements, especially during forced inspiration, in diplegia laryngis they are found to sink downwards in the larynx. In this disease tracheotomy alone can restore the animal's usefulness.

Starting with the hypothesis that the cause of paralysis is situated in the intrathoracic portions of the nerve, MacDonald attempted to unite the ascending (cervical) portion of the recurrent to the spinal accessory, and thus to "short-circuit" the nervous impulses. Both he and Haslam claim to have had good results. Other operators, however, have been unsuccessful, partly because of the necessarily delicate manipulation involved, but more probably because of the serious changes that had already occurred in the nerves and muscles.

\section{(3.) FRACTURE, DEFORMITY, AND STENOSIS OF THE TRACHEA.}

Injuries to the trachea having already received attention as complications of wounds in the neck, it only remains to consider those subcutaneous solutions of continuity which, when affecting the cartilaginous rings, are described as fractures. These injuries result from the forcible action of blunt bodies on the air tube; in horses are caused by the impact of the carriage pole, by rushing against projecting bodies, or by falling whilst haltered tightly. Fractures of the cartilages, or rupture of the inter-annular ligaments, in dogs are caused by their being captured with a wire snare.

Bru noted rupture of the ligaments between the first three tracheal rings in a mule. Walley saw a fox terrier in which the trachea was torn across; death occurred very rapidly. Cajöry found the trachea almost completely torn from the larynx in a horse which had died in the stall after a sudden attack of dyspnœa. Von Bockum-Dolffs saw a horse suffer from emphysema of the neck and severe dyspnœa, in consequence of having run against the pole of a passing carriage. Tracheotomy removed the difficulty in swallowing and breathing, and the animal again became fit for work.

But such injuries cannot always be successfully treated; deformities of the trachea sometimes result; extensive cicatrices produce stenosis of the trachea, accompanied by dyspnoea. Schwanefeld describes a horse in which the trachea was flattened about the middle of the neck and greatly narrowed. The presence of a stallion in the stable excited it and induced suffocation. Some deformities of the trachea are clearly congenital. Occasionally the posterior ends of the tracheal rings are 
bent inwards, or flattened so that the lumen forms merely a narrow fissure.

Gurlt saw a dog with marked flattening of the air tube. Harms and Hagen note similar cases in cows. Johne describes two instances of dilatation in horses, one about 24 inches in length, and a similar condition was recorded by Bartenstein. Vegezzi found the dorsal surface of the air tube of a horse presenting a furrow produced by bending inwards of the ends of the cartilages and rupture of the inter-annular ligament. In this furrow lay the œsophagus, carotid, vagus, and sympathetic. Eberbach describes a horse in which dyspnœa was caused by compression of the trachea, due to a large sarcoma.

The intact condition of the mucous membrane and the extension of the change throughout the trachea shows the disease to be congenital. Compression, with narrowing of the trachea, may also result from goitre, from enlargement of the bronchial glands, or from tumour formation. Johne relates such a case in a giraffe, and Dietrich in a foal which had died with symptoms of suffocation. Between the first pair of ribs was a diseased gland, which had compressed the trachea and caused suffocation.

Stenosis of the trachea is not an infrequent result of tracheotomy, especially in foals, or where the tube, worn for a long period, does not fit well. Chronic perichondritis resulting from the continued irritation, induces formation of cicatricial tissue, which sometimes ossifies, and narrows the lumen of the tube, firstly by pressing on it, and afterwards by contracting around it. Thus Tiede found the trachea narrowed to the size of a goose quill. Stenosis is not always a result of unskilfulness in performing tracheotomy or selecting a tube; stenosis sometimes recurs in horses in which tracheotomy has been performed below a previous contraction, it therefore seems likely that some animals are predisposed to such conditions.

The symptoms of injury to the trachea are difficulty in breathing, and emphysema on the neck, with localised inflammation, swelling, and pain. The degree to which the lumen of the tube is narrowed in consequence of hæmorrhage, dislocation of its cartilages, or inflammatory swelling, determines the extent of the respiratory disturbance, which may become so great as finally to produce suffocation. In other cases a loud sound is heard both during inspiration and expiration. Very slight changes in the trachea are sufficient to produce it. Sometimes the noise may be increased or diminished by pressing on a particular part of the trachea. Laying the ear on the wind-pipe, the position of the stenosis may be more exactly fixed, but this method does not always prevent mistakes. During the next few days the emphysema usually spreads over the body, especially when coughing exists, but disappears later, generally without bad results. 
The course of the disease depends on the extent of the injuries and of the resulting bleeding. The animal may be completely restored to usefulness, or difficulty in breathing may remain (asthma tracheale). The dyspnoea depends partly on the degree of stenosis, partly on the character of work performed. In spite of marked deformity, dyspnoea may be absent, whilst apparently unimportant changes sometimes produce greatly disturbed respiration; indeed, Schwanefeld's case shows that they may cause death. These diversities are explained by the fact that the respiratory difficulty is determined, not by the external deformity of the trachea, but by the degree of stenosis. The gravity of such cases is gauged by the state of the respiration during severe work. In stenosis of the upper portions of the trachea, tracheotomy usually affords relief, but is not available when the lower portions in the neck or thoracic cavity are affected. Such conditions, however, are often aggravated by lapse of time.

Treatment. Where great dyspnœa follows fresh injuries of the trachea, tracheotomy not only removes the threatened danger of suffocation, but, if a tampon-canula is used, also prevents the entrance of blood into the trachea. A tampon-canula may be extemporised by carefully wrapping the stem of a Barthélemy's tracheal tube with cloth, but care must be taken that the bandage material, tow, wadding, or piece of sponge used for this purpose does not fall into the trachea. Severe emphysema, although seldom endangering life, may sometimes necessitate tracheotomy.

Stenoses of the trachea are only occasional reasons for direct treatment. In man it is possible to dilate the trachea with elastic tubes introduced from the larynx. In animals the use of tubes is more difficult, the time for tardy healing would be grudged, while frequently the cicatrices, as well as portions of the tracheal rings, are ossified. The position and extent of the stenosis must determine whether tracheotomy is applicable, or likely to be successful. It is generally serviceable when carried out below the stenosed spot. Richolson, by removing a dislocated portion of cartilage, permanently relieved the dyspnoea. Similar cases are rare. The interesting communication of Lafosse and Sticker show that "tubage" of the trachea is possible, and may be successful. Lafosse divided the trachea below the narrow point caused by tracheotomy, thrust a cork cylinder, bound round with tow, into the trachea, and fixed it with tape. In ten days the cylinder was replaced by a tube, the latter was removed after six weeks, the trachea being then so far dilated as to admit of easy respiration without a canula. Sticker dilated the trachea and larynx by means of a metallic spiral, which was left permanently in position, and is said to have finally been covered by the tissues. 


\section{(4.) FOREIGN BODIES, TUMOURS, AND PARASITES IN THE TRACHEA.}

Foreign bodies seldom enter the trachea during life. Owing to the excessive sensibility of the mucous membrane of the larynx, they immediately produce coughing, and are ejected. Masses of food have nevertheless been repeatedly found in the trachea both in oxen and horses. Tumours of the pharynx, which are common in cattle, sometimes interfere with swallowing, and favour the entrance of food into the trachea. In horses fluid medicines unskilfully administered occasionally pass into the trachea and bronchi. Fatty oils are particularly dangerous in this respect. Linseed oil, formerly much used as a laxative, has been thought to produce a specific pneumonia, but its effects merely depend on its entering the trachea. Dust enters with the inspired air, and may produce mechanical pneumonia, especially in horses, the subjects of severe disease, which lie continuously. Abscesses in the walls of the pharynx or trachea, perforating the mucous membrane, may pour their contents into the air passages, and produce fatal pneumonia. Rost saw a cow die thus from suffocation. In animals suffering from tetanus, saliva and medicine not infrequently find their way into the respiratory passages, and produce the same effect. Blood derived from wounds or operations in the mouth, trachea, or neck may enter the air passages and clot there, producing suffocation. Badly-made tracheal tubes sometimes break, and a portion falls into the trachea. This is common with Barthélemy's canulæ, where the tube is not always firmly fitted to the shield. Henderson reports such a case. During tracheotomy, if care is not taken, the piece of divided cartilage may easily fall into the trachea. Other foreign bodies rarely enter the trachea, though a case is reported where a horse died, in consequence of a stem of Robinia pseudacacia 12 inches long passing into the right bronchus, penetrating the pleura and producing plemro-pneumonia with hydro-thorax.

Tracheal tumours are rare, though they have been seen-principally in oxen. They are usually pedunculated, probably in consequence of being continually moved by the air stream. Gurit, Gerlach, Hink, and others found tracheal polypi in oxen, Rieck and Hink sarcomata. Siedamgrotzky describes a colloid cyst in a horse's trachea. It was found embedded in loose connective tissue just below the cricoid cartilage. Benjamin discovered in the anterior wall of the trachea a tumour, which had formed after an attack of sore throat, and produced difficulty in breathing. Besides the varieties of strongylus, usually occurring in quantities in the bronchi of ruminants and swine, acari have been seen in the trachea. Paulicki found them in a long-tailed monkey.

The symptoms are very varied. Dust or fluids obtaining entrance into 
the bronchi cause mechanical pneumonia sometimes with fatal issue. Larger objects remain in the trachea or. bronchi, and may produce death from suffocation. A cow described by Grül, which, till the moment of seizure had been perfectly healthy, suddenly died in the stall with symptoms of suffocation, and post-mortem showed the bronchi to le filled with food. Similar cases have repeatedly been observed. But Leisering has drawn attention to the fact that, in animals with incomplete closure of the cardia, and especially in ruminants, portions of the food after death may be forced through the oesophagus into the pharynx, thence into the trachea and bronchi, completely plugging them. This is most likely to occur where the stomach has been full, or its contents in a state of fermentation, or the carcase has been moved. A case described by Werner was clearly produced in this way. The distinctive features are absence of inflammatory appearances and complete stoppage of the trachea, which could never have been so packed had the material entered during life.

Dyspnoa results immediately the foreign body in any way narrows the lumen of the trachea. The movement of the foreign body can sometimes be heard or felt from without. The narrowness of the rimaglottidis renders it difficult for solids that have entered thus far to make their exit. In Henderson's case, as reported, the tube of a tracheal canula, which had slipped into the windpipe, was said to have been ejected through the mouth; but such a conclusion is very exceptional. Tumours in the trachea only attract attention when they become large enough to obstruct the air passage and produce dyspnœa. A loud sound is then heard, both during inspiration and expiration. It is particularly characteristic of tracheal tumours that they cause a marked sound during expiration. The position and form of the new growths explain this peculiarity, and their slow development accounts for the gradual increase of dyspnœa, which may finally cause suffocation. The presence of a tumour can only be directly detected when it originates on the outer surface of the trachea, and thence breaks through the tracheal wall, as malignant new growths generally do. Otherwise the position of a tumour may be determined by palpation, pressure at a particular spot increasing or decreasing the dyspnœa and noise. This symptom is quite peculiat: to tumours.

Treatment. Prophylactic treatment requires that in giving fluids to dogs and horses particular care should be taken, especially if the animals are restive. The head must neither be raised too high, nor turned on its own axis. It is easy to judge of the action of such abnormal positions of the head by attempting to swallow whilst the head is either turned much towards the right or left. Such positions are, however, less dangerous in oxen. Very great care is required if the drugs are not 
in complete solution, or if they contain fatty oils or irritants. Should the animal cough during the administration of fluids, the process should be stopped. Rubbing the neck is not only useless, but also dangerous, as coughing is thus easily induced. In operations, during the course of which blood may find entrance into the air tubes, a tampon-canula (fig. 81) should be used, or the operation performed with the head pendent -a procedure which has, however, the disadvantage of allowing the animal to move.

Large, firm objects can usually be removed from the air passages only after tracheotomy. Removal is sometimes possible with the help of suitable forceps. If necessary, the fissure in the trachea can be lengthened, when the body may be forced out by the pressure of the expiratory current. Tumours can sometimes be removed in a similar fashion.

\section{(5.) TRACHEOTOMY.}

Where obstacles to the passage of air exist in the upper air-tract (nose, larynx, or upper portion of the trachea), a direct entrance to the lungs may be provided by opening the trachea. This operation (tracheotomy), formerly wrongly termed bronchotomy, has been carried out in man from the earliest times (Asclepiades); has, however, repeatedly fallen into disuse, and has recently again come into fashion in men and animals. Viborg especially, showed its simple character. Tracheotomy consists in surgical opening of the trachea, in which is generally placed a tube or canula permitting passage of air. The operation is adopted for the following purposes:-

(1) To ward off suffocation resulting from swellings in the schneiderian membrane, in the larynx, or from other obstacles in the upper air passages.

(2) To restore to usefulness animals suffering from chronic dyspnœa produced by stenosis of the air passages. To this category belong horses suffering from hemiplegia laryngis.

(3) To remove foreign bodies from the trachea, and prevent the entrance into it of blood or inflammatory products.

(4) More rarely to carry out direct treatment of the larynx and mucous membrane of the trachea.

Tracheotomy is almost invariably restricted to horses, in which animals impaired respiration is commonest, and interferes most seriously with usefulness. In ruminants diseases of the larynx are rarer, and when they occur the animals are generally slaughtered. Owing to the comparatively long neck, and exposed position of the trachea, the operation offers less difficulty in horses than in cattle, where the trachea is covered by the dewlap. The isthmus of the thyroid glands in the horse 
is so slightly developed that no particular notice need be taken of it. In man, however, the operation is distinguished as superior, inferior, or medial, according as the operation is performed above, below, or through the isthmus.

In animals, and especially in horses, there is, therefore, a wide field for operation, comprising the space from the upper end of the trachea to near its entrance into the thorax. The upper third is covered by the panniculus of the neck, by the subscapulo-hyoideus, sterno-thyro-hyoideus, and sternomaxillaris, but the last named passes on to the lateral aspect of the neck at the lower border of the upper third, thus leaving the anterior wall of the trachea covered only by the first-named muscles below this spot. Günther, therefore, prefers for the operation a point about one-third of the distance from the larynx to the chest, though he admits that it may be carried out higher or lower without disadvantage. Laafosse chooses the space between the third and fourth tracheal rings; Krieshaber, that between the larynx and trachea. As a rule, the space between the upper and middle thirds of the neck is the point selected, because it is convenient, interferes less with the appearance of the animal, and in the event of stenosis supervening, the operation may be repeated at a lower point. The prejudice existing in human surgery against tracheotomy as a dangerous operation has led to modifications, few of which, however, are practical. The various complicated tracheotomes and bronchotomes proposed by Brogniez, Marty, Thompson, and others, and intended to simplify and facilitate the operation, are not needed by practised operators, and are of little use in unpractised hands. Two operation methods can be distinguished: (1) Puncture ; and (2) Incision of the trachea.

(1) Puncture of the trachea was recommended by Pilger, Gowing, Hayne, and others. Hayne's method met with most approval, and consisted in passing a trochar, provided with a canula having lateral openings, transversely through the trachea (fig. 82). This operation required some skill and care, particularly in horses having well-developed necks,

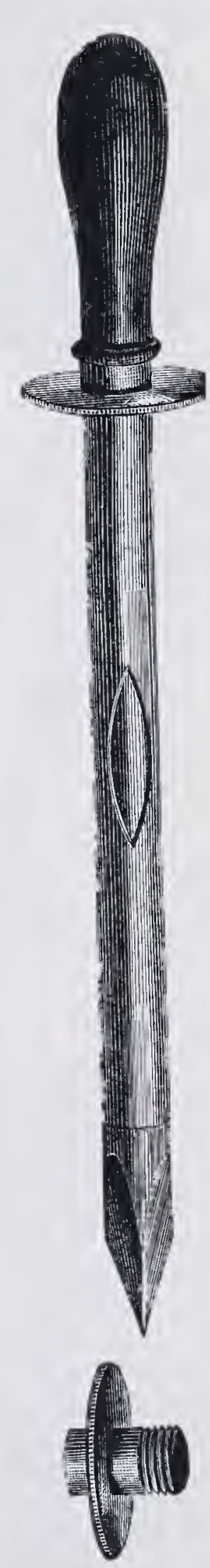

Fig. 82.Hayne's tracheal trochar. in order to avoid injuring the jugular and carotid; while even the large canula, used for heavy working-horses, did not admit sufficient air. The origin of this procedure, which does not possess even the advantage of being rapidly carried out, could only have been fear of using the knife. Thompson's tracheotome was designed to simplify operation, 
though its value is not great. It consists of a special forceps (see figures 83 to 87), with jaws bent at right angles to the limbs and externally ground to a sharp cutting edge. When closed, the head of the instrument is readily thrust through the skin and into the trachea; the jaws are then opened and the canula slid into position between them. The figures very clearly show the instrument and the mode of using it.

(2) Incision into the trachea is most generally practised. The trachea may either be split or a portion excised from one or more rings. The splitting, although it increases the difficulty in introducing the canula,
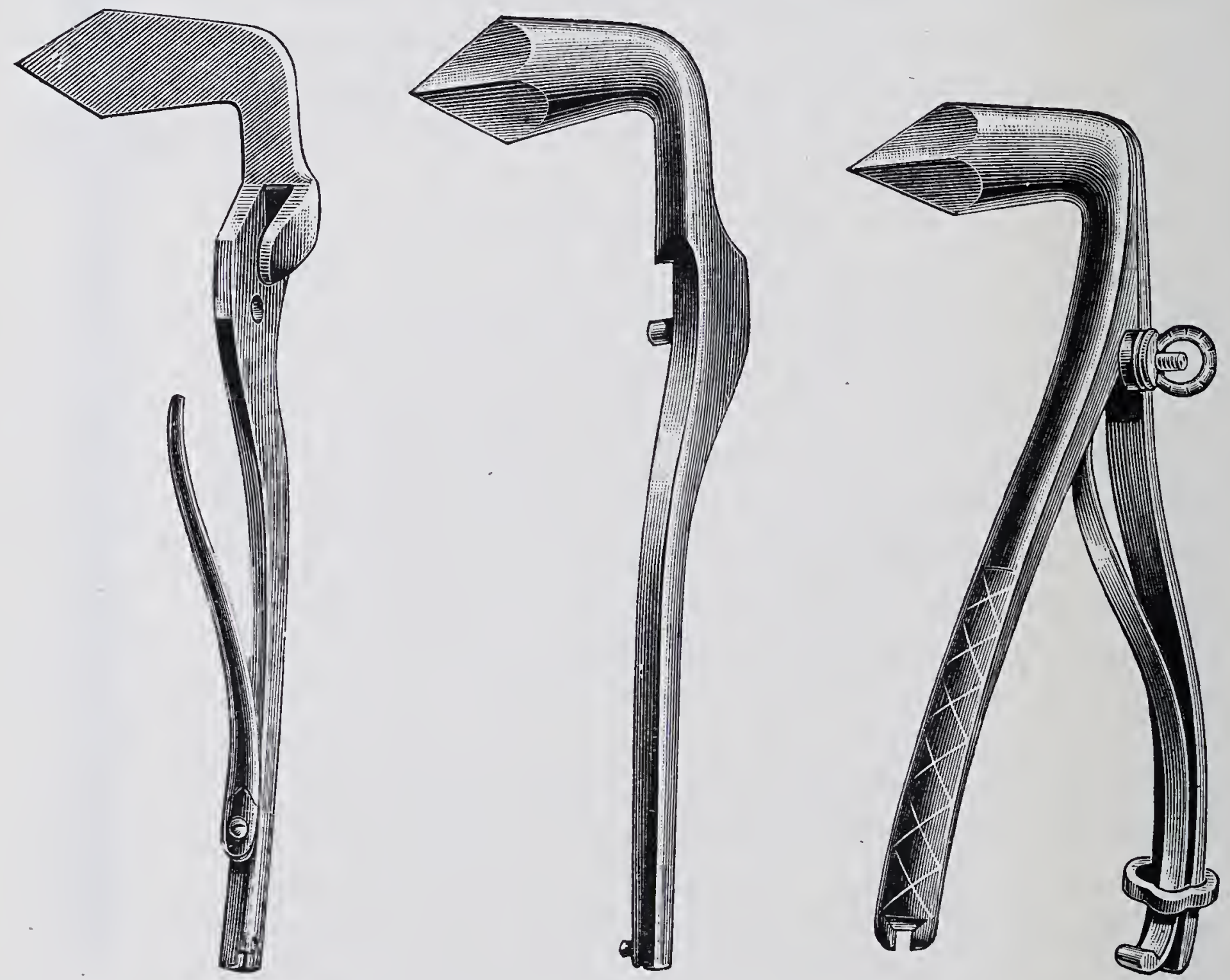

Fias. 83, 84, 85.-Thompson's tracheotome.

is sometimes preferred where the tube is likely to be only temporarily used, is less apt to cause deformity and stenosis, and hence is generally commended in acute diseases like strangles for foals and race-horses, in which even slight stenosis is dreaded. The excision of a portion of the trachea facilitates the introduction and removal of the canula for cleaning, and is specially desirable in such chronic cases as paralysis and contraction connected with the nose, larynx, or trachea. Experience does not justify the belief that stenosis is more apt to result when the cartilage is excised than when it is split.

Tracheotomy is conveniently performed whilst the horse is standing; casting usually aggravates any dyspnœa present. The animal should 
be placed so that the front of the neck is well lighted. A twitch is applied, and the horse, if still restless, has each ear held by a strong

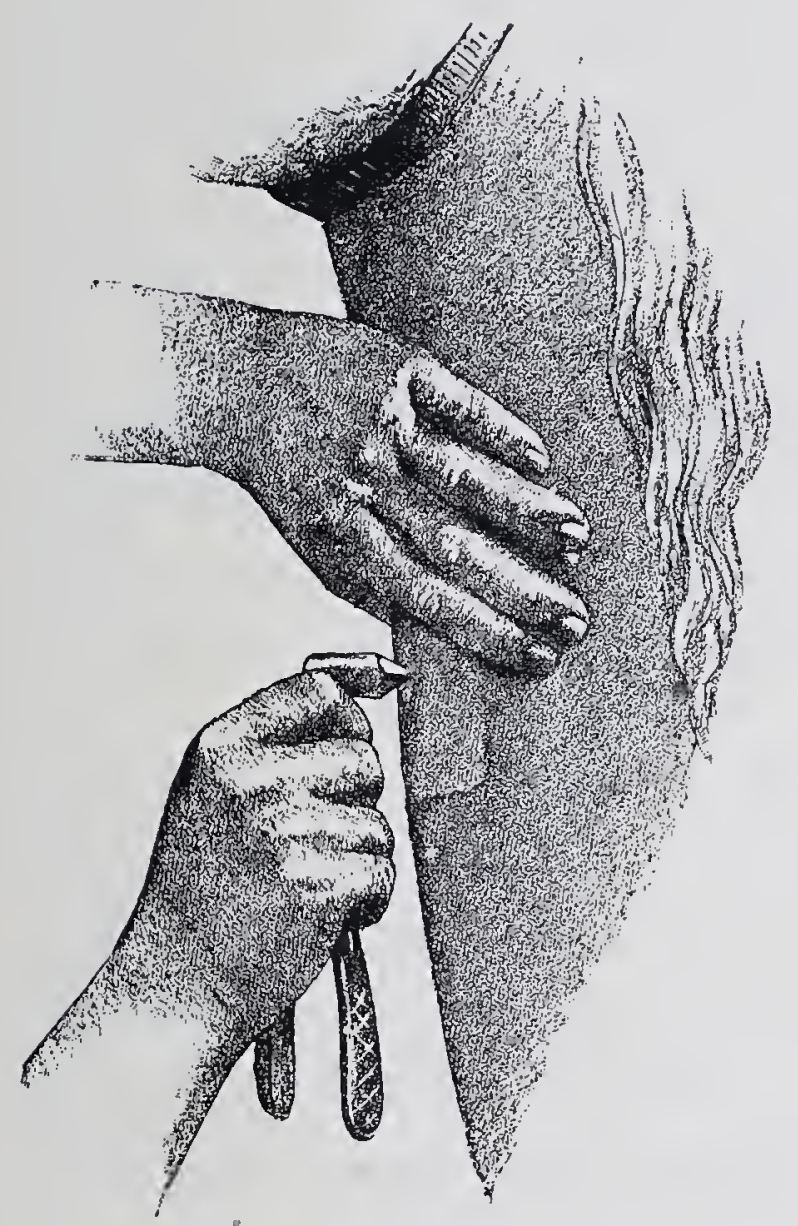

FIG. 86.-Making the incision.

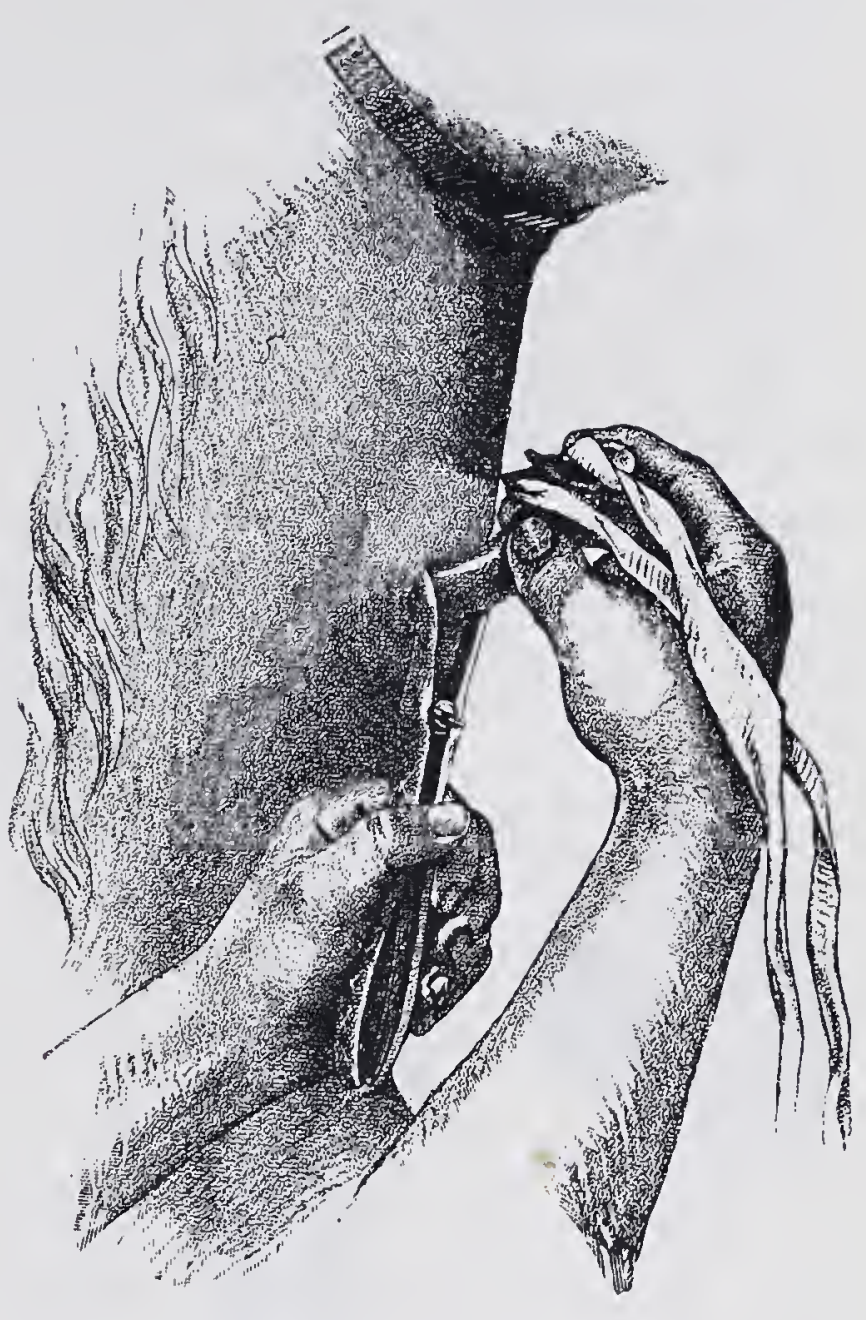

FIG. 87.- Introducing the canula.

man. The head is moderately raised, and the hair clipped from the seat of operation in a narrow strip, 3 to 4 inches in length, in the
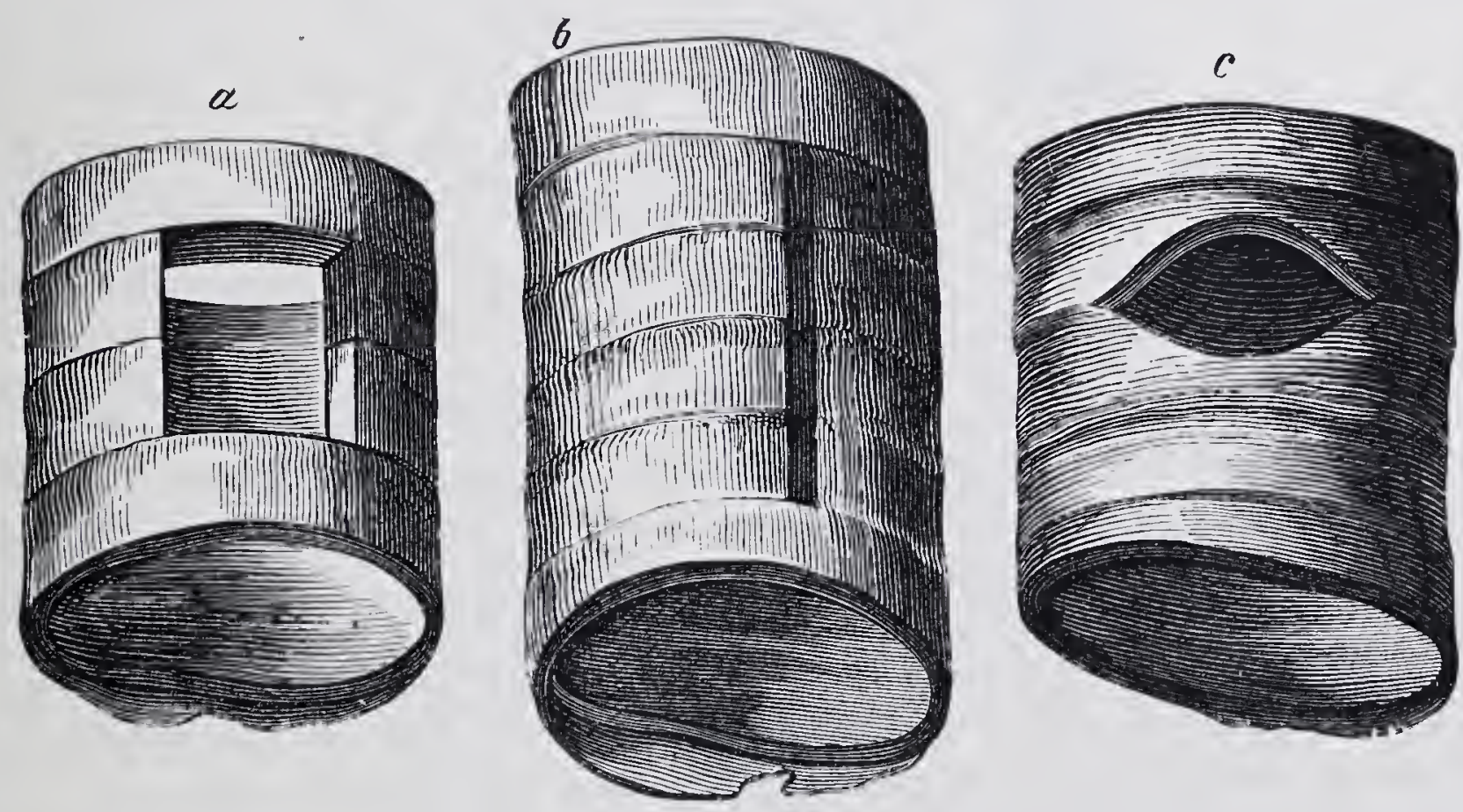

FiG. 88.-Method of performing tracheotomy.- $a$, Square opening; $b$, simple incision ; $c$, oval opening. (Hering.)

middle line of the neck. The operator grasps the skin with the left hand, and with the aid of an assistant lifts a horizontal fold about $\frac{1}{2}$ inch high, and exactly in the centre of the shorn patch; an incision is then carried through the fold. This prevents the skin wound being 
made larger than intended, in consequence of the animal suddenly stretching the neck, though in phlegmatic horses, and those suffering from severe illness or dyspnoa, the precaution is not so necessary.

The skin being divided, and any bleeding vessels ligatured, the panniculus is cut through, and the pairs of muscles lying below it are separated in the middle line, the proper point being indicated by its lighter colour (connective tissue). Where the middle line is exactly encountered scarcely any bleeding results, and the trachea is exposed. In thin horses with slightly developed necks this procedure is very simple; but in fat horses with thick necks there may be a little more difficulty, though no danger. The edges of the wound are held apart with a pair of broad hooks, with the fingers of the left hand, or with

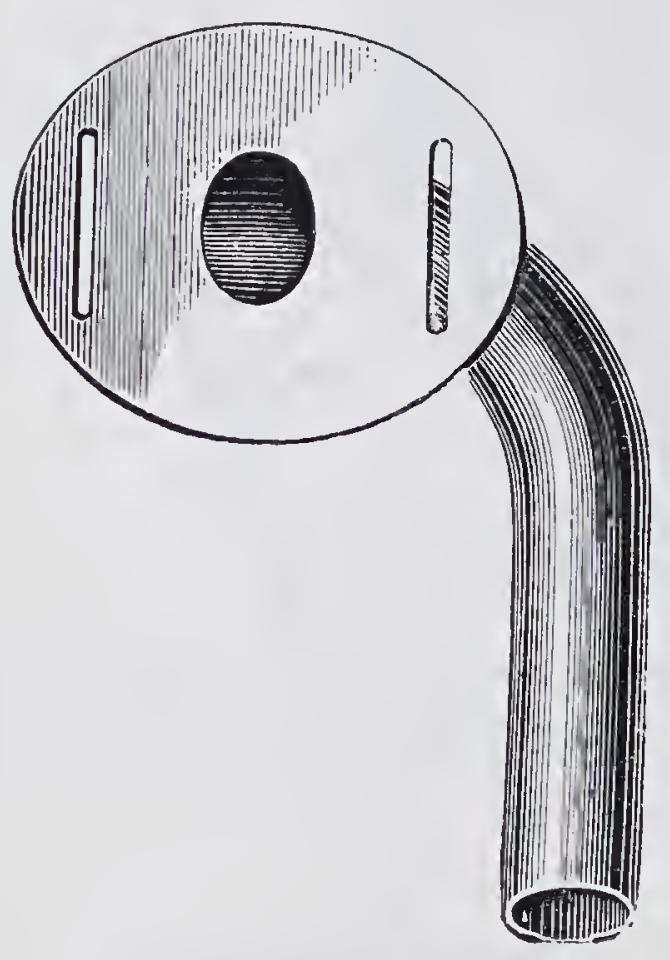

FIG. 89.-Barthélemy's canula. a retractor. Up to this point the procedure is the same in both methods.

In the operation without removal of tissue, the trachea is now divided either perpendicularly through the third and fourth rings, or a horizontal cut made in the intervening ligament; or a vertical incision providing more room is made by thrusting the knife, with the cutting edge upwards, into the trachea at the lower edge of the wound, and carrying the cut upwards through three or four tracheal rings (fig. 88, b). The hooks are now introduced into the trachea, or the index and middle fingers of the left hand hold the edges of the wound apart, and the canula is inserted. The trachea in the horse being of considerable size, little difficulty is met with, even when dealing: with the firm textures of old subjects.

When the canula is to be worn for a considerable period, and must, therefore, be removed every few days to be cleaned, a square, oval, or occasionally a round piece is excised from the anterior wall of the airtube. Viborg, who first recommended the square opening (fig. 88, a), directs the knife to be inserted between two cartilages, and a horizontal cut made about $\frac{3}{4}$ inch in length. This cut should extend an equal distance on either side of the middle line. From each of its ends a vertical cut is next made downwards, and the piece of cartilage which now hangs by the intercartilaginous band below is grasped with dissecting forceps and cut away, care being taken to prevent its falling into the trachea. The canula is then placed in position. Lafosse, Brogniez, and Günther have expressed themselves in favour of the oval opening, which may be produced by first dividing the intercartilaginous ligament and 
then excising a half-round piece from the cartilages above and below, without, however, quite dividing these (fig. 88, c). Bending of the tracheal rings and stenosis of the trachea, which result from completely cutting through the cartilages, are thus avoided. Brogniez's broncho= tome likewise produces an oval cut, but can be replaced by a bistoury, deftly manipulated. The same remark applies to other instruments,

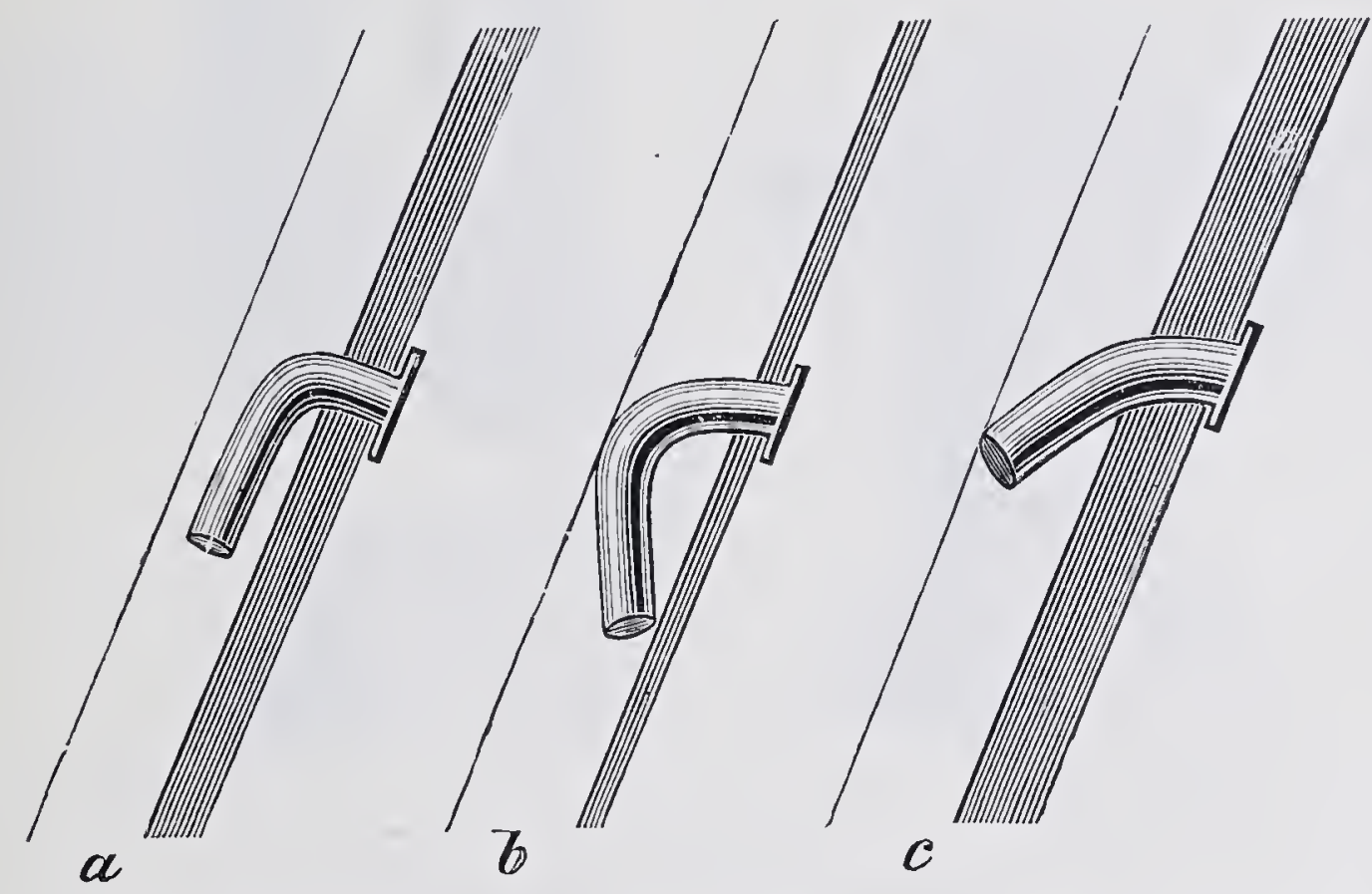

Fig. 90.-Position of tracheal canula. $\alpha$, Normal ; $b$, when too much ourved; $c$, when insufficiently curved.

such as Marty's tracheotome, which cuts a circular piece from the two cartilaginous rings, like a trephine.

Tracheotomy is one of the simpler of equine operations, and is easily carried out with a little care. Its success depends greatly on the canula used.

Many of those recommended can only be employed in connection with their appropriate tracheotomes, and, therefore, may be disregarded. The oldest (Barthélemy's) consists of a metal tube, 4 to 8 inches in length and $\frac{3}{4}$ to 2 inches in width, bent at one end, and provided with a plate to prevent its entering the trachea too far. The edges of the plate and those of the lower end of the tube must be carefully rounded off to prevent their abrading the skin or mucous membrane. The tube, while nearly filling the tracheal space, must not press on the mucous membrane, nor must the soft tissues of the neck be pinched. The bend must be confined to the upper end, and must be suited to the thickness of the parts between the skin and front wall of the trachea. Where the bend is too short, not only will the skin and muscular tissues be bruised, but the lower end of the tube will press against the posterior wall of the trachea and produce inflammation with proliferations, leading to stenosis (fig. 90, c). Where the bent portion is too long, there is undue space between the plate and the skin, and if such a tube be pressed home by tapes or straps, it becomes displaced and liable to injure both walls of the trachea (fig. 90, b). Tracheotomy tubes, formerly made of tin plate, are now much better manufactured of German silver.

The objection to Barthélemy's canula, viz., that it falls out easily, may partially be obviated by straps (with buckles), fastened in the eyes of the plate and passed transversely round the neck. They should not be carried 
tor far forward, as the tube is then liable to fall out during coughing or violent expiratory movements. Barthélemy's tube somewhat disfigures the animal; expectoration of mucous, pus, or blood is rendered more difficult; and where

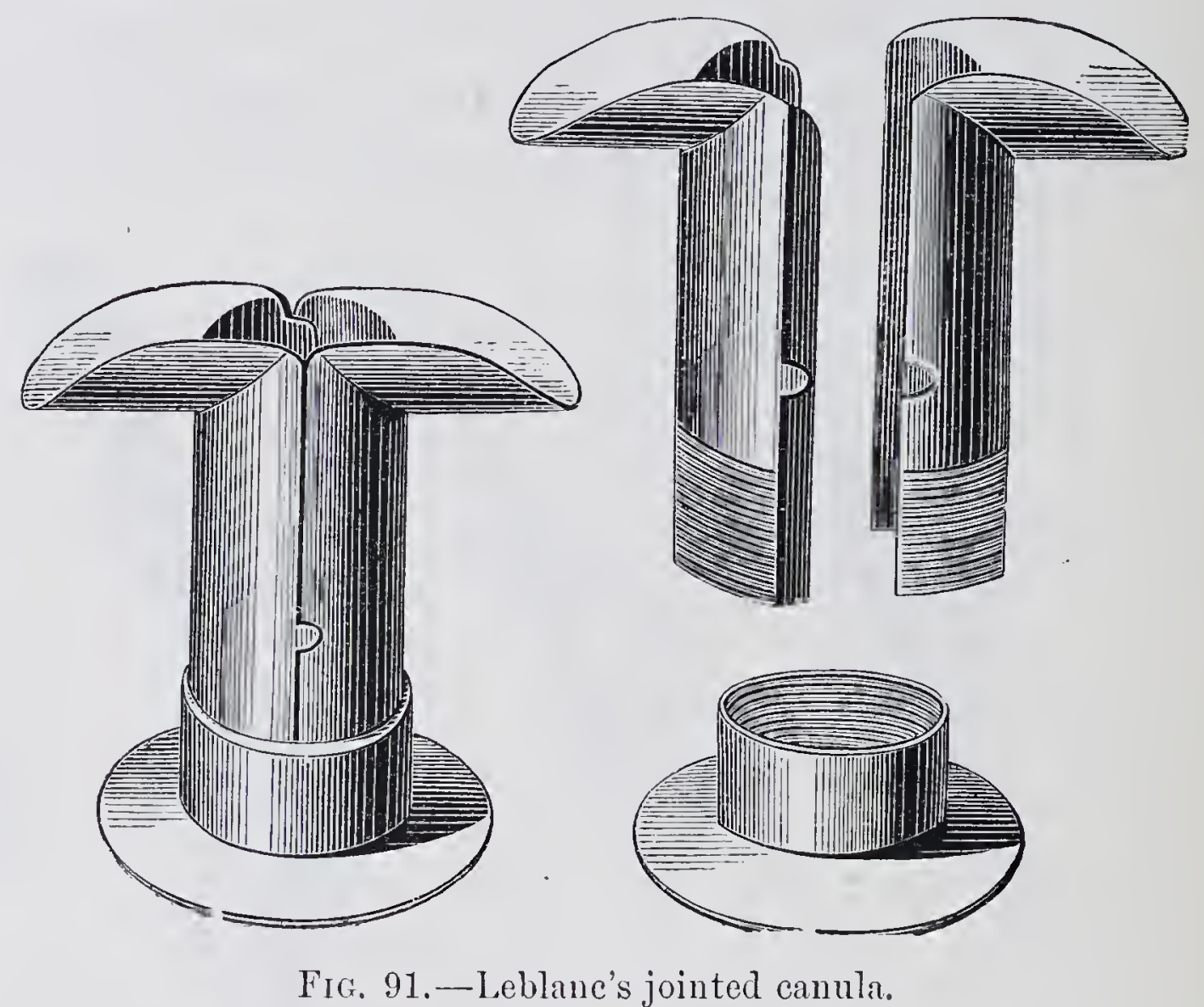

used for long periods very careful fitting is necessary to prevent thickening and narrowing of the trachea. This tube is therefore now seldom used. To overcome the above disadvantages models have been designed by Leblanc, Degive, Vachetta, Peuch, Field, Coleman, Jones, and others. Doing

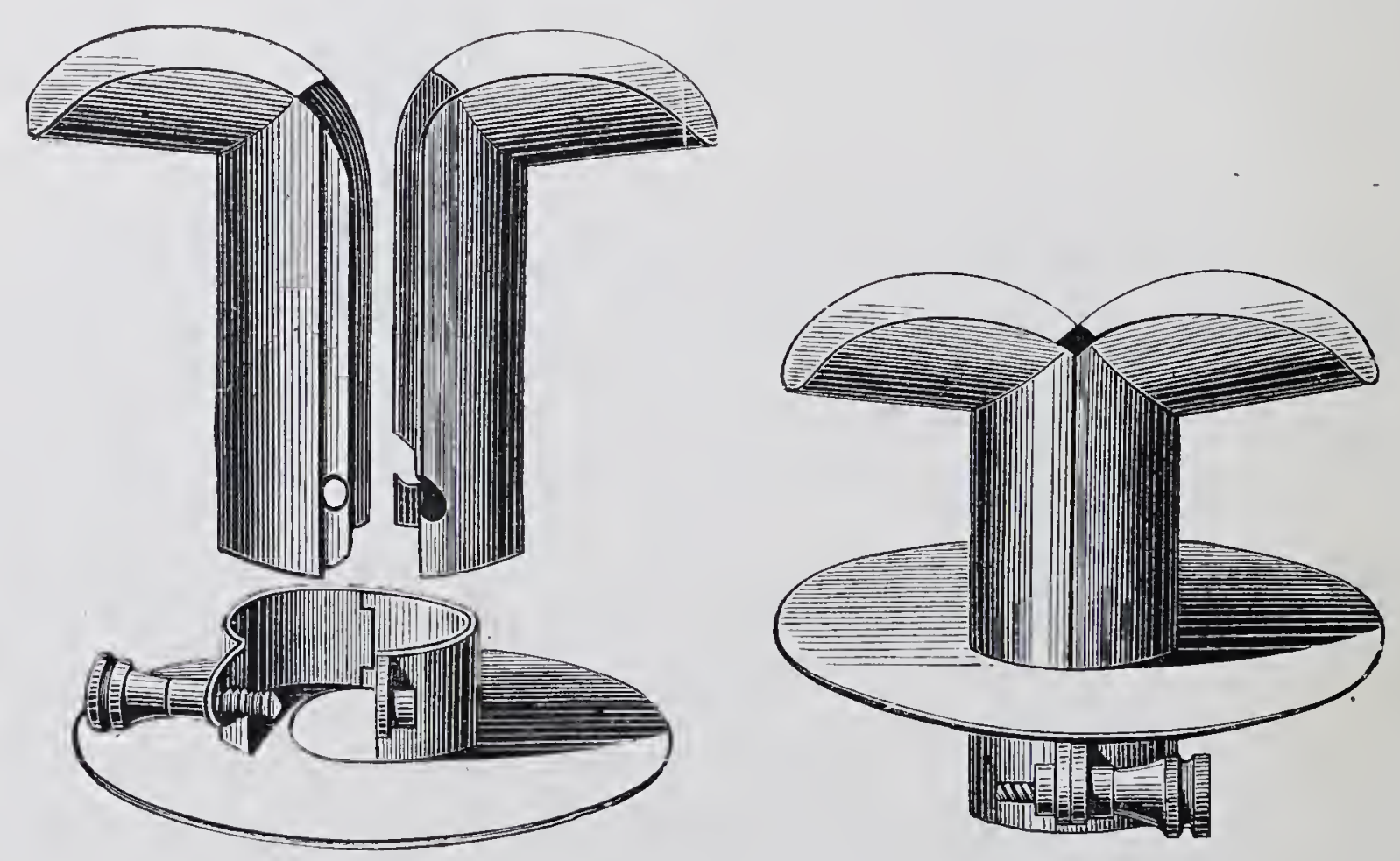

FIG. 92. - Leblanc's jointed canula.

away with the straps lessens disfigurement without interfering with the secure fixing of the canula, and diminishes risk of bruising and irritation. The trachea remains unobstructed in an upward direction as far as the larynx, whilst the canula is very easily cleaned. Leblanc has constructed a jointed tube said to be adapted to every form of neck (figs. 91 and 92), but one of the halves sometimes becomes detached and drops into the trachea. Modern 
experience shows that the tracheal tubes of Field, Peuch, Jones, and Coleman, which correspond in principle, are amongst the best (figs. 93 to 95). They consist of two or three pieces, which are inserted singly by first pushing the wider tube into the trachea and afterwards the narrower one, so that the projections point upwards and downwards in the lumen of the trachea without pressing on its mucous membrane. Provided it is sufficiently long to enter easily, the tube with the shortest projections is to be preferred, because its hold is more
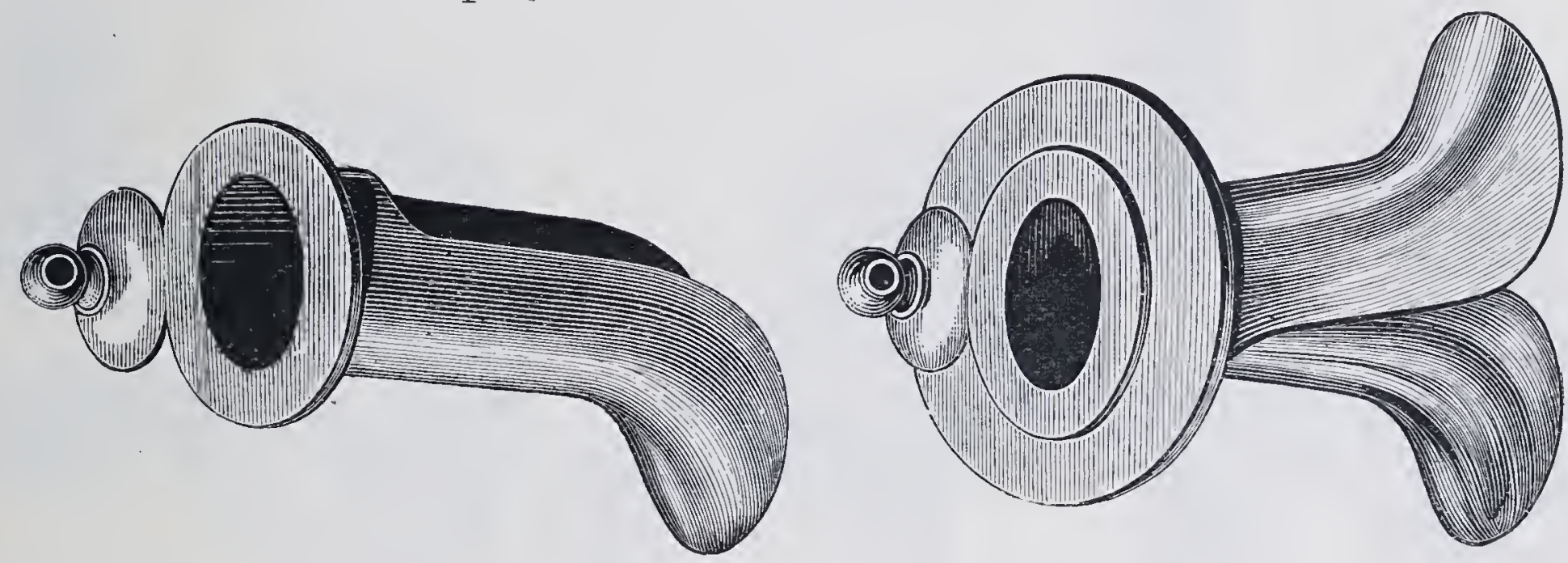

Fic. 93.-Peuch's canula,

secure and it is less liable to injure the posterior wall of the trachea. Naturally, the length of the canula, that is, the distance between the plate and the curvature of the projections, must correspond to the thickness of the anterior wall of the neck. Such tubes may be worn by horses for several years without

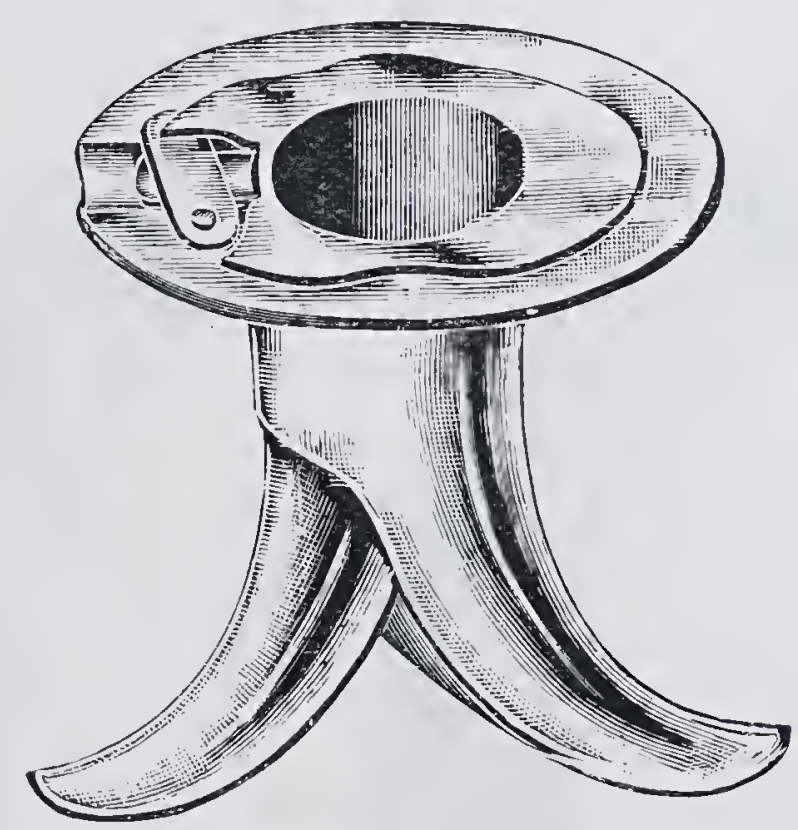

Fra. 94.-Field's tracheotomy tube.

producing stenosis or difficulty in application. Hauptner has recently somewhat modified Peuch's tube. The inner portion is made lighter and can be more easily introduced; but, on the other hand, it has not so wide a basis, and, therefore, more easily produces proliferations. To introduce the tube the index finger of the left hand should first be passed into the trachea, when the tube itself may easily be slid along it, the finger acting as a guide. Afterwards the second portion may be inserted.

Field's tube is largely used in England, and is convenient to insert and withdraw as well as to clean. The shields and half tubes should, however, be either in one piece or be very secureiy united in order to prevent accidents. 
Coleman's tube is also to be recommended as light, simple, and convenient. The parts are very firmly united when the tube is in position, and there is less danger of their being displaced by the patient than in the case of Field's tube.

Canulæ must be light and durable, and their edges, as already stated, well
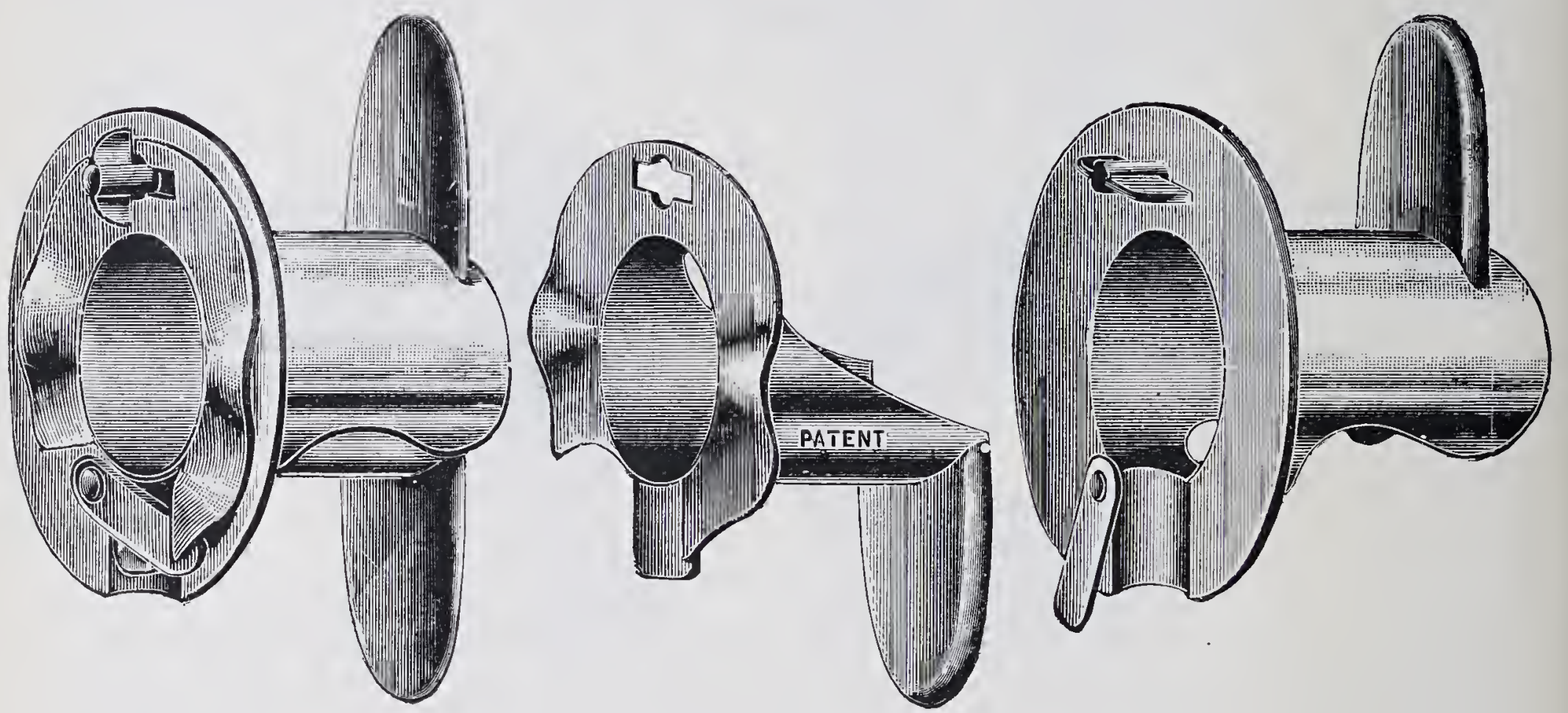

FIG. 95.-Coleman's tracheotoiny tube.

rounded off. They are usually made of German silver, but their weight may be reduced by using aluminium, which has been employed in making canulæ on Peuch's model. These seem to be well suited for race-horses. Where it
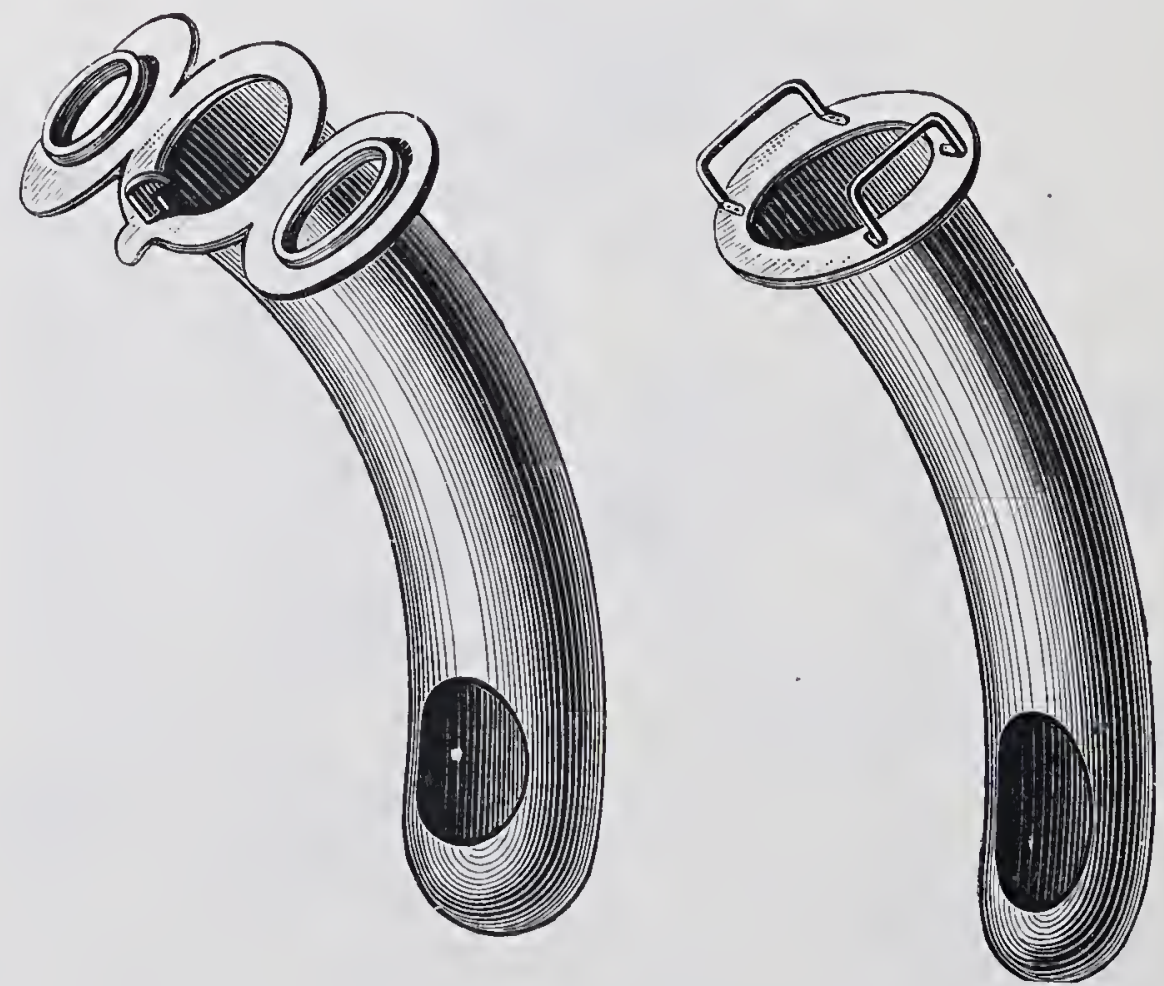

Fig. 96.-Donble-tube canula.

is necessary to combat suffocation occurring in diseases of an infectious character, a canula of a long form is selected, in order to avoid bruising the swollen soft parts in the neighbourhood of the point of operation. In anticipation of swelling supervening, the steadying of the canula may be effected by placing one or more leather shields below the plate, or enveloping the projecting portion of the tube in jute 
The tube gradually becomes stopped by secretion from the mucous membrane and the operation wound, and must, therefore, be frequently cleansed, at first, indeed, daily. As the secretion diminishes, cleansing is required at intervals of three or four days. Hence it is well to have two similar canulæ, so that whilst one is in use, the other can be cleansed or, if need be, repaired. Such precautions are necessary, because the wound contracts very rapidly; indeed, after the lapse of an hour or two the introduction of the canula may be difficult, or even impossible. Whilst the horse is in the stable, the opening of the instrument may be stopped up with a cork, which will check secretion and blocking of the tube. Double tubes have been recommended to obviate the necessity for changing the canula. They are generally used in man (fig. 96). Whilst the outer tube lies in the trachea, the inner can be cleaned from time to time. But this form, shown in fig. 96, must be condemned, because the curvature of the upper part is too slight, and that of the lower part too great; the lower portion, as already stated, should be quite straight. In double tubes this curvature cannot be avoided; hence they are unsuitable, and can only be used where the trachea is very near the surface. This model also possesses another fault-its opening lies at the side. Injuries from its lower border are certainly thus avoided, but the lateral opening coming in contact with the mucous membrane, the passage of air is interfered with.

To obviate the necessity for a canula, Strauss recommends cutting out an oval piece from the trachea, with precautions against injuring the mucous membrane, which is sutured to the skin. But Hering doubts whether this operation can be successfully done, and Möller's experience tends in the same direction. In the absence of a canula, hooks may be introduced on either side into the tracheal opening, and held apart by tapes passed round the animal's neck.

As a temporary expedient, the neck of a bottle, or a pair of strong wires bent to a spoon-handle shape, may be inserted. To save animals from threatened suffocation, there is often insufficient time to proceed according to rule. Most practitioners have on occasion been forced to operate in a dark stable, and, furnished merely with a bistoury, to find the middle line of the neck as they best could, and insert a tube. Despite these difficulties, the parts usually heal well.

Krieshaber has recommended an operation under the title of "trachéotomie sous-cricoidienne," in which an incision is made immediately under the cricoid cartilage; the crico-tracheal ligament, which is about 1 inch in breadth, divided, and a double-limbed canula introduced. The position of this ligament is easily discovered by palpation, especially in horses, where the muscles of the neck are not greatly developed. With the head extended, the operation is not difficult in quiet animals. This method has the advantage of only slightly impairing the animal's appearance, and facilitates direct examination and treatment of the larynx, while the action of the tube, moreover, widens the larynx, and it is said that, after wearing the canula for a time, animals sometimes cease roaring altogether. This may perhaps result from the irritative processes produced by the canula causing anchylosis of the articulation of the arytenoid cartilage on the paralysed side. Bouley fears that horses thus operated 
on may injure the larynx by violently bending the head. This seems doubtful, provided the canula fits well to the neck.

The evil results of tracheotomy comprise-

(1) Mechanical pneumonia, produced by aspiration of inflammatory

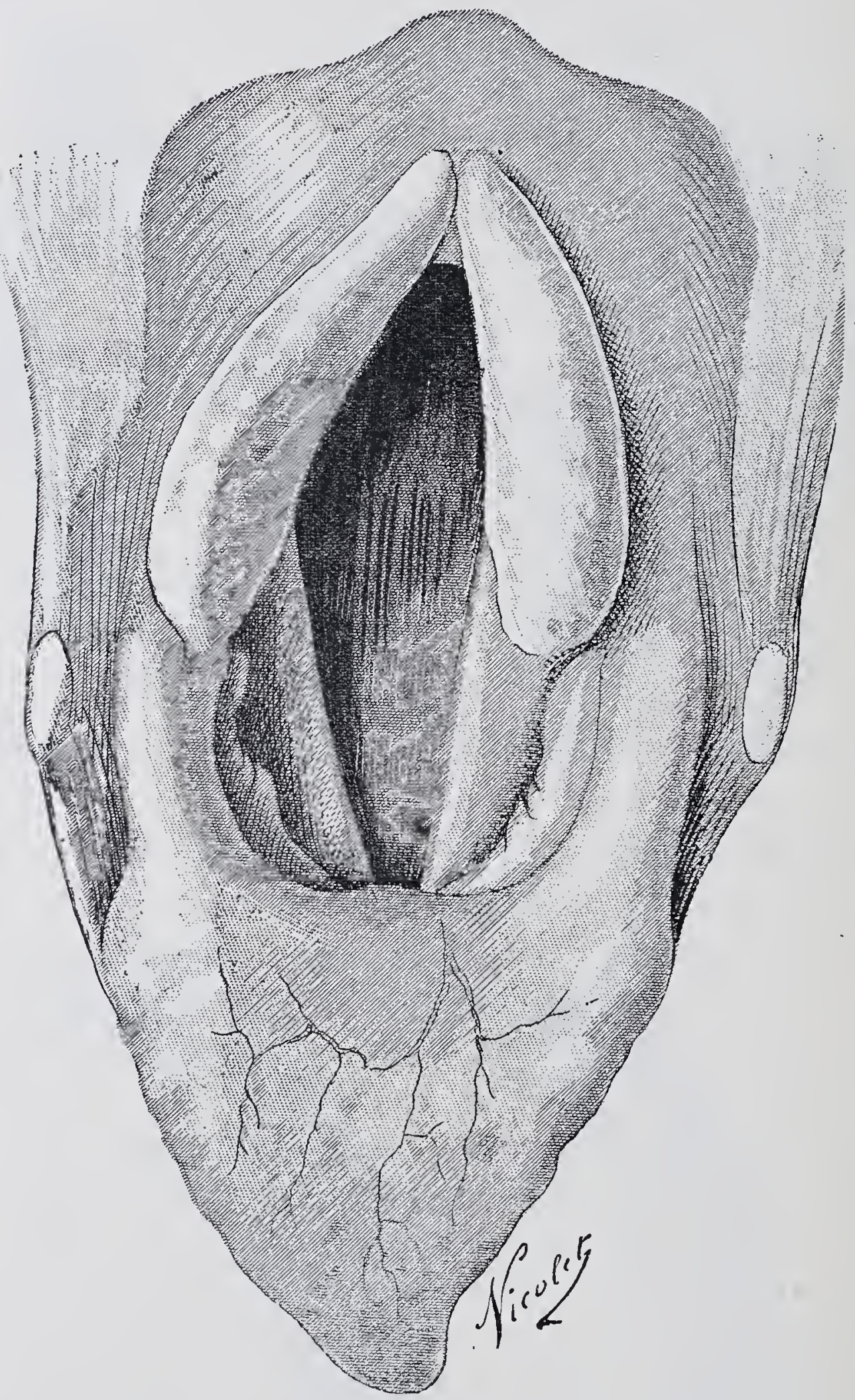

Fig. 97. - Superior orifice of the larynx and glottis of a horse affected with chronic roaring.

secretions in such diseases as petechial fever and acute laryngitis, or from abscesses breaking into the pharynx or trachea. These complications are prevented by the use of the tampon-canula, made on the pattern of Trendelenburg and Hahn's human canula. The former carries an indiarubber balloon, which, after being placed in position, may be inflated with a pair of bellows (fig. 105). Where foreign materials are present in the trachea, they should, as far as possible, be removed with a feather, 
a soft bottle-brush, or a sponge carefully fastened to a wire. Such implements may without danger be passed as far as the division of the trachea. The tampon-canula may then be placed in position and moderately inflated, that is to say, until the balloon fills the lumen of the trachea without exercising much pressure on the mucous membrane, which might produce necrosis. Another pattern is Hahn's tamponcanula, which carries a sponge tent. A substitute may be improvised by wrapping Barthélemy's canula with tow, jute, or sponge, such substances being carefully secured with string or thread, so that they shall not become loose and fall into the trachea.

(2) Proliferation and thickening of the mucous membrane with narrowing of the lumen of the trachea (tracheal stenosis). Sclerotic inflammations occasionally occur in the skin and the soft tissues lying beneath, and usually follow cellulitis. Proliferation producing tracheal stenosis results from faulty formation of the canula, from its being too heavy, badly made, or not fitting the form of the neck. The canula cannot fit well if it be too long, too narrow, too little, or too much bent, or if the opening in the trachea be too large. A pronounced tendency to such growths often exists in heavy horses, and though the canula is perfect in every respect, the contraction recurs after each operation. Such stenosis gradually renders the introduction of the tube more and more difficult, and finally impossible. The tracheal opening must then be increased, a procedure sometimes rendered-difficult by ossification of the newly-formed materials. Where there is room, a second opening may be made lower down. In view of such a contingency, tracheotomy, especially in young horses, should be performed in the upper third of the neck.

\section{(6.) LARYNGOTOMY. LARYNGO-FISSURE.}

Section of the larynx in man has lately been much employed, particularly for the removal of tumours. As already stated, such growths in the larynx are rare in animals. But the operation has been practised on horses in hemiplegia of the larynx, otherwise termed roaring:

K. Günther, who made the first experiments, hesitated to divide the cricoid cartilage, because he feared stenosis, and therefore operated from the trachea. Stockfleth later recommended dividing the cricoid cartilage, thus introducing larnygotomy into veterinary practice. Thyroidotomy and cricotomy are now distinctive terms, designating respectively division in the median line of the thyroid and cricoid cartilages. Where the upper rings of the trachea are also divided, the operation is styled cricotracheotomy; while the division of the cricoid and thyroid cartilages, together with the connecting crico-thyroid ligament, is entitled cricothyroidotomy. 
Crico-tracheotomy, as adopted in the treatment of hemiplegia laryngis, was fully described in the brochure published by Möller in 1888.

The animal is chloroformed and placed on its back; the operator kneels on the right side of the neck, shaves the hair from the larynx and upper portion of the trachea, and disinfects the site of operation. The position of the cricoid cartilage is easily determined by palpation. An incision, exactly in the middle line, is made through the skin, extending from the body of the cricoid cartilage as far as the first two or three rings of the trachea. After ligaturing any bleeding vessels, the muscles lying below the skin are divided exactly in the middle line. The trachea and cricoid cartilage are thus exposed with scarcely any bleeding, but any vessel spurting must at once be ligatured. A

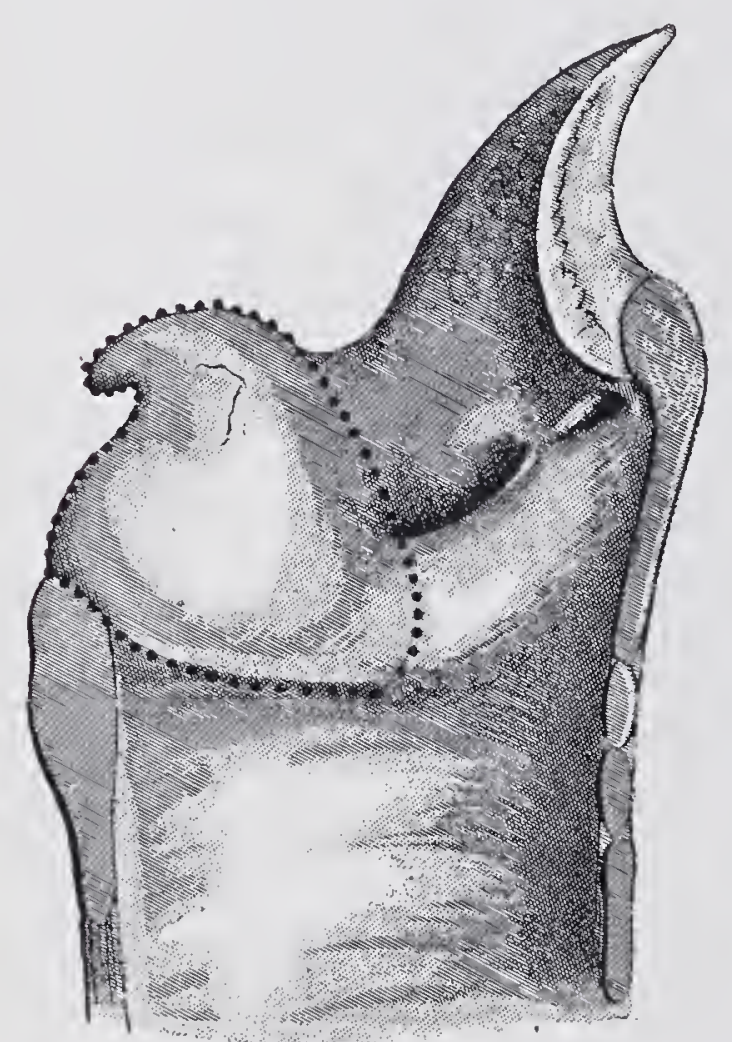

Fič. 98.-Möller's operation. Tertical and antero-posterior section of the larynx. The dotted line represents the mucous membrane covering the edges of the arytenoid cartilage. tampon-canula (Trendelenberg canula) provided with a bellows is held in readiness. An incision is made through the first two rings of the trachea with a pointed bistoury, turning the cutting edge towards the animal's head, and extending the opening by carrying the knife up to the thyroid cartilage. In case of vessels bleeding at this stage, the tampon-canula should be inserted, inflated with air, and the vessels ligatured. Entrance of blood into the trachea must in any case be prevented. The wound is now held open with a pair of blunt hooks or retractors, and after removal of any blood, the interior of the larynx can be seen.

Where tumours have to be excised, little difficulty is encountered, if their bases are not broad. Resection of the vocal cords has already been described. Where there is paralysis of the recurrent, the arytenoid cartilage on the paralysed side is removed. While Günther and Stockfleth practised partial resection, Möller proposed total removal of this cartilage, and still prefers this method. With a specially-constructed scalpel (fig. 99), the mucous membrane at the periphery of the arytenoid cartilage is divided. Beginning at the point of union of the arytenoid cartilages, the knife, carried through the mucous membrane and the interarytenoid ligament in an upward direction, close beside the middle line, follows the posterior border of the arytenoid cartilage upwards as far as the vocal process (in fig. 100 the dotted line shows the course of the incision). The vocal cord is divided with scissors at its point of union with 
the arytenoid, and the cartilage separated from the muscles covering: its outer surface with the fingers, scissors, or scalpel. By reeping close to the cartilage the bleeding is slight. The mucous membrane is then divided from the anterior upper border of the arytenoid cartilage, care being taken to spare it as much as possible. For this purpose the index finger of the left hand is introduced into the sacculus

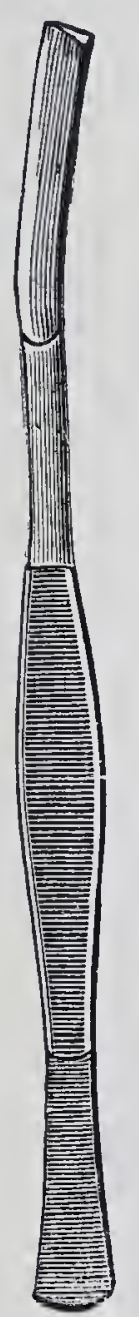

Fig. 99.Scalpel for resection of arytenoid.

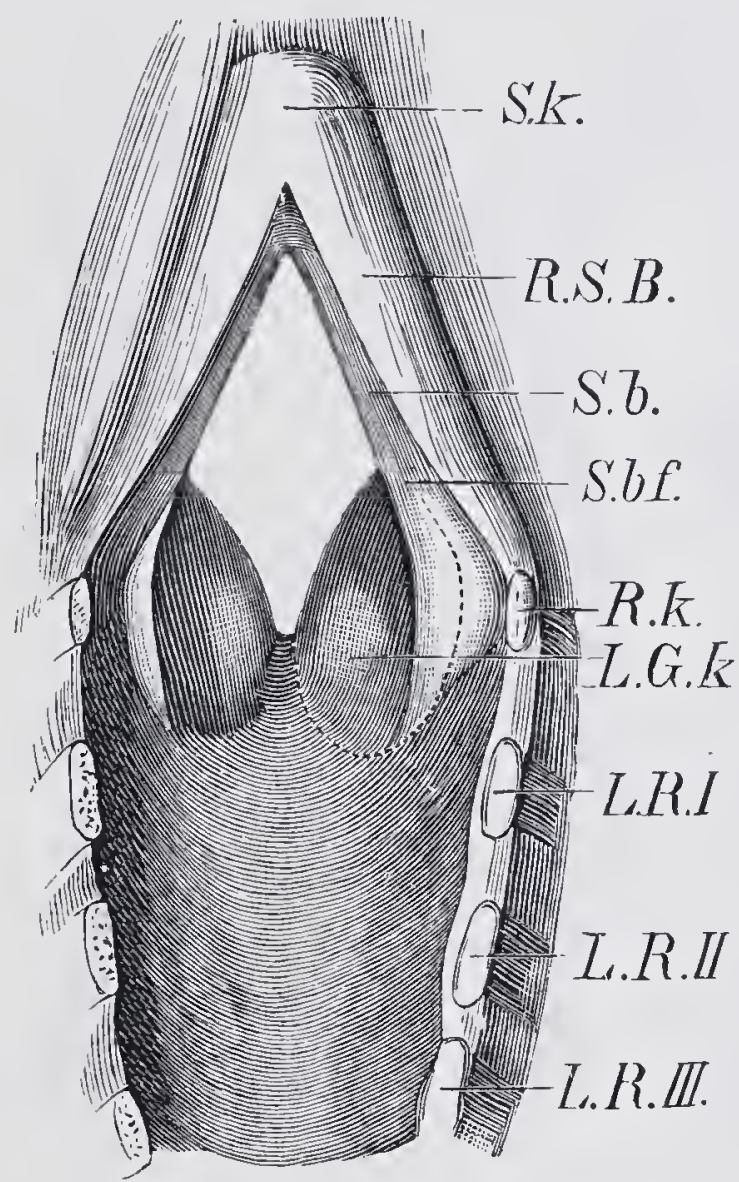

Fig. 100.-Course of incision.

Horse's larynx (seen from below). Sk., Thyroid cartilage; R.S.B., cricothyroid ligament; $S . b$. , vocal cord ; S.lf., vocal process of arytenoid; R.K., cricoid cartilage; L.G.K., left arytenoid; L.R. (I., II., III.), first, second, and third rings of the trachea.

laryngis, and the membrane cut through with scissors close to the border of the cartilage. In this procedure one is specially guided by the indications afforded by the left hand. The arytenoid cartilage is now cut through with the scalpel, as near as possible to its articular surface, so that only a thin fragment of cartilage remains. This portion of the operation is the most difficult; especially in old horses, where the articulatory portion of the cartilage is often ossified. The knife must be inserted within and below, and the cut made upwards and outwards. As considerable force is often necessary in dividing the cartilage, it requires care to prevent the knife slipping off unexpectedly. Although frequently performing the operation, Möller never, however, had an accident of this kind. As soon as the arytenoid cartilage is divided it may be easily freed from the neighbouring soft parts, and removed with the help of a long and sharply-bent pair of scissors. In this case, also, 
it is necessary to follow carefully the borders of the cartilage, holding the latter with a pair of lion forceps (fig. 102). Throughout the operation the larynx must be cleared of blood by the use of small sponges fastened to thin sticks, and wielded by an assistant; and in the same way the field of operation must be cleared after removal of the arytenoid itself. Bleeding is usually slight, though in rare cases a spurting vessel may require to be closed by torsion. After bleeding is somewhat checked, the surface of the wound is swabbed with 10 per cent. chloride of zinc

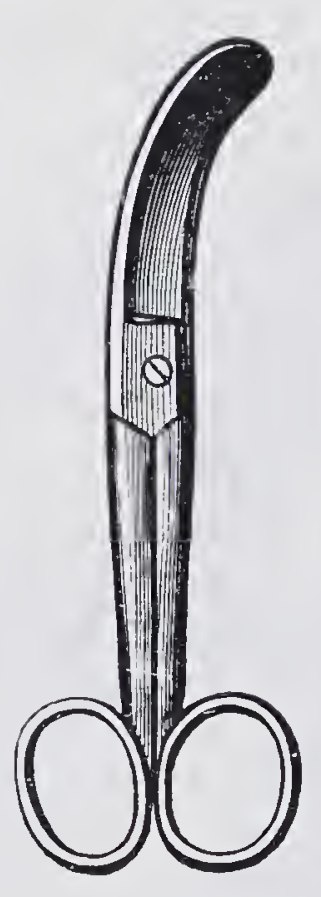

FIG. 101.-Curved scissors.

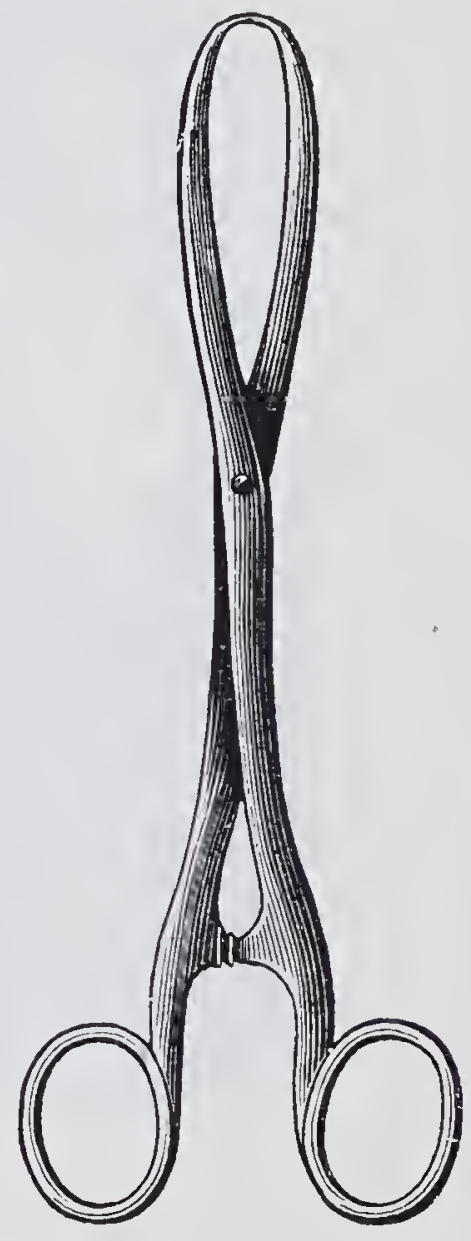

Frg. 102.-Forceps for grasping the arytenoid.

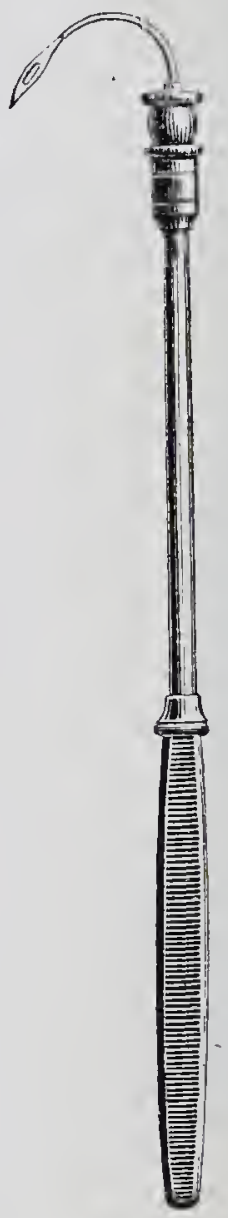

FIG. 103.-Curved needle for suturing laryngeal mucous membrane.

solution applied with a sponge, and is then powdered with 1 of iodoform to 3 of tannin.

More recently Möller attempted to stitch the mucous membrane, using a curved needle (fig. 103). It can be very well managed after a little practice. The needle, threaded with catgut, is first passed through the upper fold of the membrane, which is then drawn downwards and fastened to the under border of the wound. Silk should be avoided, as in the larynx it remains unabsorbed for months. Three sutures usually suffice to fix the membrane in position, to assist cicatrisation, and prevent the loose plicæ aryepiglotticæ interfering with breathing after healing. Removal of the vocal cord is unnecessary and injurious. A tampon properly fitting the larynx, or a Trendelenberg canula, is introduced and adjusted. To secure the tampon and tube a couple of temporary sutures are passed through the skin, and two pieces of tape over the seat of operation and round the neck. 
The horse is now placed on its side and the hobbles removed; while, to prevent straw or other foreign bodies being inspired through the tracheal tube, a clean cloth should be laid under the neck. The animal is allowed to lie until it rises of its own accord, when it should be placed in a box, receive only soft hay and water, and not be tied up. It is unnecessary to remove the tube and tampon after twenty-four hours, as was formerly done ; both may remain in position three to four days without bad conse-

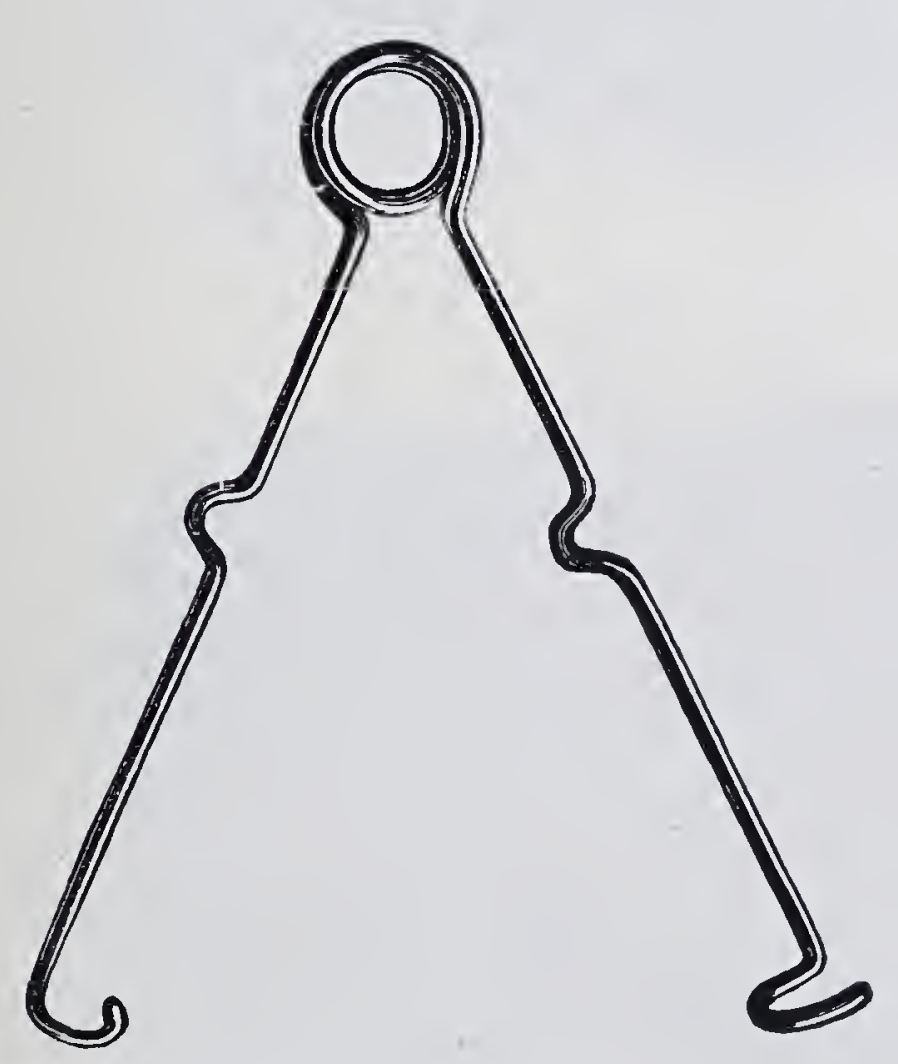

FIG. 104.-Spring hooks.

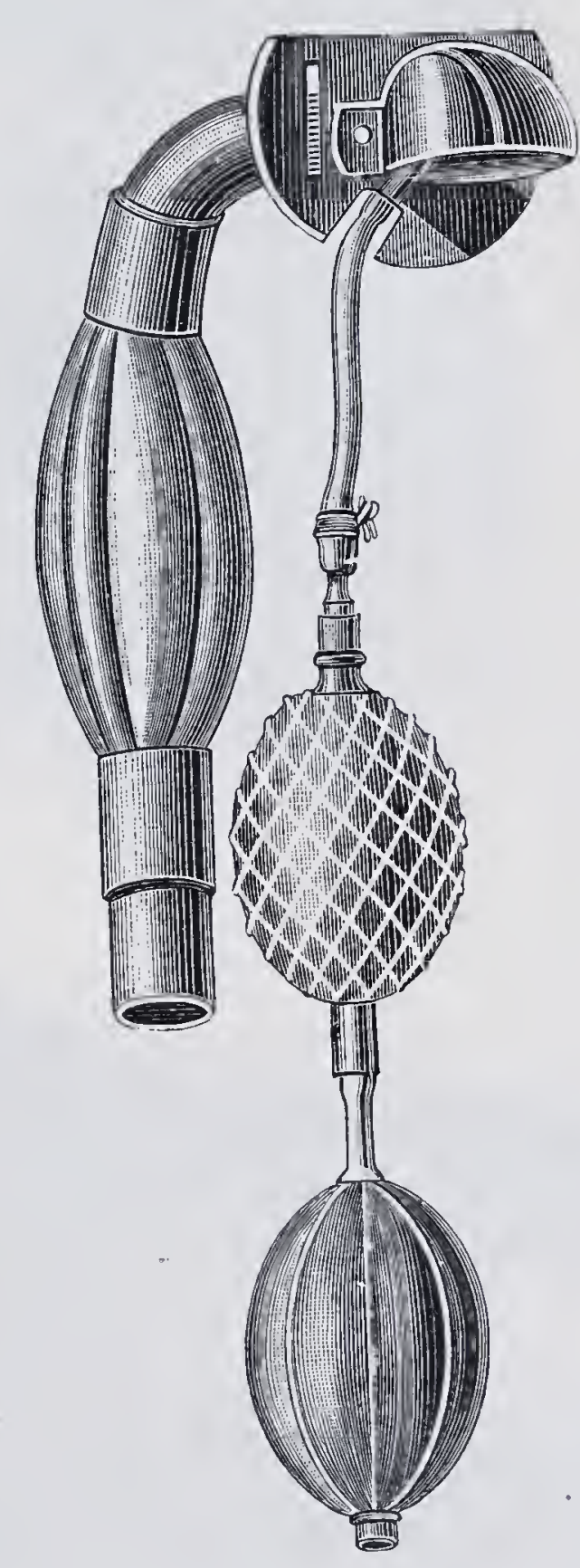

FIG. 10\%.-Trendelenberg canula.

quences, provided no marked fever results. This method, indeed, is to be preferred. Any discharge may be soaked up with a piece of sponge, some wadding or wood wool, and the wound, if dry, moistened with carbolic lotion. Drinking water should be kept constantly in the box, and given in a pail placed rather low or on the ground. The diet should consist of soft hay and a small quantity of oats mixed with bran.

On the fourth day the surface of the wound is carefully cleansed, and the stitches in the skin loosened. The tampon can then be removed, and the larynx swabbed out with small sponges wrung out of carbolic solution; but the sponges must be nearly dry to prevent fluid running V.S. 
down the trachea. Any secretion or blood found above the tube must be carefully soaked up. The canula is then removed, and the animal's head
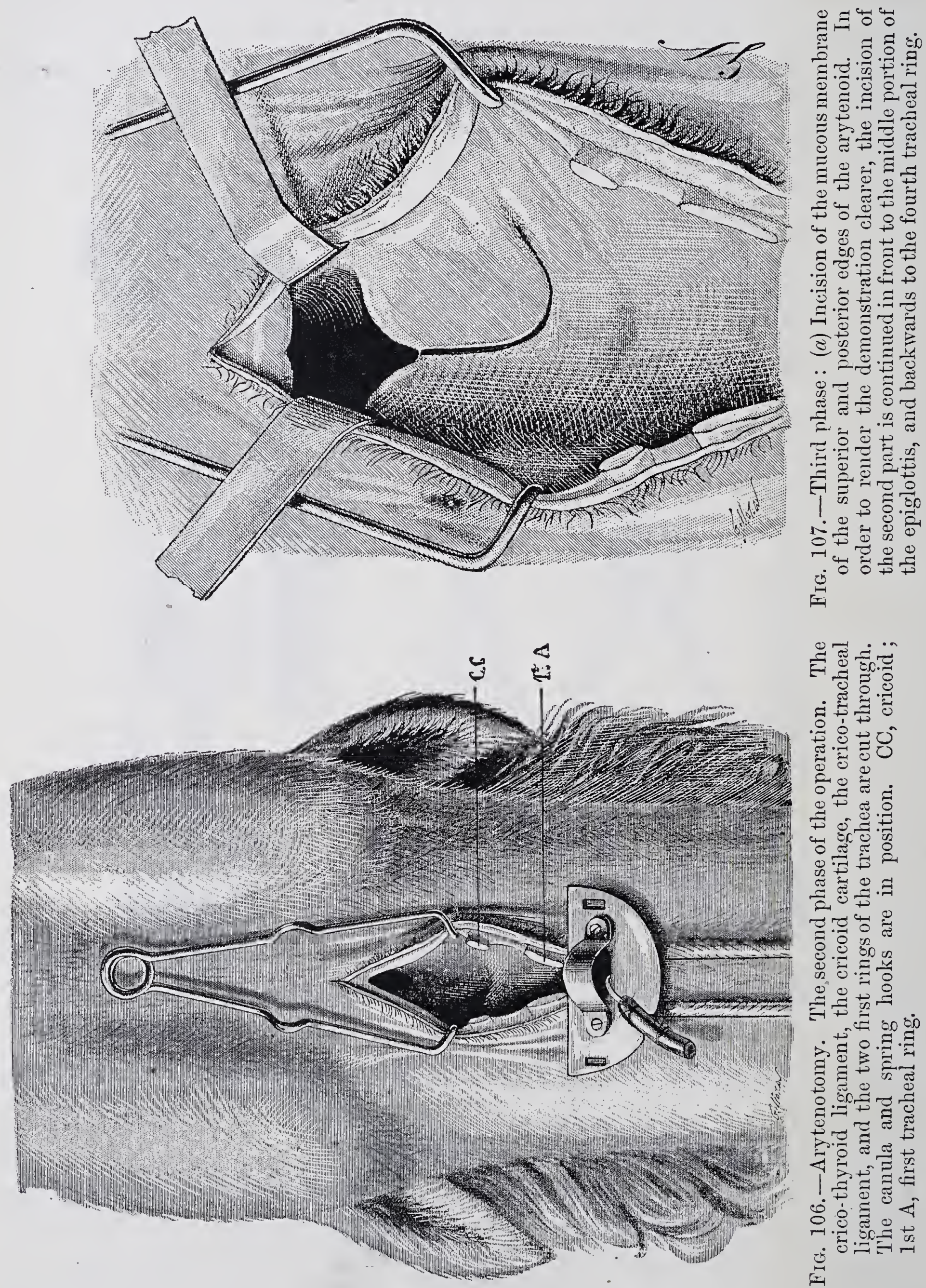

tied up high. After cleansing the larynx several times, fresh water may be given from a pail held about 2 feet from the ground, and note taken whether much water returns through the nose. If in drinking, say a 
quarter of a pailful, not more than a half-pint returns by the nose, the tube may be discarded. On the other hand, if fluid flows freely from the
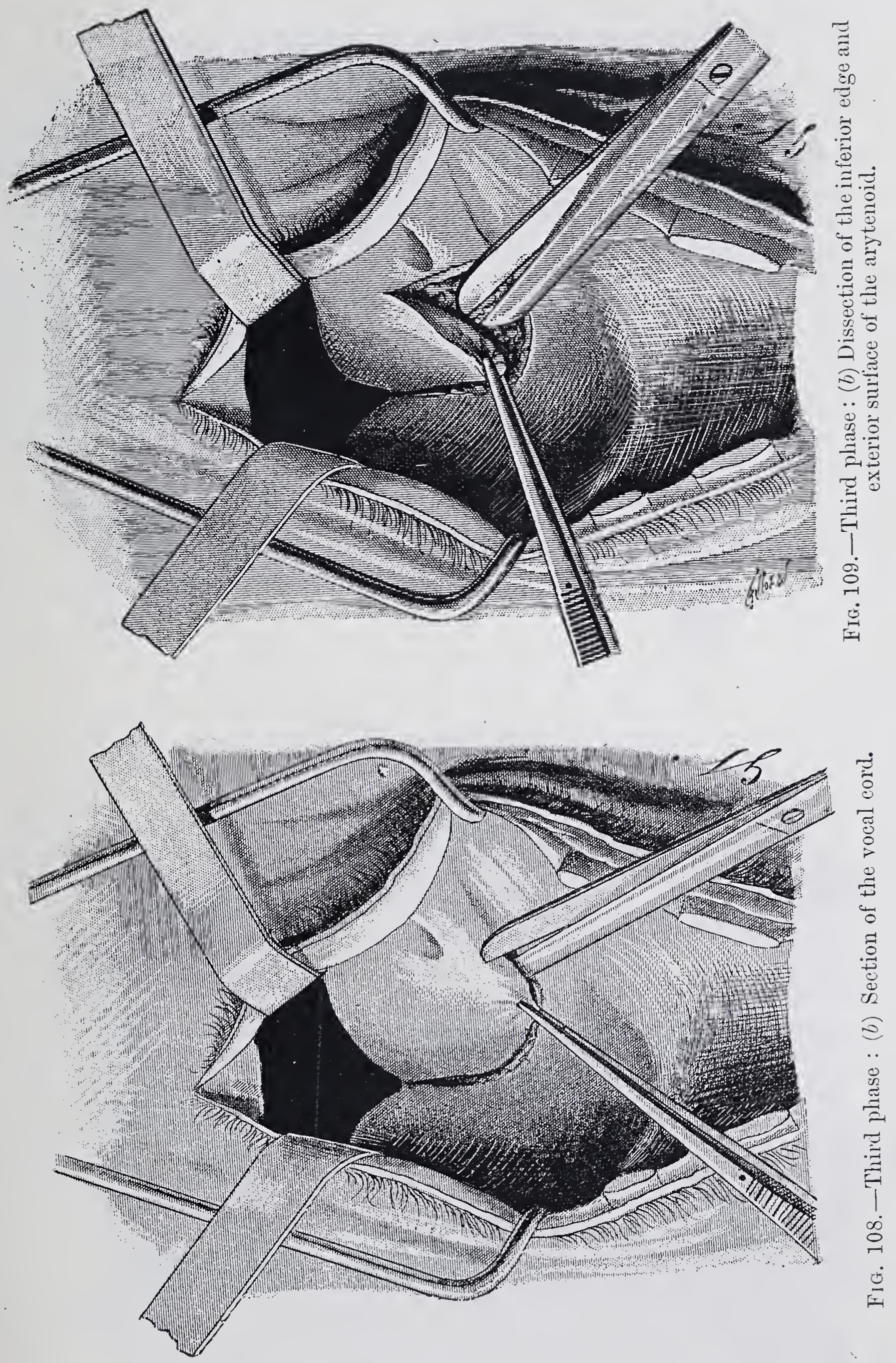

nostrils, or if dyspncea appear after the tube is removed, the latter must be replaced. The skin wound should be cleansed from discharge daily until a dry scab forms, when, unless such complications as fever and 
dyspnœa appear, neither skin wound nor larynx will require treatment. The wound, as a rule, after eight days requires no special attention. The
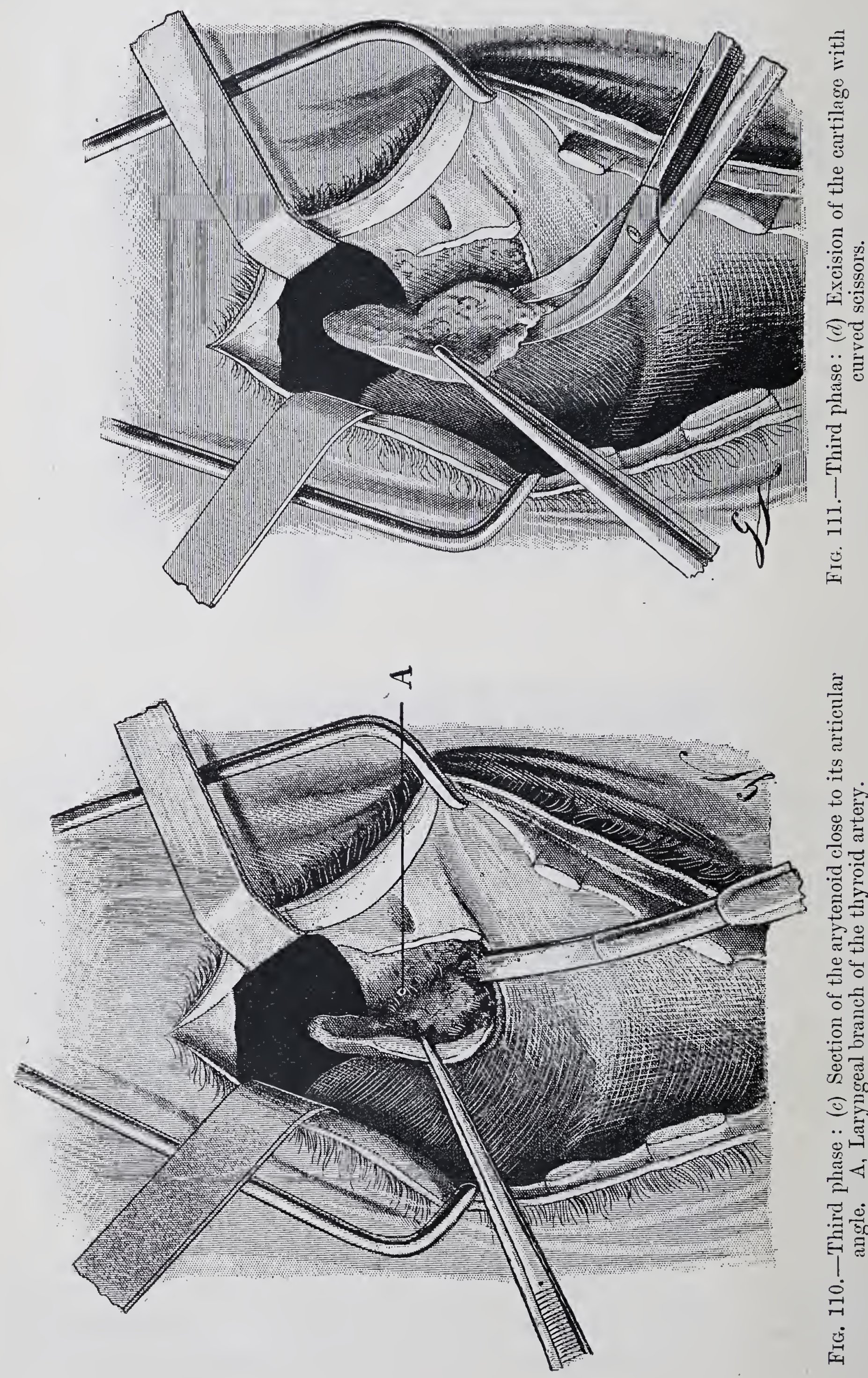

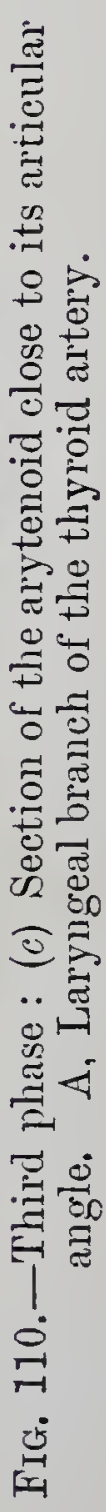


horse, however, should still be confined in a well-ventilated box, and receive easily-digestible food.
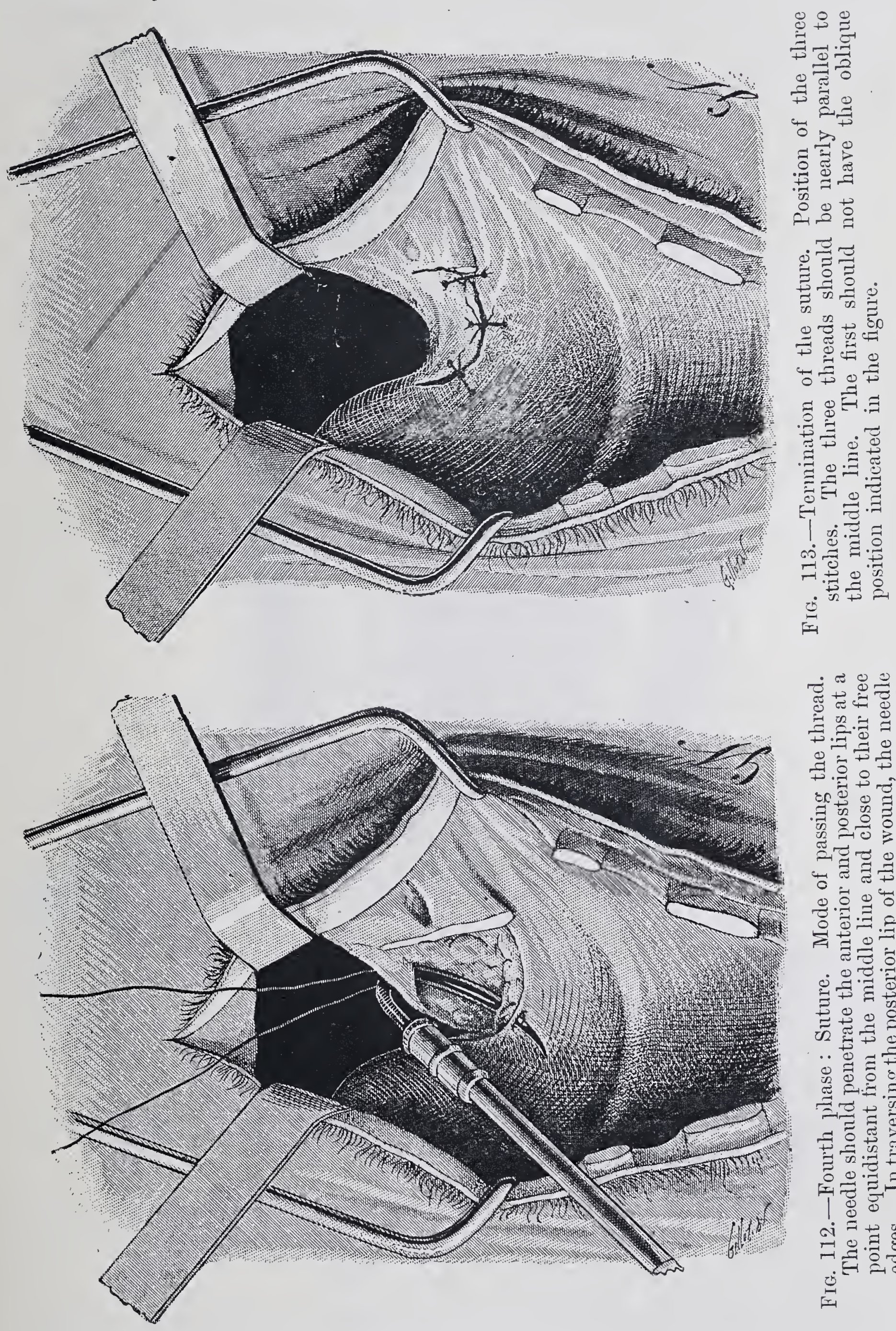

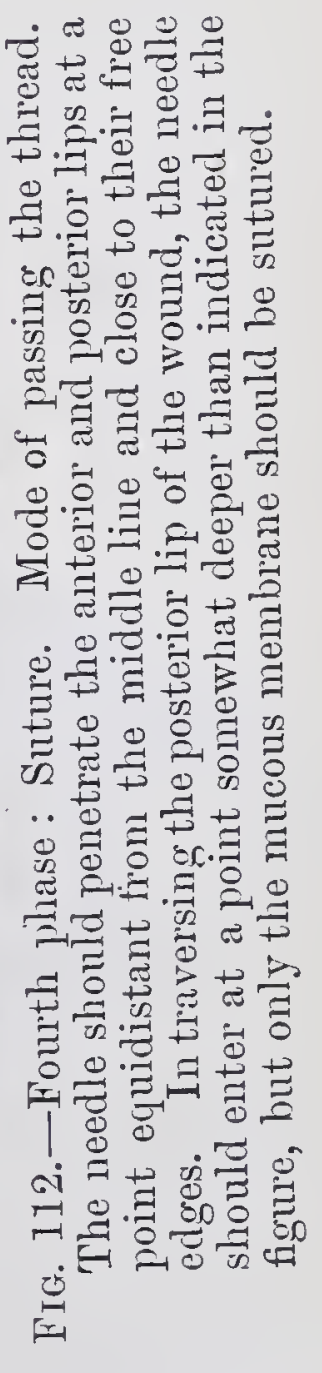

The external wound usually closes completely in three to four weeks. It leaves a cicatrix, which contracts so far in five to six months that even an expert has difficulty in discovering it. In about four weeks after the 
operation, provided the weather be favourable, the animal may be exercised daily, though for another month it must be liept from work of every description. After this time it may be ridden or driven until breathing becomes audible; but, as the forcible entrance of air stretches the cicatricial tissue, and may endanger the success of the operation, it

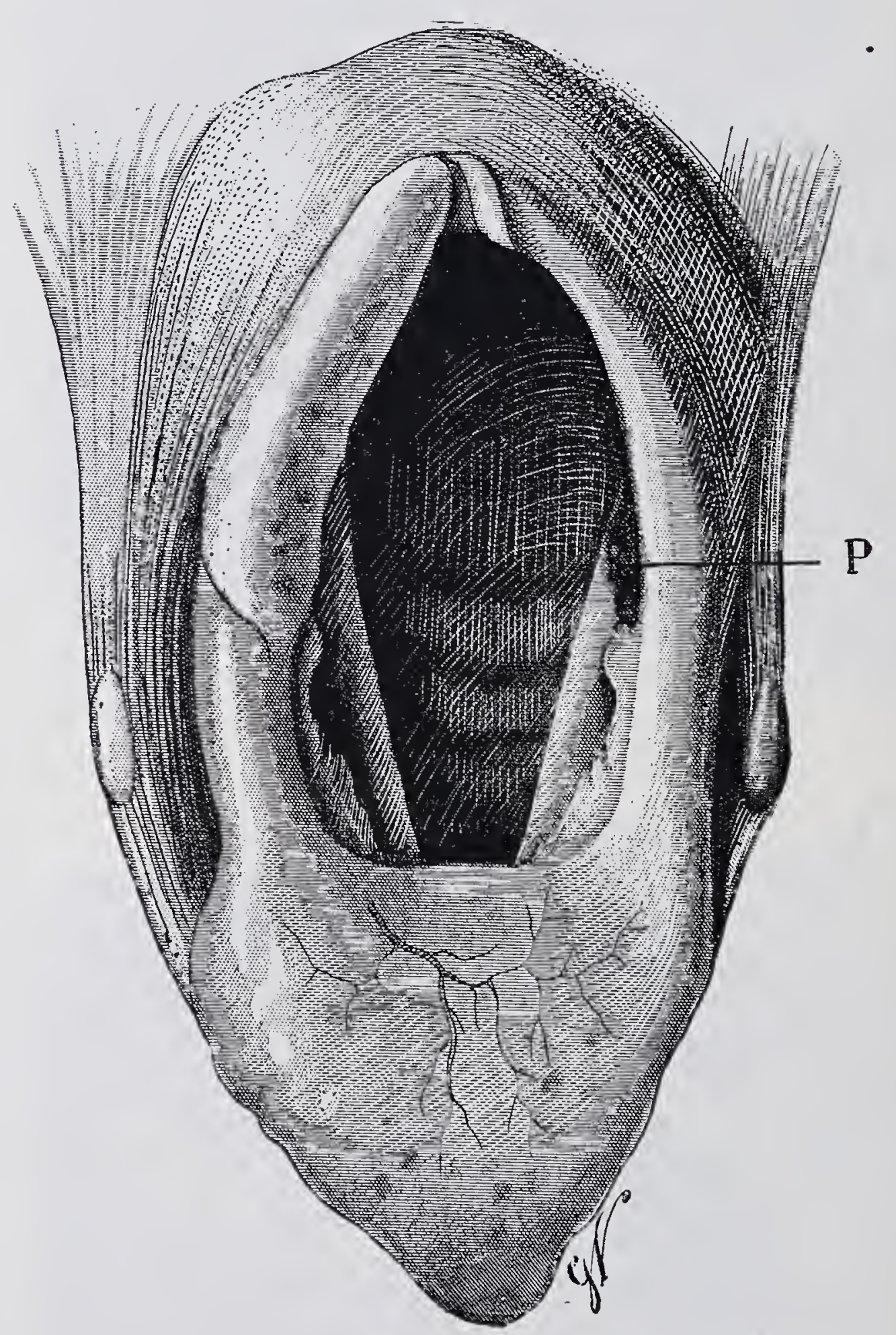

Fra. 114.-Superior opening of the larynx and glottis of a "roarer" that died of pneumonia eighteen days after the operation. "The lips of the round resulting from ablation of the arytenoid were not sutured. P, Partly healed wound.

should not be pushed beyond that point. Too early or injudicious work injures the animal and may produce failures. In summer it is well to turn the patient out to grass for six or eight weeks.

Dyspnœa sometimes disappears in about eight weeks after the operation, but the process of cicatrisation often takes longer, and sometimes roaring only ceases in four to six months after operation.

'This operation must not be expected to do more than render valuable 
horses, which are practically useless owing to roaring, again capable of work. It will, however, do this in a certain proportion of cases. A number of horses operated on by Möller worked for several years. Some were

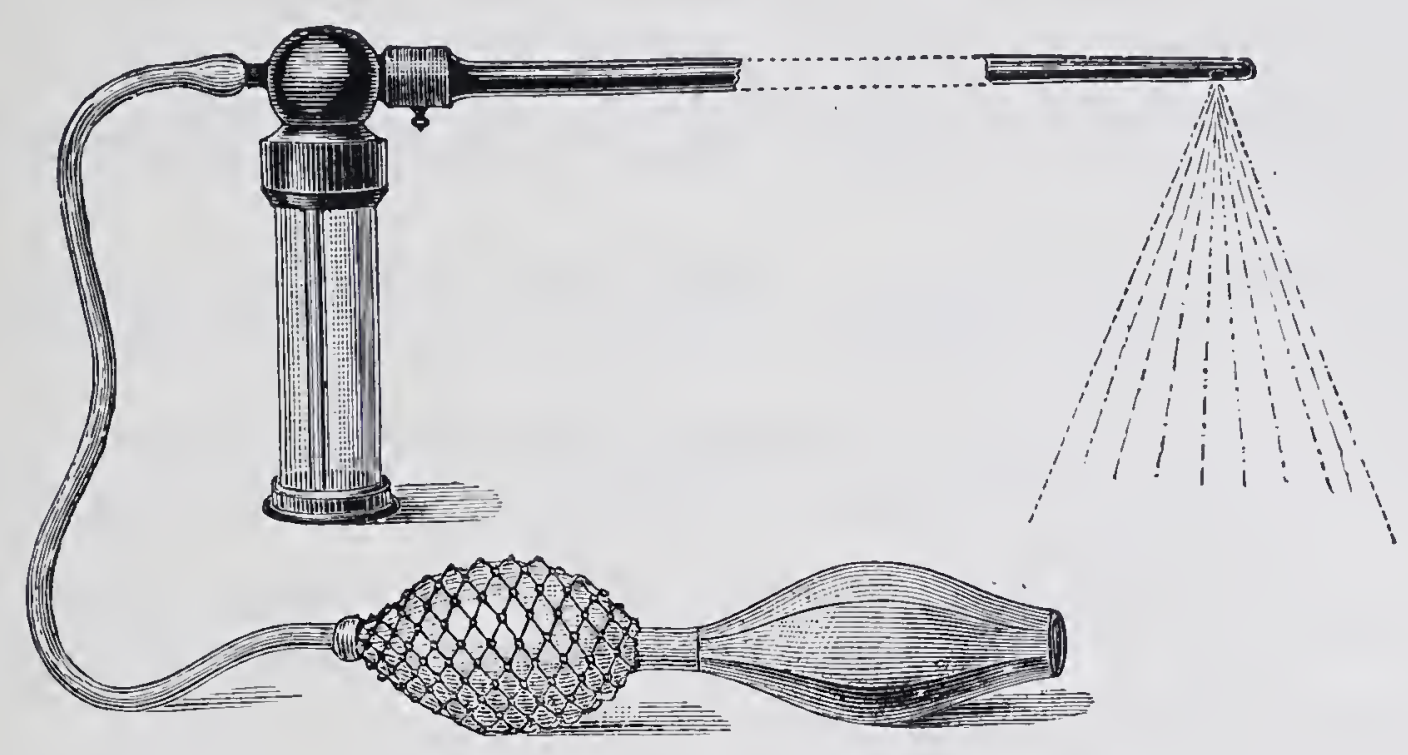

Fig. 115. - Pulveriser for disinfecting the laryngeal wound.

used for riding, and even for racing. A race-horse, operated on in 1891, won $£ 1,500$ the next year, the roaring having completely disappeared. It cannot be denied that success is sometimes remarkable. No other

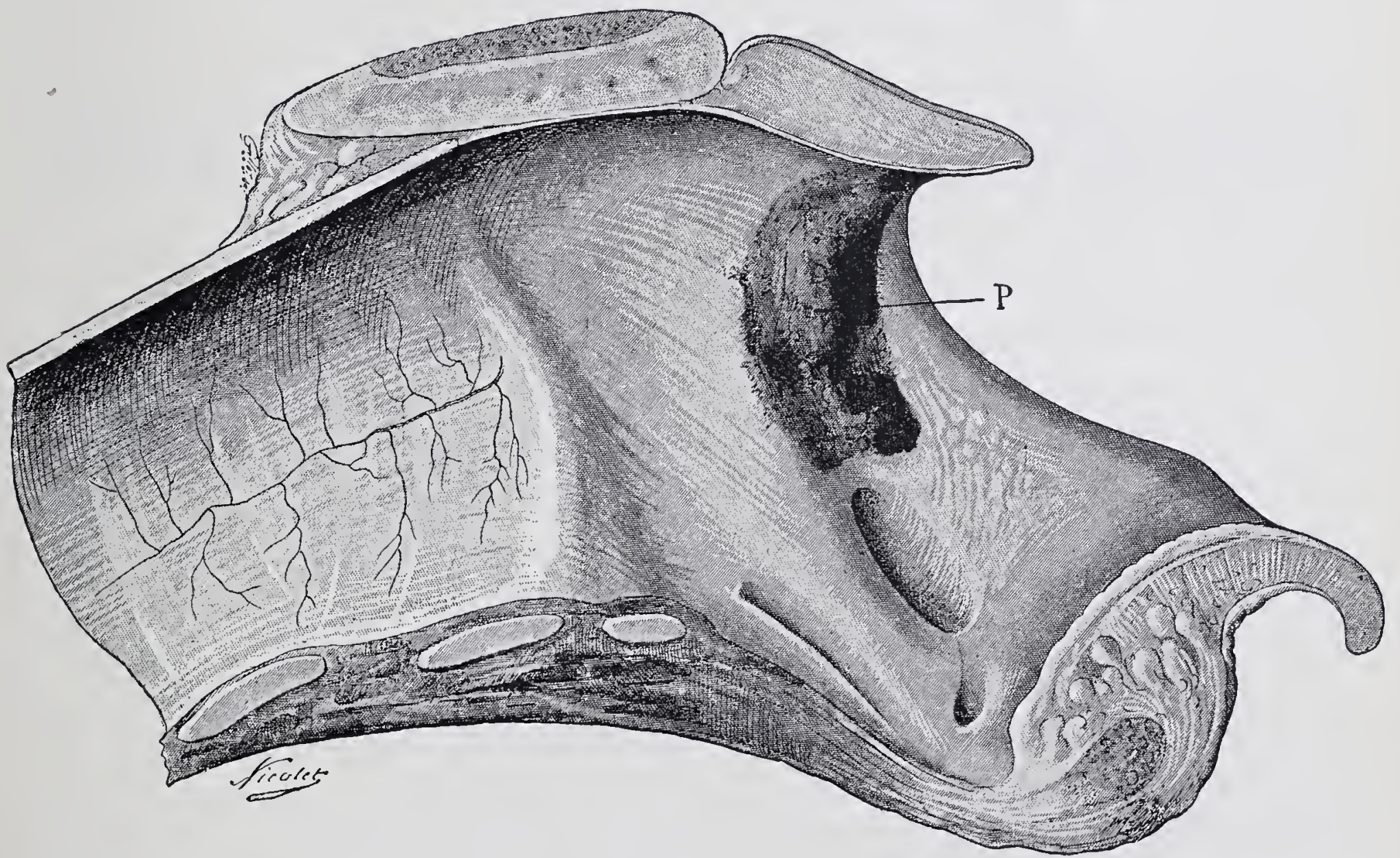

Frg. 116.-Antero-posterior section of the preceding larynx. P, Wound.

remedial measures are known. The use of a tracheal canula, apart from the discomfort it causes, has certain serious disadvantages; on the other hand, the operation only succeeds in a certain proportion of. cases, and does not ensure removal of the loud breathing. Cadiot, adopting 
the method described, operated on a large number of animals, and considered the results encouraging. In those cases which succeeded, recovery was not only complete, but lasting. Of four operations performed by Siedamgrotzky, one was completely successful, and three rendered the horses capable of work. Labat operated on five horses by this method. The first two died; of the remaining three, two completely recovered, and one was much improved.

Plosz operated five times; three cases recovered completely, one incompletely, one failed entirely.

Lanzillotti-Buonsanti used a canula padded with gauze; in one case pulmonary gangrene and death occurred, in the other distortion of the left side of the epiglottis.

In six cases Blanchard removed a portion of the cricoid cartilage, and claims to have seen improvement.

Liautard sutured the arytenoid cartilage to the crico-thyroid ligament and excised the vocal cord; four cases are said to have recovered, and two to have been improved.

A large number of cases of operation for roaring will be found described in Cadiot and Dollar's "Clinical Veterinary Medicine and Surgery," pp. 30 to 34 , and p.p. 347 to 353.

[NoTE.-To prevent misapprehension, I wish to state here that eight of the preceding figures after Cadiot, the British copyright in which is my property, have recently been reproduced, without acknowledgment of my rights, and contrary to my expressed wish, by the publisher's of a work on veterinary surgery.-JNO. A. W. DOLLAR.'] 


\section{DISEASES OF THE THORAX.}

\section{I.-FRACTURES OF THE RIBS-FRACTURÆ COSTARUM.}

Fractures of the ribs are usually produced in the larger domesticated animals by external violence, by thrusts from carriage-poles, kicks, falling on uneven hard ground, unskilful casting, and in the case of runaways by collisions with obstructing objects; in ruminants, from blows with the horns; and in smaller animals from treads with the foot, or falling from considerable heights. Contused fractures are most common. Breakage seldom results from excessive muscular action, though Stockfleth has seen fracture of the first ribs produced in horses drawing heavy loads. Fractures of the ribs seem most frequent in swine, but are usually discovered only after death. Charpentier and Lafourcade, from observations in the slaughter-houses, found that 15 per cent. of swine had suffered fractures of the ribs, many of which had completely united. Simple transverse fractures are most common; but sometimes the fracture is incomplete, the concave surface bending inwards, and the periosteum occasionally remaining uninjured (subperiosteal fracture). Less frequent are compound fractures, involving injury to the skin. In such fractures the pleura and lungs, or the peritoneum and abdominal walls, are some. times injured. In a case of Grosswend's, a horse's last rib having been fractured, the fragments perforated the omentum and stomach.

The course taken is determined by the form and variety of the injury. Partial and simple subcutaneous fractures of the anterior ribs usually unite completely without marked symptoms. The great mobility of the posterior ribs interferes, however, with their union; a callus fibrosus, or pseudoarthrosis forms, but does not impair health, and, indeed, is often only discovered after death.

Compound fractures, involving perforation of the skin, may lead to pus formation and necrosis of bone; but healing, even though long delayed, occurs. Should the sequestrum not be removed, a costal sinus may, however, develop (see page 219).

When the pleura, lung, or peritoneal cavity is involved, the consequences are much graver. In penetrating injuries of the thorax, air from the lungs frequently enters the pleural sac, but is commonly absorbed as quickly as the emphysema ; for it has been found that air does not contain infectious materials in as large amount as was formerly believed, and portions of 
what it does carry are deposited on the surface of the respiratory mucous membrane. Hence, both in man and animals, unexpectedly good results not infrequently occur, especially where the skin is not perforated. A further danger occurs from pleurisy and pneumonia, or from injury to thoracic blood-vessels (arteriæ intercostales et thoracicæ internæ), which may lead to fatal hæmorrhage; in this connection fractures of the ribs are especially dangerous, as other large vessels may easily become damaged from this point. In a case in which a runaway horse struck against a tree, broke several ribs, and died in a few minutes, the postmortem showed not only rupture of the blood-vessels at the anterior opening of the thorax, but injury to the heart by fragments of bone. Collisions with the carriage pole cause similar injuries.

Köhne treated a horse with complicated fracture of the ribs caused by collision with the pole of a carriage; though a large wound existed in the skin, both fracture and wound united in two months. Six months later a new swelling appeared, which broke, and discharged freely; the finger, introduced between the 9 th and 10th ribs, discovered a large cavity from which the pus had come. The animal recovered, but was troubled later with chronic cough.

Injuries and ruptures of the diaphragm occasionally happen as complications of costal fractures. When broken ribs perforate the abdominal walls, fatal peritonitis is apt to ensue.

Symptoms. In partial and subcutaneous fractures there may only be somewhat hurried breathing, without further disturbance. Crepitation may sometimes be heard during breathing, and is the most reliable evidence of fracture. When the pleura is injured, there is a tendency to cough, and more rapid respiration: whilst where the lung is involved, the cough may be accompanied by blood-stained discharge from the nose or mouth, and subcutaneous emphysema. Fractures of the first ribs are accompanied by marked disturbance in the movement of the forelimb and greater difficulty in breathing, because of the attachment of the muscles of the limb, and those of respiration of this point. Rogers, Vennerholm, and many later observers have described such cases. Rogers was probably the first to point out that fracture of the first rib is frequently associated with symptoms of radial paralysis. Hunting's view, however, that all cases of "dropped elbow" (the chief symptom of radial paralysis) are due to fractured first rib is evidently too sweeping; and has been corrected by later observations. Rogers' case is reported in Cadiot and Dollar's "Clinical Veterinary Medicine and Surgery," p. 35़7. Frick, in a dog with fracture of the left first rib, noted paralysis of the left recurrent nerve, hoarseness, a symmetry of the vocal cords, and alteration in the voice. Recovery occurred in six weeks. When the skin is perforated the broken fragments can often be directly seen. Dogs with multiple fractures of the ribs usually show more pronounced disturbance, 
especially if many ribs are broken. The severity of the symptoms increases with the number of ribs broken and the degree of displacement.

Treatment in simple and partial fractures consists in keeping the animal quiet, and guarding it against work and movement. Even in complicated fractures, with injury of the pleura and lungs, nothing further may be necessary. Reduction in such cases is difficult. Hertwig recommends placing large animals with the sound side against a tree, and endeavouring to bring about reposition by drawing the hind-quarter's towards this side. If the skin be broken, fragments of bone dislocated inwardly may be replaced by pushing a finger or hook under the anterior border of the rib, thus avoiding the vessel and nerve which pass down the posterior border. Antiseptic precautions should be observed, and a dressing afterwards applied to guard the wound against infection. Injury of the skin greatly increases danger, and under no circumstances should a wound be artificially produced for the object of reposition; it is much better to adopt an expectant treatment. Charpentier and Lafourcade, in 100 slaughtered swine found 15 with united fracture of the ribs, 10 without further injury, 5 with adhesion of the lungs, and 3 with pleuritic thickening. Cases of one broken rib were rare; and two or three were usually affected. The fracture occurred in the middle of the rib or in its lower portion. According to Stockfieth, the first ribs seem especially prone to break at their upper end.

\section{II.-COSTAL SINUS.}

Chronic inflammatory processes in the thoracic wall, with formation of sinuses, are more frequent in horses than in other animals. They usually result from necrosis of one or more ribs due to direct injuries or to cellulitis, occasionally to burrowing of pus in fistulous withers, or formation of strangles or glanders abscesses. When depending on necrosis of bone, the sequestrum, if allowed to remain, causes chronic inflammation, with pus formation and thickening of the ribs and of their surroundings, which may persist for months and even years. The condition seldom leads to pleurisy, the fascia endothoracica and pleura becoming thickened, and preventing complication.

The symptoms consist in a swelling more or less extended, seldom sharply defined, but hard and firm, without evidence of acute inflammation. A narrow opening exists in the centre of the swelling, and a probe passed through this is arrested by the hard, rough surface of the rib. General disturbance is commonly wanting; symptoms of pleurisy only occasionally occur, but when they do, almost invariably result in death. 
Prognosis. 'The sequestrum prevents healing; whether it can be removed depends on its size and position, and to ascertain this, operative interference is usually necessary. Prognosis is more favourable where the process is confined to the external surface of the rib; but where it extends to the inner surface the condition becomes chronic, and pleurisy may supervene. The latter condition is indicated by the difficulty in breathing and the relatively copious discharge. But even with chronic necrosis of the outer surface, animals may remain useful for a long time.

Treatment. The direction and extent of the sinus has first to be discovered, bearing in mind that the middle point of the swelling is the spot beneath which the chief alterations exist. The sinus should be laid open freely, especially if it trends downwards, care being taken not to wound the external thoracic vessels. The exposed rib is scraped with a curette, the necrotic bone removed, and healing encouraged. In sinuses which cannot be laid open setons may be used, or they may be treated with antiseptics and astringents, such as liquor Villati, ${ }^{1}$ carbolic, or sublimate solutions. When the sinus extends to the inner surface of the rib, non-irritant substances are selected. After removal of large pieces of bone, deep scars often remain in the chest wall. The resection of a piece of rib, although not easy, and involving danger of perforating the pleura, was effected in the horse by Ferguson fifty years ago, and may occasionally be necessary. In such case the necrotic portion is exposed by a long incision through the skin and subcutaneous tissues and periosteum. The periosteum is separated from the deep face of the rib, great care being taken not to perforate the pleura nor to injure the intercostal artery, which passes down the posterior face of the bone. The necrotic part is then removed by strong bone forceps. A drain is inserted in the lower part of the wound, which is sutured and covered with a dressing.

\section{III.-STERNAL SINUS AND FRACTURE OF THE STERNUM.}

Sternal sinus is a condition almost entirely confined to the horse, in which, however, it is not uncommon, and is due to suppuration dependent on local necrosis of the sternum.

The sternum consists of a mass of spongy bone, and in horses is especially liable to be injured by falls on uneven hard ground, by the

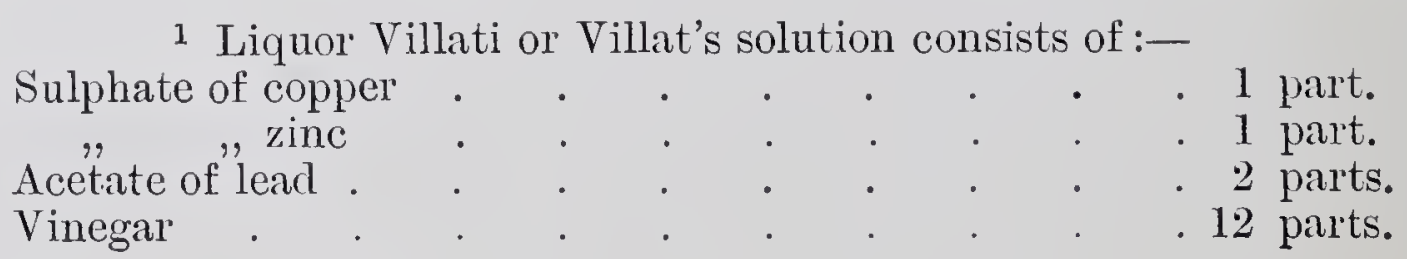


sharp heels of a front shoe, or by the animal being run into. It is either directly injured, purulent periostitis established, and necrosis ensues, or it is indirectly affected from cellulitis extending to it from neighbouring textures. The thick muscular covering of the breast bone renders the removal of any necrotic portions difficult and tedious; pus meanwhile burrows, and new necrotic points are formed, thus protracting the process for months or indefinitely. From time to time acute inflammation sets in, and pus forms; the inflammation then either disappear's or the substernal thickening becomes greater.

Brill described a sternal sinus with two openings below and three at the height of the costal cartilages in a foal $2 \frac{1}{2}$ years old. Similar conditions have occasionally been seen in cattle. Delle mentions that a cow swallowed a splinter of glass, which perforated the rumen and lower wall of the chest, and produced sternal abscess and sinus. The glass was removed, and recovery occurred in five months.

Symptoms. A semi-soft, slightly painful swelling appears between the forelimbs, sometimes exhibiting sinuous openings, from one or several of which ill-smelling bone pus discharges. A probe introduced far enough strikes on the roughened surface of the necrotic bone. When the movements of the forelimbs are interfered with by the severe swelling, lameness may be noted, the animals straddling, while inflammatory action or acute pyrexia may exist.

Treatment. The disease is always chronic, recovery rare. Laying the parts open, scraping necrotic portions of the sternum, careful washing out, followed by the use of iodoform and other materials, have usually been found useless. Günther testifies to the inveteracy of the disease. It is seldom worth while to carry out a tiresome, costly, and rarely effectual treatment. Isolated recoveries may, however, occur. Marcoux reports a case which, after prolonged and fruitless treatment with liquor Villati and the actual cautery, was said to be healed by the use of opodeldoc. Landel tried opodeldoc without effect, but recovery followed the repeated use of the actual cautery, a farrier's polier being heated white hot and thrust into the opening, which was then washed out with spring water ; the report does not indicate whether recovery was lasting. In a long-standing case treated by Lari, the inflammatory process at last extended to the pleura and pericardium.

The prognosis is therefore unfavourable. If treatment is attempted, it can only be of an experimental character, and be based on general principles. Many practitioners confine themselves to dealing with the attacks of cellulitis.

Treatment. Free exit for pus must be provided. Where it is necessary to lay open the lumen of the sinus, this should be effected as far as possible in the longitudinal axis of the body. Disinfectant injections 
may assist healing. Where possible, the diseased bone should be scraped with the curette. For this purpose it will often be necessary to cast the animal. The actual cautery under certain circumstances may be useful, as Landel's case shows. Where acute inflammation occurs, warm antiseptic poultices will hasten recovery and minimise swelling; abscesses must be opened. In the acute stages, the animal, having difficulty in walking, must remain idle; but in chronic cases, it is generally better at slow work.

Fracture of the sternum, which is rare in horses, was observed by Sand. The horse had run away and fallen violently to the ground. It rose, however, and after galloping a further distance of about 400 yards-suddenly fell once more, and immediately died. Death was found to have been due to injury to the internal thoracic artery, which had been torn by a fragment of the sternum. Sand thinks that the hor'se had fallen on its shoe.

\section{IV.-WOUNDS AND BRUISES OF THE SOFT PARTS OF THE CHEST-WALL.}

\section{(1.) WOUNDS OF THE CHEST-WALL WITHOUT PERFORATION.}

Wounds associated with injuries of the sternum have already been discussed, and consideration has now to be given to those of the soft parts; injuries due to collar pressure will be considered elsewhere. Such wounds in horses are caused by collisions with the pole of the carriage, by two animals meeting violently; in the army by lance thrusts, or by falling on uneven hard ground or on the rider's spur. When the injury is on the anterior surface of the chest, the trachea, the great vessels, and the first ribs may be endangered. Injuries to the sides may consist of extensive gashes in the chest and shoulder muscles, or an offending body may force its way between chest-wall and shoulder, severely damaging the larger blood-vessels and nerves.

Thienemann records that a horse, which had received a wound over the elbow and died two days afterwards, had the axillary vein torn through. Hübner describes a case in which the horse fell; a fluctuating swelling immediately appeared, in consequence of rupture of the axillary artery, gradually extended over the neck and chest, and proved fatal forty-five minutes later.

Prognosis depends chiefly on the position and degree of the injury. Wounds involving only the skin and superficial layer of muscle usually heal well, especially if the secretions find ready exit; but deep-penetrating wounds to the lower border of the neck are very grave; the 
discharges are liable to burrow in the loose connective tissue which surrounds the great vessels, the trachea, and oesophagus, and may extend into the chest, causing septic pleuritis. Wounds complicated with fracture of the first rib often produce similar results. The great vessels of the neck and the nerve-stems (vagi) may, moreover, be affected, endangering fatal bleeding or paralysis of the larynx. Violent shocks to the chest-walls may injure the axillary vessels and nerves and produce paralysis, while similar conditions also occur in fractures of the scapula and humerus. Wounds complicated with fracture of the scapula and humerus are usually fatal, septicæmia soon appearing, either in consequence of direct infection of the wound or of the animal continuously lying down. Coldness and paralysis of the affected limbs point to thrombus formation in the vessels, while paralysis alone suggests injury of nerve stems. Such serious results are, however, in great part prevented by the protection afforded by the stout thoracic fascia. Where the shoulder-joint is not injured, mere muscular wounds often heal well. Even where the wound passes under the shoulder, as far as the anconei, recovery usually occurs without affecting the movement of the limb.

The lameness is important in forming a prognosis: as long as it is not marked, and no severe symptoms of fever exist, healing may be expected, even where the muscles are extensively lacerated. Danger of pleurisy is not great. The emphysema sometimes occurring in these muscular lesions must not be supposed to depend upon the thorax being perforated; nor must moderate acceleration of breathing, determined by injury of the accessory respiratory muscles, be mistaken for pleurisy.

Treatment. After bleeding has ceased, the wound should be carefully examined to discover its extent and the possible presence in it of foreign bodies, which, if present, should be carefully and thoroughly removed. The parts are then cleansed; in deep injuries of the anterior surface of the chest, extending towards the middle line, particular provision must be made for the escape of wound discharges in order to prevent the formation of sternal fistulæ. It is well to lay open any pockets or make counter openings. Drains or setons are introduced if required. Where larger vessels, especially veins, are exposed, after-bleeding may result during the first three days, but seldom later. It is, therefore, advisable, whenever possible, to ligate injured vessels, to fill large wounds with antiseptic tampons, and to watch valuable animals continually. Permanent irrigation with lukewarm antiseptic solutions should be continued for several days (sublimate 1 in 3,000, carbolic acid or creolin 1 in 50). Cold solutions should only be used while bleeding persists. Where continual irrigation is unattainable, the wound must be rinsed out carefully every hour or two with a disinfectant. Similar methods of 
examination and checking hæmorrhage are applicable in wounds extending under the shoulder. Arrangements must be made for the easiest exit of discharges, and incisions, counter openings, drains, \&c., combined with effectual irrigation, adopted as above indicated.

\section{(2.) WOUNDS OF THE PLEURA AND THORACIC CAVITY.}

Punctured or penetrating wounds of the chest are usually caused by thrusts with the bayonet, lance, or sabre; in war by projectiles; by falling on harrow teeth, or on the rider's spur ; in oxen by horn-thrusts, by splinters of wood, or by the aspirating needle used to drain the pleural sac. Such wounds may extend through the pleural costalis, and even injure the thoracic or abdominal organs. Besides the dangers of bleeding and infection, such injuries may lead to pneumothorax, hæmothorax, pleurisy, and pneumonia.

(1) Pneumothorax. Air may enter the pleural sac either from without, or from the lung. As soon as a considerable wound penetrates the pleural costalis, the distended elastic lung generally collapses, air is drawn in through the opening in the pleural sac, and breathing ceases in the affected portion of lung. The walls of the chest continuing their respiratory movements, however, the air streams outward during expiration, and re-enters during inspiration. Should the opening in the thoracic wall be partly or entirely closed during expiration, emphysema results from the retiring air being forced into the loose connective tissue in the neighbourhood of the wound. Injury to lung or a bronchus also causes pneumothorax, usually attended with severe bleeding and collapse of the affected portion of lung.

Air in the pleural sac is not necessarily fatal, so long as both lungs do not collapse, though in the horse such a complication is to be feared on account of the cribriform nature of the mediastinum behind the heart. It is reabsorbed in a comparatively short time, as has long been known in man; and more recently been demonstrated in horses by the experiments of Trousseau, Leblanc, and Perosino. Perosino opened the pleural sac in a horse between the 10th and 11th ribs, and allowed air to enter through a tube until the difficulty in breathing threatened death from suffocation; but, on the evening of the day of experiment, all untoward appearances had vanished. Pneumothorax is, nevertheless, a very grave condition, because infectious material, entering with the air, may produce severe inflammation. Air, entering the thorax through wounds, is more apt to be infective than that admitted through the lungs, which is purified as it passes over the respiratory mucous membrane, much in the same way as air entering a bottle may be filtered by a 
cotton-wool plug. The emphysema in the neighbourhood of chest wounds is usually of little importance.

(2) Hæmothorax, or the presence of blood in the pleural sac, arises either from rupture of vessels (intercostal) in the wall of the thorax, or from injury of the lung or heart. Large quantities of blood in the thorax may certainly produce sudden death by compressing the lungs and inducing suffocation; but even a small quantity is dangerous, because organisms may reach it either through the chest wall or lung, and produce septic pleurisy. The simultaneous existence of hæmothorax and pneumothorax accordingly render prognosis doubly grave.

(3) Pleurisy results from the injury, and from the wound becoming infected. It may thus arise either from the external or the lung injury, and will evidently be favoured by foreign bodies remaining in the wound, and by difficulties in carrying out antisepsis. Pneumonia only results in cases where the lung is simultaneously injured. and occasionally happens in cases of complicated fracture of the ribs. Cadéac describes a case of pyothorax in the horse, which was treated by washing out the thorax. During the operation a glass tube about 4 inches in length and $\frac{3}{16}$ inch in diameter slipped into the cavity. Seven ribs were resected, allowing the entire hand to be introduced and even the heart to be felt, near which the glass tube was found. It was removed and the wound healed. On subsequent post-mortem examination the affected half of the lung was found entirely adherent to the pleura costalis; the lung itself was healthy.

Symptoms. In determining whether a penetrating wound of the chest exists, one must not immediately have recourse to probes, nor should one, under any circumstances, proceed to explore with uncleansed fingers. As perforation of the still uninjured pleura costalis may be produced with a probe, such examination may introduce infectious materials into the thoracic cavity where the wound has not been first carefully cleansed. The question must therefore be answered by first considering the other symptoms. One should make-

(1) An examination of the position and condition of the wound and of the body which has produced it, as well as the method of its production. This may suffice to indicate whether the chest-wall has been penetrated.

(2) The breathing must be watched. Dyspnœa may be the result of pneumothorax, hæmothorax, or pleurisy, and occasionally in slighter degree, of injury to the muscles of respiration. Septic pleuritis is associated with high fever. Percussion determines the existence both of pneumothorax and of hæmothorax, and allows of their being distinguished.

(3) Blood-stained discharge from the nose or mouth generally points V.S. 
to injury of the lung, though its absence does not establish the contrary.

(4) 'The passage of air through the wound during breathing is strong evidence of a penetrating wound of the chest; but, as above stated, passage of air, as well as emphysema of neighbouring parts, occur in some non-penetrating wounds of large extent.

(5) Pulmonary prolapse sometimes occurs after large openings in the thoracic wall, and the piece of lung is liable to be strangulated and become necrotic.

(6) If these observations do not determine whether the wound is a penetrating one, and it is considered absolutely necessary to determine the question rather than to leave it uncertain and merely to adopt the treatment usual in penetrating wounds, the parts may be disinfected, and further examined with the aseptic finger or probe. In default of an accurate diagnosis, it is, however, better to adopt the treatment for a penetrating wound. Bleeding vessels should be ligatured, and any fragments of broken ribs replaced under antiseptic precautions.

Animals with such wounds sometimes bend the hind-quarters instinctively towards the injured side, and thus prevent the wound gaping.

Prognosis. Every penetrating wound of the chest should be viewed as a possibly fatal injury, but all do not necessarily take a fatal course. They are more grave in horses than in cattle, but less so in carnivora. The prognosis chiefly depends on whether or not pneumothorax or hæmothorax exists and pleurisy seems imminent. According to general experience, the risk of pleurisy is greatest in horses. When thoracic wounds are of a serious character, most horses require to be supported in slings, otherwise they sometimes die in two or three days. Incised wounds produced by scythes or.lances heal more easily than penetrating bruised wounds made by manure-forks or the tines of harrows. Complications materially affect prognosis; pneumothorax, hæmothorax, or pleurisy is unfavourable. Moderate fever from wound infection does not necessarily prevent recovery, but symptoms of pleurisy generally forebode death.

Numerous recorded cases testify that such injuries in animals sometimes take an unexpectedly favourable course. Jacobin records the satisfactory recovery of a horse from a penetrating wound caused by a spur, which had perforated the thoracic wall and injured a rib. Recovery is not impossible even in cases in which the finger can be introduced into the pleural cavity, and the movement of the lung distinctly felt. Thus Thierry describes a case of shot-wound, with prolapse of the lung through the thoracic opening, in a sporting dog. Recovery occurred in three days. Esvelt saw a horse whose chest had been penetrated by a carriage pole between the 11 th and 12 th ribs. There was prolapse 
of the lung. After reducing this and applying an antiseptic dressing the parts gradually healed.

Noguiès, called to see a mare which had been "horned" by a cow, found a mass of prolapsed lung protruding from a thoracic wound three inches long. The mare was cast, the torn mass of lung ligatured, and a piece, weighing 20 ounces, was removed with a single sweep of the knife. The ligature was afterwards removed, the parts were disinfected, the hair was clipped away, the wound sutured, and a blister applied over all. Recovery occurred without complications.

On the other hand, apparently slight wounds may prove rapidly fatal. This is especially true of insignificant-looking penetrating wounds, like those produced by manure-forlis, in which danger of infection occurs during the first three or four days. When pus forms, if meanwhile no threatening symptoms have appeared, the prognosis becomes more favourable.

The principles of treatment are clearly apparent, the first and most important being thorough antisepsis. After checking the flow of blood by ligaturing with sterilised material the injured vessels, the wound is carefully disinfected, and where healing by first intention seems possible, sutured. Where much discharge is to be expected, drains must be inserted and counter-incisions made. Over the ordinary dressings an air-tight bandage should be applied. For the larger animals moss wadding is suitable, but where this is not obtainable a straw mat, or a cloth folded several times, can be substituted, and the compress kept in place with a roller. In small animals, as in man, bandages are usually serviceable, but must be renewed immediately they become moist with discharges.

Where proper dressings cannot be improvised, arrangements may be made for continuous irrigation with disinfectants; but if penetrating wounds exist, care must be taken that fluid and air do not enter the pleural sac. Sometimes both the dry and moist methods are associated or alternated by allowing disinfecting fluids to pass through the dressing and wound, from whence they are suitably drained. Such irrigation, or frequent moistening with antiseptic solutions, is useful. Dressings must be changed as soon as soaked with discharge, or where high fever has set in. Little can be done in combating pneumothorax; and in hæmothorax the blood, being already clotted, is difficult to remove. In septic pleuritis in horses and carnivora the thorax is sometimes rinsed out with 1 part of corrosive sublimate to 3,000 of water ; for ruminants, a solution of 1 in 700 of boric acid, or a lotion of alum. acet. of similar strength may be used. But such treatment is seldom effectual. Weisner, experimenting on two horses with 1 per cent. solution of salicylic acid, was not successful. 


\section{Y.-SHOULDER ABSCESS.}

Hertwig describes two varieties of shoulder abscess-one superficial, consisting of inflammation of the subcutis, and only presenting the same features as similar swellings in other parts of the body; another of a special type, almost peculiar to horses employed for draught.

Symptoms and progress. A sharply defined, hard, slightly painful tumour, as large as a child's head, lying either in or below the mastoidohumeralis muscle (levator humeri) suddenly appears in harness horses. The skin is sound and movable; lameness is scarcely ever present, though Günther states having observed it.

For some time no marked changes occur in the swelling, except possibly an increase of circumference, and it remains stationary for weeks or months, during which steady work may be done. Usually after some months, fluctuation appears at the most prominent part, and on incision thick fluid pus is discharged. The earlier the swelling is punctured the more quickly it disappears. If operation is long delayed permanent thickening may remain.

Opinions differ as to the origin of these tumours, though it is characteristic of them that soon after their formation a small suppurating point may always be found at their centre.

Franck believes that they are associated with injury of the prescapular glands. Hertwig, who is supported by Esser, considers that they are caused by continuous pressure of badly-fitting collars, as indicated by their occurring only in draught horses, usually in those drawing heavy loads. Viborg views them as complications of strangles. Every practitioner must have seen many draught horses with this shoulder abscess, but riding horses never seem to be so affected. Stockfleth, however, reports a case in a horse which had been confined for several months to the stable on account of lameness; but this may be regarded as the exception which proves the rule. It is specially noteworthy that, even from their earliest beginning, these abscesses contain pus, in which pyogenic cocei are almost invariably present. This fact has been amply proved by the large number of these abscesses containing staphylococci opened in the Berlin clinique during the last few years, though streptococci were sometimes found instead.

But how does the infection occur? Though it is clear that infection with cocci must be associated with the mechanical-injury caused by the collar, it is by no means clear how the cocci penetrate to the deep-lying seat of abscess through uninjured skin. Schimmel urges that they proceed from the lower portions of the limbs by the lymphatic vessels; and in support of this view adduces the case of a horse which suffered from a suppurating sanderack, from which discharge of pus was prevented 
by applying a bandage, when abscess on the shoulder was shortly developed.

Similar swellings are produced by a peculiar parasite, named by Rivolta Discomyces equi. This organism has also been frequently found in cases of scirrhous cord, in swellings due to harness pressure, in mammary growths, in growths about the tail, and even in the lung and in bone.

Cadiot and Dollar relate two such cases. (See. pp. 477-479, "Clinica] Veterinary Medicine and Surgery.") Iodide of potassium was unsuccess-

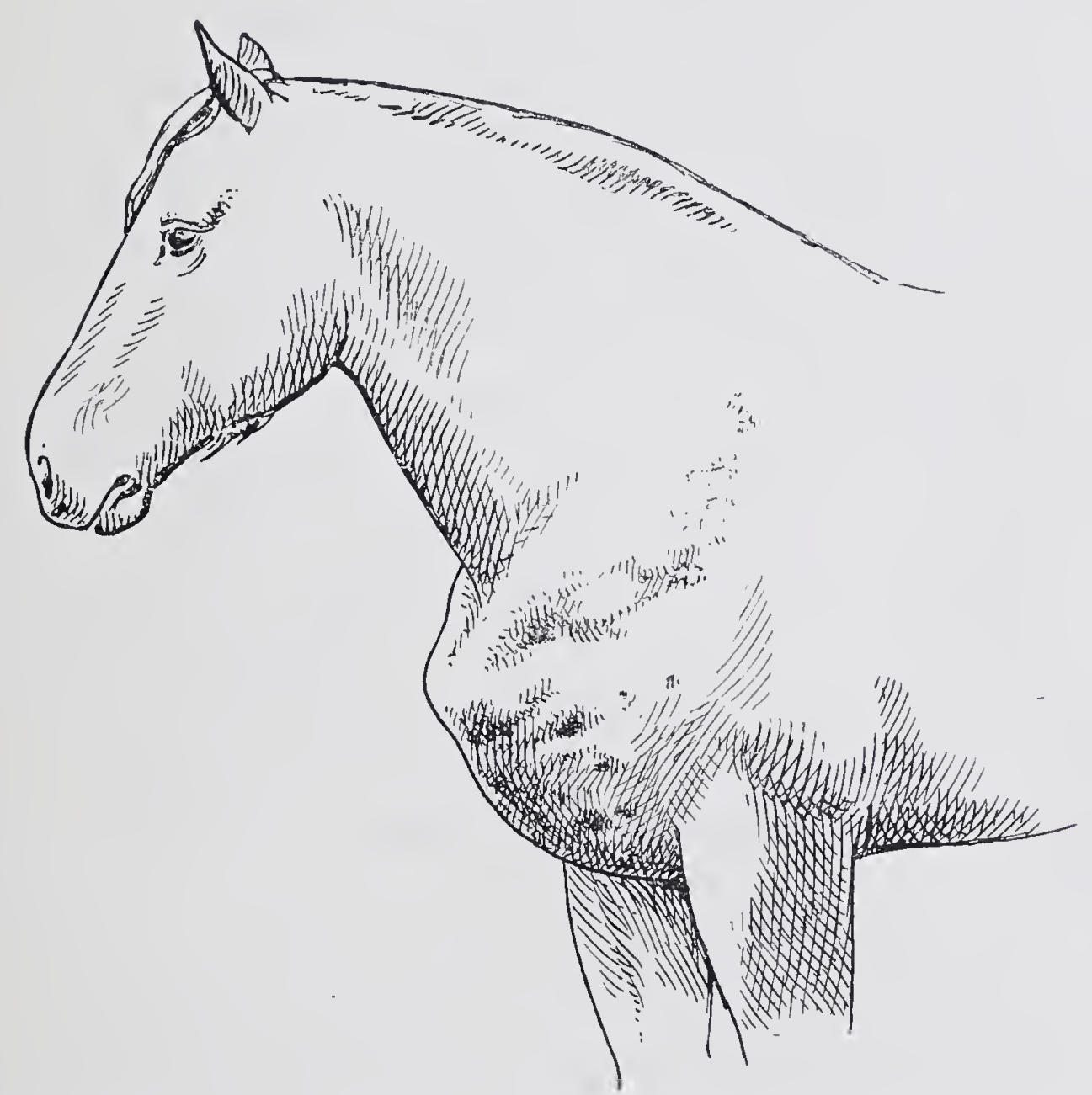

Fig. 117.-Multiple abscess formation in the shoulder-region due to Discomyces equi.

(Bothryomycosis.)

fully tried in these and in 22 other cases of bothryomycosis. In almost every instance, however, recovery followed ablation.

Two factors are at work-collar'-pressure and infection. The former certainly favours development of micro-organisms in the mastoidohumeralis muscle or prescapular glands, but the method of infection is less clear. It seems possible that micro-organisms may enter the injured lymph-glands with the lymph-stream, or that they may be poured into the disease-focus along with the extravasated blood. Schmidt inoculated several oxen with Kitt's symptomatic anthrax lymph, but only one animal, which had been struck with a stick, developed anthrax and showed the characteristic swellings. Rosenbach broke the tibiæ of rabbits, into whose blood-stream he had previously injected cultures of bacteria, and noted severe inflammation at the injured spot. Still it is 
possible that infective materials may set up inflammatory changes and swelling in the lymph glands directly, and thus render them more liable to bruising.

In many of Möller's cases the disease has started in the prepectoral glands, and shown the character of a lymphadenitis chronica suppurativa. The process either arises from the common mastoido-humeralis muscle, and is of the nature of a myositis apostematosa, or the underlying: lymph glands are the primary seat of disease, and in such cases it should be described as lymphadenitis suppurativa; while from this, the inflammation extending to the muscles produces a myositis chronica fibrosa. Other growths, however, occur here; thus in a grey horse Möller saw a melano-sarcoma, which had been mistaken for abscess on the shoulder, and Günther reports a similar case. The slow development of melanosarcoma, however, sufficiently differentiates it from the condition now in question. The disease is usually chronic, especially when not treated. Resorption of the pus may even occur, but is very rare.

Prognosis is favourable where treatment is early adopted. When delayed, imflammation becomes chronic, firm masses of connective tissue are produced, and when these have existed for months complete extirpation, which is always difficult, is the only remedy; otherwise the swelling is again bruised by the collar and becomes inflamed, leading to further formation of fibrous tissue. Whatever the cause, the earlier the abscess is opened and drained, the more rapid and thorough is resolution. Extirpation of the glands is the only means of dealing with chronic lymphadenitis.

Treatment. Formerly blisters were recommended as the first application, and the abscess was punctured in eight to ten days. Stockfleth inserted the actual cautery into the cavity of the abscess after dividing the skin. Others allow the abscess to mature. Block has lately recommended the following compound: Ungt. cantharid. off., 30 parts ; tinct. cantharid. and tinct. euphorb., of each 15 parts (evaporated to half their bulk); ol. croton, 1.20; hydrarg. biniod., 4; fresh butter, 30 . After clipping the hair and applying the ointment, Block holds a red-hot iron a short distance from the skin so as to heat it. The animal is then fastened up for three days. The best treatment is to immediately open the abscess and evacuate the contents. The abscess sometimes lies as much as 4 inches below the surface, but this should not prevent operation. By thrusting the knife towards the centre of the swelling dangerous bleeding is avoided and the abscess opened with certainty, though the knife must not pass beyond this point on account of endangering large vessels.

As in other such operations, the hair is removed, the skin cleansed, the position of the swelling determined by palpation, and the horse twitched. 
A pointed bistoury, with its cutting edge directed upwards, is inserted from 2 to 3 inches. The pus usually then discharges; but if not, the finger is introduced, the fluctuation localised, and the wall of the abscess divided with either knife or finger. After discharge of the pus, the swelling should be laid open in its entire length, if possible, by a vertical incision, thus completely exposing the cavity of the abscess, which is then freely curetted. Bleeding may be checked by means of tampons, and single vessels ligatured.

To promote dispersal of the swelling, moist warm applications, wetted from time to time with hot water, are employed for eight to fourteen days; they are kept in position with a cloth doubled two or three times, and fastened to the neck by a bandage. Jacobs punctured the abscess with a trochar, and injected 2 per cent. lysol solution, followed by Lugol's solution of iodine. In one case the abscess could not be found. Lugol's solution was injected three times in eight days, and healing occurred. Schmidt recently recommended the injection into the swelling of a saturated solution of common salt. Active suppuration and perforation are said to follow; but a case treated in this way by Schilling soon afterwards died from gangrene. Injection of common salt certainly hastens suppuration, especially if not sterilised, but as it possesses the disadvantage of exposing the animal to grave infection the use of the knife is always preferable.

Where these abscesses have existed for several weeks, and contain no large cavity, extirpation becomes necessary. This may be either (1) partial, which is only adopted where no sharp margin divides the swelling from the sound textures, and is seldom ineffectual; or (2) total, which is specially indicated when the duration of the case has been considerable, and there is hence no prospect of reabsorption of swelling after opening. In most cases free opening of the abscess cavity is sufficient, as above mentioned. In old growths as much as possible of the anterior part should be removed. Some operator's practise local anæsthesia by Sleich's method (see Dollar's "Operative Technique ") ; Malkmus applies a twitch and the hippo-lasso. Bayer recommends strict antiseptic precautions, and after carefully curetting and disinfecting the abscess cavity applies a dressing which is fixed to the button sutures used to close the wound. Malkmus has shown that these excessive precautions are unnecessary, and that by using interrupted sutures, inserting a drainage-tube, and dressing with glutol, the same end is attained. He also insists, however, on the importance of curetting the cavity and of observing antiseptic precautions. The horse is afterwards kept for a fortnight on the pillar reins, and if great swelling occurs the parts are kept moist with 3 per cent. selution of acetate of alumina. If the covering of glutol separates from the surface of the wound it is renewed; in a week the drainage-tube 
is removed, and in eleven days the sutures. The operative wound usually heals by primary intention in three weelss, and the horse can resume work in one to two weeks later. This method can be recommended as practical and efficacious.

Before attempting total extirpation the horse should be cast, for even if quiet, bleeding or other complication may ensue, which cannot be properly dealt with if he is standing. The animal is laid on the healthy side, placed in a good light, and the hair removed from the swelling. Spring artery forceps, thick and thin ligatures, and some tow tampons must be provided. An incision is made in the direction of the neck over the tumour; the skin on either side is drawn back, which, on account of the looseness of the subcutis, is not difficult, and any portion of skin adherent to the swelling taken away. In order to exert traction on the tumour, a piece of tape is passed through it, and the loose connective tissue surrounding it is separated with the fingers or scissors, beginning at the lower and inner side in order to avoid the carotid and its branches, or in case of injury, to facilitate their being ligatured. At the upper portion of the swelling this may become impossible, and grave bleeding may accordingly result. Similar danger occurs if the swelling is cut into, or a portion of it is allowed to remain. Vessels cut through sometimes retract into the masses of connective tissue, their ligation becomes impossible; and as the actual cautery often belies its reputation, the operator may see the animal bleed to death before his eyes. This danger is avoided if the operator carefully separates the loose connective tissue as far as possible with the fingers, and at once ligatures the exposed vessels in two places. The tumour secured, if necessary, with a strong thread, is then cut away. After rinsing out the cavity with a disinfecting fluid, and ligaturing any bleeding vessels, the surfaces should be powdered with 1 part of iodoform to 3 of tannin, the cavity plugged with carbolised jute, the skin sewn up, and the animal allowed to rise.

In twenty-four hours the sutures may be loosened and the wound rinsed out with sublimate solution. A couple of stitches may be inserted into the margins of the wound, on either side, and fastened to a roller or to the mane, thus allowing free entrance of air. Dressing with iodoform and tannin is persevered with. To prevent interference with the wound, the animal should be placed on the pillar reins, or a side-stick applied in the recognised manner. After a few days a dry black scab covers the surface of the wound, and, if it become loose in spots, should be removed and the antiseptic powder reapplied. Towards the edges of the wound a small quantity of discharge may appear, which can be dried up with wadding or wood wool and the surface again powdered with iodoform and tannin. During the first six days the wound shows no marked change 
under this treatment, but gradually becomes smaller, and in about three weeks the cavity, which was as large as a child's head, will have completely filled up.

An abscess of the shoulder, extirpated on July 16th, left behind a cavity 7 inches long, 4 inches broad, and $2 \frac{3}{4}$ inches deep. Until the 22 nd no marked change had occurred, but subsequently the dimensions were reduced as follows :-

\begin{tabular}{|c|c|c|c|c|}
\hline $\begin{array}{l}\text { Day. } \\
\text { July } 23 .\end{array}$ & $\begin{array}{l}\text { Length. } \\
6 \frac{1}{2} \text { inches. }\end{array}$ & $\begin{array}{l}\text { Breadth. } \\
3 \frac{1}{2} \text { inches. }\end{array}$ & & $\begin{array}{l}\text { epth. } \\
\text { ches. }\end{array}$ \\
\hline , 24 & $6 \frac{3}{8} \quad$, & $3 \frac{1}{8} \quad$, & $2 \frac{1}{8}$ & ,. \\
\hline 25. & $6 \frac{1}{8}$ & $2 \frac{7}{8}$ & 2 & $"$ \\
\hline 26. & 5 & $2 \frac{3}{4}$ & 2 & " \\
\hline 27. & $4 \frac{3}{4}$ & $2 \frac{5}{8}$ & $1 \frac{7}{8}$ & , \\
\hline 30. & $3 \frac{7}{8}$ & $1 \frac{7}{8}$ & $1 \frac{3}{4}$ & ", \\
\hline 31. & $3 \frac{3}{4}$ & $1 \frac{3}{4}$ & $1 \frac{\overline{8}}{8}$ & $"$ \\
\hline
\end{tabular}

In such cases open wound treatment is not only the quickest, but the most convenient. When a dry scab is produced over the entire wound nothing further is necessary. The margins of the wound being laid back obviously prevents their being drawn inwards by cicatricial contraction, which is apt to interfere with healing.

\section{VI. -SADDLE AND COLLAR GALLS-INJURIES FROM PRESSURE.}

The saddle and collar exercise heavy pressure on the tissues below, and may produce injuries to the skin or subjacent textures. These are usually of the nature of bruises, seldom of excoriations; and as those produced by the saddle possess the greatest interest, they will be first considered.

\section{(1.) INJURIES PRODUCED BY THE SADDLE- SADDLE-GALLS.}

To prevent the pressure of the saddle injuring the back, the following precautions must be observed:-

(1) Pressure should be distributed as evenly, and over as large a surface, as possible.

(2) Pressure must be confined to parts fitted to bear it.

(3) Those portions of the saddle which bear on the body must be properly stuffed, or the back covered with a folded rug, \&c. Weight can only be borne where muscles or fat underlie the skin, as is the case along both sides of the vertebral column in the dorsal region.

In the middle line of the back and on the withers, where the bones are not cushioned, any considerable pressure causes bruising. Saddles are, therefore, made to bear on. the protected parts only. 
The anatomy of that portion of the horse's back carrying the saddle may be briefly described as follows (fig. 118) :-

(A) In the middle line of the wither's and back, under and closely adherent to the skin $(a)$, lies the fascia of the dorsal panniculus $(b)$. Below it extends the scapular fascia $(c)$, which is applied closely to the termination of the ligamentum nuchæ, covering the superior spinous processes of the 5th-10th dorsal vertebræ $(d)$. This is not covered with muscular padding.

(B) On either side of the vertebral column, in the "saddle-bed," or surface on which the saddle rests, the skin is provided with a strongly developed subcutis, and covers the fascia of the dorsal panniculus (b), which is attached by soft connective tissue to the scapula fascia $(c)$.

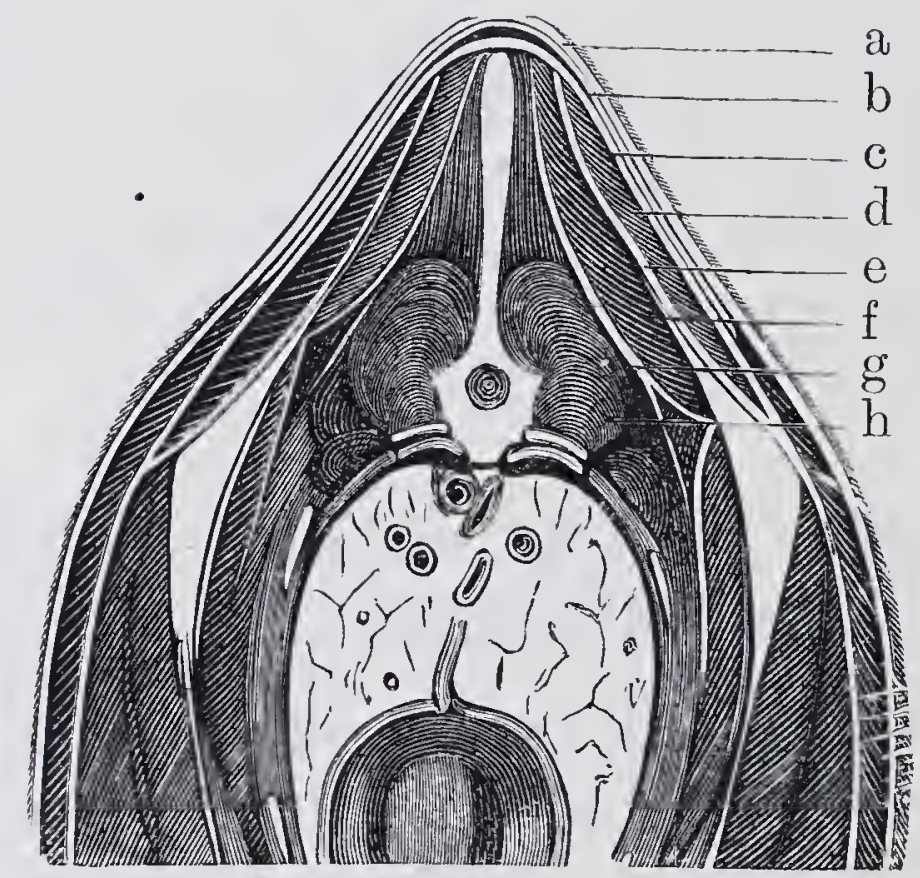

FIG. 118. - Transverse section through horse's thorax (semi-diagrammatic, after Franck). $a$, Skiu ; $b$, fascia of panniculus ; $c$, scapular fascia ; $d$, muscular layers ; $c$, external fascia of withers ; $f$, muscular layers; $g$, subscapular fascia.

Below this lies a pad of muscle, comprising the latissimus and longissimus dorsi, the spinalis and semispinalis dorsi, the trapezius, rhomboideus posterior and the ilio-costalis (retractor costæ) muscles.

Injuries in the saddle-bed occur most frequently from irregularly distributed pressure continued for a considerable time. Limited areas of skin are compressed, leading to vascular distension and rupture; but while pressure continues little extravasation occurs. So soon, however, as the saddle is taken off, blood and lymph are passed from the ruptured vessels into the perivascular spaces. As in other bruises, if the skin were not pigmented, reddening might be noticed. At first a serous fluid accumulates in the interstices of the cutaneous tissue, and at a later stage, more and more white corpuscles appear, with plastic infiltration. Where serious bruising occurs, plasma and blood are effused. The gall consists of a circumscribed firm swelling, not unlike that of urticaria, caused by extravasation into and infiltration of the cutis. It may be soft, occasionally 
fluctuating, and withont sharp borders, as when blood and lymph are freely poured into the loose subcutis. Should this condition develop under the fascia, the swelling is less sharply defined and more tense.

The lesion, when situated on the wither's or spine and caused, as indicated, by bruising of the skin covering the superior processes of the vertebræ is less sharply defined, more dispersed, and often shows fluctuation. Although it may not be painful at first, it becomes so if the vertebræ are injured. While lesions of the withers and spine are generally subcutaneous, those of the saddle-bed are generally cutaneous. From pressure and friction of the girths and other parts of the harness, hair and epidermis are often rubbed off. If bruising also occurs, cutaneous swelling ensues. The condition is one of cutaneous or subcutaneous bruising, which sometimes consists in rupture of the tissues and smaller vessels, less frequently in injury of larger vessels and consequent extravasation.

Symptoms. Saddle-galls are best discovered half an hour to one hour after unsaddling; it usually takes this time for their appearance. They generally vary in size from a sixpence to a five-shilling piece; are tense, hard, somewhat painful, warm, and sharply marginated. The larger' are always flat. Should the hair over the saddle-bed be wet, these points of pressure dry first, producing isolated dry spots. Not infrequently they are itchy, and the animal bites or rubs them. Bruises of the subcutis are diffuse, sometimes odematous, sometimes fluctuating, and are usually more painful, especially on pressure. Saddle-galls are best detected by passing the hand over the withers, spine, and the saddle-bed, when thickening, firmness, or tenderness may be discovered. When the tender spot is touched or pressed the animals bend the back or move away. This is specially noteworthy in the region of the withers, for the detection of such swellings in this site is otherwise somewhat difficult.

Disease implicating the ligamentum nuchæ is characterised by great swelling and profuse pus formation, swelling of the neighbouring lymph vessels, and of those on the shoulder and over the ribs. Suppuration and necrosis in the depths is indicated by exuberant, flabby, dark-red granulations, which bleed easily; whilst implication of the superior spinous processes is shown by the greater degree of pain. Necrosis of these processes, or of the cartilage covering them, can often be detected by palpstion. Where the skin has become necrotic, it is hard and leatherlike, and the hairs on the affected spot are usually erect. Excoriations produced by rubbing are easily seen.

Course. Where extravasation has been slight, cutaneous swellings usually disperse rapidly. The blood serum and lymph poured into the insterstices of the cutis are reabsorbed; the aggregated leucocytes re-enter 
the circulation, and the ruptured membrane undergoes repair. The status quo ante is thus restored. Proper treatment may remove such a swelling within a few days; but, as formed elements gradually replace the earlier infiltration of blood or serum, the longer such swellings have existed the longer will be the time occupied in their reabsorption.

Subcutaneous extravasations of blood and lymph do not become absorbed so rapidly, and frequently last eight to fourteen days or longer. The larger the fluctuating swelling, the longer it persists. Sub-fascial extravasations are still more tedious, and hence those on the withers are always more difficult of removal than those on the saddle-bed.

Cutaneous swellings are frequently followed by necrosis of the skin. Owing to defective local nutrition, it is most common on the site of old cicatrices, and also where the surfaces, while still tender, are again subjected to severe pressure. The living processes are thus arrested, the fluids are to a great extent pressed out of the tissues and vessels, and mummification or dry gangrene results. When this occurs, demarcation generally occupies two to three weeks, sometimes even longer; it is first completed in the loose subcutis, later in the corium.

The third termination is suppuration : it seldom or never occurs in the cutaneous swellings, but is common in the subcutaneous, especially in those associated with extensive extravasation. This termination is met with, when the skin is broken as well as bruised, and also when the knife has been unnecessarily used. Fluctuation sometimes exists, and should it be mistalien for a sign of suppuration and incised, grave consequences are likely to result, especially in the region of the withers. Pus formation may lead to necrosis of the neighbouring fascia of the ligamentum nuchæ, and even of the superior spinous processes, producing fistulous withers. The burrowing pus excites inflammation, which increases the difficulty of free discharge, brings about a circulus vitiosus, and greatly delays recovery. Hübner, during such a case affecting the occiput and the shoulder, counted 200 abscesses, from the size of peas to that of a child's head.

In severe injuries, like bruising of the slin and soft parts and crushing of the spinous processes, putrefactive processes and other grave conditions may occur with fatal results, which, however, are not very frequent. Disseminated pus formation and lymphangitis are liable to ensue as complications, sometimes spreading in the subcutis, and not infrequently also under the fascia. Necrosis of the ligamentum nucha, of the superior spinous processes, and even of the deeper portions of the vertebræ may occur, and in exceptional cases necrosis of the ribs and of the shoulder-blade is seen.

These conditions must not be confounded with certain forms of eczema, which are particularly apt to attack riding horses in regions 
where there is much chalk dust. They affect the back, especially behind the saddle-bed, but the extent of surface involved, the trifling amount of swelling, and the thick scurf formed sufficiently differentiate them.

In a case described by Möller, pus formation had extended along the fascia of the back as far as the pelvis, producing necrosis, and also invaded the under surface of the longissimus dorsi, separating the latter to a great extent from the bodies of the ribs. Several ribs afterwards became necrotic. Pus also burrowed under the serratus anticus major muscle.

The prognosis depends on various factors. It is modified by-

(1) The position of the swelling. The cutaneous is more hopeful than the subcutaneous, especially if situated in the withers.

(2) The duration of the swelling. The longer swelling has existed, and the harder and more resistant it appears, the further has plastic infiltration proceeded, and the more tardy will be resolution. Fistulæ of the withers of long standing are usually difficult of cure.

(3) Cellulitis or lymphangitis adds to the gravity of the case. The condition is of little gravity provided it remain aseptic; the occurrence of suppuration renders it serious, and doubly so if the withers be the part involved.

(4) In prognosing fistulous withers, particular note must be taken of the degree of swelling and pain, and the amount of pus. Swelling indicates burrowing of pus, if the discharge takes place only on pressure from without, or during movements of the animal. Intense pain points to injury of the superior spinous processes.

Causes. Certain horses suffer more than others, depending on greater vulnerability of the skin, or on the presence of cicatrices or scabs, or on peculiarities in form interfering with the fitting of the saddle. Amongst these may be included abnormally high, low, or short withers, flatness of the ribs, keel-shaped breast and short sternum, and distension of the abdomen, causing the girth to slip easily forwards or backwards. Old horses sometimes have the muscles in the saddle-bed atrophied, and are therefore more liable to contract saddle-galls. Vicious, irritable, and restless horses which cannot be carefully saddled often suffer, as also do those which have irregular action. Lame horses frequently have saddlegalls, especially on the withers, on account of the unequal incidence of the body-weight shifting the saddle towards the side of the sound foot. Saddle-galls are often caused by horses sweating profusely, or by the skin being soaked in rainy weather. The mechanical causes may be divided into three groups-

(1) Faulty construction of the saddle. The best preventer of saddlegalls is a skilful and careful saddler. Though it appears easy in principle to distribute the pressure equally over the largest possible surface, in 
practice it is really very difficult. The saddle must neither be too narrow nor too wide, and provision must be made for changes in the condition of the animal. A saddle, when too wide, endangers the withers; when too narrow, it bruises the bodies of the ribs with the outer edges of the bearers. The front portion must conform to the height of the wither's, which are injured if it be too low. Fractures or bends in the bearers or branches of the saddle-tree, badly-stuffed panels, or repeated soaking of them, easily lead to saddle-galls. Parts of the saddle or collar, which do not fit closely to the body of the animal, sometimes produce skin excoriations.

(2) Faults in saddling, or in putting on the collar. Folds or foreign bodies in the padding, however small, often produce bruises. The padding, if it shifts upwards, may injure the withers. The practice of applying the saddle-blanket so that it shall be pushed slightly backwards when the saddle is placed in position is recommended, because the hair of the saddle-bed will thus be smoothed in its proper direction. The saddle, if too far forward, may directly bruise the withers; if too far back, it works forward, the girth becoming loose and easily causing bruises. Loose girths, and irregular tightening of the component parts of the girth in the German saddle, also gives rise to saddle-galls.

(3) Errors in riding. An awkward position in the saddle, sleeping on horseback, and continual shifting forward, displacement of the saddle in mounting, and injudicious riding, are frequent causes of injury. These injurious influences must, however, be in operation some considerable period before serious saddle-galls are produced; they seldom result from short excursions.

Treatment. To prevent the condition, general experience suggests leaving the saddle on for half to one hour after dismounting. Where an injury has taken place, the vessels are compressed and almost bloodless. If pressure be now suddenly and completely removed, blood is vigorously forced into the paralysed vessels, and may thus rupture their walls. On the other hand, if the saddle is allowed to remain some time in position, circulation is gradually restored without injury. The fact that the swelling first appears after removal of the saddle supports this explanation.

In cutaneous swellings further extravasation is most surely checked by cold, which causes the vessels to contract; while reabsorption of extravasation which has already taken place, is most favoured by moist warmth. The use of cold is indicated in fresh cases-that is, in those seen immediately after the appearance of the swelling. In twelve to twenty-four hours, on the other hand, moist warmth deserves preference; and, where cold cannot be carefully and continuously applied, Preisnitz's poultices should be employed. The use of cold may be associated with gradual slight pressure, or, even better, with massage. A cloth folded several times, or a bag filled with little pieces of ice, is very useful in 
fresh cases. The old practice of binding a flat stone on the swelling leads to necrosis of the skin ; the application of a turf soils the back, and thus predisposes to new bruises. Massage, in the form of gentle stroking with the fingers, greatly hastens reabsorption. Most cutaneous swellings of recent appearance can be removed in twelve to twenty-four hours by cold, associated with massage. When of longer standing, moist warmth in the form of Preisnitz's poultices deserves preference, and can be assisted by massage. Where necrosis does not result, this method of treatment always succeeds. Tincture of arnica, often used, has no effect; even its antiseptic properties are nullified on account of the excessive dilution to which it is generally subjected.

Where necrosis has appeared, separation of the dead portions is facilitated by Preisnitz's poultices, or warm cataplasms of linseed meal, to which an antiseptic is added. Portions of necrotic skin, as they become loose, should be removed with dressing forceps. Some power may have to be exerted. Should this not succeed, the heat and moisture must be persevered with. Extraction of the mummified piece of skin does not greatly assist healing, because the line of dissection seldom coincides exactly with the division between living and dead tissue. After separation of the piece of skin, the wound is gradually filled up by granulations. The general principles of asepsis must be strictly enforced. As soon as a dry scab has formed, interference should be avoided. Where the wound has filled up, cicatrisation advancing, and the neighbouring swelling disappeared, the saddle may again be used, with such precautions as a smooth piece of waxed linen sewed into the flaps, where they overlie the damaged spot. Where a felt numnah is used, a piece corresponding in size and position to the injury may be cut out; where a padded saddle is in use, a portion of the padding can be withdrawn. This protects the tender surface while it is denuded of hair and only covered with a thin epidermis.

Injuries to the withers, particularly those complicated with effusion and extravasation into the subcutis, require quite different treatment. In fresh cases cold is useful, but without pressure and without massage. Any considerable pressure usually causes pain, while massage favours further bleeding into the subcutis. After twenty-four hours, moist warmth is to be preferred. To prevent infection and pus formation, an antiseptic can be added to the poultices, which may be prepared with 1 per 1,000 sublimate solution. Where the tenderness is less, and large hæmatomata exist, massage may later be used. Artificial removal of the contents may here be recommended, using a Pravaz's syringe, though antiseptic precautions must in such case be observed very carefully. Both the skin and instrument must first be strictly disinfected; and, after removal of the extravasation, which is frequently lymph, moderate 
pressure should be maintained by means of antiseptic dressings to prevent recurrence. Particular care is required in using the knife, and fluctuating swellings must only be opened when it is quite clear that pus exists. It is preferable, in doubtful cases, to employ a blister of cantharides, sublimate, or biniodide of mercury, by which a swelling is caused and moderate pressure produced on the extravasated material, bringing about resorption.

Pus formation in the region of the withers renders treatment very difficult. Removal of pus and necrotic material, and prevention of fresh pus formation and necrosis are the chief objects to be sought in these cases. The knife must often be used freely. Fistulæ should, if possible, be laid open to their extremity, pockets exposed, and necrotic pieces of lig. nuchæ, of fascia and of superior spinous processes removed as soon as possible. Setons and drainage-tubes assist the escape of the discharge, and prevent fresh burrowing of matter. In the event of pus forming beneath the muscles, these are laid open, but care must be taken not to divide the skin crosswise over the middle line of the back, because in this way wounds result, which heal with difficulty on account of their edges turning inwards. Sometimes suppuration extends to the muscular tissue of the neck, which must then be laid open, usually as deeply as the lig. nuchæ, which, as in poll-evil, requires to be divided. Necrosis of the ligament itself necessitates free removal of the affected parts. In such exceptional cases Hertwig and many others recommend dividing the ligament an inch or two in front of the necrotic portion (desmotomie cervicale). A mass of new tissue forms at this point, constituting a barrier to the further spread of necrosis. Once this is produced the fistula is freely laid open, and all the necrotic portions of the ligament behind the cicatricial tissue removed.

The greatest difficulty is experienced where necrosis has attacked the superior spinous processes of the vertebræ. Separation takes a long time, and during this process new pockets may form and fresh structures become necrotic. Should the diseased parts be removed with the curette or saw, fresh necrosis usually occurs on the surface of the bones, so that with this operation no progress is made. Removal of the diseased spinous process is only successful where permanent irrigation can be provided for, and perfect asepsis attained; but, in such cases, the results are often astonishingly successful. Unfortunately the inflammation and pus formation only occasionally allow of this. Good recoveries can only be expected where pus formation has almost entirely disappeared, and where no marked swelling exists.

Cadiot and Dollar describe a case of fistulous withers complicated with necrosis of the supra-spinous ligament, and at a later stage with necrosis of the ligamentous tissue covering the sides of the superior spinous process of 
the third dorsal vertebra. The necrotic parts were removed with the bistoury and curette, and the wound dressed with creolin, and at later stages with iodine tincture and iodoform. Progress was very slow, and supplementary operations became necessary. Spraying with warm creolin solution, followed by dressing with traumatol, finally proved successful, but the case lasted between four and five months. ("Clinical Veterinary Medicine and Surgery," p. 354.)

Still graver is burrowing of pus under the scapula. In such cases necrosis of the scapula and its cartilage readily occurs, and great difficulty is found in effecting the escape of pus. The process may involve the inner surface of the scapula on account of the extension of purulent process and necrosis of the fascia of the serrati muscles, and the disease thus become further removed from the possibility of direct treatment. In such cases counter openings at the posterior border of the scapula, trephining the scapula, and the insertion of drainage-tubes, may be tried, though such treatment often fails. Portal removed the entire cartilage of prolongation of the scapula through a $V$-shaped incision, the point of the $V$ being above. A portion of the upper margin of the scapula was also removed. Several divided arterioles were ligatured. The wound was dressed for three weeks with Villat's solution, and for a fortnight with Rabel's solution; cicatrisation was then complete. Such success is, however, exceptional.

In bruises of the withers, inflammatory disease of the bursa mucosa lying on the superior spinous processes of the 5th to 7 th cervical vertebræ, may occur. In such cases a flat fluctuating swelling appears on the withers close to the middle line, sometimes on one, sometimes on both sides; often attains the size of a small cheese-plate, and is attended by moderately developed symptoms of inflammation. The condition consists of bursitis with extravasation of serum or blood (hydrops or hrmatoma of the bursa), and may be easily mistaken for extravasation or abscess. Its slow progress, and its appearing on both sides, distinguish it from lymph or blood extravasations, which usually develop rapidly. It is easier, but more dangerous, to regard it as an abscess, because incision always produces pus formation, which it is desirable to avoid. The hard swelling produced by infiltration, appearing around an abscess, distinguishes it from the soft surroundings usually met with in bursitis. Should difficulty occur in diagnosis, an exploratory puncture may be made under antiseptic precautions. After carefully disinfecting the skin, a sterilised needle or an exploratory trochar may be passed. Should serum or blood alone be discharged, it is clear that the case is one of bursitis or extravasation. After removing the contents, compresses should be applied to keep up moderate pressure on the diseased spot, and prevent recurrence of the exudation; while to avoid infection, disinfectants are used in the dressings. Where these cannot be applied, infriction with sublimate ointment ( 1 to 8 ) may be adopted.

V.S. 
Excoriations of the skin must be carefully cleansed, and the surface powdered with iodoform conjoined with tannin or starch flour, or smeared with a neutral fat or ointment (ungt. vaselini). The latter should be applied when swelling attends the excoriation, and after applying the fat or ointment, either cold or Preisnitz's poultices may be used. When such places have healed, the collar must be provided with a soft covering (hare skin) or a small piece of waxed cloth, in order to prevent injury. The active movements of the muscles of the slin sometimes prevent the healing of surfaceinjuries. This occurs especially in summer, when flies abound. In such cases the application of blistering ointment in the neighbourhood of the affected spot is of excellent service.

\section{(2.) BRUISES PRODUCED BY THE COLLAR OR OTHER PORTIONS OF THE HARNESS.}

The horse collar consists of the housing, the two limbs, usually formed of iron, the padding, and the upper and lower open groove. The collar must lie parallel with the scapula without touching it, which it is apt to do if too wide, producing excoriation of the skin over the scapular region. Most frequently the upper groove causes injury; if too narrow it bruises, if too wide the skin is rubbed. As a rule, when the collar is in position, there should be sufficient room to allow of the hand passing between it and the withers. The collar, besides, must be wide enough to be easily pushed over the horse's head, and, moreover, fit the shoulder, so that it is neither pushed towards the left nor right.

Injuries caused by the collar: are in all essentials similar to those caused by the saddle. Those in the neck region have the same significance as injuries to the withers, that is, they are only grave when the subcutis is injured. As long as the swelling is movable and remains sharply defined, the cutis alone has been damaged. In diffuse swellings pus formation easily extends to the lig. nuchr, producing cellular inflammation and necrosis, and burrowing of pus between the cervical vertebræ-a condition which offer's great difficulty in treatment. This is particularly true of necrosis of the membraniform portion of the lig. nuchæ. Such diseases are always obstinate, sometimes incurable; because: cellulitis, set up between the muscles of the neck, leads to abscess formation, and renders it impossible to make counter openings for exit of pus. Extensive swelling and profuse pus formation are amongst the gravest symptoms.

Treatment must be based on the same principles as are laid down for fistulous withers. The skin must not be divided transversely across the top of the neck, but the lateral surfaces need not be spared, and 
muscles undermined with pus must be freely laid open. Cutaneous swellings produced by collar pressure are to be diagnosed and treated in the same way as similar injuries caused by the saddle. In harness horses, and particularly in the heavier breeds, a swelling is often seen at the base of the neck, and about 3 or 4 inches in front of the withers, caused by bruising of a mucous bursa existing at this point; the swelling varies in size between that of the clenched fist and a man's hand. Such swellings should not be opened unless it is clear that pus has formed, as fistulæ of the withers and necrosis of the ligamentum nuchæ may thus be produced. This bur'sa is occasionally the seat of sarcomatous or other new growths. The injuries produced by traces, tail straps, girths, and surcingles usually consist of excoriations of the skin, are caused by the harness not fitting accurately and, during the animal's work, moving continually over the surface of the skin, and so rubbing off hair and epidermis. Such injuries are naturally favoured by a hard and rough condition of the harness. To protect the injured spots, the portions of the harness causing the injury should be covered with smooth waxed linen, which is preferable to hare or rabbit skin, sometimes used.

In cattle similar injuries are caused at the upper part of the neck, by the collar or yolie. The diagnosis and treatment must be as above indicated. In those animals deep-seated suppuration occurs in the region of the poll, giving rise to intermuscular inflammation and further pus formation, and sometimes producing conditions which are very difficult to treat. Bruises on the head in cattle are also caused by yokes, especially if the horns are placed low, or the forehead is markedly convex. Such inflammatory processes readily extend to the periosteum, lead to necrosis of the temporal bone, and may extend to the horn core, producing loosening and loss of the horns. Swelling, increased warmth, and pain occur to a varying extent and degree. The prognosis, however, is usually favourable, if the animals can be laid off work for some time. As to treatment, the general principles of surgery must be pursued. Necrotic bone and loosened horns are to be removed, and the spread of inflammation checked by antisepsis.

\section{VII.-TUMOURS OF THE BREAST.}

In draught horses, tumours occur in and under the skin, chiefly from friction of the collar. They are either single or multiple, and may attain considerable size. Those in grey horses are generally melanotic; in horses of other colours they may be mycofibromatic, or result from infection with bothryomyces, staphylococci, and other pyogenic cocci, sometimes introduced by dirty collars. Wilhelm found enlargements in the skin of the breast, each containing a small pus centre. They had 
been treated with iodine and preparations of mercury without effect. Pflug has lately described certain diseased processes of the skin of the shoulder as tylomata. The cut surface in recent cases often appears cedematous, and on casual examination may easily be mistaken for a myxomatous growth; the older swellings are firm and hard (tylomata fibrosa, Pflug). Nor is it astonishing that new growths often rise from the cutis or subcutis, considering the chances of infection here on account of the epidermis below the collar being macerated and not infrequently injured. Many horses in the same stable have been seen to suffer from this disease, the tumours appearing under the collar and producing inflammatory swellings from bruising. In horses, local centres of inflammation occur in the shoulders, forming little nodules. They originate in the sebaceous and hair glands, and are commonest during warm weather. If they remain unnoticed, and the animals be kept at work, inflammation spreads and abscesses form in the site of the glands.

Prognosis of such enlargements depends on their extent and position, usually determined by careful palpation. Small tumours are extirpated with the scissors, large with the knife; when taken in hand early recovery is usually complete, although recurrence is not infrequent. If the neglected swelling attains a considerable size, possesses a broad base, or extends to the pre-scapular lymphatic glands, removal is more difficult, When convinced that these glands were implicated, Möller has repeatedly excised them without particular trouble or danger. The "heat bumps" require the animal to be kept from work, or the collar to be eased. Moist warmth favours their absorption. Those suppurating must be opened early.

\section{VIII.-TAPPING THE CHEST (PARACENTESIS THORACIS, THORACOCENTESIS).}

This operation, practised in man since the earliest times, was first introduced into veterinary surgery by Lafosse in 1772. It is adopted to remove fluids, especially pleuritic effusions, seldom to get rid of air. The diagnosis of such conditions is explained in the text-books on special pathology, and can usually be determined without the explorateur. In detecting empyema, however, the exploring needle is serviceable. Pleuritic effusions compress the lungs and diaphragm, and, interfering with respiratory movements, and the heart and great vessels, impair circulation. Tapping relieves these untoward conditions, but does not always arrest outpouring of fluid or save life. Hence the operation is often regarded as an experiment, and is only occasionally performed. Its timely adoption is frequently useful where the pleural cavity contains pus or septic materials, especially if the pus can be washed out with 
disinfectants. No permanent benefit results from removing blood from the pleural sac, because if the membrane is not seriously diseased absorption rapidly occurs. In perforating wounds of the chest, the early injection of antiseptics, and performance of paracentesis, may prevent injurious effects from decomposition of extravasated fluid; but in chronic hydrothorax, which has resulted from impaired circulation or change in the composition of the blood, it only hastens death.

\section{(1.) Puncture of the Thorax for Removar of Serous or Pleuritic} Exudate.-The trochar used for the larger animals has a diameter of $\frac{1}{4}$ inch; but one of smaller calibre is usually preferable. Side openings in the canula are not of particular value. Various precautions require to be observed :-

(1) Carefully disinfect the instrument and site of operation.

(2) Prevent entrance of air into the thorax.

(3) Remove contents slowly, to minimise interference with circulation.

The patient, if possible, should stand, as the recumbent position only adds to the difficulty of the operation. This applies equally to the smaller animals especially if there is much dyspnoea. Dogs with hydrothorax sometimes die in a few minutes from being placed on the side. The operation is not now performed as low down as formerly. In all animals the opening should be made either slightly above or slightly below the subcutaneous thoracic vein, keeping clear of the large pectoral muscle, which lies still lower. The upper position deserves preference. Where both pleural sacs contain fluid, the operation should be performed on the right side, to prevent injuring the heart. The vertical boundary for punctures lies in the horse between the 5 th and 8th ribs; in oxen, between the 6 th and 8 th; in swine, between the 7 th and 9 th ; and in carnivora, between the 5 th and 9 th. In actual practice the ribs are often disregarded, and in the horse the puncture is made a span behind the point of the elbow. (For relations of the ribs to thoracic contents, see figs. 143 and 144.)

The hair is first shaved from the site of operation, the skin washed with soap and rinsed with rether, and finally saturated with such disinfecting fluid as diluted sublimate. Similar care must be taken in disinfecting the trochar. In private practice this is most easily and safely effected by boiling the instrument in water, or laying it in a 3 to 5 per cent. solution of carbolic acid. To facilitate the entrance of the instrument, often difficult in animals with thick skins, an incision is first made with a bistoury or lancet. The trochar, grasped with the handle in the hollow of the right hand, is supported with the thumb and fingers, while the index finger, more or less extended, is fixed on the canula at the point to which the trochar is to enter. This 
distance varies according to the thickness of the thoracic wall, and in the larger animals varies from $1 \frac{1}{8}$ to $2 \frac{1}{4}$ inches. Should the skin be movable on the sublying tissues, it may be pulled forward, and the trochar introduced perpendicularly to the chest-wall until the index finger meets the skin. The stilette is now withdrawn with the right hand, whilst the left supports the canula, and the left index finger is held ready to close the opening against entrance of air, which may occur
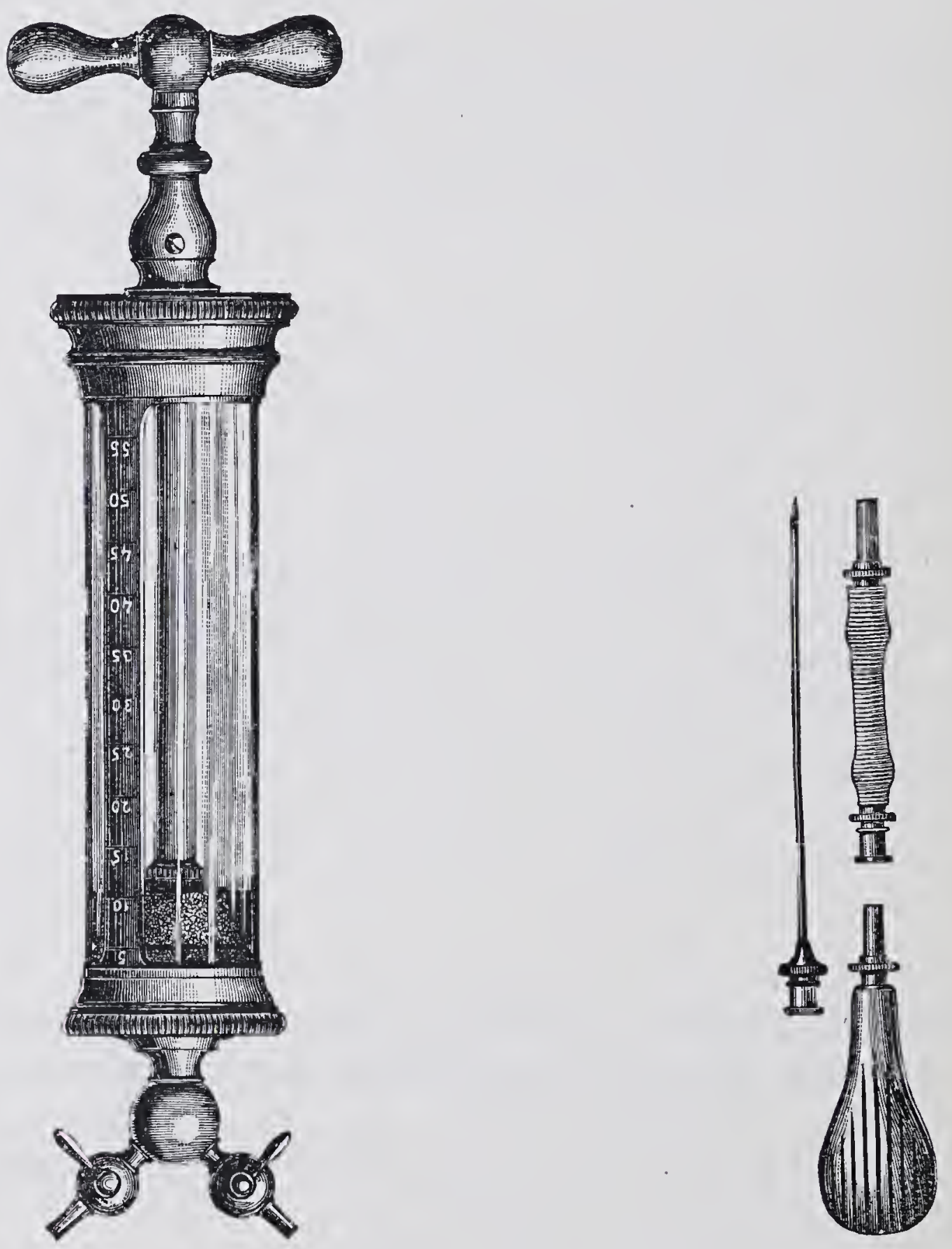

FIG. 119.-Dienlafoy's asprirator, with handle for introducing the needle and flexible commection for syringe.

when the flow of fluid ceases, when inspirations are specially deep, or when coughing ensues. If the discharge of fluid is prematurely checked by clots of lymph or blood, or by apposition of the lung, a few slight lateral movements should be made with the canula, and the stilette or a carefullydisinfected probe passed through it. To facilitate the discharge of fluid and prevent access of air, various arrangements have been proposed.

Schuh has devised a trochar, carrying at its outer end a reservoir (trough or bath), so that the opening of the canula is always immersed in fluid; but this arrangement is not reliable, and is little used. The 
so-called aspirator (fig. 119) consists of a rubber tube, hollow needle, and large syringe provided with a tap. The hollow needle is inserted, the piston raised, and when the syringe has become full, the tap is turned, and the contents discharged. The process is then repeated. The aspirator, however, has been little used in veterinary surgery. The same object is more easily attained by pushing one end of a suitable indiarubber tube over the free opening of the canula, and dropping the other end into water. This syphon-like arrangement exerts an aspirating action on the contents of the thorax, but has the disadvantage that the outflow cannot be exactly controlled, while forcible aspiration may rupture pleural vessels.

While using the ordinary instrument, if it be required to check the outflow of fluid, or prevent entrance of air, and especially if excitability, dyspnœa, and coughing set in, the trochar must be removed. The necessity of removing pleuritic effusion gradually, points to the

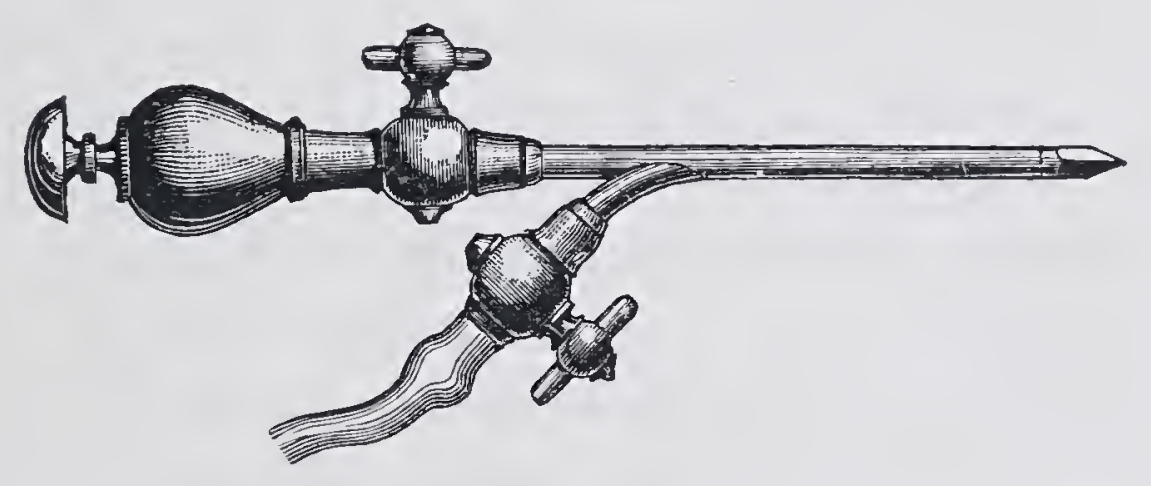

FIG. 120.-Special trochar and canula. The second limb is provided with a long rubber tube, allowing the instrument to be used as an aspirator.

use of trochars of moderate calibre. In carnivora, and to a less extent in horses and oxen, rapid discharge of large quantities of fluid is liable to produce sudden changes of intra-thoracic pressure, determining grave disturbances of circulation, which may occasion not only fresh extravasation, but pulmonary bleeding, syncope, or even sudden death. The danger is greatest in left-side extravasations, by which the heart has been pushed towards the right side. It is, therefore, advisable in horses and cattle to remove only 10 to 15 pints at one time, and a further quantity some hours later, or on the following day. From the position of the tapping, the complete evacuation of the pleural sac is impossible; but even if it were possible, as already pointed out, it would not be desirable. In too many cases fresh extravasation soon takes place. When sufficient fluid is believed to have been removed, the stilette, previously cleansed, is again introduced, and the canula carefully removed, the wound rinsed with a disinfectant, powdered with iodoform, and closed with wound gelatine or collodion. A simple application of tar may be sufficient, but a suture is sometimes inserted. The benefits of paracentesis mainly result from its modifying intra-thoracic pressure, 
relieving compression of the lungs, and improving conditions for absorption.

(2.) Puncture of the Thorax for Removal of Purulent Fluid.Thoracic puncture for purulent or putrefactive collections, or for abscesses in the lungs, is not often employed, and rarely proves successful. The procedure is the same as in pleuritic effusion, particular attention being paid to antiseptic precautions. Exploratory punctures may sometimes be needful for diagnosis.

After complete removal of the contents, it may be desirable to wash out the cavity, or the abscesses between the layers of the pleura, or in the lung. For this purpose Lafosse used aromatic fluids. Hoffmann employed the tube used for carrying off the fluid, for introducing into the thorax chloride of sodium, 5 per cent.; sublimate 1 in 5,000 ; carbolic acid 5 per cent., or concentrated salicylic acid, or thymol solutions, warmed to $98^{\circ} \mathrm{Fahr}$. What success was attained is not recorded. In man, an incision is sometimes made into the upper part of the thorax (thoracotomy), a portion of a rib removed at a lower point, and the sac freely irrigated. Such operations are far less hopeful in our patients, in which treatment is generally a question, not so much of saving life, as of restoring usefulness. Under these circumstances the operation is burdened with conditions which it is unable to satisfy, and which render it difficult to carry out in private practice. The same is largely true of the surgery of the lung, lately introduced in man, which aims at direct operative treatment of diseased processes in the lungs and in other organs of the chest. As a last resort in the treatment of a valuable patient thoracotomy might, however, be tried.

(3.) Tapping the Pericardium in the Dog.- - In the dog, tapping the pericardium becomes necessary in the treatment of exudative pericarditis, which occurs in the acute and chronic forms. Attention is always first attracted by the physical symptoms.

As soon as the pericardium contains a certain quantity of liquid, the heart is thrust upwards and a little forwards, the degree of displacement depending on the amount of exudate. The pericardial sac becomes distended, especially about its base, lifting the pulmonary lobes, and pushing them upwards towards the vertebræ, though to a much less degree than in pleurisy. Thus on palpation of the precordial region the heart's impulse appear's weak, or seems lost. On auscultation the normal sounds, clearly detected in health even in very small patients, are dull, distant, and smothered, or completely inaudible. When the heart is but slightly compressed the pulse preserves its character; but as soon as pressure becomes marked the pulse 
grows small, fugitive, and weak, sometimes almost imperceptible and uncountable.

Compression of the auricles-the portions of the heart which collapse most readily-impedes the flow of blood, produces cyanosis, venous pulse in the jugulars, and mechanical dyspncea owing to stasis of blood in the lungs. The symptoms of oppression at first seen are undoubtedly of reflex origin, due to the pain in the inflamed pericardium:

If not treated, acute pericarditis may rapidly lead to death, sometimes in less than a week. It may also (though exceptionally) terminate in recovery, the exudate becoming reabsorbed, the symptoms gradually diminishing and finally disappearing. In certain cases it assumes the chronic form.

The variety of pericarditis to which the term hæmorrhagic has been applied is characterised by a reddish, sanguineous exudate, and occurs somewhat frequently in the dog. It is not infrequently of tuberculous origin, and is always very grave in character.

In general, when the veterinary surgeon is called on to examine a dog affected with exudative pericarditis, the disease has already been in existence for some time, occasionally for several weeks; and, provided he makes a complete examination of the patient, and does not forget the heart, a careful consideration of the signs furnished by palpation, percussion, and auscultation should enable him to arrive at a correct diagnosis. Ascites is often the most striking symptom, and puts one on the right path. It is usually easy to differentiate between pericarditis and pleurisy. In pleurisy with moderate exudation, resembling that of pericarditis, the zone of dulness changes with the animal's position. By standing the animal on its hind legs the heartsounds and vesicular murmur become readily perceptible, while the upper part of the thorax is resonant; in the normal standing position the resonance disappears or becomes dulled.

When, despite treatment, the exudate increases and the symptoms become more marked and alarming, or when even on first examination the general condition appears dangerous, the pericardium can be punctured.

Operation is as follows:---The precordial region is prepared by clipping away the hair, and shaving the skin a little below the centre of the zone of dulness for a distance of two or three square inches. The parts are afterwards washed with alcohol, and with a $\cdot 1$ per cent. solution of sublimate. The aspirator is provided with an indiarubber tube. The air being exhausted from the cylinder of the aspirator, the instrument is passed to an assistant; the point of the needle is then introduced at the centre of the prepared surface, through the fifth 
intercostal space three or four fingers' breadth above the lower margin of the thorax. As soon as its end has fairly entered the thoracic wall the tap connected with the aspirator is opened and the needle very gently pushed forward until liquid appears in the glass index of the rubber tube. Operating in this way the needle need only just enter the pericardium, and with a short point, injury of the heart (which is always pushed upwards and shortened in its vertical axis) need not be feared. Furthermore, by using a small needle, fluid is very slowly withdrawn and danger of syncope prevented. In the absence of an aspirator, puncture may be effected with a fine trochar. The injection of warm 1 per cent. boric acid solution or normal salt solution has been suggested after withdrawal of the fluid contents of the sac, but is difficult to perform and of doubtful utility. After operation the wound is closed by painting with collodion, and the parts covered with a cotton-wool dressing and a bandage.

During the following days the patient is fed on milk, milk preparations, meat juice, or fragments of raw meat. When appetite returns, more substantial food and tonics may be given.

When pericarditis is complicated with ascites it is not always necessary to tap the abdomen. Once the pericardium is relieved, the peritoneal exudate tends naturally to become absorbed. 


\section{SURGICAL DISEASES OF THE ABDOMEN.}

Introduction. The abdominal walls consist mainly of the skin, yellow elastic tissue, a fibrous aponeurosis, particularly strong in herbivora, the muscles, a layer of fat, and the peritoneum. The muscular fasciculi partly overlie and cross one another, and have numerous tendinous expansions. In herbivora the stout, thick abdominal walls, which always appear distended, have to support the bulky digestive organs, and effectually protect them from external injury, but render examination of them almost impossible. The abdominal walls are externally clothed with skin, possessing a well-developed subcutis, whilst their inner surface is covered with peritoneum, which both lines the cavity and invests its contained organs. The uninjured peritoneum is smooth and shining, allowing the abdominal viscera to play easily over each other; but when inflamed, exudation occurs, and the surfaces may become adherent. Except as a result of penetrating wounds (more rarely of bruises), the abdominal organs are seldom injured from without. Voulton (Stockfleth) found, however, the rumen of an ox ruptured by a blow from a horse's foot. Death rapidly followed from peritonitis.

In horses, ruptures of the spleen have been observed.

The abdominal walls receive their blood from two sources: the lumbar arteries enter between the lumbar muscles, pass obliquely across the abdomen, between the outer and inner oblique abdominal muscles, and between the latter and the transverse abdominal muscle, and communicate frequently with the vessels of the abdominal parietes. The anterior abdominal artery arises from the internal thoracic artery and passes backwards. The posterior abdominal artery, arising from the prepubic artery, takes a course close below the peritoneum in the middle line, passes the inner abdominal ring in a forward direction, and anastomoses with the anterior abdominal and with the lumbar arteries.

\section{I.-BRUISES OF THE ABDOMINAL PARIETES.}

These occur rather frequently in the domestic animals, and are caused by kicks, thrusts with the horn, treads, by animals rushing together, or by collisions with vehicles. The nature of the injury mainly depends on the character of the injuring body, and the force with which it is applied. Sometimes the walls are ruptured,-a condition described later; whilst the skin, on account of its greater elasticity, remains intact. Sharp objects produce surface wounds, sometimes perforate the abdominal walls, and may even penetrate the internal organs; these last accidents will also be considered later. Bruises of the soft portions of the abdominal parietes from horn-thrusts, \&c., are frequent in herbivora, on account of the tension of the abdominal walls; and, as in other regions, are characterised by a subcutaneous solution of continuity in the vessels and tissues. When 
small blood-vessels and limited tracts of tissue are ruptured, more or less hard, firm, inflammatory swellings result. When larger vessels are ruptured, swellings (hæmatomata) resembling herniæ are produced. In large animals extensive extravasations commonly develop in the subcutis, or under the panniculus, but are rarely seen under the slightly elastic, yellow abdominal tunic. The tunica abdominalis may likewise be divided, and the rupture mistaken for a hernial opening. But such faulty diagnosis is less serious than when a hernia is taken for an extravasation, and treated as such.

Progress. Simple inflammatory swellings, even when of moderate size and situated on the yellow abdominal tunic, are more easily reabsorbed than large hæmatomata. But when the swelling disappears, a portion of the abdominal contents may present itself under the skin. From its fluctuating character this new swelling may be mistaken for an abscess. An abscess, however, is distinguished by its periphery of firm solidified tissue, and softening centre. When an abscess forms, it generally perforates outwardly, seldom discharges into the peritoneal cavity or into the intestine, and, when opened, usually heals. Inflammatory processes invading the peritoneum cause thickening, and occasionally lead to adhesions limiting the movements of the bowel, the walls of which may give way, as Curdt has noticed in the horse. Hæmatomata undergo a course similar to that of inflammatory swellings, but their resorption is less probable. On their perforating or being opened, they discharge offensive fluids and sometimes gas.

In cattle, less frequently in the other domestic animals, "cold abscesses" occur in the walls of the abdomen. The cause may escape observation, the injury remaining unrecognised for a considerable period; like those of strangles, "cold abscesses" may have a metastatic origin. In cattle especially, months may elapse before any change is noticed, but then the swelling suddenly and rapidly increases, becomes painful, sometimes contains gas, may produce high fever, and, if not opened, soon perforates.

The appearance of the swelling, as before stated, may vary. It may be firm, hard, and painful : sometimes it is fluctuating. ' When recent, it is often associated with odema, especially in deeper lying neighbouring parts, under the belly. As pointed out, it may be mistaken for a hernia, and the rule should be observed not to operate before being perfectly convinced of the absence of rupture. The compressibility of the hernia is certainly characteristic, but nevertheless mistakes easily occur. In doubtful cases, an exploratory puncture may be made with antiseptic precautions.

Treatment. Newly developed swellings are first treated with cold applications to prevent further extravasation; subsequently moist warmth is 
used to assist dispersal. Disinfectants, conjoined with the other applications, will check infection and pus formation. Where this has already occurred, early opening is advisable; indeed, the sooner this is done the sooner will the swelling disappear, but care must be exercised in diagnosis. Owing to the strains thrown on the abdominal muscles in working animals the parts are more liable to tear and produce hernix, and therefore rest is essential in such cases. "Cold" or chronic abscesses are best opened as soon as it is clear that no hernia is present, and that incision can be made without fear of severe bleeding.

\section{II.-WOUNDS OF THE ABDOMEN.}

Wounds of the abdominal parietes may, from a clinical standpoint, be thus divided :-

(a) Surface wounds; those that do not divide the abdominal walls.

(b) Penetrating wounds, dividing the parietal portion of the peritoneum.

(c) Abdominal wounds, with prolapse of internal organs.

(d) Abdominal wounds, with injury to internal organs.

(a) Surface wounds, if not of great extent, and not likely to be followed by further laceration of the abdominal walls, merely require to be kept clean, and are treated on antiseptic principles. A tar plaster is sometimes useful. Bandages are difficult to retain in position, and can generally be dispensed with. Clean straw must be provided for bedding. Where the wound discharges freely, it is dressed with disinfecting fluids. Such wounds generally heal well if freely dressed with iodoform-tannin or glutol. If pus burrows, counter-openings, drains, or setons are resorted to. Where the walls are extensively lacerated, and further laceration is possible, the wound should be carefully sutured, and supported with a bandage. Strong and deeply-inserted stitches are required, pin sutures may be necessary, and thorough rest should be enforced. Burrowing of pus sometimes occurs between the abdominal muscles. This condition is treated in the same way as sinus-formation in the thoracic region.

(b) Penetrating wounds are usually caused by thrusts with the horns, by sharp instruments like stable-forks, lances, bayonets, knives, or scissors, by gun-shots, by injuries from leaping over hedges or fences, or by falling on sharp objects, as harrows, spurs, \&c. The bites of dogs may also penetrate the peritoneal cavity. The perforating character of the wound can sometimes be determined from the appearance of the body producing the wound. The probe should not at once be used, as there is risk of its conveying infectious material into the peritoneal cavity. Besides, its value for diagnosis in such cases is small, owing to the layers of muscle being often displaced, and thus occluding 
the channel of the wound. Where perforation is suspected, the case should be dealt with as if it were proved to exist. Penetration of the peritoneum may shortly be demonstrated by symptoms of peritonitis, the distension of the abdominal wall, small, wiry, frequent pulse, paleness or dirty red colour of the visible mucous membranes, slight or continuous colic, and, in carnivora, vomiting. Perforation, however, may occur without peritonitis. It may be disclosed by protrusion of abdominal organs, or discharge of the contents of the bowel.

Every penetrating wound of the abdomen must be regarded as dangerous. The abdominal cavity is now, however, frequently opened, with greatly reduced risk, in surgical operations undertaken for the relief of enteric tympanites, abdominal ascites, and the castration of females and cryptorchids. Numerous accidental injuries with perforation often heal, even in circumstances apparently unfavourable to recovery. (For illustrations showing the relative position of the abdominal contents see figs. 143, 144 and 145, section on "Puncture of the Bowel.")

Prognosis principally depends on whether prolapse of the intestine and peritonitis can be prevented. Unlike men, animals cannot be kept for any considerable period lying on their back, or even quietly in the recumbent position, and there is thus greater danger of prolapse of the bowels. Peritonitis can be prevented by antiseptic treatment of the wound. So long as asepsis is maintained, recovery need not be despaired of. Even where pus formation has occurred, this is not impossible, provided the inflammatory disease has not extended to the peritoneum. Retention of septic fluids and their entrance into the abdomen are the chief dangers, and the prognosis largely depends on whether they can be prevented or not. The peritoneum varies in sensitiveness in the various classes of animals. Wounds penetrating it are less serious in carnivora and ruminants, but more so in horses, in which the risks of peritonitis are greater.

Treatment. The chief indications are to maintain asepsis, and prevent prolapse of internal organs. The first is attained by careful cleansing and disinfection of the wound and its neighbourhood. If necessary, bleeding must be checked, by ligaturing injured vessels, and blood prevented accumulating in the wound, but tampons are only employed in case of need. Foreign bodies, and loose shreds of tissue, likely to become necrotic, should be removed. The wound is washed out very carefully with disinfectants, one of the best of which is, corrosive sublimate. It may then be sutured with sterilised silk; cat-gut is not sufficiently strong. The abdominal muscles and the skin should be brought together separately with deep stitches, so that should washingout afterwards become necessary the outer stitches need alone be loosened. On account of the great strain which the stitches must 
bear, particularly in herbivora, it is better in closing wounds of the abdominal walls to employ quilled sutures. Where pocketing of considerable quantities of wound discharge is probable, drains must be inserted, and the parts once more rinsed with disinfecting fluid. The sterilised material (wadding, wood wool, or jute, sc.) may, in larger animals, be held in position with soft cardboard or a straw mat; where these are not available, a sack or woollen rug may be used. The several dressings are kept in place by bandages or girths passed between the animal's forelegs, and over its neck in the long axis of the body. Bandages also are sometimes introduced between the layers of dressings. In small animals, to prevent displacement from breathing movements, the bandages are wound around both the thorax and abdomen.

Should it subsequently become necessary to cast a large animal, in order to insert stitches or adjust dressings, care will be required to prevent prolapse and to protect prolapsed organs. For these purposes it may be necessary to apply a provisional dressing. Under certain circumstances anæsthesia is of assistance. After adjusting the dressing, the animal must be kept as quiet as possible. Dogs are often troublesome, and as they try to tear off the dressing, must be continually watched, or receive a full dose of morphine, so that they shall sleep for several hours. 'The larger animals may, if necessary, be placed in slings.

For several days the food chosen should be digestible, nutritious, and small in amount, and water given only in small quantities. During this time. fever may supervene; if not marked and if symptoms of colic are absent, a favourable termination may be expected. The dressing is left undisturbed, unless it become saturated with discharge, or fever or other untoward symptom appear, when it is removed, the wound cleansed and disinfected, and the dressing replaced. Where from any cause dressings cannot be employed, the wound, after being cleansed and sutured, is irrigated with antiseptic fluids. Danger of wound infection and peritonitis is greatest during the first three days, but for some time afterwards the same precautions regarding the wound must be observed and care taken to facilitate drainage. A rise in temperature points to peritonitis, and necessitates fresh examination of the wound, or at least the renewal of the dressing.

(c) Abdominal Wounds, with Prolapse of Internal Organs. The prolapse of portions of omentum, bowel, uterus, or other abdominal organ, constitutes a dangerous complication. The prolapse per se is not so dangerous, but there is much difficulty in returning and retaining it in position, preventing soiling and injury and the entrance of infectious materials into the abdominal cavity. Walthard showed by experiments on dogs and cats that the injurious action of the air on the peritoneum 
depended on the abstraction of moisture, and not on direct chemical irritation, as had previously been supposed. Early reposition is therefore the most important indication.

Omental prolapse is least dangerous, because, in the event of its return being impossible, a large piece may be removed without bad consequences. This is often done with impunity in castrating horses and other animals. Where the omental protrusion is large and contains important blood-vessels, a portion is withdrawn, a sterilised ligature applied to the healthy part, the portion below cut off, and the remainder thrust back in to the peritoneal cavity, or, still better, into the abdominal wound, to which it becomes attached, preventing egress of other organs and entrance of infective wound secretions. The portion of omentum thus detained in the wound shows no great tendency to set up inflammatory processes. If the protruded omentum has become soiled or necrosed, its removal is still more imperative. All experienced practitioners recommend its being further withdrawn and cut off. Möller has repeatedly seen abdominal wounds in dogs and cats, in which the prolapsed omentum was converted by injury into a black greasy mass with an offensive smell, but when this was excised, healing quickly ensued.

Prolapse of the bowel is more dangerous, because the viscus must be returned to the peritoneal cavity. Recoveries neverthless occur in all animals, provided the protruded portion be carefully cleansed and replaced, and fresh prolapse prevented. It is important to effect reposition before the serous covering has become dry (and necrotic). The introduction of a few germs though risky is not particularly dangerous, as the healthy tissues are quite capable of destroying them. When the serosa is injured, however, its vitality is lowered and the conditions are all in favour of the growth of such organisms, which then become a very serious menace. Prolapses of the large intestine are generally least troublesome. In the horse, prolapse of the large intestine or cæcum, following injury to the under portion of the abdominal walls, is more easily reduced and kept in position than that of the small intestine, because the colic mesentery is shorter than that of the small intestine, and the viscus projects less, hence there is less risk of infectious organisms being introduced into the abdominal cavity. Drolshagen treated a foal, which had been wounded by a horn thrust; the omentum and bowel were prolapsed, the extruded bowel, which was as large round as a bee-hive, hindered the animal's movements, and compelled it to lie down frequently. After thorough cleansing the bowel was returned, a portion of the omentum cut off, the wound sutured, and a dressing applied. Though it had a few slight attacks of colic, the patient appeared restored to health in three weeks, but four months afterwards it died. Post-mortem showed that the small intestine had 
passed through a rupture in the mesentery, and become strangulated. Kleinpaul saw a penetrating abdominal wound in the umbilical region of a mare, caused twelve hours previously by injury from the teeth of a harrow. A portion of the small intestine, as large as a man's fist, was prolapsed, odematous, and swollen. This was replaced, an iodoform dressing applied, and food being withheld for four days, perfect healing resulted. Fourteen days later the mare gave birth to a foal and did well.

In treatment of prolapsed bowel, uterus, or other abdominal viscus, the chief point is to prevent injury and soiling. For this purpose, if large animals have to be cast, a broad cloth soaked in disinfectant fluid is first passed round the body. The wound and its neighbourhood; together with the protruding portion, are then carefully cleansed with a lukewarm disinfecting fluid (carbolic, salicylic, or sublimate solution), and endeavours made to replace the prolapse, which effected, the wound is once more cleansed, any foreign bodies carefully removed, and the parts stitched up. In some cases it may be necessary to enlarge the wound before reduction can be effected. The after-treatment is similar to that described in penetrating wounds. Should the protruded bowel have become necrotic recovery is rare, and the formation of an artificial anus or resection, which is worth attempting, offer the only chances of saving life (compare "Foreign Bodies in the Bowel"). Small doses of opium check the action of the bowel and diminish danger of peritonitis.

(d) Abdominal Wounds with Injuries to Contained Organs. In herbi= vora the colon is most frequently injured, on account of its great area, its distension with hard food, and its fixed position. The more mobile small intestine being generally filled with fluid frequently evades the object producing the injury. In this way, as experiments have shown, pointed instruments, and even projectiles, may penetrate the abdomen deeply without injuring this intestine. The escape of the dog transfixed, as above described, is thus explained. Horses frequently recover from injuries of the colon and crecum, although their peritonem is much more sensitive than that of the ox, dog, or fowl.

Guilhelm (Stockfleth) saw a horse with a wound $5 \frac{1}{4}$ inches in front of the umbilicus, produced by a horn thrust, and through which the under portion of the double colon protruded in a mass as large as a man's head. A wound in the bowel, $3 \frac{1}{4}$ inches in length, discharged food material. This was stitched, the bowel cleansed and replaced, the wound in the abdominal wall sewn, and a dressing applied over all. In spite of great swelling, recovery occurred in twenty-five days.

In this connection, Herbet made experiments in pigs. In castrating a sow, he intentionally thrust his finger through the colon, drew the injured spot into the skin wound, and sewed it up with waxed thread. The animal received no food for several days, and recovered. The extent of the recuperative power's is further illustrated by a case reported by Richter. Whilst endeavouring to

V.S. 
insert a seton under the belly of an excitable horse, he was unable to separate the skin with his finger and forced to use a probe-pointed bistoury and a seton needle. Immediately the operation was completed, a yellow discharge of food issued from the wound. Examination with the finger showed that the cæcum had been divided to the extent of $\frac{3}{4}$ of an inch. Within a short time 6 to 7 quarts of fluid were discharged, the horse during several days having received only gruel. Richter expected that the animal would die, but the owner determined not to lose its service, and yoked it into a heavy manure waggon. Eight days later Richter found his patient working in a plough, and perfectly well; in twenty days the seton was removed, and the horse remained perfectly healthy. Such favourable conclusions are unfortunately exceptional, but they emphasise the importance of always attempting treatment.

Treatment consists in suturing the injured bowel or uterus with sterilised cat-gut or silk. (For further particulars, compare "Bowel Suture.") The injured organs must then be carefully cleansed and disinfected, the wound and its neighbourhood being next attended to. Sometimes it may be possible to cleanse the peritoneum by washing out the cavity with lukewarm, previously boiled water. It might be useful in certain cases to arrange for drainage by inserting strips of iodoform gauze, or by packing the parts with the same material, as is done in luman surgery. The subsequent treatment has already been described. The rumen may be incised without dangerr, as in rumenotomy and puncture; but, in horses and dogs, injuries of the stomach are serious; more so, in fact, than those of the bowel and uterus. In carnivora they are attended with vomiting, but, although serious, are not always fatal. The successful use of Haynes's stomach trochar also testifies that horses sometimes survive wounds of the stomach. The treatment of injuries of the stomach is similar to that in wounds of the bowel.

Abdominal wounds, complicated with injury of the kidneys, are distinguished by the passage of bloody urine, and are generally accompanied by paraplegia. In such cases early slaughter is advisable. Injuries to the bladder allow urine to enter the abdominal cavity, and are recognised by anuria, sometimes by urine flowing from the abdominal wound. They almost invariably result in death; but recovery occasionally occurs, especially in carnivora, as shown by Rodloff's case. Wounds of the bowel are sometimes accompanied by the passage of blood-stained freces. In the dog, vomiting of blood indicates injury to the stomach. A dog had a penetrating abdominal wound, associated with prolapse of the bowel, which was replaced and the wound sewn up by a layman. In subsequently operating for the rupture, the bladder was cut into. After it had been sewn up and a ligature passed round the hernial sac, both healed. Ponfik's latest researches show that, although most injuries to the liver end in death, yet they are not necessarily fatal.

Stockfleth has collected statistics concerning the progress of abdominal wounds, complicated with prolapse of the bowel. In twenty-seven abdominal 
wounds, prolapse was noticed fifteen times. Of fourteen horses, two mules, two cows, and one dog, two horses, one cow, and one mule died. This result gives, however, no indication of the relative gravity of these abdominal wounds, the successful cases apparently having been selected for publication. The course is usually less favourable. Jewsejenko saw thirty-one abdominal wounds in the horse during the Russo-Turkish war. Those caused by large projectiles were the gravest. The smaller the projectile, the less the danger. Of eight horses with shot-wounds injuring the bowel, five died with peritonitis ; three recovered after suturing the wound. Two wounds of the stomach, produced by splinters of granite, led to death. One wound of the stomach from a ball was sutured and healed. Of four wounds of the liver, three were fatal; one case recovered. Of three wounds of the spleen, two ended fatally; one recovered after the projectile had been removed with dressing forceps.

After penetrating wounds of the abdomen, animals, like men, sometimes die rapidly from collapse. It was formerly believed that this peracute progress was owing to shock; but more recently it has become evident that the active agent is profuse bleeding into the abdominal cavity. This must be borne in mind, and in recent injuries the pulse and mucous membranes must be examined before giving a prognosis, or attempting operative interference, which may be blamed for the animal's unlooked-for death.

In order to study the effect of injuries produced by pistol bullets discharged into the abdomen, Parkes experimented on thirty-seven dogs; three died immediately from bleeding from the aorta, or from the renal or splenic arteries; twelve lived for one day, and then died from bleeding, with appearances of shock. The others lived longer; but in almost all the bowel contents obtained entrance into the peritoneal cavity, though they could not be detected in the skin wound. Of the twenty-one which did not immediately perish, two died from peritonitis. Of twenty-one treated by laparotomy, ten died in from three days to three weeks, and nine were cured.

Chaput also produced experimental abdominal wounds and injuries of the abdominal organs in dogs. Of those subjected to immediate treatment the whole recovered; where treatment was expectant only 32 per cent. Chaput therefore recommended performing abdominal section in the middle line, drawing forward and fixing the bowel by passing a director through the mesentery, and seeking out and suturing the injured spot. LanzillottiBuonsanti collected a large number of clinical records referring to various forms of abdominal injury, from simple perforating wounds to such as involved severe injuries of the contained viscera. Of eighty-three cases in solipeds, sixty-five recovered and eighteen died; five cases in cows all recovered, as did four cases in pigs. Numerous recoveries from similar injuries have more recently been recorded, thanks to antiseptic treatment.

\section{III.-BOWEL FISTULA (ANUS PRETERNATURALIS).}

'I'he term bowel fistula is applied to a direct communication between the lumen of the bowel and the external air through the intestinal wall. A probe introduced into the fistula passes through the abdominal walls directly into the bowel. The opening may occur at different points, but 
is generally found on the lower surface of the abdomen, in horses often close behind the last rib.

Bowel or gastric fistulæ-not infrequently produced for physiological objects-may also result from accidental injuries penetrating the abdominal coats, and Curdit related cases of the kind both in the horse and the ox. Howard produced fistula of the colon in a sucking: pig, which was suffering from atresia ani, in order to save the animal. If, in penetrating abdominal wounds, the intestine is laid open, its edges may unite to the abdominal wound and external skin, and produce a bowel fistula. Arndt, Lindenberg, Dammann, and others have described such cases in horses and oxen. The injury, however, sometimes originates in the gastric or intestinal mucous membrane. The serosa becomes inflamed and firmly adherent to the wall of the abdomen, and if now abscess formation occurs, the abdominal walls may be perforated and a bowel fistula produced. Körber saw a horse suffer in this way after an attack of colic. Perforation had occurred close to the middle line of the abdomen behind the umbilicus. Urban reported a similar case in a foal, in which an umbilical hernia had been opened, producing bowel fistula. Bayer noted a like accident after dressing an umbilical hernia with nitric acid. Fürstenberg describes a fistula of the abomasum in a cow. Seven to ten minutes after receiving water, a stream of fluid mixed with food was projected several feet beyond the wound. Flourens produced fistulæ of the rumen artificially for the purpose of studying rumination in oxen and sheep, and Haubner saw gastric fistulæ in sheep result from giving arsenic insufficiently powdered. Foreign bodies swallowed by cattle often perforate the wall of the abdomen, or that of the thorax close behind the elbow, and produce gastric fistulæ, but these generally heal. Strecke found one half of a pair of scissors in the abscess. Dammann describes a case complicated with hernia in a nine-year-old mare. The fistula had resulted from an external injury.

Symptoms and course. Animals, with intestinal fistula, may survive a long time, and, if liberally fed, may even remain in good condition, though the constant discharge constitutes a blemish. Urban kept a foal under observation for two years. In spite of generous feeding it remained thin, and was finally killed on that account. In another case recovery took place.

Treatment should be directed to preventing discharge of bowel contents, which is the chief obstacle to healing. Körber succeeded by passing a red-hot wire into the fistula, after which cicatrisation occurred in twelve days. Lindenberg recommends a purse-string suture, inserted as deeply as possible so as to bring together the inner end of the fistula, i.e., the opening into the intestinal wall. It is not sufficient to close the external opening by bringing the skin together, as new abscesses continually form. 
Dammann used sutures in one case, but attacks of colic occurred and caused the ligatures to tear out, and as the animal was no better after five months' treatment, it was killed as incurable. Where the opening is small, dressing with irritants, or the use of the actual cautery, may cause swelling of the edges and union; but should this fail, a strong thread may be passed through the muscular walls of the abdomen. Dammann cured the second case in two months by using liquor Villati, applied on a mass of tow and kept in position with a bandage. Should the injured portion of bowel have become adherent to the abdominal wall, a section may be excised and the divided ends united by sutures, or in the case of small animals by using Murphy's bution or tubes of decalcified bone.

\section{IY.-PARACENTESIS ABDOMINIS (PUNCTURE OF THE ABDOMEN).}

Serous fluid collects in the peritoneal sac in consequence of chronic inflammation of the peritoneum, or more frequently of disease of the kidneys, liver, or disturbance of circulation, and may require to be removed by tapping. The peritoneum certainly possesses the power of rapidly taking up large quantities of fluid and returning them to the circulation, as clinical and experimental observations have shown; and these facts accord with our conception of the peritoneal cavity as a great lymphsac. When, therefore, fluids remain for long unabsorbed, some special cause must be at work, and as that cause is not removed by draining away the fluid, the latter usually returns after a short time. For this reason the operation has, at the most, only a symptomatic importance. It may, by relieving pressure on the diaphragm, lessen the danger of suffocation, and mitigate difficulty in breathing, but it seldom produces lasting improvement. In ascites the operation must generally be repeated after a short time, and as the already weakened constitution loses more albumen on each occasion, it often only hastens a fatal termination.

Diagnosis of ascites is seldom difficult where so much fluid is present as to call for puncture. The disease is commonest in dogs, whose soft abdominal walls allow the internal organs to be readily examined. Such an examination gives valuable information as to the causes of dropsy. One often finds chronic changes (tumours, \&c.) in the liver, which render exceedingly improbable any lasting favourable results from operation. The same is true where the dog suffers from heart mischief. In such cases the abdomen often shows unmistakable distension, which is sometimes attended with dyspnoa. When the animal is standing, the wave-like movement of the fluid may be felt by placing the left hand on one side of the abdomen and tapping the other side with the right. 
This phenomenon, termed "Sucussio Hippocratis," is the most reliable symptom of dropsy.

Tapping the peritoneal sac - a very simple operation-is effected with a trochar of the same strength as is employed in puncturing the intestine. There being no danger here, as in puncture of the intestine, of infecting the peritoneal cavity. from the bowel, and it being easy to disinfect the skin, the operation, when carefully carried out, is quite simple, and not likely to give rise to peritonitis. Indeed, in former times, when the principles of strict asepsis were not understood, the operation was usually performed quite successfully without antiseptics.

The puncture was formerly made at various points. Those through the rectum and vagina were soon given up because of the risk of infection, and the operation is now performed either in the neighbourhood of the umbilicus, close to the linea alba, or on one side of the abdominal wall, the former being usually selected in small animals. In large animals, which are operated on in the standing position, the side of the abdominal wall is found a more convenient spot. In cattle the right side is chosen, so as not to injure the rumen, which lies on the left; in horses the left side, because the crecum is on the right.

The bowels, being partly filled with gas, float on the fluid, and the latter therefore occupies the lower portion of the abdominal cavity, which is consequently the point selected for puncture. To make sure, the proposed seat of operation should first be percussed, when portions of bowel will be recognised by the hollow sound, and fluid by the completely dull tone which they respectively give.

In large animals the operation is performed as follows:- The hair being shaved from the point of operation, and the latter disinfected (washed with soap and rinsed with a disinfectant), an incision is made through the skin with a pointed bistoury or lancet to assist the passage of the trochar. This precaution is most useful in excitable animals and those with thick skin. Puncture is then effected in the same way as puncture of the thorax. The trochar is carefully disinfected and held with the handle in the operator's palm, whilst the forefinger, lying on the canula, limits the distance to which the instrument may penetrate. In larger animals, $1 \frac{1}{4}$ to 2 inches may be allowed; in smaller ones, $\frac{3}{4}$ to $1 \frac{1}{4}$ inches. As soon as the trochar has entered, the stilette is withdrawn, and the fluid allowed to discharge. Sometimes a check occurs, and the canula requires to be moved in various directions, or cleared of clots by passing the stilette or a probe. The precautions required in paracentesis thoracis are not so imperative here, and as soon as the fluid ceases to flow, the trochar may be removed, and the wound treated as in operating on the chest.

It is better to lay dogs on the side for operation, as when placed on 
the back, portions of the bowel may come in contact with the lower: part of the abdominal wall. Large dogs, if quiet, may be operated on standing. Either side may be chosen, provided one keeps close to the middle line. The posterior portion of the abdomen is usually preferred, and the puncture made between the umbilicus and the anterior portion of the os pubis, it being thought that this position offered less danger of injuring the stomach or liver, which is often increased in size on account of disease. The under surface of the abdomen is disinfected, and the operation performed as above described.

A bandage may be applied before operation, so that by tightening it as the fluid escapes intra-abdominal pressure may be sustained and syncope prevented. After operation, bandages prevent secretion of fresh fluid, though unfortunately in dogs they can seldom be kept in place, but slide backwards, even if passed over the chest.

To prevent the return of ascites, the injection of spirit or solution of iodine into the peritoneal sac has been recommended, after allowing the fluid to discharge ; but the uselessness and danger of this procedure have already been shown in human surgery, and it is now scarcely ever used. In animals it is not infrequently necessary to repeat the operation.

\section{V.-HERNIA.}

Hernia or rupture is a condition in which portions of the abdominal contents have passed through the abdominal walls, and lie under the skin. Should they pass through the skin, the condition is termed pro= lapse. Under the older system of nomenclature; the passage of abdominal organs through the diaphragm into the thorax was also described as hernia (diaphragmatic hernia), and in cattle strangulation of the bowel by the spermatic cord was termed pelvic hernia, con= ditions which will be noticed here, although in the true sense of the word they belong less to the herniæ than to the incarcerations.

The cause of hernia is sometimes a congenital defect, like too wide an inguinal ring or an open umbilicus. Such herniæ are, therefore, either congenital, or develop soon after birth. Increase of intra-abdominal pressure, frequent coughing, dyspncea, pressure on the abdomen, or development of gas in the bowel (tympanites), all assist their formation, while rupture of the abdominal wall directly produces them. Such rupture may be produced during parturition, or by excessive exertion, severe pressure on the abdomen, or the violent impact of blunt bodies. In men external influences, such as the pull of lipomatous new growths on the abdominal walls, may induce local pocketing and prepare the way for ruptures; but such cases do not appear to have been seen in animals. 
The essential constituents of a rupture are:-

(1) The opening in the abdominal wall through. which the viscus has left the abdominal cavity, and which may either be a normal opening like the umbilicus, or one abnormally dilated, as the inguinal canal sometimes is, or a rupture in the abdominal coats, without solution of continuity in the skin.

The rim of this orifice is termed the hernial ring. Its form and size vary greatly. Sometimes it will only admit the little finger, but in large animals it may be the diameter of a man's fist. Sometimes it is round, sometimes oval or slit-like. In umbilical and inguinal herniæ it is lined with peritoneum. In fresh ventral herniæ the edges of the ring are formed by the abdominal coats.

(2) The hernial swelling, which may vary from the size of a hazel nut to that of a man's head or more, consists of the portion of protruded viscus, the hernial "contents," and its coverings, the hernial "sac." The latter is divided into neck and base. The hernial sac consists of skin and subcutis, the latter usually thickened, sometimes of layers of muscle and abdominal fascix. Under certain circumstances other coats may exist, as, for instance, in inguinal hernia, the tunica vaginalis communis and the scrotum. Such special coverings are also termed accessory coats of the hernia.

The sacs of umbilical, inguinal, and femoral hernix, and those whose openings consist of abnormally dilated physiological apertures, are lined with peritoneum. In those produced by tearing of the abdominal walls, the peritoneum is usually divided; the internal organs may then lie under the subcutis, and only be covered by the latter and the skin.

The contents consist either of a loop of intestine or of omentum constituting respectively enterocele or epiplocele. Only very occasionally are other internal organs like the stomach (gastrocele) or liver found in the hernial sac. Sometimes the sac also contains a serous fluid (hernial fluid).

In reducible ruptures the contents lie free in the sac, and may be returned to the peritoneal cavity (reposition, taxis); but sometimes the hernial contents and sac become adherent to one another, and complete reposition can only be effected after division of the attachment. This forms irreducible hernia. The irreducible character may also be due to strangulation or other causes, like excessive distension of the protruded portion of intestine by hard masses of freces.

Diagnosis. A hernia is seldom difficult to recognise, provided it be reducible. In such case it has the following characteristics:-

(a) It is free from inflammatory symptoms (especially pain), is soft, elastic, and compressible, that is, it may be diminished by pressure.

(b) It varies in size from time to time: coughing or severe exertion 
increase abdominal pressure and render it larger; but it decreases when intra-abdominal pressure again falls.

(c) It is reducible, that is, the swelling may completely disappear on pressure, or on placing the animal in a suitable position, but it returns on removal of pressure or alteration of position.

Diagnosis only becomes absolute when the hernial opening is discovered. It can generally be felt by thrusting a finger into the depth of the sac, especially after reducing the hernia. Examination per anum is sometimes useful in horses and oxen; the hernial ring and the contained bowel may be felt; by traction on the bowel the hernia can sometimes be reduced. Exploratory puncture may also be resorted to in otherwise doubtful cases. Some care is required to differentiate hernia from abscess or hæmatoma, an error which might have grave consequences, as already pointed out in speaking of bruises of the abdomen.

Prognosis. Hernia itself is not fatal, but immediately strangulation occurs it becomes exceedingly dangerous. Prognosis chiefly depends on the probability of strangulation, a factor which will be considered in speaking of the various hernix. The smaller the hernial aperture compared with the size of the sac, the greater the danger of strangulation. Small intestine becomes more easily strangulated than colon or omentum. The size of the aperture, and the use to which the animal is put, must be considered, whilst it should be remembered that strangulation occurs more easily in working-horses, and that large hernix may interfere with usefulness.

Many herniæ, and especially umbilical and inguinal herniæ in young animals, disappear without treatment, and others may last the animal's whole life without interfering with its use.

Irreducible herniæ are more dangerous than reducible, recent than old, and intestinal than omental, because in each case the former are more likely to become incarcerated than the latter. We shall speak further on this head in describing the various hernix.

Strangulation or incarceration is the condition in which the hernial ring presses on the contents and interferes with normal circulation of blood and passage of ingesta through the intestinal loop. It consists, then, in ligation of the contents by the hernial ring, producing stasis of frees and disturbance of circulation and nutrition. In consequence of the pressure of the hernial ring on the contents, return of blood through the venous. vessels is first checked. In them blood-pressure is lower, and the walls weaker, and therefore circulation is more easily interfered with than in the arterial vessels, whose strong walls and high blood-pressure oppose considerable resistance to compression. Blood continues, therefore, to enter by the arterial vessels even after strangulation, and soon 
produces a severe venous congestion, usually associated with more or less extensive rupture of small vessels and hæmorrhage. Blood-stained serum is exuded, the hernial fluid increases, and in consequence of mixture with blood becomes red in colour. The hernial contents, especially the serosa of the intestine, is dark red or black, and this coloration is distinctly and usually sharply bounded by the ring-like circle of compression, which is grey and anæmic. Both this anæmia and the venous congestion impair nutrition in the strangulated parts, and may finally produce necrosis.

The frecal stasis adds to the disturbance thus produced, because the imprisoned intestinal contents putrefy, irritate the mucous membrane, and co-operate with defective circulation in producing necrosis of the mucous membrane. Infectious materials and their products possibly play a certain rôle here.

Necrosis first shows itself at two points, viz., in the deepest portion of the hernial contents, where circulation suffers most, and at the line of strangulation. It is well to remember, from the clinical standpoint, that dark colour is not always a proof of necrosis. The latter is usually typified by the presence of grey spots or patches on the surface of the peritoneum, or of ulcerative changes in the hernial contents. Necrosis is suggested by the presence of stinking fluid or gas in the hernial sac. The anterior portion of the bowel- - that is, the part nearest the stomach -appears greatly distended with food, whilst the posterior portion is empty and narrower than normal.

Causes. Strangulation was formerly believed to be produced by spasmodic contraction of the hernial ring; but, apart from the fact that in most ruptures such contraction seems scarcely possible, it is not to be imagined that active contraction could continue so long. Such views are no longer entertained, and the cause of strangulation is to be sought rather in the character of the hernial contents. Inflammatory swelling near the neck of the hernia may in exceptional cases cause incarceration, but is exceedingly rare. The primary factor in incarceration is usually increase in the hernial contents; conversely this increase may be a result of incarceration. Let us imagine a case: A horse affected with hernia suffers from tympanites; the increased intra-abdominal pressure thrusts a large portion of bowel and omentum from the abdominal cavity into the hernial sac; on account of the increase of the hernial contents the ring becomes too narrow; it presses on the enlarged contents and interferes with circulation in the parts which it surrounds, and incarceration results (figs. 121 and 122). Or let us fancy that in the posterior portion of the intestinal tube-that is, in the portion through which the intestinal contents are returned from the hernial sac into the abdominal cavity-some check occurs, causing fæcal stasis. Necessarily the hernial 
sac must become more or less filled with intestinal contents, which enter through the anterior portion of the intestinal tube, but cannot escape. Distension may finally become so great that the hernial ring acts as a ligature, and incarceration results after this fashion. The forward movements of intestinal contents may be variously impeded. Thus the posterior portion of intestine not infrequently becomes twisted either in the hernial sac or in the peritoneal cavity, particularly at its point of exit from the ring, and the twist, which acts like a kink in an indiarubber tube, may produce fæcal stasis in the hernial sac and incarceration. In the same way gradual distension of the anterior portion of the loop of intestine lying in the hernial sac may displace or compress the posterior portion, and bring about fæcal stasis and incarceration. According to Rose, folds of mucous membrane in the
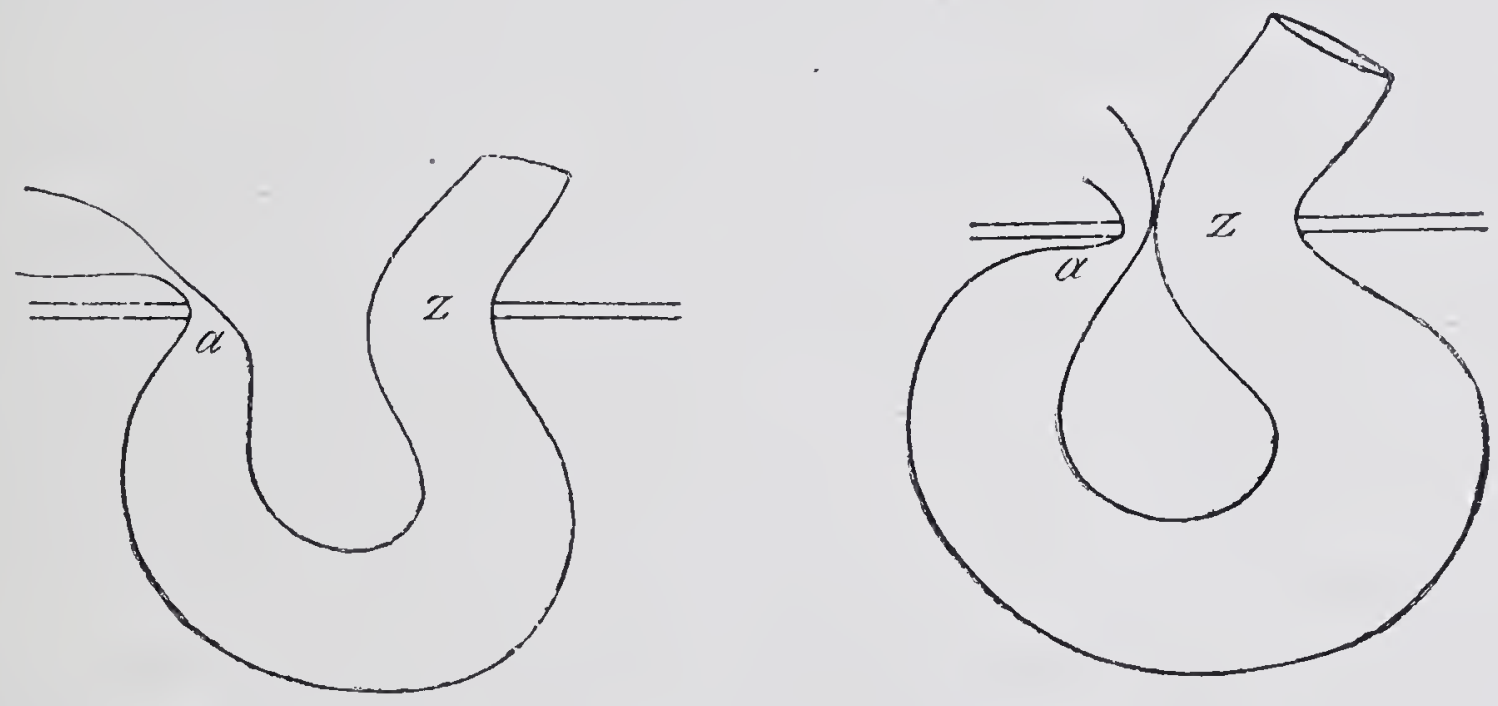

Figs. 121, 122.-Showing mechanism of incarceration of intestine(a) Efferent, ( $\approx$ ) Afferent, portion of intestinal loop.

posterior portion of the loop diminish the passage under certain circumstances, and produce similar results. In man extensive statistics have been accumulated on the mechanism of strangulation, and attempts have also been made to solve the question of its origin by experiments on animals. But both in men and animals the first causes may be so varied that they cannot usually be explained, and therefore the question is here of no great importance.

The views on omental strangulation are just as varied, though all coincide in declaring that omental hernix are much seldomer strangulated than intestinal. Some, like Rose, altogether doubt its occurrence, and have supported their opinions by experimentally ligaturing the omentum. Others allow its occurrence, and insist that its symptoms are similar to those of strangulation of the intestine, but less pronounced, because the circulation alone is interfered with, and frcal stasis does not occur.

Diagnosis is seldom difficult. As a rule, symptoms of colic appear, and in carnivora vomiting. The pain is regular and lasting. In cases of colic in ruptured horses care should therefore invariably be taken to 
first examine the hernial sac. If strangulated, the hernia will be found to have increased in size, become hard, painful, and tenser than formerly. Sometimes symptoms of peritonitis set in, and the animal soon dies if reposition is not effected. The appearance of albuminuria in strangulated herniæ is certainly interesting from a scientific standpoint, but not clinically.

Prognosis. Strangulated herniæ usually kill in a short time, not infrequently in twenty-four hours, and as reposition is often difficult or comes too late-that is, when necrosis has already set in-the condition must always be viewed as dangerous. Spontaneous reduction is exceedingly rare, partly on account of the accumulation of fæcal material in the sac, which increases with time, and partly because the incarcerated section of bowel soon becomes paralysed in consequence of defective circulation, and is then unable to discharge its abnormal contents, even when the causes of strangulation have been removed.

Treatment of non-strangulated hernix. In many cases, particularly in slight hernix in young animals, no treatment is required, because spontaneous recovery is usual, strangulation rare. Peculiarities of treatment will be separately described in connection with each of the various herniæ. One differentiates a palliative cure, and a radical cure. The first consists in bringing about a gradual diminution in the hernial sac ; the radical cure in closing the hernial opening.

The palliative cure depends on suitable dietetic precautions, supplying concentrated and easily digested food, avoiding hard work, and in larger animals on the preservation of a suitable position, that is to say, a position in which, whilst the animal is in the stall, the viscera will be as far as possible removed from the position of the hernia. These measures are supplemented by the following:-

(1) Hernial truss. Although much used in men, trusses cannot be employed to the same degree in domestic animals. Trusses for inguinal herniæ in animals, other than foals, are impracticable, and it is only in exceptional cases that they can be continuously worn in other abdominal hernix, though the male hippopotamus in the Berlin Zoological Garden wore such a truss for a considerable time. These appliances will be described in speaking of inguinal hernix.

(2) Diminution in the hernial sac and return of the hernial contents into the abdomen, by inflammatory and cicatricial processes in the skin of the sac produced by infrictions with sulphuric, nitric, or chromic acid, or the application of the actual cautery, or subcutaneous injections of common salt, alchohol, \&c. The artificial inflammation is accompanied by swelling in the skin and subcutis, and the contents of the hernia are said to gradually return to the peritoneal cavity. Cicatricial contraction of the cutis follows, and thus diminishes the size of the hernial sac, 
and even of the hernial aperture. The cautery, lately recommended in man, has the same effect. Radical treatment consists in removal of the sac and closure of the hernial aperture by causing the hernial ring or the neck of the hernial sac to unite, or by other methods. This procedure presents much greater difficulties in animals than in men, because it is neither so easy to attain asepsis, nor to keep the animal in the necessary dorsal position after operation, and when the many other dangers incidental to such operations are considered, few cases justify interference.

The following methods of radical cure are employed:-

(1) Ligation of the hernial sac. This can only be resorted to where the sac possesses a narrow neck. To prevent displacement of the ligature, it has been recommended to pass needles transversely through the neck of the sac, and to apply the ligature over them. It need scarcely be said that the hernia must first be reduced.

(2) In herniæ with broad bases interrupted ligatures may be employed. After returning the hernia, strong threads of sterilised silk are passed through the neck of the sac in the neighbourhood of the hernial opening, The procedure consists thereafter in multiple ligation; the skin of the hernial sac can then be cut through and separated from underlying tissues, the other coats of the hernia similarly treated, and the skin brought together over them. This method is frequently employed, and usually allows of healing by first intention, which greatly tends to ensure success. Sometimes, after dissecting away the skin, it is possible to close the hernial sac by ligatures applied round its neck.

The hernial sac may also be opened, and after multiple ligation of its neck be removed, following which the skin should be closed with sutures. Healing is often completed without pus formation. But in large animals, and especially in herbivora, suturing the hernial ring often presents insurmountable obstacles, because the abdominal walls are too firm and resistant to allow of approximation and union. Where the aperture is slit-like, further trouble is often caused by the hernial contents having become adherent to the abdominal walls, and dissection is both difficult and very dangerous. After freeing the bowel, or cutting off the omentum, or, if necessary, ligaturing it, the hernial contents are returned to the abdomen and the ring sutured. To assist union of the cicatrised edges of the hernial ring, they can be pared with the knife or scissors, or numerous superficial incisions made. In inserting sutures, the left hand holds back the viscera to protect them from the needle. The edges are brought together with strong, carefully sterilised silk, the hernial ring being as far as possible closed. Where tension is great, quilled sutures are useful. The skin is afterwards brought together with strong material, a deep hold being taken. 
It is scarcely necessary to add that the strictest antisepsis must be observed, without which there is always danger of peritonitis, and closure occurs far less rapidly and certainly. The special precautions necessary will be mentioned in speaking of different ruptures.

Finally, a radical cure may be effected by applying clams to the neck of the hernial sac, a method generally employed in umbilical herniæ, which will, therefore, be described under that head.

Treatment of strangulated herniæ. It has already been stated that incarceration is always dangerous, and that treatment should at once be resorted to. The latter consists primarily of attempting reduction. For this purpose the animal should be placed so that the hernia is as elevated as possible, as the contents then tend to return to the abdominal cavity by their own weight. The restlessness of horses, and the tendency they have when cast to contract the abdominal muscles, often cause difficulty in reposition. To avoid this, anæsthesia should be produced, without which reposition cannot be effected in larger animals. In horses, chloroform, in dogs, morphine is usually employed. In oxen it is often preferable to slaughter the animal, because the use of chloroform may make the flesh unfit for consumption.

It should be remembered that the obstruction always lies near the hernial ring, and therefore that any attempts at reduction must be directed to this point, pressure at the base of the hernial sac being mere waste of time. In extensive hernix, the sac is grasped with both hands, the points of the fingers lying near the hernial opening. Attempts are then made to diminish or return the contents lying immediately over it, and by gradually applied pressure, to get rid of the obstruction. Cold applications often have a favourable influence, and greatly assist in effecting taxis. In the large omnibus depots in Paris, where stallions are extensively employed and strangulated hernia correspondingly common, the employés have orders in such cases to keep a cold jet of water turned on the parts until the arrival of the veterinary surgeon. It is said that when so treated the majority of cases can be reduced by taxis.

Where the bowel is thought to be twisted on the abdominal side of the ring, the sac should be moved sideways, so as to lift the twist and set free the bowel contents. This must be done slowly but continuously and in different directions, because it is impossible to tell exactly where the obstacle lies. A gurgling sound may then sometimes be heard, indicating the evacuation of the imprisoned intestine. Diminution of the swelling under the fingers is a still more favourable sign. Reduction may sometimes be favoured by an assistant passing his arm into the rectum and exercising very cautious traction on the affected portion of bowel. The greatest care is, however, required. Attempts should be 
made to again bring the animal into the position in which these symptoms have occurred, and to further diminish the size of the sac.

Failing reduction by this method within half an hour, an incision must be made, or, under certain circumstances, may be resorted to at first. Should it be clear that the displaced intestine is already seminecrotic or is ruptured in the hernial sac, one dare not proceed to reposition on account of setting up peritonitis. The longer, therefore, strangulation has continued, the more careful should we be in attempting it. Inflammation of the hernial sac and emphysema of the skin clearly point to rupture of the bowel having taken place, a condition which is usually fatal in animals.

Incision (herniotomy) is resorted to, to remove strangulation, and may be carried out in various ways. In human surgery a distinction is made between hernial incision without opening the peritoneal cavity, so-called herniotomia externa, and a similar operation with division of the peritoneum (herniotomia interna). External herniotomy offer's great difficulties, but was formerly more resorted to than at present, because now-a-days the use of antiseptics has greatly diminished danger from peritonitis. The same is true in animals. The procedure in performing the external operation is as follows:--The seat of operation is carefully disinfected, the animal anæsthetised and placed in a suitable position, and an incision, which must extend the entire length of the sac, carried through the skin covering the hernial swelling, in the longitudinal axis of the body. After dividing the panniculus to a similar extent, both skin and panniculus are thrust to one side, and an attempt made to attain the hernial ring. Large vessels are ligatured to keep the field of operation clear, firm portions of connective tissue divided with the scissors or knife. By introducing the finger into the depths, one can discover the narrowest, that is, the strangulated, spot, which is then widened with a herniotome or tenotome without injuring the peritoneum. This effected, taxis becomes easy, and the wound is at once carefully cleansed and stitched up.

Internal herniotomy requires similar preparations. The incision is made through the skin in the same way, and the hernial coats lying beneath divided with a knife as far as the peritoneum. A little fold of the latter, at the base of the hernial sac, is then raised with forceps, and cut through close below the forceps with a linife held horizontally, producing a small opening. By means of blunt-pointed scissors this opening is enlarged, the index finger pushed into the hernial sac, and the peritoneum incised with scissors as far as the neck of the sac, the finger meanwhile pressing back the hernial contents and protecting them from injury. The finger is now passed into the hernial opening, the herniotome introduced alongside it, and the ring or neck of the hernial sac 
divided at the point of strangulation. A slight incision suffices to enable the contents of the sac to be returned to the abdomen, unless the hernia is adherent.

In umbilical and abdominal hernix, hernia knives are replaced by blunt-pointed tenotomes, which have been constructed of various forms. A special herniotome is only necessary in inguinal herniæ in the horse.

Internal herniotomy has the advantage over the external operation that one can determine the condition of the strangulated bowel, and should the latter prove to be necrotic, can either proceed to further treatment or resection of the necrotic portion. Reposition, under these circumstances, would destroy any chance of recovery. It should, how. ever, be repeated that dark coloration does not always indicate necrosis.

It is necessary in such cases to promptly decide whether to attempt resection of the necrotic bowel, a proceeding which, though certainly offering greater chance of success in carnivora than in herbivora, especially in horses, is even in them eminently fatal. Incarcerated portions of omentum, which, however, are seldom met with, are first ligatured with aseptic material and then cut off, and the wound treated as before described. It is of the highest importance to secure asepsis and prevent prolapse of the intestine. Perfect cleanliness ensures the first, and carefully-inserted quilled sutures lead to proper union of the edges of the wound.

Should resection of necrotic portions of intestine be determined on, it is very important to avoid infecting the peritoneal cavity with intestinal contents, and to carefully provide for union of the ends of the cut portions. The intestine is drawn forward as far as necessary, and protected by layers of cloth dipped in warm disinfectant solutions.

After separating the necrotic portion with scissors, the ends must be rinsed with carbolic or sublimate solution, and their contents, as far as possible, removed without soiling the wound. An assistant compresses the intestine to prevent further passage of contents from the anterior end, and the parts are then so brought together that the two surfaces of serosa are in apposition. To effect this, the free edges of the posterior portion are turned inwards, the anterior portion pushed into this, and the two sewn together with closely applied stitches. After once more carefully cleansing the intestine, it is returned, the wound disinfected, and stitches inserted as above described. Experiments made many years ago with the simplest apparatus in cases of invagination show that under some circumstances, especially in oxen, resection of the intestine may prove successful (compare with the section on "Suturing the Intestine"). 


\section{VI._UMBILICAL HERNIA (OMPHALOCELE, EXOMPHALOS).}

By union of the visceral plates in the linea alba, the abdomen closes during uterine life as far as the annulus umbilicalis, which remains open for the umbilical cord, and after birth ceases to exist on account of obliteration of its vessels. In new-born animals the umbilical ring not infrequently appears abnormally large, so that the umbilical cord does not completely fill it, in consequence of which the skin and peritoneum either immediately or in the first few weeks after birth, yield to the pressure of the abdominal contents, and allow the latter to pass through under the skin. This condition, which occurs in different classes of animals, though most frequently in horses, oxen, and dogs, has been termed umbilical hernia, and is divided into two forms,-viz., congenital, which is apparent at birth, and acquired, which appears during the first few weeks thereafter.

As a rule, a portion of the colon or cæcum, and sometimes of omentum, is found in the hernial sac, which is composed of skin and peritoneum, and which varies in size from a hazel-nut to that of a man's head. Pathe saw an umbilical hernia in a calf, containing a portion of the abomasum.

The division usual in human surgery between umbilical herniæ and herniæ of the umbilical cord has at present no practical importance in animals. In the latter, the viscera lie in a dilatation of the umbilical cord, and are only partially, or not at all, covered by the skin, and prognosis is therefore usually less favourable than in the ordinary form of umbilical herniæ. When cases occur no treatment is possible, and even in man they generally lead to death.

Causes. The disease is always due to abnormal size of the umbilical ring. The larger this is the more easily does rupture occur. This explains the hereditary character of umbilical herniæ, as observed by Viborg and Eléonet in horses, and Benkert in dogs (Stockfleth). In congenital umbilical herniæ, the abdominal viscera have often never been in the abdominal cavity, because they have not been surrounded by the visceral plates. After birth, umbilical herniæ result either from over-stretching of the skin covering the umbilicus, e.g., from violent tearing away of the umbilical cord; or they may be caused by any circumstance producing increase of intra-abdominal pressure, such as tympanites, excessive action of the abdominal constrictors during constipation (e.g., atresia ani), severe exertion and continuous coughing. They seldom develop in old animals.

Symptoms. Umbilical hernia is recognised by the presence of a swelling of the size above mentioned, which lies below the umbilicus, is elastic, soft, sharply defined, and free from inflammatory symptoms. It usually V.S. 
takes the form of a larger or smaller hemisphere, and seldom possesses a neck. By thrusting one or two fingers upwards into the swelling, the umbilical ring can be discovered in the depth. Sometimes it scarcely admits a finger, sometimes the entire hand can be thrust into it. By laying the animal on its back, or pressing on the swelling with the open hand, it disappears.

No mistake can well occur, except in young animals with inflammatory disease of the umbilicus; but caution is required in using the knife, because such hernir have often been incised with the idea that they were abscesses. Where the umbilical ring cannot be felt, and its complete closure ascertained, every fluctuating swelling must arouse suspicion of umbilical hernix. In dogs, and occasionally in oxen, a swelling the size of a walnut is seen in the umbilical region. It is not a hernia, but is due to thickening of the subcutis, or to remnants of the umbilical cord, which has ruptured or been divided at too low a point. The swelling is not affected by pressure, and the umbilical ring is closed. In oxen this thickened tissue sometimes becomes inflamed and suppurates.

Prognosis. Incarceration of umbilical herniæ is rare, apparently because the contents are formed by colon and omentum. For a similar reason, spontaneous recovery is common, and is favoured by small size of the rupture, narrowness of the ring, and youth of the animal. In herbivora, small umbilical herniæ generally disappear as soon as the animals begin to receive more hard food, because this produces not only distension of the colon, with firm masses of food, but also dilatation of the abdominal walls and tension of the peritoneum and skin, by which the hernial contents are pressed into the peritoneal cavity. As these causes act principally during the first short period after birth, spontaneous healing in foals and calves occurs during the first six months of life if at all, seldom after the age of one year.

Prognosis depends, first, on the age of the animal, and then on the size of the sac and of the umbilical ring. Incarceration is only exceptionally seen in animals, and then only in such as suffer from tympanites, in greedy feeders, in those inclined to digestive disturbances associated with the formation of gas, or in horses in very heavy draught.

Treatment. The most varied applications, most of them useless, have been recommended in umbilical hernix. Amongst these are local astringents and the subcutaneous injection of solutions of common salt. Such treatment is only followed by recovery where the latter is almost a foregone conclusion, as in small ruptures in young animals and particularly in herbivora. Amongst the palliatives most resorted to are-

(1) Trusses, which are, however, only of real use in cattle and horses, and even then to a limited extent. A tampon of tow, smeared with Venice turpentine to retain it in position, is placed on the hernia and 
fastened by a girth passed round the body. Kölling in this way cured umbilical herniæ in foals in six weeks. Marbot states having cured ten cases of the kind in thirty-two days by means of bandages. As a substitute for the bandage, a pitch plaster may be applied, or the sac painted with collodion after reducing the hernia, but neither method is reliable, especially on the very elastic skin of dogs, and plasters seldom remain a sufficient time in position to have any permanent good effect.

(2) Caustic and blistering substances, like sulphuric or nitric acid or cantharides ointment, applied to the hernial sac, have been recommended in the larger animals (horse and ox). In France nitric acid was first recommended in 1848 by Dagot, and about the same time it was used in Germany in common with sulphuric acid. The latter is generally diluted with 3 to 5 parts of water or spirit, and rubbed into the hernial sac daily for five to eight days. Concentrated sulphuric or nitric acid is applied with a glass rod in the form of lines. The lines must be at least $\frac{3}{8}$ to $\frac{3}{4}$ of an inch apart, and the acid can seldom be used more than twice. Others apply the concentrated sulphuric acid with a brush to the entire surface of the sac twice, beginning at the periphery, and taking care that the lowest portion of the sac does not receive too much. Particular care is required in applying concentrated nitric acid, and it should never be left to the owner, but be used by the practitioner himself. The irritant effect is sometimes too great, and, owing to excessive swelling and tension, the skin tears through or becomes necrotic before union has taken place at the neck of the hernial sac. Prolapse of the bowel then occurs, as noted by Weber in foals and Roche-Lubin in dogs. The thinner the sac and the skin covering it, the greater the care required in applying concentrated acids. Duriaux and Cagny have recently recommended sinapisms instead of acids, stating that mustard produces the same effect as acids, but is less dangerous. Acids are useful for this purpose, because they do not soften the structure of the skin like alkalies, but give a firm scab. Nitric acid produces a stronger effect than sulphuric, and is, therefore, more painful and dangerous, while chromic acid and bichromate of potash ( 1 to 3 ) often affect deep-seated structures too much. The swelling which appears is the best guide, and immediately it seems sufficient, further applications should be avoided.

The actual cautery has been recommended with the same object, and is applied in the form of lines. In France and Belgium bandages and blistering ointments have been simultaneously used, but this "mixed" method is not commendable.

Degive recommends a bandage constructed by Martin for use in the horse, which carries a plate of tinned iron, 14 inches long and 4 broad, whose form responds to that of the lower surface of the belly, upon which it is 
fastened by two rollers. The plate has a rounded prominence about 2 inches high on the side next the abdomen, which fits into the hernial ring and keeps back the contents. This truss is worn for four to eight days, when swelling of the ring occurs, and it may be removed to see whether the hernia returns. Should this happen, the parts can either be dressed all over with diluted nitric acid or be fired, after which a second bandage is applied, whose girth carries a similar plate 14 inches long and 9 inches broad, covered with a tarred linen compress. Three days later Martin blisters the parts with cantharides oil to assist the separation of the eschar, which is said to occur eight days afterwards. The girth is then loosened a little and reapplied, after smearing the rupture with tar. Martin states having cured umbilical herniæ in this way within twenty days.

Without doubt the pressure of the bandage on the inflamed hernial sac assists its contraction and the return of the contents, but necrosis of the sac is apt to occur, and the truss must, therefore, be frequently examined.

Imminger recommends injecting subcutaneously 1 to $1 \frac{1}{2}$ ounces of a 15 per cent. salt solution about an inch in front of, and also behind the rupture. One injection is said to be sufficient to cure small herniæ in four to six weeks. Thickening remains for some time, but this is thought to close the ring. Lucet injects from 2 to 7 ounces of a similar solution all round the ring.

These methods assist return of hernial contents to the abdominal cavity by the swelling they cause, an action which is continued by the subsequent cicatricial contraction of the skin. Acids are preferable to blistering ointments, as, e.g., cantharides; and Hupe states that inguinal hernia in foals can be cured with certainty during the first six months of life by smearing with acid. sulphur.

This treatment is only suitable to small herniæ in young animals; in larger herniæ it is of no value, and Degive restricts its use to cases where not more than two fingers can be introduced into the hernial opening, and where the sac is not greater than a hen's egg. Where irritants have failed, the hernial contents often become adherent to the sac, and may later cause difficulty in operative treatment.

(3) The following methods of radical treatment have been employed:- -

(a) Simple or multiple ligation of the sac. This is best performed in spring or autumn and at a time when the animal is otherwise in good health. The limits of the sac should be marked before casting. In hernix not possessing too broad a base the parts are first carefully replaced (the animal having been placed on its back and the hind legs bent), and a ligature passed around the neck of the sac. Esser recommends for this purpose the elastic ligature. When, ten to twelve days later, this falls off, together with the hernial sac, a cicatrix has formed in the position of the latter, which prevents prolapse of the bowel. The elastic ligature should, however, not be drawn tight enough to cut rapidly. To prevent its sliding off, a strong needle may be passed through the sac below it.

Degives takes up as much as possible in the ligature, and draws this quite tight. According to him the method is very successful in ruptures 
where the abdominal opening is not of greater diameter than the thumb. Where the base is broad the cord easily slips off; removal by multiple ligature, i.e., ligation in sections, is then preferable, and in foals, and even in older horses, gives good results. Procedure is as follows :-The animal is cast, placed on its back, the hind legs bent and drawn outwards, the hair shaved from over the hernial sac, and the latter washed and rinsed with a disinfecting fluid. After complete reduction, the folds of the sac are gathered together in the direction of its long axis, and, beginning at one end, transfixed close to the abdominal wall with a slightly bent needle, provided with a strong sterilised double silk thread. The threads, when drawn through, are divided close to the needle, and the ends of the one half tied tightly together, whilst an assistant draws the saic gently upwards. The needle carrying the second half of the thread is again passed through the sac about $\frac{3}{4}$ of an inch from the first point of incision, and this section ligatured in a similar way. The same process is continued until the entire sac is ligatured. Should the animal struggle whilst the needle is being passed, the fingers of the left hand are

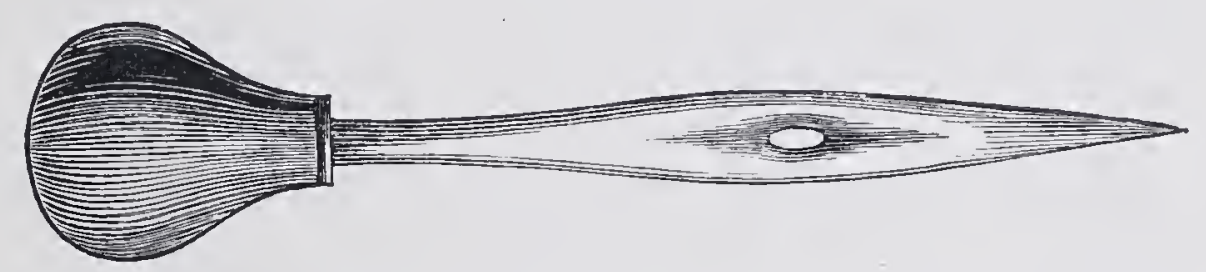

Fig. 123.-Kühn's needle for ligation of the sac in umbilical hernia.

placed on the umbilical ring to prevent the intestine protruding and being transfixed, or one waits until the animal is again quiet. The entire sac is then once more rinsed with sublimate or carbolic solution, and the ligatures on both sides of the sac powdered with a mixture of iodoform and tannin ( 1 to 3 ), or the entire sac may be painted with wound gelatine and covered with wadding.

The animal is now allowed to rise and placed in a stall provided with good clean straw. Should the above described precautions be taken, the wound generally heals aseptically. In from ten to fourteen days the ligatured portion of the sac sloughs away, and the wound appears healed. The operation is performed in a similar way at Brussels, but, instead of silk, strong waxed cords or hemp threads are employed. To facilitate the work of ligation French veterinarians use an iron clam, which is applied over the empty hernial sac close to the abdominal walls. It prevents the portions of bowel which enter the sac when the animal struggles being penetrated by the needle. This may just as easily be avoided by chloroforming the animal, or by carrying out the above described method.

'To prevent prolapse of the viscera during multiple ligation, Mangot employs a long rectangular plate of lead, which has an opening in the 
centre through which the empty sac is drawn and then ligatured. 'Two wooden pegs are then thrust through the skin below the thread, the sac is cut off, and the plate of lead fastened over the animal's back by four straps, which pass through suitable holes at the corner's of the plate. Imminger has lately introduced this method in foals, but he employs silver wire for ligation. If the wire is not too thin, and not too strongly pulled, prolapse of bowel does not occur.

(b) Removal of the sac by clams. The"clams act like multiple ligatures

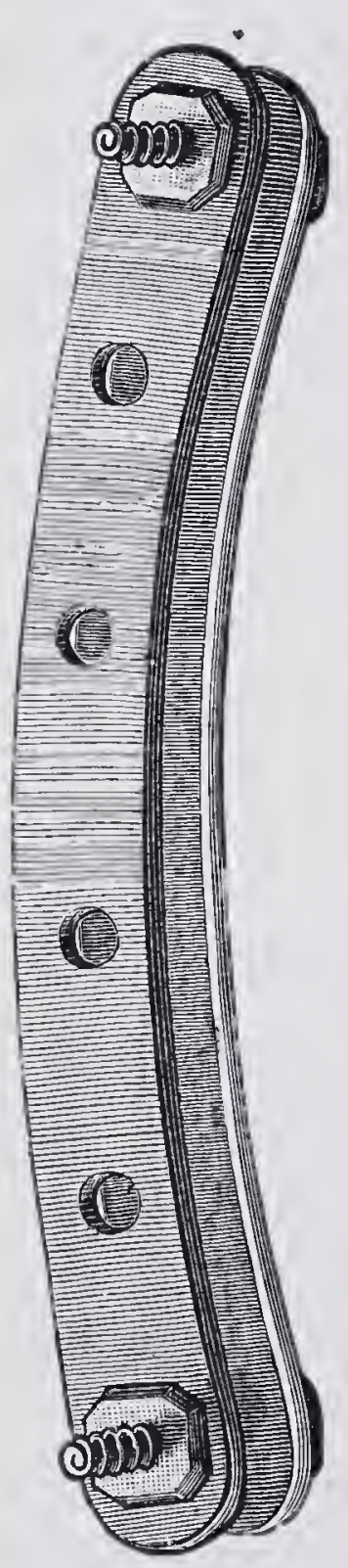

FIG. 125.-Combe's clam.

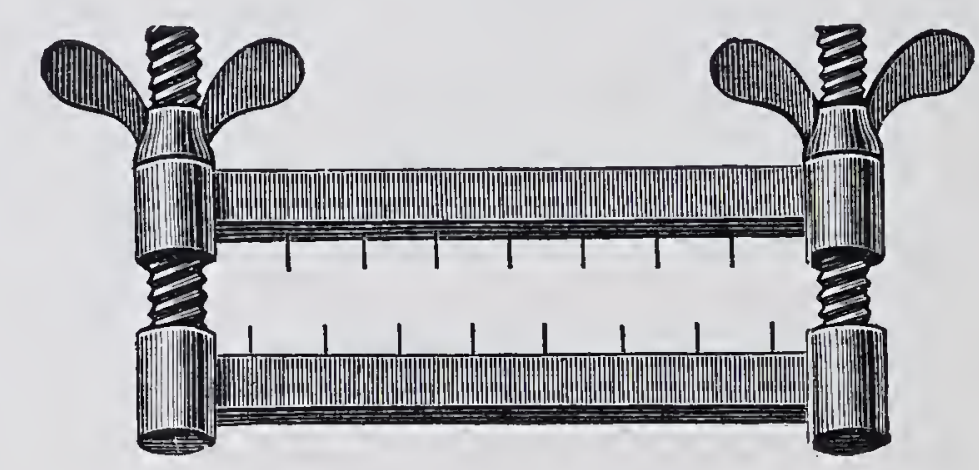

FIC. 124.-Bordonnat's clain.

and are most useful in herniæ with broad bases; but, although this method is simpler than ligation, it usually prevents healing by first intention. A further difficulty in using clams is the fact that they easily fall off. To prevent this, pegs are thrust through the sac below the clam, or the clam itself penetrates the sac. Bordonnat constructed for this purpose the iron clam shown in fig. 124, and Combe invented a perforated clam (fig. 125) allowing the passage of ligatures or strong needles. After reducing the hernia, the clam is applied to the neck of the sac, as near as possible to the under surface of the belly, and in the direction of the linea alba, and fastened by screws, or, as in castration, by a cord. Whatever the method employed, the following symptoms almost always appear:-During the first few hours after operation there is slight colic. Next day signs of inflammation become apparent: the umbilical region is swollen and painful; the hernial sac is cold, purple in colour, and insensitive; appetite is in abeyance and fever is present. Towards the fourth day the skin becomes necrotic; the clams separate and fall about the twelfth to the fifteenth day, leaving a considerable wound and much local swelling, which disappears slowly. In three weeks to a month the umbilical opening should be obliterated. In cases of very wide umbilical ring, however, recurrence of the hernia was noted even after the above treatment.

The chief points in using clams are to grasp sufficient skin, and to fix the clam firmly and evenly; and therefore wooden clams fastened with cords are less satisfactory than iron ones with screws, because, 
on account of their one-sided fastening, pressure cannot be so evenly applied.

In using wooden clams one is sometimes applied over the other to prevent the first falling off. Degive recommends this, especially in large hernir, when the upper clam should be about 12 inches long, the under: one some 4 inches less. The upper is applied as close as possible to the belly, so that after the animal rises, the sac appears to have vanished. Hernir as large even as a man's head may be completely cured by this method. Demesse states having thus cured a case where two hands could be passed through the umbilical ring together. Should the sheath in male animals reach so far forward that it would be touched by the clam, the latter may be applied at right angles to the long axis of the body.

(c) Herniotomy. When, as in very large herniæ, the above methods fail, a cure may possibly be effected by suturing the hernial ring. Cordelier gives the following indications for this operation :-1. Strangulation. 2. Large size of the hernia. 3. Long existence of the hernia. 4. Impossibility of reducing the condition. 5. Adherence of the contents to the hernial sac. 6. Inflammation of the hernia, or the existence of colic. The operation is carried out as previously described, and under antiseptic precautions. The animal is chloroformed, laid on its back, the entire surface shaved, rinsed free of soap, and disinfected. The slin is then divided in the long axis of the hernia or of the hernial ring. From this point the operation may be completed either with or without opening the peritoneal cavity. Siedamgrotzky prefers the latter way, and next separates the skin from the hernial sac, which he thrusts into the hernial opening. The edges of the umbilical ring are then freshened, brought together with strong silk threads, and the flaps of skin, after being shortened to the necessary extent, united above them. An antiseptic dressing is secured over all by means of bandages or a few threads, and healing takes place completely in from twenty to thirty days. Catgut is here unsuitable, as it is too quickly absorbed. Guttmann had five relapses among twenty cases from this cause.

Storry proceeded in a similar way. After dividing the sac from the skin, he sutured it with a strip of white leather, then cut it off and sewed the skin together.

This method is to be preferred to herniotomia interna (which necessitates opening the peritoneal sac), if for no other reason than that the chances of peritonitis are slighter, and success is not endangered by prolapse of portions of intestine or omentum. It must, however, not be forgotten that in many cases the peritoneum cannot be separated from the surrounding tissues, especially at the base of the hernial sac, and that the peritoneal cavity is apt to be opened in making the attempt. Nevertheless barl results seldom occur. 
The peritoneum may be incised without grave risk, as shown by various observations: Degive operates in this way; Wiesner lately reported such a case, though it is clear that healing is not so simple as in Siedamgrotzky's method. The foal operated on was very unwell for the next few days, whilst in herniotomia externa, scarcely any feverish disturbance was noticeable. Fröhner operated under strict antisepsis, sutured the abdominal wound, and effected healing by first intention in ten days.

Should the hernia be adherent, which is not uncommon after the use of acids or other irritants, it is better to limit the opening in the peritoneal cavity. Degive proceeds as follows:-The hernial sac is opened under antiseptic precautions and the peritoneum cut through only far enough to admit the finger, so as to separate the adherent portions; scissors may also be used. The sac is afterwards ligatured in sections, as above described. (For another method see section on "Ventral Hernia.") The cutting operation is not suitable in large herniæ, especially if the rupture be circular, because of the difficulty in uniting the edges of the ring; in such cases it is better to use irritants supplemented by bandages.

The animal should be prepared for operation by reducing the diet, giving easily digestible and concentrated food, and administering a purgative to empty the bowel. Similar dietetic precautions should be continued for some days after operation. The bed should be of good clean straw, and it may be necessary to tie up the horse for several days.

In small animals (dogs) irritants cannot be employed on account of their being licked off. Operative interference is here easier, but it must not be forgotten that many animals will not endure bandages, clams, threads, \&c., but will at once tear them off. In such animals the radical operation is therefore preferable, and as far as possible one should avoid opening the peritoneal cavity. Dogs may receive a strong dose of morphine to quiet them, following which they generally sleep for twenty-four hours. The greatest pain has then disappeared, but it may occasionally be necessary to watch the animal continuously.

\section{VII.-INGUINAL HERNIA, SCROTAL HERNIA (HERNIA INGUINALIS, HERNIA SCROTALIS).}

Passage of abdominal contents through the inner abdominal ring into the processus vaginalis constitutes inguinal hernia. Should the viscus attain the scrotum, the condition is termed scrotal hernia. As the inner abdominal ring only remains open in male animals and in bitches, they alone suffer from inguinal hernix. The horizontal position of the body in domestic animals is less favourable to the production of inguinal hernia than the perpendicular one in man. It occurs in horses and 
pigs, less seldom in ruminants and dogs, and the sac usually contains intestine, less frequently omentum. As inguinal hernia exhibits certain peculiarities in the different classes of animals, it will be considered separately in each.

\section{(1.) INGUINAL HERNIA IN THE HORSE.}

In stallions and geldings the inguinal canal consists of a flat funnel-shaped passage about 4 inches in length, whose upper opening (annulus abdominis) is from $\frac{3}{4}$ to $1 \frac{1}{2}$ inches in length, but occasionally much wider. It extends in an oblique direction from behind outwards and forwards, is situated 1 to 2 inches from the oblique branch of the os pubis, and 4 to 6 inches from the linea alba. Anteriorly and inwardly the abdominal ring is bordered by the posterior edge of the inner oblique abdominal muscle, posteriorly and outwardly by the cremaster or spermatic cord. The vessels supplying the posterior portion of the abdominal walls pass about $\frac{3}{4}$ of an inch from its posterior angle, and on its inner side. The inguinal ring or outer abdominal ring (annulus inguinalis) consists of a slit between the inner and outer tendinous heads of the external oblique abdominal muscle, whose posterior inner angle lies $1 \frac{1}{4}$ inches in front of the branch of the os pubis, and somewhat further from the middle line of the abdomen. Its normal length is about 4 inches; it likewise passes forwards and outwards, and opens when the thigh is directed backwards and abducted. The anterior inner wall of the inguinal canal is largely formed by the inner oblique abdominal muscle, the outer by the crural arch or Poupart's ligament.

Causes. In stallions with abnormally wide abdominal rings, and less frequently in geldings, the small intestine or omentum may enter the inguinal canal. According to Berdez, Vogel, Stockfleth, and others, the condition is always caused by excessive width of the ring; whilst Lafosse, H. Bouley, and Gerlach declare that inguinal hernia may occur even where the aperture is of normal width. But even if we allow this, there is no doubt that in by far the greater number of cases the condition is due to abnormal width of the ring, which sometimes measures 4 to 6 inches. Möller has, however, also seen inguinal hernia in hor'ses in which the inner ring scarcely allowed the entrance of two fingers, though, as a rule, two easily pass in addition to the spermatic cord. It is therefore difficult to fix a normal width and settle this question, which requires numerous measurements to be made in different races of animals. Inguinal hernia is generally congenital, or appears during the descent of the testicle. The tendency to it seems to be inherited. It has even been seen in the foetus. Its production is favoured by all circumstances which cause increased abdominal pressure, such as tympanites, severe exertion, hard drawing on soft ground (where the action of the muscles in lifting the feet also produces dilatation of the abdominal ring), or struggling in hobbles. Hammerschmid's case shows that difficult labours may produce inguinal herniæ in the foal, particularly 
if in delivery the hind-quarters present; the foal in question showed an inguinal hernia as large as a child's head on the third day after birth. All movements which cause dilatation of the abdominal ring, like violent kicking or slipping, especially slipping outwards and backwards, may give rise to inguinal hernix. The same result may be produced by dragging on the spermatic cord during castration, by the pull of heavy clams, or of largely developed testicles in old stallions. In the latter, inguinal herniæ sometimes result during copulation, the erect position

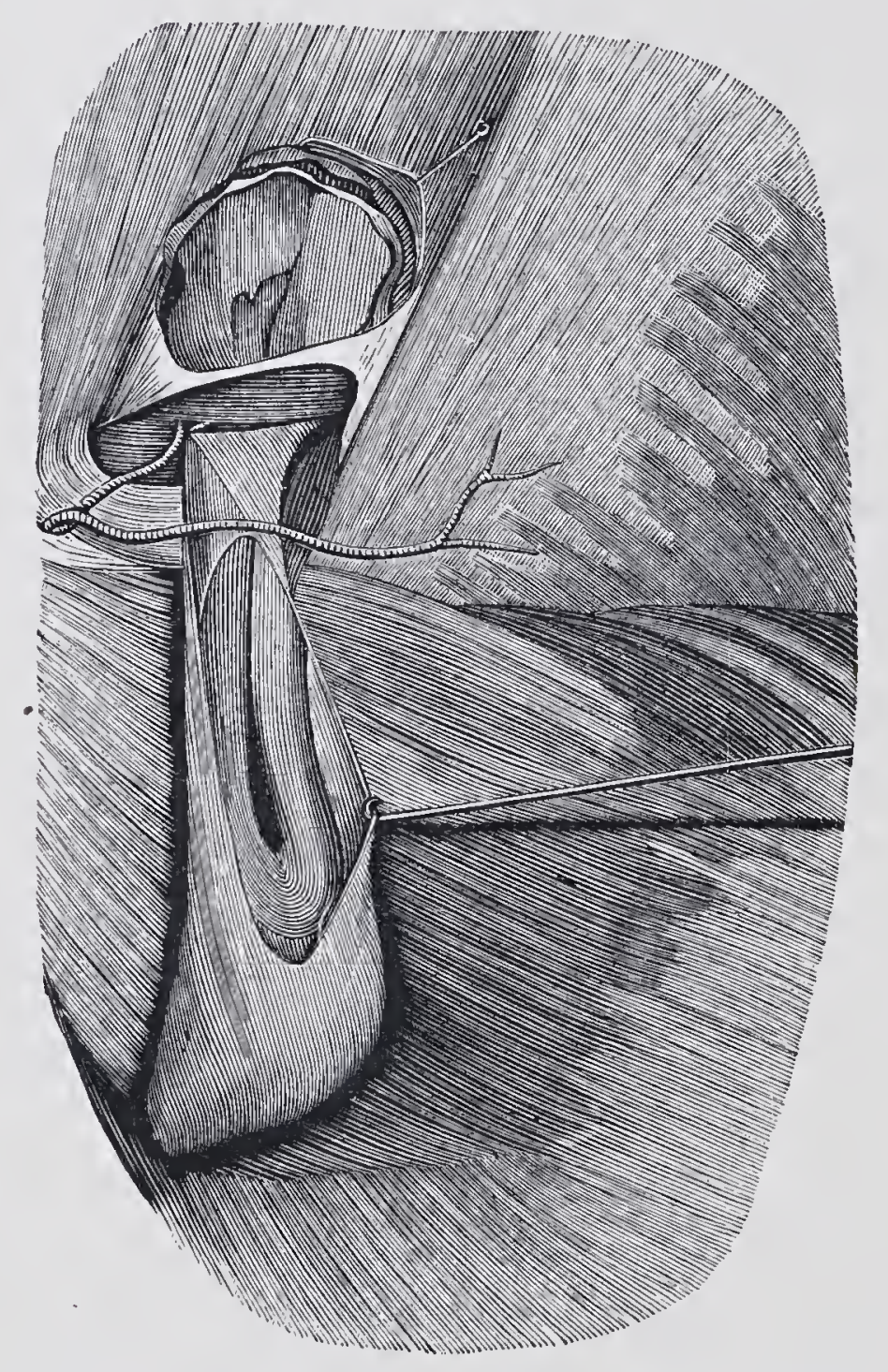

FIG. 126.-Incomplete inguinal hernia. (The fig. shows the position of the imner abdominal ring and the course of the external pudic artery.)

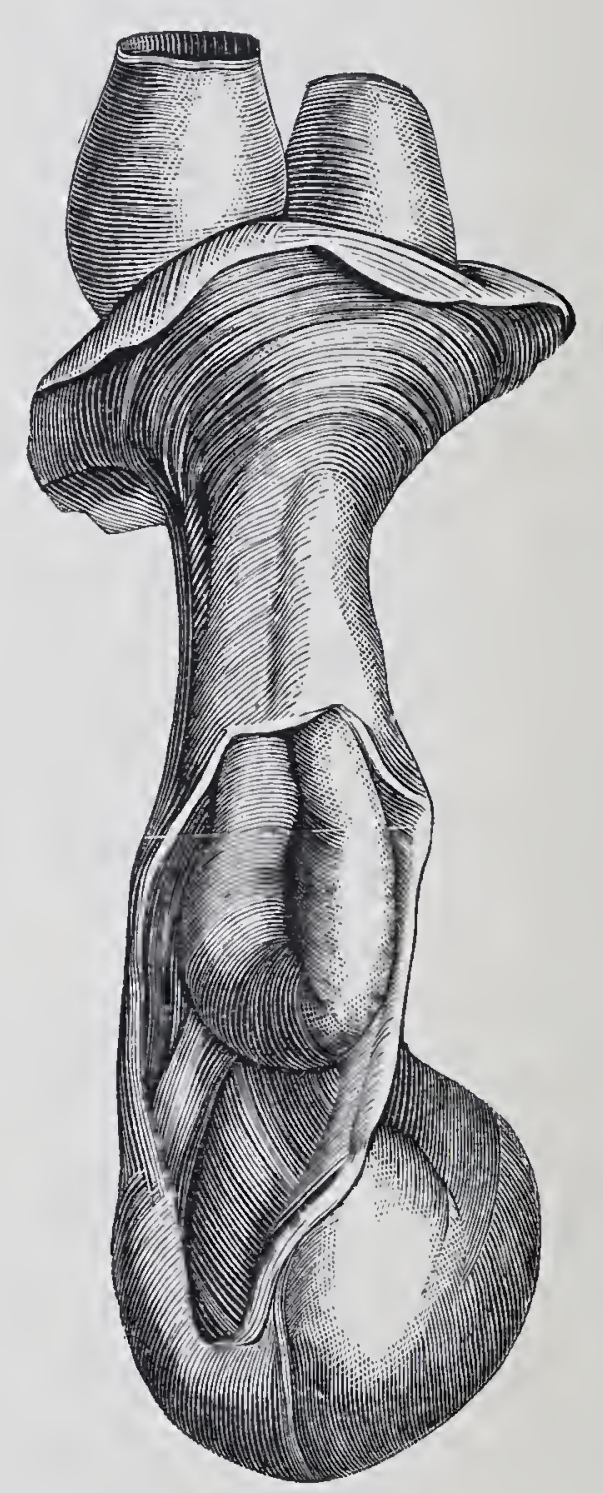

FIG. 127.--Inguinal hernia of the horse. (Hering.)

causing the viscera to be pressed towards the pelvis and into the inguinal canal, which is dilated in consequence of the thighs being turned outwards.

The condition occurs more seldom in geldings, because it is either cured by castration or leads to the death of the animal, and because dilatation of the abdominal ring very seldom happens late in life. The earlier the gelding is castrated the smaller does the inner abdominal ring become, and this explains why it is so small in most geldings, though the latter also suffer from inguinal hernia at times. The swelling of the spermatic cord after castration distends the canal and favours hernia; the contents are usually omental, but intestinal herniæ also occur, and in stallions 
constitute the rule. Sometimes both intestine and omentum are present in the sac, which is formed by the processus vaginalis, tunica dartos, and skin.

Symptoms. The scrotum is increased in size by the entrance of viscera, but to a very varying degree; in cases a careful search may be required to detect the rupture, while in others the swelling extends below the hocks, and attains the size of a sugar loaf. Degive saw a hernial sac, 16 inches in depth and 8 in width, containing 27 pints of fluid, and others as large have been seen.

The swelling has the general characters of a rupture, is elastic, soft, and, if omental, somewhat doughy. It is yielding and disappears on pressure, or on the animal being placed on its back, leaving the sac empty except for the testicle, which, in old inguinal herniæ, is usually atrophied and flaccid. A coil of intestine may sometimes be detected in the scrotum, and, according to Boulet, always lies on the inner side of the spermatic cord. The swelling is increased by exertion, by the action of the abdominal muscles, or by coughing. A tympanitic tone on percussion and rumbling betray the presence of air or gases. In geldings the neck of the hernial swelling is of considerable size, and appears firmer, in consequence of being surrounded by omentum.

The detection of so-called incomplete inguinal herniæ is very difficult. In these cases the spermatic cord is usually abnormally thickened, either from sdema following pressure by the hernial contents on the vessels of the cord, or from hypertrophy of the cremaster in consequence of increased work. The former is therefore commoner in recent inguinal herniæ, the latter in old ones.

Doubtful cases can be cleared up by a rectal examination.

In recent inguinal hernix with commencing strangulation, the hindlimbs are generally moved stiffly, the toes dragged, and the limbs turned outwards, the breathing is rapid, the animal stretches out, shows slight colic, and draws up the testicle of the affected side; Girard regards the latter symptom as pathognomonic. These signs either vanish in a few days, or symptoms of severe strangulation set in.

In inguinal, as in other hernix, strangulation is generally first signalised by attacks of colic, and therefore it is a good practical rule always to examine the scrotum in stallions suffering from colic. The horse, under these circumstances, usually stretches out or sits on his hind-quarters like a dog. Jessen saw vomiting, and though, as a rule, this only occurs in rupture of the stomach, it must not be forgotten that such rupture is tolerably frequent in this disease.

A second group of symptoms indicative of incarceration are found in the changes undergone by the scrotum. The hernial swelling becomes larger, harder, tenser, and more painful, and can no longer be compressed 
or replaced. In several of such cases the epididymis has appeared very prominent close to the testicle.

Diagnosis of inguinal and scrotal herniæ and of strangulation is therefore seldom difficult. The conditions most resembling it are :-

(1) So-called interstitial inguinal herniæ. This disease will later be referred to.

(2) Sarcocele, though the greater hardness of the entire swelling scarcely allows of mistake.

(3) Hydrocele. Collection of fluid in the vaginal sac may be recognised by the swelling being easily displaced and more confined to the lower portions of the scrotum. Hydrops of the spermatic cord may be at once recognised by its greater firmness and immobility, and can only be mistaken for incarcerated hernia.

(4) Hæmatocele, that is, distension of the scrotum, or of the tunica vaginalis, with blood, presents the same appearances as hydrops, but is often associated with odema of the scrotal skin.

(5) Inflammation of the skin covering the scrotum may, under certain circumstances, give rise to suspicion of inguinal hernia, and in all such doubtful cases examination per rectum must be resorted to, when it is at once easy to determine both the size of the abdominal ring and the condition of viscera which have entered it.

(6) Tumours of the spermatic cord and testicle develop slowly, and are not dissipated by pressure.

(7) In geldings fistulæ or other diseased swellings of the spermatic cord may lead to mistakes, unless a careful examination be made.

Course. In foals, spontaneous recovery often occurs, though seldom later than the first year of life. Gross believes that good feeding favours recovery, by producing a better muscular tone; and distension of the intestine with firm contents, consequent on hard diet, certainly assists reduction. On the other hand, the rupture may gradually increase, particularly if work is heavy and the food bulky, and in such cases incarceration often occurs. Cases like Brinhall's in a three year old stallion are, however, very rare. He saw a scrotal hernia suddenly develop, and two weeks later break, in consequence of necrosis of the sac. An intestinal fistula was thus produced, but closed again in a month without any assistance. Perhaps this was a case of strangulation, but the result was certainly very extraordinary. In exceptional cases inguinal hernia appears in old animals ("hernies inguinales récentes," in opposition to the " hernies inguinales anciennes" of the French) and generally soon becomes strangulated. Such cases are probably often due to a simple inguinal hernia developing into the scrotal form, the former having been overlooked. In stallions scrotal herniæ may become so large as even to interfere with movement of the hind-limb, or be injured 
by it. As the disease is difficult to treat and endangers the animal's life, inguinal hernia must always be regarded as a grave condition. When the bowel is strangulated the only hope of recovery lies in skilled interference.

Prognosis. Inguinal hernia is always dangerous, because treatment is difficult, and death often follows strangulation. The most important elements in forming a prognosis are the age of the animal, and the size and character of the hernia.

Spontaneous recovery is rare in adults though common in young animals. The condition produces difficulty in castrating foals, and renders this generally simple operation dangerous, whilst fresh cases of inguinal herniæ in old animals are doubly grave, on account of the risk of incarceration. Extensive ruptures, and especially those with large apertures, not only injure the appearance of the animal and interfere with its use, but at the same time offer the greatest difficulty to treatment.

Omental are usually less grave than intestinal hernix, and on this ground geldings are more hopeful subjects, though even in them the intestine sometimes descends and becomes strangulated. In a gelding Möller replaced an incarcerated inguinal hernia four times during a period of six weeks. Irreducible hernix are doubly dangerous, because of the difficulty in operating. Finally, it should be remarked that incarceration does not necessarily depend on the size of the hernia. Small and incomplete inguinal herniæ are more frequently strangulated than extensive herniæ with wide abdominal ring.

Treatment. Operation is rendered difficult by the length of the inguinal canal and the position of the hernia. Hering very shrewdly advises leaving well alone, and not operating unless obliged. This, however, becomes necessary when a stallion has to be castrated, and incarceration not infrequently necessitates operation. As, in the latter case, procedure differs from that in non-strangulated ruptures, the treatment of the simpler condition will first be dealt with.

\section{(A) TREATMENT OF NON-STRANGULATED INGUINAL HERNIA.}

Various methods have been proposed; amongst the most important are-

(1) Closure of the inner abdominal ring by inducing inflammation of the spermatic cord and adhesions with neighbouring structures. If, after successful reposition of the hernia, the spermatic cord be caused to swell, it may completely fill the abdominal ring, or so far occlude it as to prevent the passage of intestine or omentum.

(a) In Spain, according to Stockfleth's description, the scrotum and 
spermatic cord are continuously rubbed. 'The stallion is starved for two or three days, the rectum emptied by means of clysters, the animal cast, placed on its back, and the hind-quarters drawn up. The testicle is now drawn forward with one hand, whilst the thumb, index, and middle finger of the other are employed in rubbing the spermatic cord until marked swelling appears. The animal is allowed to rise quietly, and during the first three days is sparingly fed, and only walked when exercised. Animals are said to be cured in twelve days; but though this is possible, it is certain that failures often result.

(b) Bagge's method is similar. After replacing the hernia, a woollen band is tied round the scrotum, in the neighbourhood of the inguinal ring, and allowed to remain on for eight hours, when a swelling is found to have been produced, and the band is removed. Recovery occurs in six to eight days. Bagge states having thus cured nine stallions in one day (?).

In this connection, Kruckow's procedure may be referred to even though the method be not usually possible. In a fresh case of inguinal hernia Kruckow thrust the displaced portion of intestine, together with the testicle, back into the abdominal cavity, after which the intestine did not return. The scrotum was rubbed with diluted sulphuric acid. This report is open to question, for two reasons-firstly, it is certainly only in exceptional cases possible to thrust the testicle into the abdominal cavity through the inguinal canal, and even if this were always practicable, the absence of the spermatic cord from the abdominal ring must greatly favour recurrence.

(2) Treatment by closure or narrowing of the processus vaginalis.

(a) Foelen has recommended the application of irritants to the scrotum in the neighbourhood of the inguinal ring, as in treating umbilical hernix. The ointment he uses consists of 1 part of cantharides, 2.5 parts of euphorbium, 15 parts of fat, and 1.5 parts of verdigris ; it may be applied without casting the animal by drawing the testicle downwards, thus rendering the skin of the scrotum tense. The application should be made once daily, until the epidermis becomes loosened, which usually happens in ten to twelve days. The parts are then washed with lukewarm water, and when the inflammation has disappeared the infrictions are recommenced, until in four to six weels the rupture has disappeared. Degive also recommends this method, though it is of little use in animals more than four to six months old.

(b) The application of clams. This is very generally practised, and resembles the covered method of castration. The hernia is reduced and the clams applied as high, that is, as near to the inguinal ring, as possible, and over the tunica vaginalis and spermatic cord. In order to be able to apply the clams very high it has been recommended to give them a bent form, but this is not desirable, because the centre portion does not press sufficiently on the tunica vaginalis. A simpler 
and more effectual way of closing the tunica vaginalis above the clam is to give a half turn, which may later be made a complete one, to the tunica vaginalis and the spermatic cord before applying the clams.

Displacement of the abdominal viscera is said to be prevented by the closure of the processus vaginalis, but this is dependent on the size of the abdominal ring; and where the latter is large the viscera not only interfere with closure, but may even cause rupture of the processus vaginalis. Though this danger is certainly decreased by twisting the processus vaginalis, it is not entirely removed, and, therefore, the process has been somewhat modified. The operation should be performed antiseptically under chloroform. As the chief difficulty in applying the clams high is occasioned by the outer skin and soft parts lying below it, a short but carefully disinfected pair of clams is selected, and an incision made through the skin, large enough to allow the clams to be pushed to the bottom of the wound, and to lie just under the inguinal ring. The skin is then brought over them and suturen, thus retaining them in the wound. If neither fever, swelling, nor other disturbance is marked during the next few days, the clams are allowed to remain in position for a week. On removal, healthy granulations will be found unaccompanied by pus formation, and the wound heals in a short time under aseptic precautions.

J. F. Stockfleth operated in a similar way. He drew the lappets of skin over the clams, by means of purse-string sutures, but loosened the latter next day, and applied a ligature around the processus vaginalis and above the clams. If this be not done, it is well to fasten the clams to the neighbouring skin by a ligature.

The clams must not be removed too soon. The longer they remain, the better the union between the surfaces of the processus vaginalis. Jessen found three days too little, and in his later cases left the clams on for five days. In the above operation they may remain in position still longer without disadvantage, and need not be removed, even though pus forms, provided fever does not set in. In any case, the clams should not be removed before the fourth or fith day. A bandage and dressing similar to that used in castration of cryptorchids (compare with this) can be applied, to assist in supporting the weight of the clams and preventing the tunica vaginalis tearing.

Dieterichs recommends using a sterilised sponge to temporarily close the processus vaginalis. He opens the latter, ligatures the spermatic artery, and then thrusts a sponge, provided with a central hole, over the spermatic cord, and as high up as possible, so as to occlude the abdominal ring. The clams are then applied to the spermatic cord, but removed again after twenty-four hours, whilst the sponge is left in position until it comes away spontaneously. The disadvantage is, that should the sponge not be carefully sterilised, which is very difficult to ensure, peritonitis often occurs, as shown by the experience 
of Bassi and others, and for this reason this method cannot be recommended; that above described is certainly to be preferred.

Sörensen (Stockfleth) and others apply a ligature above the clams for a similar purpose. The clams are removed next day, but the ligature is left in position until it falls off. Tetanus is, however, said to be a common sequel.

(c) The method of ligaturing the tunica vaginalis, together with the spermatic cord, has not found many supporters; though a few recommend it. It certainly cannot produce such perfect occlusion as the clams, though, if aseptic materials like sill be employed it may render it possible to close the processus still higher in the inguinal canal.

(d) Bouissy's plan of multiple ligation of the scrotum is inadvisable, because of the danger of including a portion of bowel, and to prevent this (even when using the clams over the processus vaginalis), it has been suggested to first incise the processus, and, by introducing the finger, to make sure that no intestine is present. This may be useful in doubtful cases, but an external examination generally suffices.

(3) Closure of the abdominal or inguinal ring. Many operators describe having sutured the inner abdominal ring, but as the modus operandi is never sufficiently well described to convince one of the correctness of the assertion, the point must remain doubtful. In old stallions the narrowest portion of the inguinal canal lies $\frac{3}{4}$ to $1 \frac{1}{4}$ inches below the abdominal ring, and presents an almost insuperable difficulty. If the inner abdominal ring has ever been sutured, an abnormal condition of the parts has clearly existed, to begin with. On the other hand, the position of the inguinal ring (i.e., the outer ring) would easily allow of its being sutured, but the rigid character of the edges (tendinous head of the external oblique muscle) effectually prevents union. Certainly the same success could not be obtained as by the above described methods, and this probably explains its slight popularity.

In the gelding, operative measures must be somewhat modified. Here the processus vaginalis must first be found, which is most easily effected in the following way :-The horse is cast, chloroformed, and laid on its back; the castration cicatrix discovered, and an elliptical incision made through the skin around it, so that the skin which is adherent to the base of the processus or to the end of the spermatic cord can be lifted, together with the sublying tissues, by means of a narrow tape passed through it. The processus vaginalis is then separated as in the operation for scirrhous cord, and exposed for a considerable extent in an upward direction. The clams are now applied over the processus and spermatic cord in the manner above described, provided no intestine is present in the processus; but as one must, in the gelding, always be prepared for adhesions, it is best where the slightest doubt exists to open the processus 
vaginalis, and by introducing the finger to make sure of the absence of intestine or omentum. To prevent injury, the intestine must be separated with the greatest care. For this purpose the scissors are most useful; and if possible the border of the adherent bowel should be found, and where this cannot be clearly made out, a portion of the processus can be left adhering to the surface of the bowel and the two replaced together. Pieces of omentum may be ligatured with sterilised material and cut off, or they may be included in the clams without danger. It is also advisable to partially or wholly rotate the processus vaginalis, or to apply a small pair of clams as high as possible within the wound, allowing their weight to be carried by the skin. Strecker, in operating on a gelding, used a ligature to close the processus vaginalis.

\section{(B) TREATMENT OF INCARCERATED INGUINAL HERNIA.}

This must be carried out as early as possible, and in conformity with the general precautions mentioned under "Treatment of Strangulated Herniæ."

The horse is carefully cast, chloroformed, and laid on its back, the hind quarters being higher than the fore, which Jessen effects by laying the fore-part in a hollow; the hind-legs are bent, and, if possible, the leg of the affected side is drawn outwards and backwards. One then endeavours, by pressing on the neck of the hernial sac, and by drawing and turning as described (vide loc. cit.), to effect taxis, the testicle meanwhile being drawn forward. Reposition may be effected by introducing the hand into the rectum and cautiously pulling on the parts in the inguinal canal, but caution must be used in manipulating the hernial sac, and the older the strangulation the greater the care required.

After complete reduction, the animal is allowed to roll on to the sound side; the hobbles are loosened, and if the animal remains quiet, it may be allowed to lie for a quarter to half an hour, though it must not be forcibly prevented from rising.

If, after trying for half an hour, reduction cannot be effected, herniotomy is usually the only resource. For this purpose anæsthesia should be reinduced or rendered complete; the hoofs of the hind-legs carefully cleansed and covered with moistened cloths, and the hair and undersurface of the legs also moistened to prevent dust falling on the point of operation. If the latter has been cleansed, disinfected, and all the requirements of antisepsis complied with, the skin and tunica dartos are carefully cut through as far as the base of the scrotum and separated from the processus vaginalis with the fingers, assisted by scissors, up to the spot where the neck of the hernial sac appears most markedly strangulated. This point lies in the inguinal canal an inch or so below 
the internal abdominal ring, as Hering and French veterinarians have noted. Reduction may now sometimes be effected by introducing a finger into the inguinal canal and pressing and lineading this point, especially if

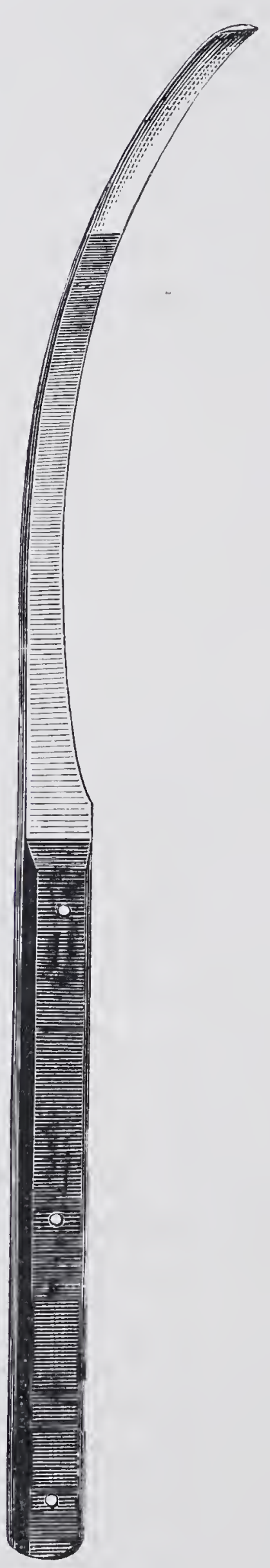

FIa. 128.-Herniotome.

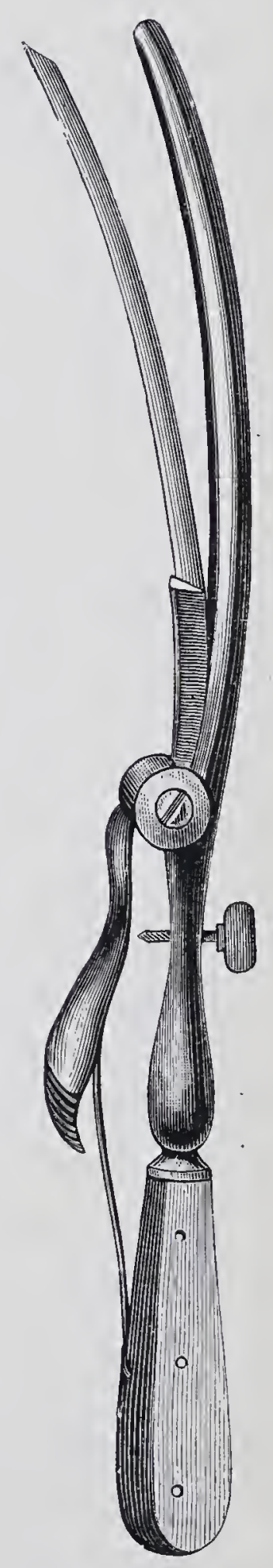

Fig. 129.-Herniotome.

an assistant pass his hand into the rectum and pull gently on the bowel. Möller prefers to carry out this accessory manipulation personally, because, whilst it is important to exercise a steady and regular pull on the portion of bowel in the inguinal canal, the spermatic cord must not be grasped, as it would limit the pull. With a little practice the parts may easily be 
distinguished through the rectal wall. Should these attempts come to nothing, the operator must proceed to herniotomia interna, that is, incision of the processus vaginalis together with the abdominal ring. The seat of incarceration may generally be felt by introducing the finger, and division must be made at that point. The intestine generally lies in front of and above the testicle.

According to Möller's experience, in old stallions strangulation occurs about an inch below the internal abdominal ring. In geldings, on the other hand, he has generally found the annulus abdominalis the narrowest point. Possibly the heavy pull of the testicle on the spermatic cord, which becomes fan-shaped in the abdomen, exercises some influence on the funnel-shaped, or, as the French call it, the "hour-glass" dilatation of the processus vaginalis, which is most noticeable towards the inner abdominal ring. The continuous pull can without doubt produce a dilating influence of that kind, which would explain the greater predisposition of old stallions to protrusion of the bowel at the point indicated.

Girard constructed for this operation a pair of long hernia knives, one in the form of a greatly enlarged straight tenotome, and the other in that of a bent tenotome. The ordinary herniotome (fig. 129) may also be employed, or in case of need, a blunt-pointed bistoury. The abdominal ring must be incised near its anterior angle, and the cut be made in an outward direction. Towards the middle line, and in the neighbourhood of the posterior angle, are the vessels of the abdominal walls, which must be avoided, and any lengthening of the anterior angle is apt to be followed by tearing of the inner oblique abdominal muscle. Even when the point of incarceration has only been incised to the extent of a line or two, reposition becomes easy, and indeed usually results from the pull of the abdominal viscera themselves. Paty recommends pouring extract of opium and belladonna dissolved in oil into the processus vaginalis. This certainly lubricates the contents of the hernial sac and assists reposition; but plain oil sterilised by boiling would serve the same purpose. After reduction, treatment is the same as in non-strangulated rupture, that is, a pair of clams are adjusted as high up as possible over the spermatic cord and tunica vaginalis, which latter should be rotated once on its long axis. ${ }^{1}$

Where it is important to preserve the testicle, the subcutaneous operation, recommended by Bouley, can be carried out, though the strictest asepsis must be observed. The scrotum and tunica vaginalis are cautiously divided at the outer side, in the neighbourhood of the inguinal ring, so that a grooved director may be introduced into the narrowest part of the processus vaginalis, which is then divided with the

1 A full description of several cases of acute inguinal hernia treated by operation will be found on pp. 55 and 371 of Cadiot and Dollar's "Clinical Veterinary Medicine and Surgery." For details of the "covered operation" of castration, see Dollar's "Operative Technique," p. 217. 
hernia knife. Others use the fingers instead of a probe, and pass the hernia knife along these to divide the abdominal ring. Siegen states having thus rendered a horse fit for work in twelve days. The second wound is sewn up and a suitable antiseptic dressing applied. Unless strictly antiseptic, this method is open to grave objection, as Peuch has already pointed out.

A bloodless method of operation which aims at preserving the testicle, but could only be used in very recent cases, is described by Bagge and Grünwald. The stallion is cast, rolled on its back, and anæsthetised. A mass of cotton wool saturated with chloroform is laid on the scrotum. The evaporation is said to cause rapid diminution in volume of the gases enclosed in the strangulated portion of bowel, and to allow of reduction in a few minutes.

In other respects the operation for inguinal hernia is not so grave. Bouley states having effected a cure after strangulation lasting twentyfour hours. According to Stockfleth, of 55 horses, 13 died after dilatation of the abdominal ring; in Alfort, 8 out of 20 died; but Benjamin only lost 5 out of 28 operated on.

Reported cases of the successful use of hernial trusses are rare. Klingan states having succeeded in curing foals by means of a complicated bandage; but in all probability much was due to the intervention of Nature.

\section{(2.) FALSE INGUINAL HERNIA (HERNIA INGUINALIS INTERSTITIALIS).}

This term is used to describe inguinal or scrotal hernix where the hernial contents lie outside the processus vaginalis. The small intestine or rectum, or very occasionally a portion of omentum, pass into the inguinal canal or scrotum, not through the inner abdominal ring, but through a pathological opening which usually lies close in front of the abdominal ring. At this point a lacuna exists in the abdominal muscles of the hor'se, which is filled with connective tissue, and is therefore less resistant. This section of the abdominal wall is lined with peritoneum, and is considered by Franck to be a tendinous expansion of the oblique abdominal muscle; by Schmalz it is termed the inguinal ring.

After rupture of the peritoneum and of this connective tissue, intestine or omentum may pass into the inguinal canal outside of the processus vaginalis, a condition which is termed peritoneal-scrotal hernia (fig. 130). The condition clearly has a great resemblance to genuine inguinal hernia; but the swelling appears higher up, close under the inguinal ring, because the hernial contents are not confined by the processus vaginalis, in consequence of which the base of the hernial sac appears more pointed, and may take the form of a peaked night-cap. Taxis is more difficult 
than in the previous condition. In doubtful cases examination per anum may give reliable information as to the hernial character of the swelling.

The condition was discovered by Hildach during a post-mortem on a stallion, and has been described by $\mathrm{H}$. Bouley in France as "hernie extra-vaginale." Sometimes it is first recognised when performing the operation for inguinal hernia, the contents of the hernia, and not the processus vaginalis, coming in view on incising the skin of the scrotum and the tunica dartos. Stockfleth saw it in boars, but it seems unknown

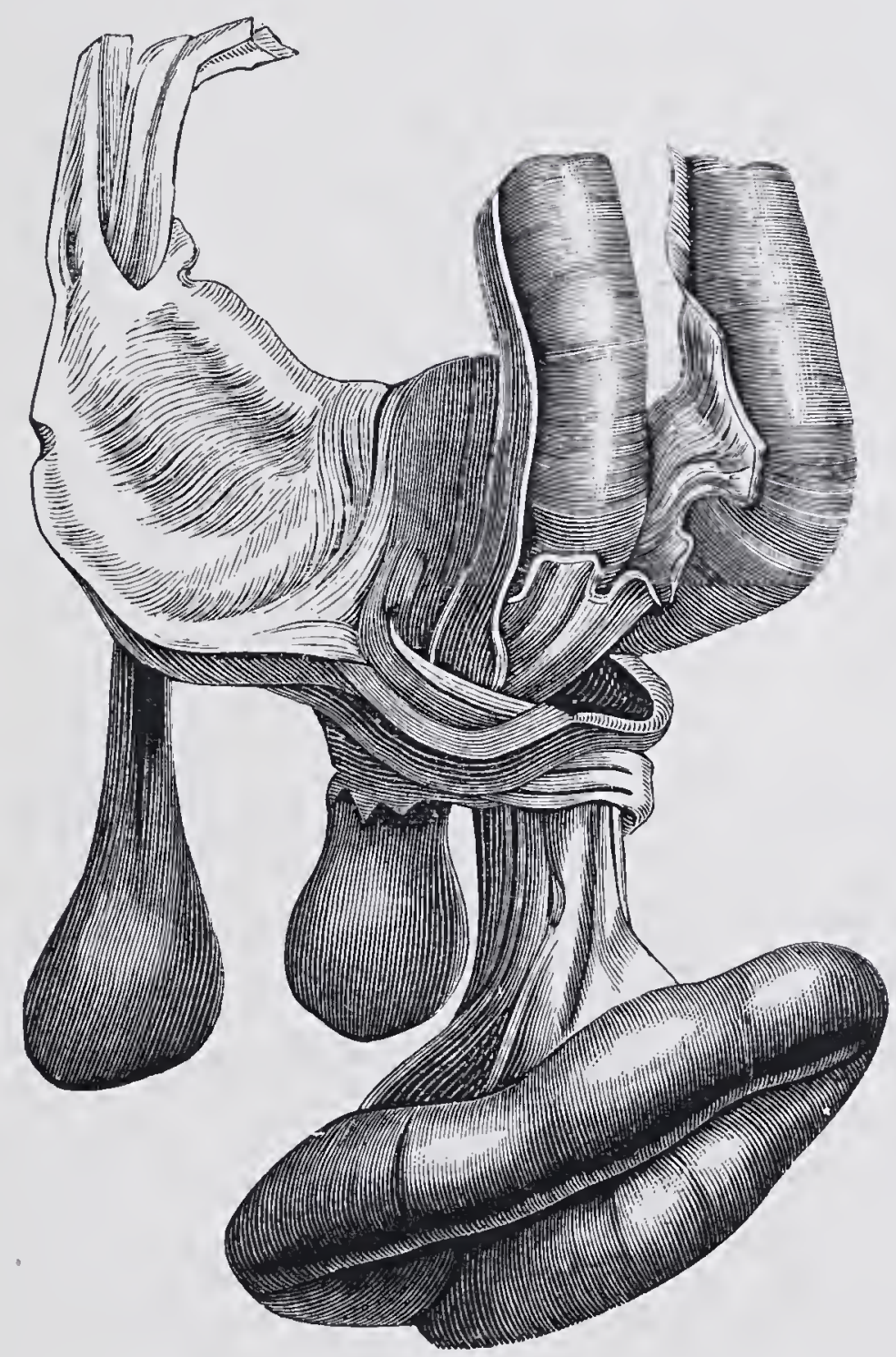

FIG. 130.-Peritoneal-scrotal hernia. (Hering.)

in other animals. The first change consists in rupture of the abovedescribed portion of the abdominal wall, resulting from abnormal increase in intra-abdominal pressure, or possibly from violent movement. Hildach saw this form of hernia suddenly occur after the animal had been ridden.

Prognosis is even less favourable than in true inguinal hernia, the danger of incarceration being greater, and recovery attended with more difficulty, because the processus vaginalis cannot be drawn forward to effect closure of the hernial opening.

Treatment. Where strangulation has not set in, operation should be avoided. Reduction of incarcerated herniæ may be attempted in the standing position, but usually fails, even when assisted from within by a 
hand introduced into the rectum. But under no circumstances should operation be attempted until every effort to effect taxis has failed. For this purpose the same measures may be adopted (casting, dorsal position, narcosis) as in true inguinal hernix. Should herniotomy prove absolutely necessary, the scrotum is cautiously opened under antiseptic precautions. After dividing the skin and tunica dartos, the hernial contents appear, and must be protected from soiling by cloths dipped in disinfecting fluids. The hernial opening is then sought for with the index finger, which is guided by the processus and the hernial contents; and should it prove impossible to dilate the ring with the finger, and return the intestine to the abdominal cavity, the opening must be widened with a herniotome, after which taxis becomes easy. An attempt may then be made to suture the abdominal ring with sterilised catgut or silk. The skin is closed with numerous button sutures, the surroundings of the wound cleansed, dusted with iodoform, and covered with a dressing, as after castration of eryptorchids. Möller states that, in the case of cryptorchids, sutures of the kind described may be inserted in the inguinal canal. Lund operated on an interstitial inguinal hernia with success ; but Hering noted prolapse of the bowel, and death after operation.

\section{(3.) INGUINAL HERNIA IN RUMINANTS.}

Inguinal hernia is rare in ruminants, and only a few recorded cases exist. Youatt saw it in a steer, Löble in an ox, Hess in a calf, Rychner in a cow, Lafosse in a male goat, and Lenguenard in a lamb.

The symptoms and course of the disease are similar to those in horses, though the condition is far less grave than in the latter-the value of the animal not being diminished to the same extent, and it being possible, even where the hernia has become incarcerated, to slaughter without very great loss.

Treatment is much as above described. Should the hernia become strangulated, Löble's method of operating through the right flank may be tried. He thrust the ox against a wall, cut through the skin and muscle below the external angle of the ilium, passed the hand into the abdominal cavity, and found a double loop of intestine strangulated in the left inguinal canal. As this could neither be freed, nor could a finger be introduced into the inguinal canal, Löble inserted a straight bistoury (whose point was guarded with a strip of plaster), holding it between the index finger and thumb, and thus enlarged the abdominal ring. A tenotome is recommended for this purpose, which should be fastened by a cord, so as not to be lost in the abdominal cavity. After freeing the bowel the abdominal wound was sutured, and recovery 
occurred in six weeks. It should not be forgotten, however, that the hernia can often be reduced viâ the rectum, without opening the abdomen.

\section{(4.) PERINEAL HERNIA IN SWINE.}

Both boars and castrated swine often suffer from perineal hernice. The hernia is generally one-sided, very seldom double-sided, and its contents usually consist of a portion of bowel which, in castrated animals, is often adherent to the sac. Omental herniæ are rarer. In swine perineal hernia is usually congenital and hereditary, or develops during the first few weeks of life, seldom later.

Symptoms and course. In sucking pigs the swelling may be the size of a man's fist. The testicle lies at the bottom of the scrotum, which, in consequence of the thin and yielding character of its skin, may be greatly enlarged. Raising the fore-quarters increases the swelling, raising the hind-quarters diminishes it; whilst thrusting the finger upwards into it discovers the existence of dilatation of the abdominal ring. Sometimes the rupture is so small as only to be detected on castration. The little animals fail to develop, whilst the rupture gradually increases in size; but spontaneous recovery is sometimes seen. Though strangulation is not frequent, it occasionally happens both in boars and castrated animals, and is associated with the same symptoms as in other animals-colic, increased hardness and pain in the swelling, and difficulty in replacing the hernia.

Prognosis is more favourable in pigs than in horses, the peritoneum being less sensitive, and not resenting operative interference to the same extent, though castration of a ruptured boar requires particular care, and sometimes ends fatally.

Treatment. In castrating ruptured sucking pigs, the animals are placed for some days on short rations, and starved for ten to twelve hours beforehand. The covered operation is selected and the ligature, which should be rather thick, applied as high as possible, and drawn moderately tight, but not sufficiently to cut through the tunica vaginalis, which might lead to prolapse of the bowel. To avoid this danger Stockfleth sutured the skin, whilst Gerlach inserted a deep interrupted suture.

Should the hernial contents be adherent to the processus vaginalis they must be carefully separated, injury to the bowel being avoided. A case of Eberhardt's shows that even incision into the bowel is not always fatal in pigs. Meyer, in cutting through an adhesion, left a portion of processus vaginalis adherent to the bowel, but had good results. On account of such adhesions the operation is usually more 
difficult in castrated animals than in boars. The second testicle may be removed at the same time. Stockfleth recommends examining the abdominal ring first; when this is too small to admit the finger, the usual method of castration may be adopted, otherwise the covered operation is preferable. In double-sided perineal hernix in sucking: pigs, both testicles may thus be simultaneously removed.

Imminger describes the following method which he has practised for thirty years. In uncastrated sucking pigs the scrotum is opened as in the covered method, and under antiseptic precautions; the testicle, together with the spermatic cord and common vaginal tunic, is then twisted around its long axis, forming a spiral, whilst the left hand, inserted into the canal, assists the operation. As soon as the twisting has extended as far as the outer abdominal ring the hernial contents will be found to have returned to the abdominal cavity, and the spermatic cord, still covered by the common vaginal sheath, is ligatured with silk as close to the outer abdominal ring as possible. The ligatures should not be drawn too tight. The skin wound is sutured.

Imminger uses a similar method in inguinal hernia in bitches. Should the hernial contents be adherent to the sac, or should the pig have been castrated and an abscess have formed in consequence, the common vaginal tunic is opened, the adhesion broken down or the abscess opened, and the operation completed as above.

old ruptured boars are castrated by the covered operation, clams being used, and either left until they fall off spontaneously, which occurs in one to two weeks, or after a similar time removed. The clams, which should be rather small, and applied as high as possible, must be used on both sides, even though the rupture be only one-sided. Degive opens the processus vaginalis to make sure of the complete return of the bowel, and applies the clams as high as possible.

After operation, the animals are placed in a dry stall provided with clean straw, and for some time receive short rations. Local treatment is not necessary, nor, on account of the excitement of the animals, is it to be recommended.

Stockfleth saw a case of peritoneal scrotal hernia in a boar; on operating, the bowel was found outside the processus vaginalis. He therefore enlarged the skin wound, replaced the hernial contents, and ligatured the inner coats of the hernia (which consisted of firm connective tissue) at the same time as the tunica vaginalis. The animal made a good recovery. The case shows that, in opening the hernial sac, care must be taken to avoid injuring the bowel, in case a rupture of this sort should exist. Storch describes a hernia, involving the uterus, one of the Fallopian tubes, and one of the ovaries, in a sow ; the hernia (inguinal) formed a long, oval swelling as large as a child's head. 


\section{(5.) INGUINAL HERNIA IN DOGS.}

Inguinal hernix, although rare in dogs, is common in bitches, in which the abdominal ring remains open to give passage to the round uterine ligament covered by its peritoneal sheath. The hernial contents generally consist of uterus, sometimes containing embryos. Stockfleth found four fully-grown puppies. Sometimes, however, portions of intestine and omentum, and even of the urinary bladder and spleen, are found in the sac, which is formed by peritoneum, udder, and skin.

Inguinal herniæ are only seen in bitches, which have already borne young, the increase in length of the round uterine ligament, and the widening of the abdominal ring, necessary to hernia formation, being effected by muscular action during delivery.

Symptoms and progress. The hernial swelling is least characteristic in bitches, being covered by the udder. It is harder, but less sharply defined than in males, is often as large as a man's fist, lies towards the back of the udder, and appears, both when single and double-sided, as a regular diffuse swelling. In thin animals, and where the udder is only slightly developed, the uterus may sometimes be felt in the depths; in fat animals, having well-developed mammæ, diagnosis is often rather difficult. The possibility of reduction, and the disappearance or decrease of the swelling in the dorsal position, are the most important symptoms.

It is only possible to mistake this condition for tumour of the udder on superficial examination, and it should be remembered that tumours scarcely ever lie so deep as does the hernial swelling. It is more difficult to distinguish it from thickened round uterine ligament, though the latter appears as a hard cord connected with the anterior border of the os pubis.

In doubtful cases the vagina must be examined. Where the uterus lies in the hernial sac the vagina appears lengthened, narrowed or drawn towards the affected side or downwards, and often scarcely admits the finger, whilst the anterior portions of the vagina are less movable than usual.

Incarceration and other complications threatening life are seldom met with. Even delivery is usually easy. Strangulation, however, when occurring, produces symptoms similar to those in other animals. Vomiting is occasionally seen.

Treatment. Unless incarceration (the symptoms of which should be made known to the owner) occurs, treatment is, as a rule, confined to regulation of diet. In France, the injection of iodine was formerly tried, though Lafosse had doubtful success with it; severe inflammation 
occurred, leading to death. When surgical interference becomes absolutely necessary, the radical operation is recommended, though it is more difficult to carry out than in male animals. It consists in ligation of the hernial sac, or of its inner coat, and of the peritoneum and surrounding connective tissue. Narcosis and antiseptic precautions are necessary. An incision is made through the skin and udder; the inner coat of the hernia is then separated and ligatured as near as possible to the hernial ring. This is facilitated, as in swine, by rotating the hernial sac. Should it prove impossible to replace, the uterus can be amputated. Operation should be avoided during pregnancy, soon after delivery, and during œstrum.

\section{CRURAL HERNIA (HERNIA CRURALIS).}

Crural hernia is so rare that Girard altogether doubted its occurrence in animals, and in spite of an extensive experience, Möller has never seen it. Lafosse, however, states having several times had to operate for crural hernia; and usually it is said to have been seen in horses and dogis.

The crural canal (canalis cruralis) consists of a triangular space between the sartorius and iliacus muscles and Poupart's ligament, and lies somewhat nearer the mesial line of the body than the inner abdominal ring, but close behind it. It is usually occupied by the crural artery and vein, by lymph glands, and connective tissue, and is covered by the outer tendinous head of the inner oblique abdominal muscle, and by the peritoneum, by which the passage of abdominal viscera into the canal is prevented. Should, however, this covering become dilated and a portion of bowel or omentum enter the canal, a crural hernia results. The latter consists of an abdominal hernia, to whose covering the fascia cruris further contributes; sometimes a slit exists in the fascia through which the hernial contents have passed.

Symptoms. The hernial swelling, which is usually small and flat, is found on the inner aspect of the thigh, at the point where the vena saphena leaves the surface. It can be replaced in the dorsal position, often causes straddling gait, and when incarcerated, may produce lameness, colic, \&c. Examination per rectum leaves no doubt as to its existence.

Causes and progress. The condition is either congenital or arises during delivery, from the hind-quarters of the fotus remaining a long time in the maternal passages, and the abdomen being powerfully compressed. In later life it may be caused by slipping, drawing heavy loads, or by very severe labour pains. Dandrieux saw a cow develop crural hernia during labour. Spontaneous recovery scarcely ever occurs, and incarceration, which produces the same symptoms as in other hernia, is not uncommon. Crural hernia must, therefore, be considered as at least as dangerous as inguinal hernia. 
Treatment. Lafosse attempted taxis after casting the horse, but if the hernia be strangulated and taxis fail, he recommends operation. An incision is made through the sac large enough to enable Poupart's ligament to be sewn with the sartorius muscle after reduction of the hernia.

In non-strangulated hernix the use of irritants, like cantharides ointment, has been frequently recommended. After operation the animal should be tied up for a week, and fourteen days later may be quietly exercised.

Tidborn opened an incarcerated hernia in a foal, replaced the loop of small intestine, which had already become adherent, and sewed up the wound; the animal, however, died. Lafond states having cured crural hernia in the she ass by using clams. In Germany the disease appears to be very rare. Whether the different food or the heavy work is the cause of its more frequent occurrence in France is uncertain.

\section{IX.-PERINEAL HERNIA.}

The posterior wall of the pelvis possesses in male animals only one dilatation-the excavatio recto-vesicalis; but in female animals there are two-the upper the excavatio recto-uterina, the under the excavatio vesico-uterina. Extension of one or other of these boundaries of the pelvis and entrance of abdominal viscera into the space so caused is termed perineal hernia.

The disease is most frequently seen in dogs, less in the cow, ewe, and bitch, in which animal it usually consists of a dilatation of the excavatio vesico-uterina, which is more exposed to strain on account of its lower position. In male animals either the intestine or urinary bladder is found in the excavatio recto-vesicalis, in females the hernia consists of small intestine. Frick, in the case of a pregnant cow, saw a portion of the gravid uterus in the sac.

Symptoms. As a rule only one side is affected. In dogs a swelling, varying from the size of a man's fist to that of a child's head, and possessing the known characteristics of a hernia, occurs close to the root of the tail and just over the ischial tuberosity. In ewes it may be as large as a goose's egg, and appears alongside the vulva. In the cow a round swelling, sometimes as large as a man's head, arises close under the vagina, and presses forward the labir. The hernia can be reduced and the swelling dispersed by pressure, by raising the hind-legs, or by walking the animal down-hill; whilst it is increased by raising the fore-legs, or by any cause which increases intra-abdominal pressure. Incarceration very seldom occurs, though Siedamgrotzky noticed strangulation of the urinary bladder in a dog. 
Causes and progress. Continuous contraction of the abdominal muscles, difficult labours, heavy draught, walking on the hind-legs (dog), and standing with the hind-quarters low (cows), are known to favour production of perineal hernia, but its immediate cause is a mystery. It may exist for long periods without causing trouble, for, on account of the great width of the hernial opening, incarceration is exceedingly rare, though, according to Stockfleth, it sometimes impedes delivery in ewes, because the vagina is pressed into the hernial sac during the pains, and becomes bent at an angle. Cows with this disease should not be used for breeding. In Frick's case (see p. 299) assistance was

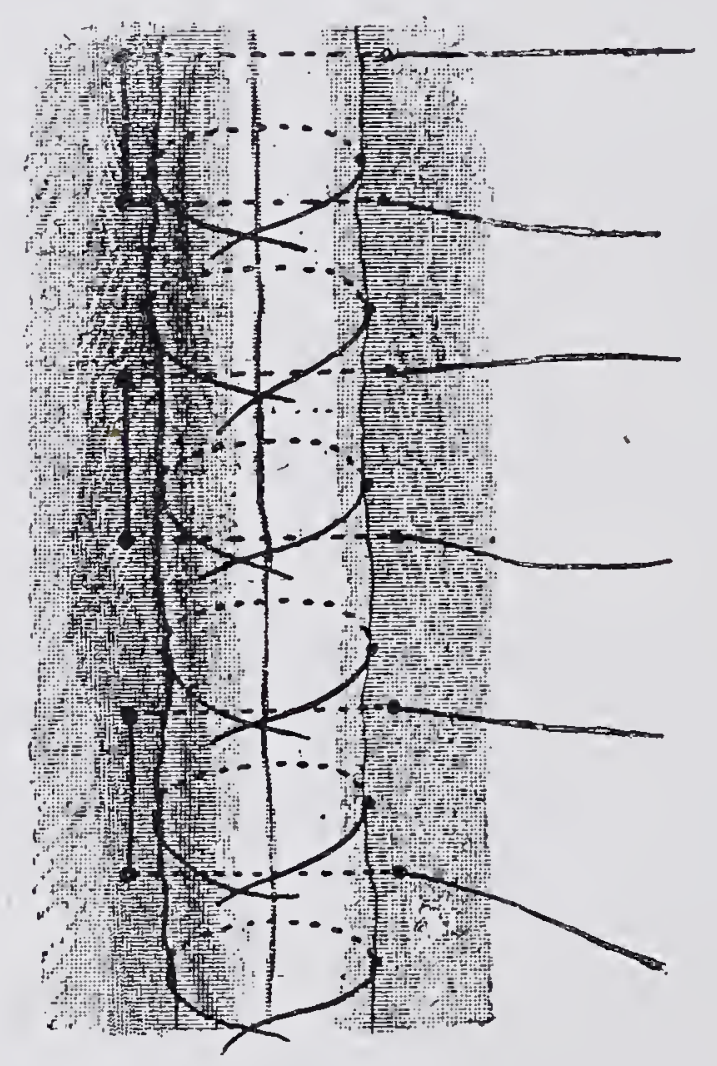

Fig. 131.

required to deliver the calf as the fore-legs became fixed in the hernial sac.

Treatment. As a preventive measure, cows should not be placed with the fore feet higher than the hind. Unless strangulation occurs, all treatment should be avoided; the difficulties during labour are said to be lessened by dilating the vagina. Another reason for avoiding operation is that the hernial contents are often attached to the sac, a condition indicated by the latter being marliedly drawn inwards when the hind-quarters are raised. Should incarceration necessitate operation, or the owner desire it, the animal is chloroformed, an incision made under antiseptic precautions, and the hernial sac ligatured in sections, that is, if the contents of the sac are clearly non-adherent. Otherwise the inner coats must be cautiously cut through, the intestine or bladder separated; the sac brought together with several stitches, and the skin first shortened sufficiently and then sutured. A case treated in the Dresden clinique shows that it is possible to effect a cure, even 
under unfavourable circumstances. The urinary bladder had been incised, but was immediately sutured, and recovery followed. The pursestring suture may sometimes be used to advantage.

A case of rupture of the perineum in a mare consequent on difficult labour, and successfully treated by operation, is described on p. 426 of Cadiot and Dollar's "Clinical Veterinary Medicine and Surgery." The wound was of old standing and had been sutured, but failed to unite. It extended from the superior commissure of the vulva as high as the anus, but without involving the sphincter ani. The edges of the wound were freshened and re-formed and two series of sutures (see fig. 131) were inserted. A second operation became necessary on account of some of the deep sutures tearing out, but recovery was complete within six weeks of the first operation.

\section{X.—VENTRAL HERNIA (HERNIA VENTRALIS).}

Whilst the herniæ hitherto described are due to dilatation of openings normally present, all others caused by solutions of continuity in the abdominal walls (but not of the skin) are described as ventral herniæ. Such breaches are either caused by external injuries, like kicks, treads, thrusts, the impact of blunt bodies, collisions with the carriage pole, staking of the abdomen, and falling on blunt objects, or they may result from excessive muscular contraction during parturition, \&c. Sometimes they attain very large dimensions, as shown by Eberhardt and Dette's cases, and by a mare Möller had under observation (fig. 132). Hertwig believed that congenital fissures in the walls of the abdomen sometimes caused ventral herniæ.

As a rule, the sac consists of skin and and panniculus, the contents (intestine or omentum, or both) having passed through a rupture in the abdominal muscles, to which they later become adherent, the peritoneum is generally torn through. The hernia usually contains intestine, though Noack reports two cases in the cow where it was above the udder, and contained portions of the uterus. One, operated on by Guitard, was in the right flank, and contained the abomasum. Gerlach and Schmiele have seen hernix containing portions of the liver. A pig described by Frick, which had died of double-sided hydronephrosis, showed just in front of the os pubis a hernial opening through which the bladder had slipped and become bent backwards.

Symptoms. In recent ventral herniæ two sets of symptoms exist, those of hernia proper, and those of rupture and bruising of tissue; the latter may, indeed, appear the more important and mask the hernia. Ventral hernix are found most frequently near the last rib, in cattle on the right, in horses on the left side. The parts are acutely inflamed, 
painful on pressure, and, in consequence of adema, either firm or doughy; if much blood has been poured out, there may be fluctuation. Older hernix fail to display such symptoms, but will be recognised by their compressibility, fluctuation, softness, varying size, \&c. In the depths, the hernial opening may usually be felt as a round or elongated aperture.

The swelling varies within wide limits; in small animals being often only the size of a pigeon's egg, while in cattle and horses it may exceed

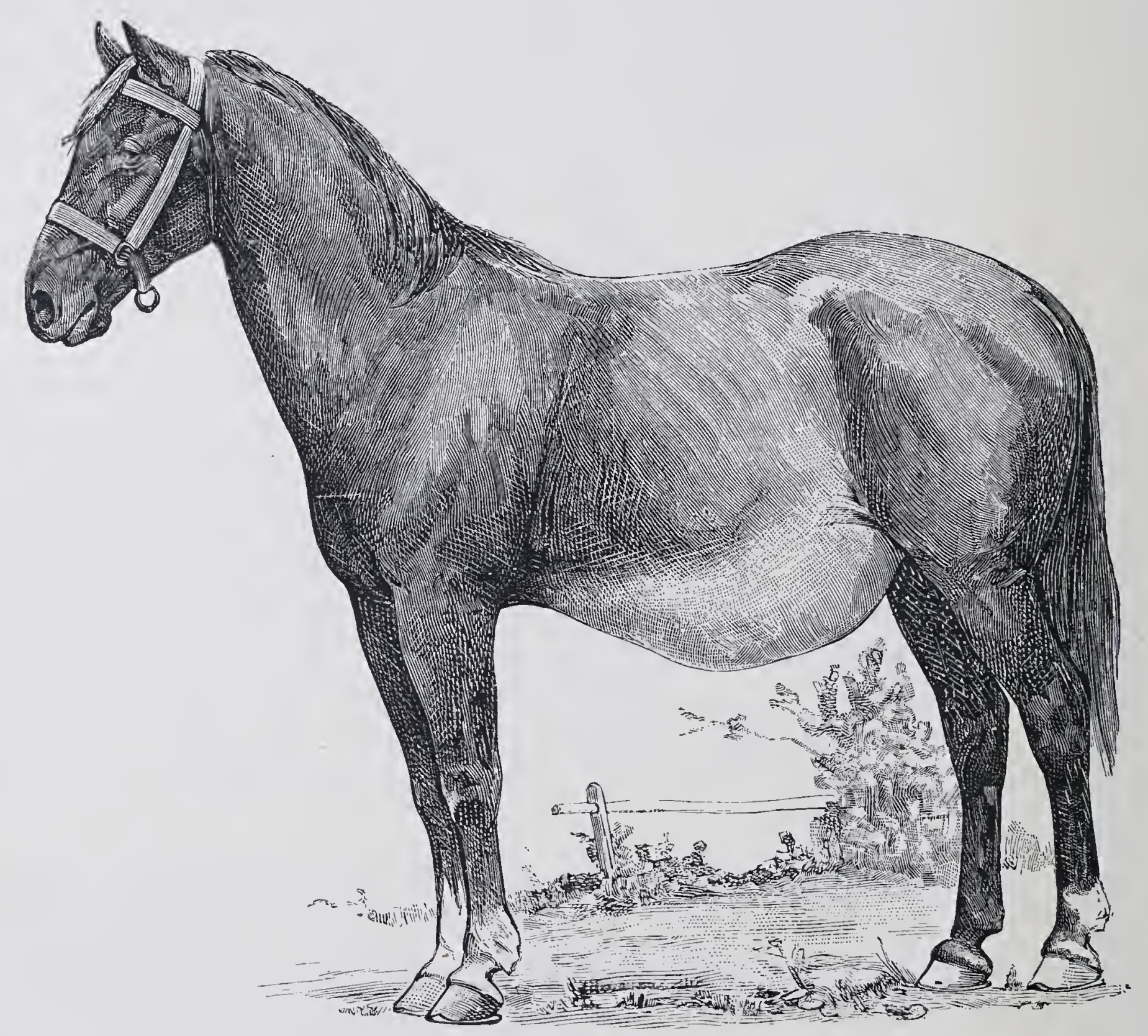

Frg. 132.--Ventral hernia in a mare (from a photograph).

that of a sugar loaf. In the horse the hernial swelling seldom exceeds the size of the clenched fist or, at the most, of a man's head, but greater dimensions are occasionally reached, as in the case of the mare shown in fig 132. According to its position, it is termed hernia lineæ albæ, hernia iliaca, or hernia perumbilicalis. The contents are usually formed by intestine or omentum, seldom by other abdominal organs.

Differential diagnosis. Recent cases may be mistaken for inflammatory swellings or hæmatomata in the abdominal walls. In those of old standing the hernial aperture can always be discovered by palpation, provided accidental inflammatory processes are absent from the neighbourhood of 
the sac. The sudden appearance of the swelling and its compressibility must arouse suspicion of a rupture. In any case, caution is required in operative interference, and, in doubtful cases, sharp instruments should not be used before making certain of the absence of hernia. Rectal examination is sometimes useful, and, if needful, the parts can be punctured with a trochar, under antiseptic precautions.

Particular caution is indicated in presence of colic, which may proceed from incarceration, but in fresh injuries may also be caused by the pain associated with peritonitis; though the latter is the exception, the former the rule.

Strangulation is not at all common; some have denied its occurrence altogether; but undoubtedly both recent and old ventral hernire do occasionally become strangulated, though the danger is much less than in either the umbilical or inguinal forms. The symptoms are similar in all. The necessary conditions are a small opening and a large sac. The immediate cause may be heavy work, tympanites, colic, or parturition, and it is common experience that the small intestine becomes more readily strangulated than the colon. Small ventral hernix sometimes disappear spontaneously, and even large ones diminish with lapse of time.

Ventral are thus usually more hopeful than inguinal hernix, though they may seriously interfere with the use of hard-worked horses, and are always a danger in the event of the animal suffering from colic or tympanites, or becoming pregnant; and, for this reason, female animals with ventral herniæ should not be used for breeding.

Treatment is best confined, in recent cases, to counteracting inflammatory symptoms, unless strangulation has occurred. Under favourable circumstances, the hernial contents may be retained, or further egress prevented, by a carefully applied compress; but it must not be forgotten that any considerable pressure may cause necrosis of the skin and favour prolapse. Old ventral herniæ seldom receive treatment, unless they interfere with the animal's work, when the same means may be employed as were formerly described (vide "Umbilical Hernia"). Trusses can seldom be used, and treatment is then practically confined to the following :-

(1) Application of irritants or mineral acids. The same principles apply here as were described in umbilical herniæ. Rademacher recommends sulphuric acid.

(2) Simple or multiple ligation, or the application of clams. This method presupposes the absence of adhesion, which, as already stated, is somewhat rare. Simple ligation is seldom successful, on account of the large base of the hernia, and therefore recourse must be had either to multiple ligation or to clams, after making sure of the 
absence of adhesion. Either wooden clams (castrating clams) or the iron ones shown in figs. 124 and 125 may be used, and the process is the same as in umbilical hernia.

Degive has used the following method extensively in cases of ventral and umbilical hernia. He first opens the hernial sac under antiseptic precautions, in order to break down any existing adhesions, and then transfixes the skin and edges of the hernial ring with pack needles about 8 inches long. Above these he adjusts a clam, which is closed by means of a screw and firmly secured. The pack needles are then replaced by

Fig. 1

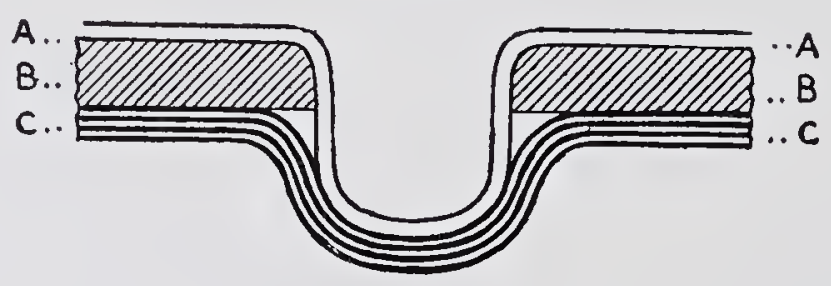

Fig. 2.

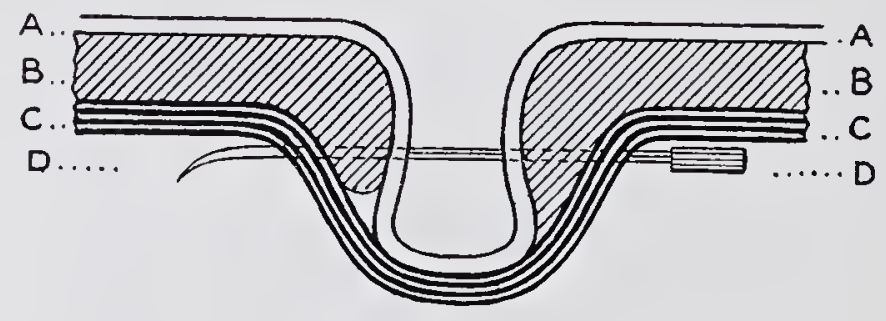

Fig.3.

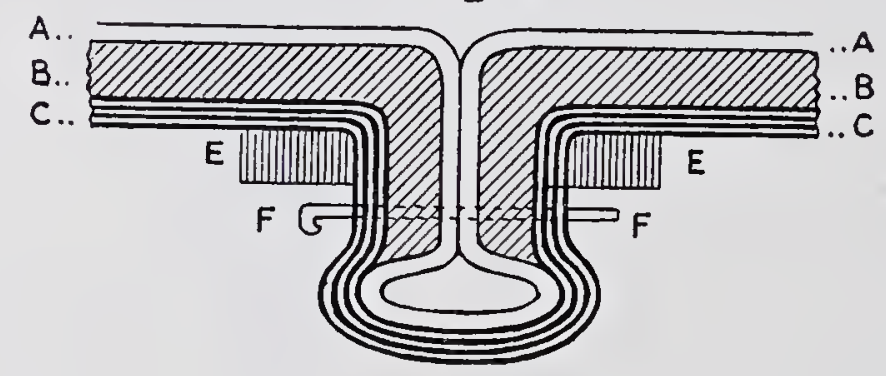

FIc. 133. - Schema illustrating Degive's operation for umbilical and ventral herniæ. A, Serous; B, musculo-aponeurotic, and C, cutaneous coats of the hernia; D, the special needle in place; $\mathrm{E} \mathrm{E}$, clams; F F, nails. The three figures show the successive stages of the operation.

horse-shoe nails, the points of which are bent round. In about a week the necrotic tissue falls away, and recovery occurs even in severe cases in which previous treatment had failed.

(3) Herniotomy. This only succeeds where the rupture is slit-like, and can be sutured. Jürgens reported several successes-in one the cow calved without any difficulty soon after operation; in another cow, showing symptoms of incarceration, Meyer sutured a recent ventral hernia in the neighbourhood of the udder. The colon and a convolution of the small intestine were replaced, and both the abdominal ring and the skin brought together. It is, however, often very difficult to suture tissues already saturated with blood and inflammatory products, and such treatment is much less successful in horses than in cattle and carnivora. In old ventral herniæ extensive adhesions often exist 
between the contents and the sac, and occasion great difficulty to the operator; and whilst he must be prepared for this, he should use the greatest care in endeavouring to break them down. The external operation (vide "External Herniotomy") sometimes succeeds, but not always, the inner hernial envelope, which has been retained in the hernial opening by means of the purse-string ligature, sometimes failing to become adherent and the hernia reappearing later. In general, the larger the aperture the slighter is the hope of cure.

Incarcerations may sometimes be reduced by steady pressure from without, and assistants may even be entrusted with the treatment. It has been stated that in ruptures occurring in the posterior portion of the abdomen, reposition may be assisted in large animals by passing the hand into the rectum.

Gerlach and Schmiele operated on a dog suffering from fractured ribs, and a hernia containing part of the liver. A firm but compressible swelling, almost as large as a man's fist, had suddenly appeared in the left subcostal region. The hernial sac was divided, one lobe of the liver, which was found between the ribs, was replaced, the wound sutured, and a cure effected.

The mare shown in fig. 132 foaled without any help, in spite of the extensive nature of the rupture, the animal instinctively lying on the abdomen ; it was then, however, killed, and the post-mortem showed that the straight abdominal muscle was ruptured a hand's-breadth in front of the os pubis, and that an aperture existed, measuring 20 inches in one, and 24 inches in the other direction. Both the oblique and transverse abdominal muscles were torn away. In the rupture lay a great part of the cæcum, its point directed backwards. Connective tissue had formed in all directions in large quantities, and the fascia of both thighs was greatly thickened as far down as the hocks; lameness had not been observed during life.

Guitard operated on a ventral hernia containing a portion of the abomasum, by incision through the flank. After thrusting back the abomasum the muscles were brought together, a dressing and compress applied, and the case terminated successfully.

Cadiot and Dollar describe a ventral hernia in the left flank caused by a blow from the handle of a windlass. The abdominal tunic was ruptured opposite the stifle, and slightly above this point the muscles were torn through, leaving the herniated small intestine only covered by skin. After incision the hernia was reduced, the muscles and aponeuroses united by two separate lines of silk sutures, the skin was brought together and a dressing applied. In less than a month the wound had healed and the hernia was cured. ("Clinical Veterinary Medicine and Surgery," p. 369).

\section{XI.-PELVIC HERNIA, INTERNAL ABDOMINAL HERNIA,, PERITONEAL HERNIA, "GUT-TIE" (HERNIA INTERNA ABDOMINALIS).}

This affection occurs most frequently in hilly regions, like Switzerland, but has been seen in England and Denmark. Though first described by Oesterlen, it was left to Anker to explain the nature of

$$
\text { V.S. }
$$


the condition and its mode of origin. It almost always affects two to three year old oxen, occurs on the right side, and results from a portion of the colon, or less frequently of the small intestine or omentum, becoming strangulated by passage through a rupture in the peritoneal covering of the spermatic cord. The bowel usually becomes fixed in an aperture of the peritoneum in the neighbourhood of the sacrum and strangulated, though in other cases the free end of the spermatic cord returns into the abdominal cavity, and may encircle portions of the bowel in the neighbourhood of the abdominal ring.

Causes and progress. The immediate cause is a solution of continuity in the peritoneum covering the cord, or displacement of the cut end of the cord, though this is rare. Both conditions are due to unskilful castration, especially to pulling on the cord, without previously grasping it above, as in forcibly tearing off the testicles, a custom still common in the south of Europe.

The initial rupture is also favoured by heavy work in hilly districts. In climbing slopes, the viscera are pressed backwards, part may pass through the rupture existing in the peritoneum of the cord, or may possibly even cause rupture, and then become incarcerated. As the size of the rumen, which lies on the left, ensures it against strangulation, it is easy to explain the frequent occurrence of the condition on the right side. Failing early assistance, the animals die in four to eight days from incarceration, rupture of the bowel, and peritonitis. Prognosis is only favourable during the first few days, but as long as symptoms of peritonitis are absent, proper treatment generally leads to recovery.

Symptoms. The condition is announced by colic, loss of appetite, striking with the feet towards the abdomen, moving backwards, frequent lyingdown followed by suddenly springing up, and general restlessness. Where much green food is given, tympanites may also be observed.

After twelve hours an apparent improvement sets in. The animals are quiet, again masticate, and possibly take food. During the next few days, however, they relapse; colic returns, the animal passes blood-stained fæces or mucus, has difficulty in breathing, and the pulse becomes small and frequent. As soon as these symptoms of peritonitis have appeared, the condition is in the highest degree threatening.

Sometimes pain is shown on pressure in the right flank, and on examination per rectum, a swelling about as large as a man's head, which is painful and doughy, at first soft, but later becomes hard, may be detected close in front of the entrance to the pelvis, and near the wall of the abdomen. Towards the middle line it is bordered by a tensely stretched cord. In stangulation by the free end of the cord a similar swelling may be recognised on the lower abdominal wall, near the abdominal ring.

These symptoms leave little doubt as to the nature of the disease, 
though occasionally it may be confused with invagination of the bowel (compare with this), or with other kinds of incarceration. Anker saw strangulation caused in one case by a hole in the round uterine ligament. Lindenberg states having seen similar symptoms from rectal obstruction. Examination of the rectum always gives the necessary information.

Treatment. In fresh cases reposition may be attempted by raising the hindquarters or walking the animal down-hill, but little time should be lost in such attempts, because taxis becomes more difficult and dangerous the longer the malposition exists.

(1) Reicherter and others have suggested performing taxis through the rectum. When the incarcerated portion of bowel is not much distended, this may succeed, but later becomes impossible. Whilst Reicherter states having thus cured sixteen animals; others have altogether questioned the efficacy or possibjlity of the operation. In making the attempt, the animal must be placed with the fore legs lower than the hind; the loins pressed upon, to prevent arching of the back, the hand introduced into the rectum, and efforts made to thrust the strangulated bowel forward and so to free it. To prevent the animal lying down during operation a sack may be passed under the body and held on either side by one or two men; full-grown animals can be pressed against a wall by means of a long stout pole laid along the body. Lösch claims to have reduced the hernia in two cases by throwing the animal down, tying the fore and hind limbs respectively together, and thrusting a pole between them in the long axis of the body. The abdominal contents were then vigorously kneaded and moved about, the legs meanwhile being raised and lowered by means of the pole.

(2) Others recommend rupturing the spermatic cord from the rectum. Schenk states having grasped the

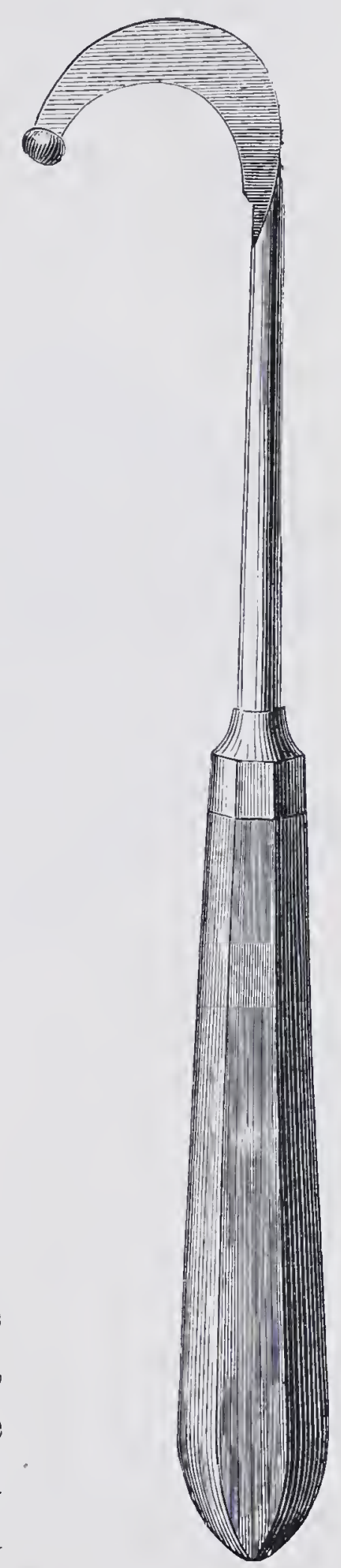

FIG. 134.-Anker's guarded knife for pelvic hernia. cord with the extended left hand, and having drawn it so far backwards that it broke in two; some operators recommend placing the thumb against it and pressing forwards to effect rupture. In any case, it is of importance to protect the rectum, which is best effected by Schenk's method owing to the broad surface employed.

(3) Whilst Anker states having thus invariably succeeded, others have failed to produce the desired rupture, and have proceeded to open the 
abdomen. For this purpose an incision, about 4 to 5 inches in length, is made in the skin of the right flank, following the direction of the outer oblique abdominal muscle. After dividing the other muscles to the same extent, the peritoneum is broken through; the hand introduced into the peritoneal cavity, the omentum perforated, and a search made for the incarcerating spermatic cord, which is divided either with a bistouri caché or with a specially constructed knife in the form of a hook. When the operator has convinced himself of the absence of a similar condition on the other side, the wound is sutured and treated according to general principles. If performed early, the operation is stated not to be dangerous. Strauss lost only 11 animals out of 110 subjected to operation; Kuhmann cured 112 cases in ten years; von Weil claims only to have lost 4 cases out of a total of 200 ; Anker records 44 recoveries.

\section{XII.-DIAPHRAGMATIC HERNIA (HERNIA DIAPHRAG- MATICA).}

Rupture of the diaphragm is commonest in horses, but also occurs in dogs and other animals, and is caused by sudden falls, by rearing and falling over, and by severe tympanites; in exceptional cases, it occurs as a complication of fractures of the ribs. Abdominal organs may then pass into the thorax, a condition termed diaphragmatic hernia. Lehnhardt saw the disease in a cow; the recticulum had entered the thorax. In dogs the stomach, liver, or spleen may be found in the thorax; in horses the small intestine or, more rarely, the colon. Provided the bowel does not become strangulated in this opening, respiration is little affected.

Symptoms and progress. Large masses of abdominal viscera entering the thorax may cause sudden asphyxia by compressing the lungs, whilst strangulation produces the usual symptoms, and, taxis being impossible, soon leads to death. In the absence of such fatal complications, difficulty in breathing is marked, the animals behaving as though broken-winded, owing to interference with the diaphragm, and ruminants show digestive disturbance.

It has been stated that the condition may be diagnosed with certainty by auscultation, but such is seldom the case. It is always difficult to say whether the peristaltic sound originates in the abdomen or the thorax, and, therefore, beginners may easily form an erroneous diagnosis. Nor can a tympanitic percussion sound be regarded as evidence of the disease, because this accompanies a series of changes in the lungs, and in short no absolute diagnosis can be founded on clinical examination. The character and course of the symptoms and the absence of fever, though never pathognomonic, are perhaps the most reliable guides, though an 
exploratory puncture with Dieulafoy's aspirator may settle the diagnosis. Should the prolapsed viscus be penetrated, fluid, partially-digested food will be drawn off. No doubt can then remain as to the condition.

Prognosis is always unfavourable and treatment unavailable, because even though reposition could be effected by performing laparotomy, the condition would probably recur, it being impossible to close the diaphragmatic opening.

Schrader saw an uncommon complication in the horse. The 7th rib was broken and a fragment had perforated the diaphragm, producing an opening

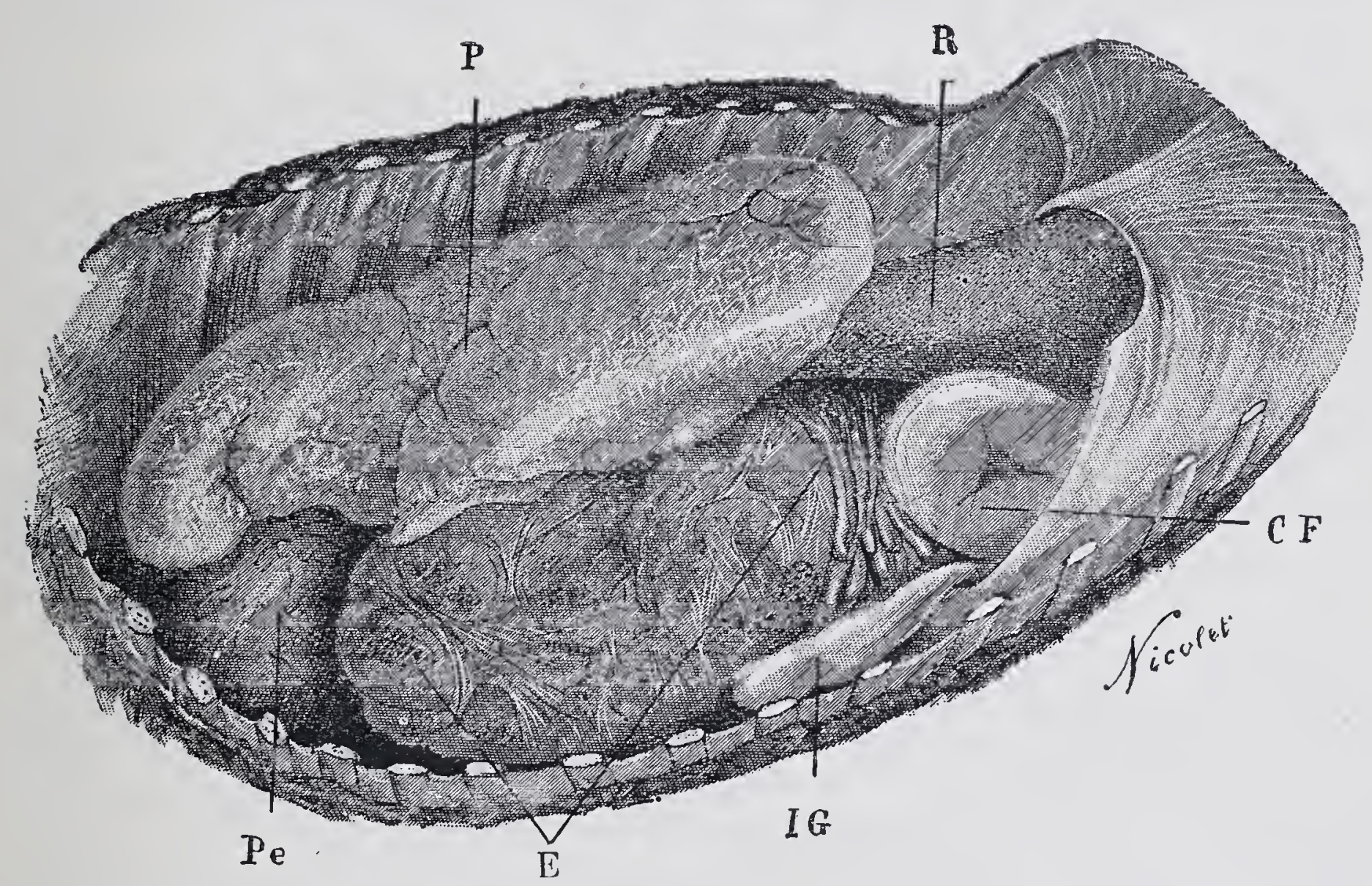

FIG. 135.-Diaphragmatic hernia. E, Epiploon ; I G, loop of small intestine; CF, floating colon; $\mathrm{R}$, spleen ; $\mathrm{P}$, lung ; $\mathrm{Pe}$, pericardium.

through which portions of bowel, omentum, and spleen had passed into the thorax, and making their way through the thoracic wall and under the skin, had produced a hernia as large as a man's head just below the left elbow. The horse lived for several years, but finally died in consequence of the hernia becoming strangulated.

A case of diaphragmatic hernia is described in Cadiot and Dollar"s "Clinica] Veterinary Medicine and Surgery," p. 373, while the condition is fully treated on pp. 44 et seq. of the same work. The case referred to is here illustrated (fig. 135). The rent in the diaphragm had probably existed for many months, judging from the condition of its edges, but at first had only given passage to a few loops of small intestine and epiploon. Seven days before death the horse showed colic, but apparently recovered. Twelve hours before death, however, it had violent pain, which continued until the end. The gastric and splenic hernix were probably produced at that time; soon after becoming herniated the stomach ruptured. 


\section{SURGICAL DISEASES OF THE STOMACH AND BOWELS.}

\section{I.-FOREIGN BODIES IN THE DIGESTIVE TRACT.}

Sharp substances, like needles, wire, nails, \&c., accidentally present in the food are sometimes swallowed by oxen, less often by sheep and goats, and give rise to injuries both of the digestive tube and of other parts, like the pericardium and heart. Horses are not so often affected, though in one case a quantity of nails, buttons, and screws were found in the colon of a horse, their abraded condition showing they had already lain there for a long time.

Dogs often swallow stones, corks, balls, or coins which they have picked up or had given them to carry; whilst in the stomachs of oxen and horses portions of probangs or balling-guns are sometimes found.

Hahn, while making a post-mortem of a horse, found an abscess in the spleen, containing a piece of wood 13 inches in length and $\frac{1}{2}$ an inch in thickness. In one case a pig swallowed a castrating knife 4 inches in length, which remained lying in the stomach for two months without producing any marked disturbance. A similar experience in the dog is related by Iwersen. A sporting dog swallowed a pocket-knife while carrying it to its master, but vomited it again nineteen days later. Seven stones, of a collective weight of $5 \frac{1}{4}$ ounces, were found in the stomach of a Newfoundland dog. The organ was greatly distended, its mucous membrane thickened and covered with warty growths. The dog had been accustomed to play with stones, tossing them into the air and again catching them, and this had at length proved fatal, a piece of coal having blocked the ileo-creal valve and occluded the bowel. A cat swallowed a glass-headed hair-pin, $4 \frac{1}{2}$ inches long. The head had entered the stomach, but the sharp end remained in the oesophagus, and led to perforation and death.

The danger thus occasioned is of a double nature: sharp foreign bodies, like needles, nails, \&c., perforate the wall of the stomach or bowel, and lead to fatal peritonitis, or they penetrate the diaphragm and produce septic pericarditis. This is the rule in cattle, where such bodies enter from the reticulum. It often happens that, at the point of injury, he stomach or bowel becomes adherent to the abdominal wall, leading to perforation outwardly and escape of the foreign body. The discharge of needles, hair-pins, and portions of wire has often been observed in cattle, and usually occurs on the left side, close behind the elbow. Avril removed 
a tobacco pricker, which had lain for six months in the ox's rumen, from this point. Recovery is generally perfect, stomach or bowel fistula being seldom produced. Sometimes the foreign body enters other organs, such as the spleen, and induces abscess formation, and death from peritonitis. Sheep and goats are less frequent victims, though cases of perforation of the abdominal walls and fatal peritonitis have also been seen in them.

Smooth foreign bodies, in consequence of their weight or size, remain lying in the stomach or lumen of the bowel, though heavy objects, like metal balls, knives, \&c., often fail to pass the stomach. Sometimes they produce no discomfort, as in the case of a dog which swallowed a grapeshot, and in Nichoux's case, where the animal carried a silver five-franc piece and a large sou in his stomach for twelve years; but sometimes digestion is disturbed. Lighter foreign bodies enter the bowel and are apt to become fixed at the ileo-creal valve. Bottle corks are particularly dangerous, on account of their swelling in the bowel.

Symptoms and course. Patients are seldom directly observed when swallowing foreign bodies, and the presence of the latter in the digestive tract can only be discovered by the symptoms they give rise to. In cattle, the first sign of injury to the stomach is sudden unaccountable disturbance in digestion, with periodic colic and tympanites; dyspnœa soon follows, in consequence of injury to the diaphragm and lungs; irregularity in the action of the heart sets in later, the cardiac beat becomes laboured and intermittent; auscultation reveals pericardial murmurs, whilst the heart sounds themselves still appear normal. To these are added rubbing, scraping, buzzing, or creaking sounds, or fluid sounds, like gurgling and bubbling. Circulation is impaired, cdema is present below the breast and in the dew-lap, and the jugular vein betrays pulsation (venous pulse). And lastly, palpation of the abdomen on the left side, close to the ensiform cartilage towards the site of the reticulum and in the heart region, may cause pain.

In dogs, obstruction of the bowel caused by foreign bodies shows itself by vomiting and complete loss of appetite. The animals vomit all kinds of nourishment-even pure water-soon after receiving it. Severe febrile symptoms, which generally accompany inflammatory diseases of the mucous membrane of the stomach or bowel, are here wanting. Under such circumstances diagnosis can scarcely be doubtful.

By palpation of the bowel the foreign body may sometimes be felt. For this purpose the dog is placed on his hind legs, the body grasped from above, and the viscera allowed to glide between the fingers of the two hands, by moving these with slight pressure from the under portion of the abdomen towards the vertebræ. But such manipulation often requires to be frequently repeated to discover the foreign body. Sometimes the latter is not felt on the first attempt, but may be discovered 
after a short interval, say an hour; sometimes it is so far forward, and so sheltered by the ribs, as to altogether escape palpation. Examination by Röntgen rays gives immediate information as to the position and character of the foreign body, and has been successfully resorted to in dogs.

It is easy to avoid mistaking masses of fæces in the rectum, or the kidneys or abdominal tumours, for foreign bodies. The first will be recognised as long sausage-shaped cords of a softish character. A foreign body is differentiated from a kidney by the fact that it may be pressed towards the lower abdominal wall, which cannot be done with a kidney. The greatest difficulty is in distinguishing foreign bodies from tumours, but as these are very rare in the anterior part of the abdomen, they may almost be left out of account.

Dogs with stoppage of the bowel generally die in eight to ten days, with symptoms of extreme weakness; oxen with injuries to abdominal and thoracic organs after an illness of varying length. Recovery is rare, except where the foreign body perforates the abdominal wall.

Treatment usually comes too late. In oxen, repeated attempts have been made to remove sharp foreign bodies by rumenotomy. Obich cured four cases out of thirteen; in three, abscesses formed, and in five help was too late. Meyer has operated with success. After making an incision into the rumen in the usual way, the operator inserts his arm in a forward and downward direction to discover the reticulum, which lies on the right, searches this carefully, and endeavours to remove the foreign body. The chief difficulty is the impossibility of certain and early diagnosis. Provided the foreign body has not injured the diaphragm and pericardium, there is little to indicate its presence. Disturbance of digestion and breathing, caused by injury to the wall of the stomach and to the diaphragm, are not sufficient to warrant operative measures, whilst, should the pericardium be already injured, operation can scarcely procure recovery, and slaughter is generally preferable. Meyer, however, states having succeeded, even after pericarditis had set in. Eppele and Seloz removed a piece of probang and gloves from a cow's stomach by rumenotomy (see that heading).

Dogs are sometimes seen to swallow the foreign body, and in such case an emetic should first be tried, but not before giving a considerable quantity of firm food (flesh), so as to distend the stomach. Frecal stasis, if already existent, may be overcome, and the foreign body brought away by injecting lukewarm water into the rectum. Plenty of fluid should be used, so as to distend the bowel and open the way for the foreign body. Sometimes the hypodermic injection of eserine produces powerful peristaltic action and passage of the offending object, but purgatives given per os are useless, and arealways vomited. 
As a last resource, laparotomy may be tried, though it generally comes too late, the animal's strength being gone before operation is determined on. Siedamgrotzliy, however, was successful with a dog which had swallowed a large flint stone. Adam removed a grape shot from the stomach by

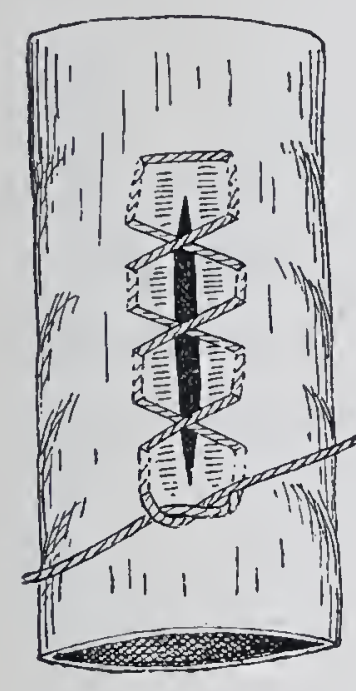

Fid. 136 . Gely's bowel suture.

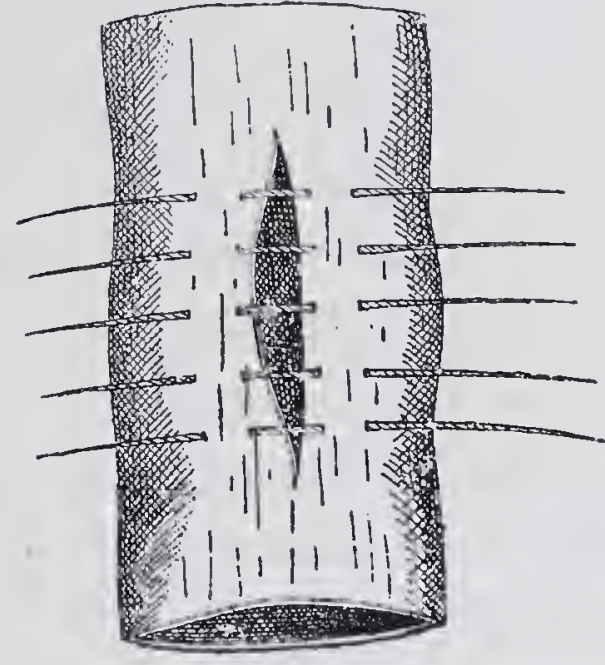

FIG. 137.Lembert's bowel suture.

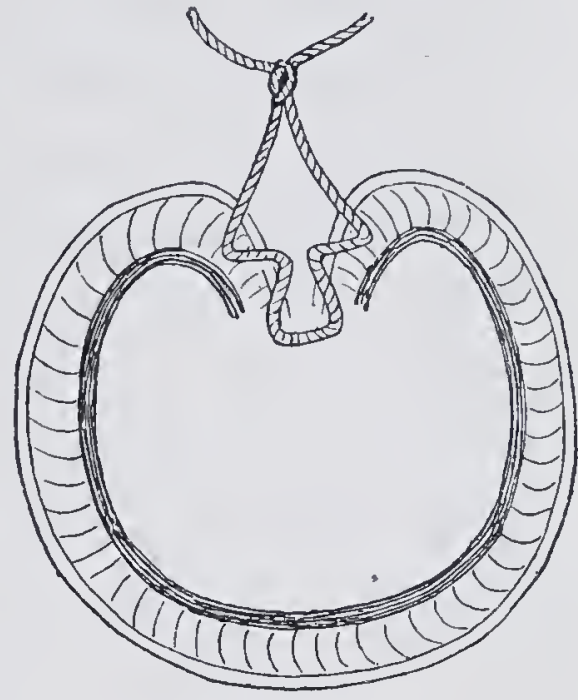

Frg. 138.-

Wölfler's bowel suture. gastrotomy, and effected a cure. The strictest antisepsis is, however, necessary. After narcotising the dog with morphine, followed by rether, or simply with chloroform, the lower surface of the abdomen between the umbilicus and sheath or umbilicus and pubis is shaved and disinfected. The skin is then cut through close to the linea alba, and the muscular tissue divided for a distance of 2 to 3 inches. The peritoneum is next incised (vide "External Herniotomy"), the finger introduced into the cavity

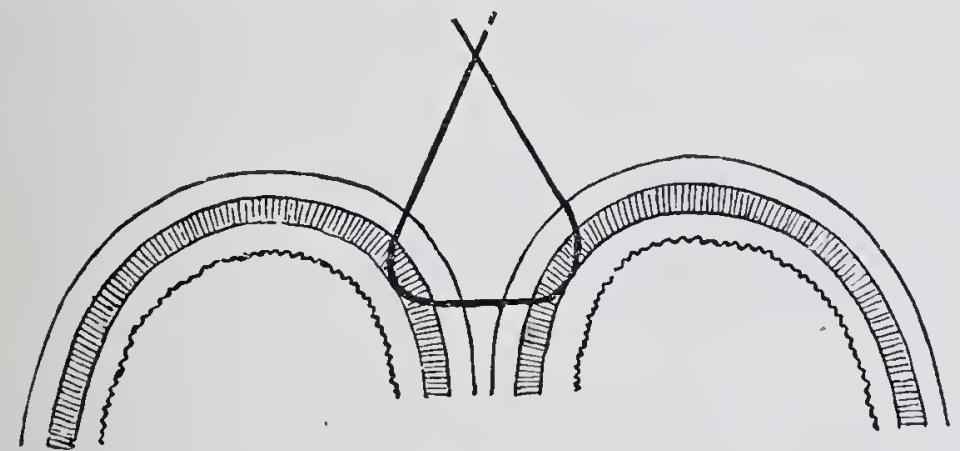

FIG. 139.

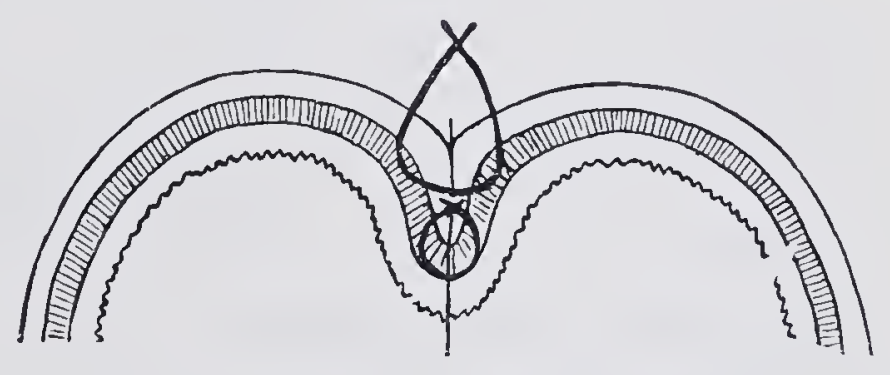

Fig. 140 .

to prevent injury to the bowel, and the peritoneum finally divided to the same extent as the abdominal walls. Two or three fingers are now introduced into the peritoneal cavity, and the foreign body sought. To prevent prolapse of the bowel, the left hand, or, preferably, a cloth saturated with disinfecting fluid, is laid on the wound. Frick passes one or more silk sutures through the entire thickness of each lip of the wound (including the skin, muscles, and peritoneum), and uses these sutures to hold apart or to approximate the edges.

It often takes some time to discover the affected piece of bowel, and one must not lose patience. As soon as the part is felt, it should, if possible, be drawn through the abdominal wound. The portion of 
bowel coming from the stomach (afferent portion) is immediately recognised by its being distended, and should be kept closed by an assistant pinching it. The bowel is now incised immediately over the foreign body, and in its long axis, as far as seems necessary. After taking away the foreign body, the wound in the abdominal wall is closed. At this time it is important to keep the afferent portion of bowel closed to prevent advancing ingesta soiling the wound, and, in case of need, a provisional ligature may even be applied, but must not be drawn very tight. It can be replaced by a twisted strip of sterilised gauze applied with moderate tension around both the afferent and efferent portions of bowel. To soak up discharge a thick mass of sterilised gauze may be loosely applied around the bowel on the inner side of each of these ligatures. Finally the intestinal wound is closed with bowel sutures (figs. 136 to 138). As it is very important to bring the serosa of both sides into contact, and to make the closure as perfect as possible without piercing all the intestinal coats, which would perhaps lead to peritonitis, one of the methods recommended by Gely, Lembert, or Wölfler may be employed. Thereafter the surface of the intestine and of the wound is once more cleansed, the abdominal muscles and skin are sutured with sterilised material, and a proper dressing applied. Where the animal is particularly valuable continuous watching may be necessary to prevent the dressing moving or being torn off. A dog operated on by Möller, pulled off the dressing and loosened the sutures, occasioning prolapse of the bowel and death.

In the horse laparotomy has often been successfully performed for the castration of eryptorchids, and McQueen, in a paper on "Abdominal Surgery" read before the National Veterinary Association in 1895, gives a long series of cases in which accidental wounds penetrating the peritoneal cavity, and with or without prolapse of abdominal organs, have been successfully treated. He himself surgically opened the peritoneal cavity in four experimental cases. In three he withdrew and handled portions of intestine, incised the floating colon, and closed the wound with sutures. In the fourth he divided and afterwards united the jejeunum. The first case died of septic pneumonia; the two succeeding cases recovered, but for research purposes were afterwards killed. The last case died in 39 hours.

In practice, Dollar, Rickards, and Rogers unsuccessfully resected a portion of floating colon. Smith and Garry have also performed laparoenterotomy for obstructed bowel, but the horses died. Félizet in 1849 is said to have cured a miller's horse in France by removing a calculus through a flank incision, but no such successes have been reported in England. Where operation has been attempted it has usually been postponed until too late. The success of treatment of accidental 
abdominal wounds in the horse and the failure of surgical laparotomies for bowel obstruction are probably explicable on this ground alone.

Should the portion of bowel surrounding the foreign body be necrotic or in process of becoming so, it may as a last resort be resected, care being taken to operate in sound tissue. A triangular piece of mesentery having the bowel for its base is at the same time removed. The ends of the bowel are then turned inwards and the opposed serous surfaces united by means of numerous fine silk sutures. McQueen used cambric needles and fine sterilised silk. The best form of suture is probably Lembert's. A second series of sutures is then inserted so as to bring as large a surface of the serous coat as possible into contact. As shown by Dollar's experiments, adhesion of the serous surfaces is remarkably rapid. To shorten and simplify the process of uniting the serous coats Murphy introduced a "button" in two parts, one of which was inserted in each end of the bowel and secured by passing a tobacco purse stitch around the periphery of the bowel. When pressed together the two extremities of the button automatically engaged and kept the two serous surfaces of the bowel firmly in contact. In four to five days the button became loosened in consequence of those portions of bowel includer within it undergoing necrosis, leaving a circular line of union around the bowel. The button was passed per rectum.

Murphy's button has often been successfully used in dogs; Murphy himself made more than 600 experiments, but McQueen's experiments on horses failed, and in these animals the method cannot be recommended. Decalcified bone tubes might be useful in the larger animals.

\section{II.-PUNCTURE OF THE BOWEL IN HORSES (PUNCTIO INTESTINI).}

In horses, colic is often accompanied by active production of gas in the colon and cxcum, by which the diaphragm is pressed forward and respiration impaired, in consequence of the lungs being pressed on and the posterior ribs fixed. Suffocation may even be threatened, and circulation in the abdominal viscera so affected as to endanger life. The animal's recovery, therefore, depends on speedy removal of the gas. The more marked the respiratory disturbance, the shallower and more frequent the breathing, the greater is the danger. In extreme cases the animals become unsteady on their legs, stagger about, fall down, and die in a few minutes. Crib biting induces the same symptoms, though in a minor degree, and seldoms leads to death, but tympanitic colic, produced by fermentation in the intestinal canal, often takes a fatal course, the reason being that in the one case air ceases to be swallowed as soon as the animals experience discomfort or pain, whilst 
fermentive changes and production of gas still continue, even under considerable pressure.

Internal medicaments, supposed to neutralise or absorb intestinal gases, act too slowly, and, like those given to prevent fermentation, enter the colon too late to be of benefit. As the natural passages for discharge

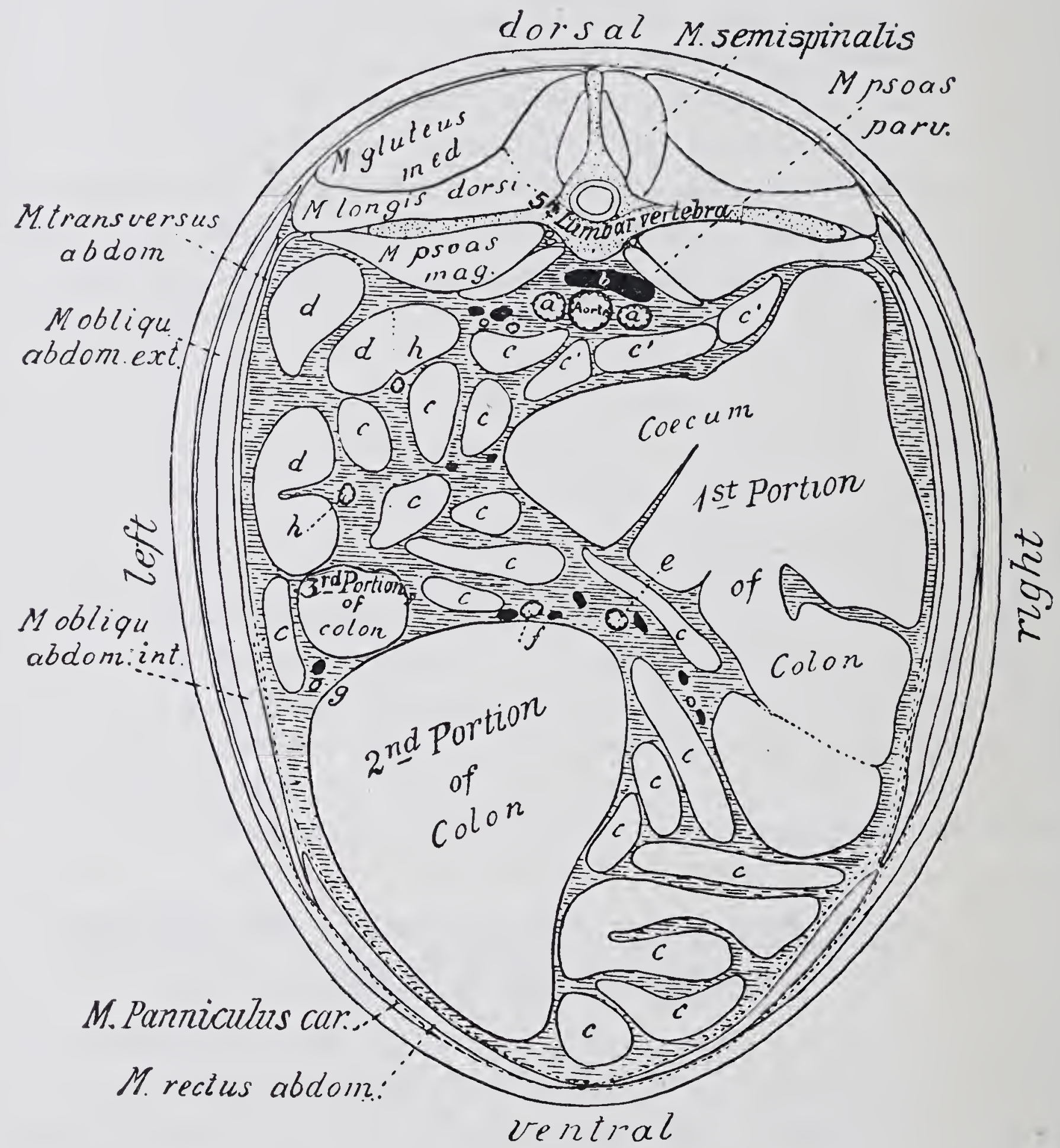

FrG. 141:- Vertical section of the horse's body between the last rib and external angle of the ilium.

$a$, Femoral artery ; $b$, posterior rena casa ; $c$ and $c^{\prime}$, loops of small intestine; $d$, rectum ; $e$, colic artery; $f$, inferior colic artery ; $g$, superior colic artery ; $h$, arteries of the rectum ;

$i$, ureter. (After Ellenberger and Baum.)

of gas may be filled with solid matter, the only method of staving off suffocation often resolves itself into removal of gas by trochar.

The operation acts like puncture of the rumen; but puncture of the bowel in the horse is more dangerous, even though recent cases, like Friedberger's, show that risk of peritonitis is greatly diminished by careful antisepsis. The precise cause of the greater vulnerability of the peritoneum in horses is still unknown, though it is clear that peritonitis 
is only caused by the introduction of infective materials into the peritoneal sac. It therefore becomes the chief task of the operator to prevent such infection; but though it is easy to guard against soiling from without, it is impossible to prevent it from within, i.e., from the bowel itself, at the moment of withdrawal of the trochar. To minimise risk, the instrument must be of small calibre, as small, indeed, as will allow of free discharge of gas. Friedberger's model has met with wide approval (fig. 142). Canulæ with side openings are to be avoided on account of their favouring infection of the peritoneal sac.

As, however, puncture is never without danger, it is only justified by such pressing necessity as dyspnœa threatening life.

In the horse a large portion of the colon is not directly in contact with the abdominal wall, but is covered by coils of small intestine, and is, therefore, not easily reached in the erect posture; though, as animals thus suffering soon die of suffocation when lying, puncture of the bowel is usually only undertaken in the upright position. It must also be remembered that the lower lying portions of the colon usually contain firm masses of food, while the gases collect in the upper parts, whence their discharge, by means of the trochar, is both easier and less dangerous. For these reasons, the seat of operation should be in the cæcum, as its base is fixed at the height of the external angle of the right ilium, and can be found with certainty with the trochar. The field of operation, which is fairly extensive, is triangular in form, bounded above by the transverse processes of the lumbar vertebræ, in front by the posterior edge of the last rib, and below by a line drawn from the middle of the last rib. The point to select is that which projects most prominently, and returns a tympanitic sound on percussion, but should these signs for any

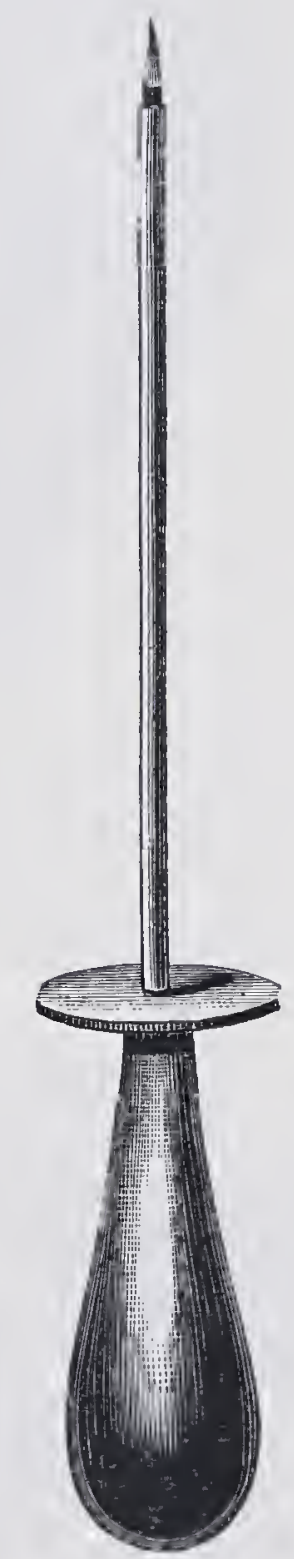

FiG. 142.--

Friedberger's intestinal trochar for horses. reason be wanting, or appear more distinctly at another spot, the latter may be selected without disadvantage. The important matter is to alight on a fold of bowel filled with gas; the larger this is the better.

The chief difficulty lies in observing antisepsis, and, therefore, both the trochar and skin round the point of puncture must first be carefully disinfected, provided there is time. The instrument should be boiled for five to ten minutes in water or soda solution, the hair shaved off with a razor, the skin washed with soap and rinsed with a disinfectant (such as sublimate solution). If possible the field of operation should be washed with æther or spirit preparatory to washing with the disinfectant. 
The trochar, prepared as described, is then thrust through the abdominal wall at the point indicated. To render its passage easier,

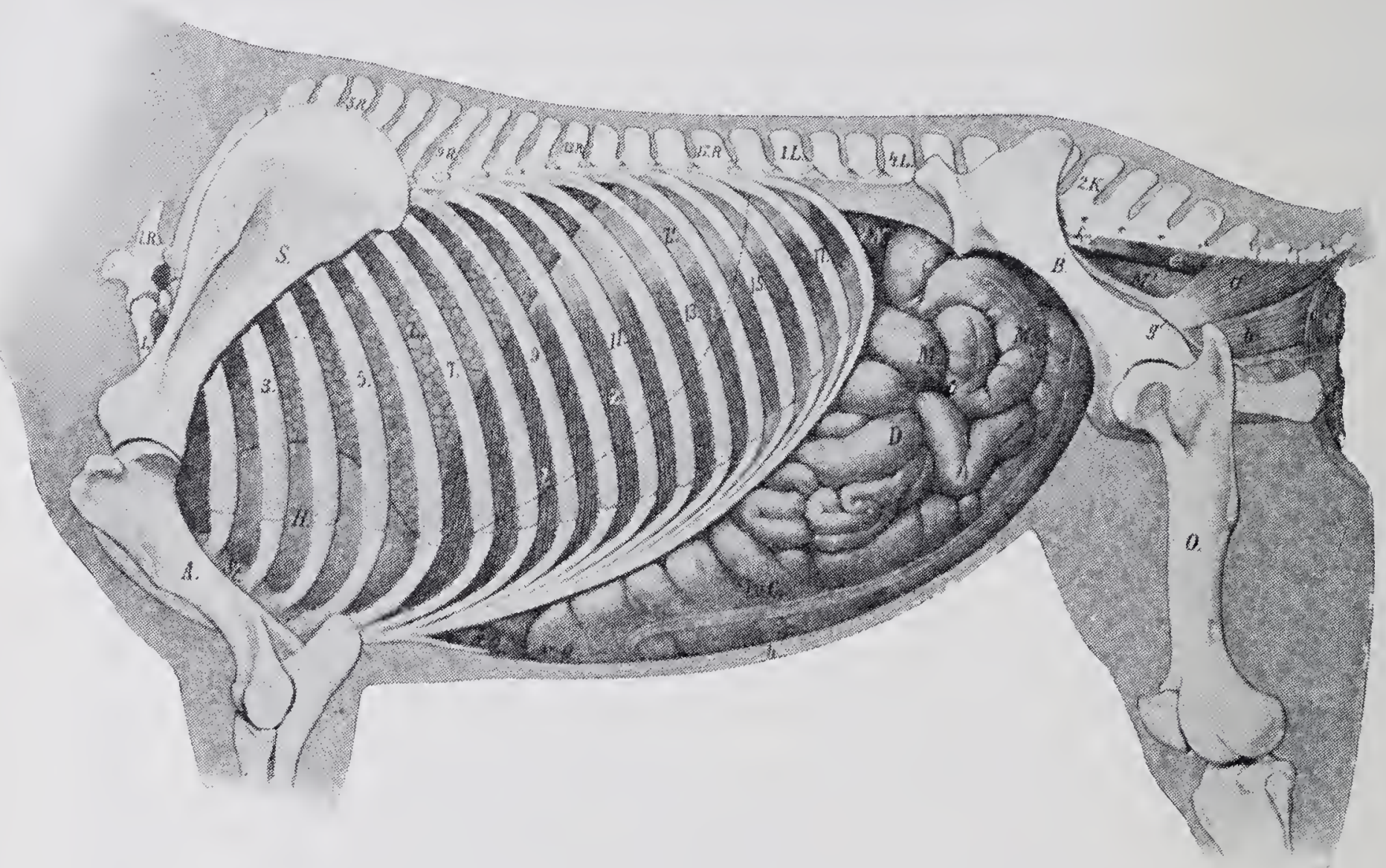

Fig. 143. - Showing the relative positions of the thoracic and abdominal organs. Left side. (After Leisering.)

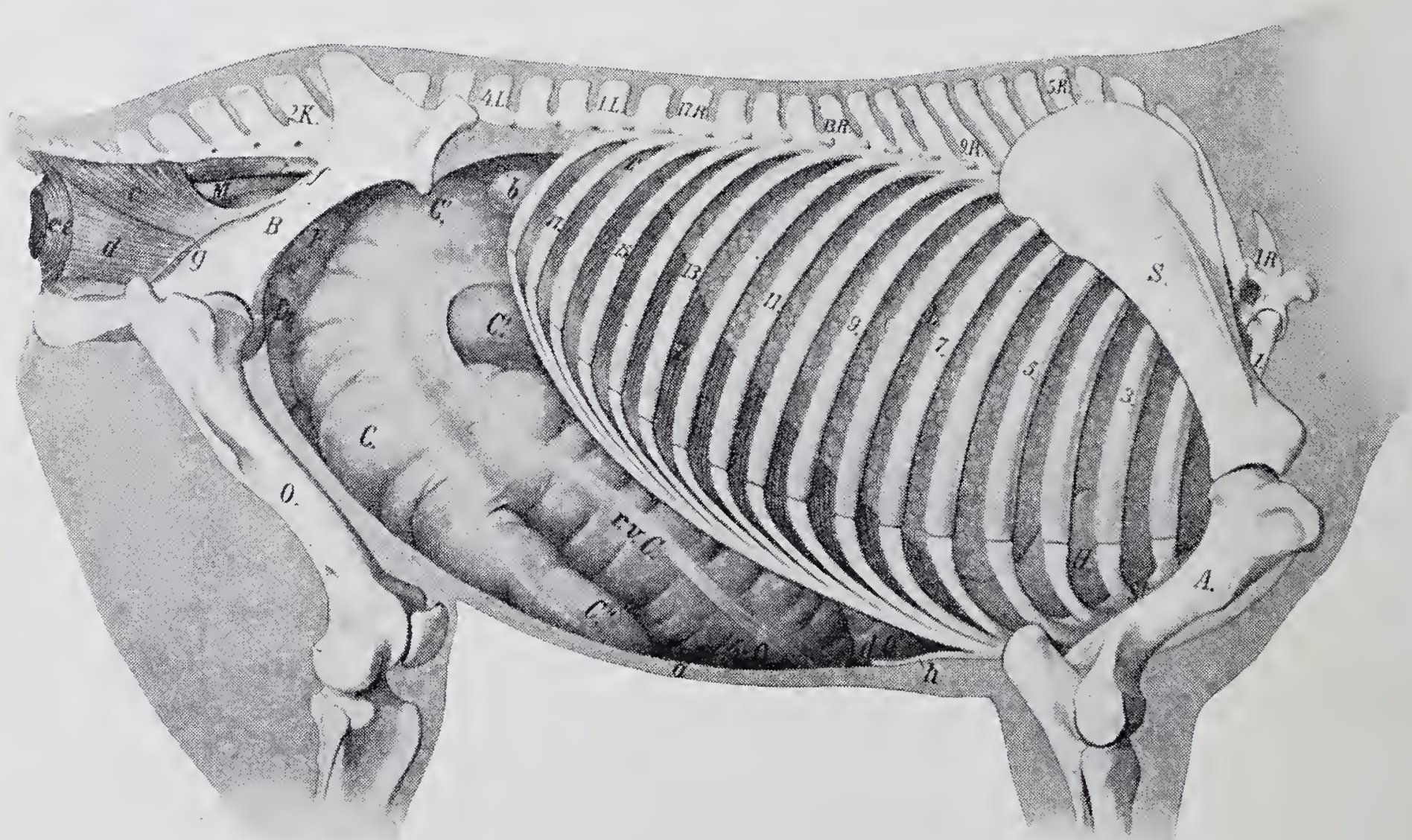

F1G. 144.- Showing the relative positions of the thoracic and abdominal organs. Right side. (After Leisering.)

an incision may first be made through the skin with a pointed bistoury, and when distension is not so great as to prevent it, the skin should be drawn a little to one side, and the puncture made so 
PUNCTURE OF THE BOWEL THROUGH THE ABDOMINAL WALLS. 319

that the abdominal wound will afterwards be covered by the skin. In passing the instrument it is directed perpendicularly to the surface and slightly towards the left elbow, and introduced far enough to be sure of perforating the bowel. The distance depends on the thickness of the abdominal wall. As a rule, the trochar can be introduced for $3 \frac{1}{2}$ to

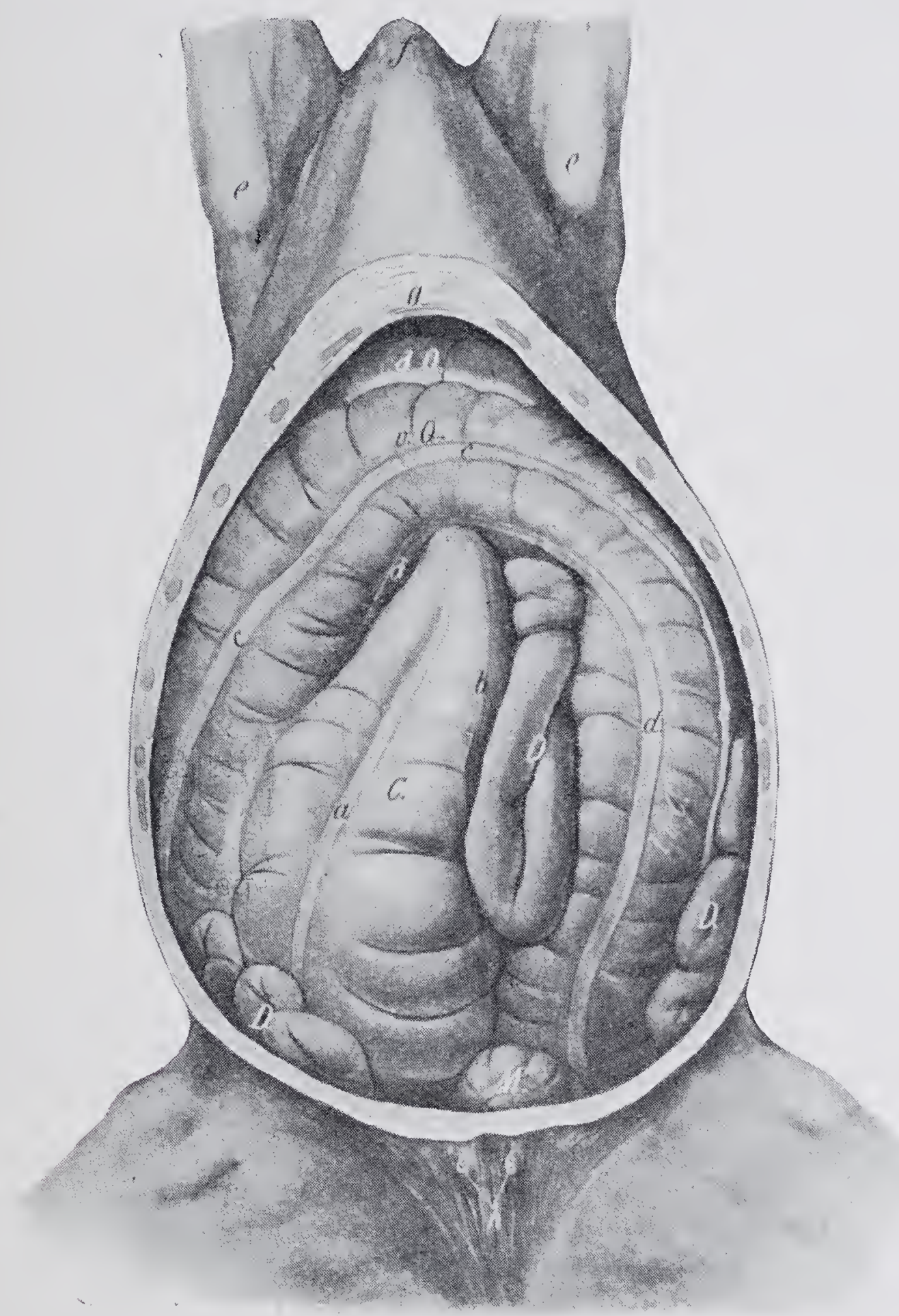

FIG. 145. - Abdominal organs seen from below.

C, Cæcum ; r. v. C, 1st portion of colon ; v. Q, suprasternal flexure ; 1. v. $C, 2$ nd portion of colon; d. Q, diaphragmatic flexure; $D$, loops of small intestine; $M$, loops of floating colon.

4 inches, or even further, without danger. The stilette being removed, the abdominal gases discharge through the canula with a whistling noise; the canula is left in position until the distension subsides and gas fails to reaccumulate. To test this point the canula should be stopped with a cork. If tympanites does not recur, and the animal shows no further restlessness, the instrument may be removed. The procedure is similar to that in puncture of the rumen. To prevent 
infective material escaping into the peritoneal cavity during withdrawal of the canula, some antiseptic fluid may be injected through it into the lumen of the bowel. One or two fluid ounces of creolin may be so employed, and will also assist in checking fermentation and further development of gas.

The wound is now cleansed, disinfected, powdered with iodoform, and covered with adhesive plaster or collodion. As long as the canula remains in position, the animal must be watched to prevent it rolling, or the instrument falling out.

Both the skin and intestinal wounds generally close by first intention, if antisepsis has been carefully carried out.

To prevent the bowel falling away from the canula, and to check the entrance of bowel contents into the peritoneal sac, Brogniez has constructed the so-called enterotome, which consists of a trochar, whose canula is provided with a pair of projections capable of being opened by pressure after insertion; but, as Hertwig has already pointed out, it is unnecessary, as the wall of the bowel can only fall away on account of other portions "of bowel becoming insinuated between the punctured bowel and the abdominal wall, or because of gas forming in the same position, an accident which can scarcely occur if, after removing the stilette, the canula is thrust far enough in. As Brogniez's enterotome is of great diameter, and its surfaces are not smooth and continuous like those of the trochar, the instrument is not only inefficient, but positively dangerous, on account of its favouring the passage of intestinal contents into the peritoneal cavity and wound, and increasing the risk of peritonitis. In some cases the wings of the trochar have refused to collapse, and removal of the instrument from the peritoneal cavity has been attended with great difficulty.

The method proposed by Bourgelat, Chabert, and others, and recently revived by Föhringer and Imminger, of puncturing the bowel from the rectum, presents a great risk of infecting the peritoneum from the mucous membrane of the bowel, a danger which cannot be entirely overcome, even by careful antisepsis.

In the case described by Imminger, rotation of the colon on its long axis possibly existed, and after discharge of the gas, underwent spontaneous reduction. In such cases, reposition should certainly be first attempted, and only where this fails does puncture of the colon appear indicated, though even then I should prefer the abdominal walls to the rectum, especially as the position of the colon can generally be discovered from the rectum.

If for any reason puncture through the rectum be considered unavoidable, the bowel should as far as possible be emptied, and most carefully rinsed out with sublimate solution, as recommended by Imminger. The left hand is then passed into the rectum, whilst the right introduces the trochar (with the stilette drawn back), and, guided by the left hand, places the instrument ou the pelvic flexure of the colon, which will be found distended with gas. The stilette is then thrust forward with a slight jerk, and the trochar caused to enter the colon. For this operation a long curved trochar is indispensable. The one used by Imminger has a length of nearly 9 inches, and a diameter of $\frac{1}{8}$ of an inch, and corresponds to Flourant's instrument, except in being somewhat thinner. Further procedure is similar to that in puncturing through the abdominal wall.

Pus sometimes forms at the point of operation, by which the danger of 
peritonitis is increased and recovery delayed, though not necessarily prevented. In cases seen by Rainard and Schaak, pus burrowed as far as the scrotum. Brogniez lost a case after enterotomy, owing to injury of, and fatal bleeding from, a cæcal artery.

Even at the present day the best authorities hold very conflicting views as to the admissibility of puncture of the bowel: and as operation does not remove the primary diseased condition, it must always fail in some cases. In the Lyons clinique the results were bad, but that this was not the fault of the operation is shown by the fact that twenty-five horses experimentally operated on were little the worse. Sometimes local peritonitis, with abscess formation, occurs at the point of operation, and may only produce death after an illness of several weeks.

\section{III.-PUNCTURE OF THE RUMEN AND RUMENOTOMY.}

In sheep and oxen gas often increases rapidly in the rumen, distends the abdomen, and presses so strongly on the diaphragm as to interfere with respiration and endanger the animal's life. This is generally due to rapid consumption of large quantities of fermentescible materials. Red clover, eaten whilst covered with dew, or in a withered or heated condition, and rapidly grown juicy green food, particularly that grown on heavy ground, are especially dangerous. But any other food which easily ferments, like brewer's grains, wet bran, and roots, \&c., may lead to rapid development of gas and distension of the stomach. A frequent cause of tympanites is the presence of foreign bodies in the osophagus, which prevent the regular discharge of gases formed in the rumen.

Reiset's experiments on oxen, rendered tympanitic by feeding on clover, show that these gases consist of 74 per cent. of carbonic acid, 24 per cent. of carburetted hydrogen, and 2 per cent. of nitrogen; in wethers of 76 per cent. of carbonic acid gas. Lungwitz's analysis gave 80 per cent. carbonic acid, a certain quantity of marsh gas, nitrogen, oxygen, and traces of sulphuretted hydrogen. Lungwitz thinks that the composition of the gas depends not only on the nature of the food, but also on the stage which digestion has attained. At first more carbonic acid gas is found. The small quantity of gas found in the stomachs of hungry animals consists principally of marsh gas with some nitrogen and oxygen, but contains little carbonic acid.

The symptoms of acute tympanites are unmistakable, the most striking being more or less rapidly developed swelling, particularly in the left flank, which, under certain circumstances, rises above the level of the lumbar vertebræ. The abdominal walls are often distended to the utmost, and on percussion give forth a hollow sound, feeding ceases, the animals are restless, show colic, and dyspnœa keeps pace with the advancing distension ; the respirations become shallow, the countenance is anxious, the veins about the head, neck, and abdomen (milk vein) are greatly distended, the pulse grows more frequent and smaller, the action of the 
heart tumultuous, and, after staggering movements, the animal falls to the ground and rapidly dies.

The course of the disease is acute; death may occur within an hour; sometimes the attack continues for twelve to twenty-four hours, seldom longer. The more rapid the rate of distension, the greater the danger. The condition is particularly grave where the right flank also appears distended, a token that the colon is filled with gas. Slight cases may recover of themselves, but severe ones are always fatal if speedy help be not afforded. Large numbers of animals when under similar conditions may simultaneously become affected.

Treatment. Internal remedies, such as lime water, spirits of ammonia, oil of turpentine, petroleum, chlorate of potassium, hyposulphite of soda, \&c., have been recommended to assist absorption of gas and to

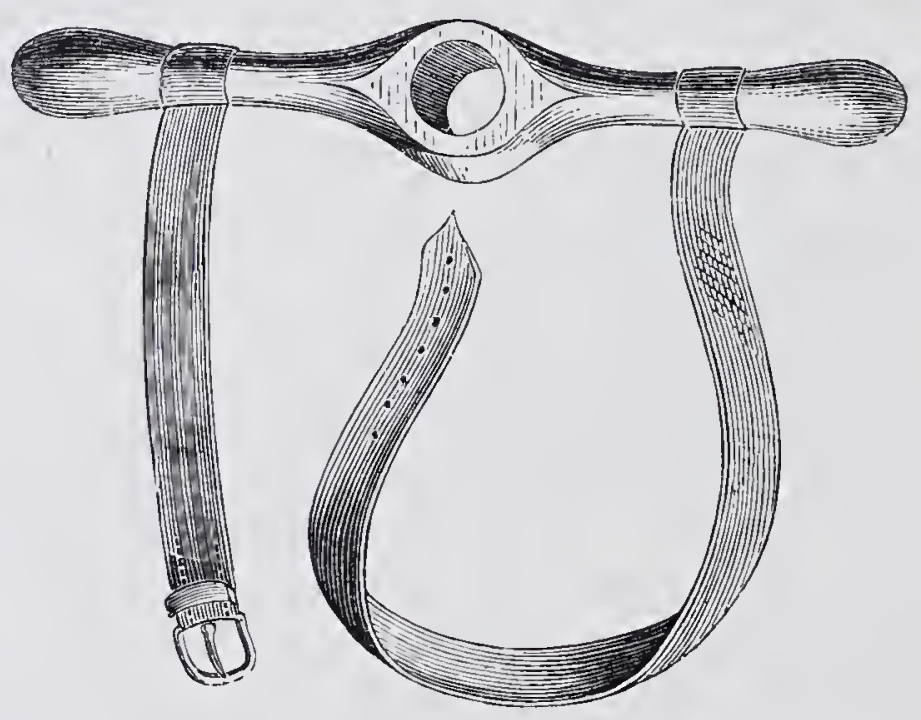

Fig. 146.-Mouth gag for oxen.

prevent its further development. Lungwitz, on the basis of his laboratory researches, recommends 4 per cent. magnesia usta suspended in water, milk of lime, and 2 per cent. of spirits of ammonium, or, in emergencies, soapy water. These materials are seldom of much service and are only used in semi-acute tympanites.

Fastening a piece of plaited straw smeared with tar in the mouth, inserting the mouth gag (fig. 146), or drawing the tongue strongly forward, is said to assist eructation and give relief, and the use of the probang has been recommended.

Strong pressure on the left flank is more effective. The gases are directly discharged, and movements of the paunch at the same time excited.

Though such measures may prove sufficient in slight cases, time should not be lost in making trial of them in severe attacks; and as soon as dyspnoa is marked, the probang or trochar ought at once to be employed.

The probang for cattle generally conists of a spiral of steel provided with a coating of leather (fig. 74), though the simple steel spiral is 
sometimes used. In sheep the vesical catheter used for horses forms a sufficiently effective probang. As the patient cannot be cast, passage of the probang in an excited animal suffering from severe dyspnoea is often no easy matter, but cattle are usually better subjects than sheep.

To ensure the instrument taking the right direction, a piece of wood provided with an opening (mouth gag) is first inserted in the mouth, and through this the tube is passed. The animal's head is then extended and the rounded end of the probang pushed along the palate into the pharynx, whence it glides along the superior pharyngeal wall, and enters the osophagus. Care is required to prevent it passing into the larynx and trachea, an accident which is announced by violent coughing and dyspnoa; in such case the tube must immediately be withdrawn. Should it have safely gained the cosophagus, it passes easily downwards without any untoward symptom, and can be felt on the left side of the neck. As soon as the end reaches the stomach the stilette is removed, and the accumulated gases allowed to escape through the hollow tube. But this does not always follow; sometimes the tube becomes stopped up and the probe must again be introduced; often the gases are not free on the surface of the contents of the rumen, but are mixed up with masses of fermenting food, and this explains why even the probang has not always the desired effect.

Puncture of the rumen forms another means of treatment, the rumen being pierced in the left flank with the trochar to allow exit of gas. The operation is very simple, and is often carried out by laymen when danger of suffocation threatens. In the case of cows and sheep even a pocket knife can be used, should a trochar not be at hand.

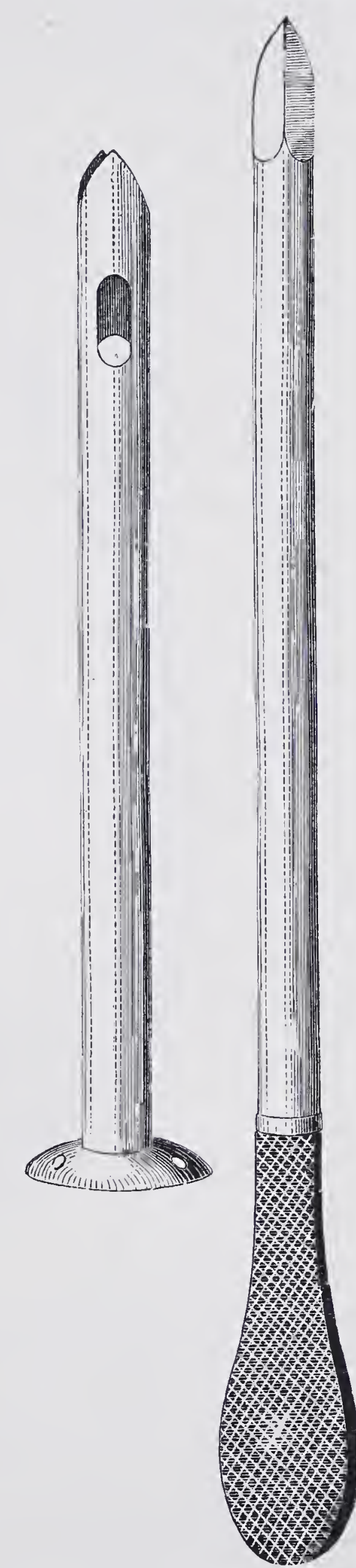

FIG. 147.--Trochar and canula for puncture of the rumen.

At the present time round trochars without side openings are almost exclusively used; the largest, having a diameter of 2 to 4 lines, is used for oxen, and a somewhat smaller one, 1 to 2 lines, for sheep. 
The seat of operation is the centre of the left flank, which becomes very prominent in the tympanitic animal. In fixing the spot, one imagines a line drawn forwards through the outer angle of the ilium parallel with the vertebre. In cattle the trochar is inserted on this line about 4 to 6 inches, and in sheep about 2 to $2 \frac{1}{4}$ inches, in front of the

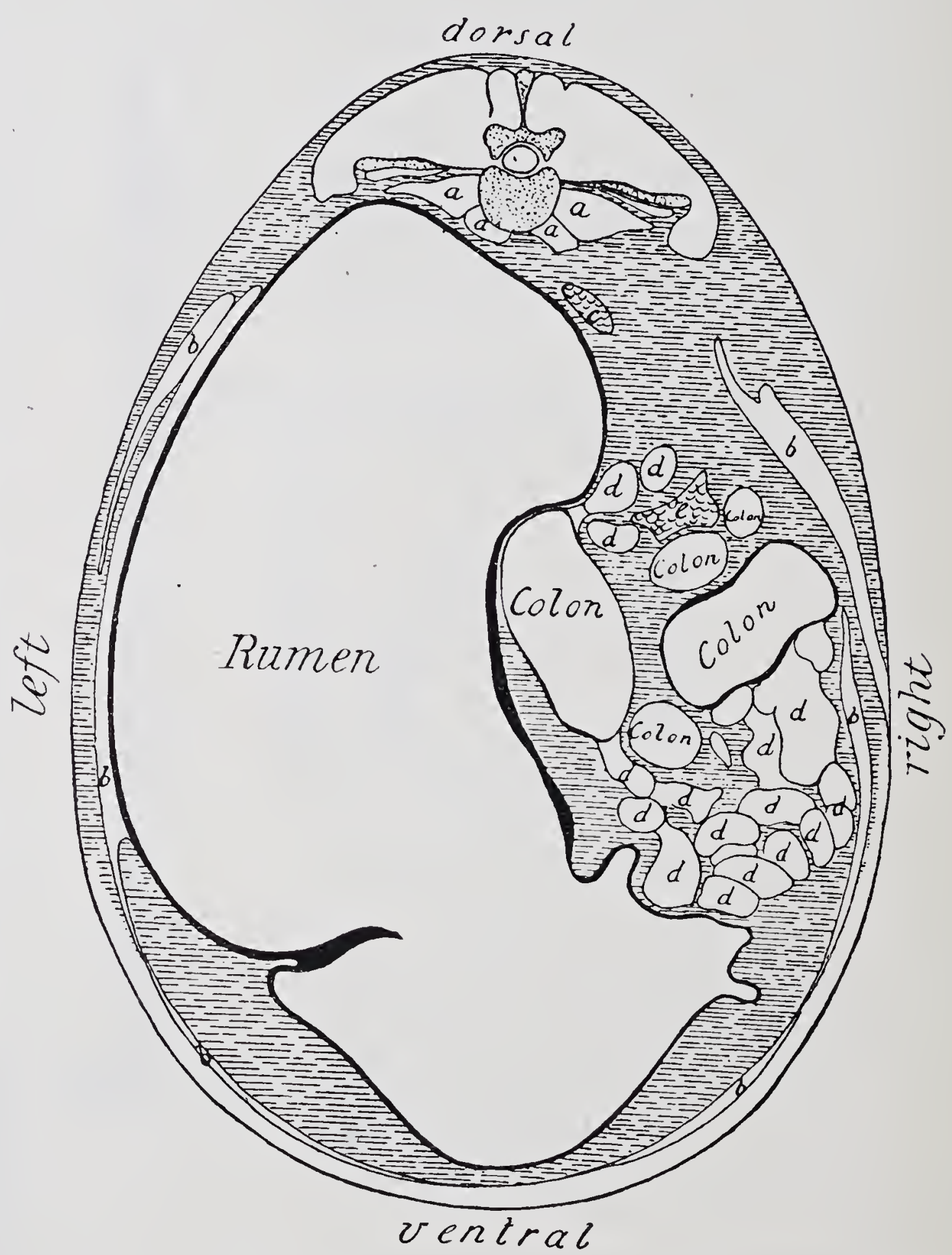

F1G. 148. - Vertical section of the ox's body, showing relative position of rumen and other organs.

$a$, Lumbar muscles; $b$, abdominal muscles; $c$, glands; $e$, portion of the pancreas. (After Ellenberger and Baum.)

outer angle of the ilium. As delay is often dangerous, there may be no time for cleansing the point of operation, otherwise the usual precautions are taken. Where the skin is thick, it is best to make the primary incision with a bistoury; older practitioners were in the habit of using the fleam for this purpose. The trochar is now moistened and thrust through the walls of the abdomen and rumen, being directed slightly towards the right. Considerable force is required, and a slight rotary 
movement should be made. If the operator is of slight stature, it may sometimes be easier to give a smart blow on the instrument with the open hand. On account of the size and distension of the rumen, it is of no importance how far one thrusts the trochar; no injury is likely to result, and therefore it may be allowed to enter almost up to its shield.

When the stilette is removed the gas rushes out, sometimes under high pressure and mixed with particles of food, which are apt to block the canula, and require to be removed with the stilette or a probe.

The canula remains in position until fermentation ceases and danger of suffocation disappears. To make sure of this the canula is closed with a cork and the animal watched; if tympanites fail to recur, the instrument can be removed. Before doing so, however, a few ounces of creolin or other active but unirritating antiseptic may be injected; the stilette is then reintroduced, the skin is held in position with the fingers of the left hand, and the whole instrument slowly withdrawn.

The skin wound requires no particular treatment, though it may be cleansed and covered either with a pitch plaster, some tar, or collodion. When time serves it is best to disinfect the skin and trochar before operation.

Bad results seldom follow the use of the trochar in oxen, though digestion may sometimes be impaired by the rumen becoming adherent to the abdominal wall.

The operation is more dangerous in sheep, though it becomes necessary in cases of threatened suffocation. If possible, the long wool should first be clipped away. Where the rumen is punctured with a linife, a thin tube, an elderberry stem, or even a couple of strong straws may be used as a canula to allow of escape of gas, being introduced into the rumen alongside the blade. The animal must be watched during the whole time these remain in position to prevent their being displaced.

Rumenotomy. In dealing with a rumen distended with masses of fermenting food containing much gas, when the trochar is no longer of value, rumenotomy, which cattle tolerate very well, can alone give relief.

The animal is placed with the right side against a wall and fastened up short. A piece of wood held in a sloping direction in front of the left hind-foot, or a stake driven into the ground, will shield the operator from injuries by this foot.

The hair over the left flank is now clipped, the skin cleansed, and a strong bistoury introduced at the point where the rumen is usually punctured, with its back towards the animal's vertebræ (Hertwig's method); the blade should be thrust in as far as the handle. With a 
strong, drawing movement, the wound is now carried downwards, in oxen for a distance of 4 to 6 inches, in sheep $1 \frac{1}{2}$ to 4 inches. To prevent food entering the peritoneal sac, it is necessary to avoid making the skin wound smaller than that in the wall of the rumen.

The operation may also be carried out by first cutting through the skin at the point indicated, dividing the abdominal muscles, and finally incising the peritoneum and wall of the rumen.

Immediately the rumen is opened gas and fermenting masses of food often rush out with considerable force. To prevent the rumen collapsing after evacuation and allowing food to enter the peritoneal cavity, a handkerchief, or serviette, is so placed that one portion extends from the lower angle of the wound into the rumen, and the other lies on the outer surface of the abdominal wall. With the same object, Horsburgh and Roche-Lubin stitched the rumen to the abdominal wall. The latter operates like Hertwig, by thrusting a bistoury through the walls of the abdomen and rumen, a little above where the trochar is inserted. After allowing gas and some food to escape, the incision is carried downwards about 4 inches; tapes are passed through both rumen and abdominal wall on either side, and about 1 inch from the edge of the wound, by means of a bent needle. The tapes are introduced from within outwards, and each carries at its end a mass of tow, which acts like a knot; the free ends, being drawn apart, open the wound and prevent the rumen collapsing.

Whichever method is adopted, the rumen should be emptied as far as possible, either with the hand or a pair of forceps; but this must be done gradually to prevent the animal becoming unconscious and falling down. Certain operators state that stimulants, like wine or brandy, produce a good effect in such cases, and recommend pouring through the opening in the rumen four to six pints of wine, or a suitable dose of æther, alcohol, \&c.

After the rumen has been emptied, the wound cleansed, and care taken that no food has entered the peritoneal cavity, or that what has entered has again been removed, the wound in the rumen is closed with catgut or silk, interrupted sutures being used. These are placed so close that no food can pass, and it is very important that the edges of the wound lie in close apposition. The opening in the abdominal muscles is sutured in a similar way, though that in the skin may be left patent without disadvantage. If, however, healing by first intention is desired, the skin should also be sutured. Sometimes the wound heals in a few days, but may take weeks and even months, or leave behind a fistula of the rumen.

Obich recommends suturing the wall of the rumen to that of the abdomen, and leaving the stitches in for seven or eight days. Meyer 
is opposed to this, and states that it causes tearing. Extensive adhesion of the rumen to the wall of the abdomen certainly interferes with digestion.

To prevent food infecting the peritoneal cavity, Sajoux had as early as 1839 employed a trochar with movable wings by which to fix the rumen to

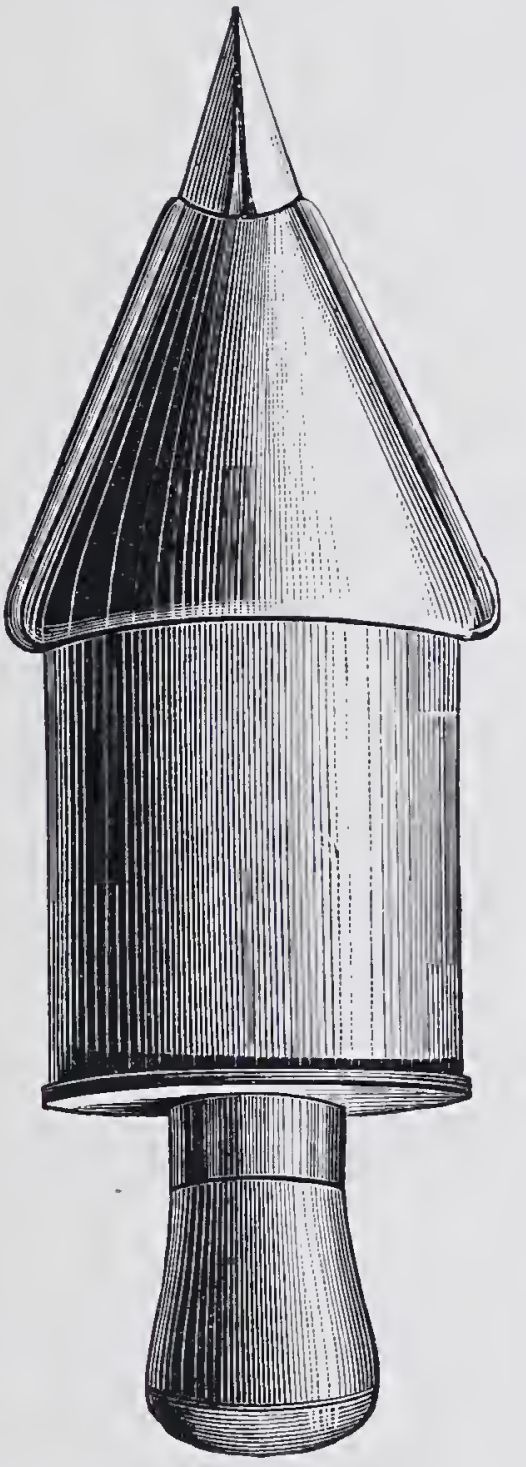

Fig. 149.-Bräuer's trochar.

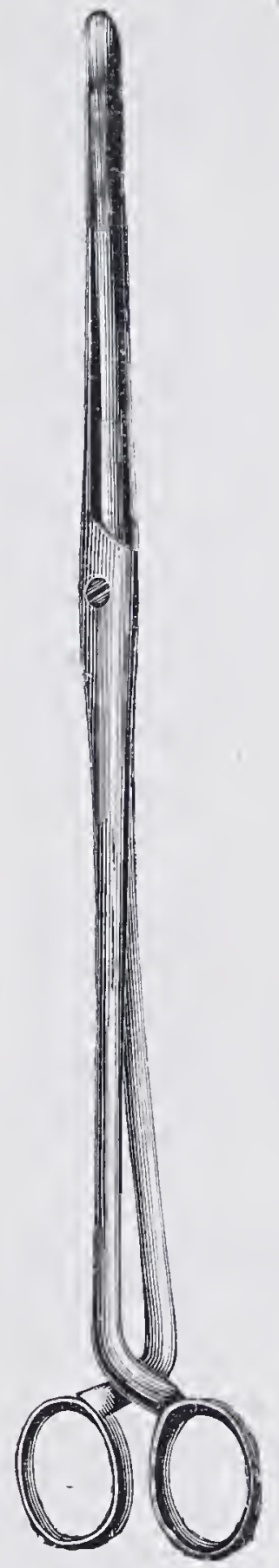

Fig. 150.-Forceps for use with Bräuer's trochar.

the abdominal wall (Hering) ; about the same period three instruments termed "gastrotomes" were constructed by Brogniez.

In Germany this complicated piece of apparatus was never much used, because it by no means absolutely prevents infection of the peritoneal cavity; on the contrary, as it requires great care and cleanliness to keep it in perfect condition, it is frequently unavailable when most wanted.

The trochar constructed by Bräuer (fig. 149), on the contrary, appears simpler and more practical. Its canula is so wide that the food may be removed through it from the rumen by

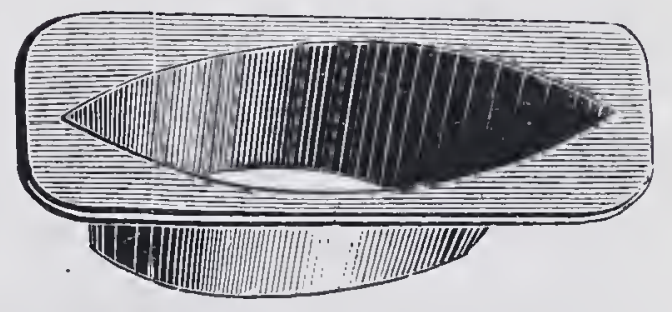

Fig. 151,-

Sheath of Bräuer's trochar. using a pair of forceps. The handle and the greater part of the stilette consist of wood; the latter is flattened, and ends in a cutting part, which is formed by the union of two knife-shaped portions of steel, which come 
together in a point. The canula is of tinned iron, and has at its upper part an opening $4 \frac{1}{2}$ inches long in one direction and $1 \frac{1}{2}$ inches in the other; below, the aperture measures 4 inches in the one, and $1 \frac{3}{8}$ in the other direction (fig. 151). The hair is cut from the seat of operation, the skin cleansed, and the instrument applied like a trochar, but as it requires considerable force to thrust it into the rumen, a moderately heavy hammer or mallet is employed.

After removing about a third of the contents of the rumen with a pair of spoon-shaped forceps, whose blades are $\frac{1}{4}$ inch $(6 \mathrm{~mm}$.) broad, and 16 inches long, Brïuer attaches a funnel to the canula, and pours in 10 to 15 quarts of salt water.

Treatment of the wound, in rumenotomy and after the use of Bräuer's instrument, is conducted on general principles.

As this operation is most frequently performed in summer, when flies are common, it is best to apply a dressing which guards against insects and injury and soiling by the tail or mouth. Smearing the parts with tar serves a similar purpose. To prevent relapse some care is required in feeding after operation. For further information on this point, handbooks on special pathology should be consulted.

Heyne, in 1836, recommended "punctio ventriculi" in the horse to remove gases from the stomach. Apart from the fact that diagnosis is very difficult in these cases, gastric tympanites very seldom occurs in the horse, and generally only as a consequence of stasis in the small intestine, in which case the operation cannot have lasting results. Add to this that puncture of the horse's stomach is always dangerous to life, and it will be understood why it has not been practised.

\section{IV.-INTUSSUSCEPTION OR INVAGINATION OF THE BOWEL.}

In oxen invagination of the bowel forms one of the most frequent causes of fatal colic. In horses and dogs it is of rarer occurrence. While operation for this condition has not hitherto been attempted in horses, it has been invoked with considerable success in oxen and dogs. Excessive and irregular peristalsis may cause several feet of the small intestine to become intussuscepted. The outer (invaginating) portion strangulates the inner (invaginated), and disturbs circulation; the onward movement of ingesta is stopped, and, broadly viewed, the same conditions obtain as in strangulated hernia. Oxen may survive for five to ten days, or even longer, but horses die rapidly. In exceptional cases the invaginated portion is said to become necrotic, to pass forward through the invaginating piece, and be discharged with the frces. Invagination is commonest in the rectum or small intestine, but in the horse Merten, Hübner, and other's have observed passage of the cæcum into the colon.

Symptoms. The disease begins with a severe attack of colic, which may last twelve hours, and is followed by subsidence of pain, though 
appetite is wanting. Rumination ceases, tympanites and discharge of blood-stained mucus set in, or obstinate stoppage of the bowel occurs; peristalsis is in complete abeyance; the pulse becomes frequent and small, but the temperature seldom rises. On examination per rectum, the invaginated spot may sometimes be felt as a cord-like, painful swelling. Slight colicky symptoms, straining to pass fæces, and discharge of small quantities of mucus or blood continually recur. Merten says the animals show a desire to lie on the back.

Dogs show no signs of colic. They move about restlessly, and usually lie down flat on the belly. Appetite completely disappears, but water is often taken greedily. Water, food, and medicine are, however, usually vomited at once. The vomit is bile-stained. No frces are passed, though there may be straining. Local examination (palpation) of the abdomen reveals the invagination as a sausage-shaped, painful swelling.

Diagnosis is often difficult; in oxen and dogs it may be necessary to perform an exploratory laparotomy under antiseptic precautions.

Treatment. Medical treatment is worthless, and purgatives cannot, of course, reduce the invagination.

Siebert attempted reduction by generating carbonic acid gas in the body. After giving aloes with sulphate of soda in linseed tea, he injected 25 ounces of bicarbonate of soda suspended in water into the rectum, which had previously been emptied as far as possible, with the hand and tobacco clysters. Diluted hydrochloric acid was then passed in, and the anus closed with the hand. In a short time the right, and later the left, side became greatly distended, and the animal strained so much that it was difficult to keep the anus closed. After a time the hand was removed, a large quantity of carbonic acid gas and fæces escaped, and the animal recovered. Siebert states having thus cured a cow of invagination of five days' standing; but his treatment is scarcely recommended by the fact that he afterwards found the cast-off portion of bowel in the dung. If invagination had really existed, recovery was due less to the treatment than to the vis medicatrix nature. The method may, however, be tried when operation is out of the question, and other means are ineffectual.

Surgical treatment consists in opening the peritoneal cavity from the right side with antiseptic precautions, and reducing the invagination or resecting the affected piece of bowel. The animal is placed with the left side against a partition, and is fastened with cords and planks. The hand is introduced into the abdomen, the diseased spot discovered, drawn forward, and an attempt first made to reduce the invagination, but this often fails because the opposed surfaces of serosa have become firmly adherent. Degive's experience shows, however, that when the union is not intimate there need be no hesitation in effecting it. The abdominal wound should then be closed with the usual precautions.

Reduction failing, resection becomes the only alternative, and has been performed by Meyer with success in oxen. In his "Manuel opératoire 
pour l'espèce bovine," Guittard describes it as quite an everyday affair. Bleeding, after cutting through the bowel or mesentery, can be stopped by torsion, or the vessels may be ligatured with sterilised material. Great difficulty is caused by the continual passage of ingesta through the anterior section of bowel, which accordingly should be compressed by bowel clamps, or lightly ligatured during operation. After removing the invaginated portion, the ends of the bowel are brought together by the bowel stitch, the abdominal walls and skin are sutured each to each, and the wound antiseptically treated.

Taccoen operated on two cows, from one of which he removed 10 inches of bowel, but had no bad consequences. Thirty-five days later the external wound was healed, and, on slaughter, the incision in the bowel was found to be completely cicatrised. In a second case, an incurable anus preternaturalis formed, but did not impair the animal's health.

Riedinger treated, during 1890, ten cases of invagination of the bowel in oxen. Seven animals had to be slaughtered on account of the operation being done too late; in the other three, laparotomy was carried out and the invagination reduced. The portion of bowel was cleansed with $\cdot 1$ per cent. of sublimate solution, replaced, and the wound closed with button sutures. After-treatment consisted in giving purgatives. Five to six hours after operation, action of the bowels occurred. In one of the animals peritonitis occurred five days after operation, rendering slaughter necessary; the other two recovered in fourteen days.

In dogs laparotomy can be performed in the linea alba and the invagination reduced or the bowel resected as above described. In the latter case Murphy's button can be used.

\section{Y.-TWIST OR ROTATION OF THE COLON IN HORSES.}

At the Natural Science Congress at Bremen in 1890, Jelkmann first indicated the possibility of recognising during life and of surgically treating torsion of the colon, which not infrequently occurs in horses, and always leads to death. The importance of this question is shown by the constant occurrence of the disease. According to Jelkmann, 70 out of every 192 horses dying of colic in Munich had twist or displacement of the colon. Of 23 post-mortems after colic, made in the year 1887-8, twist of the colon was found in 10; Jelkmann, therefore, concludes that the disease occurs most frequently in Middle and South Germany, which may perhaps be referred to the heavy breed of the horses. According to the statistics given by the Veterinary Sanitary Reports of the Prussian army, in 1886, 13 horses ; in 1887, 27 horses; in 1888, 37 horses ; and in 1889, as many as 84 horses died from displacement or twist of the colon. Great credit must be given to Jelkmann for having directed attention to this point, and, though his statements have in certain 
quarters been met with distrust, this may be explained in part by the fact that practitioners had not made themselves sufficiently acquainted with the anatomical conditions or methods of surgical treatment. In 205 colic patients Jelkmann found displacement 13 times, and effected recovery by retroversion. During 1890,63 cases of rotation of the colon were met with in the horses of the Prussian army, and during 1891, 52 cases.

Jelkmann says the twist is usually towards the right, and is produced by distension of the upper portion of the colon and its displacement from the left lower wall of the belly at the same time that portions of the rectum are forced towards this spot. If the animals rise after lying on the right side, the upper portion of the bowel, which has been displaced towards the middle line of the belly, is thrust downwards and finally twisted around its long axis. This explains many cases; but just the opposite sometimes occurs, and twist takes place towards the left, as is shown by the reports of post-mortem examinations in the Pathological Institute of the Berlin College. Sometimes the upper layer of the colon is displaced towards the centre line, sometimes towards the left abdominal wall. The comparatively great length of the portion of bowel filled with food, and its freedom to move, explain the frequent occurrence of rotation. (For the normal position of the abdominal contents see figs. 143,144 \& 145.)

The symptoms are not characteristic, but a rectal examination generally removes any doubt. When colic, at first slight, is accompanied by continued pain and becomes worse hour by hour, the bowel sounds weaker, the pulse smaller and more frequent, and some form of stoppage of the bowel seems certain, a rectal examination will generally clear up the point. In front of the anus, one feels the distended colon, which may for the moment be mistaken for the over-filled urinary bladder, but careful examination reveals its real nature. The longitudinal muscular bands can be distinctly felt, and show, not only that we have to deal with the colon, but also in what direction torsion has occurred. When the bowel is in its proper position, the bands run nearly parallel with the long axis of the body; but in twists, a change in their course is distinctly appreciable. In torsion towards the right, they run backwards and inwards ; in torsion towards the left, backwards and outwards. According to Jellimann, the rectal mesentery, whose fixed border can be felt below the lumbar vertebræ, appears greatly stretched, and in right rotation does not pass perpendicularly downwards, but towards the left, and pressure on it causes the animal pain. Careful examination of the direction of the bands of the colon seems of more importance in diagnosis, and no doubt can exist either as to the presence or direction of the torsion if they can be discovered, but the posterior bands of the crecum, 
which can be distinctly felt when the latter is distended with food, must not be mistaken for those of the colon. Such an error is avoided by remembering that normally the cæcum runs from the outer angle of the right ilium in a bow directed backwards and ends near the left stifle.

By removing that portion of the left abdominal wall lying between the last rib, the outer angle of the ilium, and the tranverse processes of the lumbar vertebræ, whilst the dead subject was supported in an erect posture, Möller confirmed the anatomical relations of the organs lying in the posterior section of the abdomen and in the pelvis; the colon can be greatly inflated in situ, or after successful rotation, filled with water. The hand introduced into the rectum allows of the experiment being easily controlled, and the experimenter may convince himself which portion the hand is touching. Carried out before students, such a demonstration is exceedingly instructive.

After artificial rotation the change in position of the organs could be recognised, and replacement attempted from the rectum. The experiment shows that the position of the bands is very important, especially as torsion of the posterior sections of the colon displaces the attached border of the great and rectal mesentery only very slightly. Examination of the bands left no doubt as to the displacement, or the facility with which diagnosis should be effected in cases met with in practice.

Prognosis. In very exceptional cases torsion may be reduced by the animal rolling, but, as a rule, the only chance of recovery lies in early manual treatment. Though the question whether reduction is possible in every case, or how often it may be effected, cannot yet be settled for want of published observations, it is clear, from Jelkmann's communica. tion, that it often succeeds, and Möller considers it practicable, though it certainly requires considerable muscular power in the arms and ability to withstand fatigue. This would probably become less after practice.

Treatment is commenced by giving a clyster of lukewarm water in order to clear the rectum as far as possible, and to obtain sufficient room for introducing the hand. Jelkmann inserts the left hand, presses forward towards the left abdominal wall, and endeavours to thrust the left portion of the colon with the convolutions of the rectum forwards from this point towards the middle line of the abdomen. Once the bowel is brought into this position, Jelkmann passes the hand slowly upwards, when the colon falls back over it into its normal position; he considers that the convolutions of rectum, displaced towards the left lower abdominal wall, having been thrust upwards, leave room for the colon to return to its normal position. Möller's experiments tend to support this explanation.

Möller replaced a left rotation of the colon in the following way :After emptying the rectum, the right hand was introduced, and discovered the bands of the colon running from in front backwards and outwards or towards the left. The bands of the colon lying above were now employed to bring about reposition. Whilst the hand in the rectum 
was strongly adducted, its volar surface or the fingers were laid against the bands, and after repeated careful attempts finally succeeded in drawing these so far towards the right that the colon again took up its position parallel with the middle line of the body. As had been shown by the experiments on dead animals, in torsion towards the left, the bands of the lower section of the bowel offer a purchase for retroversion. After effecting this, the pelvic flexure of the bowel, until then filled with gas, at once collapsed, the symptoms of colic disappeared, peristaltic action, which had almost completely ceased, again set in, the small, frequent pulse altered its character, and half an hour later the recovery of the animal could be confidently foretold.

It is clear that all torsions of the colon cannot be treated by one and the same method; differences in displacement will render modification necessary; but when attention has been directed to the point, experience will give valuable indications for procedure. Puncture of the overdistended colon might possibly assist reduction (see "Puncture of the Bowel "). Jellkmann was compelled to puncture the cæcum five times in thirteen cases, after which retroversion succeeded; the use of the trochar caused no bad results.

Before attempting manipulation, Gultmann evacuates the gas by puncture. Imminger and others recommend puncture, and claim to have seen reduction of the twist in consequence. Whether puncture is always necessary can only be settled by more extended observation. It is desirable that reduction should be attempted oftener than at present; those who have tried it speak of the method in favourable terms.

\section{Möller describes the following case :-}

On June 8th, 1890, I was called to see a heavy cart-horse which had been suffering from colic for twenty hours. There was moderate but continued restlessness; the animal lay down frequently, but soon rose again, moved about in various directions, and showed all the symptoms of obstruction of the bowel. The pulse was sixty-five per minute, small and weak, the mucous membranes dirty red, respirations thirty and shallow, the flank moderately distended with gas, peristalsis occasionally slightly audible at the right side, general perspiration. Defæcation had been in abeyance for twenty hours, only three hard portions of dung having been passed, the appetite had decreased during the same period, and drink was only taken in small quantities. Examination per anum discovered the pelvic flexure of the colon greatly distended with gas, which caused it to be pressed towards the posterior wall of the pelvis. On the outer surface of the colon a tense cord could be felt which passed from above downwards and inwards, from behind upwards and outwards. A similar cord passing in the same direction could be detected on the inner surface of the colon; the urinary bladder was only partially filled.

The hand introduced into the rectum, was laid in the above described manner against the outer cord, and after several attempts it was found possible, by very considerable exertion, to move the bands of the colon towards the middle line of the abdomen. Active peristalsis and passage of gas at once 
followed, after which firm excreta were passed. The restlessness decreased, and after a short time entirely disappeared, whilst the pulse recovered its normal condition, and the mucous membrane lost its redness. In an hour, pain was entirely gone.

Other forms of displacement of the bowel in horses have not yet been surgically treated, if we except Meschkow's case, in which the rectum was strangulated by coils of small intestine. Meschkow performed laparotomy, reduced the strangulation, and the horse recovered. 


\section{SURGICAL DISEASES OF THE POS- TERIOR PORTIONS OF THE RECTUM AND OF THE ANUS.}

In large animals the posterior end of the rectum to the extent of 8 to 12 inches, and in smaller ones to a correspondingly less extent, is not covered by peritoneum, but attached to the surrounding structures by loose connective tissue. This portion lies, therefore, beyond the peritoneal cavity, and is connected above with the sacrum, and below in males, with the bladder, in females, with the uterus.

The thin and hairless skin of the anus possesses many sebaceous and sweat glands. In carnivora a small gland, about the size of a hazel-nut, exists on either side of the anus, and is surrounded by the sphincter ani ; it contains a greenish fatty fluid. In addition to sebaceous glands, carnivora possess flaskshaped anal or perineal glands which contain a yellow substance.

\section{I.-CONGENITAL MALFORMATIONS. ABSENCE OF THE ANUS AND FORIMATION OF CLOACE (ATRESIA ANI ET RECTI, FISTULA RECTO-VAGINALIS).}

During early intra-uterine life, the anus is formed by invagination of the outer coverings. In the horse and ox this happens during the eighth week, in sheep, goats, and swine in the seventh, and in carnivora in the fifth. In dogs and swine, less frequently in ruminants and horses, the process sometimes remains incomplete, and in consequence the new-born animal possesses no anal opening. This vitium primae formationis may be confined to the anus (fig. 152), or the posterior portion of the rectum may also be closed (atresia recti, fig. 153); less frequently the anus appears open, but a portion of the rectum closed. The sphincter ani then develops regularly, and a depression occurs at its middle point in place of the anal opening.

In females the rectum sometimes opens into the vagina, a condition described as recto-vaginal fistula (anus vaginalis or cloaca formation); Rotter saw it in a six months pig. Less frequently a communication exists with the bladder (anus vesicalis). Varoldi saw atresia recti with cloaca formation and open urachus in a calf.

Symptoms. The want of an anal opening is seldom noticed in animals immediately after birth; as a rule, it is only discovered when the results of suppressed defxcation become well marked; the little animal's 
abdomen appears distended, colic sets in, the patient stops sucking, strains and attempts to pass frees, and when the anus is examined it is

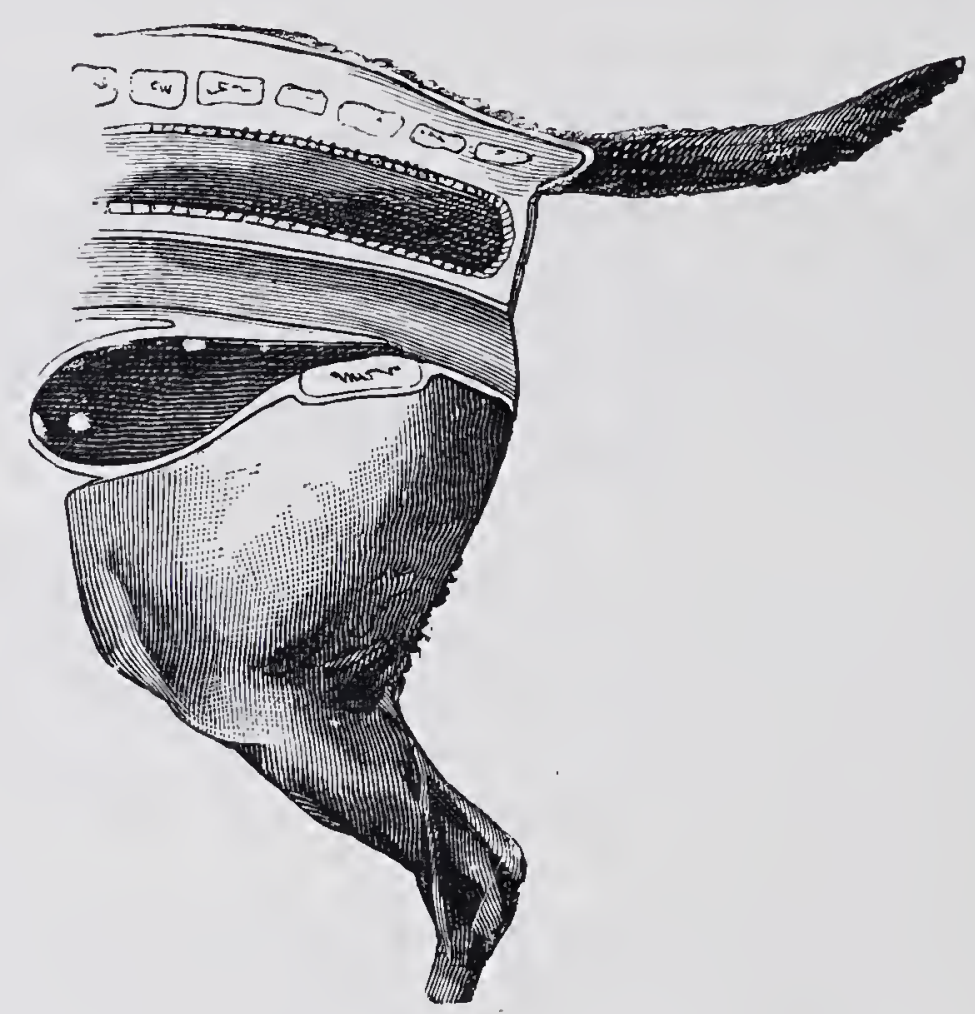

FIG. 152. - Congenital malformation-atresia ani in the bitch (semi-diagrammatic).

found there is no opening. If the anus alone is imperforate, the skin projects at the centre of the sphincter, rectal contents may even be distinctly felt beneath it; but, when the walls of the rectum are also

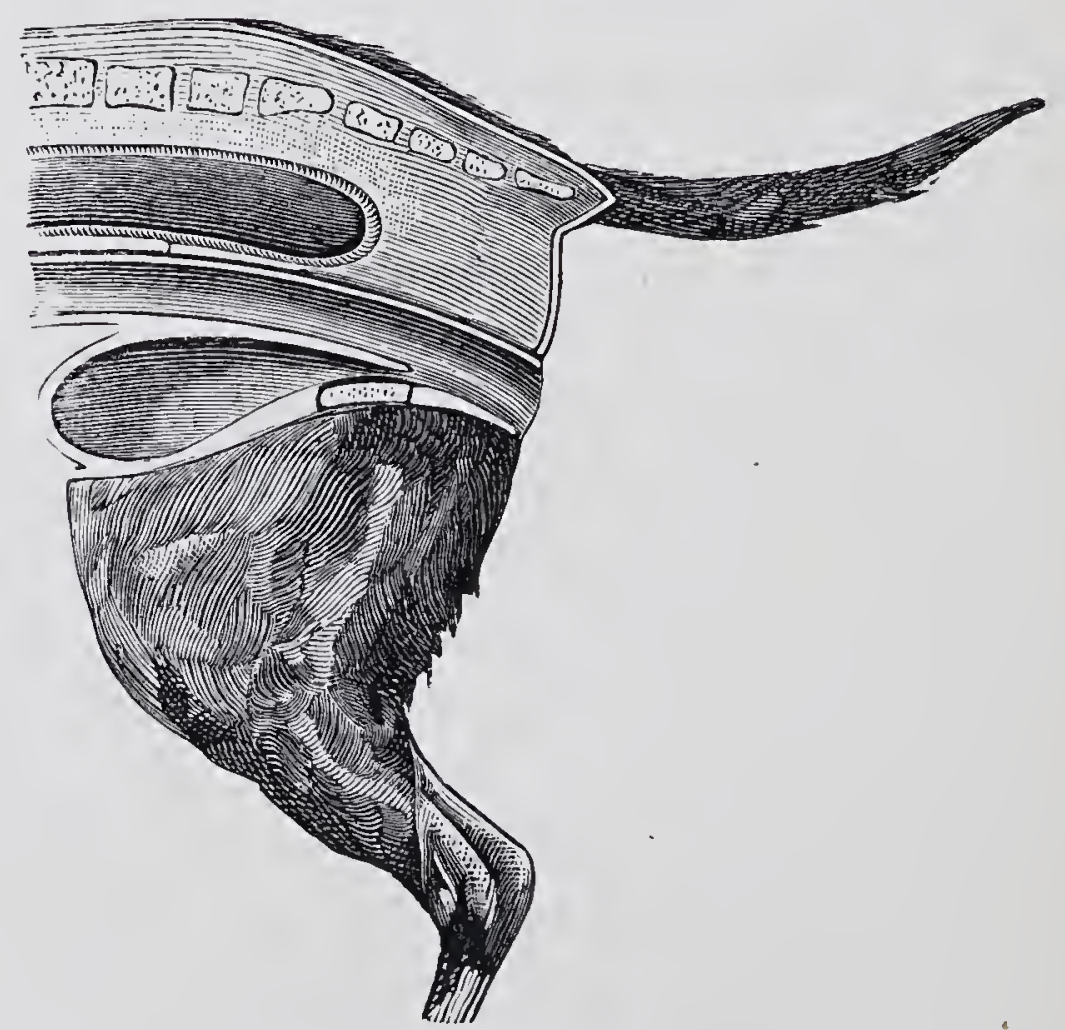

FIG. 153.-Congenital malformation-atresia ani et recti in the bitch (semi-diagrammatic).

adherent this projection is wanting, and either the anus is visibly closed or, on introducing the finger or a probe, an obstruction is felt not far from the external orifice. In recto-vaginal fistula excrement escapes by the vagina, and the communication between the upper vaginal wall and rectum can usually be discovered with the finger or probe. 
In the latter case the animal may live, provided the ano-vaginal opening is large enough to allow discharge of bowel contents. Burmeister saw an ano-vaginal fistula of the size of a straw in a six weeks pig. Piéperbrok found recto-vaginal fistula and imperforate anus in a pregnant sow.

In atresia ani or recti the animals sooner or later die, if provision be not made by operation for discharge of excrement, though, as the milk forms comparatively little fæces, the animals may continue to live for several weeks. Atresia ani was seen by Waltrup in a twenty-six days sucking pig, and by Möller in a puppy of the same age. Ruminants seldom live so long; in them severe disturbance results from suppressed defæcation after four to eight days; in a case described by Bull, a calf lived five days : the post-mortem showed the walls of the rectum adherent for a distance of 5 to 6 inches.

Prognosis is in general favourable, provided operation is not too long deferred. It depends principally on whether the anus alone or both anus and a considerable piece of rectum are simultaneously closed; in the latter case there is always great difficulty in laying open the bowel, and in keeping it patent. The artificial anus tends strongly to contract and interfere with defæcation, whilst the rectum becomes paralysed on account of severe distension, and may even be ruptured; in such case operation gives no relief. In man, clysters of tobacco smoke have been used, and may be tried in animals. Where they fail eserine may be given, though in cautious doses if the animals are already weak.

Treatment. Operation is the sole resource. Some recommend delaying it for a day or two after birth, because moderate distension of the rectum is rather advantageous, but as a rule the surgeon's attention is not demanded at this time, often not till much later, when death is unavoidable. In atresia ani the anus is only closed by a layer of skin which is simply grasped with forceps and cut through with the scissors. Frees are usually discharged at once : if not, the subcutaneous tissue, which sometimes contributes to the obstruction, must be thrust on one side. Reunion of the edges may generally be prevented by smearing the parts with some mild ointment. For a similar purpose the mucous membrane of the rectum is in man united with the outer skin by incising the skin from above downwards right over the anus, dividing the subcutis on either side of the projecting end of the rectum, cutting through the mucous membrane in the same direction, and uniting it with the outer skin by several interrupted sutures. Stenosis is thus prevented.

The operation for atresia recti is more difficult, particularly if the rectum is extensively adherent to neighbouring parts. The less prominent the anus from pressure of bowel contents, the more unfavourable

V.S. 
the condition. In such cases the adhesions must be broken down, taking care in males to protect the urethra and bladder, and in females the vagina. In males a catheter should be introduced into the urethra, in females the index finger of the left hand inserted in the vagina during operation. The first incision is then made, as above described, by either removing a piece of skin with scissors, or making a cross-shaped cut through the skin over the anus, and gradually thrusting a finger or probe into the depths until the lumen of the rectum is attained. The position of the probe or finger indicates the proper direction.

In animals the trochar has been recommended for opening the occluded rectum, but the above method deserves preference. After discharge of fæces, an attempt should be made to suture the mucous membrane to the skin, and reunion may be prevented by frequently smearing the parts with oil. Clysters assist the bowels in acting for the first few days.

Howard produced a colic fistula in a sucking pig with atresia recti. The same operation has been practised in man, but being as a rule of no practical value, is now seldom adopted. Treatment of recto-vaginal fistula is described later.

\section{II.--INJURIES TO THE RECTUM AND ANAL REGION.}

Injuries to the rectum in mares and cows are often caused by the penis during the act of coition, while perforations result from incautious exploration of the rectum with the hand, and by the attempts sometimes made by laymen to remove blood from the mucous membrane of the rectum in order to cure colic. The incautious use of clyster syringes may produce wounds; and injuries are occasionally inflicted on the rectum by persons desiring to revenge themselves on the owner of the animal.

Less frequently the rectum is ruptured by abnormally large and hard masses of freces, but in mares and cows during parturition, injuries caused by the fotus, or by violent and awkward attempts at assistance, are very common, the colt's feet sometimes penetrate the upper wall of the vagina or uterus, and the lower wall of the rectum.

Finally, any hard substance accidentally consumed with the food may penetrate the mucous membrane of the rectum or anus, and produce proctitis. In dogs, animal or fish bones are a frequent cause. In a horse which had swallowed a large number of fir-needles, Kirchner found inflammation of the rectum caused by the needles penetrating the mucous membrane. Stockfleth removed from the anus of a cat a 
fish-hook, which had passed through the entire digestive tract. Jansen found the skull of a fœtus in a sow's rectum; the skull had, during parturition, penetrated from the vagina into the rectum.

The anus is also liable to be injured during delivery. The perineum and, under certain circumstances, the sphincter ani may be simultaneously ruptured; in oxen, horn-thrusts are often responsible for these accidents.

The course of such injuries depends principally on their seat and extent. Wounds penetrating the peritoneal cavity always end fatally; and those caused in mares and cows by pressure of the penis are nearly as dangerous, though a few cows have been seen to recover. On the other hand, sudden death attributable to shock has sometimes been noted. Mares usually die within twenty-four hours; cows may survive longer. Passage of the penis into the rectum is not, however, always followed by rupture-injury depending chiefly on the degree to which the rectum is filled with fæces. Wounds from the clyster syringe generally end fatally, and particularly if drugs are at the same time injected into the peritoneal cavity.

Injuries of the posterior portion of the rectum lying beyond the peritoneal cavity, though certainly not so dangerous as the above, are yet apt to lead to septic cellulitis in the connective tissue around the rectum (paraproctal connective tissue), which may be followed by fatal consequences. But Hüppe's case shows that in the horse even extensive injuries at this point may heal. Injuries of the vagina are less dangerous, though sometimes followed by formation of recto-vaginal fistulæ.

Diagnosis is at once settled by examination of the rectum, to which the colic, tenesmus, and blood-stained discharge draw attention. But before proceeding to thus determine the seat and extent of the injury, it should be ascertained whether manipulation of the parts has already been practised, and the owner should be informed of the possible existence of a fatal injury, so that the operator may not be suspected of having caused it. Complication with peritonitis is characterised by fever, small and frequent pulse, and slight but continuous colic, and in horses death occurs in a few days, often even in a few hours.

Wounds of the anus are only grave if the sphincter or perineum is divided. If no difficulty occurs in closure of the anus, wounds of this kind heal easily and completely; only exceptionally does anal fistula result. The healing of ruptured perineum offers greater difficulty and, in mares, may result in sterility, but a cure is usually effected by suturing the wound early and cautiously, or even at a later period if the cicatrix be freshened.

Treatment. Treatment of perforating wounds of the rectum is seldom 
desirable. Oxen and sheep are best killed at once. Should the attempt be made, the rectum must first be carefully emptied, food withheld, and water given only in small quantities. Clysters are better avoided, because they favour the entrance of bowel contents into the peritoneal cavity and peritonitis. Opium might possibly be of service on account of its checking the movement of bowel contents towards the injured spot.

In injuries of the pelvic portion treatment is more hopeful; the diet should be as above. The wound may be cleansed by clysters (which at the same time wash out the contents of the rectum), followed by disinfecting materials like salicylic acid, carbolic acid, or creolin; in horses, by diluted sublimate solution (1 in 5,000). Röder successfully sutured a recto-vaginal rupture; recovery followed.

Wounds in the neighbourhood of the anus may sometimes be sutured and bleeding vessels ligatured. Cold water clyster's serve to check bleeding from the anterior parts of the bowel. It has also been suggested to introduce a bladder or rubber balloon into the rectum, and to exercise pressure on the bleeding vessels by inflating it or filling it with water; but its use is much limited in animals on account of its causing severe straining, and thus often proving more dangerous than useful. The same is true of tamponing the rectum, though in extreme cases one might certainly try it.

As regards abscess-formation after injury to the rectum, see succeeding pages.

\section{III.-INFLAMIMATION OF THE IMUCOUS MEMBRANE OF THE RECTUM AND ANUS (PROCTITIS).}

Apart from inflammation of the rectum and anus, produced by grosser injuries, inflammatory processes are seen in severe intestinal catarrh, in dysentery, and after continuous diarrhoea, particularly in young pigs and dogs. The same result may be produced by clysters of too irritating a character, or administered too hot, and by very large masses of fæces. Schwanefeld found a piece of broomstick, 8 inches in length, in the rectum of an ox. In dogs, bones and firm masses of frces often produce inflammation of the mucous membrane.

Inflammatory disease of the anus in the horse has been seen after tearing away the larvæ of cestridæ, in carnivora in consequence of rubbing the anus to allay the irritation of pruritus ani. In long-coated dogs the hairs in the neighbourhood of the anus sometimes stick together, close the anus, and produce inflammatory irritation, or the animals may suffer from inflammation of the anal glands.

Symptoms. Inflammatory disease of the mucous membrane of the rectum is characterised by tenesmus, that is, repeated but unsuccessful 
attempts to pass frees. The animals stand with the back arched, and the continuous severe straining often leads to prolapsus ani or recti. The mucous membrane is more or less intensely reddened.

Injuries to the anus may be directly seen; and where the hairs have become adherent and occluded the orifice, the neighbouring skin appears reddened and often excoriated.

Disease of the perineal glands may be recognised by inflammatory swelling; defæcation is painful and often repressed; after some time fluctuation and perforation occur, and the swelling subsides, though relapses are common and sometimes cause laymen to suspect hæmorrhoids.

These conditions are seldom dangerous, but occlusion of the rectum and of the anus may result from chronic catarrh in young animals. Injuries sometimes lead to inflammation of the perineal or paraproctal connective tissue and thus cause trouble.

Treatment. Inflammation of the rectal mucous membrane is treated with mucilaginous and oily clysters; in larger animals starch paste is suitable. In dogs, lukewarm oil may be used, and when tenesmus is marked, opium can be added. Foreign bodies and hard masses of freces should be removed cautiously. The long adherent hairs about the anus must be cut away with scissors, the anus cleansed, and powdered with some material like iodoform and tannin.

Suppurating swellings of the anal glands must be opened, the contents removed, and, after thoroughly cleansing, the surfaces of the wound strewed with iodoform powder. For inflammation produced by removal of cestrus larvæ, lukewarm lotions and dusting powders are recommended. In all these diseases it is of importance to render defæcation as easy as possible. For this purpose suitable nourishment should be given, and clysters and laxatives administered.

\section{IV.-INFLAMIMATION OF THE CONNECTIYE TISSUE SURROUNDING THE RECTUM (PERIPROCTITIS OR PARAPROCTITIS APOSTEMATOSA).}

This disease, though not common, is sometimes seen in large animals, and is caused by wounds of the posterior portion of the rectum becoming specifically infected. Metastatic abscesses have been seen here during the course of strangles. In females peri- or para- proctitis may result from injury of the vaigina.

The loose connective tissue surrounding the end of the rectum appears particularly liable to cellulitis, and it depends principally on the action of the infecting material what course the disease takes. In general, however, septic cellulitis is rarer than one would expect, perhaps because 
septic infectious material is destroyed in the digestive tract. Most of the published cases have been due to simple purulent cellulitis, leading to formation of abscesses. In man, howevèr, by far the commonest cause is tuberculous infection.

The course of the disease depends principally on where the abscess perforates; should it discharge into the peritoneal sac, death from purulent peritonitis is inevitable; but when perforation occurs into the rectum, or the abscess is punctured from this point, recovery often follows. The case is still more favourable where the discharge occurs outwardly beside the anus. Wilke succeeded in opening the abscess from the vagina, a method which deserves preference, as avoiding the bad results of perforation outwardly or into the rectum. In the latter case there is always danger of the formation of an anal fistula.

Symptoms. Periproctitis is first announced by interference with the passage of frees, caused by swelling and abscess formation. The animals show slight but continued symptoms of colic; defæcation is difficult and painful, and is either suppressed or accompanied by groaning. There is usually slight fever. Only where cellulitis extends to close under the outer skin does swelling occur in the neighbourhood or at the side of the anus. From here pus may burrow along the muscles of the thigh, and cause emphysematous swelling and lameness.

Exploration per rectum determines the extent and position of the disease. The posterior portion is empty, but in front of this the bowel is swollen and its lumen narrowed. In a case of Möller's in a horse, a painful fluctuating swelling, almost as large as a child's head, could be detected on the upper wall of the rectum, about 8 inches from the anus. This had so diminished the passage that there was scarcely room below to pass two fingers. The rapid development of the symptoms, the soft, fluctuating, painful character of the swelling, and the moderate fever, distinguish the condition from tumour formation.

Treatment. When an abscess has already formed, and attention been called to its existence, nothing usually remains but to give exit to the pus, and as it is of importance to effect this in the least dangerous way, the path usually chosen is that through the vagina or the skin and tissue lying around the rectum. The latter is preferable, if the abscess be close to the anus, and the puncture must be made as low as possible to favour drainage. If at all possible, division of the sphincter ani must be avoided. In females the vagina forms a convenient and safe route for arriving at abscesses lying below the rectum. Wilke perforated the abscess wall through the vagina with the finger, and emptied an abscess the size of an ostrich egg, which lay about 12 inches from the anus.

In purulent cellulitis affecting the upper wall of the rectum, drainage into the bowel should only be chosen when the abscess cannot be 
punctured from without. In the above described case, Möller opened the abscess with a bent trochar (Flourant's), and gave exit to about 2 litres of thick offensive pus, containing fragments of necrotic tissue. As the abscess had refilled next day, the opening was lengthened with a button-pointed tenotome, introduced into the rectum with its cutting surface covered by the hand. Complete recovery took place in three weeks.

After incising the abscess, the cavity must be carefully washed out with a strong stream of disinfecting material, so as to remove necrotic portions of tissue, and precautions taken for securing drainage of pus. Relapses, however, are common; in a case described by Verlinde pus burrowed downwards as far as the Achilles tendon; nevertheless, the animal eventually recovered.

\section{Y.-PROLAPSE OF THE RECTUIM AND ANUS (PRO- LAPSUS RECTI ET ANI).}

Permanent protrusion of the mucous membrane of the rectum through the anus is described as prolapsus ani. As the membrane appears after each act of defreation, prolapsus ani really consists only in the abnormal continuance of a physiological condition. This protrusion of the mucous membrane is most distinctly seen in horses, and in them prolapsus ani is rather frequent, but it also occurs in dogs and other domestic animals.

When not merely the mucous membrane but the entire intestinal wall passes the anus, the condition is termed prolapsus recti. The posterior end of the bowel can only pass the anus after rupture of the periproctal connective tissue, but those portions of bowel normally clothed with peritoneum sometimes pass through the pelvic portion and anus, after becoming invaginated in the last part of the rectum, constituting a third condition-prolapse with invagination. We therefore distinguish-

(1.) Prolapsus ani.

(2.) Prolapsus recti.

(3.) Prolapsus recti cum invaginatione.

Prolapse of the anus and of the rectum generally results from severe diarrhœea, particularly if accompanied by tenesmus. The disease is favoured by weakness, with relaxation or paralysis of the sphincter ani. It is commonest in young animals, but in swine it also occurs at a later period.

As a rule, the immediate cause is increased abdominal pressure, though inflammatory swelling of the mucous membrane of the rectum may cause prolapse, and both these have been seen after use of irritant 
or excessively hot clysters. They also appear during colic or obstruction of the bowel, sometimes in difficult parturitions; in cows in connection with prolapse of the vagina, and in horses which struggle violently when cast with hobbles. In two cases noticed by Mauri, which occurred during castration, the prolapsed rectum was ruptured, and coils of small intestine protruded through the opening.

Symptoms. In prolapsus ani, a red, slightly painful, hemispherical swelling of the mucous membrane appears behind the anus, which shows at its centre a shallow depression; sometimes only a few folds appear at one side. In simultaneous prolapse of portions of the rectum (prolapsus recti) the swelling is larger and harder, but even then does not attain the size usual in prolapse complicated with invagination, where portions of ${ }_{\iota}$ bowel a yard in length may protrude through the anus. In dogs,

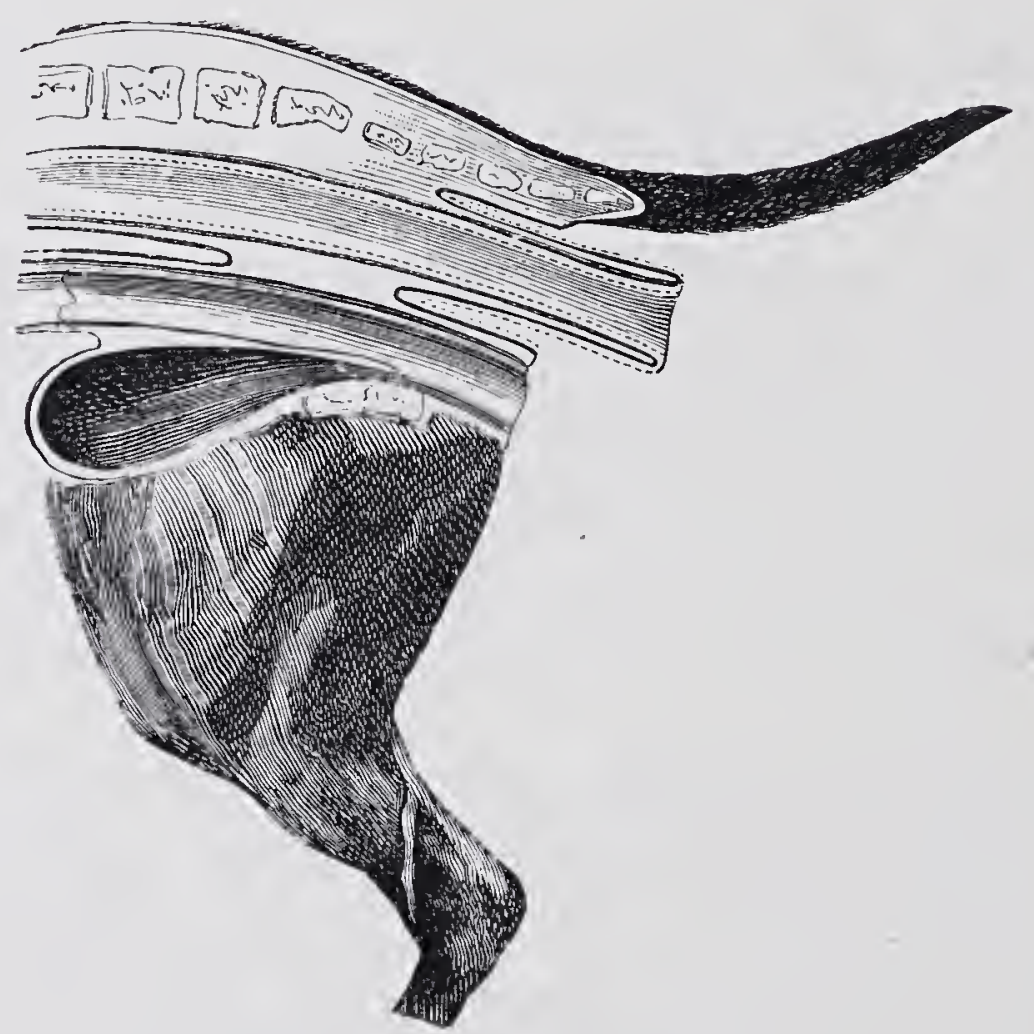

FIG. 154.-Prolapsus recti with invagination in the bitch (semi-diagrammatic).

Möller has repeatedly seen prolapses 12 to 20 inches in length. On account of the tension on the mesentery, the prolapsed portion sometimes becomes twisted upwards in horses (fig. 155).

When the prolapse attains such dimensions no doubt can exist in diagnosis, but it is otherwise when only small portions of bowel project beyond the anus. In simple prolapsus ani et recti the ring-shaped swelling shows an opening at its centre, through which freces are discharged; at its periphery it is impossible to pass the finger towards the pelvis. The case is different where prolapse is complicated with invagination; then the finger, and in large animals the entire hand, can be introduced alongside the prolapsed part into the pelvic portion of the rectum. It is of importance to note this, as it at once differentiates the two conditions, and affords important indications for treatment. 
Progress. Prolapse of the anus is usually reduced by laymen without skilled assistance, and only when it continually recurs and the mucous membrane becomes greatly swollen, or when it has persisted for a long time, does it become the subject of skilled treatment. Frey saw a horse which had difficulty in defreation in consequence of prolapse; others have erroneously described chronic prolapses as hæmorrhoids. The mucous membrane undergoes change from continued exposure to the air ; it becomes thickened, dry, necrotic, and may even slough, thus eventually bringing about spontaneous recovery from the prolapse.

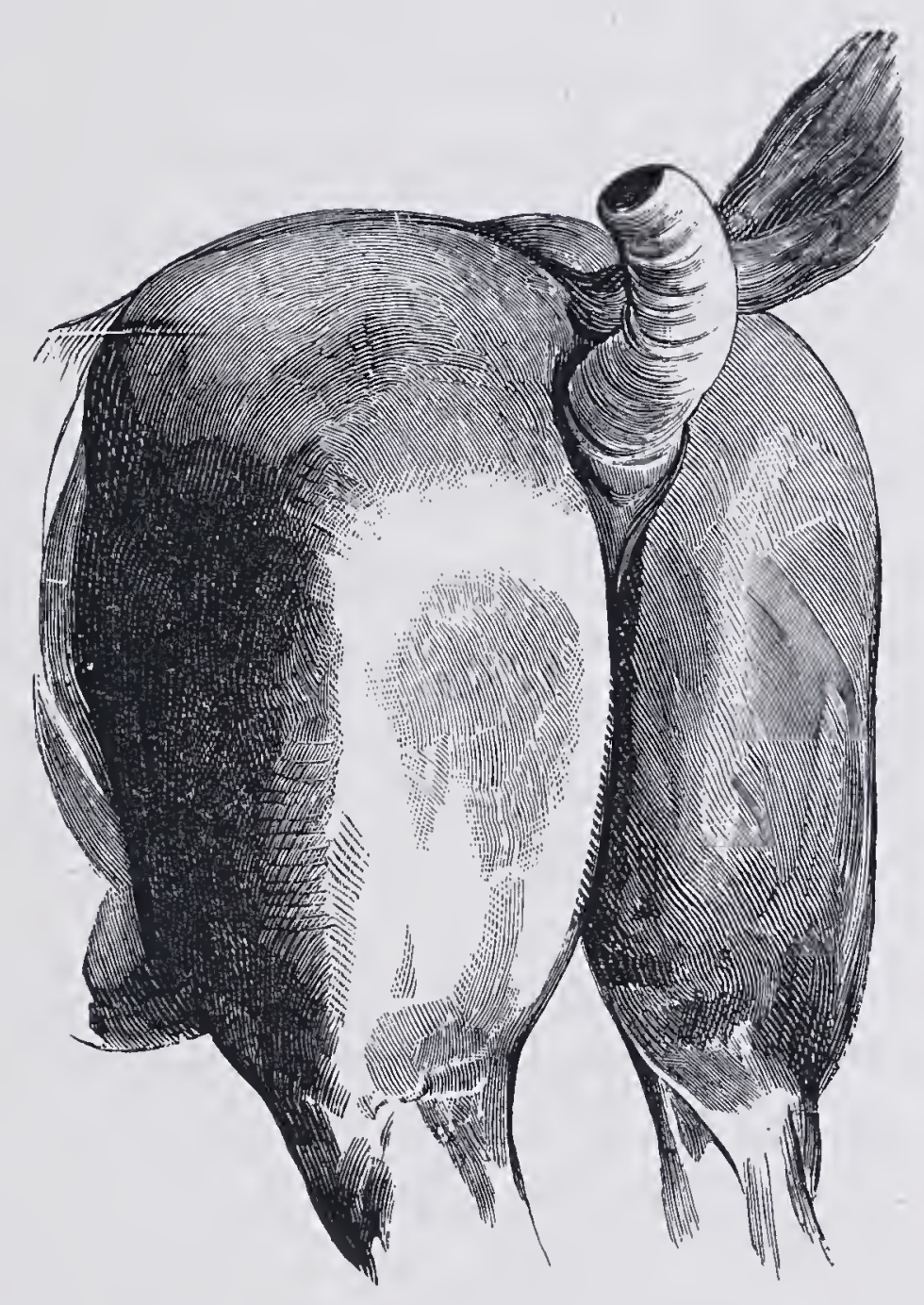

FIG. 155. - Prolapsus recti with invagination in the horse.

Groll saw a pig with prolapse; twenty days later the protruded part sloughed away spontaneously, and recovery followed.

The above is also true of prolapse of the rectum with invagination, except that as a rule defæcation is difficult, the passage of material being interfered with in the invaginated portion of bowel. The animals strain severely, causing further portions of intestine to protrude, these soon become œdematous, the folds in their mucous membrane disappear, and the surface of the prolapsed portion consequently appears smooth. The surface of the bowel, which is dirty and not infrequently injured by the animal rolling or lying, gradually becomes dry and presents a dark red or black colour. Pigs sometimes bite off the prolapsed portions in their fellows. 
Death is generally due to the consequences of complete obstruction of the bowel acting on animals which are already weakened. Only exceptionally has rupture of the paraproctal connective tissue and prolapse of other portions of the bowel been seen; it is differentiated from prolapse of the rectum by the surface of the protruded bowel exhibiting a serous covering.

It requires no particular demonstration to show that prolapsus ani involves less danger than prolapsus recti, especially when the latter is complicated with invagination, and though spontaneous healing sometimes occurs, by the necrotic portion of bowel sloughing, it is very exceptional. Prolapses of the anus are most easily healed, when they have persisted for a short time only, and the mucous membrane. of the protruding part is not much altered. Should prolapsus recti be complicated with invagination, and have existed for several days, reposition becomes impossible, because the peritoneal surfaces have already become either adherent or united.

Herbivora withstand the condition far better than carnivora. In dogs, invagination is very general, and the commonest subjects are weakly animals, or those whose constitutions have been lowered by continued diarrhoea, as Haubner has already noted. Pigs bear prolapse better, and the larger animals still less frequently die of it. Horses and oxen generally recover completely, though Tetzner saw a horse with prolapsus recti die with symptoms of colic; the post-mortem showed the cause to be a leiomyoma.

Treatment. In recent prolapse the mucous membrane is cleansed and replaced by regular and steady pressure with the hand, or in small animals with a finger. Sometimes this requires to be repeated, and the owner or attendant may be instructed how to carry it out. Tenesmus is combated by clysters of mucilaginous or oily fluids, containing, if needful, opium; cocain may also be worthy of trial. Diarrhœa must be treated by suitable diet and internal medication. 'Should the prolapsed mucous membrane be swollen, scarification and bathing with such astringents as 2 to 5 per cent. alum solution will facilitate reposition. Continued recurrence or structural change in the mucous membrane may necessitate surgical removal of the protruded part.

The procedure is the same in simple prolapsus recti, but prolapsus recti with invagination offers greater difficulty. In such case reposition must be effected as soon as possible, for the longer prolapse exists, the greater the difficulty and the less the chance of success, though even in these cases cure is occasionally effected. Larsen immediately reduced a prolapse, about 32 inches in length, which occurred during the castration of a horse, caused an assistant to keep the anus closed, and completed the interrupted operation. When the animal rose, the prolapse had 
disappeared and did not recur. In reposition care must be taken to simultaneously reduce the invagination. For this purpose it is not sufficient to thrust the protruded bowel through the anus, but the extreme end must be carried forward at least twice the length of the prolapsed part. In large animals this is best effected with the arm, in the smaller (dogs) by means of a tallow candle. Stockfleth used a stick; the end covered with tow and rubbed with fat. Infusions of warm water injected whilst the hind-quarters are raised may also assist reduction. The animal should be laid with the hind-quarters high; if small it may be lifted by the hind legs. When the animals strain violently, narcosis must be resorted to, for which purpose morphine is very useful. The greatest obstacle to reposition and permanent cure lies in this severe straining. Large animals should, therefore, be watched for some hours, and if it sets in, pressure should be exerted over the loins. Mild clysters and the application of cold combat inflammatory symptoms about the anus and rectum.

To ensure retention, the anus may be sutured. Strebel inserts two strips of leather in the neighbourhood of the ischial protuberances, and crosses them over the anus; by applying tow or sponges below these the intestine is held back; in horses, the tail has been fastened so far forward with a pair of straps as to exercise pressure on the anus. André's "tobacco-pouch" suture has been recommended. This is a continuous suture, made by passing a narrow tape in and out under the skin, and working in a circle; it should be left in position for twentyfour to thirty-six hours. To allow of defrcation the tape is, when necessary, loosened, and afterwards again drawn tight and knotted. It is clearly not to be employed in cases of invagination where its use would be irrational. Cocain ointment injected into the rectum may be tried in severe training:

When prolapse with invagination has already existed for several days, reposition becomes impossible, nor should it be attempted if structural change has occurred. Nothing, then, remains but to remove the protruded portion, for which purpose one of the following methods may be employed, viz. :-

(1.) The use of irritants. Jessen powder's the protruded parts four or five times a day with sulphate of copper and pulverised charcoal in equal parts; inflammation results, and in a few days a scab forms (necrosis), after which the prolapsed part sloughs off in eight to fourteen days. Danish practitioner's have used this material with success in large animals and swine, but others consider it useless; Weber, after trying it in foals, was finally forced to operate. It can, of course, only be used in prolapsus ani et recti without invagination. Apart from the uncertain action of this treatment, it must be remembered that it is 
often followed by extensive contraction of the anus and difficulty in defæcation, and most practitioners, therefore, prefer amputation.

(2.) Ligation has been adopted by Viborg and Stockfleth in the case of pigs, dogs, and foals. As it is necessary to keep the anus open for the passage of freces, Stockfleth binds a ring of wood, 1 to $2 \frac{1}{2}$ inches in

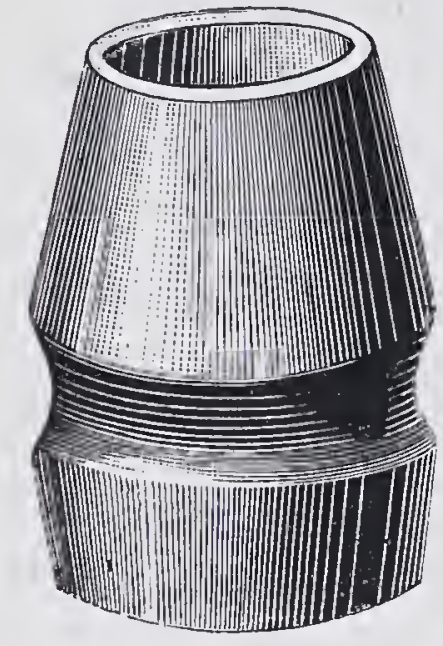

FIG. 156. Stockfleth's wooden ring. diameter, in the anus; to prevent the ligature slipping off, the ring has a shallow groove on its surface (fig. 156). The prolapse is divided up to the anus, the ring then thrust in, and a stout ligature passed around it and the prolapsed bowel, which slowly cuts through, until finally the portion of intestine beyond the ring is cut off. During the next few days defæca. tion must be assisted by clysters, and bulky and indigestible foods avoided. The tube falls away spontaneously in five or six days, and as a rule healing is then complete. Sorrensen in this way amputated a piece of bowel, weighing 23 ounces, in the horse. A case of Johne's, however, in the pig, shows that after ligation stenosis, and even complete occlusion, may occur.

(3.) Multiple ligation. If the layers of bowel are not completely united to one another, or at any rate not firmly adherent, there is considerable risk of opening the peritoneal cavity when practising this method.
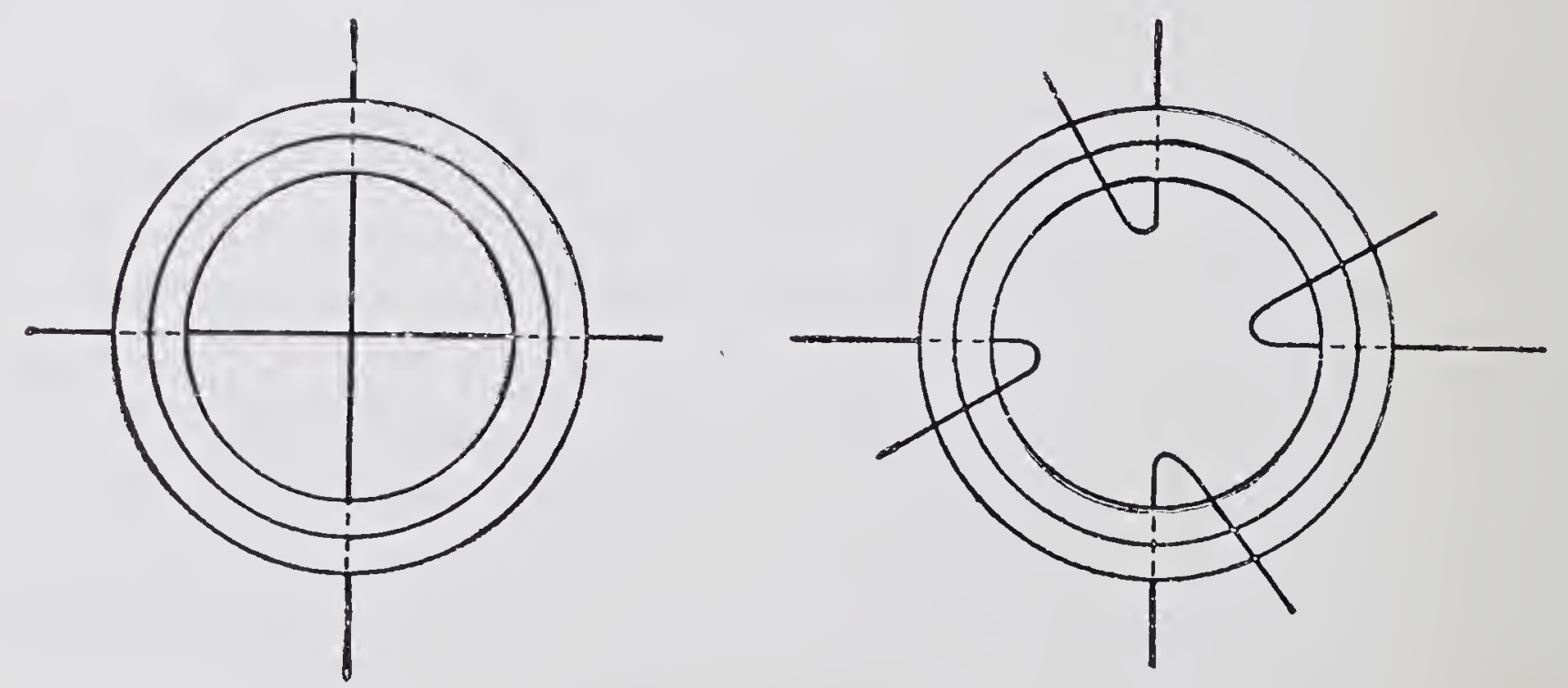

FIGS. 157, 158. - Method of ligation in prolapsus recti.

The simple cobbler's stitch is often recommended. A transverse incision is made through the upper half of the protruded bowel, close behind the anus, and the two layers of tissue in front of the incision united by closely applied ligatures. The lower half is then divided and the ligaturing continued, until finally the protruded portion of bowel is completely divided and removed. The simultaneous division and ligation prevents the end of the bowel not yet firmly fixed by sutures slipping back into the peritoneal cavity, and producing fatal peritonitis. 
In dogs the following method can be employed. 'The protruded piece of bowel is transfixed close behind the anus with two needles arranged crosswise, and provided with two long threads (fig. 157). The prolapsed part is then cut through, about $\frac{1}{2}$ to $\frac{3}{4}$ of an inch behind the threads, which are then drawn forward out of the intestinal opening. By dividing these in the centre, one has four threads (fig. 158), which when united form four stitches, and are generally sufficient. Should it be seen on cutting away the posterior part of the bowel that union is still incomplete, another pair of stitches can be inserted between each two of those previously existing, thus forming eight ligatures, which are sufficient even in large animals. Savournin saw a horse in which the rectum had already become necrotic, but by ligaturing it in two portions he effected complete healing in twelve days. In the case of a mare, Grammlich gave chloroform and then performed multiple ligation, cutting off the prolapsed portion of bowel about half an inch behind the ligatures. The bowels did not act naturally (i.e., without assistance) for six weeks. It is scarcely necessary to add that, both in selecting the ligature and in carrying out the operation and after-treatment, it is necessary to practise antisepsis as far as possible. To facilitate ligation Reuter recommends thrusting into the lumen of the prolapsed bowel a conical piece of carrot, turnip, or even of soap, before commencing the actual insertion of the ligatures. In swine Olivier divides the prolapsed bowel in its long diameter and ligates the two pieces separately.

(4.) In old cases of prolapse, where union between the layers of peritoneum is complete, and no chance exists of opening the peritoneal cavity, simple removal of the prolapsed part is sufficient. This method has been used in swine, but caution is required; simple or multiple ligation is greatly preferable.

\section{VI.-DILATATION, STENOSIS, AND PARALYSIS OF THE RECTUM AND ANUS.}

After operation for atresia ani, amputation of prolapsed portions of the rectum, and injury to the anus, cicatricial contraction sometimes occurs, causing marked stenosis and difficulty in defæcation. Sometimes the anus is completely closed, as in Johne's case, in a six months old pig after ligation of the prolapsed rectum, or in Volk's, in a pig, where the same accident followed spontaneous sloughing. Occlusion of the rectum occurs, both in pigs and dogs, in consequence of chronic diarrhoea, particularly during the first year's of life; the epithelium is lost, the opposing mucous surfaces become adherent, and finally unite.

Cicatricial contraction of the rectum may also result after injury or ulceration of its mucous membrane, and cause stenosis, or new 
growths, within or without the bowel, may compress it and narrow its lumen.

Johow saw a cow which stood with the back arched and continually attempted to defrcate; a ring-shaped stenosis of the rectum existed at the entrance to the pelvis, and was barely large enough to admit two or three fingers; the rectum was greatly distended in front of the spot, which was about half an inch wide. After incising the stenosis and using clysters, a cure was effected. The nature of the case seems obscure.

Rogerson diagnosed in a mare and a foal, which both suffered from stoppage of the bowel, well-marked stenosis of the rectum about 20 inches in front of the anus; the post-mortem examination showed the rectum to be greatly thickened, of cartilaginous consistency, and to some extent ossified. Johne saw the same condition in a cow, Meyer in a horse. Gurlt found the rectum of a foal so narrow that only a good-sized goose-quill could be passed through it.

Pathological dilatations of the pelvic portion of the rectum are not infrequent in horses. They are seldom partial-so-called diverticulabut usually the entire pelvic portion is dilated. They are oftenest seen in old horses which have long been fed on bulky food, and in dogs which have suffered from habitual constipation or enlargement of the prostate, which interferes with defrcation; dogs also show this dilatation in hernia perinealis. Old horses often suffer from extensive dilatation of the pelvic portion without showing distress, though they have difficulty in defrcation, especially if paralysis of the rectum accompanies dilatation.

Hengst speaks of an old horse which suffered from colic, and showed a rectal sacculation 12 inches in front of the anus, which was the size of two fists and filled with frees. After emptying and washing this out, the colic disappeared. Martin noted the formation of a diverticulum in a horse after injury to the rectum; 16 inches in front of the anus was a wound about 6 inches long and 2 broad, which caused severe fever and colic; it was washed out with solutions of boric acid and permanganate of potash, and later of carbolic acid. Cicatrisation occurred, but a diverticulum as large as a man's fist formed, from which the dung had to be daily removed. Stockfleth described in a horse a diverticulum which lay on the upper wall of the rectum about $5 \frac{1}{2}$ inches from the anus, and opened into the lumen of the bowel by means of a narrow slit. Möller found one about the size of a hen's egg in a Dalmatian dog on the left wall of the rectum, close in front of the anus, which was thrust forward during defæcation, and was filled with soft fæces. This was possibly an enlarged anal pouch; but as the dog was only seen during life, the point could not definitely be decided.

Paralysis of the rectum is generally associated with paralysis of the tail, or of the bladder and hind legs, and apart from the general paralysis caused by fractures of the vertebræ, \&c., occurs most frequently in the horse. It is particularly common in mares. Harms found rectal paralysis in a cow to be due to fracture between the sacrum and first vertebra of the tail. Though this paralysis is usually spontaneous, the immediate cause can often be traced to severe bruising or injury in the 
lumbar region. In the army reports, the following case occurring in a seventeen year old mare is given :-The animal showed paralysis of the rectum and bladder, with atrophy of the muscles of the quarter and thigh, and was found, on post-mortem examination, to have thickening of the dura mater spinalis from the 3rd lumbar vertebra backwards. The grey substance of the spinal cord appeared lighter in colour, and softer than usual. A considerable quantity of red fluid was found between the meninges of the spinal cord.

Friedberger saw the condition during the course of contagious plemropneumonia in the horse. We have repeatedly seen paralysis of the rectum develop spontaneously and quite gradually, and affect the rectum, tail, bladder, and, later, the hind-quarters, but only in non-pregnant mares; generally in well-bred animals. The first change appears to be paralysis of the lumbar nerves, followed by accumulation of fæces in the rectum, and gradual dilatation of that bowel.

The rectum receives its nerves from two points: the n. hæmorrhoidalis medius, a branch of the pubic nerve, gives twigs to the curvator coccygis and retractor ani; the n. hæmorr. posterior arises from the 4th and 5th lumbar nerves, and supplies the terminal portion of the rectum and the retractor penis muscle. The depressor coccygis derives its motor twigs from the 5th lumbar nerve, while the levator coccygis is supplied from the last twigs of the spinal nerves, the so-called cauda equina. In the cases noted the disease began with paralysis of the levator coccygis, but the rectum and bladder were only affected after several months. Finally, the general paraplegia necessitated slaughter or total withdrawal of the animal from work. Unfortunately, post-mortem examinations were not made, but the course of the disease points to progressive degeneration of the posterior portions of the spinal cord. The observations of others and the above-described post-mortem appearances support this view. Peters saw the disease associated with paralysis of the bladder in a mare. Schwarznecker observed the same thing in a pregnant mare, which later died from paraplegia; the post-mortem gave a negative result. Deigendesch reports a similar case; the mare died from rupture of the bladder. Dollar has seen the condition after severe attacks of contagious pneumonia (influenza).

Symptoms. Abnormal dilatation of the rectum first attracts notice on account of the large quantities of frees which are passed, and the long intervals between the acts of defæcation, which only becomes difficult when actual paralysis has occurred; the rectum is then distended with dung, which requires to be removed manually. Cases occur which require to be daily assisted in this way. Sometimes paralysis of the levator coccygis exists simultaneously, and then the tail swings to and fro as the animal walks, and the hair becomes soiled with urine and freces. After a certain time incontinence of urine sets in, the bladder becomes distended, and urine flows away continuously (ischuria paradoxa); still later sacral paralysis with atrophy of the muscles of the haunch occurs (compare with sacral paralysis). Sometimes pruritus exists about the hind-quarters. 
The disease takes a chronic course and treatment has no visible effect, but however slow its progress, the animals finally become useless, and may even die of the disease.

Dilatation of the anus is commonest in animals which have long suffered from severe diarrhœa, or been much weakened by internal diseases. Even after prolonged rectal examination, when the arm has been in the bowel for a considerable time, paralysis of the sphincter ani may persist for several days; the anus remaining open, and the air, which streams in and out during breathing, producing a loud noise.

Stenosis of the anus and rectum soon impedes defæcation and attracts notice; the position, degree, and extent of the condition is at once recognised on local examination. Hönisch saw a horse which had long shown difficulty in passing fæces; it placed the hind feet as far forward as possible and discharged single small, hard masses. The post-mortem discovered cicatricial stricture and thickening of the rectum, which extended about 5 inches forwards from the anus. It has been said that chronic inflammation in the paraproctal connective tissue may cause stenosis, but as a rule the cases published throw no light on the question.

Prognosis is guided by the above-mentioned facts. Though usually unfavourable, the animal's usefulness depends on the primary disease and the work to be done. Complete and lasting cure is seldom to be expected, but Harms's case of paralysis of the rectum in a cow, resulting from fracture between the sacrum and first coccygeal vertebra, recovered in a month. A case is also described in the annual report of the Prussian army, where a horse recovered in three weeks from paralysis of this nature following a fall.

Treatment. Cicatricial stricture of the anus and posterior sections of the rectum may be temporarily relieved by forcible dilatation; in man, bougies are employed, but their use in animals is attended with difficulty, and is only justified when, for instance, a favourite and very valuable dog is in question, for whose recovery every means must be employed. Bougies consist of cylindrical hollow or solid rods, formed of hard rubber, or of material similar to that of which catheters are made. To be successful they require to be very frequently passed. Another method is to forcibly dilate constrictions with forceps. Johow relates having, after incision, dilated with the hand a firm ring-shaped stricture in the rectum of a horse. Volk cured a pig whose anus had closed, after prolapse of the rectum, by making a cross-shaped incision over the anus and breaking down the adhesions.

Constipation, following either constriction or dilatation, is treated by suitable diet and the use of purgatives or clysters; the intestinal contents being softened by copious injections of lukewarm water. In advanced 
cases of dilatation or paralysis, the rectum requires to be emptied once or twice daily. To combat paralysis all kinds of drugs, the induced electric current, \&c., have been tried, but without success. Deigendesch tried strychnine without good result.

\section{VII.-ANAL FISTULA AND RECTO-VAGINAL FISTULA.}

All fistulæ in the neighbourhood of the anus are described as anal fistulæ. Where a communication exists between the skin and rectum, the fistula is termed "complete," where one end is blind, "incomplete." Sometimes one end communicates with the rectum, the other with the vagina (Fistula recto-vaginalis). The latter is sometimes congenital, but also results from injuries, particularly during delivery.

Injuries and cellulitis of the paraproctal connective tissue are the common causes of anal fistula, but the condition may be congenital and associated with atresia ani. Operation is the only effective treatment, and in the case of recto-vaginal fistulæ frequently fails.

Schrader, in a six year old mare, observed recto-vaginal fistula of a diameter of $1 \frac{1}{4}$ inches, about 4 inches in front of the anus. Meer found a similar one, which had appeared after delivery, 3 to 4 inches in front of the anus in a mare. Munkel observed in an ox a "complete" anal fistula, the rectal opening 6 inches in advance of the anus, the second on the lower surface of the tail. "Incomplete" anal fistulæ in horses have been seen by Hertwig; one was 12 inches, the other 16 inches in length, and both had resulted from abscess formation. Novotny describes a fistula communicating with the rectum, and extending between the semi-tendinosus and biceps femoris muscles.

Diagnosis is confirmed by passing a probe or the finger, or both, into the rectum, and discovering the opening of the fistula.

Treatment. To prevent anal fistula, proper treatment of wounds, \&c., is very important, and injuries of the vaginal walls during delivery must receive special attention.

It is seldom possible to bring about closure by injecting with irritants like liquor Villati or disinfecting fluids; and when fistulæ extend into the rectum, or far forwards in the paraproctal connective tissue, the use of irritants is dangerous, on account of the possibility of their reaching the peritoneal cavity. Operation and free exposure of the fistula are therefore preferable. If possible, the sphincter ani must be spared, though its section often produces no lasting inconvenience, and union becomes quite perfect. Munkel divided the sphincter without bad results; Hertwig endeavoured to spare it as far as possible. In laying open a "complete" anal fistula a grooved director is inserted, the end of the fistula discovered by inserting the index finger of the left hand in the rectum, and an incision then made down to the finger, care being taken to spare the soft parts as much as possible, and so regulate the cut as to T.S. 
favour wound drainage. Roupp passed a lead wire through a "complete" rectal fistula, drew one end back through the anus, and laid open the fistula by daily tightening the wire about an inch. Novotny secured healing in one case by drainage. Röder sutured a recto-vaginal fistula but only obtained partial union. Short recto-vaginal fistula may sometimes be closed by passing a seton; the larger lying near the vulva may be sutured. This treatment failing, they generally prove incurable.

\section{VIII.-TUMOURS IN THE RECTUM AND ANUS.}

In dogs fibromata, sarcomata, carcinomata, and adenomata are not infrequently seen in the paraproctal connective tissue; while horses, particularly those of a grey colour, suffer most from melano-sarcomata and melano-carcinomata. Various tumours are also found in the rectum. Kitt saw many cases of mucoid polypi of which several sloughed away, and on examination revealed the presence of Lieberkühn's glands. Kitt therefore regarded them as cedematous folds of mucous membrane (plice polypose). He also saw adenomata, adeno-carcinomata, and true carcinomata. According to his view the sarcomata originate in the lymph follicles of the rectum. Scott endeavoured to remove from beneath the sacral region a sarcoma which interfered with defæcation ; death from septicæmia resulted. Teetz saw cysts and pedunculated polypi in horses, Carogeau collections of varicose veins in dogs.

Symptoms and course. Tumours near the anus and close below the skin may be directly seen and felt, but the veterinary surgeon is seldom called in until stenosis or displacement of the rectum or anus sets in and interferes with defrcation. Tumours in the posterior portion of the rectum sometimes protrude during defæcation, and occasionally cause prolapsus ani. When further forward, they are either accidentally discovered in examining the rectum, or the continuous and gradually increasing disturbance in defæcation draws attention to them.

Manual examination determines their size, form, and position; sometimes the rectal or vaginal speculum is useful.

Inflammatory swelling of the anal glands, common in dogs, may be mistaken for tumour formation, though the local pain sufficiently indicates its nature. Rectal and anal tumours, especially when malignant, are often accompanied by secondary growths and swelling of the lymph glands in the abdomen.

Prognosis depends chiefly on the difficulty of extirpating the tumour. Not only must the nature of the new growth, and its position and size, be borne in view, but the question whether secondary growths or infection of lymph glands has occurred must be considered. 'Tumours 
near the anus may easily be removed if not adherent to the mucous membrane of the rectum, a point which can at once be settled by introducing the finger or hand. Pedunculated tumours within the rectum offer no difficulty in removal, but those with broad bases are often impossible to extirpate.

Treatment. Warts and other benign growths originating in the skin are simply removed with knife or scissors. Those growing from the subcutis or paraproctal connective tissue, if only small and not attached to the mucous membrane of the rectum, are treated in the same way. After incising the skin with the usual precautions, the tumour is drawn well forward, either with forceps or by passing a tape through it, and dissected out without injuring the rectal mucous membrane. Tumours in the rectum itself are extirpated either by ligation or "écraseur." Serious bleeding is rare, and such as occurs can be controlled by cold clysters or tampons.

Erey states having noted in the horse two sarcomata (?) as large as a hen's egg close in front of the anus, which produced prolapsus ani. Köhne removed from the rectum of a horse by ligature a polypus whose seat was 4 to 5 inches in front of the anus, through which it protruded during defæcation. In a second horse Köhne removed with the "écraseur" a polypus the size of a man's fist, which lay about 16 inches forward from the anus. Stockfleth states having seen cancer of the rectum in old cattle. Siedamgrotzky discovered in a gelding a cyst as large as a man's fist lying on the lower wall of the rectum 6 inches from the anus; it discharged a clear fluid on perforation with the trochar. After injecting tincture of iodine, healing occurred. A similar case is described by Hierholzer.

Truelsen removed from the rectum of a foal several polypi, which lay about two hands'-breadths from the anus, and had repeatedly caused constipation and colic. An improvised "écraseur" was constructed of a canula and wire, with which Truelsen removed the growth, and effected a cure.

Fröhner removed from a horse a serous cyst which had its seat on the upper wall of the rectum about 4 inches from the anus; he applied a silk ligature and snipped off the growth with scissors.

Carogeau successfully operated on a mass of varicose veins involving the rectum and lower surface of the tail in a horse. Teetz in the case of a horse laid open a cyst and obtained healing. 


\section{DISEASES OF THE URINARY ORGANS.}

\section{I.-CONGENITAL MALFORMATIONS.}

\section{(1.) FISSURING OF THE MALE MEATUS URINARIUS (HYPOSPADIA AND EPISPADIA).}

IN consequence of arrested fœtal development the meatus urinarius may fail to entirely close at some point in its course, and thus present the appearance of an open channel. When this occurs in the posterior, lower wall, the condition is termed hypospadia, when in the upper, anterior wall, epispadia. In animals, neither condition has the same significance as in men, as the patients, which are usually dogs or sheep, are either destroyed or left without treatment. Horses are seldom affected. The abnormal opening may lie just behind the glans, or in the course of the meatus nearer the bladder; where it occur's close below the anus, the animals are sometimes mistaken for hermaphrodites. Hypospadia is not infrequently associated with cloaca formation, as noted by Möller in the case of a dog which appeared to suffer continuously from sexual excitement. Guinard saw hypospadia in a three year old cryptorchid bull.

Treatment is seldom invoked unless the condition is accompanied by difficulty in urination. The natural opening of the meatus urinarius may not exist, and if the fissure is insufficient for the discharge of urine, it may require enlargement. T'o prevent reunion of the parts the meatus is divided from below upwards in the form of a "Y," and the edges attached to the skin.

\section{(2.) PERVIOUS URACHUS.}

Up to the time of birth urine is discharged through the urachus, but when that closes the urine passes through the meatus urinarius. In a few cases the urachus remains open even after birth, and urine is discharged through it. Burmeister saw this in a three weeks old foal; when staling, some urine always flowed from the navel. In a colt described by Herbet urine was only passed by the urethra in drops, but flowed in a stream from the opening in the navel, which was about $\frac{1}{8}$ inch across. Kauffmann and Blanc found the following conditions 
existing in a thirty-seven days old calf-atresia ani ; hernia, as large as a child's head, in the linea alba, between the navel and os pubis; a fissure 2 inches long, in the umbilical region, through which both the open meatus urinarius and the urachus opened; fæces were discharged by the latter; a canal, the size of a goose-quill, existing between the occluded rectum and the bladder, whence the material made its way into the urachus. The latter also received the ends of the ureters and spermatic ducts. Lancelot saw in calves and goats swelling of the navel, which to the touch gave the impression of an umbilical hernia. Several animals died because the urine found no exit through the urethra, and as the umbilical cord was also closed, the urine accumulated in its dilated end below the skin of the umbilicus. Greve found pervious urachus in a filly.

The primary cause of disease is the obstacle to discharge of urine through the urethra, and attention should, therefore, first be directed to this point. When obstruction can be detected, blistering the parts will sometimes close the urachus; Burmeister succeeded in this way. Should it prove impossible to lay open the urethra, the urachus may be further divided and the urine thus allowed to escape, a method which does well enough in animals intended for early slaughter. Herbet effected healing in five days by powdering the parts with burnt alum; March, in the case of a calf, by applying a ligature.

\section{II.-URINARY CALCULI.}

Urinary calculi have only a surgical interest when occurring in the urethra or urinary bladder. They result from materials deposited through the urine accumulating around hard substances, foreign bodies, clots of blood, masses of mucus, \&c., which have accidentally found their way into the urinary passages; catarrh or inflammation of the urinary passages, therefore, often give the first impulse to their formation. Calculi are also said to be due to an abnormally high percentage of salts in the urine, produced by food and water rich in lime and to a specific tendency (lithiasis). Of more importance is the fact that retention and decomposition of the urine are liable to cause an alkaline reaction and calculous deposit. Such conditions are, therefore, to be provided against or removed as soon as possible, and care taken to disinfect catheters, \&c., before use, and so prevent micro-organisms being carried into the bladder. Sometimes deposits occur in the pelvis of the kidney, pass into the bladder, there become enlarged, or being discharged with the urine remain fixed in the urethra. This explains why the symptoms of urethral calculus generally appear suddenly and are apt to recur, and why the stone is almost always found at the narrowest point 
of the tube. Urinary calculi are seldom single, generally they occur in large number's, or as so-called gravel.

In herbivora, urinary calculi most frequently contain triple phosphate and carbonate of calcium; in sheep, some have been found to consist of silicic acid, with phosphates of calcium and magnesium. Urinary calculi in herbivora are sometimes coloured red by iron salts; their surface is generally smooth, though those covered with oxalate of calcium are rough and uneven. In carnivora one distinguishes:

(a) Uric acid calculi, which consist of phosphate and carbonate of calcium and of urate of ammonium. These often attain considerable size, and appear smooth and white on the surface. V. Rátz believes they result from acid formation in the urine leading to deposit of calcium salts or to a basic condition of the urine.

(b) Oxalic acid calculi consisting of oxalates of ammonia are usually coloured yellow ; their surface is rough, resembling a mulberry ; Rátz considers that oxalates are excreted when the food contains quantities of oxalic salts, but they may also be found in the body by the decomposition of urinary acids. The excretion of oxalic acid in consequence of diseases of the organs of respiration and digestion, which has often been suggested, is denied by Rátz.

(c) Cystin calculi are principally formed of cystin, are yellow, soft, and when dried are friable, and feel greasy to the touch. Concretions, consisting of epithelium mixed with lime salts and fatty crystals, are sometimes found in the urinary passages. As urinary calculi are of varying importance in the different classes of animals, and from a surgical point of view require particular treatment, we shall here view them under separate heads.

\section{(1.) URINARY CALCULI IN THE HORSE.}

Urinary calculi rarely occur in the ureters or bladder of the horse, still less frequently in the comparatively wide urethra. According to Bang the circumference of the latter in male animals is as follows:- In the pelvic portion 1 to $1 \frac{1}{2}$ inches, in the abdominal portion $\frac{7}{4}$ to $\frac{5}{8}$ inch, behind the external opening $\frac{1}{2}$ inch. In mares the urethra is very wide, and calculi never remain fixed in it, and therefore in them vesical calculi alone claim consideration.

Urethral calculi may be recognised in the stallion and gelding by difficulty in urination. The animals place themselves in position to urinate, but can only discharge fluid in drops or in a small stream. Sometimes the upper sections of the urethra are abnormally distended, or may be felt to contain a stone, and the catheter, when introduced, strikes on a hard substance. Examination per anum discovers the urinary bladder to be greatly over-filled, but on pressure, urine either fails to be discharged or issues in drops.

Vesical calculi take longer to produce obstruction in staling; the urine is passed more frequently, but in small quantities, often in drops. When the animal has been driven fast, the fluid may be blood-stained on account of the stone injuring the mucous membrane. Hertwig saw sexual appetite excited in a mare by vesical calculus. Exploration per 
rectum generally reveals the stone as a firm, hard substance; in mares it can be directly felt by passing the finger through the urethra. Gravel is rarer in the horse, though cases are on record where large quantities have been removed by operation.

Treatment. Internal medication is useless for dissolving the stone, and surgical removal alone can cure. Though it is certainly easier to remove a stone from the urethra than from the bladder, neither operation is easy nor unattended with danger, especially in carnivora; herbivora bear operation much better.

Cutting for stone was first practised in man. In veterinary surgery it is said to have been introduced by Lafosse, who made the first experiment on a horse. Ercolani contends that even in the 14th century similar attempts had

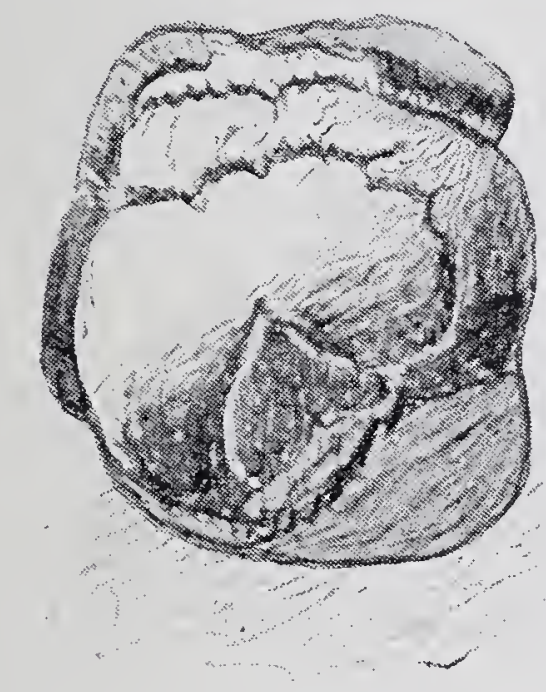

Fig. 159. - Calculus passed spontaneously. Natural size. (Mr. McFarlane's case.)

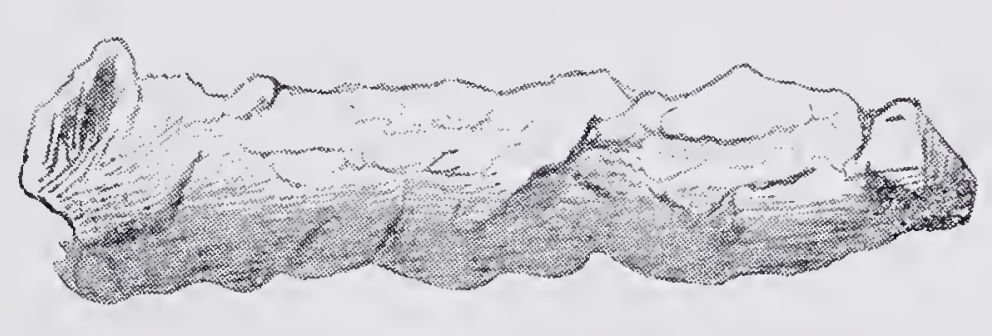

FIG. 160.-Urethral obstruction removed by $\mathrm{Mr}$. Mellis. Probably consisted of cystin.

been made; but Bouley was probably the first to successfully employ the operation in the horse as a means of treatment.

Several methods formerly employed are now obsolete, such as cystotomia rectalis, in which the urinary bladder was incised through the lower wall of the rectum, and the stone removed through the rectum. Severe cystitis often resulted, and caused this method to be given up both in men and animals.

The bladder may also be reached through the lower portion of the abdominal wall, close in front of the os pubis, a method which in man has been called cystotomia suprapubica, or sectio alta, and is still used at the present day, but as in animals this necessitates opening the peritoneal cavity there is considerable danger of peritonitis, while the horizontal position of the body favours prolapse of bowel, and it is, of course, impossible to keep the animal continuously lying on the back. It is, therefore, necessary to resort to a third method, namely, section through the perineum (cystotomia perinealis). Or as an alternative the urethra may be incised (urethrotomy) at one of various points in its course.

Urethral calculi in the horse are only exceptionally found close behind the glans. When in this position, however, they may be removed without much difficulty or bleeding, by the operator standing on the left side of the animal, drawing the penis forward by means of a cloth, or allowing an assistant to hold it, and then grasping the calculus with dressing or 
ordinary forceps passed into the urethra. Sometimes strong pressure on the penis with the hand is sufficient, or the urethral orifice may be enlarged, and the calculus thus removed. Urine then generally flows away in large quantities, if not, a catheter must be passed, to discover whether other calculi remain in the urethra.

Landvatter removed from a gelding, by means of forceps, a calculus about $1 \frac{1}{2}$ inches in length and $\frac{3}{4}$ inch thick, which lay in the terminal portion of the urethra. Two years later he took from the same animal a still larger one, which was fixed in the urethra 4 inches from the anus. Reicherter grasped a calculus, lying $1 \frac{1}{2}$ inches in front of the opening of the urethra, with a pair of dressing forceps and broke it down; fragments were afterwards passed with the urine. In a case seen by Rother, the calculus, which lay about $2 \frac{1}{4}$ inches behind the glans, had caused ulceration and perforation of the urethra, in consequence of which urine had extensively infiltrated into the neighbouring tissues. The animal died from rupture of the bladder. Müller cured a horse apparently suffering from colic by removing two chalky concretions from the urethra; one from just behind the urethral orifice, the other about 4 inches further back. Concretions of inspissated smegma around the glans may also cause dysuria in the horse.

Two cases of urinary calculi passed per urethram are related in The Veterinarian for 1896, pp. 29 and 30. A. M. McFarlane saw a horse apparently suffering from colic pass a stone weighing 20 grammes (about 5 drachms), and of the size of the illustration (see fig. 159). Thos. Mellis saw a three months old foal, which was violently straining to micturate and was evidently in great pain. On examination the body illustrated (fig. 160) was found protruding from the meatus urinarius, and was without much difficulty removed. The figure is natural size, but Mr. Mellis states that nearly 1 inch was broken off in removal, and the total size was therefore much greater. This was probably a cystin calculus.

As a rule, in the horse, the calculus lies at the height of the ischial arch, where the urethra turns downwards and forwards. As casting an animal with distended bladder is open to danger, it is usual to operate in the standing position, the patient being controlled by twitches and its hind legs extended. To prevent accidents it is best to place the animal in slings or stocks, so that it cannot lie down. Should a really dangerous horse require to be cast, every precaution must be taken, and a very thick straw bed provided. It is best to operate with the animal on the left side or back; the latter is perhaps preferable.

After plaiting the tail and cleansing the skin, a catheter is passed, and an incision $1 \frac{1}{2}$ to $2 \frac{1}{2}$ inches long made through the skin immediately over the urethra, that is, in the middle line of the body and over the point where the calculus can be felt. This is successively carried through the retractor penis muscle, the accelerator urinæ, the corpus spongiosum of the penis, and, finally, the urethra itself. The incision should gradually become smaller as it penetrates deeper, so as to favour the free exit of wound discharges and of urine. The incision into the wall of the urethra must be as small as will allow exit of the calculus, which 
may sometimes be removed by simply pressing upon it with the finger ; if not, by employing forceps, a catheter, or the end of a blunt pair of scissors. Immediately the urethra is clear, urine flows away in large quantities; should not this occur the parts must be examined with a probe to discover if a second calculus be not present at a higher point.

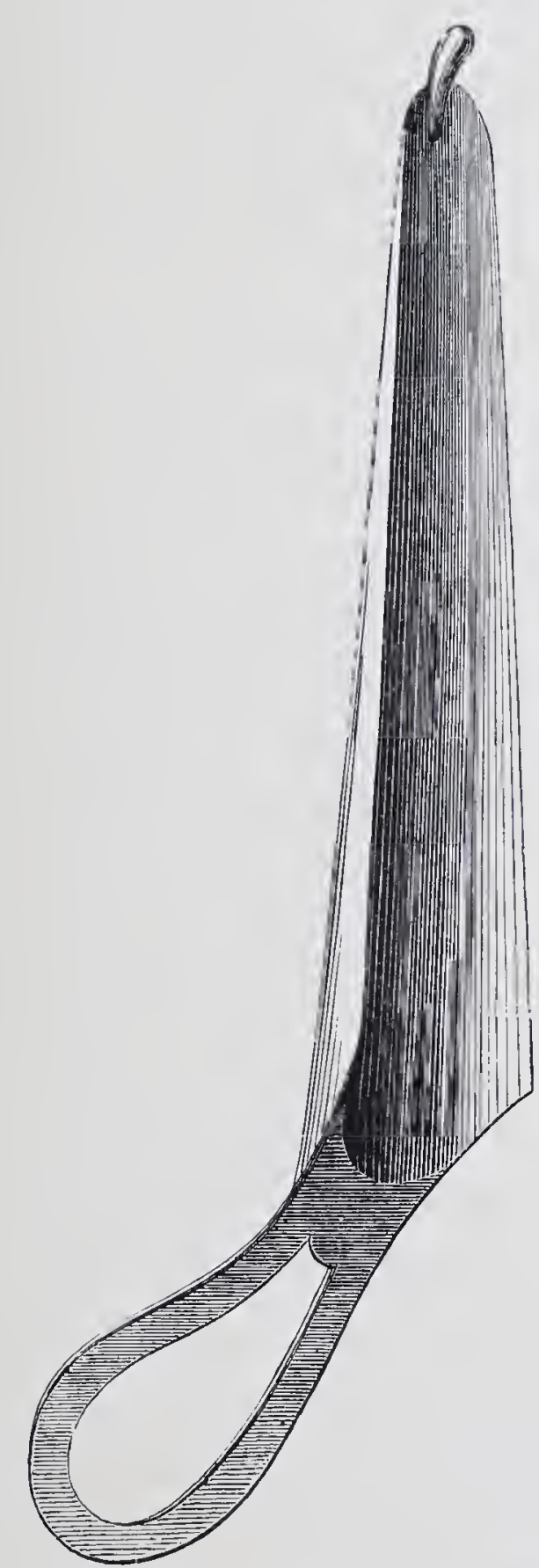

FIG. 161.--Vaginal speculum.

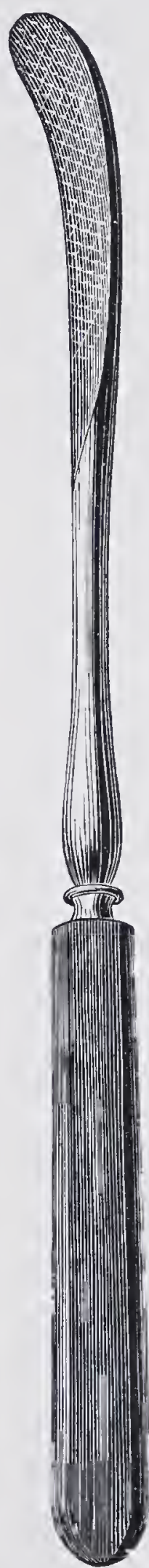

Fig. 162.-Calculus spoon.

The urethral wound may be sutured with catgut or silk, though this is not absolutely necessary, for healing also occurs without it.

Operating in this way Russell removed from a cart horse an urethral calculus $\frac{7}{8}$ inch long, $\frac{3}{4}$ inch broad, and $\frac{5}{8}$ inch thick. The surface was very rough. Three harelip sutures were inserted. Healing was rapid, the horse being sent home eight days after operation, and hardly any 
scar being visible six weeks later. There was no sign of stricture. (Cadiot and Dollar, "Clinical Veterinary Medicine and Surgery," p. 416.)

Schirrmann removed a large bean-shaped calculus from the urethra of a gelding. It lay in the neighbourhood of the scrotum. The operation necessitated casting. Fröhner performed urethrotomy in a gelding and removed two calculi as large as a hen's egg.

After-treatment is very simple; the wound is either left completely to itself, after being powdered with iodoform, or is cleansed once or twice daily. Union occurs more quickly after suturing, but even without it is complete in two to three weeks.

Bad results seldom follow this operation, though healing by first intention is very rare. Provided the incision be properly made, infiltration of urine need not be feared, nor is severe inflammation seen, unless the parts were injured before operation. Altogether progress is favourable, and if the operation be performed exactly in the middle line there is little bleeding.

In quiet animals cystotomy may also be performed in the standing: position, the procedure being as above described. Many persons recommend operating with the animal on its left side, but the general consensus of opinion is in favour of the dorsal position; chloroform is necessary. The urethra is divided over the ischial arch, as in urethrotomy, but as the urethra is not easy to find unless filled with fluid, an elastic catheter should first be passed into the bladder. After incision the catheter is withdrawn, and a grooved director inserted as far as the neck of the bladder. The probe must be introduced immediately the catheter. is withdrawn, as otherwise the urethra will prove difficult to find.

If only a small calculus or gravel has to be removed, an attempt should first be made to pass the forceps into the bladder and reach the calculus without a second incision. By gradually opening the forceps, whilst still in the neck of the bladder, the latter may without danger be so dilated as to allow removal of calculi of considerable size; if not, a tenotome or bistoury is passed along the director into the bladder, and the constrictor urethræ muscle, just behind the neck of the bladder, incised during withdrawal. A special instrument has been made for this purpose, but is not absolutely necessary. In France a lithotome, which corresponds in construction to the herniotome (fig. 129), is employed. To prevent injury, the rectum is emptied before operation, and the incision made, not exactly in the middle line, but a little to the left or right. In dealing with large calculi, the constrictor urethræ may be incised in two directions.

The forceps (fig. 163) are now passed, and attempts made to grasp the stone; the operation wound being meanwhile closed to prevent the bladder entirely emptying; otherwise the wall of the bladder is apt to 
become so closely applied to the calculus that the greatest difficulty is experienced in seizing the latter. As soon as a hard body is felt the forceps are opened, and an effort made to seize the stone. A hand in the rectum may prove of considerable assistance in grasping the stone. Once grasped, the forceps are rotated around their long axis to make sure that the mucous membrane has not been included; and if no great resistance is felt the stone is drawn forward and removed, the rush of urine which takes place assisting the process. The instrument should be reinserted or the bladder examined from the rectum, to make certain that no other calculus or gravel is present, which would require removal or washing out, otherwise one proceeds as in urethrotomy. Suturing the wound is not absolutely necessary, though it somewhat hastens recovery; but if unskilfully performed, so that the urethra is left open while the skin is closed, infiltration of urine occurs and leads to grave consequences. Healing occurs in the same time as in urethrotomy. For a short time urine escapes in part by the operation wound; but this soon closes, and only occasionally does urethral fistula result.

After-treatment consists in washing out the bladder once daily with a lukewarm saturated solution of boric acid or a 2 per cent. solution of acetate of alumina, by means of a rubber tube. This prevents decomposition of urine. If treatment must be left to laymen, a tube may be fixed in the urethra, otherwise the practice should be condemned as favouring infection of the bladder. The tube must be cleansed or placed for' a short time before insertion in the lotion to be injected.

In removing large stones, the neck of the bladder must sometimes be enlarged to such an extent as to admit the entire hand. After incision of the con-

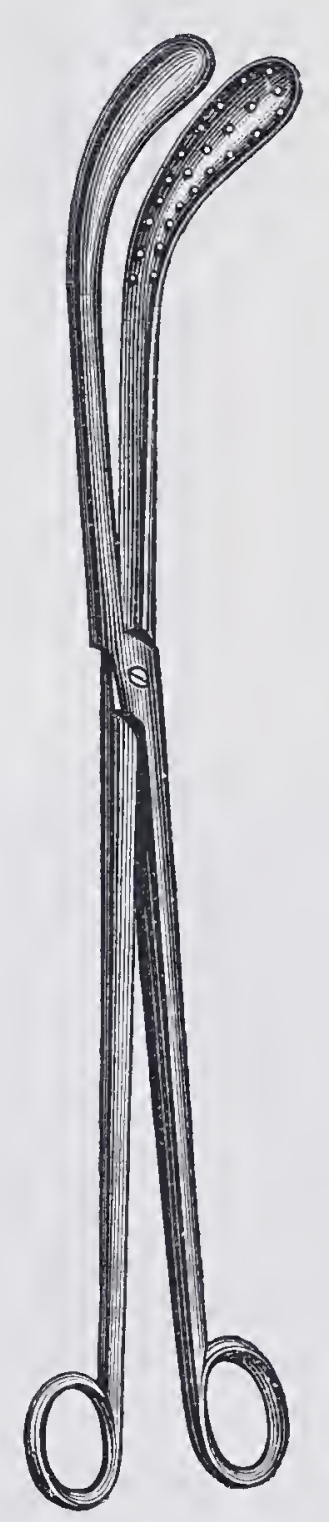

Firæ. 163.-Calculus forceps. strictor urethræ Möller once removed a cystic calculus weighing over 20 ounces. The bladder must not be completely emptied, however, as its walls cling to the stone and render extraction difficult. If such an accident has happened, lukewarm fluids, like 5 per cent. carbolic lotion, may be injected, but are not always retained, particularly where the opening resulting from division of the constrictor urethræ is of great size.

The danger of operation increases with the size of the calculus, though stones of considerable magnitude may be safely removed, as shown by Möller extracting two calculi from the bladder of a nine year old gelding, one weighing $2 \frac{1}{2}$ ounces and the other $20 \frac{1}{4}$ ounces. The operation wound took three weelis to heal. In a second horse from which he took a stone 
weighing 20 ounces gangrenous cystitis with septic paraproctitis and peritonitis resulted, from which the animal died on the fifth day after operation.

Large calculi may be crushed, though the instruments intended for this purpose are not constructed for calculi of the above size. Calculi indeed often yield to powerful pressure from the forceps and fall to pieces; Bouley's forceps (fig. 164) may be employed as a lithotrite. They have the advantage that they can be fixed after grasping the stone,

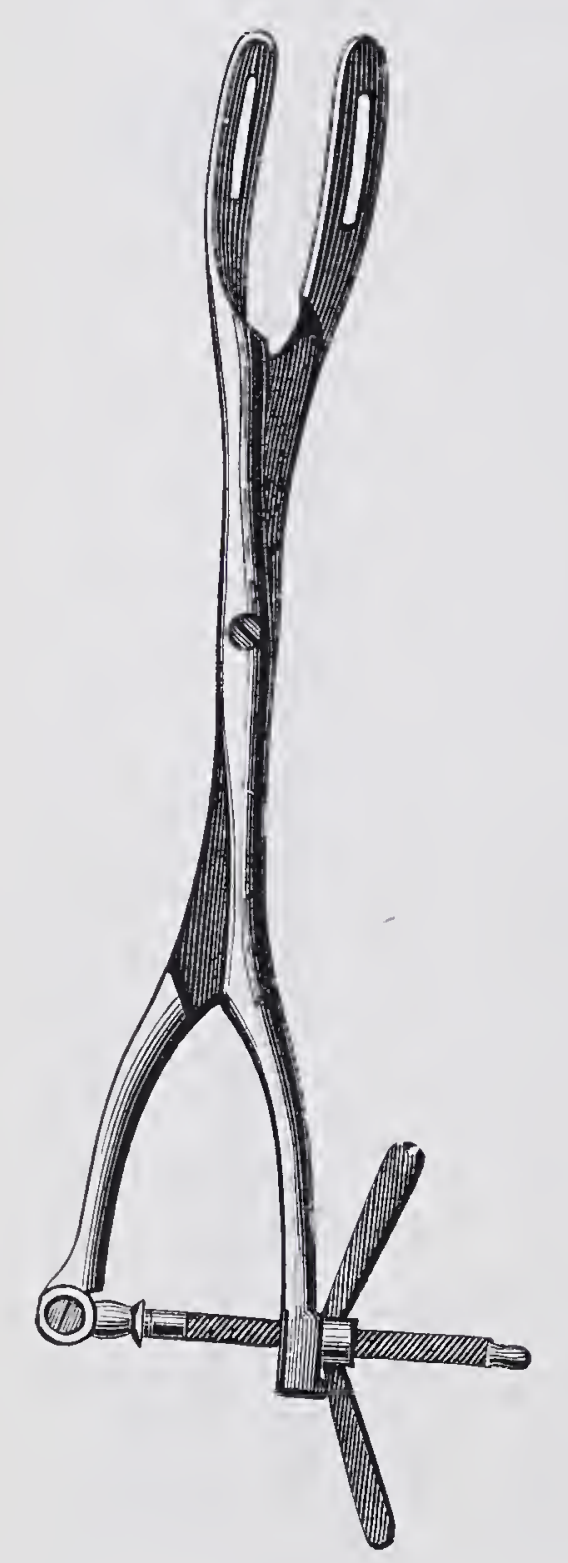

FIG. 164.-Bouley's calculus forceps. thus rendering easier the extraction of large concretions. Bouley's instrument is useful, though in most cases the older calculus forceps are sufficient (fig. 163), and a pair of farrier's tongs are even said to have been used.

Urethral calculi are rare in mares, whilst vesical calculi may generally be removed without incision; the urethra can be sufficiently dilated to admit a small hand and calculi the size of hens' eggs have thus been removed. Forceps are used, and the urethra slowly enlarged. Until the stone can pass through it, considerable exertion of power is necessary, and complete dilatation often occupies half an hour or more; an assistant may be required. Möller removed by this method from the bladder of a mare a stone the size of a hen's egg and weighing nearly $2 \frac{1}{4}$ ozs. Hertwig divided the urethra laterally, Kutzner the upper wall; Fehsenmeir, after dividing the urethra for the space of 2 inches in an upward direction, withdrew by hand a stone weighing 7 ozs. Krämer removed one of $15 \frac{1}{2}$ ozs.; the urethra was dilated throughout, so that Krämer was able to remove the stone by hand. Recovery occurred in twenty-eight days.

The stone illustrated, weighing $3 \frac{3}{8}$ ozs., and measuring $7 \frac{1}{4}$ inches in circumference, was removed by A. Chinniah, of Ceylon. It was not spherical, but convex on both surfaces, and was apparently composed, outwardly of phosphate, and inwardly of oxalate of lime. The final results of operation were not reported, though the animal was said to be doing well.

V.-Lieutenant Walker removed from a gelding an oxalate of lime calculus weighing $7 \frac{1}{4}$ ozs., with a nucleus, consisting of blood clot (see figs. 166 and 167). Instruments failed to grasp or crush the stone, so the constrictor urethræ was incised on both sides, the hand introduced into the bladder and the stone, which was of oval section and measured 
3 inches in diameter, was removed. The urethral wound was twice sutured, but the stitches tore out on each occasion; nevertheless the parts healed so far that the horse returned to duty in two months, and

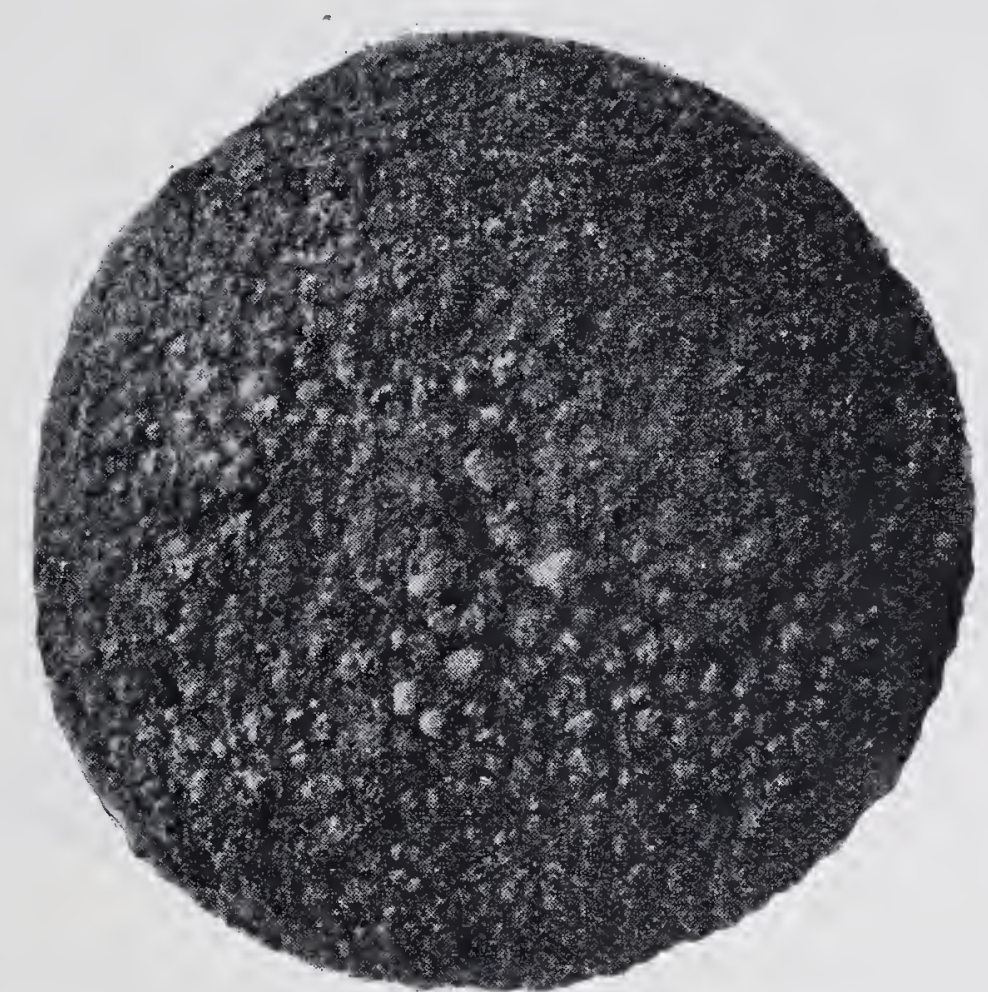

Fig. 165. - Vesical calculus composed of phosphate and oxalate of lime. Weight, $3 \frac{3}{8}$ ounces ; circumference, $7 \frac{1}{4}$ inches. (Chinniah's case.)

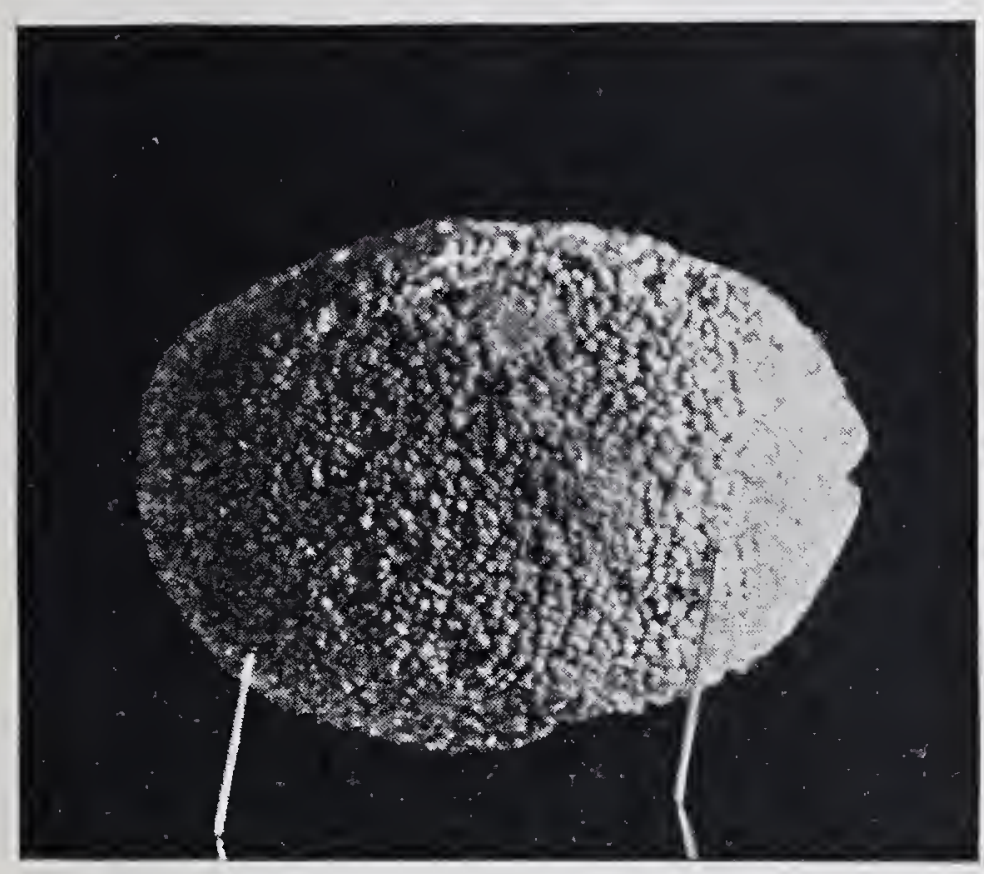

Fig. 166.-Cystic calculus removed by V.-Lieut. Walker.

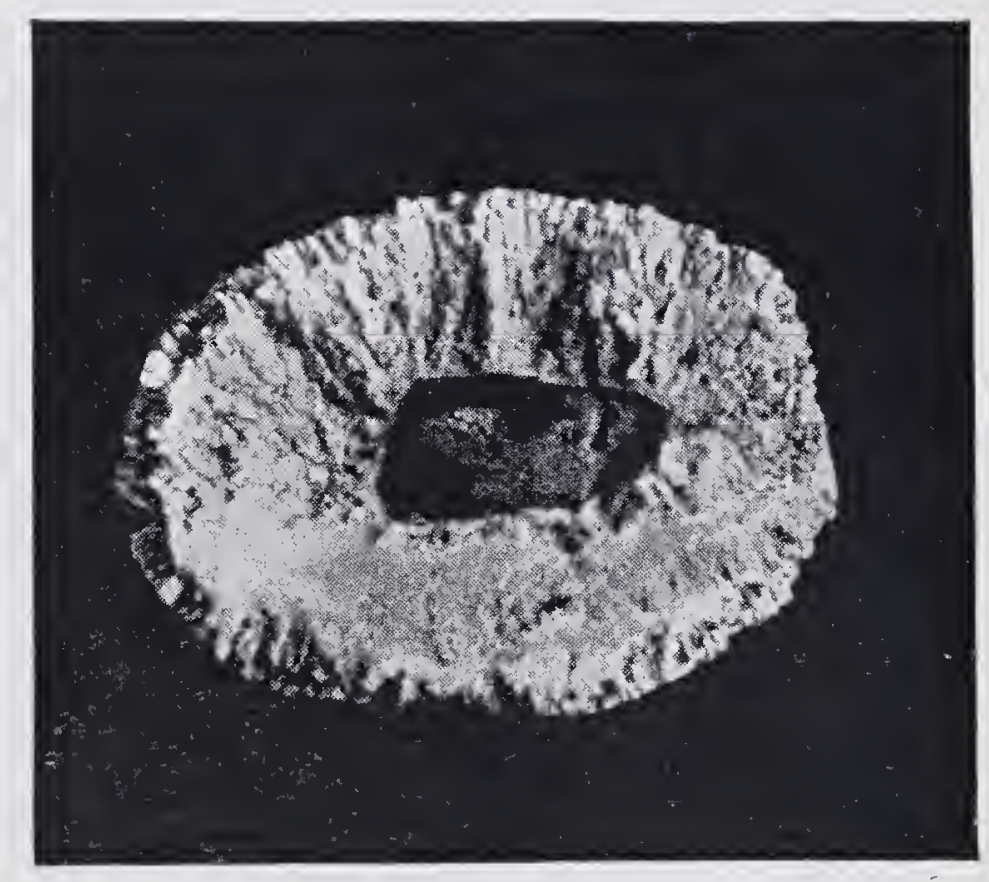

Fig. 167.- Section of above calculus.

three months later urine ceased altogether to be passed by the fistula. (The Veterinarian, p. 299, May, 1898.)

\section{(2.) URETHRAL CALCULI IN RUMINANTS.}

Of all animals the bull suffers most from calculi. Formed in the bladder, they enter the urethra during urination, and partly on account of its comparatively narrow lumen, partly of its peculiar course, remain fast (fig. 168). 
The pelvic portion resembles that in other animals and has a width of $\frac{3}{16}$ to $\frac{6}{16}$ of an inch, but in the abdominal portion the passage contracts to $\frac{1}{8}$ inch, and at its orifice even to $\frac{1}{12}$. In the neighbourhood of the scrotum it makes with the penis the so-called " $\mathrm{S}$ "-formed turn. At the first bend near the front of the scrotum $(h)$, the penis turns once more backwards, making about 3 inches behind and over the posterior surface of the scrotum a second bend $(i)$; at the height of the latter the retractor penis $(l)$, which is very strong in cattle, becomes attached. The sinuous course and slight diameter of the urethra explain why even small calculi or concretions, weighing only a few grains and not exceeding the size of a pea, may remain fixed in the urethra and obstruct it. The stone is usually lodged in the first bend $(h)$, less frequently in the second or near the end of the organ.

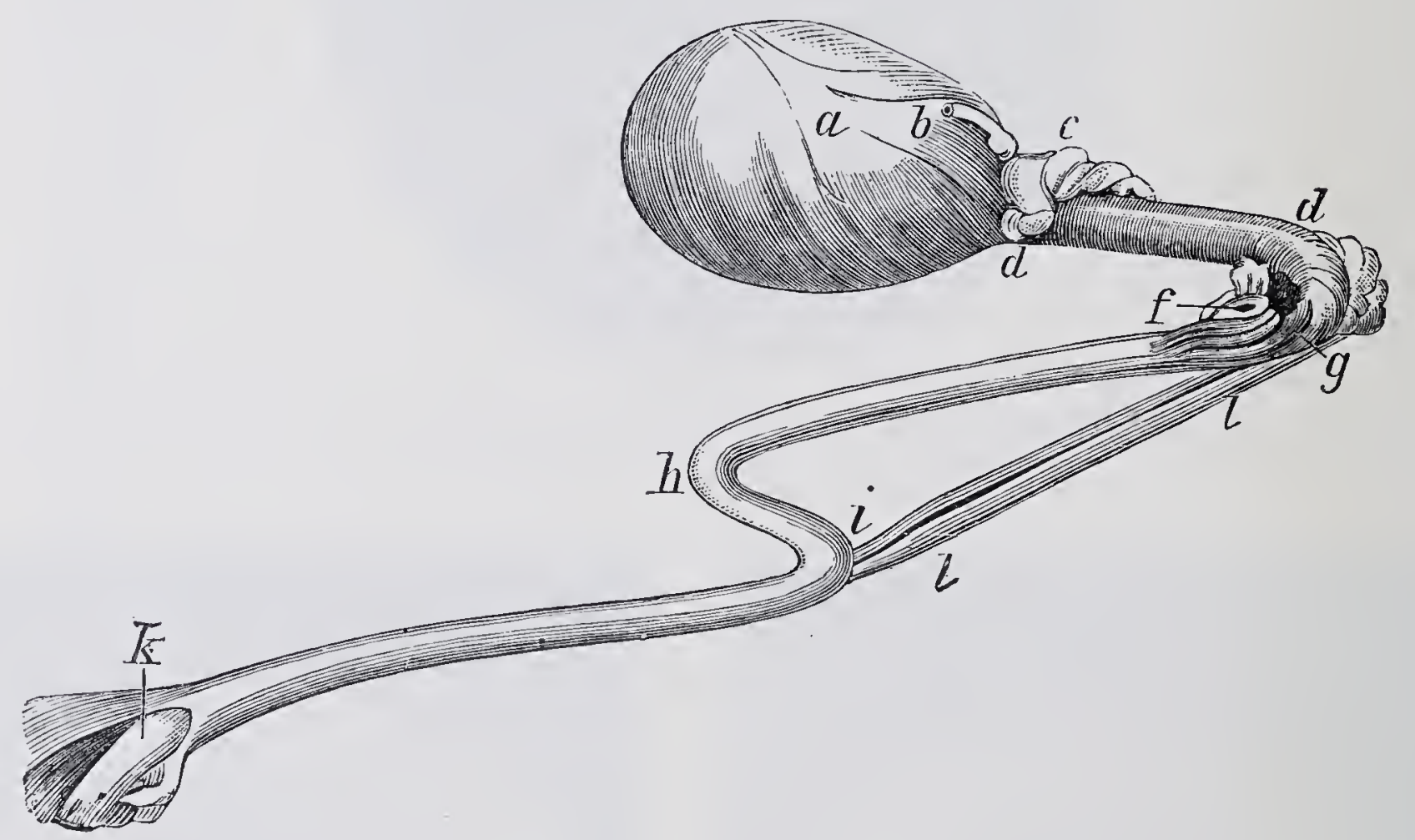

FIG. 168.-Course of the urethra in the ox. (After Hering.)

$a$, Urinary bladder ; $b$, ureter, cut off ; $c$, vesicule seminalis ; $d, d$, pelvic portion of urethra $f$, commencement of the corpus cavernosum of the penis ; $g$, ischial curve of the urethra; $h$, first bend; $i$, second bend; $k$, anterior extremity of the penis ; $l$, retractor penis muscle.

Diagnosis. Urethral calculus first attracts notice by the difficulty which exists in passing urine. The animal is fretful, stamps with the hind feet and moves to and fro, lifts the tail and makes frequent short side movements with the root of it; it lies down, but immediately rises again, and strikes with the hind feet towards the body.

In oxen slight symptoms of colic should always arouse suspicion of urinary calculus and lead to examination of the bladder and urethra. The bladder and sometimes the upper portions of the urethra are found excessively distended, and may show pulsative movements as the animal strains whilst a few drops of urine are sometimes passed. Passing the finger along the course of the urethra causes the animal to show pain and uneasiness at the spot where the stone lies, though the latter can seldom be directly felt. Rotention of urine for twelve to twenty-four hours may result in rupture of the bladder and death after a few days. After rupture the pain diminishes, the animals may even begin to feed 
again, but tympanites soon sets in, and death takes place with symptoms of peritonitis and uræmia (frequent pulse, rigors and giddiness). Cases are reported where oxen have still lived for some weeks after rupture of the bladder. Stöhr mentions an ox which lived six weeks, and Jacobi states having seen recovery after rupture, but as a rule animals in this condition are at once slaughtered, to avoid injury to the flesh, which takes on an unpleasant smell from resorption of urine from the abdominal cavity. Franck therefore recommends removing the urine by a trochar passed at the deepest point of the abdomen.

In oxen it is important to operate early, and, on account of the peculiar course of the urethra, one method alone, viz., urethrotomy, is available. With few exceptions the calculus is found at the first curve, and can be reached most directly from the anterior surface of the scrotum. As, however, it is less convenient to operate here, most practitioners make the incision behind, and about a hand's-breadth above the scrotum. For this operation the animal should be cautiously cast and laid on the left side, though quiet oxen may be operated on standing. The right hind foot is drawn forward with a cord, exposing the field of operation.

After making sure by rectal examination that the bladder is not ruptured, and having once more carefully determined the position of the stone by palpation, and found that it is not contained in the superficial portion of the urethra, the slin is incised for 2 to 4 inches in the direction of the urethra, either at the point where the greater sensitiveness or hardness of the swelling indicates the calculus to be, or a hand's-breath behind and above the scrotum. The retractor penis muscle, which is much more developed in the ox than in the horse, is then divided to the same extent. The urethra can then be felt, and if one operates at the point where the calculus is fixed the latter may be detected within it. After incising the corpus cavernosum, and the urethra itself, the stone is easily removed.

Where, however, the calculus is situated in the first bend of the urethra $(h)$ the retractor penis is divided, and an attempt made to draw forward the penis with the finger; the bulbo-cavernosus muscle and urethra cut through, and the stone removed by pressure with the fingers, or if necessary, by means of forceps. The stone when rough sometimes becomes so firmly fixed in the swollen mucous membrane that even the knife must be employed to free it. The cut in the urethra should be as small as possible, to facilitate healing and prevent stricture. Should the stone be the sole obstruction to passage of urine, an immediate discharge occurs on its removal, partly through the operation wound and partly from the orifice of the urethra, but in any case it is necessary to examine the lower portion of the urethra with a catheter, to discover whether other stones are present and require 
removal. In oxen concretions often occur in hundreds, resembling tapioca grains. The urethral wound need not be sutured, though Reichle and Pflug recommend inserting a few silk sutures to assist healing; Ciani suggests dividing the urethra from the side, and closing it with close-set sutures, to prevent infiltration of urine. The penis is then allowed to return to its natural position. To prevent infiltration the outer wound is not sutured, but it often occurs in spite of this precaution. Esser, therefore, provides for escape of urine and wound discharges by passing a drainage-tube forwards, and allowing its anterior end to project in front of the scrotum. He says this prevents infiltration of urine, which not only checks healing, but constitutes a grave danger to the animal's general health. The operation wound heals in about fourteen days.

When the calculus cannot be discovered, Ciani recommends completely dividing the urethra above the obstruction, producing fistula, to allow passage of urine, and fattening the animal.

Urethrotomy in front of the scrotum.

As already remarked, the calculus almost always lies in the first bend of the urethra, just in front of the scrotum, and, therefore, next to the skin. This position is inconvenient for operation, and is not often selected in Germany; it seems to be more in use in France. According to Peuch and Toussaint, the animal is laid on its left side, the right hind foot drawn towards the shoulder, the operator's left hand passed into the sheath, and the penis drawn forward so as to extend the "S". shaped bend. Should the stone now be felt, a longitudinal incision is made in the urethra and the calculus removed with forceps or a hollow probe. If not, the hair is cut away in front of the scrotum, a cross fold of skin pinched up, and together with the prepuce is divided. The index finger of the left hand is passed into the opening, the penis grasped with the bent finger and drawn forward through the wound. The rest of the operation is as above. Dupont divides the urethra immediately after cutting through the skin. If, after removing the stone, no considerable quantity of urine is discharged, the urethra must be explored, and, if necessary, the operation repeated again at a higher point. Bouley thinks infiltration of urine need scarcely be feared, and if it should appear, can be cured by making deep incisions; abscesses, when occurring, are similarly treated.

Urethrotomy in the ischial region.

On account of the considerable diameter of the urethra, calculi seldom become fixed at this point, and operation is usually performed at the ischial arch only to give relief more rapidly and remove the imminent danger of rupture of the bladder. Operation here is also easier for the unpractised. After providing an exit for the urine, the concretions, which lie at a lower point, are allowed to remain. The operation is, therefore, 
most useful in animals nearly ready for slaughter, though it may be replaced by puncture of the bladder (see section her'eafter, "Puncture of the Bladder", ).

This method may also be resorted to if the stone cannot be found, or if found, cannot be removed, or if such changes have occurred in the urethra as are likely to prevent passage of urine.

The general procedure and after-treatment are similar to those in the horse. If the animal is not destined for early slaughter a tube should be fastened in the urethra to keep the wound open, but this measure is only of temporary benefit on account of inflammatory swelling setting in and preventing discharge of urine. Dupont therefore recommends, in the event of a calculus being found at this point, to thrust it back into the bladder, thus obviating the necessity for such a large urethral wound.

As relapses are not uncommon it is usually advisable to fatten the animal for slaughter as soon as possible.

Bader, who performed one hundred lithotomies in the ox, almost always found the calculus in the first (lower) curve of the urrethra. Most of the animals were in the first or second year of life, a few in the third, and only one in the fourth. In 24 out of 25 cases, Deisinger found the stone in the lower curve of the urethra. Diani states having seen 300 cases, and having found the stone in the upper curve, or between it and the lower one, in 98 per cent. of these. In 2 it lay in the pelvic portion, and in 5 or 6 near the glans.

\section{Urethrotomy in the sheep.}

The ram's narrow urethra lies embedded for the most part in fatty tissues, and is therefore still more difficult to find than that of the ox. Calculi and deposits are not common in sheep, and when occurring are usually found close behind the opening of the urethra, which extends beyond the penis, is free, and as a rule curved into a shape somewhat resembling that of an "S." Dammann has described several cases of the kind.

The symptoms are similar to those in cattle. Retention of urine renders the animals restless, they stand with the back arched straining to pass urine, and examination per anum shows the bladder to be greatly distended. By closing the nostrils with the hand a healthy sheep can be caused to pass urine, but here the attempt is unsuccessful, or only a few drops are passed.

According to Peuch, it is usual in France, as in Germany, to remove the free end of the urethra in cases where calculi or concretions cause retention of urine. The same is recommended in England; Read saw a case where 11 to 12 pints of urine were afterwards passed.

If the obstruction lie further back, Peuch recommends, in valuable animals, passing a catheter or sound. The sheep is placed on its back and the penis drawn forward out of the prepuce so as to obliterate the v.s. 
"S"-formed curve. A flexible metallic sound is then passed into the urethra, which has previously been laid open transversely close behind its free end. It is said to be thus possible to displace the sediment and effect an exit for the urine.

Others recommend laying open the urethra at the ischial arch, a method which also admits of the removal of vesical concretions. Luthens opened the urethra of a ram at the " $\mathrm{S}$ " bend as is done in the ox ; the animal, however, died from further formation of calculi.

\section{Complications of urethrotomy in herbixora-}

(1) Unless the bladder is ruptured before or during operation, an abundant discharge of urine follows removal of the calculus. Sometimes, however, the detrusor urinæ having been greatly over-extended and failing to contract, the bladder appears paralysed. In such cases, in oxen, the hand should be introduced into the rectum and attempts made to empty the bladder by exercising slow and steady pressure upon it. Meisel recommends moving the animals, but altogether considers the condition very grave.

(2) A second unfavourable complication consists in the formation of stricture of the urethra at the point of operation, though this only occurs if the animal lives for a considerable time afterwards, which is seldom the case in oxen. Relapse being probable, the animal should be immediately prepared for slaughter. Such strictures are common in the horse, and necessitate the re-performance of urethrotomy at a spot lying nearer the bladder. Räber suggests keeping a metal tube in the urethra, but this is of no permanent service, the irritation produced causing fresh stenosis.

(3) Abscesses which form at the point of operation are laid open and treated on general principles.

Cystic calculi are rare in ruminants, and are generally found quite accidentally after slaughter, not having caused any disturbance during life. They seldom produce noticeable symptoms, as the average life of oxen is too short to allow of their attaining any considerable proportions. But all the necessary conditions for their production exist, as is shown by the frequent occurrence of urinary calculi. Hermann found in the bladder and urethra of a three months calf calculi of a collective weight of 5 drachms.

The symptoms in the ox are similar to those in the horse. In operating, the same general principles are observed in both animals.

\section{(3.) URINARY CALCULI IN SWINE.}

In swine this disease is of less interest. Serious cases are best slaughtered. As in oxen, calculi and concretions are sometimes found 
in the bladder after death; Schell met with a collection of white earthy concretions weighing 13 drachms in the urinary bladder of an eighteen months pig that had always appeared healthy.

\section{(4.) URINARY CALCULI IN THE DOG.}

The urethral calculi not infrequent in old dogs are usually formed in the bladder. They almost always become fixed in the gutter of the os penis or close behind it. 'Straining to pass urine, passage of a few drops, uneasiness, distension of the bladder, \&c., sufficiently indicate the nature of the condition.

A metal catheter, which is easily introduced into the urethra, will at once reveal the presence of the calculus and its position. In this examination the dog is laid on its back, with its left side towards the operator. The prepuce, which should be grasped about an inch behind its termination, is then thrust back with the fore-finger and thumb behind the corona glandis, the latter firmly pressed upon, and the penis thus caused to protrude. The catheter is then introduced into the opening of the urethra, which is easily seen, and passed onwards until checked by contact with the calculus, readily recognised by its hardness. By now removing the left hand from the prepuce, the end of the catheter and the stone may at once be felt below the skin.

In spite of this the operation offer's greater difficulties than in other animals, is often followed by severe strictures, and not infrequently proves fatal. It has therefore been recommended, when possible, to thr'ust the stone back into the bladder, which may sometimes be effected with the help of an elastic catheter; but if, in consequence of the rough nature of the stone and the already existing swelling of the urethral mucous membrane, this prove impossible, early operation alone offers a chance of saving life, for rupture of the bladder in dogs leads to death in twenty-four to forty-eight hours, and is the more to be feared the more completely the passage of urine appears to be interfered with.

Urethrotomy is carried out in dogs in the above described dorsal position. A catheter is passed into the urethra and held by an assistant; the skin is then incised for a length of $\frac{3}{4}$ to $1 \frac{1}{2}$ inches immediately over the stone, and a second incision made exposing the stone. The difficulties only begin at this point. As the gutter in the os penis is narrower below, and forms a lind of tube split along its inferior margin, it is often impossible to remove the stone, even when completely exposed. It is often necessary to employ a powerful but narrow pair of forceps, so as first to break down the calculus and then to remove the fragments from the swollen urethra, a proceeding often entailing injury to the latter. Several more calculi may be lodged behind the first and cause 
great difficulty in removal ; and therefore, if urine is not at once voided in considerable quantities after removing the stone, the urethra should be examined with a sound, or elastic catheter.

More serious embarrassment is caused by the infiltration of urine, which regularly happens in dogs, and is almost always followed by gangrenous inflammation. Possibly the acid reaction of the urine in carnivora plays an important part in effecting this, or it may be caused by decomposition products resulting from alkaline fermentation of the urine. Simon has noted that though acid human urine injected into the subcutis produces no inflammatory change, it is quite otherwise when alkaline fermentation has set in. Whatever the cause, the fact remains that infiltration of urine in the dog always causes inflammation, which tends to take on a gangrenous character. In such cases good results often follow from vegetable diet and the free administration of alkalies. Repeated washing of the wound with alkaline lotions like 5 to 10 per cent. sod. carb., or powdering with a mixture of sod. bicarb. and iodoform, also appears useful. Should these complications be safely escaped, cicatricial contraction of the urethra occurs after some time, and greatly impedes the discharge of urine. As a rule, operation in carnivora for urethral calculus must be classed amongst the least thankful exercises of the healing art.

Of cystic calculi in the dog the same may be said as of cystic calculi in ruminants and swine. In spite of an extended experience in canine practice, Möller never found opportunity to carry out lithotomy in these animals, though he repeatedly met with cystic calculi in making postmortem examination. Hendrickx and Liénaux successfully removed a cystic calculus in the dog by laparo-cystotomy; Malzeff has also performed the operation.

Malzeff anæsthetises the dog, which is then laid on its back. The bladder having been emptied, washed out with 2 per cent. boric solution, and the seat of operation thoroughly disinfected, an incision, 2 to 3 inches long, is made over the linea alba. The incision commences at the symphysis pubis and is carried down to the peritoneum, the penis meanwhile being pushed towards the left side. The peritoneum is next opened, and (the hands having been carefully disinfected) the urinary bladder is sought for by introducing the forefinger. When discovered it is grasped with forceps, drawn forrard into the wound, and incised for a short distance to permit the stone to be withdrawn. Bleeding is slight. The wound in the bladder is next closed with boiled or carbolised silk, that in the abdominal coats cleansed with $2 \frac{1}{2}$ per cent. carbolic solution, powdered with iodoform, and brought together with button sutures. A dressing is useless, and even hurtful, as it makes the patient restless. Eleven cases out of twelve recovered. Rubay claims to have opened the kidney without penetrating the peritoneal sac, removed the calculi, and washed out the pelvis of the kidney and returned the organ to its position. 


\section{III.-PUNCTURE OF THE BLADDER (PUNCTIO VESIC $\not E)$.}

When the discharge of urine by the natural passage is hindered, the urinary bladder becomes enormously distended, and puncture by trochar is resorted to to prevent rupture. The operation was first practised in man, and is still employed in dysuria, particularly when following enlargement of the prostate. The trochar used for the horse is an enlarged copy of that constructed for the above purpose by Flourant (fig. 169). Pilger described the operation more exactly, and later it was frequently employed in oxen which suffer from urethral calculi. Before attempting it, the diagnosis of over-distended bladder must be verified by rectal examination. In the horse the distended bladder extends downwards and forwards, that is, towards the abdominal cavity. In oxen and bulls the extension takes place more towards the sides. Even in the horse, however, the bladder does not reach the lower wall of the abdomen; this condition is only seen in swine and carnivora, in which the bladder, when filled, lies to a great extent in the abdominal cavity. It need scarcely be said that, before making the attempt, all simpler means, as, for instance, the use of the catheter, should have been tried.

The bladder may be reached from different points if Flourant's form of trochar, which is moderately bent (fig. 169), be employed. These different methods of puncture are distinguished.

(1) The punctio vesicæ suprapubica, which is now most generally used in man, is impracticable in herbivora, because in them the lower part of the bladder is not attached to the wall of the abdomen, and in the horse and ox the bladder would be only reached through the peritoneal cavity. In swine and dogs it

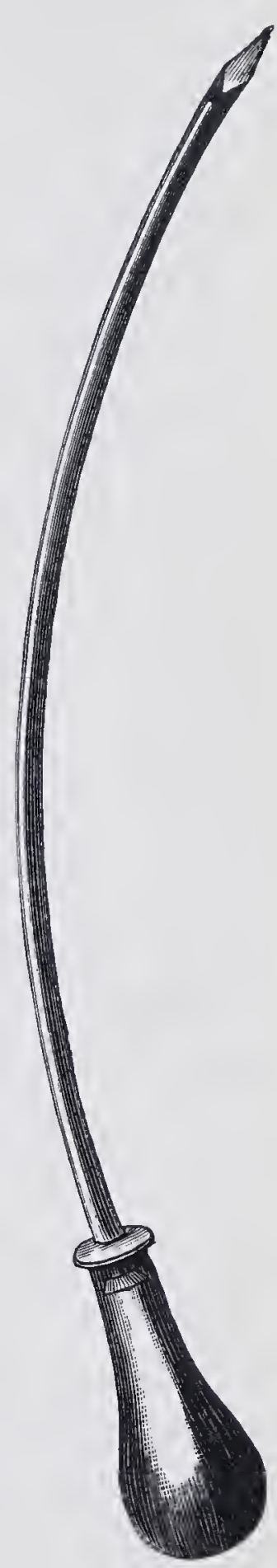

FIG. 169.Flourant's trochar. can, however, be approached from the lower abdominal wall, and this path is to be preferred because the more convenient method of operation through the rectum employed in larger animals is here attended with difficulty. Hering, who recommends this operation for dogs, places the animal on its side, and, after making an incision through the skin, passes a thin trochar into "the tensest portion of the swelling in the lower region of the flank." In man the operation is done in the dorsal position and close to the linea alba, and the same procedure has been recommended 
in dogs. Hering states having completely cured a dog by his method, though he does not say what caused the difficulty in urination.

(2) In large animals puncture is effected from the rectum (punctio rectalis).

This is the easiest method and that usually followed when it is desired, as often happens in oxen, promptly to remove the danger of ruptured bladder, and it is intended to slaughter the animal in a short time. The canula, guided by the left hand and with the trochar drawn back (covered), is introduced into the rectrum, and directed as nearly as possible perpendicularly to the surface of the bladder. It is well not to go much beyond the neck of the bladder, but rather to pierce it close behind the neck, so as to avoid opening the peritoneal sac.

(3) Punctio perinealis deserves preference in large animals unless when destined for early slaughter. Inflammation of the urinary bladder is less likely than in punctio rectalis, where it readily occurs in consequence of infection from the rectum. The skin is incised close below the anus and somewhat to the side of the urethra, and efforts are made to penetrate thence as far as the neck of the bladder by working with the fingers, assisted when needful with knife and scissors. As soon as the distended urinary bladder is felt, a straight trochar with the point covered is introduced, placed on the bladder and the stilette pressed forwards. On withdrawal, urine is discharged through the canula, which is slowly thrust forward so as to follow the movement of the collapsing walls of the bladder. Should a single evacuation suffice, the trochar is at once removed; it is not necessary to suture the skin wound. Hering recommends, after making the cutaneous incision, to thrust the trochar directly forwards in the direction of the urethra, as far as the bladder, without dividing the connective tissue. When considerably distended, the bladder can scarcely be missed. Should the prostate or vesiculæ seminales be injured little harm results, especially in castrated animals. On the other hand, however, there is no reason against providing, as far as possible, a safe passage for the trochar, especially if antiseptic precautions be taken. If it is desired that the animal shall live for a considerable time after operation, this method certainly deserves preference, as, in the event of a repetition of the operation becoming necessary, it will be much easier.

The canula may remain in position for one to two days; in oxen intended for slaughter this is to be recommended, and an additional advantage is that the trochar may here be more easily fixed in position than in the rectal operation.

In quiet animals the above operations may be performed standing, by extending the hind feet and applying a twitch. Rychner recommends the dorsal position, because it allows the bladder to be still more easily 
discovered from the rectum. It has already been remarked that where the bladder is much distended, casting must be effected cautiously.

\section{IV.-INJURIES, INFLAMIMATION AND STRICTURES OF THE URETHRA.}

Save by operation, the urethra is seldom wounded. Adam describes a case of injury in the horse by an iron hook. Healing was difficult. Fuchs saw a severe case in a horse that had fallen in front of a tramway car. The urethra was almost completely torn out of the penis by a hook: a small portion remained hanging to the glans; the rest was only connected with the bladder. About 16 inches was cut away and the wound disinfected. Some hours later bleeding occurred from the corpus cavernosum, but was checked by ligature, and though severe swelling occurred it disappeared in eight days. Recovery was sufficiently advanced in four weeks for the horse to return to work. The urethra opened somewhat below the perineum.

Inflammation of the urethra may be caused by foreign bodies entering it accidentally, or being introduced by way of treatment.

Should foreign bodies like awns of wheat obtain access, the minute spines they possess cause them gradually to pass upwards and produce injuries of the mucous membrane and inflammation. Specific conditions like gonorrhœa of man have not yet been recognised in animals, if we except dogs, which occasionally suffer from chronic purulent urethral catarrh. Many cases are really only purulent preputial catarih (sees "Inflammation of the Prepuce in Carnivora").

Stricture of the urethra is commonest after operations like urethrotomy and amputation of the penis, but it may also result from accidental injury.

Perforating wounds of the urethra are recognised by urine escaping through them during micturition. They are often associated with symptoms of infiltration of urine, such as inflammation, severe swelling, and a tendency to gangrene. Such a complication is most to be feared when the wound in the mucous membrane is greater than that in the skin, or when the latter is not divided at all, as in bruises.

Swelling of the mucous membrane of the urethra consequent on inflammation produces symptoms like those of urethral calculus. In oxen the urethra is said to be sometimes ruptured by the passage of urethral calculi.

Foreign bodies in the urethra produce a like train of symptoms. Bluhm describes the case of a horse which suffered from colic and retention of urine, and allowed the penis to protrude from the sheath. Careful examination discovered in the urethra an oat-head 4 inches in length with awns. 
Strictures are recognised by the gradually increasing difficulty in urination. The stream of urine gradually becomes smaller as time elapses, the act is more and more protracted; finally fluid can only be discharged in drops. Passage of the catheter reveals the position and extent of the stricture. The symptoms produced by urethral calculi generally appear suddenly.

Purulent catarrh of the urethral mucous membrane in dogs can be recognised by laying the animal on its back, drawing forward the penis (see "Urinary Calculi in the Dog"), and passing a sound over it with moderate pressure from the corona glandis to the opening of the urethra, when, if urethritis exist, a purulent secretion will be discharged.

The prognosis is very varied. In injuries, it depends on whether urination is impeded, and whether infiltration of urine exists or is to be expected. Impeded urination may be due either to inflammatory swelling or stricture. The less the inflammation and infiltration of urine, the more favourable the prognosis. Injuries of the urethra in carnivora are graver than in herbivora, because they readily lead to gangrenous cellulitis.

Intra-urethral injuries, caused by careless management of the catheter, are seldom dangerous, for the wound opens forwards and hence is not favourable to infiltration of urine. On the other hand, injuries by foreign bodies easily induce retention of urine and inflammation in the mucous membrane, with the formation of urethral calculi, because such bodies carry with them organisms likely to determine decomposition of urine. Stricture is always grave, especially in horses, as it becomes aggravated with time; the greater the interference with urination, the greater the danger.

Treatment. The chief indications are to prevent retention and infiltration of urine, and to assist union. The more regularly and rapidly healing proceeds, and the less cicatricial tissue produced, the slighter the chance of cicatricial contraction and stricture formation. The wound is treated on general principles, and protected as far as possible from discharge of urine. Clean-cut wounds of the mucous membrane may be brought together with catgut or silk, and in herbivora sometimes heal by first intention. In carnivora it is still more important to suture the mucous membrane, in order to prevent infiltration. For the same reason the skin wound is best left open. Under these circumstances healing by first intention occurs in exceptionally favourable cases. If not, the skin wound, when not already larger.than that in the mucous membrane, may be increased in size. In carnivora the injured spot should be washed every hour or two with an alkaline fluid (5 per cent. carbonate of soda), or powdered with iodoform and bicarbonate of soda (equal parts), and vegetable diet enforced until the wound 
is healed. The greatest care is necessary during the first few days; after granulations have formed, infiltration of urine is less likely to occur. Injuries produced by the catheter must also receive close attention. If during the first twenty-four hours great swelling and retention of urine result, urethrotomy or puncture of the bladder may become necessary to prevent rupture. Foreign bodies should be removed as soon as possible from the urethra, and the injury they may have caused treated according to existing circumstances.

When urine has infiltrated the tissues, careful provision must be made for its exit, either by inserting setons, or, better still, drainage-tubes. Scarification may be of assistance.

Though strictures of the urethra may be dilated by passing the catheter or a bougie, and urination thus assisted, the effect is seldom permanent, and the method not of practical value. Nor is urethrotomy much more reliable. In large animals it may temporarily relieve the difficulty, but new strictures soon form. Strictures near the free end of the urethra may sometimes be cured by freely laying them open.

Urethral fistula is caused by an injury healing incompletely, and leaving a little opening in the wall of the urethra, through which urine is from time to time discharged. It may sometimes be closed by applying irritants or the actual cautery; but often it gives much trouble to the practitioner, and ends by producing stricture of the urethra. Moussu saw such cases in dogs after injuries to the urethra. Fröhner improved the appearance of a horse suffering from urethral fistula by performing urethrotomy at a point above the fistula where there had been a dilatation of the canal. Urine was afterwards discharged by the artificial opening.

\section{Y.-PARALYSIS OF THE URINARY BLADDER (CYSTO- PLEGIA). CATHETERISM.}

Long-continued retention of urine, due sometimes to urethral calculus, causes over-distension of the bladder, paralysis of the detrusor urinæ, and inability to eject the urine.

Paralysis of the sphincter vesicæ produces involuntary discharge of urine (enuresis, incontinentia urinæ). Not infrequently retention and involuntary discharge exist together, e.g., in severe internal diseases, puerperal apoplexy, brain diseases, hæmoglobinuria, \&c. In severe internal complaints, like post-partum paralysis, brain disease, hæmoglobinuria, and in diseases of the spinal cord and fractures of the dorsal and sacral vertebrx, paralysis of the bladder may occur, and is sometimes associated with paralysis of the tail and paraplegia. Calculi and gravel may also produce paralysis of the urinary bladder. 
Symptoms. When the detrusor is alone paralysed, the bladder gradually fills, and urine is discharged in small quantities (ischuria). If paralysis of the sphincter is also present, involuntary discharge occurs, the bladder overflows like an over-filled vessel, and the condition is described as ischuria paradoxa. The bladder can then be emptied by pressure from the rectum, or by contraction of the abdominal muscles, as happens during defæcation. At each act a variable quantity of urine is discharged. When the sphincter is completely paralysed urine also flows away in an uninterrupted stream, even when the bladder is incompletely filled (enuresis) ; nevertheless, in male animals the bladder always fills to a certain extent.

The prognosis depends on the active cause. In general it is the

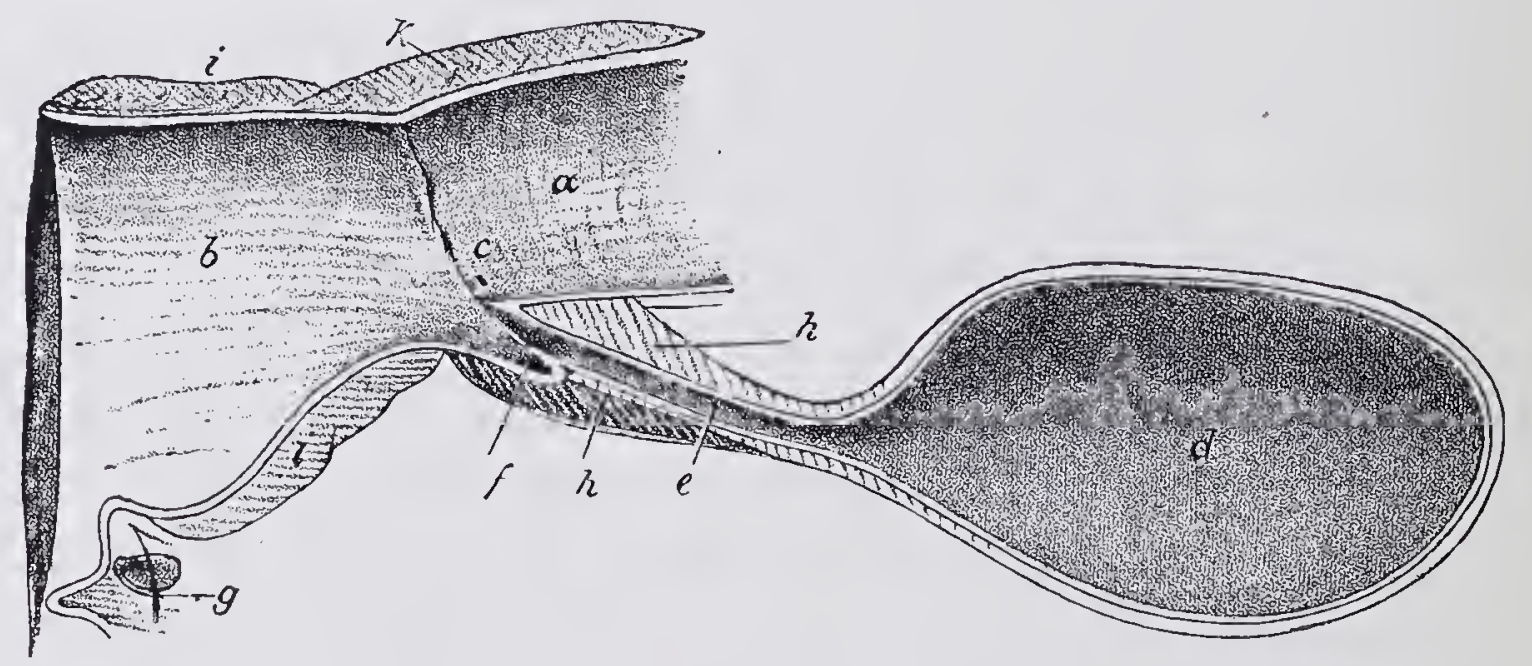

FIG. 170.-Median section of the bladder, urethra, vagina, and vulva of a cow. a, Vagina; $b$, vulva ; $d$, bladder ; $e$, urethra ; $f$, valve of the urethra ; $g$, clitoris.

more unfavourable the longer the condition has existed without visible improvement.

Treatment. At first an attempt should be made to remove the cause. Strychnine, cantharides, \&c., may be given internally; when, in consequence of paralysis of the detrusor, the bladder is greatly distended, it may be emptied by pressure through the rectum; or should this fail, by the catheter.

The use of the catheter (catheterisation) calls for two precautions: the urethra must not be injured internally, nor must infectious or decomposing material be introduced into it. Failure to observe the latter precaution may result in decomposition of the urine, inflammation of the bladder (cystitis), or even often fatal pyelonephritis, on account of the inflammatory processes spreading as far as the pelvis of the kidney.

In Germany elastic rubber tubes are generally employed, whose outer opening is somewhat dilated, whilst the opposite end possesses one or two lateral openings (eyes), by which the urine may enter the catheter. A suitable stilette serves to assist the introduction and cleansing of the instrument. 
In large male animals one large catheter generally suffices; in dogs, catheters of varying calibre must be kept. For mares and dogs a straight or slightly bent tube with a well-rounded end is used, though in case of need the index or middle finger may replace the catheter. The introduction of the catheter is seldom difficult in these animals, if it be borne in mind that the outer opening of the urethra lies on the lower wall of the vagina, over which the instrument is allowed to glide forwards until it enters the urethra. For cows Imminger uses a special instrument about 13 inches in length and $\frac{3}{16}$ inch in diameter, with a pointed end and a small round head; he also employs a metal catheter 12 inches long and $\frac{1}{4}$ inch thick, slightly bent towards the rounded point, which is $\frac{3}{4}$ inch long. Before commencing the cow's head is firmly tied to a ring or the animal is thrust against the wall. The hand is wetted or oiled and passed into the vagina, where the opening of the urethra is soon discovered and is slightly dilated by passing the forefinger into it. The catheter is then passed in and slipped beneath the finger into the urethra and so into the bladder. As, in young animals, the forefinger can scarcely be introduced into the urethra itself, the fore and middle fingers are passed as far as the opening and the catheter introduced between them. A blind sac of varying size exists below the opening of the urethra in cows, and sometimes renders the passage of the catheter difficult, especially when the urethra opens on the upper wall of the sac. In stallions and geldings the operator stands at the side of the abdomen on the animal's left, and draws the penis forwards out of the sheath. In order to grasp it more firmly a cloth is passed round the penis, and the catheter is then introduced. The instrument must, however, have been previously carefully cleansed to prevent the introduction of organisms into the bladder, and, to allow of its easy passage, the surface smeared with oil or water. This is absolutely necessary. In the anterior sections of the urethra the catheter glides easily forwards under slow, steady pressure. The first difficulty is on passing the bend of the urethra at the ischiatic arch. Here an assistant must direct the end of the catheter forwards towards the bladder by pressing on it, whilst the operator thrusts the instrument cautiously on, and gradually retracts the stilette. Violent movements at this time are liable to injure the urethra, and must be strictly avoided.

Immediately the front end of the catheter has passed this point it again glides easily forwards, and finally attains the neck of the bladder, which seldom opposes any great resistance to its entrance. The stilette is then withdrawn and the urine allowed to escape.

In bulls and oxen only the forward end of the urethra as far as the "S"-shaped curve can be catheterised, and even this seldom becomes 
necessary. The same is true of rams, though in them the entrance is rendered still more difficult by the free portion of the urethra. In case of need, the end may be cut off or the urethra incised behind this point (see "Urethrotomy in Ruminants").

Catheterisation is easiest in the dog. The animal is placed on a table in the dorsal position, with the left side towards the operator. The penis is then exposed by the method described under "Urinary Calculi in the Dog," and the catheter, previously carefully prepared, passed into the urethra. T'o prevent injuring the canal at its curve over the ischial arch, the instrument is passed as far as this point, and the steel stilette then drawn back at the same rate as the catheter advances; when the bladder is reached, the stilette is removed entirely.

By means of the catheter not only can decomposed urine, \&c., be removed, but fluids may, if necessary, be introduced into the bladder and its mucous membrane directly treated. Baertz, in a case of strangury, injected infusion of belladonna with good results. Grintzer states having cured contraction of the neck of the bladder which impeded catheterisation in a horse by clysters of chloral hydrate, renewed at intervals of a quarter of an hour.

\section{VI.-INFLAMIMATION OF THE URINARY BLADDER (CYSTITIS).}

Inflammation of the mucous membrane of the urinary bladder usually results from decomposition of the urine (alkaline fermentation), and irritation produced by products of such decomposition; or from mechanical lesions produced by hard bodies liké cystic calculi. In females infectious processes, e.\%., diphtheritic or septic inflammation of the uterus and vagina, extend to the bladder, especially soon after birth. Röder saw such a case in the cow. Finally, irritant substances, cantharides, oil of turpentine, and the like, may be excreted from the blood through the medium of the urine, which if long retained may cause inflammation of the cystic mucous membrane. Cystitis may perhaps be produced in this way during the course of infectious diseases.

Inflammation of the outer surface of the bladder, that is, of the serosa covering it (pericystitis), occurs as a diffuse peritonitis after castration and similar causes. It may, however, appear locally in consequence of chronic inflammation spreading from the uterus, rectum, or spermatic cord.

In a horse described by Oeben, which had suffered for four days from colic, and had died after passing large quantities of fæces and exhibiting continuous straining, the wall of the urinary bladder was 
found to be nearly 2 inches thick, the walls of the rectum and of the iliac arteries were as thick as a man's finger, but showing no diminution of their lumen; probably the inflammatory process had started from the rectum.

Siedamgrotzky was able, by washing out the bladder, to remove a great quantity of sediment from a horse which had suffered from enuresis paralytica, and which, though continually straining to pass urine, could only eject small quantities in jerks, whilst at other times discharge occurred involuntarily. The horse, however, died, and on postmortem was found to have suffered from purulent diphtheritic cystitis, pyelonephritis, and peritonitis. Friedberger reports a case of chronic hæmorrhagic purulent cystitis in the horse, caused by cystic calculus. Catarrhal diseases of the cystic mucous membrane may probably also be produced by chills.

Symptoms. Repeated attempts to discharge urine and the passage of small quantities, sometimes pathologically altered, point to the presence of the disease. The animals often stretch out and draw the testicles upwards; male animals may exhibit erections, and females contraction of the vagina. Pressure through the rectum on the almost empty bladder causes acute pain. Carnivora exhibit pain on pressure over the posterior portions of the abdomen, and often seem to suffer from slight colic. The urine discharged is turbid, and shows on microscopical examination much cystic epithelium, blood, pus corpuscles, flakes of mucous membrane, and not infrequently crystals of triple phosphate in the well-known coffin-lid shaped form. In carnivora the urine gives an alkaline reaction.

Pus in any considerable quantity produces a yellow sediment, whilst in diphtheria of the bladder croupous membranes are discharged with the urine. Infectious disease of the bladder is usually associated with fever, the intensity of which indicates the nature and significance of the disease. Cystic calculi are often indicated by discharges of blood, especially after work (see "Urinary Calculi in the Horse").

Chronic pericystitis seldom produces marked symptoms, but leads to gradual thickening of the bladder wall; and post-mortem shows a condition greatly resembling those hypertrophic processes resulting from long-standing obstruction to urination (dysuria), which are associated with abnormal distension of the bladder itself. Fünfstück, when maling: the post-mortem of a goat, found the bladder of enormous size and containing 52 pints of fluid.

The course of this disease varies; for whilst purulent diphtheritic cystitis, either directly or in consequence of complication with pyelonephritis, soon proves fatal, pericystitis and chronic inflammation, caused by cystic calculi or sediments, may endure indefinitely if the cause of the 
disease be not removed. Catarrhal cystitis usually disappear's in a few days.

Prognosis, and indeed treatment, therefore, depend on the indications furnished by a careful examination of the urine, which must be considered in conjunction with the symptoms. It should be remembered that many of these diseases have a great tendency to recur.

Treatment. Some benefit results from the administration of medicines which, excreted with the urine, exert a curative effect on the cystic mucous membrane, but direct treatment is always more effectual. Boric acid, salicylic acid, biborate of soda, chloride of potash, tannin (which is excreted as pyrogallic acid), and other materials have been used, and may prove of service in simple catarrhal inflammation.

But immediately putrefactive changes, especially alkaline fermentation of the urine, appear, local treatment must be resorted to. The bladder should be washed out by means of the catheter-in mares and cows through a rubber tube,-either with salicylic acid (concentrated watery solution), carbolic acid $(0.5$ per cent.), sublimate $(0.5$ per 1,000$)$, boric acid (2 per cent.), creolin ( 1 to 3 per cent.), nitrate of silver $(0.5$ to 1 per cent.), or tannin ( 1 to 2 per cent.), at least once a day. The chief difficulty is, that in male animals the treatment can only be carried out by experts. The lotions should, of course, be warmed to body temperature. Concretions and sediments are removed by vigorously washing out the bladder, or by surgical operation. Chronic cystitis in man has lately been treated with salol, which renders the alkaline urine once more acid, clear, and free of offensive smell. It is well borne by the stomach, and is decomposed by the pancreatic juice in the small intestines into salicylic acid and phenol.

\section{VII.-PROLAPSE AND INYERSION OF THE URINARY BLADDER IN FEMALE ANIMALS (PROLAPSUS ET INVERSIO VESICE).}

In cows and sows, infrequently in bitches, the lower wall of the vagina is ruptured during delivery, the urinary bladder passes through the opening, and may even project beyond the vulva (prolapsus vesicæ). The condition is distinguished by the prolapsed viscus being covered with serosa. Gradually it becomes distended, and assumes an almost spherical form; small quantities of urine are discharged on pressure. By introducing the hand or finger into the vagina close to the prolapsed portion, the rupture in the lower wall can be felt.

Diagnosis of prolapse is seldom difficult; but as the condition occurs during parturition, the swelling might possibly be mistaken for the 
presenting fœtal envelopes, and grave results ensue if perforated under this erroneous idea.

Inversion of the urinary bladder is a condition in which the bladder is turned inside out, passing through the urethra something like a reversed pocket; the disease has up to the present almost always been observed in mares during or soon after parturition. Grüger saw it in a mare which had shortly before aborted, and suffered rupture of the perineum. Lönnecker noted inversion in mares both during pregnancy and after parturition. Esser diagnosed the condition in sows, also after

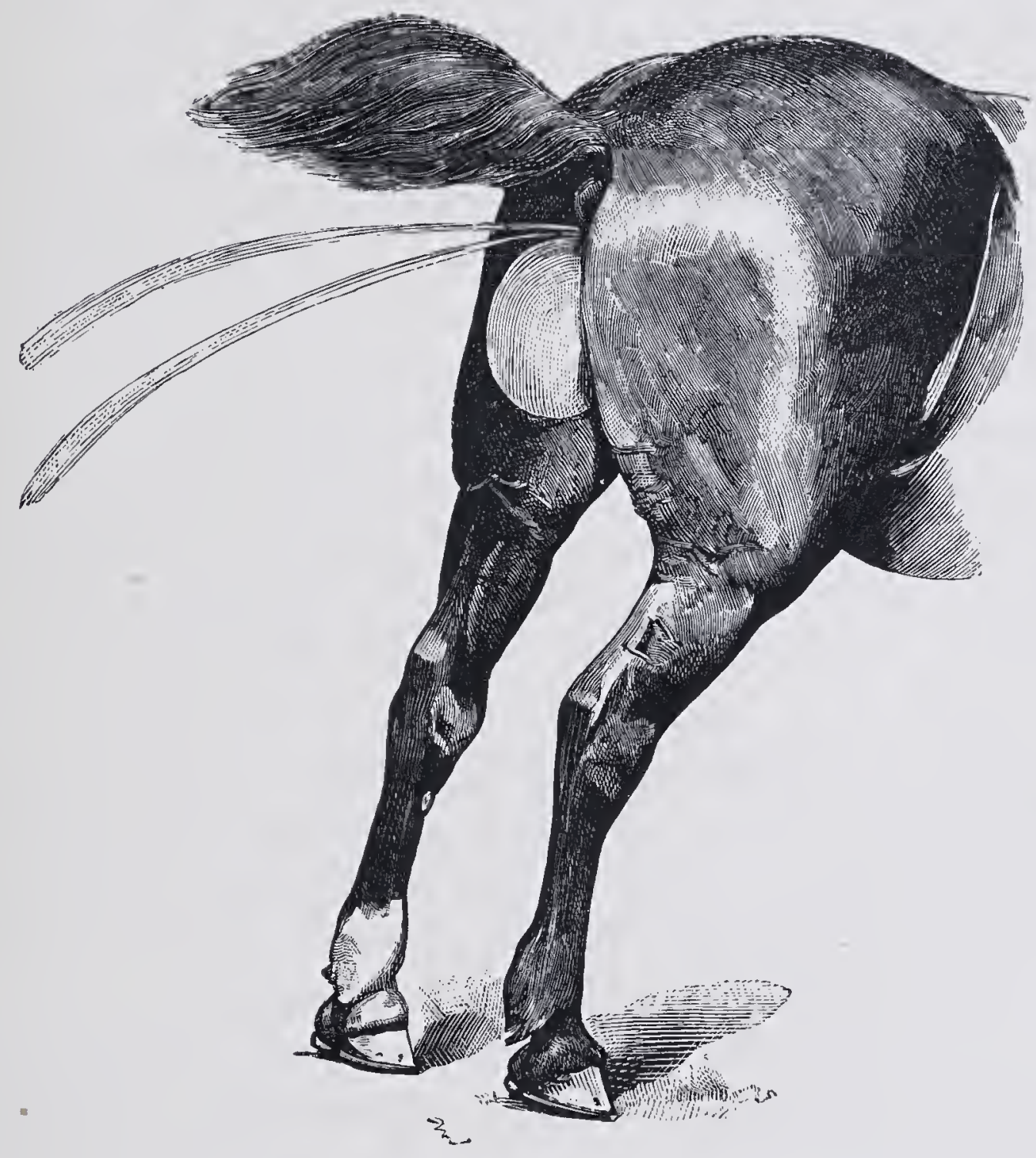

FIG. 171.- Inversion of the bladder in a mare.

parturition. It therefore seems that about this time the urethra is abnormally dilated, a condition necessarily antecedent to inversion of the bladder. Mann saw a mare in which the disease did not occur until three weeks after parturition. Rauscher speaks of a two-and-a-half year old filly which suffered from inversion. Forcible dilatation of the urethra in mares, practised for the removal of cystic calculi, may occasion the disease if the constrictors of the abdomen contract powerfully. In geldings Möller has repeatedly dilated the neck of the bladder sufficiently to admit the whole hand for the purpose of removing cystic calculi, but in spite of this has never noticed the condition.

Inversion of the bladder is distinguished by the presence of a spherical 
swelling, either in the vagina or protruding beyond the vulva (Fig. 171). The swelling appears elastic, owing to the inverted organ containing: peritoneal fluid, is covered by the mucous membrane of the bladder, and two folds appear in it, the orifices of the ureters. By lifting up the swelling, or pressing on it, or by movement on the part of the animal, urine (from the distended ureter's) is discharged in two streams (fig. 171).

The mucous membrane gradually dries by continuous exposure to the air, is soiled by dirt and dust, and may finally become necrotic. When the condition has existed for a long time stenosis of the urethra may lead to strangulation of the bladder, which is shown by severe congestion, and sometimes ends in rupture. Injury to its walls causes the swelling to subside.

Prognosis. Prolapsus vesicæ is evidently graver than inversion, as the peritoneal cavity is opened and peritonitis is liable to occur, a danger which is absent in inversion. Reposition is only possible in recent prolapse, where the serosa of the bladder has undergone no considerable change. Otherwise septic peritonitis occurs, and invariably proves fatal. The prognosis is more favourable in inversio vesicæ; the mucous membrane of the bladder possesses greater resistance, and even when, after successful reposition, irritation is marked, cure is not impossible. The animal's progress depends principally on the condition of the mucous membrane and on the calibre of the urethra. As long as these allow of reposition, the operation must be attempted, as, in the event of its failing, nothing remains but amputation of the bladder. In distinguishing the inverted bladder from fotal membranes, the directions given under "Prolapse of the Bladder" must be borne in mind. Failure to distinguish inversion of the bladder from fotal membranes has sometimes led to awkward consequences both for patient and operator.

Treatment. Provided the serous coat of the prolapsed bladder is little changed, it should be carefully cleansed with disinfectants and attempts made to replace the viscus. At the same time the wound in the vagina must receive attention. Unless the animal strain much, a repetition of the prolapse need scarcely be feared, and can be provided against by injecting lukewarm fluid into the replaced bladder. Rivière diagnosed prolapse of the bladder in a cow soon after birth. The rupture in the vagina was $3 \frac{1}{4}$ inches in length. After discharge of the contents, the bladder was replaced by using a piece of hollow elder stick, and though severe straining ensued, the condition did not recur. Recovery was complete. When the rupture in the lower wall of the vagina lies not far from the vulva an attempt might be made to suture it, to prevent recurrence and to guard against the passage of urine into the abdominal cavity.

When the bladder becomes inverted, the first point is to effect reposition 
before the mucous membrane is much altered. After expelling the peritoneal fluid from the inverted bladder by pressure and cleaning the prolapsed part, the animal is placed with the hind-quarters high and a twitch applied. The urethra is sometimes so wide that the displaced bladder can be passed through it with the hand, but should it prove too narrow, a blunt stick, well rounded off at one end to prevent injury to the bladder, may be employed. Lönnecker employs a probang for this purpose. The hand or stick is placed on the base of the bladder and the latter thrust through the urethra. One proceeds here in the reverse order to that observed in replacing a prolapse. By injecting a lukewarm fluid like saturated boric acid lotion, diluted alum lotion (1 to 5 per cent.) or tannin ( 1 to 2 per cent.), recurrence may be prevented and inflammatory reaction checked; such solutions also favour contraction of the urethra. By slow exercise, pressure on the loins, or by watching the animal, the straining and consequent eversion may be obviated.

Lönnecker replaced an inversion, but as it reappeared five days later he sewed up the orifice of the urethra, grasping and raising the mucous membrane with the left hand, and passing a lead wire through it, after which recovery took place. Holgen successfully effected reposition five weeks after the first appearance; probably the bladder was not continuously exposed. The same applies to the case related by Degive, in which reposition or reversion was successful fourteen days after the viscus first protruded.

As a rule, however, the surface of the mucous membrane is so much injured during prolonged exposure as to become necrotic, in which case reposition is contra-indicated, and amputation of the bladder offers the only chance of recovery. Though this certainly deprives the animal of the natural reservoir for the urine, which, therefore, flows continuously or is discharged in small quantities from the vagina, yet as it does not render ordinary working-horses and cows useless its employment is sometimes justified.

Amputation of the bladder is generally effected by ligation, best by using the elastic ligature. As the ligature tends to slide forwards off the spherical swelling and over the orifice of the ureters, two different methods of operation have been suggested. Some recommend transfixing the bladder and ligaturing on both sides, that is, applying two ligatures; in this case the peritoneal sac is opened and the danger of peritonitis increased. Others prefer the simple ligature, transfixing the bladder with a metal needle in front so as to secure the ligature in position. Bang passes a second ligature in front of and at right angles to the first, and after cutting away the bladder carrying the ligatures backwards and tying them in the form of a cross. The same result is more simply attained, without transfixing the bladder, by laying a tape with its two 
ends above and below the neck of the bladder, the rest of the tape extending over the bladder in the middle line; the ligature is then applied, embracing the tape, and the two free ends are carried backwards and tied at the back of the swelling. If the bladder be not immediately cut off after ligation, it sloughs away in some days, though, unless elastic ligatures are used, it is necessary to tighten the ligatures a few times during the next few days; the elastic ligature is, however, much preferable. When adhesions have already occurred between the layers of peritoneum covering the bladder, it may be simply cut away, as was done by Gaullet in the case of a mare. The bleeding was insignificant and recovery soon occurred. As, however, it is never certain that the adhesions are firm, the ligature deserves preference. The portion of bladder not included in the ligature gradually retracts into the vagina after the free portion has sloughed, and the external appearance of the animal is not much injured.

\section{VIII.-TUMOURS IN THE URETHRA AND BLADDER.}

New growths in the urethra soon impede urination; hence the animals are either slaughtered or die of the condition, and opportunities of noting or treating tumour of the urethra during life are rare. Matthias, however, found a polypus of the urethra in a gelding. Vorberg, 10-12 polyp-like new growths in an ox; they were $\frac{1}{4}$ to $1 \frac{1}{4}$ inches in length, and closely packed together at one spot. The polypus noted by

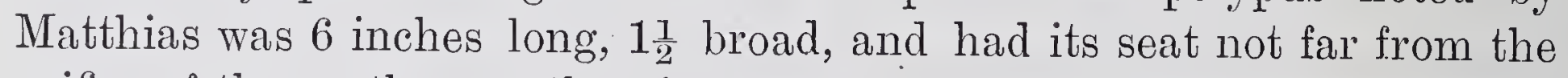
orifice of the urethra, so that it could be grasped with dressing forceps and removed.

New growths in the bladder are more frequent; they may either be innocent, like fibromata, lipomata, myxomata, or malignant (cancer).

The collection in Copenhagen contains the bladder of a cow with " fibrous cancer" (Bang). Pflug has seen carcinoma of the bladder in a horse. Siedamgrotzky described epithelioma of the bladder in the horse; the disease had extended to the peritoneum and caused secondary growths in the omentum. He also found in oxen leucocythæmic infiltration of the wall of the bladder, the uterus, and the ligaments of the uterus. Esser was able to diagnose by manual examination from the rectum during life a large papilloma in the bladder of a cow. Wolff and Leisering discovered myxomata in the same region. Cows appear to suffer from new growths in the bladder oftener than horses, not infrequently from carcinomata, probably tuberculous growths also occur; tuberculous tumours are common near the openings of the ureters in the Trigonum Vesicæ Lieutaudii. Barnick discovered a tumour twice as large as a man's head in the bladder of a horse which had died with symptoms of difficulty in urination and colic. Tright found a myoxma of the bladder in a dog.

Demeurisse diagnosed cancer of the bladder in a bitch suffering from 
cancer of the mamma; the growth could be recognised as a painful swelling on examination per anum. The animal showed progressive emaciation and died in a short time; on post-mortem a perforation was found in the upper wall of the bladder, through which urine had flowed into the abdominal cavity. Near the neck of the bladder lay a carcinoma which had prevented the passage of urine.

Symptoms. Tumours in the urethra are recognised, like strictures, by their gradually causing increased difficulty in urination. During the act the stream of urine becomes smaller and smaller, until finally drops alone are passed. Displacement of the tumour, slight swelling of the mucous membrane, or the formation of a blood clot may tend to the sudden development of symptoms. On passing the catheter the obstruction is found to be more or less soft, which differentiates a tumour from a urinary calculus.

The growth of tumours in the bladder gradually diminishes its capacity and causes urine to be passed more frequently. New growths sometimes cause profuse bleeding; the urine takes on a bloody character, usually blood clots of various sizes are discharged; dysuria may also occur, especially if the tumour lie near the neck of the bladder. Purulent inflammation in some cases accompanies new growths; the urine appears turbid, and on standing deposits a sediment. The presence of tumours in the bladder is often signalised by the deposit of salts and the formation of precipitates, hence microscopical and chemical examination may be necessary to discover the origin of turbidity. These symptoms having suggested tumour formation, the diagnosis can be verified by examination per rectum or per vaginam. Not until the new growth attains a considerable size does it give rise to disturbance; a soft mass may be felt in the bladder, just as a hard mass may be felt when a calculus is present. In mares and cows the swelling may sometimes be felt from the urethra.

Inflammatory swellings of the mucous membrane of the urethra and calculi may sometimes be mistaken for tumours.

The prognosis is usually unfavourable. Only when the new growth is near the exit of the urethra and can be removed is prognosis hopeful. Though tumours in other parts of the urethra may occasionally be removed by performing urethrotomy, yet their existence and position can seldom be so exactly determined as to justify operation, especially as more or less serious consequences always ensue. Removal of tumours from the bladder is likewise very difficult, and this of itself sufficiently explains the unfavourable character of the prognosis.

Treatment. Pedunculated tumours near the free end of the urethra may be torn away with dissecting or dressing forceps. Where in male animals the growth cannot be grasped, the lower wall of the urethra is 
laid open. The position of the tumour and the practicability of operation having been ascertained, removal may sometimes be effected by opening the urethra. Urethrotomy may be resorted to as a palliative when animals have to be kept alive for some time to gain condition or require to be sent on a journey. Cystic tumours are seldom removed, being generally recognised too late, and success appearing doubtful. Schmidt and Mogford have, however, recommended everting the bladder in mares to remove tumours. Schmidt states having carried this out in the horse, and having removed a tumour weighing 40 ounces. Mogford declares eversion of the bladder to be easy in horses. After removing the tumour the bladder is washed out, and the concretions which often occur here removed. Mogford, however, does not give any satisfactory description of his method.

Nevertheless, both in mares and cows, it is worth trying to dilate the urethra, so as to be able to enter the bladder with polypus forceps or the hand. Though the method is certainly not easy, there seems no doubt that where it succeeds tumours may be removed and recovery effected. Levens thus removed a fibroma as large as a goose's egg, which lay at the neck of the bladder in a cow. Eversion of the bladder would probably often follow dilatation of the urethra if the animal were not chloroformed. Anæsthesia is therefore advisable in horses, and even in oxen, especially if not intended for immediate slaughter.

Schmidt removed a cystic polypus from a gelding by performing urethrotomy at the sciatic notch, and breaking down intervening tissues as far as the neck of the bladder; he was then able to excise and extract the pedunculated tumour ; the operation wound healed in eighteen days. Heyne describes a case of fistula between the small intestine and bladder, a rather rare condition. The small intestine was adherent to the base of the bladder, with which it communicated by an opening $\frac{3}{4}$ to 1 inch in width. The animal had suffered from weakness, defective appetite, diarrhœa, and great emaciation. 


\section{DISEASES OF THE MALE ORGANS OF GENERATION.}

\section{I.--INFLAIMIMATION OF THE PREPUCE (BALANITIS).}

The prepuce, consisting of a duplicature of the skin, is liable to inflammatory changes, which present very notable differences in appearance, course, and results, in the various classes of animals.

\section{(1.) INFLAMMATION OF THE PREPUCE IN THE HORSE.}

In the horse the covering of the penis is peculiar in that it consists of a double involution of the skin, that is, it is formed of an inner fold (prepuce in the narrow sense of the word) and of an outer, the so-called sheath. The visceral portion of the first covers the end of the penis, clothing it closely, and lying, when the penis is not erected, in folds, but these disappear on erection. The outer fold forms a swelling as thick as the finger, attached to the under surface of the member by the inner fold of the sheath; the latter presents a second considerably thicker covering, which is reflected backwards at the entrance to the sheath, to become continuous with the skin of the scrotum. The inner lining of the sheath and the outer fold of the prepuce are provided with numerous sebaceous and sweat glands, the secretions of which, together with the loosened masses of epidermis, form a blackish-grey fat-like material (smegma preputii).

In horses inflammation of the prepuce is not infrequently produced by the animals failing to extend the penis during urination, and passing the urine into the prepuce. This produces continuous irritation, thickening and subsequent contraction of the folds of skin, and finally stenosis of the outer folds of the sheath, which renders it impossible for the animal to expose the penis (phimosis). As in this condition more smegma always accumulates, the escape of urine may finally be seriously interfered with. Sometimes a large quantity of the material accumulates around the corona glandis, exerts pressure on the urethra, and renders urination difficult. Esser saw a case of the kind in a gelding. Colic, distension of the bladder, and ineffectual straining to pass urine, set in; sometimes urine is only discharged in drops. As a rule, geldings alone suffer from this condition.

Local examination discovers contraction of the opening of the sheath and accumulation of preputial smegma. Cadiot saw a horse in which 
the sheath had become so enormously enlarged that it hung down as low as the hocks; the condition had been brought about by a wound of the prepuce, which was followed by adhesion between it and the penis; urine was then passed into the sheath. Partial removal of the sheath was followed by recovery.

The condition may be cured by early treatment, but if delayed the animals either die from rupture of the bladder consequent on inability to pass urine or from exhaustion.

In many districts horses suffering from colic or difficulty in urination are treated by rubbing the penis and sheath with irritant substances like pepper, which afterwards produce extensive painful swelling of the penis and sheath, exudation from the surface, and finally ulceration. If applied freely and energetically they may even cause necrosis of the skin; the penis then protrudes from the sheath in a more or less swollen condition, while the preputial fold may be as large as a goose's egg. The inflamed parts are doughy, painful, of increased temperature; and if the penis itself, or the prepuce covering it, is greatly swollen, the pressure on the urethra may interfere with urination. Inquiry generally reveals the nature of the substance employed.

The inflammatory symptoms generally disappear without having caused permanent damage, though the sequel depends largely on their degree and extent. Provided urination is unimpeded, no very bad results need be apprehended, but when paraphimosis sets in, as is sometimes the case, treatment becomes difficult.

Treatment consists in cleansing the prepuce, particularly the corona glandis, so as to allow free exit for urine. Injections of lukewarm soap and water assist the removal of smegma. As a rule, this causes temporary improvement, and if the sheath be regularly cleansed the case usually does well. When symptoms of violent inflammation are already present, the injections should consist of mucilaginous materials, oil, or astringents like alum; while to combat excessive swelling of the sheath a suspensory bandage may become necessary (see "Paralysis of the Penis" hereafter).

Inflammation of the prepuce and penis produced by irritants like pepper calls for careful cleansing of the parts and bathing with astringents such as the above-mentioned alum lotion. Excoriated parts may be covered with a non-irritant fat, with zine or lead ointment, or powdered with iodoform, tannin, lycopodium, or similar materials. To assist resorption and removal of swelling, a suspensory bandage may be applied, and the animal placed in a clean dry stall.

Where the opening in the sheatl is much diminished in calibre, it may require to be laid open, which in quiet animals can be done standing. To prevent the edges of the wound reuniting, they can be 
held open by sewing them to the neighbouring skin, an operation which is not difficult if the sheath be divided on its under surface.

Hering divided the cutaneous portions of the prepuce in a stallion which had difficulty in erection; four months later he completely excised them; but as the prepuce contracted and caused the penis when in a state of erection to be directed backwards the animal had difficulty in covering, which was only removed by once more dividing the cicatrices and preventing reunion.

\section{(2.) INFLAMMATION OF THE PREPUCE IN OXEN.}

The prepuce of the ox, formed by a simple infolding of the skin, presents a long narrow sheath, the entrance to which is small, and is provided with a bunch of long strong hairs. The inner fold consists of mucous membrane, presenting some resemblance to the cutis, and displaying numerous follicles and papilli. It lies in longitudinal folds. The prepuce is moved by two muscles, so as to render the act of urination easier.

The chief sufferers from inflammation of the prepuce are oxen, bulls are very seldom affected. Oxen do not usually extend the penis during urination, especially if the opening of the prepuce has already become painful and constricted on account of inflammation. The smegma, usually decomposed and soaked in urine, accumulates in increasing quantities under the prepuce, until finally it impedes urination. Accidental injuries to the sheath may also prevent protrusion of the penis, and give rise to this disease. Gurlt found a head of wheat in the inflamed sheath. An abnormally irritant condition of the urine has also been regarded as a cause.

Dotter saw enzootic outbreaks of this form of inflammation which he regarded as due to contagion. Röbert had a similar experience ; he regarded the straw, which was very bad, as the offending material. After provision of fresh bedding and disinfection of the paving the outbreak ceased.

Albrecht noted a case of croupous inflammation of the visceral layer of the sheath; he laid open the parts and removed a mass of fibrous material and broken down tissue saturated with urine and of very offensive odour. Recovery followed the free use of lysol and creolin in six weeks.

Symptoms and course. A longish swelling, which is hot and painful, first appears at the front of the sheath, and may extend as far as the scrotum. Urination is difficult, and the urine is passed in drops or in a fine stream. The prepuce cannot be drawn back over the penis ; phimosis exists. In the prepuce a grey-black smegma is found, the removal of which gives pain. The bladder, when examined per anum, is found greatly distended and painful on pressure. Colic soon sets in. The animals stop feeding, may show fever, and if help be not soon afforded may die.

The risks are numerous. There is, firstly, the chance of necrosis of 
the prepuce and penis; sloughing of large portions of the penis has often been observed. Then there is the danger of infiltration of urine into the subcutaneous tissues, causing septic cellulitis, gangrene, and severe general disturbance from absorption of inflammatory and septic products. Finally, rupture of the bladder may be threatened. The condition is, therefore, in no way trivial, and imperatively calls for early treatment.

Treatment consists in cleansing and making patent the orifice of the prepuce to allow of urine escaping; if the entrance is too much swollen and no urine is passed, the prepuce must be laid open. As the animal strikes out furiously when the penis is handled, it should be placed in stocks, or some restraint applied. If stocks are unavailable, restive animals may be placed against the wall and held in this position by several strong men; Pflug recommends casting such animals. On account of the great pain, it is often impossible to free the entrance to the sheath in the standing position. The bunch of hairs must first be cut away, and lukewarm oil injected into the prepuce, whilst its outer surface is rubbed with the same material. If urination is moderately free, the animal may then be allowed to rise, and the task of emptying the prepuce left until next day. To facilitate this, Pflug first injects lukewarm water, solution of acetate of lead or Goulard's solution. Others recommend a saturated solution of chloride of lime. Permanganate of potash $(3-5$ per cent.), sulphate of zinc, tannic acid (3-5 per cent.), are also useful. The points to be kept in view are to cleanse the sheath as completely as possible, to remove decomposing material, or destroy its injurious properties by disinfectants, and to combat the inflammation. Bathing the sheath, or frequently washing its surface with lukewarm water, is therefore first indicated. Necrotic parts should be removed, or their separation assisted by bathing with lukewarm aromatic fluids, or frequently washing them
with warm carbolic lotion.

In most cases it is necessary to lay open the prepuce. A strong probepointed tenotome is introduced, and the cut made either downwards or to one side. Pflug prefers the latter direction on account of the parts not being so easily moistened by urine, which is likely to produce-fresh
strictures.

When the subcutaneous tissue about the sheath is infiltrated with urine, incisions are made, the parts pressed to remove the contents, and the skin and wounds frequently cleansed.

\section{(3.) INFLAMMATION OF THE PREPUCE IN SWINE.}

The prepuce of the boar consists of an inversion of the skin, but the inner Surface is formed of mucous membrane containing numerous lymph follicles. "un the upper wall of the sheath, and close in front of its opening, is the 
when distended it often attains the size of a hen's egg. It is lined by squamous epithelium, and contains numerous sebaceous and sudoriferous glands, hence smegma not infrequently accumulates at this point, and, becoming inspissated, may produce "preputial calculi."

In swine, as in the ox, only the castrated animal seems to suffer from inflammation of the prepuce; the causes are similar to those in oxen. They consist in accumulation of smegma in the prepuce, or in the abovementioned appendix; if swine were not slaughtered so early in life, the condition would probably be seen more frequently.

Symptoms. The prepuce is swollen, painful, hot, its orifice contracted, and not infrequently there is difficulty in urination. A stinking sebaceous material escapes on pressure. This material sometimes stops up the prepuce, and fills the umbilical pouch, causing it to protrude as a round swelling above the end of the penis.

Treatment is on the same principles as in cattle, and consists in evacuation of the sheath and pouch, cleansing and disinfection as far as possible. The same materials may be employed as in oxen; sometimes it is necessary to lay open the sheath.

\section{(4.) INFLAMMATION OF THE PREPUCE IN CARNIVORA}

Presents somewhat different features to the same condition in other animals. Apart from accidental injuries, preputial inflammation is rare in these animals, but dogs suffer frequently from chronic catarrh of the inner surface of the prepuce; indeed, most suffer from it to a limited extent.

Symptoms. The hairs clustered round the opening of the sheath appear gummed together, and a purulent material discharges from the prepuce. Animals are inclined to lick the affected parts, but apart from this there is little disturbance, and no difficulty exists in urination. Neither the mucous membrane of the prepuce nor of the glans is much altered, and the disease seldom requires treatment except in house-dogs. The causes are unknown. The popular idea that the disease is related to similar affections in man appears unfounded.

Treatment demands a good deal of patience. Injections of astringents (sulphate or sulphocarbolate of zinc, 1-2 per cent.; tannic acid, 3-5 per cent.) diminish the secretion, which, however, returns as soon as the treatment ceases. Even painting the inner surface of the prepuce with 1-5 per cent. nitrate of silver solution is seldom of permanent benefit. The owner or attendant may be entrusted with the application of the solution, and instructed to use it three or four times a week. 


\section{II.-PHIMOSIS, PARAPHIMOSIS, PARALYSIS OF THE MUSCLES OF THE PENIS, INJURIES TO THE PENIS.}

Phimosis consists in an abnormally narrow condition of the preputial opening preventing exposure of the glans. This contraction may finally cause difficulty in urination, or render it impossible. Phimosis seldom occurs congenitally in animals, but has been seen in dogs, and is produced by inflammatory swelling of the prepuce or cicatricial contraction (compare with "Inflammation of the Prepuce"). Under certain circumstances, it may form the primary disease, and lead to retention and inspissation of smegma with formation of so-called preputial calculi. Miller saw phimosis in a bull with adhesion between the penis and prepuce, caused by a layer of strong connective tissue. After dividing the adhesion, the bull was incapable of coitus.

Paraphimosis is a condition in which the glans cannot be retracted into the prepuce, either because the opening is too narrow or the glans too large. The peculiar formation of the penis and prepuce in the dog explains why it is so frequent a sufferer. In horses the outer fold of the sheath presents a wide opening through which the penis is easily retracted, though, when the preputial folds are greatly swollen, its return is sometimes impossible, - a condition which has been described indifferently as paraphimosis or paralysis of the penis. Whether simple paralysis of the muscles concerned in withdrawing the penis into the prepuce ever occurs is doubtful. In such case the retractor penis, which derives its motor filaments from the 4 th and 5 th lumbar nerves and from the N. hæmorrhoidalis posterior, would probably be affected. In disease of the spinal cord, we certainly sometimes see paralysis of the muscle in question. More often the condition is due to injury of the penis, causing swelling of the glans and hindering its withdrawal into the sheath. Several reported cases also point to thrombosis of the vessels of the penis itself. Bang saw a gelding with thrombosis of the veins of the sheath.

In the horse, inability to retract the penis is rarely caused by contraction of the outer folds of the prepuce. Their width and disposition are unfavourable to the production of paraphimosis; but swelling of the inner folds may in these animals cause prolapse of the penis and inability to retract it Such swelling, incorrectly described as paralysis of the penis, occurs temporarily during inflammation of the sheath and neighbouring parts, as, for example, after castration or injury to the penis. Slight wounds are sometimes followed by cellulitis in the sheath, as Haase noted after a blow from a whip. Möller has repeatedly seen this condition after irritant substances like pepper had been rubbed into 
the penis and sheath. Bang noted it in a gelding, following thrombosis of the veins of the sheath.

Swelling of the prepuce often remains after subsidence of the inflammation and interferes with the return of the penis. Inflammatory symptoms being absent, such cases have erroneously been looked on as paralysis of the penis. Cases do occur, however, where nothing abnormal can be detected either in the penis or prepuce, and yet, despite this, the glans cannot be retracted, or if replaced within the prepuce, at once falls forward. In such case the retractor penis may be paralysed. Paralysis may also afford an explanation of the cases which follow severe infectious diseases like purpura hæmorrhagica (Cagny); but at present we have no accurate information on the point. Injury and ulceration of the penis often occur as sequelæ of paralysis, as the penis occasionally protrudes to such an extent as to be struck by the hind feet and wounded during movement.

Local examination does much to differentiate the nature of the case. When no change is visible, and the penis is insensitive to pricks or pinches, paralysis may be surmised. In paralysis of the retractor penis, sensibility remains unimpaired. Particular attention must be paid to the prepuce; it suffers more frequently than is generally supposed.

Ruminants and swine very seldom suffer from paraphimosis, because in oxen the glans is very small, and in sheep and swine is altogether wanting. Tapken, however, saw a bull which, in attempting to cover a cow at grass, received several injuries to the penis. Recovery occurred in twelve days.

In dogs the condition is favoured by the presence of long hair's around the prepuce, and it is often seen after coitus. Should the hairs become adherent to the penis during erection, their ends are apt to be afterwards drawn back into the prepuce. The edge of the latter is rolled round its outer surface, is turned inwards, and the further return of the penis prevented. The glans then begins to swell, which, of course, aggravates matters. Swelling of the glans from inflammation or new growths may also produce the condition. Bang found a ribbon twisted round the penis of a dog; the parts were much swollen.

The glans is exposed, and shows more or less oedematous swelling; its surface is shining, bright red, sometimes of a darker tint, often covered with dirt and dust, and not infrequently dry. The prepuce is tightly applied behind the swollen glans, strangulating it. When the disease has existed for some time, the parts may be injured or ulcerated. Haubner once had to remove the glans and os penis on account of necrosis; recovery followed in fourteen days. Apart from those caused by malignant new growths about the glans or by severe wounds to the penis 
the greater number of cases progress favourably without very elaborate precautions.

Prognosis depends on the nature of the disease. Paralysis of the penis or of the retractor penis are generally incurable. Only those forms occurring during severe infectious diseases disappear with the lapse of time. The longer, therefore, the condition has existed, the less the chance of recovery. Swelling of the prepuce is often very obstinate, and old standing cases are most difficult of treatment. Hard, firm swellings are less easy to get rid of than those which are soft and cedematous.

Treatment. After thoroughly cleansing the glans and prepuce, the displaced hair must be withdrawn or cut off with scissors. A few drops of oil can then be placed on the glans and strangulated prepuce, the animal placed on its back, the prepuce grasped on each side with the forefinger and thumb, and attempts made to draw it over the glans. The attempt generally succeeds; slight pressure with the finger on the glans is sometimes of assistance. Failing reposition in this way, the lower border of the prepuce may be laid open, when the penis will return of itself. Any subsequent inflammation is combated by bathing with solution of acetate of zinc, alum, \&c.; this is, however, seldom necessary.

Inflammation of the prepuce is to be treated as already described. Should the outer coats of the sheath be already contracted, they may be divided. Application of a suspensory bandage will assist absorption and removal of cedema, and is also useful in swelling of the prepuce. The bandage is formed of a triangular piece of linen similar to that used in dressing the wound after castration of cryptorchids. One angle lies on either side of the penis, and is continued by a broad strip of linen, which is passed over the animal's back and tied in a knot. The angle of the bandage, directed backwards, is stitched to two pieces of linen, which are passed between the hind legs, brought upward and forward, and finally united with the transverse strips.

It is advisable to place a pad of tow, jute, or wadding next the penis to prevent injuries. The pad must be renewed when soiled with urine, and the linen cleansed or replaced from time to time. The pad may be soaked in acetate of lead or alum solution, and occasionally moistened with the same fluids.

In old painless swellings, especially swellings of the prepuce, massage is recommended. The swelling can be kneaded daily for five to ten minutes at a time; but to avoid injury, the parts should previously be smeared with fat. Attempts can then be made to return the penis, and if it again prolapses it may be held in position by passing a couple of stitches through the sheath. 
To lessen the size of the glans and thus facilitate its return, the elastic bandage may be used. The parts are cleansed, several turns of the bandage applied to the glans, and left in position for a few minutes. In very severe swellings this may need to be repeated two or three times. The glans is then either drawn back or, after being laved with cold water, may be returned to the prepuce. This method can be recommended.

In case the preputial swelling still persists, benefit sometimes results from scarification and bathing with such astringents as solution of alum or acetate of lead. Needless to say, a suspensory bandage must be worn during massage treatment. Failing improvement by any of these methods, a portion of the prepuce may be amputated; this operation has repeatedly been performed with the best results. The animal is placed on its back, the penis drawn forwards as far as possible, the prepuce cleansed, and the preputial swelling isolated by multiple silk ligatures in the manner usual in ruptures and new growths; the swelling itself is then removed with knife or scissors. Haase and others have frequently seen the best results from this treatment; the penis remained in position, and the inflammation consequent on operation was so slight as to require no treatment.

In paralysis of the penis, massage of the retractor penis muscle between the anus and scrotum may be tried; injections of veratrin, strychnine, or similar nerve stimulants might possibly prove useful. The paralysed penis is shielded from external injury by applying a suspensory bandage. In case of need, it can be amputated.

In the horse, injuries to the penis are most frequently caused during prolapsus by accidental blows, as with the whip. Extensive wounds are sometimes caused in stallions during coitus. In bulls, injuries may likewise be caused by the penis failing to enter the vagina and striking against the ischia of the cow. In such cases laceration sometimes takes place, the corpora cavernosa are ruptured, bleeding occurs, and marked bending and distortion ensue; the condition has also been seen in the horse. Similar injuries have also been caused by ignorant castrators applying clams to the penis, instead of the testicle. Lambert and Kobert report such cases, in one of which the testicle lay in the abdomen. As the horse suffered from colic on the following night, and made fruitless efforts to pass urine, the clams were removed, and recovery occurred, but amputation of the penis was found necessary. In dogs, injuries of the penis are caused by bites and violently separating the animals during coitus.

The first serious symptom which occurs is difficulty in urination. Either the injury itself or the inflammatory swelling consequent on it compresses or displaces the urethra and produces obstruction. Such injuries sometimes produce cicatricial strictures. Lacerations of the 
penis generally do well, that is, the distortion or bending disappears along with the inflammatory symptoms, though cases have been seen where it persisted, and rendered the animal useless for stud purposes. Gallimore reports a case where the end of the penis no longer became erect.

Treatment must be based on general principles. To assist circulation, and repair and prevent the wound becoming soiled, a suspensory bandage is employed, and may be made the vehicle for the application of drugs, cold astringent lotions, creolin, \&c.; the swelling which usually remains will thus be most rapidly removed, and for the same purpose massage will be found of service.

\section{III.--TUMOURS ON THE SHEATH AND PENIS. AMPUTATION OF THE PENIS.}

Fibromata. (warts) are often seen on the horse's sheath; according to my own experience, carcinomata also occur here, and melano-sarcomata and melano-carcinomata have been reported. Piovesau removed a fibrosarcoma as large as a child's head from a stallion's sheath. Warts occur on the prepuce of the dog and of other animals, not infrequently in great numbers. They are generally situated on the inner folds, but frequently on the penis also. Whether condylomata occur here, as Bayer believes, seems questionable.

The simultaneous appearance of warts on the mouth does not prove the specific nature of the new growths, because warts are also frequent about the lips. Cadiot removed a large papilloma weighing $12 \mathrm{lbs}$. from the glans penis of a dog by amputating the penis itself with an elastic ligature. Francesco used the écraseur to remove an ulcerating angioma. Fellenberg saw a "linobby" swelling on the penis of the dog; - Rubinski found in this situation cancer in geldings; Laurent a melanoma weighing about $50 \mathrm{lbs}$. in a horse; Johne and Eber primary tuberculosis in an ox. Schwenk removed a papilloma from the glans of a stallion; it had interfered with copulation.

The significance of these conditions depends chiefly on their nature and extent, which require to be carefully considered in delivering a prognosis. I'umours on the prepuce rapidly produce stenosis and phimosis or paraphimosis; they may also press on the urethra, or attack its walls and impede urination, whilst malignant tumours lead to disseminated secondary growths.

Treatment. Pedunculated warts are snipped off with scissors, any slight bleeding being checked with the cautery or with lunar caustic, which at the same time tends to prevent reproduction. Large pedunculated tumours may be ligatured or removed with the écraseur. The 
elastic ligature is also recommended. Warts and other benignant tumours with broad bases may, unless very large, be destroyed by irritants such as chromic, nitric, or sulphuric acids; the larger, and particularly those suspected to be malignant, are better excised. Malignant growths on the penis may necessitate amputation, to save the animal or to render it capable of work for some time.

\section{AMPUTATION OF THE PENIS.}

The greater part of the penis consists of the corpora cavernosa, which, together with the urethra and blood-vessels, are surrounded by the firm fibrous tunica albuginea. At the lower border of the penis lies the urethra in a furrow, surrounded by the corpus spongiosum, cavernous bodies and muscles. The arteria and vena dorsalis penis form the large blood-vessels. In dogs the os penis must also be considered in operating.

In amputation of the penis, three factors are to be reckoned with, viz. :-

(1) The bleeding.

(2) The after-contraction of the urethra.

(3) The pain.

Amputation may be rendered necessary, as described, by the presence of tumours, by paralysis, and by inflammation and necrosis of the penis. The following methods have been proposed:-

(1) Ligation. This gives the greatest security against bleeding, is, however, very painful, and requires particular care to preserve the urethra. To prevent its occlusion from pressure of the ligature, the urethra must previously be dissected out, or a metal tube thrust into it to keep it open. The elastic ligature is particularly valuable, and will be considered later. Hertwig recommends the castrating loop. By daily tightening the cord, the penis is cut through in from six to eight days. When the ligature has to be applied high up the prepuce must previously be laid open.

Ligation is, of course, useless in dogs over the region of the os penis.

(2) The actual cautery. To prevent bleeding, a knife-shaped cautery has been recommended. Its application is very painful, but the pain may be avoided by narcosis. The urethra is exposed, and the penis cut through with the cautery. In order to fix it conveniently, two ligatures are passed around it, and the division made between them; particular care is required in severing the dorsal vessels. Nocard recommends the galvano-cautery for small animals, though, as it is a very expensive piece of apparatus, it is not always to be found even in large cliniques.

(3) Simple section. It has often been remarked that even in large animals simple section produces no dangerous bleeding. Some operators 
first ligature the dorsal vessels, others cut through them at once; Barthélémy and Charlot operated in this way in the horse, Fellenberg in the dog. Two ligatures are applied, between which the amputation is made; the upper ligature prevents bleeding. That even this is not always necessary is shown by a case of Hunting's, where a pony's penis was simply cut through, the stump compressed by an assistant for half an hour, and no particular bleeding ensued. If the horse is cast for operation, that portion of the prepuce covering the penis may be drawn together with sutures in front of the stump. Haase operated in this way, using catgut, and states having had healing by primary intention. In such cases it is necessary to expose the urethra, and divide its mucous membrane, which is stitched to the tissues on either side to prevent stenosis.

If the animal is cast, the actual cautery may be employed after section to check bleeding. It is better to ligature each bleeding vessel separately, and in the horse to draw the prepuce over the stump and suture it in that position.

(4) The écraseur. This method and removal by galvano-cautery or ligature are the most painful, but the operation only lasts a short time, and an anæsthetic can be given.

After cleansing the penis and sheath, a piece of tape is tied around the end of the former to furnish a good hold. The urethra is then dissected out for 1 to $1 \frac{1}{2}$ inches and cut through, after which the écraseur is applied at the point to be divided and slowly turned. There is little resistance whilst the corpora cavernosa are being compressed and cut through, but towards the end of the operation it becomes greater, because the tunica albuginea is then undergoing division, and at this period the chain may occasionally be broken. In such cases one may pass a ligature around the remainder, and simply remove the end of the penis with a knife; experience has shown the operation to be perfectly successful, and healing has occurred rapidly and uninterruptedly. Möller always operates in this way. Not only is injury to the instrument avoided, but bleeding is very slight and the wound heals rapidly. The urethra may be sutured to the tunica albuginea, and if sterilised catgut or silk be used its edges soon become adherent, and the danger of stricture is reduced to a minimum.

Nevertheless, whatever method be employed, strictures form the most frequent and most troublesome sequelæ of amputation of the penis.

The insertion of a metal tube in the urethra during the first week or two certainly obviates difficulty in urination consequent on inflammatory swelling, but later it operates in exactly the opposite way, and it is, therefore, better to avoid such tubes. Stricture may even lead to rupture of the bladder, as Cagny has shown. 
Contraction of the urethra may be avoided by one of the two following methods.

The first consists in cutting through with the bistoury or theimocautery all the tissues of the penis except the urethra, which, after having been carefully dissected out, is divided about three-quarters of an inch nearer the free end. This kind of artificial urethral tube is afterwards divided vertically and transversely, so as to form four flaps, each of which is fixed to the wound surface by means of a suture. In the other method, derived from human surgery, where it was first practised by Richet and Ricord, and afterwards skilfully modified by M. Guyon, a reversed V-shaped incision is made immediately above the line of amputation, and on the lower surface of the penis, the skin

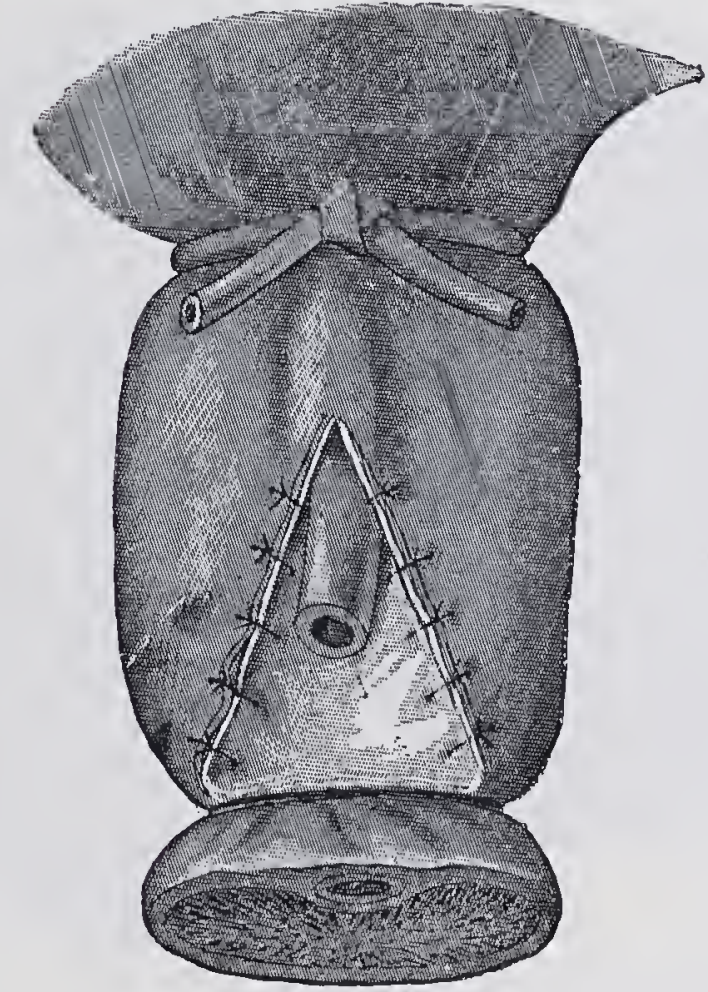

Fig. 172.-Amputation of the penis by the elastic ligature.

being first divided, then the subjacent tissues, the suspensory ligaments, corpora cavernosa, and erectile tissue; the urethra is next cut through transversely, opposite the base of the $\mathbf{V}$, its inferior surface dissected free from all the exposed tissues, the edges of which are afterwards sutured to those of the cutaneous wound; lastly, the penis is cut through opposite the point where the urethra was divided, and the chief vessels closed by means of ligatures or forceps. Or, again, a preferable method, so far as checking hæmorrhage is concerned, consists in applying an elastic ligature opposite the base of the wound, and amputating the penis an inch or so below.

The procedure is as follows :-

The animal having been cast on the left side, the right hind limb is lifted, carried forward, and fixed to the corresponding forearm, as in castration; the penis is disinfected, together with the posterior abdominal and scrotal regions. 
Having introduced a catheter into the urethra for a distance of 10 to 12 inches, an assistant covers the free portion of the penis with a cloth, and draws it gently forwards. Another assistant, placed behind the patient, draws the skin covering the base of the penis towards the perineum. A ligature is then applied to the base of the penis.

A little above the point where amputation is to be performed two lines, starting from above the urethra and diverging towards their free ends (an inverted $\bigvee$ in fact), are traced on the inferior surface of the penis, the, extremities being about $1 \frac{1}{4}$ to $1 \frac{1}{2}$ inches apart. The base of these incisions is united by a transverse incision, and the triangle of skin thus delimited is removed. The subjacent tissues are then excised, layer by layer, over the space covered by this wound until the urethra is

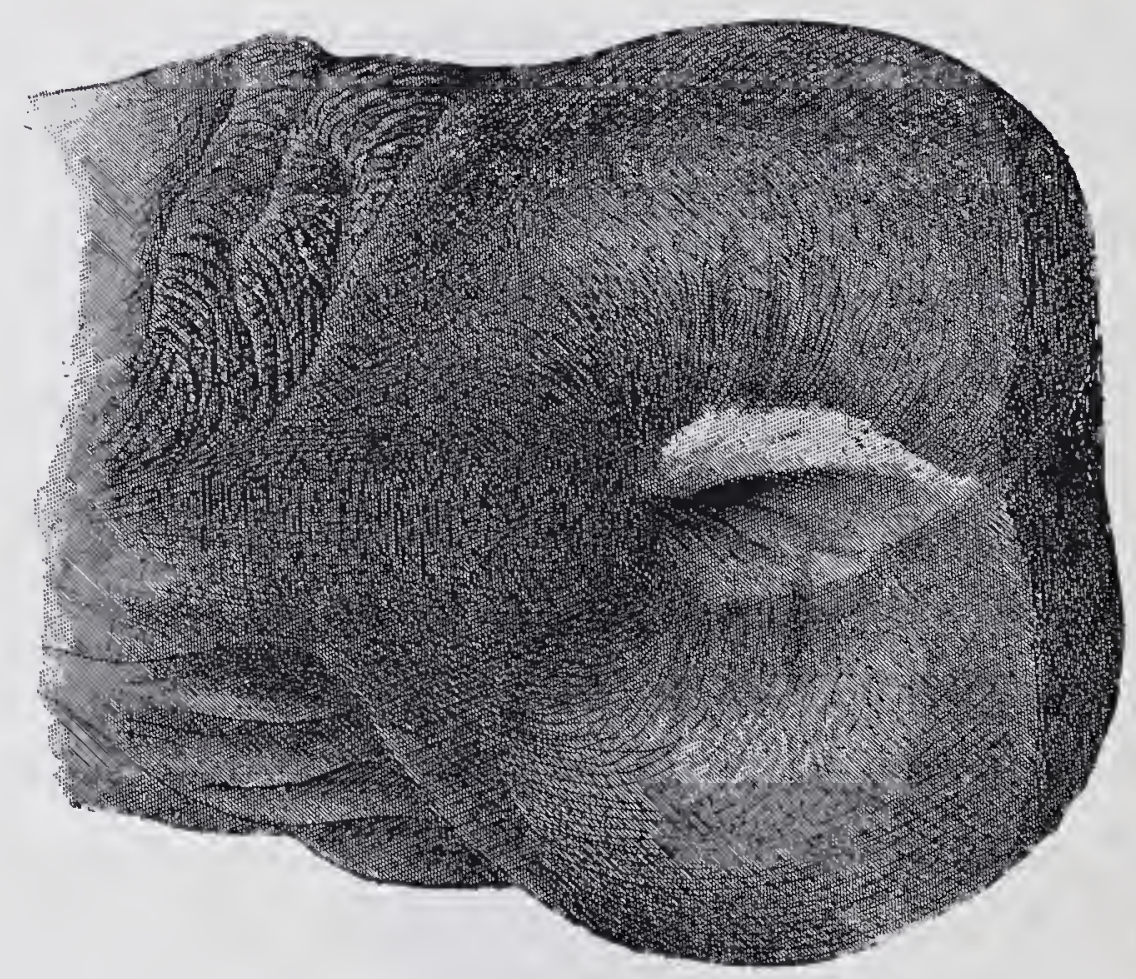

Fig. 173.-Amputation of the penis showing stump and urethral orifice.

exposed. This is opened at the lower margin of the wound by a transverse section. The catheter is then removed, and a grooved director passed into the exposed end of the divided urethra, the groove being directed towards the lower surface of the tube, and the urethra is divided with the bistoury along its middle line throughout the entire length of the part exposed. It is next divided transversely, and each flap of the mucous membrane of the urethra united to the corresponding flap of the skin of the penis by silk sutures. The operation is completed by applying, just at the base of the wound, four or five turns of a tightlystretched rubber cord. The ends of the cord are fastened together, and the penis divided an inch or so below with a single cut (fig. 172).

With the method referred to a mass of dead tissue remains for several days adherent to the end of the penis. Any risk of infection is prevented by antiseptic irrigation. The dead mass and ligature fall away between the sixth and tenth days. The stump is then usually 
much tumefied, but the swelling and odema rapidly diminish. The wound suppurates little. A layer of granulations soon covers it, becomes hard, and contracts, gradually drawing the skin over the stump until towards the end of the third month, the cicatrix is comparatively small. As for the muco-cutaneous wound, when the sutures hold, and the mucous membrane does not cut through, the apposed lips rapidly unite. Most frequently, however, the mucous membrane cuts through at one or more points and becomes separated from the skin; vegetations occur on the exposed tissues, often becoming so abundant as partially to obstruct the meatus. There is, however, no cause for alarm. Excessive granulation soon ceases, the parts heal; like the wound on the extremity of the penis, cicatricial contraction results in drawing the mucous membrane towards the skin, and the urethral opening resumes and permanently preserves the shape given to it by the operator. In both cases the final result is the same; when the surgeon has done his part well, patency of the urethral opening is ensured. As soon as inflammatory symptoms disappear, the animal stales with the same ease as any other horse.

Of five cases operated on in the Alfort clinique, during a period of two years, not one showed after-contraction; and in those treated between 1890 and 1895 the results-except for hæmorrhage-were not less satisfactory.

A case of necrosis of the penis in the dog, successfully treated by operation, is described in Cadiot and Dollar's "Clinical Veterinary Medicine and Surgery," p. 409. For cases in the horse, see pp. 411, 412, and 413 loc. cit.

In dogs, amputation can be effected either behind the os penis by using the knife or écraseur, or the bone can be sawn through. After the latter method strictures and closure of the urethra are certainly commoner, though, in my hands, amputation behind the os penis by ligaturing blood-vessels, and exposing and sewing the urethra to the neighbouring parts, and amputation by sawing through the os penis, have been equally successful. A dog operated on in the latter manner died a year later from disease of the bladder. On post-mortem, the stump was found cicatrised without any considerable stricture of the urethra. After-treatment is in all methods the same, and consists in washing or syringing out the sheath with disinfecting fluids.

\section{IV.-DISEASES OF THE PROSTATE.}

In the horse the prostate gland consists of two lobes about 3 inches long and 2 broad. The gland is little developed in oxen and swine, but is large in carnivora, in which it appears to surround the urethra. In 
animals disease of this gland is much rarer than in man, but both acute inflammation and abscesses occur, and chronic thickening, with formation of new growths, has been seen in dogs. The first clearly results from inflammation spreading from the urethra. Haubner opened an abscess in the gland through the urinary passages; Reinemann (in a steer) operated from the anus. Bang saw acute prostatitis in the horse; Frauenholz tuberculosis of the gland in oxen.

Hypertrophy of the prostate, which often occurs in elderly people, and consists of new growth of glandular and muscular connective tissue, is, amongst animals, almost entirely confined to dogs, and even in them is seldom seen; Forster has, however, examined several cases of the kind. Sickert made the post-mortem of a horse in which the prostate weighed $2 \frac{1}{2} \mathrm{lbs}$.

Tumours are common in the prostate ; Cadiot found cancer.

In sheep, Gurlt described a. so-called prostatic calculus, consisting partly of a precipitate from the secretion of the gland, partly of gravel from the bladder.

The gravity of these conditions depends on the fact that the urethra is pressed upon and urination rendered difficult. Acute inflammation may extend to the urinary passages, cause disease of the urethra, and prove fatal from pyelonephritis.

Symptoms and progress. Difficulty in defæcation and urination first directs attention to the disease. T'he animals strain, sometimes without result, sometimes with the discharge of urine in a thin stream or in drops, sometimes urine is discharged involuntarily. If in dogs thus suffering urethral calculus is absent, a rectal examination must be made, when the swollen prostate will be felt close behind the bladder. When acutely inflamed, the swelling is soft and painful, but in chronic conditions it is hard and painless. Prostatic calculi sometimes cause the gland to feel almost like a shot-pouch. Abscesses occasionally discharge into the urinary passages under the pressure of the finger. Haubner examined a dog in which the prostate gland was as large as a hen's egg; pressure caused the discharge of a purulent, ill-smelling fluid through the urethra, after which recovery occurred; but in the case noted by Reinemann in a steer the animal died from cystitis.

Hypertrophy of the prostate and tumours generally cause death by interfering with urination, or they may necessitate slaughter of the animal. Linéaux saw cases of hypertrophy of the prostate in dogs; in one there was intermittent bleeding and difficulty in urination; in the other these symptoms were accompanied by secondary cystitis and hydronephrosis. Buth cases ended fatally. A third case was complicated with perineal hernia; this also died.

Treatment. In acute cases, laxatives and clysters remove the pressure 
on the urethra resulting from a distended rectum. Abscesses in the gland can be evacuated through the rectum. Disinfectant fluids may be injected through the catheter. In chronic disease in man extirpation has been practised. Castration was warmly recommended as a cure for enlarged prostate in man by $J$. William White, of Philadelphia. A number of cases were operated on with marked success, cases where the catheter had been used for year's showing immediate improvement, and patients being able to urinate without assistance in five days after operation. Nevertheless, final judgment should be reserved, though the operation is more likely to prove satisfactory in dogs, which occasionally suffer from enlarged prostate, than in men.

R. H. Clarke operated on an eight year old bull dog, in which the prostate had become enlarged to the size of a billiard ball, a portion as large as a walnut being ossified. The symptoms consisted chiefly in straining as if to pass frees, and were only marked for three months before death. Mr. Clarke purposed forming a perineal fistula, but as the conditions appeared unfavourable when operation was advanced, the dog was killed with chloroform. The description of symptoms, treatment, and especially of the post-mortem examination and microscopical appearances of the growth, are fully given in Cadiot and Dollar's "Clinical Veterinary Medicine and Surgery," p. 391.

\section{Y.-DISEASES OF THE SCROTUM.}

(a) Apart from those caused by operative interference, injuries, wounds, and bruises are comparatively rare in animals, in consequence of the sheltered position of the scrotum. In dogs, however, the scrotum is sometimes bitten, and injuries may be caused in other animals by fragments of glass and wood, and, during attempts to jump hedges, by thorns. In horses the scrotum is sometimes injured by the carriage-pole, and decubital gangrene readily occurs in dogs after lying long in one position, as during paraplegia or distemper. In delivering a prognosis, it should be particularly noted whether or not the injuries are of a penetrating character, and have affected the testicle. By penetrating wounds are meant those which extend to the processus vaginalis or peritoneal cavity. But even in penetrating wounds the prognosis and chance of recovery are favourable, provided there is no diffuse peritonitis, though the testicle can seldom be saved, on account of the danger of infection extending to the peritoneum. Severe bruises and injuries followed by extensive gangrene must be viewed as dangerous. Superficial wounds, on the other hand, generally heal readily.

Treatment. The parts are first thoroughly cleansed, and then carefully examined, to determine the area of the injury. If the testicle is to be saved, strict antiseptic treatment will be required. In large 
animals the suspensory bandage already described should be used. In deep wounds penetrating the processus vaginalis, pus formation and peritonitis are likely to ensue unless the parts are at once rendered aseptic. Castration is often necessary, and in such case the scrotum should be freely divided to give the freest possible exit to discharge, otherwise the usual principles are followed. Superficial injuries are treated like similar wounds in other parts.

(b) Inflammation of the scrotum occurs in northern countries, from travelling bulls in snow-drifts. On reaching the stable the skin of the scrotum becomes red and swollen, and the folds disappear. Exudation soon sets in, and superficial necrosis of the skin may take place.

Though the course of the disease is almost always favourable, yet at times a troublesome eczema remains, which bids defiance to treatment, especially when the discharge is profuse. When large areas of the scrotal skin have sloughed, cicatrisation occurs with difficulty.

In exceptional instances, inflammation extends to the scrotum from neighbouring structures. Rainard saw such a case follow puncture of the bowel.

In dogs an acute eczema sometimes extends over the entire scrotum, and is characterised by swelling, reddening of the skin, exudation, pain or itchiness, causing the animals continually to lick the parts, and thus interfere with treatment and healing. The swelling sometimes causes a peculiar straddling gait.

Treatment of frost-bite of the scrotum consists in bathing the parts with disinfectants and applying dry dressings. Wheat flour, tannic acid, or tannic acid and iodoform are all useful. In eczema the powder may consist of lycopodium, tannin, and iodoform, which will prevent the animal licking the diseased spot. When exudation is not excessive, the parts may be brushed over with iodoform collodion with advantage; cocain might also be tried.

(c) New growths are not infrequent in the skin of the scrotum. Rosenbaum found a swelling on the scrotum of a steer, and Eberhardt describes a tumour nearly 16 inches in length in an eighteen months bull. The nature of these new growths cannot, however, be ascertained from the description given. Fröhner cured a case of bothryomycosis of the scrotum in a horse by operation. Varicose conditions of the slin in $\operatorname{dog}$ (diffuse dilatation of the veins) have been described, attended with ulceration of the skin, profuse bleeding, and a tendency to recur.

In dogs, a pendulous condition of the scrotum and failure to retract the testicles when the scrotum is grasped is a grave symptom seen near the termination of severe internal diseases, and may sometimes be observed in other animals.

The treatment is almost self-evident. New growths, unless possessing 
too broad a base and extending to the tunica vaginalis, are generally easily removable. The mobility of the tumour gives sufficient indication on this point. If needful, the entire scrotum can be removed along with the testicles without danger, as the primitive methods of castrating ruminants clearly show.

Various parasites, which also occur in the peritoneal cavity, are seen in the scrotum and tunica vaginalis, such as Sclerostomum armatum, Filaria papillosa, \&c. (Gresswell). They have no particular significance, and are accidentally encountered in castration or when making postmortems.

\section{VI.-DISEASES OF THE TESTICLE. CASTRATION OF CRYPTORCHIDS. ${ }^{1}$}

\section{(1.) CONGENITAL DEFECTS AND RETENTION OF THE TESTICLES.}

In the horse and dog, less frequently in other species, the testicles are sometimes either absent or in a state of rudimentary development. Leisering found the testicles of a stallion which had ineffectually covered forty mares almost normal in size but flabby in texture wanting the tense normal character. Their arteries were distended, their connective tissue thickened, the semen watery, transparent, and containing many round cells, but only isolated spermatozoa. Testicles which have been retained in the abdominal cavity often show similar appearances. This condition (retentio testis) is not infrequent in stallions of the coarse, heavy variety, but is also seen in other animals. Leisering and Gurlt found the testicles of a dog still in the abdominal cavity, Preusse has seen the same thing in pigs, and Kaiser in bulls. Imminger considers the cryptorchid condition as common in bulls as in horses, and he was able to establish the hereditary character of the condition in certain cases. This abnormality is termed retentio abdominalis when the testicle lies near the upper wall of the abdomen, retentio iliaca when it is near the inner abdominal ring, and retentio inguinalis when it is within the inguinal canal. The apparent absence of one or both testicles thus produced is termed monorchismus or cryptorchismus. During the first few months of life in the foal the testicles certainly lie in the inguinal canal, but towards the end of the first year they again descend into the scrotum.

Gurlt saw a horse in which the testicles occupied a very rare position, viz., in contact with, and adherent to, the diaphragm. Sometimes they

1 ['This section has been retained as being perhaps useful to students. Those who desire a fuller treatment of the subject are referred to my "Operative Technique," the first volume of this series, pages 237 to 250 , where the varions operations are fully illustrated.-JNO. A. W. D.] 
lie outside the abdominal cavity, but not in the scrotum (ectopia of the testicle); thus the testicles have been found below the diaphragm or in the crural canal : the first condition is termed ectopia abdominalis, the latter ectopia cruralis. In dogs one or both testicles have been found lying beneath the skin next the glans penis; in a bull, one testicle was met with in the subcutis of the flank.

Supernumerary testicles are said to have been seen in horses and mules. Cox found three testicles in a certain horse, and Olivier as many as four in a mule. The accuracy of these observations may, however, be questioned, as a thorough description of the supernumerary organs is wanting, and one cannot help thinking that these were cases of mistaken diagnosis, or that the supposed accessory testicle was really the epididymis which, in cryptorchids, sometimes lies in the inguinal canal, whence it is removed, while the testicle remains behind in the abdomen.

Absence of the testicles (anorchidism) is extremely rare. Mauri describes an attempted operation on a cryptorchid in which the testicles seemed to be absent.

The significance of all these conditions is self-evident. Both anorchidismus and defective development of both testicles makes the animal useless for stud purposes. The same is usually also true of cryptorchismus, for testicles when retained in the abdominal cavity are generally atrophic, lax, and either contain degenerated spermatozoa, or none at all; on the other hand, more than one operator has removed retained testicles which yielded abundance of spermatozoa. The question of the fertility of cryptorchids was first raised by Gurlt when studying the function of the spermatozoa: his experiments seem to deny fertilising power under such circumstances, for he was unable to discover spermatozoa in the retained testicle. Since then the same question has been variously answered. Peters considers such animals not fertile, though quite capable of coitus. Wesche, on the other hand, states having seen fertile cryptorchids ; he refers, however, to a case of cryptorchismus inguinalis. A final answer can scarcely be given. The animal's fertility clearly depends on the development of the testicles. The great majority of retained testicles certainly appear degenerated, and contain no spermatozoa, though many persons of acknowledged authority describe having removed from the abdominal cavity testicles which were fully developed and contained numerous motile spermatozoa. Paugoué speaks of a stallion in which both testicles were retained, and whose progeny numbered amongst them five cryptorchids or monorchids; thus apparently proving the condition to be hereditary.

The retained testicle is often the seat of disease and not infrequently of malignant new growths like sarcoma, carcinoma, \&c. Leisering, in the 
case of a dog, found the retained testicle attacked with cancer ; the same condition has been seen in horses. In man, such testicles still more frequently become diseased, so that early removal is generally necessary.

The same necessity does not exist in the horse, though removal often becomes desirable on account of sexual excitement (particularly in spring), often greatly interfering with the animal's usefulness. Many cryptorchids are too vicious for use. The flesh of cryptorchid swine has usually a repulsive taste, which, according to Koch, is retained even by the salted meat, and is more intense the more completely the testicles have developed.

Castration of cryptorchids requires some practice and careful antisepsis. Since it has been so much practised by Stockfleth and Nielsen in Denmark and Degive in Belgium, Bang and Cadiot have taken it up. At one time Hering considered it altogether unjustifiable. With antiseptic precautions the operation loses much of its danger, and is often of great service.

In former times, and occasionally even of late years, the flank operation was recommended, but almost all surgeons now operate by the inguinal canal. In large cliniques special arrangements are possible, but when operating in the country a field is usually the most convenient spot to select. The straw used as a bed should be spread out the evening before to prevent causing dust. The operation is easiest in horses which have been kept low for some weeks and are in thin condition. Some days before operation a dose of physic is given to empty the bowel, and from that time the animal receives only a limited amount of concentrated food, like oats, with a little hay, but no straw.

Thus prepared, the horse is cast on the side opposite the seat of operation; the hind-quarter's are half-rolled over, and lie somewhat higher than the fore. 'The upper hind-foot is then drawn forward and fixed in a position of abduction and flexion, as in ordinary castration. By previously cleansing the hoofs and surrounding them with moist cloths, and by moistening the hair on the upper part of the thigh, the point of operation is protected against infection.

Three dishes, each containing 2 to 3 per cent. carbolic solution, are necessary. The instruments are placed in one, the ligatures and needles in the second, the third is used by the operator for moistening his hands before introducing them into the abdominal cavity; in a fourth empty dish, pledgets of tow are in readiness. It is also well to have at hand a vessel with a lip, to use as an irrigator.

All being ready, the animal is chloroformed, during which time the seat of operation is washed with soap and disinfected with carbolic or sublimate solution. As soon as narcosis is complete, the assistants, holding the dishes, place themselves near the operator, who has carefully 
disinfected his hands and thrust his sleeves back above the elbow. The position is the same as in castration. The operation is divided into the following stages :-

(1) Incision. An incision is made through the skin, about 2 to 3 inches long, over the outer inguinal ring (perhaps slightly nearer the middle line) and parallel with it, and the subcutaneous tissue and fascia lying at this point are divided to the same extent. Any bleeding vessels. are at once ligatured, and blood removed with sterilised pledgets of tow.

(2) Opening the inguinal canal. The loose connective tissue in the inguinal canal must now be thrust on one side, both index fingers and possibly the thumbs being employed, and an entrance effected in the direction of the inner abdominal ring. The index and middle fingers of the hand corresponding to that particular side of the animal are then passed into the inguinal canal to make sure whether the processus vaginalis and remainder of the spermatic cord lie there. Should the testicle be met with at this stage (retentio inguinalis), the operation becomes very simple, being, in fact, just like ordinary castration. Sometimes a more or less degenerated process of the tunica vaginalis, perhaps as large as the finger of a glove, together with the end of the epididymis, may be felt in the inguinal canal. In that case the inner abdominal ring must be examined by introducing the index finger, and its width discovered. Generally it is very narrow, and would not even permit the passage of the rudimentary testicle. As it is difficult to dilate, it is better in such cases to ignore the process of the tunica vaginalis, and seek an entrance to the abdominal cavity alongside of it. When the inner abdominal ring appears wider, the processus vaginalis is to be divided, and the operation continued as after perforation of the abdominal walls.

(3) Perforation of the abdominal walls. By passing the fingers, nearly to the last knuckle, into the inguinal canal, the abdominal wall can be felt in the depths. The posterior border of the obliquus abdominis and the fibres of the obliquus internus can be clearly distinguished. Close behind the latter is a mass of loose connective tissue. Degive makes his perforation at this point. Bang prefers entering through the inner oblique abdominal muscle at the inner wall of the inguinal canal, somewhat nearer the median line than the inner abdominal ring. This produces a "button-hole wound," which does not gape, but, on the contrary, soon comes together again and prevents prolapse of the bowel. A vigorous thrust with the index and middle fingers during inspiration carries them through the abdominal wall into the peritoneal cavity. Different animals, however, present great differences in this respect; in some perforation is easy, in others it requires considerable force. 
(4) Finding the testicle or spermatic cord is always the most difficult part of the operation for the unpractised; nevertheless, it can generally be effected with the two first fingers, and without introducing the entire hand. As soon as the abdominal wall is perforated, search is made on all sides for the testicle, epididymis, or spermatic cord. The testicle may easily be mistaken for an empty loop of bowel, but is distinguished by its sharper outline and greater firmness; pressure on it causes struggling. The end of the epididymis is recognised as a soft mass, containing harder, firmer cords. The spermatic duct can be felt as a hard cord, and is very useful for discovering and drawing forward the testicle, for its slight size and thread-like hardness render it easily recognised. It can, moreover, be brought forward with much less difficulty than the testicle itself, which, when grasped and pulled on, causes struggling. The object to seek, then, is a very small body, possessing a hard, string-like cord (spermatic cord). As a large mesenteric artery might be mistaken for it, the cord should be examined to detect pulsation. It is much easier to discover the spermatic cord than the testicle. When drawn forward, the spermatic cord is recognised by its light colour. By gently pulling on it the testicle is drawn upwards through the opening, though, in exceptional cases, the testicle may be so large as to offer considerable resistance. Difficulty is sometimes experienced when the spermatic cord is grasped above the epididymis, and the epididymis itself is found to be firmly united to a rudimentary tunica vaginalis, which cannot pass the inner abdominal ring. In such case the testicle cannot be drawn forward by pulling either the upper or lower parts of the loop, and the operator is forced to search for a still lower portion of the cord or to use the écraseur. As soon as the testicle appears outside the wound, two, or if it be very large three, carefully disinfected catgut or silk ligatures are passed round the spermatic cord, which is then cut off half an inch below the point of ligation, and allowed to return to the inguinal canal. The wound is closed with 5-8 threads of silk or hemp powdered with iodoform-tannin (1-3), and covered with a layer of wood-wool or carbolic wadding. Only when the spermatic cord or wound has been soiled is it necessary to wash out with sublimate solution.

This completes the operation, and the animal can be allowed to rise. Once on its feet, a bandage like the suspensory described under the heading "Paralysis of the Penis" is applied, but may be omitted if the animal be restless. The patient is tied up for four days (except during its daily period of exercise) and is kept on half rations, after which it may be allowed to lie down, still wearing the dressings, which are removed, however, after six to eight days.

As.a rule, little or no pus is formed; but should it appear, the front 
and back stitches in the skin wound may be loosened, and the latter washed out once daily with carbolic, creolin or sublimate solution. Provided fever or loss of appetite is not marked, after-treatment is unnecessary, and the animal may return to work in about fourteen days.

It is sometimes difficult and requires patience to find the testicle. If needful, the other hand can be introduced into the rectum, and attempts made to force the testicle through the opening. Thus exposed, it is secured with a cord, covered with sterilised cotton-wool, the hand which has been introduced into the rectum is at once cleansed, and the operation proceeds as above described.

When the spermatic cord is very short, it renders ligation particularly difficult. Under such circumstances the écraseur must be used.

In one case of Möller's the testicle could not be found. He therefore introduced the entire hand into the abdominal cavity, and discovered a soft body the size of two fists. This he at first took to be the urinary bladder, which further examination showed, however, to be in its normal position, whilst the soft body lay near the inner abdominal ring, was movable, and carried at one end a firm object which resembled a testicle. Convinced that he had to deal with a degenerated testicle, he extended the opening in the skin and abdominal walls sufficiently far to allow the testicle and spermatic cord to be ligatured. After removing the testicle and suturing both the skin and abdominal walls with strong silk (interrupted sutures), recovery occurred without complication.

A closer examination showed that the testicle had almost entirely disappeared, and a cyst containing 16 ounces of serum, and having a circumference of 14 inches, had formed in the spermatic cord. At the lower end of the cord lay a lipoma, about the size of a duck's egg, and partly ossified. This tumour was thought to be the degenerated testicle, until attention was directed by Degive, who has repeatedly seen similar cases in his extensive practice, to hydrocele of the spermatic cord. Degive scratches the hydrocele with the finger-nail until it discharges into the abdominal cavity, when the testicle can easily be removed. Tapken operates on bulls from the left side. The animals are starved for a day or two to prevent the rumen being distended. Tapken sometimes found the testicles just below the spinal column enclosed in a fold of peritoneum.

Castration of cryptorchid boars is similar to that of horses, with the one exception, that a flank incision is preferable. Williams and Tapken castrate from the flank. Tapken recommends operating about the second or third month, and by the same method as is employed in spaying sows; the testicle, however, is sometimes difficult to find. Levens describes a case where the castrator had removed the boar's kidney instead of the testicle, as was discovered on slaughtering the animal. The other kidney had undergone compensatory hypertrophy. Lungwitz, in a boar, found both testicles adherent to one another in the abdominal cavity. Vennerholm operated on dogs by making an incision about an inch on one side of the prepuce, introducing the forefinger and drawing the testicle forward into the wound. The cord was then ligatured. 


\section{(2.) INFLAMMATION OF THE TESTICLE AND EPIDI- DYMIS. ORCHITIS, PERIORCHITIS, EPIDIDYMITIS.}

Inflammation of the testicle is certainly not frequent, though it has been seen in different animals. Inflammation of the epididymis, common in man during gonorrhoea, is seldom seen in animals.

Orchitis sometimes results from external violence, from bruises and injuries, sometimes from inflammation extending from the urinary passages to the spermatic ducts, and thence to the testicles; metastatic inflammation of the testicle is met with in pyæmia, glanders, \&c. Walraff insists on having seen enzootic orchitis with abscess formation in horses and oxen, but nothing specific is known of its cause. Ludwig found in a horse suffering from brain mischief abscess of both testicles; Knese saw a similar affection in pigs. Inflammatory processes and caseous nodules have been found in the testicles of tuberculous horses. Hess describes a case of tuberculosis of the epididymis in a breeding bull. The end of the epididymis formed a swelling as large as a goose's egg, which was distinctly divided from the testicle by a circular depression.

Symptoms and progress. The first symptọm consists in swelling, sometimes slow, sometimes rapid, but always very painful, especially in acute inflammation; the gait is, therefore, often straddling, and sometimes the pain produces symptoms of colic. The scrotum escapes as long as the processus vaginalis remains intact, but, if periorchitis also occur, œedema of the scrotum sets in. Infectious orchitis is always accompanied by fever.

Aseptic inflammation of the testicle resulting from bruises generally takes a favourable course. Bleeding may certainly occur, and produce hæmatocele, but the blood seldom fails to be readily absorbed, and normal function to be restored. But that form of orchitis conveyed through the medium of the urinary organs or produced metastatically often produces pus formation or necrosis. The testicle is especially prone to necrosis, as is seen after subcutaneous ligation of the spermatic cord for castration. In ruminants and horses, calcification and ossification of the testicle have also been seen. These processes, however, are not dangerous to life in the same degree as pus formation, which readily extends to the spermatic cord and produces fatal purulent peritonitis. The commonest result is periorchitis, adhesion of the testicle to the tunica vaginalis, or perforation outwardly through the skin of the scrotum. Hess saw periorchitis plastica hremorrhagica in a sucking-pig and in a stud bull. Periorchitis often results in hydrocele or hrmatocele.

Treatment. Rest is very necessary, especially if serious swelling: interferes with the animal's movements. In such cases a suspensory bandage may be applied. In fresh traumatic orchitis, applications of 
cold water, solution of acetate of lead, or similar fluids may be made. In human medicine, orchitis is treated by compresses of adhesive plaster, which may be tried in larger animals, or in dogs can be replaced by painting the scrotum with collodion. Scarification, though often recommended, is of doubtful value; if only applied to the surface it is without effect, if it reaches the processus vaginalis it is identical with castration. The latter may certainly appear indicated, especially in infectious orchitis, with simultaneous disease of the spermatic cord. Should signs of abscess formation appear in the scrotum, an incision must be made; and if high fever, \&c., result, castration must be resorted to, and can be carried out in the usual way. As far as possible, any diseased portions of spermatic cord should be removed. Hertwig has seen recovery from spontaneous orchitis in the horse after low diet, withdrawal of blood, and the use of laxatives. Mercurial ointment with iodide of potassium and belladonna poultices were applied externally.

\section{(3.) TUMOURS OF THE TESTICLE (SARCOCELE) AND OF THE EPIDIDYMIS.}

Since olden times every abnormal swelling of the testicle has been termed sarcocele. The condition is seen in various domesticated animals. In swine and cattle tuberculous growths occur in the testicle; in horses sarcomata, carcinomata, myomata, dermoid cysts, with hair and teeth, have been met with, and in dogs both sarcomata and carcinomata. Hess saw tuberculosis of the epididymis in a steer; the end of the epididymis formed a swelling as large as a goose's egg, and was divided from the testicle by a distinct furrow. Schmidt saw tuberculosis of both testicles in a bull; Arens in a boar; the scrotum showed numerous fistulous openings communicating with abscesses containing caseous pus; the two testicles weighed nearly 20 lbs. The horse's testicle, affected with medullary cancer, which Puntigam described, weighed 13 lbs. Lothian found atheromatous cysts in a horse's testicle.

Symptoms and course. One or both testicles slowly increase in size, the swelling appearing painless. Sometimes the surface of the testicle retains its smooth character, sometimes it appears lobulated or linotted. The swelling may become very considerable; at Alfort a horse was seen that had a testicle weighing 20 lbs.

Maligant new growths often spread to the spermatic cord, causing it to swell and become hard, uneven, or lobulated. Where carcinomata exist, the lymph glands of the lumbar region also become swollen, and in the case of carcinoma of the testicle shown in fig 174, they were so greatly enlarged as almost to prevent the hand passing into the abdominal cavity. Provided new growths remain confined to the 
testicle, recovery follows castration, which, so long as if stills appears practicable, should not be delayed if the swelling rapidly increases, or takes on a malignant character, even if the spermatic cord is already affected.

Treatment. In such cases castration is the only resort, and even where the growths are of a benignant character it is to be recommended if they have attained a large size. As the spermatic vessels are often abnormally dilated, clams should be used, as they most surely prevent bleeding. If, however, the upper part of the spermatic cord be much

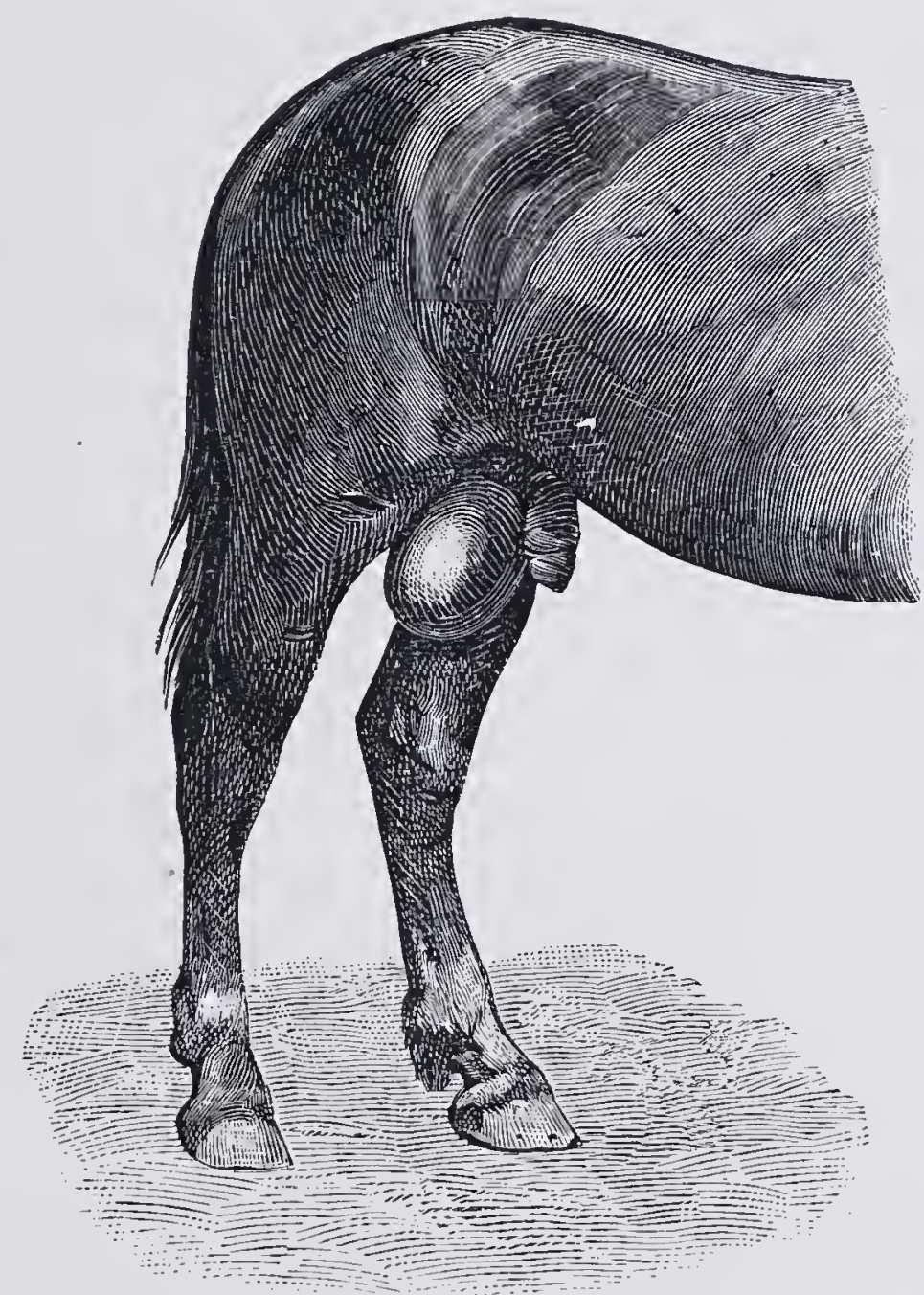

Fig. 174. - Carcinoma of the testicle in a horse. (After photograph.)

diseased, the application of clams is sometimes impossible, and it is better to ligature the cord with catgut, and allow the ligatures to hang from the wound, removing them after five or six days. Should the new growth have already reached the abdominal cavity, the animal must be prepared for slaughter, or worked until advancing disease renders it useless.

\section{VII.-HYDROCELE, HÆIATOCELE, AND VARICOCELE.}

On account of its physical appearances, dropsy of the processus vaginalis has, since olden times, been described as hydrocele, i.e., a watery swelling. Instead of the few drops of fluid usually present, one finds large quantities of serum between the parietal and visceral coats of the tunica 
vaginalis. The condition, therefore, in its nature resembles hydrops ascites, and may result from inflammation of the tunica vaginalis. Sometimes the fluid accumulates in the spermatic cord, and then lies between the folds of the tunica vaginalis propria, a condition which has been termed hydrocele of the spermatic cord.

Both conditions have repeatedly been seen in horses, oxen, and rams. Steiner found hydrocele of the spermatic cord in young geldings; the serous fluid was contained in several small sacculations. Richter has described the same disease in goats and horses. Möller met with hydrocele of the spermatic cord containing more than 16 ounces of a reddish serum in castrating a cryptorchid. This complicated the operation and rendered it much more difficult, but nevertheless it succeeded. Steffen saw hydrocele of the processus vaginalis in a ram; Puntigam noted dilatation of the lymph vessels of the spermatic cord.

Causes. Hydrocele may result from inflammation of the processus vaginalis of the testicle or of the spermatic cord, and may take either an acute or chronic course. In a sucking-pig and a stud bull Hess found not only serum, but fibrin and blood in the tunica vaginalis. External injuries, like bruises, are doubtless sometimes responsible for disease of this character. When they lead to severe bleeding from the spermatic cord or processus vaginalis, we speak of hrematocele. Hydrocele of the processus vaginalis occurs in connection with hydrops ascites and hydrothorax as a local symptom of the general dropsical condition. Martin concluded that the occurrence of filaria papillosa in the dropsical vaginal process was a cause of hydrocele, but this view cannot be upheld, as the parasites are seen in by far the greater number of cases unaccompanied by hydrocele.

Symptoms. Hydrocele with hæmatocele of the processus vaginalis sometimes presents a striking resemblance to inguinal hernia. The swelling is often very marked (Steffen saw a goat in which the scrotum reached nearly to the ground), but is soft, elastic, painless, and free from inflammatory symptoms. The condition is distinguished from rupture by the fact that swelling is confined principally to the lower part of the scrotum; above, the scrotum tapers away, and shows no abnormal distension, because the fluid is easily displaced. By compressing the upper part of the scrotum fluctuation may easily be detected at the base.

In large animals the presence of inguinal hernia can also be determined by examination per anum. In hydrocele of the spermatic cord such examination may be necessary to confirm the diagnosis, as the form of the swelling often greatly resembles that of a hernia. If absolutely necessary, an exploring needle may be passed into the scrotum.

This disease is distinguished from sarcocele by the less firm swelling, and by the smaller size of the testicle, which as a rule appears atrophied. 
Prognosis is favourable in as much as life is not threatened, but recovery with conservation of the testicle is generally doubtful. Early treatment of hæmatocele and hydrocele is often successful ; complication with inguinal hernia renders the condition grave.

Treatment. Velpeau was the first to employ injections of iodine for hydrocele, and they are still used in man. Simple puncture seldom succeeds. Stephen emptied the tunica vaginalis of a ram fourteen times, but it always filled anew; nor was puncture and injection of iodine solution more successful. Antiseptic precautions must, of course, be observed. When employing this method, the tincture of iodine should be freshly prepared, the processus vaginalis emptied of its contents, and the solution injected and allowed to remain. Great swelling follows, but subsides after six to eight days.

Castration is more certain if the diseased portions of the spermatic cord be removed together with the testicles; if properly performed, healing generally results. Operation must, however, be delayed until acute inflammatory symptoms have disappeared. In castrating four sucking-pigs affected with periorchitis and hydrocele, Hess lost two; it was remarlsable that of five of a litter, four suffered from hydrocele.

Varicocele, or cirsocele, consists in abnormal dilatation and lengthening of the veins of the spermatic cord. It is commonest in old animals, but only exceptionally calls for treatment; it is usually only discovered on castration, and even then requires no particular precautions. Aneurysmal varix has been observed in oxen, the spermatic arteries emptying into the spermatic veins. The dilatation of the plexus pampiniformis, associated with varicocele, is sometimes so severe that the scrotum appears as much enlarged as in inguinal hernia, or in the above described conditions. Aneurysmal varix is sometimes denoted by pulsation in the scrotum, or the passage of blood under the skin may be felt; both symptoms disappear, however, immediately the scrotum is compressed above; Meyer was able to feel the artery. Castration is the only treatment, and care is required to prevent rupture of the greatly distended vessels : the vessels can either be ligatured with stout cord, or clams employed.

\section{VIII.-INFLAMIMATION OF THE SPERMATIC CORD. SCIRRHOUS CORD. FUNICULITIS CHRONICA.}

On account of its sheltered position the spermatic cord rarely becomes inflamed, unless the scrotum be opened, though bruising or hyperextension may produce acute inflammation and hrmatocele. It has already been stated that chronic inflammation may occasion hydrocele. But none of these diseases compare in frequency with scirrhous cord, V.s.' 
a condition due to infection of the cord after castration. In horses it is comparatively common, and in oxen by no means unknown.

A portion of the cord is removed in castration, and the remainder then swells, partly in consequence of disturbed circulation, partly of inflammatory irritation. This swollen condition generally disappears completely in eight to fourteen days, sometimes a little later. In horses, and occasionally in oxen and other animals, the disappearance, however, is sometimes incomplete; the swelling increases, and the stump of the spermatic cord may become as large as a man's head. The enlargement may be confined to the extremity, but generally extends further upwards, even beyond the inguinal canal and into the abdominal cavity. Ringk noticed an extraordinary development in a horse, the left spermatic cord on post-mortem weighing $24 \mathrm{lbs}$. and the right $30 \mathrm{lbs}$. In such cases the operative wound refuses to heal; a slight opening remains, from which small quantities of pus are discharged, and through which a probe can be introduced for a distance of 4 to 8 inches into the spermatic cord.

It was long known that the disease was due to chronic inflammation of the spermatic cord, though its cause was not discovered until a comparatively recent date. Rivolta, in 1871, suggested that it consisted in infection with microparasites (discomyces equi), and a short time afterwards Johne independently came to the conclusion that it was a funiculitis actinomycotica. Later, however, he declared micrococci to be the cause, named the disease myliodesmoid or mykofibroma, and ascribed it to infection with a micrococcus, which Johne described as M. ascoformans, and Rabe as M. bothryogenus.

From this it is clear that the disease is of an infectious nature, and may possibly be caused by more than one organism. (The scirrhous cords removed in the Berlin clinique were generally found to contain bothryomyces and staphylococci.) Its varying progress also points to infection. Sometimes the growth and thickening occur rapidly, and the spermatic cord may attain the diameter of a goose's egg in a few months; sometimes the condition may exist for indefinite periods without marked increase in size.

Scirrhous cord is commoner in certain districts, perhaps because the necessary conditions for infection are more favourable; perhaps because the style of castration there adopted favours infection.

The following indirect causes of scirrhous cord are recognised :-

(1) Exposure of the cord after castration, and its projection beyond the tunica vaginalis or skin wound. Sometimes this is the result of applying clams too low, using too heavy clams, dividing the "non-vascular" "portion of the cord before applying the clam, or cutting off the vas deferens. More frequently the spermatic cord is not carefully returned to the processus vaginalis after removing the clams. Pulling on the cord 
or violently tearing away the testicle ruptures the peritoneal folds which support the cord within the abdomen and allows the cord to protrude below the wound. Exposed to the air the cord swells, and sometimes becomes strangulated in the narrow skin incision and in the processus vaginalis. The portion lying beyond the castration wound then begins to proliferate, and a condition results which has been described as "Champignon," and which often leads to scirrhous cord (fig. 175). In other cases the infected cord is returned to the tunica vaginalis, but in consequence of the presence of micro-organisms, becomes inflamed, and swollen.

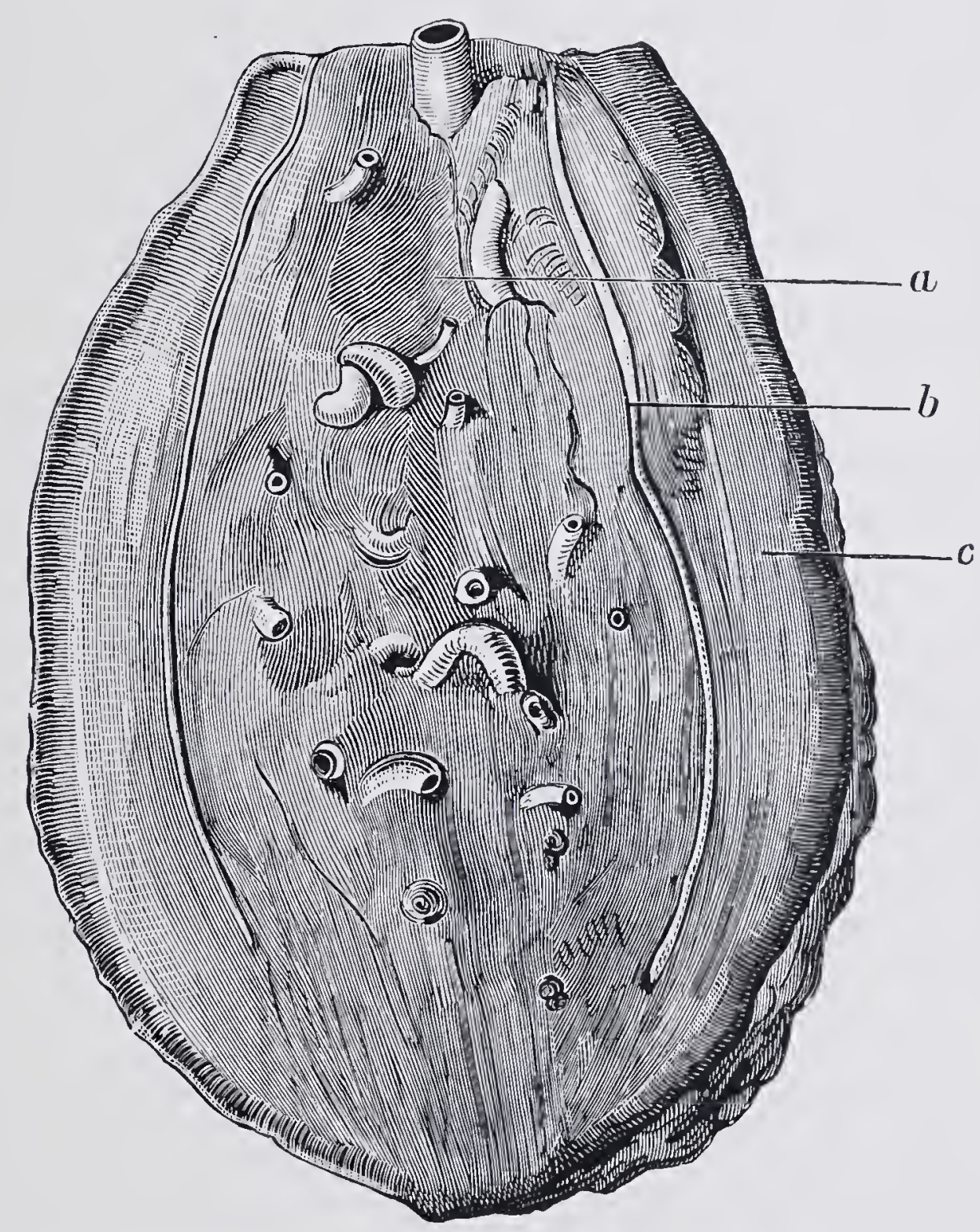

FIG. 175. - "Champignon" formation in the horse. $a$, Thickened spermatic cord ;

$b$, processus vaginalis; $c$, thickening external to processus vaginalis.

Unless strict asepsis be observed, which is almost impossible, scirrhous cord may result, and for this it appears unjust to hold the castrator alone answerable.

(2) All influences favouring pus formation and inflammation in the operation wound favour development of scirrhous cord. These comprise, too small an incision, or an incision too far to one side of the scrotum, both of which conditions impede drainage; too small a wound also favours strangulation of the cord and formation of "Champignon," as shown by Trianon's experiments. Castration by ligature is doubly dangerous if performed with unsterilised materials. The ligature often remains in 
position a long time, favours pus formation, and therefore increases the danger of infection.

Inflammation spreads to the inner surface of the processus vaginalis, which soon becomes adherent to the spermatic cord; but the connective tissue lying between the tunica vaginalis and tunica dartos is seldom affected.

Symptoms and course. The swelling of the testicular cord does not completely subside after castration, but continues to discharge pus and slowly increases in circumference. This seldom receives early attention, and expert assistance is only called for when the owner fancies the discharge has lasted too long, or when several attacks of acute cellulitis have occurred. As soon as the fistulous aperture has closed, the swelling suddenly increases, spreads to neighbouring tissues, including the connective tissue around the processus vaginalis, often even to the sheath itself, and only subsides when a new opening has been established. This acute cellulitis returns at varying intervals. It may, however, never occur, and the disease exist for an indefinite time without making much progress or occasioning disturbance, indeed without ever being recognised. The swelling, which is firm, hard, and slightly painful, sometimes remains confined to the lower end of the spermatic cord, but sometimes extends further upwards, and may even reach the abdominal cavity. Less frequently the lower end of the cord is not attacked, and the swelling begins an inch or two above. At the point where the swelling is in contact with the skin, it is always attached to the base of the scrotum.

The fistulous opening is small and funnel-shaped, and discharges a slight quantity of purulent fluid; a probe can be passed 2 to 4 inches into it.

Cadiot and Dollar (p. 40 of "Clinical Veterinary Medicine and Surgery") describe a case of deep-seated inguinal abscess after castration The condition simulated scirrhous cord. There was diffuse induration in the left inguinal region, extending over the abdomen beyond the sheath. The horse showed fever and was lame on the left leg, which was abducted during movement. Examination per rectum revealed a diffuse, rounded, smooth swelling, larger than a man's fist and about 4 inches thick, in the prepubic region, opposite the left inguinal ring, which was diagnosed as an abscess. The animal was cast, the inguinal canal examined, and a large abscess containing two quarts of pus was opened. By drainage and antiseptic dressing the abscess cavity closed so far that five weeks after operation the horse was able to return home.

In another case described by the above writers (p. 408 loc. cit.) the growth, by pressing on the infero-lateral surface of the penis, caused difficulty in micturition. Extirpation was followed by recovery.

Prognosis. As cure depends on complete excision, it is necessary to ascertain whether operation is possible, how far the cord is diseased, and then whether all diseased parts can be removed. Extension of 
diseased processes into the abdominal cavity renders the condition incurable. Examination from without and from the rectum will determine the dimensions of the growth; of 100 cases submitted to Möller, all proved amenable to treatment.

When disease is less extensive, the swelling being small and confined to the lower sections of the cord, the new growth can be destroyed by caustics, but where inflammation and new tissue formation have attacked the outer surface of the processus vaginalis, greater difficulty is encountered. Such changes are indicated by extensive adhesions between the skin and new growth, and extension of the growth towards the inguinal region.

Treatment. Preventive measures are of great importance; the clams should be properly shaped, and, in applying them, the posterior part of the cord should not be cut through; ligatures are better avoided; if used they should be sterilised. After removing the clams, the spermatic cord should be completely returned to the tunica vaginalis, and exit of discharges favoured by moving the animal.

When inflammation has become chronic, neither potassium iodide internally, iodine injections, setons dressed with sublimate, nor the use of the actual cautery, commonly succeed, though where operation is impossible they may be tried. Sublimate setons and the cautery are perhaps more efficient than injections; but when the spermatic cord is extensively diseased, removal of the diseased part is alone useful. Though the period of acute celluiitis must be avoided, there should be no hesitation in performing the operation, which is simple, and, if precautions against bleeding are taken, not dangerous.

The horse is cast, the feet of the affected side fastened together, and the animal placed on its back. The operator then kneels behind it. The seat of operation is cleansed, and an elliptical piece of skin, sufficiently large to allow of the growth passing easily through it, removed with the knife. Any skin adherent to the growth must also be taken away, and care taken to provide for drainage.

A tape, passed through the subcutis of the isolated piece of skin and through the growth, is handed to an assistant, who pulls gently on the spermatic cord. The operator now breaks down the tissues surrounding the diseased cord, which is covered by the processus vaginalis. Any bleeding vessels are at once ligatured. The tunica vaginalis, recognised by its bluish colour and smooth surface, soon comes in view, and is divided from the connective tissue surrounding it by thrusting away the latter with the fingers, assisted by the knife and scissors. The processus vaginalis must be exposed at least as far as it is adherent to the spermatic cord, and, if a pair of clams are to be applied over it, up to the highest point the disease has attained. This method is preferable, 
and as soon as the upper end of the swelling is exposed, a strong pair of clams is applied over the spermatic cord and tunica vaginalis. One then proceeds as in castration, by the covered method. In dealing with extensive swellings, a short pair of clams, which can be introduced into the operative wound, should be applied to the cord, so that, after removal of the degenerated part, they may remain, just as in the operation for inguinal hernia (which see).

Where the disease extends so far upwards as to prove beyond easy reach, the tunica vaginalis is divided where it seems movable over the swelling; the spermatic cord can then be drawn down, and the clams applied above the diseased portion. In other cases the ligature or écraseur must be used. It is best to apply a stout ligature, tying it as tightly as possible, and allowing the ends to hang below the skin wound, so that it may be removed if it does not fall away of itself. The elastic ligature is also valuable. Carefully used, the écraseur and the torsion method also prevent bleeding. Degive combines the two ("torsionbornée"-which see). 'I'o prevent bleeding, the wound, when cleansed, is powdered with iodoform tannin and stuffed with carbolised jute, a few sutures being inserted to retain the tampons in position. The same means will control after-bleeding caused by the altered position of the blood-vessels when the horse rises.

Dressing and clams remain in position for forty-eight hours; after removing them, the parts are washed out with sublimate or carbolic solution, again powdered with iodoform tannin, and treated as an open wound. After about eight days the ligature may be removed, if it has not previously come away. Daily exercise favours drainage. Recovery generally occurs in fourteen days to a month, without complications. Möller operated in the manner described on 100 horses, and always had good results.

If the new growth has spread outwards beyond the processus vaginalis, the subcutaneous connective tissue, which is infiltrated with small abscesses, must, as far as possible, be removed. Any remaining abscesses or sinuses should be scraped out with the curette, or dressed with concentrated solution of chloride of zinc.

Mallimus has recently proposed a modification of this procedure. Starting from a spot, if possible behind the growth, he breaks through the tissues in the direction of the inguinal canal with the fingers, in order, from that point, to destroy the adhesions of the growth to surrounding parts. The most resistant portions of connective tissue are divided by the linife, scissors or écraseur, blood-vessels being, if necessary, ligatured beforehand. For dividing the spermatic cord he uses a special écraseur with a very strong chain.

New growths of the spermatic cord outside the processus vaginalis, 
termed "Champignon," can sometimes be removed by ligature and caustics. It is often possible to cut through the growth with a ligature or to scrape it away with the finger, though this is not satisfactory unless the cause, i.e., the strangulation of the cord by the scrotum or processus vaginalis, be at the same time removed. Fig. 175 shows a "Champignon" formation caused by the protruding spermatic cord being strangulated by the scrotal wound. It was removed by ligature three months after castration. As a rule, however, it is best to remove the lower end of the spermatic cord in the manner above described, otherwise scirrhous cord almost always develops. Degive passes a metal rod through the swelling after the écraseur has almost cut through the spermatic cord, turns that portion of the cord lying within the écraseur chain once or twice around its long axis, and then completes the operation. This he terms "torsion-bornée."

When the morbid growth extends as far as the inguinal ring or into the abdomen, the inguinal canal may be dilated and the chain of the écraseur passed even into the abdominal cavity. Dollar has successfully operated on several cases of scirrhous cord extending as far as the inguinal ring, using the écraseur. He has never had bad results or troublesome bleeding, the secret of success being to observe all possible antiseptic precautions and to divide the cord very slowly. Cases are on record of growths weighing as much as 120 lbs. Necessarily they could not be removed.

Soon after potassium iodide had been proved efficacious in actinomycosis it was tested in the treatment of scirrhous cord. The recorded results have been conflicting, probably because scirrhous cord is not a specific disease and the organisms producing the changes may not be the same in every case, and also because swellings of the cord occurring soon after castration may have been mistaken for the essentially chronic disease in question. In support of the first contention it may be noted that some observer's have found scirrhous cord due to malignant growths like sarcoma. Fröhner records two cases, one of which received 10 ounces of potassium iodide per os and $6 \frac{1}{2}$ ounces of sodium iodide intratracheally, the other nearly 25 ounces of iodide of potassium per os within a few weeks. Neither showed any improvement. The first horse had to be killed. The second was operated on, and recovered sufficiently in three weeks to return home. In seven weeks from operation recovery was complete. 


\section{DISEASES OF THE FEMALE ORGANS OF GENERATION.}

As the greater number of these diseases are considered in works on special pathology and obstetrics, only a short account of those which necessitate surgical interference is here given.

\section{I.-INJURIES AND INFLAMMATION OF THE VULVA, VAGINA, AND UTERUS.}

In cows, the labia pudendi are most frequently injured by hornthrusts; in mares, occasionally, by the shafts of vehicles; in all species of animals by violent and unskilful manipulations during delivery. In such cases ruptrre of the perineum is common. In cows, the vagina is sometimes wounded, though seldom seriously, by the application of the vaginal truss for the purpose of retaining the prolapsed vagina or uterus; sometimes, however, severe croupous inflammation results, and extends over the vagina. These conditions seldom require surgical interference, and must be treated on general principles. Sometimes wounds require incising to allow of better drainage; sometimes healing can be assisted by carefully applied stitches. Wounds in this region most frequently result from rupture of the perineum. When fresh they should be accurately sutured; when of old standing the edges may require to be freshened.

Extensive wounds and excoriations of the mucous membrane of the labia pudendi may lead to occlusion of the vulva. Apropos of this, the following case may be quoted:-An eighteen months filly exhibited occlusion of the vulva from its upper angle as far almost as the clitoris, where an opening the size of a goose-quill remained, from which urine was discharged in a thin stream. The adhesion was divided; but as it recurred several times, it was found necessary to pass the hand into the vagina daily and dilate the vulva. Under this treatment perfect recovery occurred.

Occlusion of the vagina, due either to persistence of the hymen or to adhesion of the surfaces of a vaginal wound, has been seen in the cow and mare. In either case the os uteri is not attainable, and a 
muco-sanguineous fluid collects behind the obstruction. Occlusion from imperforate hymen is of little consequence, as it can readily be cured, but extensive union between the opposed vaginal walls is a serious matter.

A case of the former kind in an Ayrshire "grey" was cured by incision. The animal had shown a temperature of $104 \cdot 5^{\circ} \mathrm{F}$., and severe abdominal pain. Four pints of offensive fluid were evacuated. (Cadiot and Dollar's "Clinical Veterinary Medicine and Surgery," p. 424.)

Geyer noticed occlusion of the vagina in three calves two to three weeks after birth; in two cases it was possible to break down the adhesions. After the discharge of several quarts of a reddish, turbid, ill-smelling fluid, Geyer washed out the vagina with 1 per cent. creolin solution; recovery followed. In the third calf the entrance to the vagina was too narrow to admit even a single finger. After repeatedly passing the trochar, the animal was fattened for slaughter.

Schmidt describes a calf which eight days previously had been bulled, and had immediately bled freely; the vagina was closed by a membrane in front of the opening of the urethra. Excessive dilatation of the vagina rendered the passage of fæces difficult. After perforating the membrane with a trochar, increasing the opening with the finger and hand, and washing out the parts with creolin solution, healing occurred in fourteen days. In this case also four to five quarts of a whitish, ill-smelling fluid were discharged. The hymen was probably unusually resistant.

Wounds of the vagina in cows are commonest after prolapse or after parturition, especially in primiparx. Sometimes the surface alone is injured or the mucous membrane excoriated, though deep tears in the tissues, and even perforating wounds of the vagina, are also seen, particularly in the large animals. More than one observer has seen perforating wounds of the vagina caused by the penis of the stallion during coitus. It must be remembered in these cases that the posterior part of the vagina is surrounded by loose connective tissue, which connects it above with the rectum, and below with the os pubis. In the mare, this connection extends about 5 inches in a forward direction, and below for a rather longer distance. Perforating vaginal wounds, therefore, lie farther forward, and must always be regarded as serious, both on account of the danger of peritonitis and of prolapse of the bowel. In mares, peritonitis is most to be feared. Prolapse of the bowel is particularly favoured by straining, which is almost always present after such injuries, and by the entrance into the peritoneal cavity of air, which sometimes causes very great distension.

Hæmorrhage is less grave, unless blood passes through a perforating wound into the peritoneal sac, where it decomposes, and leads to purulent peritonitis. Surface injuries require attention only in pregnant animals, in which decomposed infectious substances, always developed in the after-birth, readily initiate such disease as septic metritis. The danger is greater in summer than in winter, particularly if the after-birth 
is long retained. For the same reason, wounds of the lower vaginal wall are graver than those of the upper; but even perforating wounds generally heal without bad results, as is well shown by the results of castrating animals after Charlier's method.

An extremely severe injury, sometimes produced during delivery of a very large foetus, or by the fore legs of one of ordinary size, is rupture of the upper wall of the vagina and lower wall of the rectum. Sometimes the parts are only perforated, and a recto-vaginal fistula results; but in others the parts are torn for a distance of 10 to 12 inches from the opening of the vagina. Fæces then pass from the rectum into the vagina, setting up vaginal catarrh, which may extend to the bladder and uterus. Breeding from such animals is out of the question. The condition is not fatal, but implies such unpleasant complications as to render the animal almost worthless. For the surgical treatment of rupture of the perineum and recto-vaginal fistula, see "Perineal Hernia" and "Anal Fistula." Humbert and Beaufils cured four cases of perineal rupture by inserting double rows of tape sutures. In the most serious case the vulva, anus, and recto-vaginal septum were implicated.

The treatment of non-perforating wounds of the vagina requires particular care in pregnant animals, and in those having just been delivered. The foetal membranes must be removed as soon as possible, and the vagina and uterus repeatedly washed out with creolin, carbolic or alum solution; in mares, diluted sublimate solution $(1$ in 5,000$)$ may also be employed. One of the best disinfectants is a dilute solution of iodine and potassium iodide of about the colour of sherry. It has the advantage of not setting up violent expulsive efforts, as do most of the other fluids named. Injuries to the vulva or its neighbourhood should be swabbed with 10 per cent. solution of chloride of zinc, so as to form an eschar, and diminish the chance of absorption through the wound. It is better to avoid washing out perforating wounds, because of the danger of thus causing peritonitis. If within reach, as when affecting prolapsed organs, such wounds may be sutured before reducing the prolapse.

Wounds of the uterus are caused, during parturition, by instruments or by the little animal's feet, and in cases of prolapse, by external violence; rupture of the pregnant uterus has also been seen. When diseased the uterus naturally tears more readily than when healthy, and such accidents are therefore commoner after metritis. Whether, as has been stated, rupture is produced by dropsical conditions of the membranes seems doubtful; more frequently it results from violent interference when the fœetus is in an awkward position.

Buhl found the uterus of a young pregnant cow completely torn away from the vagina, and adherent to the rectum, between which and the displaced 
uterus a communication existed; fæces had entered the uterus, whilst the bones of the fœetus were almost completely exposed by decomposition of the soft parts; some had penetrated into the rectum.

Hess saw a similar case : a three year old cow which had been covered in February, did not calve by November, and was therefore fattened; in the following spring, on its being slaughtered, the neck of the uterus was found to have been ruptured, and to have again united in a globular form. The uterus containing the calf lay in the left lower flank, suspended by the broad uterine ligament. It was 20 inches in length, and weighed nearly 40 pounds. The skin already showed growth of hair, and the two nipping teeth were distinctly developed; the fœetus, when separated from the uterus, was therefore about eight months old. Hess thought that torsion of the uterus was responsible for the rupture.

The principles laid down in speaking of wounds of the vagina apply equally to the prognosis and treatment of uterine injuries. As long as the cervix uteri remains closed to infection, injuries like ruptures of the uterus seldom produce grave results. Certainly they are sometimes followed by abdominal pregnancy and death during parturition, while extensive ruptures may also talie a fatal course in consequence of excessive bleeding. It is quite otherwise in injuries occurring during parturition. Even slight wounds of the uterus then become grave; and in mares, perforating wounds almost always prove fatal.

The gravity of injuries to the prolapsed uterus is determined by their extent and character, and the degree of general disturbance. When colic, fever, and continuous severe straining exist, and when in carnivora vomiting occurs, little can be done, though even such cases should not be abandoned.

The prolapsed parts should be carefully cleansed, bleeding checked as far as possible (for the greater the quantity of blood which accumulates in the uterus when replaced, the greater the danger of decomposition), any remaining after-birth removed, wounds sutured, and the organ then replaced. (See "Prolapse of the Uterus.")

Inflammation of the vagina or uterus most frequently follows parturition, and is treated of in the study of obstetrics. Certain forms of purulent inflammation occur, however, in non-pregnant animals, especially soon after coitus, and in young cattle, and are apt to take a chronic course. Such conditions have repeatedly been described by English veterinarians. Recovery is stated to rapidly follow washing out the vagina and uterus with carbolic solution or other antiseptic fluid. In bitches fatal septic inflammation of the uterus is not infrequent, even when the animal is not pregnant and has not recently been delivered.

Cases of infectious fluor albus in oxen (vaginitis, kolpitis purulenta) have often been described in Germany. Martens found the disease throughout a certain district, and believed it was spread during coitus. He recommends 
irrigation of the vagina with dilute acetate of alumina, which is also said to be a useful prophylactic against infection, and for this purpose is syringed into the bull's prepuce. Wilhelm gives 75 grains of salicylic acid in about $1 \frac{1}{2}$ pints of linseed tea once daily, discontinuing it on the fifth or sixth day, and later repeating the course two or three times. Frick saw in cows an epizootic catarrhal disease of the vagina which was spread by coition, and was frequently followed by failure to conceive. Recovery followed vaginal irrigations with $\frac{1}{2}$ per cent. liq. alum. acet., but only after a long time.

Dieckerhoff noted a severe infectious vaginitis in cows and mares. Cows affected by it invariably perished; and though the disease was less severe in mares, some of them also died. The disease (kolpitis perniciosa, D.) set in with fever, and the vaginal inflammation was associated with a tendency to the formation of blisters and ulcers. A blood-stained exudation was found in the abdominal cavity on post-mortem. The disease developed after a short incubation period (twelve to twenty-four hours), and generally proved fatal in a few days. Disinfection of the vagina with carbolic or creolin solutions had no visible effect. In this case also the disease must principally be combated by protective measures.

\section{II.-PROLAPSE (INVERSION) OF THE VAGINA. PROLAPSUS (INVERSIO) VAGINÆ.}

As above indicated, the term "prolapse," though sanctioned by custom, is incorrect; strictly speaking, the condition is that of inversion, as is recognised in the French title, "renversement du vagin."

Prolapse of the vagina is most frequent in cows and sows, rarer in mares.

The disease depends on displacement of the uterus backwards and stretching of its ligaments - conditions usual after repeated pregnancies. It is favoured by tympanites, distension of the abdomen, continuous stall-feeding on distillers' grains; distension of the abdomen, pressure of the fœtus, severe straining on the part of the animal, and particularly by standing in sloping stalls; it is seldom seen when animals are in the open. It is commonest in pregnant subjects, especially during the latter period of pregnancy, though it also occurs after parturition. In pregnant animals the vagina appear's beyond the vulva when the animals are lying down, but disappears immediately on rising. Strictly speaking, the disease consists of eversion of the vagina, in which the upper wall is most frequently displaced. When the vagina only attains the vulval opening, a round red swelling of varying size, covered with mucous membrane, appears (incomplete prolapse). Less frequently a large portion of the vagina protrudes beyond the vulva, in which case the opening of the uterus is visible in the middle of the swelling, which is about as large as a man's head, and displays, on its lower surface, the opening of the urethra. In prolapse of some duration, the surface of the mucous membrane, previously of a rose-red colour, gradually dries, and not 
infrequently meets with injury from the animal lying down, or from the parts being trodden upon, sometimes from attempts at replacement. During this time the patient strains continuously, and frequently passes urine. Prolapse occurring after delivery seldom disappears of itself, the vagina remaining continuously displaced until it receives proper attention.

Prognosis. Incomplete prolapse is usually only of importance in breeding animals, but here demands attention on account of its recurring after every pregnancy, and interfering both with the animal's condition and milling qualities. Injury may also cause inflammation of a dangerous character. In rare cases severe straining may be followed by rupture of the vagina and prolapse of bowel and bladder. This condition has oftenest been seen in mares, and is rarely curable. Under such circumstances the uterus may become prolapsed; cases of the kind have been reported in goats and sows.

Complete prolapse is always grave, doubly so in pregnant animals, because of its almost invariably interfering with parturition. Prolapse in non-pregnant animals often occasions injury and inflammation of the vagina, which reduce the production of milk, and greatly lower the breeding value of the animal.

In swine, the prognosis is still more unfavourable, because of the difficulty in treatment.

Treatment. As a preventive, the animal must be placed in a proper stall, so arranged that the hind quarter's are at least as high as the fore. Most other precautions necessitate increased outlay, thus rendering their adoption out of the question. The most important is, of course, to prevent the animal breeding.

In reducing a complete prolapse-the incomplete form generally returns of itself-the cow is placed with the fore limbs lower than the hind. By introducing the finger into the urethra, the bladder is first emptied, the parts then cleansed as far as possible, any existing wounds sutured, and endeavours made to return the vagina through the vulva by placing the open hand against the swelling and gradually pressing it forwards. On attaining the vulval opening, the hand should be closed without relaxing the pressure, and the prolapsed vagina thus returned to its normal position. The operator takes advantage of the intervals between straining, and retains the hand in the vagina until these efforts stop. With the arm in the vagina the operator places his closed fist against the os uteri, which he pushes back as far as possible; this manouvre stretches the walls of the vagina and removes the folds. When reduction is complete it is important to make sure that the mucous membrane is equally distributed.

Where the prolapse is very extensive and the parts greatly swollen it 
often assists reposition if the lips of the vulva are held open by assistants during the attempts to return the tissues.

To ensure retention in pregnant animals, trusses are employed, which partly close the vulva. Sauberg's vaginal ring is of the same character as West's clamp, though less perfect; it consists of a metallic ring, which is passed through the lips of the vulva. When these are not available, a

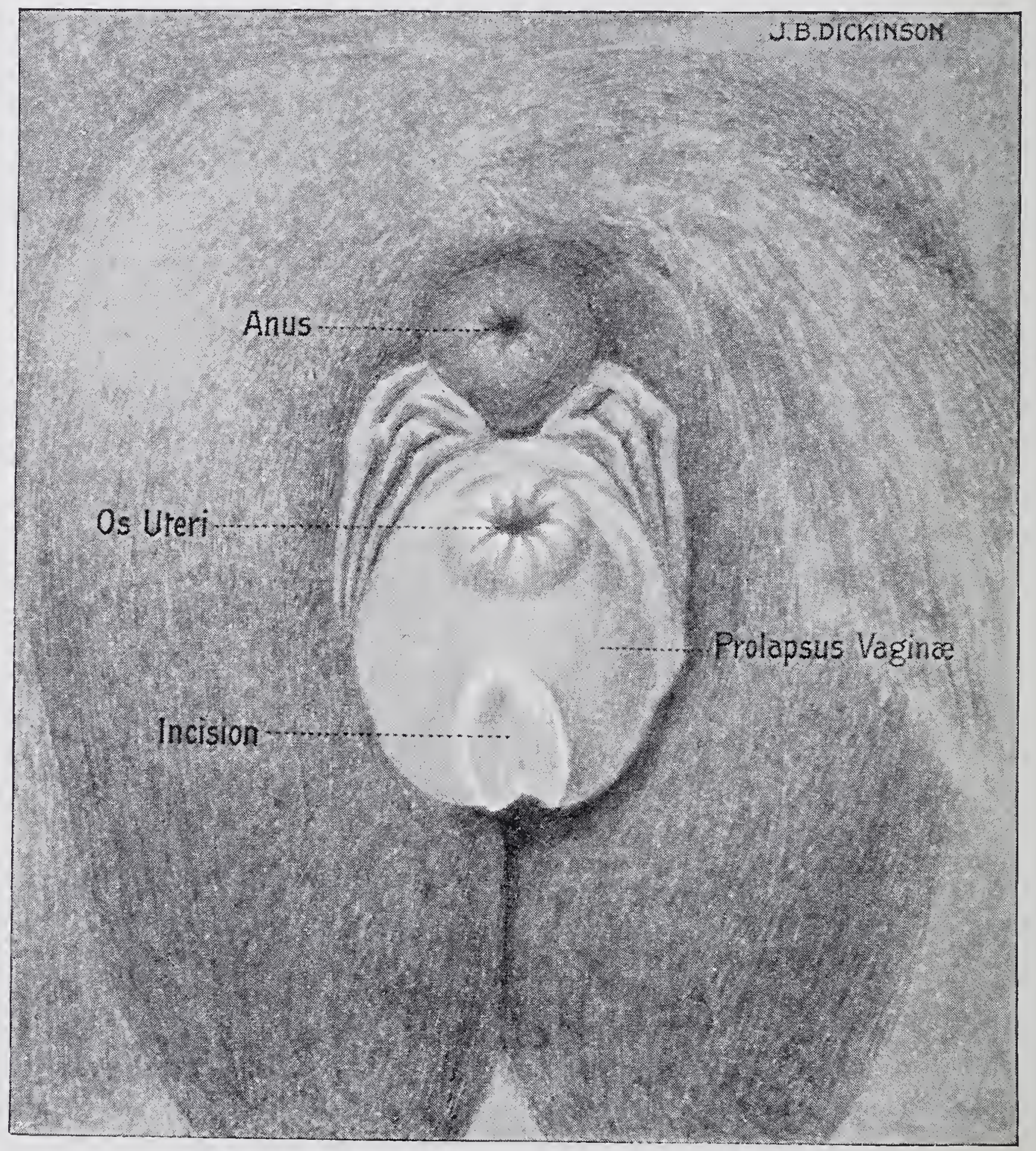

Fra. 176.-Prolapse (inversion) of the vagina.

couple of wire sutures may be passed through the vulva. The wire must be thick enough to prevent tearing out or injuring the vagina when it is pressed forwards. Tape is still better. In any case a strong hold should be taken of the skin, as the lips of the vulva very readily yield.

Koepke saw a prolapse of the vagina, as large as a man's fist, in a three year old filly. It occurred suddenly and disappeared without treatment.

Hewetson saw the prolapse figured (fig. 176) in a two-and-a-half year old Clydesdale mare. The vagina had several times appeared prominent previous 
to the actual accident. Reduction could not be effected owing to struggling, so the parts were bathed three times a day with disinfectant or astringent solutions, and on the third day they returned spontaneously. A fortnight later the prolapse again occurred. The former treatment failing, the vaginal wall was scarified. Improvement was slow, and the mare was turned out during the day. Three weeks later the wound shown was healed and the vagina could be replaced. West's prolapse clamps were applied and left in position for a month. Recovery appeared complete.

In sows, reposition is assisted by holding up the hind legs. The prolapsed parts are cleansed, and an attempt made, during an interval when the animal neither strains nor cries, to thrust back the swelling. The vulva can be closed with a couple of sutures.

If the prolapsed vagina be greatly swollen, bathing with 2 to 3 per cent. solution of alum will assist reposition.

Bitches often suffer from partial prolapse of the upper wall of the vagina, which is very frequently mistaken for a tumour. A ligature can be applied to the base and the prolapsed part removed.

\section{III.-PROLAPSE (INVERSION) OF THE UTERUS. PROLAPSUS (INVERSIO) UTERI.}

This disease, frequently seen in cows and swine, but very seldom in other animals, really consists in inversion of the uterus, the surface of the prolapsed organ appearing covered with mucous membrane. The condition, therefore, is somewhat like inversion of the bladder, but can only occur when the cervix uteri is relaxed, and in consequence its occurrence is almost exclusively confined to the period of parturition. Partial displacement of the uterus is certainly imaginable, even with a closed cervix, if the extremity of one or other horn should become intussuscepted (like the finger of a glove). Though the process cannot be detected in the living animal, it probably represents the first step towards so-called prolapse, the straining it occasions finally causing displacement of the entire organ.

Such invagination may remain after prolapse if the uterus and its cornua are not completely returned. In mares, the anterior portion of the displaced uterus is sometimes strangulated in the cervix uteri, generally producing a fatal issue. A similar accident has been seen in cows.

In any case, prolapse of the uterus presupposes elongation of the uterine ligaments, and therefore the factors predisposing to prolapse of the vagina predispose to this disease. They consist in too high a position of the fore limbs, repeated pregnancy, \&c.; the immediate causes are generally non-occurrence of post-partum uterine contraction, severe straining and excessive labour pains. Retention of the after-birth is one of the chief, partly because the cervix then remains 
open for a long time, partly because the manipulation necessary to remove the after-birth favours inversion. Violent interference during parturition may also give rise to it. Relaxation of the cervix is the second necessary condition; the disease is therefore rare, except soon after delivery.

Symptoms. As already remarked, incomplete prolapse, i.e., inversion of a portion of the uterus, is seldom directly observed, though it sometimes causes metritis and death; usually, however, it may be detected by introducing the hand into the uterus.

In complete prolapse of the uterus, a swelling, covered by mucous membrane, and similar to that in prolapsus vaginæ, appears beyond the vulva. In cows, it resembles a half-filled sack; in mares, its form is more cylindrical, the organ sometimes reaches as far downwards as the animal's hocks; the surface is not infrequently covered by after-birth, but if the latter has already been removed, the cotyledons, which in cattle have a mushroom-like form, are exposed. The surface gradually dries, and often becomes soiled and wounded. In swine, the prolapsed uterus is not infrequently bitten by the other occupants of the sty.

The prognosis is most favourable in cows, provided the uterus is not much injured and has not been exposed more than three or four hours. Reposition, though certainly not easy, is yet generally possible, and if not, the animal's life can, as a rule, still be saved by amputation. In other animals, and particularly in mares, the disease takes a graver, and usually fatal, course, especially if the uterus is much damaged. Fenner, however, cured a mare after suturing a wound about 4 inches in length, involving the mucous and muscular coats. Körner removed the uterus in a mare by ligature, and had good results. Violent pains and delirious attacks certainly occurred, but only lasted one day. Goats and pigs have also been saved by amputation.

Not infrequently the uterus or vagina is ruptured, leading to prolapse of the rectum, in which case the animals always die unless assistance is promptly afforded, and often even when it has been given.

Treatment. The organ must first be protected from injury by applying a clean cloth. Provided the parts are not much injured, and the tissues have undergone no great change, reposition should be attempted. The operation is assisted by placing the animal with the fore-quarters lower than the hind; but if it cannot stand, it is placed on its back, with the hind limbs as high as possible.

Anæsthesia greatly assists reposition. Bang reconmends giving the cow subcutaneously 7 to 20 grains of morphine, and a further dose of 6 to 10 drachms of chloral hydrate, by the mouth or in a clyster. Failing this, 1 to 2 pints of brandy may be given. Morphine often acts as an excitant; chloral is preferable. To diminish the severe straining as much as 
possible, continuous powerful pressure must be exercised over the loins; if the animal is standing, a sackful of sand may be laid on the back. Where the prolapsed portion is large the rectum and bladder should first be emptied. T'o provoke micturition it is often sufficient in cows, \&c., to lift the protruding mass and pass one or two fingers into the urethra; should this fail the catheter must be passed.

After carefully cleansing the prolapsed parts with 3 per cent. creolin, dilute iodine solution, or, if much swelling exists, 2 per cent. alum solution, and removing any traces of fotal membranes, the operator must discover whether any deep injuries exist. It may be necessary to insert sutures. The size of the prolapsed mass often presents a serious obstacle to reduction. In such cases the late Professor Robertson used to effect methodical compression by passing a long, broad strip of linen around the uterus and causing assistants to pull steadily on the ends. A broad strip of Macintosh cloth may be used for the same purpose, being applied first at the point furthest from the vagina. If the patient be tympanitic puncture of the rumen or cæcum may be necessary.

The first portions to be replaced are those lying nearest to the vulva, particularly the upper sections, the other prolapsed parts being meanwhile supported; an attempt is then made to thrust these through the vulva into the vagina, and to pass the entire mass as far forward as possible. To prevent ruptures and to assist replacement, the manipulations are made when the animal is not straining.

The flat hand should be used; not the tips of the fingers or the fist. By stretching out the fingers within the uterus, the folds may be completely got rid of-the same object is served by injecting lukewarm water; in cows, several bucketsful may be required. Instruments should be avoided. They may cause perforation when the animal strains.

Becker uses Günther's parturition crutch. With the half-closed hand, he thrusts the lowest portions of the uterus as far as possible into the vagina and pelvis, then introduces the previously well-oiled rounded end of the crutch along the arm as far as the hand, and, whilst retaining the crutch with the other hand, withdraws the arm from the vagina. An assistant holds the crutch firmly, without thrusting it further forward, while the operator, by using both hands, passes any portion of the uterus still in view into the vagina, reintroduces the right arm, removes the cruth, and endeavours to bring the uterus into its proper position. The assistant then passes his left arm into the vagina or uterus alongside the operator's right, closes his hand, and whilst the operator removes his arm, that of the assistant remains for an hour in position. The cow is watched for the next ten to twelve hours to prevent straining, in which case the hand should be pressed against the vulva. If possible, the animal should, after reposition, be kept standing. Operation is facilitated

V.S. 
by previously giving a couple of ounces of chloral dissolved in a pint of water.

In order to ensure retention, some operators recommend keeping the arm for some time in the vagina, and only withdrawing it when straining stops. Others, thinking this encourages straining, replace the uterus, give an anodyne, and apply a vaginal clamp or truss. To entirely reduce the invagination of the uterine horns, which often keeps up straining, cows may be walked downhill. The animal is then placed with the hind parts higher than the fore; it is even better if it be kept standing, or be slowly moved. By introducing small pieces of ice or a little cold water, contraction of the uterus is excited, and fresh prolapse prevented. The measures recommended in prolapse of the vagina may be tried; for example, the truss, the vaginal ring, and the suture, though they are seldom of much use. Complete reposition, and the return of the entire uterus and its horns to their proper position, is of much greater importance. Closure of the vulva is only of value in preventing injury to the prolapsed parts, and is most easily and securely effected by using West's clamp or brass-wire sutures. A thin trochar can be passed from right to left through the lips of the vulva. After drawing back the stilette, a brass-wire suture, provided at one end with a leather button, is passed from left to right through the canula, the canula removed, and a leather button adjusted on the other end of the wire to prevent its tearing out. Two or three sutures of this sort are sufficient.

In cases where the violence of the expulsive efforts renders reduction very difficult some operators have had excellent results from slinging. The hocks are fastened together and the hind-quarter's raised from the ground by means of a block and tackle until the animal rests on the sternum. In this nearly vertical position the prolapse is spontaneously reduced, and the animal is cautiously lowered until the hind-quarters rest on a bed of straw considerably higher than the surrounding parts. 'This manipulation can be repeated if necessary.

When reposition is impossible, or the tissues of the prolapsed organ are much damaged, amputation is the only resort. In cows it is simple, and not particularly dangerous, but in no animal should it be attempted until all other means have been tried or at least considered. St. Cyr lost six animals of a total of twenty-five; Franck describes thirty cases of amputation, of which four (two cows and trvo goats) had to be slaughtered. Lanzillotti-Buonsanti has collected records of 140 cases. Of 86 cows, 77 recovered and 9 died; of 8 mares, 6 recovered and 2 died: of 27 sows, 22 recovered and 5 died; of 9 bitches, all recovered; of 4 goats, all recovered; of 3 sheep, 2 recovered and 1 died; of 3 cats, all recovered. Nevertheless, these figures are not to be implicitly relied on. They truly represent the records, but successes are 
more often recorded than are failures. When the animal is not cast, precautions must be taken against its lying down or kicking the operator. The surface is then cleansed, and care taken to discover by palpation that no portion of bowel or bladder is included in the inverted uterus. A strong tape or cord, in the form of a surgical snare, is then passed round the neck of the uterus, avoiding the urethra, and drawn very tight. In this case, as in all where large masses of tissue have to be ligatured, it is necessary to proceed slowly. The ends of the cord are tied to pieces of stick to ensure a better purchase, and the ligature is drawn tight; the operator then waits a few seconds to allow the tissue fluids to filter away, and again tightens the cord; if the prolapsed uterus is much swollen, the procedure requires repeating several times. The elastic ligature has been recommended and successfully used on sheep and swine by several operators. After applying the ligature, the organ is cut away.

In large animals, and where swelling is great, amputation by multiple ligature is to be recommended, or the mucous membrane can be divided and the ligature applied to the muscular and serous coats. These methods, however, have no particular advantage over that above described. Bang also prefers the ligature; the portion thus tied off can at once be cut away, but should movement of the ligature be feared, or further tightening become necessary, amputation may be postponed till next day. In such cases care must be taken that the weight of the uterus does not cause it to tear away, as such complications are often difficult to deal with. Hering recommends, where the animal is able to stand, to lay the prolapsed parts on its quarter. Without question, however, it is better to remove them at once, if for no other reason than the fact that this allows of reposition of the vagina. To remove inflammatory products, discharges from wounds and from the ligatured stump, the vagina must be repeatedly washed out during the following days. If swelling is severe, it may be necessary to pass the catheter. Should further prolapse of the vagina result from severe straining, either the truss, West's clamps, or a few metallic stitches may be passed through the vulva. The operation is not without danger ; loops of bowel may protrude, and cows often show great nervous excitement for some time.

In bitches, the uterus has repeatedly been amputated with success. In them also the displacement of the pregnant uterus, in inguinal hernia, may render the operation necessary. Antisepsis must, as far as possible, be practised.

In small animals, and especially in goats, prolapse of the uterus has been reduced by injecting water. The animal's hind legs were held up, the smaller horn of the uterus reduced and filled with lukewarm water. The weight of the water carried back the horn or the uterus to its 
normal position. After the second horn has been replaced by the same method (though great difficulty is often met with here), the uterus is also filled with water, and after replacement, the fluid is allowed to escape by placing the animal in the horizontal position. The lips of the vulva are closed with a loose button suture left in position for three days.

\section{IY.-TUMOURS OF THE VAGINA AND UTERUS.}

As a rule, diseased new growths in these organs are commoner in the human species than in animals. Fibromata in particular seldom attain the extensive development here which they do in the human subject, though fibromata, carcinomata, sarcomata, and especially retention cysts have repeatedly been seen in the vagina of cows. Such tumours also occur in bitches (fibromyomata), but are rare in other animals.

In bitches, partial prolapse of the upper wall of the vagina sometimes occurs, attended with severe swelling in the prolapsed part, and is generally regarded, and even treated, as a fibrous new growth. A pale red ovoid swelling suddenly appears in the vulva, generally attains the size of a hen's egg, and cannot be returned, or if returned, soon appears again. The disease is commonest during the time of heat, and after successful coitus.

Heckmann found in the vagina of a bitch a pedunculated polypus, which arose from the right wall, and was removed by ligation. Zipperlen describes a polypus on the cervix of a cow; Leblanc a similar growth in a bitch.

In cows, the most common growths are cysts, which occur in the vestibule of the vagina, particularly on the left side. They result from blockage of Bartholin's glands, while those occurring close behind the opening of the urethra are due to occlusions of Wolff's duct. Eggeling detected, in a cow's vagina, a sarcoma telangiectodes, which led to death by bleeding during parturition. Ulcerating carcinomata have repeatedly been found both in cows and bitches; Bang saw carcinoma of the vestibulum vaginæ in a cow.

Sand saw severe bleeding from vaginal varicose veins in a mare. The bleeding could not be stopped, and in consequence of progressive anæmia the animal had finally to be slaughtered.

Gilruth describes an epithelioma of the vulva and vagina in an aged cow. The external growth was about 7 inches long and 6 inches wide, implicated both labix and the lower part of the anus, and extended about 8 inches into the vagina. The animal was killed. The pelvic and posterior mesenteric glands contained small, greyish soft nodules (Cadiot and Dollar's "Clinical Veterinary Medicine and Surgery," p. 425).

Apart from tuberculosis, the urethra is seldom the seat of tumours, and still less frequently does it call for surgical treatment on their account. The changes, often described as scirrhous, probably result from endometritis chronica, though polypoid new growths occur, and are sometimes of considerable extent; Bedenberg found in the uterus of a cow a tumour which weighed between $28 \mathrm{lbs}$. and $30 \mathrm{lbs}$. Carcinomata and sarcomata have repeatedly been seen in bitches; Esser found a fibroid of the uterus in a cow. 
In a cow which had suffered from periodical bleeding from the vulva, Eckhard found a fibroma of the uterus, weighing $52 \mathrm{lbs}$. Gratia, in a cow, discovered a large fibromyoma that had existed for several years, and had repeatedly caused profuse bleeding. As the new growth rendered parturition difficult,

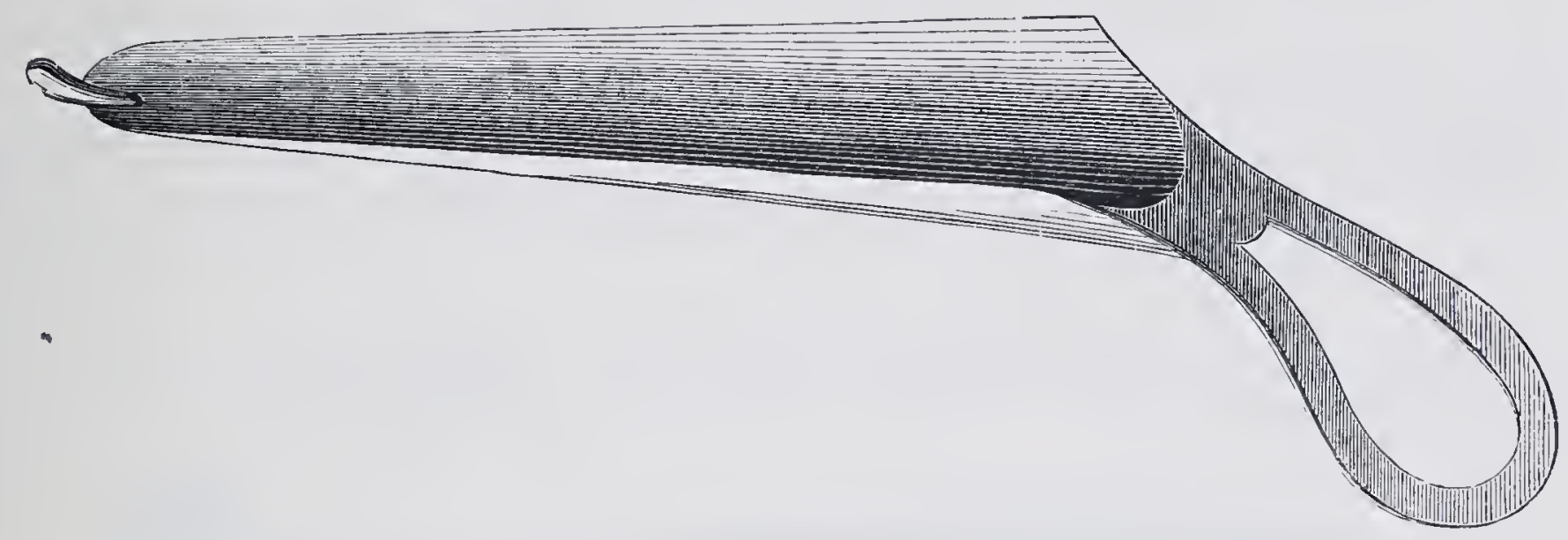

Fic. 177. - Simple vaginal speculum.

it was removed by ligature, but the animal died from acute peritonitis. Von Bockum-Dolffs found tuberculosis of the uterus in a cow which had been regarded as pregnant. The animal was found on slaughter to have general tuberculosis, the uterus being greatly distended with caseous material.

Symptoms. Tumours of the vagina sometimes appear beyond the vulva during urination, and becoming strangulated, are at once recognised; their position and extent are detected by palpation. The same

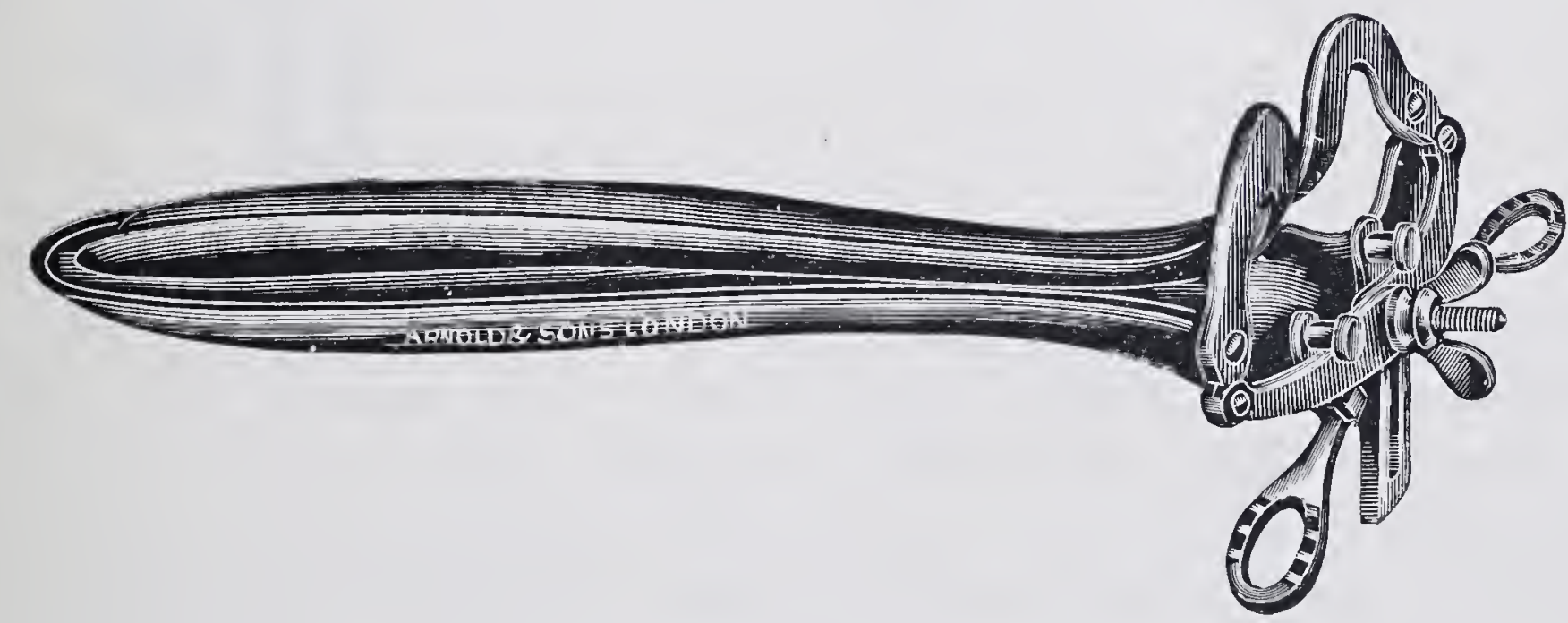

Frg. 178. - Expanding vaginal speculum (closed).

is true of new growths within the vagina. They seldom give trouble unless when of large size, and then because they injure the animal's appearance, or interfere with parturition or urination. They frequently produce bleeding or chronic discharge from the vagina, which, in the case of ulcerating new growths, assumes a purulent character. The cysts which occur in cows sometimes protrude from the vulva, appearing as bladders about the size of a hen's egg, and containing clear or turbid fluid.

Tumours of the uterus are seldom recognised until they produce difficulty in parturition; they often render animals barren. In the 
larger species they can be discovered by rectal examination. Tuberculosis sometimes produces great increase in size, either in one horn or in large tracts of the uterus. The Fallopian tubes then appear like hard cords as thick as a man's finger, arranged in curves, and there is usually a muco-purulent vaginal discharge mixed with masses of firmer consistence and containing tubercle bacilli.

Prognosis depends chiefly on whether the growth can be entirely removed. This is, of course, easier in vaginal than in uterine tumours. In pregnant animals uterine tumours become much more dangerous; and those which produce severe straining, and thus favour prolapses, are particularly troublesome. Vascular tumours may cause death by bleeding.

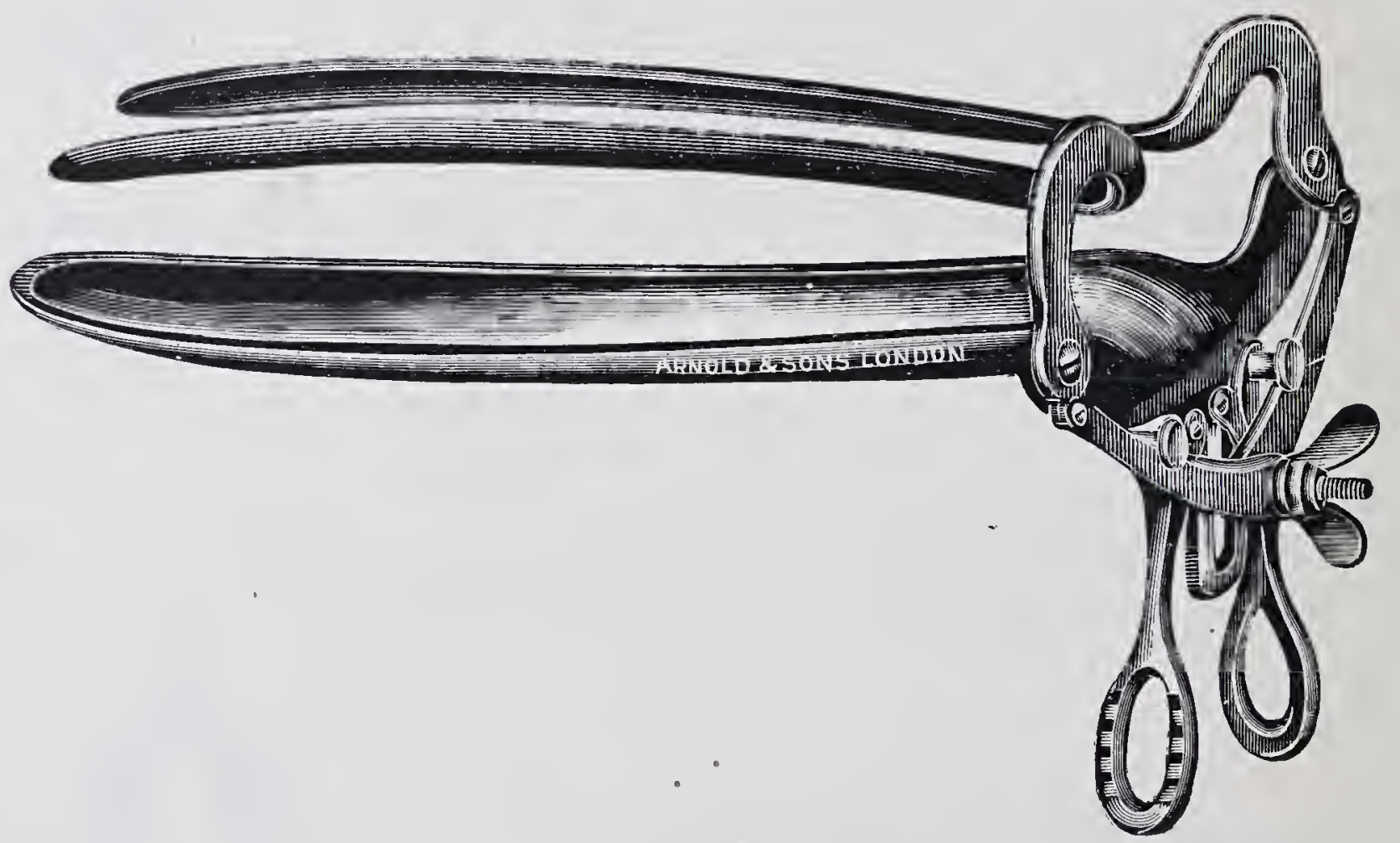

Fic. 179.-Expanding vaginal speculum (open).

Treatment consists in operative removal. Pedunculated tumours can be ligatured, and multiple ligation is also the best method of dealing with partial prolapse of the vagina in bitches. Sessile tumours may be removed by enucleation. Tumours lying further forward may sometimes be grasped with the écraseur, cysts are burst by the pressure of the hand; if necessary, the operation can be repeated, and the base of the tumour cauterised.

Cadiot and Dollar (p. 423, "Clinical Veterinary Medicine and Surgery") describe a case of myxoma of the uterus in a bitch. The swelling projected slightly beyond the vulva, was ovoid, smooth, of fairly firm consistence, and reddish in colour. It measured 5 inches in length and 6 inches in circumference. Removal, by ligature, of the pedicle and section on the peripheral side was followed by recovery.

Müller treated a bitch with fibromyoma, complicated with prolapse of the vagina, by injecting ergotin (extr. sec. corn. grs. vii., spirit. dil. et glycerin. a. m. Ixxv.) : of this fluid m. xv. were hypodermically injected at the most convenient spot. In four weeks the swelling had greatly diminished, and complete recovery finally occurred, but whether as a result of the injections is not altogether certain. 
Malignant tumours of the uterus are rare in all domesticated animals, and are still more rarely diagnosed. Where, for reasons of sentiment, the owner attaches unusual importance to the animal's preservation, ablation of the uterus may be performed, but the operation is only practicable in small animals.

Atresia of the neck of the uterus or closure of the os sometimes renders animals sterile and calls for operation. The entrance to the uterus can usually be dilated by introducing the fingers or a sponge tent.

\section{V.-DISEASES OF THE MAIMIMARY GLAND.}

The mammary gland consists of parenchymatous tissue and stroma. The formation of the former is partly alveolar, partly tubular. In the connective tissue of the stroma lie variously-shaped spaces, lined by a membrana propria, which (like the alveoli of the lung in the smallest bronchi) open by gland ducts. Both are lined with low cylindrical epithelium, and together form the milksecreting tissue. The milk flows thence into the milk ducts, to pass either directly outwards through the teat (man and carnivora), or in herbivora, into a large cavity at the base of the teat, termed the galactophorous sinus. The ruminant's udder possesses only one galactophorous sinus and teat for each division; in the mare's udder every teat is supplied by two sinuses. From the galactophorous sinus a narrow canal, lined with mucous membrane, leads to the outer air, and in mares two of these, therefore, exist in every teat, in ruminants only one. In swine, each teat may sometimes have two delivery ducts. In herbivora, then, each teat corresponds to a particular division of the udder ; in cows, to a quarter; in sheep and goats, to one-half of the udder. In carnivora the divisions of the udder are, externally, not so sharply bounded, and each is connected with the eight to twelve openings on its corresponding teat.

The stroma consists of connective tissue and elastic fibres, which form a capsule under the skin, and surround the udder. From this capsule the stroma is continued inwards into the gland, filling the space between the alveoli and ducts of the gland, and is analogous to the interstitial connective tissue of the lungs. The quantity of stroma varies. During lactation it diminishes, and in place of it alveoli develop. In the udder of young animals, connective tissue predominates. Between the larger divisions of the gland and their ducts lie considerable masses of connective tissue, which often contain elastic fibres and fat.

A double system of lymph vessels exists in the udder; the one lying near the surface arises from the capsule, and is connected with the lymph vessels of the skin; the other invests the alveoli, which are surrounded by fine lymph vessels. Fürstenberg distinguished between the surface lymph vessels arising close under the skin of the udder, which pass into the depths along with the chief veins, and those which arise from the plexus in the interior of the gland, and accompany the deeper-lying venous branches. Fürstenberg states that during lactation the lymph vessels appear to be distended with lymph. . He also succeeded in filling them with air from the milk ducts, and discovered a valvular arrangement in them.

The alveoli are directly surrounded by lymph capillaries, which in their turn are surrounded by capillary blood-vessels; according to Rauber these lie partly in the lymph spaces, into which they pour fluid contents and leucocytes, 
particularly during lactation. The white blood-corpuscles are stated to pass hence even into the alveoli of the gland. This intimate relationship between the alveoli and the blood and lymph vessels is of the greatest importance in studying the development of disease of the udder.

The skin covering the udder shows no special peculiarity; it possesses few or no hairs, but, on the other hand, has in places well-developed sebaceous glands and large papillary bodies.

In cattle, the skin of the teats is free of glands, and has no hair. In the teat of the mare, especially near the opening of the milk canal, many glands are found. In sheep and goats, the skin of the teat is covered with a soft hair, and is rich in glands, particularly at its lower end. In swine, the teat is devoid of hair and glands; but in dogs, single hairs and sebaceous glands exist.

\section{(1.) WOUNDS AND BRUISES OF THE UDDER. LACTIC CALCULI.}

Wounds, \&c., of the udder and teats are most frequent in cows, and are caused by treads; animals with large pendulous udders may even tread on their own teats when rising. Similar injuries are also caused by bites (from dogs) or by sharp objects like thorns, when the animal leaps through or over hedges. Sometimes the skin is divided, sometimes only bruised.

Surface wounds-that is, such as neither penetrate the glandular substance nor the milk ducts-are of no particular importance, and may be treated on general principles. Considerable bleeding sometimes occurs at the base of the teat; and should the wound open into the gland, there is some danger of the formation of milk fistulæ. Though healing then appears to proceed satisfactorily, cicatrisation fails to occur, on account of the milk continuously flowing away. The wound nearly closes, but leaves a little funnel-shaped opening, termed a milk fistula. This is, however, only to be feared during lactation. Injuries of the teat may produce cicatricial contraction, and consequent difficulty in milking.

Bruises of the udder cause bleeding, either into the gland ducts, the milk then being mixed with blood, or into the tissue of the gland, the skin, and subcutaneous tissue. The blood may be absorbed, as in other soft parts, or lead to formation of pus or to putrefactive changes.

Treatment. In all deep wounds of the udder the first object should be to obtain healing by first intention, which will most certainly prevent the formation of milk sinus. Provided the injury be still fresh, and seem to warrant such an expectation, the edges of the wound are carefully disinfected and sutured. It must not be forgotten, however, that during lactation milk fistulæ often resist the most careful treatment, though they readily heal when the animal becomes "dry." The gaping of wounds on the teats and escape of milk can sometimes be prevented by applying a well-fitting indiarubber ring, which, however, must not be too tight; 
adhesive plaster, collodion or wound gelatine may be employed for the same purpose on the hairless or previously-shaven udder. Hertwig recommends a liniment of alum and albumen, which should be applied to the thickness of an eighth of an inch or more. During lactation, however, every means may fail, because the udder is continually filling and emptying, and the skin changing in position. The use of a milk catheter, by allowing the mill to discharge from the affected section, often gets over the difficulty and ensures healing by primary intention. If healing by first intention be no longer possible, treatment must follow general principles. By observing careful relative antisepsis, the burrowing of pus and cellulitis can be prevented. Closure of fistulæ may be sought by using caustics or the actual cautery; during treatment a milk catheter should be inserted. All other methods failing, the end of the lactation period must be awaited, when the fistula can generally be closed by cauterising with nitrate of silver.

Bruises, accompanied by the passage of blood-stained milk, require the udder and the teats to be most carefully lept clean, so as to prevent infection. The removal of the blood and mill' is best effected by catheter. Moist warmth in the form of poultices assists resorption. So-called resorbent applications like mercurial ointment rarely effect any good, though they serve to pacify the owner. If licked by the cow or calf they may prove positively dangerous, and under any circumstances are apt to get into and foul the milk.

Calculi sometimes form in the mammary gland or galactophorous sinus. They consist chiefly of organic substances, but usually contain a considerable quantity of phosphate of lime, form rounded masses varying in size and number, though they are not often numerous, and can be detected by palpation of the udder or teat. Those which enter the teat can usually be removed by manipulation from above downwards with the finger and thumb. Failing success by this method the teat must be laid open, the calculus removed, and the wound closed by sutures. The parts (which should have been carefully disinfected before operation) can then be thickly painted with iodoform collodion, or sublimate gelatine. Calculi which do not enter the teat seldom prove troublesome.

\section{(2.) ACUTE INFLAMMATION OF THE UDIER (MASTITIS ACUTA). ITS PATHOLOGY AND VARIETTES.}

Authors have adopted the most varying views as to the origin and classification of the various forms of inflammation of the udder, some based on anatomical, other's on clinical and pathological grounds. All of these have some justification, but none equally satisfy the practitioner and the scientist. Were it possible, classification should follow ætiological 
lines, but further study and the discovery of the exact cause in each case can alone enable us to divide clinically similar conditions. Until such discoveries have been made we shall still remain ignorant of why one attack remains of a trifling character and recovery soon follows while another leads to gangrene and death. Despite the numerous and deeply interesting researches of the last few years an ætiological classification still seems impossible.

We are therefore compelled to adhere to the clinical standpoint. The old view, however, that there was only one mastitis with varying causes and results is no longer tenable, and the division here adopted is based on the course and results of the different attacks. Needless to say it must immediately yield place to any mode of grouping which better satisfies practice and theory.

During lactation acute inflammation of the udder is most common, but at other times isolated cases are seen, and under very rare circumstances inflammation of the udder has been noticed in virgin cows. Guillebeau says that cows. most frequently suffer from mammitis between the 5th and 6th year, and that the majority of attacks (nearly two-thirds) occur in the first four months after parturition; the greatest number of these soon after the act.

The physiological activity of the milk glands in many respects favours the development of inflammation. The early processes of lactation, particularly the congestive stage, so closely resemble acute inflammation, even in their outward manifestations, that it is difficult to draw a sharp line between the two, and the inflammatory non-infectious œdema noticed by Kitt is probably of this nature. Both the vascularity of the gland and the processes necessary for the development of secretory activity clearly favour the appearance of disturbances which readily take on an acute inflammatory character. It is, therefore, very natural that most diseases of this lind occur during the years of parturition.

The anatomical formation also favours acute inflammation. The milk forms an excellent cultivating medium for bacteria, to which the ducts offer an easy point of entrance. Finally, the position of the udder is exceedingly favourable to the entrance of infectious material; and as injuries to the skin of the udder, and especially to the teats, are frequent in animals, they easily form the point of origin for infectious inflammation.

The causes of acute inflammation may be divided into mechanical, chemical, thermal, and specific. In cattle the first takes the form of horn thrusts and similar injuries; large udders may be trodden on by other animals, or by the cow herself when rising. This division therefore includes bruises and wounds, as already described.

Chemical or thermal irritants are much less frequent, though the milk may undergo changes within the udder; irritant substances form 
and produce acute mastitis: our linowledge of such conditions is, however, still very limited, though such irritants are very probably of bacterial origin. Frost-bite seldom attacks more than the skin, though it is possible the udder may sometimes "catch cold." As we find in other mucous membranes that inflammatory processes are often caused by chills, it may possibly be the case in the udder also.

But more important than any of these are the specific irritants. After Franck had directed attention to the point, others described different bacteria as the cause of acute inflammatory processes in the udder. The number is very large. Some appear to be of a specific nature, others produce mastitis when injected into the udder. Of the former may be mentioned: Bacterium phlegmasiæ uberis (Kitt), Micrococcus mastitidis ovis (Nocard), Streptococcus agalactir contagiosa (Nocard, Mollereau, Guillebeau, Z/schokke), Galactococcus versicolor (Guillebeau), Staphylococcus mastitidis (Guillebeau), Galactococcus fulvus, albus (Guillebeau). Many other microbes are capable of producing mastitis if introduced into the udder. Such are: the streptococcus of strangles, the pneumococcus, the black quarter bacillus, pyogenic cocci, bacillus of malignant cedema, and bacteria found in such septic disorders as metritis and omphalitis of foals.

This diversity in the microbes discovered constitutes no valid objection to the parasitic theory; on the contrary, it probably explains the great variations in intensity, duration, and gravity of the local and general symptoms, as well as the disagreement in regard to the efficacy of any single line of treatment. Infection may occur by three paths.

(1) The slin, which, as already remarked, often suffer's slight injuries sufficient, however, to form the starting-point of infection. We see, then, why cows are more frequently affected with disease of this character than mares, the reason being that their teats are more often injured and simultaneously infected during milking. Disease once produced spreads, especially in the course of the superficial lymph vessels of the udder, and therefore first affects the slin and subcutaneous tissue. This method of spread (lymphogenous mastitis, Kitt) is, however, less frequent than the next in order, as Johne has shown.

(2) Infection from the mammary duct. In many cases a drop of milk remains hanging at the mouth of the duct, and forms a favourable cultivating medium for pathological bacteria, which pass hence into the galactophorous sinus, and finally into the smallest divisions of the affected portion of the gland, producing, according to their degree of virulence, a more or less violent inflammation of the parenchyma of the udder (galactophorous mastitis, Kitt). At the first glance it appears astonishing that inflammatory processes are not more frequently produced in this way. The apparent rarity is explained, however, by the fact that an effectual closure mechanism exists in the milk duct, and 
that the milk seldom remains pent up in the udder for any long time. Numerous experiments, in which pure cultures of particular bacteria have been injected in to the galactophorous sinuses, show, however, that inflammation may start here. Kitt even produced mastitis by painting the teat with a pure culture.

Of late it has been much disputed whether inflammation of the udder can be caused by not milking the animal. Whilst this was formerly regarded as a frequent cause of mastitis, the general view nowadays is, that retention of the milk alone never produces disease of this description. It is clear that neither view is quite right. The retention of milk, as such, certainly does not produce mastitis, but it favours its production, because of the readiness with which decomposition and the growth of bacteria occur in the retained milk, and secondarily produce inflammation.

This explains why mastitis is most common during hot weather; Strebel says most cases occur between March and September. Other factors may also be at work, such as the animal's condition, the attention it receives, and the time during which the calf is with it; but this much is unquestionable, that warm weather favours decomposition of the milk, and the multiplication of bacteria in it.

(3) Finally, infection through the blood stream appears possible (hæmatogenous mastitis), though it is probably very rare.

It also seems possible that when the disease is widely spread, or conditions are favourable for the transmission of the specific microbe, mastitis may take an epizootic form; the infected material being easily carried from animal to animal during milking. It is not astonishing, considering, the varying virulence of pathogenic microbes, that many different forms of udder infection occur, so that sometimes only slight irritation, sometimes severe inflammation, and sometimes, gangrene results. We divide the acute inflammatory processes in the udder into two groups, viz. :-

A. Acute inflammations of the udder.

(a) Phlegmonous mastitis.

(b) Catarrh of the udder.

(c) Purulent inflammation of the udder.

(d) Gangrenous inflammation of the udder.

B. Chronic inflammations of the udder.

(a) Infectious catarrhal mastitis.

(b) Tuberculosis of the udder.

(c) Actinomycosis of the udder.

(d) Bothryomycosis of the udder.

These varying forms can be clinically differentiated, and yet in a general sense the classification is in harmony with the atiology of the 
disease. It is not suggested, of course, that this classification in each case corresponds to a strongly marked anatomical and rtiological entity; for, needless to say, a phlegmonous mastitis may be complicated with abscess formation or even gangrene, and, in consequence of a mixed infection, a catarrhal mastitis may assume the purulent character.

Considering the intimate relationship between the parenchyma and stroma of the gland, it is scarcely surprising that acute inflammatory processes often extend from one to the other, that in inflammation of the parenchyma the stroma, and in disease of the stroma the parenchyma, often suffers. The former occurs the more readily, because the bloodvessels of the udder ramify without exception in the stroma. No sharp line, therefore, can be drawn between diseases of the parenchyma and those of the interstitial connective tissue.

\section{(A) ACUTE INFLAMMATIONS OF THE UDDER.}

\section{(A) PHLEgMONOUS Mastitis. ${ }^{1}$ Mastitis PHLEgMonosa.}

This disease, previously described as superficial inflammation of the udder, affects the skin, subcutis, and interstitial connective tissue. It generally starts from the teats, which in the cow very frequently suffer from surface injuries, the rough hands of the milker abrading the skin, and possibly conveying infectious material; the straw may also cause injury and infection. The micro-organisms first enter the skin and subcutis and set up inflammation, which generally extends to the capsule and the stroma of the gland.

The process, therefore, seldom remains confined to one quarter, but extends to several, or may even attack the entire udder. The isolated course of the lymph vessels of the separate teats, however, sometimes causes the disease to remain confined to single sections of the gland. The skin and subcutaneous tissue are at first oedematous, and later become infiltrated with plastic material; slight exudation of blood is not uncommon. We have to deal, then, with an erysipelatous or phlegmonous disease, of which the skin or subcutaneous tissue forms the special seat.

Causes. We clearly have to deal here with phlegmonous or erysipelatous inflammation, caused by infection through slight fissures and cracks in the skin, particularly of the teats. The disease is probably caused by pyogenic cocci or other microbes; Kitt produced the disease in cows by infecting with the bacillus of malignant oedema.

\footnotetext{
1 The term "phlegnonous inflammation" is no longer used in human medicine. It is, however, so convenient that I retain it here to indicate inflammation of the subcutis or of connective tissue, frequently associated with pus formation.-[JNo. W. D. $]$
} 
Symptoms and course. The condition is announced by swelling and redness, which is generally diffuse, and seldom limited to particular portions of the gland. It appears suddenly: the skin is injected; at first it appears bright red, afterwards bluish. The swelling is then soft and doughy, but later becomes hard and firm, and requires some force to indent it. The skin is often exceedingly hot, but pain, as in subcutaneous inflammation, is seldom severe, nor does the mill show much change in quality, though the total quantity certainly falls off. At the commencement, slight fever is present; the lymph glands of the udder swell, and the tumefaction spreads.

In four to eight days the symptoms may gradually recede, the redness disappear, the swelling subside, and the secretion of mill become normal. In other cases abscesses form. In or just beneath the skin, less frequently in the superficial portions of the gland, several fluctuating points develop, which after a short time break and discharge pus. Such abscesses are distinguished from those occurring during parenchymatous mastitis by their superficial position (antemammary abscesses) and slight size. This disease may also lead to other complications, like gangrenous inflammation.

Differential diagnosis. The present affection is distinguished from disease of the parenchyma by the slight change in the milk, by the involvement of several sections of the udder, and by the marked simultaneous changes in the skin. It is more likely to be mistaken for the œdema occurring in the udder at the time of parturition. In the latter condition, however, the well-developed inflammatory symptoms, such as increased warmth, reddening, and pain, are wanting. Nevertheless, it cannot be denied that this cedema (before calving) is sometimes so intense as to be difficult to differentiate from mastitis. In occasional cases, indeed, mastitis follows it.

The prognosis depends on the virulence of the micro-organisms, as shown by the violence with which the process develops, and by the degree of the general disturbance, particularly of fever. As a rule, the process is benign, but abscess formation prolongs it.

Treatment should, in the first instance, be of a prophylactic character. Considering the possibly contagious character of all forms of mastitis, the operator's hands and all instruments, like milk catheters, should be carefully disinfected before and after operation. The animal's bedding should be clean and frequently renewed, and the floor of the stall be washed down and sprinkled with a disinfectant. Direct treatment is similar to that in erysipelatous and phlegmonous conditions in other parts of the body. Cold applications are contra-indicated, and the best results follow infriction with mild fats (like borated or camphorated vaseline oil or butter), or with carbolised oil, mercurial ointment, \&c. 
The parts may also be washed with lukewarm solutions of disinfectants, particularly if the teats are injured. The udder must be kept warm, and may be covered with wadding to assist resolution. Some practitioners inject into the udder weak antiseptic solutions like 1 per cent. boric, carbolic, or creolin solutions. Full doses of the sulphates of soda or magnesia combat constipation, and stimulants sustain the animal's strength.

Local injuries must be kept as clean as possible by washing with a disinfecting fluid, after which they are smeared with any suitable material, like boric acid ointment (acid boric and ungt. paraffin, 1 to 10 ).

Johne saw in cows furunculosis of the udder, which spread rapidly in the affected herds, and lasted for several months. Hard, red, painful swellings, as large as a hazel- or wal-nut, occurred in the skin of the udder, generally at the base of the back teats. The neighbouring tissues were also inflamed, often appeared livid, and the diseased centre soon increased to the diameter of a child's fist. After three to four days it pointed and broke, when a necrotic plug was discharged along with the pus, in which, on microscopical examination, a dead hair-follicle could be detected. Granulation and healing soon occurred.

As a precautionary measure, Johne made the attendant wash both the udder and his hands twice daily with carbolic solution. Inoculation experiments were without result.

\section{(B) CATARRH OF THE UDDER. II. CATARRHALIS.}

This disease consists of inflammation of the lining membrane of the milk ducts. Sometimes the galactophorous sinuses alone suffer, but generally the lar milk ducts are also attacked. The process may also extend to the smaller milk ducts, and even into the alveoli of the gland. If the process is confined to the galactophorous sinus and larger milk ducts, the quantity of milk secreted is little diminished, though, as inflammatory exudate from the diseased mucous membrane is mixed with it, its quality may be considerably altered. The more the small ducts and alveoli are involved, the greater the diminution and change in the milk. In consequence, the disease by no means offer's a constant train of symptoms.

Causes. The fact that the disease is generally confined to one section of the udder indicates its origin through the sinuses. It is probably due to invasion by micro-organisms of slight virulence, which find a favourable soil in the milk. Opportunities for such infection are very frequent, and this disease is often seen widely disseminated, being carried by intermediate bearer's (milkers) from one animal to another. Finally, microbes have been directly recognised in the diseased udder or in the milk, and the disease produced by injections of pure cultures. Kitt thus produced catarrh of the udder with various microbes; amongst others, those of blue milk, oidium lactis, arthrococcus lactis, and the 
bacillus of malignant cedema. Zürn found micrococci, mycothrix threads, spores of mucor mucedo, and yeast-like cells in the milk. Nocard and Mollereau recognised streptococci as the cause of the disease, Bang staphylococci and various bacilli. Hock saw the affection during the course of foot-and-mouth disease; several portions of the udder were often simultaneously affected.

Symptoms and progress. The surface of the udder is little changed; swelling is often entirely wanting, while the redness may be slight, but pain in the depths can be detected on pressure, and by the sensitiveness shown during milking. General condition is little altered.

The principal changes are to be found in the milk, which contains clots of varying sizes, and often drops of fat. Eberhardt found the milk exhibited a yellowish covering of fat after standing; or sometimes it appeared reddish, from admixture of blood. The reaction is often normal, but it may prove acid or excessively alkaline.

The process sets in suddenly, and attacks sometimes only one, sometimes several sections of the udder. In most cases it disappears in a few days, the milk becoming normal; sometimes, however, it takes a chronic character, the milk retains its abnormal appearance, and may be mixed with pus, whilst the mucous membrane of the galactophorous sinus gradually becomes thickened, and feels on palpation like a firm cord; while the swelling extends from the sinus into other parts of the gland. Should the process in the smaller milk ducts become chronic, it occasions localised thickening and induration of the udder. In other cases the inflammation leads to stenosis or occlusion of the milk ducts, with change in the affected sections of the gland, the induration extending as far as the skin. Abscess formation, however, is not often seen until the disease has become chronic, i.e., until it has reached a stage when it can no longer be properly described as catarrhal.

Prognosis depends principally on the course of the disease; the longer it has lasted the less the chance of recovery. In some cases the function of the udder may be destroyed for the whole of one lactation period. The outlook becomes very grave when extensive thickening and hardening take place, and even small thickenings may cause trouble on account of their forming new points of origin for the disease at the next milking period.

Treatment. With a view to prevention, the greatest care should be taken against introducing infected probes, bougies, milk catheters, \&c., into the mammary ducts and galactophorous sinuses. In enzootic outbreaks it is best to segregate the disease, and provide against transmission by the milker's hands. Beyond combating inflammation, little more than this can be done. It is very important, however, to withdraw the milk frequently, if possible hourly, and at such times 
massage will be found to assist discharge of secretions from the finer milk ducts. The diseased udder must be kept war'm, and may be rubbed with fat and covered with cotton wadding. Where the disease is clearly infectious, which may be discovered by microscopical examination of the milk, disinfectants may be injected. Weak solution of iodine in potassium iodide is most efficacious. The animal is milked dry, and the fluid injected with a syringe or a teat syphon provided with a rubber tube and funnel. The udder should then be massaged to distribute the fluid and the latter removed by milking.

(c) PURUlent inflamintion OF the UdDer. Mastitis apostenitiosa.

In this form the inflammation also originates in the milk ducts and alveoli, but, instead of remaining confined to their surface, spreads to the depths and to the interstitial connective tissue.

The purulent catarrh affecting the lining membrane of the milk ducts is accompanied by phlegmonous inflammation of the connective-tissue substance of the stroma of the gland. Franck was the first to produce the disease experimentally, by injecting a decomposed meat extract into the galactophorous sinuses in cows. Next day that portion of the udder was inflamed, the milk was curdled, puriform, containing numierous pus corpuscles and micrococci, and when injected into the udder of a healthy cow reproduced the same disease. Purulent mastitis is clearly produced in a similar way, and therefore always remains confined to single quarters of the udder. Kitt described the disease very thoroughly: he found "excessive cellular infiltration of the entire connective tissue of the udder, emigration of cells, even into the lumen of the alveoli, necrosis and shedding of the epithelium of the gland, ectasia of the milk ducts, and concretions, consisting of gland secretions, which had undergone necrobiotic change, and exudate, hyperplasia of the connective tissue produced by extensive formation of fibroblasts, and atrophy of the tubuli of the gland.".

Causes. There is no doubt that this form of mastitis is caused by infectious materials entering through the mammary ducts (galactogenous mastitis, Kitt). Experiments have shown that normal milk contains no organised ferment, and therefore, when such is found, it must have entered from without; further, as the disease process always appears in a single section of the gland, the natural conclusion is that infection follows through the mammary duct. Although infection through the blood stream is possible, it is unquestionably very rare. On the other hand, opportunities for infection through the milk canal are frequent. The little drop of milk left hanging on the teat after milking may facilitate it, or milk catheters or other instruments introduced into the mammary duct also act as bearers of infection. The experiments of

V.S.

G $G$ 
Franck, Nocard, Mollereau, and others have shown beyond cavil the possibility of this mode of infection.

Numerous experiments have been made regarding the nature of the infectious material. Guillebeau found ten different microbes (staphylococci, galactococci, streptococci, and several bacilli), possessing various degrees of virulence. Guillebeau believes the constituents of the milk, particularly the milk sugar, are decomposed by the microbes, and irritant materials produced which cause inflammation.

McFadyean and Nencki showed that one of the streptococci isolated by Guillebeau from a case of mastitis was capable of decomposing milk sugar into lactic acid, albumen and peptone into an iodoform-producing substance, acetic acid, butyric acid and ammonia.

Zschokke, who has made a special study of the streptococcus mastitis contagiose, states that it occurs in two forms, viz., in long and in short chains. He believes that the gravity of the disease is very closely associated with this question of length. When the chains are short the disease ceases by itself; it is only necessary to avoid milking the affected quarters. The disease then remains confined to these, the secretion is re-established at the next calving, and the milk is then normal-the streptococcus has disappeared. On the other hand, when the long chains are present the disease is incurable. Even though the affected quarter gives milk at the next succeeding calving such mill is purulent, and contains numerous streptococci in long threads. For further particulars see Zschokke's article in the "Schweitzer Archiv für Thierheilk.," 1897, p. 145.

Symptoms and progress. The disease sets in suddenly, with pain and the other symptoms of mastitis. The swelling originates in the interior of the udder, but spreads as far as the skin, which is abnormally warm and reddened. Functional disturbance soon sets in; the secretion of milk diminishes or entirely disappears, the milk is curdled, and later appears yellowish from admixture of pus, or red from bleeding; finally, it may become more and more puriform, and contain curdled material, which blocks the milk ducts.

'These symptoms are supplemented by fever, loss of appetite, excessive thirst, and depression. The swelling may interfere with the movement of the corresponding hind leg and cause lameness; sometimes complications like paraplegia, or inflammation of joints or tendon sheaths are seen.

Microscopic examination reveals numerous white and red corpuscles, pus elements, and fattily degenerated gland-cells (colostrum bodies) in the milk. Chemical examination shows albumen and water to be in increased quantity, sugar and casein to be diminished.

Either resolution occurs, or the disease goes on to abscess formation. In the first case, after four or five days, sometimes a little later, the symptoms gradually begin to recede, whilst the secretion more and more recovers its normal character. In some cases no permanent disturbance is left, though, as a rule, the affected section of the udder does not completely recover its functional activity until the next lactation period. 
Abscesses, however, frequently form. Those which occur in the interstitial connective tissue either perforate into the milk canals or through the skin. They generally lie deep, and when developing in the connective tissue above the udder (retro-mammary abscess) may lead to the destruction of a large section of the gland. Permanent thickening and milk fistulæ are not infrequent results of this process.

Hard swellings sometimes result from the accumulation in the milk ducts of clots, which gradually become inspissated, and form yellowish crumbling masses, surrounded by a capsule of connective tissue. They also result from abscesses, and not infrequently form the point of origin of disease at a later period.

Such suppurative processes may entail grave consequences, and at times lead to pyæmia or septicæmia. Haase found pulmonary emboli in a cow which had died from this disease of the udder.

Prognosis. There is little hope of completely restoring functional activity to the udder during the existing period of lactation. Any further opinion must depend on the progress of the disease. When large tracts of tissue become indurated, full function will not be restored even during the next lactation, and in aggravated cases life may even be endangered. For treatment, compare with next section (D).

(D) GANGRENOUS INFLAMMATION OF THE UDDER. IIASTITIS GANGRENOSA.

Considering the varying virulence of the infectious material, it is not surprising that gangrene sometimes occurs. This form of the disease is commonest in sheep, though it also occurs in cows and goats, but in the latter it does not take the enzootic form generally seen in sheep.

The nature of the specific poison is at present little understood. Nocard isolated from the udder of a sheep suffering from mastitis gangrenosa a micrococcus, pure cultures of which, injected into the mammary duct of healthy sheep, always reproduced the disease, but in other animals only caused slight swelling.

Symptoms and course. The disease starts as a peracute parenchymatous mastitis. Severe general symptons, high fever, loss of appetite, great weakness, severe pain, and stiff straddling gait first direct attention to the udder. Local examination reveals bluish-violet spots on the skin, which on palpation are found to be exceedingly soft, insensitive, and often abnormally cool. These spots quickly spread. They are surrounded by an inflammatory swelling, which may extend to the lower part of the abdomen and the thigh. The animals stand with the back arched and the hind legs straddled, exhibit acute pain, groan, and after a short time display well-marked symptoms of septicæmia; not infrequently they die within twenty-four hours. 
In exceptional cases the local process is limited. A dissecting inflammation sets in which separates the necrotic tract and may result in recovery. This is, however, a rare exception; the greater number of animals perish with symptoms of septicæmia. The disease is not infrequently complicated by septic metritis, which may either have been produced simultaneously, or may even have occurred as the primary condition, but for the time have escaped notice.

Esser saw an enzootic outbreak of the disease in sheep. Twelve to twenty-four hours after the appearance of the first symptoms, comprising pain, and refusal of the dam to let her young suck, local manifestations set in, and generally affected one-half of the udder. The skin was dark red and showed discrete violet spots about the size of linseed, which rapidly increased in size, and felt soft and cold. Finally, the affected parts became insensitive, and the animals died in three to four days when suitable treatment was not very early resorted to. During the disease the lambs suffer from sores on the lips.

The prognosis is unfavourable, for in many cases not even the sacrifice of the udder can save the animal's life.

In treatment of the purulent and gangrenous forms of mastitis, the first point naturally is to prevent infection, or combat it if already existing. The greatest care is required in using milk tubes, probes, catheters, and other instruments introduced into the milk canals.

If the disease becomes enzootic, and especially if it assumes the gangrenous form, segregation of the diseased and disinfection of stalls is absolutely necessary. Esser bathed the udder and placed the animals in fresh stalls under the care of a special attendant. This prevented any further cases. He draws attention to the danger in the gangrenous form of transmission by the shepherd.

In purulent mastitis occurring enzootically, which Jouquain believes to be intimately connected with the use of old, bad straw, similar precautions are required. Dieckerhoff disinfects the stalls, causes the walls and floors to be washed with hot lye and strewn with lime. He also attaches great importance to careful and regular removal and destruction of the diseased milk, and to the cleansing of milk pails and pans.

A second precaution, frequent milking of the diseased quarter of the udder, is just as necessary, for the increase of infectious material occurs chiefly in the milk. Provided the udder is not too sensitive, it should be gently rubbed and kneaded to favour the discharge of inflammatory products from the acini of the gland. The milk catheter is of little service, on account of its becoming stopped by clots.

The diet should be of such a character as to diminish milk secretion to a minimum. For this purpose, in cows, purgatives like Glauber's salt may be used, and under certain circumstances bleeding may be resorted to. 
The remaining points are to destroy or render innocuous infective material in the udder and to combat inflammatory changes. The former may be effected by injecting disinfectants, though practitioners hold very different views as to their action; and while some advise their use, others consider them of no particular value. In gangrenous inflammation they are generally too late and without effect, but, on the other hand, they are often exceedingly useful in the purulent form. Their effect clearly depends on what tissues require disinfection. Though they can penetrate the galactophorous sinuses and large milk ducts, they fail to reach the fine milk ducts and alveoli.

Four per cent. boric acid is generally recommended; Eggeling advises injecting with a rubber canula a one-fifth per cent. to one-quarter per cent. sublimate solution into the galactophorous sinuses, distributing it as much as possible by cautious manipulation, and removing it in ten to fifteen minutes by milling. Franck uses 2 per cent. alum or 2.5 per cent. carbolic solution. Kielihäfer had good results from injecting 4 per cent. solution of boric acid and washing the udder externally with 5 per 1,000 sublimate solution. When the healthy quarters still give milk, carbolic acid must be avoided, because it makes the milk unpotable. Very weak solutions of iodine in potassium iodide are worth trying. The solution should only be of a very light sherry colour. It can then be freely injected. Some operators, however, use Lugol's iodine solution (iodine 1 , pot. iodid. 5, water 100) in quantities up to 4 fluid drachms. The udder should be stripped beforehand, the fluid injected, the parts freely massaged, and the fluid withdrawn.

In purulent mastitis warm poltices or fomentations and wrapping the parts in wool will help to diminish inflammation. Bang recommends linseed or oatmeal poultices, and the application of linen or woollen cloths wrung out of hot water and kept warm by covering with mackintosh. Fomentation with diluted alkali or soap and water have often been recommended. Once tension and pain diminish, the parts may be smeared with flour paste and covered with wadding. Vogel uses a mixture of two tablespoonfuls of water, four of starch, twenty of glycerine, and one of turpentine. When warm the mass forms a paste, which is applied thickly and left on for fourteen days. From time to time it may be removed to allow of massage, which is of considerable assistance in this disease. Cold is considered by experienced practitioners to be badly borne, a conclusion warranted by the nature of the disease. Infriction with unirritating fats or unguentum hydrargyri is useful. Johne employs a mixture of equal parts of blue ointment, potash soap and lard. Franck recommends an ointment of ol. hyoscyam. 8, liquor ammon. caust. 2, camphor 1 . As soon as the abscess appears under the skin it should be opened and the cavity cleansed and disinfected. 
Local induration can often be dissipated by cautious massage, though it is of little value when the swelling is of old standing.

In purulent and gangrenous mastitis medical treatment is seldom of much value, and operation offers the only hope of saving life. The operator, however, should first thoroughly satisfy himself that the results are likely to pay for the trouble and expense. Among the questions he has to answer are: Is the functional activity of the udder likely to be preserved? If not, is the animal's life absolutely dependent on operation? An affirmative answer in either case would justify operation. Single abscesses in the udder should be opened and treated on general principles; multiple abscesses may necessitate operation to save the animal's life, but not to preserve the function of the gland. Partial or total amputation of the udder is usually only of value as allowing the animal to be kept for fattening or for work.

Partial amputation becomes necessary when necrosis occurs during the progress of an attack of mastitis. Practitioners should, however, be cautious, and only operate where necrosis is circumscribed. Unless signs of demarcation are visible partial amputation is very liable to prove disastrous. The operation succeeds best in cows. In them onehalf of the udder can be removed without septic processes extending to the other half, the suspensory ligament forming a very effective septum between the two portions.

Total amputation is justifiable in acute forms of mastitis if thereby the process can be terminated. This is the case in the severe septic forms in ewes and cows, where, save for operation, death is almost certain. Strict antisepsis is necessary. In such cases an oval incision is made, embracing the whole mamma. All vessels, including the veins, must be ligatured to prevent secondary bleeding. After dividing the skin, dissection is carried on with the fingers, a director or other blunt instrument, until the udder only remains adherent to the suspensory ligament, which is then divided. The cavity is freely powdered with iodoform, filied with sterilised tampons of tow or cotton wool, and the edges brought together with sutures, which may be left in position for forty-eight hours. After this time sutures and tampons can be removed and the parts treated as an open wound. Healing sometimes occurs under a dry scab if iodoform-tannin be freely used.

In gangrenous mastitis early incision into the necrotic parts and disinfection may effect some good. If the process has made much progress, even this treatment seldom suffices, and the animal's life can only be saved by amputation of the diseased portion or of the whole udder.

Esser amputated the diseased portion or the entire organ in several sheep at the beginning of the disease. After checking hæmorrhage, the surface of the wound was powdered with iodoform and smeared with tar. 
Of five sheep thus treated, only one died. Nocard also emphasised the usefulness of injections; he recommends amputation, and subsequent cauterisation with sulphate of copper.

\section{(B) CHRONIC INFLAMMATIONS OF THE UDDER,}

\section{(A) INFECTIOUS CATARRHAL MASTITIS.}

This disease has been seen in sporadic and apizootic forms, affecting cows, sheep and goats in various parts of Switzerland, France, and Italy. It consists in a chronic infectious catarrh of the udder, which, without causing any particular anatomical lesions, leads to atrophy.

Guillebeau and Hess regard the cause as a streptococcus which they found in the milk and in the cells and interstitial connective tissue of the gland. It is easy to cultivate and stain, and by inoculation reproduces the disease,

The clinical appearances consist in diminution in quantity and change in character of the milk, withaut any particular pain or swelling of the udder. Kitt has therefore termed the disease Agalactia contagiosa catarrhalis. No loss of bodily condition usually occurs.

Diagnosis depends on finding the specific organism in the mill on microscopic examination.

The prognosis is favourable so far as life is concerned, but unfavourable in regard to the restoration of function of the udder, which is usually lost. Moreover, in those cases where the disease seems to have been arrested it often reappears during the next lactation period.

Treatment has hitherto been unsuccessful. Preventive measures, like disinfection of the sheds, \&c., seem most promising.

\section{(B) TUBERCULOSIS OF THE UDdER. MASTITIF TUBERCUlOSA,}

Tuberculosis of the udder can scarcely be regarded as a surgical disease. It is usually of a secondary character. In Denmark it appears to be common.

The clinical appearances consist in slow enlargement of the udder, without acute inflammatory symptoms. The supramammary lymphatic glands become simultaneously enlarged, sometimes to the size of a man's fist. At first the milk seems unchanged, but afterwards contains tubercle bacilli (sometimes in great numbers). Still later, it diminishes in quantity and becomes watery, flocculent, or lumpy.

Diagnosis is assured by the discovery of the tubercle bacilli in the milk, though the clinical appearances are often quite sufficient to settle the character of the disease.

The prognosis is unfavourable, and treatment is of no value. 


\section{(C) ACTINONYCOSIS OF THE UDDER.}

This disease occurs in cows and, much more frequently, in sows, but, owing to the peculiar mode of attack, is in the latter seldom diagnosed.

Actinomycosis appears in two forms. In one, hard swellings develop, consisting of a fibrous exterior and a central abscess-cavity clothed with granulations; in the other, the little actinomyces growths are found distributed through firm but apparently normal gland tissue. Both forms appear in the animals mentioned. The last form, however, sometimes goes on to the formation in the diseased tract of extensive abscesses, containing thin fluid pus and numerous masses of the actinomyces fungi.

The cause is the presence of the above-mentioned fungi. Their exact mode of entry is unknown, but that they may grow if introduced into the milk duct has been shown by Johne, who experimentally injected cultures.

The clinical appearances consist in slow enlargement of the udder, which shows either discrete hard swellings or a diffuse, hard, slightly nodulated enlargement. Sometimes the abscesses break externally and are succeeded by fungating masses of granulation tissue, or by fistulæ, or, again, by cicatricial depressions on the surface of the gland.

Diagnosis is at first difficult. The disease is distinguished from tuberculosis by the normal condition of the supramammary lymphatic glands. The recognition of the specific fungi confirms the diagnosis.

The prognosis is unfavourable to the continuance of the millk supply, as the gland gradually undergoes atrophy. Sucking pigs sometimes die in consequence of the sow being unable to supply proper nourishment.

Treatment is often uncalled for, as the disease makes slow progress and the animals can be fattened. The use of potassium iodide in mammary actinomycosis has not yet been properly tested. As a rule, it is preferable to prepare animals for slaughter.

\section{(D) BOTHRYOMYCOSIS OF THE UDDER.}

Bothryomycosis is commonest in the mare, though it has also been seen in cows. The udder becomes converted into an indurated mass of connective tissue strewn with hard "tubercles" containing pus, in which are found the bothryomyces.

Clinically the disease appears as a chronic, gradually-extending, painless swelling of the udder, which becomes hard and knotted, and from time to time is the seat of circumscribed acute inflammatory swellings. These break and discharge pus containing the specific fungi. The wounds may heal, or may be succeeded by sinuses, but the udder steadily increases in size. Gradually the process may extend to neighbouring structures producing like changes.

Diagnosis is easy, provided the pus be microscopically examined. 
Prognosis depends on the degree of the disease. If the udder alone be invaded, recovery may follow operation. Otherwise, nothing can be done, and the animal must eventually be slaughtered.

Treatment generally necessitates amputation of the udder. Potassium iodide treatment has hitherto proved unreliable.

\section{(3.) STENOSIS AND CLOSURE OF THE MAMMARY DUCT.}

In cows-and these alone usually demand our attention-the mammary duct only possesses one opening, at which point the skin is reflected to cover the lower end of the duct. At the upper end of this canal, which is from $\frac{3}{16}$ to $\frac{1}{4}$ inch in length, the mucous membrane begins, and lines the galactophorous sinuses up to their termination. At the point where the skin. becomes continuous with the mucous membrane, i.e., about $\frac{3}{8}$ of an inch above the end of the teat, lies a sphincter or valve, which, like the greater part of the teat, consists of muscular fibres.

Stenosis of the mammary duct in cows results either from proliferation of the well-developed epithelium lining it, or from contraction of cicatricial tissue. The former of these conditions occurs while the animals are "dry," and stenosis only appears after next calving.

Occlusion of the duct is sometimes congenital in cows, or results from inflammation at the lower end of the teat. Diseases of the skin, cowpox, aphtha, injuries, \&c., may cause adhesion and obliteration of the opening of the duct and of a considerable proportion of its length.

In the upper sections tumour formation occurs, and sometimes folds of mucous membrane project into the lumen of the duct and produce closure. More frequently chronic inflammation, occurring during mastitis, causes stenosis or complete occlusion; finally, the opposing walls of the mammary duct and lower portion of the galactophorous sinuses may become adherent.

Tumours sometimes form in the upper portions of the milk duct, or folds of mucous membrane may be so disposed as to obstruct the flow of milk. The constriction or valve above referred to is not constant in character ; sometimes it is represented by a fold of mucous membrane, but in other cases there is only present a circular arrangement of musccular fibres of the nature of a sphincter.

Jensen examined 2,048 teats (cow's), with the following results :

In 84 cases there were accessory portions of gland within the wall of the galactophorous sinus.

In 7 cases there were circular folds of mucous membrane.

In 28 cases the walls of the teat were very thick, and the sinuses small.

In 53 cases there was a nodular epithelial thickening at the margin between the duct and sinus. 
In 3 cases there was a vertical dividing wall in the sinus.

In 1 case there was doubling of the teat (congenital).

In 1 case there were small accessory sinuses.

In 6 cases there were traumatic injuries.

In 9 cases there were ulcerations in the mucous membrane of the sinus.

In 221 cases there were cicatrices in the mucous membrane of the sinus.

In 97 cases there were papillomata in the mucous membrane of the sinus.

In 9 cases there were fibrous enlargements in the mucous membrane of the sinus.

In 37 cases there was thickening of the mucous membrane of the sinus.

In 16 cases there was formation of septa in the sinuses.

The diagnosis of this condition offers no difficulty, though the discovery of the cause is sometimes a hard task. Congenital closure will be recognised on the first calving by the excessive distension of the udder and teats, and by the fact that no milk flows when the parts are com. pressed. The little piece of skin covering the opening of the teat visibly protrudes, and on attempting to pass a probe, no opening can be found in the teat; the same is the case in adhesions, in which, however, indications of an opening exist.

In stenosis or occlusion of the duct the probe either passes with great difficulty or not at all. The milk escapes in a thin stream, while the animals evince pain by lkicking or moving about. Stenosis, caused by thickening of the epithelium or proliferation of the mucous membrane, can be felt as a cord-like swelling when the teat is rolled between the fore-finger and thumb. Induration in the upper portions of the teat may similarly be detected, whilst, in closure by folds of mucous membrane, palpation gives a negative result, and the probe meets with a somewhat yielding obstruction in the depth. This valve-like closure is occasionally indicated by those portions of the galactophorous sinus above the valve gradually becoming filled with milk and increasing in diameter, whilst those below appear small, and are generally occupied by a clear watery fluid, which, according to Larsen, always contains bacteria (cocci or bacilli). A small quantity of this fluid may from time to time be discharged. Sometimes the valve can be thrust on one side with the probe, and, for the time being, the milk allowed to flow freely away.

Treatment. The stenosed mammary duct may be dilated by repeatedly introducing bougies; thick catgut threads, 1 to $1 \frac{1}{2}$ inches long, provided with a knot at one end, so that they cannot slip completely into the mammary duct, can be passed a couple of hours before or immediately 
after milking, and allowed to remain in position for some hours. They can be used more than once, but, to prevent infection and severe mastitis, the bougies should be carefully cleansed and dried on each occasion. The same is true of all other instruments, such as mill catheters, probes, \&c., which must be carefully cleansed and rendered absolutely sterile. Particular care is required where the teat is inflamed, as in cowpox, aphtha, \&c.

If for any reason the mammary duct cannot be dilated in this way it may be laid open, or the end of the teat amputated. For the former purpose a narrow, straight tenotome, or specially-constructed "milk needle," consisting of a slender double-edged fistula knife, is employed. The operation is easiest with the udder distended. The animal is twitched (with "bulldogs"), the teat grasped with the left hand, and attempts made to distend it with milk. The instrument is then introduced $\frac{1}{2}$ to $\frac{3}{4}$ of an inch, and the milk duct divided. As the milk afterwards flows away in an oblique direction, it is best to divide the anterior teats in a backward and the posterior ones in a forward direction, to direct the stream in each case towards the milking-pail.

The result of division is often unsatisfactory, the wound growing up again, and stenosis becoming, if possible, still more pronounced. Inflammation of the udder may also follow, in consequence of infection with micro-organisms always to be found in the mammary duct below the sphincter. Inflammation therefore sometimes occurs, despite antisepsis. For this reason the cavity should first be disinfected by injecting a 1 in 1,000 sublimate solution, and the obstruction then divided with a sterilised "milk needle." Congenital atresia is most easily dealt with; here it is often sufficient to make a cross-shaped incision or a puncture with an inoculating needle or similar instrument.

In acquired stenosis which resists other treatment, Bang recommends amputating the lower end of the teat just over the mammary duct; the sphincter can be distinctly felt here, and its position easily determined. The animal's hind feet are fastened, and the end of the teat cut away with strong scissors, the skin being as far as possible preserved. In Denmark special forceps are used, and the teat grasped, so that the parts can be removed with one powerful cut just below the forceps. Bleeding is slight. The parts must be kept as clean as possible, to prevent inflammation in the affected division of the udder. For a short time milk flows away continuously, but this can be prevented by applying a suitable rubber ring: During the healing process, the parts gradually contract, involuntary discharge ceases, and the animal can again be milked in the usual manner.

In closure of the upper parts of the teat by indurated masses of tissue, a passage may be made by passing a thin trochar, the canula being left 
in position for some time, so as to prevent adhesion, whilst the milk is prevented flowing away by stopping the canula with a cork. The trochar is also useful in occlusion of the duct by folds of mucous membrane, though success is often only temporary. It has been suggested to remove such obstructions by dividing them with the "mill needle." In this case also relief is rarely lasting.

Madsen eradicated papillomatous growths from the galactophorous sinus by disinfecting the parts carefully, slitting the teat, removing the growth and suturing the wound.

Septa in the sinus or duct have been ruptured by force applied from without. A portion of the udder is grasped between the fingers, and the contained milk suddenly pressed against the dividing membrane until the latter gives way. Some operators use special forceps with wide rounded jaws or even two rounded wooden staves covered with rubber tubes, between which a portion of the gland is grasped and the milk so forced against the septum.

\section{(4.) TUMOURS OF THE UDDER.}

Tumours of the udder are oftenest seen in bitches, less frequently in cows and mares. In cows and bitches they usually take the form of warts, and are much commoner on the teats than on the udder. Frequently they are pedunculated, and from 1 line to $\frac{1}{2}$ an inch or more in length. Those on the surface of the udder are usually larger and have broader bases; in cows, they have even been seen as large as a man's head. Their surface is rough and horny, sometimes moist and inflamed from injury during milking. Kunze found the udder of a four year old cow covered with numerous warts 4 inches long and $\frac{3}{4}$ inch thick, so that it resembled a large cauliflower growth; the warts were removed by ligature, and by swabbing with pure sulphuric acid.

Less frequently polypi occur on the mucous membrane of the mammary or milk ducts, and may interfere with the discharge of millk.

Cysts, varying from the size of a hazel-nut to that of a hen's egg, are sometimes discovered in the udder after slaughter. They are generally retention cysts, caused by closure of single gland ducts; their contents are sometimes clear, sometimes turbid from contained masses of fat. They are difficult to detect during life, only those which are superficial exhibiting fluctuation; those in the depths can scarcely be differentiated from indurations.

Sarcomata are seen in cows and bitches. Carcinomata and enchondromata are a frequent source of trouble in bitches. Stockmann has described two cases of adenoma of the udder in bitches, in both of which 
there was fatal pulmonary metastasis. One case, which was not operated on, had been visibly unwell for a week before death; in the other the mammary growth was excised; death followed by gradually increasing dyspnoea on the fourteenth day. Stockmann regards adenomata as the commonest malignant neoplasms in the bitch, and states that their primary seat is usually in the mammary gland. Carcinomata are also stated to have been seen in the mare. Such malignant tumours occur in a multiple form, and carcinomata are usually accompanied by swelling of the lymph glands. In bitches, the skin is often under great tension, the swelling sinks and forms a pendulous tumour, and its position then favours injury and ulceration.

In forming a prognosis, the nature, seat, and size of the new growth must all be taken into account. In cows, warts on the teats give pain during milking, thus rendering the animals restless, and do not disappear spontaneously as in other positions, like the mouth.

Operative removal is not difficult, but the pain during milling is not diminished but rather increased on account of the wound. It is therefore best to wait until lactation ceases. If this cannot be done, pedunculated warts must be removed close to the skin, using scissors, and the surface cauterised with nitrate of silver, which will also check any slight bleeding. In case of subsequent pain during milking, the milk must be removed from the affected quarter by the teat syphon.

Warts on the surface of the udder only require treatment when they interfere with milking. In attempting enucleation, the gland substance must be carefully avoided, and therefore it is well to defer operation until after lactation, or remove the milk by syphon, bearing in mind the precautions mentioned in the section on "Stenosis of the Mammary Duct."

In bitches, malignant tumours, even if extensively developed, may generally be removed without danger to life. The general rules for such cases are to operate in the loose connective tissue, remove secondary growths as far as possible, and suture the skin, so that after applying a dressing, rapid union may occur. A dose of morphine prevents the animal loosening the stitches. Should primary union not be obtained, the parts are treated as an open wound. The fact that the dog licks the wound usually renders cleansing unnecessary, and healing almost always occurs without difficulty, even after removal of malignant tumours. Secondary growths certainly often occur, but operation may be repeated. Even though recovery is not complete, the owner is often contented if the animal's life is prolonged for some years.

Up to the present, diseases of the ovary in animals have received little attention; the ovary seldom appears to become inflamed, whilst tumours 
are only discovered on slaughter. Various writers recommend, however, in nymphomania of cattle, to crush the cysts sometimes met with in the ovaries by operation through the walls of the rectum, from which point also the diagnosis is made.

The radical cure is ovariotomy or oöphorectomy, for a description of which see the first volume of this series ("Operative Technique," by Jno. W. Dollar, p. 250). 


\section{DISEASES OF THE SPINAL COLUMN AND PELVIS.}

\section{I.-FRACTURES, LUXATIONS, AND SUB-LUXATIONS OF THE DORSAL AND LUMBAR VERTEBRÆ. COMIMOTIO SPINALIS.}

IN the horse, fractures of the dorsal and lumbar vertebræ are not uncommon, either single processes being broken off, or the body and arch of the vertebra fractured. The first are rare and of no great consequence, though fractures of the superior spinous processes of dorsal vertebræ have been described. Fractures of the transverse and oblique processes rarely occur apart from injuries to the body of the bone. Fracture of the body is serious, because bleeding takes place into the vertebral canal and causes pressure paralysis. Such accidents are commonest in the horse, and may be produced in various ways, but are oftenest caused by the animal getting below some fixed object which prevents its rising. Vertebral fractures may also be caused by collisions, by the animal rearing and falling over backwards, and being violently stopped or started. Fracture of a lumbar vertebra has been seen to result from a horse striking out violently with both hind feet, from excessive muscular action in galloping, from a collision with a tree, \&c., \&c. It is still more frequently produced by muscular action when horses are cast, hence such fractures often form complications after important operations. In France and Belgium the general opinion is that fracture is produced at the moment of casting by excessive curvature upwards of the spinal column, but German and English opinion inclines to the belief that it most frequently occurs after the animal has been cast and is awaiting operation. A dull, crunching sound is often heard at the moment. Two movements are especially dangerous, and should, if possible, be prevented:-

(1) Violent arching of the back. When the animal's head is placed against the sternum, the longissimus dorsi is passively extended. If it and the ilio-psoas now contract energetically, the pressure on some one of the dorsal or lumbar vertebræ may be so severe as to cause fracture ("crushing fracture"), which is usually comminuted; the body of the bone is almost always broken. 
(2) The second action likely to produce this accident is excessive lateral thrust on the spinal column produced by unilateral contraction of the dorsal muscles. It occurs from lifting the hindquarters from the bed, and is probably favoured by anchylosis of the vertebræ or ossification of the inter-vertebral discs. Frcolani and many others have drawn attention to the predisposing influence exerted, under these circumstances, by bony union of the vertebra. This is probably the chief reason why old horses so often suffer from fracture of the vertebræ. Such fracture is commonest during dental operations and the castration of thoroughbred horses. The necessity for dental operations is greatest in old horses, whose bones are less resistant than those of younger animals. In castration fracture generally occurs at the moment when the clams are applied to the spermatic cord, and in this case is due to the violent muscular movements. Fractures of the vertebræ occur most frequently when the animal

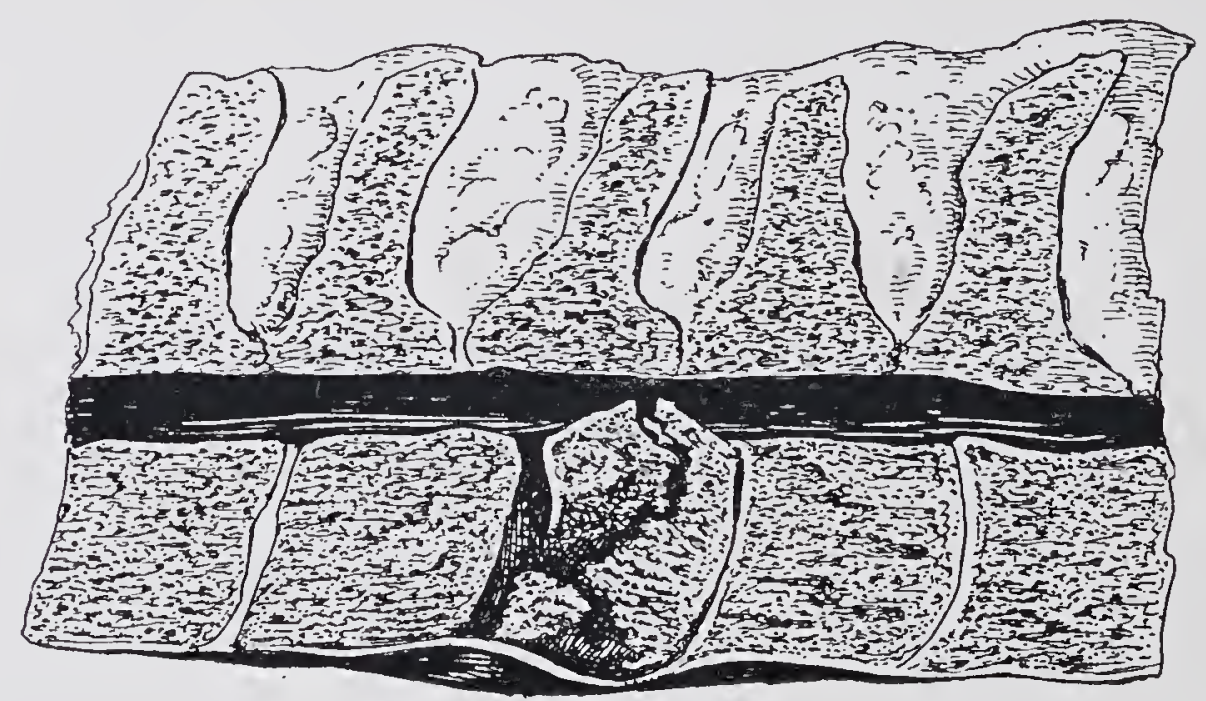

FIG. 180.-Fracture (by crushing) of the body of a vertebra.

is lying on its side, seldom when on its back; Möller only met with one of the latter, and of Dieckerhoff's fourteen cases only one was produced in the dorsal position.

Fractures may perhaps be favoured by disease of the spinal column. Thümmler describes abnormal fragility of the ribs and vertebræ in a ten year old mare; Lehnert, a case of vertebral fracture, favoured by caries, resulting from an abscess. Paltz mentions a case of osteomalacia in a horse; after several minor fractures had occurred, the 3rd lumbar vertebra was broken, with fatal results. On post-mortem examination, all the bones were found excessively soft-the transverse processes of the lumbar vertebræ particularly so. Degive also recognises such a predisposition.

With few exceptions the fracture occurs either in the last dorsal or first Iumbar vertebra, which are not only the weakest, but lie in the middle of the mobile column, which, like a stick, if excessively bent, breaks most readily in the centre. But falling over backwards, and other accidents, may also cause fracture of the anterior dorsal or lumbar vertebræ. 
Vertebral fractures in oxen occur during coitus, fracture in the cow being caused by too heavy a bull, and in the bull by falling. Small animals may be run over, or violently kicked or struck.

Luxation, that is displacement of the articular surfaces, and subluxation (displacement of the vertebra from rupture of the inter-vertebral ligaments), cannot clinically be distinguished from fracture. Voigtländer and Gillmeister saw subluxation between the last dorsal and first lumbar vertebræ in a cow, Thieme in a horse.

Symptoms and course. Fractures of the vertebral processes produce no particular general disturbance. Swelling occurs at the spot, and may sometimes lead to pus formation; while fracture in the region of the withers of the superior spinous processes, which give attachment to various muscles of the fore limb, greatly interfere with movement, as Bouley mentions in describing a case in the horse. The head and neck, and also the back and loins, were held stiffy as in tetanus; the position of the fore feet suggested laminitis; swelling, pain, and crepitation existed in the region of the withers. The animal could not lie down for a month; and although the ability to move gradually returned, yet for some time the feet were not properly lifted.

Fractures of the body of a vertebra are generally followed by displacement of the broken fragments and injury to the spinal cord, or by bleeding into the vertebral canal, and paraplegia. The animal cannot rise, and even when able to move the fore limbs, has no control over the hind. Under some circumstances injury to the spinal cord is delayed, and it is certainly possible for vertebræ to be merely fissured. Many observers have seen cases where the animal could still move, though the back was held stiffly, and where symptoms of paraplegia only occurred after several days. Spinola saw an animal which could still carry its rider and could perform heavy work, though he believed it to be suffering from fissure of a vertebra. Straube describes a horse with a similar fissure, or fracture without displacement, which could nevertheless be hunted and jumped. In rare cases the animals can stand, but cannot move, as when the ischiatic nerve is paralysed and the crural escapes. Bombach saw this condition after fracture of the second lumbar vertebra; Moller found functional activity of the crural retained after fracture of the fifteenth dorsal vertebra.

Local examination gives negative results in large and well-nourished horses. Crepitation, pain, or displacement of the superior spinous process of the affected vertebra can seldom be detected, except in small, thin animals with slight muscular development. Paraplegia is accompanied by anæsthesia of the hindquarters, sharply marginated in the lumbar region. Convulsive attacks have also been noticed. Correctdiagnosis is the more important because prognosis is always unfavourable, and slaughter must be recommended as soon as fracture of a vertebra is recognised.

v.s. 
In horses, the condition is oftenest mistaken for hæmoglobinuria. The history generally clears up this point, otherwise the urine must be examined, though it should not be forgotten that in hæmoglobinuria the urine occasionally appears normal; the presence of blood, colouring matter, or albumen would, however, point to the latter disease. In hæmoglobinuria the muscles are abnormally hard and firm, especially in the dorsal region, but in pressure paralysis there is pronounced relaxation. Again, in hæmoglobinuria the visible mucous membranes are more or less reddened, which, during the early stages at least of fracture of the vertebræ, is certainly not the case. Diagnosis is more difficult if the animal is unable to rise, and is suffering from fever.

Plugging of the posterior aorta or of the iliac arteries produces similar symptoms, but, at first, is usually accompanied by clonic and tonic contractions; at a later stage, the interference with movement occurs gradually, and is only seen when the animal is exercised. In doubtful cases, examination per rectum will settle the point.

Fractures of the vertebræ are more difficult to distinguish from injuries to the cord, produced, for example, by violent concussion. In the latter case the cord may be ruptured, or bleeding into the vertebral canal may be produced, without the vertebræ being displaced; finally, concussion of the medulla may exist without visible lesions or anatomical changes, a condition clinically described as commotio medullæ spinalis. This has repeatedly been seen in dogs and cats, which have fallen out of windows, or been run over, or struck with heavy sticks. When injuries of this sort are accompanied by rupture of the cord or hrmorrhage into the vertebral canal they take the same course as vertebral fractures, and their exact diagnosis is, therefore, of no especial importance. It is otherwise in commotio medullæ spinalis, which is generally followed by symptoms of complete paraplegia, but disappears again in a short time, sometimes even within a few hours. In many cases, therefore, the diagnosis must be extremely cautious, and an expectant attitude should be assumed. The symptoms due to concussion of the cord may disappear in eight to fourteen days, and during this time recovery is not out of the question. It should be borne in mind that concussion of this kind is rare in the horse, but fairly frequent in small animals like dogs and cats.

Schmaus's observations and experiments show that in concussion of the cord, bleeding, softening, swelling, and degeneration of single axiscylinders occur. It is evident that the course of such injuries depends on the kind and extent of the anatomical changes; and as these cannot be exactly defined during life, prognosis is always doubtful.

In fracture of the vertebra the last hope of recovery vanishes as soon as paraplegia is complete. Provided the animals can stand, treatment 
may be attempted, although, as paraplegia may still occur, recovery is uncertain; Körber saw it set in as late as five days after injury. As, however, the diagnosis in such cases can never be perfectly certain, it is advisable to wait, particularly if the animal's slaughter value is slight, or if it be valuable for breeding. Isclated cases are said to have recovered, though the descriptions given throw some doubt on the correctness of the observation. But though possible, recovery (in horses) depends on so many circumstances that it can never confidently be anticipated.

In these cases prevention is better than treatment. Stables should be

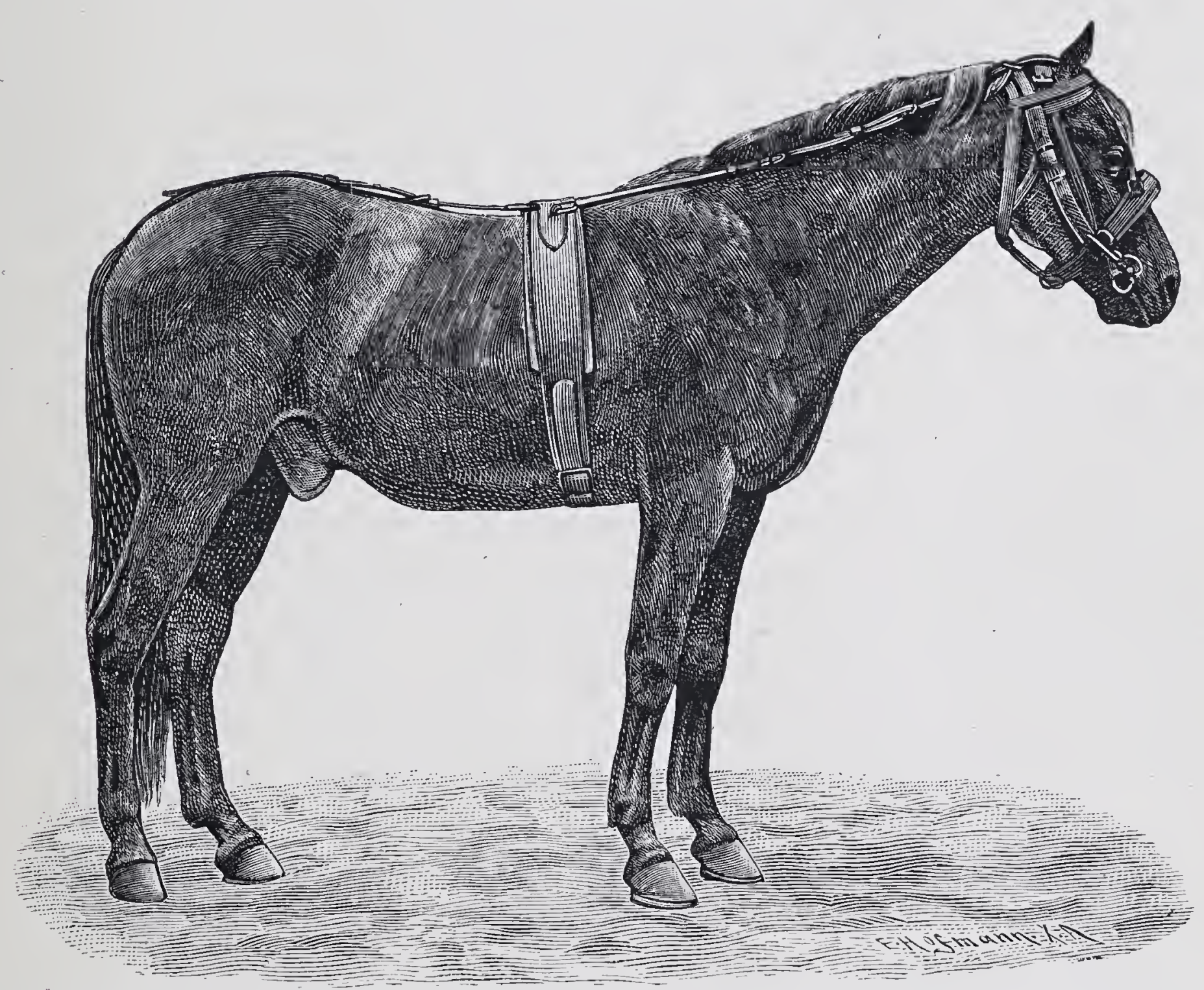

Frg. 181. - Bernardot and Butel's apparatus for prevention of broken back when casting.

so constructed that animals cannot injure themselves in rising, and great care must always be taken in casting. The first matter is beyond our province; but in regard to the second, the following points must be observed :- In casting the larger animals, a soft bed, preferably of straw, is necessary to prevent fractures of the ribs and of the bones of the pelvis. The horse should first fall with the chest towards the earth, and whilst lying the head and neck should be liept extended by several powerful assistants. As a further precaution, especially in well-bred horses with strongly-developed vertebræ, Bernardot and Butel's apparatus (fig. 181), consisting of a strong head-collar with padded straps, and of a girth and crupper, may be employed. The head-collar and girth are 
connected by thick straps, rendering it impossible for the horse to bend the head and neck beyond a certain point.

Hirzel employs for the same purpose a girth provided with a ring above the animal's back, from which leather straps pass to a strong head-collar. He considers this absolutely prevents any danger of fracture of the vertebræ.

The necessity for casting has been greatly diminished since the perfecting of the operating table. The best table now in use is that first invented in 1890 by M. Vinsot, and since considerably improved. For a description of this and other means of restraint, see the 1st volume of this series ("Operative Technique"), pp. 22 et seq.

To prevent side-movements of the spinal column when the horse is cast, a man should seat himself on the quarter, and, with the same object, it is well to place the animal with the quarters at a lower level than the feet. In painful operations like castration, particularly in well-bred horses, chloroform should be used. It is also best to avoid castrating aged race-horses when in full condition, as the muscular development is then at its greatest. Such animals should previously be rested for eight to fourteen days in the stable and put on low diet.

In suspected fissure of a vertebra, the animal must be kept from work, and prevented lying down for a month, either by being placed in slings or fastened up. In commotio medullæ spinalis treatment is expectant. The animals should have a deep straw bed, and be turned from time to time to prevent decubitus. ${ }^{1}$ In simultaneous paralysis of the rectum, the fæces must be periodically removed; and if the bowels are confined clysters of luke-warm soap and water may be resorted to.

\section{II.-FRACTURES OF THE SACRAL AND CAUDAL VERTEBRÆ.}

Fractures of the sacrum are commonest in cows, and are caused either by external violence, falling on hard objects, or falling from a height, and in the fotus, by clumsy, violent efforts at assistance during parturition. Albrecht saw fracture between the sacrum and last lumbar vertebra in a bull after mounting a cow. A horse suffering from fracture of the first and second. lumbar vertebræ after collision was seen in the Vienna clinique. In an army horse a fall was followed by fracture of the fourth and fifth lumbar vertebræ.

Fractures of the caudal vertebræ in dogs and cats are most frequently produced by the animals being run over or having the tail nipped in a

\footnotetext{
1 Decubitus is a convenient medical term used to describe the ill effects attendant on the animal continuously lying in one position. It includes the formation of bed-sores, the production of hypostatic congestion, \&c. [JNO. A. W. D.]
} 
door ; in larger animals, by falling over backwards, if at the moment the tail is lifted. To induce oxen to move, the tail is sometimes violently twisted, with the result that the caudal vertebræ are fractured. In the tail region the inter-articular cartilages are sometimes torn through. Treatment is very similar to that of fracture.

Symptoms and course. The plexus, formed by the last lumbar and two first sacral nerves, provides the muscles of the hind limbs with motor fibres; and fracture of the first sacral vertebra or of the lumbar vertebræ is therefore followed by paralysis in that region. The plexus pubo-coccygeus, which leaves the vertebral canal through the third and fourth sacral notches, gives motor and sensory fibres to the rectum, penis (N. dorsalis penis), and perineum. Fractures of the sacrum are therefore always attended with paralysis of the tail and rectum, and sometimes of the bladder, in addition to paraplegia. The symptoms depend on the position of the fracture and the changes consequent on it. The fact that blood is often poured into the vertebral canal, explains why fractures occurring further backwards than the last sacral vertebra are often attended with extensive disturbance. Most fractures of the sacrum in cattle are accompanied by weakness in the hindquarters, some with complete paralysis. The fragments of bone are generally displaced downwards, voluntary defæcation is always and urination sometimes impossible. Fractures of the caudal vertebræ only paralyse the tail when the first vertebræ are affected. The tail is generally exceedingly mobile, and crepitation may be audible; not infrequently the skin is injured (compound fracture); sometimes the end of the tail remains hanging by a few tendinous shreds of the caudal muscles.

So long as an animal with fracture of the sacrum is able to stand recovery may occur, but cure is out of the question in complete paraplegia, especially in horses ; in cattle and carnivora paraplegia renders prognosis doubtful. Paralysis of the urinary bladder is a very unfavourable symptom, as, on account of the necessity for emptying the bladder by catheter, infection may occur, the urine decompose, and severe cystitis setin. A cow, however, with paralysis of the rectum, consequent on displacement between the first caudal and last sacral vertebræ, recovered completely (Harms). Fractures of the upper caudal vertebræ may also cause deformity and limitation of movement in the tail. Möller saw a calf in which, in consequence of fracture, the tail was twisted to one side and could no longer be freely moved. Compound fractures of the tail vertebræ are readily followed by necrosis; this always happens when the vertebræ are crushed; the tail then feels cold, showing that the blood-vessels are thrombosed. Permanent paralysis is another frequent consequence, and is especially troublesome, because the tail becomes soiled by the frees, and in cows by the urine. 
An army horse, the subject of fracture between the fourth and fifth sacral vertebræ, showed a swelling as large as a man's fist over the sacrum, and suffered from rolling gait, paralysis of the tail, and difficulty in passing fæces. After five weeks' rest in slings, the swelling increased to the size of a child's head, and became harder ; seven months later the animal could carry a light rider; but as sacral paralysis gradually set in, the animal had finally to be killed. Post-mortem examination revealed the presence of a callus as large as a child's head, and fresh bleeding into the vertebral canal.

Treatment. Treatment is seldom justifiable in fracture of the sacrum accompanied by complete paralysis, or if attempted, must be confined to preventing decubitus. When, in consequence of complete paraplegia, the animals lie continously, the usual precautions must be taken. The discharge of frees and urine requires to be assisted. In repeatedly using the catheter, the precautions recommended on p. 379 must be borne in mind.

Fractures of the caudal vertebræ, with severe local bruising or crushing, necessitate amputation of the tail; in compound fractures it is generally the quickest method of cure. Only in valuable horses is it worth while resorting to antisepsis or the application of dressings.

\section{III.-CURYATURE OF THE SPINE.}

The most frequent form of this disease is hollow-back, a condition known as lordosis. ${ }^{1}$ Curvative upwards is called kyphosis, ${ }^{2}$ and towards the side skoliosis. ${ }^{3}$ Simultaneous curvature upwards and sideways is termed kypho-skoliosis.

One class of these abnormalities arises during intra-uterine life. Animals born crippled are generally at once killed. Marked curvature of the spine, immediately after birth, has been often seen in pigs ; Rehrs noticed curvatures (hump-back) during rhachitis. Pigs also suffer from curvature of the spine in consequence of being trodden on by other occupants of the sty. Pütz describes kypho-slioliosis in an eighteen months' foal. The dorsal vertebræ were bent towards the right, the lumbar vertebræ towards the left, and the back was depressed in places. The condition described by Preusse (kypho-skoliosis) in a foal appears to have been congenital. Vives noticed a horse in which the lumbar vertebræ were bent downwards almost at a right angle, and the thorax and abdomen were so depressed that the latter almost touched the ground. A tree had fallen on the animal's back, causing symptoms of vertebral fracture. After some time recovery took place, but the vertebral column remained bent.

(1) $\lambda o ́ \rho \delta \omega \sigma \iota s=$ curvature.

(2) $\kappa u ́ \phi \omega \sigma \iota=$ clookedness.

(3) $\sigma \kappa \circ \lambda i \omega \sigma \iota s=$ crookedness. 
T'he causes of such abnormalities cannot always be identified, but the conditions may be grouped as follows:-

(1) Hereditary deformities.

(2) Results of fractures and luxations of the spine.

(3) Rhachitis, osteomalacia, caries, and other local diseases of the spine.

The first are of the greatest interest, and are typified in the horse by lordosis. Although little amenable to treatment, this condition has a special interest from the prophylactic point of view.

Lordosis develops during the first year's of life, though occasionally it appears later. The predisposing cause is weakness due to abnormal length of the back. The extrinsic causes include all influences leading to continuous or excessive strain on the spinal column. Amongst these are the weight of the rider, distension of the colon by bulky food, or enlargement of the uterus consequent on pregnancy; mares which have repeatedly been bred from frequéntly suffer from lordosis.

In foals, the condition may result from feeding from high manger's and racks, which throws increased strain on the extensors of the back and depresses the spinal column.

The collective result is to bend the spinal column downwards. The ventral surfaces of the vertebræ become extended, the dorsal surfaces compressed. The animals being young, the lower portions of the vertebræ grow, while in the upper part growth is checked by the increased pressure, and therefore, as time elapses, the spine tends more and more to assume that particular form. This explains why in man work leads to kyphosis, in animals to lordosis. Special disease processes, like rhachitis, may favour the development of such deformities, but at present are little understood in animals.

A second factor in the production of spinal curvature (Pott's disease) is local disease of the vertebra, like caries, i.e., some chronic process accompanied by inflammation (osteomyelitis). Tuberculosis is, perhaps, the principal cause of such disease in man, but actinomycosis has also been recognised. Hess in a steer saw kyphosis, in consequence of tuberculous disease between the second and third lumbar vertebræ.

The greatest number of cases have been seen in oxen as a result of tuberculosis. Morot described twenty. Treatment is practically useless. Distortion following suppurating ostitis of one or more vertebræ is occasionally seen in animals, including the horse. The first cause is usually a deep-seated abscess, or a wound implicating the vertebral column. Treatment then consists in puncturing the abscess, removing loose fragments of bone, and continuously irrigating the parts with a disinfectant. So long as no signs of paralysis occur there is hope of recovery, though such cases are always prolonged and wearisome. Fractures and luxations 
may also be responsible for spinal curvature, but as the animals are usually killed, such cases seldom have an opportunity to develop. Finally, disease of the intra-vertebral dises may cause deformity.

A twenty-five year old horse which had suffered for many years, and in. increasing degree, from hollow-back was found on post-mortem to have marked convexity of the spine, extending as far as the third lumbar vertebra. The aorta, which was aneurysmal and had very thin walls, followed this curvature; the articulation between the last lumbar vertebra and the sacrum showed signs of arthritis. The centres of the intra-vertebral cartilages, between the dorsal and lumbar vertebræ, had disappeared; their circumferences were ossified, and exostoses existed both in the vertebral canal and on the under surface of the bones. The superior spinous processes of the dorsal vertebræ also showed signs of rarefying ostitis, which had diminished movement. The bodies of the lumbar vertebræ were firmly united by bony material.

Symptoms and course. Only when the above described changes become greatly developed do they excite attention. The rate of development of lordosis is very varied. When it appears suddenly it may be followed by difficulty in respiration. As a rule, however, it occurs gradually, and only in exceptional cases becomes so well marked as to interfere with the animal's usefulness. Most of the dorsal and lumbar vertebræ are usually involved in the curvature. Where single vertebræ are diseased, e.g., where the deformity is due to caries, flexion develops slowly, whereas that resulting from fracture is of sudden onset. This fact generally enables the nature of the disease to be distinguished.

Treatment. In animals reduction of displaced vertebræ is seldom possible. The only means (orthopædia) is difficult and tedious, so that results are robbed of their value by the time and expense involved. Prophylaxis is more important, especially in foals. Up to a certain point lordosis can be prevented, by proper precautions. Young horses with long backs should never be heavily loaded. It is best to give concentrated food, and to place it in low mangers or on the earth. Grazing exercises a beneficial influence, on account of the back having to be bent upwards as the animal crops the grass.

\section{IV.-FRACTURE OF THE PELVIS.}

Fracture of the pelvis is commonest in the larger animals, particularly in horses, and is caused by falls on smooth pavements, by collisions, sometimes by the animal's legs slipping from under it, or by the horse making a sudden turn when being ridden. Since asphalt has been extensively laid down fractures of the pelvis in horses have greatly increased in frequency, and often occur even without the animal falling. Trasbot saw fracture of the ilium in a race-horse produced while galloping. The pelvis may also be fractured by casting the horse on hard ground, if the pelvis falls first; the animal's chest should first touch 
the bed. Stockfleth noted a case of fractured pelvis from violent struggling: in hobbles. Fractures of the outer angle of the ilium are also caused by passing through narrow doorways, by kicks or by heavy objects falling on the bone; the skin is often simultaneously injured, and a compound fracture results; with few exceptions, other pelvic fractures remain subcutaneous. In the mare, however, Möller saw perforation of the vagina by a splinter of the ischium.

In cows fractures of the pelvis may be caused by violent attempts to effect delivery. Similar injuries occur during coitus and after falls.

Whilst in large animals the weight of the body or violent muscular

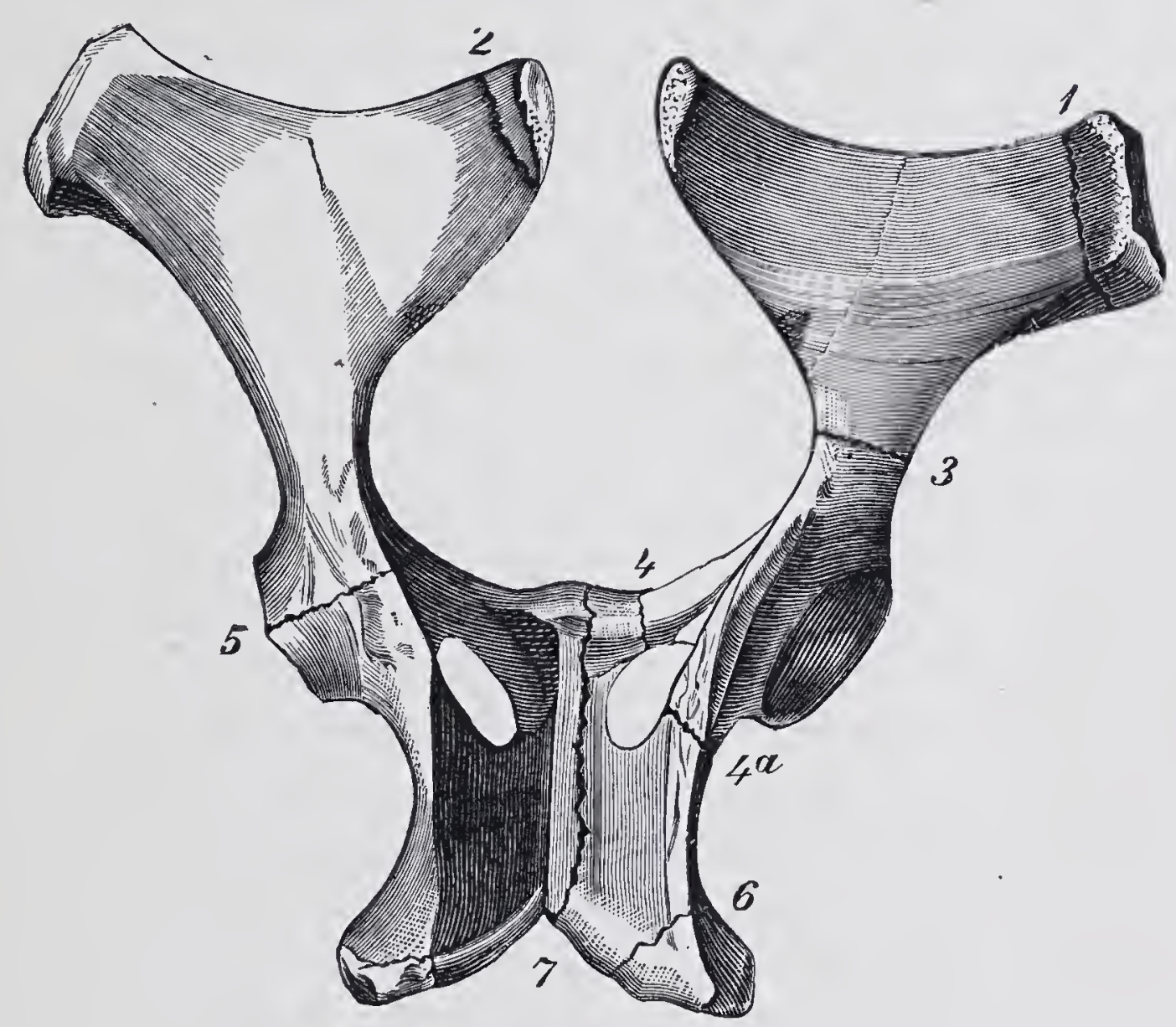

FIG. 182.- Schema illustrating fractures of the pelvis in the horse.

Fracture of (1) external iliac angle; 2 internal iliac angle; (3) shaft of the ilium; (4) transverse portion of the os pubis ; $(4 a)$ external portion of the ischium; (5) cotyloid cavity ; $(6)$ tuber ischii; (7) symphysis pubis.

action is responsible for these fractures, in smaller animals and in man they generally result from external violence, as from being run over, severely kicked, or crushed in narrow passages or in doorways. This explains why multiple fractures of the pelvis are frequent in small animals, but comparatively rare in large ones.

For the purposes of diagnosis and prognosis, fractures of the pelvis may be divided into two groups:-

(1) Pelvic fractures without division of the pelvic girdle (fig. 182). These comprise fractures of the external (1) or internal (2) iliac angle, fractures of the tuber ischii (6), transver'se portion of the os pubis (4), and external portion of the ischium $(4 a)$. The external angle of the ilium is the most frequent seat. It need scarcely be said that such injuries 
are less grave than the following group, though they may, nevertheless, cause incurable lameness-as, for example, when they involve the acetabular margin of the hip-joint. Jess mentions a case where a horse fell several times and then suddenly died. On post-mortem examination the tuberosities of the ischium were found to be broken, the posterior portions of the bodies of the pubic bones and the shaft of the ilium fractured. Death had resulted from hæmorrhage.

(2) Fractures of the pelvis with division in the pelvic girdle. These include fractures of the shaft of the ilium (3), through the cotyloid cavity (5), through the obturator foramen, i.e., simultaneous fracture of the oblique branch of the os pubis and of the external branch of the ischium (4 and 4a), and finally, fractures through the os pubis and os ischii, parallel to the symphysis pubis (7). Multiple or comminuted fractures of the pelvic bones sometimes occur. Fracture of the ilium complicated with fracture through the obturator foramen is often seen; and one reported case showed simultaneously double-sided fracture of the inner angle of the ilium and fracture of the femur. It must not be forgotten that such multiple fractures do not always occur together, but may only be developed by moving the animal. Immediately the pelvic girdle is divided, there is danger of further fractures occurring from the entire weight being thrown on the diseased side; this is commonest after injury to the inner angle of the ilium. Huth saw one case, post-mortem, with four completely united fractures.

Symptoms. Diagnosis of fractured pelvis is easy when crepitation exists. Otherwise careful search is required, including examination by the rectum or vagina. The most important symptoms are:-

(1) Lameness. This sets in suddenly, is seldom absent in recent pelvic fracture, but varies greatly in character. In fractures in front of the cotyloid cavity, particularly in fracture of the external angle of the ilium, there is marked interference with movement of the swinging limb, ${ }^{1}$ and the forward stride is shortened. This is ascribable to the fact that one of the muscles extending the thigh is attached to the external angle of the ilium. Similar lameness is noticed in fracture of the tuber ischii. In either case equal weight is placed on each foot. Fractures behind the cotyloid cavity with division of the pelvic girdle produce marked lameness when weight is placed on the leg (supporting leg lameness); those into the cotyloid cavity produce lameness, both when the leg is swinging and when weight is placed on it (mixed supporting and

1 I have, somewhat reluctantly, introduced two new terms into English veterinary phraseology. German veterinary surgeons employ two very convenient words, which have no equivalent in English, to differentiate lameness most marked when weight is thrown on the limb, and lameness most marked when the limb is carried. The former term is in German Stützbeinlahmheit (literally, "supporting leg lameness"), the latter Hangbeinlahmheit (literally "hanging or swinging leg lameness"). [JNo. A. W. D.] 
swinging leg lameness). In fractures of the ischium and pubis there is often a tendency to place the thigh in a position of adduction. Even when these fractures are uniting, and in fractures of the ischium, supporting leg lameness and abduction of the thigh is not infrequent. In fractures into the cotyloid cavity, especially when complicated with rupture of the ligamentum teres, the thigh is more or less relaxed, whilst during one stride the foot is abducted, and during the next adducted, causing the animal to stumble.

Supporting leg lameness also follows fracture of the foramen ovale and of the transverse branch of the os pubis. In three cases seen by Möller lameness was absent: once in a fracture of the transverse branch of the os pubis, once in fracture through the os pubis and os ischii parallel to the pubic symphysis, and once in fracture of the external branch of the os ischii. The two latter fractures may not produce lameness; if then the animal is worked, the pieces of bone are repeatedly thrust apart, and union is attended with great difficulty, or may never occur.

Nocard describes a peculiar lameness consequent on fracture through the foramen ovale. The foot was advanced stiffly (tout d'un pièce), the thigh alone was normally moved, whilst the other parts of the leg seemed passively to follow it. The foot described a circle outwards, the stride being greatly shortened. This lameness, which Nocard ascribes to pressure of the callus on the nerves in the obturator foramen, shows a great similarity to that described by Möller as paralysis of the internal popliteal or of the obturator nerve. It is not surprising, under the circumstances, that the nerve should suffer disturbance of function.

A mare had fallen six days before, and on being brought for examination, showed marked supporting leg lameness and abduction of the thigh. In this case the right hind foot was abducted even when at rest. On post-mortem, a - fracture was found involving the inner and outer branches of the os pubis, and extending over the symphysis pubis to the opposite side. The outer branch of the right os ischii was fractured close behind the capsule of the joint, the inner branch was divided from the os pubis, from which point the fracture extended forward towards the symphysis pubis.

In a gelding, slight lameness, accompanied by very distinct crepitation, occurred after a fall. Even when trotted, the only point observable was slight stiffness of the back and moderate abduction of both hind feet; but by placing the hand on the quarter and causing the slightest movement, distinct crepitation could be produced. Examination per anum showed the case to be fracture parallel to the symphysis pubis.

(2) Crepitation. This is particularly noticeable in fractures of the pelvis with division of the pelvic girdle, and may even be audible several steps away. In fractures of the ischium and pubis it is less marked, though sometimes quite audible; in those of the external and internal angles of the ilium it is absent. In maling the examination, one hand is placed on the outer angle of the ilium, the other on the buttock in the region of the tuber ischii, and attempts made to move the rump from side to side so as to throw the weight from one hind foot to the other, or 
the hand is placed on the outer angle of the ilium and the horse made to move. In mares and cows, crepitation, especially in fractures of the os pubis and os ischii, can be felt from the rectum or vagina by laying the hand on the ischiatic notch, and as the sensation is better conveyed by the bones than by the soft parts, it is best to examine from this point. Very slight crepitation can also be detected by applying the ear against the quarter. When the animal is lying down, crepitation may sometimes be produced by pressing on the outer angle of the ilium, or by moving the hind leg forwards; the latter is more successful in fractures of the tuber ischii.

In fractures extending through the cotyloid cavity, crepitation can even be heard when the horse is standing by merely pressing on the muscles of the quarter, or by causing weight to be placed on the affected leg. This is explained by the fact that the muscles of the quarter are inserted partly in front of and partly below the point of fracture, and, in alternately contracting, displace the pieces of bone-a symptom that is of value both in prognosis and diagnosis.

(3) Apparent deformity of the quarter is, as a rule, only seen in fractures of the ilium. In fractures of the outer angle, the loose piece of bone is drawn forwards and downwards by the oblique abdominal muscle, and by the tensor vaginæ femoris and lies in the abdominal wall, so that the outer iliac angle seems to have disappeared. To detect this, the animal is placed with the hind feet level, and the operator, standing behind, compares the relative contours of the outer iliac angles. The larger the broken portion, the more marked, of course, will be the asymmetry.

In fractures of the shaft of the ilium, the entire ilium, and especially its outer angle, sinks; this symptom can almost invariably be detected if the horse be made to stand equally on both feet.

A peculiar change in form follows fracture of the external branch of the ischium; the buttock is flattened at the height of the tuber ischii, whilst the region of the hip-joint appears considerably swollen, and the ilium slightly depressed. The abnormality is discovered by standing a few steps behind the animal and regarding the pelvis. Fractures through the acetabulum or os pubis are not generally followed by changes of form in the quarters, though in fracture of the acetabulum a slight depression may sometimes be seen over the hip-joint.

(4) The bones of the pelvis are seldom abnormally mobile, except when the external and internal angles of the ilium are simultaneously fractured, though in exceptional cases mobility is present after fracture of the shaft and body of the ilium. In such cases the outer angle of the ilium yields when pressed on, and crepitus may sometimes be detected.

(5) In fractures of the pubis, cdematous swelling sometimes occurs 
under the belly in the region of the udder or scrotum, but its absence does not exclude the possibility of such fracture. Fractures of the os pubis are often followed by swelling about the vagina; fragments of bone from the pubis and ischium may penetrate the vagina and produce bleeding from the vulva. After fractures of the tuber ischii, swelling may also occur in the neighbouring thigh muscles. On the other hand, in fractures of the ilium, swelling is seldom externally visible, but can be discovered by rectal examination.

(6) Unusual mobility of the thigh. All fractures which divide the pelvic girdle behind the cotyloid cavity, as well as fractures into the acetabulum, with rupture of the ligamentum teres, are characterised by abnormal freedom in abduction, sometimes also in adduction, of the thigh of the affected side.

(7) Examination per anum or per vaginam affords further information in fractures of the ilium and pubis, particularly when the shafts are broken. Local swelling can be detected; and if the animal's quarters be moved to and fro, a hand inserted in the rectum easily follows the movements of the fractured pieces of bone.

(8) In fractures of the pelvis, as in other fractures, fever may occur, or symptoms of bleeding follow injury to large blood-vessels. This danger is perhaps greatest in fracture through the obturator foramen. The following are the distinctive symptoms of the most commonlyoccurring fractures of the pelvis:-

(1) Fractures of the external angle of the ilium (fig. 182-1), deformity, adduction of the stifle, swinging leg lameness, absence of crepitation, sometimes swelling and pain on pressure.

(2) Fracture of the shaft of the ilium (3). Depressed position of the affected quarter. Swinging leg lameness, crepitation when standing on the foot of the affected side. Examination per anum gives more information. This fracture is seen in cows during parturition or after falls.

(3) Fracture through the acetabulum (5). Severe swinging leg and supporting leg lameness, often accompanied by painful groaning. Crepitation without moving or placing weight on the limb, deformity slight, sometimes sinking of the hip region and abnormal mobility in the hip-joint.

(4) Fracture through the obturator foramen (4 and 4a). Supporting lameness, crepitation, no deformity, sometimes anæmia.

(5) Fracture of the os pubis (4). Supporting leg lameness, with a tendency to adduction of the limb, pain on forced abduction, produced by moving backwards or sidewards. Crepitation sometimes absent, sometimes slight, sometimes distinct. Swelling below the abdomen may be wanting. Examination per anum gives further information. 
(6) Fracture of the ischium (a); of the tuber ischii (6). Swinging leg lameness, swelling of the vagina or rectum, frequently of the muscles of the thigh. After union is complete the gait often remains shuffling, the feet being dragged and the toes excessively worn, as in animals suffering from partial lumbar paralysis. Deformity of the point of the buttock sometimes exists, the latter when seen from behind appearing abnormally broad; when from the side, not reaching so far back as the sound buttock. Crepitation on moving the hind limb.

(b) In fractures of the lateral branch of the ischium (4a), the vagina may be perforated, and bleeding occur from the vulva. Crepitation on moving the hind limbs is often marked. Slight lameness, or (if the pubis remain intact) absence of lameness, accompanied, however, by marked crepitation, point to fracture of this bone, especially if the above-described pelvic deformity exists.

A knowledge of these facts, assisted by careful rectal or vaginal exploration, will in the larger animals usually ensure accurate diagnosis, not only of the position but also of the extent of fractures of the pelvis. When crepitation is marked and the femur known to be intact, broken pelvis can be confidently diagnosed. In small animals examination by Röntgen rays may also prove useful.

The prognosis varies exceedingly, according to the position and extent of the fracture and the nature of existing complications. The danger increases somewhat in the following order:-

(1) Fractures of the angles of the ilium. In fracture of the internal angle, provided the pelvis does not separate from the sacrum, movement is not interfered with and recovery always occurs. The significance of fractures of the external angle depends on the size of the broken off portion and the amount of deformity of the quarter. When only the anterior or posterior tuberosities are broken, lameness disappears completely in three to six weeks; and even after fracture of both or detachment of a large fragment of bone, the lameness may also subside in six weeks, but not infrequently recurs after work. Fractures involving the flat portion of the ilium may sometimes permanently interfere with movement, the swinging leg lameness that remains preventing the animal going beyond a slow walk. Compound fractures of the ilium, i.e., those associated with injury to the skin, generally reunite under proper treatment, though sometimes only after a considerable time (six to ten weeks). The form of lameness frequently described as rupture of the M. tensor fasciæ latæ may perhaps be sometimes due to fracture of the extermal angle of the ilium.

Pfeiffer saw a horse with fracture of the internal angle of the ilium; the fractured part was as large as a cheese plate. The animal showed severe lameness whilst the leg was swinging forward. 
(2) Fracture of the shaft of the ilium without much displacement may in quiet animals be followed by complete restoration to usefulness; otherwise lameness remains, or continually returns after severe exertion, and restricts the animal to walking work. As the result depends on the amount of displacement and the nearness of the fracture to the joint, prognosis should be preceded by rectal examination. Prognosis is, however, generally doubtful, and is the more unfavourable the more patent the deformity of the croup, the greater the depression of the ilium, and the nearer the fracture to the acetabulum. Marked atrophy of muscle is also an unfavourable symptom. Stockfleth saw continuous lameness due to formation near the joint of a large callus, which interfered with movement of the upper trochanter.

(3) Fractures of the transverse branch of the os pubis or external branch of the os ischii are also grave. Union proceerts slowly, and often remains incomplete, resulting in the formation of a callus fibrosus; fractures of the pubis often recur, especially if the animal soon afterwards becomes pregnant. Fracture of the external branch of the ischium often fails to unite, and the animals are only of use for slow work. In a case of Möller's there was still marked crepitation after eight weeks, but complete union occurred in four months, and the animal could be put to trotting work. The fact that pain is not severe favours movement and displacement of the fragments, and interferes with recovery.

(4) Fractures of the tuber ischii generally require months to unite, and sometimes result in the toe being dragged and the M. biceps femoris thrust out of position during movement.

(5) Fractures through the obturator foramen are most dangerous on account of the risk of injury to the larger blood-vessels and of fatal bleeding. Otherwise they are rather more hopeful than the following, because, though rare, yet union may occur; and the animal become capable of slow worl. Many horses, however, are unable to bear the continual standing on the other foot, and if not killed, die from decubitus.

Jivision of the pelvic girdle close to the symphysis pubis and parallel with it sometimes produces little disturbance; but union is always slow and incomplete, while in many cases it altogether fails to occur. Laser saw a case where the bladder had been nipped between the pieces of bone. Such fractures tend to recur, especially if the animal be moved too early, or give birth to a foal.

(6) Fractures of the acetabulum are the most dangerous, because as a rule the margin of the cotyloid cavity is broken into several pieces; hence prognosis is always unfavourable.

Fracture of the pelvis through the sacro-iliac symphysis is also 
generally incurable, the danger being that, although only one side may be at first affected, the body-weight is apt to break down the union between the sacrum and the ilium on the other side, or that the inner angle of the ilium may become fractured; the animals are then unable to stand, and always die from decubitus. Complete fracture in or near the ilio-pubic symphysis in horses also appears to be incurable.

Treatment. If treatment is attempted, complete rest is a primary necessity. In fractures of the external angle of the ilium, it is sufficient to keep the animal from work for a time; if unable to stand on the foot of the affected side, the patient should be slung. This precaution is very necessary, because of the grave risk of further fractures or displacement of the broken pieces occurring when the horse lies down. In fracture through the obturator foramen, special care is required to prevent fatal bleeding.

In four to six weeks the patients may be moved cautiously, but if unable to bear weight on the foot, should be returned to slings. Stiffness in movement is less important, because it depends to some extent on the long rest, and gradually disappears with exercise. Provided pain is not excessive, the muscles of the quarter and thigh may be massaged to prevent that rapid atrophy which follows severe lameness. Daily kneading of the parts will promote nutrition of the muscles. Atrophy, being only due to inactivity, disappears with return to regular work.

Cold poultices are superfluous, indeed injurious; they soften the skin, and so favour its being rubbed by the lining of the slings (which irritates the patient), and have no effect in relieving pain. An adhesive plaster or a charge is often beneficial.

\section{Y.-DIASTÁSIS OR LUXATION OF THE SACRO-ILIAC ARTICULATION.}

The union between the sacrum and ilium is formed by a stiff, almost immobile, joint, the short capsular ligament of which extends from the upper surface of the wing of the sacrum to the under surface of the ilium. The inferior sacro-iliac ligament, assisted by the upper and lateral sacro-iliac ligaments, contributes to this union.

In horses, this ligamentous apparatus unites the bones so firmly that they are very seldom dislocated, it being easier to break the ilium. In cattle the union is less firm, the upper sacro-iliac ligament being sometimes absent. For this reason these animals occasionally suffer incomplete or complete disunion of the sacrum from the ilium, especially during parturition. Stockfleth and Berdez have thoroughly studied the condition, and their description is here followed. Berdez, considering the mode of origin of this dislocation, lays particular stress on the atony of the abdominal muscles which occurs at the time of parturition. Of 
these the rectus abdominis appears to act in opposition to the ligamentous apparatus described, and fixes the joint. When, therefore, it is relaxed, as during parturition, danger of displacement becomes immi. nent. Stockfleth believes that movement in the symphysis pubis favours displacement. If division occurs in the symphysis pubis, the sacro-iliac joint is endangered, hence the simultaneous occurrence of the two conditions as related by Golis. It will also be clear that dislocation on one side may readily lead to a similar accident on the other. It is even the rule to meet with double-sided dislocation, as noted by Albrecht, Guillebeau, and others.

According to Stockfleth, incomplete luxation is often occasioned in pregnant cows by pulling on the tail to assist them in rising, and complete luxation then follows. It may also be caused by violent muscular exertion. The ilio-psoas and longissimus dorsi, together with one group of the thigh muscles, draw the sacrum downwards, whilst the glutei and other muscles of the thigh move the inner angle of the ilium outwards. Powerful contractions in these muscles, produced by the animal rising or walking, may thus occasion dislocation of the bones.

In incomplete luxation, the union between ilium and sacrum is not completely broken down. The muscles are partly torn through, and, like the sheaths of the great nerve stems, become infiltrated with blood, but the ligaments are only partly divided. In complete luxation, on the other hand, all the ligamentous structures are ruptured, and the sacrum sinks between the inner angles of the haunch, its anterior portion especially, so that the articular surfaces may sometimes be 2 to 4 inches below the inner angle of the ilium. In consequence, the abdominal organs are compressed and sometimes injured; even the passage of fæces may be interfered with.

Symptoms and course. After incomplete dislocation, the animal is inclined to rest continuously, rises with difficulty, and when moving, takes short stumbling steps with the hind legs. Sometimes large quantities of frees are passed, a symptom which Stockfleth considers characteristic of dislocation. Pressure between the inner angles of the ilia produces pain, and the separation can sometimes be felt through the rectum.

Complete luxation is characterised by marked depression of the sacrum, and by the anterior edge of the ilium being unusually sharply defined. The swelling and tenderness in the middle line, and the inability to rise or to stand, render the condition unmistakable. On examination per rectum, the pelvic cavity is felt to be narrowed, both in its perpendicular and transverse diameters. Defæcation is sometimes interfered with, in consequence of compression of the rectum.

In incomplete dislocation, recovery may occur in three to four weeks, V.S. 
but double-sided dislocations take much longer, and many never reunite. Paraplegia generally sets in on the second or third day. The patient is unable to stand, and if parturient, will require active assistance, because labour pains, are generally weak. Not infrequently fatal peritonitis occurs.

According to Stockfleth, complete luxation is less to be feared, for the animals sometimes recover sufficiently to be useful for dairy purposes, even though, on account of the narrowness of the pelvis, they cannot be bred from. With sufficient rest the bony union is replaced by a fibrous joint, which enables the animal to walk well enough for grazing purposes.

Treatment is essentially similar in both conditions. In incomplete luxation, rest is above all else necessary. A good bed must be provided, and any open drains covered up to prevent the animal stepping into them and producing further injury. Where there is difficulty in rising, assistance must be afforded, but the tail should never be used for this purpose; assistance is also required during parturition.

Complete luxation also calls for a lengthened rest, and cases always do best if the animals lie continuously. Plenty of straw should be given, and help only afforded if the animal attempts to rise. The patient must not be driven for at least two to three months, nor again used for breeding. Complications often occur during recovery, and carry off the animals.

\section{VI.-LUXATION OF THE SYMPHYSIS OSSIUM PUBIS.}

This disease, almost invariably confined to cattle, is still rarer than the last named. The luxation most commonly appears during or as a sequel to parturition. Golis saw it in connection with luxation of the sacrum.

An eight year old cow, nearly at full term, suddenly became lame during work, and a few hours afterwards could neither rise nor, when lifted, stand on her hind legs. On attempting to move, the inner angle of the ilium on the affected side rose and sank. Displacement of the os pubis and os ischii at the symphysis pubis could also be felt. The cow was slaughtered, and the post-mortem showed luxation of the sacrum and separation of the bones forming the symphysis pubis.

Prietsch saw a similar accident in a two year old heifer, which slipped with her hind legs on a smooth pavement. The anima was unable to rise, and on post-mortem the symphysis pubis was found separated throughout its entire length.

In horses, the symphysis pubis becomes completely ossified, and therefore in them fracture is commoner than separation.

The diagnosis becomes absolute on examination per anum. The incurability of the disease renders immediate slaughter advisable. 


\section{VII.-HYGROMA OF THE SUBCUTANEOUS BURSA OF THE TUBER ISCHII IN CATTLE.}

In cattle, which often rest with the hind-quarters against walls, \&c., chronic dropsy of the subcutaneous bursa on the point of the ischium has been observed. A longish, ill-defined, painless, or only slightly painful, fluctuating swelling, the size of a man's fist or even larger, develops on the point of the buttock, and on incision discharges a serous fluid; the cavity often contains corpora libra as large as a pigeon's egg.

In some recorded cases an incision about 3 inches long was made, the corpora libra were removed, and a tampon of tow saturated with iodine tincture was inserted. Recovery occurred in two months.

\section{VIII.-PARALYSIS OF THE HIND EXTREMITIES. PARAPLEGIA.}

Strictly speaking, paraplegia means doubie-sided paralysis, and in general is used to describe disease of the spinal cord, though paraplegia is occasionally the result of brain injury. Two forms, complete and incomplete paraplegia, are recognised. The former is seen :-

(1) In injuries to, or pressure on, the spinal cord, following fracture of vertebræ in the dorsal, lumbar, or anterior sacral regions (see "Fractures, \&c., of the Dorsal and Lumbar Vertebræ"). Hess saw paraplegia in a bull caused by caries between the first and second lumbar vertebræ. The spinal column presented an elbow directed upwards, and the vertebral canal had become narrowed. As the animal was suffering from tuberculosis of the epididymis these changes were regarded as tuberculous. Stroese saw a case of pachymeningitis spinalis interna purulenta in a cow, caused by streptococci and bacterium coli communis. Matthiesen identified as the cause of paralysis of the hind-quarters in a cow an actinomycotic growth, which, growing from the condyloid foramen, had exerted pressure on the medulla oblongata. Dörrwächter found a gliosarcoma of the first dorsal vertebra in a cow. In a horse which, after falling, had shown gradually progressive paralysis from behind forwards, Fröhner discovered a circular belt of connective tissue surrounding and strangulating the spinal cord between the fourth and fifth cervical vertebræ.

(2) In inflammation of the spinal cord and its membranes in the regions indicated, usually a sequel of some other disease, especially of an infectious disease like parturient fever, influenza, strangles, or distemper. The enzootic paraplegia, so common in America, belongs to a group of infectious diseases which are constantly in evidence in some countries. Paraplegia is also one of the symptoms of sunstroke. 
On the other hand, that form of paralysis affecting the hind-quarter's during hæmoglobinuria is dependent less on change in the spinal cord than in the muscular tissue. The fact that hæmoglobinuria is always accompanied by paralysis of the hind-quarters has not only led to the incorrect description "rheumatic paraplegia," but also to the erroneous view that disease of the kidney may produce paraplegia.

Idiopathic inflammation of the spinal cord and of its membranes has also been seen in horses. Friedberger found two areas of softening a little in front of the lumbar swelling of the spinal cord, and a watery fluid in the sub-arachnoid space in an eighteen to twenty year old horse which for some days had been affected with incomplete paraplegia, and later had shown symptoms of paralysis about the head (lips and tongue). Dieckerhoff describes a case of meningitis spinalis purulenta in a horse. Axe discovered, on making a post-mortem of a horse which, after recovering from strangles, had suffered from paraplegia, œdema and capillary hæmorrhage in the pia mater spinalis and-infiltration of the spinal cord with pus corpuscles, whilst the spinal column itself was quite intact; possibly in such cases metastasis may sometimes have occurred. Johne reports having seen hæmorrhagic pachymeningitis and leptomeningitis of the lumbar portion of the cord in a horse which, whilst standing in the stable, became completely paralysed in its hind-quarters, and died twenty-four hours later. The spinal cord was more than half torn through between the first and second lumbar vertebræ, though the vertebræ themselves were free of injury. The epizootic paraplegia described in books is probably to a great extent only hæmoglobinuria. This seems also true of the condition described by Comény, which occurred in isolated divisions of a troop, and appeared mostly to affect mares. Signol reports an epizootic form of paraplegia amongst horses of Arabian blood, which also generally affected mares. The post-mortem gave no indications of the cause.

In dogs affected with paraplegia, anæsthesia of the hind-quarters, and paralysis of the bladder, Kitt and Stoss found circumscribed pachymeningitis externa chronica, with formation of cartilaginous material, thickening of the dura, dilatation of arterioles, accumulation of spinal fluid, leading to excessive distension of the dura mater; though in the spinal cord itself no change whatever could be detected.

(3) In consequence of tumour formation in the vertebral canal. Pfister found in a cow, which had shown gradually increasing symptoms of paraplegia, a lipoma $3 \frac{1}{4}$ inches long and $1 \frac{1}{4}$ thick in the anterior end of the sacral portion of the vertebral canal, which was dilated, whilst the spinal cord appeared flattened. Johne detected meningomyelitis tuberculosa in the spinal cord of an ox, whilst in a horse which had died with symptoms of paraplegia, Hertwig found a melanotic tumour. 
It had originated in the lymph glands in the lumbar region, had partly destroyed the last three lumbar vertebræ and the sacrum, and had penetrated into the vertebral canal.

(4) As a secondary symptom, paraplegia accompanies general paralysis caused by meningeal inflammation and other inflammatory changes in the brain, medulla oblongata, and spinal cord. Dieckerhoff found a gastrus larva in the cervical portion of the spinal cord in a horse.

The reports of psychic paraplegia or nervous apoplexy of the spinal cord seem completely inexplicable. Failure to discover diseased conditions on post-mortem examination of animals dead of paraplegia in no way proves that such have not existed, and this is especially true of paraplegia due to concussion of the spinal cord. Aruch describes three cases of psychic paralysis in dogs following punishment. Hagen saw paraplegia in an old horse after fright by a locomotive. The condition disappeared in three days. Probably other unexplained causes were at work in these cases. The same is true of those cases in which paraplegia is said to have resulted from the presence of parasites (worms) in the bowel. Complete paraplegia has been seen in horses after powerful electric shocks produced by lightning. Paraplegia and general severe nervous depression follow shocks by high-tension electric currents (500 volts). Professor Cadiot recently published an interesting report on the subject. (See "Jour. Comp. Path.," and The Veterinarian, March, 1903.)

Paraplegia may also follow changes in the nerves, vessels, or muscles of the hind limbs. As these will afterwards be considered separately it is only necessary here to mention lesions of the lumbo-sacral plexus and plugging of the aorta.

Since olden times every permanent disturbance of function in the hind limbs has been described as incomplete chronic paraplegia. As a rule such disturbances are accompanied by peculiar irregularities in movement and rolling gait, but these symptoms cannot be viewed as characteristic of any one disease; they may be caused in various ways. Without doubt change in the spinal cord plays a chief part, but unfortunately in this disease thorough post-mortems have not been made. The difficulty in movement and the course of the disease point to chronic morbid processes in the spinal cord. In horses, there is usually a difficulty in co-ordinating movement termed ataxia, similar to that in tabes dorsalis in man. The separate groups of muscles do not act together; harmony of function is wanting.

The diseased conditions, however, to which these symptoms are due cannot merely be, as Dieckerhoff believes, over-extension of the fascix. In "maladie du coit" which is followed by progressive paralysis of the hindquarters, changes have been found in the spinal cord. Hæmorrhagic 
myelitis with formation of cavities in the spinal cord (syringomyelitis) and degenerative processes were met with at isolated points. In other varieties of paraplegia changes could doubtless be found in the spinal cord, provided examinations were made in advanced stages and by proper methods. Wolff saw the hind legs trailed in horses in consequence of fracture of the caudal vertebræ; here the change had clearly extended to the spinal cord from the point of fracture. On the other hand, it must be allowed that incomplete paraplegia is not always the result of primary disease of the cord, nor even of changes produced in it by infectious conditions (like influenza or strangles); it may also follow diseased processes in the vertebræ. In France, paraplegia is generally considered to result from injury to the spinal column in the dorsal or lumbar region, and is described, therefore, as vertebral displacement (entorse dorsolombaire). Vatel has given a thorough description of the post-mortem of a horse affected with this disease. According to Peuch and Toussaint, the inter-vertebral dises in the dorsal or lumbar region sometimes appear yellowish-green and partly destroyed; in advanced stages they quite disappear, granulations form on the ends of the individual vertebræ, become adherent, ossify, and so lead to the formation of a synostosis. Sometimes exostoses form in the vertebral canal and press on the spinal cord. These changes may be associated with rupture of the muscles. Rigot states having found hæmorrhages in the psoas muscles, and Goubaux abscesses in the longus colli. In these cases it certainly seems that the condition has been confused with other diséases.

In horses, a condition simulating incomplete paralysis often occurs from catching cold, but disappears after a short time. In such cases muscular rheumatism is probably the cause.

Periodic paralysis of the hind limbs, owing to obstruction of the blood-vessels of the thigh and pelvis, is not uncommon. 'Tumours may also develop in the vertebral canal or extend into it, press on the cord, and produce paraplegia. In a horse brought to the Alfort clinique, carcinomata were found to be pressing on the spinal cord and causing paralysis. Secondary carcinomata existed in the abdomen. Kampmann saw paraplegia in a foal from perforation of the second lumbar vertebra by a cyst which had discharged its contents into the vertebral canal.

In cows, a condition which occurs during the last period of pregnancy, but generally disappears after parturition, has been described as incomplete paraplegia. It is really due to insufficient muscular porwer to raise the greatly increased body load, and is favoured by advanced age, defective nutrition, very fat condition, or continuous confinement to the stable. In these animals, lesions in the spinal column and 
pelvis (fissures) resulting from parturition occasionally cause complete or incomplete paraplegia. Whether so-called "reflex paralysis" occurs seems doubtful. "A great number of bovine diseases described as paraplegia are clearly due to mistaken diagnosis of painful conditions in the hind legs and digits.

In sheep, paraplegia results from the presence in the medulla of cœnurus cerebralis, a parasite, which has also been seen in the ox. In the later stages of sturdy, sheep always suffer from creeping paralysis of the hind-quarters and irregular movements of the hind feet.

In dogs, paraplegia is sometimes caused by injury (concussion) of the spinal cord or fracture of vertebræ, but generally results from distemper, and depends on chronic disease of the brain or spinal cord. Acute or chronic myelitis and atrophy of the cord have been detected; Siedamgrotzky found œedema of the cord, and Johne yellow points of softening in it. In three dogs and one rabbit Mauri noted paralysis and rolling movements; red softening of the cerebellum was discovered on post-mortem.

Complete and incomplete paraplegia have also been seen in swine. Complete paraplegia is commonest in sucking-pigs on account of the little animals being trodden on by the mother ; in older swine rhachitis may produce it. Paraplegia has also been seen in birds; Siedamgrotzky noted it in parrots. Its cause is little understood.

Symptoms and course. It is clear that conditions differing so much in their anatomical causation are not likely to agree in their symptoms. Nor do the clinical appearances usually exhibit such wellmarked peculiarities as to admit of definite anatomical diagnosis. The nature of these conditions has already been determined to some extent in man, but in animals it still remains obscure. Researches are specially needed in incomplete paralysis; exact diagnosis of the causative, condition in complete paralysis of the hind-quarters is less interesting on account of the animals being killed as incurable, or dying in consequence of decubitus (a complication which can seldom be prevented), though exceptions occur even here, as, for example, in commotio medullæ spinalis, and the course of the disease must, therefore, be carefully watched. The sudden appearance of symptoms, however, does not necessarily indicate acute disease; an army horse which had suddenly shown paralysis of the hind-quarters was found to be suffering from an osteo-sarcoma of the spinal column. Complete paralysis of the hindquarters is distinguished :-

(1) By inability to stand. The animals lie, and are unable to raise themselves or to walk. In exceptional cases, the function of the crural nerves, and therefore the ability to stand, is preserved, though the animal is unable to walk. This may be due to the fact that the solution 
of continuity in the spinal cord occurs behind the exit of the nerve referred to (fourth to sixth lumbar vertebræ). The same peculiarity also occurs in fracture of the spinal column in the dorsal region, as has been noted in horses. Dogs sometimes learn to walk on the fore legs, as related by Nocard; they lift the hind-quarters into the air by powerfully contracting the longissimus dorsi, \&c., after the manner of circus dogs.

(2) Reflex irritability is completely in abeyance, and the animals make no resistance to the operator's manipulations. This is always the case where the seat of disease is in or behind the lumbar portion of the cord. When in front of this spot, that is, in the dorsal region or further forward, reflex irritability is not only retained, but may often be abnormally pronounced, so that stimulation of the skin, ligaments, or bones (slight blows or pricks with the needle) cause active contractions in the paralysed muscles. Continuous contraction (cramp) may thus be caused (spastic paralysis). Nocard saw increase of the tendon reflexes in a dog.

(3) In grave lesions of the cord, sensibility appears to be completely lost; in myelitis spinalis it is at first not much impaired; its continuance points to injury of the posterior columns and of the grey posterior cornua. In pressure paralysis, sensibility may sometimes be increased, as shown by Nocard's reported cases in dogs ; muvement of the paralysed hind-quarter produced acute pain (paraplegia dolorosa). The examination of large animals is more difficult, because sensation cannot be exactly gauged, reflex movements being so difficult to distinguish from those caused by painful sensations.

(4) To the above cardinal symptoms of paraplegia are added those of paralysis of the bladder, rectum, and tail (see "Diseases of the Tail"). There is often incontinence of urine, and frees cannot be discharged without assistance. This grouping of symptoms generally accompanies pressure paralysis from fractures of vertebræ or of the sacrum, from extravasation of blood into the vertebral canal, and in rarer instances from tumours, but may also occur in concussion of the spinal cord. The diagnosis must be based on the history of the case, its manner of origin and course. In fractures of the vertebræ, displacement of fragments or crepitation may be detected.

The symptoms of incomplete paralysis show still greater variety; but even though it is not possible, in every case, to form an accurate diagnosis, it is well, from the clinical standpoint, to distinguish two kinds :-

(1) Paraplegia incompleta vera, vel spinalis (true incomplete or spinal paralysis); and

(2) Par. incompleta spuria (incomplete spurious paraplegia): The 
causes of the first lie within, those of the second without the vertebrai canal. Paraplegia of the first kind, due to acute or chronic inflammation of the spinal cord and its membranes, or to pressure and 'degenerative processes in the medulla spinalis, declares itself by the following symptoms :-

1. Disturbance in movement, distinguished :-

(a) By loss of strength, and

(b) By irregularity in movement. The animals show weakness, and rapidly become tired; the hind limbs, though able to sustain the weight of the body, only slightly assist in forward progress, and, during walking, make irregular ataxic movements.

Ataxia is a form of interference with movement arising from defective co-ordination. The proper innervation of the separate groups of muscles is interfered with, in consequence of which their contractions are not harmoniously combined. Single groups contract too much or too little, others too early or too late.

In horses and dogs, such symptoms accompany incomplete paraplegia. The hind feet are lifted hesitatingly and incompletely from the ground; the toe is dragged, and gradually becomes worn. In the next stage the foot is lifted suddenly and to an abnormal height, is set down awkwardly and with a tapping movement, and often describes a semicircle outwardly; the feet are placed crosswise over one another, while the hind-quarters roll first to one side and then to the other (plaiting the legs). These ataxic movements are particularly well seen when turning; the animal has difficulty in backing.

2. Sensibility and reflex irritability are generally retained, the latter may even be increased, causing exaggeration of the tendon reflexes. In general the same remarks apply here as were made in reference to complete paraplegia.

3. Muscular atrophy. This is seldom seen in spinal paralysis, or is confined to gradual slight atrophy of both sides in consequence of inactivity. Degenerative atrophy and signs of degeneration never occur.

4. The paraplegia of dogs, due to disease of the spinal column, is almost always associated with spastic contraction of the extensors, and, according to Dexler, with anæsthesia of the testicle.

The non-appearance of atrophy in the spinal form of incomplete paraplegia shows that the cause lies in front of the trophic centre of the muscles, that is, in the posterior columns of the medulla, as paralysis due to brain injury generally takes the form of hemiplegia.

The several causes of paraplegia incompleta spuria lie outside the spinal cord or spinal column. They sometimes consist in fissuring of 
vertebræ, disease of the inter-vertebral discs, or still more frequently muscular disease, which may be of a rheumatic character, due to chill, or of purèly mechanical origin. In the horse the latter is more frequent; because in it violent, intrinsic or extrinsic, over-extension of muscle produced during heavy and unwonted exertion is common. When animals are continuously overworked, permanent interference with muscular function in time results, causing the mechanical efficiency of the muscle to become impaired: The resulting disturbance has been referred to disease of the fasciæ, but the truth of this statement has never been satisfactorily established. The pathological changes would here be similar to those in tendons, but, as the disease is so much more obdurate, it appears doubtful whether the fascia plays such an important part.

Vollers describes a form of paraplegia which suddenly developed in a mare after parturition, simultaneously with deformity of the spinal column, between the 14 th and 15 th dorsal vertebræ. The animal could stand, but could only walk with difficulty; it made rolling movements with the hind-quarters. At first, movement was greatly interfered with, but afterwards improved, though the spinal deformity continued. Johow saw a fifteen year old horse which had suffered from weakness of the hind-quarters for a period of six months. The difficulty was greatest in the morning on getting up and making the first few steps. One day complete paraplegia suddenly set in. The body of the first lumbar vertebra was found, on post-mortem, to be one-third thicker than that of the others. The thickening encroached on the vertebral canal and pressed on the spinal cord, which had correspondingly diminished in thickness.

Harms found the connection between the anterior ends of the glutei maximi and the longissimus dorsi torn away on both sides in a four year old horse. When resting, nothing remarkable could be seen, but during movement the hind-quarters rolled from side to side. A large cavity could be seen on either side of the spinal column, at the point where the large gluteus arises from the longissimus dorsi. In front of the ilium an elevation was noticeable. After movement, the anterior end of the large gluteus returned to its normal position, as could easily be seen. No improvement occurred.

Prognosis in complete paraplegia is unfavourable, and only those cases due to concussion of the cord have any chance of recovery. In large animals the prognosis is much less favourable than in small ones, the former always dying at an early stage from decubital gangrene, and horses even sooner than cattle.

In incomplete paraplegia, the prognosis naturally depends principally on the nature of the causative disease process. Recent and sudden cases, especially those resulting from chill, offer more hope of recovery than the more chronic, which are generally due to some incurable disease. All forms of paralysis, distinguished by degenerative changes of tissue and well-developed muscular atrophy, are unfavourable; in these recovery is exceptional. The condition usually becomes gradually 
worse, though at times it seems to remain nearly stationary, and allows animals to be used for considerable periods at a walking pace.

Treatment. In paralysis, the animal must have a suitable soft bed and be frequently turned over to prevent decubitus. Easily digested food is indicated. The bladder and rectum must be emptied from time to time, and sometimes purgatives are allowable. Further treatment must depend on the nature of the cause; rest and cold applications are indicated in superficial mechanical injuries; hot moist packs or applications of hot sand or bran in rheumatism; at the same time the muscles may be rubbed with stimulant lotions, \&c.

In incomplete paralysis, treatment must be based on a knowledge of the original cause; when this is mechanical, the animal should be placed in slings. Rheumatic conditions are to be treated as above. When inflammation of the spinal cord or its membranes is suspected, irritants like mustard poultices or cantharides can be employed. To combat atrophy of the muscles, gentle exercise should, as far as possible, be given, and the parts massaged by kneading or striking (tapotement). In horses good results have been said to follow subcutaneous injection of veratrin.

In dogs, the continuous or induced electric current may be employed. The induced current can scarcely be used in horses, on account of their great sensitiveness to it and the resistance they offer.

In dogs, Zwicker recommends injecting 7 to 10 minims of a solution prepared as follows: Eserin, $\cdot 6$; pilocarpin, $1 \cdot 2$; water, 80 . He gives carbonate of potash solution in syrup internally.

Mourot claims to have cured paraplegia in a horse in three weeks by subcutaneous injections of testicular extract from a goat. After removing its envelopes the testicle was rubbed into a paste with water, and a quantity subcutaneously injected each day. Until confirmed from other quarters, such reports will be received with caution.

\section{IX.-DISEASES OF THE TAIL.}

(1) Putting on one side operative wounds, injuries of the tail are most frequent in dogs and oxen. In the former they are caused by blows and treads, by the tail being struck against the walls of the kennel or other objects, or by its being run over. Defective sensibility seems to play a certain rôle here. Paræsthesia, that is, abnormal subjective sensibility, sometimes causes dogs to gnaw the point of the tail so as to lay bare the vertebrx; Prietsch saw this in a lion.

In cows, bruises are produced by blows from sticks, by violently bending the tail to make the animal move, or by squeezing it between two sticks, which is done for the same object, and often in a very rough 
way. In former days the root of the tail was often injured by operations destined to eradicate disease. Such injuries were sometimes inflicted on swine for the purpose of bleeding. In oxen, pleuro-pneumonia inoculation sometimes produces severe inflammation in the tail, especially if impure material is employed. Sometimes the tail whilst soiled with dung is accidentally wounded. Injuries of this kind in oxen, when not promptly treated, readily produce cellular inflammation, due to infection, or ulceration, and may extend to the vertebræ, producing a chronic disease which was formerly viewed as specific. Ulceration, caries, or necrosis, resulting from cellulitis, may set in and lead to sloughing of portions of the tail. The outer skin being closely applied to the caudal vertebræ, severe swelling at the root of the tail is liable to be followed by necrosis, just as in other organs, e.g., the tongue; the pressure exercised by the greatly distended skin impedes and finally stops circulation. The accident is common after inoculation with instruments or lymph conveying septic substances, or in cases where animals are not well looked after. Its wide distribution in certain stables formerly led to its being regarded as a specific disease. In Finland, official precautions have even been taken against it.

Symptoms and course. Injuries to the tail are easily detected. Cellulitis is marked by diffuse swelling, which often extends to the body, causing feverish symptoms, and even death. In other cases the inflammatory swelling leads to partial gangrene of the tail, or to necrosis of skin, liable to be followed by caries of the caudal vertebræ.

Cancer of the tail has been seen in the horse. Cadiot and Dollar describe a case (p. 427, "Clinical Veterinary Medicine and Surgery"). 'T'wo growths as large as walnuts, and with firm, reddish, excoriated surfaces, appeared on the lower surface of the tail about 4 inches from its base. 'There were other, smaller, growths. Removal and cauterisation produced temporary improvement, but the growths recurred, and the horse was eventually slaughtered. On post-mortem examination the tissues in the pelvic region, as well as the spleen, liver, and lungs, were found to be invaded by the new growth.

In dogs, the point of the tail is often raw from continually striking against neiglibouring objects; it may either be covered with a dry scab, or with fluid discharges. The animal may lick or even gnaw or bite the parts, continually exposing the last caudal vertebræ. The condition is commonest in short-haired or large dogs. Recovery may follow proper treatment, but frequently amputation of the extremity of the tail is necessary, and even this may fail to stop the process, for the animals gnaw the exposed stump, and the difficulty arises de novo. In a lion, Prietsch twice amputated portions of the tail before healing ensued. 
Treatment. Preventive treatment consists in cleanliness; where the parts are already infected antisepsis should be adopted.

To check cellulitis it may be necessary to scarify the skin of the tail lengthwise, and to follow this by the application of an antiseptic surgical dressing. Block scarifies as deeply as the periosteum, and rubs in common salt or turpentine.

In dogs it is best to smear the wound with iodoform, collodion, or tar, or to cover it with a dressing to prevent the animal licking and gnawing the point of the tail. Should this fail, the part must be amputated. Sometimes it is useful to cauterise the stump.

The so-called "rat-tail" produced in horses by gradual loss of the long hairs of the tail can only be referred to shortly. Up to the present the exact cause is unknown, though it has been thought due to psoroptic invasion; nor is there any sure means of preventing the loss of hair, or of assisting its return. Perhaps the best treatment consists in periodic thorough washing and the use of sulphur iodide ointment. Popow recommends scarification of the skin of the tail, but its efficacy requires further confirmation.

(2) Paralysis of the muscles of the tail. Difficulty in moving the tail may be caused by fractures of the sacrum, and by inflammation and new growths, but primary nerve disease of the caudal muscles also occurs in horses and dogs, both as an accompaniment of paraplegia and independently of it. The disease is often seen in mares, and judging from recorded cases it seems to affect them particularly, though it sometimes attacks geldings. It generally commences slowly; at first the tail is not carried in the ordinary way, but hangs more or less limply between the hind legs, swinging to and fro as the animal moves, and becoming soiled by urine and fæces. After the lapse of several months, paralysis of the bladder (incontinentia urinæ) and habitual constipation may appear, and manual assistance become necessary to empty the rectum, the last portion of which is gradually dilated. If relief be not afforded, the animal suffers from colic. Finally, paraplegia incompleta appears, with atrophy of the muscles of the quarter and of the hind leg.

In 1890, Dollar saw several cases as sequelæ to influenza. In one the symptoms were preceded by extravasation of lymph into the posterior chambers of both eyes, and the animal became temporarily blind. All of the cases seen had finally to be slaughtered.

Dexler found the following appearances on the post-mortem examination of a case: The sacral portion of the muscles of the tail showed marked fatty degeneration, and the muscular bundles were of a pale yellow colour. The . muscles of the tail proper resembled the flesh of a fish, were pale, transparent, and as if soaked in water. The rectum was greatly dilated. The mucous membrane of the bladder was swoilen, yellowish-red in colour, and uneven. 
From the last lumbar vertebra onwards the epidural fat rapidly diminished in quantity and finally disappeared. from the first sacral vertebra to its termination backwards the entire spinal canal was filled with a greyish-white, firm growth of such dimensions that it could scarcely be extracted, except in fragments. Microscopically, this growth consisted of firm connective tissue, partly interposed between the nerve bundles and partly surrounding the nerve stems. This connective tissue hypertrophy also affected the part of

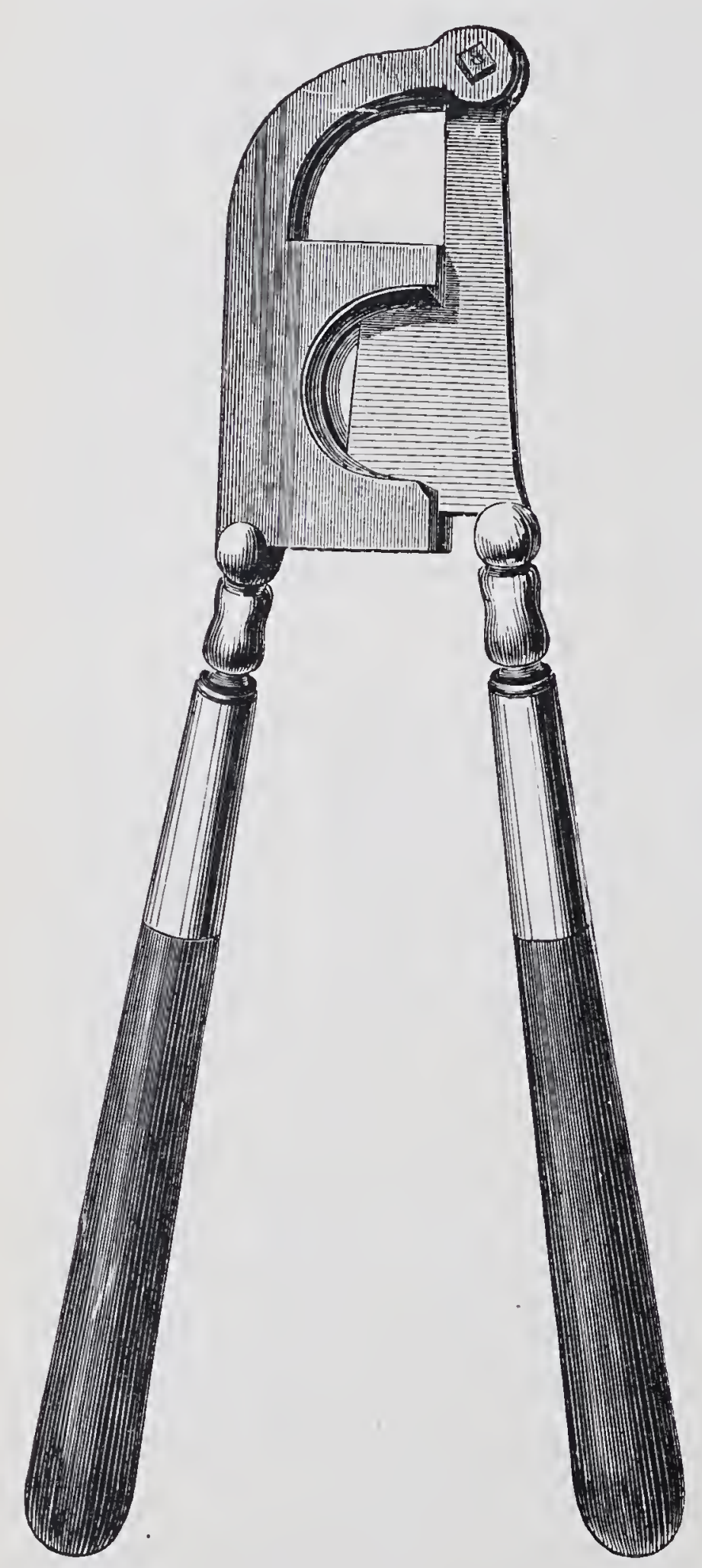

FIG. 183. the efferent nerve trunks and their ganglia. The calibre of the vessels was greatly diminished in consequence of thickening of the tunica intima. The nerve elements had undergone partial fatty degeneration. Secondary, ascending degeneration of the tracts in the spinal cord could not be detected. The spinal ganglia in the lumbar and sacral regions displayed marked increase of the interstitial material and complete degeneration of the nerve elements. Of the peripheral nerves the pudic nerve, the coccygeal plexus, and the nerves supplying the tail showed degeneration. The condition, therefore, consisted in an inflammation of the cauda equina. The process secondarily affected the nerve trunks and spinal ganglia, and led to degeneration of the muscular groups served by them.

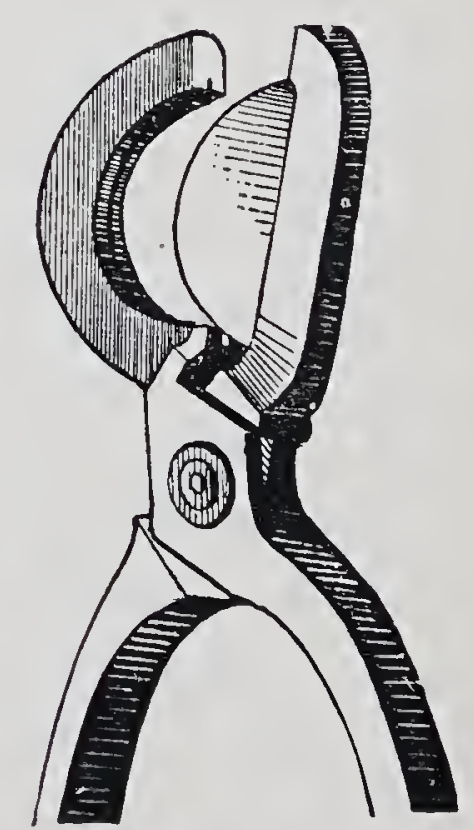

FIG. 184.

Prognosis is unfavourable, as treatment has hitherto proved powerless against this gradually progressive disease. In most cases neither stimulants nor the use of strychnine, nor of electricity, are of any use. Röder gave strychnine without success. The animal gradually becomes worse, finally unworkable, and has to be killed. A mare seen by Deigendesch died from rupture of the bladder.

(3) Tumours on the tail occur in horses, oxen, and dogs. Carcinomata and sarcomata, and in grey. horses the so-called melanomata, are not 
uncomimon. Lehnhardt and Rosenbaum have seen vascular new growths on the tails of oxen. These sometimes start from the skin, sometimes

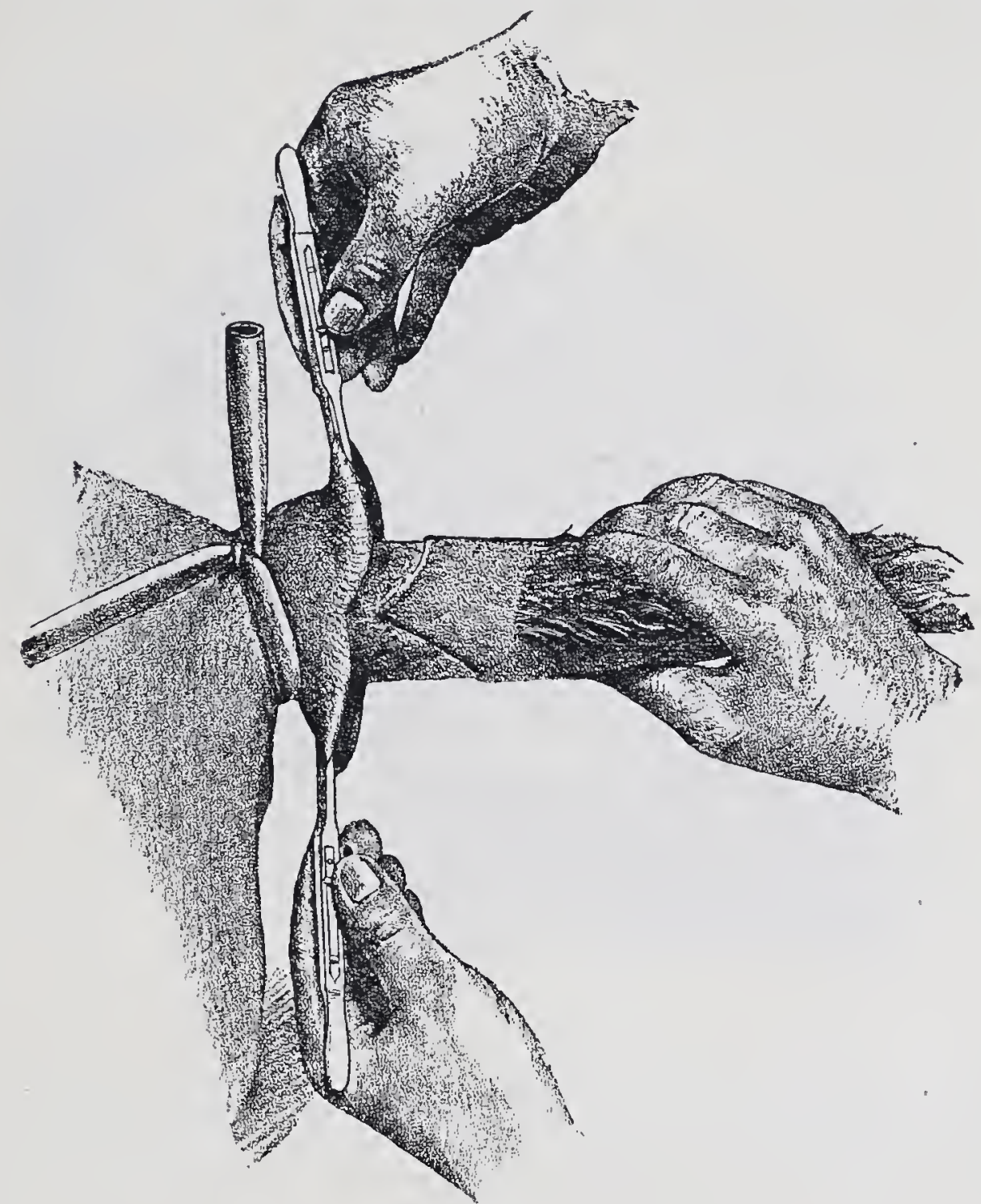

FII 185.

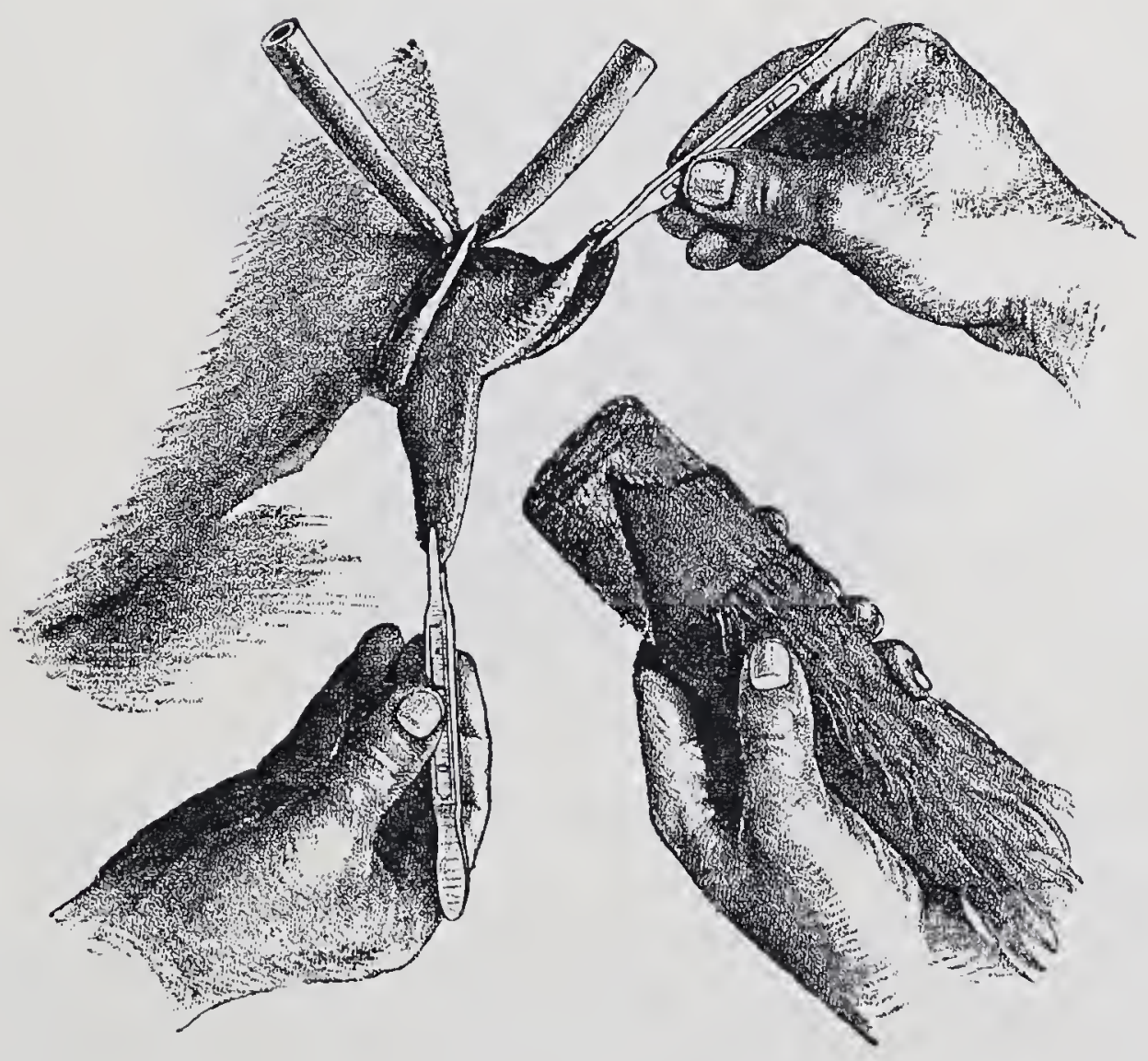

FIT. 186.

from the vertebræ of the tail, and produce ulceration, or they extend to the perineal connective tissue, and lead to obstruction of the bowel. 
Diagnosis is easy, but it is otherwise with treatment. Superficial tumours, that is, those having their seat in the skin, may easily be removed with the knife, but if they arise from the vertebræ amputation

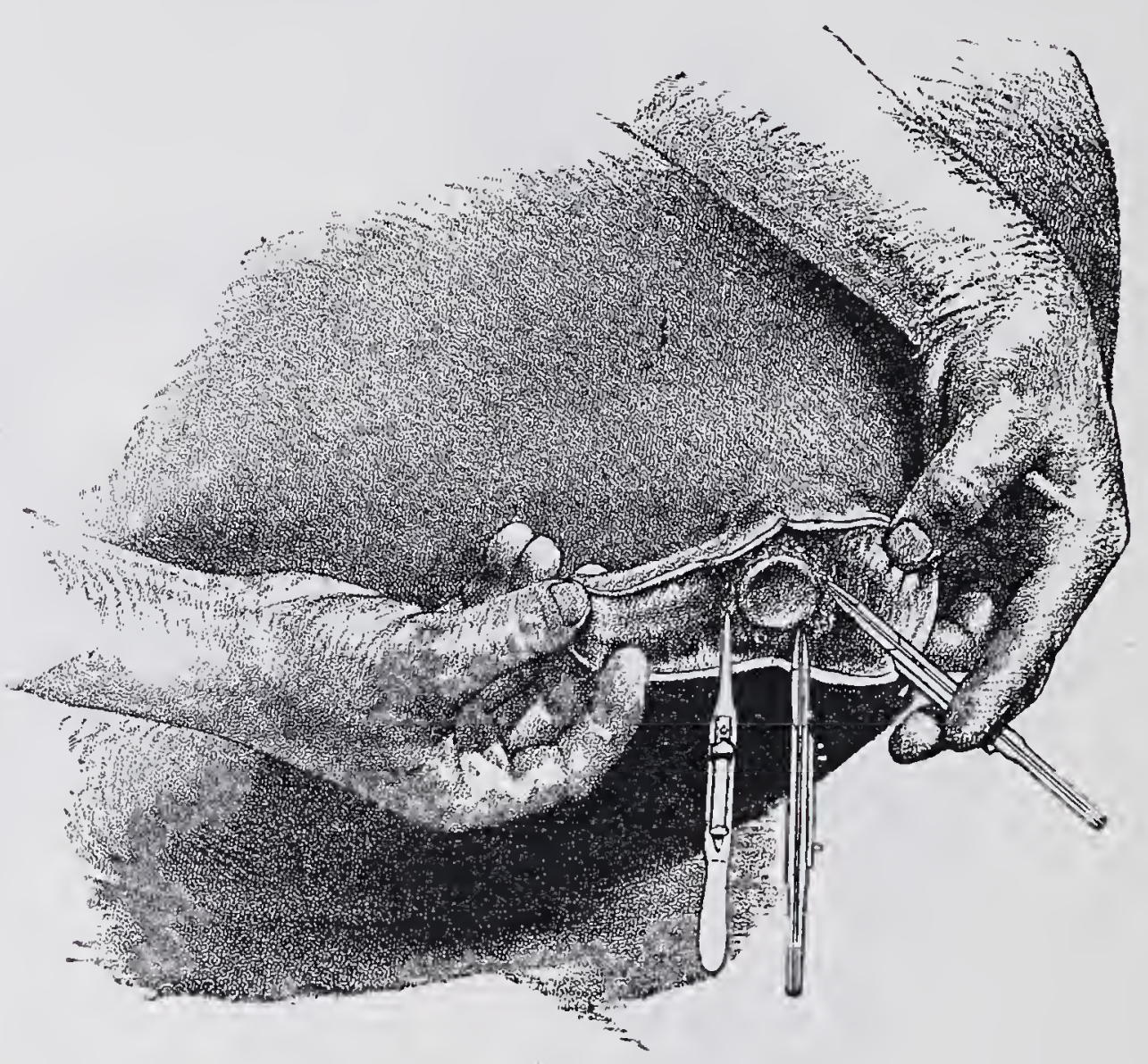

FIG. 187.

of the tail is the sole resource. Tumours near the end of the tail are dealt with by "docking"; those near the base are, however, more

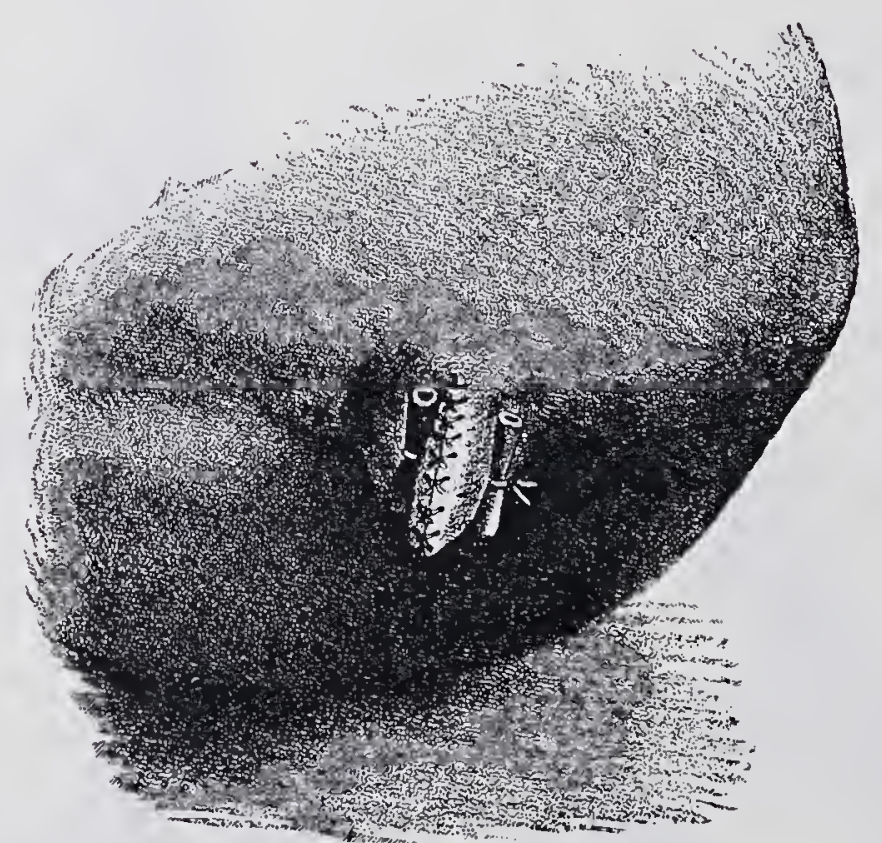

FIG. 188.

serious, and necessitate amputation, a double-flap operation being that generally employed.

Bayer proceeds as follows: After shaving and disinfecting the skin, a rubber cord is tightly applied round the base of the tail. Two flaps are then formed by semi-elliptical incisions of suitable length so as to ensure sufficient skin being left to cover the stump without tension. The tail is then divided close to the base of the flaps, by disarticulating 
one of the caudal joints. The rubber cord is then cautiously relaxed, any bleeding vessels are picked up and ligatured, the wound is thoroughly cleansed, and the two flaps are carefully brought into contact. Should the initial incisions have been skilfully made, the flaps will cover the stump without difficulty, and without being either tightly stretched or inclosing too large a cavity. It is best to form the flaps rather longer than absolutely necessary in the first instance, as they can afterwards be easily reduced with scissors or the linife. The edges are then brought into exact contact with closely inserted silk sutures, and should any cavity remain, a couple of "tension sutures" (see Dollar's "Operative Technique," p. 137) are used to obliterate it. The several stages of the operation are well shown in the foregoing illustrations (figs. 185 to 188$)$.

\section{X.-NECROSIS OF THE LUMBO-DORSAL FASCIA.}

The lumbo-dorsal fascia (fascia lumbo-dorsalis) lies just beneath the panniculus in the region of the back, and covers the dorsal extensors like a sheath. Its superficial portion arises from the outer angle of the ilium, becomes attached to the superior spinous processes of the lumbar and dorsal vertebræ and to the ligamentum nuchæ, is continued backward in the fasciæ of the abdominal muscles, and forward in the direction of the ligamentum nuchæ, to become attached to the scapular fascia. Its deep portion is confined to the lumbar region. It connects the transverse processes of the lumbar vertebræ to one another, and to the outer angle of the ilium. The dorsal fascia serves as a point of attachment to various muscles, and forms the medium of connection between the extensors of the back and the muscles of the hindquarter. It is best developed in horses.

Suppuration in the skin of the back, produced by pressure, sometimes causes necrosis of the dorsal fascia; external injuries and cellulitis may also produce it. The great strength of this fascia renders dissection of necrotic parts tedious, whilst new portions are continually attacked, and die on account of progressive necrosis. Sooner or later, therefore, large tracts are destroyed. Möller has repeatedly seen this in horses; cases last for weeks, and give the greatest difficulty in treatment. Recovery is quickest after surgical removal of the diseased portion; pus formation is checked by continuous irrigation with disinfectants. Schmidt saw a foal in which the fascia was the seat of purulent necrosis extending from the withers to the pelvis. On incision, necrotic pieces of tissue and ill-smelling pus were discharged. Although the ribs could be felt on introducing the finger, recovery occurred in six weeks by using carbolic lotion, hut few cases prove so amenable to treatment.

v.s. 


\section{DISEASES OF THE FORE LIMB.}

\section{A. DISEASES OF THE SHOULDER REGION.}

\section{I.-FRACTURES OF THE SCAPULA.}

ON account of the sheltered position and slight mobility of the scapula in domesticated animals, it is comparatively seldom broken, though fractures are described as having resulted from blows with blunt bodies, from collisions, kicks, treads, and falls, particularly from falls on the shoulder, or with the legs widely straddled. Trasbot saw the scapula fractured by struggling whilst in hobbles; oxen occasionally fracture the bone when carelessly lying down. The accident is also at times caused by violently reining-up animals, the neck of the bone being then generally broken.

Sometimes the cervical or dorsal angle is broken across, sometimes the spine or body, not infrequently the neck or glenoid cavity. The greater number of these fractures are subcutaneous; only occasionally is the skin perforated. Such fractures are commonly fissured, but comminuted fractures also occur. Complications are commonest after fractures of the spine, the fragments of bone becoming necrotic and causing abscess formation. Möller saw one in a horse which was working in a gravelpit during the winter. The scapula was crushed into more than eighty pieces by the falling of a mass of frozen earth.

Diagnosis. In well-nourished animals, notably in horses, and in cases where severe swelling has occurred, diagnosis is difficult. Displacement of the broken fragments is often slight, and crepitation may be entirely absent. It is, therefore, clear that every marked, rapidly-produced lameness, which is evident both when the animal stands on the limb and when it moves, must awaken suspicion of fracture of the scapula, pro-
vided there is no other self-evident cause.

Fracture of the scapular spine is the only one of the above conditions in which the foot may be placed on the ground in the usual way, though movement is then in the highest degree painful. In fracture of the scapular spine, and of the anterior or posterior angle of the scapula, the fragments can be displaced with the hand. Fractures of the neck of the scapula are attended with excessive mobility of the foot, especially in ab-and ad-duction. Fractures of the body are most difficult to diagnose, but may always be surmised if crepitation be present whilst the humerus
is known to be intact. 
Course and prognosis. The nearer the fracture is to the lower end of the bone the graver the condition. This principle, established by Binz, must be borne in mind ; it rests on the fact that, in fractures of the upper portion of the scapula, the fragments are not so easily displaced, and the pain is less.

The prognosis of fracture through the glenoid cavity is most unfavourable in working animals, and especially in horses. Fracture near the neck of the scapula does not necessarily prevent the animal again becoming useful, especially if the fragments are not much displaced; sometimes perfect usefulness is restored. This is oftener the case in fractures of the body, and almost always so in fractures of the spine and upper portions of the scapula, provided they remain subcutaneous. Compound fractures are always dangerous, because of the burrowing of pus in the fascia of this region, though compound fractures implicating the spine only often heal if sufficient time be given for the separation and discharge of the fragments. The less the pain and lameness the greater the chance of healing; but when, in horses and cattle, such symptoms are marked, it is better to slaughter the animals unless they are of particular value for stud purposes, as they suffer severe loss of condition during recovery. Marked pain forces the animal to rest entirely on the other foot, and there is then danger of laminitis and descent of the pedal bone, especially if the patient stands continually. Increased pulsation in the metacarpal artery, and attempts to rest the foot, point to this complication, which is so painful that the animal generally lies down and cannot be moved. Death then occurs from decubitus.

In small animals, particularly in carnivora, subcutaneous fractures of the scapula generally unite in four to six weelss, butlameness sometimes remains. In a few cases necrosis of the entire scapula has been seen after fracture. ${ }^{1}$

Treatment. Displaced fragments must, as far as possible, be replaced, which is easiest with the patient in the prone position and under chloroform. In large animals, however, the operation is always difficult, and even when successful, does not ensure that the fragments will remain in position. The bandaging and plasters so often recommended are of little use except in small animals. When pain is not sufficient to compel the animal to keep the diseased limb quiet, and especially when weight cannot be borne on it, early slaughter is preferable to treatment, with its doubtful results. Animals which will lie down should have a thick straw bed, otherwise they may be placed in slings, and kept as quiet as possible. If in compound fractures treatment be resolved on, attention should first be directed to rendering the wound aseptic, and to keep it so, a dressing is indispensable.

1 Cadiot and Dollar's "Clinical Veterinary Medicine and Surgery," p. 430. 


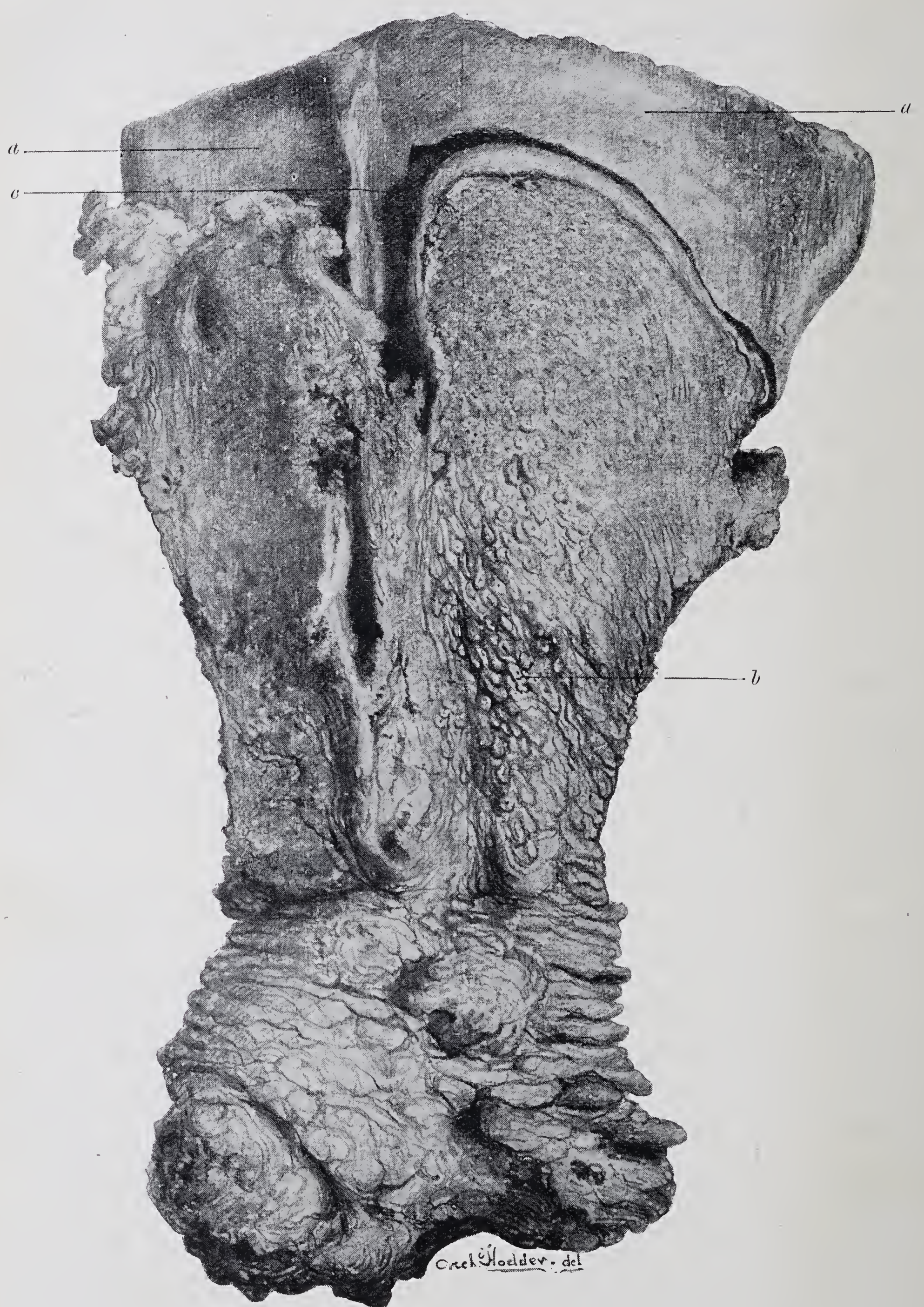

FIG. 189. - Total necrosis of the scapula. a, Sequestrum ; $b$, case of bone formed by the periosteum ; $c$, space filled with pus in fresh specimen. (Hodder's case.)

In small animals suitable bandages are more easily applied. They should include the thorax and lower part of the neck, so as to ensure their remaining in position. 


\section{II.-FRACTURES OF THE HUMERUS.}

Though fractures of the humerus are as a whole uncommon in animals, they nevertheless occur with some frequency in dogs, in which one or other condyle breaks away from the lower end of the humerus. Peuch has seen double-sided fracture of the humerus in horses. Fracture occasionally follows muscular exertion, as in rearing, or even in trotting. Horses at grass may be kicked on the arm. Drosse saw fracture of the "outer and upper protuberance of the humerus" (ridge), and removed the piece of bone; recovery occurred in fourteen days. Six or seven weeks later the animal stumbled and broke the humerus in the direction of its length.

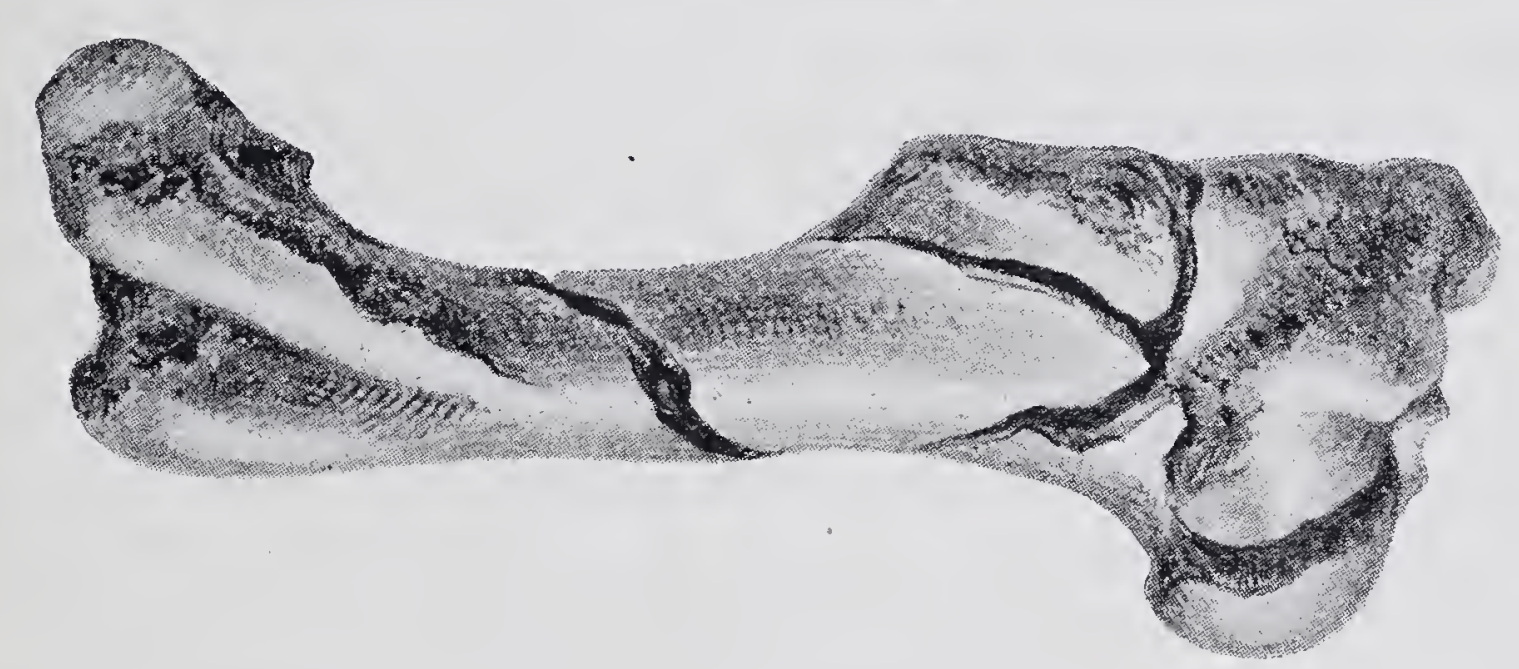

Fig. 190.-Fracture of the humerus. (Hodder's case.)

Fractures of the diaphysis of the humerus are commonest in cattle and dogs, and are produced by external violence, kicks, collisions, treads, or accidental falls; occasionally in horses by the animals being suddenly wheeled round whilst being ridden. The figure shows the humerus of a horse which ran away and came in collision with a wall.

Fractures of the condyles are caused by treads, notably in dogs, though similar cases have been seen in horses. Sometimes the external, sometimes the internal condyle is broken off in consequence of the supporting leg making a violent rotary movement, or the free leg being excessively ad- or ab-ducted. In dogs such fractures are produced by awkwardly jumping or falling from a chair or table. Of twenty-six fractures of the humerus in dogs treated by Stockfleth, seven were through the diaphysis and nineteen through the condyles.

As a rule, diagnosis of fracture of the diaphysis offers no difficulty. There is severe pain both when the animal stands on the limb and when it moves; swelling and abnormal mobility and crepitation when the scapula is fixed ; the other bones of the limb are found intact. In such cases there can be no doubt. It is more difficult to detect fracture of the condyle. Sometimes the disconnected condyle is movable, and there may be crepitation; diagnosis is easiest in the dog. On account of the anatomical 
construction of the elbow-joint in dogs, the external condyle when broken off is usually drawn upwards, whilst the internal becomes visible on the inner side of the elbow-joint, and appears lower in position than usual. This increased prominence of the internal condyle seems to have given rise to a belief that in the dog it (the internal condyle) is the more frequently fractured. In horses there is severe lameness when the limb is carried, and inability to place weight on the foot. The leg is adducted (turned in) in fracture of the extensor condyle, and abducted (turned out) in that of the flexor. In the former case the lower joints of the limb are excessively flexed, so that the front of the hoof is in contact with the ground, owing to the relaxed exterisor muscles being loosened from their upper points of insertion. During recovery, this abnormal position of the limb often suddenly disappears, and if, in fractures of the flexor condyle, weight be then placed on the foot, the knee is excessively extended, so that the anterior surface of the limb appears concave; this is particularly noticeable during trotting, at the moment when weight is placed on the foot. When accompanying abnormal flexion of the elbow-joint, this symptom is explained by the relaxation of the flexor metacarpi, consequent on loosening of its upper point of insertion. The same is true of the flexor tendons in fractures of the inner condyle. Otherwise this symptom only occurs in rupture of the flexors, or in fractures of the pisiform bone, and is, therefore, of particular diagnostic value. It does not occur in carnivora.

Fissuring of the humerus can scarcely be diagnosed, though it may be suspected.

Course. In carnivora, fractures of the condyles almost always unite in six to eight weeks, though some interference with movement of the elbow may remain. Stockfleth and Möller in dogs have seen such fractures occur in both legs simultaneously, and yet reunite. Möller's case was in a sporting dog, which afterwards regained its usefulness. Opinion should, however, be guarded, as the condyle sometimes fails to heal, and in old dogs, even when this occurs, movement of the elbow-joint is often very limited on account of callus formation. In another case, in a horse, recovery took between two and three months, and yet the animal was finally quite able to work. Williams appears to have had less favourable results, for he declares that union of the broken condyle seldom occurs, and that in consequence lameness is permanent.

Fracture of the diaphysis is less hopeful in all classes of animals, especially in draught-horses, and as a rule, no good comes of attempting treatment. It is otherwise with valuable breeding-horses, particularly if of quiet temperament, and in the smaller domestic animals healing is quite possible so long as displacement is not great, and the other foot is able to bear weight. Under these circumstances recoveries have been 
noted. Warnecke saw fracture of the humerus in a stallion, and Meredith compound longitudinal fracture in a horse, reunite in ten weeks. Numerous fragments of bone were removed and an antiseptic dressing applied. Number's of similar cases have been reported. Vogt relates a case where in a cow the broken shaft of the humerus united. A serious point for consideration is whether the marked shortening of the limb, which always occurs (even after union) when there has originally been much dislocation, may not make the horse or ox useless. Prognosis is more hopeful in small animals, especially when the fragments are not much displaced. Not only is reduction easier, but in carnivora the humerus may be supported with bandages, particularly if the fracture is near the lower end.

Treatment. In horses and cattle suitable bandaging can scarcely be effected, and recovery must, therefore, be left to nature. The complicated dressings recommended in France do not seem to assist much in retaining the fragments in position. In carnivora, on the other hand, the lower portion of the humerus may be bandaged, and in them it is also possible to completely fix the broken condyle. A plaster bandage is applied extending from the metacarpus as high as possible, and care taken that the elbow-joint is moderately flexed, so as to avoid a bad position afterwards. The lower part of the limb should neither be fixed in a position of excessive supination nor of pronation. In three to four weeks the animals commence to place weight on the foot, and the bandage may then be removed. At first the elbow-joint cannot be freely moved, but the amplitude of movement gradually increases, especially if passive exercise be given. In one case Frick attempted to fix the displaced condyle in position with bone sutures, but the bone proved too friable and the sutures tore out. Such operations, however, are perfectly practicable provided careful antisepsis be observed; the fragment may be fixed in position with wire sutures, or, as in human practice, with ordinary wood screws which have been silvered.

Large animals, and notably horses, require slinging. After prolonged slinging, however, the animal may show signs of acute laminitis in the foot on which it stands. Despite the risk of displacement, there is then no other course open but to allow the animal to lie down, trusting that union may be sufficiently far advanced to prevent bad consequences. The bed must be soft and the box roomy. If the animal refuses to lie down, there is little hope of recovery.

\section{III.-DISEASES OF THE SHOULDER-JOINT.}

Anatomy. The shoulder-joint, which in all domesticated animals is an enarthrodial or ball-and-socket joint, possesses a lax capsular ligament. The lateral reinforcement of the capsule by strong connective tissue and the application of muscular substance on both sides limit movement in the joint, 
and only allow slight abduction, adduction, and rotation, though considerable flexion and extension. Flexion of the shoulder-joint is limited by the biceps, extension by the caput muscles, abduction by the subscapularis, adduction by the antea and postea spinati, so that the shoulder-joint in domesticated animals possesses far less mobility than in man. The oval flat glenoid cavity is much smaller than the head of the humerus. In swine and carnivora, the size of the former is increased by a cartilaginous disc (Labrum glenoideum scapulæ). In these animals the shoulder-joint is also connected with the bursa intertubercularis.

(a) Luxation of the Shoulder-Joint.-Incomplete luxation of the shoulder is very rare in animals, there being nothing to keep the head of the joint in position on the sharp edge of the glenoid cavity. Complete displacement is also much rarer than in man, though it occurs both in horses, ruminants, and dogs. In swine and carnivora, the position of the caput humeri is ensured by the labrum glenoideum; in the horse, by the tendinous biceps muscle.

Luxation can generally be referred to excessive flexion of the joint, and the humerus is, therefore, always thrust forwards and upwards, the head of the bone being discovered in front of and above the glenoid cavity of the scapula. As the biceps brachii muscle antagonises this movement, displacement can only occur when that muscle is partially relaxed, as it would be, for instance, during simultaneous flexion of the elbow-joint. For this reason luxation of the shoulder-joint results most frequently from falls. A sudden check to the movement of the lower portions of the leg may also bring it about. Hertwig saw several such displacements, on account of the feet striking against some obstruction in leaping. Excessive movements of abduction may also result in the head of the humerus being displaced inwardly beyond the glenoid cavity of the scapula.

Symptoms. The accident is distinguished by excessive lameness, which appears suddenly, and is most marked when the leg is being moved. Passive movement of the joint is also interfered with. Whilst there is much difficulty in flexing, and still more in extending, the joint, abduction and adduction are abnormally easy, and the leg appears shortened. Provided swelling be not excessive, dislocation of the head of the bone can be discovered by palpation. Pressure on the parts produces pain. Complications, like fracture of the scapula or humerus, often exist.

Prognosis and course. The results of many observations seem to indicate that even complete luxation, if early reduced, can be cured in two to three.weeks. Smith, after reducing a supposed dislocation under chloroform, caused the horse to be moved, and it then showed no distinct lameness; eight days later it was nearly sound. The diagnosis, however, in this case is not beyond question. In other cases chronic lameness remains. In working-horses, it is best to wait for eight to fourteen days after reduction, and if in this time no improvement is manifest, 
the animal should be slaughtered. Reduction being easier in small animals, the prognosis is correspondingly more favourable.

Treatment. In large animals, which stand quietly, attempts may be made to reduce the dislocation without casting. Siedamgrotzky advises that one man should hold the animal's head, another draw the leg forward, and a third press on the knee-joint to extend it, whilst the operator endeavours to return the head of the humerus to the joint cavity by strong pressure. Should this fail, the animal must be cautiously cast and the attempt repeated. A noose is passed round the leg, and two or three powerful men effect extension by pulling on it. Counter-extension is provided by a girth passed round the animal's body. The operator stands on the joint and supervises the direction of extension, whilst endeavouring to replace the head of the bone. Successful reduction is notified by the production of a loud click, and by restoration of free movement to the limb. Failing reduction by this method, the elbowjoint is strongly flexed, and fresh attempts made; if the head of the humerus lies in front of the glenoid cavity, this way is more likely to be successful. The shoulder muscles retain the parts in position, so that bandaging is not usually necessary. Lodezzano, however, in the case of a cow, cut away the hair from the shoulder, smeared the parts with a mixture of pitch, turpentine, and wax, and applied a stiff piece of cloth; when the mass became hard, fresh layers were applied. In pronounced inflammation, cold applications are indicated. Absolute rest is necessary, and should be continued as long as any trace of lameness exists. To prevent return, which is liable to follow attempts to rise, the patient should be placed in slings for one or two weeks. Blistering also diminishes free movement of the shoulder and tends to prevent recurrence. Bourgelat employed an iron splint like a horse-collar to assist retention. The apparatus is generally unnecessary, unless the animal be allowed to lie down.

(b) Contusions and Distortions of the Shoulder-Joint.-These accidents are in general rare. The absence of firm ligamentous structures tends to prevent distortion occurring, while the muscles of the shoulder, especially the biceps, shield the joint from contusions. As long as the limb remains in its normal relationship to the trunk, even the violent concussion resulting from falls is not injurious; the displacement being guarded against by the well-developed cartilages at the head of the joint. On the other hand, both excessive abduction, adduction, and rotation, produced by slips, falls, or attempts to free the foot which has become fixed in some obstruction, may result in distortion of the joint. The greater number of diseases described under this head consist, however, in injuries to the biceps and to the intertubercularis bursa. 
Symptoms. Inflammatory disease of the shoulder-joint is recognised by sudden lameness, when lifting the leg, by the limb being abducted, and by the animal being unwilling to place weight on the foot. The forward stride is shortened, the movement of the limb is slow, the backward movement impaired, so that the hoof is dragged along the ground. Inflammatory swelling, accompanied by increased warmth and pain, appears about the shoulder. Diagnosis is often doubtful, and the disease then falls under the category of shoulder lameness (see "Shoulder Lameness").

Prognosis and course. The degree and extent of the injuries associated with distortion being very varied, prognosis is equally uncertain. It must, therefore, be principally guided by the degree of lameness and the character of the local symptoms, as well as by the time during which these have existed. If pain is slight, complete recovery may occur in three to four weeks; gradual improvement during this time points to a favourable issue. On the other hand, chronic arthritis may sometimes cause lasting and incurable lameness, The more severe the lameness, and the longer it has existed, the less favourable the prognosis; marked atrophy of the shoulder muscles is, therefore, an exceedingly grave symptom.

Treatment. Complete rest must always be the first measure. Cold applications, irrigation, or poulticing combat inflammation. At a later stage irritants, blisters, and setons can be employed. The subcutaneous injection of atropine and morphine has been recommended by some but condemned by others. It might be worth trying.

(c) Wounding and Inflammation of the Shoulder-Joint (Omarthritis). -On account of its sheltered position, the shoulder-joint is seldom wounded. Romant describes a torn wound of the joint in a mule, in which perfect recovery followed the employment of sublimate solutions. As a general rule, these injuries call for cautious prognosis and treatment.

Spontaneous inflammation of the shoulder-joint is seen in oxen and dogs under the form of rheumatic arthritis; in foals, calves, and lambs, as a complication of so-called navel-ill. The shoulder-joint is, however, not particularly prone to this disease. The knee and hock are much more frequently attacked.

'That form of omarthritis recurring during septicamia cannot here be referred to. Abscesses in the neighbourhood of the joint seldom break into its cavity.

Chronic omarthritis may either be a sequel of distortion and luxation, or be of rheumatic origin. Williams gives two illustrations of chronic omarthritis in horses, showing the edge of the joint covered with 
exostoses; the synovial membrane was thickened, and to some extent ossified; in one case synostosis had even occurred.

Prognosis. The variations in the pathological conditions are so great that no fixed principles can be laid down either for prognosis or treatment. The degree of local change, the severity of the lameness and the time it has existed, form the chief indications. Excessive muscular atrophy and obstinate lameness are always grave. The symptoms are equally varied (compare with "Shoulder Lameness").

\section{IV.-INFLAMMATION OF THE BURSA OF THE BICEPS, FLEXOR BRACHII OR CORACO RADIALIS MUSCLE (BURSITIS INTERTUBERCULARIS).}

Anatomy. During flexion and extension of the shoulder-joint the tendon of the flexor brachii glides over the bicipital groove of the humerus, which is covered with cartilage, and forms one of the boundaries of an extensive bursa (bursa intertubercularis). The bursa extends from the periphery of the cartilage-covered tuberosities, over the tendon, clothes a portion of its surface, then its sides, and finally its under face. The sides of the bursa are covered by the lower points of insertion of the supraspinatus muscle, and its cavity is divided from the capsule of the shoulder-joint by a mass of fat. In swine and carnivora, the bursa is continuous with the shoulder-joint. Its position is nearer the central line of the body than in the horse.

In horses, the extensive intertubercular bursa is sometimes the seat of acute or chronic inflammation, in which the biceps tendon shares, and which almost always results from severe bruises, such as are caused by collisions when the shoulder is struck and the biceps receives the full shock. Wounding of the bursa may be followed by acute septic inflammation. Runaway horses frequently suffer.

A chronic bursitis (which may be bilateral) is produced in tramway and omnibus horses by continuous rapid driving.

As in acute inflammation of other tendon sheaths, serous and fibrous exudates occur; in affected wounds and metastatic diseases purulent inflammation of the sheath of the tendon may set it. Dieterichs saw symptoms of acute inflammation, hyperæmia, and exudation in horses which had shortly before fallen ill. Necrosis of the bursa in the horse was seen by Möller. The tendon was partially necrotic, the gliding surface on the humerus deprived of cartilage and eroded.

Chronic bursitis produces abrasion of cartilage, formation of exostoses on the humerus, and ossification of the biceps. Williams and Dieterichs found the muscle almost entirely ossified, though in Dieterichs's case the biceps had become adherent to the humerus. The gliding surface of the biceps is frequently roughened from friction against the roughened 
bicipital groove. Villate has also seen ossification of the muscle, with abrasion of its gliding surface.

Symptoms. Acute bursitis intertubercularis is shown by excessive lameness when the leg is advanced (swinging leg lameness). On attempting to move the animal, the foot is not carried forward, but remains at a point behind that of the other side, giving the impression

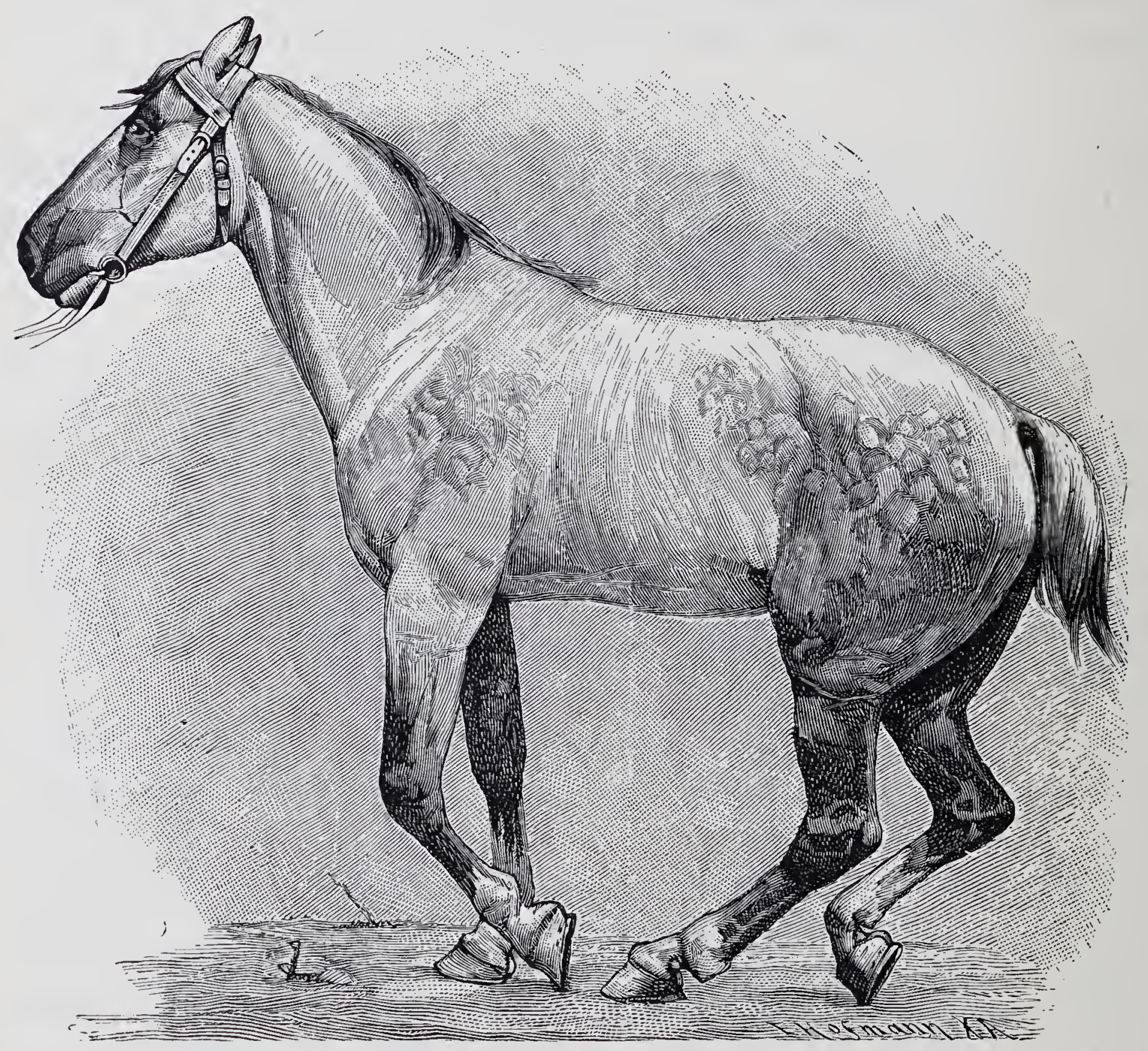

FIG. 191. - Lameness from bursitis intertuberenlaris (the horse is being led).

that the foot cannot be lifted from the ground. When forced to move, the horse places absolutely no weight on the lame leg (fig. 191). It moves back without much difficulty, and may then be able to lift the foot a little. Even after pain diminishes, the foot still remains behind when moving, and the forward stride appears much shortened. During rest, the foot is placed behind its neighbour, and weight may even be borne on it, a symptom seen by Brauell and K. Guinther even after cutting through the biceps. Inflammatory symptoms (increased warmth, swelling, and pain), sometimes appear in the muscle and neighbouring parts, though they seldom bear any direct relation to the degree of lameness. 
In chronic bursitis intertubercularis, inflammatory symptoms are wanting, though there is inability to place weight on the leg, and the forward stride is shortened. When the disease appears bilaterally, the animal seems "tied at the shoulder." In some cases both atrophy and contraction of the muscles occur.

Prognosis and course. Acute bursitis occasionally takes a favourable course, but is prone to become chronic. When severe, the animals die from continuously lying in one position, otherwise recovery occupies several months, and chronic lameness is apt.to remain; treatment is, therefore, only advisable in valuable horses. If the local symptoms are slight, if weight is still placed on the foot, and if lameness is not severe, recovery may be looked for in six to eight weelis, but sometimes takes several months.

In chronic bursitis, prognosis is even less favourable, though the disease takes a slower course, and the animals continue to some extent useful.

Treatment must follow general principles, being guided by the existing changes. In acute bursitis, it comprises absolute rest, cold applications (best in the form of ice poultices), or permanent irrigation with cold water; as the pain diminishes, warm moist applications, at a later stage irritants and blisters, and finally setons.

Dieterichs says that in horses lameness is sometimes caused by dislocation of the biceps brachii muscle. Such a case never occurred in Möller's practice, nor did Hertwig, even in his extensive practice of so many years, see one. Even Dieterichs's description leaves it doubtful whether the condition occurs, and the same may be said of Becker and Dominic's cases. From the latter's statement, it is clear that the scapula was displaced, but probably from muscular rupture.

Rupture of both biceps muscles was seen in a fourteen year old gelding which had suddenly gone lame. The neighbourhood of the shoulder-joint was swollen and painful. The condition somewhat improved, and the horse was put to light work; but four months later, after being driven about two miles, became suddenly much worse, and appeared to have lost control of both fore limbs. It was placed in slings, but gradually wasted, whilst the thorax sank between the shoulders, so that four weeks after the last attack the withers were $4 \frac{1}{2}$ inches lower than before. The sternum reached to the lower half of the fore arm, and the scapula lay in an almost horizontal position. Both shoulder-joints were greatly swollen, but not painful. The animal's movement was passable, though it often stumbled. Post-mortem showed extensive periostitis around the shoulder-joint. Instead of the coracoid process, there only existed on the scapula a roughened spot, the biceps muscles of both sides were torn away from their scapular insertions, and their tendons thickened (Nesbit). 


\section{Y.-INFLAMIMATION OF THE TENDON OF THE POSTEA- SPINATUS MUSCLE AND OF ITS BURSA.}

On the external tuberosity of the humerus, above the point of insertion of the postea-spinatus muscle, is a bursa, which, together with its tendon, sometimes becomes inflamed. K. Günther first drew attention to this affection, and showed that it may be caused by bruises, kicks, collisions, falls, or by strain of the tendon. When horses with narrow chests and closely-placed fore legs are used for fast-trotting work this tendon may become strained on account of increased abduction of the shoulder necessitated by the special conformation.

Symptoms. The disease produces lameness during the period when weight is placed on the limb (supporting leg lameness) and abduction of the entire limb. Weight is certainly put on the foot, but the latter is placed as far as possible outwards, by which abduction of the shoulder, and consequentiy painful extension of the diseased tendon, is as far as possible avoided. 'There is pain on pressure over the affected spot, together with increased warmth and swelling. Crepitation may sometimes be detected by applying the hand (tendovaginitis crepitans).

Prognosis and course. The nature of the disease renders a rest of at least four to eight weeks absolutely necessary, but recovery always occurs if the patient is given sufficient time.

Treatment. Acute inflammatory processes are combated by cold applications, later moist warm applications can be used, or, if necessary, irritants, setons, or the actual cautery.

\section{VI.-PARALYSIS OF THE SUPRASCAPULAR NERVE.}

This disease was first observed in 1785 by Rohlwes. In 1864 Bouley suggested that it was due to rupture of the tedinous insertion of the postea-spinatus muscle. It was first correctly diagnosed and described by K. Günther; of late years it has been repeatedly seen in horses. Frick saw a case in an ox resulting from the animal being frightened and springing forward into the manger; lameness was at once apparent.

The suprascapular nerve, an important branch of the brachial plexus, takes its origin chiefly from the seventh cervical nerve, and passes between the supraspinatus and subscapularis muscles on to the lateral aspect of the shoulder, to supply the supraspinatus, infraspinatus, and teres muscles. It therefore supplies with motor twigs the above-named muscles, which form lateral ligaments to the shoulder.

The cause of paralysis of the suprascapular nerve is violent backward movement of the shoulder or of the leg whereby the nerve is overstretched. The general causes are running against trees, against the 
manger or other firm objects, or against another horse, as in cavalry attacks. It is therefore common in cavalry horses, in runaways, or in animals which have been struck by the pole of a carriage. Cadiot has seen it follow casting for operation ; the horse had been kept down for a long time. At the end of two months wasting of the postea-spinatus was very marked. Reported cases seem to indicate that it sometimes accompanies hrmoglobinuria and rheumatism. Hansen saw a case produced by a door slamming and striking the animal. The causes are therefore similar to those of bursitis intertubercularis. The difference consists in this, that violence to the unloaded limb thrusts it back, and is apt to cause this paralysis, whereas violence to the limb when supporting the body causes contusion of the shoulder, backward movement being then impossible. This paralysis is therefore more likely to be produced when the shoulder is struck by a heavy, slow-moving body than where the blow is given suddenly; in the latter case the biceps and its bursa are endangered.

The symptoms are explained by the loss of function in the muscles which act as lateral ligaments to the shoulder-joint. Whilst nothing abnormal can be remarked as long as the limb is rested, sudden abduction occurs immediately weight is placed on it, and at the moment when the foot is perpendicularly below the body. The scapula and humerus are then jerked away from the wall of the thorax. This movement is best seen when the horse is slowly walked in a straight line.

The disease then is distinguished by lameness when weight is placed on the limb (supporting leg lameness), by abduction of the limb, and by jerking of the shoulder outwards at the moment when the leg is perpendicular. Atrophy of the paralysed muscles occurs later, and is most marked in the supraspinatus, infraspinatus, and two teres muscles, whilst the deltoid, which receives its innervation from the circumflex nerve, remains intact. The atrophy is rendered more noticeable by the increased projection of the spine of the scapula.

Roloff saw a horse which had suddenly exhibited double-sided shoulder lameness during heavy work, and found marked atrophy of the supra- and infra- spinatus muscles. The shoulder-joint was thrust outwards. In this case probably there was double-sided paralysis of the suprascapular nerve. In cattle, on the other hand, a somewhat similar condition is caused by overextension or relaxation of the adductor groups (MM. subscapularis and teres major). It is seen in particular races, such as the Holsteiners, particularly in winter ; in summer, when the animals are pastured, it often disappears.

Prognosis and course. Paralysis caused by mechanical influences is known to be less favourable than the rheumatic forms, and treatment is often unsuccessful. As a general rule, prognosis is much less favourable than in paralysis of the radial nerve, though recovery sometimes occurs in six to eight weeks. Otherwise, and especially if the lameness 
continue severe-that is, if the difficulty in movement has not begun to disappear-there is little hope. Of five cases seen at the Berlin school, three alone recovered, though Kattner effected a cure in six weeks. Of ten cases of suprascapular lameness seen between 1875 and 1890 , three were discharged improved and four uncured. The case in a bull before mentioned remained uncured. Marked atrophy of the paralysed muscle is an unfavourable symptom, though one case mentioned by Lesbre recovered completely and the atrophied muscle was restored after an interval of eighteen months. The return of irritability under the faradic current is a reliable sign of improvement. While animals affected with this lameness cannot be employed in rapid draught, yet they can still do slow work in a breast-collar; the ordinary collar favours the disease, because it tends to displace the shoulder.

Treatment must follow general principles. Little can be done to check the progress of already-existing pathological changes in the nerve. At first the patient must be rested, and attempts made to increase local nutrition, and thus prevent atrophy, by kneading or striking the muscles for 15 or 20 minutes twice daily. Massage may possibly favour removal of already-existing anatomical changes in the nerve; for the same purpose veratrin may from time to time be injected. One and a half grains of veratrin (the variety insoluble in water) is rubbed down with about 75 minims of water without adding alcohol, and the mixture injected beneath the skin over the region of the muscle. Good results have been seen from subcutaneous injection of oil of turpentine.

On account of the excessive sensitiveness of the horse, the use of electricity, especially of the induced current, is generally too troublesome to be of service, though it is one of the most effective means of restraining loss of muscular and nervous irritability, and may perhaps be tried in valuable animals. When similar symptoms follow bruising, cold applications are preferable.

In the spring of 1875, three horses with this lameness were sent to the Berlin school in rapid succession. Two showed traces of violence in the shoulder region : one had run away and struck the affected shoulder against a tree, the other one had also been in collision. Symptoms of lameness appeared shortly after the accidents. One of the three horses soon became sound the two others appeared incurable. Later two more cases were seen to recover, one in spite of the fact that there had been considerable rupture of muscle.

Williams describes, under the name of "shoulder-slip," a disease said to occur in plough-horses, which are obliged to walk with one foot at a lower level than the other. According to Williams's description, this appears to be paralysis of the suprascapular nerve, though he describes pathological changes in the spinati and teres muscles, as well as in the 
shoulder-joint. He especially mentions abrasion in the bursa of the postea-spinatus muscle, and rupture of its tendon. It can be proved that rupture of this tendon, which sometimes occurs after inflammation of its sheath, produces the same disturbance in movement as paralysis. Section of the tendon of the postea-spinatus results in precisely similar lameness. Professor Dewar has pointed out that the foregoing description of paralysis of the suprascapular nerve is exactly applicable to the condition known to horsemen in Scotland as "slipped or prised shoulder," the most common form of which is atrophy of the subspinatus muscle in three year old horses being trained to agricultural work. The muscles of both sides are often affected. As these animals worls in traces, and have not to back, the cause in their case can scarcely be a backward movement of the shoulder.

The disease may, of course, be accidentally complicated with other injuries. Thus, in a riding-horse which had run away and struck its shoulder against a tree, the levator humeri and pectoralis transversus were ruptured and there was paralysis of the suprascapular nerve. Rupture had probably occurred in other of the breast muscles. Soon after the injury the hair fell away from a particular point on the skin, and severe eczema developed. The longish oval spot, which began about a hand's-breadth below the shoulder-joint, extended perpendicularly upwards almost to the middle of the neck. At the centre, and just over the shoulder-joint, it was about 8 inches broad, and became smaller both in an upward and downward direction. The hair which afterwards grew on the spot was of a lighter colour, and if the animal was excited or slightly pushed active secretion of sweat occurred here whilst all the rest of the body remained dry. The spot was also insensitive to the prick of a needle. Without doubt this was a case of simultaneous paralysis of the superficial scapular nerve, a twig of the sixth cervical. The condition improved, but very slowly.

\section{VII.-PARALYSIS OF THE RADIAL NERVE (PARALYSIS NERVI RADIALIS.}

The radial (or musculo-spiral) nerve derives its fibres from the seventh and eighth cervical, and from the dorsal roots of the plexus, and passes downwards and backwards on the subscapularis and teres major muscles, and some little distance behind the axillary vessels, from which it is separated by the ulnar nerve. On reaching the deep humeral artery, it disappears in front of the large head of the triceps, and is continued round the humerus in the musculospiral groove, where it rests on the brachialis anticus, and afterwards, on the posterior or outer border of that muscle. Before the nerve disappears behind the humerus, it gives branches to the great and small heads of the triceps, and a long branch which passes backward to divide under the scapulo-ulnaris for the supply of that muscle. Behind the limb it supplies the medium head

V.S. 
of the triceps and the anconeus, and furnishes a few cutaneous branches, which perforate the caput medium. In the fore arm the nerve supplies the extensor muscles and the flexor metacarpi externus.

Paralysis of this nerve used often to be seen, though not accurately diagnosed. Harms states having recognised this paralysis in a cow. In 1875 Möller saw the disease repeatedly in horses, and then for the first time carefully described it; it has since been frequently diagnosed. During the last few years Möller has often seen this lameness in horses and in one case in the dog.

Even at the present time little is known of the causes of radial paralysis. Sometimes it appears in horses which have been cast for a long time, especially if lying on sand or on the earth. But it is doubtful whether such lameness is not principally myopathic and produced by continued pressure on the triceps muscle, interfering with circulation; its rapid disappearance supports this view, which seems in accord with similar observations in man. Fröhner has suggested that the condition in question is a myopathic paralysis of the caput muscles, and supports his view by the results of examinations of the muscles in two slaughtered horses suffering from the disease. These changes, however, were probably secondary in character. Lanzillotti-Buonsanti, who found similar lesions, pointed out that they were strictly confined to muscles served by the radial nerve. Moreover, the nerve itself was hyperæmic. There can be little doubt that in cases of long duration the nerve, and not the muscles, is the structure primarily affected. Lustig saw radial paralysis lasting thirty-eight days after a tedious dental operation. Since casting on soft mattresses has become common, such cases have seldom occurred.

As a rule, radial paralysis occurs suddenly during heavy draught, clearly pointing to a traumatic origin. During a very short period in the summer of 1887 Möller saw many horses with this disease, which is generally rare. It, therefore, seemed as though a specific cause were at work, a view confirmed by the peculiar clonic spasms of the triceps muscle seen in other horses. These spasms ceased during worls, but returned with rest, and could be produced by flicking the muscles with the finger. Bormann in horses twice diagnosed radial paralysis which set in in the same sudden fashion. One case seen by Werner, and described as paralysis of the extensor pedis, occurred after violent attempts to free the foot, which had been caught in some obstruction. Bräuer saw this lameness result from long exposure to rain during work. Lübke also saw double-sided partial radial paralysis caused by chill; the disease was accompanied by fever, and proved fatal.

In the year 1890 Möller saw a case of diplegia of the radial nerve in the horse. The disease suddenly appeared in the left limb during a 
journey, and, being painful, caused the animal to lie a great deal, following which the radial nerve of the right limb became affected. Friis describes a case complicated with paralysis of the hind leg of the opposite side (hemiplegia cruciata).

A series of interesting clinical records and a full description of this disease will be found in Cadiot and Dollar"s "Clinical Veterinary Medicine and Surgery," p. 64.

Willis, Rogers, Hunting and others have drawn attention to the frequency with which pronounced radial paralysis is associated with fracture of the first rib. For a time, indeed, it was believed that radial paralysis was always caused by fracture of the first rib, but a more careful research has disposed of this view. Rogers in 1894 described the following case in an eight year old well-bred chestnut mare. The animal had been worked for three hours in a victoria, and returned home with great difficulty, exceedingly lame in the off fore leg. The near fore heel showed signs of a recent overreach. The animal was in great pain, and supported the weight of the body mainly on the hind legs; the off fore leg was flexed, the knee and fetlock bent, and the outside of the toe just touched the ground. The elbow was lower than normal, but was not unusually " dropped." By forcing the knee backwards, and thus straightening the leg, the animal was enabled to take one step with the sound limb; but immediately the knee of the injured limb became in the least degree bent the leg collapsed, and the animal nearly fell. Progress was facilitated by pulling forward the lame leg as far as possible, and then pressing on the knee until a step had been taken with the sound leg. The must marked symptoms were absolute inability to advance the leg, and great difficulty in keeping

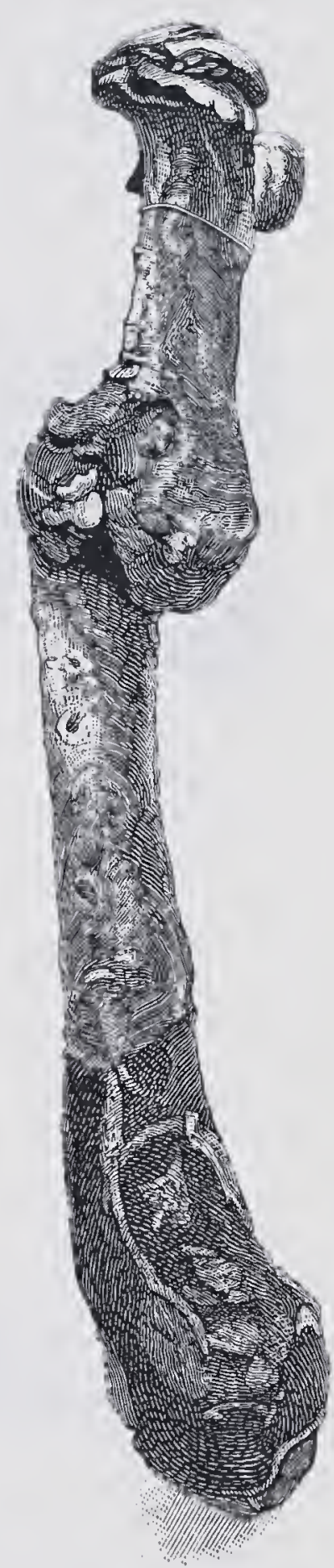

FIG. 192 . it perpendicular. No fracture of any of the leg bones could be distinguished. Crepitus was entirely absent.

Fractured first rib was diagnosed. The animal was placed in slings.

During the three weeks and three days it was kept, the limb was always flexed and turned slightly inwards, so that the outside toe of the foot became worn.

Post-mortem examination showed fractured firstrib (see fig. 192); the surrounding muscles were not lacerated or ruptured. Only a small provisional callus had formed. The broken and overlapped pieces of 
bone were freely movable, and not (as might appear from inspection of the figure) rigidly fixed together.

The reason why fractured first rib and marked radial paralysis are so often associated is to be found in the proximity of the brachial plexus, from which the radial nerve originates, to the site of fracture. Even in incomplete fracture a sufficient effusion of blood may occur to seriously interfere with the brachial plexus.

The symptoms vary according to the degree and extent of the paralysis. When complete, the limb takes a position resembling that in very painful

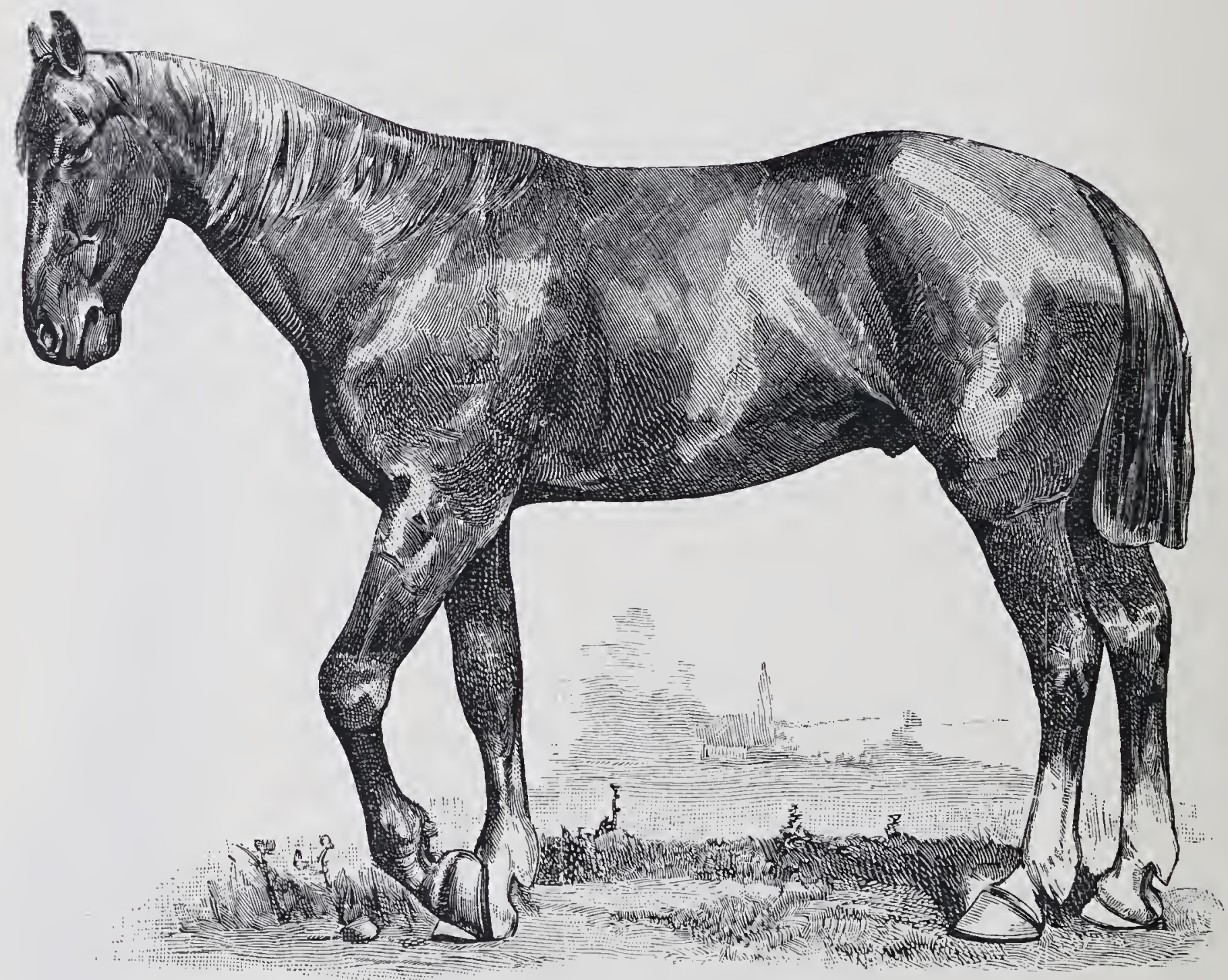

FIG. 193. - Complete paralysis of the radial nerve.

affections of the foot. The shoulder and elbow are extended, while all the other joints are fixed in a position of volar flexion, those of the phalanges often to such a degree that the anterior surface of the wall of the foot may be almost in contact with the ground (fig.193), the limb under such circumstances appearing to be too long. In attempting to move, the upper portions of the limb are carried forward in the ordinary fashion, but cannot support the weight of the body on account of inability to bring the joints into the necessary position. The limb collapses at every attempt to place weight on it.

The disease is, therefore, characterised by lameness when weight is placed on the limb (supporting leg lameness), flexion of all joints from the elbow downwards, and inability to bring the foot sufficiently forward 
to enable it to carry weight; when passively extended, lowever, the limb is able to sustain the body. On moving the horse the triceps muscles appear relaxed. They fail to contract, and after some time atrophy.

In incomplete radial paralysis the weight of the body can be supported provided sufficient muscular power exist to extend the joint. When, therefore, the animal moves slowly and on a smooth surface, nothing particular is visible, though lameness appear's immediately the horse meets with a slight incline or with any obstacle. In such case the foot is not sufficiently extended, it strikes against the obstacle and the leg collapses, so that the horse may fall. Depending on the degree to which the nerve or muscle is affected, this stumbling occurs more or less frequently; sometimes it is only seen after exertion on soft or uneven

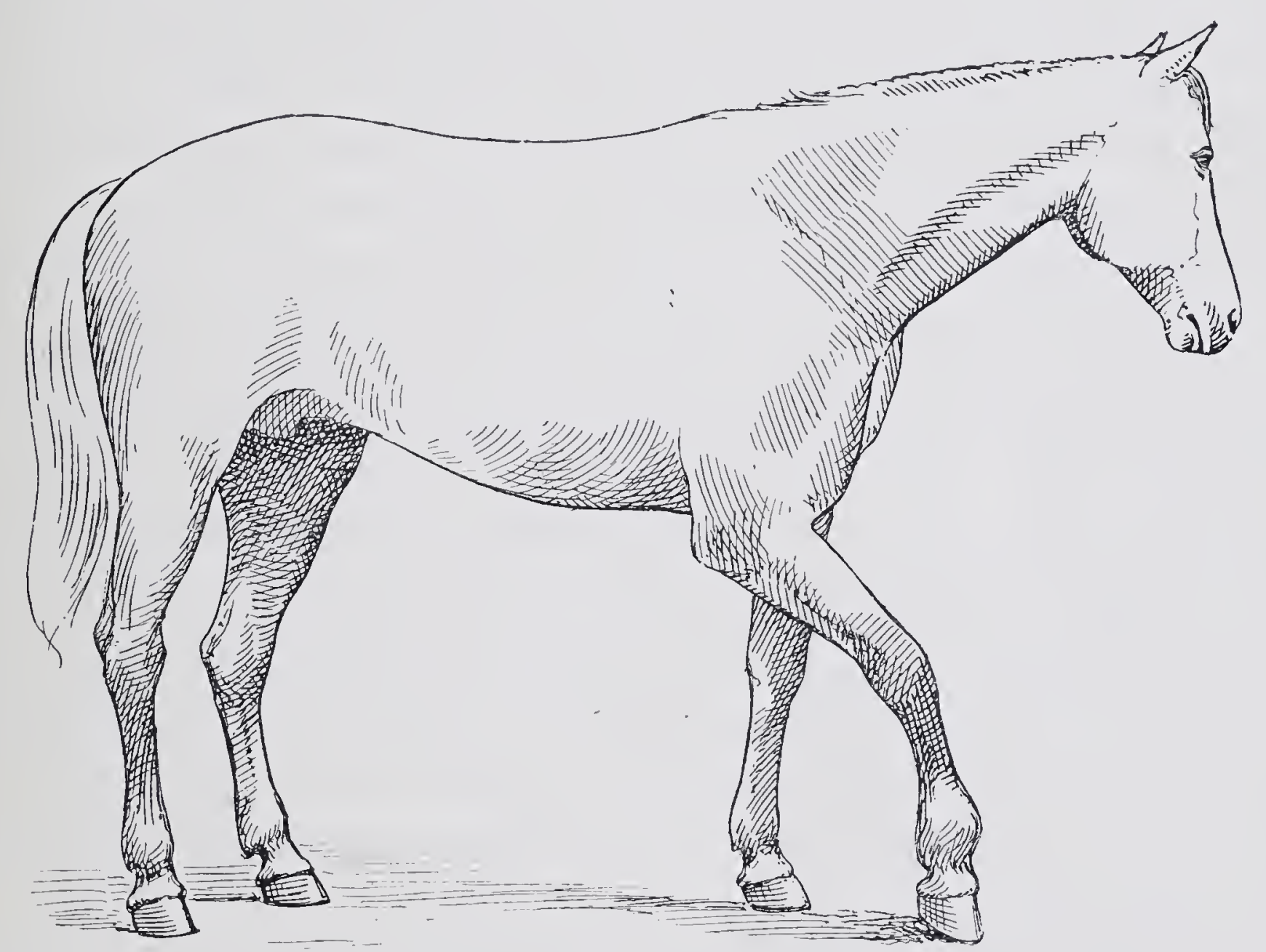

FiG. 194. - First portion of the stride in a case of paralysis of the radial nerve. The animal was recovering.

ground. When the lameness diminishes after having long existed, the animals instinctively adopt a peculiar way of moving. In stepping out they slide the foot forwards with exaggerated action of the shoulder muscles, so as to compensate for the defect in that of the extensors. The lameness is characterised by stumbling, particularly on uneven ground, and later by a sliding motion of the foot when the limb is extended.

Cases of radial paralysis due to fractured first rib can sometimes be diagnosed by drawing the affected limb towards the opposite side of the body, and sharply returning it to its former position. By applying the 
ear over the scapula while this manipulation is performed by an assistant crepitation may be detected.

Not infrequently paralysis is partial. The greater number of cases seen by Möller in the summer of 1887 , and certain cases observed later, were distinguished by the fact that the function of the caput medium (M. anconeus externus) and of the extensors lying in the region of the fore arm was clearly retained, whilst the other portions of the caput muscle appeared relaxed; when weight was placed on the foot, these contracted in the usual way. For this reason the lameness has a peculiar character: during the period when weight is placed on it, and at the moment when the limb is perpendicular, the shoulder is suddenly jerlied forwards; the scapula and humerus move with a visible jerk, causing the disease to present a certain similarity to suprascapular lameness. But as these involuntary movements take place in a forward direction (best seen by moving the horse slowly and viewing it from the side), the disease is readily distinguished from the above lameness, where the shoulder moves directly outwards (see "Paralysis of the Suprascapular Nerve"). The condition is characterised by "supporting leg lameness" and jerking of the shoulder forwards at the moment when the limb is upright.

The reason of the caput medium being sometimes unaffected in this lameness must be sought in the distribution of the nerves. From the point of origin twigs are first given off for the heads of the caput magnum muscle; these are comparatively short fibres, whilst the main stem distributed to the caput medium and parvum and extensors of the foot lying in the fore-arm region is much longer, and is, therefore, not involved to the same extent in any strain occurring here. The correctness of this view is supported both by the fact that partial paralysis occurs accidentally after mechanical injuries, and that in such cases function is always retained in the extensors of the knee, of the fetlock, and of the foot.

As already stated, paralysis in these groups of muscles can be detected during movement both by sight and feeling. Muscular atrophy occurs sooner or later, and to an extent varying according to the amount of functional disturbance. Sometimes, though not invariably, sensation is lost in the skin covering the anterior and external surface of the fore arm.

The diagnosis of radial paralysis is therefore easy.

Differential diagnosis. The disease may. be mistalien for myopathic lameness of the caput group of muscles, or for any of those conditions in which they partially or completely fail to act. 'This is particularly true of oblique fracture of the ulna-the lower point of insertion of these muscles-and of rupture of the extensor pedis. Hertel saw all the extensors attached to the ulna torn away, but in such cases local examination at once dispels any doubt. 
Diagnosis and course. The twenty cases of radial paralysis seen by Möller all eventually recovered, though not with equal rapidity; incomplete paralysis usually disappears in fourteen days, but the greater number of cases of complete paralysis last for five to six weeks. Certain severe cases, possibly due to fractured first rib, require six to nine months for complete recovery. Three cases relapsed after some weeks' severe work, but finally did well. Prognosis is, therefore, generally favourable. Uncertainty of movement sometimes remains even after disappearance of lameness, but generally disappears in time. The longer lameness continues, the less the chance of cure. Well-marlied muscular atrophy is therefore unpropitious, but return of irritability to the Faradic current must be regarded as a favourable sign.

Treatment. In the greater number of Möller's cases no particular treatment was required. Electricity is only worth trying in valuable horses, but massage, especially in complete paralysis, is certainly of the greatest value in assisting nutrition of the muscles. When paralysis is incomplete, daily exercise on soft ground and at a slow pace is for the same reason advisable. If thought advisable, subcutaneous injections of veratrin and strychnine may be employed. The application of powerful douches can only be regarded as a kind of massage. Bormann effected recovery in five to six weeks by giving strych. nitr. $\cdot 3$ to $\cdot 6$ grain subcutaneously; but in this time the disease would generally disappear without any treatment whatever.

A less frequent variety of intemittent radial paralysis has been seen by Möller. A young carriage-horse, which showed nothing unusual in the stable, or when beginning work, displayed symptoms of radial paralysis in the right fore leg as soon as it had gone about two miles. The condition was first incomplete, as shown by stumbling, but gradually became complete, with the above-described characteristic symptoms. By resting the animal, the symptoms disappeared in about half an hour, to reappear, however, as soon as work was resumed. Möller suspected thrombosis, either in the arteries supplying the triceps muscle or in one of their nerve stems. Although the post-mortem was conducted with the greatest care, nothing could be detected, so the cause of this rare disturbance remained unexplained. There was no muscular atrophy.

\section{VIII.-PARALYSIS OF THE BRACHIAL PLEXUS.}

Holmann saw paralysis of the entire muscles of the fore limb in a horse, following on a fall, and found on post-mortem severe bleeding around the brachial plexus. From his description it is, however, clear that simultaneous rupture of the serratus magnus existed, for the horse was not only unable to move the foot, but the body sank several inches 
at each attempt to place weight on the limb. The limb was also abnormally abducted.

Trasbot describes a case of paralysis of the brachial plexus in which hemiplegia appeared later. Recovery occurred in fifteen days. This case is also not quite clear. Wilson saw hemiplegia in a horse after rolling over backwards, and Anacker has seen it in a sucking-pig.

In dogs it is commoner, and occurs both as peripheral paralysis and as hemiplegia. In the latter condition Möller repeatedly saw well-marked spastic lameness in all the muscles of the shoulder, most notably in the triceps group; this was followed by long-continued disturbance in movement. Paralysis of the brachial plexus in horses is sometimes of central origin; in these cases other troubles precede, accompany, or follow it.

A seven year old mare showed in December, 1887, right-sided facial paralysis ; in January, 1888, left-sided hemiplegia. The toe of the left fore foot was dragged along the ground. Soon afterwards a small cataract appeared in the right eye, and gradually extended over the entire lens. The post-mortem showed a tumour as large as a walnut between the petrous portion of the right temporal bone and the cerebellum; it extended to the pons varolii and restiform bodies of the medulla. Its interior was occupied by a small quantity of pus.

In another horse, lameness of the right hind leg suddenly appeared, followed in two days by paralysis of the right fore leg. The post-mortem showed hæmorrhagic spots in the right half of the cerebrum and cerebellum. Similar symptoms were seen in another case, but disappeared in twenty-five days.

Shocks from the high-pressure currents used to operate street trams or railways may produce brachial paralysis, which, however, passes away, provided the shock has only been momentary. Lightning stroke produced paralysis of the brachial plexus in a Prussian army horse, but the condition disappeared again in a few days.

Another case occurred in the Alfort clinique. A horse was cast and kept down for a long time. On rising it showed complete paralysis of one fore limb. Post-mortem examination showed that the brachial plexus had been bruised and paralysed by a bony tumour on the second rib.

Many cases of brachial paralysis are produced in this way, resulting from bruising, subscapular bleeding, deep-seated abscess formation, the growth of tumours on the inner surface of the shoulder, or even from direct injury like that due to the deep penetration of a carriage shaft.

The symptoms which accompany paralysis of the brachial plexus depend on whether paralysis is complete. Complete paralysis of the entire axillary plexus prevents all voluntary movement in the limb. Provided, however, the foot be placed in the proper position, the limb, 
in the horse and dog at least, is still able to support weight, but again collapses when the weight is removed. The body load is better sustained so long as the radial nerve remains unaffected, but there is difficulty in advancing the foot. When contraction occurs in the triceps muscles, the leg, from the elbow downwards, is continuously fixed in extreme extension. This position is retained even during movement.

Prognosis and course. The prognosis must be based on general principles, as our knowledge of this lameness is still very incomplete. As would be expected from its cerebral origin, spastic paralysis of the brachial plexus in dogs generally does badly.

Treatment is similar to that of radial lameness. Massage, which improves nutrition, and prevents atrophy of muscle, is of more use than drugs.

\section{IX.-SHOULDER LAMENESS.}

Besides those above described, other lesions occur in the shoulder region, some of which evade even the most careful examination, and cannot be exactly diagnosed, or their results foretold. Lameness is the sole symptom, and for want of clearer knowledge these conditions have been included under the general term "shoulder lameness." As the chief muscles for the movement of the limb are found in the shoulder region, it is not surprising that these lamenesses show a certain agreement in symptoms. They are almost always marked by lameness when the limb is carried, i.e., movement appears chiefly or solely interfered with during the forward stride, and the affected limb is advanced slowly and incompletely. The lameness varies according to the position, nature, and extent of the disease process; and therefore, in speaking of each condition, we shall go more fully into these points. The following are some of the forms of shoulder lameness :-

(1) Disease of the shoulder-joint and its surroundings. As a rule, this consists in chronic inflammation, which cannot be directly recognised by clinical means. Arthritis chronica, with erosion of cartilage and periarticular exostoses, is met with in old horses. In cattle and dogs rheumatic diseases of the shoulder-joint occur, and often produce lameness. Sometimes passive movements of the shoulder, raising and lowering the foot, occasion pain, and by relaxing the muscles of the shoulder render the head of the humerus more prominent. Atrophy follows long-continued lameness. As a rule, there is pain when weight is placed on the limb, and especially when the animal is turned in short circles.

In a horse which had suffered from chronic shoulder lameness, Siedamgrotzky found great thickening of the anterior surface of the joint, and chronic inflammation of the bursa intertubercularis and biceps brachii. The synovial 
membrane was thickened and covered with numerous thread-like proliferations, and the articular surface of the scapula increased to the extent of about an inch by a circular bony growth. The cartilage covering the articular surfaces, both of the scapula and humerus, was abraded.

(2) Disease of the muscular tissues of the shoulder is sometimes traumatic, sometimes rheumatic, in origin. External violence, or violent falls or leaps, may produce inflammation of the shoulder muscles. Gerlach erroneously assigned these conditions to the biceps; but although we are not in a position to absolutely deny their occurrence in this position, yet they are exceedingly rare. Nesbit reported a case in which both biceps brachii muscles were torn away from the coracoid process (see "Inflammation of the Bursa of the Biceps Muscle"). Inflammatory symptoms, such as increased warmth, pain on pressure, or on extending the diseased muscles, can almost always be detected on careful examination, but care and judgment are required in order to avoid error.

Most horses flinch under strong pressure on the shoulder, nor is tenderness a constant symptom even in painful lamenesses, so that it is quite erroneous to consider that it is simply a question of unequal sensitiveness. Moreover, the affected spot has often been made more sensitive by manipulation, or by the application of irritants. On the other hand, inflammation occurs in single muscles, like the levator humeri, and even in entire muscular groups, such as the pectoral muscles, in consequence of bruises and sprains, and in young horses from severe exertion, and can only be detected by careful palpation. On drawing the foot backwards, the animals show pain.

These conditions are usually accompanied by lameness while the limb is being advanced, though none is shown when weight is placed on it. After some time muscular atrophy sets in, and is most marked in the region of the diseased muscles.

Rheumatic disease of the shoulder muscles, which is especially common in the levator humeri, produces similar symptoms. Pain generally diminishes during movement, and may entirely disappear when the animal begins to sweat. Rheumatic disease is indicated by remittent or intermittent lameness, and by unusual pain after slight sudden movement of the muscles, such as that produced by flipping the parts with the finger, whilst powerful pressure is less painful. Sometimes extensive contraction can be induced in single muscles, e.g., in the entire levator humeri, by a light blow with the finger. In disease of this character in the levator humeri, the animal's neck is drawn downwards and towards the diseased side (torticollis), and the muscle appears very tense and prominent.

Pütz saw a peculiar shoulder lameness: movement was difficult, though the animal showed no pain on examination. The muscles were swollen but 
relaxed, the body sank between the limbs, and the shoulder-joint was pressed away from the thorax. Post-mortem examination discovered a myositis chronica fibrosa, produced by the presence of parasites.

Blenkinsop, in Australian horses, repeatedly observed atrophy of the pectoralis anticus. A deep depression was present in the pectoral region, especially noticeable when the limb was drawn backwards. The diseased leg was advanced when moving, without, however, being extended. The shoulderjoint was turned outwards, the elbow inwards; below this point the limb was abducted, and trailed during forward movements.

Rupture of shoulder muscles has often been seen in horses. Di Nasso had a case of rupture of the serratus anticus major, subscapularis and pectoral muscles from collision with a tree. On placing weight on the limb the shoulder-joint was rotated outwards-a sign of paralysis of the suprascapular nerve. A similar case has already been described under the heading "Paralysis of the Suprascapular Nerve." In horses shoulder lameness may also be produced by over-extension and partial rupture of the superficial pectoral muscles, due to falling or slipping.

Rigot several times noted rupture of the subscapularis muscle in horses, in consequence of the limb slipping outwards. As a rule, the tear occurred near the lower insertion, and was followed by formation of exostoses on the humerus. The following case was due to rupture of the triceps. A ridinghorse suddenly fell lame on the off fore leg when turning sharply. The lameness increased with movement, and was distinguished by the lame leg being so much adducted that it crossed the other leg. Seven weeks afterwards the animal was killed as incurable.

A horse which four months previously had suffered from the thoracic form of influenza, showed cramp of the muscles of one side of the neck and of the fore limb of the same side, best seen in the stable when the animal moved the affected parts. It made sudden nodding motions with the head, lifted the affected fore limb, and after some seconds again set it on the ground. Oscillating movements, lasting for five minutes at a time, sometimes occurred in the muscles of the left side of the neck, particularly in the levator humeri. Repeated injections of veratrin were followed by recovery in three weeks.

There is no difficulty in diagnosing such cases, but the prognosis can only be based on a thorough knowledge of the local anatomical changes.

(3) Shoulder lameness may be caused by disease of the scapula or humerus. Though fractures are generally easy to recognise, fissures may elude the closest examination. Periostitis and the formation of exostoses are common on the scapula and humerus.

Gerke, in making a post-mortem of a horse which had suffered for two years from shoulder lameness, found an exostosis on the inner side of the head of the humerus. The connective tissue surrounding it appeared thickened, and formed, along with the exostosis, an "extraordinary articular surface." The radial nerve was smaller than on the sound side. The horse moved the limb in circles, but could still place weight on it. The lameness only occurred at a trot, but did not disappear with work. Similar cases have repeatedly been seen. 
(4) Thrombosis of the brachial artery produces lameness, which regularly recurs with work, and can, therefore, be easily recognised. It is rare, but has several times been seen in horses.

An old Percheron mare appeared lame on being rapidly trotted or worked in a cart. At first there was only stiffness, but in about ten minutes both fore limbs began to tremble, and thereafter to give way, the animal threw the head and neck violently upwards, and seemed doubtful which leg to stand on; the respiration and pulse were increased, and sometimes general sweating followed, though the fore limbs remained dry. These symptoms disappeared after a quarter to half an hour's rest, but always recurred on movement. Post-mortem examination showed thrombosis of both brachial arteries, which were much thickened.

Möller saw a similar case in a seven year old mare. When resting she showed nothing whatever unusual, though careful examination revealed hypertrophy of the heart. After ten minutes' trotting the mare began to place the fore feet abnormally far forward and outward and to stumble. The off fore limb was especially affected; the toe often struck the ground, causing the animal to stumble and fall ; on rising, the legs were propped out and the muscles trembled, particularly the triceps. All these symptoms disappeared after five to ten minutes' rest, to return again on exertion.

Many similar cases have been seen and verified by post-mortem. A horse which suffered from obstruction of the femoral artery began to show symptoms of radial thrombosis; during movement, the radial symptoms set in later than the femoral, but lasted longer, persisting for even half an hour. Postmortem showed thrombi in the arteria subclavia, arteria axillaris, brachialis and radialis, and even in the metacarpal arteries. The muscular coats were thickened, and the intima altered in character (endoarteritis).

Martin saw thrombosis of the axillary artery followed by gangrenous inflammation of the muscles of the limb, hypertrophy of the heart, and fatty liver. The disease appeared suddenly, with severe feverish symptoms, and proved fatal in eighteen hours. The case closely resembled septic cellulitis or malignant œdema.

(5) Inflammatory swelling and new growths sometimes interfere with movement. Thus acute or chronic swelling of the prescapular and axillary glands, resulting from glanders, strangles, or infectious local diseases of the fore limbs, may all cause shoulder lameness. Gerke reports several cases of the kind.

A horse, lame for two years and a half, showed on post-mortem a swelling as large as a man's fist beneath the shoulder, surrounding the axillary plexus. Another, which had suffered from shoulder lameness for four years, exhibited swelling and degeneration of the axillary glands. In both cases there was excessive atrophy, not only of the muscles, but even of the hoofs.

In these conditions the limb is often abducted, both during movement and when weight is placed on it. Periodically recurring shoulder lameness may be caused by swelling of the axillary glands. Chronic inflammation and suppuration in the skin of the shoulder may produce such swelling of the lymph glands. Scholz found an abscess near the axillary artery in a horse which had suffered from metastatic strangles, and subsequently from lameness. 
(6) In addition, shoulder lameness may undoubtedly be produced by nervous disease, though reliable observations on this point are not to hand. The case of intermittent lameness reported under the heading "Paralysis of the Radial Nerve" cannot be viewed as of nervous origin without further evidence.

The diagnosis of "shoulder lameness" chiefly depends on the negative results of local examination; the more careful the local examination, the rarer will be the diagnosis shoulder lameness. The practitioner should avoid basing his opinion on single symptoms, or attaching too great weight to the indications of palpation. As the striding movement is chiefly dependent on the action of the shoulder muscles, it is naturally much interfered with, and therefore shoulder lameness is generally characterised by difficulty in advancing the swinging limb. The stride is shortened, and, as a rule, uncertain. The foot is imperfectly lifted; it tends to strilie the ground, especially when the surface is uneven, or when the foot meets with obstacles. In leading the hol'se uphill, or in circling (especially if the lame leg is outwards), lameness is generally more marked. The limb, when being advanced, is not infrequently turned outwards. Lameness appear's equally on hard and soft ground-in fact, may even be more marked on the latter. When trotting, the head is often nodded in a pronounced way, but even this symptom is not constant. It is generaliy present, however, in disease of the levator humeri, and of the other levators of the shoulder, because of the pain induced by extension of the affected muscle. In moving backwards, the lame leg is often not lifted, but slid over the ground. Disease in the levators of the fore arm produces marked pain when the foot is passively moved either forwards, backwards, inwards, or outwards. In chronic lameness, too great stress must not be laid on atrophy of muscle or of the hoof. Both are secondary symptoms, and do not always indicate the seat of disease.

Prognosis and course. Our ignorance of the nature of this lameness renders prognosis uncertain, and the chief indication is the course which the disease takes. The longer lameness has existed and the greater the atrophy of muscle, the more serious the condition. Inflammation of a single muscle generally takes a favourable course, but rheumatic disease is often obstinate. None of the articular diseases offers much hope of recovery, especially when of old standing. As soon as some improvement can be noted, a more sanguine prognosis may be delivered. Many cases of shoulder lameness recover in two or three weeks, whilst other's may continue for an indefinite time.

The treatment is as varied as the prognosis. In acute inflammation and mechanical injuries, rest and cold applications in the form of irrigation or cold poultices are most useful. Failing marked improvement 
in eight to fourteen days, a powerful douche may prove serviceable. At a later stage, irritants may be tried, and in the event of their failing, setons. The long rest thus ensured has a very important influence in determining recovery. When massage can be properly applied it should have preference, especially in disease of soft parts, as it assists resorption of inflammatory products, increases nutrition of muscles, and prevents atrophy.

Antiseptic poultices are also useful, particularly in the inflammation following injury or rheumatic disease. Sometimes both methods may be used together. Subcutaneous injections of veratrin are also worth a trial. There is no doubt that veratrin affects the functional activity of muscle, and assists metabolism. In shoulder lameness due to muscular rheumatism exceedingly good results often follow veratrin injections. Veratrin that is insoluble in water should be used, because it is less rapidly absorbed, and therefore produces a local effect. Wonderful results are said to have been produced in some cases by the subcutaneous injection of the following solution: Atropin sulphate .75 grain, morphine hydrochlor 3 grains, water 300 grains. Failures, however, are not infrequent, and in some cases fatal colic is said to have occurred, which has been attributed to the atropin. If this treatment be tried, the animals should be fasted for 12 hours beforehand or the dose of atropin diminished. Möller saw no good results from subcutaneous injection of common salt. A pure solution produces no visible effect, whilst if the solution or syringe is dirty, extensive pus and abscess formation may ensue; whatever efficacy the treatment has, apparently, depends on its causing purulent inflammation. The same objection applies to injections of turpentine and ether; and as they occasionally produce violent local action and necrosis of the soft tissues, their employment is dangerous.

When the stride remains shortened for a long time, Von Chelchowski recommends moving the horse in a small circle, with the lame foot outwards.

\section{B. DISEASES OF THE ELBOW AND FORE ARM.}

\section{I.-FRACTURE OF THE ULNA.}

Though seen in all species of animals fractures of the ulna are rather rare except in horses. In horses the olecranon is generally broken, in other animals the strongly developed body, as a consequence of external violence, in the form of kicks, treads, or falls. Jansen reports three cases, one caused by falling and sliding along the ground and two by kicks. Double-sided fracture of the ulna was seen in a horse which had 
passed the fore feet through a hay-rack. In general the olecranon breaks completely off, loosening the lower point of insertion of the extensors of the elbow, and producing symptoms like those of radial paralysis.

Birrenbach saw complete fracture about $1 \frac{1}{2}$ inches below the joint. When, as is generally the case, the fracture is lower and extends into the elbow-joint, lameness and pain are severe when weight is placed on the limb. The displacement of bone can sometimes be directly observed.

Mann noticed permanent lameness and symptoms of radial paralysis in a foal which had been kicked on the elbow. Post-mortem showed that the ulna was completely broken off from the radius, and that a space of nearly two inches existed between them. In this case the ligamenta transversa and the ligamentum interosseum must have been ruptured.

Prognosis and course. Union of transverse fracture of the ulna is rare; most horses suffering from it have sooner or later to be killed. Günther says foals recover. In man, transverse fractures of the olecranon only reunite by fibrous callus, because, it is said, of the displacement of broken fragments by the pull of the muscles, and of the failure to form bony callus on account of the absence of periosteum. On the other hand, A. Cooper has shown by experiments on dogs that in longitudinal fracture of the olecranon a bony callus always forms. The unfavourable

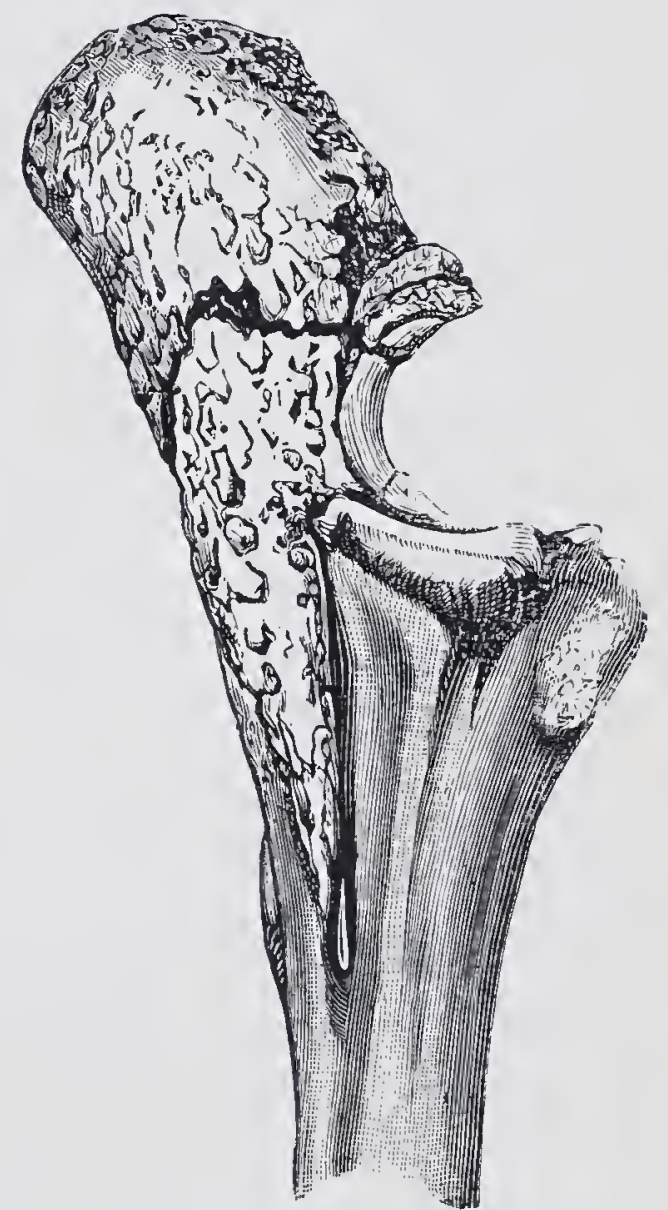

Fig. 195.-Fracture of the ulna in a horse. course which transverse fracture of the ulna takes in the horse must be referred to the great dislocation produced by the pull of the triceps muscle, and to the fact that the fracture almost always extends to the articular surface. Fig. 195, drawn from nature, shows a typical fracture, such as is usually seen in horses. Möller never had a recovery. Under exceptionally favourable circumstances, fracture of the ulna may reunite. In pigs, dogs, and cats, in which the ulna forms a bone distinct from the radius, recovery is much commoner, the radius acting to some extent as a splint for the broken ulna.

Treatment. As in horses and ruminants no effective bandage can be applied at this point, treatment is confined to keeping the animal quiet, or possibly to placing it in slings. The action of the triceps muscle interferes with healing, and therefore it is best to prevent animals lying 
down, which in horses of little value is most easily effected by fastening them up short, in others by slinging them. In carnivora a plaster bandage assists recovery, but is somewhat difficult to apply. To ensure its remaining in place the bandage should be passed over the back and between the fore legs, reversing the direction alternately. (For information on Bandaging and Dressing, see Dollar's "Operative Technique," p. 177.)

\section{II.-FRACTURE OF THE RADIUS.}

In the horse, fracture of the radius is not usually accompanied by fracture of the ulna. In other animals it is sometimes seen with, sometimes without, fracture of the ulna. In dogs the fore arm is most frequently fractured by the animals being run over, or being squeezed or kicked; in larger animals, it results from kicks, falls, and slipping whilst getting up, \&c. Freer, in a horse, saw fissuring of the radius which, a fortnight later, was succeeded by fracture.

In ruminants and carnivora, diagnosis is only difficult when the ulna remains intact, and crepitation is absent. Careful examination, however, shows abnormal mobility, crepitation, swelling, and pain. Not infrequently the broken ends perforate the soft tissues and produce a compound fracture.

Prognosis and course. Subcutaneous fractures of the radius with intact ulna unite, in carnivora and ruminants, in three to six weeks. Should the ulna be simultaneously fractured, proper treatment may be succeeded by recovery in four to eight weeks; and although in rumimants and swine repair is more difficult, it is by no means impossible. In horses, however, complete fracture of the radius must be viewed as relatively incurable, particularly when near the epiphysis; in the diaphysis, recovery may occur if the animal can be kept perfectly quiet, and if able to stand long enough on the other leg.

An English thoroughbred mare with transverse fracture of the radius and rupture of the carpal ligaments recovered sufficiently for breeding purposes.

Compound fractures of the radius in the horse must be viewed as incurable, though in small animals they are rather more hopeful.

Treatment. Reduction is effected according to the general principles already laid down, and succeeds best under anæsthesia. Before applying the plaster bandage, the operator must make sure that the leg occupies its normal position, and is neither in a condition of supination nor of pronation. The position of the other leg should guide the dresser. In carnivora, the plaster bandage is applied directly to the skin, and includes both the elbow- and knee- joints. In larger animals it must reach at least to the knee, to prevent its sliding down for want of a fixed point. 
Large animals should be liept in slings. Ordinary splints are seldom of much use, but felt and poroplastin have been used with advantage. In treating an oblique fracture, the animal was placed in slings on the second day, reduction effected, and after applying wadding and gauze bandages, a piece of moistened felt was fixed in position by linen bandages. Complete recovery followed in three months. A number of successful cases in the horse have been reported by French practitioners. In most instances after reducing the displacement the limb was enveloped in masses of tow, saturated with white of egg and alum, or in bandages treated with a resinous solution. Over these were applied wooden splints, retained in position with a further series of bandages. In some cases it was found needful to pass the bandages over the shoulder in order to prevent the dressing slipping down. Some operators used plaster bandages. The dressings were left in position for one to two months. A more or less extensive callus remained, and the horse usually went lame, but in the majority of reported cases became sound again after firing. It must not be forgotten, however, that the failures are less often reported than the successes, and the former probably exceed the latter at least tenfold. Complicated fractures are treated on general principles.

\section{III.-LUXATION OF THE ELBOW-JOINT.}

In large animals, luxation of the elbow-joint is impossible without previous rupture of the powerful lateral ligaments; in the horse, fracture of the ulna is then usually produced. According to Rigot, luxation occurs during flexion, on account of the force acting on the ulna. It is exceedingly rare in animals. Such cases have, however, been described in the horse. In one the internal lateral ligament was ruptured in consequence of a fall. The limb could be abducted to an abnormal degree. The horse recovered almost perfectly; in five weeks only a slight "swinging leg" lameness was noticeable.

A mule carrying a very heary load of barley slipped and fell. The left leg, from the elbow downwards, was doubled under the body, and when the animal was lifted the head of the radius and the ulna were thrust to the inner side of the inner condyle of the humerus; that is to say, the lower extremity of the humerus overlapped the upper end of the radius.

In another case a horse fell on its left side, with the corresponding leg in a doubled-up position; on rising the fore arm pointed obliquely forwards and outwards, the limb below the fetlock hung limply downwards. In this instance the head of the radius was outside the condyle of the humerus.

Luxation of the radius outwards in dogs has been referred to the fact that the articular surfaces slope downwards and inwards, and the external lateral ligament is particularly strong.

Distortion is very uncommon, on account of the effective limiting V.S. 
apparatus of the joint-the strong lateral ligaments and beak of the ulna. Hertwig insists that in carnivora both complete and incomplete displacement may occur in the elbow-joint, and either inwardly or outwardly, without fracture. Möller only saw such displacements as congenital conditions, occurring simultaneously in both limbs, and associated with deformity both of the elbow- and linee-joints. The animals are generally killed as hopeless cripples.

The diagnosis of displacement is not difficult, as there is always extensive rupture of soft tissues.

Prognosis and course. In carnivora, cases do well provided they are treated early and the joint is not involved. The mule and one of the horses above mentioned are said to have recovered after reduction of the luxation, but the statements require confirmation. Congenital luxation is unpromising, and in direct proportion to the amount of deformity present. Even if the bones can be replaced, retention is very difficult, and treatment can scarcely be advised.

Treatment. Reposition and retention are effected under anæsthesia and in accordance with general principles. In carnivora, the joint may be supported by plaster bandages; in large animals this is impracticable.

\section{IY.-INFLAMIMATION OF THE ELBOW-JOINT.}

Acute inflammation of the elbow-joint is seen in all species of animals, sometimes from wounds of the joint, sometimes from metastatic disease, like "navel-ill" of young animals. In dogs the joint may be opened by bites, in hor'ses by kicks or by injuries from stable-forks. Disease may also be caused by injury to the sheath of the flexor metacarpi externus, which communicates with the elbow-joint. Franck states that the sheath always opens into the elbow-joint by a slit about 1 line in breadth. Lothes saw chronic inflammation of the elbow-joint in a horse, apparently produced in the first instance by an injury to this sheath. Lanzillotti treated a horse which suffered from necrosis of the radius in consequence of a kick. During operation for removal of the sequestrum the elbow-joint was opened, but under antiseptic treatment healing occurred.

Chronic inflammation of the joint is rare. Roloff noticed fungous arthritis of the elbow-joint in the horse; Dammann, a cystenchondroma. As a rule, tumour formation is not frequent. Arthritis chronica is difficult to diagnose with certainty, and is generally included in "shoulder lameness."

Percival noted a form of chronic lameness in the horse which presented considerable resemblance to laminitis. Post-mortem showed chronic inflammation of both elbow-joints, a portion of the articular surface being worn away and covered with granulations.

Symptoms. Acute inflammation of the elbow-joint due to wounds 
produces excessive pain on movement. The patient may sustain weight on the limb, but carefully avoids any movement of the joint, particularly at the commencement, when acute synovitis alone exists. Afterwards fever and marked swelling of the whole joint set in.

The course is almost always unfavourable, acute inflammation of this joint often ending fatally, and chronic inflammation being followed by incurable lameness.

Treatment is, therefore, chiefly of a prophylactic character, and follows general principles, including careful antisepsis of wounds involving the joint or tendon sheath above mentioned. In suppuration involving the joint, little can be done. The thick muscular tissue and unyielding capsular ligament present great difficulties to washing out the cavity, and usually render such treatment useless. The form of arthritis chronica seen by Percival was also incurable, and, after long and unsuccessful treatment, the horse was killed.

\section{V.-CAPPED ELBOW IN THE HORSE.}

The structure corresponding to the bursa olecrani of man is found in the horse and dog at the posterior surface of the elbow-joint, and though not constant, generally forms below the skin a bursa mucosa as large as a walnut or apple. Injury caused by the heel of the shoe may result in local infection (discomyces) and produce a swelling, vulgarly termed "capped elbow."

Pathological anatomy. Bruising leads to acute inflammation of the bursa; sometimes bleeding takes place into the bursal cavity ; sometimes bursitis sero-fibrinosa vel hæmorrhagica occurs. If pus cocci enter, bursitis suppurativa may result. In any case, however, the connective tissue around the joint also suffers (parabursitis), though in the horse this condition is not marked. At first, cedematous swelling of the connective tissues sets in, but later gives place to plastic infiltration, and finally to extensive formation of fresh connective tissue, which may even become ossified.

The disease, therefore, consists of a bursitis and parabursitis olecrani. The swelling is chiefly caused by the latter.

Symptoms. The clinical symptoms differ somewhat according to the pathological changes. The only constant feature is the swelling, which is more or less sharply differentiated from its surroundings. When recent, it feels abnormally warm, is doughy and slightly painful. It afterwards becomes harder, depending on the degree of plastic infiltration and induration of parabursal tissue. Occasionally distension of the bursa is the more prominent symptom, and the swelling is then fluctuating, and on being opened discharges blood-stained fluid. The bursa may become as large as a man's fist and contain fibrinous clots. 
If not opened, its contents may be reabsorbed, and whilst the parabursitis disappear's, the over-stretched skin forms a loose fold. Lameness is rare, and the condition can only be mistaken for inflammatory changes in the subcutis, which, however, are never so sharply defined as is the diseased bursa.

Causes. The disease is produced by bruising, often due to lying on the heels of the shoe, though sometimes to contact with the floor.

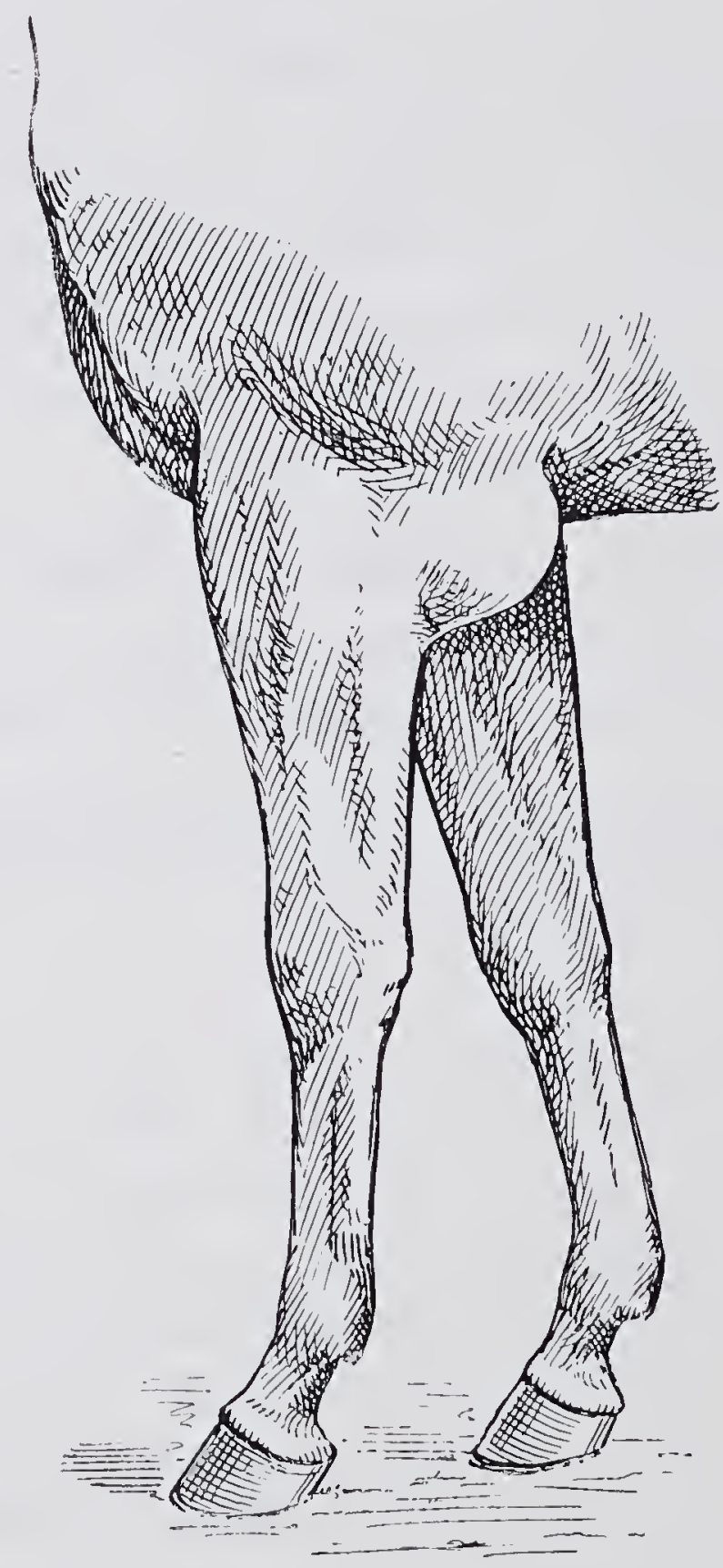

FIG. 196.-Capped elbow.

Weakness, too short a halter rein, too small a box, and in brokenwinded horses, difficulty in breathing, may all cause the animal to rest on the sternum, with the legs tucked under it; in animals with short fore arms the posterior surface of the elbow then comes just against the posterior part of the hoof, and is readily bruised by the heels of the shoe. Capped elbow, however, is not infrequently seen in horses shod in "tips," in which, therefore, the shoe cannot be blamed. The position assumed when resting, is of great importance in determining the condition. Metastatic disease of the bursa is sometimes seen during strangles.

Course. The condition is generally produced in a single night. By 
immediate treatment the swelling may sometimes disperse, but more frequently it becomes chronic, resorption being incomplete, and induration occurring in the parabursal tissue. When a large swelling disperses after existing for some time, a loose fold of skin remains, which detracts from the animal's appearance. Resorption, however, is not infrequently prevented by repeating bruising. The animal may continually lie in the position described, injuring the bursa and its neighbourhood, and chronic thickening may occur in the parabursal connective tissue, rendering resorption impossible.

When the skin is injured suppuration may occur in the bursa, and after some time be followed by fluctuation and perforation. Metastatic bursitis produced during strangles is always of this character. As the disease seldom causes lameness, prognosis in working-horses is always favourable as far as continued usefulness is concerned. The older the condition the less the chance of complete recovery, but firm swelling s may be removed with the knife.

Treatment. An excellent preventive consists in causing the horse to wear a stout cushion extending across the chest just behind the elbows. This cushion, if applied regularly every night, sometimes causes small capped elbows to disappear. Another mode of prevention is to apply a well-padded "boot," covering the heels, or to strap a thick straw pad extending above and below the linee to the back of the limb. This checks the animal when it attempts to flex" the knee, and prevents it taking up the undesirable position. Fresh swellings are treated by cold applications, and if further bruising be prevented, complete dispersal often results. Resorption is assisted by repeatedly applying irritants, which favour resolution. At the same time they render the skin sore, and thus prevent the animal lying on the diseased spot and renewing the injury. A 10 per cent. iodide of mercury ointment may be applied every two or three days.

The bursa should not be opened except when pus is present. Where the lining has been destroyed by suppuration, recovery may occur, otherwise granulations do not form readily, on account of the peculiar nature of the bursal surface. More frequently, serous fluid is actively secreted, prevents the granulations around the incision healing, and finally leads to sinus formation. It is therefore necessary, when the bursa is opened before suppuration has occurred, to destroy the membrane by irritants.

Stockfleth recommends swabbing out the sac daily with 1 part of caustic potash to 4 of water; the other parts of the leg must, of course, be protected against the irritant. The use of the actual cautery seems simpler. Stockfleth employs it to open the diseased bursa, thus preventing the emphysema which sometimes follows incision. The bursa should 
only be opened either when pus exists in it, or when its walls are very thin, and the swelling is principally bursal. Hardness of the swelling shows that it principally consists of indurated connective tissue, and treatment is then limited to destroying or excising the newly-formed material. At one time the first method was largely adopted, arsenic or other irritant, in the form of powder or of small particles, being passed through an incision as far as the centre of the swelling. Gangrene occurred in the bursa and its surroundings; after eight to fourteen days, the swelling separated from the skin and sloughed, and the wound was filled by granulations and healed. Though this method has the advantage of not stopping the horse's work, yet it may cause serious complications, and therefore operative removal, either by dissection or by ligation, has latterly received preference.

Ligation is a simple and easy method, and is almost always successful. When the tumour is sufficiently pedunculated to allow a ligature to be applied, it is the method most used by Continental operators. The elastic ligature is most useful. After cutting away the hair and cleansing: the skin, a rubber cord, about the thickness of a quill, is passed round the base of the swelling, and drawn as tight as possible. The ends are tied together with string. The ligature soon begins to cut in, and in four to ten days, according to the thickness of the tumour, it falls away. In large tumours it may be necessary to remove and reapply the ligature after two or three days. The surface of the wound should meanwhile be cleansed daily and washed with disinfectants such as sublimate, creolin, or carbolic solution. On account of its deodorising qualities, permanganate of potash is especially useful. Cases of tetanus are said to have occurred after ligation, but they are not common. As a preventive measure, however, careful disinfection is advisable, while at the same time it destroys the unpleasant smell of the mass of dead tissue. Möller prefers passing the ligature over the skin, which is not previously cut through; the skin is thus drawn together, and cicatrisation reduced to a minimum. Should the application of an elastic ligature prove difficult, on account of the tumour having no neck, a thin cord may first be applied, as it does not so readily slip off as the elastic ligature. In a few hours the tumour swells, when an elastic ligature can be put on with ease. In case of need, slipping may be prevented by passing a long needle completely through the swelling in front of the ligature.

In using the ligature, the remainder of the tumour must not be cut away with lnife or scissors, as it always contains large arterial vessels, and bleeding of an unpleasant if not positively dangerous character may result. When a cord is used, it will be necessary to tighten or renew it after a couple of days.

After-treatment is simple. As soon as the swelling has fallen off 
(which may be somewhat assisted by torsion slowly performed), the surface of the wound should be cleansed daily with disinfectants until a dry scab has formed, under which healing occurs in eight to fourteen days, without leaving any considerable cicatrix. Formation of a scab will be promoted by powdering the parts with iodoform combined with tannin.

Enucleation is not so simple as at the first glance it appears, but it is eminently "surgical," and Dollar prefers it in the majority of old-standing cases. In this operation the horse must generally be cast, and if restless, placed under chloroform, though it is possible to remove large growths without casting by previously injecting a few minims of 5 per cent. cocain solution at a number of points around the base of the tumour. If the horse has been cast, the foot is loosened and drawn forward by two cords, or fastened by a modified "side stick," and a ligature passed through the swelling to afford a better hold. In shaving the parts an oval-shaped "island " of hair may be left to indicate the area of skin subsequently to be removed; otherwise the skin may be displaced and the operator may make an asymmetrical incision (see p. 544). The tumour is next separated in the ordinary way. Care is required, however, to avoid injuring the fascia of the fore arm and the muscular tissue, which might easily produce cellular inflammation, nor should the point of the elbow be exposed. After removing the swelling, the wound is treated on general principles. As asepsis is not always possible, large vessels should be tied, and a firm tampon of carbolised jute sewn up in the wound to check bleeding. The tampon is removed next day, and openwound treatment proceeded with. But, provided care is taken, relative asepsis can often be attained and much better results ensured. In this case Dollar carefully cleanses the interior of the wound, rinses it with 1 in 1,000 biniodide of mercury solution, and powders it with iodoform, leaving the flaps open for six to twelve hours. When all bleeding has ceased and the surfaces are covered with a serous discharge, he unites the flaps with two series of sterilised silk sutures; one set being used to bring together the edges of the skin, and the second set, consisting of two or three sutures only, being passed further from the edges and used to obliterate any "dead space." If thought necessary, a drainage-tube is inserted. The horse is rested, and liept standing for eight days, or may be placed in slings.

A similar disease occurs in large, heavy dogs, which, when lying on hard ground, support themselves on the sternum and elbows. The skin alone may be injured and thickener, or callosities form at the affected spot, but occasionally the bursa olecrani becomes diseased. The disease differs from that in horses, inflammation being almost always confined to the bursa, and parabursitis occurring very rarely, consequently the 
swelling is soft and fluctuating. Symptoms of inflammation (warmth and pain) are in rare cases present; but, as a rule, there is only a fluctuating swelling of an oval or round form, varying from the size of a hazel-nut to that of a hen's egg.

The swelling is produced by distension of the bursa olecrani with serum, which sometimes contains fibrin (bursitis sero-fibrinosa); blood is rarely met with. It never interferes with movement, but is a blemish which is difficult to remove, the cause being always at work. Animals accustomed to lie in the position described cannot be broken of the habit, especially as the swelling is painless. Simple puncture and removal of contents are therefore not sufficient, and, owing to the flatness of the swelling, a ligature cannot be applied. If necessary, the enlarged bursa must be surgically removed, though operation is difficult, and, as a rule, cicatricial thickening remains. Before operating, it is best to give a dose of morphine. The hair is removed and an incision made over the swelling in the long axis of the limb. The bursa is then separated from neighbouring parts, care being taken not to incise it, which would greatly increase the difficulty of the operation. The greatest precaution is required in separating the bursa from the elbow, with which it is firmly united, and the bone must not be exposed, as this would delay healing, and prevent the formation of a cicatrix. When excision has been successful, it is sometimes possible, by observing antisepsis, to produce healing by first intention. The operation wound is carefully dressed, and thick layers of wadding or wood-wool applied to the posterior surface of the elbow to prevent further bruising when the animal lies down. When healing by first intention is impossible, the wound must be treated on general principles. A soft bed is particularly requisite. Remnants of the bursa, which remain after enucleation, may be destroyed by irritants like nitrate of silver, and a proper granulating surface so produced.

\section{VI.-WOUNDS AND BRUISES OF THE FORE ARM.}

Anatomy. In the horse, the fore arm (antibrachium) has, as a basis, the radius and ulna, which are surrounded by numerous muscles, tendons, and fasciæ. The following surface-markings should be noted:-Towards the outside lower end of the fore arm a long depression, slightly inclined backwards, and marked at its centre by a prominent cord, the tendon of the extensor suffraginis. In front of this runs another cord about one inch in breadth, the tendon of the extensor pedis. Towards the front of the limb, and at the boundary of the external and anterior faces, another depression, and still further forward and inward, the tendon of the extensor metacarpi magnus, about $1 \frac{1}{2}$ inches in breadth.

Behind the first-named depression lies the flexor metacarpi externus, which is bounded behind by the flexor metacarpi medius and the flexor metacarpi internus. 
The inner surface of the fore arm displays another depression, at the bottom of which can be felt the radius, here only covered by skin. Behind it lies the flexor metacarpi internus, likewise readily felt below the skin; in front of this furrow, the bone is margined by the extensor metacarpi magnus. Between the two depressions, and quite clearly visible, runs the internal subcutaneous or radial vein.

Two fascia cover the fore arm, $(a)$ a thin superficial and $(b)$ a deeper, better developed layer, the fascia antibrachialis, which arises from the various muscles of the humerus and shoulder, is attached partly to the subcutaneous portions of the radius, partly becomes continuous with the tendons of the extensors and flexors, and partly extends to the ligaments of the knee. Between the skin and fasciæ pass the thin cutaneous nerves; those on the posterior surface of the fore arm arising from the ulnar nerve, those on the exterior from the radial nerve, and those on the anterior partly from the median. The cutaneous vessels, viz., the internal and anterior subcutaneous veins, correspond to these.

Vertical wounds confined to the skin of the fore arm are of no particular consequence, but horizontal wounds are more apt to cause trouble, on account of their gaping. If carefully disinfected, primary union may result from applying a proper dressing, which, however, is more difficult in large than in small animals.

Injury of the muscles and fascix of the fore arm is frequently followed by burrowing of pus and other dangers, for which reason such wounds must be dressed antiseptically or freely laid open. Sometimes setons or drains are useful, especially in penetrating infected wounds accompanied by much swelling and pain. Fever is a particularly dangerous symptom.

The edges of horizontal muscular wounds are apt to gape excessively. The animals generally walk lame, though they may be able to stand on the limb, especially when the chief seat of injury is in the extensor muscles. Gaping of the wound and contraction in the divided muscles alike impede union; and as, in large wounds, the skin soon retracts, it is best, even when asepsis seems out of the question, to suture such wounds as soon as possible. Sterilised tape about $\frac{1}{8}$ to $\frac{1}{4}$ of an inch broad forms a good suture material, and an attempt should be made to fix the skin and prevent it retracting. By passing the sutures through the bodies of the muscles a better hold is ensured, and there is less chance of their tearing out, whilst the tapes may be used for fastening on a dressing. Any tendency to excessive granulation must be repressed early by using astringents and dry dressings.

Bruises of the muscular tissue and fascix of the fore arm are commonest in cart-horses, in consequence of kicks or of blows inflicted with the pole. They are easily recognised by the pain, swelling, and difficulty in that phase of movement when the limb is carried. Resolution generally occurs and lameness disappears in eight to fourteen days. In other cases abscesses form, and should immediately be incised to allow free exit of pus, which may otherwise burrow and cause necrosis of the 
fasciæe of the fore arm; extensive counter-openings are therefore at times necessary. The radius is sometimes injured just above the knee. In this case also it is important to provide for drainage, either by counterincision, drainage-tubes, or setons. Slight swelling with great pain points to disease of the bone (periostitis or necrosis), a complication which may occur at any time before pus formation has completely ceased.

In horses, the inner surface of the elbow-joint may be injured by straddling across bales, poles, or similar objects. The pain is often so acute that the animals are unable to place weight on the limb. In these cases the median nerve is bruised; and although the effect of the injury may only last a few minutes, it may also continue for a couple of days. The pathognomonic symptom is insensibility below the elbowjoint, especially at the posterior surface of the limb. The affection soon disappears.

Bassi in a mule saw movements of the left fore foot simulating stringhalt. The front foot was lifted unusually high, and the animal often stumbled; the symptoms were particularly noticeable when the animal moved backwards. On the external surface of the fore arm, just below the elbow, was a thickening, the result of a fall ; it was not inflamed, and had existed for some weeks. The difficulty in movement had first appeared twelve to fifteen days after the fall. Permanent recovery followed division of the flexor metacarpi externus.

\section{DISEASES OF THE KNEE.}

Anatomy. The knee, a complicated joint, consists of three portions, -

(1) The radio-carpal articulation.

(2) The intercarpal articulation.

(3) The carpo-metacarpal articulation.

The first two are incomplete ginglymoid joints, because, in addition to flexion and extension, they allow of slight lateral movement, and, in the flexed position, of some amount of rotation, most marked in carnivora. The lower row of bones forms with the metacarpus an arthrodial joint, the movement of which, in all animals except carnivora, is exceedingly limited.

Each of these three joints is surrounded by its own synovial membrane, though the lower is connected with the middle by a narrow slit. The common capsular ligament arises from the radius, is applied to both rows, and ends on the metacarpus. Two strong lateral ligaments attach the radius to the metacarpus, while the bones of the carpus itself are united to one another and to the radius and metacarpus by interosseous ligaments which limit the movement of the knee-joint.

The fascia covering the carpus and metacarpus, which is an extension of the deep portion of the fascia of the fore arm, forms, on the anterior surface of the knee-joint, sheaths for the extensor tendons, and is in close connection with the capsule of the joint. On the outer surface of the carpus the fascia becomes continuous with the ligaments of the joint, with the periosteum of the external surface of the pisiform bone, with the tendon of the flexor metacarpi externus, and with the extensor pedis. On the inner side of the carpus 
the fascia becomes attached to the ligaments of the joint, to the lower extremity of the radius, and to the inner small metacarpal, and covers the flexor pedis tendons, with which it becomes continuous.

The skin lies immediately over the fascia, and, on the dorsal side especially, is thick, tense, and provided with a moderately well-developed subcutis. Subcutaneous bursæ are sometimes found on the os magnum, as well as on the lower anterior surface of the radius towards the inner side of the sheath of the extensor pedis. The discharge of synovia after injury to the front of the knee is therefore no proof that the knee-joint has been opened, although it always calls for special precautions in treatment.

\section{I.-FRACTURES OF THE BONES OF THE KNEE.}

Fracture of these bones is in general prevented by their small size and sheltered position. In large animals like horses, fractures are commonest from falls on hard ground. They usually occur in conjunction with wounds and injuries of the joint, i.e., they are compound, and the fragments seldom unite; but when this does occur movement of the joint is more or less limited, or altogether prevented by formation of synostoses.

On account of its isolated position the pisiform bone is an exception to the above rule, and Möller has repeatedly seen fracture of it in racehorses. A case occurred in a draught-horse during heavy work, the immediate cause being excessive contraction of the flexors of the knee. One or other ligament of the pisiform bone may also be ruptured.

The symptoms are, inability to bear weight on the limb which is flexed, as in complete radial paralysis, and local pain. Abnormal mobility of the pisiform bone and crepitation under pressure reveal the character of the fracture beyond doubt. On the other hand, fractures of the other carpal bones produce very ill-defined symptoms; though in most cases the sudden appearance of severe lameness ("supporting leg lameness") and local pain about the knee-joint sufficiently indicates the seat if not the exact nature of the lesion.

Course. Union of fractures of the pisiform bone is difficult. The fragments of bone are drawn upwards by the pull of the attached muscles, and, as in fractures of the ulna, the conditions are unfavourable to callus formation. A fibrous callus forms, generally of insufficient strength, and if put to work too soon the animal relapses. In other cases the knee shows marked dorsal flexion. Even favourable cases last for two to three months; but many horses have to be destroyed in consequence of laminitis and descent of the os pedis, and of the resulting acute pain, fever, \&c. Compound fractures of the knee bones often end in septicæmia and death. Should recovery occur, there is usually extensive callus formation and loss of function in the joint, so that the animals are of little use except for stud purposes.

Treatment. The animal should be placed in slings and reposition 
attempted, though it is seldom successful. In quiet animals a dressing may be applied, but it is useless in fidgety patients, which can only be given a roomy stall or box, with plenty of bedding, and allowed to lie down, thus preventing laminitis of the other foot. Complete rest is always the first indication in treating fractures of the carpal bones. Compound fractures call for the strictest antiseptic precautions; though as a rule the animal has eventually to be slaughtered.

\section{II.-INJURIES TO THE ANTERIOR SURFACE OF THE KNEE.}

In consequence of the anatomical and physiological peculiarities of the knee, its anterior surface is much exposed to injury by bruising, especially in horses and ruminants. In horses, injury results from falling on hard, uneven ground ; in cattle, from the continual pressure of the body-weight when lying on hard surfaces, and from the manner in which the animal lies down and rises. Such peculiarities explain the varying character of the injury in different species of animals.

\section{(1.) INJURIES TO THE KNEE IN THE HORSE.}

When horses fall during movement, they almost always strike the knee, and sometimes produce bruises, i.e., injuries not involving a skin wound, but consisting in rupture of blood-vessels in the subcutis, easily recognised by their fluctuating character. Lacerated wounds are more common, and vary in gravity according to their extent. Defective formation, senile degeneration of muscular tissue, \&c., render animals uncertain on their fore limbs, and, therefore, inclined to fall; in such case both knees are often injured. The commonest injury is superficial bruising of the skin. When animals are going fast and only fall for a moment, excoriations and loss of hair may occur, but are of little consequence unless the skin is perforated. Serious abrasions, however, require rest and careful treatment, in spite of which thickening and hairless spots often result. Repeated bruising often produces hygroma of the knee, or "capped knee" (see fig. 197), an indolent, diffuse, uniformly fluctuating swelling, extending over the whole anterior surface of the joint. In time the walls of the swelling become hard and thickened. The contained fluid often shows fibrinous clots or myriads of rice-like bodies. Occasionally the swelling becomes inflamed and the contents purulent.

Swelling of the soft tissues in front of the knee at once interferes with movement. If the linee is then forcibly flexed, as for example by the animal falling, the cutis and subcutis, infiltrated with inflammatory products, may easily be ruptured and a horizontal wound produced, which heals very badly. 
Injury or exposure of the fascia is also grave. The wound is then much deeper, and contains shreds of the injured fascia. In such cases healing is slow, though finally arrived at by proper treatment. Provided the sheaths of the extensor tendons are not laid open, and swelling and pain remain slight, recovery usually occurs, but exposure of the bursæ constitutes a grave complication. Swelling becomes severe, and extends both upwards and downwards; jelly-like synovia discharges from the wound, the limb is very painful when flexed, though weight may be placed on it, and in walking it may only be carried stiffly.

Falls on sharp stones, or on rough, hard ground, may injure the extensor tendons, or even one of the three main divisions of the joint. Although the knee-joint is included in the category of "indolent" joints, i.e., such as only slightly resent mechanical injury, yet severe symptoms may follow such accidents. Pain is sometimes so excessive that weight can only be borne on the limb intermittently, sometimes not at all. The swelling extends over the whole joint, and often far beyond it, and is accompanied by fever. The finger or a probe can often be introduced into one or other of the joints, and in the later stages it may be possible to feel the articular surfaces, which are rough and denuded of cartilage. Sometimes crepitation can be detected when the joint is bent.

Course. Provided the tendon sheaths are not affected, bruises disappear in from two to three weeks, though they often leave local thickening. Otherwise, and especially if the

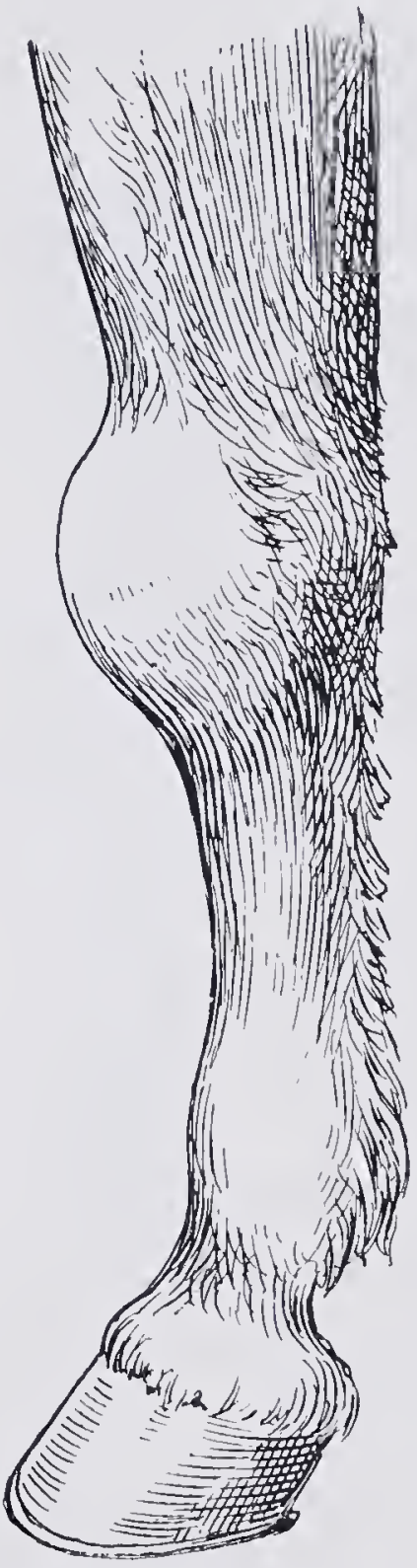

Fig. 197.-Hygroma of the knee resulting from bruising. parts cannot be kept aseptic, recovery may extend over four to six weeks. Injuries to the joint may heal (with formation of exostoses), but generally produce so much disturbance and fever, and are followed by such obstinate lameness, that the animal is either killed or dies of septicæmia.

The prognosis therefore depends chiefly on the extent of the injury. If the parts can be kept aseptic, which is seldom the case, there is, of course, a much greater chance of recovery, and blemishing is minimised.

Treatment. Provided the injury is confined to the skin, it is sufficient to cleanse the abraded parts and smear them with some neutral fat like vaseline or zinc ointment. "Capped knee," when recent and of small 
dimensions, often yields to repeated friction with mild stimulants like turpentine and ammonia liniment or camphorated alcohol, but when large and of old standing is best treated by laying open the swelling at its lowest point, clearing out fibrinous clots, \&c., inserting a drain, and injecting twice daily a 5 per cent. carbolic solution or diluted tincture of iodine. Gentle compression with a rubber bandage assists recovery. Needle firing has also proved successful (see Dollar's "Operative Technique," p. 119). Failing success by these methods, excision may be attempted, provided the animal's value justify operation (see succeeding pages).

Wounds perforating the skin require complete rest, to prevent formation of large cicatrices, and in addition general antiseptic treatment. Very careful asepsis is required where the fascix are injured or exposed. A circular bandage provided with a dressing should be passed around the knee, being left rather looser above and below, and the animal placed in slings or tied up short to prevent its lying down. Sutures should be used where practicable. Wounds which cannot be rendered aseptic should be left open. Continuous irrigation with lukewarm dilute sublimate $\frac{1}{2}$ to 1 per cent. zinc chloride or 1 to 2 per cent. Goulard's extract lotion is useful during the first few days, especially when there is much discharge. At a later stage dry dressings like iodoform and tannin, glutol, amyloform, boric acid, \&c., may be used to form a dry scab, under which healing proceeds. Excessive proliferation may be checked by the occasional use of 5 per cent. chloride of zinc solution.

The treatment of open joint calls for all the resources of antisepsis. Provided purulent or septic disease of the joint has not yet set in, the wound must be carefully washed with sublimate or creolin solution, and an antiseptic dressing applied; but when such changes have occurred, the only chance consists in carefully cleansing the parts and providing for permanent irrigation. This may at least be tried, and any special complications treated on general principles. The chief points are to immobilise the joint and observe the most careful antisepsis. In animals: of little value, and in cases where there is great pain, further treatment is scarcely advisable.

Where a valuable horse has, by falling, produced a permanent cicatrix, denuded of hair, it may be worth performing the operation proposed by Cherry, revived by Hunting, and perfected by Vinsot. The object of the operation is to permanently remove the scar left by accident, substituting at first a mere linear cicatrix, which, once covered by hair, is almost imperceptible, even to the trained senses of an expert.

To render operation successful the strictest asepsis or, failing this, antisepsis is necessary, and for a considerable time the personal attention of the operator is required. 
The operating table described on p. 22 of Dollar's "Operative T'echnique" is almost indispensable for operation. Under any circumstances, it facilitates the antiseptic precautions, and avoids the need for a straw bed, a fruitful source of wound infection.

The instruments and materials required are-clipping machine, razor, scalpels, artery forceps, lion forceps, suture needles, "silkworm-gut" ligature, carbolic gauze sponges, indoform gauze, pieces of freshly-boiled linen, absorbent wadding, tarlatan bandages, cotton bandages, plaster bandages, nail-brush, soap, boiled water and several vessels for holding it, $2 \frac{1}{2}$ per cent. carbolic solution, 1 per cent. permanganate solution,

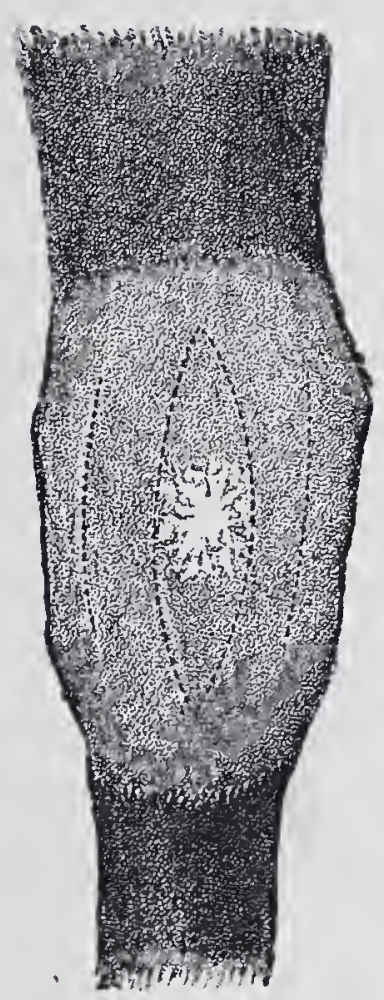

Fig. 198.

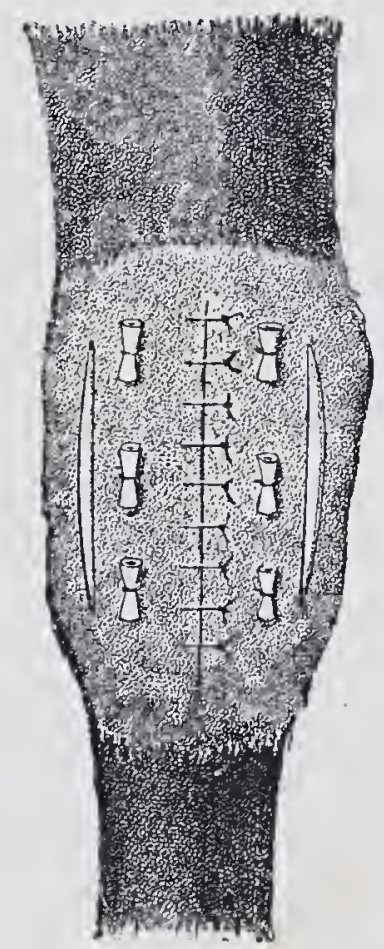

FIG. 199

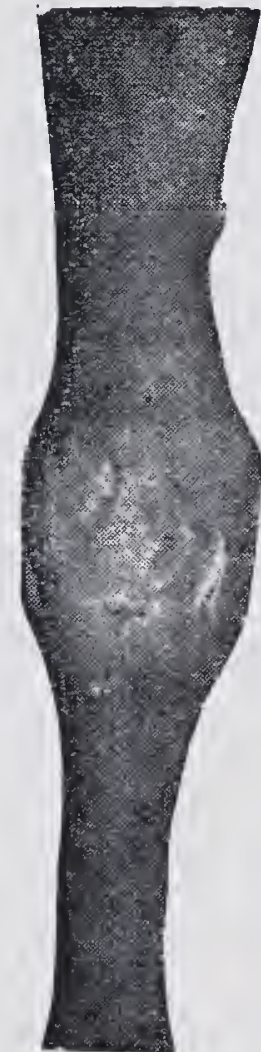

FIG. 200.

$12 \frac{1}{2}$ per cent. bisulphite of soda solution, sulphuric ether. All dressing materials must previously have been sterilised.

All the instruments should be of metal and have metal handles. They should be boiled for at least twenty minutes, and be placed in sterilised, shallow, enamelled iron trays containing a little boiled carbolic solution.

The operation is divided into four stages:

1. Control of the animal and aseptic precautions.

2. Excision of the scar.

3. Suturing the operative wound.

4. Adjustment of the antiseptic dressing and splint.

1. A dressing saturated with 5 per cent. carbolic solution is applied to the seat of operation overnight. Next morning the animal is placed on the operating table and chloroformed. The limb intended for operation is freed, drawn forwards as far as possible, and fixed to the lower bar of the table by means of broad strips of webbing. To ensure the limb being fully extended another piece of webbing is passed round 
the fore arm and drawn forcibly backwards; the knee is thus fully extended. An Esmarch bandage is applied above the knee, to control bleeding at a later stage.

The limb is clipped, the seat of operation and a liberal space on eithẹr side (about twelve inches above and below the scar) are shaved, a lozenge-shaped area corresponding to the part to be excised being left, however, so as to guide the operator at a later stage. The parts are then rinsed carefully with permanganate of potash solution, followed by bisulphite of soda solution, which removes the discoloration caused by the permanganate. (A variation of this method may be made by substituting for the permanganate and bisulphite solutions, boiled water, tinged a deep sherry colour by the addition of iodine tincture.) Finally the parts are washed with ether, and immediately covered with pieces of linen removed from boiling water.

In the meantime the operator and his assistant have thoroughly cleansed their hands, arms, and finger-nails in a similar way, and with similar solutions. The operation, properly so called, then begins.

2. The importance of the unshaven area now becomes apparent. Being symmetrical, the edges of the wound traced round it will afterwards exactly coincide, forming a straight line. It is advantageous to trace this area in a line inclined obliquely to the long axis of the limb. The final linear cicatrix is then extremely difficult to discover. In this way it is possible to remove scars as large as a five-shilling piece, leaving a very trifling cicatrix. Scars of a larger size may be greatly diminished, though not entirely removed.

The operator makes the first incision without stoppage, as it is extremely important to produce clean-cut edges; the scalpel should penetrate the entire thickness of the scar, stopping just short of the synovial sacs. The second incision is made in a similar manner. He then carefully dissects away the flap from the underlying tissues, removing at the same time as much of the thickened (cicatricial) material as possible, but taking great care not to open the synovial sacs. This operation finished, the remaining fibrous tissue must be removed, so as leave the base of the wound perfectly level, and any trifling hæmorrhage checked by picking up and ligaturing bleeding vessels. If the piece of tissue thus removed is so broad as to render it difficult to bring together the edges of the wound, the skin may be dissected from subjacent tissues over the entire front of the knee and two lateral skin incisions made (figs. 198 and 199). It will then glide easily, and offer much less resistance to the coaptation of the wound edges.

3. When bleeding has ceased the surface of the wound is carefully dried with pledgets of aseptic or carbolised gauze, care being taken to avoid the formation of blood-clots, which check healing. In a few 
minutes the wound is dry, and the edges can be brought together with interrupted sutures of silkworm gut, each inserted about $\frac{1}{4}$ inch from the line of incision, and about $\frac{3}{8}$ inch from its neighbour. It is better to commence at, say, the upper extremity of the wound, and proceed downwards, rather than to commence at the centre or at an intermediate point. The surgical knot should be employed. Silkworm gut is the best material to employ; it is solid, aseptic, non-porous, does not irritate the tissues, and is readily removed at a later stage.

4. The wound being perfectly closed is powdered with iodoform, covered with iodoform gauze, and with a large pad of surgical cotton wool retained in position by tarlatan bandages, previously boiled. The slightly moist tarlatan readily adapts itself to the form of the limb, and is preferable to any other material. Bandaging should commence low down on the cannon bone, and be gradually continued upwards over the knee.

It being absolutely essential to prevent all movement in the limb after operation, some form of splint is necessary. Plaster bandages have been tried with good effect, but a much simpler and more effective apparatus consists of a "legging" of very stout leather, reinforced by ribs of spring steel, and capable of being tightened by straps. This serves all the purposes of a plaster bandage, is slightily flexible, will not crack, and is less likely to injure the skin. Moreover it permits of the occasional inspection of the dressing, should this become necessary. The legging extends from just above the fetlock to 8 inches, more or less, above the knee.

All going well, the "legging" and dressings are removed about the twelfth day after operation, when, if all details of the operation have been thoroughly carried out, the operative wound is found cicatrised without the most trifling formation of pus. The threads may then be cut with scissors and removed with aseptic forceps. A light bandage of iodofor'm gauze, retained in place by tarlatan bandages, is applied for another week to permit healing of the orifices left by the sutures, after which time the animal may be exercised in hand or put to light work. Slight swelling over the seat of operation occasionally persists for a week or two, but soon disappears, the hair grows again, and, instead of a large conspicuous blemish, an almost imperceptible linear cicatrix remains. This is soon covered by the growth of hair, and nothing can be distinguished except by the closest examination.

Painful swelling of the limb, or discharge or smell from the dressing during the progress of treatment, points to suppuration. In such case the dressing must be removed, the parts irrigated thoroughly with some antiseptic solution, and the dressing renewed. Where local suppuration V.S. 
occurs around one or two sutures, it is sometimes sufficient to touch these lightly with nitrate of silver.

\section{(2.) TUMOURS OF THE KNEE IN CATTLE.}

When lying down and again when rising cattle are apt to bruise the knee, and to produce chronic inflammation of its anterior surface, which often leads to great swelling and thickening of tissue. The same result occasionally follows falls on rough, hard ground, in which case inflammation is acute.

Tumour on the knee cannot be regarded as a definite diseased con-

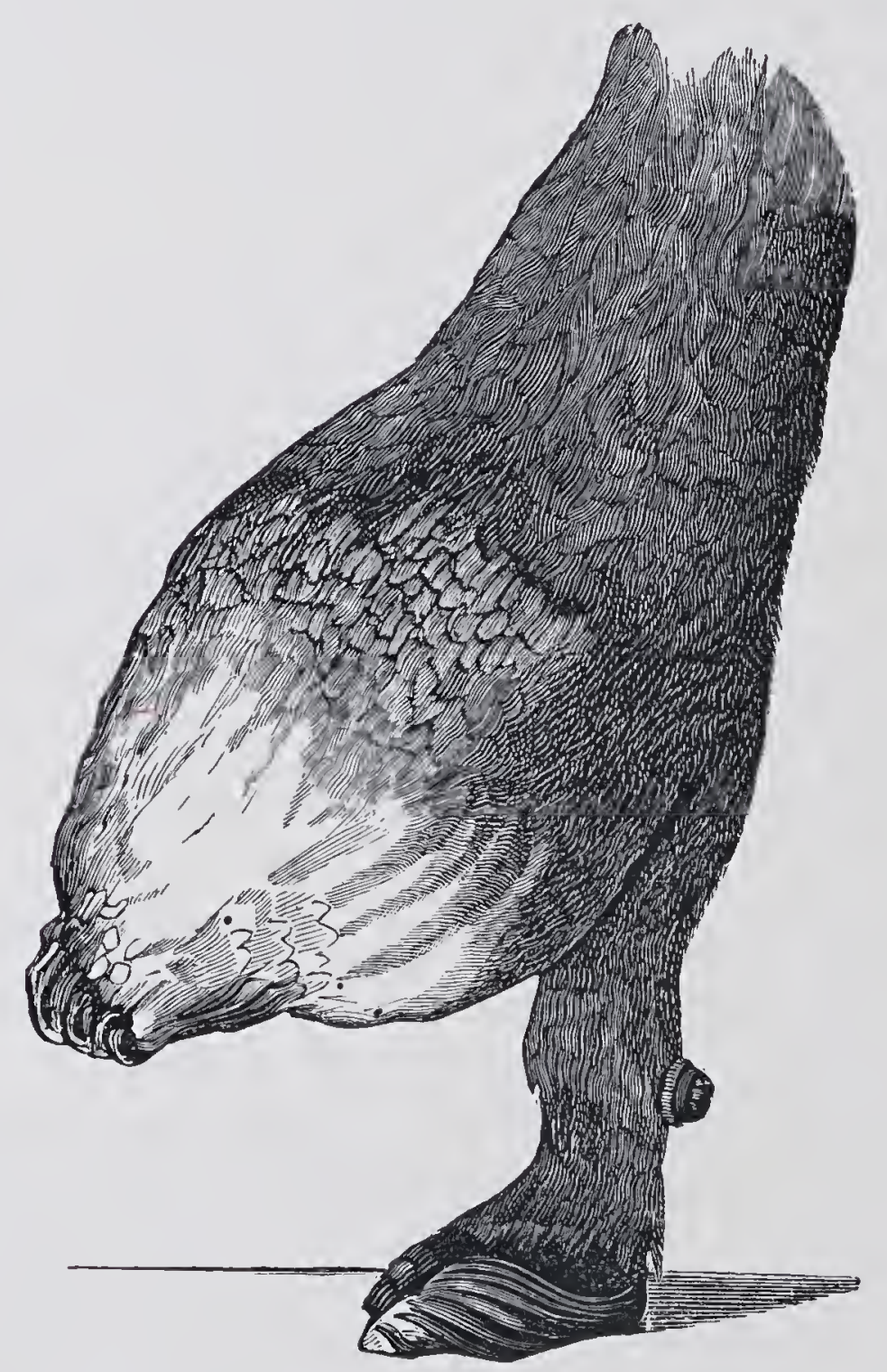

Frg. 201.-Knee tumour (cutaneous form), after Stockfleth.

dition like " capped elbow," but results either from chronic inflammation and thickening of the skin or subcutis, or from inflammation in the sheaths of the extensor tendons. In exceptional cases, the swelling consists of a "tumor albus," due to chronic inflammation of the connective tissue lying around the carpal joint, producing fibrous thickening. These swerlings were formerly divided into hard and soft forms. A better classification is :-(a) Cutaneous, (b) synovial, and (c) articular or periarticular. This classification, though it cannot always be observed clinically, facilitates the study of the condition. 
The cutaneous form consists either in excessive thickening of the skin and subcutis covering the front of the knee, not infrequently associated with active increase in the epidermis covering it (fig. 201), by which a hard swelling, often as large as a man's head, results, or in the development in the subcutis of cavities filled with serous fluid, which often contains lymph flocculi. Hence the condition is sometimes regarded as "capped knee" and is compared with the retention cysts. The descriptions given indicate that in many cases cysts form in the cutis and subcutis at the same time that the epidermis undergoes active proliferation. Johne describes this condition as "dermoid cyst, with diffuse keratosis.",

The synovial form consists in a tendovaginitis chronica serosa or fibrosa, which sometimes affects the tendon sheaths of the extensor pedis, but more frequently the sheath of the extensor metacarpi magnus. The swelling is sometimes chiefly formed by the sheaths of the tendons distended with serum (tendovaginitis chronica serosa), sometimes by the greatly thickened connective tissue of the sheath and its surroundings (tendovaginitis chronica fibrosa). This disease of the tendon sheaths has often been found due to tuberculosis.

A soft and hard form can be distinguished. The tendon sheaths are often greatly distended; in one Gurlt found 17 lbs. of fluid. Small papillæ form on the inner surface of the sheaths, and when rubbed off appear like grains of rice; this condition is termed hygroma proliferum. Pus formation may also occur.

The articular form presents a true tumor albus, that is, progressive increase and induration in the peri- and para- articular connective tissue, and is alvays associated with exostosis formation on the bones of the knee, fore arm, and metacarpus. The swellings are of variable hardness, and result from chronic inflammation of the knee-joint. Ossification in the new connective tissue increases the hardness of the swelling.

Causes. The chief causes are injuries sustained in lying down and rising in badly-paved stalls and on insufficient bedding. The repeated bruising may cause extravasation of blood in or under the skin, or into the sheaths of the tendons, and proliferation of connective tissue. That bleeding often occurs is shown both by the presence of clots in the serous fluid, and by the pigmented condition of the newly-formed connective tissue. A single severe bruise from falling on uneven, hard ground, as occurs in working-oxen, may produce the disease. Chronic processes, like tuberculosis, invading the carpal joint, may also induce such tumours, but the nature of the cases hitherto reported is seldom fully explained.

Symptoms and course. The chief symptom consists of swelling of the front of the knee, which sometimes increases rapidly, sometimes 
slowly, but is continued. The tumoui may become immense (as much as a yard in circumference), and even reach to the ground. The degree of pain varies, sometimes being considerable, but chiefly depending on the mode of origin of the disease. When caused by a single violent bruise it is generally painful: but the slower the development the less the pain. The swelling, though at first soft and fluctuating, at a later stage becomes firm, and is often covered with thick masses of horn. In cutaneous "capped linee" the tumour remains movable on the subjacent tissues for a long time; in the synovial form it takes an elongated shape corresponding to the direction of the sheaths of the tendons, and extends over the metacarpus from the region of the extensor pedis tendon. The articular form is more diffuse; it often covers the sides of the knee, and is firmly attached to the underlying parts. Ossification is denoted by unusual hardness, and by greater difficulty in moving the joint than in the two previous forms. Lameness is usually slight; only when the disease is acute and causes great pain, or when the swelling becomes excessive, is movement much interfered with.

The treatment of "capped linee" is as varied as its causes, and must depend on the nature of the injury and the anatomical changes. Fresh bruises and acute inflammation, accompanied by great pain, are best combated by cold applications, followed later by warmth and moisture. In skin injuries search must be made for foreign bodies like splinters of glass, fragments of gravel, \&c., and the dressings should be saturated with disinfecting fluid; in any case plenty of clean straw must be given, and it is well to cover the bottom of the stall with sawdust or sand instead of leaving the stones exposed.

Where superficial fluctuation can be detected, cavities should be opened at the lowest point. The animal is tied up securely, and an assistant directed to grasp it by the nose. The swelling is then opened with a bistoury or pointed tenotome. As the leg is generally lifted at this moment, the cutting edge of the linife must be directed upwards and outwards to prevent deep-seated structures being injured. 'The cavity is freed from clots with the fingers, the animal placed on soft, clean straw, and the wound cleansed daily. Recovery generally occurs in three to four weelis. Hertwig recommends passing a seton through the swelling, leaving it in position for fourteen days, and following up this treatment by blistering.

Although similar treatment may be employed in the synovial form, it is better, if the parts are already aseptic, to puncture with the trochar, using antiseptic precautions, and afterwards inject a solution of iodine in iodide of potassium. Should this fail, or should purulent inflammation of the sheath of the tendon set in, it may be necessary to lay the sheath open and thoroughly cleanse it. Having regard, however, to the fact that this 
form of the disease is frequently of a tuberculous nature, treatment is seldom of much value, and animals should be prepared for the butcher.

As a rule, little can be done for the hard form of "capped knee," unless the swelling is marginated, of slight size, and has not too broad a basis, in which case it may sometimes be extripated, but it is difficult to keep the parts aseptic. Perhaps, by repeatedly applying a blister, the parts might be rendered so painful that the animal would be obliged, in lying down and rising, to spare the diseased leg, and absorption might then occur, as it sometimes does in "capped elbow." The introduction of euphorbium or caustics like sublimate and acids into the swelling, is liable to injure the articulation.

\section{III.-CHRONIC INFLAMMATION OF THE KNEE-JOINT.}

Chronic inflammation of the knee, arthritis chronica, occurs oftenest in horses, and has long been recognised. Cherry described it under the title of "knee spavin." Schrader found the articular surface more or" less "degenerated," the cartilage worn away, and exostoses on the bones. He also described ulceration of the cartilage of the joint, caries of and exostoses on the bones, and anchylosis. According to Cherry, the os lunare and head of the large metacarpal may alone suffer, but the inflammation generally extends further.

Causes. Horses with narrow, imperfectly-formed knees, short fore arms and upright shoulders, appear most predisposed to the condition, possibly on account of the peculiarly exaggerated movement in the knee-joint which such formation favours. Russian trotters display this form and action most conspicuously, and also very frequently suffer from chronic carpitis. Not infrequently the disease is bilateral; it is certainly more common in coarse than in well-bred animals, and is favoured by putting young horses to work too soon. Inflammation may extend from the periosteum to the joint, particularly in horses with defective formation, which are thus liable to "brush" in going, as is often the case in Russian trotters.

Symptoms. Swelling is the chief symptom, and is sometimes so great that the entire joint may appear deformed. The swelling is usually hard and firm, consisting principally of exostoses and new connective tissue, but when the sheaths of the tendons are involved, and become distended, it is soft and fluctuating. Free movement of the joint is often interfered with. When resting, the animals lean forward, and if forced to move go stiffly. Bending the knee either produces pain or is attended with difficulty; but in applying this test to the two limbs care should be taken to have the elbow-joints in the same position.

Lameness may or may not be present. It occurs oftener than is 
believed, and is shown by the limb being advanced more slowly and the stride shortened, whilst the knee-joint is imperfectly flexed and the limb abducted when weight is placed on it. If both limbs be affected, or if the process be confined to the lower portion of the joint, which in the horse is only slightly movable, there may be no lameness. According to Cherry, such a horse gives the rider the feeling that the thorax is pressed out of position and rises with every stride. The condition is most liable to be mistaken for navicular disease. The limb is extended, and turned slightly outwards. The quarters of the foot often reach the ground first, and on account of the peculiar gait the condition was in England termed "chest founder." The pace naturally assumed is a short, unpleasant gallop, which shakes the rider. The forward stride of the diseased limb is shortened, giving a certain resemblance to shoulder lameness. The difference consists in-

(a) The circular sweep of the limb when moved forward.

(b) In the horse bringing the quarter of the hoof to the ground first.

(c) In the production of pain by forcibly flexing the joint.

Course. The nature of the disease explains why it is so often chronic or incurable. The most favourable cases last from four to six months, and it is therefore of great importance to know whether or not the horse will afterwards be useful. The smaller the exostoses, and the lower they are situated, the more favourable the prognosis. The disease is, of course, not so serious in heavy working-horses as in hacks.

Treatment. Treatment is principally confined to blistering or the use of the actual cautery, and though usually ineffective, sometimes decreases the lameness in two or three months. Where the growths arise from the periosteum of the metacarpus the pointed cautery is of use. Division of the median nerve sometimes removes the lameness; Möller so far cured several cases by neurectomy that the animals became perfectly useful even for carriage work.

\section{IV.-INFLAMIMATION OF THE BURSA OF THE FLEXOR PEDIS.}

The large bursa of the flexor pedis perforans and perforatus, lying at the back of the knee, may become either acutely or chronically inflamed. Acute is commoner than chronic inflammation, and is caused by bruises or wounds. The sheath of the tendon may be opened by the horse putting its foot into the manger, or by a stab with a fork or other sharp instrument. Sometimes cellular inflammation extends from the metacarpus to this point; the septic inflammation following tenotomy is most dangerous. The chronic form generally starts from the check ligament of the flexor pedis, and often produces distension of the bursa, extending 
6 or 8 inches below and as far above the knee-joint (fig. 202). As a rule, the swelling appears on the outer side of the joint, both above and below, but in the latter situation may involve the inside; in the former it is often as large as a child's head, in the metacarpal region usually smaller. Palpation reveals the connection between the different parts.

Whilst, then, the chronic form appears as a fluctuating swelling in the region covered by the sheaths of the tendons, and is neither accompanied by lameness nor pain, the acute variety shows the following symptoms:-

(1) Marked lameness; the limb is held stiffly, and is incompletely extended.

(2) Increased warmth; pain on pressure; more or less firm, hard swelling, which surrounds the entire joint, and extends for some distance beyond it. In purulent inflammation, periarticular abscesses may form.

(3) Discharge from the wound of large quantities of synovia, afterwards mixed with pus (then appearing of a straw-yellow colour), sometimes with necrotic fibres of the flexor tendon.

(4) Usually fever; in septic disease, the temperature may be very high.

Course. The acute form generally takes an unfavourable course, and when caused by extension of septic cellulitis or by wounds, is always dangerous; little can then be done, and slaughter is advisable. The appearance of fever is, therefore, always threatening. Simple inflammation is less serious, and recovery commonly occurs, though some swelling may be left. Though more difficult to treat, the chronic form, associated with formation of large swellings, does not as a rule interfere with the animal's working powers.

Treatment. In the chronic form blisters or firing may be tried, but are rarely successful. Nor is compression or massage of much service. If thought desirable, bandages may be firmly applied above and below the joint, and changed from time to time. Better results, however, attend the injection of weak iodine, sublimate or carbolic solution after the synovial sac has been emptied with a fine trochar or aspirating needle. When the tendon sheaths are wounded, every effort must be made to render the parts aseptic, and after inserting sutures, a dressing must be applied. In purulent synovitis, the bursa may be frequently rinsed out with antiseptics, though such treatment is seldom successful. The horse must, of course, be slung. Where an antiseptic dressing cannot be applied, the parts may be permanently irrigated with boiled water or antiseptics. If performed cautiously, there is little danger in puncturing the swelling and giving exit to contents, though the gain is slight, but particular care should be taken not to make large incisions, 
unless they can be liept aseptic, as purulent synovitis generally results, and eventually proves fatal.

\section{Y.-ENLARGEMENT OF ARTICULAR AND TENDINOUS SYNOVIAL CAYITIES IN THE FORE LIMB.}

In the fore limb several of the joint cavities and tendon sheaths are so situate that distension is not visible externally. This is the case in the shoulder and elbow. On the other hand, other joints, tendon sheaths, and bursæ, when excessively distended with synovia, form swellings or "galls." To prevent repetition the most important will here be collectively dealt with. We may distinguish three varieties:(A) Distension of joints; (B) Distension of the sheaths of flexor tendons; (C) Distension of the sheaths of extensor tendons.

(A) Distension of articular synovial sacs.

1. Of the knee. Though the construction of the knee is unfavourable to the production of "galls," yet in working-horses distension of the capsular ligament lying between the radius and upper row of carpal bones is occasionally seen. This constitutes a knee gall, and either appears as a roundish swelling, divided into several parts by the extensor tendons on the front of the knee, or occasionally as a tumour the size of a hen's egg, lying on the outer surface of the limb, behind the radius and just over the pisiform bone.

2. Fetlock galls. These consist of roundish swellings between the metacarpus and suspensory ligament, caused by distension of the synovial membrane of the fetlock-joint. They are divided into two parts, are seldom larger than a duck's egg, and extend towards the front of the fetlock, appearing on either side of the extensor pedis. When the foot is lifted they may disappear, but immediately weight is placed on the limb they return. In exceptional cases they appear at the posterior part of the fetlock-joint and close below it. Hoffmann says they are particularly noticeable when the fetlock is upright.

(The reader is advised to refer to "Diseases of the Tendons and Tendon Sheaths in the Tarsal and Metatarsal Regions," and to study it in connection with the present section. It contains some illustrations throwing light on conditions here described.)

3. Of the hoof. These are of rare occurrence. They appear above the coronary margin at the toe as tense, painful swellings as large as hens' eggs; their real nature is often unrecognised. Frick has seen a number, and has satisfied himself of their true character by post-mortem examination.

(B) Distension of the sheaths of flexor tendons.

It is now generally allowed that distension of the sheaths of flexors 
calls for greater care in treatment than that of extensor's. The first variety may be divided as follows:-

1. Knee galls. At the back of the knee the flexor pedis has a large bursa, which begins about 4 inches above the joint and extends about one-third down the metacarpus (fig. 203, c). Eichbaum says that above and below the joint its lateral walls are unprovided with fibrous strengthening coats, and thus explains why the swelling appears above and below the knee, and sometimes on the inner sometimes on the outer side. The enlargement may become excessive (fig. 202), but generally takes the form of a longish, soft swelling, the outline of which corresponds with the borders of the bursa. When the limb is lifted, the communication between the upper and lower portions of the swelling is easily detected by palpation.

2. Wind galls. The above-described tendon sheath only extends as far as the beginning of the middle third of the metacarpus, the next portion of the flexor pedis being surrounded by loose connective tissue. At the lower third another tendon sheath begins and extends downwards behind the fetlockjoint to the centre of the coronet bone (fig. 203, d). Its posterior wall is connected with the flexor pedis tendon, whilst below the fetlock a fibrous extension surrounds it so firmly that

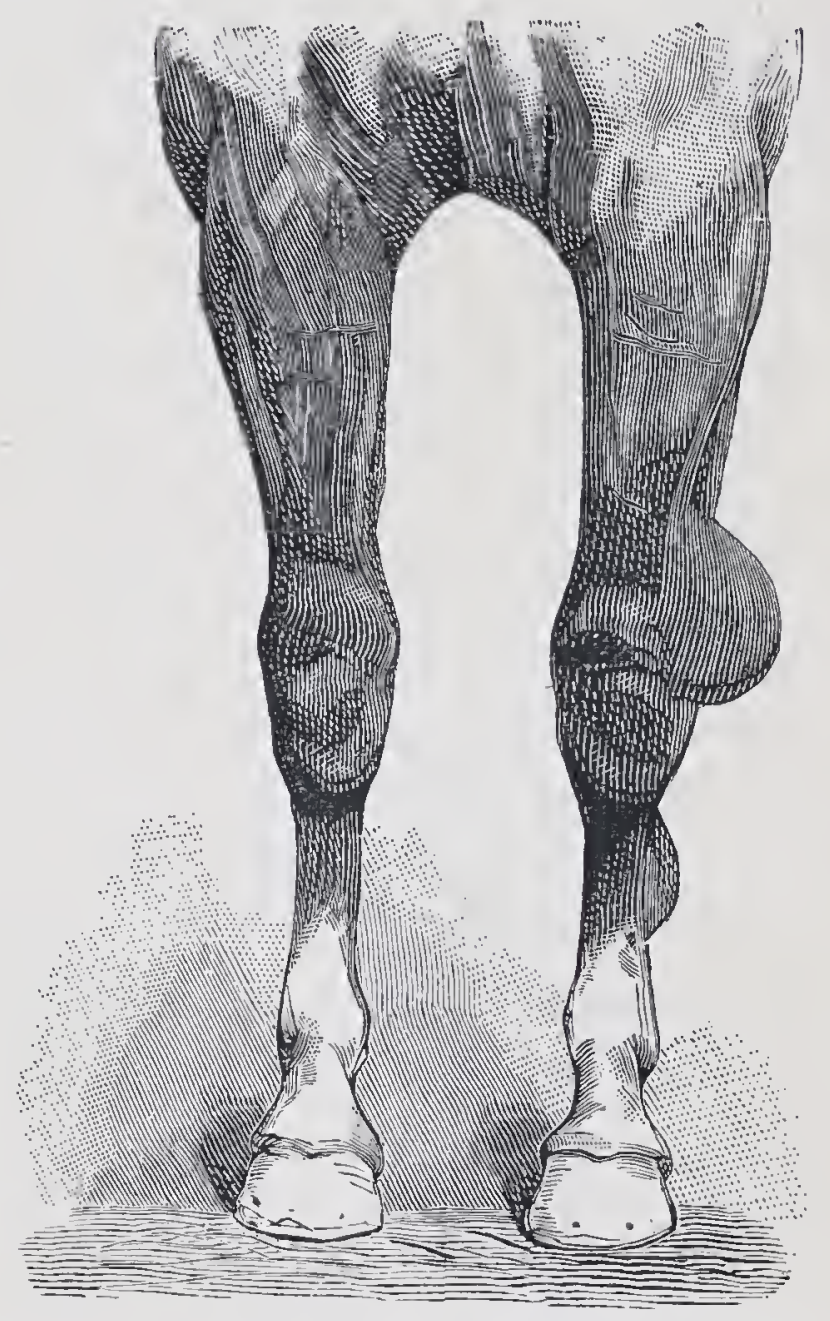

FIG. 202.-Distension of the flexor pedis bursa (most marked on the outer side of the limb). After a photograph. swelling can only occur above the fetlock-joint, and (on account of the position of the flexor tendons) on either side. At this point the sheath is covered with loose connective tissue, which favours the production of the two characteristic longish swellings lying on either side of the lower end of the metacarpus, close to the flexor pedis tendon, and extending upwards towards the middle third of the cannon bone. Their size varies greatly; sometimes they can only just be detected, sometimes they are as large as a goose's egg. Occasionally they appear below the fetlock in the pastern region, and are then of a more flattened form. Wind galls are very common. They generally result from chronic synovitis, though in the hind feet a peculiar form, termed indurated wind galls, are produced by a tendovaginitis chronica fibrosa. 


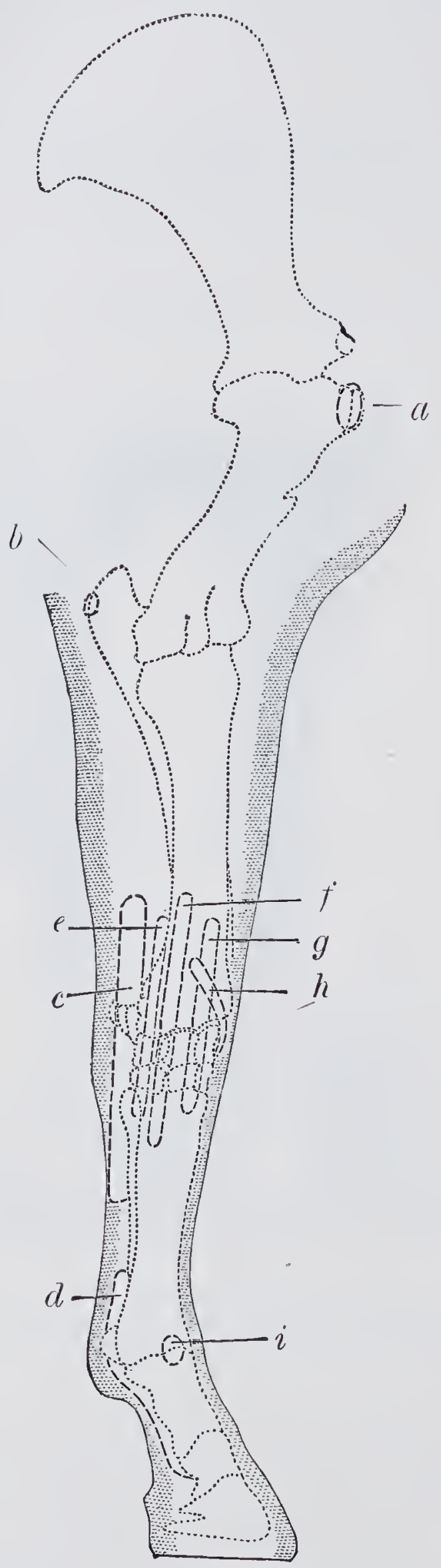

FIG. 203. - Schema of the more important tendon sheaths and bursæ of the fore limb, seen from in front and without. $a$, Bursa intertubercularis ; $b$, bursa olecrani ; $c$, upper tendon sheath of the flexor pedis muscles (carpal sheath); $d$, lower tendon sheaths of the flexor pedis muscles (metacarpo-phalangeal or great sesamoid sheath); $e$, sheath of the flexor metacarpi externus; $f$, upper sheath of extcusor pedis; $g$, sheath of extensor metacarpi magnus; $h$, sheath of extensor metacarpi oblicuus ; $i$, bursa of extensor pedis.

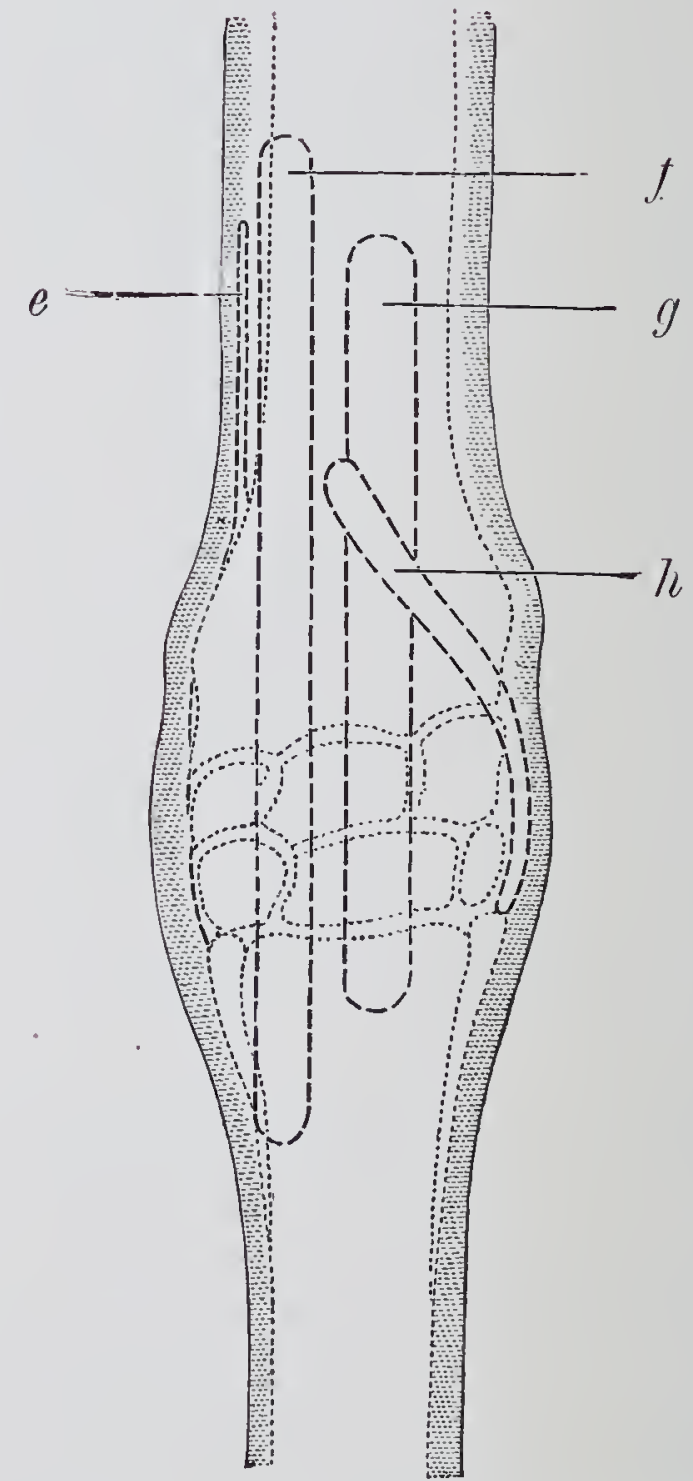

Fig. 204.-The same, as seen from the front. 
(C) Distension of the sheaths of extensor tendons. Of the many varieties the most important are:-

(1) Distension of the sheath of the extensor suffraginis in the carpal region. This consists of a swelling as large as a goose's egg, which appears 3 to 4 inches above the linee, and beginning on the outside, extends downwards; in some cases it may even encroach on the front of the joints (figs. 203 and 204,e).

(2) Distension of the sheath of the extensor tendons in front of the carpal joint. The extensor pedis, extensor suffraginis, and extensor metacarpi are provided with sheaths in front of the knee. These sheaths may be mechanically injured, as already pointed out (see "Injuries to the Knee"), but often become distended. The following" are the chief seats of such "galls":-

(a) The sheath of the extensor pedis, which begins 6 inches above the knee-joint, passes through the outer furrow in the lower end of the radius, and over the front of the carpus as far as the upper end of the metacarpus (figs. 203 and 204,f). On account of its being clothed in a strong fascia, dropsy of this sheath seldom occurs.

(b) The extensor metacarpi has, at the same height, a sheath which passes through the middle furrow in the radius, and extends as far as the lower row of carpal bones (figs. 203 and 204, g).

(c) The sheath of the extensor metacarpi obliquus begins on the outside, 3 inches above the knee, passes obliquely downwards and inwards over the anterior surface of the joint as far as the head of the inner small metacarpal (figs. 203 and 204, $h$ ). Distension of this sheath produces a sausage-shaped swelling, extending in an oblique direction as described, and divided by the extensor pedis into several portions.

(d) The bursa for the extensor pedis lies above the fetlock-joint on the anterior surface of the metacarpus. Its upper or anterior wall is formed by the tendon of the extensor pedis (fig. 203, i). When healthy it is from $\frac{3}{4}$ to 1 inch long, and $\frac{5}{8}$ to $\frac{7}{8}$ inch broad, but when diseased, may form a swelling larger, than a goose's egg, lying on the anterior surface of the metacarpus, just above the fetlock-joint. This bursa sometimes communicates with the fetlock-joint. To shorten description, the following classification has been adopted:-

(I.) Bursal enlargements on the front of the knee-joint and on the fetlock-joint.

(II.) Bursal enlargements on the outer surface of the knee and socalled "wind galls."

(III.) Enlargements of sheaths of extensor tendons in the carpal region. These last may again be divided into-

(a) Superior-sheath of extensor suffraginis above the knee-joint.

(b) External-sheath of extensor pedis. 
(c) Internal-sheath of extensor metacarpi magnus.

(d) Oblique-sheath of extensor metacarpi obliquus.

'Those on the anterior' surface of the metacarpus and fetlock-joint will

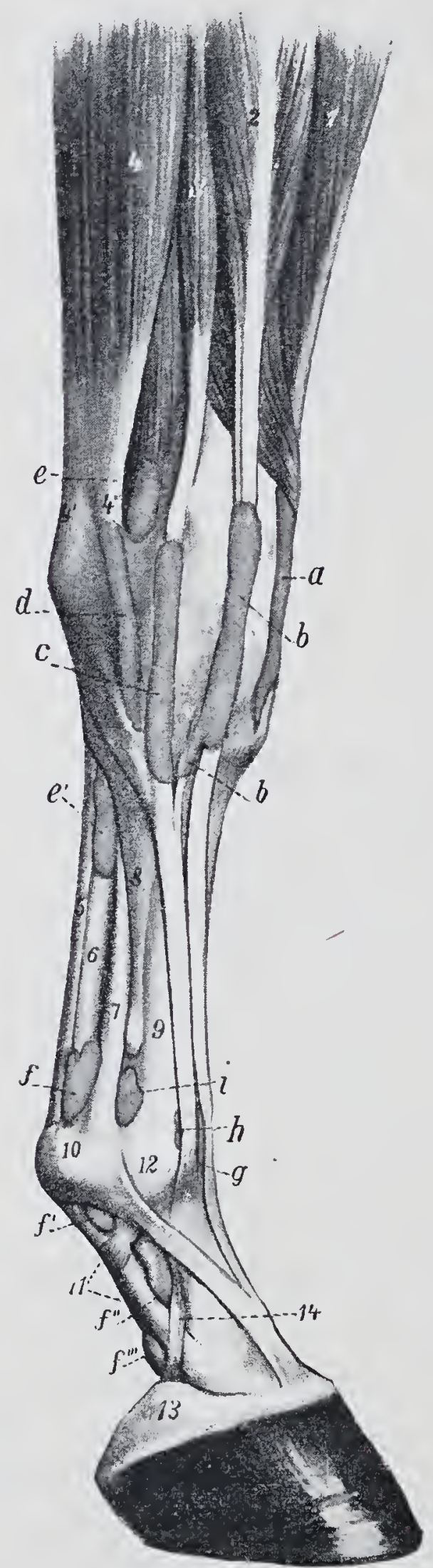

Fig. 205.-Outer surface of the fore limb; to show synovial sheaths. a, Tendon sheath of extensor metacarpi magnus; $b$, tendon sheath of extensor peris ; $c$, tendon sheath of extensor suffraginis; $d$, tendon sheath of flexor metacarpi externus ; $e e^{\prime}$, superior and inferior pouches of the synorial membrane of the carpal sheath; $f, f^{\prime}, f^{\prime \prime}$, and $f^{\prime \prime \prime}$, superior, middle, and inferior pouches of the synovial membrane of the metacarpo. phalangeal sheath; $g$, bursa beneath the extensor pedis tendon; $h$, bursa beneath the extensor suffraginis tendon; $i$, protrusion of the synovial capsule of the fetlock-joint.

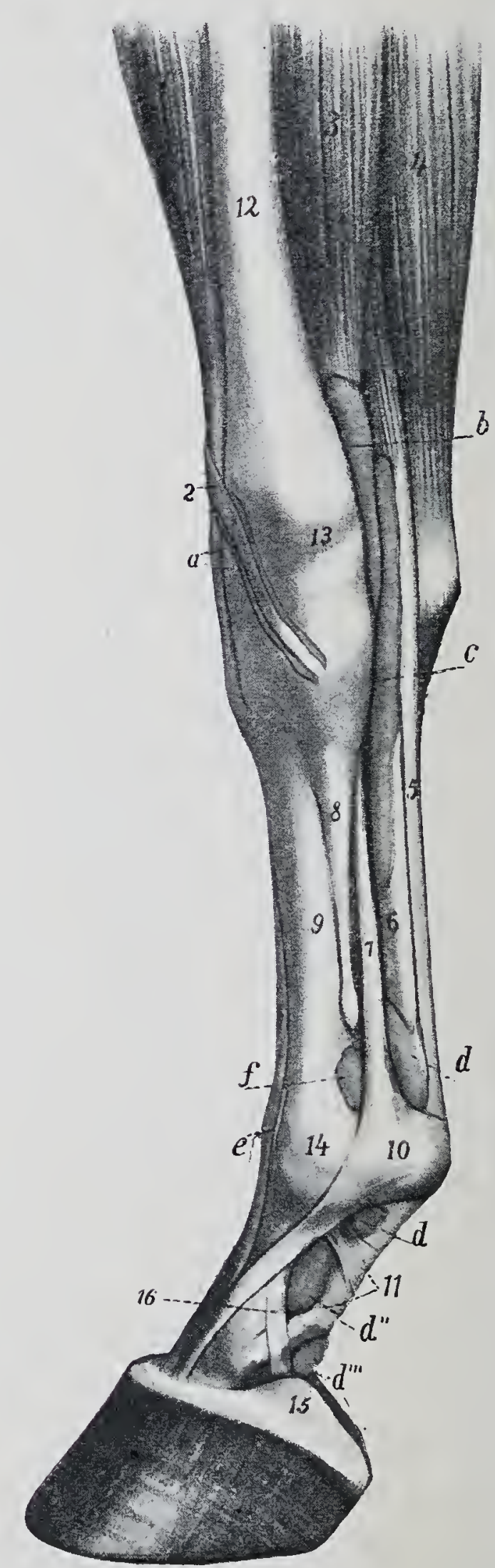

Fig. 206. - Inner'surface of the fore limb; to show synovial sheaths. a, Tendon sheath of the extensor metacarpi obliquus ; $b$, tendon sheath of flexor metacarpi internus; $c$, carpal sheath; $d, d^{\prime}, d^{\prime \prime}, d^{\prime \prime \prime}$, superior, middle, and inferior pouches of the metacarpo-phalangeal (great sesamoid) sheath; $e$, bursa beneath the extensor pedis tendon in front of the fetlock; $f$, distended synovial capsule of the fetlock-joint. 
be considered under the head "bursal enlargements of the extensors in the fetlock region," and may be divided into upper-those of the extensor pedis; and lower-those of the extensor suffraginis.

Causes. In the horse, these swellings are, with few exceptions, produced by chronic irritation caused by severe continued work, leading to dropsy of the joints or tendon sheaths, and are, therefore, almost always confined to working-horses.

A predisposition to them, depending on individual peculiarities which are not infrequently inherited, exists in coarse-bred horses and certain other animals. The cause is clearly some defect in the tissue, though its character is not yet clearly understood. The commonest causes are of a mechanical nature, such as strains, partial rupture of tendons or tendon sheaths, or subluxations of joints. The synovial membrane becomes inflamed or blood is poured into the joint cavity. In exceptional cases, external influences, like displacement or contusion of the joint, occasion enlargments of bursal cavities. Thus, bruising may be followed by bleeding into the capsule of the joint or tendon sheath, and years after the accident, flakes of fibrin may be found in the diseased joint or tendon sheath.

Symptoms. The symptoms need scarcely be enumerated after what has gone before; and if the anatomy of the parts is borne in mind, diagnosis presents no difficulty. Bursal enlargements, both of the kneeand fetlock-joints, diminish, however, when the limb is relieved of weight or is flexed, whilst those of tendon sheaths become more distinct and appear more tensely filled. Swellings due to distension of articular sacs are generally horizontal, those of tendon sheaths more or less vertical. The presence of acute inflammation is shown by heat, swelling, and lameness.

Prognosis and course. Chronic dropsy of the synovial cavities of joints and tendons results in the horse from chronic irritation; in occasional instances from acute inflammation; bursitis of the sheaths of the extensor tendons is generally preceded by lameness. Enlargements of the upper sheath of the extensor suffraginis often contain fibrinous clots-a result of their hæmorrhagic origin-even after having been in existence for some time. On the other hand, enlargements developing: either from joints or from flexor tendon sheaths are, with few exceptions, due to chronic irritation of the synovial membrane. Sometimes the cavity is filled with light-coloured, clear serum; less frequently it contains small bodies resembling grains of rice; occasionally the tendon sheath is more or less thickened (tendovaginitis chronica fibrosa) (indurated galls). In such case the tendon is generally involved.

For the most part, such enlargements in horses are only regarded as blemishes, and in old animals are seldom entirely absent. When of 
fresh growth and small size, they may, if properly treated, be cured; but, on the other hand, may persist for long, and resist all forms of treatment, yet without impairing the animal's usefulness.

Enlargements of the extensor tendon sheaths seldom cause lameness, are more accessible to operative interference, and are, therefore, of less moment. Distension of flexor sheaths, especially when near joints, is more difficult to remove, still more so are enlargements of the capsular ligament of the joint.

Galls on the flexor pedis perforatus, particularly in the hind limbs, are apt to become chronic and indurated. They are even more troublesome if accompanied by inflammation of the tendon of the flexor pedis and by lameness, complications which are also more frequent in hind than in fore limbs. As a rule, the swelling appears distinctly above the sesamoid bones.

Treatment must follow general principles, and it is here only necessary to remark that recent painful conditions are best treated by cold poultices or irrigations, or by immersing the parts in cold water. This should, when possible, be supplemented by bandaging and compression. As soon as acute inflammation subsides, moist warm applications may replace the cold ones, compression being continued, and, if the parts are not painful, massage may be tried. Light work also promotes absorption. Though moderate recent swellings may sometimes be dispersed in this way, the effect is seldom permanent, for distension generally recurs with work, and the practitioner is forced to resort to irritants like cantharides ointment, ungt. hydrarg. biniodid. 1:8, sulphuric acid, sublimate, \&c., or, better still, to blistering-plaster, cantharides collodion, or the firing-iron. The effect of these applications is to be ascribed to the regular and lasting pressure produced by the swelling: acting on tendon sheaths or capsules of joints, and assisting absorption. On account of the pressure it exercises, blistering-plaster acts more energetically than blistering ointments. The firing-iron produces its effect by cicatricial contraction. When freely used it is most effective, though, as it leaves scars, and only substitutes one blemish for another, it should not be lightly resorted to; nevertheless it is one of the most valuable remedies in such cases.

The effect of artificial drainage has been much overstated. In France, however, drainage and the subsequent injection of iodine solution, has long been a favourite method of treating chronically large synovial cavities. Simple extraction by trochar is in nowise dangerous if performed with antiseptic precautions, but its effect is not lasting, and the tendon sheath or joint refills in a few hours, though, after repaated abstraction of fluid secretion sometimes seems to stop. Cure is always uncertain, and even the after-injection of iodine does not ensure it. Sometimes the tendon 
sheath undergoes slow thickening and its contents become absorbed, success or failure appearing particularly to depend on the degree of inflammation produced by the injection. It is best to use a freshlyprepared solution of iodine, to see that it is completely removed after injection, and to follow this with a blister or with firing; when blistering is contra-indicated, a tight bandage may be substituted. To test the relative danger of iodine injections, Leblane and Thierry made a series of thirty-two;-fifteen into joints, seven into mucous bursæ and ten into tendon sheaths, without in any case having bad results. Needless to say, they observed full antiseptic precautions. In general, this treatment is uncertain, and when the enlargement is indurated, impossible. Masses of fibrin can only be removed by operation, and although in theory such operation should not be dangerous if performed under aseptic precautions, yet there is no certainty that dressings will remain in position; even when the preliminary dressing, applied for the purpose of sterilising the parts, has not been interfered with, dressings applied after operation are often violently rubbed off. The same is true in regard to animals other than the horse. The opening of distended joints or flexor tendon sheaths is in general dangerous, though there seems no particular risk in opening extensor sheaths, even when the parts cannot be kept aseptic. The risk need not prevent operation being tried, but attention should certainly be previously directed to the danger, and the strictest antisepsis should be observed. With this object, twenty-four hours beforehand, the hair is shaved from the point of operation, the skin washed, and a dressing moistened with 1 per 1,000 of sublimate applied. After casting the horse for operation and removing the dressing an incision is made, under antiseptic precautions, at the highest point of the swelling; the finger is then introduced, the condition of the sheath examined, and any masses of fibrin, \&c., removed. The cavity is washed out with a solution of iodine and iodide of potassium in water $(1: 3: 16)$, followed by sublimate or carbolic solution. The loose distended skin can then be partly cut away in an elliptical form, the wound closed with silk, and a dressing applied to keep out air and to exercise pressure. This dressing should not be changed for about eight days. The wound probably closes in three to four days, though the cicatrix is not then sufficiently firm to prevent re-opening if the animal be moved. Operating in this way Ries cured a large wind gall (enlarged sesamoid sheath) and an enlargement of the precarpal sheath of the extensor pedis tendon which had resisted all ordinary methods of treatment. In enlargements of the extensor sheaths setons may be used, but Möller prefers operation.

Bosco has recommended opening enlarged tendon sheaths with the red-hot iron, and favourable results have since been announced from other quarters. 'The glowing point of a firing-iron is thrust into the 
enlargement, allowing its contents to be discharged. More or less severe inflammation results, and recovery may possibly follow. As the point of operation itself is sterilised and an aseptic wound produced which soon closes by inflammatory swelling, opening by the actual cautery is less dangerous than by the knife, but the effect is not always reliable. When the exact degree of inflammation necessary to prevent aftersecretion of fluid is produced, a cure may result, but this degree is very difficult--indeed, usually impossible-to secure at will.

The same criticism applies to drainage of synovial sacs. This treatment consists in puncturing the enlargement at the highest point, passing a director and making a counter-opening at the lowest point, inserting a drainage-tube, and irrigating the sac with 1 in 1,000 sublimate solution. Active inflammation follows and persists for three or four weeks. In favourable cases, the endothelial lining of the sac, though at first replaced by granulations, is said to be restored. The treatment has hitherto been confined to enlargements of the extensor sheaths in front of the linee, hock, and fetlock.

To sum up: enlarged synovial cavities should at first be treated by rest, cold applications, massage and compression. If they cause lameness a blister can be tried; in the event of this failing the parts are fired in lines or points, or the swelling may be punctured with the cautery and the contents évacuated, but only with full antiseptic precautions. For enlargements of old standing which resist other forms of treatment and cause permanent lameness neurectomy may be performed.

\section{DISEASES OF THE METACARPUS.}

The metacarpus in solipeds consists of the so-called os metacarpi tertium, the cannon bone, and the two small metacarpals. The tendons of the extensores pedis et suffraginis cover its anterior surface; behind it lie the two flexor tendons, to some extent enclosed by the two small metacarpals. The whole metacarpus is covered with a tensely-stretched skin, which is only slightly movable on the underlying parts.

In ruminants the two cannon bones become united soon after birth and form only one bone, on whose posterior and outer surface lies the rudimentary small metacarpal, which is articulated to the fused metacarpal by means of a small joint. This rudimentary metacarpal does not contribute to the formation of the knee.

In swine the two middle or true cannon bones form, with the external or false metacarpals, the metacarpus. Carnivorous animals possess five metacarpals, those of the third and fourth toes being the strongest.

These peculiarities in the formation of the metacarpus cause considerable diversity in local disease processes in the different species of animals. Especially is this true of fractures. 


\section{I.-FRACTURES OF THE METACARPUS.}

In solipeds and ruminants fracture generally involves all the bones of the metacarpus. The small metacarpals seldom escape, still less frequently are they alone fractured. In other animals, particularly in carnivora, a single bone may be injured, though it is more common for several to be broken at one time. Owing to the thin covering of soft parts the closely-applied skin is readily perforated by fragments; compound fractures are thus produced, which unite with great difficulty.

Causes. The most frequent causes are kicks, falls, and in small animals, blows with heavy sticks, and injuries from carriages. Where all the bones of the metacarpus are broken, diagnosis is seldom difficult, the abnormal mobility of the parts, the inability to place weight on the leg, and the crepitation pointing clearly to fracture.

It becomes, however, more difficult where single bones are involved, but even then careful palpation is generally sufficient, and crepitation can almost always be detected. The most difficult to detect are fractures confined to one or other of the small metacarpals, or such as have already produced severe swelling. Fissuring of the cannon bone can seldom be more than guessed at. Such cases have, however, been reported. Like similar accidents in connection with the pastern and tibia, fissuring of the cannon bone is apt to culminate, a few days after its occurrence, in complete fracture.

Course. In all the fractures enumerated recovery is possible, and in small animals is fairly rapid. Compound fracture of the cannon bone is, however, seldom hopeful, and in the large domestic animals even subcutaneous fracture must be viewed as relatively incurable. Only in colts or valuable and quiet horses is treatment worth attempting, but that complete usefulness may be regained is shown by many reported cases. The course of fracture of the metacarpal chiefly depends on whether the animal can bear its whole weight sufficiently long on the other foot without producing laminitis and descent of the os pedis. Union is readier in cattle than in horses, and in them the lameness occasionally left causes no inconvenience. In the small ruminants and in carnivora subcutaneous fractures unite in three to six weeks. Fractures of the diaphyses of the metacarpal bones unite more easily than those in the neighbourhood of joints, but prognosis is unfavourable when a joint is. involved.

Treatment. Reduction is seldom difficult. Before applying a dressing care must be taken to bring the limb into a proper position, and especial attention should be given to the position of the hoof or claws. In the horse the toe of the hoof, in the ox the interdigital space, is the best guide. A plaster bandage is very useful, especially in small animals.

V.S. 
Stolz employed an ointment, now nąmed after him, composed of two parts of resin and one of wax, which is smeared on linen, and used as a first dressing, over which a splint is applied. Horses must generally be slung. Pujos saw transverse fracture of the cannon bone, just above the fetlock, in the horse, which united after applying a plaster bandage. Eight days (?) after the accident the animal began to place weight on the limb. Forty-five days later, when the bandage was removed, the limb could be moved as usual. In sixty-six days the horse was again put to work.

\section{II.-WOUNDS AND BRUISES OF THE METACARPUS.}

On account of the skin being so tightly stretched and firmly united to the underlying structures, wounds in this region, especially in large animals, often take an unfavourable course. It sometimes happens that bones, tendons, and tendon sheaths are all simultaneously injured, and such cases demand the most careful antiseptic treatment. The hair should be removed, along with any foreign bodies or loose shreds of connective tissue, and the wound and its neighbourhood carefully washed with a disinfectant. If possible the parts should be immersed in a bath of sublimate, carbolic or creolin solution, and the wound irrigated with a powerful stream of sublimate until its edges are thoroughly saturated with the solution. Gaping wounds must be sutured with aseptic material, and a dressing applied over all. To complete the disinfection it is well for some time to moisten the dressing two or three times daily with sublimate solution.

Immediately the dressing becomes wet through with discharges or blood it should be changed, under antiseptic precautions. Thus treated the wound may unite by primary intention, even when tendons, tendon sheaths, or bones are damaged, as shown by reported cases. Similar precautions are required in all injuries complicated with much loss of skin, or: where large flaps are threatened with necrosis. In such wounds, cicatrisation is difficult, for in consequence of its firm structure and fixed position the skin camnot stretch; and even when the defect is filled up, the epidermis is not replaced. Exuberant granulations, liable to result after operation or extensive necrosis of slim, must be kept back by the early use of astringents (iodoform conjoined with tannin, glutolite), or by applying a well-fitting pressure dressing, or they may be destroyed by suitable caustics. In all cases the skin must, as far as possible, be preserved, further necrosis prevented by antiseptic dressing, and the fact borne in mind that wounds which fail to heal by first intention always produce well-marlied cicatrices.

In hol'ses, the tendons and tendon sheaths in the metacarpal and 
metatarsal regions are often injured, either: by the horse lashing out, or by its falling on sharp objects like broken bottles, scrap iron, sickles, or scythes. The flexor tendons are oftenest injured, the extensor pedis less frequently; wounds of the latter are seldom serious, and heal when treated on general principles. Injuries to the flexor tendons are, however, very dangerous.

There is no difficulty in diagnosis, though it is not always easy to discover the exact extent of the wound. As a rule, blood-vessels are divided, and bleeding may prove dangerous, though it is generally checked by the attendant applying cloths. The degree of lameness varies. When the flexor pedis perforatus, which is most exposed, is alone divided, it may only be slight; and weight may be placed on the limb, though the toe is directed somewhat upwards, and no longer touches the ground. Division of the flexor pedis perforans results in excessive dorsal flexion of the coronet-joint on every attempt to stand on the limb. When the flexor tendons and suspensory ligament are cut through, all the lower joints are in this condition, and distortion is often so marked that the fetlock comes in contact with the ground.

After cessation of bleeding, the divided ends of the tendons may be seen in the wound, and the extent of injury be determined by palpation or by probing with the finger. The position of the wound generally indicates whether the sheaths of tendons have also been injured.

Bruises of the metacarpus most frequently occur just below the knee, and may lead to extravasation of blood or lymph. The swelling varies in size, between a pigeon's and a hen's egg, is fluctuating, but not often acutely inflamed, and seldom causes lameness.

Course. Healing chiefly depends on the parts being kept aseptic. When this is possible, wounds heal in two to three weeks, though if pus formation occurs they may take four to six weeks, or even months. Injuries confined to the flexor pedis perforatus naturally heal more readily than those affecting both the flexors. Wounds involving the sheaths of flexor tendons are exceedingly dangerous, purulent or septic tendovaginitis being liable to set in, especially in horses, and, therefore, wounds close above the fetlock or in the pastern region are more dangerous than those in the middle of the. metacarpus, where such sheaths are absent. In the ox the conditions are similar to those in hor'ses, though healing sometimes occur's without any particular precautions. Gerlach saw complete division of the flexor pedis perforans and perforatus in the cow, followed by cicatrisation in four weeks. For some time there was very marked dorsal flexion, but this disappeared in three months. In small animals injuries of this class generally do better, because the limb can be better protected.

Lymph extravasations due to bruising are usually indolent and persist 
for long periods. This is particularly true of extravasations over the head of the internal small metacarpal bone, where the subcutaneous bursa is often involved.

Treatment. Recent wounds require the strictest antisepsis. In many cases, unfortunately, they are already infected by the bandages applied to check bleeding, but can generally be efficiently disinfected, even twelve to twenty-four hours after injury. Bleeding must be checked by ligaturing vessels with sterilised material. The limb must then be carefully washed with soap and water, the wound and its surroundings rinsed with sublimate or carbolic solution, all foreign bodies removed, and the wound washed out with a powerful stream of antiseptic fluid. If asepsis seems possible, the wound, and even the ends of the tendon, should be sutured, and a dressing moistened with antiseptics applied, and repeatedly wetted during the first day or two with sublimate solution. Provided it is not sodden with blood or wound discharge, the dressing is left in position until the animal begins to place weight on the limb, or the wound is healed, otherwise it must be renewed, under antiseptic precautions.

Failing asepsis, the separation of necrotic portions of tendon must be assisted, and excessive proliferation of tissue around the wound checked. Portions already partially separated can be removed with the scissors and knife. Over-prominent growths may be checked by astringents, such as glutol, tannoform, amyloform, tannin mixed with equal quantities of iodoform, or by alum or sulphate of zinc in powder; a well-fitting pressure dressing serves the same purpose. Sometimes new growths may require to be removed with the knife or curette. All such surgical wounds must be kept very clean to prevent infection. During recovery the patient should be rested.

Where tendon sheaths are also injured, extreme attention must be given to antiseptic precautions. Reported cases show that careful antisepsis may secure healing even in severe injuries of tendons and tendon sheaths.

Bruising of the metacarpus, due to kicks, \&c., often causes formation of hæmorrhagic or lymph extravasations of the size of a walnut, or hen's egg. If soon opened troublesome bleeding may result; on the other hand, absorption takes a long time, particularly in injuries about the head of the inner small metacarpal, accompanied by extravasation into the subcutaneous tissue lying here. Lameness is rare, and animals can be worked, whilst reduction of swelling is encouraged by continuous compression and daily massage. If, in valuable horses, operation be attempted, strict antisepsis must be observed, for pus formation may be followed by subfascial cellulitis and troublesome consequences. Extravasations of lymph sometimes lead to sinus formation, and therefore should not be incised too early. 


\section{III.-RUPTURE OF THE FLEXOR TENDONS AND SUSPENSORY LIGAMENT.}

Rupture of the flexor tendons is produced by external violence, especially by violent over-extension when weight is thrown on the fore limb; it is therefore almost entirely confined to solipeds, the great strains necessary for its production only occurring during movements like jumping or galloping. In ruminants a ligament, extending from the knee to the claws, assists in preventing such accidents. Rupture is favoured by changes in the nutrition of the tendons, and after infectious diseases, or even after long rest, often results from slight exertion.

Schellhase and Comény saw simultaneous rupture of the inferior sesamoidal ligaments of both fore limbs in horses which had been rested for a long time. Johne also observed rupture of this ligament in a foal which had been in hospital, and as the rupture was accompanied by tearing away of portions of bone, suggested it was due to change in the bone substance. In another case the superior sesamoidal ligament was ruptured near its bifurcation in a horse which had fallen. Smith describes a case in the horse where, in consequence of the animal jumping, both flexor tendons and the suspensory ligament of one fore limb were suddenly ruptured, the metacarpus penetrating 4 inches into the earth.

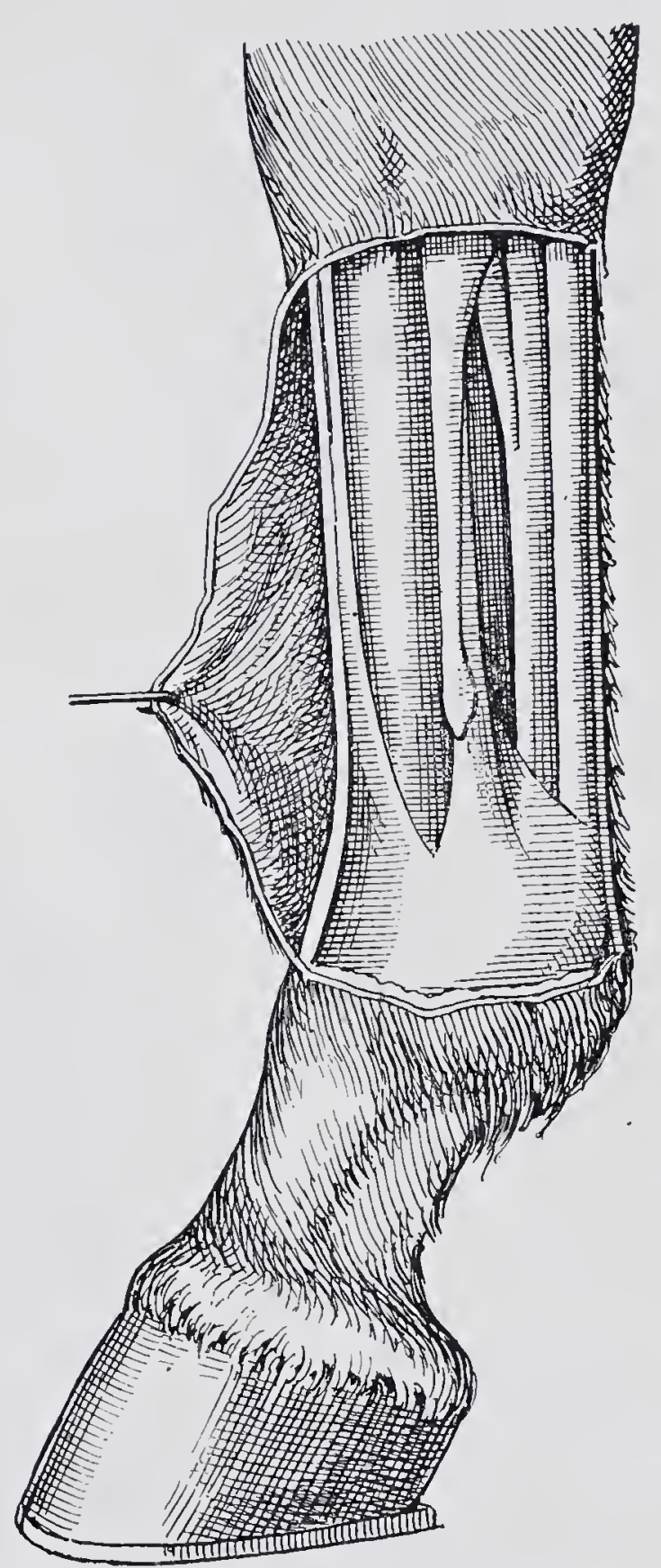

Kay, in a horse, noted rupture of both Fra. 207. - Showing relative positions of flexor tendons and the suspensory ligament in three separate limbs. The flexor pedis perforans was torn away from the os pedis and the suspensory ligament from the sesamoid bones, while portions of the bone were also loosened. The horse had previously been lame, and for some time incapable of work. In the year 1890 a horse was sent to the Berlin clinique suffering from recent and sudden lameness; the animal could scarcely stand even for a few moments. When forced to do so, the hind feet were placed far forwards and the fore feet disposed as in laminitis. Post-mortem showed rupture of the flexor pedis perforans in all four feet at and above its point of insertion into the os pedis. No exact 
explanation of the cause could be discovered further than that the horse had previously been confined to the stable for some days on account of lameness in one limb. A similar case was reported a year later; in another the flexor pedis was torn away from the os pedis in both hind limbs, and the sesamoid bones of both front limbs were fractured. In these cases, also, the animals had previously been unworkable. Maillet, in a horse, saw both the flexor pedis perforans and perforatus ruptured. Some old-standing disease, such as partial rupture of the tendons, had probably been in existence. Rodet noted

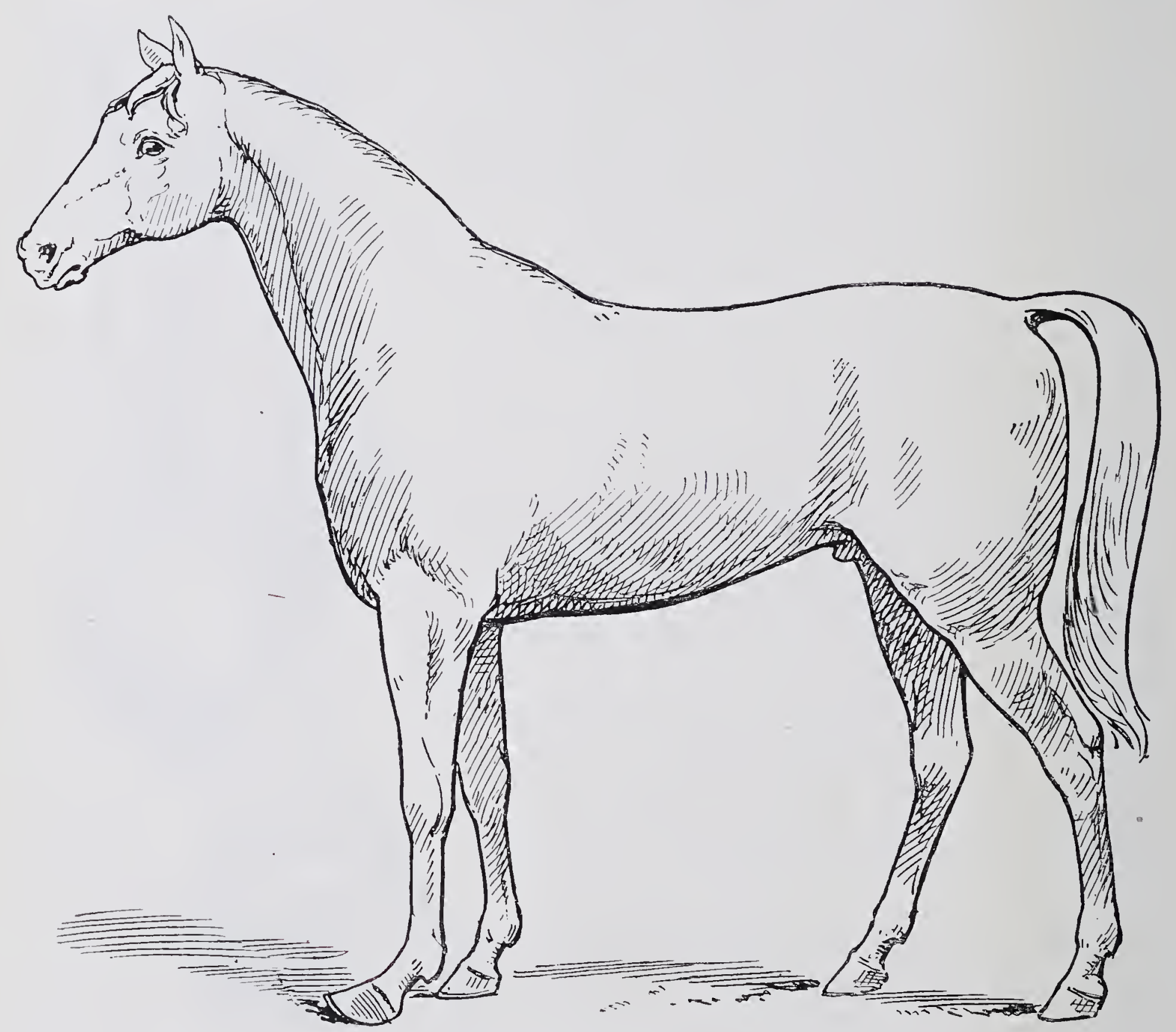

FIG. 208. - Rupture of the flexor pedis perforans and perforatus tendons.

a similar accident, affecting both fore limbs, occur during galloping. Schraml describes tearing away of the flexor pedis perforans from its insertion into the os pedis, portions of which remained adherent to the tendon; the navicular bone was broken into many fragments, which Schraml ascribes to the excessive weight thrown on it after rupture of the tendon. Dégive saw rupture of the flexor pedis perforans in all four limbs after an attack of laminitis.

Möller has repeatedly seen similar cases. The suspensory ligament is almost always torn away from its lower attachment to the sesamoid bones, whilst portions of the bone are loosened. In van-horses the suspensory ligament is sometimes ruptured, even in the hind limbs. In front, this rupture is commonest in race- and steeplechase-horses, and is 
generally caused by jumping obstacles. Sometimes the flexor pedis perforans is affected, though the suspensory ligament may also be ruptured. Fig. 210 is drawn from a case of rupture of the suspensory ligament in a race-horse which fell lame on the race-course and had been unsuccessfully treated for a long time.

A careful inspection of instantaneous photographs of moving horses (like those of Marey or Muybridge) shows that in galloping and in landing from a jump the animal's whole weight rests for an appreciable time on one of the fore limbs. At this moment the fetlock is supported by the contraction of the flexor pedis perforans and perforatus muscles, which are elastic, and by the passive resistance of the suspensory ligament, which is comparatively inelastic. Should, then, the strain thrown on the limb exceed the elastic resistance of the muscles, either they or their tendons must necessarily yield. One of two results may follow. If the miscles yield, the excess strain falls on the comparatively inelastic suspensory ligament, which may be partially or wholly ruptured. If the tendons yield, a similar result may follow, the flexor tendons and the suspensory ligament then suffering together. A single very violent shock is therefore more likely to affect both the tendons and ligament (because the muscles being untired do not yield), while long-continued exertion is favourable to strain of the suspensory ligament alone, on account of the muscles relaxing and the pull on the tendons never becoming sufficient to overcome their tensile strength. In the latter case the excess strain necessarily falls entirely on the suspensory ligament. The fact that strain of the suspensory ligament is practically confined to the front legs in all riding horses is explained by the fact that an unduly large proportion of the rider's weight falls on the fore limbs, and that this proportion tends to increase with increase in speed.

Rupture of the flexor tendons often coincides with disease of their surface at the point where they respectively pass over the sesamoids or navicular bone, a change which commonly accompanies chronic navicular disease and suppuration in the fibro-fatty frog, terminating in necrosis of the flexor pedis perforans. Rupture of the flexor perforans and perforatus often follows purulent inflammation of their tendon sheaths and of the gliding surface of the sesamoid bones. In such cases rupture is due to inflammation in the sheaths of the tendons; it is favoured by neurectomy. Fig. 209 shows an almost complete rupture of the flexor pedis perforans behind the fetlock, consequent on purulent disease of its sheath. During navicular disease the gliding surface of the navicular bone becomes rough and produces erosion of the perforans tendon; the peculiar cases following neurectomy result from violent over-extension of the tendon.

Möller describes cases where the perforans and perforatus tendons in 
hind limbs have become totally ruptured on account of advanced necrosis. Purulent or septic cellulitis extending to the flexor tendon sheaths sets up purulent tendovaginitis, which leads to necrosis of the tendon. The animal then stands continuously on the other foot, this sometimes causing descent of the pedal bone. Pain then increases to such a degree that weight is placed on the first affected limb in spite of the disease of the tendon. This apparent improvement is, however, suddenly cut short

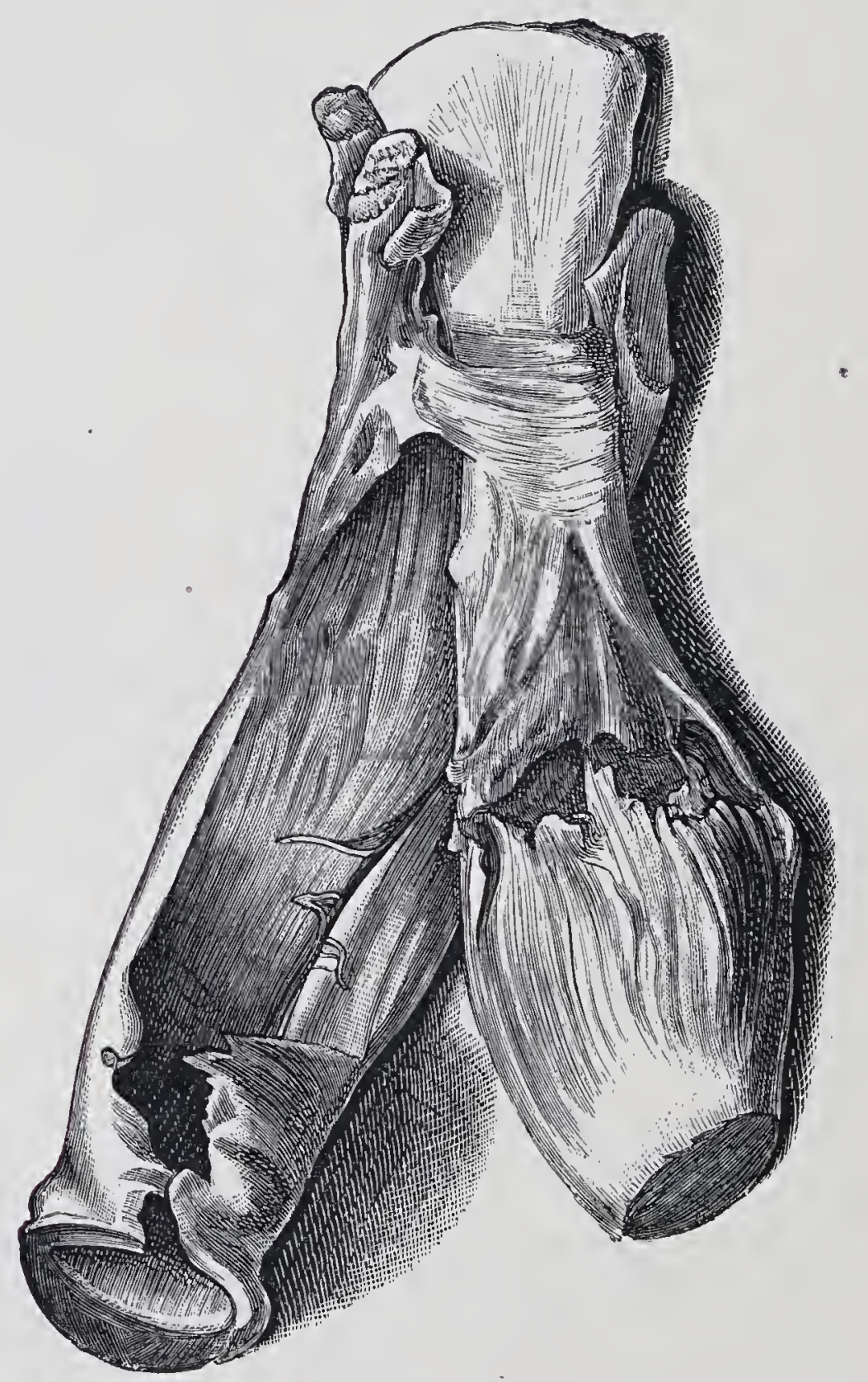

FIG. 209. - Rupture of the flexor pedis perforans behind the fetlock in consequence of suppuration in the tendon sheath.

by rupture of the tendon. The change in the position of the hind limbs is generally noticed in the morning.

Necrosis of the ruptured tendons is indicated by the dull yellow colour of the structures, and (in cases where separation of the dead parts has already set in) by the red granulations bordering the normal tendon tissue.

Gramlich describes a horse which, during the course of influenza, suffered from inflammation of the tendons of all four limbs. In one front leg the perforans and perforatus tendons were both ruptured, and on post-mortem examination were found partly necrotic. 
Rupture of fasciculi occur in almost all cases of inflammation of the flexor tendons produced by severe strains.

Symptoms. Rupture is at once followed by lameness. Either no weight can be borne by the limb, or if weight is placed on it for a moment the affected joint is abnormally flexed; the symptom, however, is less marked in rupture of the perforatus tendon. In consequence of the dorsal flexion of the coronet-joint, the toe of the foot is directed upwar:ds. Rupture of the superior sesamoidal ligament produces abnormal dorsal extension of the fetlock (fig. 210), but the toe retains its normal position. The same is true of ruptures of the inferior sesamoidal ligaments. In rupture of the flexor perforans, all three phalangeal joints show abnormal dorsal flexion, the hoof sometimes only touching the ground at the heels. Unless swelling has become pronounced the rupture may be detected by palpation. The degree of pain varies greatly; usually it is slight.

Course. Ruptures of the flexor tendons unite regularly and completely, provided-

(1) The ends of the tendons are not too far apart, and

(2) Rupture has occurred outside

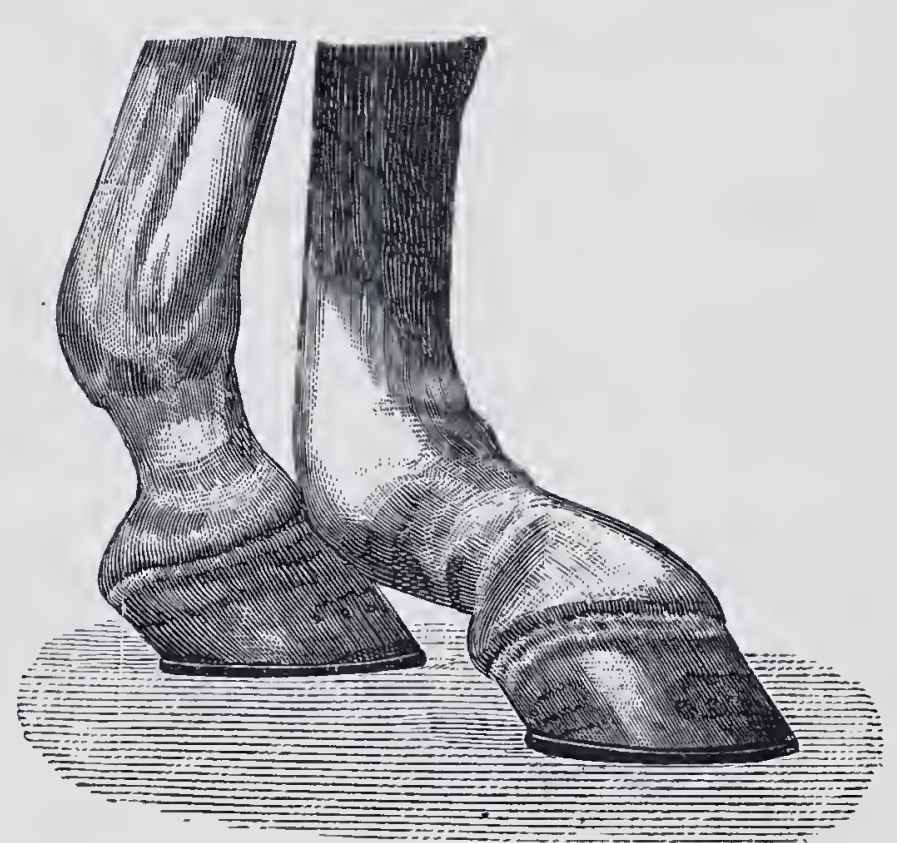

FIG. 210.-Rupture of the superior sesamoidal suspensory ligament (from a photograph).

the region of the tendon sheath. Ruptures within the tendon sheath unite exceedingly slowly, and only incompletely, because here the paratendineum (connective tissue surrounding the tendon), which mainly contributes to union, is wanting. The extent to which the ends of the tendons are displaced may be estimated from the change in position of the fetlock. Under favourable circumstances, rupture of the flexor perforans or perforatus unites in six weeks, though two to three months may elapse before the animal is fit for use. A race-horce which had suffered rupture of the suspensory ligament, with fracture of a sesamoid, in one fore limb was no better after six weeks. It was killed, and on post-mortem it was found that though union had begun, yet there was no visible callus formation between the displaced fragments of the sesamoid bone.

Ruptures of one or other insertion. of the suspensory ligament unite in from six to eight weeks, usually without leaving any lameness. The condition becomes grave when the rupture occurs over the sesamoid bones. Where rupture of the tendons is accompanied by other: diseased processes like acute or chronic navicular disease or necrosis, the prognosis is quite hopeless. 
Treatment of complete ruptures is generally useless, because in the majority of cases it is accompanied by extremely grave degenerative changes in the tendons themselves. Even in the most favourable cases where the tendon substance is normal it is confined to bringing the divided parts as near together as possible and fixing them, for which purpose plaster bandages are useful. If the animal be quiet, an iron splint fixed to the heal of a bar shoe, and of a curve corresponding to the normal position of the foot, is useful in rupture of the flexor perforatus. After covering the foot with a pad to equalise pressure, the splint is padded and fixed in position by means of circular bandages. Slinging is generally necessary when using this apparatus.

Rupture of the extensor tendons is very rare, and is usually brought about by efforts to liberate a foot which has been caught in some obstacle like railway points, or by a direct mechanical injury severing the tendon. Healing is slow, the chief obstacle being the excessive volar or plantar flexion ("knuckling over") which occurs. A suitable iron splint may, however, overcome the difficulty.

\section{IY.-INFLAIMIMATION OF THE FLEXOR TENDONS (TEN- DINITIS) AND OF THE SUSPENSORY AND CHECK LIGAMENTS.}

Inflammation of the flexor tendons is very frequent in the horse, and generally occurs in the fore limbs, on account of their being much more exposed to the violent strains which form the general cause of the disease.

Causes. Horses with weak flexor tendons, heavy bodies and powerful muscles, are most predisposed to tendinitis. The structure of the tendon, and consequently its powers of resisting strain, are of the greatest importance; its absolute thickness bears no direct proportion to its strength. On the contrary, tendons of considerable size and surrounded by abundant paratendineum suffer more frequently than the more sharply defined, and those which are firm to the touch. The latter peculiarity shows that the tendons are well provided with firm fascicular tissue, whilst in the first case they consist more of connective tissue. As mentioned in the last chapter ("Rupture of the Flexor Tendons"), continued rest seems to lower the resistance against strains. Rupture and inflammation following severe diseases, are, in part at least, to be referred to this fact. Further, all conditions which increase the load on the limb and the strain on the flexor tendons favour tendinitis.

Long, weak, and slender fetlocks are especially liable to it. The strain on the tendons is increased by increased length and obliquity of the fetlocks. The more slender the fetlock-joint appears when seen from the animal's side, the greater the mechanical disadvantage at 
which the tendons are placed. For' a similar reason, horses with "tiedin" knees are disposed to tendinitis, this formation being generally accompanied with small size of the fetlock. Further, everything which favours dorsal flexion of the joint increases risk of inflammation of the flexor tendons. Low heels and long toes are therefore apt to cause it. Long toes throw a powerful strain on the flexor tendons during the latter phase of movement, just before the limb is lifted from the ground. The kind of work required of the horse is also important. Thus race-horses and hunters, and horses required to trot at a sharp pace for long periods, are the most general sufferers. Animals with considerable powers of endurance suffer most. The greater the muscular development of the hind-quarters, the greater the shocks produced in the fore limbs by the violence with which the weight of the body is thrown forward on to them. Spirited animals suffer more than phlegmatic. For similar reasons, cart-horses show strain more frequently in the hind limbs. Those which work in two-wheeled carts and have oblique pasterns are specially liable to strain of the hind limbs, particularly when descending hills with heavy loads.

Strains of flexor tendons are produced either by the body-weight suddenly falling on the front limbs when the horse is jumped or quickly pulled up (passive strains), or by continued violent action of the flexor muscles (active strains). In the first instance, the flexor perforatus is principally involved, its less length causing it to suffer first from forced dorsal flexion of the phalanx. The flexor perforans is less likely to be injured. The effective length must be reckoned as the distance between the lower insertion and the upper one, and in the perforans and perforatus tendons from the upper insertion of their so-called check ligaments.

Inflammation of tendons produced by excessive muscular action (active sprains) have, with few exceptions, their seat in the flexor perforans, because the muscular head of this tendon is by far the stronger, and its tendon is therefore exposed to the greater strain. But passive strains also occur, as shown by the frequence with which the check ligament, which extends to opposite the middle of the metacarpus, becomes diseased. With regard to strain of the check ligament, which is particularly common in cart-horses, the remarks made in the previous chapter re strain of the suspensory ligament in large measure apply. The conditions, however, are not precisely the same, because owing to their respective mechanical arrangements strains do not affect the suspensory and check ligaments in exactly the same way. Judging from their course and attachments, it would appear that while the suspensory ligament is always more or less in tension, the check ligament is frequently entirely relaxed, and that stress, when thrown on it, is liable to be of the nature of a sharp jerk. Such a jerk would follow any miscalculation of distance 
on the horse's part, as for instance when the animal unexpectedly steps into a hole. Heavy work is another well-recognised cause of strain of the check ligament in cart-horses. In this the case injury is probably inflicted in starting the load. The animal leans forward in the collar, and before actually commencing to pull causes excessive dorsal flexion of the fetlock. At this moment the perforans muscle is relaxed and a heavy strain thrown on the check ligament, which may be strained. Closely similar conditions prevail when the horse is drawing a load up a steep hill. The injury occurs during relaxation of the perforans muscle. In the hind limbs the portions of the flexor pedis perforans tendon which suffer oftenest are those lying just above the fetlock or below the hock.

Bruising rarely causes inflammation of tendons, though the condition is seen in the flexor perforatus, its surperficial situation more readily allowing of injury from kicks, \&c. This kind of injury has been repeatedly seen in riding-horses, particularly in race-horses, where the tendon is struck by the hind foot of the same side. Inflammation sometimes extends from the tendon sheaths or articular surfaces to the flexor tendons; and the form associated with infectious diseases, and occurring during convalescence from thoracic influenza, always starts in the tendon sheath. Schrader, who carefully described this disease, found that the first sign of inflammation is a peculiar semi-soft swelling just above the fetlock, which extends thence to the perforans and perforatus tendons. One-sixth of all his cases convalescent from thoracic influenza showed it, but the disease disappeared again in twelve to twenty-four hours; Möller has often seen a similar appearance, and looks on such cases as due to secretory metastasis. In convalescents the inflammation sometimes originates in the perforans tendon, particularly when animals are worked too early. The interference with general nutrition produced during the disease is probably the predisposing cause.

The greater number of cases described as inflammation of the tendons are, doubtless, really due to reparative processes consequent on partial rupture, as shown by their course and termination. The persistence of thickening points to extensive damage, and is therefore unfavourable. This extra deposit is necessary for recovery, but tendons thus thickened never attain their primary strength or condition.

A number of Spiroptera reticulata were found in the suspensory ligament of a horse which had been chronically lame in consequence of swelling of the ligament. No treatment had proved of any service.

Symptoms. Inflammation of the flexor tendons is accompanied by the following symptoms :-

(1) Lameness, which varies according to the position and extent of injury. The form seen after influenza is usually the most painful, that following mechanical injury of the perforans less so. In inflammation of 
the suspensory ligament, lameness is slight. When only one division of the ligament is affected, lameness may be absent. Lameness is usually deferred until some hours after the strain.

Lameness is only shown when weight is placed on the limb. Attempts are made to shorten that phase of movement during which the phalanx is in a position of volar flexion, i.e., is upright and under the body. Neither passive extension nor rotation appear very painful, a fact which distinguishes the disease in question from disease of the joint. The symptoms are then, supporting leg lameness; shortening of the period when the limb is upright, volar flexion of the phalanx, and absence of pain when the limb is rotated. Sometimes animals try to relieve the weight on the diseased limb by setting the heels on an eminence, e.g., on a stone, and, by thus bringing the fetlock into an upright position, minimise the tension on the tendons. This is well seen in the hind limbs during disease of the flexor perforans. When lameness is severe, the animal never stands so completely on the foot, even when at rest, as it occasionally does in disease of the joint.

(2) Local examination detects pain, swelling, and increased warmth, which latter, however, is only seen early in the disease, and even then indistinctly. It is difficult to distinguish pain in the reinforcing band of the flexor perforatus (superior carpal ligament), though it also occasionally suffers. In applying pressure to the tendons, it is important not to be deceived by mere general sensitiveness:

The swelling varies in degree and extent, that accompanying strain of the flexor perforatus or sub-carpal ligament being, in "clean" legs, sometimes visible from a distance, and in other cases only to be detected by palpation. At first it is soft and diffuse; later it becomes harder and sometimes sharply defined. In examining for pain and swelling, the foot is lifted; the reinforcing bands can only be properly examined in this position.

(3) The symptoms enumerated are afterwards followed by shortening of the diseased tendon, continuous volar flexion and upright position of the hoof. This is differentiated from the volar flexion produced by placing weight on the leg, by the fact that it continues even when the animal stands fairly on the limb; the point is at once settled by lifting the other foot. Whilst contraction of the flexor perforans produces volar flexion in all the lower joints, contraction in the flexor perforatus and suspensory ligament only affects the obliquity of the pastern; the hoof remains in its normal position.

In race-horses a large number of fasciculi of the flexor perforans may be ruptured, producing abnormal dorsal flexion of the phalanges and so-called "break-down." This is oftenest seen when both limbs suffer, or when the animal is forced to stand continuously on the diseased limb. 
Similar stretching of the flexor tendons is sometimes seen after neurectomy performed whilst the tendons are inflarned. The ordinary weight of the body may then cause stretching of the tendon, or of the cicatricial tissue newly formed within it. Möller has seen this after neurectomy of the tibial nerve while the tendons were inflamed.

Course. The disease always occurs suddenly, and the anatomical structure of the tendons explains its subsequent chronic course. After strain or rupture, isolated hæmorrhages and hyperæmia of the tendon set in, and are followed by serous infiltration into the interfascicular

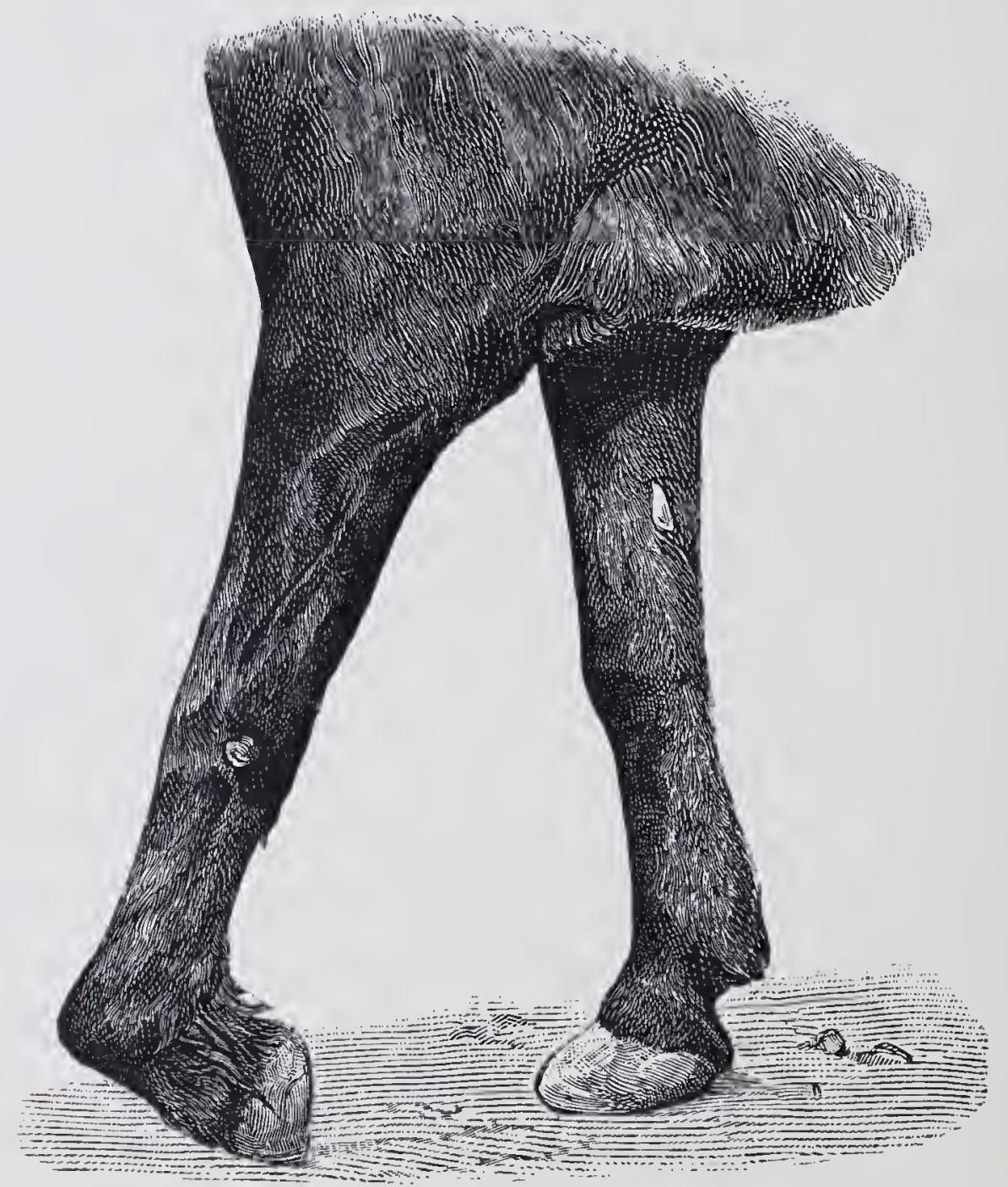

FIG. 211. - Excessive "knuckling over" in a horse (from a photograph).

connective tissue and paratendineum. The fasciculi of the tendon are thus thrust asunder, and predisposed to further rupture. At the seat of serous infiltration numerous lencocytes afterwards appear (plastic infiltration), causing the swelling, which is at first soft, to assume a harder character. On section the parts display greyish white, sometimes greyish red, masses of connective tissue. In consequence of extravasation of leucocytes into the spaces of the firm connective tissue thickening results, which may lead to shortening of the tendon, as the newly-formed tissues contracts, whilst the paratendinitis leads to adhesion with neighbouring tissues. The tendon gradually becomes permeated with blood-vessels; ossification has sometimes been seen and parasites met with in inflamed tendons. 
Zscholke describes a case in the horse, in which the sesamoid ligaments were ossified. Roloff found the flexor perforatus changed into cartilage. In these thickenings has been found filaria cincinata, which has also been discovered in the subcutis of horses.

"Knuckling over" may either be brought about by shortening of tendons, in consequence of their cicatricial contraction, or of contraction of muscles. In the first case, only the lower joints take up the position of volar flexion; when muscles are involved, the linee-joint is also bowed over. All cicatricial tissue, whether in tendons or elsewhere, is inclined to shrink, but excessive contraction is only seen when the limb is rested and entirely relieved of weight. Fig. 211, which is from a photograph, shows to what extent shrinkage may occur. If weight is placed on the limb, even occasionally, it never becomes so excessive. After contraction the fibres of the tendons no longer run in a straight direction, but take a tortuous course.

On account of the peculiar character of the pathological processes, tendinitis varies greatly in course and results. Complete resorption of inflammatory products occurs in eight to fourteen days when the case is favourable, and when rest and early careful treatment are adopted, but in most instances some thickening remains. Though lameness usually disappear's in three to four weeks, the newly-formed tissue has not the strength of normal tendon, and a point of less resistance results, which explains the frequent relapses. Continuous severe pain points to serious local change, and often causes "lnuckling over" ; the prognosis therefore depends chiefly on the degree of pain. The results of strains of the flexor tendons depend very largely on the region involved; the most serious being those which affect the flexor perforans and its check ligament, in which complete resolution is rare. Next comes the suspensory ligament. Inflammation of the flexor perforatus is less troublesome. The animal's usefulness is not impaired, even though considerable thickening remains. Needless to to say, the older the condition the less the chance of recovery; when lameness is of old standing the outlook is bad. The condition is still graver when both limbs are affected. In hacks, prognosis is less favourable than in light van-horses. When the joints are much shot-over, tenotomy is sometimes successful, but this depends on whether the flexor tendons are adherent to one another or to their lower sheaths. Adhesion of the flexor perforans to the perforatus is always troublesome, though cure is not entirely out of the question. Tendinitis occurring during infectious diseases is more obstinate than inflammation mechanically produced in otherwise sound animals. Great pain and resistance to treatment point to an infectious origin, though such swellings occasionally disappear after twelve to twenty-four hours, when the sheath of the tendon is only 
slightly involved. Möller saw a horse in which the affection changed its seat several times in a few days: leaving the hind, it attacked the front limbs, and vice versâ (rheumatic tenositis).

Treatment. The most important point is to stop work; where possible, absolute rest should be given, and the shoe removed. Fresh cases, due to mechanical injury, are treated during the first twenty-four to forty-eight hours by cold applications, the best form being immersion or irrigation, but after this time little good results from the employment of cold. Moist warmth, supplemented by proper bandaging, is then preferable, and is applied as follows:-After carefully cleansing the foot, two longish rolls of tow rather thicker than a man's thumb are moistened and laid lengthwise on either side of the diseased tendon, close to the metacarpus, and fixed there by a linen or cotton bandage, wetted and applied pretty tightly. Over this a dry woollen bandage is placed, so as to exercise regular pressure on the material below and on the diseased spot; when available, a piece of rubber tissue or waxed linen may be placed between the two bandages. Some practitioners envelop the limb thickly in cotton-wool, over which a calico bandage is tightly applied. This dressing is changed every four hours; the moist warmth and regular pressure favour reparative changes; excessive extravasation and cellular proliferation are checlied, and resorption assisted. The rolls of tow or cotton-wool must exercise even pressure on the diseased tendon.

When pain disappears massage may be resorted to during the interval of changing the dressing, the injured tendon being rubbed from below upwards with the thumb and fore finger, using moderate pressure, for about five minutes at a time, the parts being meanwhile covered with a piece of paper (see Dollar's "Operative Technique," p. 190). The operation must not be persevered with if pain or swelling follow.

When early and carefully used, massage cures and removes the swelling in cases where, with the antiphlogistic methods formerly employed, thickening almost always remained. The slight vascularity of tendons in no way supports the theory of the action of cold; on the other hand, moist warmth and methodical compression assist absorption, and are certainly not so likely to impede repair as cold. Experience shows this treatment to be by far the best.

Infriction with ointments or fluids act like massage, though the specific resolvent effect ascribed to preparations of mercury and iodine causes them to be most widely used. A mixture of unguentum hydrargyri and sapo viridis in equal quantities can be used, with double the amount of lanoline where massage seems called for. Warm baths of soap or weak potash solution considerably assist absorption.

The action of blisters is largely due to the mechanical effect of 
infriction and the pressure of the cutaneous thickening on the inflamed spot; and when the above-described treatment proves impracticable, it is good practice to apply a blister or, better still, a blistering plaster. The use of "charges," though now almost discontinued, is of considerable value in many cases of strained tendons. Roborans plaster, liquefied by heat, is spread thinly over linen strips and applied with gentle pressure from the fetlock to within an inch or two of the flexure of the knee. Occasionally the liquid plaster is applied to the skin and is covered with chopped tow; but the former method is the better. The charge is left on for six to eight weeks, provided no complications ensue. This method is less likely to blemish than the use of blisters. Pötting assists the action of the blister by a pressure dressing. About the third day, when exudation has ceased, he applies moist wadding to either side of the diseased tendon, and passes a woollen bandage over all. The first dressing remains on for two days, and is then renewed and left in place for three to five days.

Old thickenings are best treated by firing. In this case the chief effect is produced by the mechanical action of the inflammatory swelling and cicatricial shrinkage in the cutis. The lines, running obliquely from the front downwards and backwards, should not be wider apart than half an inch, nor should they meet at the back of the perforatus tendon. After the scab has fallen, the above-described pressure dressing can be again applied.

If, in old thickening of the tendons, firing is out of the question, a compress dressing saturated with "water glass" solution is useful, especially in small swellings, and where the animal cannot be rested, but precautions must be taken against dirt or sand getting under it and causing chafing.

The animals must be kept from heavy"work for some time, but can be put to exercise as soon as lameness has quite disappeared. During this stage riding-horses must be kept from jumping and continued rapid trotting, nor should they ever be suddenly pulled up.

In shoeing horses with disease of the perforans or perforatus, the heels should be spared, the toe shortened as much as possible, and shoes with calkins or thick heels used. For many years it has been disputed whether raising the heels by calkins exercises any influence on the angle of the phalanges towards the ground; it now seems agreed that this is certainly the case, for the pedal and coronet joints at least, and for the former to a greater extent than for the latter. The position of the fetlock-joint and the fetlock angle are, however, scarcely affected by the dorsal flexion so produced in the pedal and coronet joints; raising the heels, therefore, has no effect on the suspensory ligament, but it is useful in inflammation of the tendons. 
Tenotomy is the only method of treating severe "knuckling over" due to contraction of the tendons, but is only useful if no joint be involved, and if the contracted tendon be not adherent to its sheath at some point below the seat of operation, because, in order to allow the fetlock to resume its normal position, the lower end of the divided tendon must retract. Where the knee is simultaneously bent, the upper portion of the tendon must also be able to retract to allow the knee to straighten, but the operation is then seldom of use, because the bent position is due to shortening in the flexor muscles of the knee and metacarpus. Before operating, lameness must be allowed to subside, one of the conditions of success being that the limb shall afterwards carry some weight, without which the tendon further contracts.

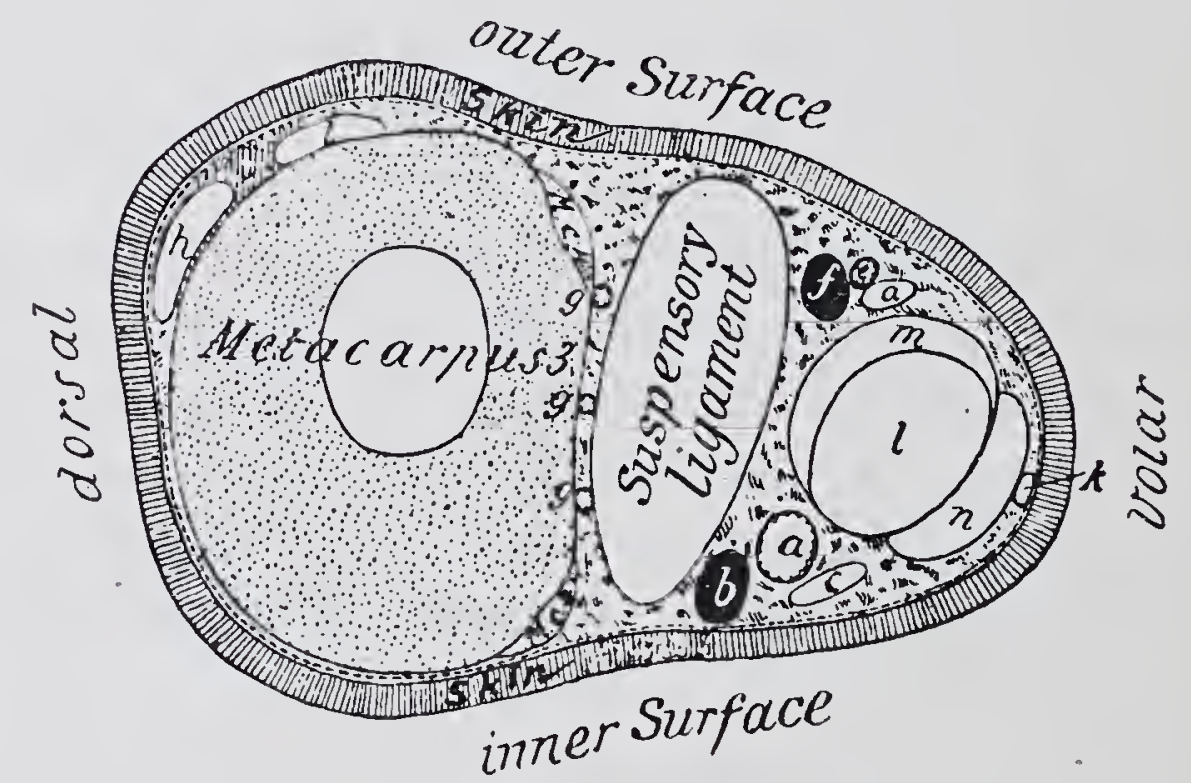

Fig. 212. - Section through the centre of the metacarpus.

$a$, Large metacarpal artery ; $b$, metacarpal vein ; $c$, inner plantar nerve ; $d e f$, corresponding external structures; $g g \mathrm{~g}$, interosseous arteries; $k$, oblique branch from intermal to external plantar nerve; $l$, tendon of flexor perforans ; $m$, its reinforcing band; $n$, tendon of flexor perforatus.

Tenotomy aims at lengthening the contracted tendon. It may be performed in various ways, though the subcutaneous is the only really successful method. Some operate from the outer side, and direct the incision either towards the suspensory ligament or towards the skin. The inside is preferable, because of the absolute guarantee this gives against injury of the metacarpal artery. The operation is, however, only valuable in young and high-priced horses, because recovery and restoration to work require six to eight weeks or even longer.

At the inner side of the leg, about the middle of the metacarpus, the hair is shaved, and the entire metacarpus washed with soap and water and rinsed with sublimate solution. A ring of sublimate woodwool is then laid round the point of operation, fixed by a bandage, and moistened three times a day with sublimate solution. In this way in twelve to twenty-four hours the metacarpus is rendered completely aseptic, so that aseptic healing may be reckoned on with certainty 
provided the other rules of asepsis are carried out. Hands and instruments must be disinfected, and sterilised dressings employed.

The horse is cast on the affected side, the foot to be operated on fastened to the cannon bone of the upper hind leg, and the dressing removed. If a stout rod is handy it may be used like a side-stick to

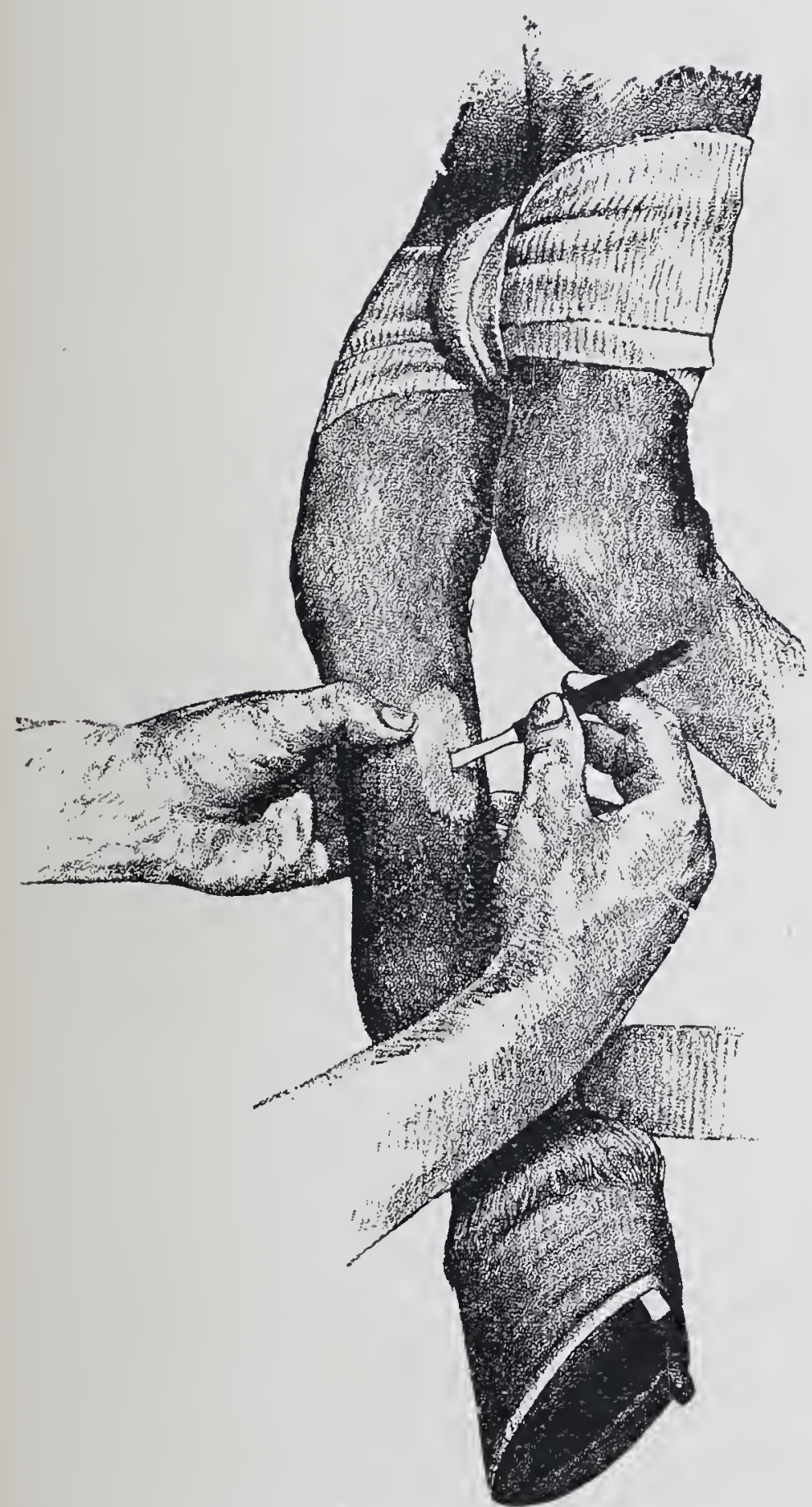

FIG. 213.-Tenotomy-first stage.

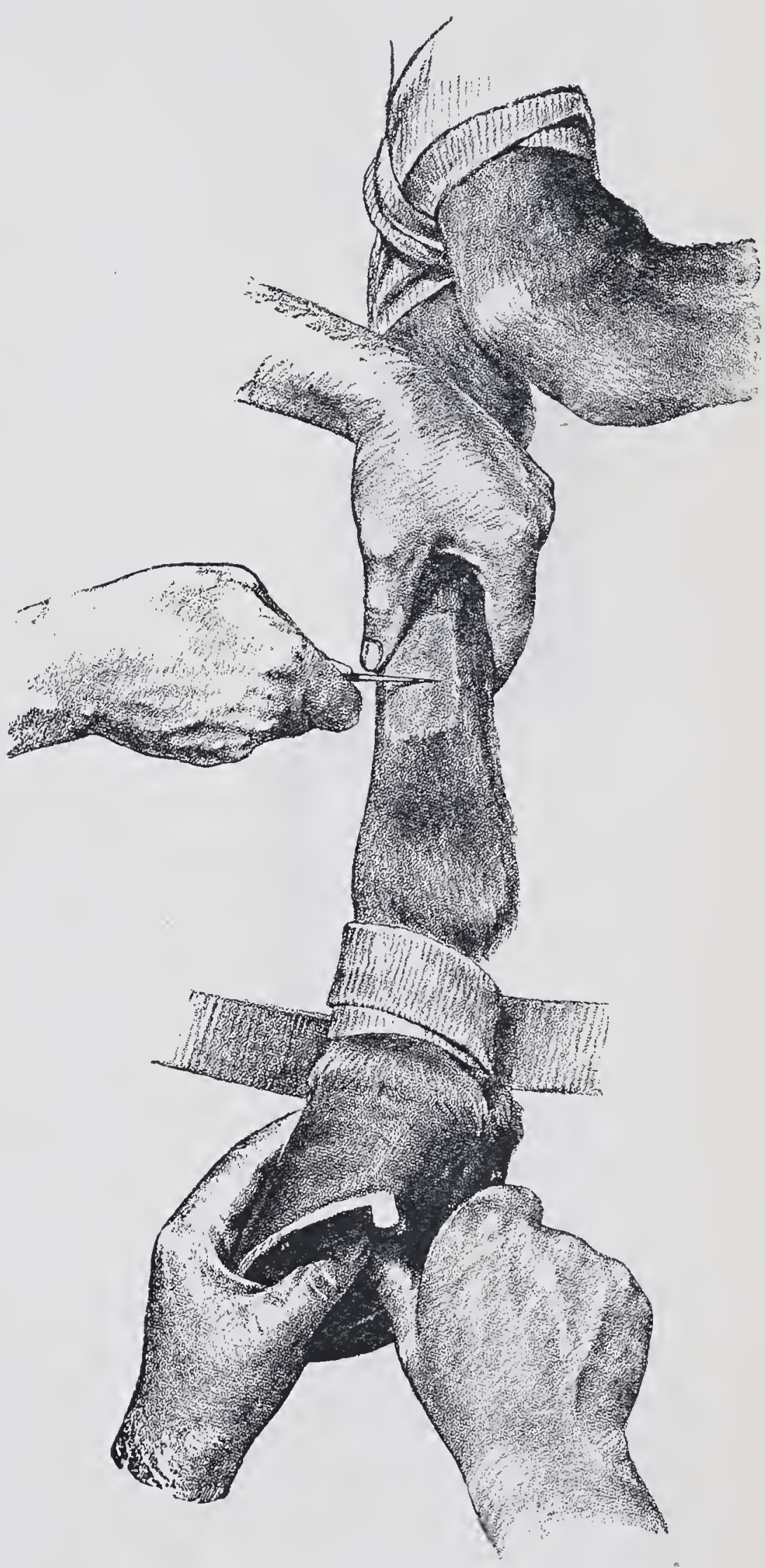

Fig. 214.-Tenotomy-second stage.

keep the limb extended. An incision through the skin is made with a pointed bistoury midway between the knee and fetlock, just above the fiexor pedis perforans, and is afterwards prolonged into it. If the tendon is thickened or adherent at this point to the flexor perforatus, the operation is repeated somewhat lower, though the lower tendon sheath must not be opened. A blunt tenotome is inserted, with its 
surface lying close to the tendon of the flexor pedis perforans, and is pushed between this and the suspensory ligament until it can be felt at the other side. Its cutting edge is then turned against the flexor perforans, the phalanx extended by a rope or by an assistant pulling it forward, and the tendon divided by slowly lowering the hand in the direction of the cannon bone. Should the ends of the tendon not retract after complete section, adhesions with the perforatus tendon probably exist, and must then be divided in a similar way.

By introducing the tenotome close to the perforans tendon the danger of injuring the large metacarpal artery is avoided. Sometimes small

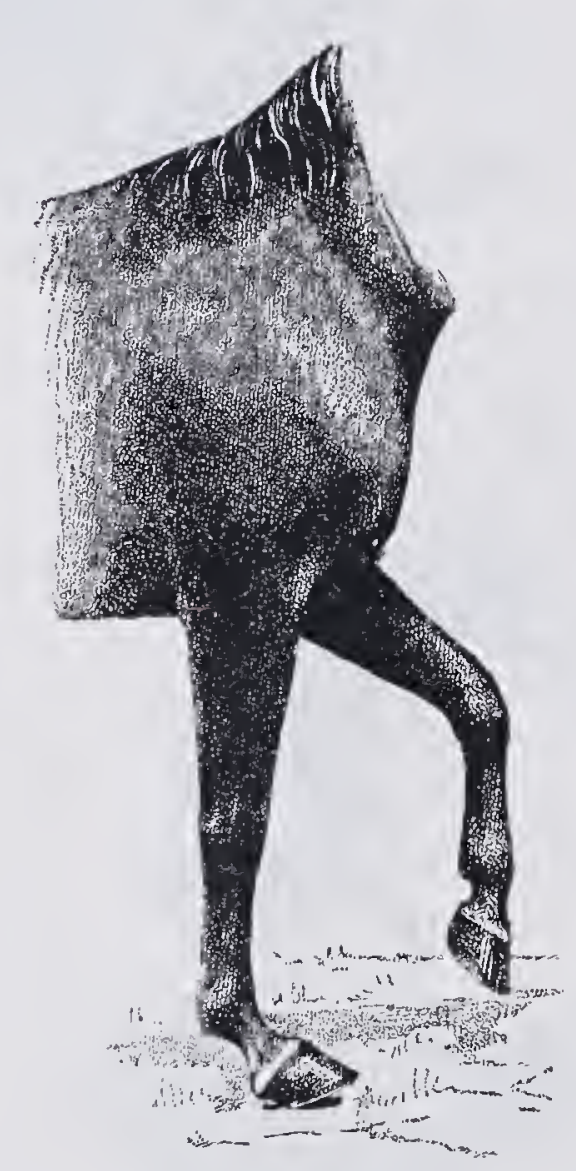

FIG. 215. - To illustrate the peculiar gait after tenotomy. The horse treads on the bulbs of the heel. cutaneous and subcutaneous veins are divided, causing a little bleeding, but this is of no consequence. The surface of the wound is then washed with sublimate solution, a tampon of jute applied to the little incision, the limb surrounded with sublimate wood-wool or similar material, a circular bandage applied, and the horse allowed to rise. If the operation is to prove successful, the fetlock-joint ought now to appear moderately extended.

Provided the dressing is not wet through by discharge, it may be left in position for a week, when the skin wound will be found completely closed. The dressing may also be saturated with sublimate solution and changed the next day; it must, of course, be changed should it become wet through with blood. After removing it the leg should be rinsed with sublimate, carbolic or creolin solution, any blood-clots washed away, and a fresh dressing applied.

With these precautions antisepsis is maintained without difficulty, even if the metacarpal artery has been cut through. In the event of such an accident, the dressing must be very tightly applied, moistened with sublimate, and changed, with proper precautions, in twenty-four to forty-eight hours.

As a rule, healing proceeds much better where only the flexor perforans is divided. The animal's usefulness is sooner restored, and, in consequence of weight being placed on the limb at an earlier period, excessive contraction of the cicatricial tissue is less to be feared. Where the flexor perforatus is also divided excessive dorsal flexion may occur, or the animal may prove unable to stand on the limb, and fresh contraction take place. For this reason tenotomy often fails; but it is 
undesirable to follow Fogliata, who first divides the perforatus tendon, and when this is not sutficient, cuts through the perforans. If the horse, when quietly standing, places sufficient weight on the leg, it may be placed in a stall, better still in a box: otherwise, on the second day after operation, it should be slowly walled for a quarter to half an hour, when possible on soft ground. T'his is almost indispensable to prevent cicatricial contraction and ensure restoration of the normal fetlock position. The frequent relapses noted are due to neglect of this precaution. For a similar reason, operation must be delayed until pain has almost disappeared, otherwise the requisite weight cannot be put on the limb. Experiment shows that mechanical extension of the cicatricial tissue is necessary to produce the characteristic spindle-formed cellular elernents, i.e., to give newly-formed tissue the character of tendon substance.

It almost goes without saying, that, either before or soon after operation, the foot must be restored to its proper form by judicious paring. Animals can sometimes be returned to work in ten weeks; sometimes, however, pain continues, preventing them being put to rapid work for a longer interval. When it persists, the lameness may generally be removed (in animals which cannot be kept idle) by dividing the median nerve. Möller repeatedly performed neurectomy and tenotomy simultaneously in horses belonging to a large horse-owner, who loaded his horses heavily, and worked them hard; and where lameness was still marked, has treated both contracted tendons and shot-over fetlocks, due to contraction of the suspensory liga-

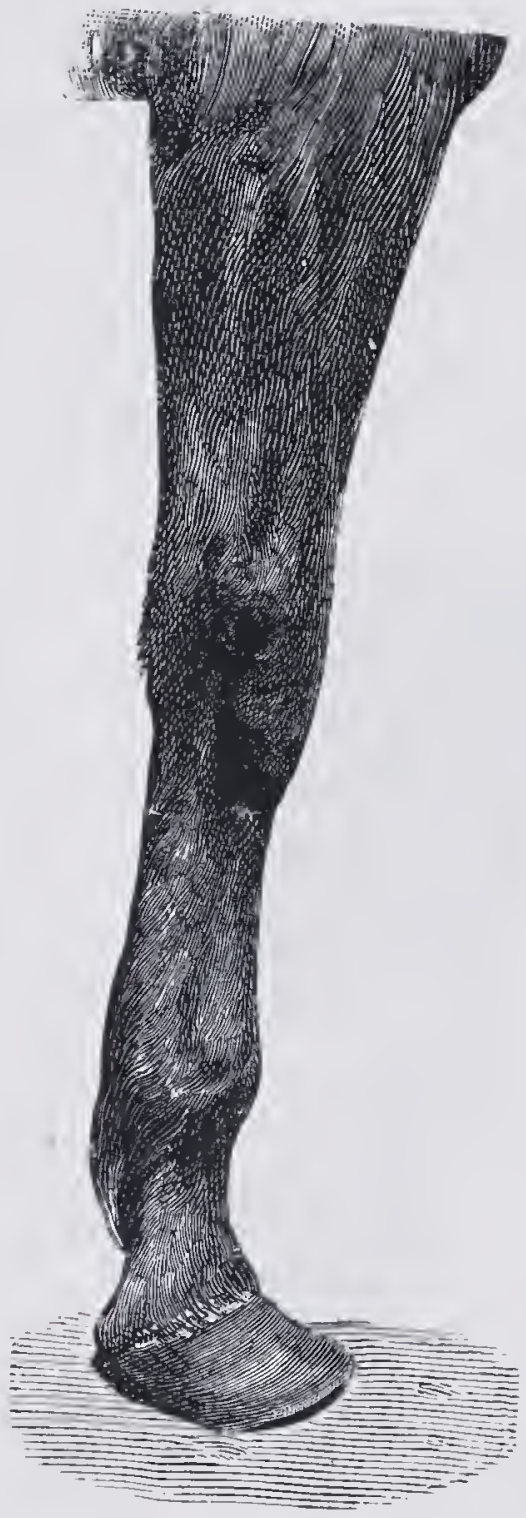

FIF. 216. - Excessive dorsal flexion after section of the perforans and perforatus tendons-the "knuckling over" at the fetlock is clue to contraction of the suspensory lioament, and has not been affected by the operation (from a photo. graph). ment, at the same time, and rendered horses workable again even in four to five weelis. ${ }^{1}$ Median neurectomy is first performed, then tenotomy. For a description of median neurectomy, see the section on "Ringbone" hereafter. As the neurectomy wound rarely heals by first intention, it must, during the first few days, be frequently cleansed to prevent discharges running down and infecting the lower point of operation. The excessive dorsal flexion (fig. 216) following simultaneous

1 Möller's own statement: "Wurde . . die Pferde oft schon nach 4_5 Wochen arbeitsfähig." 
section of the perforans and perforatus tendons, particularly in heavy horses, may be removed by wearing a long bar-shne. If needful, an iron crutch, with fork-shaped arms reaching to a point just below the fetlock-joint, may be affixed to the heels, to afford the joint a surface on which to rest. The apparatus termed an "orthosom" is of similar" character. Sometimes the suspensory ligament is contracted, causing the fetlock to remain upright, even after complete division of the perforans and perforatus tendons, a condition which experience tends to show is incurable. It occurs not only after strain and thickening of the suspensory ligament, but also after severe and old-standing "Trnuckling over."

Horses which are "knuckled over," if worked slowly and on soft ground, may be made useful for a long time by applying a long-toed shoe, though this will not cure or even effect improvement in the original

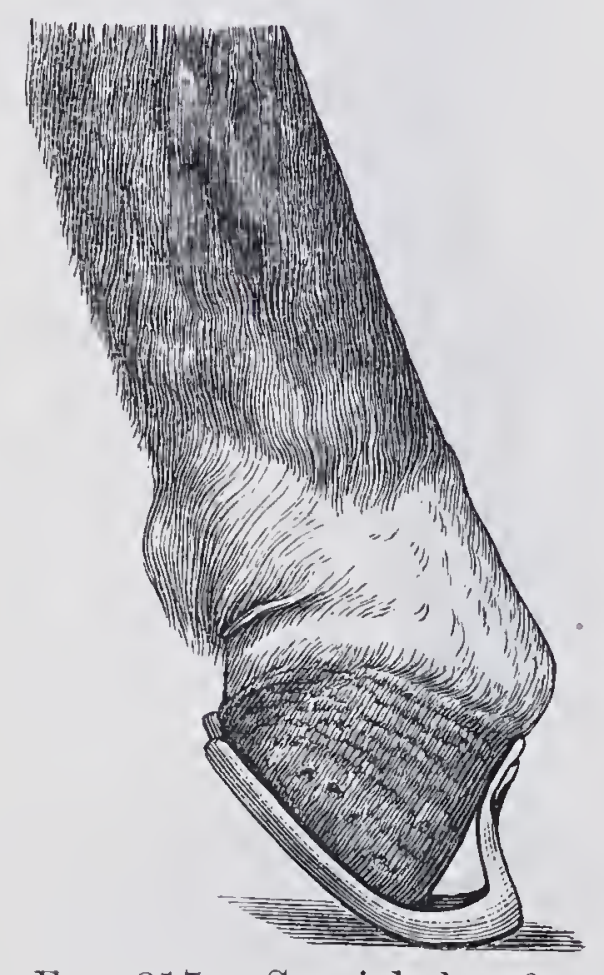

FIG. 217.- Special shoe for "knuckling." disease. Tendinitis occurring during infectious disease, especially during influenza, requires special attention, while, to prevent relapse, such animals should not be too soon put to work, or otherwise exerted; during the convalescent stage particular caution is called for, and the tendons may be bandaged with advantage.

If, during the course of any of these diseases, inflammation of the tendons or tendon sheaths occurs, mild stimulation and bandaging constitute the best primary treatment. When pain is severe, luke-warm disinfectant foot-baths often relieve it. In other cases, warm moist applications and moderate pressure on the diseased tendons may be resorted to. Sometimes the condition disappears with unexpected rapidity, but generally returns in the same or some other spot, and may often involve both limbs. The degree of swelling and pain forms an index to the gravity of the condition, which sometimes resists blistering, and even firing, and not infrequently proves incurable. In other cases, and especially where there is no marked anatomical change, recovery may occur after many months.

\section{Y.-CONTRACTED TENDONS ("KNUCKLING OVER") IN FOALS.}

In English pure-bred stock, less frequently in other breeds, new-born foals are sometimes unable to move on account of excessive "knuckling" on the fore limbs. The upright position of the fetlock prevents the flexors taking their proper part in sustaining weight. This peculiar deformity 
generally affects both front legs, often causing the animals to walk with the front of the phalanx on the ground, and preventing their standing upright. It also develops, though rarely, during the first two years of life, and never in such a serious form.

The cause is at present unexplained. Franck considers it due to congenital contraction of the flexor tendons existing at the time of birth. Others ascribe it to abnormal position of the foetus in utero, whilst Lafosse thinks it arises from disproportion between the stallion and mare, the bony framework being excessively developed when the stallion is too large. As large animals are especially affected, and as, in general, only the phalanges suffer displacement, the cause must lie between the point of insertion of the flexor perforans and its reinforcing ligaments. There are two possibilities: either the tendons are too short or the metacarpus is too long. The first may be referred either to defective development or subsequent shortening of the tendons. Whether, in such case, the development of the flexor tendons is interfered with by the position of the fœtis in utero, or whether contraction occurs, or whether the long axis of the metacarpus is excessively developed, so that the tendons are relatively too short, or whether again the point of origin of the muscles is abnormal, is for the time at least undecided. Perhaps the condition may be caused in more than one way.

Abnormal position in utero would probably interfere with development of the joints and muscular tissue. Increase of pressure on the posterior portions of the joints, caused by continued volar flexion, would check the growth of bone, while diminution in the anterior portions of the joints would favour its production, and thus tend to thrust the phalangeal joints more and more into a position of volar flexion, favour the development of the disease, and cause it to assume a certain resemblance to "pes equinus" in man. The rapidity with which the condition yields to proper treatment, however, seems to contradict this theory. Whether the cause is too little space in the uterus (too little liquor amnii), or too narrow a maternal pelvis, need not be considered here; but the frequent occurrence of the disease in pure-bred animals appear's very remarkable.

Symptoms. The most important symptom is the abnormal volar flexion in the fetlock-joint. No disease of the tendons or muscles can be detected, though they appear tensely stretched when the fetlock is upright. Where the condition is well developed, the limb is incapable of bearing weight. The new-born foal rears itself up, but cannot stand, and may die from local gangrene produced by lying (bed-sores), or is killed as a hopeless cripple. Sometimes the animals stand on the front of the fetlock-joint, and thus produce serious injuries. Where the appearance of the disease is delayed the animals can stand, but move with difficulty, 
and after some time show excessive uprightness of the hoof. The condition is distinguished from contraction produced by tendinitis by the absence of changes in the flexor tendons.

Course and prognosis. In newly-born foals the abnormal position is usually seen on the first attempt to stand, but disappear's under proper treatment in eight to fourteen days. Not infrequently these apparent cripples develop into very useful animals. Sometimes improvement occurs first in one limb, the other meanwhile retaining its

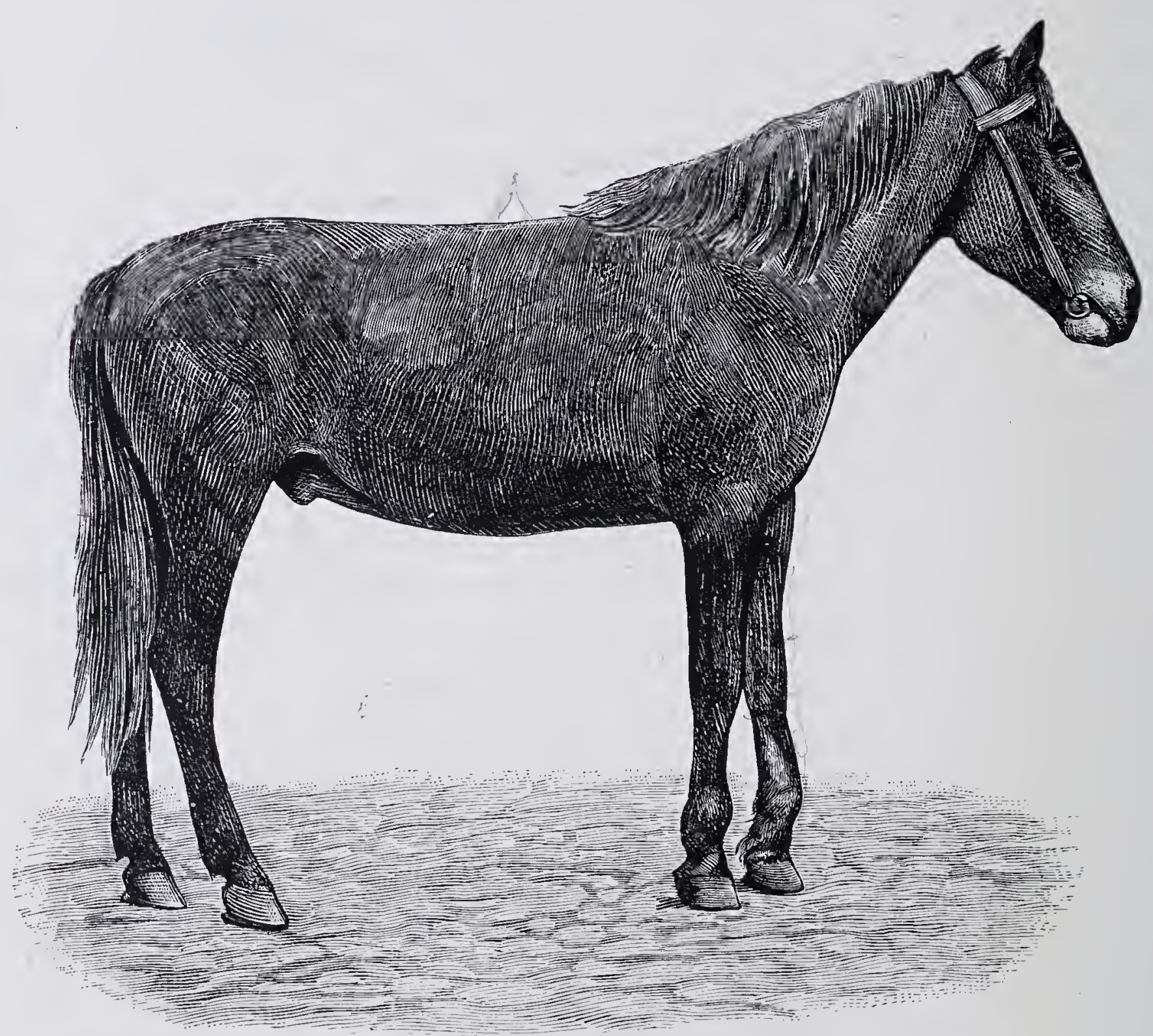

FIG. 218. -Double-sided "knuckling" in a two year old colt (from a photograph).

abnormal position. In calves the disease usually takes a favourable course.

When appearing a couple of years after birth the disease takes a less favourable course, and may, indeed, never entirely disappear. In other cases improvement follows proper treatment, though complete cure, i.e., recovery of the normal position, is rare.

Treatment. The great extensibility of the tendons in foals favours early and complete recovery. It is, therefore, important to practise forcible extension, and to such a degree that the tendons are stretched and the fetlock brought into an oblique position. For this purpose the foal is cast, the phalanges extended as far as possible and fixed 
in this position, either by using a plaster bandage or applying a strong leather splint. Ehrle uses a plaster bandage or a glue bandage and splints. The parts are freely padded with wadding to distribute pressure. The degree of success is usually astonishing, especially when treatment is early resorted to. Weight can often be placed on the limb after a few days; the bandage should then be removed, because the weight of the body acting through the oblique position of the pastern will produce further extension of the tendons, and is quite sufficient to prevent contraction. Friebel's extension apparatus (fig. 219) appear's very practical. It consists of a small leather shoe, provided in front with a well-padded iron splint. By means of a screw the splint can be
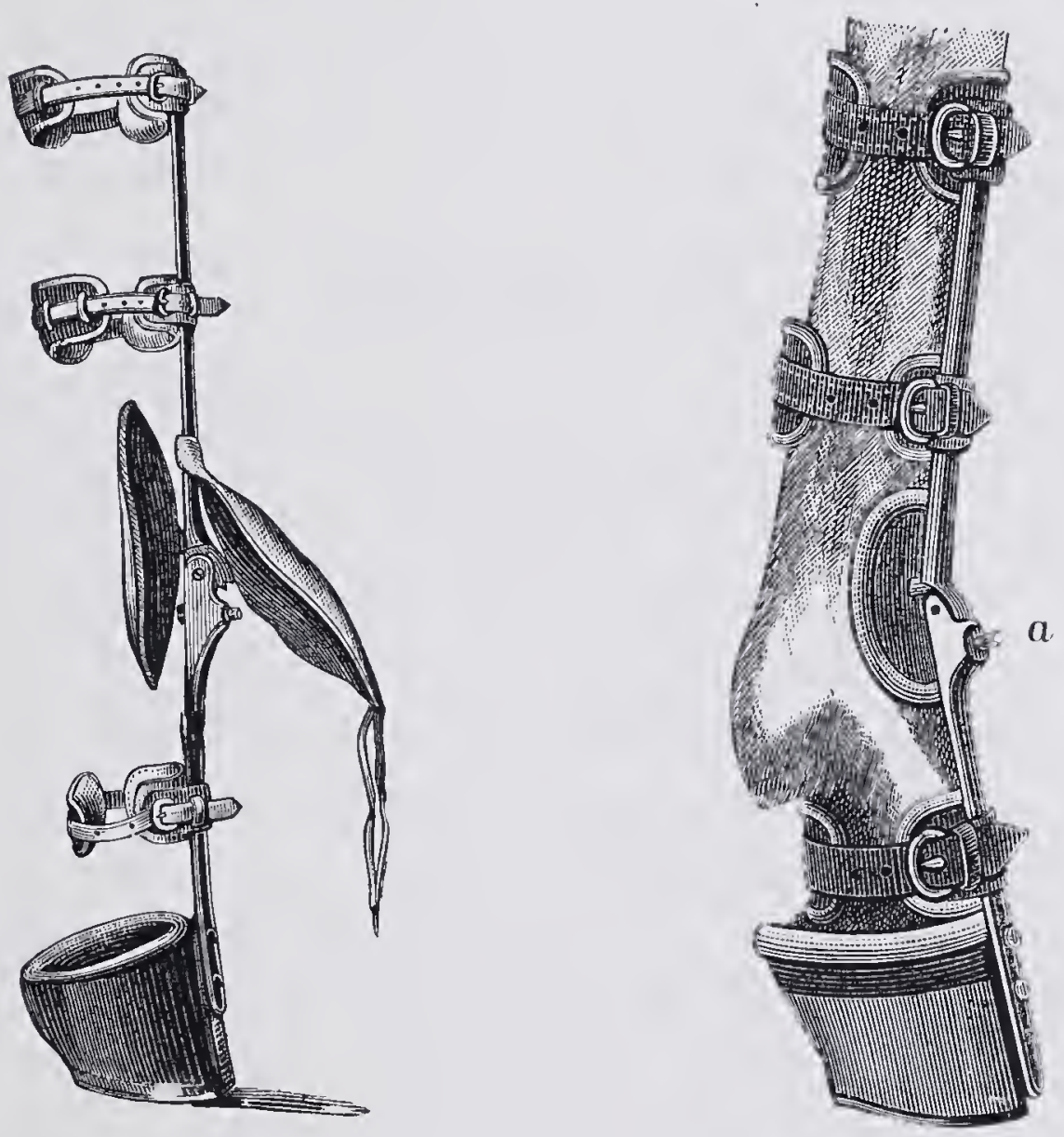

FIG. 219.-Friebel's extension apparatus.

so fixed as to exercise pressure on the anterior surface of the fetlockjoint and thrust the latter backwards, i.e., into its normal position.

Eassie saw "knuckling over" in all four limbs in a five months old foal. In front the disease was cured by tenotomy, behind by proper shoeing. Ostertag cured congenital contraction of the tendons in a foal in four weeks by applying a wooden splint and plaster bandages, which were changed several times. As the dressing accommodates itself to the gradually improving position of the fetlock, Ostertag considers it necessary to change it two or three times.

Hofer had a gutter-shaped splint made from pine wood which reached to the fetlock; he states having seen recovery occur in one to three weeks. "Knuckling," which sets in two years or so after birth, even when unaccompanied by inflammatory disease of the tendon, can seldom be so 
completely removed as to restore the animal's working power's. Trager, however, states having cured cases by section of the perforans and perforatus tendons.

Eichbaum suggested the use of a long-toed shoe; Bombach, who regarded the disease as a secondary matter resulting from uprightness of the hoof, cured it by freely lowering the heels. Matz applied blisters and recommended turning the animal out.

Brunet used a peculiar extension apparatus, which started from the shoe and exercised pressure on the front of the knee-joint. Experience shows that treatment should depend on the degree of displacement; when slight, it is sufficient to freely lower the heels, but should this be unsuccessful, a long-toed shoe may be used. If needful, tenotomy can be performed, it being sometimes sufficient to divide the flexor perforans. Tenotomy is, however, seldom necessary, and can only be recommended as a last resort.

\section{VI.-SPLINTS.}

Pathology. Splints are due to a form of periostitis which is frequent in horses though rare in ruminants, and which develops between the large and the inner small metacarpal, or on the inner, rarely on the outer, surface of the large metacarpal itself. On account of its frequency - for splints are met with in most horses - and of its interference with usefulness, it is not surprising that even in the earliest times this condition received attention both from practitioners and authors. Jordanus Ruffus described splints as supraossa, and Solleysel distinguished five varieties of them. At first they were regarded as a secretion from the bone, and it was only in the present century that their dependence on periostitis was clearly demonstrated. The real disease, i.e., the condition causing lameness, is a periostitis ossificans, associated with formation of exostoses. Two periods ean, therefore, be distinguished: the first that of inflammation-of periostitis; the second that of complete development, in which inflammation is absent. During the first, lameness is therefore very general, but disappears during the second.

The process usually starts in the periosteum or in the interosseous ligament, which fixes the internal small splint bone to the cannon bone. Irritation in its deep layers produces growth of bone and swelling, the permanence of which led older writers to view it as an exudate. The process of splint formation is completed by ossification of the newlyformed material. The size of the splint depends on the extent of surface involved and the duration of the periostitis, and varies between that of a threepenny and a five-shilling piece. The splint itself generally assumes an elongated form, lying parallel with the small splint bone. When periostitis has passed away, and the formation of the splint is 
complete, pain and lameness generally disappear, though the splint itself remains as a blemish. In many cases, however, especially in young horses, severe work is again and again followed by lameness, causing the disease to persist for a long time. The mature exostosis gradually diminishes, sometimes by becoming flattened, sometimes by the neighbouring bone becoming thickened, and under certain circumstances may so far recede as to require a careful examination to detect it. The late H. G. Roger's drew attention to the frequent occurrence of exostoses on the metatarsal bones, and suggested the possibility of their causing lameness. Though they undoubtedly do so in occasional cases, as Dollar has convinced himself, the proportion of such cases is probably small.

The cause of periostitis consists in traumatic irritation of the interosseous ligament, periosteum, and bones. On account of their almost invariably occurring on the inner side, the view was once held that splints were produced by striking. Havemann was the first to expose this error, and to point out the longish shape of the splint, and the fact that injury of the skin is very rare. He directed attention to the construction of the carpus, and especially to the position of the small trapezoid bone (os multangulum minus hom.), and viewed this as a cause of the disease. Whilst on the outside the unciform (os hamatum hom.) rests partly on the outer small splint bone, partly on the metacarpal bone, on the inside the small trapezoid rests almost entirely on the inner small splint bone. This explains why faulty action or unequal distribution of pressure in the knee may throw an excessive load on the inner small metacarpal, and cause ruptures in the interosseous ligament fixing the small to the large metacarpal. The incomplete union between the bones in young animals explains the frequent occurrence of splints during early life. At a later period union is ossific, and the disease therefore seldom occurs.

This explanation of Havemann's was generally viewed as correct, until recently Dieckerhoff raised a doubt about it, and stated that the disease of the periosteum was produced by the pull of the fascia of the fore arm. He describes splints so produced as "spontaneous," in contra-distinction to those caused by blows, which he terms "traumatic." The lower insertion of the fascia of the fore arm is partly into the metacarpal bones, especially the inner small metacarpal, partly into the subcutaneous connective tissue. Ruptures of it, and especially of its point of insertion into the bone, are said by Dieckerhoff to give rise to disease in the subfascial connective tissue, which disease extends to the periosteum. The facts adduced by Dieckerhoff in support of this modification of Havemann's view seem insufficient, and by no means conclusive. There is much evidence in support of Havemann's showing. The disease generally begins in the interosseous ligament, between the small and large metacarpal bones, whilst no disease processes of any kind can be detected in the fascia. Nor can œdematous swelling of the subcutis be regarded as an infallible sign of the condition referred to,

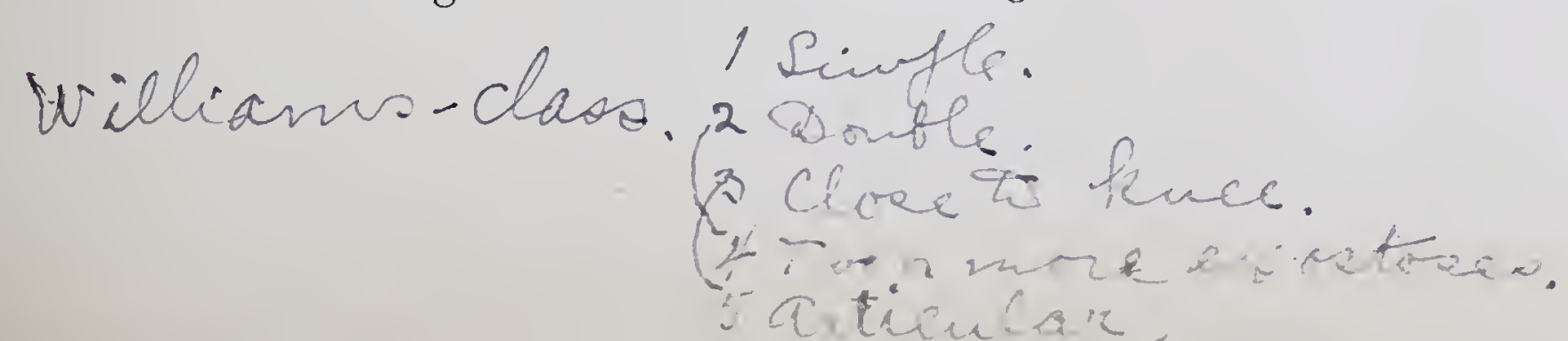


for it is scarcely ever absent in periostitis in other positions. To this must be added that inflammation may also be caused by violent tension on the other ligaments, which become attached to the inner small metacarpal bone, and may lead to disease in the spot in question. For prognosis and treatment, it is, of course, of little importance which view one takes. The most important difference is that, according to Havemann's view, pressure, according to Dieckerhoff's, tension, is the cause of the periostitis.

In any case we may divide splints into two classes-those due to unequal distribution of pressure, and those due to blows. The two kinds admit of easy clinical distinction. The first is generally of a long shape, and is always on the small metacarpal; not infrequently several lie one above the other, having been formed simultaneously or soon after one another. Those produced by blows are generally further forward and on the large metacarpal, i.e., they appear in front of the small metacarpal, are generally of a rounded shape, and are discrete. Cicatrices or injuries of the skin cannot always be detected.

Horses with bad action often strike themselves. The weight is irregularly distributed in their joints, and therefore such animals have a double disposition towards the production of splints. This is particularly true of those which turn the toes either outwards or inwards excessively, as well as of those which stand with the legs widely extended or too close together. Defective shoeing may lead to splint formation by its favouring striking and unequal distribution of weight in the joints. During military evolutions injuries are often caused by striking, by unequal distribution of weight, especially in restive horses, and also by the animal being tired or constitutionally weak. Continued severe work on stones in large towns favours the production of splints. Coarsely-bred animals suffer oftener than well-bred, and the splints are usually larger. Inasmuch as defective formation is often a cause of their production, the inheritance of a disposition to them can be explained without resorting: to Didfield's idea that the drinking-water has some influence.

Many peculiarities of the disease still require explanation, such as its simultaneous and exactly symmetrical appearance on the metacarpus of both fore legs, even in old horses, and the more frequent appearance on the left fore limb. The reports of the Prussian army for 1891 show that exostoses occurred twice as often on the left fore limb as on the right. It is possible that the frequently performed "right gallop" may afford an explanation of this.

Symptoms and course. (1) Lameness. The pain resulting from periostitis produces lameness, which is therefore a regular accompaniment of the development of splints. It is seldom absent, but its character depends partly on the position, extent, and intensity of the process, partly on the work done and on the animal's sensitiveness. 
Lameness is less common in coarse-bred than in well-bred horses. When periostitis is caused by mechanical injury, lameness disappears with its subsidence, and only returns if the injury be repeated. In such cases it lasts about three weeks. Partial rupture of the interosseous ligament recurs very easily, causing the lameness not only to continue for longer periods, but also to return, and therefore splints of a longish shape occurring on the small metacarpal continue to interfere with the animal's usefulness for a long time, frequently for many months. In periostitis near the linee there is danger of inflammation extending to the knee-joint; the lameness then continues for longer periods, sometimes indeed becomes chronic, from changes in the knee producing arthritis deformans, and from mechanical interference with the mobility of the joint by large exostoses in its immediate neighbourhood. Splints at this point following on injury often produce chronic lameness.

Lameness may therefore be caused-

1. By periostitis.

2. By inflammation of the knee-joint.

3. By formation of extensive exostoses near the knee, which interfere with free movement. The idea that lameness is caused by the splint pressing on the flexor tendons is erroneous.

In general, splints produce lameness, in the free limb (swinging leg lameness) and abduction of the limb; the knee is not fully flexed. The lameness is better seen on hard than on soft ground, is much more marked at the trot than at the walk, and is aggravated by exercise. Weight is generally placed on the limb, and the hoof in most cases is put down firmly and in the usual manner. The excessive tension in the fascia of the fore arm during the last phase of movement, just before the limb is lifted and during its extension, causes painful pressure on the inflamed periosteum.

Abduction is particularly marked when the knee-joint is also involved. Extensive exostoses interfering with movement of the knee can be detected by passive movement; attention is sometimes drawn to them by the knee being held stiffly, and only partially flexed during movement.

(2) Local examination. The development of periostitis is characterised by slight swelling, increased heat, and pain on pressure. The swelling shows a certain degree of hardness, but in consequence of cedema of the skin is at first of doughy consistence. This gradually disappears, the swelling becoming firmer and harder. To detect pain the opposite foot is lifted and the hand placed with the thumb resting on the outside, the fingers on the inner side, of the affected metacarpus. By passing the finger's from above downwards, it is easy to estimate the tenderness of the periosteum. To prevent mistakes, the same process should be gone through with the sound limb. In practised hands this 
examination is perfectly easy, though in some cases care is required to avoid prominence of the "button" on the lower end of the small metacarpal with periosteal swellings. On the other hand, inflammation of the skin is sometimes difficult to distinguish from periostitis, though, as a rule, painful lameness is here absent.

It has been said that splint lameness is easily mistaken for that of navicular disease, because the lameness produced by periostitis usually increases with movement; this, however, seems erroneous, for navicular lameness decreases, and often disappears entirely with exercise. Nevertheless, the other portions of the limb should always be carefully examined, in order to confirm the diagnosis. This is particularly necessary when the pain produced by pressure is slight.

Prognosis is generally favourable, especially where the position and character of the periostitis point to mechanical injury. Exostoses of a longish form occurring on the small metacarpal and in young horses are apt to be troublesome. The chance of recovery is less if, in consequence of defective formation of the limb, the parts are likely to be struck by the other foot. Prognosis is doubtful when the knee is coexistently diseased, or when extensive exostoses have formed close to it, and opinion should be reserved even when the patient, after a long rest, shows no lameness. In cases of this kind in young horses, the splints which remain not infrequently cause renewed lameness on every attempt to work, and, as a rule, never disappear completely.

Treatment may be called for by the presence either of periostitis, lameness, or exostosis. The treatment of periostitis first demands the removal of the cause. Skilful shoeing is important to prevent striking and to effect a proper distribution of weight, but scarcely comes within our present province. Rest is essential, particularly when the disease is clearly not of mechanical origin. The splints so common in young foals and yearlings often disappear spontaneously, and it is better to wait a reasonable time rather than produce permanent blemishes by undue haste in treatment.

Local applications of cold water, mercurial ointment, tincture of iodine, \&c., are seldom of use. In adults a blister may be indicated provided the skin is uninjured. Cantharides ointment, though often recommended, has less effect than caustic mercury preparations, especially sublimate, employed in concentrated solution. This was a favourite application of the older practitioners, but its use demands caution. One part of sublimate dissolved in four parts of spirit is applied with a feather, and sharply rubbed in. The cutaneous and subcutaneous swelling produced exercises general pressure on the inflamed periosteum, and limits exudation therein. Firing answers the same object, though, without doubt, the subsequent rest is of great importance. 
When objected to, these methods may be replaced by a pressure dressing. A mixture of equal parts of mercurial ointment and lanolin should be rubbed in smartly once a day, and a proper pressure bandage applied.

Periosteotomy, though revived from time to time, has not been much practised. The operation sometimes shortens the duration of the pain and tension in the periosteum which causes lameness, but usually it neither diminishes the morbid changes nor lessens the lameness, while it sometimes occasions considerable thickening, and even necrosis of bone. Perhaps these troublesome complications might be avoided by strict antisepsis, but the recorded cases are insufficient to settle the question.

Needle firing has also been recommended and deserves trial. The best method is probably that described by Dollar as " antiseptic firing," where the parts are prepared and afterwards treated like a surgical wound (see Part I. of this series, "Operative Technique").

Ablation of splints by the use of the gouge or chisel and mallet has been practised since Lafosse's time, but seems a barbarous method of removing a growth which very frequently denotes the need for local increase of substance. It could only be justified where the splint formed a mechanical impediment to movement, as, for instance, when the animal was in the habit of striking it with the opposite foot.

Removal of exostoses is assisted by massage and methodical compression. The former can seldom be continued sufficiently long, though, when the necessary assistance is available, the splint may be diminished by rubbing it daily with a firm leather pad or similar object. The treatment must, however, be persevered with for months. A better plan is as follows:-A piece of lead (a leaden bullet, beaten flat) or thick soleleather is fixed over the exostosis with moderate firmness by a bandage. The pressure thus produced, if continued for some weeks, often brings about considerable diminution in size. Care is needed to avoid causing necrosis of skin, and it is therefore well to change the dressing frequently, where possible daily, and examine the skin. By employing massage, resorption is hastened.

A peculiar periostitis on the large metacarpus (occasionally on the metatarsus), termed "sore shins," is seen in young race-horses. It always appears suddenly after violent gallops, and is usually confined to the lower half of the bone. The affected limb is rested, or if both limbs suffer, the weight of the body is alternately shifted from one to another. When moving, the leg is not properly lifted, and is extended stiffly; the horse goes "short." Soon after, a hot, very painful swelling (periostitis) apear's on the front of one or other, sometimes of both shin-bones. It usually disappears in a few weeks, though necrosis of bone has occasionally been observed-perhaps, in consequence of the treatment. 'The disease occurs 
almost solely during the first two years of life, is seldom seen during the third and fourth years, and never occurs in old horses. Subcutaneous periosteotomy, formerly recommended, is of very questionable value. Unless performed with strict antiseptic precautions it may lead to suppurative periostitis - a still more dangerous condition than "sore shins." Complete rest, low diet, and warm moist applications are usually sufficient in the early stages. Later on a mild blister is useful.

If pus form, an ample depending opening must be made and the fullest antiseptic precautions taken. Warm antiseptic baths, in which the leg is immersed above the knee, may be continued for several hours every day until pus formation and pain diminish. Dry dressings are then useful. The horse should afterwards have several months' rest and be very slowly brought into training.

\section{E. DISEASES OF THE PHALANGES.}

Anatomy. In solipeds, the phalanx consists of the pastern, coronet, and pedal bones, with the two sesamoid bones and the os naviculare. In ruminants, two completely developed and two rudimentary phalanges exist, with four sesamoid bones and two navicular bones.

The pig has four phalanges-two true and two rudimentary; eight sesamoid and four navicular bones.

In the five phalanges of carnivora, the first consists of two, the others of three joints. Each has two sesamoid bones, whilst the navicular bone is wanting. On the other hand, a small lenticular bone exists in the tendons of the extensor pedis, on the anterior surface of the joint between the metacarpus and first joint, as well as between this and the skin.

In the horse, the three joints of the phalanx, i.e., the fetlock, coronary, and pedal, form partly incomplete and partly complete ginglymoid joints.

The fetlock, a complete ginglymoid joint, possesses a capsular ligament strengthened laterally by fibrous bands, its synovial membrane has a dilatation behind which extends, for a considerable distance upwards, between the two portions of the suspensory ligament and the fetlock, to unite with the tendon of the flexor pedis perforans. The strong lateral ligaments, in conjunction with the cylindrical form of the articular surface, prevent any side movement. The fetlock-joint is further provided with two lateral sesamoidal ligaments, which, reinforced by the crossed ligaments, connect the sesamoids with the cannon bone and the first bone of the phalanx.

The intersesamoidal ligament connects the two sesamoid bones. The fetlock-joint is so fixed by the upper and lower sesamoidal ligaments as to prevent excessive dorsal flexion and assist the function of the flexor tendons. The superior sesamoidal (suspensory) ligament passes in an upward direction from the superior border of the sesamoid bones to the lower row of carpal bones and upper end of the metacarpus. The inferior sesamoiden ligaments are three in number. The superficial is fixed below to the glenoidal fibrocartilage, behind the articular surface of the os coronæ, ascends behind the os suffraginis, and is inserted into the base of the sesamoids and intersesamoid ligament. The middle ligament is fixed to the back of the os suffraginis and inserted into the base of the sesamoids. The deep consists of a few crossed fibres which arise from the back of the os suffraginis and are inserted into the sesamoids. 
The pastern is an incomplete ginglymoid joint lying about an inch above the upper margin of the hoof. On account of its flat articulatory surface, it allows of limited side movements, especially when in a position of volar flexion. Its tense capsular ligament is attached anteriorly to the extensor tendon, laterally to the lateral ligaments; behind it is expanded, and lies in contact with the branches of the inferior sesamoidean ligaments. At the

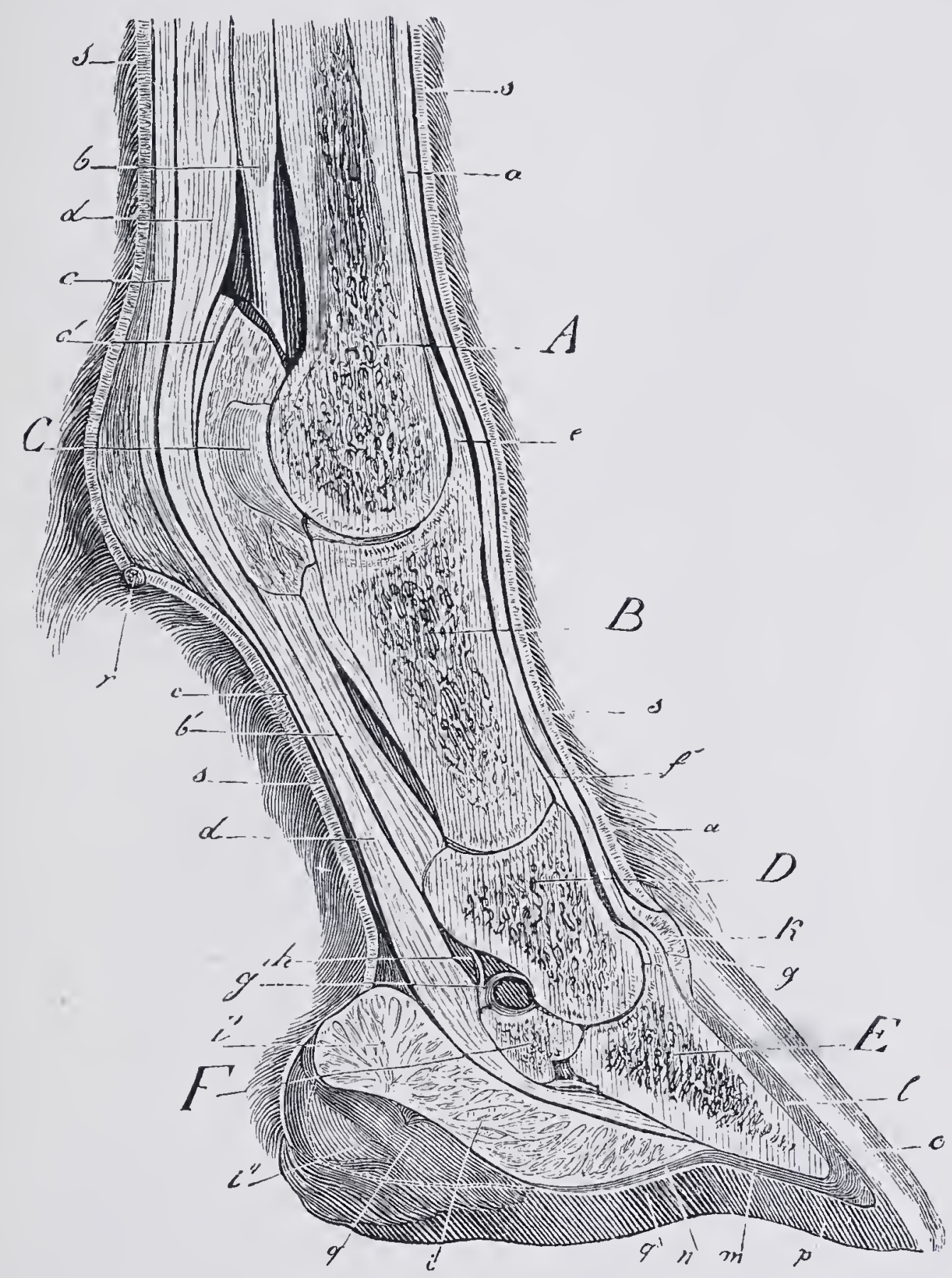

Fig. 220.-Perpendicular mesial section of right fore foot (the position of the lower bones is shown rather too upright). $A$, Lower end of great metacarpus; $B$, suffraginis or pastern bone ; $C$, inner sesamoid bone (to render the bone visible, a portion of the intersesanoidean ligament has been removed); $D$, coronet bone ; $E$, pedal bone; $F$, navicular bone ; $a$, extensor pedis tendon; $b$, superior sesamoidean or suspensory ligament; $b^{\prime}$, iníerior sesamoidean ligament ; c, flexor pedis perforatus tendon ; $c^{\prime}$, great sesamoid sheath ; $d$, flexor pedis perforans tendon; $e$, capsular ligament of the fetlock-joint; $f$, capsular ligament of pastern-joint; $g$ and $g^{\prime}$, capsular ligament of coffin-joint; $h$, bursa of flexor pedis perforans ; $i$, plantar cushion ; $i^{\prime}$ portion of plantar cushion forming the bulbs of the heel $k$, coronary band; $l$, sensitive wall ; $m$, sensitive sole ; $n$, sensitive frog ; $o$, horny wâll ; $p$, horny sole ; $q$, horny frog; $r$, ergot at base of fetlock; $s$, skin.

sides are two short, stout lateral ligaments, whilst behind, the joint is supported by the inferior sesamoidal ligaments in the same way as the fetlock is by the suspensory ligament; the inferior sesamoidal ligaments run from the posterior or lateral borders of the pastern-joint to the under portion of the sesamoid bones. The posterior articulatory surface of the os coronæ is enlarged by the glenoidal fibro-cartilage attached at this point.

The pedal-joint lies within the hoof near the lower border of the coronary

V.S. 
band. Its synovial capsule extends from the margin of the articulatory surface of the os corone to the os pedis and os naviculare. Though in front tensely stretched, it expands and becomes thinner in a backward direction, and is in connection with the tendon of the flexor pedis. Its two lateral ligaments render the pedal an incomplete ginglymoid joint. The postero-lateral ligaments are physiologically identical with the superior and inferior sesamoidal ligaments. They arise from the upper border of the navicular bone, and are inserted on the lateral aspects of the os coronæ and the lower portion of the os suffraginis. The tendon of the extensor pedis passes over the anterior, those of the flexor pedis perforans and perforatus over the posterior surface of the phalanges, which here are almost entirely surrounded by tendon sheaths. In company with the sesamoidal ligaments, these tendons act in fixing the fetlock-joint; with the posterior coronary ligaments, and assisted by the postero-lateral ligaments, they also fix the coronet and pedal joints, and thus sustain the weight of the body. The larger blood-vessels and nerves pass down the outer and inner sides of the phalanges.

The terms dorsal and volar flexion are hereafter used to describe the movements occurring in the phalanges: the first indicates the movement given by the extensor, the second that given by the flexor, muscles.

\section{I.-LUXATION OF THE PHALANGES.}

The union of the three phalangeal joints is so strong that displacements (luxations) are exceedingly rare, and must always be preceded by extensive rupture of ligaments or tendons. Luxation of the fetlockjoint is seen in race-horses after complete rupture of the flexor tendons; the lower end of the metacarpus then passes downwards over the posterior margin of the articular surface of the os suffraginis, causing excessive dorsal flexion of the fetlock-joint. Less frequently, the metacarpus is displaced anteriorly ; this indicating rupture of the lateral and sesamoidal ligaments. Lateral displacements are exceedingly rare, and are necessarily accompanied by rupture of the lateral ligaments. They are sometimes produced by violent struggles to free the foot which has accidentally become fixed, as, for example, between railway-metals. Wilhelm saw lateral displacement in the hind leg of a horse which had run away, and had fallen. The joint was directed outwards. Siedamgrotzky has described three similar cases. In other animals luxations of the phalanges are still less common.

The diagnosis is easy. The altered position of the juint, and the changes discovered by manual examination, leave no room for doubt.

The course of luxations is almost always unfavourable, on account of the excessive rupture of tissue. The larger animals either die from decubitus, or more or less marked lameness remains which prevents the animal working. Reported cases, however, show that recovery may occasionally take place.

Wilhelm records recovery in eight weeks in the above-described case. He placed the horse in slings, and after reducing the dislocation, applied 
a wooden splint fastened to the metacarpus by straps. Treatment was directed to moderating inflammation, and later a dressing and wooden splint, provided with a hinge.over the fetlock-joint, were applied.

Schellhase has seen similar cases. In one it was possible not only to place the os suffraginis at a right angle with the large metacarpal, but a
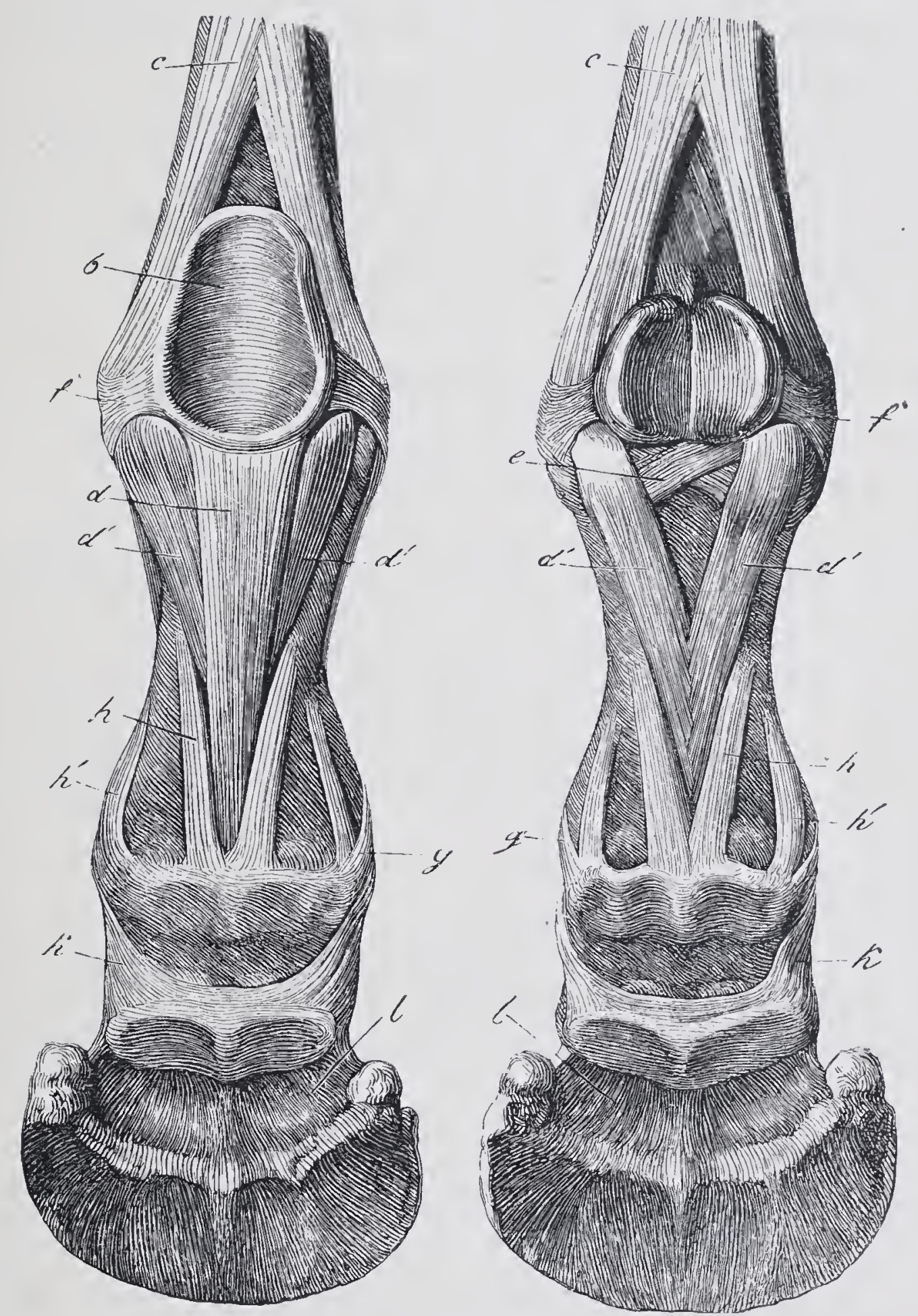

Firs. 221-222.-a, Extermal lateral liganent of pastern-joint; $b$, intersesamoidean ligament; $c$, superior sesamoidean ligament ; $d$, middle limb of inferior sesanoidean ligament ; $d$, lateral limb of inferior sesamoidean ligament; $e$, cruciate ligament; $f$, lateral sesamoidean ligament; $g$, outer lateral ligament of the pastern-joint; $h$ and $h^{\prime}$, posterior corono-suffraginal ligaments: $i$, outer lateral ligament of pedal-joint; $k$, postero-lateral ligaments of navicular bone ; $l$, fibrous sheath of synovial membrane of coffin-joint.

wound existed which admitted the finger between the os suffraginis and metacarpal bone; nevertheless, recovery occurred. Such cases, are, however, quite exceptional. As a general rule the horse must be destroyed.

Luxation may occur spontaneously, or without any particularly violent effort. A pure-bred stallion showed very severe lameness alternately in 
both hind legs. Rheumatism was suspected and treatment directed accordingly. One morning the horse was found lying down with the left hind fetlock luxated. On being approached to apply a bandage, the horse attempted to rise and luxated the other fetlock. On post-mortem, the extensor and flexor tendons were found intact, but the synovial capsules and lateral ligaments were ruptured. In cases of this kind it is almost certain that inflammation or degenerative changes must have previously occurred in the lateral ligaments.

Johne gives the result of a post-mortem on a horse after luxation of the os pedis, where the lower end of the os coronæ had been displaced backwards and outwards, and lay above the os naviculare. The capsular ligament was consequently torn, and the flexor perforans ruptured and inflamed.

Treatment follows general principles. Reposition and control of inflammation are, however, very difficult. Anæsthesia is generally necessary before reduction can be effected.

\section{II.-SPRAIN OF THE CORONARY JOINT.}

Whilst luxations of the phalanges are rare, sprain is more common, particularly in the coronet-joint. It occurs either from excessive dorsal or volar flexion, or forced lateral movement in the joint, caused generally by slipping to the side, and consists in violent stretching, sometimes in partial rupture of the ligaments.

Such accidents are favoured by-

(1) Defective formation of the joints. Small articular sufaces offer less secure support, and are generally furnished with weak ligaments. This is particularly true of joints which appear narrow when seen from in front.

(2) Faulty position of the limb. The most important of such faults is turning the toes outward or inward. If the axes of the joints are not perpendicularly under the centre of gravity, unequal stress must be thrown on the ligaments every time weight falls on the limb; in addition, unequal weight being thrown on the inner and outer portions of the joints, the gait becomes insecure.

(3) Narrow hoofs and excessive lowering of the wall. Where one side of the wall comes in contact with the ground sooner than the other, the load is unevenly distributed, and the articular ligaments are apt to suffer. Horses which have worked for a long time under such conditions generally show excessive lengthening of the ligaments, best recognised in a position of volar flexion. The immediate causes of sprain are slips, falls, or injuries sustained in rising. Such accidents. are favoured by irregular pavement, high callins and heels, narrowness of the chest, and lameness. The condition is therefore commonest 
in large cities, and during the winter. Less frequently the animal is caught in deep ground, or between fixed objects like railway-metals. The commonest complications are fractures of the os coronæ; next to these come fractures of the pastern. The lateral ligaments of the coronet-joint are most exposed to sprain.

Symptoms. Lameness appears suddenly following a slip ; the animal avoids placing weight on the limb, and movement is often painful. The foot is rested, and held in a position of volar flexion. Pain is evinced, both on passive rotation of the joint and when the animal is turned round. At a later stage, weight may be placed on the limb when the animal is in the stable or on level ground, though attempts to turn are followed by distinct recurrence of lameness. This is due to the fact that moving on level ground produces little tension in the articular ligaments. The affected part is warm and swollen, and there is often marked pain on pressure.

Careful examination of the separate joints usually reveals the position of injury, especially if the fetlock-joint be fixed and the coronet rotated, or vice versâ. Passive rotation of the fetlock-joint, however, does not produce so marked an effect as that of the two lower joints, because the form of its articular surface effectively limits movement. It is often difficult to discover whether the pedal or coronet joint is affected, but luckily the question is of no practical moment.

Course and results. Sprains and the inflammation resulting from them vary greatly in extent and intensity. A slight slip may produce violent momentary pain, in consequence of strain of the ligamentous apparatus, though such usually disappears with proper rest in twentyfour to forty-eight hours, when the condition may be regarded as cured. When sprain is complicated with rupture of ligaments, great pain is shown, but often diminishes rapidly, and may even disappear for a short time. It returns after some hours, however, and then continues. The second attack results from inflammation, and is in direct relation to its intensity and extent. When the strain and rupture are still more severe, pain continues longer, and inflammatory symptoms (increased warmth and swelling) appear early: Slight cases completely recover within a period varying from a few days to several weelis, according to their gravity, but a disposition to fresh injury exists, which is directly proportional to the changes in the joint. Cases of extensive and intense inflammation of the joint, consequent on injury to ligaments (periarthritis), generally last four to six weeks, but may be followed by complete recovery. In severe cases the diseased ligaments are apt to contract and impede free movement of the joint, which is also distorted. Excessive volar flexion may then occur, especially after inflammation of the fetlock-joint. Limitation in the movement of the joint is shown by 
lameness, and by the upright position of the os suffraginis. Inflammation of ligaments often extends to bones and produces exostoses, particularly where the ligaments are inserted into the os suffraginis and os coronæ.

The synovial membrane may also be affected, causing distension of the cavity of the joint with inflammatory exudate (hydrops articuli) and chronic enlargement. In other. cases blood may pass into the joint (hæmarthrosis), producing on movement a peculiar sound which has been likened to that made by squeezing snow in the hands " snowball sound"). Necrosis of bone occasionally occurs, and produces severe symptoms, or even death.

Siedamgrotzky noticed in horses three cases of rupture of the lateral ligament of the hind fetlock, which recovered in three to eight weeks.

Percival made some anatomical researches on the production of articular diseases, and found the fetlock-joint anchylosed in five cases, the coronet-joint in forty cases, and the pedal-joint in sixteen cases. When implicating the coronet-joint, the condition is termed ring-bone (see section on that disease).

The rare occurrence of sprains in the fetlock-joint must be referred to the form of its articular surfaces which acts as an effective check on movement. Of the ginglymoid joints, the coronet suffers more frequently.

Prognosis depends on the extent and duration of injury. The degree of pain is generally a fair criterion of the mischief, though the early appearance of inflammation is more significant. The immediate pain which accompanies "treads" is of less importance than that due to strains. Although in the former cases pain may be so severe as to cause sweating and loss of appetite, it disappears almost as rapidly as it sets in, provided no grave complication occur. The delayed pain, due to inflammation, is of most importance in determining the prognosis; when appearing twelve to twenty-four hours after the accident it shows that grave injury has taken place, and if accompanied by marked fever is a grave symptom. The older the condition, the more slowly does resolution proceed. Hence the old rule: a sprain uncured in two weeks will require not less than four, and if then uncured will last at least a further four, and so on. Long duration predisposes to fresh attacks.

Treatment. The chief indication is perfect rest, but in large animals is unfortunately difficult to provide for. All movement should be avoided, and the limb supported or fixed by firmly applying a strong linen bandage. During the first three days cooling applications keep down inflammation and modify pain, but after this time the only symptom justifying employment of cold is continued severe pain. 
After the fourth day, absorbents like mercurial ointment generally act better. Warm moist applications are of the greatest service, and should be supplemented by pressure, which conduces alike to local rest and resorption. In many cases this treatment is sufficient. If, after diminution of inflammatory symptoms, severe swelling remain, the parts may be massaged with a mixture of equal parts of mercurial ointment and soft soap (B.P.), and walking exercise be given. If this prove unsuccessful, blisters of cantharides or sublimate may be applied; point or line firing is even better. In obstinate cases this treatment may with advantage be alternated, blisters being followed up by moist warmth and methodical compression, which is especially useful for chronic thickening.

Rest is necessary throughout the treatment, and even to test his soundness the horse should not be moved more than necessary. To prevent further strains, the floor of the box should be flat and be covered with a layer of sawdust, sand, or peat. The bedding should be abundant, but if straw is used it should be cut into eighteen-inch lengths to prevent it winding round the animal's legs. When lameness disappears, the animal may be slowly exercised on soft ground-if possible on sand-and precautions taken against fresh slips.

The long-toed shoe, formerly recommended against "knuckling," should be carefully avoided, on account of its aggravating any existing inflammation in the injured ligaments. Contraction is best combated by slow, cautious exercise during the period of convalescence.

The diseased joint may sometimes be fixed by a plaster bandage. In the fetlock the best application is a splint formed of several thicknesses of stout canvas sewn together.

As the coronet-joint can scarcely be fixed in this way, however, strict rest and the application of a blister may be substituted.

\section{III.-FRACTURES OF THE PHALANGES.}

In the horse, fissures and fractures are commonest in the os suffraginis, comparatively rare in the os coronæ and os pedis. Such fractures may be transverse, longitudinal or comminuted, simple or compound. They are often the result of violent slips or sudden turns, and therefore are especially frequent in race-horses. They may also be caused by jumping or falling in races, but in addition occur in heavy van-horses employed for slow draught, particularly in winter when the roads are frozen. In riding-horses a frequent cause is sudden turning, simultaneous fracture of the os suffraginis in more than one limb being produced in this way. Röder saw in a horse, transverse fracture of both front and of the right hind pastern bones following a fall. Wentworth describes fracture 
of both front pastern bones in a mare. Whether a peculiar predisposition existed, or whether the cause was the sudden strain thrown on the still intact bone in consequence of the first fracture, could not, of course, be decided. Tuson, in the above-described case, found a want of inorganic substances in the fractured os suffraginis, but, somewhat characteristically, gives no particulars of any analysis. In solipeds generally, and race-horses in particular, fissure of the os suffraginis is not infrequent. It was first noted in England, and Robertson and Williams described split pastern as quite a common occurrence. The condition is sometimes overlooked, being mistaken for strain of the joint. Prolonged rest in the stable seems to favour its occurrence; many of the cases of multiple fracture, i.e., fracture of the pastern in more than one limb, were seen in horses which had just returned to work after long idleness. Peters made a series of observations, and explained why fissures of the os suffraginis almost always start from the middle depression (figs. 223, $224,225)$. According to his view, the split is produced by the prominence on the lower articular surface of the metacarpus at a time when weight is unequally distributed over the two articular surfaces of the pastern lying on either side of it. Violent rotary movements of the supporting limb act similarly, and explain why fissures follow sudden turning on the fore limbs. Sometimes the split extends through the entire bone as far as its lower margin, sometimes it passes towards one side. Not infrequently it is very short, and ends close below the upper articular surface, and is followed by periostitis and the production of an exostosis (fig. 224).

Transverse fractures are also common in the horse, and affect sometimes the upper, more frequently the lower, end of the os suffraginis. They likewise result from unequal distribution of pressure during slips. In one case Möller was able to detect during life that the inner border of the upper articular surface had been broken away by the violent pull of the internal lateral ligament, the outer border by the pressure of the metacarpus (fig. 226). Cases of transverse fracture, i.e., cases where the fracture started from the external lateral ligament and extended to a varying distance downwards (fig. 227), are not uncommon. That figured had been caused by suddenly throwing the horse on his haunches. Comminuted fractures of the os suffraginis are sometimes seen. Johne saw a transverse fracture unite in eight weeks without much callus formation; Dressler found a race-horse's pastern broken into nineteen pieces; Bonnard counted thirty-four pieces in a similar case.

In complete fractures diagnosis is seldom difficult, on account of there generally being some crepitation. When the lateral prominences of the bone are broken off, the fetlock-joint shows abnormal lateral mobility. On the other hand, careful examination is required to detect 
fissures. The sudden severe lameness, associated with volar flexion of the fetlock, is apt to give the impression that the case is one of distortion, and therefore the limb should always be examined for

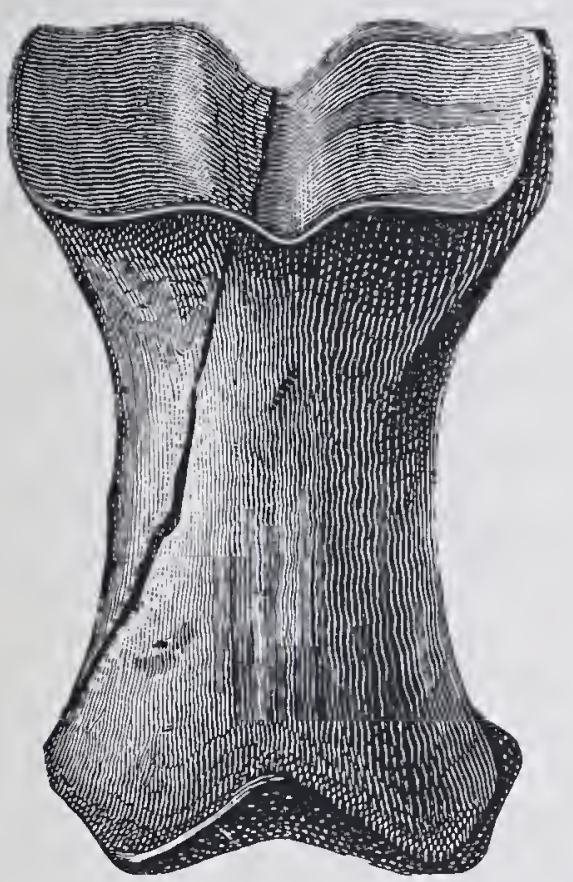

Fig. 223. - Fracture of os suffraginis.

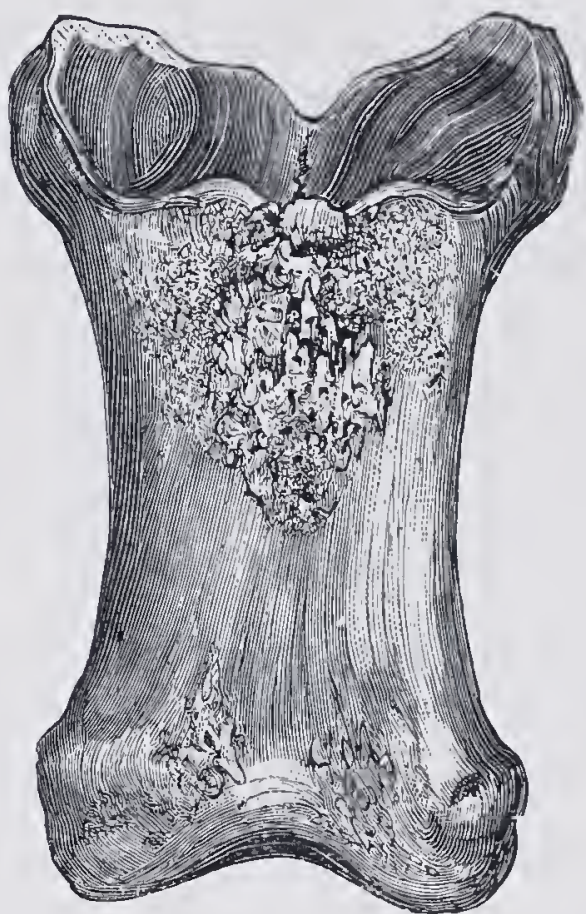

Fig. 224.-Fracture of os suffraginis with formation of exostosis.

fracture wherever lameness is of sudden onset and severe. When passive rotation of the foot produces no crepitation, the anterior surface of the pastern should be firmly manipulated, beginning at the centre of

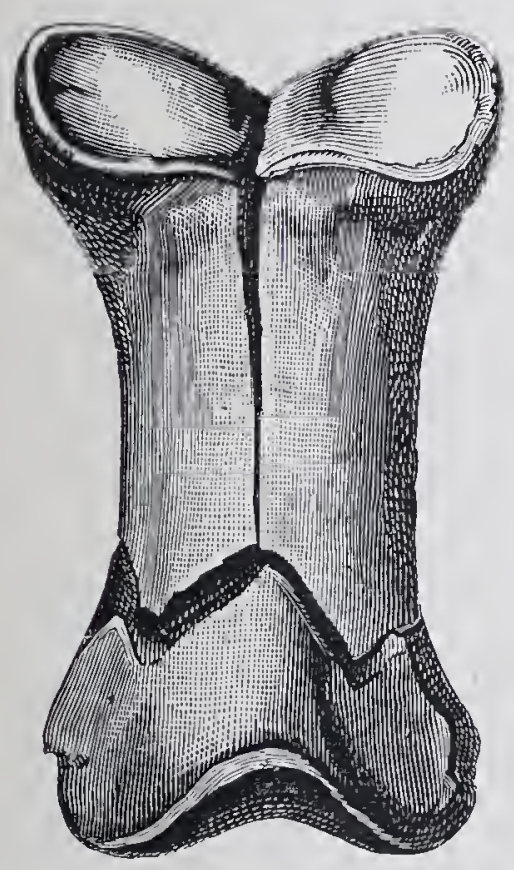

Fig. 225.-Transverse fracture of os suffraginis.

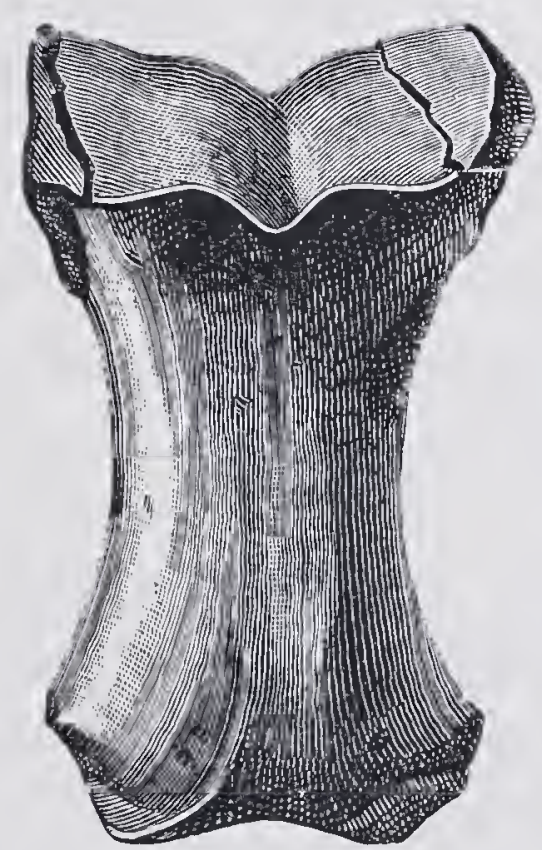

Fig. 226.-Donble fracture of upper end of os suffraginis.

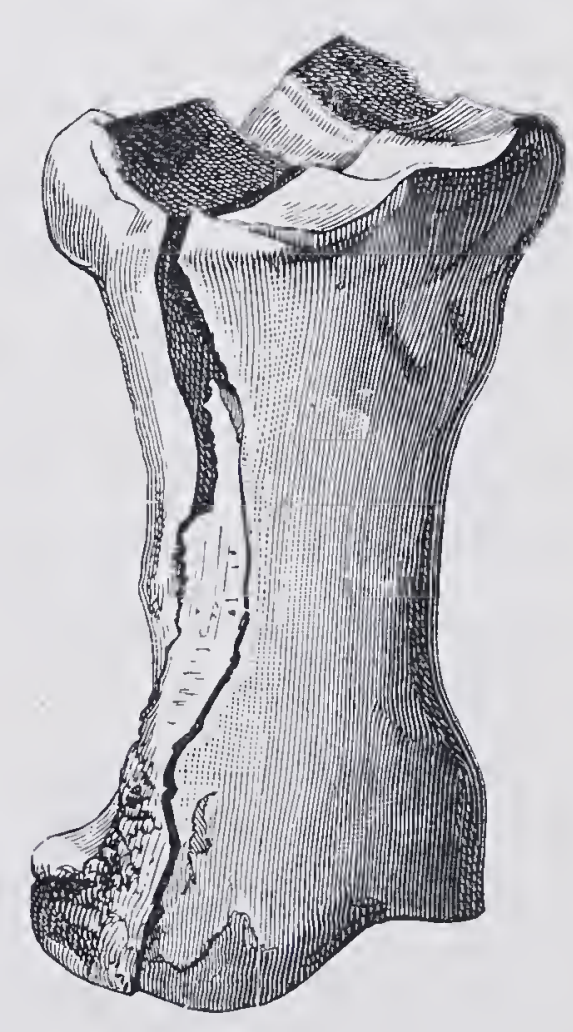

FIr. 22\%,-Longitudinal fracture of os suffraginis.

the upper end. Starting from here, a line of increased sensibility may be traced nearly corresponding to the course of the extensor tendon, and passing towards one or other side of the bone. In most cases the fissure runs towards the outer side - a fact perhaps due to the smaller size of the outer division of the articular surface. During this examination 
the limb must of course be at rest, otherwise it would be impossible to properly estimate the degree of local pain. It is only after the lapse of some time that periostitis or exostosis can be detected at the upper end of the bone.

In solipeds fractures of the os coronæ are caused in the same way as those of the os suffraginis. They are a common sequel of the foot being caught between railway-metals. The bone is either split longitudinally, or may be broken into several fragments. Henon saw the os coronæ broken into seven, Schrader into six, and Lafosse into twenty pieces. Henon saw a horse with fracture of the os coronæ in all four feet; sometimes only the ligamentous prominences are torn away. Möller mentions a case in which the bone was fractured into a number of pieces by a nail penetrating through the frog. Fissure and simple fracture are rarer in this bone than in the os suffraginis.

Fracture is detected in the same way as in the suffraginis, and when complete offers no difficulty. Crepitation in this region points to fracture of the os coronæ, provided the suffraginis is known to be intact.

Fracture of the os pedis is comparatively rare, though it has been seen by a number of observers. It is caused in the same way as fracture of the other phalanges, i.e., by slips, particularly when animals are suddenly reined-up; but fracture of the pedal bone also follows injury by picked-up nails. Fracture is common in horses which have been "unnerved." In this case its occurrence is partly explained by loss of sensation in the foot producing abnormal action, partly by alteration in the nutrition of the foot and changes in the bone. It is well known that after chronic lameness the nutrition of the bones of the affected limb generally suffers. According to Williams, fracture also results from the heels of the shoe being caught in railway-points, and, in heavy horses, from falls. Lemhöfer saw fissure of the os pedis produced by the horse striking the foot against a wall when jumping. This fracture united in four months, Schrader also noted fractures of the ossified lateral cartilage.

Diagnosis is very difficult, crepitation being rare. Only occasionally can it be produced by rotating the foot or pressing the heels together, and in its absence the diagnosis must be arrived at by a process of elimination, and by considering the history. Fracture of the os pedis may be surmised when severe pain is shown on attempts to place weight on the foot, when the lameness is of sudden onset and attended with marked volar flexion, and when, at the same time, there is no acute inflammation of the flexor apparatus or in the loof. Increased pulsation in the arteries does not occur until twenty-four hours after fracture. Wüstefeld found the superficial veins of the foot greatly swollen. Examination with farriers' pincers generally, though not invariably, 
causes pain. Slesarewsky saw fracture of the os pedis in consequence of a large portion of the hoof being torn away by the foot catching in some obstacle. Several pieces of bone were pulled off with the horn, but recovery followed the use of carbolic dressings.

Prognosis is most favourable in fissures of the os suffraginis, after which many animals recover their usefulness if rested for six to eight weeks, provided laminitis does not attack the other foot from the continuous standing. Complete fractures of the os suffraginis have also been known to reunite. Popow describes five cases of fracture of the os suffraginis which recovered under the use of the plaster bandage, though in two cases the fetlock-joint became anchylosed. In two cases the coronet-joint was affected, and in one both joints. In the case described by Halder, the animal could be put to light field-work after a month's rest, though slight lameness and thickening of the os suffraginis remained. Both Kretowicz and Wilhelm saw transverse fractures of the os suffraginis of hind feet unite completely in a ferw months. T. A. Dollar successfully set the fractured near fore pastern of "Sceptic" when the animal was six months old. The bone was broken into four or five fragments, and there was considerable displacement. "Sceptic" afterwards won thirteen races, many of importance. Foals and yearlings are much more hopeful subjects than full-grown animals, and even though a large callus forms and lameness persists for a time, the animal may eventually be rendered useful by neurectomy. As a rule, it is only worth while treating complete fractures when the horses are young, are valuable for breeding, or can be kept for a small sum. Compound fractures are generally incurable, though a few have been successfully treated by antiseptic methods.

Fractures of the os coronæ are still less favourable, recovery being incomplete, and lameness remaining even in quiet animals, and where the fracture is simple. As a rule, the bone becomes greatly enlarged, and excessive volar flexion, particularly of the fetlock-joint, sets in, and is followed by permanent lameness.

Fracture of the os pedis is less dangerous, because the parts are held in position by the hoof. Recovery chiefly depends on whether the other foot endures the strain of continued standing without suffering from laminitis. The prognosis is therefore ruled by the degree of pain. In Wüstefeld's case the hoof became enlarged from pressure exerted by the displaced fragments.

Compound fractures of the os pedis or os coronæ, caused, for example, by picked-up nails, are generally incurable, on account of the impossibility of securing asepsis, though, when the case is quite recent; and disinfection thorough, healing sometimes occurs in a comparatively short time. 
The treatment of fractures of the phalanges consists principally in perfect rest and the use of slings. In fissures of the os suffraginis a well-fitting linen bandage is generally sufficient, though a plaster bandage may be applied if the horse be slung. Plaster bandages should extend well above the fetlock and as far down as the hoof in order to limit movement of the limb. Considerable care is required in padding the limb previous to applying the bandage itself, otherwise troublesome sores may form. Stolz saw complete union of fracture of the os suffraginis follow the employment of his ointment dressing (see "Fractures of the Metacarpus"). In valuable horses this method may be tried after successful reduction. Cold-water applications are usually valueless, and have the positive disadvantage of softening the skin and favouring excoriation by the bandage.

Fractures of the os coronæ are similarly treated, but, as bandaging is here of less value, one is often confined to merely resting the animal. Excessive pain may be reduced by cold baths or applications. Stripping the sole, formerly practised in such cases in France, is to be carefully avoided, as inflammation and suppuration are thereby greatly favoured.

The lameness which follows fracture of the phalanges may sometimes be removed by neurectomy of the median nerve, and the animal thus rendered useful for a time.

In slinging animals in the above and other conditions, the following points should be borne in mind. The slings serve principally to prevent the animal lying down, and to temporarily support it in painful conditions like lameness; but as in severe lameness of one limb the opposite limb is continuously loaded, laminitis unfortunately too often occurs, and it is therefore better when the animals are in good condition, and able to lie down without injuring the diseased foot, to favour this by giving plenty of straw and abstaining from the use of slings. Many practitioners prefer sawdust to straw as a bedding in these cases. It is clean, gives a secure foothold, does not wind round the legs like straw, and when fresh is slightly antiseptic.

Laminitis and displacement of the pedal bone may be recognised by pain and increased pulsation in the metacarpal artery. Immediately it occurs the patient should be removed from the slings, provided with a soft bed, and allowed to lie down. The practitioner must be careful not to mistake the symptom of weight being placed on the diseased foot as a sign of improvement. 


\section{IV.-RING-BONE.}

The term "ring-bone" has been used since olden times to indicate many chronic diseases about the coronary joint attended with swelling. At first such swellings were regarded as resulting from a single cause, but careful anatomical examination shows that they may be due to very different disease processes. The following forms of ring-bone have been differentiated:-

(1) Articular ring-bone. This consists in an arthritis chronica. Sometimes it consists of an arthritis chronica deformans in which the articular cartilage forms the point of origin of the disease, sometimes it follows synovitis. The articular cartilage of the os suffraginis or os coronæ is

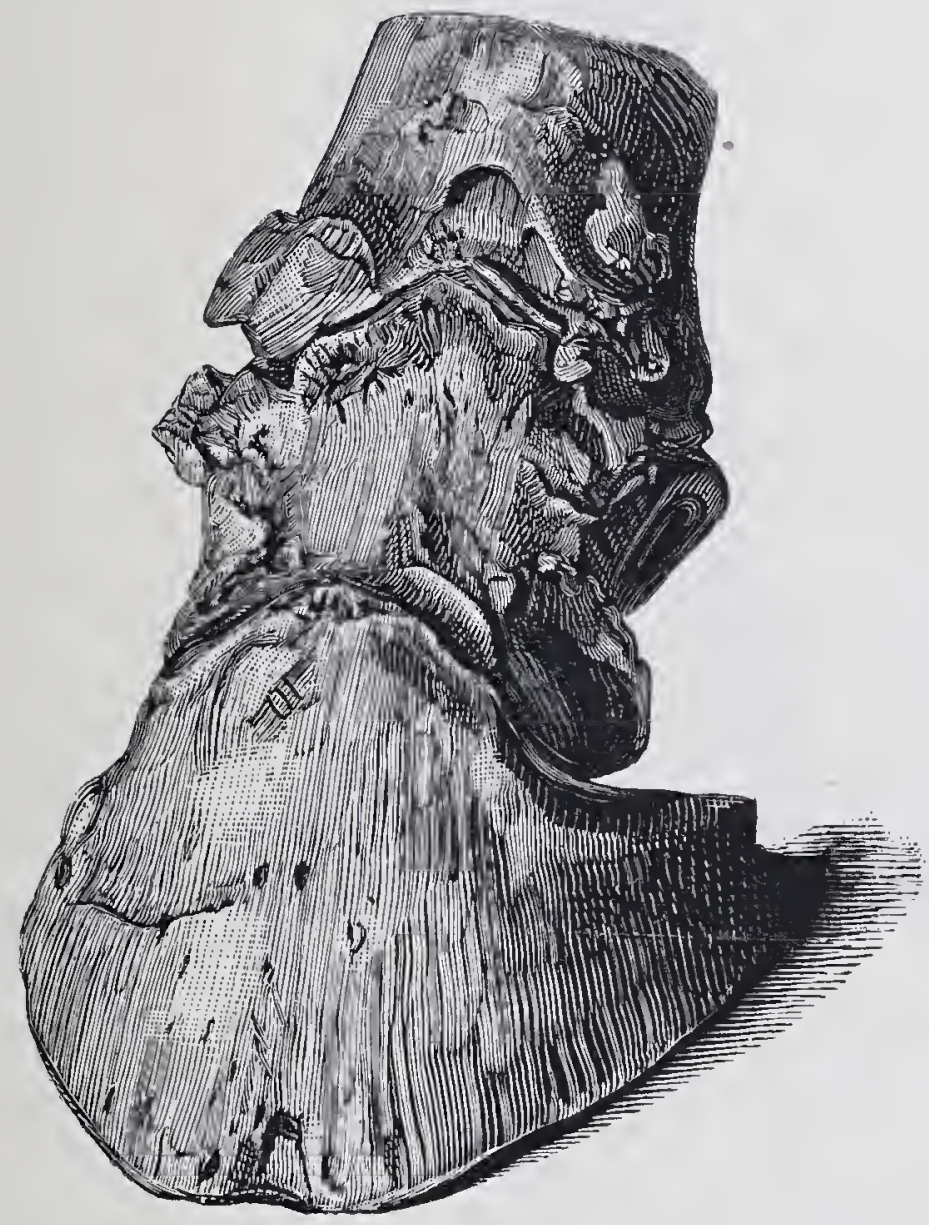

FIG. 228.-Articular ring-bone.

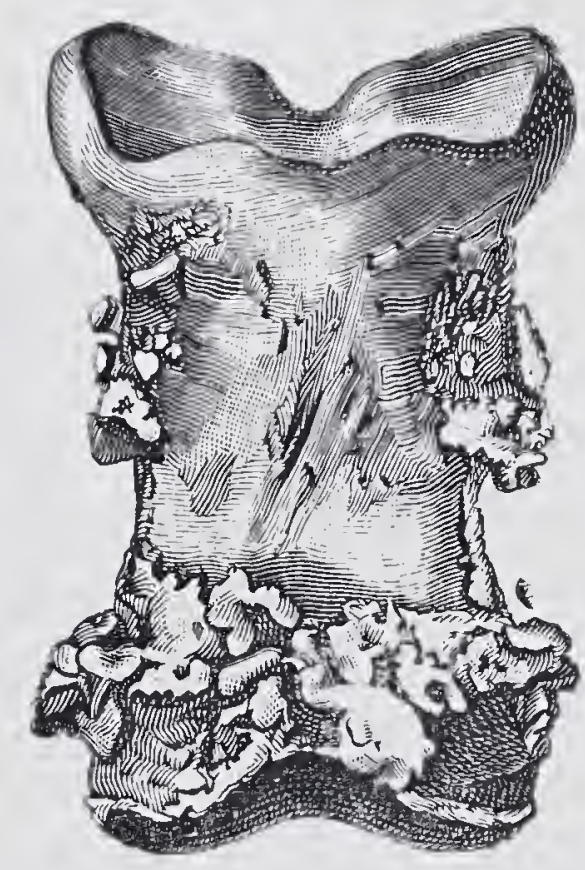

Frg. 229.-Periarticular ring-bone.

found to be more or less altered, according to the age of the disease, whilst exostoses occur on the borders of the joint (fig. 229). We, therefore, have to deal with an arthritis sicca vel deformans. In the later stages the suffraginis and coronet bones become covered with extensive ossific deposits, whilst the articular cartilage is totally destroyed; obliteration of the joint (synostosis) may even occur, as shown by numerous reported cases.

(2) Periarticular ring-bone. In consequence of chronic periarthritis produced by displacement, or by continued strains on the limiting apparatus of the joint, thickening occurs, particularly at the points of insertion of the lateral ligaments, and produces swellings on the inner 
or outer side of the joint. Post-mortem examination shows the articular surface to be healthy, but the ligamentous apparatus is thickened, and more or less well-developed exostoses exist at its points of insertion, particularly at the points of insertion of the lateral ligaments, though they also occur at the spot where the posterior ligaments of the coronetjoint become attached to the suffraginis. Sometimes the insertion of the synovial capsule is also the seat of exostosis (fig. 229). As the articular cartilage is free from disease, this condition has by some been named false ring-bone.

(3) Phachitic ring-bone. In young animals suffering from rhachitis, thickenings sometimes persist on the lower extremity of the suffraginis

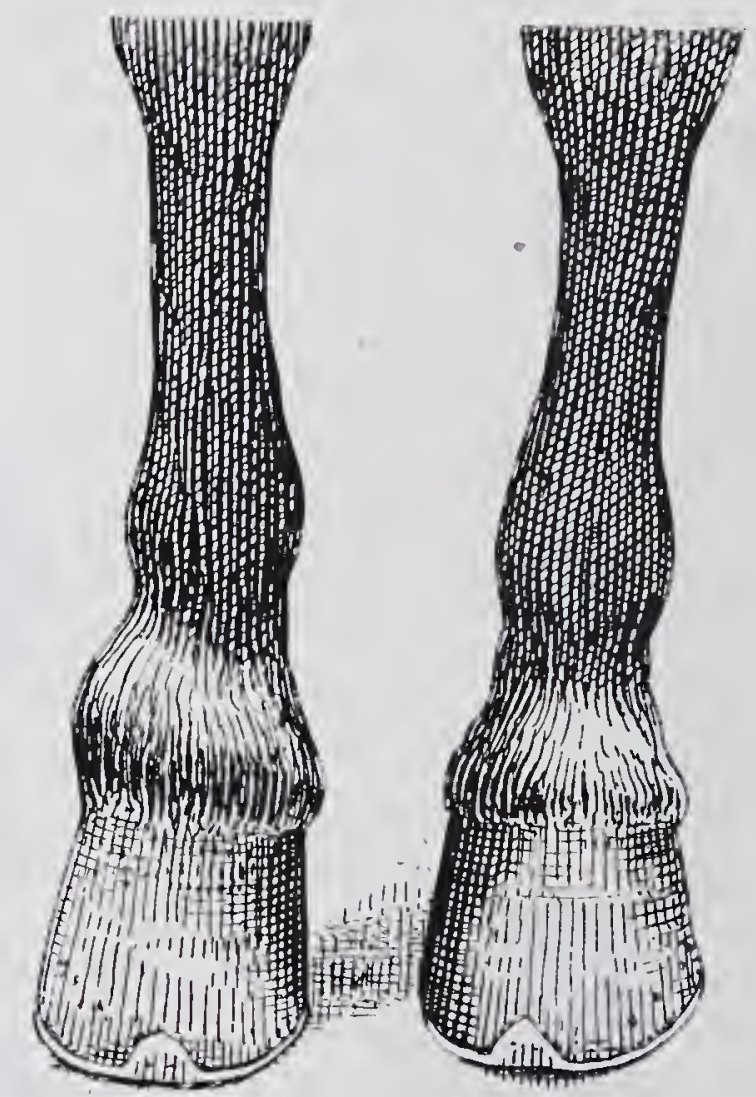

FIG. 230. - The outside of the right coronet shows ring-bone formation; the left is normal.

and at the upper extremity of the coronet bones, and, as they cause swelling around the joint, have been described as ring-bones. In such cases, however, there is no pathological change either in the joint proper or in its ligaments.

(4) The term "traumatic ring-bone" includes all enlargements about the coronary joint produced by perios titis due to other (usually traumatic) causes, whether following treads on the coronet or acute inflammatory disease of neighbouring parts, gangrene, \&c. 'This classification meets practical requirements, for, in clinical diagnosis, it is quite possible to distinguish the different forms, and to modify the prognosis according to circumstances.

Causes. The cause of the coronet-joint being so frequently the seat of disease lies in its anatomical formation. To act as an incomplete 
ginglymoid joint, its area must necessarily be limited, and its articular surface flat. This, however, favours strain of the ligaments and unequal distribution of weight over the articular surface, circumstances which lie at the root of the morbid changes, while they also explain why the periarticular form of ring-bone is the most frequent, and why the disease only occurs in solipeds.

Considering the varied forms of ring-bone, it is not surprising that equal variety exists in the immediate causes. The existence of the disease in two or more feet suggests hereditary predisposition, and may often be traced to small and badly-shaped joints, or defects in the formation of the limbs. The greater weight borne by the front limbs explains their so frequently becoming diseased. 'T'urning the toes in or out causes unequal distribution of weight in joints and strain of ligaments, and therefore often produces periarticular ring-bone. Such conformation being perpetuated in the progeny, renders it easy to understand why the disease is often inherited. Havemann drew attention to this, and condemned the use of animals with ring-bone for breeding. There seems no reason for believing that coarse-bred animals suffer oftener than others; but if the statement be true, the fact is probably due, in part at least, to the less care taken in selecting mares for breeding.

Both Peters and Williams state that ring-bones are commonest in animals with long, weak pasterns, and refer this to the greater strain on the ligaments. Peters says the process begins at the middle of the dorsal or anterior surface of the joint, as an inflammation of the capsular ligament. Percival believes, on the other hand, that upright pasterns favour formation of ring-bones, because of the greater shock to which the bones are subject in moving. The abnormal formation above noted is, however, of more importance. The predisposition to ring-bone in young animals is a result of the greater vulnerability of the bones and joints.

The external causes are sprains and all influences favouring sprain, such as defective formation of the limb, unequal paring of the hoof, using animals at a rapid pace and on rough ground. Unskilful shoeing plays an important part. If one side of the foot-wall is left higher than the other, that side will first come in contact with the ground, the weight will be distributed unequally in the joints, and the external ligaments of the opposite side may be strained. The "camber" of modern roads acts in the same way. The causes of rhachitic ring-bone are little understood, and the same is true of the reported cases of ringbone following strangles, and its greater frequency in certain breeds. It has not yet been shown that it is due to deficiency of calcium salts. Traumatic ring-bone is produced by inflammation extending from neighbouring parts to the periosteum. 
Dieckerhoff says that ring-bone results from inflammation of a small bursa mucosa lying between the branches of the perforatus tendon and the postero-lateral ligaments of the navicular bone. It is quite true that swelling of this bursa may be mistaken for ring-bone; but it seems very doubtful whether disease of the joint or lameness is ever caused by it.

Symptoms. The common basis of these conditions is chronic enlargement around the coronet-joint, which is sometimes so slight as only to be detected by careful examination and comparison of the two feet. In such cases, of course, the limbs must be placed as nearly as possible in similar positions. Exostoses on the lateral surfaces of the joint are best seen from the front; those on the anterior surface from the side, and from the height of the affected foot. Diagnosis is also assisted by palpation and comparison of the two joints.

In articular ring-bone the exostosis extends from either side over the anterior surface of the joint, and appear's more or less circular, from which circumstance it has received its name. The posterior surface is covered by the ligaments and flexor tendons, and therefore cannot well be examined. The exostosis sometimes arises chiefly from the suffraginis, and is then rather higher placed, sometimes from the coronet, when it is found just above the hoof. A high and low form of ring-bone have therefore been distinguished. The swelling is hard, painless on pressure, and the skin covering it is movable.

The periarticular form is similar in appearance, except that the enlargement is usually confined to the lateral surfaces of the suffraginis and coronet bones, and the anterior surface is usually normal. As in the former case, the swelling may appear higher or lower, and in general what has been said above also applies to this form.

Lameness is an almost constant feature of both these forms of ringbone. It occurs principally when weight is placed on the limb, and is sometimes considerable, sometimes only slight. Lameness is absent in rhachitic ring-bone, and usually in the traumatic form. After a long rest it may partly disappear, and in spirited animals slight lameness is often lost during movement. When the condition has become chronic, the muscles of the limb atrophy, wasting being proportioned to the chronicity and severity of the lameness. As a rule the latter phase of the stride is considerably shortened, and the phalanges show volar flexion, i.e., the fetlock is upright. In the periarticular form, lameness is best seen when the animal turns in short circles towards the diseased side. It is a consequence of inflammation of the articular surfaces or of ligaments, though at times it results from mechanical obstruction to movement in the coronary joint, due either to the formation of exostoses or to contraction of ligaments, and adhesions between the articular 
surfaces. Such adhesions are often accompanied by excessive volar flexion. By lifting the foot the anchylosis of the joint is discovered immediately an attempt is made to rotate, to flex, or to extend it. From the above it will be clear that the degree of lameness in no way depends on the size of the exostosis; a large exostosis may only produce slight lameness, and vice versî.

The diagnosis of ring-bone is very difficult unless the enlargement is well developed. In foals the phalangeal epiphyses appear well developed, and the coronet-joint lies high in consequence of incomplete development of the hoof. The proper distribution of weight on all four feet and the absence of lameness show this condition to be normal. Thickening of the skin may be recognised by palpation, and ossification of the lateral cartilage can easily be distinguished from ring-bone if palpation is practised with any approach to care.

It is more difficult to distinguish ring-bone from strain of the coronary-joint, though the latter is accompanied by acute inflammatory symptoms (increased warmth and pain on pressure), which sufficiently distinguish it. As, however, chronic disease consequent on strain also falls under the designation ring-bone, conditions occur which may be described by either term. The disease may be masked by thickening of the skin around the joint, a condition induced for purposes of deception; the trick succeeding the more readily as laymen are pre= disposed to refer lameness to the skin injury. It is always safer to take the opposite view.

Course and prognosis. As a rule, the disease is chronic, though the different forms of ring-bone exhibit many peculiarities. The articular form generally sets in slowly, though at times it results from acute synovitis, and is then difficult to differentiate from synovitis itself. As complete recovery cannot be expected, the prognosis depends on the degree of lameness and the trork required of the horse. Lameness of sufficient severity to prevent work can only be removed by neurectomy. Exostoses, involving the front of the joint, offer little chance of cure, and those fixing the joint none at all.

Periarticular ring-bone sometimes results from acute periarthritis of the coronet-joint, or from repeated strain of its ligaments. Improvement or cure depends on the nature of the pathological processes, and the possibility of removing the active cause. When the joint is much enlarged and the position of the pastern altered, recovery is not to be expected. The more nearly. the enlargement approaches the joint, especially the front or back of the joint, and the nearer it is to the hoof, the more serious the condition; high ring-bones are therefore less dangerous than those in the coronet region. The prognosis is also less hopeful where conformation is bad. When, on the other hand, errors in 
shoeing, such as unequal paring of the foot, are the cause, their removal will be followed by diminution or disappearance of lameness, but the exostoses are never completely absorbed. The prognosis is naturally unfavourable in anchylosis of the joint. The rhachitic form always develops slowly, and is permanent, though it never causes lameness; if limeness exists, it may safely be ascribed to other changes, which must therefore be considered in delivering a prognosis.

Traumatic ring-bone, consequent on local inflammation near the coronet-joint, only causes lameness in the early stages, and as a rule this afterwards disappears, but large exostoses near the joint may produce permanent lameness.

The forecast is unfavourable in severe chronic lameness; marked atrophy of muscle is likewise a bad sign.

Needless to say the prospect is less hopeful in hacks than in ordinary worling-horses, though horses with large ring-bones have been known to perform excellently even as steeple-chasers. Special care is required in young patients, particularly if ring-bone develop before the animal has done much work. If, under such circumstances, the lameness is obstinate, there is little prospect of the animal ever proving useful. On the other hand, old horses which have shown ring-bones for a long time without going lame are likely to continue sound, unless exceptionally severe work is demanded of them. It is common experience that ring-bones in hind feet less frequently cause lameness than those in front.

Treatment. Ring-bone can be prevented by care in the selection of breeding animals, by proper use and treatment of the young animal, by careful paring of the hoof, and later by proper shoeing. In the articular form, nothing whatever is gained by ordinary treatment; neurectomy alone is likely to remove lameness, but even it fails when the joint has become fixed.

In periarticular ring-bone attention must be directed to preventing sprains and to securing a proper distribution of pressure in the articulations. 'The hoof should be carefully pared to allow the weight to be distributed regularly in the coronet-joint, and to avoid strain of its ligaments. The parts of the wall which first meet the ground when moving should, therefore, be shortened. Though attention must be directed to the sides of the wall, yet the bearing of the toe should not be overlooked. Over-long heels should be shortened; some practitioners recommend plain shoes (i.e., without calkins), thin at the heels; or, if the heels be too low, a shoe with callins, or better with thick heels. As a rule, it is sufficient to properly pare the foot. Care must be taken that the pastern lies parallel with the walls of the hoof; if not, it must be caused to do so. This is necessary on two grounds: firstly, because the position of the pastern is often changed in the disease in 
question; secondly, because such change in position may lead to sprains of the ligaments of the joint. Observance of these precautions is of far greater value than any local treatment. In this way Möller often cured old-standinglameness, which had resisted every other method of treatment attempted. Cold poultices and absorbents are only of use on account of the rest they render necessary; in many cases the apparent success is due to errors in diagnosis. As long as inflammation of the ligaments or periosteum continues, stimulation of the skin may be successful. Cantharides and biniodide of mercury ointment, \&c., are of little value unless rest is given. When possible, the patient should be kept quite quiet for four to six weeks. Failing success by these methods, firing may sometimes be successfully resorted to. Puncture firing in two or three rows is most efficacious. Sometimes a few punctures over the swelling are sufficient. Of late year's deep (needle) - firing has come to be regarded as the most efficient means of dealing with ring-bone. Deep firing has been recommended in France, but care must be taken not to open the joint. For this purpose Dollar uses and strongly recommends Graillot's zoö-cautery, or Déchery's automatic cautery with fine points (see p. 121 of Vol. I. of this series, "Operative Technique "). To secure the best results he proceeds antiseptically (vide loc. cit.). Periosteotomy has been suggested, but is of no real value.

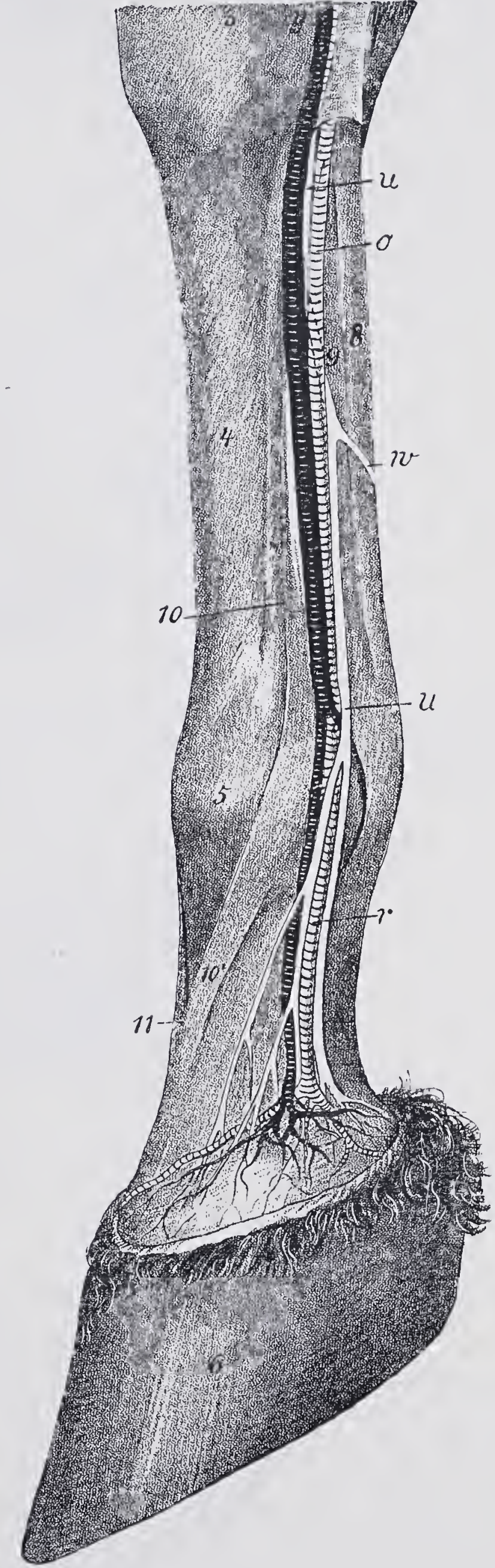

FIG. 231.- $\iota$, Internal plantar nerve, which, below the fetlock, divides into the anterior, middle, and posterior digital nerves ; $n$, oblique branch from intermai to external plantar nerve ; $o$, large metacarpal artery; $r$, digital artery ; $y$, internal metacarpal vein, which, below the fetlock, is represented by the digital vein. 


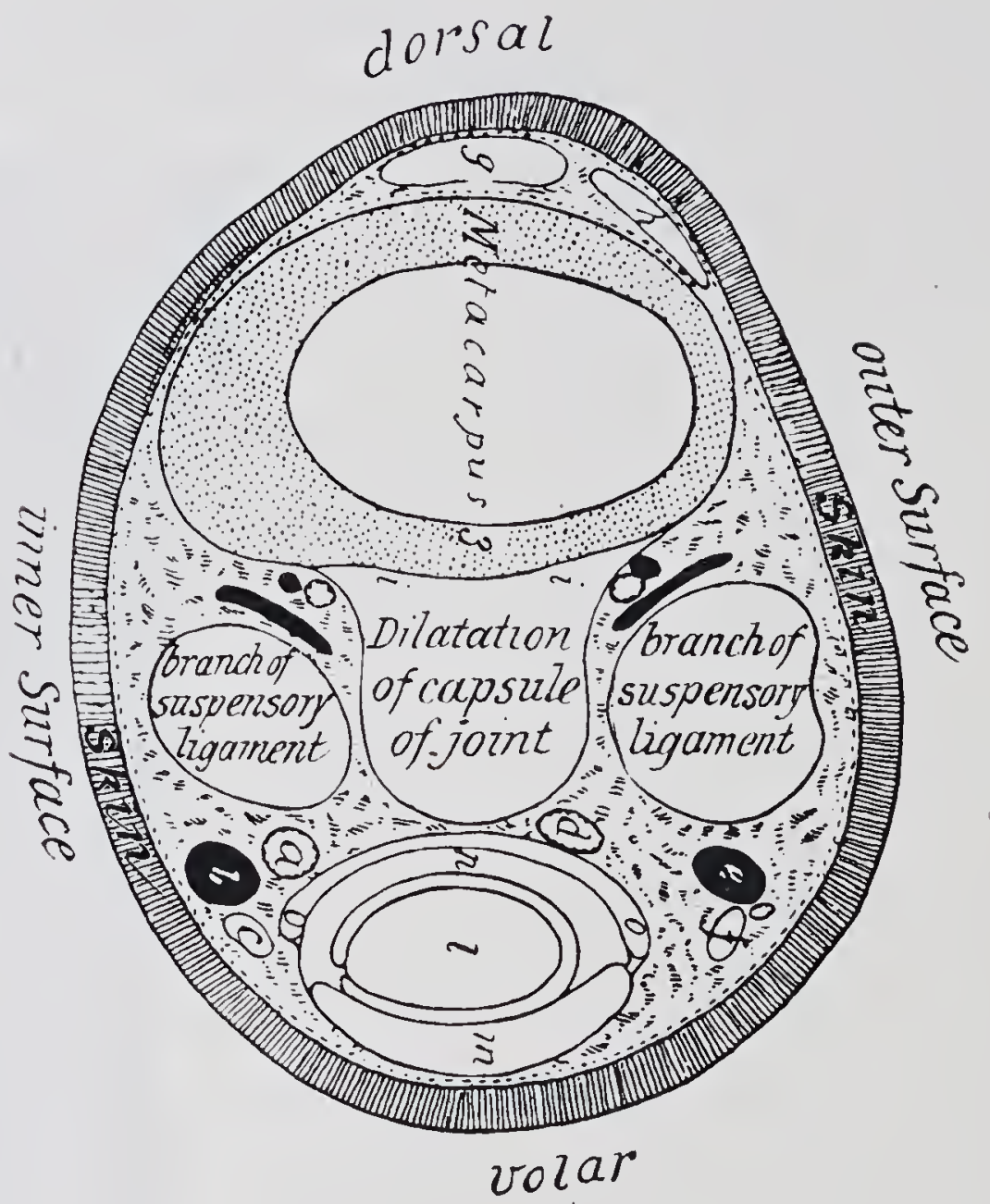

Fig. 232.-Section through the metacarpus about 3 to 4 iliches above the fetlock-joint. $a$, Digital artery; $b$, digital vein; $c$, digital nerve; $l$, flexor pedis perforans tendon: $m$, flexor pedis perforatus tendon ; 0 , tendon sheath.

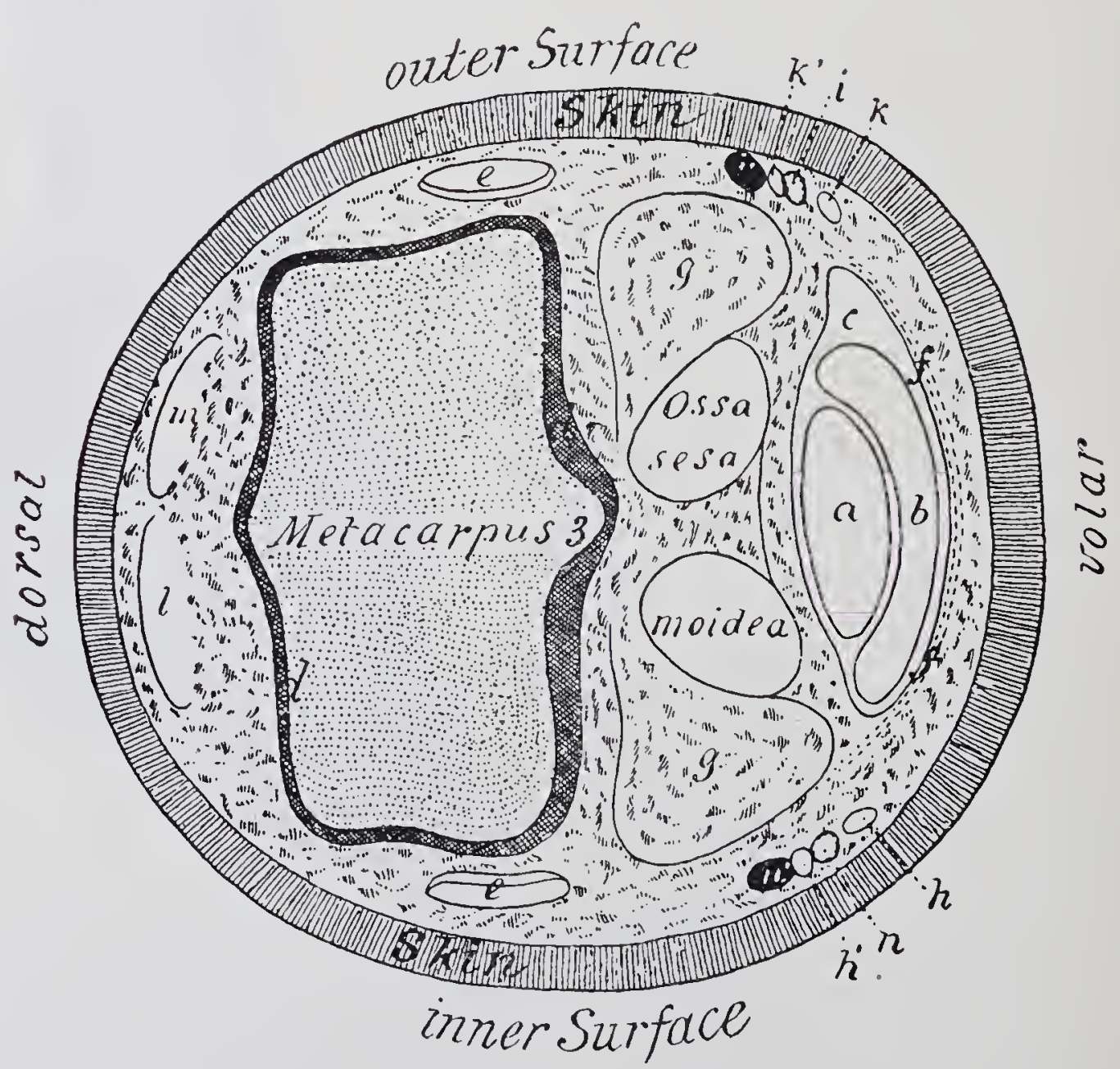

Fir. 233.-Section through the fetlock-joint. a, Flexor vedis perforans tendon; $b$ flexor pedis perforatus tendon; $c$, tendon sheath; $d$, capsule of the fetlock-joint; $h$ and $h^{\prime}, h^{\prime}$ and $k^{\prime}$, branches of the posterior digital nerves; $i, n$, digital arteries. 
If after such treatment lameness still persists, or if the animals are of little value, and long-continued treatment is objected to on the score of expense, neurectomy may be tried. As a rule, the plantar, digital, or median nerve must be divided, and the operation is generally successful unless mechanical obstruction to the movement of the joint exists.

As the treatment of chronic ring-bone often calls for every remedy in the répertoire of the practitioner, and as the various neurectomies usually

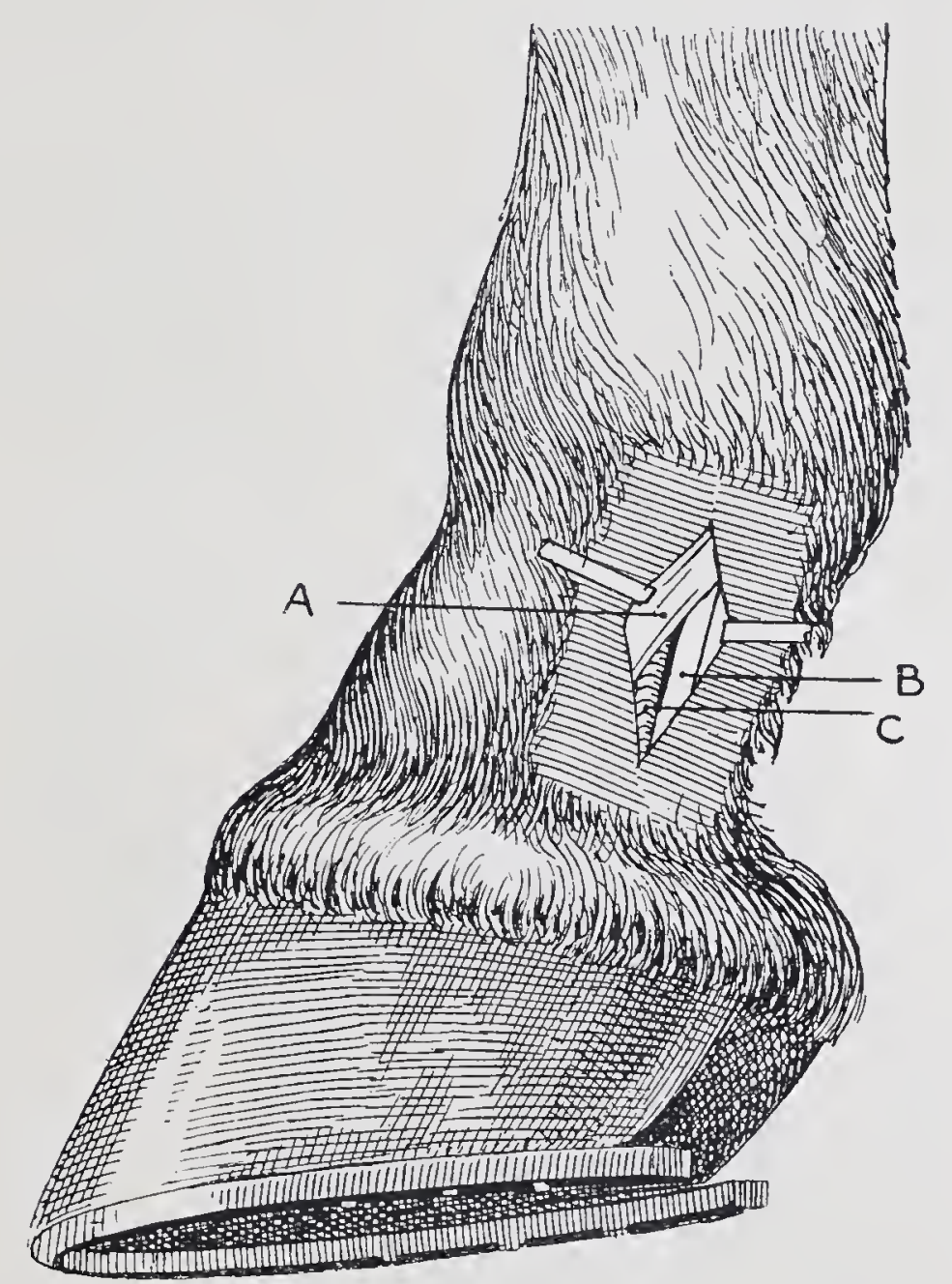

Fig. 234.-Neurectomy below the fetlock. A, Band from the suspensory ligament to the extensor pedis tendon (this includes the tendon of the interosseous muscle); $\mathrm{B}$, digital nerve; C, digital artery.

represent his last resource, it has been found convenient to shortly describe those more commonly performed at this place.

Before utilising any of these operations, however, it is necessary to clearly ascertain two points-viz.: (1) That the diseased and painful parts are exclusively supplied with sensory fibres by the nerve to be divided; and (2) that the lameness is due solely to pain and not to such changes as adhesions in or around the joints themselves or contraction of tendons or ligaments. If these conditions can be satisfied the lameness will be removed by neurotomy or neurectomy-not otherwise. Needless to say, it is unwise to operate on riding-horses, especially when the front limbs are placed far under the body and the pasterns are upright; such animals are prone to falls under any circumstances, and 
to deprive them of sensation in the lower part of the limb is calculated to aggravate the defect.

The first operation to be considered is that of

\section{PLANTAR NEURECTOMY:}

The operation can be performed at one of several points: either 2 to $2 \frac{1}{2}$ inches above the fetlock, where the nerve lies immediately in front of the flexor tendons, or on the lateral surface of the fetlock-joint itself, or, again, an inch or so below the fetlock-joint. In Germany and

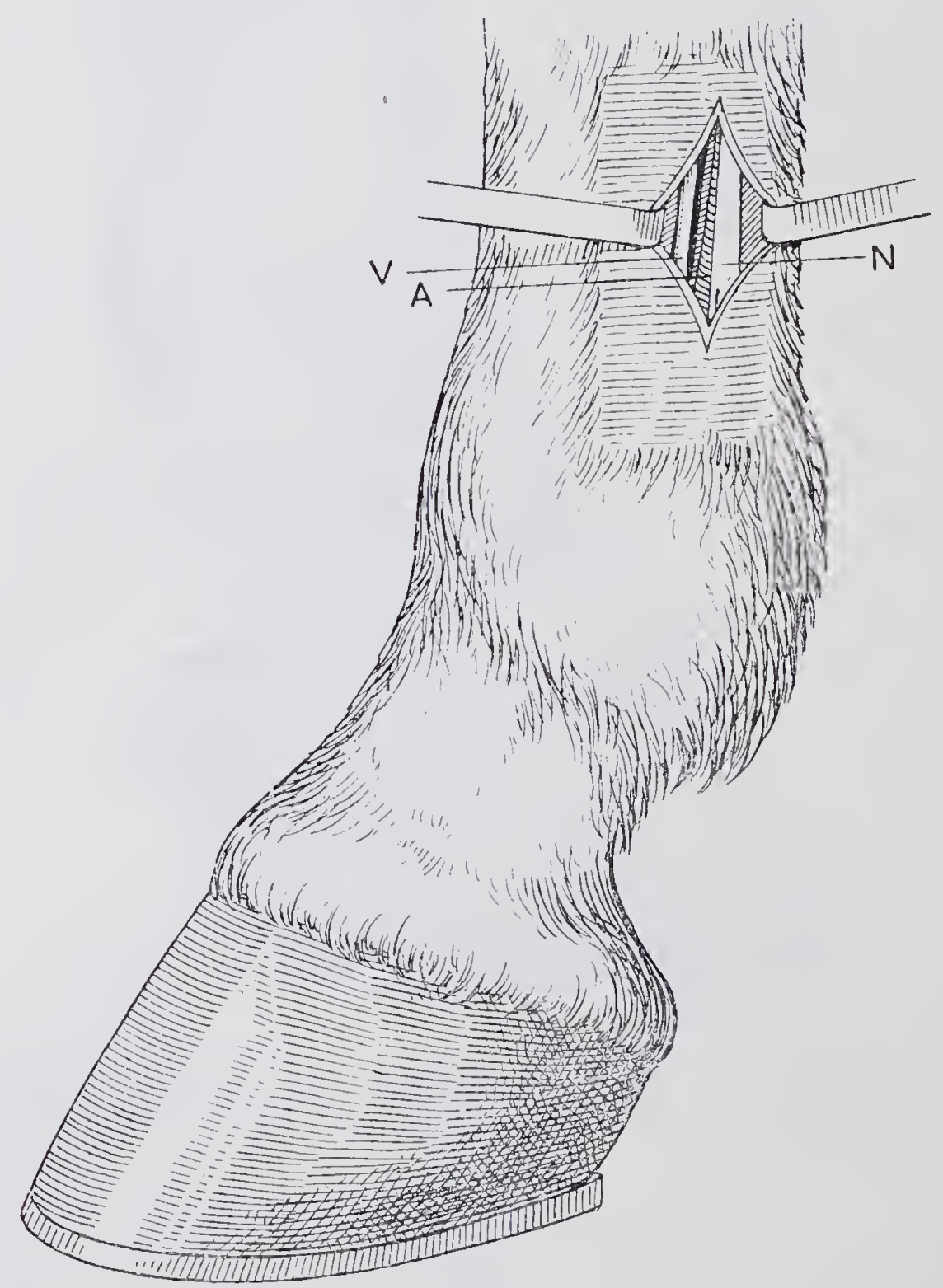

FI:. 235. - Neurectomy above the fetlock. V, Vein ; A, artery ; N, nerve.

France the high operation is usual; in England the high operation is usually performed on the inside of the limb, the low operation on the outside, the reason being that there is less chance of the wound on the inside being injured by the opposite foot if it be made clear of the fetlock-joint. The animal is cast, the limb to be operated on is released and drawn forwards, the skin shaved from the seat of operation, and the parts cleansed and disinfected. To prevent troublesome bleeding it is

1 For a review of the value and results of plantar neurectomy, see pp. 86 et seq. of Cadiot and Dollar's "Clinical Veterinary Medicine and Surgery." 
very convenient to apply a rubber bandage from the fetlock to the knee and to pass a stout rubber cord tightly round the limb just above the knee itself: the bandage is then removed, leaving the parts practically bloodless and partially anæsthetic.

The operation is usually performed first on the inner side, as the wound can then be better guarded against soiling than in the opposite case. By observing antisepsis primary union can be obtained. After ascertaining the position of the nerve by palpation an incision about 1 to $1_{2}^{\frac{1}{2}}$ inches long is made directly over it. The nerve is readily recognised by its fibrous appearance and lighter colour. A skilful operator will expose the nerve with one strolie of the linife, and the skin incision should certainly not require more if good union is to be obtained. Any trifling bleeding is checked with tampons of cotton-wool and the nerve freed from surrounding tissues with a few touches of the linife. A thread is then passed under the nerve with a curved needle, and a portion of the nerve about half an inch long freed and one limb of a pair of scissors passed under it. The nerve is then divided at the upper angle of the wound, by smartly closing the scissors. The animal usually struggles at this stage. Division of the lower part of the nerve is unattended with pain. Any blood is removed with tampons of cotton-wool and the parts cleansed, if necessary, with sublimate or carbolic solution. The skin is brought together with one or two interrupted silk sutures and the parts covered with iodoform collodion, wound gelatine, or a small cotton-wool dressing.

The operation on the outside of the limb is performed in a precisely similar manner, the point of incision only differing. The nerve can readily be discovered at the point where it passes over the posteroexternal surface of the fetlock-joint as a small hard cord which rolls under the finger. Fgures 231 to 235 clearly show the position.

The wound usually heals in five to eight days, provided antisepsis has been observed and the incisions skilfully made. It is best, if possible, to avoid washing out the wound with disinfectants, as the tissues are thereby injured and healing is slower. In a word, asepsis is preferable to antisepsis.

During the last year or two neurectomy has been performed without casting by injecting 30 to 60 minims of a 10 per cent. solution of cocain into the subcutis immediately within the area of operation. By applying an Esmarch bandage and rubber cord the operation can be performed with out bleeding. The horse's foot can be placed on a farrier's shoeing block so as to bring it to a convenient height for operation.

Sometimes the horse becomes sound immediately after operation, sometimes lameness only disappears by degrees. In other cases improvement is effected, but the animal does not become sound. Such cases are usually due 
to recurrent sensibility, or to mechanical interference by bony growths with the play of the joints. The horse should be rested for several weeks and closely watched, in order to note any possible signs of degeneration in the tendons or inflammatory changes in the foot. Neurectomy should never be performed during the existence of local inflammation in or near the foot, and it is best only to remove $\frac{3}{8}$ to $\frac{7}{2}$ an inch of the nerve so as to permit of sensibility gradually returning after an interval of several months. The object of the operation, in fact, should not be to permanently destroy sensation, but only to remove it for a sufficient time to allow chronic inflammatory processes to subside and to render the animal useful during this interval.

Nocard has shown that the uprightness of the foot often diminishes and the size of the bony growths becomes less after plantar neurectomy.

\section{MEDIAN NEURECTOMY.}

The operation of median neurectomy is as follows :-

The median nerve is readily discovered on the inner surface of the fore arm, running obliquely downwards and slightly backwards just behind a ridge on the head of the radius, into which is inserted the internal lateral ligament of the elbow. It crosses the posterial radial artery at a very acute angle, and passes with it towards the posterior surface of the radius. The posterior radial vein (or veins) is situated in front of the nerve. The operation is performed (under general or local anæsthesia) opposite the lower portion of the elbow-joint, or immediately behind the upper extremity of the radius towards the upper point in the depression between the radius and the flexor muscles of the fore limb.

The skin having been shaven and disinfected, the limb is drawn well forward and an incision about an inch in length is made, traversing successively the skin, subcutaneous connective tissue, and the prolongation of the posterior superficial pectoral muscle. Any bleeding vessels are carefully twisted or closed with Wells' forceps, and the seat of operation is cleared of blood. 'The operator then assures himself by palpation that the nerve lies in or near the middle of the incision. Should this not be the case, the limb is moved slightly to and fro until the nerve is brought into the desired position. Towards the lower angle of the wound the fascia of the fore arm is slightly incised, a grooved director passed under it and pushed upwards, parallel with the nerve. By passing the bistoury along the groove the fascia is then laid open. Another method consists in using a probe-pointed bistoury. Certain operators even go so far as to snip away with scissors an elliptical fragment of

1 For fuller details of complications after median and ulnar neurectomy, see p. 447 of Carliot and Dollar"s "Clinical Veterinary 11 edicine and surgery." 
fascia on either side, thus more freely uncovering the nerve. The skin wound is then held open with retractors. The next step in operation consists in dissecting free the nerve (sometimes a rather tiresome process), and raising it on a grooved director or tenaculum. The nerve is cut through as high up as possible, and again near the lower limit of
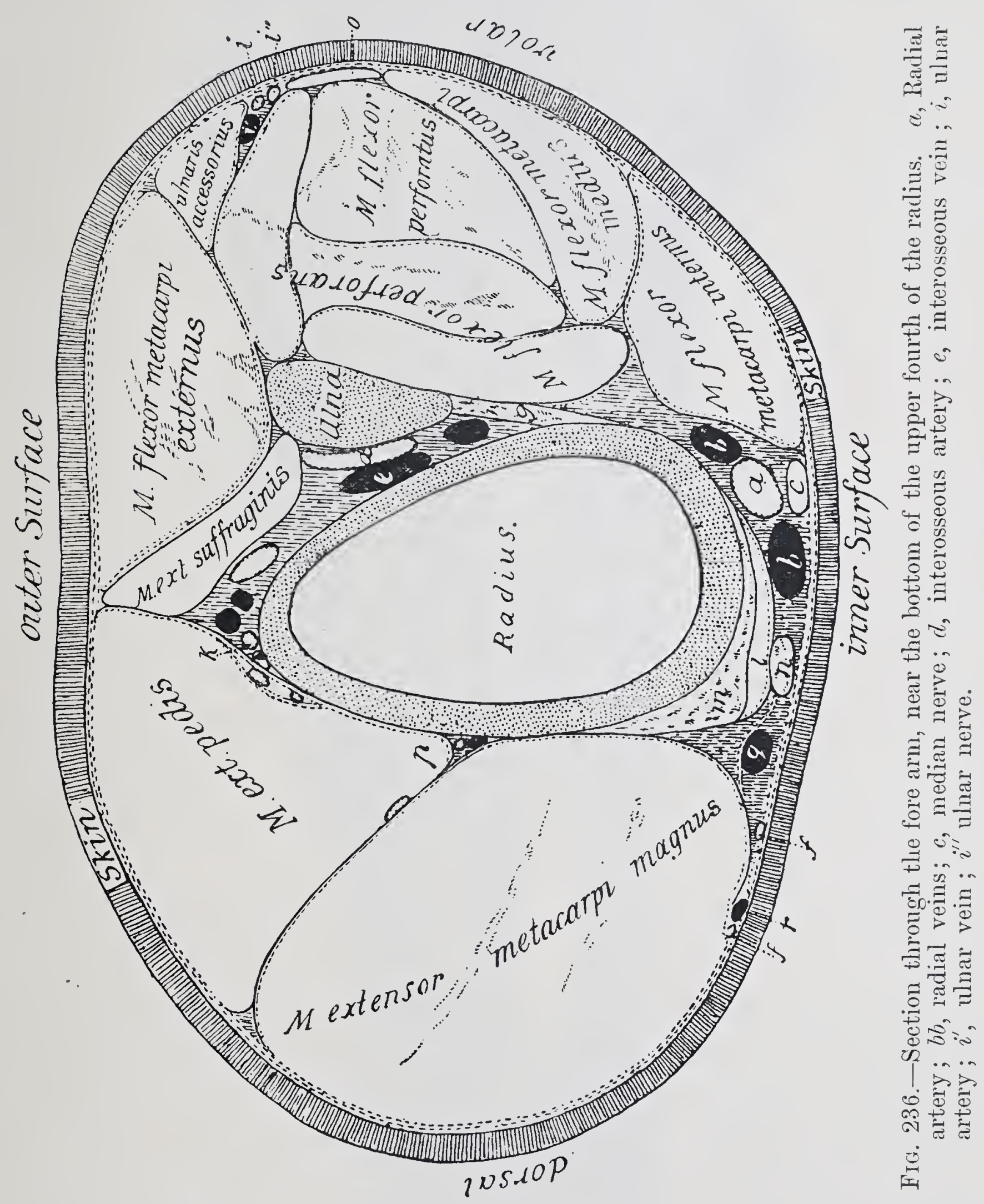

the wound, a piece about three-quarters of an inch in length being removed. The operation is concluded by wiping the wound dry, dusting with iodoform, suturing the slin, and applying a little iodoform collodion or similar dressing.

When the incision is skilfully made at the proper point, and the antibrachial aponeurosis opened, the nerve often appears immediately as a flattened whitish cord, which has a tendency to become thrust forward 


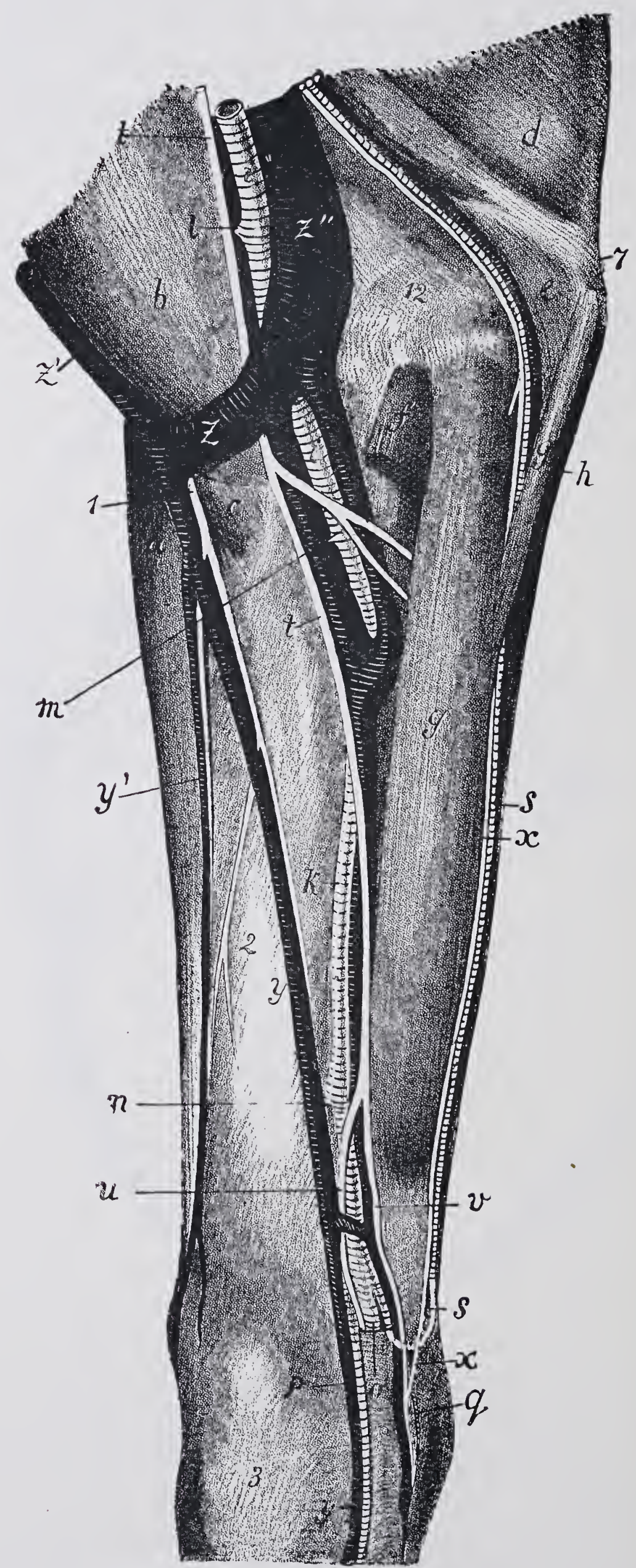

FIG. 237.- $k$, Radial artery ; s, ulnar artery ; $t$, median nerve ; $x$, ulnar nerve. 
and to protrude between the lips of the incision in the aponeurosis. When the nerve does not appear, it is usually sufficient to slightly alter the position of the limb. The most serious difficulty to be feared is from injury to the veins in this neighbourhood, and every care must be taken to avoid such a complication.

In certain animals operation is rendered difficult by an abnormal arrangement of the radial veins, but this is rare.

Peters, and after him others, showed that division of the median nerve alone may remove lameness resulting from bilateral lesions-that is to say, occupying both sides of one of the lower parts of the leg, or encircling these regions. The results are explained by the preponderating influence of the median nerve in the innervation of structures below the

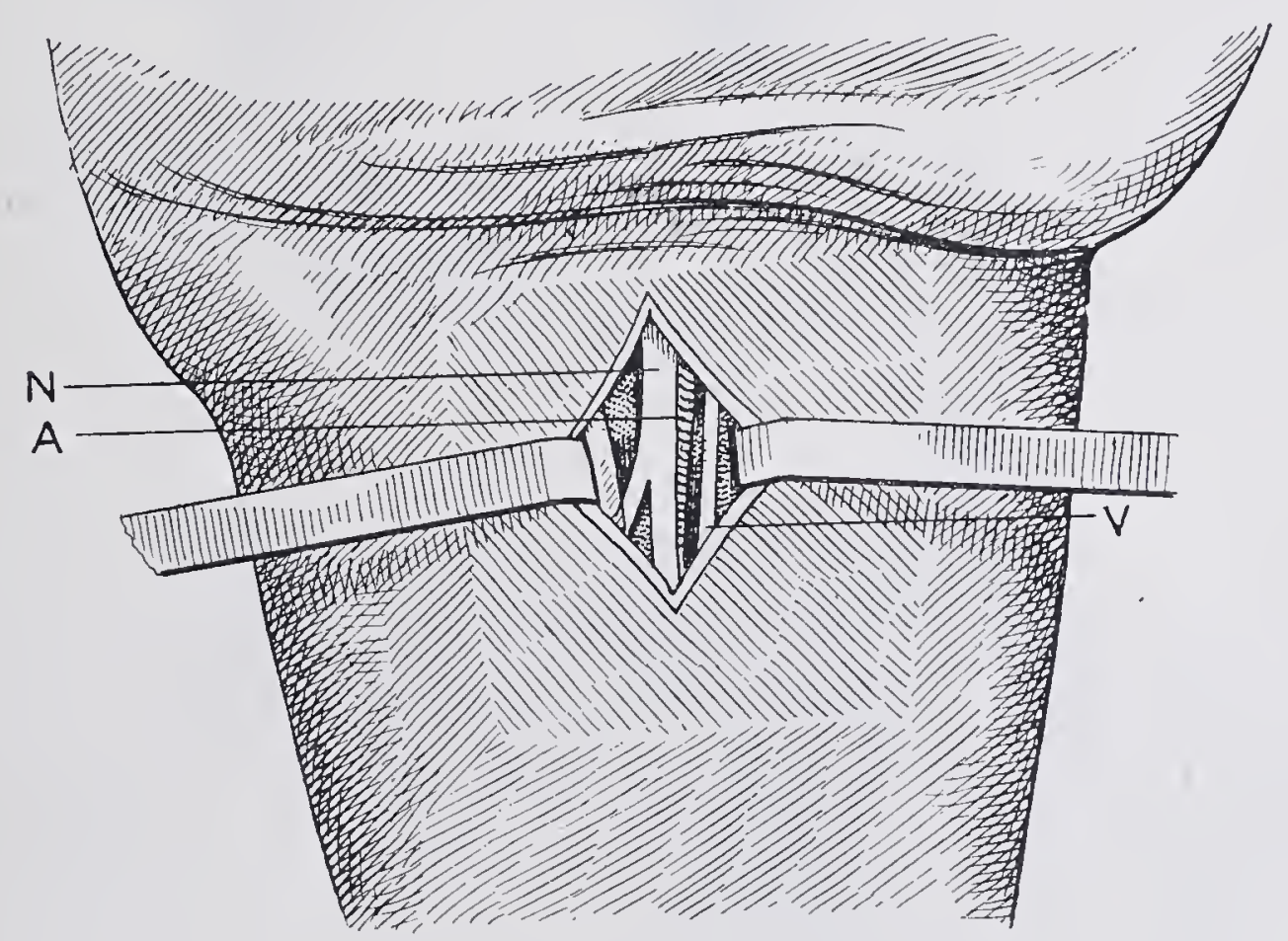

FıG. 238-Median neurectomy (semi-diagrammatic). N, Median nerve; A, posterior radial artery; V, one of the post-radial veins.

knee, a preponderance due to the fact that at a variable point in the fore arm the nerve terminates by dividing into two branches, one of which is continued as the internal plantar nerve, while the other joins the ulnar at the upper border of the pisiform bone, beneath the tendon of the middle flexor, and is continued as the external plantar nerve:

Cadiot and others have seen horses with various chronic affections, like strained tendons, splints, cartilaginous ring-bones, and periostitis of the phalanges, in which lameness has been removed, or certainly diminished, by dividing the median nerve.

But others occur in which section of the nerve fails to remove lameness caused by lesions on the outer side of the limb, or at times even on the inner. The persistence of pain and lameness in the latter case is explained by the existence of recurrent fibres. Under such circumstances, 


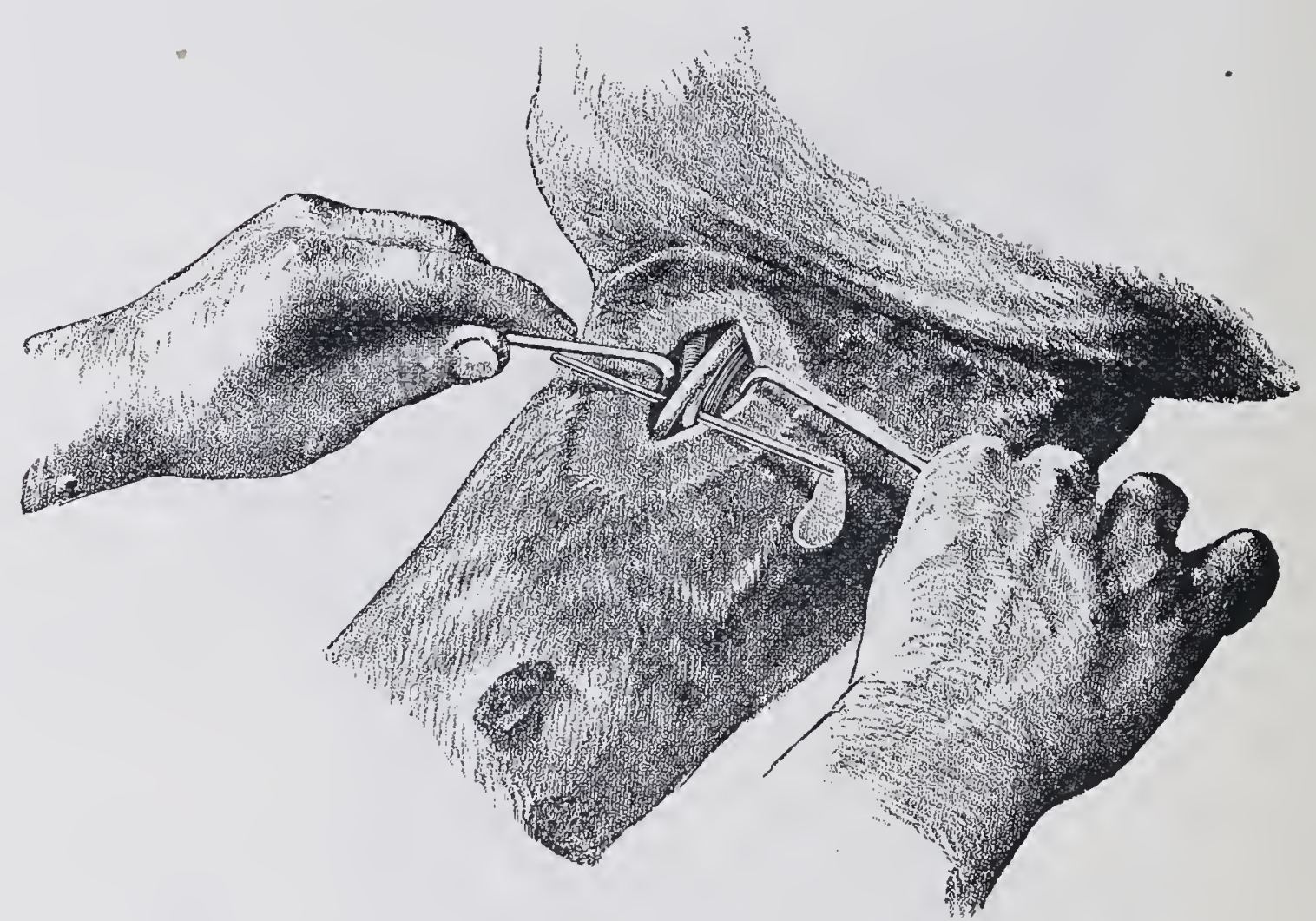

Frg. 239.-Operation of melian neurectomy. The nerve exposed.

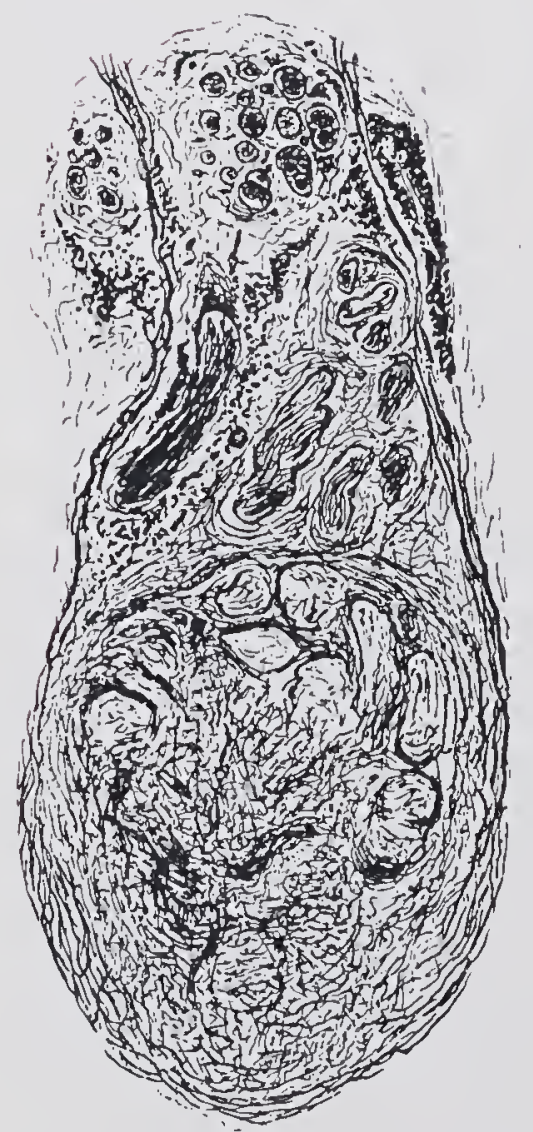

Fir. 240. - Section of neuroma from the divided end of the median nerve, three months after operation.

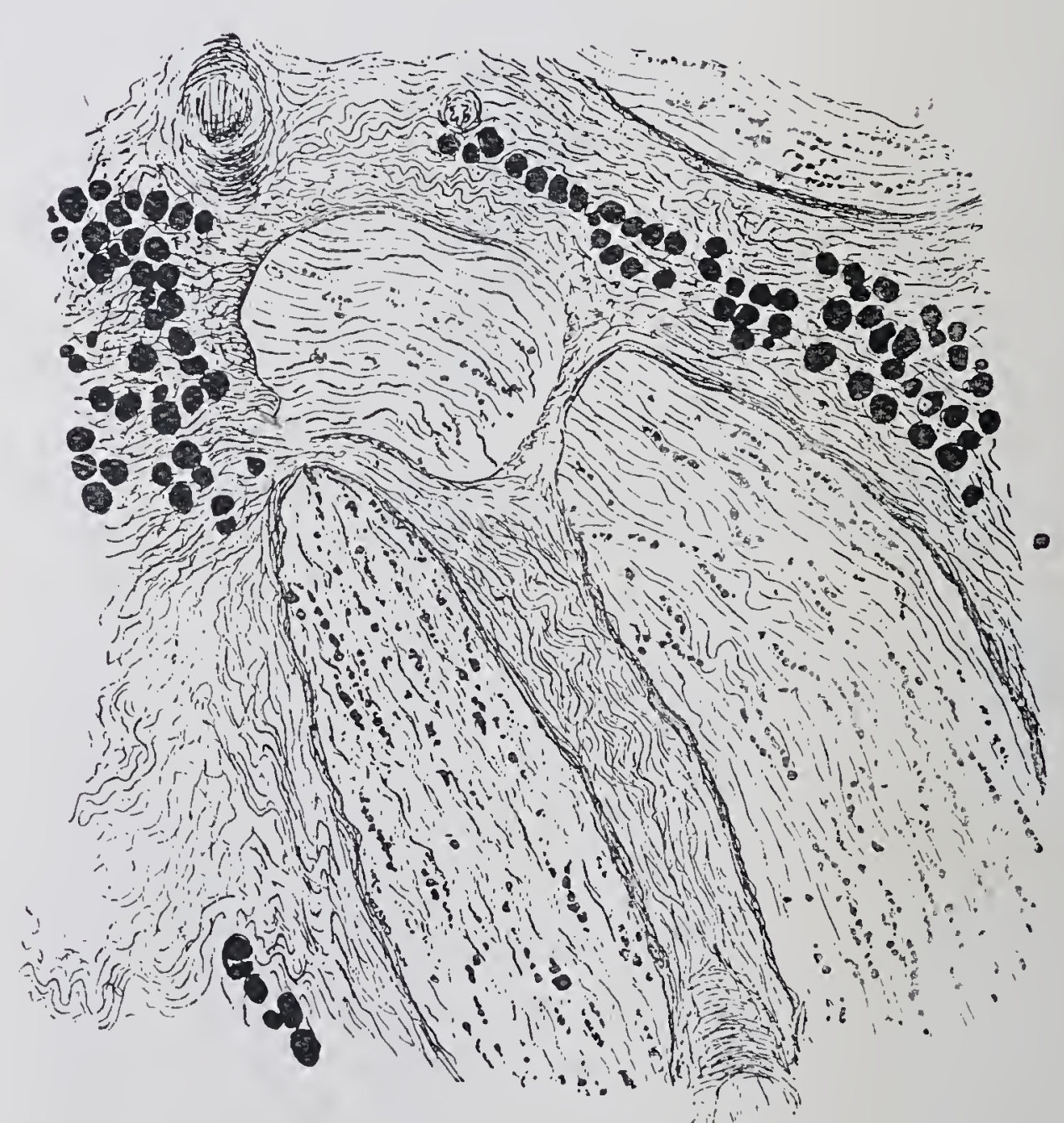

Fig. 241.-Longitudinal section of the median nerve, 6 inches helow the point at which neurectomy was performed. The remains of the nerve bundles contain the degenerated myelin cylinders (stained black). The loose connective tissue surrounding the divided nerves contains fat as indicated by the smaller black dots. 


\section{ULNAR NEURECTOMY}

may prove useful. Throughout the whole extent of the fore arm the ulnar cutaneous nerve, accompanied by the ulnar artery and vein, is situate between the oblique and extemal flexors of the metacarpus, and immediately under the fascia uniting them. By palpation with the finger tips the muscular interspace which fixes the line of operation is readily discovered.

In performing this operation the horse is cast on the sound side. The affected limb is left in the hobbles, but drawn tense by means of two strips of webbing, one fixed on the upper portion of the cannon bone

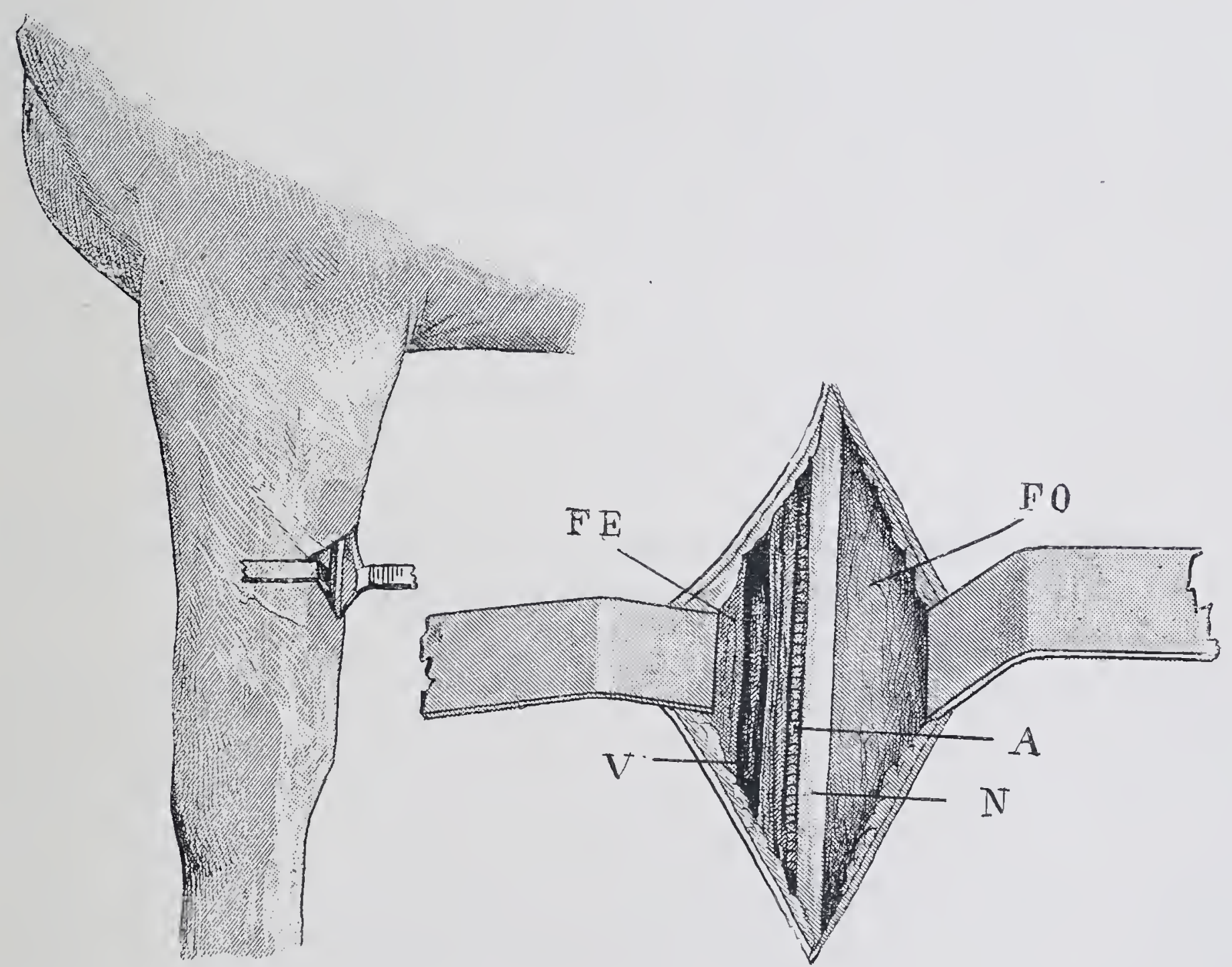

Figs. 242 AND 243. - Neurectomy of the ulnar nerve. FE, Flexor metacarpi externus ; FO, flexor metacarpi obliquus; $\mathrm{N}$, ulnar nerve; AV, nlnar artery and nerve.

being pulled backwards, the other, attached to the coronet, in a forward direction. Two assistants, holding the free ends, keep the parts steady.

The operator kneels in front of the upper part of the fore arm; the point selected is 4 to 6 inches above the knee.

The parts having been prepared, he makes at the point just indicated, an incision about $1 \frac{1}{4}$ to $1 \frac{1}{2}$ inches long through the slin. The subcutaneous connective tissue, the antibrachial aponeurosis, and the fascia which unites the aponeurotic covering of the two muscles are then divided.

With forceps and bistoury the connective tissue surrounding the nerve is dissected aray, following the direction of the wound, and carefully 
avoiding injury to the ulnar artery and vein which accompany the nerve:

The nerve being isolated is divided at the upper angle of the wound, and a fragment about $1 \frac{1}{4}$ inch in length is excised. The wound is cleansed and the skin brought together with a few small sutures.

Double neurectomy of the median and ulnar nerves is not often performed. Neurectomy of the median alone often has serious drawbacks. It should be reserved for old-standing chronic affections of the fore limb, and-particularly in dealing with valuable animals-should not be tried until all other methods of treatment, especially firing, have proved unsuccessful.

Loss of the hoof, thickening of the subcutis, fracture of the os pedis, and gelatiniform degeneration of the flexor tendons are not uncommon sequelæ; the first most frequently results when there are large exostoses on the front of the joint, near the coronet. Perhaps loss of the hoof is due to the coronary band being bruised between the exostosis and the hoof. To avoid this contretemps, the toe of the hoof should be freely pared, whilst the heels are preserved. If needful, shoes with thick heels or high calkins are applied. Loss of the hoof also follows neglected corns, or pricks in shoeing; the injury not being detected owing to the animal showing no signs of lameness.

\section{Y.-INFLAMIMATION OF THE POSTERIOR LIGAMENTS OF THE CORONET-JOINT.}

The deep sesamoidal and the postero-lateral ligaments correspond physiologically to the suspensory ligament of the fetlock-joint. In common with the capsular and lateral ligarnents they fix the coronet-joint; the latter also fix the pedal-joint when the limb is loaded. On account of the slight development and flattened shape of the upper articular surface of the os coronæ, the fixation of the pastern and coronet bones throws a severe strain on the limiting apparatus of the joints, which is greatest in the case of the lateral ligaments. These, therefore, become diseased, forming ring-bone; the posterior ligaments of the coronet-joint also become affected, and more frequently than is generally supposed.

Causes. The condition is produced by over-extension, sometimes accompanied by partial rupture, resulting from excessive dorsal flexion of the coronet-joint. Attention should, therefore, first be given to the position of the hoof. Low heels and long toes are almost always the active causes. Heavy horses in mounting hills bring about a similar condition of things; the leverage of the toe becomes greater. If the general direction of the phalanges (phalangeal axis) be not parallel with that of the walls of the hoof (hoof axis), but more upright, there is danger of straining, not only the different portions of the inferior sesamoidal ligament and the antero-lateral and postero-lateral ligaments, 
but the tendon of the flexor pedis also. Such strain also results when the heels of the shoes become worn down. The condition is favoured by work on hard rough ground, especially in riding-horses; animals with upright pasterns and narrow hoofs seem particularly predisposed. Gutenäcker has recently studied it carefully, and has found osteophytes at the points of insertion of the ligaments into the os suffraginis.

On post-mortem examination the ligaments on the posterior face of the joint are found thickened and ossified at their insertions (fig. 229). In aggravated cases false anchylosis may occur.

Symptoms. The disease is in the early stages signalised by lameness when weight is placed on the limb, and by the foot being rested. As the ligaments lying behind the coronet bear most weight, and are subject to most strain at the time when the limb is directly under the body, the horse avoids this position, and in the last phase of movement lifts the foot rapidly from the ground. The stride is therefore markedly shortened during this last period, and the lameness acquires a certain resemblance to laminitis, and especially to chronic navicular disease, in which the pastern is also upright.

A careful local examination will at once dispel any doubts. The postero-lateral ligaments, and those lying beside the posterior ligaments of the coronet-joint, may be manually examined to detect pain. The foot is lifted as in shoeing, and pressure exercised alternately with the thumb of either hand. The ligaments lie partly under, partly alongside, the flexor pedis perforans at the back of the pastern. To prevent mistakes, it is well to examine both feet. Sometimes thickening of the ligaments can be detected; occasionally this is of considerable extent, and may then best be recognised by allowing the horse to stand on the limb; a comparison should be made with the other foot. The diagnosis may further be assisted by throwing a certain strain on the ligaments. To do this the foot is lifted and the toe powerfully thrust forwards, i.e., in the direction given it by the extensor muscles. Fambach found in some cases where the toes were turned in that the external postero-lateral ligament was alone diseased.

In chronic navicular disease pain is produced by pressure in the heel, over the navicular bone, but is altogether absent in inflammation of the posterior coronary ligaments. Contraction of the hoof is also wanting. The fact that symptoms of laminitis, especially increased pulsation, cannot be detected, disposes of the third hypothesis.

When standing, the animal avoids placing weight on the affected limb. To prevent stretching the inflamed ligaments, the foot is sometimes rested with the heels on the wall of the opposite foot; or, when pain is excessive, it may be held off the ground with the joints flexed.

Course and prognosis. Inflammation of these ligaments occurs 
almost exclusively in fore feet, sometimes affecting one, sometimes both. It has also been seen in the hind feet in heavy draught-horses. The symptoms may either appear suddenly or quite gradually, the course taken varying according to the severity and extent of the pathological changes. As in practice these cannot be directly observed, their gravity must be estimated from the amount of tenderness or lameness, and from. the duration of the disease. Experience shows that severe cases generally last six to eight weeks, those of recent origin, in which pain is slight, recovering, on the other hand, in a few days if the cause be removed and the horse properly shod. It is best, before formulating a prognosis, to await the results of the new method of shoeing.

Treatment. The most useful measures consist in shortening the toe, equalising the bearing in the joint, and raising the back of the foot by calkins or by thick heels. The animal must be rested. Absorption is assisted by bandaging or by warm moist applications. Firing and blistering are less useful, the. deep-seated position of the ligaments preventing their action extending sufficiently far. For some time after lameness disappears rapid or heavy draught is to be avoided, and the horse should, as far as possible, be worked on soft ground.

\section{VI.-FRACTURES OF THE SESAMOID BONES.}

Though rare, these fractures have repeatedly been seen in horses. Williams found the sesamoid bones broken in several places after galloping in deep sand. Howell saw one of the sesamoid bones brolien into five portions under similar circumstances. The sandy shore of Portobello is stated to be notorious for producing such accidents. Transverse fracture has also been seen. As it is produced by the pull of the upper and lower sesamoidean ligaments, this method of fracture appears very natural. Rutherford saw a horse which after making a few bounds went very lame; the sesamoid bones of all four feet were afterwards found transversely fractured. Möller has several times seen fracture of the sesamoid bones accompanied by tearing of the suspensory ligament. Partial rupture of the suspensory ligament, with loosening of larger or smaller portions of the sesamoid bone, is seen in racehorses. Cadiot is of opinion that the sesamoid bones of the fore limb may be fractured by a direct blow from the toe of the hind foot. Such an accident seems possible, but Cadiot's suggestion would not explain bilateral fracture, still less fracture in hind limbs.

Möller witnessed fracture of both sesamoid bones and partial rupture of the superior and inferior sesamoidean ligaments in a working-horse which had been kept in the stable for some weeks on account of lameness. Schöneck describes a similar case in a Russian trotting-horse. A cart-horse, which had been rested for a long time on account of disease of the tendons, 
suddenly fell in the street the first time of going to work, and could not be got up. On post-mortem, the sesamoid bones of both fore limbs were found horizontally fractured, and in each of the hind feet the flexor pedis perforans tendon was torn away from its point of insertion into the os pedis.

Continued rest appears to cause change both in bones and tendons; the view that such accidents are solely caused by the animal capering about is scarcely defensible.

The symptoms are generally pronounced. In horizontal fractures the fragments are drawn apart and the fetlock-joint sinks to the ground, being no longer fixed by its suspensory ligaments. The accident is distinguished from rupture of the flexor pedis perforans or perforatus by the normal position of the hoof, the toe of which, in rupture of the tendons, is directed upwards, so that it no longer touches the ground. Rupture of the suspensory ligament produces the same change in position, but is easily detected on palpation. In fractured sesamoid there is excessive lameness, pain on pressure over the fractured bone, and sometimes slight crepitation. In perfectly fresh fractures a depression may be felt between the pieces of bone.

The prognosis is very unfavourable. Apart from the fact that reposition and retention are scarcely possible, it must be remembered that the sharp edges of the fragments may not only injure the posterior articular surface of the metacarpus, but may wound the flexor tendons which are worn through by the raw edges of the broken fragments; in addition, callus formation is difficult.

Möller unsuccessfully treated a race-horse for several months, and found on post-mortem examination that the broken pieces had not united, and that the condition was substantially as above described. Field claims to have seen recovery follow longitudinal fracture of both sesamoid bones. On account of the great pain accompanying fracture, excessive volar (upright pastern) or dorsal flexion (oblique pastern) may later result. The first occurs when pain is great and the limb is rested for a long time; in other cases, as when pain is slight and weight is placed on the foot during recovery, excessive dorsal flexion results. Restoration of normal position and of usefulness being very rare, treatment is only justifiable in valuable breeding animals.

Treatment. If treatment is to be attempted, the pastern-joint must be fixed in a position of moderate volar flexion by a plaster or strong linen bandage. Complete rest is necessary. Light horses, which have no difficulty in lying down and rising, do best on a soft bed; other patients must be slung.

\section{VII.-SESAMOID LAMENESS.}

In solipeds, both the anterior articulatory surface of the sesamoid bones in contact with the metacarpus and the posterior surface over which the flexor tendons glide are often the seat of acute or chronic

V.S. 
inflammation. (For the anatomy of the parts, see figs. 221 and 222 , p. 595.) Brauell, in 1845, first drew attention to inflammation of the posterior surface, and pointed out that it was similar in character to that of chronic navicular disease. Ten years later Mascher described the disease, for which Günther introduced the name sesamoid lameness. Schrader afterwards gave a thorough pathological and anatomical description of it. Acute inflammation of the

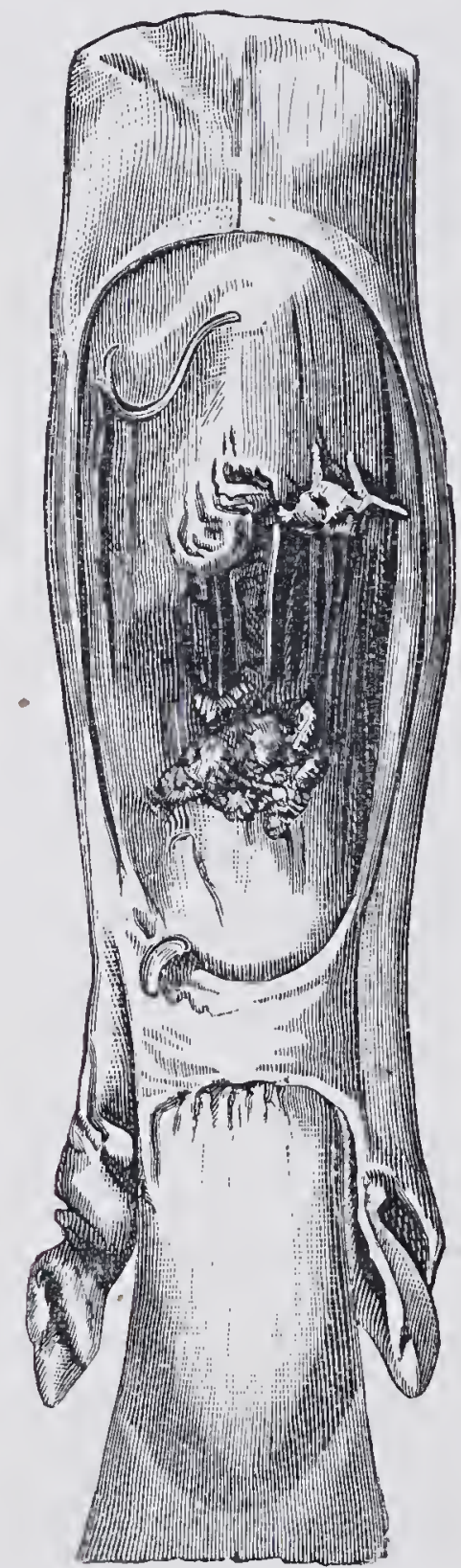

Fig. 244.-The flexor pedis perforans and perforatus tendons in a case of sesamoid lameness; at the point where it passes over the sesamoid boues the perforans tendon is fibrillated (after Brauell). posterior gliding surface of the sesamoid bones sometimes occurs in consequence of acute tendovaginitis of the flexor apparatus.

James states having found an abscess about the size of a walnut (?) in the sesamoid bones (?) of a draught-horse. It was supposed to have been caused by injury, and was accompanied by acute inflammation of the tendon sheaths. Probably the condition was purulent tendovaginitis. In two horses treated for local gangrene of the skin of the hindlimbs, suppuration occurred in the perforans tendon sheath, with rupture of the flexor tendon. Post-mortem examination showed necrosis of the tendon, of the posterior surface of the sesamoid bones, and of the navicular bone. The process had extended downwards in the track of the tendons, and had attacked the navicular bursa. From a clinical standpoint such acute inflammation is of less importance than the chronic processes (identical in character with navicular disease), i.e., which consist of an arthritis chronica, and which at times are complicated with disease of the sheath of the flexor pedis perforans just above the fetlock.

Pathological anatomy. Changes characteristic of arthritis chronica are found affecting the gliding surface of one or other of the sesamoid bones; the cartilage becomes eroded, and its margins proliferate. When the anterior surface is diseased, both it and the posterior articulatory surface of the metacarpus exhibit abraded and eroded spots. In disease of the posterior surface of the sesamoid bones, the flexor tendons appear fibrillated (fig. 244), or are sometimes completely ruptured. Brauell saw a few cases of adhesion of the flexor tendons to the sesamoid bones. The sides of the bone show osseous deposits. The tendon sheaths and sesamoid ligaments are also thickened; sometimes detached pieces of bone are found in the sheath. In the cases reported by Levens, the sesamoid bones had probably been fractured.

As a rule, both sesamoid bones are affected; Mascher, however, in some cases found disease of a single sesamoid and of the corresponding metacarpal articular surface.

Causes. Any violent strain of the flexor apparatus or increase of the pressure normally exerted by the flexor tendons on the sesamoid bones 
may produce this disease, and therefore both Brauell and Mascher regard long, weak fetlocks as the chief predisposing factor. This formation not only throws a greater strain on the tendons, but increases the pressure on the sesamoid bones, on account of the more oblique angle which the tendons make with the metacarpus (compare with section on "Navicular" Disease"). This probably explains Mascher's statement that where the toes are turned in, the outer sesamoid is affected, and vice rersi, for such defect of conformation must necessarily lead to unequal pressure on the sesamoid bones. Heavy bodies and weak fetlocks predispose to the disease, which is therefore commonest in hackis and heavy draughthorses, and occurs without exception in the fore limbs. Other causes are jumping, especially from a height, and suddenly reining up. In such cases, partial rupture of the flexor tendons probably occurs and starts the disease; Siedamgrotzky reports an observation of the kind. The disease may then set in with acute inflammation, but as a rule it develops slowly, the flexor sheaths being often simultaneously affected at a point close above the fetlock-joint.

Symptoms. In the chronic forms, lameness is the most apparent symptom. As in all affections of the flexors, pain is greatest when weight is placed on the limb, the phalanges are flexed and the limb is rested. Volar flexion is in most cases well marlied. Lameness is most distinct during the first few steps, but may disappear with exercise; it is decreased by long rest, increased by exertion, is more marlied on rough, hard ground than on sand or grass, and is sometimes so slight as only to be detected by carefully watching the animal whilst trotting, though in severe cases it is apparent at a walk.

Palpation of the back of the fetlock gives pain. After some time a bony swelling appears, corresponding in position to the sesamoid bones. If the anterior surface of the sesamoids is involved, the swelling is close to the metacarpus, i.e., at the side of the joint; in disease of the posterior surface, it is further back on the volar aspect of the joint. After a further variable period, the flexor tendons become swollen in the neighbourhood of the sesamoid bones, though such swelling may occasionally appear before the other symptoms. The acute form is distinguished by local heat, \&c., which is absent in chronic cases. During the later stages there is "lnucliling over" at the fetlock-joint, from shortening of the tendons. In one case Branell found the sesamoids, in consequence of the marked volar flexion, thrust upwards beyond the articular surface of the metacarpus, and adherent in their new position. Movement of the fetlock-joint is often distinctly limited. Crepitation can rarely be detected.

The course is chronic, inflammation seldom subsiding, but continuing for long periods, and necessitating slaughter. Lameness becomes worse 
after severe exertion, but tends to disappear with rest. Complete recovery is, however, very rare once the disease has become well developed.

Diagnosis is seldom difficult, the character of the lameness being often sufficient, provided there is no other disease of the flexors or chronic navicular disease. The swelling is less to be relied on. Thus exostoses sometimes occur on the sesamoid bones, particularly on their lateral surfaces, without causing a trace of lameness, and, from their symmetrical appearance in both limbs, often give the impression of being congenital. Before delivering a prognosis, it is best to thoroughly examine the flexor tendons, and to make sure whether the fetlock-joint can be fully flexed.

Treatment. During the stage of acute inflammation, and particularly when the flexor tendons exhibit recent swelling, the animal should be rested, and the fetlock-joint, as far as possible, fixed by a firmly applied bandage, which may be moistened with warm water, or, in case of great pain, with a continual current of cold water. Good results sometimes follow firing and blistering, though six to eight weeks' rest is then necessary. Old-standing cases are not often benefited by such treatment. Neurectomy is the only means of removing lameness; and, considering the seat of disease, it is better to divide the median than the plantar nerve.

\section{VIII.-OSSIFICATION OF THE LATERAL CARTILAGE. SIDE-BONE.}

The term side-bone is applied to a condition in which the lateral cartilages of the foot become partially or entirely ossified. The wings of the pedal bone are prolonged backwards and upwards by two plates of cartilage about a quarter of an inch thick and approximately rhomboidal in shape, each of the faces being some three inches in length (see figs. 245 and 246). They are only found in solipeds. Under the action of the body-weight the plantar cushion, to which they are attached, aiternately expands and contracts, and so long as the cartilages remain normal they respond to this movement. When ossification commences, however, movement in the anterior portions is limited, and the posterior sections of the cartilage, which usually resist disease for the longest period, tend to be excessively displaced at each step. This aggravates the already existing inflammation in the anterior parts, and causes pressure on the sensitive structures of the back of the foot, producing well-marked lameness. For this reason the disease is often progressive, and is much more serious in animals with upright, narrow heels, where the horn cannot yield, than in the opposite formation.

The entire cartilage does not always become ossified, however'; nor do 
both cartilages of one foot or the cartilages of both feet always become diseased together.

Lungwitz, who examined 1,251 horses, states that side-bones occurred as shown in the annexed table:-

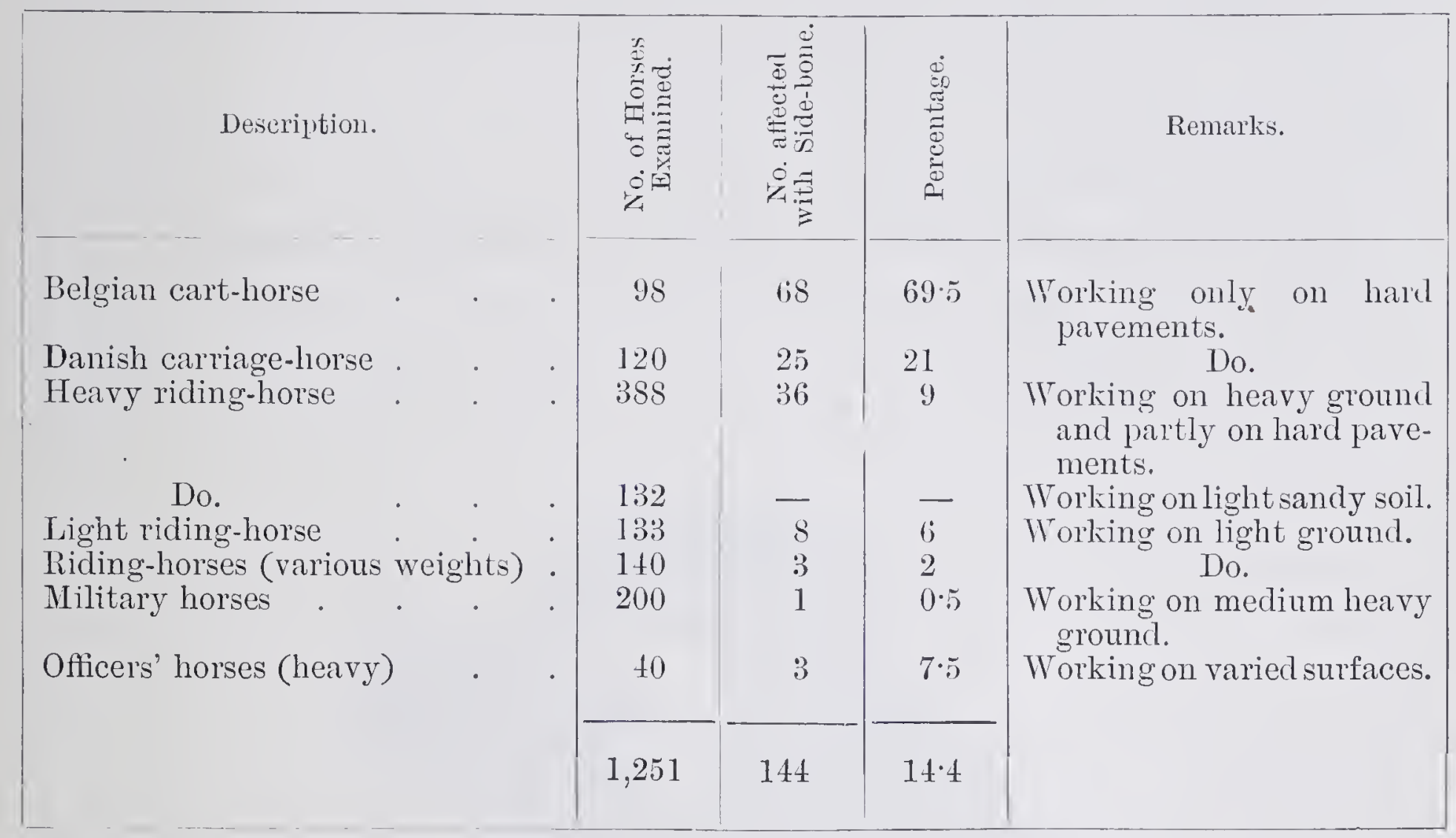

1. Ossification of the lateral cartilage occurs principally in heavy, coarse-bred horses. 2. The fore feet suffer more frequently than the

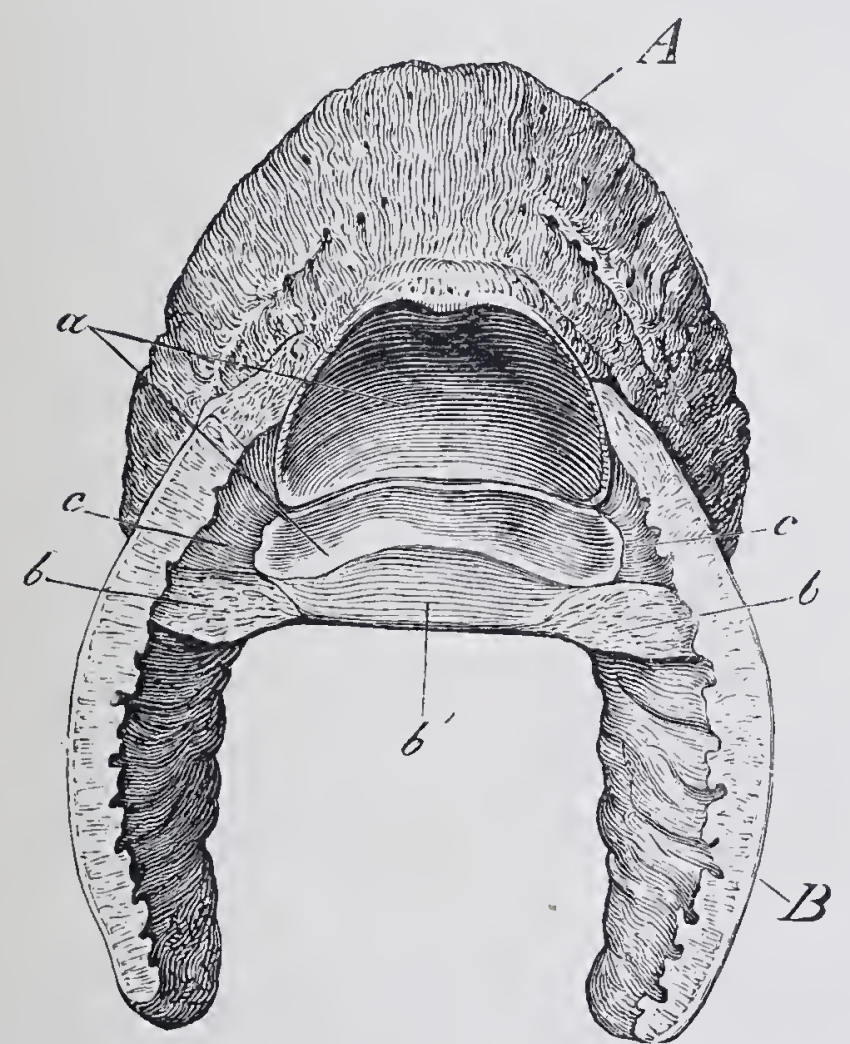

Fig. 245.- $A$, Pelal bone; $B$, lateral cartilage cut through horizontally at the level of the pedal-joint; $a$, surface, formed by pedal and navicular bones, for articulation with coronet bone; $b$, postero-lateral ligaments of navicular bone cut through; $b^{\prime}$, portion of above which are attached to back of navicular bone ; $c$, the lateral ligament of navicular bone.

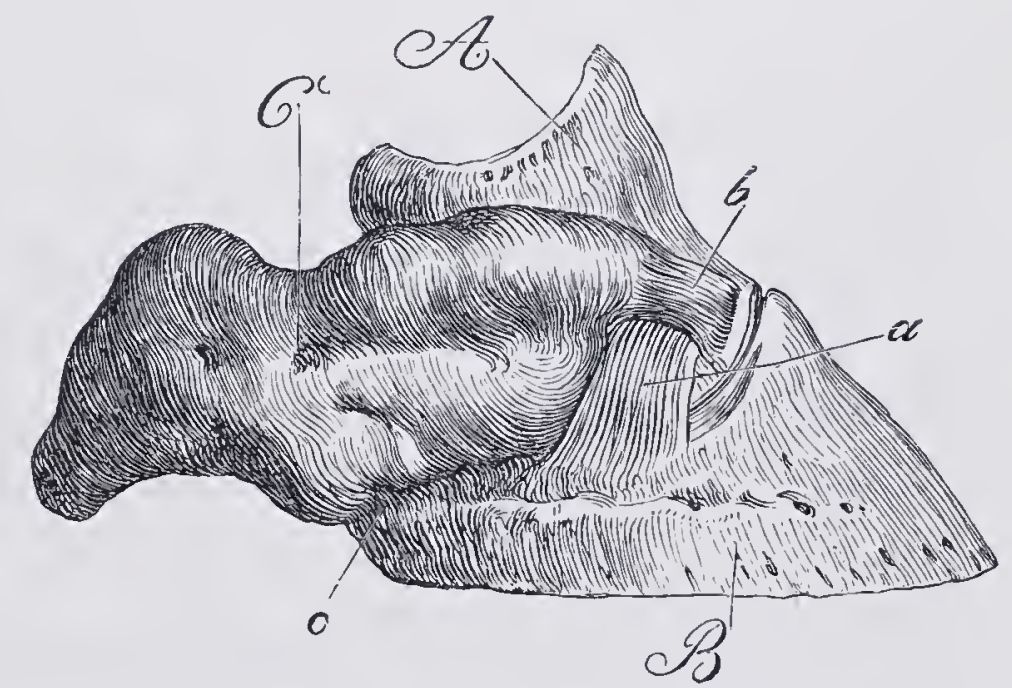

FIG. 246.-Right fore foot. A, Coronet bone ; $B$, pedal bone; $C$, outer lateral cartilage; $a$, onter lateral ligament of pedal-joint; $b$, ligament comnecting lateral cartilage to coronet bone; c, ligament connecting lateral cartilage to pedal bone. 
hind; 3 , the cartilage of the left hoof suffer's more frequently than that of the right; and 4 , the outer cartilage suffers more frequently than the inner. 5. Ossification sometimes occurs at an early age, usually when the animal is first put to work. 6. Well-bred animals suffer less frequently than others. 7. The use of animals, of heavy build, on hard roads favours the occurrence of side-bone.

The symptoms vary according to whether one or both cartilages are affected, and whether ossification is partial or complete. Expansion of the hoof is always diminished, and may be entirely suspended. Sidebones produce a clumsy, constrained action, and at times marked lameness. The symptoms are aggravated by rapid work on hard roads, by allowing the hoofs to become dry, and by using high calkins. When,

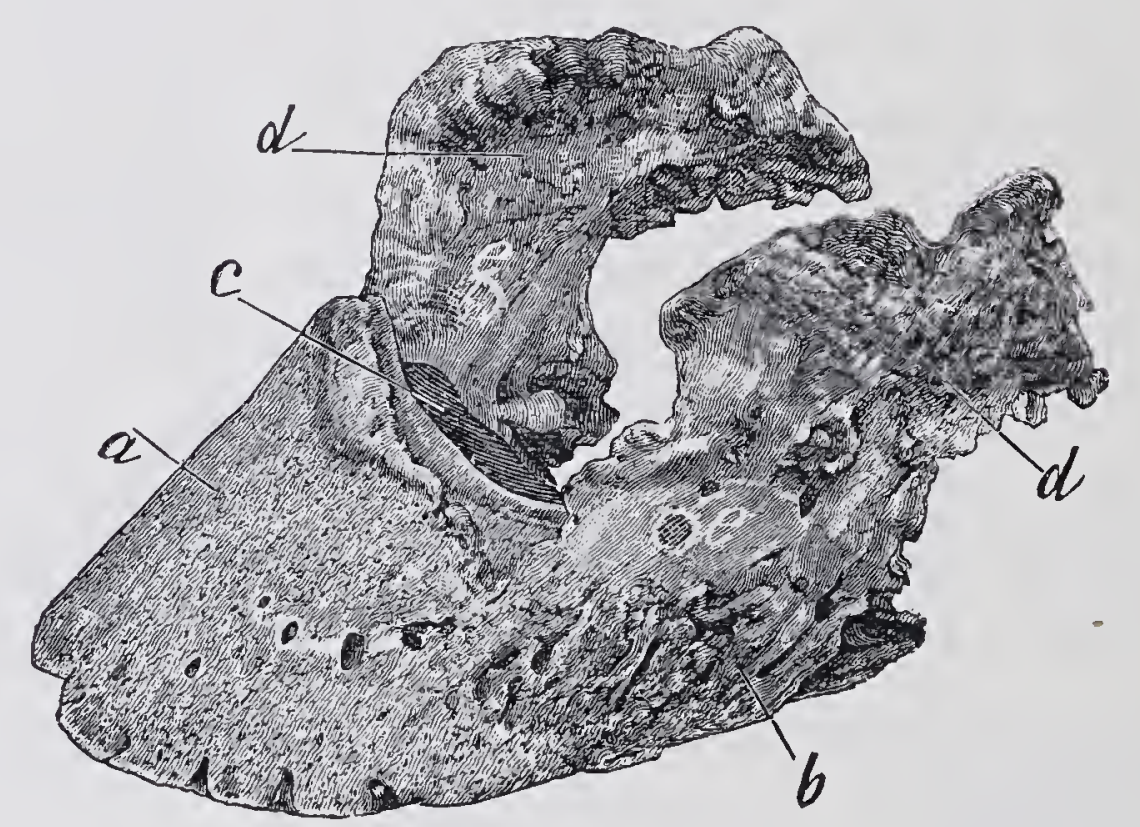

FIG. 247.-Pedal bone, with almost complete ossification of the lateral cartilages. a, Pedal bone; $b$, wing of pedal bone, from which point, as a rule, ossification commences; $c$, articular surface; $d$, rongh, uneven surfaces of the ossified cartilages.

however, the hoof is kept moist, animals may work for a long time without inconvenience.

The diagnosis of side-bone is easy as soon as the upper margin of the cartilage has become ossified; it then feels hard. On the other hand, when ossification has only attacked the lower portion of the cartilage lying within the hoof, it is either very difficult or absolutely impossible to detect the change, though the form of the hoof sometimes gives valuable indications.

The causes are-(1) congenital predisposition, in heavy, coarse-bred horses; (2) excessive concussion produced by work on hard roads, for which reason the disease is frequent in large towns; and (3) shoeing with calkins, by which the frog is prevented taking any bearing on the ground and the lateral cartilages are subjected to a continuous inward and downward dragging strain. This is concentrated at the fixed, i.e., anterior, end of the cartilage, where disease usually commences. Mechanical injury is another undoubted cause. This, and the fact that the outer 
half of the foot suffers the greater shock during movement, explains why the outer cartilage more frequently becomes ossified than the inner.

The prognosis depends on the horse's work, weight, and breed, on the form of the hoof, and on the extent of ossification. Heavy horses with completely ossified lateral cartilages are of little use for rapid work on paved streets. When only one cartilage is affected, or when the animal is worlied on soft ground, side-bones are comparatively unimportant. While ossification is in active progress the animal goes tender, if not actually lame, but as soon as it becomes complete the lameness tends to disappear, though it readily returns in consequence of bruising: or strain if the tread is not level. Lameness is usually temporary, but the diseased cartilage can never be restored to its primitive condition.

After ossification is complete, lameness may be produced by bruising of the plantar cushion and of the sensitive wall, which are then enclosed between two hard, unyielding structures; the plantar cushion, being confined by the ossified cartilages, can expand neither towards the right nor left at the moment at which weight is placed on the foot and the sensitive wall being similarly placed between the horny wall and ossified cartilage. A partial improvement may occur when the plantar cushion diminishes in volume. If, in addition, the wall is contracted at the heels, the condition is even more serious.

Treatment consists in resting the horse, removing the shoes, and placing the animal on tan or other soft bed; thinning the wall over the affected cartilage, and applying cold poultices. When lameness disappears shoe as directed below. Should lameness persist, a smart mercurial blister may be applied, or the lateral cartilage fired in points, and the animal be given two or three months' rest.

Col. Fred Smith isolates the portion of the wall covering the sidebone by deep incisions through the wall and sole, and so relieves the inflamed parts of pressure, but the results are not invariably satisfactory.

In shoeing animals with side-bone it is important to know whether the condition is uni- or bi- lateral. In side-bone of the outer heel the wall of that side is comparatively immobile, and the corresponding quarter and heel of the shoe is excessively worn. On removing the shoe the outer wall is found much higher than the inner. The external heel of the shoe is thin, the internal comparatively little worn. The hoof is either unchanged in form or the wall of the outer heel is contracted, and sometimes covered with rings. The outer portion of the coronet is more prominent, and the outer limb of the frog smaller than the inner. Bruises or strains in the wall not infrequently exist.

The shoe should be flat, the outer limb broader than usual, the seatingout should terminate behind the last nail hole, so that the entire breadth of the heel surface may form a horizontal plane. 'The outei' wall should 
be lowered more than the inner, and the shoe so formed that its inner limb fits as close as possible, the outer being left sufficiently wide to meet a perpendicular line dropped from the coronet. The supporting surface is thus widened towards the outside, and, in consequence of the level tread, more even wearing of the shoe produced.

When both lateral cartilages are ossified, a thick leather sole materially assists in diminishing shock. Special deeply-fullered shoes with rope inlay are also of value, but pads and bar shoes seem (in theory at least) contra-indicated, and, at first, cause pain by pressing on the frog and so tending to thrust asunder parts that are now unyielding.

Side-bones are often accompanied by corns, which are usually extremely persistent. As, however, these are not primary but secondary conditions, they deserve less attention when choosing and fitting the shoe than the ossification. Under these circumstances "springing" the heels, which is frequently practised in order to relieve corns, produces local strain and pain, and should be avoided.

\section{IX.-FRACTURE OF THE NAYICULAR BONE.}

Fracture of the navicular bone is rare, though it has been seen after suddenly throwing a horse on his haunches, and after movements which throw great strain on the flexor perforans, and therefore on the navicular bone. Uhlich found the bone broken in three pieces. When studying at Alfort, Dollar saw three cases of fractured navicular bone in the horses used for surgical exercises. The strength of the navicular bone is lessened, and fracture favoured by chronic inflammation like that accompanying navicular disease; the danger is greater after neurectomy. In such cases the action of the limbs is in no way modified by the diseased condition of the navicular bone, which is then readily fractured, while the flexor pedis perforans may be torn asunder. Fracture of the navicular bone not infrequently results from treading on nails. In cellulitis affecting the sensitive frog, suppuration often extends to the perforans tendon and navicular bone, and, if not at once checked, may cause necrosis both of the tendon and bone. Schraml saw fracture of the navicular bone and rupture or tearing away of the perforans tendon from the os pedis, and thinks fracture is caused, after rupture of the tendon, by the excessive pressure of the os coronæ on the navicular bone. It is much more probable that the fracture had caused rupture of the tendon.

Diagnosis principally depends on the peculiar lameness, though in acute inflammation of the gliding surface of the os naviculare the movement is similar. The foot is continuously rested, and if weight is unavoidably placed on it for a moment, the phalanges show excessive 
volar flexion. The toe is directed obliquely backwards and downwards. Marked paid is caused by lifting the foot and extending the coronetjoint (dorsal flexion) by pressure on the toe. Inflammatory swelling occurs later in the hollow of the heel.

This fracture is distinguished from acute disease of the navicular bursa by the sudden appearance of lameness, and, at the commencement at least, by the absence of swelling from the hollow of the heel.

Prognosis is unfavourable, particularly in compound fractures, caused by picked-up nails. There is always danger of the perforans tendon being injured, and, later, ruptured, by the sharp edges of the fragments, or by the callus, or of chronic lameness appearing, hence complete recovery is extremely unlikely.

Treatment is only advisable in valuable animals, like those used for the stud. Complete rest is of first importance if the fracture is subcutaneous. At a later period plantar neurectomy might be performed. In complicated fracture caused by picked-up nails, resection of the perforans tendon may be attempted. Humbert, in such a case, claims to have completely resected the navicular bone with success. The foot was irrigated night and day for a month after operation. In two and a half months the animal could trot sound. Möller, who made experiments in this direction, failed to obtain good results, and he seems to entertain considerable doubt of the possibility of cure by resection of the navicular bone.

\section{X.-NAVICULAR DISEASE.}

In England, during the latter half of the eighteenth century, attention was first directed to a disease which had become unusually common in thoroughbred horses. At first it was regarded as a disease of the pedal-joint, or was mistaken for contraction of the foot. It is now impossible to say whether the disease described by Bridges and Gibson as "grain of the coffin-joint" was identical with that now under consideration. Moorcroft states that he had previously seen the disease, though he had not directly recognised it. In a letter dated 1804, Moorcroft warns his correspondent against mistaking simple contraction of the foot for coffin-joint lameness. He describes the first condition, and states that it only occurs secondarily in this disease. Coleman and 'Turner further observed and described the condition, at first under the title of "coffin-joint lameness," and later of "navicular disease," or" "navicular lameness." The French "maladie naviculaire" is derived from this.

In Germany the disease was also first termed "coffin-joint lameness." Renner endeavoured to more particularly identify the seat of the disease by distinguishing an anterior and a posterior coffin-joint lameness, and only applied the latter term to the disease now in question. Sticker proposed the term "podarthrocace." At that time "arthrocace" was a term applied to various chronic joint diseases. Brauell first described the disease thoroughly, and named it "podotrochilitis." Both names - " navicular disease" and "pozotrochilitis" - have the merit of at least indicating the seat of the 
disease in the tendon bursa formed by the perforans tendon at the posterior surface of the navicular bone just before its insertion, which Eichbaum named "bursa podotrochlearis."

Anatomy. At the posterior surface of the navicular bone, over which it glides, surrounded by a sheath, and just above its insertion into the os pedis, the perforans tendon becomes expanded, forming a tendon sheath, which sometimes suffers from acute or chronic inflammation. Acute inflammation is most commonly the result of some mechanical injury to the hoof, either by picked-up nails or by stones becoming fixed under the shoe. In the case of picked-up nails, the bursa podotrochlearis is either directly wounded or

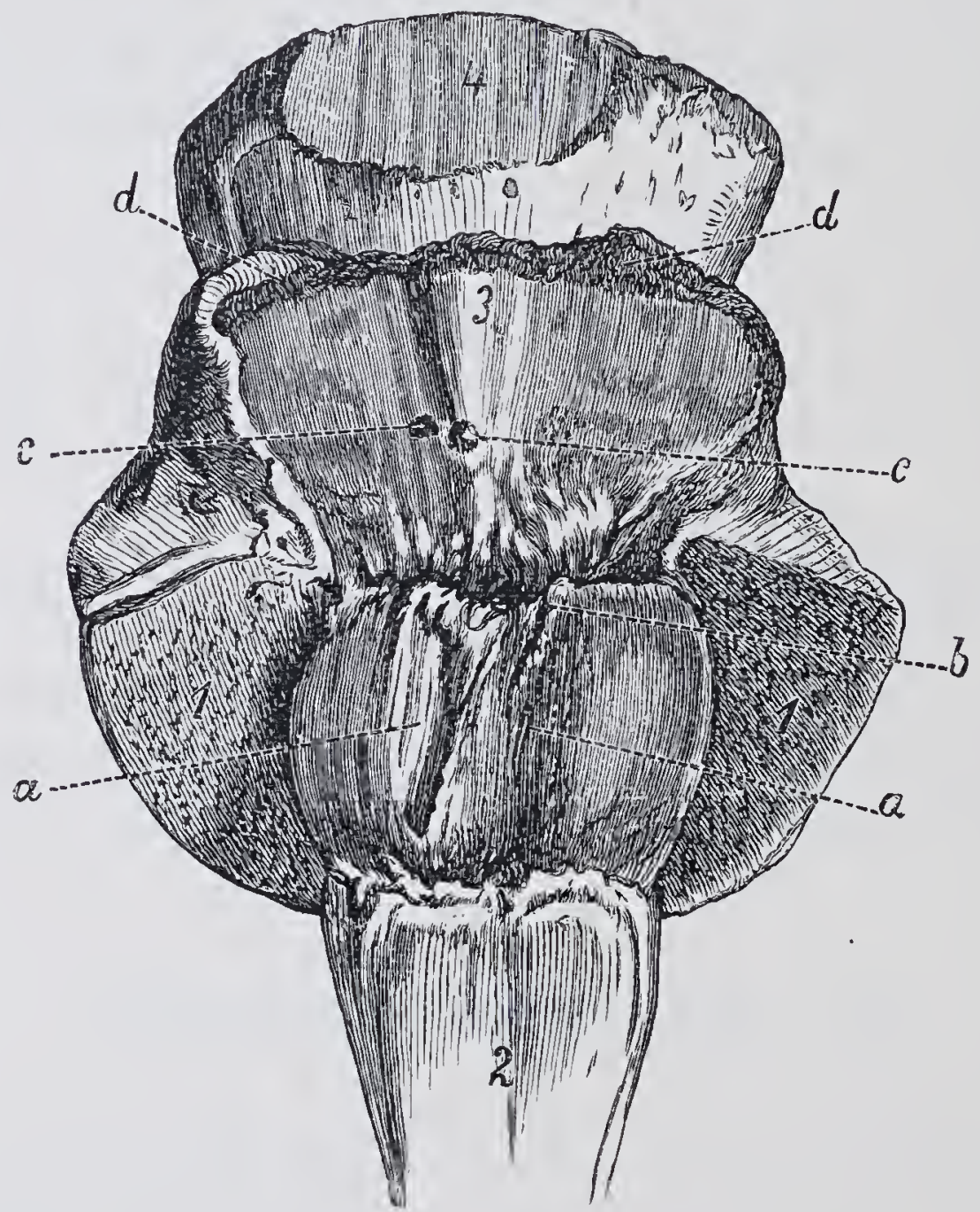

F1G. 248.-Preparation illustrating navicular disease. 1, Os pedis; 2, flexor pedis perforans tendon drawn downwards; 3 , lower (gliding) surface of the navicular bone ; 4 , coronet bone; $a$ and $b$, roughened, abraded spots on the flexor perforans tendon ; $c$, eroded spots on the uavicular bone; $d$, proliferations from periosteum on the upper margin of the os pedis.

inflammation extends to it from the sensitive frog. Such acute conditions are described under "Diseases of the Hoof."

The conditions included under the title to this section are all chronic, and vary greatly in origin, intensity, and extent. As, however, there is no satisfactory method of clinically separating them, we are forced to regard them under the one head, which therefore includes all aseptic inflammations in this position, and especially those of a chronic character.

\section{Pathology and Causation.}

The following structures are affected:--

(1) The navicular bone.

(2) The perforans tendon.

(3) The bursa podotrochlearis. 
Opinions are divided as to the point of origin of the disease. Turner places it in that portion of the bursa surrounding the tendon, Fambach considers the disease starts from rupture of fasciculi in the tendon. Schrader and others locate the primary disease in the gliding surface of the navicular bone. Brauell, however, maintains that the point of origin is not constant, and that sometimes one, sometimes the other tissue first becomes affected. The fact that the perforans tendon is always found diseased does not prove that the first changes occur in it, for immediately the surface of the navicular bone becomes roughened the tendon is endangered. The gliding surface of the navicular bone seems to be the commonest point of origin. Both the course and character of the process support this view. Little spots no larger than a millet-seed are to be found here, generally about the centre of the middle elevated portion, over which the cartilage appears coloured (figs. 248 and 249). Smith views these as calcareous deposits, but microscopic examination reveals the same conditions as in arthritis

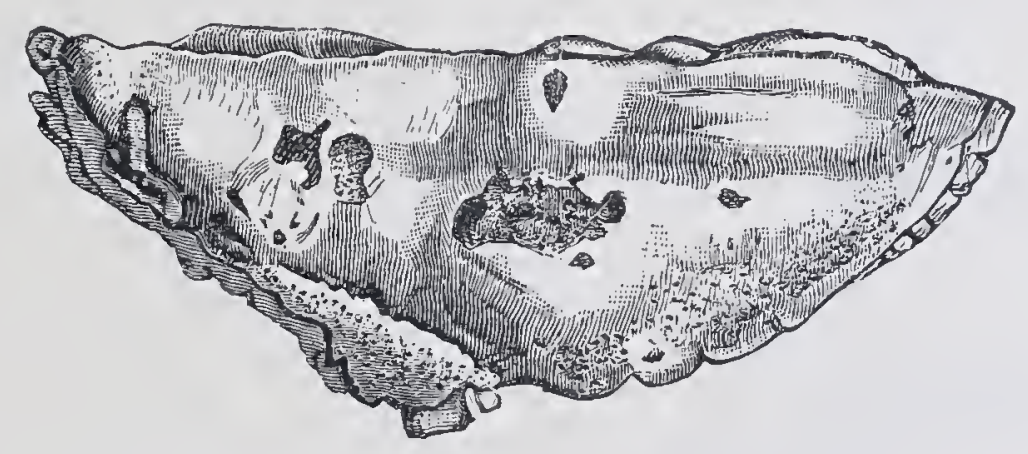

FIG. 249. - Navicular bone in chronic navicular disease (after Brauell).

chronica deformans, i.e., proliferation of cartilage cells, with fibrillation of the intercellular substance. The cartilage thus altered loses its consistence and becomes eroded (fig. 249).

As soon as the substance of the bone is attacked, granulations form, causing destruction of neighbouring portions of cartilage and of bone. Rarefying ostitis sets in ; the compact tissue, normally of a yellowishwhite, assumes a reddish colour, and disappears more and more in consequence of increasing proliferation of the medullary tissue. The bone may thus be so much weakened as to readily fracture under pressure of the tendon. Smith states luaving seen, in certain cases, change in the compact tissue precede that in the cartilage: this is more frequent where both feet suffer. As a rule, these peculiar osteophytes, which resemble those of arthritis deformans, and appear as though poured out on the surface, can be found on the margins of the navicular bone, and especially on the upper margin, where the bursa extends from the tendon on to the bone. 'The ligaments of the navicular bone become thickened, and sometimes ossified. The upper surface of the bone, which contributes to the formation of the coffin-joint, is never thus affected. 
When the condition is preceded by bursitis, which Williams considers common, the parts are more or less reddened and injected, and the bursa is thickened and its surface roughened. Such disease must, of course, affect the tendons and navicular bone.

Finally, inflammation may extend from the flexor perforans to the navicular bone; Fambach, indeed, regards this as the rule. Isolated fasciculi of the tendon are ruptured by excessive strain, and the process commences. But the course of the disease, and especially its long period of development, contradict this view, though it is a fact that, with few exceptions, the tendons are always affected. Smith found disease of the tendon in 99 per cent. of cases. The portion of tendon in contact with the navicular bone at first shows brown-coloured spots,

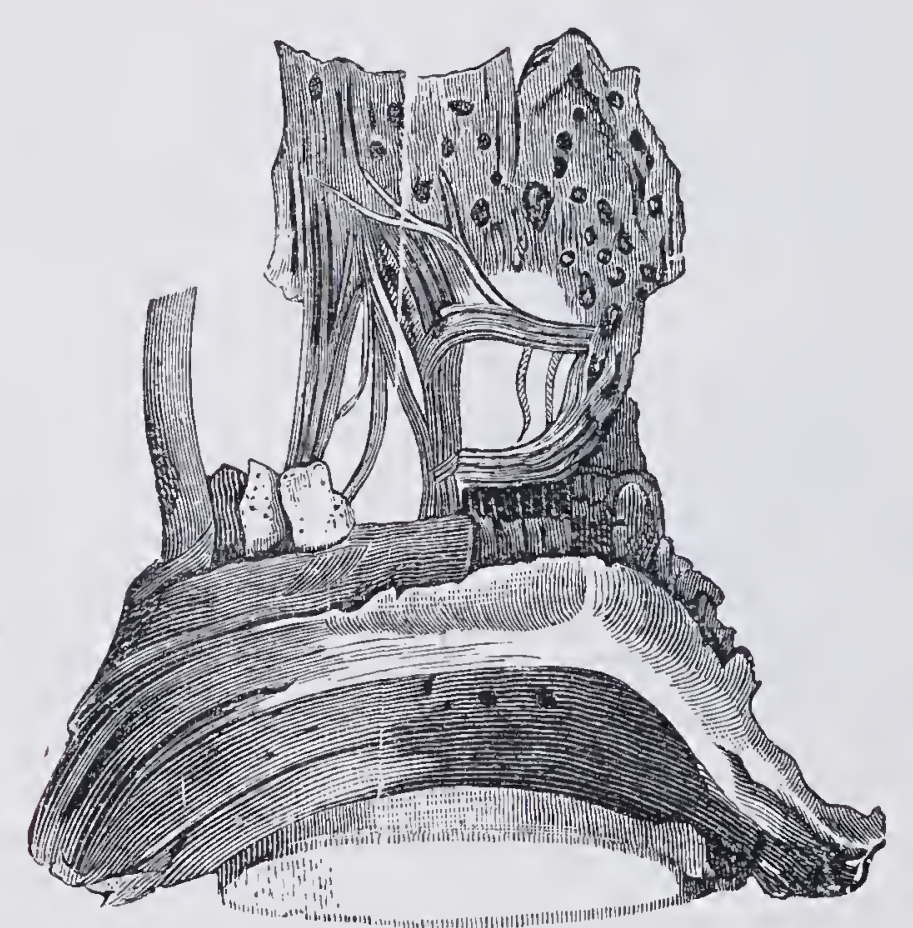

FiG. 250.-Flexor pedis perforans tendon in chronic navicular disease (after Brauell). its surface loses its glistening character, and soon shows abrasion. Single fasciculi on the surface of the tendon become ruptured (fig. 250), partly torn away, and rolled upward. Microscopic examination shows them to have undergone fatty degeneration (Smith). In old cases the tendons may be extensively eroded, and may finally rupture. Almost all such changes in the tendons are, however, secondary. Immediately the surface of the navicular bone becomes rough from loss of the cartilage, a rubbing action, similar to that between the articular surfaces in arthritis deformans, occurs between the rough navicular surface and the tendon. The fact that the portion of tendon most frequently affected is that exactly over the navicular bone supports this view, though granulation on the navicular bone may not be the sole cause of change in the tendons: rupture of fasciculi may perhaps at times be a primary condition, and seems indicated where the disease appears suddenly with severe lameness. Under favourable circumstances, and with long rest, union occurs between the perforans tendon and the navicular bone, being favoured by the granulations of the latter. The portions of tendon thus altered later become ossified.

Causes. The tendency to navicular disease probably depends largely on peculiarities of conformation; hence the hereditary character of the disease, and its tendency to attack more than one foot at the same time. Smith seeks to explain the disease by referring it to faulty development of the bony tissue of the navicular bone. Theoretically nothing can be 
adduced against this view, but further investigations can alone determine to what extent such peculiarities act as causes of the disease. One argument against it is, that the disease almost invariably occurs in well-bred horses, especially in those with narrow, high feet. In coarselybred animals it is only seen exceptionally, and under very special circumstances.

Only two cases are reported of the disease also affecting the hind feet. In both cases the investigators (Wrainsford and Fambach) confirmed their diagnosis by post-mortem examination, and there is no doubt that chronic inflammation of the gliding surface of the navicular bone and of the perforans tendon had occurred, but no explanation of the cause is given. In Fambach's case the horse was lame for nine months; as much as possible it avoided placing weight on the limb, and both during movement and at rest kept the limb flexed.

The almost constant occurrence of navicular disease in the flexor apparatus of the fore limb and in the bursa podotrochlearis is partly due to the greater weight borne by the front feet and to the violent strains thrown on the flexor tendons during rapid movement on hard ground or under the weight of the rider. Under these circumstances the navicular bone is exposed to severe pressure on the one side from the coronet bone, on the other from the flexor tendon; and though disease of the joint is seldom caused by bruises from below when moving over uneven ground, yet such an accident is conceivable. It is still very doubtful how strains, \&c., act in producing the disease. The variety of opinion on this point is to some extent due to confusing cause and effect. In no lameness are errors of diagnosis so common as in navicular disease.

The older English authors considered that a small, narrow condition of the hoof often caused this disease, the strongly arched sole pressing on the navicular bone. This view is due to confusing navicular disease with contracted sole, as Brauell has pointed out. Williams regards the upright position of the fetlock as a predisposing factor, a view which is pertinent, so far as the position of the fetlock is in some degree referable to that of the hoof.

Peters and Fambach first explained the connection between this conformation and the disease. Excessive lowering of the heels causes the axis of the hoof to become more oblique than that of the pedal bone, and throws excessive strain on the flexor perforans at every movement. The pressure on the navicular bone thus produced is increased by the flexor tendon describing a sharper curve in its passage over the navicular bone.

Leaving the toe too long may similarly cause the disease, or induce inflammation of the flexor tendons. The upright position of the pastern 
is not the sole factor, but acts in conjunction with faulty trimming of the hoof. Williams' view, that the most important factor is the failure to neutralise shock, appears incorrect, because under these circumstances the tendons would escape injury. Finally, it must not be forgotten that uprightness of the pastern may not only be a cause, but also a consequence of the disease.

Peters explains the fact that horses which stand "over" on the front feet more often suffer from this disease, by drawing attention to the sharper angle made by the flexor pedis when passing over the navicular bone, and directs attention to the circumstance that when the limb is loaded, and the os pedis in consequence sinks, that tension in the tendons increases, and, consequently, that greater pressure is exercised on the os naviculare.

The frequent occurrence of navicular disease in horses with long pasterns is explained by the greater stress thrown on the tendons. On the other hand, obliquity of the pastern is in some respects an advantage, because the flexor perforans takes a straighter course over the navicular bone.

Günther thinks that turned-in toes predispose to the disease. Hertwig's view, that high heels favour its production, is based on a misconception, though raising the frog from the ground may throw increased strain on the flexor tendons. The same applies to contraction of the foot, often blamed as a cause of the disease: it occurs during the development of the condition.

Excessive pressure on the navicular bone accompanying sudden partial rupture of the perforans tendon may give rise to the disease, and was regarded by Fambach ảs the commonest cause. Möller inclines, however, more to Peters' opinion, viz., that navicular disease is due to slowlyacting influences consequent on change in position between the bones of the limb or between them and the hoof axis, an idea which receives support from the slow character of the change. In riding and carriage horses, initial injury may be occasioned by sudden reining-in, jumping, continued sharp trotting, galloping, and occasionally even by slipping.

English authors, among them Turner and Goodwin, think it may be caused by long rest, desiccation destroying the elasticity of the hoof and favouring the disease. Möller does not agree with Smith in thinking that continued standing on a particular foot causes the disease. $\mathrm{He}$ has often seen laminitis and descent of the os pedis under such conditions, but never navicular disease. Some of the above views may be due to faulty diagnosis and mistaking "contracted sole" for navicular disease, especially considering the favourable course noted by the older English authors, who report 90 per cent. of recoveries. Bruises of the sole and frog are only occasional causes of navicular disease. 
In rare cases the disease occur's metastatically after influenza, and then generally in badly-bred and flat-soled horses, a fact which perhaps explains Brauell's statement that horses often suffer from the disease after having had diarrhoa. This was possibly due to secretory metastasis, disease of the bursa occurring in consequence of specific micro-organisms obtaining access to it.

Symptoms and course. The more important symptoms are-

(1) Lameness. On account of its slow development the beginning of the disease is generally overlooked, and attention only directed to the parts after pain and lameness are present. Lameness begins gradually: the first few steps, particularly if taken on hard ground, are painful, but this often disappears completely with exercise. When both feet are affected diagnosis is still more difficult. There may only be a tendency to stumble (Smith). The seat of disease explains why pain should be most marlied when weight is placed on the limb. Even in the stable the diseased foot is rested. As pressure on the affected navicular bone is greatest when the limb is directed backwards, at which time, of course, the flexor perforans is in greatest tension, the animal endeavours, as far as possible, to shorten this phase of movement, and, to remove weight from the tendon, the foot is held in a position of volar flexion with the pastern bone upright, sometimes to such a degree that the heels scarcely touch the ground. 'The gait then becomes stiff and insecure, and the animals readily stumble. When both feet are diseased the animal tries to shorten those phases of movement in which weight is borne exclusively by one foot, and the limb is therefore carried stiffly and rapidly forwards. Spooner says the animal moves as though it had a wooden leg. Owing to the pain the horse moves slowly, sweats, and soon becomes exhausted.

Sometimes the difficulty in movement disappears after a few steps; sometimes it is permanent, or may even increase with exercise, depending on the position, nature, and extent of the disease. It is usually more marked after great exertion. It occasionally disappears completely after a long rest-a fact which renders diagnosis difficult, and may give the impression of recovery. To sum up, the symptoms are as follows:-Lameness when weight is placed on the limb, increased after considerable exertion, decreased by long rest; volar flexion of the phalanges and extension of the foot, particularly during the first few steps.

(2) Changes in the hoof. The local inflammation may be detected by exercising pressure on the navicular bone. One jaw of the pincers is applied over the centre of the frog, the other over the wall of the toe. Hertwig's special pincers are unnecessary; but if the sole and bars are very thick, they should be thoroughly pared before using the pincers. Pain can sometimes be detected by pressing with the thumb in the 
hollow of the heel.' In doubtful cases Brauell recommends working: the horse after applying a bar-shoe, the bar of which covers the centre of the frog. This increases the lameness in real cases of navicular disease.

In some cases forcible dorsal flexion gives pain. The foot is lifted as in shoeing, and the toe pressed slowly and powerfully downwards, or the horse is so placed that its toe rests on an elevation, thus causing the heels to be abnormally low when weight is placed on the foot. The same thing often occurs accidentally when the horse is moved on uneven ground, and produces very marked lameness.

As a rule, there is no increased warmth or pulsation. Brauell and Haubner have drawn attention to the erroneous view advanced by English authors, viz., that increased pulsation was a symptom of this disease; Hausmann, indeed, views absence of puisation as pathognomonic of the disease in question. As already stated, other diseases associated with increased pulsation, such as contracted sole, ${ }^{1}$ are often mistaken for navicular disease; but as there is no acute inflammation, the absence of increased warmth and marked pulsation are not surprising.

During the further course of the disease the hoof contracts, and the heels become longer and narrower. These changes in size and form suggested to Sticker the invention of a podometer-an instrument, however, which is of no particular value for diagnosis, and can be easily replaced by the meter used for measuring the angle of the hoof.

As the patient no longer places weight on the foot, local nutrition and horn production suffer, the hoof contracts, and the coronary band appears more prominent, and seems swollen. The uprightness of the pastern causes the hoof to takè a more perpendicular position as time goes on. The frog is also relieved of pressure, and this, with the defective development of the hoof, leads to shrinking in of the heels and atrophy of the frog-in short, to contraction of the foot, the horn sole then appearing abnormally concave.

That all these changes are due to diminished pressure and movement of the hoof is shown by the fact already pointed out, that they gradually disappear after operations like neurectomy, which relieve the pain and remove the lameness.

Although contraction of the hoof is important in diagnosis, it should never alone be relied on, because, as Haubner pointed out, the left foot is often smaller than the right.

The quality of the horn also suffers during the disease. The wall frequently appears uneven, rough, or covered with rings, and its horm brittle.

\footnotetext{
1 For fuller particulars of the changes in the foot incidental to navicular disease, see "A Handbook of Horse-shoeing," by Dollar and Wheatley, pp. 340,355 et seq.
} 
In the later stages the muscular tissue of the limb atrophies more or less, depending on the degree of lameness (atrophy from want of function).

Since the discovery of cocain a valuable aid in diagnosis has been afforded. By injecting a few minims of a 5 per cent. solution of cocain over the course of the plantar nerves, temporary anæsthesia of the lower portions of the limb is produced; disappearance of lameness under these circumstances renders it probable that the seat of disease is in or about the foot.

The first stages of the disease generally pass unnoticed, until the gradual increase of lameness draws attention to them. The lameness may disappear with long rest, but always returns after hard work. Weeks and months pass, until at last it becomes necessary either to get rid of the animal or to perform neurectomy. Recovery is rare, and supposed cures mostly result from faulty diagnosis or from mistaling remission for recovery, as shown by the above-reported opinions of the older English authors, who put the average of recoveries at 90 per cent. Bracy Clark, an able observer, assesses them, on the other hand, at 1 in 16. From the nature of the disease, recovery must be rare, and can only occur during the first stages, i.e., while the disease is confined to the bursa of the flexor tendon. Prognosis is most favourable when, on account of the sudden onset of lameness, there is a probability that inflammation is confined to the tendon and bursa, and that the flexor surface of the navicular bone is still intact. But when atrophy of muscle or hoof has set in, improvement is not to be expected. Exceptional cases are seen, however, where recovery, or at least cessation of lameness, is produced by adhesion of the perforans tendon to the navicular bone. Gerke confirmed this by a post-mortem two years after the disappearance of lameness. Peters had a similar case, but such a termination is very exceptional.

Neurectomy, often resorted to to remove sensation, is apt to be followed by rupture of the perforans tendon. The tendon becomes more and more abraded by the rough surface of the navicular bone, until at last, in a moment of excessive strain, it suddenly tears through, leaving the horse completely useless. Temporary improvement following long rest must not be mistaken for cure.

The disease is not nearly so common as was formerly believed, and is often confused with diseases of the hoof and other lamenesses. Errors are easy, unless plenty of time is given to the examination, and diagnosis sometimes proves difficult, even to the most practised.

\section{Differential Diagnosis.}

The condition may be mistaken for-

(1) Contracted sole. The form of the hoof, the strongly concave sole, V.S. 
the curving inwards of the heels, the blood-stains in the white line and at points in the sole, are, however, sufficiently distinctive, while in contracted sole pain is more marked after pressure on the sole than on the frog, and lameness generally appears after shoeing, and disappears with careful paring of the sole and appropriate shoeing. Horses with contracted sole not infrequently go more distinctly lame on soft than on hard ground, but in navicular disease the reverse is the case.

(2) Greater difficulty is sometimes offered by cases of "wiring-in of the heels," which also at times appears as a symptom of navicular disease. Usually, however, there is then increased pulsation and pain on pressure over the quarters, though "wiring-in" of a secondary character may contribute to lameness. In such case the frog should be carefully tested with the pincers.

(3) Sprain of the posterior ligaments of the coronet-joint. This lameness is usually more severe. The posterior surface of the coronetjoint is then the seat of pain, whilst pressure on the sole causes no flinching (compare with "Inflammation of the Posterior Ligaments of the Coronet-joint").

(4) Some caution is required where thrush is present. If sufficiently extensive to involve the greater part of the frog, pressure on the parts may produce symptoms simulating those of navicular disease. The operator should then try the effect of forced dorsal flexion, which is not painful in thrush.

(5) The symptoms of sesamoid lameness present a certain similarity to those of navicular disease (see "Sesamoid Lameness") ; a careful local examination is therefore necessary.

Treatment. As above stated, recovery is only possible when the local changes are capable of repair. Disease which starts as an acute bursitis or tenositis offers some chance of cure, though inflammation soon extends to the articular cartilage of the navicular bone, and the condition becomes as intractable as if it started at this point. In recent cases, long rest and antiphlogistic treatment sometimes check inflammation and assist repair. The horse may be turned out to graze in a meadow with a clay bottom, where the soil is always moist. If kept in a box soft bedding, like sawdust, tan-bark, or peat, should be provided, and the feet kept moist; thoroughly paring the hoof, so as to assist the action of cold moist applications, is useful. Shortening the toe and shoeing with "tips" is also of value. The presumed efficacy of bleeding (recommended by Turner and Goodwin) and of frog-setons (Sewell) depends on errors in diagnosis. In recent cases, the rest rendered necessary by setoning assists repair, but Brauell rightly questions the resorbent action of setons: continued rest is known to temporarily alleviate the lameness, and is sometimes resorted to by horse-dealers 
preparatory to sale. Rest and the inflammation produced in the fibrofatty frog may, however, assist adhesion between the navicular bone and flexor tendons. Frog-setons were first recommended by Sewell, who constructed a special sharply-curved needle with a handle and sharp point (fig. 252) for the purpose of inserting them. The seton is best passed in the standing position, the limb being fixed by means of a kneehobble (see p. 13 of Dollar's "Operative Technique"), otherwise the foot

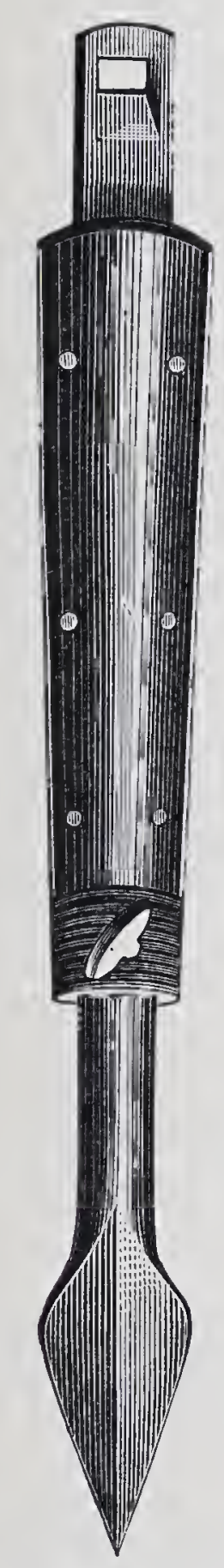

Fı. 251.--Straight seton needle.

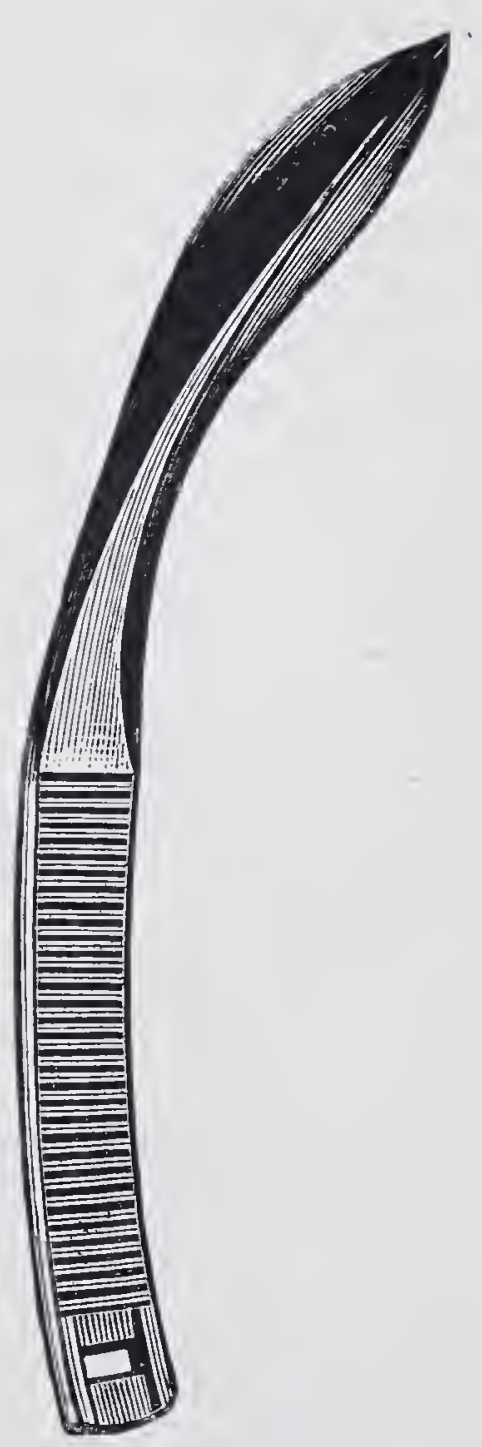

Fia. 252.-Curved seton needle.

may be moved during operation, and the needle broken. The point of the frog is first pared away, and a twitch having been applied to the nose or ear, the needle, previously threaded, is thrust into the hollow of the heel, through the plantar cushion and sensitive frog, so as to emerge at the point of the frog. The handle is then removed, the needle drawn through the frog, and the ends of the tape knotted together at the heel. The seton is left in position twelve to fourteen days, being moved daily to allow free discharge of pus. Sometimes it is saturated with oil of turpentine. 
There is no particular danger in passing frog-setons, though some care is required to avoid injuring the perforans tendon; the loop of tape must not be left too long, as it may be caught by the other foot and torn out.

Restive horses require to be thrown for operation; it is then convenient to fasten the limb to a broad plank provided with a long handle, by which it is steadied (see p. 34 of Dollar's "Operative Technique").

The seton needle must be regularly but not too sharply curved; some operators prefer a nearly straight needle, similar to that used in cellulitis of the fibro-fatty frog.

If setoning fails, nothing remains but to perform neurectomy (see section on "Ring-bone"). Möller doubts the utility of resection of the flexor perforans tendon, as in his hands it has usually left the horse either permanently lame or has necessitated so long a rest as to be of no practical service.

A valuable carriage-horse, which had for a long time suffered from navicular disease, contracted acute inflammation of the navicular joint in consequence of bruised sole. Operation was at once resorted to, and the navicular bone and perforans tendon found to be chronically diseased. The case did well, and the horse returned to work, but for several months trotted slightly lame.

Proper shoeing is of great importance, both in preventing and treating this disease. In general, it is well to shorten the toes and lengthen the heels, either by suitably paring the foot or by applying leathers or shoes with thick heels.

In marked contraction of the foot, indiarubber or cork pads or longheeled shoes may be employed. Defay's shoes sometimes diminish lameness considerably; their action is assisted by moderate exercise on soft ground and by foot-baths (see Dollar and Wheatley's "Handbook of Horse-shoeing ").

Tenotomy, recommended by Smith to assist adhesion of the perforans tendon to the navicular bone, has not proved successful.

\section{XI.-DERMATITIS IN THE FETLOCK REGION. MUD- FEYER - CRACKED HEELS - GREASE.}

The terms "mud-fever," "grease," \&c., have been used since olden times to designate a series of disease processes in the slin about the fetlock, particularly on its flexor surface, the nature and course of which are exceedingly varied. Certain characters are, however, common to all forms of these diseases, viz., their point of origin in the heel, their inflammatory nature, and spontaneous appearance. The diseases may be divided into four varieties:-

(1) An erythematous.

(2) An exanthematous, typified by "mud-fever." 
(3) A gangrenous, including " cracked heels."

(4) A verrucous form ("grease" proper).

The first three follow on acute inflammation of the skin, which, however, may be attended with complications, and may later assume a chronic course. The last form, which is usually chronic, is that generally recognised in England as "grease" proper. Dollar believes that it is often preceded by the erythematous and exanthematous forms, but, as these are of short duration and infection of the discharging surfaces almost immediate, the red-blush of the skin (erythema) and the watery discharge (eczema) only last a very short time, and are speedily merged into the chronic condition, with offensive discharge and formation of warty growths, which English veterinary surgeons recognise as "grease."

(1) Dermatitis erythematosa. This is the mildest form of dermatitis, and is commonest in horses with thin, fine slins; well-bred animals suffer more frequently than heavy working-horses. In many horses the skin of the pastern-joint seems exceedingly sensitive, so that even the presence of sand in soft soils may cause irritation, and give rise to this form of dermatitis. Similar results have been noted after long-continued exposure to direct sunlight. The unpigmented skin appear's red and rather painful, but after a few days these symptoms disappear, and active desquamation sets in. The disease consists of hyperæmia of the papillary layer of the skin, produces no permanent injury, and only occasionally requires special treatment. When, however, the condition is neglected, and the horse continues to work, this form of dermatitis may give rise to the next in order.

(2) Dermatitis eczematosa. Mud-fever. Eczema, or inflammation of the skin, appears spontaneously, is ushered in with swelling and redness, is usually soon followed by exudation from the surface, and in general takes a typical course, which, however, is less easily studied in this position than in others. The swelling and redness are often scarcely visible, though there is lameness, especially when the animals are first moved, whilst the surface of the skin becomes covered with a yellowishred fluid, which soon dries to a brownish crust. The papular stage is not well marked, though the vesicular stage, i.e., the formation of small bladders, may sometimes be noted. The weeping stage (stadium madidans) appears more distinctly, and is rapidly succeeded by the formation of crusts (stadium crustosum). The crusts soon loosen under proper treatment, and desquamation follows (squamous stage), revealing normal epidermis. In characteristic cases the process takes eight to fourteen days, and ends in complete recovery, but not infrequently complications occur, especially if the animals are worked during the disease. The movement of the skin produced by walking, assisted by 
the presence of foreign material, interferes with regular healing, and leads to the formation of folds, cracks, and fissures. Chronic proliferation then sets in around these, and leads to the formation in the hollow of the fetlock of thick cicatrices, which can only be removed by operation.

During the inflammatory stage the animals go very lame, especially for the first few steps, but improve after a short time at exercise.

Causes. Coarse-legged horses with long hair often suffer from this form of dermatitis when working on wet, ground. The epidermis is continually moistened, becomes macerated, and inflammation is then readily produced in presence of infectious substances. The absence of visible external cause, and the simultaneous appearance of disease in several feet, or in several animals in the same stable, gave rise to the idea that mud-fever was a blood disorder, and should be regarded either as a metastasis or as erysipelas. Joly and Truche regard it as contagious, although the specific organism is unknown. Truche seems to rely on the fact that the disease attacked several horses in a previously healthy stable after the introduction of some American horses suffering from it. The fact that the same causes were acting on a considerable number of animals at the same time was overlooked.

Jenner declared equine grease to be identical with cow-pox. Since his time "protective grease" has even been spoken of, though neither Jenner's experiments nor those instituted later in any way support this view. Many years ago it was denied by Leblanc, Deprato, and by other observers, and at the present time no doubt exists that eczematous grease (mud-fever) is dissimilar to variola. It is even a question whether horse-pox can be included in the same category with grease. In France medical practitioners have claimed to have inoculated men with grease, and thus proved its variolous nature. But Leblane pointed out the untenability of this view. He showed that the case of grease from which inoculation was said to have occurred had been in existence for six months, and therefore could certainly not have been variola. The description indicates the case to have been verrucous grease. Dieckerhoff has suggested that Bouley's "protective grease" was probably stomatitis pustulosa contagiosa (horse-pox). Berger declares having seen grease produced in horses by inoculation with sheep-pox, but his observations do not appear very trustworthy. The attempts made in France to inoculate horses with cow-pox and cows with grease altogether failed. In the light of recent knowledge, it is easy to understand that the secretions found in the latter stages of grease produce inflammatory processes in man, but this in no way demonstrates their specific character. There is little room to doubt that the earlier observers confused "grease" with horse-pox, an entirely different affection. Horse-pox is attended with fever, is very contagious, may affect any portion of the skin, though it is often confined to certain regions or even to one, and runs a comparatively simple, benign course, leading to recovery. When affecting the mucous membrane of the mouth and lips it constitutes the above-mentioned stomatitis pustulosa contagiosa. The confusion that has arisen between different writers owing to the loose use of the word "grease" renders it desirable that either the word be limited to the chronic verrucous form or that it be altogether abandoned in favour of the word "dermatitis," 
with the addition of adjectives like "erythematosa," "verrucosa," \&c., appropriate to the particular form under consideration.

Grease has been described by various writers, amongst others by Hertwig and Spinola, as an erysipelas. But this view is supported neither by the mode of origin nor by the course of the disease; and even though Malzef lately found, in cultures prepared from cases of equine grease, a micro-organism which appeared identical with Fehleisen's streptococcus erysipelatis, yet latter-day views of the relationship of the streptococcus to erysipelas and to suppuration in no way bear out the above suggestion.

Eczematous dermatitis, or mud-fever, is usually produced by external irritation either of a mechanical, chemical, or specific character. The disease often occurs soon after shoeing with high heels, because the changed position of the foot favours the formation of the folds in the skin just above the heels, in which sand and dirt lodge, and produce excoriation. One of the commonest causes of mud-fever and of cracked heels in town horses is to be found in the habit of washing horse's heels with strong allialine soaps (like soft soap) and hot water. The soap irritates, and the hot water produces vascular relaxation, liable to be followed by inflammation. Somewhat similar results are produced, in heavy horses, by washing the legs with a cold-water hose after returning from the day's work. Omnibus and cab horses, whose legs are only roughly rubbed dry and are cleansed next morning with a brush, seldom suffer from any of these forms of dermatitis. An excellent methor of drying and cleansing the legs simultaneously, is to rub them freely with clean pine sawdust. Animals working on stubble or freshly-laid roads or forest paths are apt to contract slight skin injuries, which sometimes form the point of origin of disease; the wound discharges macerate the epithelium, irritate the skin, and produce inflammation.

Grünald describes an enzootic dermatitis which appeared during the hottest part of summer amongst horses stalled in open sheds, and in which the lower portions of the limbs were continually exposed to the sun's rays. Horses sheltered from the sun were not affected, a fact which led Grünwald to compare the condition with erythema solare of man.

Kröpfl noticed the same thing in horses at grass. Only the unpigmented portions of skin in the hind limbs seemed to be diseased.

Bermbach believed that mud-fever was conveyed from horse to horse by using the same washing-pails, \&c. More probably the same general external influences were at work.

Amongst chemical irritants must be numbered chall-dust. In the army its action is often troublesome, on account of horses becoming affected immediately they are brought into challiy regions. Columella 
had even noticed it, for he writes- " equus, qui hieme laborat, eundo per lutum, in aestate per pulverem." The action is chemical, as shown by the fact that disease is favoured by washing the legs, the best preventive being simply to cleanse the parts with a dry cloth.

Observations made on men, dogs, and other animals, seem, however, to favour the belief that eczematous disease may be associated with constitutional changes, which are at present very imperfectly understood.

Course. Early and appropriate treatment generally results in complete cure in eight to fourteen days. When, however, the disease is neglected, recovery is impeded by the formation of fissures and of new connective tissue, which latter sometimes produces prominent thick swellings in the pastern-joint. Though simple cracks soon heal with rest and proper treatment, large masses of new tissue are very often difficult to deal with, especially if adherent to the subjacent flexor tendons and sheaths. Before giving an opinion, the parts should therefore be thoroughly examined.

The treatment of eczematous dermatitis requires in the first place rest, because movement keeps up inflammation and favours the formation of fissures and of excessive quantities of cicatricial tissue. The skin should be carefully cleansed with warm water and a neutral soap, the swellings rinsed with creolin solution, dried, and dressed with iodoform and tannin $(1-3)$. After the preliminary washing and disinfection, it is very important to avoid again wetting the parts. This is one of the chief secrets in the successful treatment of these forms of dermatitis. Astringent dusting powders or ointments are generally useful. Pyoktanin has been highly recommended, but its powerful staining properties render it unpopular. Thioform, dermatol, glutol, and amyloform (all compounds of formic acid with neutral bases) are easy to use and efficacious. An ointment of red oxide of mercury one part, with paraffin base or lanolin five parts, is useful. It may be smeared on wadding, laid on the surface of the wound, and fixed in position by a bandage. The dressing keeps out the air, and may be left in position until wet through, when the diseased spot is again cleansed and the dressing renewed.

Recovery generally follows in about eight days. Even deep fissures are soon cured by complete rest, and the use of dry dressings or of the ointment and a cotton-wool dressing.

To check excessive proliferation ("proud flesh") and cicatrisation, astringents like alum or sulphate of zinc may first be tried; and in the event of their proving unsuccessful, caustics, nitrate of silver and sulphate of copper, are often of service. Mineral acids must be applied with caution, to avoid excessive destruction of tissue and risk of opening tendon sheaths. 
Cicatrices which project above the skin, or which, from contraction, have become hard and sharply marginated, can only be dealt with by the knife, caustics being in most cases useless. The portions of growth overtopping the neighbouring slin are removed, with antiseptic precautions. The parts should be washed with sublimate, creolin or carbolic solution, and a dressing moistened with one of these materials applied and left in position twenty-four to forty-eight hours. This sterilises the point of operation. In quiet animals, the parts may be removed whilst standing; if necessary, cocain can be locally injected. Restive horses should be cast. The foot is extended as far as possible -in the case of the hind foot, it may be bound to the front one. The incisions should be smooth and regular; bleeding is generally slight, and can be checked by a pressure dressing, which at the same time prevents excessive new growth. By resting the animal and keeping the stall dry, healing is generally complete in eight to fourteen days, provided the growth is not extensive, i.e., not more than 1 to 2 inches in size, otherwise cicatrisation proves difficult, and the growth is inclined to recur. Möller repeatedly removed growths of this kind from the hind pastern, and cured cases in which medical treatment had proved useless.

(3) Dermatitis gangrenosa has a special surgical interest, because it often forms the first of a series of diseases which call for the display of surgical skill. It consists in spontaneous gangrene of the skin of the phalanges, occurs in solipeds, particularly during winter, and is oftenest seen in large towns. One of its commonest and simplest forms is known as " cracked heel."

Causes. The fact that gangrenous dermatitis occurs almost always in winter, and especially in cold countries, has suggested the idea that it is due to freezing of the skin. The fact that the disease does not occur so much during periods of excessive cold as during thaws was not held to altogether disprove that view; for it seems natural that moist cold should be particularly injurious, on account of its neutralising the protection afforded by the hair, and enabling the cold to act directly on the skin. The theory, however, is not in keeping with the local character of the disease. Were cold the sole agent, symptoms of freezing in the first and second degree might fairly be looked for around the gangrenous spot. They are, however, generally absent. The experiments of Siedamgrotzky and Jelkmann showed that the lowering of temperature, consequent on the use of salt (which is particularly common in large towns), could not be held responsible for the production of the disease. And, finally, it should be noted that at times, when gangrenous dermatitis is common, all wounds show a tendency to gangrene. It, therefore, seems tolerably clear that local freezing is not the only cause, but that other 
factors, partly of an infectious, partly of a chemical character, are also at work. Slight injuries to the lower portions of the limb lead to septic infection, which may result in inflammation, and when assisted by cold, in gangrene. Low temperatures depress the vitality of existing wound surfaces and thus favour septic bacilli in obtaining a lodgment. Whatever view may finally prove correct, thus much is certain, that as a rule infection acts along with cold in giving the first impulse to the production of gangrenous dermatitis. The cases seen about the coronet and front of the pastern are often initiated by "treads"; the horse injuring the skin with the calkin of the opposite shoe, and the wound becoming infected. That this form of dermatitis does occur, in exceptional instances, even in the warm season, only shows that gangrene of the skin may be produced independently of cold.

Symptoms and course. The disease appears suddenly, generally overnight. There is severe lameness in one or other limb, with inflammatory swelling of its lower portions, sometimes also of the upper parts. Fever is also common. Closer examination detects at a given spot a portion of skin, from 1 to 2 inches in diameter, which is soft, yielding, and swollen: pressure over this spot causes severe pain, and produces discharge of a reddish turbid fluid. The pastern is the most common seat of disease, at a point just over the bulb of the heel, or on the anterior surface near the coronet. The pain continues, the limb increases in size, and in two to three days the gangrenous portion of the slin is cast off as a soft, grey, slimy mass : the resulting wound then becomes covered with granulations, which gradually fill up the cavity. In favourable cases a cicatrix forms, and recovery is complete in one to two weeks.

But the disease does not always take so favourable a course ; sometimes the gangrenous inflammation extends downwards to the lateral cartilage, or the coronet bone, or penetrates to the sheath of the flexor tendon. In the first case a quittor forms, in the second an exostosis, which later becomes a ring-bone. And if in such cases suitable treatment often leads to recovery, i.e., to removal of lameness, yet when the sheaths of the tendons are also attacked, death usually occurs in a short time from septicæmia or pyæmia. If the necrotic process extends to the coronary vessels, pyæmia is apt to result. In the neighbourhood of the coronet, gangrene may extend to the coronary band, and produce severe inflammation, followed by loss of the hoof.

Prognosis depends on the extent, position, and character of the disease. The larger the gangrenous piece of slin, the greater the difficulty of treatment, and the danger: to deeper-lying structures. Gangrene near the coronet threatens the pedal-joint, in the pastern the sheaths of the flexor tendons. Implication of tendon sheaths at once renders prognosis unfavourable. The same is true of complications like septicæmia and 
pyæmia; in this case the swelling rapidly extends upwards, often to the elbow or knee, becomes very painful, and is accompanied by high fever. The character of the process may at times vary; in certain year's gangrenous dermatitis seems particularly malignant, and inclined to be complicated. The continuance of great pain after the necrotic portion of skin has separated is an ominous symptom.

Treatment. As a preventive measure, the feet in winter, and especially during thaws, should be kept as dry as possible, and care taken to protect them from the street mud, which macerates the epithelium, and favours infection and gangrene. The hair on the fetlock, and especially in the pastern-joint, may be anointed with a neutral fat like vaseline or lanolin, so as to lieep out mud and water. This precaution can be strongly recommended. For a similar reason, the feet should be carefully cleansed after work, and if possible dried; an excellent plan being to rub them with dry sawdust, wood-wool, or peat-moss. As urine and stable manure also have a very injurious action, cleanliness in the stable should be insisted on, and after the floor has been swept a dry disinfectant powder should be used. Holes in the floors, in which urine accumulates, must be filled up. If horses with injuries like cracks or fissures about the feet are kept at work, the wounds should be carefully cleansed and smeared with tar or creolin; and if the external temperature be below the freezing-point, a dressing may be applied.

If gangrene has already set in, its further progress may be prevented or checked by placing the parts in a lukewarm bath of sublimate or creolin solution, with which the affected spot may be frequently washed. At night a bandage should be used, moistened with camphor, or 10 per cent. chloride of zine solution. Möller had excellent results from applying 1 to 3 per cent. solution of pyoktanin to the diseased spot by means of a mass of tow, fixed in position by a bandage.

It is of importance to hasten separation of the gangrenous piece of skin; and if the entire piece cannot immediately be removed, loose portions at least should be cut off with scissors. The granulations are generally very abundant, and, being irritated by movements of the limb, are apt to become excessive and irregular. In such cases amyloform, glutol, or tannoform may be freely applied, the parts enveloped in a thick layer of cotton-wool, and a bandage applied with considerable pressure. At least once a day the dressing should be renewed, all hard fragments formed by drying of the powder and discharge being gently removed. When the wound becomes dry a little lanolin or other neutral İubricant should be substituted for the powder, the cotton-wool pressure dressing being continued. As a rule, it is more necessary to restrain than to excite the formation of granulations. If the patient be moved too soon they become excessive, and fungous growths appear, which give 
much trouble. If the coronet become swollen, the horn below must be rasped away.

A common sequel of gangrene is the formation of sinuses just above the heels, in consequence of a necrotic piece of tissue being retained in the depths, and keeping up chronic inflammation and pus formation. The passage of a sublimate seton, or the injection of liquor Villati, is sometimes sufficient to procure healing, but in obstinate cases it may be necessary to freely lay open the parts.

(4) Dermatitis chronica verrucosa. "Grease" proper. This condition is always chronic and progressive.

Originating in a small spot in the fetlock-joint, the process gradually extends until it may involve the entire carpal or tarsal joint. In other cases a considerable area may be attacked at the outset. It is due to active proliferation in the rete mucosum ; the cells produced, however, do not become horny, but break down and form a fatty, ill-smelling, grey mass on the surface of the diseased skin. In less rapidly progressive forms the cells form a grey, mealy mass. The more intense the process, the more active the proliferation, and the more abundant the exudation which bathes the surface of the skin. The papillæ at the same time increase in size, so that they appear like warts, and may generally be recognised by the naked eye; sometimes they become as large as a pea. The deeper layers of the cutis, and even of the subcutis, take part in the process, producing marked elephantiasis. The process extends to the hair follicles; the hairs are mostly shed, and the few which remain stand erect.

Leblanc in 1850 made microscopical examinations of the diseased skin, and regarded the condition as a simple hypertrophy. He found the hair follicles and sebaceous glands enlarged. In another section it has been pointed out how closely this process resembles canker. Either disease may extend to new structures, giving rise to the other ; thus grease may produce canker, and canker grease.

The disease is more frequent in the hind feet, particularly in hairylegged horses like Clydesdales; sometimes it seizes on several feet, one after the other. Whether a specific organism is the rausative agent remains for the present undecided, though such an explanation appears probable. Sometimes the disease originates from the eczematous form of dermatitis; not infrequently it is due to slight grazes or scratches.

Möller saw many outbreaks of this form of grease in certain stables; indeed, there seems to be some ground for referring them to direct infection. In large establishments animals in hospital were often attaclied after being rested for some time, and in one case the horse-keeper suffered from an eczematous disease on the hands. Every practitioner has noticed. how the disease is transferred from the affected leg to the sound one by rubbing. The local appearances and gradual spread of the disease point 
to infection, though its immediate cause is yet unknown. The variations in its method of outbreak and course suggest that it may be due to more than one cause.

Prognosis. Spontaneous recovery is very rare. As with canlier, careful and often prolonged treatment is required. The greater the extent of the disease, the more active the proliferation and consequent thickening, and the further the growth has penetrated the papillary layer of the skin the greater the difficulty in treatment. The dry form is most hopeful. When the papillæ have attained a considerable size, and folds exist in the skin, much less can be done.

Treatment. The condition being unquestionably a local skin affection, internal treatment is of little value, and the chief indications are to cleanse, and keep clean, the diseased spot, to rapidly remove decomposing secretions, or to prevent decomposition occurring. When the process is confined to one small spot, the parts may be cleansed, a white-hot firing-iron passed several times over the diseased surface, and a disinfectant applied. In more extensive attacks the limb may be bathed several days in succession with lukewarm chloride of lime solution, or with creolin or sublimate lotion, and the diseased spots repeatedly washed during the time the limb is in the bath.

A surgical dressing is afterwards applied, containing a mixture of creosote and spirit $(1-6)$, chloride of zine $(1-10)$, or creolin (5 per cent.).

Lies, of Brunswick, claims to have had excellent results from the use of sulphuric acid. The parts are carefully cleansed, the diseased surface painted with a mixture of sulphuric acid and spirit $(1-10$, or $1-20)$, and a dressing, moistened with the same fluid, applied. A little exercise should be given every day; rest favours the disease.

Dollar commences treatment with a dose of physic, and restricts the diet to bran, linseed, chaff, and green foods. After applying a very large warm bran poultice containing 2 to 3 per cent. of creolin for twelve hour's he recommends washing the parts thoroughly with $2 \frac{1}{2}$ per cent. creolin solution, and carefully drying. The application of water is afterwards carefully avoided, unless thick crusts form. Cleansing is effected with a large handful of clean cotton-waste vigorously applied, and the parts are thoroughly saturated twice a day with a lotion of zinci sulph. and alum varying in strength from 5 per cent. up to saturation point. Severe cases with the formation of "grapes" are vigorously cauterised with a whitehot iron, and the above lotion applied twice daily. Dilute sulphuric acid is only employed in very old standing cases. With this treatment Dollar has had most excellent results. Success depends on regular and vigorous treatment.

Large warts are removed with scissors, knife, or, better, with the whitehot iron. The whole surface may then be freely powdered with an 
astringent substance like iodoform-tannin, dermatol, glutol, amyloform, thioform, or a mixture of zine sulphate, alum and boric acid, and covered with a surgical dressing. The dressing must be applied with moderate pressure, and renewed daily. Ointments are to be avoided until the surface becomes dry, when it may be beneficial to apply a little red oxide of mercury and paraffin or lanolin ointment.

Treatment should be continued until the last trace of discharge has disappeared; the lower parts of the limb must then be kept as dry as possible, and any relapse treated in the above manner.

Dalrymple recommends hot poultices of wheat-flour and linseed-meal, to which acetic acid is added as the poultice becomes firm. This is applied for twenty-four hours, the surface of the skin cleansed with a dry cloth, and afterwards rinsed with chloride of zinc solution. He states having thus cured cases in fourteen days.

Straube lately recommended camphor dissolved in ether, and mixed with three times its weight of vaseline; this ointment is rubbed into the diseased skin. To check evaporation, the diseased parts are covered with carbolic wadding, and surrounded with flannel bandages. At first the dressing is changed daily, later every two or three days. When there was much itching, Straube gave arsenic internally with success. Möller seems to prefer sulphuric acid and formalin to all other materials. Their application is certainly painful, but the results are generally good. 


\section{DISEASES OF THE HIND LIYB.}

\section{A. DISEASES OF THE QUARTER.}

\section{I.-INJURIES TO THE MUSCLES AND FASCI $\approx$ OF THE QUARTER.}

(a) Strains and ruptures of the muscles of the quarter result from slipping, rearing or falling, and occasionally from over-exertion in moving heavy loads. Lameness usually follows, and may even prove incurable.

In a case of extensive tearing of the biceps femoris muscle at its origin on the ischium and gluteal fascia there was no lameness, but the animal's appearance suffered in consequence of the large depression that appeared at the site of injury. Another horse ruptured the semi-tendinosus muscle, producing a deep groove. The hind limb was afterwards carried and placed much in advance of its normal position. Lameness disappeared in two months.

A horse which ruptured the gracilis muscle by jumping, showed lameness when the leg was off the ground (swinging leg lameness); the hip-joint appeared to sink and rise to an excessive extent, and the stifle and hock joints were but partially flexed each time the leg was advanced. Lameness disappeared in a few days, but the inside of the thigh revealed a permanent falling-in of the muscle.

During a race a horse completely ruptured the semi-membranosus and adductor magnus, and to a partial extent the pectineus and adductor longus; severe lameness and marked swelling followed, and, treatment proving unsuccessful, the animal was lilled. An army horse caught one of its hind feet in the ground whilst in the act of jumping and ruptured the superficial gluteus 3 or 4 inches in front of the hip-joint. No weight could be placed on the limb, and all the joints were flexed. Six weeks later the horse was able to walk, but remained lame for a long time afterwards.

As a rule strains can only be recognised by the lameness they evoke; the limb is dragged, the movement uncertain, the limb being thrown too far backwards or forwards, or to one side. Palpation sometimes reveals local pain.

Muscular ruptures produce lameness of very varying severity and kind, 
depending on the structures involved. Sometimes the lameness persists indefinitely, sometimes it rapidly disappears. The longer it persists the more remote becomes the hope of recovery.

Treatment consists in the first stages in rest; if pain be present, hot fomentations often give relief; at a later stage massage and exercise are indicated.

(b) Bruises and wounds are commonest in horses, especially in winter, and are caused by kicks and "treads" by the sharp heels of the shoe. They also result from thrusts with the carriage-pole, from falls on rough, hard ground, or from animals slipping in front of the carriage and being run over. Amongst tramway and omnibus horses extensive injury of the muscles of the quarter is often produced in this way. In army horses wounds and bruises result from lance thrusts, sabre cuts, or falling on the rider's spur.

Blows may rupture large blood-vessels and produce extensive hæmatomata. Sometimes the skin and muscular tissue are torn through. The biceps femoris is often the seat of such injury, and in collisions the semi-tendinosus and semi-membranosus muscles are liable to be extensively injured. In heavy horses, wounds from stable-forks are often followed by septic cellulitis, which extends and produces destruction of the intermuscular connective tissue of the buttock, sometimes even necrosis of the tuber ischii. After the cellulitis has subsided, sinuses may remain and give great trouble.

Prognosis and treatment follow general principles, but one fact should always be borne in mind, viz., that the extravasation which follows bruising, and sometimes violent over-extension of muscle is seldom troublesome, if not too early incised.

In fresh cases, continuous cold irrigations and complete rest are most useful. By putting the patients to work or moving them too early, fresh bleeding and increase of the hæmatoma are favoured. To assist resorption, small extravasations may, after the third or fourth day, be treated by massage; in large ones, i.e., those containing a quart or more of fluid, such treatment is seldom beneficial, or takes longer than healing after successful puncture. Nevertheless, the knife should not be used before the fifth or sixth day after the appearance of swelling, partly because of the danger of after-bleeding, partly of infection and cellulitis. In five to six days thrombus formation is so far completed that bleeding is no longer probable, and the walls of the hæmatoma are sufficiently infiltrated with plastic material as to check the penetration of decomposition products. For the same reason, the opening should be made at the lowest point, the cavity emptied of coagulated blood as far as possible, and washed out with a disinfectant. A drainage-tube may be necessary. By adopting these precautions rapid healing is secured, and sometimes 
the walls of the hæmatoma immediately become united. Needless to say, careful disinfection is of the greatest consequence.

(c) Wounds confined to the slin of the buttock often heal by primary intention if immediately sutured with antiseptic precautions, and dressed with a disinfectant like iodoform-tannin. Union is assisted by covering the wound with a clean cloth, which is kept moistened with a disinfectant. Rest is absolutely necessary.

Muscular wounds in this position heal most rapidly under a scab. If, as is not infrequently the case, the entire wound-surface is open to the air, this will be found the best procedure. The wound is carefully cleansed, rinsed with a disinfectant, any loose fragment is removed, and, after bleeding vessels have been ligatured, the parts are powdered with iodoform-tannin. As a rule, this produces a dry scab, which is firmly adherent to the wound; nothing further is required. If the scab separates at certain points, it should be removed, the discharge wiped away with a little wadding, and the dressing renewed. Sometimes only the upper part of the wound heals under a scab; but though the lower portion is wet with discharge, granulation and healing are only delayed, not prevented. When the surfaces of the wound are in contact, or when pockets or deep injuries exist, it is difficult to maintain asepsis, and suppuration almost always occurs; in such cases, drainage must be assisted and burrowing of pus checked by a free use of the linife, by making counter-openings, and possibly by inserting setons and drains, the wound being meanwhile frequently cleansed.

Even when healing by first intention seems out of the question, recovery may be shortened by inserting stitches, particularly if the skin be separated from subjacent structures to any great extent. By bringing the flaps of skin together with thick threads, or, better still, with tape, which is not likely to cut, retraction of the cicatricial tissue in the edges of the wound is checked, and in large wounds healing is assisted.

Sinuses extending to the tuber ischii must be laid open, and if possible the necrotic piece of bone removed.

\section{II.-FRACTURE OF THE FEMUR.}

In spite of its protected position, the femur is not infrequently fractured in the different species of animals-in the smaller linds, like dogs, by treads, in horses by kicks, and in oxen by falls. Horses fracture the bone by struggling violently when cast, especially if the foot be fixed too high (that is, if the hoof be drawn up over the elbowjoint), and over-extended whilst in this position. Gurlt thinks that tying the hind limb to the cannon bone of the fore limb may cause fracture of the femur; but though German operators often fix horses in 
this way, no fractures seem to have been reported in consequence. On the other hand, Möller considers that tying the limb to the forearm or fastening it to the ring of a surcingle is dangerous. Fracture is favoured by absolutely fixing the hind limb in a position of flexion, on account of the great strength of the extensor muscles of the quarter. The less the resistance to their contraction, the less likely is fracture of the femur to occur. Lichte describes fracture of the femur in a horse affected with horizontal luxation of the patella.

Fracture of the neck of the femur, though occasionally seen, is much rarel in animals than in men, because in animals the neck is less well marked. Schrader reports a case ; the articular head had again become adherent to the femur opposite the lower trochanter. Kitt discovered post-mortem a fracture of the neck of the femur, and the formation of a false joint; the hor'se had nevertheless worked at a walking pace. Colin saw fracture of the neck and luxation of the femur in a horse. Fracture of the articular head itself is commoner; Dieterichs even saw a horse with double-sided fracture of the head of the femur. Fractures of the trochanters are rare, though Möller once saw the lower trochanter fractured by a fall on rough ground. Fractures of the lower articular processes, sometimes intra-capsular, are also infrequent, though they have been reported. In dogs the shaft is oftenest broken, usually near the lower end. Comminuted fractures may occur. Greve found a hor'se's femur broken into eighty-five pieces. In a race-horse the upper portion of the shaft of the femur was found to be broken into many pieces. Fissure of the femur in the horse has been produced by a lick; post-mortem examination revealed a transverse fracture of the femur, and it would, therefore, appear that the condition was really "green stick" fracture. Fiedler"saw a foal with separation of the upper epiphysis from the diaphysis and luxation of the femur.

The symptoms of fracture, either of the shaft, head or neck of the femur, are generally well marked. Pain is severe, the animal goes lame both when placing weight on the limb and when advancing it, the limb itself is easily moved, especially in an outward direction (abduction), and crepitation usually exists, though, if the fractured portion be much displaced, crepitation may be wanting. As a rule, the "slouching" movement of the entire limb may be remarked, even from a distance, and the only difficulty is to say whether the fracture is in the femur or in the tibia.

The diagnosis of fracture of the upper end of the femur is sometimes difficult, because of the lower portion of the bone becoming inserted into the articular cup and thus supporting the weight of the body; in such cases, however, the limb is, as a rule, visibly shortened.

Fracture of a trochanter is followed by swelling and marked lameness 
when the limb is carried, slowness in movement, shortening of the forward stride, and, usually, by abduction of the limb. Fracture of one of the lower condyles produces severe localised pain and marked flexion of the limb, similar to that of inflammation of the stifle-joint (gonitis); the other symptoms of stifle lameness develop during the next few days.

In horses and cattle the course is generally unfavourable, particularly if much lameness is shown when weight is placed on the leg. Fractures of the diaphysis may be followed by death from bleeding if the femoral artery be injured. Union never occurs in fracture of the head or neck of the femur. Hess and Schwerdtfeger kept cases of fracture of the articular head under observation for one year and for six months respectively; in neither was there any bony union between the head and shaft. Even where union occurs, the animal remains lame, owing to injury of the articular cup by the friction of splinters of bone; the articular cartilage may be completely rubbed away. Fractures of the lower condyle take a very unfavourable course. In small animals fractures of the diaphysis are more promising if there is no great displacement or shortening of the limb. Unfortunately, in dogs these two symptoms are generally marked, the upper fragment being thrust backwards through the muscular tissue, the lower one forwards; the mass of muscle thus interposed renders callus formation difficult, or altogether impossible. Recorded observations show, however, that even in large animals recovery is not entirely out of the question. Wilhelm, indeed, saw a fracture of the femur unite, though the horse was at the same time suffering from fracture of the os pubis of the other side. After ten weeks in slings the horse resumed light farm-work, though the injured limb had become 3 inches shorter than its neighbour. Fracture of the diaphysis in dogs is sometimes followed by formation of a callus fibrosus and permanent lameness, sometimes by perfect bony union and soundness. These fractures are least serious, and unite most quickly in swine and small ruminants, which are better able to rest the limb. Fractures of the trochanters are least dangerous, as they do not prevent the animal standing on the limb, and therefore are not likely to induce laminitis in the opposite foot; but even they sometimes give rise to permanent lameness, which restricts the animal to show work. 'The prognosis must largely depend on the degree of lameness associated with the fracture. Heuberger, in a horse, saw complete union of a fracture of the upper trochanter.

Treatment is confined to resting the animal, and if a horse to placing it in slings. The methods of reduction often described are usually inadmissible; even in dogs the muscles of the quarter are comparatively powerful, and oppose.great resistance to manipulation. The extension 
and counter-extension recommended often result in thrusting the fragments further apart. In dogs it is best to approximately replace the parts, and then to apply a plaster bandage, starting from the metatarsus and extending as high up the limb as possible, fastening it in position by a so-called "saddle." The bandage is passed over the back" and around the limb of the opposite side, to prevent it slipping down. This method often succeeds in dogs, but also at times fails. In man, and also in dogs, human surgeons have been successful in curing fracture by screwing together the portions of the shaft of the femur with silvered steel screws. By observing strict asepsis the wounds heal readily and the fracture reunites. Several such cases have been operated on in University College Hospital and Medical School.

In larger animals both reduction and retention are much more difficult, though the former is to some extent assisted by aniesthesia. The bandages and dressings which are often described can seldom be adjusted in such a way as to give a secure hold without setting up injurious pressure. Unfortunately, at the present day the cost of keeping horses generally renders treatment out of the question.

Ruminants and swine require rest and a comfortable stall, with plenty of soft bedding like peat-moss, and, to prevent their being annoyed by others, pigs should be placed in separate stalls.

To prevent fracture of the femur when casting horses the so-called "leg twitch" can be warmly recommended. A girth or strong cord is passed round the thigh, about 6 or 7 inches above the hock, a strong stick, 2 feet in length, pushed through it, and the cord twisted tight, so as to press the Achilles tendon against the tibia (see p. 7 of Dollar's "Operative Technique"). Any attempt to extend the limb now gives considerable pain on account of the pressure of the twitch, and the animal soon ceases to make further efforts. In addition, the twitch directly checks the action of the extensor.

It is also well to avoid rigidly fixing the hind $\operatorname{limb}$; sufficient play should be given to prevent the extensors having an absolute fixed point against which to act. It is therefore best to pass the girth or rope for fixing the limb over the tibia, between or over both fore limbs in a forward direction, under the neck and across the loins, and then back again to the limb. Then it is passed twice around the tibia immediately under the first turn, and the end of the rope led away over the body, where it is held by two assistants. The rope must not be drawn so tight as to prevent all play of the hind limb.

In an old horse with recent fracture of the lower trochanter of the right femur the forward stride was shortened about a quarter of its nolmal length. As the animal carefully avoided bending the joint, the toe grazed the ground. In advancing, the stifle described a curve 
outwards, though the upper part of the limb was adducted. When weight was thrown on the diseased limb, or when the right fore foot was lifted, the opposite quarter sank until the external angle of the left ilium was about 2 inches lower than the right.

At the point where the lower trochanter should have appeared, a depression, about as wide as three fingers, was visible. Beginning at the anterior border of the biceps femoris, and running for about 2 inches in a horizontal direction, it rose in a slight curve and lost itself at the posterior border of the tensor fasciæ latæ. On careful examination the lower trochanter could be detected just in front of the biceps femoris; a weak crepitation sound was produced by moving it. As the horse was not brought back after the first examination, its further progress could not be observed.

\section{III.-LUXATION OF THE FEMUR.}

In the horse, the depth of the articular cavity and strength of the ligamentum teres tend to prevent displacement of the femur. Rigot and others altogether deny that displacement occurs, though recorded cases clearly show that it may occasionally happen. It is certain that complete luxation cannot occur without rupture of the ligamentum teres. The pubio-femoral ligament derived from the prepubic tendon of the abdominal muscles sometimes remains intact after luxation, but the capsular ligament and surrounding muscular tissue are always ruptured. Incomplete luxation can scarcely occur in the horse, and reported cases in the cow are open to doubt.

In the horse the neck and articular head are more easily broken than displaced, but in oxen and other animals the conditions rather favour luxation, the articular cavity being flatter, and the ligamentum teres weaker. Luxation of the femur occurs in dogs. Fiedler noted a case in a foal, where the epiphysis was also separated.

In making post-mortem examinations of horses, Falke repeatedly found the ligamentum teres ruptured. In an old animal bought for dissection, which had shown no lameness prior to death, the ligamentum teres was absent. The points where the ligament is inserted into the head of the femur and acetabulum appeared deepened and smooth, but not a trace of the ligament was visible, and the pubio-femoral ligament, though normally developed, ended before entering the joint-cavity. The ligamentum transversum incisuræ acetabuli was normally developed; the posterior portions of the glutæus parvus were interspersed with cicatrical connective tissue. Probably luxation or violent distortion of the coxo-femoral joint had long before taken place, causing rupture of the ligamentum teres and of the muscle. Falke, experimenting on dead bodies, found that luxation was in one instance produced by a pull of 8 cwt., and in another of 16 cwt. In each instance the head of the bone and the cotyloid cavity remained intact, but the ligamentum teres and the capsular ligament were ruptured. It is therefore quite certain that the femur may be luxated without injury to the 
articular surfaces. Bucher's observations show that rupture of the ligamentum teres is often followed by severe lameness. Two horses which he saw with this rupture were unable to support weight on the diseased limb. One rested the point, the other the side of the toe, on the ground; the bock was excessively extended, and the phalanges fixed in a position of marked plantar flexion. It is not quite certain, however, whether these symptoms were exclusively dependent on rupture of the ligamentum teres. Wyman and Lusk saw complete luxation of the femur in the mule.

Amongst the causes are violent movement of the limb on the hipjoint, excessive flexion or extension in falling, and sometimes excessive lateral novement (ab- and ad-duction). The head of the joint, in quitting the acetabular cavity, does not always move in the same direction, but may take a variety of positions, giving rise not only to peculiarities in the symptoms, but influencing prognosis and treatment.

Symptoms. The displaced limb is sometimes lengthened, sometimes shortened, sometimes fixed in a position of adduction, sometimes in one of abduction. Its mobility in certain directions may be limited, whilst in certain others it may be abnormally increased. This resistance to manipulation distinguishes luxation from fracture, a distinction which is strengthened by the absence of crepitation. In any case, freedom of movement is lost. Luxation is always accompanied by marked lameness when the limb is carried (swinging leg lameness), though there is also more or less inability to sustain weight.

After a varying interval the hip-joint appear's swollen, though the swelling is occasionally replaced by a depression, whilst the upper trochanter appears displaced, being sometimes more prominent, sometimes sunk in the masses of soft tissue. The skin over and around the hip-joint is either very tense or abnormally loose. When the bone is displaced into the obturator foramen, its head may be felt from the rectum or sheath, and is especially distinct when the limb is moved.

Prognosis is always doubtful, and in large animals generally unfavourable. Only during the first three days is reduction usually possible, and then is effected with the greatest difficulty. If attempted early, it sometimes succeeds in small animals, but soft tissues and blood are liable to enter the joint cavity and furnish great and frequently insurmountable obstacles. After rupture of the ligamentum teres in large animals, relapses are very common; recovery is then exceptional, and the animal's usefulness is seldom restored. In old-standing cases, muscular contraction furnishes a further impediment to reduction.

Cunningham succeeded in replacing the bone in three cows; recovery followed. In the case of two horses, however, he was unsuccessful. Teetz reduced a luxation of the femur in a goat and cured the animal, although treatment only commenced three weeks after the injury. Toro claims to have reduced an ischiatic luxation in a horse and to have effected a cure. 
Treatment. Large animals are cast on the sound side and anæsthetised; and, as the operator's strength is not sufficient to extend the limb, it becomes necessary to use mechanical aids. Extension once effected, it remains for the operator to effect reposition. This may sometimes be done by abduction, sometimes by adduction and rotation of the limb, though, as a rule, the latter is impossible of accomplishment. In Ranson's case the procedure was to place a stable bracket or thick, round piece of wood between the horse's thighs, thrusting it up as near the pubic region as possible, and by pressing strongly on the tibia to convert the limb into a lever of the first order, and so raise the displaced head of the femur. The operator, with his hand on the head of the bone, then directed the limb to be moved backwards or forwards with the object of bringing the head over the cotyloid cavity. In Ranson's case a sharp movement, accompanied by a loud "click," announced the return of the head to its proper position. The animal at once regained control of the limb. In smaller animals, like dogs, extension and counter-extension may be left to an assistant, whilst the operator, having taken note of the abnormal position of the caput femoris, effects reposition,

After successful replacement, the horse should be slung to prevent it lying down, which might cause a relapse. Prolonged rest is absolutely necessary in all animals.

As the anatomical relations of the femur to the pelvis differ somewhat in animals and men, the nomenclature and methods of reduction must vary correspondingly. The following classification seems the best:-

\section{Luxation of the Feirur.}

\begin{tabular}{|c|c|c|c|}
\hline $\begin{array}{c}\text { Forwards-- } \\
\text { Luxatio iliaca. }\end{array}$ & $\begin{array}{l}\text { Backwards- } \\
\text { Luxatio ischiatica. }\end{array}$ & $\begin{array}{l}\text { Inwards- } \\
\text { (a) Luxatio pubica. } \\
\text { (b) Luxatio obtula- } \\
\text { tolia. }\end{array}$ & $\begin{array}{l}\text { Outwards- } \\
\text { Luxatio supra } \\
\text { cotyloidea. }\end{array}$ \\
\hline $\begin{array}{l}\text { The head of the } \\
\text { femur lies on the } \\
\text { illum. }\end{array}$ & $\begin{array}{l}\text { The head of the femur } \\
\text { lies below the ex- } \\
\text { ternal branch of } \\
\text { the ischimm. }\end{array}$ & $\begin{array}{l}\text { (a) I'ubic luxation. } \\
\text { The head of the fe- } \\
\text { mur lies under the } \\
\text { transverse branch } \\
\text { of the os pubis. }\end{array}$ & $\begin{array}{l}\text { The head of the fe- } \\
\text { mur lies above the } \\
\text { cup of the joint. }\end{array}$ \\
\hline $\begin{array}{l}\text { Causes.-Forcible } \\
\text { extension and rota- } \\
\text { tion outwards (sup- } \\
\text { ination). Most fre- } \\
\text { quent in cows, though } \\
\text { it occurs in horses. } \\
\text { Produced bysuddenly } \\
\text { turning in the stall. }\end{array}$ & $\begin{array}{l}\text { Causes. - Forcible } \\
\text { abduction and rota- } \\
\text { tion inwards (prona- } \\
\text { tion). Slipping out- } \\
\text { wards of the joint. } \\
\text { Commonest in cattle. }\end{array}$ & $\begin{array}{l}\text { Causes, - In oxcn } \\
\text { and holses, excessive } \\
\text { abduction (Bassi, I a- } \\
\text { fosse), passing the } \\
\text { leg over hanging } \\
\text { bales and becoming } \\
\text { caught, slipping, \&c. } \\
\text { (b) Obturator luxa- } \\
\text { tion. } \\
\text { The head of the fc- } \\
\text { mur lies in the ob- } \\
\text { turator foramen. }\end{array}$ & $\begin{array}{l}\text { Causes.-Abnor- } \\
\text { mal adduction move- } \\
\text { ments. B lows, } \\
\text { thrusts, falls. Com- } \\
\text { monest in dogs. }\end{array}$ \\
\hline
\end{tabular}


LUXATION OF THE FEMUR-continued.

\begin{tabular}{|c|c|c|c|}
\hline $\begin{array}{l}\text { Forrvards- } \\
\text { Luxatio iliaca. }\end{array}$ & $\begin{array}{l}\text { Backwards- } \\
\text { Luxatio ischiatica. }\end{array}$ & $\begin{array}{l}\text { Inwards- } \\
\text { (a) Luxatio pubica. } \\
\text { (b) Luxatio obtura- } \\
\text { toria. }\end{array}$ & $\begin{array}{l}\text { Outwards- } \\
\text { Luxatio supra } \\
\text { cotyloidea. }\end{array}$ \\
\hline $\begin{array}{l}\text { Symptoms.-Ab- } \\
\text { duction and rotation } \\
\text { ontwards. W hen } \\
\text { standing on the dis- } \\
\text { eased side the hip } \\
\text { is dropped, the tro- } \\
\text { chanter is prominent, } \\
\text { the skin covering it } \\
\text { tense, lies in folds } \\
\text { over the quarter and } \\
\text { stifle. The limb ap- } \\
\text { pears shortened, the } \\
\text { stifle turned out- } \\
\text { wards, the femur } \\
\text { almost perpendicular. } \\
\text { Stockfleth saw this } \\
\text { dislocation in both } \\
\text { limbs in a cow. }\end{array}$ & $\begin{array}{l}\text { Symptoms. - The } \\
\text { animal generally lies } \\
\text { on the belly. The } \\
\text { limb is abducted and } \\
\text { rotated in wards. } \\
\text { When standing, a } \\
\text { furrow is visible in } \\
\text { front of the biceps } \\
\text { muscle and a depres- } \\
\text { sion in the region of } \\
\text { the trochanter. The } \\
\text { skin of the quarter is } \\
\text { tensely stretched. } \\
\text { Swelling and pain } \\
\text { slight. A loud sound } \\
\text { is produced in conse- } \\
\text { quence of the head of } \\
\text { the femur striking } \\
\text { the ischium when the } \\
\text { trochanter is struck } \\
\text { or the extended limb } \\
\text { suddenly thrust back. } \\
\text { Lameness, both when } \\
\text { the limb is carried } \\
\text { and when weight is } \\
\text { placed on it. Trail- } \\
\text { ing of the limb, which } \\
\text { is carried in a circle, } \\
\text { the toe often rubbing } \\
\text { the ground. }\end{array}$ & $\begin{array}{l}\text { Symptoms. - }(a) \\
\text { In L. pubica abduc- } \\
\text { tion and rotation } \\
\text { outwards. Rolling, } \\
\text { insecure gait, troch- } \\
\text { anter indistinct, a } \\
\text { depression over the } \\
\text { hip-joint, passive } \\
\text { mobility, abduction } \\
\text { abnormal, adduction } \\
\text { limited. } \\
\text { (b) L. obturatoria. } \\
\text { Generally the same } \\
\text { symptoms. The head } \\
\text { of the bone can be } \\
\text { detected (especially } \\
\text { when the limb is } \\
\text { moved) by examina- } \\
\text { tion per anum or } \\
\text { vaginam. }\end{array}$ & $\begin{array}{l}\text { S y m pto m s, - } \\
\text { Shortening of the } \\
\text { Jimb and adduction. } \\
\text { Abduction and } \\
\text { movement limited. } \\
\text { Swelling over the } \\
\text { hip-joint. Trochan- } \\
\text { ter prominent. }\end{array}$ \\
\hline $\begin{array}{l}\text { Prognosis.-Un- } \\
\text { favourable in horses } \\
\text { and cattle. If re- } \\
\text { placement is unsuc- } \\
\text { cessful, partial re- } \\
\text { storation of move- } \\
\text { ment may follow the } \\
\text { formation of a false } \\
\text { joint, though lame- } \\
\text { ness remains. Re- } \\
\text { duction is readily } \\
\text { followed by relapse. }\end{array}$ & $\begin{array}{l}\text { Prognosis unfa- } \\
\text { vourable. Stoclifleth } \\
\text { only saw recovery in } \\
\text { two out of nine cases. }\end{array}$ & $\begin{array}{l}\text { Prognosis like- } \\
\text { wise unfavourable. } \\
\text { Reduction only pos- } \\
\text { sible in very recent } \\
\text { cases. }\end{array}$ & $\begin{array}{l}\text { Prog nosis. - } \\
\text { Only favourable in } \\
\text { dogs when seen } \\
\text { early. Later, con- } \\
\text { tinuous lameness. } \\
\text { In large animals, } \\
\text { still less chance of } \\
\text { permanent recovery. }\end{array}$ \\
\hline $\begin{array}{l}\text { Treatment.-As } \\
\text { usual in such cases. } \\
\text { Extension and thrust- } \\
\text { ing the head of the } \\
\text { joint backwards. }\end{array}$ & $\begin{array}{l}\text { Treatment.-To } \\
\text { attemptreplacement, } \\
\text { extension whilst the } \\
\text { limb is in a position } \\
\text { of abduction, fol- } \\
\text { lowed by rapid ad- } \\
\text { duction and rotation } \\
\text { outwards. }\end{array}$ & $\begin{array}{l}\text { Treat ment.- } \\
\text { Powerful extension } \\
\text { whilst the limb is } \\
\text { abducted, after } \\
\text { which reduction } \\
\text { must be effected by } \\
\text { rotation and adduc- } \\
\text { tion. }\end{array}$ & $\begin{array}{l}\text { Treat me nt.-- } \\
\text { Extension of the } \\
\text { flexed limb in the } \\
\text { direction of abduc- } \\
\text { tion. Replacement } \\
\text { to be effected by } \\
\text { movements of ad- } \\
\text { duction. }\end{array}$ \\
\hline
\end{tabular}




\section{IV.-INFLAMIMATION OF THE HIP-JOINT (COXITIS).}

With the exception of the elbow-joint, the coxo-femoral articulation seems least of all inclined to disease processes, and in horses is seldom affected, though in them, and still more frequently in cattle, displacement accompanied by partial or total rupture of the ligamentum teres and of the capsular ligament does occur. In some of Harms' collected cases (cattle) the head of the femur showed abraded spots. Eberhardt also reports cases in cattle where the post-mortem showed rupture of the ligamentum teres and of certain muscles around the hip-joint.

Contusion of the hip-joint follows falls on rough, hard ground, though the bursa trochanterica is then more apt to become diseased.

Spontaneous inflammation of the hip-joint is a rarity. In oxen double-sided purulent coxitis was seen by Noak, probably from embolism following traumatic pericarditis. In "navel-ill" in foals, and "white scour" of calves, both the result of infection of the umbilical cord in newborn animals, purulent inflammation of the joint sometimes develops. In cattle, as in men, some cases of suppuration in the hip-joint may be due to tuberculosis.

The chronic inflammation termed malum coxæ senile, so common in men, is exceedingly rare in animals, though Falke's observations show that it does occur. Schrader, who specially studied this form of disease in horses, also describes it as very rare. It is seldom recognised, even post-mortem.

Symptoms. Lameness is more or less severe, depending on the degree of inflammation. In cattle the diseased limb is extended with the toe turned outwards. The gait is of a rolling character, the limb being directed outwards and carried forward in a semi-circle. It is apt to collapse under the animal, especially when turning on the diseased side. In the horse the symptoms are similar. As a rule, there is abduction of the limb and lameness when it is carried (swinging leg lameness). Suppuration is notified by cellulitis in the region of the joint.

Course. The cases of hip-joint disease in cattle seen by Harms generally took an unfavourable course. A few improved, but none completely recovered. If lameness be marked, it is best to slaughter the animal. In horses, contusion of the hip-joint generally terminates in recovery in about fourteen days, but suppurative arthritis and arthritis chronica deformans are incurable.

Treatment. Rest is of the first importance. If necessary, the animal may be slung, otherwise it should be provided with abundant bedding. 
Recent contusions and displacements, if accompanied by severe pain, are treated with cold applications. If, after a week, there is no marked improvement, a bichloride or biniodide of mercury blister may be resorted to. Setons and "issues" are also useful, though their efficacy chiefly depends on the rest which they enforce.

\section{Y.-INFLAMMATION OF THE TENDON AND TENDON BURSA OF THE GLUTEUS MEDIUS MUSCLE. BURSITIS GLUTAI MEDII. S. TROCHANTERICA.}

Anatomy. The MI. glutæus medius, which represents an extension of the M. longissimus dorsi, is in the horse provided with two tendons, one of which becomes attached to the upper trochanter of the femur, the other passes over the ridge in front of the great trochanter, forming on its cartilaginous surface an extensive bursa, and becoming attached below to the surface of the crest. In horses, inflammation of the tendon and of its sheath on the convexity of the trochanter produces a peculiar lameness, which was first described by $\mathrm{K}$. Günther, and afterwards by Renard and others. In England the disease has been called trochanteric lameness (Williams), or false hip-joint lameness.

Causes. This disease, which is only occasionally seen in horses, may be caused by bruising or violent strain of the tendon or its sheath. Bruises are produced by falls, by collisions with the carriage-pole, by blows with heavy bodies, strains resulting from heavy draught-work and by falling on the hind limb, as often happens when the animal catches the heel of one of the hind shoes in the rack-chain. Sudden reining-up is also said to produce it. Horses with sloping quarters and slight muscular development seem predisposed to such injuries.

Course. The disease consists in a bursitis or tenositis, acute or chronic; the tendency always being towards a chronic condition, but the issue depending to some extent on the initial injury, and on the treatment adopted. Under appropriate treatment, lameness may disappear in four to six weeks; but if neglected, and especially if the primary injury be severe, the condition becomes chronic, and the animal's usefulness is lost.

The prognosis depends chiefly on the stage attained and the severity of the disease. When lameness is recent and slight, and the animal can be rested, there is good hope of recovery, but this is less in heavy draughthorses and in riding-horses than in such as are occupied in the lighter forms of draught. Marked wasting of muscle, the existence of srelling, and severe or chronic lameness render prognosis doubtful; and where there is periostitis and exostosis, with abrasion of the cartilaginous covering of the trochanter, there can be little chance of recovery. The suggestion that the bursa sometimes communicates with the hip-joint, 
and that, under certain circumstances, disease may extend to the latter, seems mistaken.

Symptoms. In recent acute inflammation there is increased warmth, swelling and pain on pressure over the great trochanter. When resting the limb is usually flexed, though sometimes no change can be detected until the horse is exercised. When moving, the forward stride is shortened; less frequently, there is a tendency to lift the limb before the last phase of the stride is complete. The animal generally moves obliquely, as does the dog, the sound side being thrust forward, and the limb kept in a position of pronation, i.e., rotated inwardly. By placing the hand on the middle trochanter during movement distinct crepitation may sometimes be detected (tendovaginitis crepitans); at a later stage the muscles of the quarter become atrophied.

The slighter, and especially the chronic, forms of lameness are often only apparent when turning or when moving leavy loads, the horse then drawing with the sound leg, and endeavouring to shorten the period of loading of the diseased one.

A similar form of lameness, however, is seen in other diseases, such as old cases of fractured pelvis; the diagnosis must therefore be based principally on the local changes. The crepitation sound is the most trustworthy, for both the swelling and pain may be caused by the upper trochanter.

Treatment. Recent cases, exhibiting acute inflammatory symptoms, are best treated by absolute rest, and cold applications, such as poultices or fomentations. Blisters may afterwards be used, and, if unsuccessful, can be followed by setons; the actual cautery has been employed with good results. Rest should be continued for some time after the disippearance of lameness; too early a return to work is liable to be followed by relapse. Some horses only recover sufficiently for light draught.

\section{VI.-PARALYSIS OF THE HIND LIMB.}

Anatomy. The muscles of the hind limb receive their nerve supply from several sources, viz. : (1) the anterior and posterior gluteal nerves, which originate from the lumbo-sacral plexus and issue from the great sacro-sciatic opening in company with the gluteal vessels and great sciatic nerve; (2) the great sciatic ; (3) the anterior crural; and (4) the obturator.

The anterior gluteal nerves supply the three gluteal muscles and the tensor vagina femoris; the posterior gluteal, parts of the superficial and deep gluteal muscles, the biceps femoris, the skin at the back of the thigh at a point 4 or 5 inches below the tuber ischii, the semi-tendinosus muscle, and give off a branch to the internal pudic, which supplies the perineum.

The great sciatic or ischiatic nerve originates from the lumbo-sacral plexus, and gives off branches in its passage down the limb to the obturator internus, pyriformis, gemelli and quadratus femoris. Its 
principal branches are the external popliteal nerve, given off opposite the small sacro-sciatic opening; a branch to the semi-membranosus, lower portion of the biceps and semi-tendinosus; and, lastly, the external saphenous nerve. It is continued by the internal popliteal, which is again continued by the posterior tibial nerve. The external popliteal (peroneus of Günther), after giving off a branch to the biceps femoris, breaks up into the the musculo-cutaneous and anterior tibial nerves, supplying the extensor pedis and peroneus and the flexor metatarsi and short extensor respectively.

The internal popliteal nerve (Tibial N. of Günther) supplies both heads of the gastrocnemius, the soleus, perforatus, popliteus, the perforans, and the flexor accessorius. It is the sensory nerve for the lower portions of the hind limb.

The anterior crural nerve is derived from the lumbo-sacral plexus, descends between the psoas magnus and parvus, and passes over the common termination of the iliacus and psoas magnus to supply the quadriceps extensor cruris. It also supplies sensation to the inner surface of the limb.

The obturator nerve is a branch of the lumbo-sacral plexus. Emerging by the obturator foramen, it supplies the obturator externus, adductor parvus, adductor magnus, pectineus and gracilis muscles.

\section{A. Paralysis in the region supplied by the gluteal nerves.}

Few recorded cases of this kind exist. Franke reports an instance of paralysis of the anterior branches caused by a neuroma. The patient, a seven year old gelding, showed gradually advancing atrophy of the gluteal muscles of the left side, which became so marlied as to result in five months in the bones being almost denuded of muscle and appearing almost like an osteological preparation; the muscles of the thigh and lower thigh also suffered severely. At first there was only insecure gait, but this symptom, always marked when the horse was turned, gradually became aggravated. Finally the lower part of the limb was kept permanently flexed, and, during forced exercise, was slid along the ground and set down with a tapping, insecure movement. The backward portion of the stride was shortened. On post-mortem examination a neuroma the size of a pigeon's egg was found on the anterior gluteal nerve 4 inches from its point of exit.

Roloff described a similar case. Here, however, the horse became lame suddenly, and carried the affected limb too near the middle line, as well as extending it too far forwards. In time the muscles of the quarter wasted to a great extent. It seems probable, however, that in this case the posterior gluteal nerves were paralysed, as suggested by the abnormal adduction of the limb.

\section{B. Paralysis in the region supplied by the great sciatic nerve.}

(a) Paralysis of the external popliteal nerve. The only well-confirmed case of this kind yet recorded is by Möller. An eight year old Belgian gelding was one morning found lame in his stall without visible cause. Seen next day, he exhibited marked uncertainty in 
moving his hind limbs, suggesting partial paralysis, but it soon became evident that only the right hind limb was affected. Whilst the left hind limb was moved and weight was placed on it in the usual way, the left leg was carried close to the middle line of the body (adducted) and was placed too far in advance and too near the right side, causing the animal to fall towards the right side; the body was only saved from coming to the ground by a rapid spring with the left foot. At the same time the stifle and hock joints were excessively extended, and the phalanges flexed (plantar flexion). The gait was clumsy and

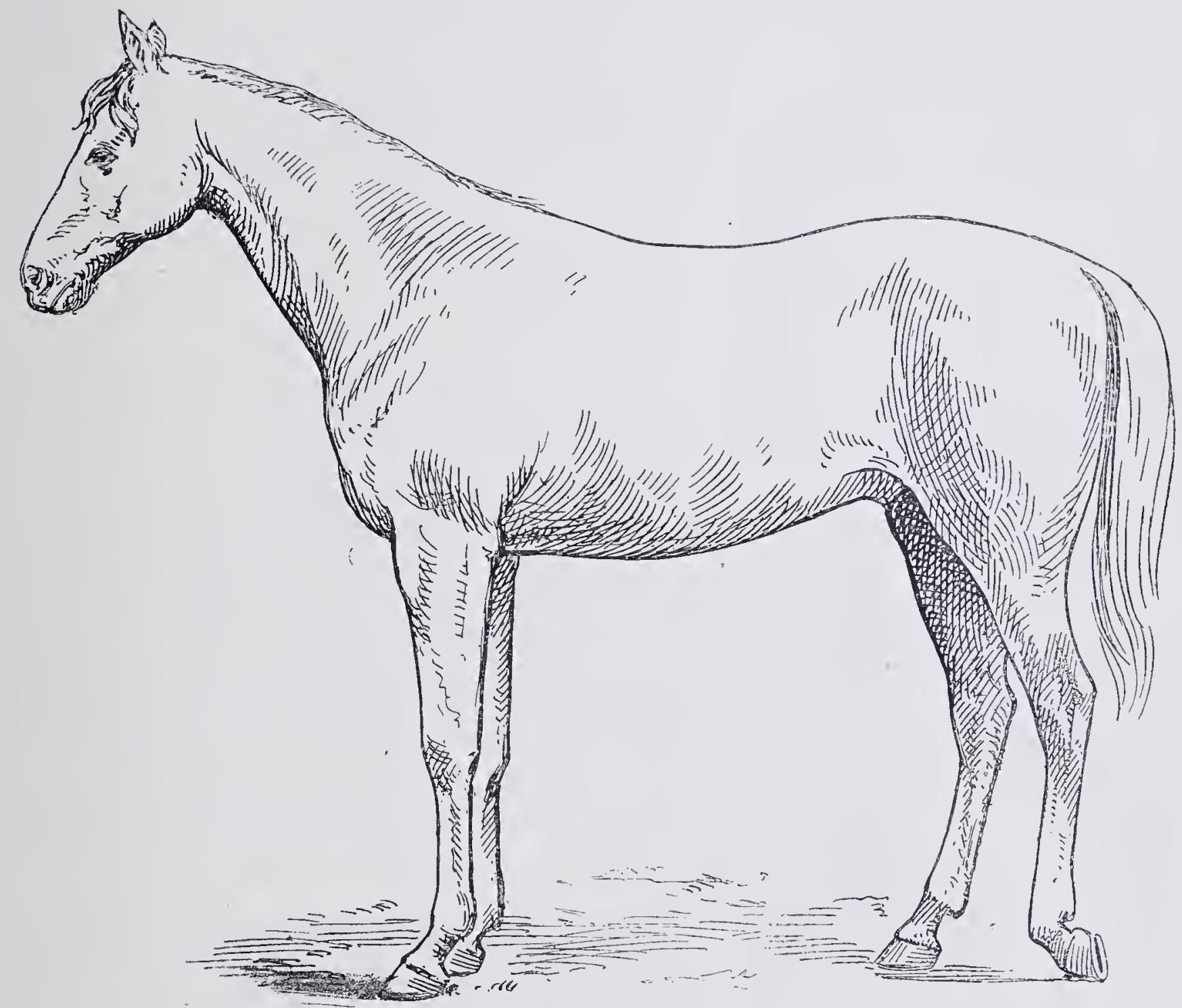

Fia. 253.-Paralysis of the external popliteal nerve.

insecure in the highest degree. These movements recurred at every step ; the animal was incapable of trotting. The uncertainty of walling was aggravated by the abnormal flexion of the phalanges, which at times caused the animal to walk on the front of the fetlock-joint itself.

By passing a rope round the right fetlock, and so drawing the limb outwards and extending the fetlock each time the animal attempted to move, walking became perfectly easy. Immediately such assistance was withdrawn, however, all the previous difficulties returned. At first no anatomical change in the limb could be noted, nor was there any sign of local pain or inflammation, but by placing the hand on the limb during movement, the muscles in front of the tibia, as well as the 
semi-tendinosus, semi-membranosus, and biceps femoris were found to remain relaxed and without movement.

Sensation in the lower parts of the thigh and about the phalanges was also impaired. The animal, however, regularly reacted to stimuli in the region of the quarter and upper thigh.

Three weeks after the first appearance of lameness an inflammatory swelling appeared between the anus and tuber ischii, showed fluctuation, and on incision discharged about two quarts of very offensive lumpy pus. After enlarging the orifice, the hand could be introduced into an extensive cavity consisting of several divisions, some lying between the muscles of the croup, some in the paraproctal connective tissue. Except for compression by the abcess, the rectum seemed to have escaped any disease change. As the abscess cavity closed, the difficulty in movement gradually diminished though the gait for long remained uncertain, especially when the horse was on uneven ground, the phalanges failing to be extended and the animal "knuckling over" at the fetlock. At this stage the animal passed from observation.

There can be no doubt that the abscess originated in the paraproctal connective tissue in consequence of infection from the rectum. It then extended between the muscles of the quarter and. pressed on the great sciatic nerve, so that both the external and internal popliteal nerves were affected. The symptoms due to injury of the external popliteal were more marked because the function of those supplied by the internal popliteal (gastrocnemius, flexor perforans, perforatus, \&c.) was partially replaced by their tendinous apparatus; this explains why weight could still be borne on the limb.

Szidon states having seen paralysis of the external popliteal nerve, consequent on injury by small shot.

Goubaux gave an excellent description of paralysis of the external popliteal nerve as early as 1848 . His case was caused by a violent contusion, and was unsuccessfully treated by repeated blistering along the course of the nerve.

Bouley cured one case by similar applications.

Cadiot has only seen one case. It recovered in three weeks by blistering and exercise.

(b) Möller has seen and described one case of paralysis of the internal popliteal nerve. In this disease the muscles lying at the posterior surface of the tibia are affected; plantar flexion is impossible, that is to say, the hock cannot be extended nor the foot flexed (in the plantar sense). As, however, in the horse flexion of the hock brings both flexors of the foot, and especially the tendon of the flexor pedis perforans, into tension, the phalanges are, in this lameness, necessarily fixed in a position of plantar flexion. This is especially noticeable when 
the animal stands on the paralysed limb, because the hock is then excessively flexed. It is still possible, however, to place weight on the limb, because the Achilles tendon fixes the hock.

The limb is advanced with all the joints excessively flexed, the foot being lifted very high, and set down with a hesitating ("tapping") movement; the action as a whole bearing some resemblance to stringhalt. The condition is clearly due to paralysis of the muscles at the back of the lower thigh. The gastrocnemii are unable to extend the hock, whilst the flexor perforatus is passive, and only affects movement through the medium of its peculiar tendinous apparatus (for an illustration of this apparatus, see "Rupture of the Flexor Metatarsi"). Trotting is impossible.

The muscles at the posterior surface of the thigh, particularly the gastrocnemii and flexor pedis perforans, are relaxed, and afterwards become atrophied.

Nocard saw fractured pelvis in the horse produce symptoms closely simulating this lameness. Masses of callus had probably formed, which, by pressing on the internal popliteal or obturator nerves, caused the peculiar lameness.

As an experiment, Möller divided a horse's internal popliteal nerve just before its point of entry between the gastrocnemii. The animal at once assumed the position above described, the fetlock-joint showing plantar flexion, and the foot being excessively raised during the forward stride, though not

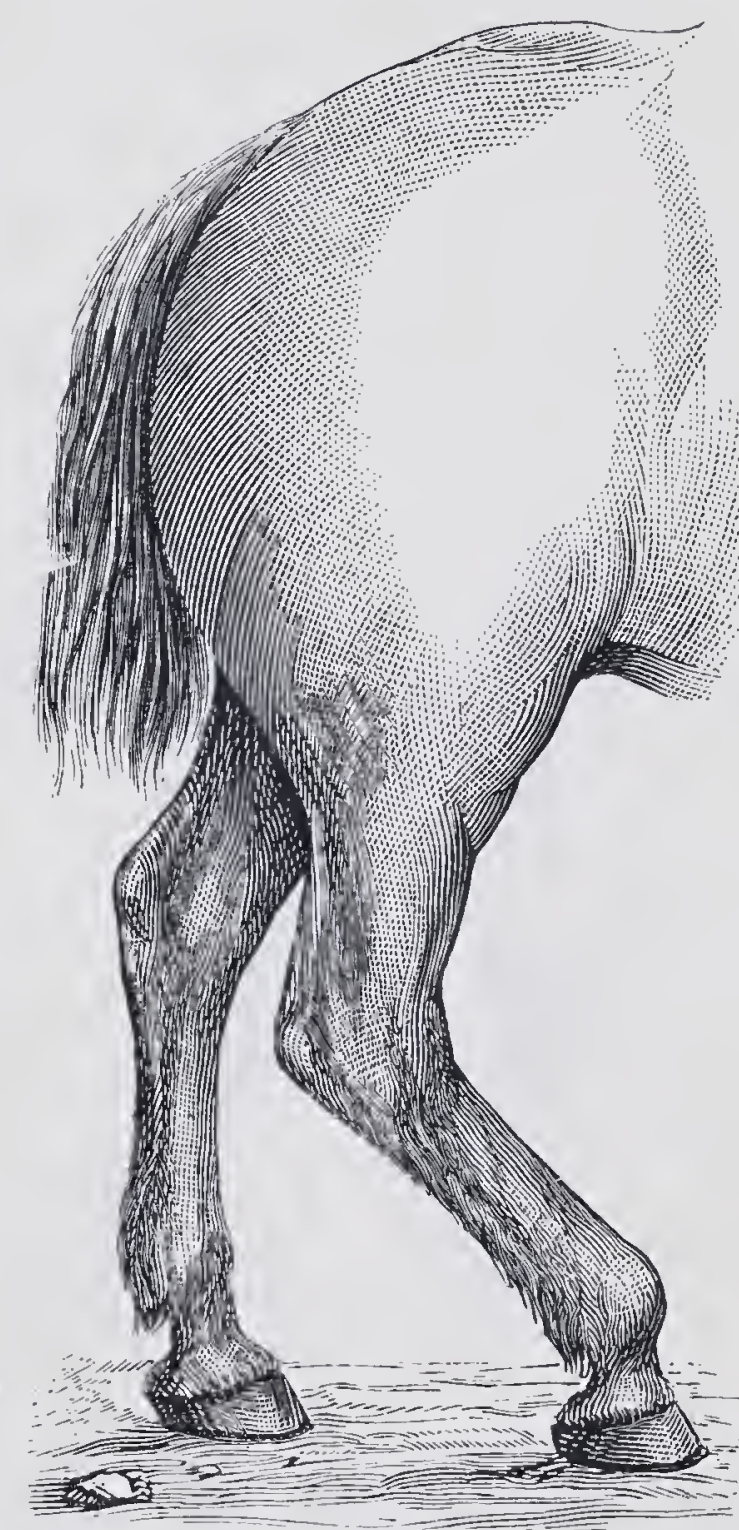

Fig. 254.-Paralysis of internal popliteal nerve. quite in the same awkward way as in the other case, in which the immediate cause of the paralysis was perhaps more centrally situated.

(c) Paralysis of the great sciatic nerve is followed by more general lameness, the muscles involved including the semi-tendinosus, semimembranosus, and biceps femoris, and the limb is no longer capable of free movement. Neither the hip, stifle, nor hock joint can be flexed, the limb hangs limply from the hip downwards, and can neither be lifted nor advanced. The skin of the limb loses its sensibility. On enforced movement the foot is dragged along the ground with the phalanges bent, and in dogs, \&c., the dorsal surface of the toes is soon rubbed raw. When, lowever, by external help, the limb is placed in 
its normal position, it is able to sustain weight in the usual way, because the extensors of the stifle fix that joint, and with it the other joints of the limb. Anæsthesia of the skin of the lower parts of the limb accompanies this condition.

Möller saw three cases of ischiatic paralysis in dogs, one in a St. Bernard, one in a bull-dog, and one in a working-dog. (In Berlin, and throughout Germany, large numbers of dogs are employed for drawing

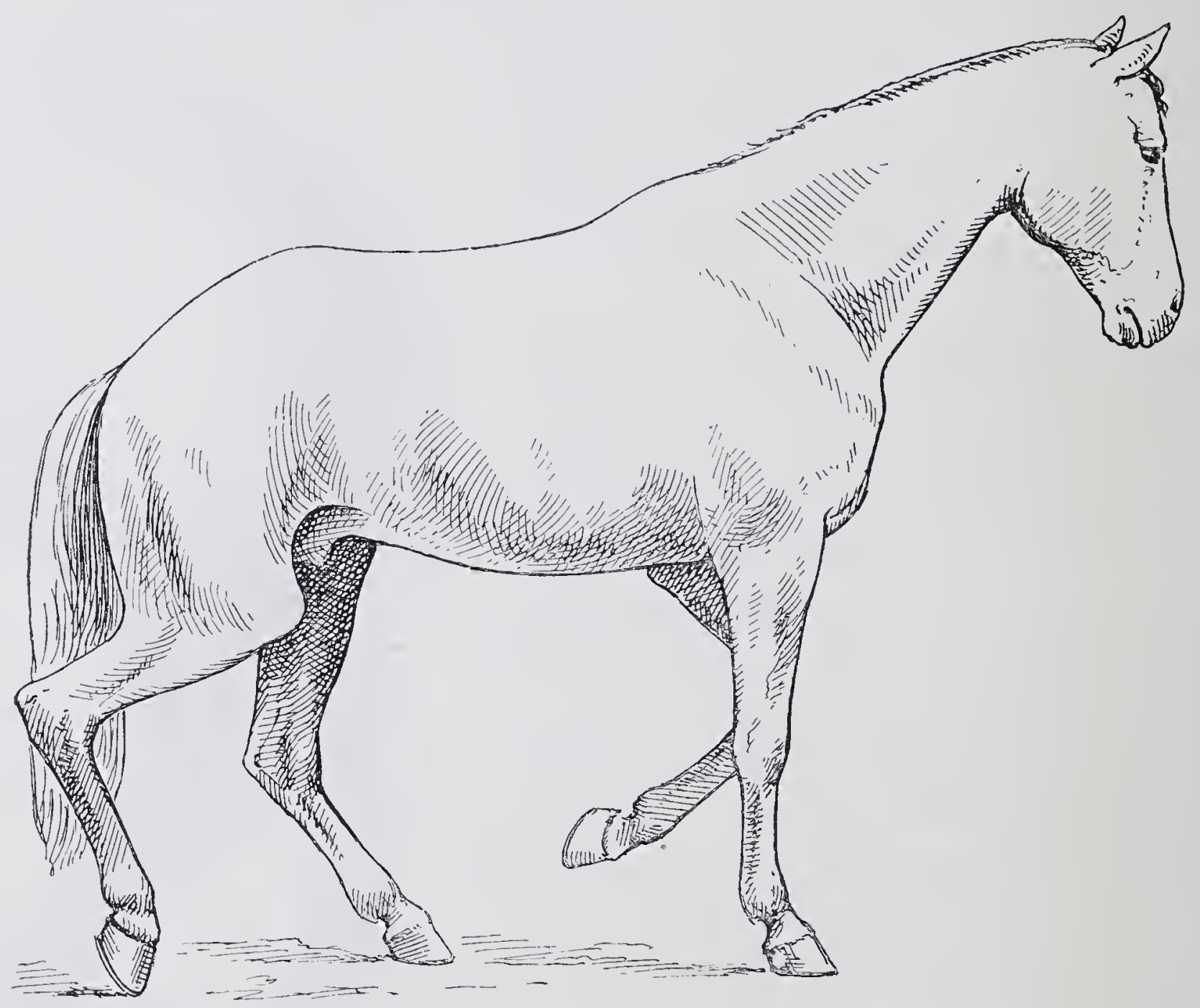

FIG. 255.-Paralysis of the anterior crural nerve.

light carts, sometimes singly, sometimes in pairs. They are of no determinate breed, and vary from the size of a collie up to that of a boar-hound.) In one case the dog had fallen from a window, and at first showed complete paraplegia, which, however, disappeared after a few days, leaving one limb affected with sciatic lameness, which also disappeared soon afterwards. The second dog became affected during convalescence from distemper, and in the third the lameness had no apparent cause.

In addition, paraplegia is by no means uncommon in dogs, and even in horses; in the hind limb the great sciatic nerve is affected, and in the opposite fore limb the axillary plexus. Such cases are clearly of cerebral origin. The condition described by Beel as paralysis of the hip nerves appears to have been paraplegia. Friis saw paralysis of the right fore and left hind limbs, which was rot improved after three months' 
treatment. The absence of anæsthesia and of muscular atrophy pointed to its cerebral nature.

Up to the present little is known of the course of these paralyses. Though Möller's case of internal popliteal paralysis appeared incurable, the case of sciatic paralysis in the dog produced by a fall improved so much in fourteen days as to warrant expectation of complete recovery. The two other cases were removed from observation, but appeared favourable, the disease not being of old standing.

The causes are: disease of the brain or spinal cord, or lesions in the course of the nerves. Disease of the spinal cord usually produces double-sided paralysis (paraplegia). The above case of internal popliteal paralysis appears to have been due to anatomical changes in the nerve during its course between the gastrocnemii. In dogs sciatic paralysis is produced both by wounds and by general disease like distemper. Albert found, on post-mortem examination of a horse which had been treated for three months without effect, the residuum of a blood extravasation surrounding the nerve at the point where it left the pelvic cavity. The tissues below this point were atrophied, and the muscles, especially the M. rectus femoris, had undergone extensive fatty degeneration.

The prognosis must depend on the condition indicated. In recent cases, where the only apparent cause is chill, and in those due to falls and sprains, the prognosis is more favourable than in old-standing cases with marked muscular atrophy. In incomplete paralysis the forecast is more favourable, but in complete sciatic lameness the outlook is bad, especially if there be local anæsthesia. Recovery is still possible where sensibility and irritability to the Faradic electric current is preserved.

\section{Paralysis of the anterior crural nerve and quadriceps femoris muscle. Rupture of the rectus femoris and vasti muscles, and of the straight ligaments of the patella.}

Though paralysis of the crural nerve is not common, yet in animals it occurs oftener than is generally believed. Sometimes it accompanies hæmoglobinuria, sometimes it is produced by strain, by partial rupture following on falls, or by violently kicking backward. Möller saw six cases in horses drawing heavy loads, and many more, as a sequel to hæmoglobinuria. Its connection with this disease is explained by the fact that the crural nerve passes through the ileo-psoas muscle, which in hæmoglobinuria seems particularly involved. Although it is possible that myopathic lameness of the rectus and vasti muscles occurs in hæmoglobinuria, yet the greater number of such cases are probably due to disease of the nerve indicated, because in nearly every instance the loss of function and excessive atrophy are common to all the

V.S. 
muscles attached to the patella, and in a similar degree, a condition scarcely to be expected in a purely muscular disease. The sudden onset and equally rapid disappearance of the disease, as well as the total want of inflammatory symptoms, support this view. Where disturbance of sensation on the inner surface of the thigh exists, there can be little doubt of the nature of the disease. Goubaux divided the nerve experimentally high up in the thigh, between the vastus internus and rectus femoris, and thus obtained all the symptoms of muscular paralysis peculiar to hæmoglobinuria. Other conditions, like psoas abscess, tumour formation, hæmorrhage, \&c., may also destroy the function of this nerve temporarily or permanently.

Though post-mortem examination sometimes shows the rectus and vasti muscles in a marked state of fatty degeneration after this disease, that fact in no way proves the condition to have been myopathic lameness, as has been suggested.

Symptoms. Weight cannot be placed on the limb, because it is impossible to fix the stifle-joint, a necessary preliminary to walking. Attempts to sustain the body on the affected limb are followed by flexion of all the joints, including the phalanges. The stifle-joint is not completely extended on the ilium, and the skin on the inner surface of the thigh loses its sensibility. At a later stage the quadriceps femoris muscle atrophies; its loss of function is immediately seen when the animal moves, and may be detected by local examination. In incomplete crural paralysis the body sinks towards the affected side, whilst all the joints become somewhat flexed (fig. 256), the last period of the stride being consequently shortened. Animals suffering from this disease learn to move better, however, in time, the ab- and ad-ductors of the limb co-operating to fix the stifle-joint. This action is easily detected when the animal is walked very slowly past the observer: it is then seen that the upper end of the tibia is not only drawn backwards and upwards, but the patella is fixed on the condyles of the femur. The last symptom to disappear in convalescence is the upright position of the os suffraginis, a consequence of incomplete action of the rectus and vasti.

Paralysis of the crural nerve or of the above muscles may be mistaken both for muscular rupture and for lateral luxation of the patella (vide loc. cit.). Ruptures in the various extensors of the patella have been seen by numbers the horse, all cansed by saw three cases of rupture of the rectus femoris in the horse, all caused by slipping or falling; two proved incurable. The symptoms resembled those of the above-described paralysis. Not infrequently a depression could be detected in the muscle. Post-mortem examination showed the muscles to be pale, as though boiled, their fasciculi shredded out and partly separated.

Schmidt describes rupture of the vastus externus. A horse had trodden in a deep rut, and, the ground being frozen, was unable to withdraw its foot 
without assistance. Severe lameness was immediately apparent, the limb was relaxed, and collapsed at every attempt to sustain weight on it; the stifle and hock were markedly flexed. At first no change could be detected in the muscles, but after some days a depression, about 4 inches deep, became visible on the outer side of the muscles attached to the patella. The horse was unable to rise for fourteen days, but in three weeks' time could again place weight on the limb. In two months it was put to light work, and in three months had entirely regained its usefulness. During its course this case also showed some resemblance to crural paralysis, and it is possible that it may have been of that character.

Hollmann saw rupture of the rectus femoris muscle in a cow, followed by permanent lameness; Haubner describes injuries to the extensors of the

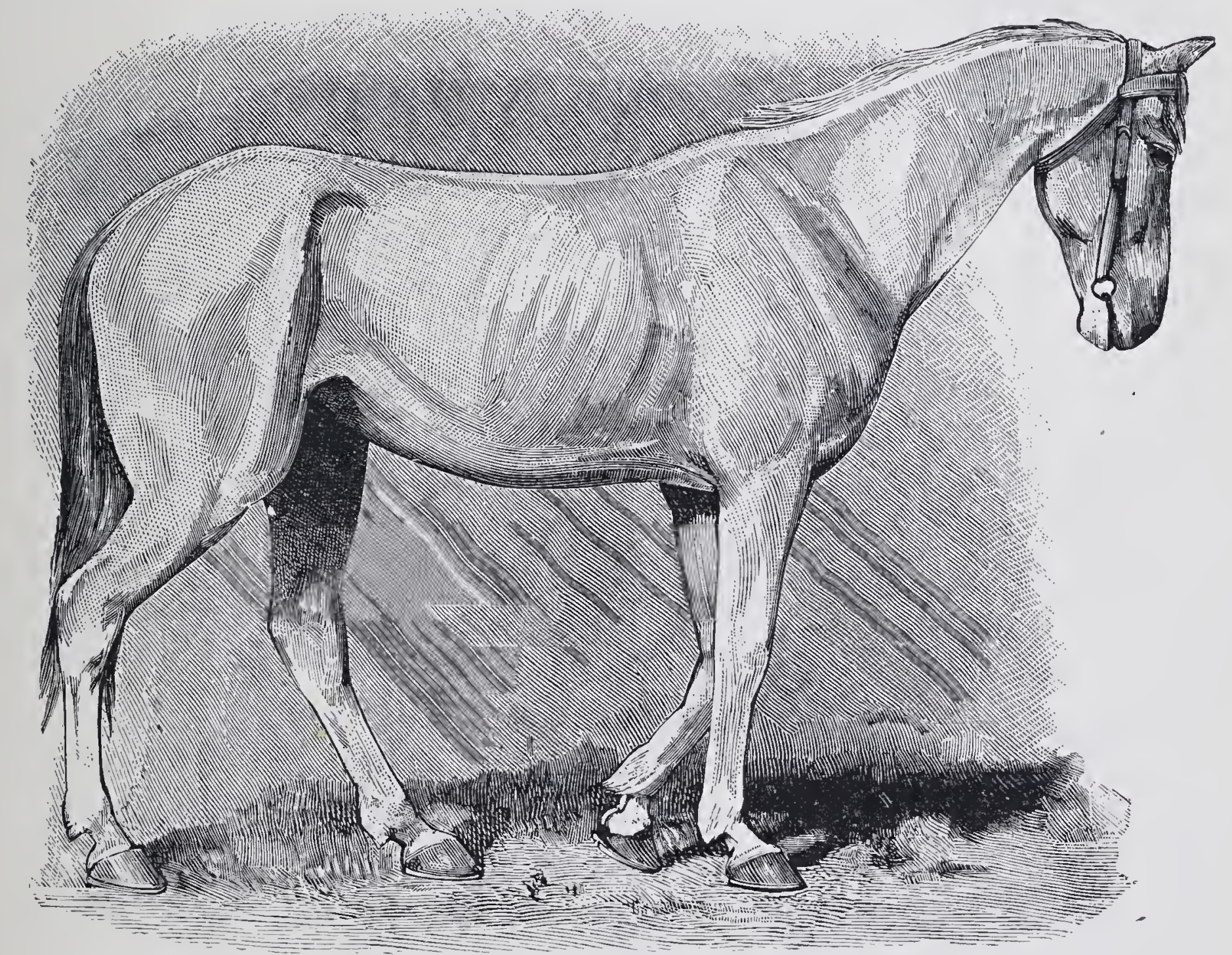

FIG. 256. - Incomplete crural paralysis (from an instantaneous photograph).

patella in a horse ; Lees a double-sided temporary inflammation of the quadriceps femoris in a horse (hæmoglobinuria?). Meyer saw rupture of the rectus femoris, vastus externus and internus muscles in a cow; a distinct depression could be detected about 8 inches above the patella. The symptoms were similar to those of crural paralysis.

Rupture of the straight ligaments of the patella has been seen in horses. Moller saw two cases of rupture of the external straight ligament-in one due to a collision with a carriage-pole, in the other, apparently to slipping in the stable. Both animals showed inability to bear weight on the limb (supporting leg-lameness). At first the animals could not stand on the affected limb at all, and later only to a certain extent. Below the stifle only the middle and inner straight ligaments could be felt; in place of the outer was a depression. Pressure at this part caused lively pain. After complete rest for 
three and four weeks respectively, the horses improved sufficiently to return home.

Voigtlïnder confirmed his diagnosis of rupture of the straight patellar ligaments in an ox by post-mortem examination. The inability to stand, severe inflammation of the stifle-joint, marked flexion of the limb, and inability to extend or bear weight on it, and the relaxation of the affected ligaments, left little doubt of the character of the injury, even during life. Voigtlïnder saw a cow with rupture of the inner and middle straight ligaments, and of the inner lateral ligament, and outward luxation of the patella. The condition had resulted from one cow attempting to mount another.

The prognosis depends principally on the severity of the symptoms. When pain is marked, and weight cannot be placed on the limb during the first few days, the prognosis, especially in the horse, is unfavourable.

The treatment of rupture consists in absolute rest, the provision of plentiful bedding, and in turning the animal with sufficient frequency to prevent bed-sores.

Course. The number of recorded cases is at present too small to guide us in laying down the general course of crural lameness, in addition to which each case varies according to its cause. Whilst the paralysis accompanying hæmoglobinuria is generally obstinate, indeed often incurable, that produced by severe mechanical strain frequently disappears in a few days. Möller saw three cases of the latter kind recover in six, twenty, and forty-two days respectively, and two others after a short interval; one case appeared incurable. Early recovery may be expected in recent and incomplete paralysis. Dollar has seen complete recovery from the paralysis following hrmoglobinuria, though the cases have lasted as long as six and eight months respectively. The horses were turned out to grass. Needless to say, some cases of hæmoglobinuria recover completely in a few weeks.

Treatment of crural paralysis must follow general principles. The use of the electric current, and especially of the Faradic current, is very difficult in the horse, and can seldom be advantageously resorted to. The position of the crural nerve also prevents its being directly reached, and treatment is therefore confined to, as far as possible, checking degenerative changes, and improving nutrition in the paralysed muscles. Massage, in the form of kneading and beating, is of great value for this purpose, and should, if possible, be performed daily. In obstinate cases Dollar has several times had excellent results from deep point firing over the affected region. Repeated blistering is useful. Exercise, in addition to favouring regenerative changes in the affected nerve, assists circulation and nutrition in the muscles. In dogs the induced electric current may be employed. Iodide of potassium and strychnine internally have also been recommended.

\section{Paralysis of the obturator nerve.}

Paralysis of this nerve has been seen, but must always be somewhat difficult to diagnose, because it presents none of the marked symptoms 
seen in the above-named conditions, and because its onset is frequently masked by the symptoms of fractured pelvis. There is loss of function in the adductors, the pectineus and gracilis muscles, and inability to abduct the foot. It is sometimes possible to directly detect paralysis of the adductors.

One recorded case was in a five year old mare, which became lame without visible cause. In walking, and still more so in trotting, the right hind foot was strongly adducted. The hip-joint was markedly flexed, and the stifle drawn upwards and outwards, as in stringhalt. When backing there was difficulty in moving the right foot, but weight was placed on the limb as usual. Recovery occurred in between four and five months, during which strychnine was administered. The case described by Nocard (see section on "Fracture of the Pelvis": sub-division, "Treatment") as due to compression of nerve trunks by the callus, resulting from a fracture through the obturator foramen, may really have been one of obturator paralysis. There was atrophy of the adductor group of muscles, and a peculiar lameness.

Obturator paralysis has several times been reported as following: tumour formation in the course of the nerve, especially in grey or white horses, the subjects of melanosis.

W. Willis has recently (1903) described two cases of obturator paralysis, and referred to others as having been seen. In each instance there existed a swelling, which later developed into a callus over the pubis at the point where the obturator nerve crosses that bone. In mares it could be detected by examination through the vagina. Willis at first diagnosed these cases as "fractured pelvis," as indeed they were. The average duration of the cases appear's to have been about three months, and the paralysis appeared to march concurrently with the development of the callus, marked abduction of the limb, its principal symptom, appearing as the soft callus increased in size and diminishing as the callus became smaller and more fully ossified. In one instance, though the animal was able to work, a certain degree of abduction continues for a year.

\section{VII.--THROMBOSIS OF THE POSTERIOR AORTA AND OF ITS BRANCHES.}

Aneurism of the anterior mesenteric artery is common in horses, and loosened particles of the thrombus may be hurried with the blood-stream into the posterior aorta and its branches, which then become thrombosed. Proeger saw thrombosis in a cow in consequence of endocarditis. Stoppage of small vessels near the femoral or pelvic arteries by emboli of 
small diameter may produce no serious results, but where large vessels are plugged, the muscles served by them, no longer receiving the quantity of blood necessary for their functional activity, are incapable of sustaining severe exertion, lameness appears, the horse comes to a standstill, and finally myopathic paralysis of the affected muscles sets in.

Whilst the animal is at rest circulation is maintained by collateral vessels, and it is only on movement that symptoms develop. The time of their appearance depends on the degree of obstruction in the vessels and the intensity of the muscular action. When the occluded vessels are small the animal may go several hundred yards, even at a fast pace, before lameness appears, and the symptoms vary in character according to the muscles involved, but thrombosis of large vessels is followed by symptoms of extensive muscular paralysis after even three to five minutes' trotting. A few minutes' rest produces an apparently perfect recovery. The intermittent lameness thus produced is quite distinctive. Thrombi extending to the aorta sometimes prove fatal, as shown by many reported cases.

A remount showed cedematous swelling and inability to stand, first in one hind limb and, on the following day, in the other. On the second night it died, and on post-mortem examination the posterior aorta was found completely thrombosed at its point of bifurcation. The thrombus. contained a specimen of Strongylus Armatus. Cases of rupture of the posterior aorta and of thrombosis of the iliac arteries are described at length at p. 387 of Cadiot and Dollar's "Clinical Veterinary Medicine and Surgery." In Rutherford's case the posterior aorta, right external and internal iliacs, and left internal iliac were all thrombosed.

The disease generally develops insidiously, attention being first directed to it by the attacks of lameness, but when large vessels are blocked other well-marked symptoms are present, like dyspncea, perspiration, and cramp in the affected groups of muscles. The thrombosed vessel never again becomes clear, though collateral circulation is sometimes established, and gradual improvement follows. In other cases the lameness increases, in consequence of the growth of the thrombus.

Symptoms.-The intermittent lameness, which soon disappears with rest, but always returns on movement, is characteristic. Its severity and extent depend on the position and size of the thrombus or thrombosed vessel. Blocking of the femoral artery is immediately followed by loss of function in the ilio-psoas and tensor fascir latæ, which it supplies with blood. Lameness is, therefore, most marked whilst the limb is being advanced. The forward stride is retarded and shortened, and the limb dragged, the reason being that these actions are normally performed by the muscles named. 
Thrombosis of the external iliac artery, which supplies the glutei and quadriceps femoris, produces a "supporting leg lameness," which closely simulates crural paralysis. The animal is unable to stand on the limb, because at every attempt the joints become flexed. Paralysis of the glutei is followed by loss of balancing power, and when double-sided by rolling to and fro of the hindquarters. In obstruction of the caudal arteries the tail hangs limply. Horses with double-sided iliac thrombosis rapidly lose control of both hindquarters when galloped. The hind limbs approach one another more and more closely, the hoofs are planted on the ground just alongside of one another, and finally the animal comes to the ground with the hindquarters first.

A rare condition was described by Haas. When first examined the horse showed symptoms of thrombosis of the left iliac artery. Fifteen minutes later, when again moved, the lameness seemed transferred to the right limb. On post-mortem examination there was found extending into the left iliac artery a thrombus, which, however, could easily be carried by the blood-stream into the right. Haas saw thrombosis of the posterior aorta in a cow. Strebel saw a cow which was only capable of standing for a few moments at a time. On post-mortem the cause was found to be thrombosis of the left iliac artery.

In bilateral thrombosis, or in thrombosis of the aorta, these symptoms are combined, and may become so severe that the animal is unable to support the hindquarters, even when at rest. Such cases often show dyspnoa, tumultuous action of the heart, cramp in the muscles of the hindquarters, and, if the animals be worked, epileptiform convulsions of the entire body. Though the rest of the body is bathed in perspiration, the affected region remains dry and cold. Sometimes even during rest the limb seems colder than its neighbour.

Stallions are sometimes unable to copulate. Möller saw one in which erections occurred, but were not followed by ejaculation of semen. The post-mortem of such animals shows emphysema of the lung, with hypertrophy and dilatation of the heart.

According to Terrillon's observations on men suffering from this disease, the attack is accompanied by a feeling of painful stiffness and severe burning. In recent cases, animals evince pain by lifting the limb when standing at rest; soon after, lameness becomes apparent. Möller once saw the primary attack of embolism. The symptoms were severe dyspnoa, laboured action of the heart, frequent pulse, and cramp in the affected muscles.

The prognosis depends on the degree of interference with movement, but the tendency is always towards aggravation of symptoms, not towards improvement, and only in slight cases can the development of an efficient collateral supply and recovery be looked for. Of eleven army horses affected during the year 1891 with thrombosis of the aorta, of its branches, or of the radial artery, only one recovered. 
Treatment. The solution or removal of the thrombus is impracticable, and internal remedies, like the alkaline carbonates which were formerly recommended, are of no value. Massage is dangerous. Though the thrombus may be loosened by manipulating the vessel through the rectum, further coagulation readily occurs, and a case thus treated died next night in consequence, the post-mortem showing complete thrombosis of the posterior aorta with fresh clots. The only treatment of value consists in exciting collateral circulation by regular work. The animal is exercised until the first symptoms of lameness appear, and then rested, or it may be put to continued light work. The increased blood circulation thus excited favours development of collateral circulation, but too much is not to be expected from this treatment.

\section{VIII.-DISPLACEMENT OF THE BICEPS FEMIORIS IMUSCLE.}

As a consequence of the differences in their respective anatomical formation, luxation of the biceps femoris is comparatively common in oxen, but very rare in horses. In oxen, the abductor of the hind limb consists of two heads, and possesses, on the spot where it passes over the trochanters, a large mucous bursa; at this point it is only loosely connected with the underlying muscles. When, therefore, the fascia surrounding it is accidentally ruptured, the upper head of the muscle glides off the trochanter, is caught behind it, and lameness is at once developed. This condition is very seldom seen in horses, though cases are reported.

Causes. The disease is almost entirely confined to mountain cattle, with sloping quarters and prominent trochanters; it is especially common in thin animals; "cow-hocks" are also said to predispose to its production. It is more immediately due to slipping, especially in a backward direction, as happens during work, and in coitus.

Symptoms. 'The lameness is usually confined to one leg. Immediately the muscle named becomes fixed behind the trochanter, flexion and extension of the limb are greatly interfered with. The limb is fixed in an extended position similar to that in upward luxation of the patella, or is thrust outwards and forwards with a kind of "mowing" movement, the claws scraping the ground. Winkler denies having seen the mowing movement in the twenty cases which came under his notice, but draws attention to the similarity between this lameness and luxation of the patella, from which it is only distinguished by the fact that the limb is not so firmly fixed when in the extended position. Sometimes the dislocation is only momentary, the muscle immediately returning to its normal position, so that the animal goes sound for a few steps, but soon 
after falls lame again. When the muscle becomes fixed in the abnormal position it appear's tense, and its outline more distinct, whilst a depression appears in front of the troclianter.

Course. Spontaneous recovery is never permanent; and unless operation be resorted to, habitual luxation results, i.e., the lameness continually recurs, or becontes lasting.

Treatment. Nyotomy is the only means of cure. The operation is usually carried out with a free incision as follows:-By drawing the sound limb forward weight is thrown on its neighbour, and a nearly perpendicular incision, about 2 inches in length, is made through the skin 2 inches below and just in front of the trochanter, in the direction of the muscle. Beginning at its anterior border, the muscle is then divided from subjacent tissues by using the fingers or the handle of a scalpel. It is next lifted, a director thrust under it transversely, and it is divided with a scalpel from within outwards. With proper treatment the wound heals in sixteen to eighteen days. Hertwig has shown that the section might be made subcutaneously. Healing would doubtless be more rapid by observing antiseptic precautions.

In the horse, the muscle does not pass over the upper trochanter, but backwards between it and the ischium, becomes attached by a tendon to the upper part of the ischial tuberosity, which it partly covers, and then blends with the middle and short abductor. The muscle, therefore, during its course, describes a curve, and one portion of it passes over the ischial tuberosity, to which it is attached by means of a tendon.

In fractures of the ischium, the tuber ischii may be displaced by the pull of this muscle, resulting in deformity of the buttock; the symptoms are similar to those in the above-described disease of cattle. Möller saw two cases of this kind.

A ten year old grey gelding had fallen in front of the carriage and.was lame, but in the stable showed nothing unusual. A careful examination of the pelvis proved, however, that the left buttock was abnormally flat at the height of the tuber ischii. Seen from the side, the right buttock projected considerably further than the left, the flattening, which was about 1 to 2 inches in size, was most marked over the tuber ischii, and lost itself above and below, as well as externally and internally. The right tuberosity could be distinctly felt, but the left was indistinguishable; a soft mass of muscle occupied its position, and the bone could only be felt in the depths. Around the trochanter the muscles of the quarter were slightly prominent, so that when seen from behind, the left quarter appeared broader than the right. Lower down the middle line of the perineum was thrust about an inch to the left.

At a walking pace there was moderate supporting leg lameness of the left limb, which was abducted both when loaded and when freely swinging. By placing the hand on the quarter, close behind the upper trochanter, during movement, it was possible at the moment the limb was relieved of weight to detect a sensation as though a cord moved from behind forwards, and then immediately glided back again. Careful observation detected this jerking movement of the biceps femoris muscle, over a region extending from a point about 4 inches above and to the side of the upper trochanter, as far as the 
middle third of the femur. There was no pain, and the lameness was found to be due to distortion of the coronary joint, which was cured in fourteen days, the above-described changes continuing, but without occasioning lameness.

The accident was probably due to old fracture of the tuber ischii, resulting in the point of insertion of the biceps femoris muscle being torn away and the muscle being displaced in a forward direction, thus differing from the condition usual in cattle, where displacement is backward.

Feyer describes a case which may have been due to displacement of the biceps femoris muscle. The horse was in heavy work. When at rest, weight was equally placed on both hind limbs, but the left limb was held a little further from the middle line of the body. On the outer side of the left thigh was a depression about 8 inches long and 2 deep, beginning in front of and below the biceps femoris muscle, and extending obliquely downwards and backwards, becoming more and more shallow as it progressed, Behind this, and corresponding to it in extent, was a swelling in the position occupied by the anterior edge of the semi-tendinosus muscle. The swelling seemed on palpation like a mass of tense muscle. The skin could be lifted and moved over the surface of the depression; pain and increased warmth were absent. In walking the leg was advanced with a circular sweep and set down in a position of abduction. The stride was shorter than that of the sound leg, and the leg was lifted and advanced soon after it passed the vertical position. The horse was not very lame when trotting, though the peculiar gait was more marked. As the horse was still able to do the slow work in which it had been engaged no treatment was carried out.

Wilhelm saw acute atrophy of the abductor muscles in a horse. There was at first abnormal sensitiveness and severe prurigo, causing the animal to bite the parts, but this disappeared in a week, and was succeeded within fourteen days by well-marked atrophy; the muscles were relaxed, but there was no lameness. It is difficult to say whether this was a case of neuritis or of localised myositis. Possibly it was due to thrombosis of the vessels of the affected muscles.

\section{IX.- HIP LAMENESS.}

The lamenesses described on previous pages may generally be diagnosed by careful examination, but not infrequently the symptoms are so illdefined that it is impossible to detect the exact cause, in addition to which pathological changes occur in the quarter and upper parts of the hind limb, especially around the hip-joint, the exact nature of which evades even the most careful examination. Owing to the thickness of the muscles, thorough palpation of deep-seated structures is impracticable, and inflammatory swellings and other anatomical changes often remain undetected, leaving the cause of disease obscure. Such cases are generally included under "hip lameness." The term comprises all forms of obscure lameness, in which, however, the symptoms point to the hip region as the seat of injury; "hip lameness," therefore, has the samie position amongst lamenesses of the hind limb as "shoulder lameness" amongst those of the fore limb.

'I'o enumerate all the diseased conditions which might produce hip 
lameness would be almost impossible, the causes being too varied. Only the most important can therefore be enumerated. Hip lameness may originate in the following structures:-

(1) In the hip-joint. Mechanical injuries, bruises produced by falls, collisions, or kicks, displacements in consequence of slipping, of the foot being caught in the ground, \&c., are all liable to produce inflammation and lameness. Sometimes the skin and surface muscles are more affected than the joint proper, and then there is more or less pronounced. inflammation over the hip-joint or external angle of the ilium.

Partial or complete rupture of the ligamentum teres and extravasation of blood into the joint are not infrequently met with. In a horse which had suffered from hip lameness for more than a year, the hip-joint was found to be surrounded by fibrous comnective tissue, and to exhibit periarticular osteophytes. The synovial membrane was about 2 inches in thickness, the articular cartilage had partly disappeared from the cotyloid cavity, and its edges were undergoing degenerative change. The case was therefore one of arthritis chronica sicca (coxitis chronica). Whilst making a post-mortem of a horse, the subject of hip lameness, Prietsch found fracture of the cotyloid cavity which had not been diagnosed during life.

In cattle, luxation of the femur may remain unrecognised. Harms states having seen subluxations in these animals. Noack found doublesided purulent inflammation of the hip-joint in an eight year old cow. The joints when opened discharged about a cupful of very thick, gruellike greyish-brown, offensive pus. Noack described the disease as metastatic, and considered it a result of the traumatic pericarditis which had simultaneously existed. It is probable that in oxen purulent coxitis is sometimes of tuberculous origin. In the specific arthritis ("navel-ill") of young animals, purulent disease of the hip-joint may be seen. The arthritis chronica sicca, known in man as malum coxæ senile, is not common in horses. Both clinical observation and the results of postmortem examination support this view, though occasionally the disease does occur in animals. In dogs, it often causes chronic hip lameness, and it has been seen in foals.

(2) Another cause of hip lameness is disease of the bones, i.e., of the lumbar vertebræ, pelvis, and femur. Old fractures of the pelvis sometimes occasion obscure lameness. In one such case Bayer detected fracture of the os pubis. On superficial examination, fracture of the external angle of the ilium may be mistaken for hip lameness, as may fracture of the trochanter of the femur. Under the title trochanteric lameness, Williams describes a disease of the upper trochanter in the horse, manifested by chronic inflammation and formation of exostoses.

(3) The cause of hip lameness is more often to be sought in the 
muscles than in the hip-joint and the bones. In lameness following external injuries, bruises, sprains, and partial ruptures (in consequence of falls, kicks, collisions, or over-exertion), the seat of disease is often in the muscles, especially in the superficial layers.

Particular attention should be directed to disease of the tendon and tendon bursa of the gluteus medius muscle, to thrombosis of the femoral or iliac arteries, and in cattle to displacements of the biceps femoris. In exceptional cases, this also occurs in the hol'se.

Kutzner, in a horse, noted gradually inereasing atrophy of the biceps femoris muscle and of the upper portions of the semi-tendinosus. Lameness only appeared after three or four months, when atrophy was far advanced; the foot was adducted just before the end of the stride, and set down too far under the body. The horse showed weakness in movement, and after lying on the diseased side was unable to rise. Kutzner referred the condition to thrombosis. Roloff noted paralysis of the gluteus maximus in a heavy draught-horse. When walking, the affected leg was advanced further than its neighbour, and was drawn towards the opposite side, causing the hindquarters to roll towards the sound side at every step. The animal had great difficulty in moving backwards, dragged the foot along the ground, and could scarcely advance it beyond that of the opposite side. During movement, the thigh was strongly flexed, but only partially extended. The animal showed no pain whatever. The muscle gradually became atrophied, and its place was finally occupied by a trough-shaped depression.

K. Günther describes a similar case, which he refers to paralysis of the lumbar plexus. In this case the limb was so excessively extended during movement that the hoof even touched the chest or elbow. It was, however, still capable of sustaining weight. At a later stage the muscles of the quarter showed marked atrophy.

Vachetta saw a peculiar lameness caused by disease of the psoas major muscle.

Noack found rupture of the tensor fasciæ latie muscle just below its insertion into the external angle of the ilium. The horse became sound in a month. Franke's case of atrophy of the muscles of the quarter in consequence of paralysis of the gluteal nerves has already been alluded to (vide "Paralysis of the Gluteal Nerves ").

(4) Until the typical peripheral nerve lamenesses were first recognised they also were included under the description "hip lameness." It is possible that certain cases described as hip lameness are due to functional disturbance of the sciatic, crural, and obturator nerves. As already stated Günther describes cases produced by paralysis of the lumbar nerves.

The descriptions of neuralgia of the sciatic nerve in animals are unconvincing; certainly the occurrence of this condition has not yet been clearly proved. The same is true of inflammation of the sciatic nerve. The lameness in calves and goats seen by Giovanoli-Soglir, and referred to inflammation of the sciatic nerve, was probably due to otler causes.

(5) Finally, disease processes near the hip-joint may produce lameness, the real cause of which either remains undiscovered, or is only 
revealed after very careful examination. Scirrhous cord, swelling of the inguinal glands, inguinal and femoral hernix, all interfere with movement, and sometimes produce lameness, simulating that due to disease of the hip-joint, or of the bones or muscles. Inflammation of the subcutis over the hip-joint may also result in lameness.

Symptoms. Although the various forms of hip lameness, being due to different causes, exhibit important peculiarities in their symptoms, yet, taken as a whole, they show certain features of general agreement, Thus in all there is difficulty in advancing the limb (swinging leg lameness), retardation of movement and shortening of the forward stride, and in many a tendency to stiffen the limb during movement, and to drag the toe. When the hip-joint itself is diseased, there is supporting leg lameness, and the animal tries to avoid throwing weight on the affected side-symptoms which are usually absent in the purely muscular forms. Lameness is marked when turning and backing, and appears in an aggravated form after severe exertion. Sometimes it is most distinct when commencing work, and gradually decreases; sometimes the reverse. 'Though rheumatic lameness usually wears off with exercise, the continuance or aggravation of lameness under such circumstances by no means points to a traumatic origin. Mechanical injury is a much more frequent cause of lameness than rheumatism.

Sometimes anatomical changes, such as muscular atrophy, swelling: and increased warmth, which can be detected, assist diagnosis, and render it approximately exact. The more thorough and complete the examination, the less common will be the diagnosis "hip lameness," which must always be regarded as a refuge for ignorance, and which we are only justified in delivering when careful examination shows no visible cause elsewhere for lameness.

In other words, diagnosis must be arrived at by a process of exclusion. Cases occur, however, which even the most practised fail to exactly trace to their source.

Course and prognosis. In forecasting the termination of a case, valuable indications are afforded by the character of the onset and course. Sometimes the disease appears suddenly, sometimes slowly; sometimes it disappear's in a short time, sometimes continues for months; sometimes it appears altogether incurable. These variations are always important in determining diagnosis and prognosis; indeed, the prognosis often depends entirely on the course. The following principles are in general true :-

(1) Sudden lameness points to mechanical injury, and usually takes a more favourable course than that which develops slowly but progressively.

(2) When improvement has once set in, complete recovery becomes 
probable in direct proportion to the rapidity with which the lameness has diminished. The gradual increase of lameness indicates pathological changes, probably of an obstinate character.

(3) The older the lameness, and the more marked the anatomical changes (muscular atrophy), the graver the prognosis.

(4) Intermittency or remittency in the lameness is also an unfavourable feature, particularly if the horse has been rested and properly treated.

(5) The duty to be performed must also be considered: thus riding and heavy draught horses offer less probability of permanent recovery than those in light work.

(6) Disease of the joint is graver than muscular disease.

Treatment. In recent hip lameness, accompanied by inflammatory symptoms, rest and cold applications, continuous cold-water irrigation, or ice bottles, are indicated. If the symptoms suggest rheumatic disease-that is, if lameness decrease with movement, if the course be intermittent, and if the disease appear without apparent cause-warm moist packs are better, and may be followed by infriction with mild irritants, like oil of turpentine, spirit of camphor, or ammonia liniment. The animal should be rested, and placed in a warm stall. In muscular disease, massage, in the form of rubbing, is valuable; but where paralysis is surmised, striking or beating the muscular tissue is better, and seems to check degenerative processes.

If no marked improvement occurs in eight to fourteen days, a blister of cantharides, bichloride or biniodide of mercury (1-8) may be applied. It is best to mark out with chalk, or by clipping the hair, the position in which the ointment is to be applied to the hip-joint, otherwise stablemen always apply it over the external angle of the ilium.

Should this treatment also fail, setons, subcutaneous injections of veratrin, or the application of the actual cautery may be resorted to. The best results follow deep firing with a fine needle point at a high temperature. Graillot's cautery is very useful for this purpose (see Dollar"s "Operative Technique," p. 121). Six to eight points may be made round the joint, and, if considered necessary, a blister may be applied. Amongst other injections, a concentrated solution of common salt has lately been recommended. Its action is uncertain and difficult to control, though when the injection produces abscess formation, it certainly acts somewhat like a seton. After recovery from long-existent lameness, particularly from lameness produced mechanically, the horse should not immediately be put to work, and heavy draught should especially be avoided.

An occasional cause of hip lameness in old horses is to be found in ossification of the fascia covering the gluteal muscles. Dollar diagnosed 
during life and removed after death, from the gluteal region, a cribriform plate of bone measuring 10 inches long, 7 wide, and at its thickest point $\frac{1}{2}$ an inch thick. Properly speaking, this plate was double, and a space existed between the external and internal layers. Laquerrière saw and removed a plate of bone about 5 inches in length and $2 \frac{1}{2}$ inches in breadth from the external crural region. The horse, which had previously been lame, was at once cured. Cadiot saw ossification of the tendon of the semi-tendinosus muscle. The bony plate was triangular; the base uppermost. It measured 6 inches in length by 3 in breadth, the point being embedded in the tendon of the semi-tendinosus muscle. (See Cadiot and Dollar's "Clinical Veterinary Medicine and Surgery," p. 442.)

\section{B. DISEASES OF THE STIFLE-JOINT.}

Anatomy. The two divisions of the stifle-joint formed by the condyles of the femur, and the interarticular cartilages crowning the head of the tibia, often communicate with one another, whilst the patella femoral joint consists of a roomy cavity above and in front of the condyles of the femur, which Franck says always communicates with one, sometimes with both of the others. The tendon sheaths of the flexor metatarsi and extensor pedis also communicate with the external division of the stifle-joint. In the horse, a bursa known as the bursa prepatellaris, varying from the size of a bean to that of a walnut, is found on the anterior surface of the patella.

\section{I.-LUXATION AND SPRAIN OF THE STIFLE-JOINT.}

The stifle-joint has broad articular surfaces, and a powerful ligamentous apparatus. Furthermore, it is surrounied on almost all sides by strong muscles and tendons, whilst the tibial spine projects upwards between the two condyles of the femur, and the relations of the two bones are so secured that in animals luxation of the joint is of excessively rare occurrence. Isolated cases have, however, been seen in cattle.

Thus Stolze describes complete luxation of the tibia forwards. The cow was unable to advance the limb, which was rigid and somewhat shortened. It was still possible, however, to place weight on the leg, and the displacement of the bone could be detected by palpation of the stifle-joint.

Reposition, though difficult, was finally effected, but luxation soon recurred, in spite of the animal being slung. After replacing the parts five times-an operation which appeared easier on each occasion - a blister was applied; the parts remained in position, and in three months the animal could move fairly well, and was able to return to grass. In the Jahresbericht for 1860 a similar case is described.

Sprain of the stifle-joint may possibly occur from violent movements, but is certainly not common in large animals. Possibly the chronic inflammation of the stifle-joint (gonitis chronica) seen in dogs is due 
to such injuries. In cows, Sand noticed spontaneous dislocation of the tibia following partial destruction of the interarticular cartilages, in consequence of chronic gonitis.

\section{II.-DISPLACEMENT OF THE PATELLA. LUXATIO PATELLE.}

The patella is retained in position by a capsular ligament strengthened by two lateral ligaments, and by its straight ligaments, of which the horse and ox possess three; most other animals, however, only one. Finally, the pull of the triceps extensor cruris and tensor vaginal femoris muscles sustains the patella above. Horses, oxen, and dogs are the commonest sufferers, and the luxation is either-

(a) Upwards, over the internal lip of the trochlea of the femur ; or

(b) To the side, and always to the outer side.

The two conditions, however, differ not only in their symptoms, but also in their prognosis and treatment, and therefore will receive separate consideration.

(a) Luxation of the patella upwards, which occurs in oxen and horses, and has been seen by Olivers in a mule, is, strictly speaking, only a displacement of the patella within its articular capsule, the bone passing upwards over the internal lip of the trochlea and failing to return. It is fixed in this position by its lower border lying against the upper margin of the inner lip of the trochlea. This luxation can, therefore, only occur after excessive extension of the stifle-joint. Violet has lately put forth the theory that the patella is retained on the trochlea of the femur by its lateral ligaments, and particularly by its internal lateral ligament. The surface of the internal lip of the trochlea describes the segment of a circle around the point of insertion of the internal lateral ligment into the femur. It can, therefore, easily be imagined that under certain circumstances the upper portions of the articular surface may lie nearer to the point of insertion of the ligament than the portions below. As a consequence the patella might easily become fixed in position on that surface of the inner trochlear lip, which Violet describes as being concave in the horse, and which in oxen is level; and require a very considerable exertion of strength to draw it down again. In consequence of this form of the articular surface of the internal lip of the trochlea, and of the tension in the lateral ligaments, the patella is sometimes arrested for a few moments, and, under certain circumstances, for a longer time at the highest point in its course, and the action of the limb thus interfered with. The condition might, therefore, be looked upon as fixation of the patella in a position of excessive extension. It has also been termed subluxation or arrest of the patella. 
Causes and symptoms. It will be seen that dislocation may be due to one of two causes, viz.:-

(1) Flattening of the articular surface of the internal lip of the trochlea in conjunction with abnormal tension in the lateral ligaments; or,

(2) Fixation of the lower surface of the patella on the upper border of the inner trochlear lip. In the first case, it is natural that the lameness should often be remittent, but only last for a few moments on each occasion (habitual luxation). This form may, therefore, be termed momentary upward luxation of the patella; and that produced by fixation of the under border of the patella on the upper border of the internal lip of the trochlea, stationary upward luxation. Movement of all the joints of the limb, with the exception of the hip-joint, is dependent, however, on flexion of the stifle, on account of the insertion into or around it of the flexor metatarsi, gastrocnemir, extensor pedis and flexor pedis perforans muscles; and therefore when the stifle is fixed the other joints are at once immobilised in an extended position, and flexion and free movement are lost (see fig. 260 in chapter on "Rupture of the Flexor Metatarsi Muscle"). In fact, any interference with the movement of the patella produces lameness: in the case of momentary luxation there is a slight check, similar to that seen in stringhalt, just as the foot leaves the ground and should begin its striding movement. Bassi, indeed, regards stringhalt as due to momentary luxation. 'The peculiar check known as "straw cramp" is often seen in the stable when the animal turns round, sometimes in one, but not infrequently in both limbs. As a rule, it gradually disappears during work, sometimes only occurring during the tirst twenty to thirty steps. 'The sudden and excessive flexion of the limb which follows the check has produced the erroneous impression that it is due to cramp in the vasti and rectus muscle. It is not uncommon after influenza and other debilitating diseases.

The degree of lameness varies greatly. Sometimes it is necessary to look carefully in order to recognise it at all, but in other cases the patella is seen to remain fast for a moment and then suddenly descend.

The symptoms are different when the patella remains contmuously fixed above the internal lip of the trochlea, i.e., m stationary upward luxation. This condition only aftects oxen and solipeds, because in other animals the internal lip is more rounded oft, and fixation of the patella above it is impossible. In consequence of violent contraction of the vasti and rectus muscles occurring when rising or falling, kicking outwards, or extending the limb, the patella passes too far upwards, and becomes fixed. Before this can occur, it is probably necessary for the straight ligaments of the patella to become elongated, as happens in pregnant animals by continual confinement to the stable, 
or to other's after passing through severe attacks like influenza. Not infrequently such elongation is congenital. Loose-jointed, weak foals with straight stifles suffer oftener than well-developed horses. Fixation of the patella on the upper portion of the internal lip of the trochlea is at once followed by extension of all the joints of the limb and inability to flex them, even with external assistance. The limb is therefore stiffly directed backvards, and cannot be advanced. If both limbs are affected at the same time, the animal stands as though rooted to the spot and cannot advance; when only one limb is affected, it can still hop forwards on the other leg. Luxation is, therefore, distinguished by lameness when the leg is carried and excessive extension of all joints.

If, whilst the patella occupies this position, the rectus and vasti muscles be relaxed, the patella may rise from the lip of the trochlea of the femur and stand upright on its lower border, a condition which in man is known as vertical luxation of the patella. 'The symptoms are so characteristic that stationary luxation can be diagnosed from a distance, and is only likely to be mistaken for dislocation of the biceps femoris muscle in oxen, in which, however, the extended position is not so well marked, and the limb can always be flexed, if only to a slight extent, while the trochanter may be felt under the slin, and the patella at least moved sideways. In luxation, on the other hand, it is absolutely immovable, and lies too high.

The prognosis depends partly on the amount of interference with movement, and therefore with usefulness, partly on the character of the lameness, the age of the animal, and the length of time the condition has existed. In weakly foals, and in animals attacked during convalescence, both momentary and permanent luxation usually cease to occur as the general condition improves. The longer, however, lameness has existed, and the more frequent and marked the attacks, the slighter is the chance of recovery:

Treatment. Young animals and convalescents should be well fed and lept from heavy work. With rest and the recumbent position, the straight ligaments of the patella return to their normal length. Everything likely to cause relapse must be avoided. In momentary luxation nothing further can usually be done, but stationary luxation calls for immediate reduction, which may be effected in one of several ways.

(1) The patella often returns to its proper position after sudden backward or side movements. Energetic contraction in the patellar muscles, produced by an unexpected blow or by drenching the animal with cold water, also induce reduction at times.

(2) Where the above means prove insufficient, an attempt should be made to free the lower edge of the patella by pressing on its upper and outer border, the pressure being directed forwards, and inwards. 
Meyer grasps the patella with the whole hand and lifts it up whilst the animal is led forward.

Reduction is assisted by passing a cord round the fetlock, lifting the toe from the ground, and drawing it forward, thus extending the stiflejoint. The animal is then thrust backward, and at the same moment the patella pushed back. Where the animal is lying, the limb can be drawn forward and fastened as for castration, replacement being attempted in this position.

After successful replacement, it is important to rest the animal for three to five weeks. The application of blisters, setons, \&c., is only useful in lieeping the limb at rest. If the patella again slips out when the animal lies down or rises, slinging may be resorted to. Another useful device is the application of a patten shoe (see Dollar's "Horseshoeing," pp. 411 to 413), by which the limb is raised from the ground, and the stifle-joint is lept flexed so that the necessary conditions for the occurrence of luxation cannot well occur.

Violet and others recommended dividing the inner straight ligament, and state having cured cases of momentary luxation which had been in existence for years. The operation is, however, by no means easy, because the inner straight ligament is in somewhat dangerous proximity to the capsule of the joint. From theoretical considerations it certainly seems feasible, inasmuch as it frees the inner surface of the patella, which is the part said to become fixed on the femur. Cavallari performed the operation with immediate and lasting success on both hind limbs of a cow, but further experience is required before a tinal judgment can be delivered.

(b) "Lateral Luxation of the Patella."

In hor'ses, oxen, and still more frequently in dogs, the patella is displaced laterally, and almost always towards the outer side. In dogs the disease is generally congenital and affects both limbs, being caused by arrest in the development of the condyles of the femur. Acquired luxations outwards are dependent on excessive strain or rupture of the inner lateral ligament. As the internal condyle of the femur is much the larger and projects further inward, luxation is very rare indeed in animals, though Stockfleth saw one case in a cow. Sometimes the patella still lies to some extent over the external condyle (incomplete luxation), though it is generally thrust on to its outer surface (complete luxation). Though in the horse the patella is certainly secured in position by a cartilaginous prolongation which curves over the inner lip of the trochlea, its displacement outwards is by no means impossible. Meyer saw luxation of this kind in two cows.

Causes. The predisposing causes of lateral Iuxation are uprightness of the limb, obliquity of the quarters, and youth of the animal. The 
condition often appears in both limbs. Another factor may consist in relaxation and weakness of the ligaments. In dogs the inner lateral ligament is much slighter than the outer.

Everything favouring rupture or strain of the internal lateral ligament may produce the condition. Meyer believed it was caused by rheumatic contraction in, or excessive action of the abductors, but failed to effect a cure by dividing them.

The prognosis is less favourable than in upward luxation, particularly in dogs. Pugs often suffer congenitally, and little can be done for them. As in other cases, the older the luxation the greater the difficulty in reduction and retention, and the less the chance of recovery. Incomplete outward luxation is clearly more favourable. than the complete form. Meyer's cases recovered sufficiently to allow the animals to be used for milking purposes.

Symptoms. The clinical symptoms of complete dislocation resemble those of crural paralysis and rupture of the extensors of the stifle-joint. They. consist of lameness when weight is placed on the limb, with excessive flexion of all the joints. Where the patella is displaced outwardly, the function of the rectus and vasti, and consequently the ability to bear weight, are lost, though the muscles named can still advance the limb, the stride being somewhat shortened. 'The capsular ligament appears distinctly below the stifle, whilst the patella can often be felt in its abnormal position. 'The symptoms are less marked in incomplete luxation, in which lameness is sometimes more pronounced when walling than when trotting.

In dogs the patella is readily replaced, giving immediate relief from lameness, but soon slips out again (habitual luxation). In horses reduction is more difficult, and the limb is afterwards held stiffly, and only slowly advanced.

Treatment. As already stated, replacement proves easy in the carnivora, but in all kinds of animals retention is difficult. Hertwig states having kept the patella in position by hand for forty-eight hours. Relays of assistants were employed, and a blister was afterwards applied. As bandages cannot be used to retain the parts, treatment is generally confined to rest and blistering, which compels the animal to keep the foot as still as possible. Large animals can be slung.

Möller saw a peculiar displacement of the patella upwards and outwards. It was impossible to bend the limb, which was extended and directed forwards, but weight could very well be borne on it. When forced to move, the animal carried the leg forward, holding it stiffly, and not moving the hip, stifle, or hock joints, and placed it far in advance. The progress of the body then brought the limb perpendicularly under the hip-joint, but the leg could not be placed behind this point, either actively or passively. Slight pressure against the outer edge of the patella sufficed to return it to its position, after 
which the horse could sometimes walk for several steps in the usual way; suddenly, however, the patella again became displaced, and all the symptoms returned.

The post-mortem showed the inner lateral and inner and middle straight ligaments to be diseased and elongated, allowing the lower border of the patella to ride up over the outer lip of the trochlea, and become fixed there. The upper border of the patella then inclined forwards (vertical luxation), and the bone itself slipped outwards to an extent of nearly 1 inch over the external condyle of the femur. When the patella was replaced, the ligaments named were seen to be distinctly relaxed. The continual pressure exercised by the lower border of the patella had caused absorption of the articular cartilage of the femur, and the formation in it of a distinct depression, corresponding in shape to the lower surface of the patella. Attempts were made during life to fix the bone in its proper position by a dressing, and for a time proved successful, but had to be given up in consequence of their causing inflammation and necrosis. The most effective appliance was a broad strap fixed to the slings, and buckled round both stifle-joints.

A splint was so applied to the tibia as to exercise pressure on the outer: surface of the patella, but was only partially successful, though, after eight days of this treatment, the patella remained in position, and the animal could stand and even walk some steps without assistance. Previously it had been unable to make even a single step without the patella becoming displaced. Unfortunately it died from an intercurrent disease.

Dollar saw a somewhat similar case, which is.described and illustrated in "Clinical Veterinary Medicine and Surgery," p. 439.

\section{III.-RUPTURE OF THE STRAIGHT LIGAMENTS OF THE PATELLA.}

Möller describes two cases of rupture of the outer straight ligament in horses, the first case caused by the impact of a carriage-pole, the second probably by suddenly springing up in the stable. Both animals showed marked supporting leg lameness. At first no weight at all was thrown on the affected limb, and later, only a certain amount. The internal and middle straight ligaments could be felt below the patella, but the place of the lateral one was occupied by a depression. There was great pain on pressure below the patella, which, however, preserved its natural position. Treatment consisted in absolute rest: one patient was sufficiently improved in three weeks, the other in a month, to allow of its leaving hospital.

Voigtlïnder saw rupture of the straight ligaments in an ox. The symptoms were: inability to stand, severe inflammation around the stifle-joint, flexion of the limb, inability to extend the stifle, and relaxation of the straight ligaments. The animal was slaughtered, and the rupture confirmed by post-mortem. 


\section{IV.-FRACTURE OF THE PATELLA.}

Fracture of the patella is rare, and up to the present has only been seen in horses. Kicks, collisions, and falls with the stifle-joint strongly flexed are the principal causes. Wollstein reports a case produced by struggling in hobbles, whilst Renault saw double-sided fracture in a horse in slings; the sling had worked backwards until the horse's hind feet were no longer in contact with the ground. Another animal is said to have broken both patellæ by slipping.

Fractures due to external violence are often comminuted, and associated with injury to the joint. Horizontal fractures are usually the result of violent muscular contraction. Vertical fractures are occasionally seen after mechanical injuries. There is then rarely any displacement, as the fibrous tissue covering the anterior surface of the bone retains the fragments in contact.

Symptoms and course. Fracture of the patella is characterised by severe lameness, inability to bear weight on the limb, violent pain on movement and pressure, and swelling in the stifle region. Provided swelling is not too great, the pieces of bone can sometimes be felt, though there is rarely crepitation. The prognosis is in most cases unfavourable. In men, although much more can be done than in horses, ligamentous union is the rule, because the fragments are considerably separated, and on account of its want of periosteum, the bone is little fitted for callus formation, while its lower portion is deprived of blood-supply, which for the most part is derived from the muscles. More successful results have been obtained in late years, since it has been found possible to reunite the fragments by means of ivory pegs, stout metal wire or steel screws, silvered on the surface. In the early days of antiseptic surgery such methods were thought impracticable, but it has since been found that foreign bodies, like screws, \&c., if sterilised before insertion, do not necessarily cause suppuration. Transverse fractures and fractures into the joint are in large animals unfavourable; the good results reported are in most cases due to errors in diagnosis. Andrieu, however, describes one case where the patella was split into three portions and the patellajoint opened, as evidenced by the escape of synovia. By introducing the finger through a wound on the front of the patella the fractured bone could be felt. The parts were subjected to continuous irrigation. In a fortnight the animal could walk slowly; in a month it did very light work; a month later still it showed only slight lameness when trotting. Such a case is quite exceptional. When, however, only a small piece, like the point of insertion of one of the straight ligaments, is torn off, recovery may occur in a month.

Treatment must follow general principles. Large animals require 
slinging, and in small ones a dressing should be applied, the limb being as much as possible extended.

\section{Y.-INJURIES AND ACUTE INFLAMMATION OF THE STIFLE-JOINT. GONITIS.}

The larger domesticated animals, and horses in particular, often suffer injuries of the patella and stifle from treads, kicks, stabs with stableforks, thrusts with lances, sabre cuts, \&c., which give rise to suppuration in the joint. Inflammation may also extend to the joint from the tendon sheaths of the flexor metatarsi and extensor pedis; and as both portions of the femoro-tibial joint communicate, suppuration extends from one to the other, and eventually to the patellar joint. Acute aseptic inflamma= tion of the stifle-joint is less frequent, but may follow violent bruises or dislocations, and lead to extravasation into the joint, or to gonitis acuta serosa (hydrops genu). Cadiot described a case of tuberculous gonitis in a dog. In oxen, and less frequently in horses, rheumatic inflammation of the stifle-joint has been seen.

The symptoms are: severe lameness; the animal carefully avoids placing weight on or moving the limb; all the joints of the limb are held stiffly in a position of flexion (fig. 257) ; there is diffuse swelling, and, when the joint is wounded, discharge of purulent synovia. The animal shows fever and loss of appetite, lies continuously, and, if not killed, dies from decubitus, and with symptoms of septicæmia. The aseptic disease takes a longer course and exhibits less severe symptoms. Cases of moderate inflammation display a considerable likeness to gonitis chronica.

The prognosis of septic or purulent gonitis acuta is almost hopeless, and in most cases slaughter is to be advised. Fat oxen should at once be killed, to avoid the loss of condition which results when disease becomes general. The aseptic form may be treated, particularly in valuable animals. The prognosis should be reserved if severe pain exist, because the continuous weight thrown on the other foot may produce acute laminitis, or the animal, unable to stand, may die from decubitus. Severe complications, nevertheless, may render even this form incurable; thus, a horse which had been struck by a carriagepole suddenly became lame; the animal died, apparently in consequence of pain, and on post-mortem, two fragments of bone as large as chestnuts were found to have been detached from the outer condyle of the femur; they lay within the joint capsule. On the other hand, a seemingly severe injury exposing the patella healed in a month. In this case the joint was probably uninjured. Sherman saw a horse die from division of the femoral artery produced by a lance-thrust in the flank. 
Treatment. Recent wounds near the joint, or involving the abovenamed tendon sheaths, require antiseptic treatment; and, as in large animals a proper dressing cannot be used, the wound must be disinfected, and in the case of small injuries a blister of sublimate dissolved in 10 parts of spirit, or the actual cautery should be applied, in order to produce swelling, and close the wound in the joint as rapidly as. possible. Large wounds may be carefully disinfected and sutured, or be liept aseptic by continuous irrigation.

\section{VI.-CHRONIC INFLAMMATION OF THE STIFLE- JOINT (GONITIS CHRONICA SICCA).}

Chronic inflammation of the stifle-joint principally occurs in heavy draught-horses and those which work continuously at a sharp trot. Omnibus, tramway, and cab horses often suffer from it. It is also seen in dogs, particularly in the larger coursing and worling breeds. In dogs it is generally unilateral; in horses it often affects both sides. Höhne regards it as a common cause of lameness in horses, and thinlis it is often mistaken for developing spavin, but the symptoms differ considerably, and should not be confused if care be taken. Postnortem examination shows changes similar to those of arthritis chronica sicca vel deformans: thickening of the margins of the articular cartilage with ostitis, particularly on the tibia. Abrasion of the articular surface is most pronounced around the tibial spine, porcellaneous deposits occur, and there is sometimes moderate dropsy of the joint. As a rule, the process is confined to the inner division of the femoro-tibial joint, the outer division and the patellar joint remaining intact. In addition to thickening of the ligaments of the stifle-joint and cauliffower growths on the articular surface of the femur, Höhne found eburnated elevations and depressions in the spongy tissue of the ends of the bone, and enlargement of the inner condyle to the extent of three-eighths of an inch. Sometimes he could only detect condensation of the spongy tissue of the inner condyle. It is, therefore, not absolutely certain that Höhne was always dealing with the disease now in question.

Symptoms and course. T'he disease sets in slowly and gradually, but generally proves so stublorn that the animal has to be destroyed. Sometimes it persists for months, or even years. At first the lameness is slight, and where the condition is bilateral may for a long time be overlooked. In the stable the diseased limb is liept flexed (fig. 257); when both are affected, they are rested alternately. In turning, the affected limb is often lifted spasmodically, as in spavin and stringhalt. When commencing to walk, the animal shows swinging leg lameness and avoids extending the limb, so that the forward stride is shortened. 
The toe of the diseased foot is apt to catch on slight prominences; at a trot the lameness shows some resemblance to spavin lameness, but the limb is not moved spasmodically, much more often it is carried stiffly.

Thongh pain cannot usually be detected, the capsule of the joint is often visibly distended below the patella, and in the later stages the internal condyle of the femur is distinctly increased in size. This examination is made standing behind the horse (of course taking proper precautions) and grasping the stifle-joints alternately with both hands, so as to compare one with the other. Dogs are examined in the same

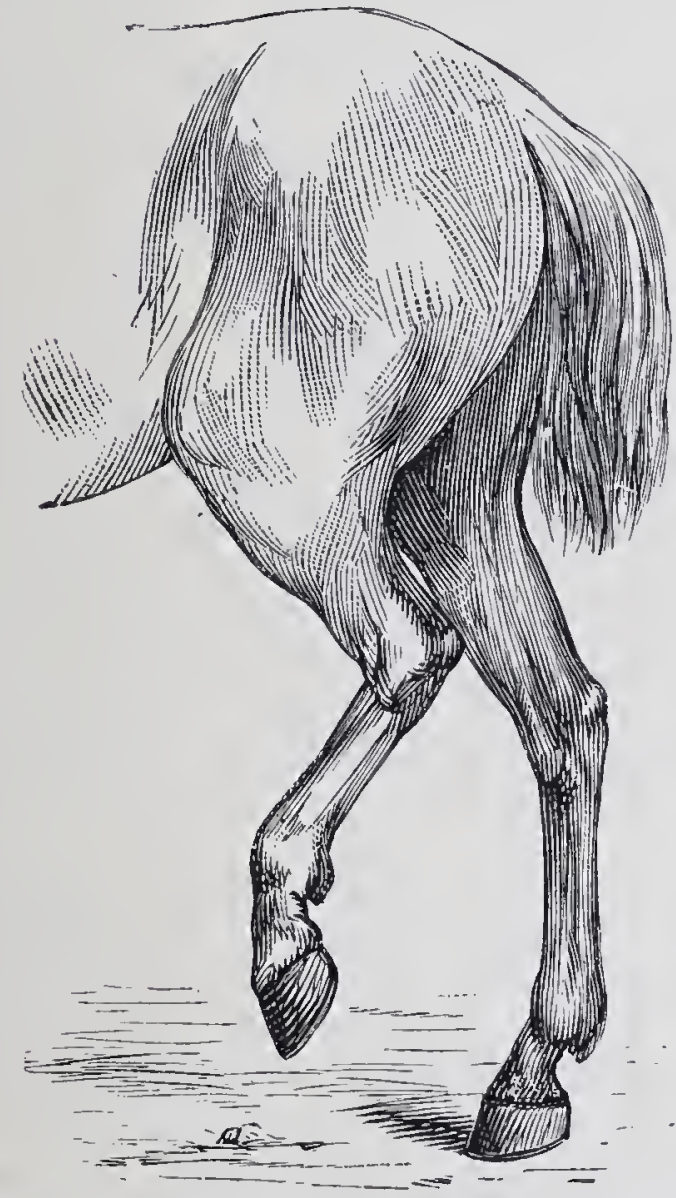

FiG. 257.--Teft-sided chronic juflammation of the stifle-joint (Gonitis chronica).

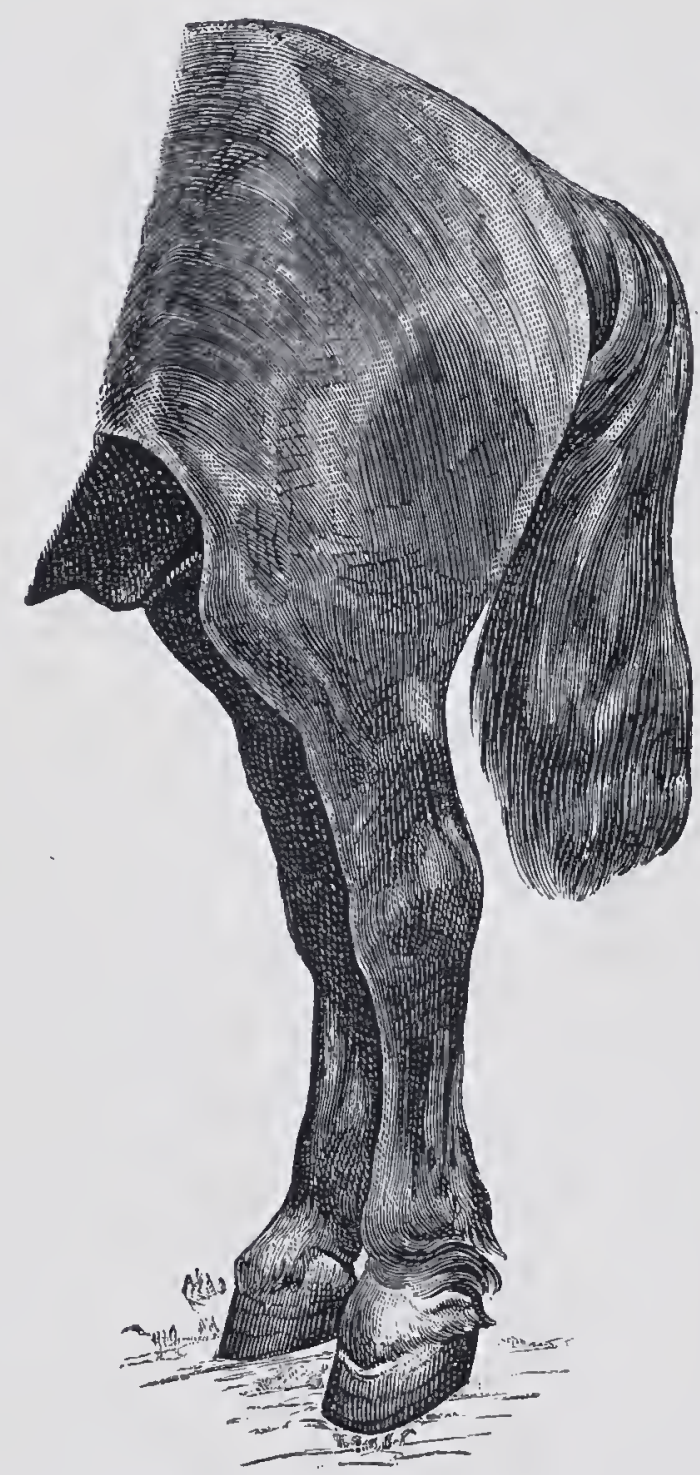

Fig. 258.--Bilateral chronic inflammation of the stifle-joint (Gonitis ehronica bilateralis) (from a photograph).

way. The animals sometimes show acute pain when the limb is abducted and rotated inwards (pronation).

The disease is incurable, and neurotomy being out of the question, nothing can usually be done. Working-horses should be used as long as possible. If the owner insists on treatment, blisters, firing, or other resorptive measures can be tried. Recovery is extremely rare, and the improvement of reported cases probably depends on mistakes in diagnosis.

A twelve year old gelding in heavy draught had for two years shown slight lameness in each hind limb alternately. The limbs gradually became upright 
(fig. 258), the movemient stiffer, and the quarters more markedly sloping. In both limbs the capsular ligament of the stifle-joint was prominent, being best defined below the patella (fig. 258). When resting, the hind legs were lifted alternately and strongly flexed, at short intervals; the horse had not lain down for a long time. In work, the hind limbs were carried stiffly and the stride shortened. The animal had become so useless that its owner caused it to be slaughtered.

The post-mortem examination showed marked proliferation of connective tissue in the muscles of the quarter and thigh, the new growth appearing in the perimysium externum in the form of broad white bands (myositis chronica fibrosa). There was chronic inflammation in the bursa glutæi radii, with great increase in the villi, which were 1 to $1 \frac{1}{4}$ inch in length and $\frac{1}{8}$ to $\frac{1}{4}$ of an inch
thick.

The patellar joint was full of a yellow viscous fluid. The capsular ligament was distended, and the synovial membrane covered with long villous growths. In the true stifle-joint the articular cartilage had almost disappeared from the internal division, and scarcely a trace remained on the spine of the tibia, though numerous eburnated porcellaneous deposits were visible. The margins of the internal division of the joint, particularly the tibial margin, were prominent and thickened. The patellar joint exhibited no particular
change.

\section{VII.-BURSITIS PREPATELLARIS.}

The bursa præpatellaris, according to Eichbaum, occurs in fully half of all horses. It lies on the anterior surface of the stifle-joint, and when injured is apt to become inflamed; the swelling, which is sometimes fluctuating, sometimes moderately firm, attaining the size of two fists. It seldom produces pain or lameness, and only forms a blemish like "capped elbow."

The disease being caused by bruises or other injuries, is sometimes accompanied by inflammatory symptoms, but sometimes occurs without them. Lameness is absent in the latter case, a fact to be remembered in diagnosing other diseased conditions. Should treatment be considered necessary, fluctuating swellings (hygromata of the bursa) may repeatedly be punctured with the hollow needle, or with a slender trochar, and emptied of contents. Injection of iodine may also be tried. As in "capped elbow," free incision leads to long-continued suppuration, and is therefore not advisable, unless the bursa can be destroyed by cautery or caustic. Blisters and firing may produce gradual contraction, but require repeated application. Care must be taken not to incise a hydrops genu in mistalie for enlargement of the prepatellar bursa. The hydrops swelling lies deeper, and cannot be so easily displaced under the skin as that now in question. 


\section{DISEASES OM THE TOUER THIOH OR IER.}

The thigh, especially in the horse, is surrounded by a tense, stretched skin, which on the external face is strong, but on the internal quite thin. Towards the front of the internal surface of the thigh, the skin and slightly-developed subcutis lie directly in contact with the tibia, which, therefore, at this point is particularly exposed to injury. About three-quarters of the bone, i.e., the entire external and the posterior portion of the internal surfaces, are covered with muscles which are singly clothed with fasciæ, and are again surrounded by the fascia lata. 'The latter' arises from the muscles of the quarter and upper parts of the thigh, and is partly inserted into the crest of the tibia, partly extends below the hock, to become continuous with the fascia covering the tiendon of the extensor pedis. In consequence of this formation, injuries to the leg present certain marked peculiarities.

\section{I.-WOUNDS AND INJURIES.}

Injuries of the tibia are commonest in horses, and are caused by kicks, and by the leg being passed over bales or over the carriage-pole. Sometimes the skin alone is inflamed, or extensively torn, but not infrequently the periosteum and even the bone are bruised. The bone is sometimes fissured by kicks, a condition which will later receive attention; or diffuse periostitis is caused, though it also follows bruising of the periosteum, without the bone itself being injured.

Periostitis of the tibia produces well-marked swinging-leg lameness and shortening of the forward stride, while the thigh is moved slowly and stiffy. It usually lasts three to four weeks, and is succeeded by thickening about the tibia. The local pain shown on palpation prevents any mistake in diagnosis. Injuries of the tibia itself can usually be detected by probing.

The presence of strong fascia covering the muscles of this region causes wounds, which would otherwise heal without trouble, to be followed by very dangerous consequences. Injury to the fascia is troublesome, because the muscular tissue protrudes through the rent (muscular hernia), and being irritated by its edges, commences to proliferate actively; - or suppuration may lead to necrosis of fascia, and, before the necrotic portions can be shed, excessive fungous granulations, the growth of which is favoured by the continuous movement of the muscle, arise and greatly impede healing. This condition is commonest on the lower portions of the biceps femoris muscle, but may occur in any region of the tibia.

A second difficulty in the healing of such wounds is the development of subfascial cellulitis. It generally accompanies perforating wounds caused by stable-forks. The symptoms are great pain when the animal stands on the limb or attempts to move it, moderate swelling, inflammation of the neighbouring lymph vessels and glands, and fever. The 
skin is not excessively swollen, but at a later stage shows fungiform swellings, produced by deep-seated abscesses breaking through the fascia and becoming subcutaneous. As the cellulitis is frequently of a septic character, it may lead to extensive necrosis and fatal septicæmia, a result favoured by the impossibility of discharges escaping through the unyielding fascia.

The treatment must be directed by general principles. Cold applications are useful in relieving severe pain; if merely bruised, the parts may be surrounded with cotton-wool, and kept continuously irrigated. Wounds, especially those reaching to the bone, are best treated by antiseptic measures. The periostitis may afterwards be dispersed by blisters. Sequestra must be removed as soon as they appear to have separated. If only the skin is bruised or excoriated, the parts should be cleansed, and dusted with iodoform, amyloform, or boric acid, or smeared with lead or zine ointment.

The exuberant granulations produced by muscular herniæ are removed with scissors, the cautery, or caustics. To prevent their return, it is often necessary to still further divide the fascia; sometimes a piece of it may be excised, and further strangulation thus prevented. Though a pressure dressing is difficult to apply at this point, benefit sometimes follows the use of a few strips of adhesive plaster. A piece of leather or strong linen is smeared with resin plaster (composed of two-thirds resin and one-third wax), and applied over a pad of gauze covering both the exuberant granulations and neighbouring parts. Absolute rest is indispensable. Subfascial cellulitis calls for early incision and disinfection, and, if necessary, drainage. Deep-seated abscesses are to be opened as soon as diagnosed. Immediately the mushroom-like swellings appear they should be divided, and the pus allowed to escape.

\section{II.-FRACTURE OF THE TIBIA.}

In the horse, fractures of the tibia rank next in point of frequency to those of the pelvis. Of 1,082 fractures occurring during four years amongst army horses, 189 were in this bone. Such fracture is oftenest caused by kicks from horses in neighbouring stalls, the point struck being the anterior and internal face of the tibia, which lies directly under the skin. In most cases the bone is at first only fissured, and the true fracture occurs later, during such acts as rising, lying down, or passing urine or fæces. It is seldom postponed more than a week or ten days from the date of original injury, though cases exist where fracture has not occurred until four or five weeks afterwards. In a case of Möller's, fracture was delayed until a month after the injury, and in an army horse 127 days passed after the primary accident before the parts 
became separated. In such cases the fracture is probably subfascial, a view supported by the commonly-observed fact that the fragments show no callus formation. Or, again, the primary injury may produce local or green-stick fracture, which weakens the tibia, but only gives rise to complete fracture under severe strains, like those occurring when rising, lying down, \&c. Abrasion of the edges of fragments either results after fracture is complete or is due to subperiosteal fracture, in which slight movement of the fractured portions is possible. The absence of abrasion is not, however, evidence that green-stick fracture may not have existed for some considerable time, a fact of much forensic importance. A kick on the inner surface of the tibia, such as would be given by a horse standing on the opposite side, may at once produce complete fracture, which is then generally complicated. The bone is occasionally broken by the animal slipping, falling, being struck with the carriage-pole during collisions, or by its struggling violently in hobbles, as, for example, during castration, though, in the latter case, the femur or vertebral column is more often fractured.

With the exception of the horse, the commonest sufferers from fracture of the tibia are dogs, in which the accident is due to being kicked, run over, \&c. Oxen and other animals are much less frequently affected; nevertheless, cases are seen in them, and the practitioner is even occasionally called on to treat parrots and canaries. F'enimore describes a case of fracture of the tibia in a foetus; the fracture had actually been produced by a kick received by the mother (a cow) whilst pregnant, and when the calf was born the fracture was united.

The symptoms vary according to the degree of the fracture. Complete fracture renders it impossible to place weight on the limb, the unusual mobility of which can be detected even from a distance; the foot, when lifted, remains dangling, and in the horse the condition shows some resemblance to rupture of the flexor metatarsi muscle. Fracture of the tibia, however, is at once differentiated by the impossibility of placing weight on the limb.

In complete fracture, crepitation can always be detected. Not infrequently the exact point of fracture is discoverable, especially if low in the leg. Perforation of the skin by splinter's of bone removes the last doubts of fracture; in such case traces of the external injury are generally apparent.

Fissuring sometimes, though not invariably, produces well-marlied lameness, both when weight is placed on the limb and when it is carried. Starting from the injured point on the tibia, the line of fissure may be mapped out by the existence of pain over it. Fissures in the subcutaneous regions of the tibia may be diagnosed with certainty, otherwise they can only be guessed at, for this linear distribution of 
pain, which is the sole reliable symptom, cannot be detected under the muiscular tissue. In the cases described in which there was no lameness, the fissure was probably subperiosteal. Incomplete fractures and local injuries are accompanied by lameness of greater or less severity, depending on the degree of periostitis; should the latter be slight, lameness is so little marked that animals, even with incomplete fracture, may be kept at work until complete fracture suddenly occurs. On the other hand, partial fracture and local injury to the bone may often be at once recognised, provided the soft parts are not greatly swollen. From the uncertainty which surrounds these cases, it is of the utmost importance, in every painful lameness resulting from mechanical injury to the inner surface of the tibia, to bear in mind the possibility of fissure or incomplete fracture. Their existence is the more probable if lameness be severe, and no marked periostitis or local pain can be discovered.

Fractures of the malleoli of the lower end of the tibia, common in man, are rare in animals, though on post-mortem examination Leisering detected a case which had caused obstinate and incurable lameness. The external malleolus was separated for a length of 1.4 inch, and to a height of $\cdot 6$ inch. The anterior portion of the middle protuberance was fractured.

Course and prognosis. Although, in the horse, complete fracture of the tibia is almost always incurable, because the patient is unable to continuously stand on the other leg until the fracture unites, and splints, $\& c .$, can only be applied to the lower portions, yet in oxen recovery has been repeatedly seen. Recovery has also been seen in young foals; in some cases a splint was applied, but in others no precaution was adopted. livolta describes a case in the ox in which a splint was applied by the local blacksmith. After six to seven weeks the animal walked sound. When the horse is valuable, and not of an excitable disposition, treatment may be attempted, especially if the seat of fracture be near the lower end of the tibia; in such cases recovery is possible. Foals and lightbred horses of quiet temperament are better able to endure the continuous lying than heavy animals, and therefore recover sooner. Montaya saw a transverse fracture of the upper third of the tibia in a mule unite, with the assistance of a splint. In France a number of cases are reported where complete fracture of the tibia with displacement in horses has been reduced and the bone has united. But these have been collected from the records of many years, and must be regarded as exceptional.

In sheep, goats, and carnivora, these fractures generally unite if not so high in the limb as to prevent a proper dressing being applied. In dogs, fractures of the tibia may completely unite in three to four weeks, even when complicated. 
In all animals, fissures and green-stick fractures unite in fourteen days to a month, provided the fracture remain incomplete. The animals require to be absolutely rested in slings for three or four weeks. 'The less the lameness, the better the chance of recovery. Fractures of the malleoli are unfavourable, especially in working-horses; and as lameness is generally severe, the continuous weight thrown on the other foot may lead to laminitis.

When instituting treatment, the above-mentioned possibility of fracture must always be kept in mind, and every injury of the inner surface of the tibia, whether extending to the bone, or causing severe lameness, should be followed by three to four weeks' complete rest, the animal being placed in slings, or tied up short. Severe lameness consequent on periostitis is best treated with cold applications, followed by blisters. Further treatment must be expectant.

Cases of complete tracture should be placed as soon as possible in slings, reposition attempted, and a plaster-of-Paris, tripolith, or adhesive plaster bandage applied. Adhesive plaster has the advantage of not slipping down so easily. Stolz recommends a mixture of 2 parts resin with 1 part wax; Hrickers, 5 parts gutta percha, 2 parts lard, and $1 \frac{1}{4}$ part wax. 'These mixtures are smeared on strong linen or leather, which is applied after the skin has been cleansed and dried. Leblanc, who successtully treated several cases, placed the animal in slings, dug a shallow pit below the affected limb, to which he attached a heavy weight. (After a time the muscles become fatigued and reposition can be effected.) Leblanc, after setting the limb, applied masses of tow and two large splints, the outer extending from the hoof to the stifle, the inner from the hoof to the middle of the thigh. The whole limb was then enveloped in bandages plastered with pitch. In oxen, with fracture of the lower part of the tibia, a plaster bandage may be used. These animals sometimes recover without treatment of any kind.

The application of plaster or tripolith bandages is easier in small ruminants and carnivora, but to be efficacious the dressing should be carried below the hock, and as far upwards as possible; by including the stifle-joint the security of the dressing is greatly increased, and the use of the "saddle" rendered unnecessary, otherwise it can scarcely be dispensed with. It is formed by passing the bandage over the back, and around the opposite limb. (See treatment of fractured humerus.)

When applying the bandage particular attention should be given to the position of the limb, to prevent the fragments uniting in faulty relation to one another. If union occurs with the parts rotated, movement is greatly interfered with. Before applying dressings to a complicated fracture, the wound should be carefully disinfected and covered with a layer of sterilised wadding. In case the parts cannot be rendered 
aseptic, a window may be left in the plaster bandage, to allow the wound to be inspected and kept relatively aseptic.

\section{III.-RUPTURE OF THE TIBIO-FIBULAR INTER- OSSEOUS LIGAMENT.}

Fractures of the fibula have rarely been recorded in animals; they are certainly very difficult to detect during life. In dogs, however, the fibula is usually involved in cases of fracture of the tibia. Behnlie, in a mare, saw rupture of the interosseous ligament, with separation of the fibula from the tibia, from the animal shying whilst being ridden. Severe lameness at once followed, and though it gradually diminished, it recurred after lying down and rising. The limb was carried stiffly, and no weight was placed on it. Post-mortem examination showed the fibula to have been torn away from the tibia; it lay amongst the muscles of the thigh, which it had severely injured.

\section{IV.-RUPTURE OF THE FLEXOR IETATARSI MUSCLE.}

In the horse the tendinous portion of the flexor metatarsi muscle forms an inextensible cord, which unites the stitle and hock joints in such a way that movement of one joint produces movement of the other, and permits the muscles of the quarter and thigh to assist in extension of the hock-joint. The tendon arises from the pit between the trochlea and the external condyle of the femur, in common with the extensor pedis, passes over the stifle-joint, lying in front of the tibia, at the upper end of which it is surrounded by a mucous bursa, and is inserted by three slips into the hock and metatarsus. In ruminants the muscle is also provided with much tendinous tissue, but arises from the tibia. In carnivora it is replaced by a tendon which starts from the internal surface of the tibia and is inserted into the bones of the tarsus, serving to prevent excessive extension of the hockjoint.

In the horse, rupture of this muscle is attended with such narked symptoms that attention was early directed to it. Solleysel described the disease, though he regarded the tendon as a nerve. At the end of the eighteenth century the disease was described by Louchard as dislocation of the hock-joint. Bouley, in 1833, correctly explained the condition, and was followed by Kigot, who made the experiment of dividing the muscle. Hertwig first thorougnly described the affection, and gave a full account of its origin.

'The flexor metatarsi becomes ruptured either in its course along the front of the tibia or at its origin from the pit between the trochlea and external condyle of the femur. In the former case the rupture is usually incomplete, the tibres appearing as though frayed but not completely torn across.

Causes. Rupture of the flexor metatarsi follows excessive extension of the hock-joint, produced either by the animal licking violently or struggling in hobbles, or from the limb being violently drawn backwards, as in shoeing in the travis, or drawn upwards by means of a cord passed 
through a ring. If, while the foot is thus fixed, the animal fall, rupture is very apt to occur. It may also be caused by efforts to withdraw the foot fixed in the travis, or caught in railway points, or even in deep, soft ground, and sometimes forms a sequel to collisions, slips, or getting over bales. It seldom follows external violence, though a case is recorded where the muscle was divided by a sharp stone. Klemm recommended section of this tendon in the treatment of spavin. Rupture seldom

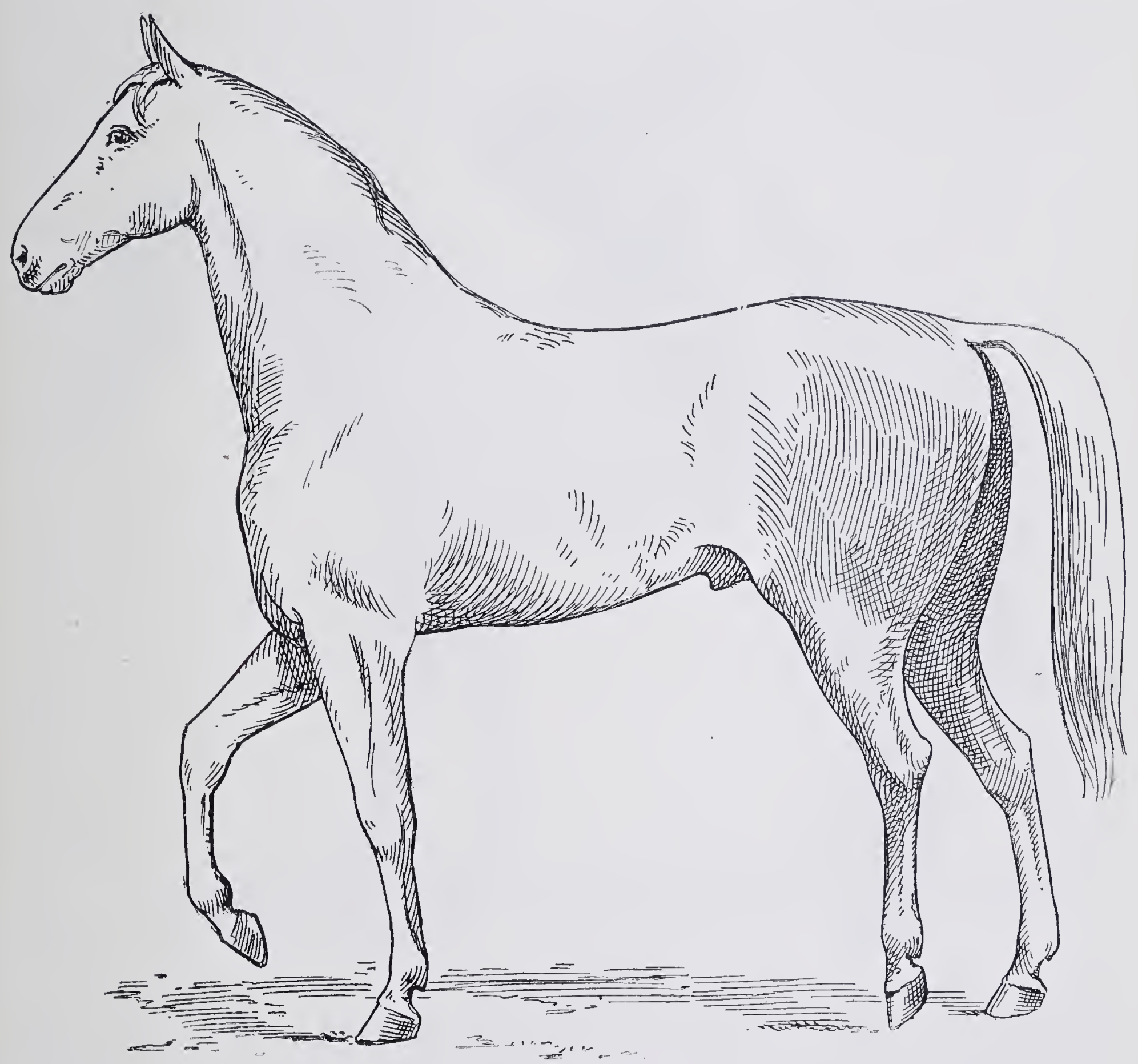

Fic. 259.-Rupture of the flexor metatarsi.

affects both limbs, and is rare in animals other than the horse, though several cases are reported in cows. In dogs, the tendon which corresponds to this muscle may be divided as a result of external injuries.

Symptoms. This rupture is distinguished by lameness when the limb is carried, marked flexion of the stifle-joint, and excessive extension of the hock. The symptoms are so marked that the condition can be diagnosed with absolute certainty, even from a distance. As the fibrous band stretching between the external condyle of the femur and the metatarsus is no longer able to transmit the movements of the femur 
to the metatarsus, and as the flexor metatarsi muscle itself is powerless to make up for this deficiency, the cannon bone is no longer flexed on the limb, but hangs inertly, and all the lower joints of the limb follow suit or are slightly flexed. That portion of the limb below the hock is not properly advanced, whilst the relaxation of the tendon favours excessive flexion of the stifle-joint. This want of harmony in the function of both

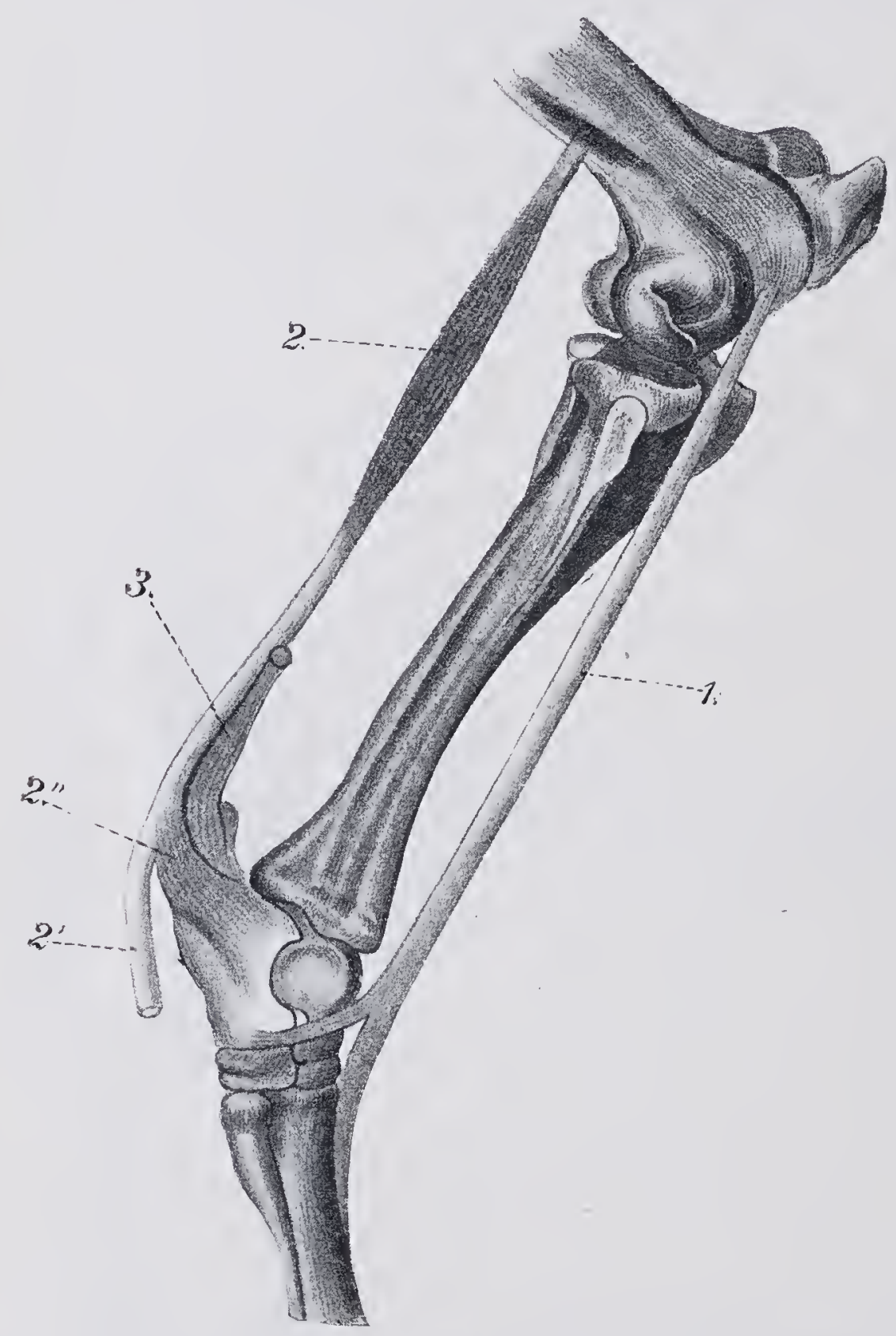

Fig. 260.-Showing the mechanism of the hock and stifle joints. The tendons of the flexor metatarsi and gastrocnemius museles unite the bones forming the joints in such a way that they are muable to move independently. 1, Superficial division of the flexor metatarsi muscle; $2,2^{\prime}$, and $2^{\prime \prime}$, flexor perforatus; 3 , gastrocnemius tendon.

joints produces an uncertain movement of the limb, which may give the impression of a broken bone, and has been so interpreted by some. The absence of fracture, however, is at once shown by the fact that the limb can still support weight. The serious symp. toms apparent during move. ment disappear when the animal is at rest. In rupture of the flexor metatarsi; the function of its tendon is in abeyance; while its antagonist, i.e., the tendo Achillis, is unopposed, appear's relaxed, and when the limb is relieved of weight and extended lies flaccid. This appearance is very charac. teristic, and only occurs in two other conditions, viz., rupture of the tendo Achillis and fracture of the tibia. It is most marked when the hock is excessively extended, as, for instance, by the farrier lifting the limb to remove the shoe. In recent cases the anterior surface of the tibia is sometimes slightly cedematous, in others absolutely nothing abnormal can be seen. Severe swelling with pain on pressure over the course of the flexor metatarsi is quite exceptional, the muscles being clothed in a strong fascia.

Course. The affection is naturally of sudden onset, and at once produces a peculiar lameness. As weight can still be placed on the leg, and the ends of the tendon retract comparatively little, union is seldom 
difficult. Judging by the many reported cases, union is almost always complete in four to eight weeks, even when both legs are affected, though one case in a riding-horse lasted for three months. In rare instances - recovery does not occur, or is so incomplete as to impair the movement of the hock. These depend on the position of the rupture and on the animal not being rested sufficiently long. Where the tendon is divided in the mucous bursa, or torn away from its point of origin on the femur (a rare occurrence), recovery is less assured, because formation of new tissue remains incomplete, the paratendineum essential to union being absent from the tendon sheath and mucous bursa. On account of the

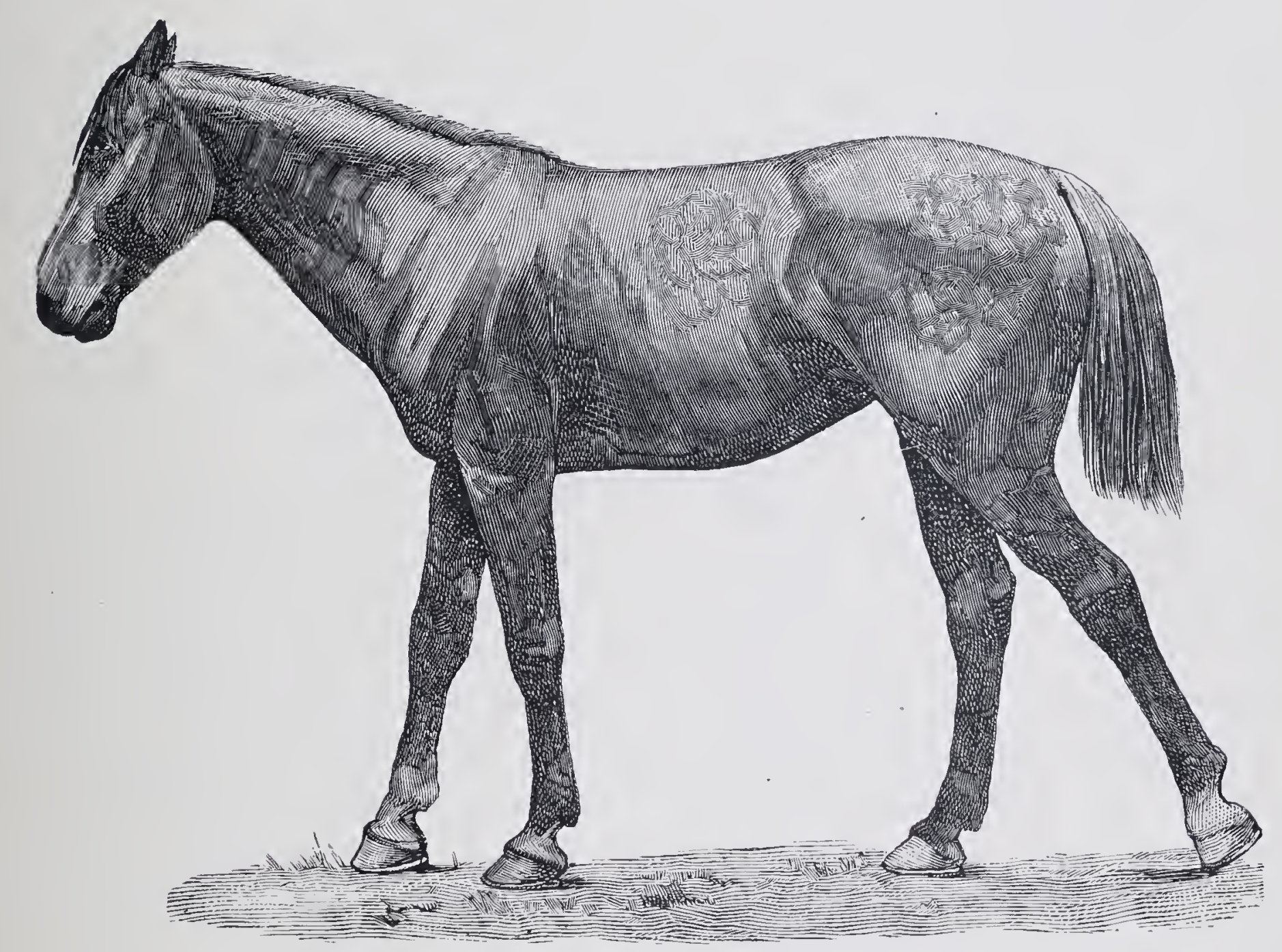

Fig. 261.-Rupture of flexor metatarsi muscle (from a photograph).

peculiar nature of its insertion into the femur, the tendon rarely tear's away, but when this occurs a fresh difficulty arises, because the new tissue consists of callus, which must grow both from the torn end of the tendon and from the femur. It is somewhat doubtful whether in Goubaux's case the tendinous or the muscular portion of the flexor metatarsi was torn away; the point is of little importance. Probably it was the tendinous portion, because muscle is very rarely torn away from its insertion into bone. H. Bouley reports similar cases. As a rule even when the tendon is torn away from its insertion the case finally recovers, though it lasts as many months as the other weeks, and slaughter is often preferable to treatment.

Partial recovery is one of the results of putting the horse to work 
before union is complete; the newly-formed cicatricial tissue gradually becomes strained and the tendon lengthened, necessarily causing permanent lameness. Excessive extension of the hock, which sometimes occurs during the early stages of union, disappear's completely. with time. A few cases have completely recovered in twenty-five days; probably the tendon was only partially ruptured.

Treatment is confined to resting the limb. The ends of the fibrous cord are rarely far displaced, and reunion is not difficult. The animal should be kept perfectly quiet for four to six weeks, should be placed on a level surface, and not receive too much bedding. Work protracts recovery, though in isolated cases union occurs despite it; it always takes a long time, however, and may remain incomplete, the ligament becoming elongated and the action permanently impaired. In horses of any value rest is indispensable. Blistering over the course of the affected muscle is unnecessary, and only serves to secure rest. Work can be resumed as soon as lameness disappears.

\section{Y.-RUPTURE OF THE TENDO ACHILLIS AND GASTROCNEMII IIUSCLES.}

Ruptures of the gastrocnemii muscles and of the Achilles tendon are much rarer in animals than rupture of the flexor metatarsi. They are oftenest seen in cows, sometimes in both limbs at once, though in horses both rupture and wounding of the tendon and muscles have been recorded. Rupture generally occurs at the lower point of insertion of the Achilles tendon, a portion of the os calcis being at the same time torn away. In cows it has repeatedly been seen at the point where the muscle becomes continuous with the tendon; but rupture of the muscle itself is known to occur both in horses and oxen. Bayer saw rupture of the tendon in dogs after bruising.

Einwächter, in a horse, found not only the gastrocnemius but also the flexor pedis perforatus torn away from the femur. Pirl saw a cow with rupture of the Achilles tendon in both legs. Swelling resulted in the neighbourhood of the popliteal space, and gradually became larger and fluctuating. The cow could only shuffle about on the ground on her hocks.

Causes. Rupture follows violent contraction of the muscle, produced in cattle by leaping one another, in riding-horses by jumping, and in draught-horses by efforts to avoid slipping. The tendon may also be strained by falling from a height, or by the animal slipping with the legs extended under it. In cows rupture sometimes follows long periods of lying with the hind limbs flexed and violent efforts to rise, as in post-partum paralysis. The injury may involve both muscles or 
the entire tendon, or may remain confined to certain portions; the latter is, however, the exception, complete rupture the rule. In small animals, like dogs, rupture may be produced by the animal being caught in a door. In the horse the Achilles tendon is sometimes cut through. Traumatic inflammation of the os calcis or tendo Achillis, or suppuration in the tendon sheath, may also lead to rupture of the tendon. St. Cyr there-

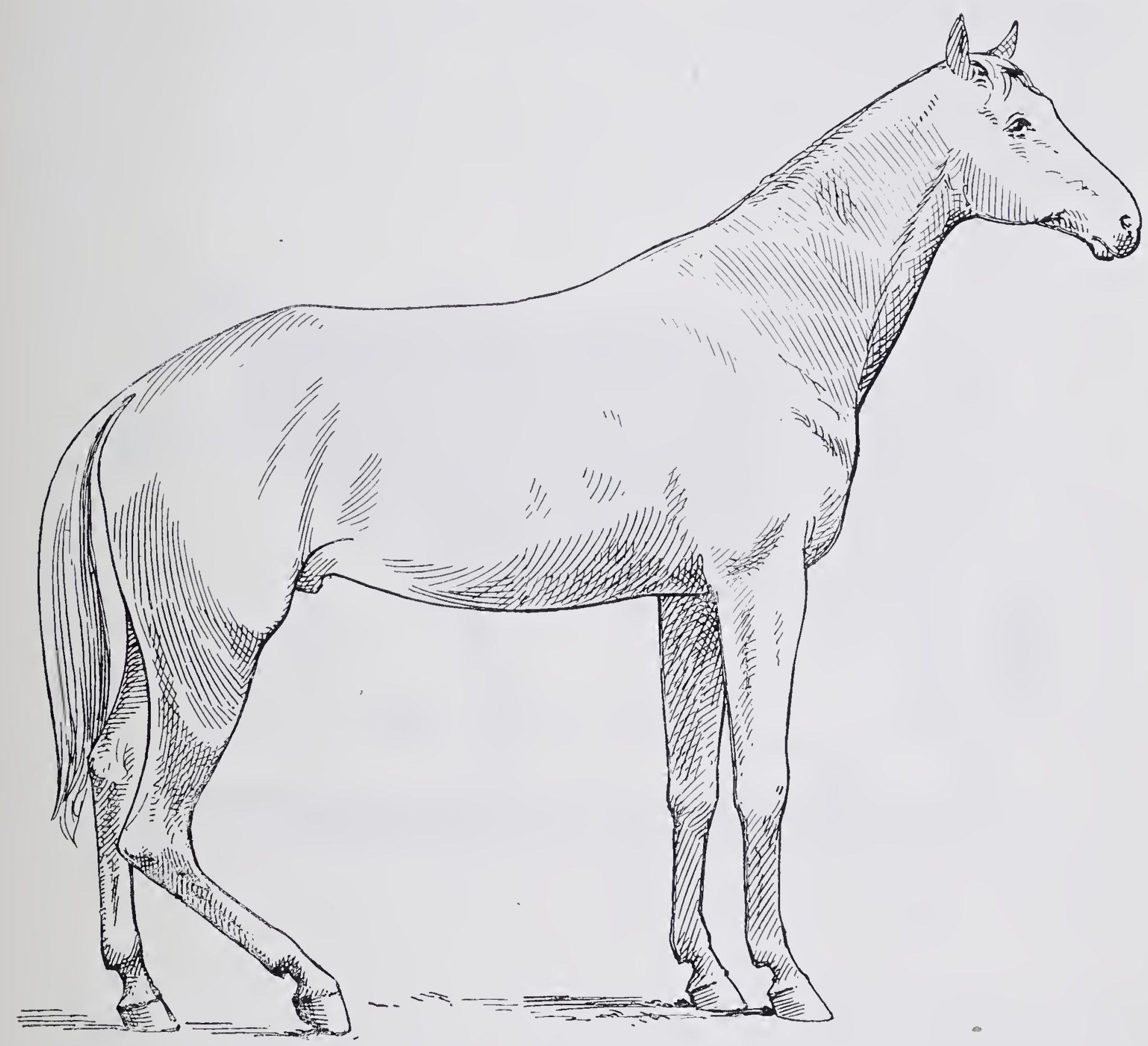

FIG. 262.-Rupture of the tendo Achillis.

fore distinguished primary and secondary rupture. Uhlich saw rupture soon after recovery from influenza.

Symptoms. When the muscles named or the Achilles tendon are completely torn away from their insertion, there is severe supportingleg lameness, inability to bear weight on the affected limb, and flexion of all joints.

The Achilles tendon extends to the point of origin of the gastrocnemii muscles, and when weight is placed on the limb serves the purpose of fixing the joints, especially the hock and stifle joints. It therefore fulfils an important part in supporting the body, and its rupture is followed by collapse of the limb-the hock sometimes descending 
sufficiently far to touch the ground (fig. 263). The excessive flexion of the hock increases the distance between the points of origin and insertion of the flexor pedis, and the phalanges take up a position of excessive plantar flexion, i.e., become "shot over." The hock can also be flexed to an unusual degree by the exertion of a little force. As a rule, a depression may be noted in the course of the tendon or swelling in the gastrocnemii muscles; the tendon appears relaxed, even when the limb touches the ground. In incomplete rupture lameness is shown when weight is thrown on the limb, and there is more or less pronounced flexion of the joints, particularly of the hock-joint.

The prognosis depends almost entirely on whether the uninjured

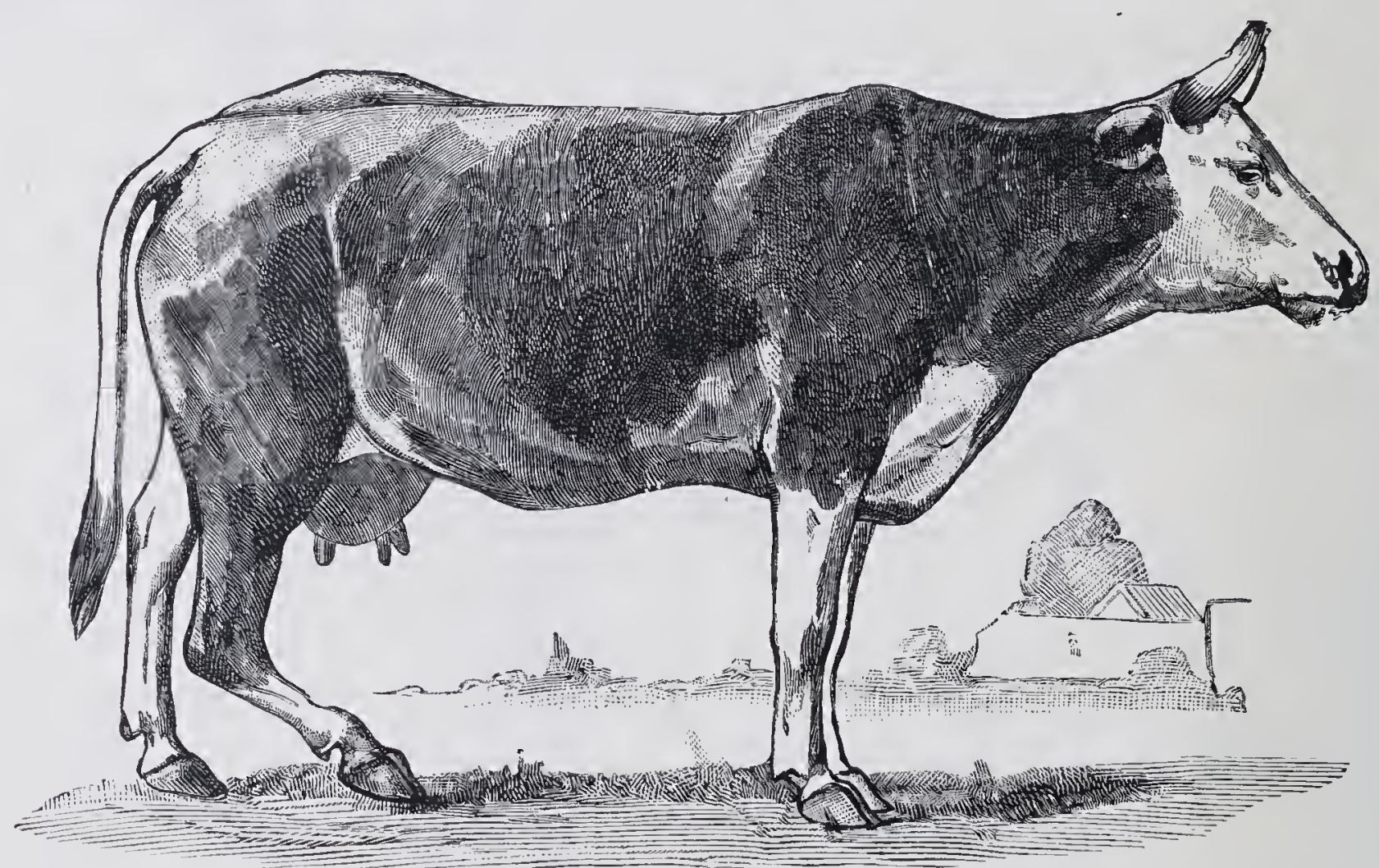

Fic. 263-Rupture of the tendo Achillis (after Stockfleth).

leg can sustain weight until union occurs. Small animals, like dogs and cats, almost always do well, but larger animals, as St. Cyr pointed out, are less favourably circumstanced. They sometimes succumb to the continued standing or lying, for union takes from four to six weeks, and under some circumstances may be protracted for several months. Oxen do best lying, but as horses are obliged to stand, it becomes a question whether laminitis may not supervene in the other foot. Nevertheless a considerable number of recoveries have been recorded in the horse. The grounds for forming a prognosis are so slight that it is generally more prudent to withhold it, and watch closely for unfavourable symptoms. As a rule, in the horse the hind feet are more liable to sontract laminitis than the front, although they bear less weight-a fact to be kept in mind when one limb is disabled. In small animals recovery is more probable, and if other means fail, an attempt may 
be made to suture the tendon. (See p. 143 of Dollar's "Qperative Technique.")

Union is more rapid when the tendon is ruptured than when it is torn away from the os calcis. Partial ruptures, in which some weight, however slight, can still be placed on the limb, are more hopeful. As a rule, the more marked the degree of flexion, the slighter the chance of recovery. Recovery is often protracted by elongation of the tendon and consequent lameness.

Treatment. In small animals the hock-joint should be as much as possible extended and a plaster bandage applied. Larger animals, like horses, are slung, so as to keep the hock continuously extended, and the ends of the divided tendon or muscle as close as possible together. In quiet horses this may be assisted by bandaging and applying adhesive plaster. Such a dressing certainly allows of some movement, but for this very reason is better borne than the rigid plaster bandage or than splints. St. Cyr stated that the latter are apt to produce excoriation, and that it is often better not to check movement. Martens saw the divided Achilles tendon unite in two months without the horse being slung, and Grüner in two and a half months. Light horses, when rising or lying down, are better able to protect the injured limb, and may be left free in a box, though even then a dressing is useful, if the animal does not attempt to displace it by violently flexing the limb. In cows, Detroye applies a gutter-shaped splint to the back of the hock-joint, and fastens it by straps to the tibia and metatarsus.

Wounds of the flexor tendons heal with still greater difficulty unless rendered aseptic, but that asepsis is quite possible is shown by Martens' and Grüner's cases. Particular precautions are necessary in injuries about the tuberosity of the os calcis.

\section{DISEASES OF THE HOCK.}

\section{I.-FRACTURE OF THE BONES OF THE HOCK.}

(a) Fracture of the os calcis. Fractures of the bones of the hock are, on the whole, rare, though cases have been seen, especially of the calcis and astragalus bones. Apart from those described above in which the Achilles tendon is torn away from the tuberosity of the calcis, fractures of this bone are produced by external violence, by kickis, or sometimes by blows with the farrier's liammer. Detroye saw three cases-one in a horse, one in a steer, and one in a calf. The horse had produced the fracture by slipping when rising in the stable. The bone was broken just above its articulation with the astragalus. In the steer 
fracture was caused by mounting a cow, and in the calf, by falling into a ditch. The same causes which produced rupture of the Achilles tendon may of course lead to fracture of the os calcis.

The symptoms and course closely resemble those of rupture of the Achilles tendon, which in this fracture also loses its lower point of insertion, though fracture of the calcis is generally more painful than rupture of the tendon. The broken fragment is drawn upwards by the tendon, and the animal is totally unable to stand on the limb, which collapses. All the joints are excessively flexed, particularly the tarsus. In moving, the limb is lifted and the metatarsus and phalanges dragged, the tendo Achillis appearing relaxed. Crepitation is sometimes heard, whilst in compound fractures splinters of bone may possibly be detected in the wound.

In horses and oxen treatment is rarely successful, but in small animals, which offer some chance of recovery, it may be attempted. Union is rendered difficult by the displacement of the fractured parts consequent on the pull of the Achilles tendon, by their defective nutrition, and by the inability of large animals to stand continuously on the other hind leg, though Bräuer says he completely cured oxen in four weeks by applying a plaster bandage. Detroye reports a similar case in a steer : the lameness completely disappeared after a time. Haase likewise reports union in the horse, though the case lasted from twelve to fourteen weeks. It would be interesting to attempt the reunion of fractured os calcis in the dog or other small animal by screwing the fragments together with silvered wood screws, or by pegging with ivory pegs. This particular operation does not seem to have been attempted hitherto. It would demand perfect technique, but appears, à priori, within the range of possibility.

The prognosis, therefore, depends, firstly, on the degree to which the fragments are displaced (sometimes dislocation is minimised by the insertion of the flexor pedis perforatus); and, secondly, on the length of time during which the animal can stand on the other foot without laminitis setting in. Compound fractures may generally be looked on as incurable.

Treatment is in general similar to that in rupture of the Achilles tendon. In these cases also it is important to fix the hock-joint in as complete extension as possible. Plaster bandages are useful for small animals.

(b) Fracture of the astragalus is also rare; it follows forced rotary movement of the limb whilst the animal's weight is upon it, or whilst it is fixed to the ground. A heavy cart-horse was seen to produce this fracture by suddenly turning round. A case of Furlanetto's in a cow was caused by falling into a ditch. 
The symptoms are: sudden and severe lameness, shortly followed by fluctuating painful swelling of the astragalo-tibial joint, similar to that in inflammation of the hock-joint. Crepitation can sometimes be detected by placing both hands around the joint and moving the lower part of the limb; but in other cases it is absent, and the condition may be mistaken for violent strain of the joint, which, however, is rare, or for severe subfascial cellulitis; but as the latter is accompanied by swelling of the local lymph vessels and by fever, it is sufficiently distinct from fracture of the astragalus.

Prognosis is absolutely hopeless, because the fracture always extends into the joint, recovery is extremely protracted, and no weight can be placed on the limb. It is therefore better to slaughter large animals without delay. An attempt at treatment may be made in cats and dogs by applying a plaster bandage, which should be put on whilst the leg is moderately extended, so as to limit as far as possible the lameness which will result from obliteration of the joint. The cases described by Furnaletto certainly recovered, but proved permanently lame.

(c) Fractures of the other bones of the hock are less frequent than those above mentioned, and are either due to kicks, to the animal being run over, or to similar violent mechanical causes, or they appear in connection with sprains and luxations. Thus Rey found dislocation of the hock-joint between the scaphoid and cuneiform magnum in a horse which had caught its hoof between railway-metals and had fallen. The external small metatarsal was torn away from the large metatarsal and the cuboid bone crushed.

Diagnosis is seldom difficult, though the seat of fracture can only be determined by post-mortem examination. There is abnormal mobility of the hock-joint, especially for movements of ad-and ab-duction ; crepitation is often audible.

The prognosis is generally bad, but depends on the degree of lameness, and is, of course, influenced by the presence or absence of other injuries and the nature of the animal's work.

\section{II.-LUXATION AND SPRAIN OF THE HOCK-JOINT.}

The strong ligamentous apparatus and the interlocking of the grooves and depressions on the astragalus and tibia respectively form so perfect a means of union that luxations of this joint must necessarily be of the rarest occurrence. In horses, it is doubtful if any have been reported, for even Louchard's case appear's only to have been a severe strain, probably accompanied by fracture of the malleoli of the tibia or of the astragalus bone. Stockfleth describes having discovered by post-mortem examination inward luxation of the astragalus in a sheep; the capsule of the joint was ruptured. He also saw displacement between the astragalus and 
upper row of small hock bones in a cat which had fallen, hind legis first, froma height; the metatarsus was thrust outwards. The displacement was immediately reduced, a rubber bandage applied, and the animal recovered. In a rabbit, which had also fallen from a height, Stockfleth found displace. ment of the astragalus. In a horse, which had passed its left hind foot through a hole in a wooden bridge, Haubner discovered rupture of the ligaments connecting the metatarsus and lower row of hock bones. The limb was excessively movable, and showed, at the seat of curb, a depression. Frick saw incomplete outward displacement of the astragalus in a dog; the lower, inwardly-projecting end of the tibia was supported by a new osseous growth covering the inner surface of the astragalus.

Schrader says that one of Havemann's pupils cured a cow suffering from luxation of the metatarsus. Townshand reports a similar case: the metatarsus was displaced in a forward direction. The animal recovered, but always went lame. In Rey's case, above cited, the union between the hock bones was divided, but there was no displacement. Stockfleth speaks of a similar case in which the astragalus and calcis were torn away from the cuboid and scaphoid bones; the horse was killed. Rüffert also saw a case in which the internal lateral ligament and portions of the interosseous ligaments were ruptured. Union appear's to be strongest between the metatarsus and lower row of hock bones; Stockfleth only gives one case of dislocation at this point. It occurred in a dog; all the ligaments and flexor tendons were ruptured, and the lower part of the limb hung by the extensor tendons. Needless to say, in all these cases recovery is usually extremely protracted and difficult, for which reason it is usually best to advise slaughter. The strong ligamentous apparatus, together with the prominences on the articular surfaces of the tibia and of the astragalus, produce so firm and secure a union of the two bones that luxation of the astragalo-tibial joint is exceedingly rare.

\section{III.-WOUNDS AND ACUTE INFLAMMATION OF THE HOCK-JOINT.}

Wounds are by far the most frequent cause of acute inflammation of the hock-joint. They may be caused by thrusts with forks, lances, or sabres, by kicks, by the animal being trodden on, and in dogs by bites, or they may be due to operation for spavin or for enlarged bursæ. Point firing.sometimes causes suppuration in the hock-joint. Inflammation of the tendon sheath of the flexor pedis at the inner side of the hock or septic or purulent cellulitis near the joint may sometimes extend to it; or, again, the capsule of the joint may be opened by gangrene consequent on lying long in one position. Acute inflammation of the tibio-tarsal joint also results from metastasis, and in horses is often seen after 
strangles and pyæmia. Inflammation of the hock-joint, probably of metastatic origin, has been observed in cows soon after calving, sometimes in both limbs at once. The cases usually followed retention of the after-birth, post-partum paralysis, agalactia, mastitis, and septic metritis. Calves and lambs often show inflammation of the hock-joint after omphalo-phlebitis ("navel-ill"). Here it is probably due to metastasis. Morot and others have seen tuberculosis of the hock-joint in cattle. Dieckerhoff describes several instances of spontaneous inflammation of the hock-joint, which proved on post-mortem examination to be of a purulent character. Whether the cases of rheumatic inflammation so often described are caused by catching cold is uncertain.

(For injuries to the point of the os calcis, compare with "Capped Hock.")

Symptoms. The local injury generally dispels any doubt as to the character of the disease, especially if synovia is being discharged. There is always more or less swelling, which extends round the joint, and both upwards and downwards; though soft at first, it later becomes hard and resistant. The distension of the tibio - tarsal capsule is often masked by the periarticular.swelling. From the first lameness is severe, and is exhibited both when the limb is being advanced and when weight is placed on it (mixed lameness). Fever is present in

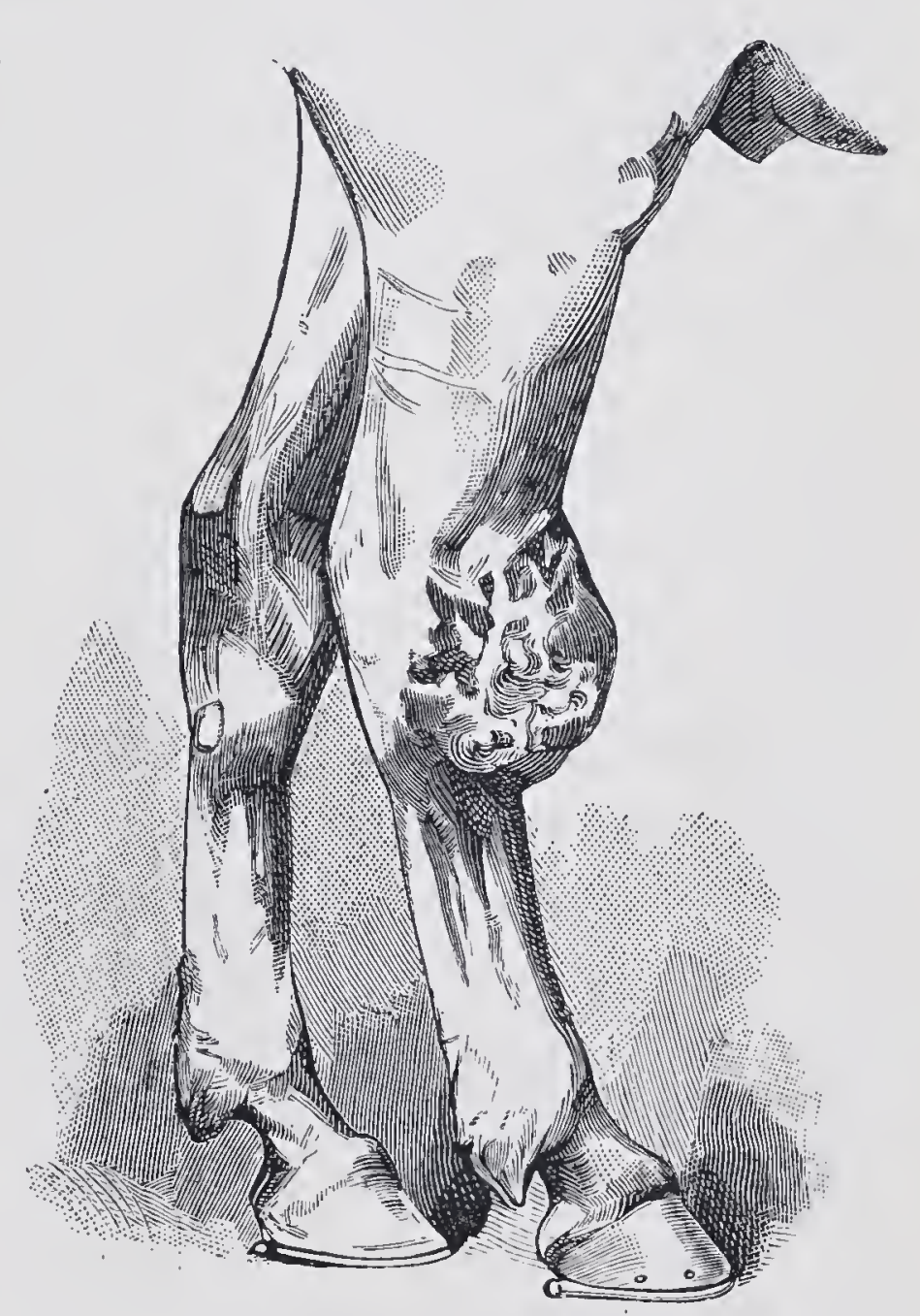

FIt*. 264.-Exuberant granulation in front of the hock.

the purulent or septic variety of disease, and periarticular abscesses always form.

The disease can only be mistaken for severe subfascial cellulitis (see "Wounds and Injuries of the Lower Thigh and Leg"), but as this exhibits all the above-described symptoms, some time may elapse before the diagnosis becomes assured. If the condition improves after the abscesses break, and especially if lameness decreases, the joint may still prove intact, but improvement is sometimes only apparent, and due to laminitis in the other foot, from the animal continually standing. Increased pulsation in the metatarsal arteries points to the occurrence of laminitis and displacement of the os pedis. 
Fractures of the malleoli of the tibia and of the astragalus cause similar symptoms, and, unless accompanied by crepitation, are very difficult to diagnose.

Injuries to the front of the hock are also difficult to treat, especially when large flaps of skin are destroyed. The unavoidable movement of this joint in horses irritates the wound, causing active granulation, which is sometimes impossible to repress. Fig. 264 shows such a condition, photographed from nature.

Course and prognosis. The course depends principally on whether the parts remain aseptic. If they do not, recovery is highly improbable, and oxen should be slaughtered before they have lost much condition. The prognosis is therefore doubtful in all cases of inflammation caused by external injury or by metastasis, though inflammation is less dangerous in the lower, less mobile portions of the hock than in the tibio-tarsal joint, in which the destruction of the articular cartilage is always followed by severe and permanent lameness, causing the patients to lie continuously, and generally proving fatal to large animals. In the smaller joints, inflammation may lead to obliteration of the joint and restoration of the animal's usefulness. Provided the wound remain aseptic, there is less danger. Such cases are more particularly seen in cows after parturition. Slight cases, i.e., those where inflammation is not marked, heal in fourteen days, but the more severe take four to six weeks.

Treatment follows general principles. If the joint be injured, efforts must be made to preserve it. Small, and especially perforating, wounds must first be carefully cleansed and disinfected, and a blister of sublimate, with lard or paraffin $(1-10)$, applied, or the firing-iron may be used. The swelling thus produced leads to rapid closure of the wound, and prevents infection of the joint. Large wounds, particularly those of an incised character, should be thoroughly disinfected and sutured. Quiet horses may be slung, and a surgical dressing applied; but when this cannot be used, and it is impossible to suture the wound on account of extensive bruising, permanent irrigation offers the best means of preventing infection of the joint. Lorenz cured a wound in a horse's hock by irrigating with 1 in 5,000 sublimate solution, and afterwards blistering.

If purulent or septic inflammation has already attacked the tibio-tarsal joint, little can be done, though irrigation with disinfectants might be tried. Washing out the joint with dilute disinfectants like 1 in 1,000 to 1 in 2,000 sublimate solution has not hitherto proved very successful, but it might be tried when the joint is opened. Syringing with disinfectants is useful in wounds in the lower portions of the joint.

Hydroxyl solution has been recommended, and success has once or twice followed its use in open hock-joint: Hohmann opened the hock- 
joint of an ox and evacuated some fibrino-purulent fluid. Healing occurred under permanent irrigation.

To prevent excessive granulation in the bend of the hock, the animal should be tied up short, and every precaution taken to limit movement of the hock-joint: the granulations themselves are treated on general principles.

\section{IV.-BOG-SPAYIN.}

Bog-spavin consists in excessive distension of the capsule of the true hock-joint. The capsular ligament and synovial membrane are attached above close to the articular surface of the tibia, below to that of the astragalus. Anteriorly and inwardly the sac presents a small dilatation, posteriorly and externally a larger one, which Franck says often communicates with the sheath of the flexor pedis perforans tendon at the inner side of the joint. Disease of this tendon sheath, however, seldom extends to the hock-joint, and vice versâ.

Distension of the capsule of the true hock-joint is commonest in young horses with upright hocks, but is also frequent in old stallions and pregnant animals. It is generally due to chronic synovitis, brought on by severe exertion; occasionally it follows acute synovitis. Chronic suppuration in this joint sometimes accompanies pyæmic inflammation of the urachus in foals, and is almost always fatal.

The most noticeable symptom is the presence of a fluctuating swelling, which may be thrust backwards and forwards.

There is seldom acute inflammation, and therefore lameness is rare. Distension of the capsule is only dangerous to life when due to acute synovitis or umbilical phlebitis. In well-developed cases the animal may show lameness after severe exertion.

Treatment must follow general principles. Recent painful and inflamed swellings are treated by cold applications, and later by blistering. As firing only diminishes the swelling, and always leaves visible scars, it merely substitutes one blemish for another; nevertheless, Stocliffeth recommends it. If resorted to, needle firing is the preferable method. In young horses, with moderately developed bog-spavin, repeated painting with tincture of iodine or massage may diminish swelling, but seldom removes it completely. In foals treatment is rarely of permanent value, and relapses often occur.

Caution is required in operating surgically. Removal of contents by Pravaz's syringe or a slender trochar is occasionally followed by benefit, and injection of iodine may be tried. In most cases the operation must be repeated several times, at intervals of four to six weeks, to be of service. Evacuation by Pravaz's syringe, washing out with 1 in 1,000 
sublimate solution, and the application of a blister, was recommended by Zimmer in 1890. Deans has since practised the above operation (evacuation and injection) in Scotland, and claims to have had considerable success. It probably constitutes the most hopeful method of dealing with this condition, but demands careful antiseptic precautions.

Horn draws off the contents with a trochar, and applies, in place of a pressure bandage, a thick layer of glue, in which finely chopped tow is mixed to increase its strength. Incising the swelling is not unattended by danger, even where full antiseptic precautions are taken, because the animal sometimes rubs off the dressing. Proceeding with full antiseptic

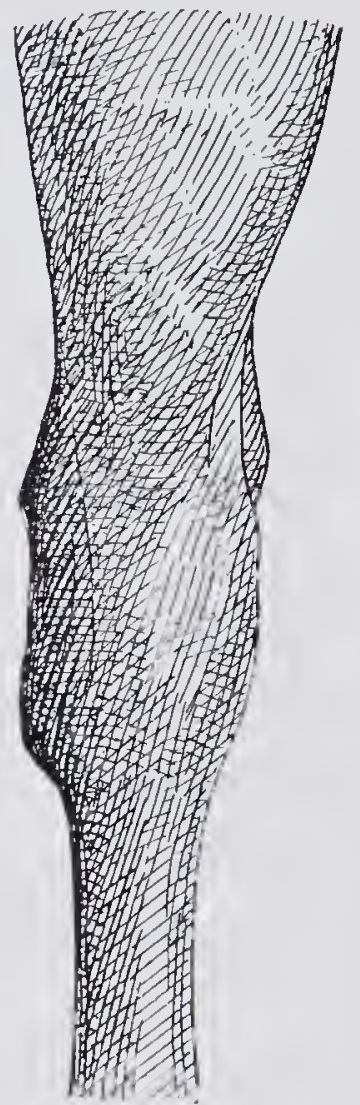

Fig. 265.--Spavin formation on left hock (seen from in front).

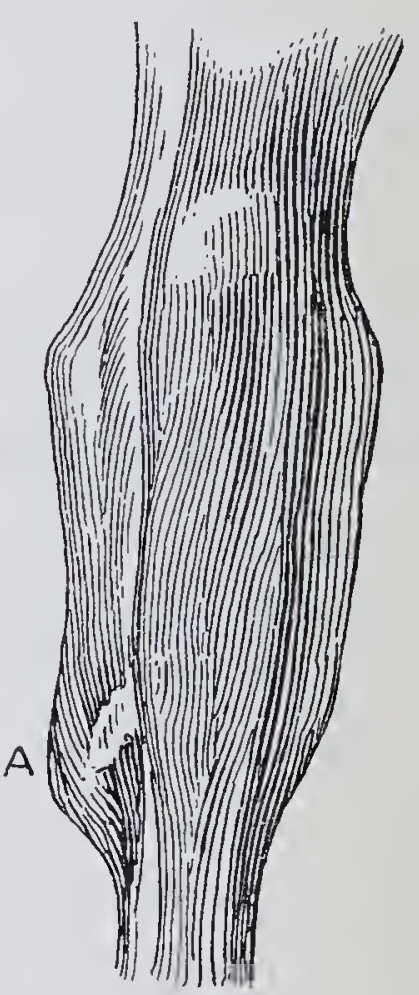

Fig. 266.-Spavin formation on right hock (seen from behind). A, Spavin.

precautions, Qualitz, in one case, removed a lozenge-shaped fragment of skin and synovial membrane about $1 \frac{1}{8}$ inch wide from the outer surface of the hock, and by suturing the parts, irrigating with an antiseptic solution, and covering the wound with a surgical dressing, was able to secure healing in a fortnight. This success, however, must be regarded as exceptional, and should not encourage one too hastily to attempt its repetition. Acupuncture has been recommended by Gloag and others. The swelling is perforated on either side to a depth of $\frac{5}{8}$ th of an inch by an apparatus composed of four needles thrust into a cork, when the fluidcontents escape. In five days the operation is repeated, iodine ointment rubbed in, an elastic bandage applied over all, and left in position for twenty-four hours. 


\section{V.-CHRONIC INFLAMIMATION OF THE HOCK. SPAVIN. ARTHRITIS CHRONICA DEFORMANS TARSI.}

It is not surprising that so common a disease as spavin should early have attracted attention. We find the disease described by Jordanus Ruffus and Mar'x Fugger, and treated of by almost every veterinary author. Owing to ignorance of pathological processes early observer's attached undue importance to outward appearances, and distinguished many linds of spavin on the ground of their physical differences. This was the basis of the terms "éparvin calleux" and "éparvin sec," invented by Solleysel, and still used in France to the present day, and of the English "bog" and "bone spavin." In Germany Kersting: distinguished five kinds of spavin. To the same cause was due the jdentification of spavin with stringhalt.

For a long time the nature of the disease remained obscure, and it was sometimes thought to be a bone affection, sometimes an affection of the ligaments. Havemann first directed attention to the disease of the articular surfaces, and Schrader and Schütz afterwards described the condition thoroughly. At the present day no doubt exists that spavin is due to chronic inflammation of the joint, and the only question is, in what structure does the disease originate?

The view that the bone tissue is the primary seat of disease is old, but has again, quite recently, been advanced by Eberlein. Eberlein's views are supported by the experiments of Gotti, and consist in regarding the bone substance as being primarily attacked, after which the cartilage of the joint, the periosteum, and the ligamentous apparatus are successively invaded. He consider's spavin to consist in an ostitis rarefaciens et condensans. Fröhner, Bayer, Hertwig, and the Günthers are among the supporters of this theory.

Dieckerhoff, Lafosse, and Hoffmann believe the disease originates in the bursa under the middle branch of the flexor metatarsi.

Anacker, Joly, Möller, Schütz, and Smith believe spavin to be an arthritis chronica deformans. The view that the articular surfaces are first invaded is held by Bouley, Dieterichs, Gurlt, Havemann, Hering, Schrader, Stockfleth, and Träger. The ligamentous apparatus is considered to be the primary seat of disease by Aronsohn, Bartels, Barrier, Hess, von Hochstetter, Roloff, and Pflug.

Höhne has advanced a theory which has hitherto won no adherents, viz., that in spavin the hock-joint is not alone invaded, but that the region of the inner tuberosity of the tibia is simultaneously affected. Höhne actually regards this affection of the tibia as more common than that of the hock, and thinks, therefore, that spavin originates, not in the hock, but in the stifle. 
An unprejudiced examination of the foregoing views can only lead us to the conclusion that to regard spavin as invariably originating in one of the regions named, i.e., to consider it as a fixed and invariable disease, is an untenable position. The variations in the anatomical changes discovered by different observers are due less to the stage at which the disease has arrived than to fundamental differences in its nature. Depending on whether the disease has arisen from injury to the bone or to the ligaments, the anatomical changes will vary. Only on this hypothesis can we explain why in one case a large exostosis is accompanied by spurious anchylosis, and in another a scarcely visible bony enlargement is found along with true anchylosis between the cuneiform bones or between the cuneiform and the metatarsus. For this reason, Frick, whilst keeping an open mind as to the essential nature of spavin, declares that the most varying forms of chronic disease of the inner surface of the hock may produce the elinical appearances which, in practice, we recognise as "spavin." Anatomically, spavin may be divided into various forms which, however, may all be regarded as having one point in common, viz., that they eventually lead to deformity of the affected joint, and may, therefore, rightly be designated as Arthritis chronica deformans. In a word, "spavin" is a collective clinical term covering a number of extremely diversified anatomical changes in the hock.

Pathological anatomy. Anatomical examination reveals:-

(1) Changes in the articular cartilage of the cuneiform, and of the upper part of the metatarsus, sometimes of the scaphoid, and other bones of the hock. Changes in the cartilage, abrasions, ulceration, and, at the borders of the articular surfaces, rugged, uneven swellings, produced by thickening of the cartilage, which later becomes ossified, can all be detected by the naked eye. Microscopically, the intercellular substance is seen to be fibrillated, and the cartilage cells to be undergoing multiplication, processes peculiar to arthritis chronica deformans.

(2) The affected bones show deposits termed osteophytes, which sometimes start from the ossified cartilaginous growth, sometimes result from inflammation attacking the periosteum of the bones named. Gotti and Eberlein state that the bone shows rarefying and condensing ostitis.

This local growth of bone is in fact the so-called spavin. It is usually only a secondary symptom due to inflammation extending from the articular surface to the periosteum.

(3) Changes in the ligaments and synovial membrane. Both the villi and borders of the synovial membrane seem thickened and vascular. Sometimes the secretion of synovia is excessive. The ligaments of the joint, and the connective tissue surrounding it, are also thickened.

(4) In advanced stages of the disease, the smaller joints may be obliterated, especially those between the scaphoid and cuneiform bones, 
and between the cuneiform and metatarsus. This anchylosis may either be true or false. The ligaments are then often ossified, and so surrounded by new growths of bone that they can scarcely be recognised. Sometimes the sheath of the flexor metatarsi muscle becomes diseased. Petit states never having found this part of the hock-joint diseased even when extensive exostoses existed.

In many cases pathological processes are confined to the lower and inner portions of the hock, though not infrequently they extend further, seize on the cuneiform bone and head of the inner small metatarsal, or even on the astragalus and external surface of the joint. The French, therefore, distinguish "éparvin tarso-métatarsien," as opposed to the disease of the cuneiform bones and metatarsus, which is termed "éparvin métatarsien."

The appearance of the above-described changes, either alone or in combination, is easily explicable on the theory that the point of origin of the disease varies. Menveux made a collection of hocks, showing widely varying anatomical changes, from horses, all of which had clinically been recognised as suffering from "spavin."

Causes. In no other disease is the division of causes into causa externa and causa interna so well warranted and so practical as in spavin. The causa interna is a predisposition partly dependent on the conformation of the hock, partly on that of other portions of the body. Whilst the complicated mechanism of the hock predisposes to disease, and especially to inflammation, this tendency is enormously increased by defective formation of the joint. It is scarcely needful to say that, under the powerful action of the muscles of the hind limb, small, cramped joints are more likely to suffer than those having well-developed, broad and ample articular surfaces. Defective development of the lower portions of the hock and of the upper end of the metatarsus, the condition described by horsemen as "tied-in hock," is particularly disastrous, while a distinct curvature (sickle-shaped hock) is almost as bad. In this formation the action of the extensor muscles of the hind limb produces excessive movement of the hock-joint and great pressure on the articular surfaces, with danger of injury to the ligaments of the joint.

But these visible peculiarities of formation are not the sole causes of spavin: the intimate structure of the bones and ligaments may predispose to disease, as shown by the inheritance of spavin, and its occurrence in entire strains, whose hock-joints appear perfectly formed. This view is further supported by the rare occurrence of disease in some races, like the Danes and Holsteiners, whose hocks are by no means irreproachable. In breeding, therefore, special attention should be paid to this disease. The exact nature of the predisposition at present eludes us, though by many it is considered to result from incomplete V.S. 
development of the elements of the joints, especially of its bones and ligaments. Working immature animals is also a potent cause. Horses which might work quite satisfactorily at five or six years old, not unnaturally develop spavin at three or four.

In considering the question of predisposition, we have to remember that the formation of other portions of the limb, and even of the body, exercises a considerable influence on the development of the disease. Experience shows that uprightness of the fetlock- and stifle-joints favours disease, though this formation is often secondary, i.e., a consequence, and not a cause, of the formation of spavin. Klemm considers that animals with cow-hocks, and those which stand with the hind-feet far back, are predisposed to the disease, and on this basis explains the curative action of shoes with high quarters and deep heels.

Peters thinks that turned-out hocks are particularly prone to spavin, and there is no doubt that this formation interferes with hock action and increases the chance of disease.

Experience also shows that very powerful horses with broad pelves and well-developed quarters are particularly liable, the reason being the great strain which this formation throws on the hock-joint. The more powerful the muscles of the hind-quarter and the freer the movement, the greater the strain on the hock. 'The same is true of over-grorvn and long-backed animals. To produce an equal output of energy, their muscles are forced to act more vigorously, and, therefore, greater strain is thrown on the hock. For the same reason powerful, spirited horses more often suffer than quiet, phlegmatic animals. The fact that spavin is commoner in young horses is principally due to the incomplete development of their bones and ligaments, and to the existing predisposition being only brought into play when the young animal is put to work; horses, when once seasoned, not being likely to develop disease unless greater demands are made on their working powers, or the hock is accidentally injured.

The causa externa is almost exclusively represented by mechanical injury. Severe, rapid work, especially under the saddle, or in front- of heavy loads, often produces slight, frequently repeated strain. Peters thinks soft, moist ground and rough pavements particularly injurious, because the unavoidable rotary movement of the hoof on the ground is interfered with, and consequently takes place, not at the extremity of the limb, but in the joints, especially in the hock-joint.

Single violent sprains, produced, for example, by wheeling round on the hind-quarters or suddenly throwing the horse on its haunches, may determine inflammation of the hock-joint, but this is far from common. Even less frequently is inflammation caused by external injuries, such as wounds or bruises. 
Frick, who lived for a long time in the mountainous parts of Schleswig, states that, with the exception of being rather "leggy," the horses there are well shaped. Nevertheless it very often happened that when worked the animals developed acute lameness, with swelling, heat, and pain on the inner side of the hock-joint. 'The lameness was similar to that of well-marked spavin. Cold local affusions and rest removed the symptoms in three to four days, but very soon after, enlargement of the inner side of the hock and lameness reappeared and the existence of "spavin" could no longer be overlooked.

Frick, therefore, regards strains as playing the chief part in the causation of spavin. The formation and function of the hock naturally favour injuries of the kind, and Frick, after careful study, is convinced of the truth of this view. As in every other form of strain, the anatomical lesions may vary, and it is this variation in clinical appearances which has caused different observers to formulate such widely differing views as to the exact nature of spavin.

The above is very far from exactly explaining the pathology of spavin ; much further investigation is required to make it completely clear. The propagation of new theories, however, does just as little for science as the recommendation of new cures for practice. What is needed, above everything, is a clear insight into the physiology of movement. Such an investigation should not be confined to considering single sections of the limb, like the hock, but should take cognisance of the action of the limb as a whole. Though by no means a light task, it would well repay study, and we hope that in the near future some one will be found to undertake it.

Pathology. Although no agreement has yet been reached as to the initial lesion of spavin, the views regarding its pathology are more in accord. Anatomy and physiology here play the chief part. The peculiar fact that the disease affects the inner side of the hock has always attracted attention, and has given rise to much speculation.

Peters sought an explanation in the anatomical formation of the parts. As the connection between the tibia and astragalus consists of a ginglymoid, or, more correctly speaking, a screw-like, joint, in which the powerful lateral ligaments limit both abduction and adduction, and only allow of movement in the direction of the longitudinal axis of the body, one would expect that the axis of the joint would be perpendicular to that of the body. This is not the case, however; the (horizontal) axis of the joint lies obliquely to it, and, starting from the inner side, runs backwards and outwards. As a consequence, the two tibiæ are not perpendicular, but converge from above downwards, whilst the metatarsal bones of the two limbs are parallel with each other when the limbs are in their normal position. It therefore results that the power does not act 
perpendicularly to the articular axis of the astragalus, and consequently, when weight is placed on the limb, the muscles do not simply extend the joint, but also tend to rotate it. This arrangement certainly assists the transference of the body-weight to the opposite limb and ensures stability; but the consequent rotary movement of the limb necessarily involves displacement of the bones of the hock-joint, and thus favours strain of the ligaments and disease of the joints. The tibio-tarsal joint is protected against injurious action of this kind by its strong lateral ligaments, and by the prominences on the astragalus; whilst the inner portion of the lower joint, which can easily be shown by anatomical preparations to possess the least power of resistance to such rotary movements, has therefore to take the chief share in them, and accordingly is most exposed to strain of its lateral ligaments and fibrous capsule. Irritation and inflammation are thus caused in the neighbouring periosteum, and produce disease of the articular cartilage. Depending on whether the cartilage and synovial membrane become affected early or late, lameness is either present from the first, or only occurs when new bony growths have formed outside the joint. This, again, depends principally on whether strain is severe from the first, or whether it is only gradually exerted.

This theory seems to explain in a satisfactory way both the manner of origin and many of the symptoms of spavin. Anything which impedes this physiological rotation of the hock-joint, such as working in heavy ground or on rough pavements, must favour the production of disease. It seems tolerably certain that spavin cannot be referred to any single cause, but that the initial disease may start in one of several structures; and therefore, while the formation of the joint on which Peters thus lays stress is probably one of the most important factors, it would be unwise to deny the possibility of the process originating in disease of the bursa of the flexor metatarsi (Dieckerhoff), or even on the surface of the joint. Möller's and Petit's investigations, however, showed the bursa to be frequently intact, even when disease of the joint was advanced. Nor does co-existent disease of the bursa necessarily prove the latter to have been the point of origin, for in many cases it becomes affected secondarily, by inflammation extending to it from
the joint.

Symptoms. (1) Spavin lameness. The diagnosis of arthritis chronica in small, "clean" hocks offers no difficulty, provided disease processes have extended beyond the joint, and exostoses exist. Until they develop, however, diagnosis remains uncertain, for the lameness is not sufficiently characteristic to form the basis of a decided opinion. It is just on this account that errors so frequently occur, and that other lamenesses, even foot-lameness, are mistaken for spavin. But other injuries to the hock, 
such as sprain, \&c., may lead even experts into error. Such mistakes often lead to doubts being cast on the value of treatment.

The lameness accompanying spavin is scarcely ever sufficiently distinctive to alone determine the diagnosis, but must be considered along with the anatomical changes in the hock-joint.

Even Knoblock, when speaking of it, says: "If one detects no swelling, how is one to know that a spavin will come?" One must not be understood to say that the character of the lameness does not afford valuable information, but the kind of lameness is less important than the manner of its appearance and its after-course, and, finally, than the absence of visible pathological changes, to which the lameness could otherwise be referred.

The onset and course of the lameness is, then, of greatest value; the style of movement, which varies greatly according to the position and extent of the inflammation and to other circumstances, much less so. In the greater number of cases the limb is incompletely extended. The last phase of the stride, while weight is still carried by the limb, is relatively shortened, apparently on account of the pain due to extension of the hock-joint. Immediately the hoof leaves the ground the limb is drawn rapidly forward. This sudden movement often resembles stringhalt, and is best seen during the first few steps, or when turning in a small circle.

A further consequence of the incomplete extension of the limb is an exaggerated hip-action, which is seldom absent. The shortening of the last part of the stride is compensated by extra movement of the quarter. Sometimes the limb is abducted, especially in double-sided spavin, in which the turning out of the limbs is often well marked. In other cases the fetlock becomes upright, in consequence of the incomplete extension of the hock causing the animal when moving over uneven ground to walk on the toe. In almost all fully-developed cases the horse wear's the toe of the shoe excessively.

As a rule, lameness develops very gradually. At first it disappear's after a few steps, and in many cases all that can be observed is a catch in the movement of the affected limb, resembling stringhalt, when turning round in the stall towards the sound side. This usually disappear's with work. The disappearance of lameness during movement, and its regular recur'rence after rest, form one of the most important peculiarities of the disease. The fact that turning towards the sound side seems more painful than towards the diseased is explained by the pressure on the inner part of the hock being then greater. Occasionally, however, lameness persists during movement, and may, indeed, become more severe, especially if it were previously well marked or of old standing. The increase in lameness when turning in small circles is clearly due 
to the rotary movement in the limb and the tendency to displacement of the bones in the affected section of the joint.

The "spavin test" is sometimes useful in diagnosing spavin lameness: it consists in lifting the limb and keeping the hock-joint flexed for one minute and then trotting the horse. Lameness is very marked.

This test, however, requires to be used with considerable care, especially in old horses, which may show similar difficulty in movement after the limb has been kept bent, even without having spavin.

Various hypotheses have from time to time been advanced as to the immediate cause of lameness. Whilst the older practitioners considered the pain to be due to pressure of the exostosis on the periosteum, and supported theil view by reference to the uneven surface of the macerated bones, we have known since Havemann's time that the changes within the joint were not only the essence of the disease, but also the cause of the lameness. Though Hering opposed this, stating that spavin lameness is sometimes seen without disease of the joint, either his observations were due to errors in diagnosis, or the process was only in a developmental stage, the articular surface not yet having suffered. As a rule, the onset and course of the lameness entirely agree with the observations made in men suffering from arthritis chronica, who at first only show lameness for a few steps. The lameness is probably due to changes partly in the articular surfaces, partly in the ligaments. Dieckerhoff adds disease of the bursa of the flexor metatarsi muscle. It seems doubtful whether chronic inflammation of this bursa ever produces lameness. Under certain circumstances, exostoses may mechanically limit free movement of the joint.

(2) The exostosis may even precede the lameness in cases where the disease develops slowly: as a rule, the two appear simultaneously, but sometimes the bony swelling does not occur until two or three weeks afterwards. Its detection requires an accurate knowledge of the normal configuration of the hock-joint, and may be effected by viewing the joint either from the front or back. For this purpose the operator takes up a position either some steps in front of or behind the horse, and compares the inner surfaces of the hocks. In this way experts can detect the slightest differences, though it is by no means possible to determine by this examination alone whether or not spavin exists, especially in English thoroughbreds, whose hocks are seldom absolutely symmetrical. But even in other breeds a want of symmetry in the hocks cannot of itself be regarded as a certain sign of spavin. The terms "coarse," \&c., used in speaking of formation, are tacit acknowledgments of this fact, and are often only circumlocutions for spavin.

(3) The above signs of spavin are often accompanied by atrophy of the muscles of the quarter, as usually happens in chronic painful 
lamenesses. In judging of this, it should be borne in mind that English thoroughbreds not infrequently show asymmetry in the breadth of the two sides of the pelvis which might easily mislead the observer.

Eberlein also states having been able to demonstrate atrophy of the diseased hock by actual measurements. The upper parts of the affected joint were sometimes 2 inches less in diameter than that of the sound limb. Even at the level of the exostosis (spavin) the measurement was lessened. Eberlein regards this as due to inaction-atrophy.

The visible changes on its exterior usually show whether the joint is or has been diseased, but alone are not proof that the existent lameness is due to spavin, for very frequently the lameness disappears after anchylosis of the joint, though other conditions may interfere with the movement of the limb. To refer such lameness to the changes in the hock-joint would be a serious error. The diagnosis "spavin lameness" is only justified when-

(1) The local changes are accompanied by lameness, the course and other peculiarities of which agree with the description above given.

(2) Muscular atrophy or other condition points to old-standing lameness, probably connected with the anatomical changes. As already stated, exostoses are more likely to cause lameness the nearer they lie to the front of the joint. Sometimes traces of past treatment are visible, pointing to a chronic condition, and supporting the diagnosis "spavin."

Acute inflammatory symptoms, increased warmth, pain on pressure, \&c., are seldom seen unless the condition has resulted from violence, but their presence is not incompatible with spavin lameness.

Differential diagnosis. By bearing in mind the above-mentioned principles, mistakes will usually be avoided, though the following: conditions present many points of resemblance to the lameness now under consideration :-

(1) Stringhalt, a disease which will be described in another place. Here it need only be remarlied that the periodical appearance and gradual abatement of lameness during work point to spavin.

(2) Hip lameness. In these cases lameness is only marked when the limb is carried (swinging-leg lameness), whilst in spavin it exists both when the limb is carried and when weight is placed on it. The same applies to-

(3) Gonitis chronica, in which also there is a marked tendency to stand with the limb flexed (see "Chronic Inflammation of the Stiflejoint").

(4) Inflammation of the tendon sheath of the flexor pedis at the inner surface of the hock-joint. This, however, can scarcely be mistaken for spavin, on account of the marked swelling (see "Diseases of the Metatarsus"'). 
(5) Tendinitis and tendovaginitis of the flexor pedis lead, in the hind $\operatorname{limb}$, to pronounced lameness when weight is thrown on the limb (supporting leg lameness), and can be detected by careful palpation.

(6) Curb is less likely to be mistaken for spavin, because it seldom produces lameness, unless when accompanying the latter.

(7) Spavin lameness is more difficult to differentiate from that due to ring-bone and sprain of the coronet-joint. It should be remembered, however, that, in a hind limb, ring-bone is less frequently followed by lameness, whilst sprain is usually distinguished by the pain (due to rotation of the joint) which occurs when the animal is sharply turned round.

(8) The absence of foot lameness is proved by a careful examination of the hoof. In laminitis there is marked pulsation of the metatarsal arteries.

(9) Double-sided spavin is sometimes difficult to distinguish from mere stiffness. In "wor'n" horses, which, as a rule, show no real lameness, too much importance should not be attached to the results of the spavin test, nor to the stiff movement.

Course and prognosis. The nature of the disease explains its chronic course. It is rarely caused by mechanical violence, but as a rule results from slight, continually repeated strain, and, therefore, develops slowly. The first symptom is usually slight sensitiveness, soon followed by lameness, though at this stage no anatomical change can be detected in the joint. Sometimes, however, disease processes are so gradual that lameness is entirely absent during the first stage, and before it develops, bony growth can be detected on the joint. In this respect spavin resembles a number of other diseases-for example, contracted hoof. When it develops slowly, contraction may become well marked, without causing lameness; whereas in cases of rapid development, the walls of the hoof exert painful pressure on the soft parts, and soon interfere with movement.

As a rule, the appearance of the exostosis is preceded for two to four weeks by lameness, which gradually increases in severity in proportion as disease processes become more pronounced. In cases where at first it was only noticeable during the first few steps, it afterwards becomes continuous. Considering the nature of the pathological process, it is scarcely surprising that lameness appear's periodically, is sometimes lost and then returns, or that in certain exceptional cases recovery takes place without treatment of any kind except complete and prolonged rest. From a purely theoretical standpoint there is no reason why the lesions in the joint may not disappear and the parts recover in every respect the status quo ante, but, in point of fact, this termination is very rare. As a rule, recovery is only relative, and is followed by obliteration of 
the joint. When the articular surfaces have undergone change and the cartilage is destroyed, repair is absolutely impossible, and recovery can only result from adhesion of the opposing diseased articular surfaces. This takes from six to eight weeks, but may be indefinitely prolonged; sometimes it never occurs, and the lameness is incurable. In actual practice it is never possible to foretell with certainty how the case will terminate, though a careful consideration of all the facts may give some indication.

'Though union principally depends on the animal being rested and placed under treatment, failing which the parts seldom unite, and lameness persists, the converse is not equally true, for even the most energetic treatment is not always successful. Resolution, i.e., the disappearance of inflammation and local change, is exceptionally rare.

Errors in diagnosis have led to the belief that absorption often occurs, and that absorptive processes may be assisted by treatment. Möller's experience is quite opposed to this view, which is due to confusing with spavin (i.e., arthritis chronica) many varied disease processes, the sole common feature of which is lameness. It is certainly difficult at times to confidently diagnose spavin on the first examination, especially if no reliable history is available. Nevertheless, an attempt at distinction should always be made, otherwise prognosis and treatment are mere gropings in the dark.

Not infrequently lameness diminishes or disappears after a long rest, but the improvement is only temporary, and as soon as the horse returns to work, lameness recurs in an even severer form. It is clear that, with rest, the inflammation may diminish in intensity, but it seldom disappears completely. Though spavin may be viewed as a typical disease of the hock-joint, yet it shows many variations in course, partly on account of the conformation of the joint, partly of the degree and extent of disease processes, but especially of the variation in external influences, amongst the principal of which must be ranked the treatment employed in the particular case.

In forming a prognosis it is necessary to bear in mind all the factors which favour relative recovery, i.e., which contribute to removal of lameness, as well as those, on the other hand, which are likely to prevent it. Such factor's comprise :-

(1) The work required of the animal. The greater the exertion, and consequently the strain on the hock-joint, the less the chance of permanent recovery. Heavy cart-horses and hacks are therefore unfavourable subjects.

(2) The degree of lameness and the time it has existed. If, in spite of proper treatment, lameness persists, it shows that the conditions are unfavourable to anchylosis, and the chance of recovery, therefore, is 
slight. For a similar reason, marked muscular atrophy is not a hopeful symptom. Severe lameness points to extensive change in the joint, and is also unfavourable, but slight lameness does not prevent the animal being used for certain purposes, even though it cannot be completely cured.

(3) The animal's conformation. The worse the conformation of the limb, and especially of the hock, the less the chance of recovery. In badly-formed hocks even moderate work produces fresh strain and prevents the bones uniting:

(4) The position of the disease. It has long been known that exostoses on the flexor surface of the joint are particularly troublesome, and that the chance of recovery is greater if the spavin lie towards the back of the joint, and be confined to the cuneiform bones. This may partly be explained by the fact that new growths of bone in front of the joint are more likely to mechanically interfere with movement, even after obliteration of the joint; while disease of the joint in this situation is less likely to be followed by anchylosis, because the amount of unavoidable movement is here greatest. As indicated by Havemann, the size of the exostosis is of less importance; horses with marlied spavins may often be seen going sound, whilst those with a small growth may prove useless for every kind of work.

(5) The animal's age. The occurrence of spavin in young animals which have not done much work points to a strong hereditary predisposition, and naturally gives an unfavourable cast to the prognosis. In such animals the lameness may disappear for a time, but returns immediately work is resumed. On the other hand, spavin lameness first developed at an advanced age is not likely to disappear, as reparative processes are then sluggish.

Prognosis, though to some extent guided by the facts given, is always uncertain. It is never possible to say confidently that lameness will disappear, even when the diagnosis is beyond doubt. The varying formation of the hock-joint and of the limb in the different breeds, the uses to which horses are put, and other factors (including errors in diagnosis), lead to the percentage of recoveries being very varyingly estimated by different experts. Taken altogether, they may number about 50 per cent.

As already stated, eight to ten weeks' rest and suitable treatment are generally necessary for the disappearance of lameness. Although recovery is not impossible, even during continued work, yet a great many of such reported cases must be referred to errors in diagnosis.

Treatment. Spavin lameness is seldom followed by spontaneous recovery, and the first essential of treatment is sufficiently prolonged rest. Havemann, Strauss, and others consider all treatment useless, but 
this is certainly too extreme a view, for every busy practitioner must know of many horses which, after treatment, have perfectly recovered their usefulness. The nature of the pathological changes renders cure only relative, for the articular surface never recover's its normal condition, and well-developed spavin lameness only disappears after anchylosis of the joint. But that it does so disappear is readily proved by post-mortem examination. Very often the lower joints of one or other, not infrequently of both, hocks are found completely united in animals which moved perfectly sound before death. Practically spealing, all modes of treatment aim at producing such an union.

This union requires, firstly, prolonged and perfect rest, i.e., stoppage of all work, and, as far as possible, of movement. Whatever the nature of the other treatment, this rest is essential, and must be continued for four to eight weeks.

Various auxiliary measures of treatment have been proposed and employed, but they all agree, on the one hand, in procuring rest of the joint, and, on the other, in evoking, in the neighbourhood of the diseased spot, an acute inflammation, which favours adhesion of the bones. The views as to their method of action, and the nature of the curative process, vary widely. Some consider that inflammation is diverted from the joint-an idea which, however, is out of harmony with the views held of such processes at the present day. The same is true of the suggestion that the artificially induced acute inflammation may terminate the changes proceeding in the articular surfaces. This is only true in the sense that it may induce union between the bones; it camnot remove the chronic arthritis. In opposition to the above view, it has been pointed out that, in man, arthritis chronica deformans shows no tendency to the formation of stiff joints; but the objection leaves out of consideration the fact that, in man, the joints affected (hip-joint, \&c.) are incapable of anchylosis; the same applies to the stifle-joint of the horse (see "Chronic Inflammation of the Stifle-joint"'). Recovery entirely depends on union between the articular surfaces of the cuneiform bones, or between these and the metatarsus. Union may be assisted by-

(1) Blister's. The action of blisters is usually too superficial. To exert much influence on adhesive processes, energetic, deep-seated inflammation must be excited, and concentrated sublimate solution, ar'senic, euphorbium, croton oil, \&c., are therefore more successful than cantharides blisters, though they are very liable to produce permanent blemishes; good results following the application of cantharides are usually due either to the rest given, or to the case not being spavin. The success obtained in the army by blisters may be similarly explained. 'To satisfy the owner Möller has often tried blisters, but seldom with good results. They may possibly be more active when subcutaneously employed, as recommended by Buch 
and Bassi. Bassi makes two or three converging incisions through the skin at the diseased spot, loosens the subcutis, and fills the pockets thus formed with blister.

Setons, formerly much used, are now no longer employed. They are easily rubbed out by the opposite foot, and leave ugly scars.

(2) The actual cautery. On account of the more intense and penetrating inflammation produced, firing is more effective than blistering, and (ceteris paribus) in direct proportion to the intensity and extent of the periosteal irritation which it excites. It is of little importance whether the firing be in lines or points; the great thing is to set up artificial inflammation in the depths. Many practitioners, therefore, prefer to perforate the skin with a pear-shaped iron; in France a long point is preferred (feu perforant). In Germany, Gerlach tried the latter and other methods, but renounced them on account of the risk of producing fatal inflammation of the joint. Perforation of the skin and of the bursa of the flexor metatarsi with the pyriform iron is usually harmless, and is unquestionably amongst the most effective methods of dealing with spavin. On the other hand, the point, when incautiously used, may cause dangerous arthritis, especially if too thick and too long, or if any portion of the joint be opened. The point used in France is from $\frac{3}{8}$ to $\frac{5}{8}$ of an inch long, and therefore seldom penetrates the joint, but when there is no marked exostosis, such an accident is quite possible. In presence of a large bony spavin, perforation is scarcely ever followed by bad results. The pointed iron produces excellent results where well-developed bony growths make it impossible to penetrate the joint. In this respect the greatest caution must be observed in well-bred horses with thin skins.

If the deposit be slight, the red-hot iron should be applied once to the highest point of the swelling, and passed through the skin into the bone by firm pressure. In dealing with large spavins, this may be done at two or even three points. As a rule, a blister of sublimate (1 to 6) may at once be applied. Sometimes the tendon sheath of the flexor metatarsi muscle becomes acutely inflamed, and severe lameness lasting several days results. This need cause no alarm, for the inflammation will pass away of itself. When the exostosis is smaller, the use of the point is somewhat dangerous.

The pointed cautery is unquestionably the best means of dealing with spavin. Dollar disinfects the parts and uses the platinum-pointed cautery, applying a surgical dressing immediately afterwards, instead of blistering; or, preferably, saturating the parts several times daily with 5 per cent. creolin solution. (See Dollar's "Operative Technique," p. 125.)

In Germany, Hoffmann recently advocated the pointed iron. He uses knitting-needles held in a pair of forceps, and introduced glowing hot. The 
animal is cast, the point of operation disinfected and rubbed with an antiseptic powder. The necessary number of knitting-needles (which can be broken in two) are placed in a basketful of burning charcoal, and, as required, are grasped with forceps and introduced into the bone. From fifteen to twenty perforations are made over a surface as large as a lemon. They enter the bone deeply enough to reach the cancellous tissue. If want of resistance to the introduction of the needle shows that the joint has been entered, the needle must then at once be withdrawn.

After firing, Hoffmann applies a thick coating of iodoform, lays the hand flat on the parts, and moves the skin backward and forward so as to displace the openings in the skin from those in the deeper-seated structures. The surfaces are then once more strewed with iodoform. After some days a slight swelling occurs, an eschar forms, and in fourteen days all symptoms are stated to disappear.

Batazzi recently recommended the treatment of spavin by subcutaneous firing, formerly introduced by Nanzio. After making an incision $1 \frac{1}{2}$ to 2 inches in length over the exostosis, the edges of the wound are drawn back and a few punctures made in the form of a triangle, with the base directed upwards. The same effect is more simply produced by firing through the skin, which has the advantage of producing smaller cicatrices.

(3) Operation for spavin. Since the times of Abildgaard and Lafosse many operations have been proposed for the cure of spavin. Abildgaard first described section of the inner terminal tendon of the flexor metatarsi, afterwards extensively practised by Lafosse.

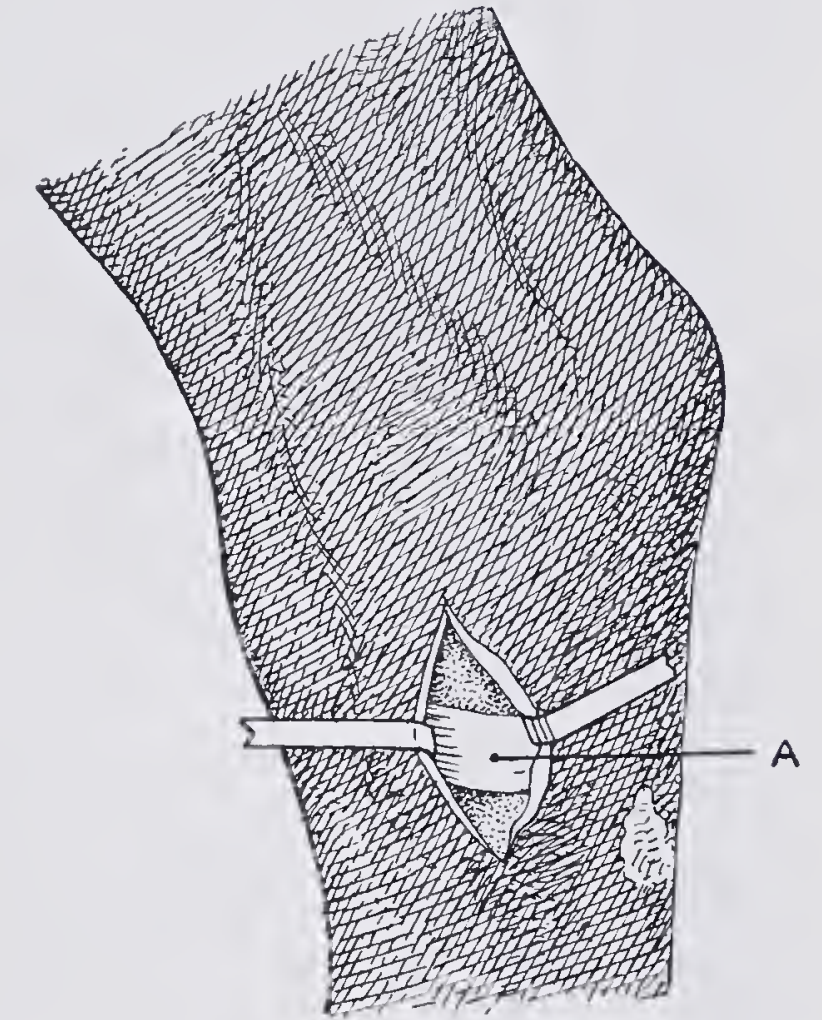

FIG. 267.--Operation for division of the inner terminal tendon of the flexor metatarsi muscle. A, The tendon.

Dieckerhoff, about the same time, suggested opening the bursa of this tendon. No doubt these operative measures may assist in removing lameness, but their usefulness probably depends on the acute inflammation which follows assisting union of the diseased surfaces, and not, as Abildgaard and Lafosse thought, on their producing relaxation of the tendon, and preventing it pressing on the diseased joint. Division of the tendon is, therefore, of much less importance than the production of acute inflammation of its bursa, which extends to neighbouring structures, and not only procures thorough local rest, but assists union by the periostitis which it gives rise to. Dieckerhoff's method is very simple, and quiet horses may be operated on standing. Restive or dangerous animals are cast, with the affected limb lowermost. A twitch is applied, the horse is placed against the wall with the sound limb lifted as in shoeing, and the operator then passes a probe-pointed listoury into the bursa of the diseased limb. After making sure that the bursa is really opened, the animal is placed in the stable. No after-treatment 
seems necessary, except to remove excessive granulations by suitable caustics. The wound generally cicatrises in three weeks, and after a further interval of a fortnight the horse can be put to work.

In many cases this method is successful, but in others recovery does not occur, while in a certain, though small, proportion acute inflammation of the hock-joint develops, and proves fatal. If in making the incision the operator cut too deeply, which is sometimes unavoidable, and alight on the boundary between the scaphoid and cuneiform bones, the joint is opened, and purulent or septic arthritis readily follows. It is, therefore, well not to make the incision too far back. Dieckerhoff recommends the centre point between the anterior and posterior borders of the joint.

No purpose is served by proceeding antiseptically, for success depends on the inflammation produced, and especially on the periostitis.

The bursa can also be opened with a rather sharply-pointed pyriform cautery. It seems of no importance which method is adopted, though in using the firing-iron care must be taken not to open the joint. The larger the exostosis, the less the danger.

By bearing in mind that union depends chiefly on the formation of exostoses around the joint, and that the articular surfaces themselves can only grow together after extensive destruction of the articular cartilage, the reason for seeking to produce extensive bony deposit will be apparent. Periosteotomy is the surest method of effecting this, and has been widely recommended in the treatment of spavin. The operations recommended do not differ in any essential point. Peters' method gives excellent results.

It is as follows:-

The horse is cast on the diseased side, and by a cord passed round the corresponding front limb, the upper hind leg is drawn far enough forward to clear the seat of operation. The hair is then cut away from the inner surface of the hock-joint, midway between its anterior and posterior borders, to the extent of about one square inch, the surface washed with soap, rinsed with sublimate or carbolic solution, and the other antiseptic precautions, such as cleansing the hands, placing the instruments in carbolic solution, \&c., complied with. By means of a probe-pointed bistoury or scalpel, an incision, at right angles to the long axis of the limin, and about half an inch in length, is then made through the skin and fascia at the disinfected spot, a pair of curved scissors are introduced through the opening as far as the joint between the blades, and the skin divided from underlying tissues in the form of a " $V$," the instrument being first thrust forward, then backward, severing the subcutis. The slightly-curved knife (fig. 268, a) is next introduced into the front pocket of skin, the cutting edge directed backwards to avoid injuring the vena saphena: As soon as it has entered up to the handle, the cutting edge 
is directed towards the joint, and the back pressed with the fingers of the left hand, while, by gently rocking the instrument, it is made to penetrate the bones of the joint. The tendon of the flexor metatarsi muscle and the periosteum part with a distinct "crunching" sound. The same process is repeated in the posterior pocket of skin, the sharp edge of the knife, however, being directed forwards. After wiping away the small amount of blood which escapes from the wound, the surface is rinsed with a disinfectant, and an antiseptic dressing applied. The bandage should be carried down as far as the fetlock, so as to obtain a firm hold. The horse is then allowed to rise, and is placed in the stable. If, during the next few days, the bandage become soaked through with blood, it should be renewed, otherwise it is left in position for six to eight days, when the skin wound will be found to have closed.

By the exercise of moderate care in operating, pus formation can be avoided; and even should it occur, it seldom entails grave consequences, for, on account of the flat position of the knife while making the incision, there is little danger of opening the joint, and thus producing dangerous arthritis.

The horse must be rested for at least four to six weeks after operation, and during this time movement, as far as possible, avoided. Some operators even recommend fastening the animal up short to prevent it lying down.

Möller discovered by experiment that both the inner tendon of the flexor metatarsi and the periosteum of the cuneiform bones are divided in this operation; not infrequently the internal lateral ligament is also partly cut through. The above method produces active periostitis and thickening of the internal ligaments of the joint, which favour periarticular exostosis and

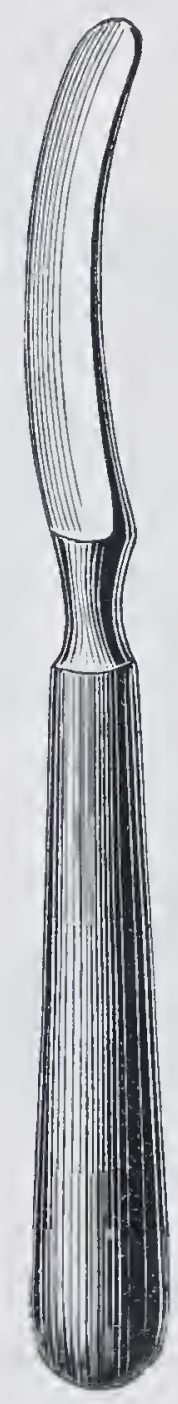

$a$

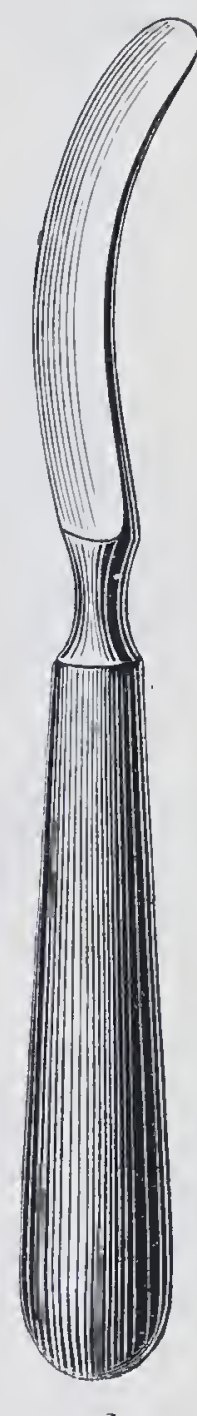

b

Fig. 268. - Perios teotomy knives. union of the smaller joints in a much higher degree than simple opening of the bursa and other operations.

Bad results are rare. For a short time exostoses are actively produced, but greatly diminish after a month or two, and may finally leave no trace of operation.

It is not pretended that this procedure cures all cases of spavin, but the objections raised against it in no way detract from its undoubted value.

'T'here need be 110 fear of using the knife, even when the exostosis is small. In such cases, to make sure of sufficiently dividing the periosteum, knives of greater curvature may be used; these penetrate more 
deeply (fig. 268, b). Möller has often broken the knife when pressing it into the bone, but never had any bad result.

A clinique offers few chances of collecting reliable statistics of the results of such operations. When the patient was not returned, Möller considered the result to have been favourable, and on that basis had no hesitation in describing periosteotomy as thoroughly effective. Very few cases were sent back as uncured; and amongst those that were traced, many had remained for years free from lameness. Failing success by this method, the pointed cautery may sometimes be found of more service. Its use is without danger, the large exostoses preventing it entering the joint.

Klemm's method of dividing the flexor metatarsi muscle 3 or 4 inches

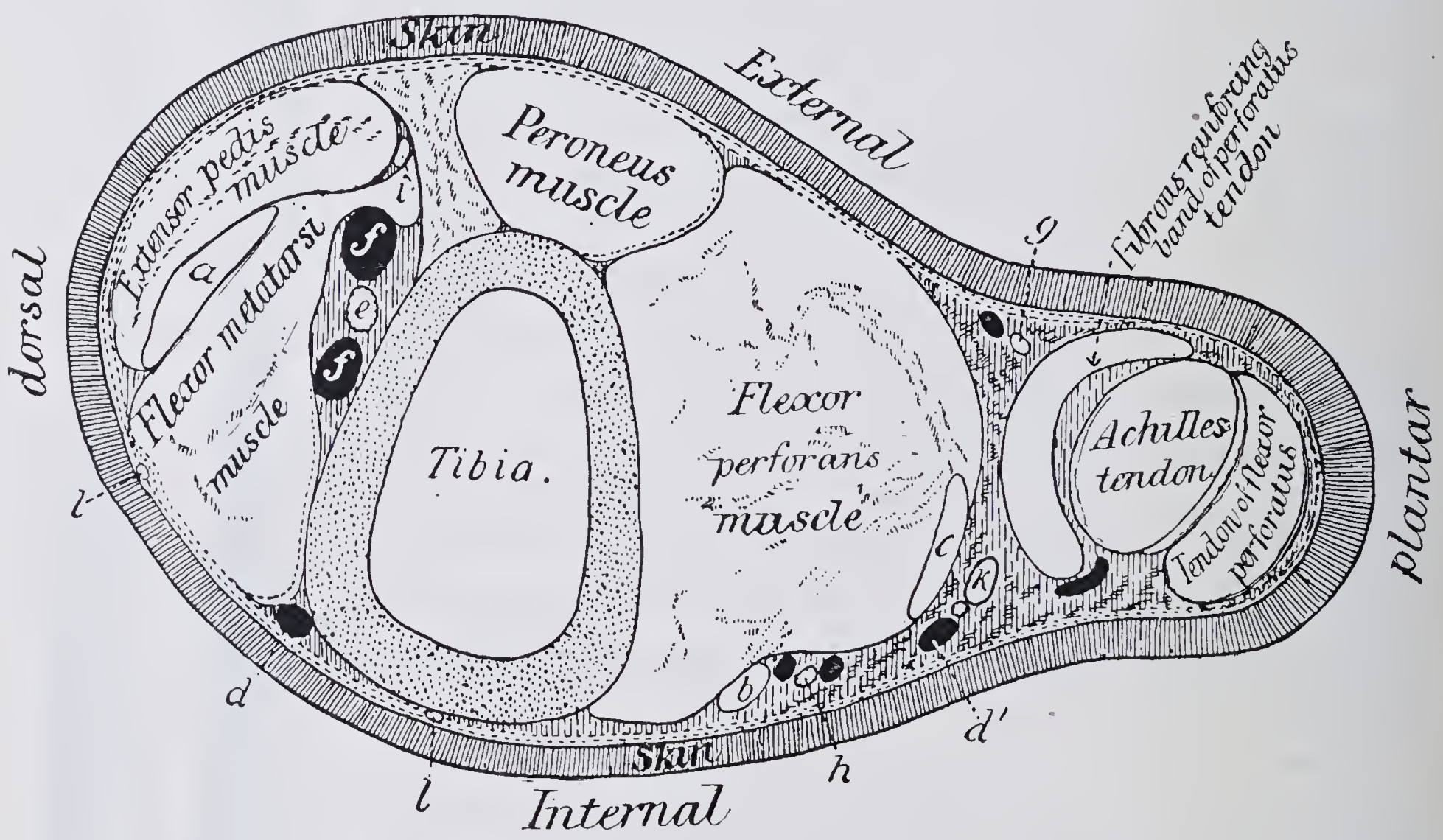

FIG. 269.-Horizontal section through the hind limb about the lower quarter of the tibia. $a$, Tendinous portion of ext. pedis muscle; $b$, tendon of flexor accessorius ; $d$, saphenous vein ; $e$, ant. tibial artery; $f f$, anterior tibial veins; $g$, ext. saphenons nerve and vein ; $h$, posterior tibial artery and veins; $i$, anterior tibial nerve; $k$, post. tibial nerve; l, branches of the int. saphenous nerve.

above the hock-joint is, in Möller's experience, useless. If divided completely, lameness follows similar to that after rupture of the muscle (see "Rupture of the Flexor Metatarsi"). By giving four to six weeks' rest, the joint may become anchylosed, and lameness disappear, but this often fails to occur. Partial section sometimes disguises the stringhalt-like lameness, but cannot cure the disease of the joint, and the owner generally returns after an interval to submit the horse to further treatment.

In the Prussian army the actual cautery has, during the last few years, been largely used in treating spavin, the successes numbering about 60 per cent. The method recommended consists in perforating the bursa with a pyriform iron, which is passed into the bone. Periosteotomy proved of less value. It must, however, be remembered that 
in no other disease are diagnostic errors so frequent as here, for even the most careful examination often leads to no definite conclusion. Serious methods of treatment are only applied to serious conditions, $i . c$. , to cases in which disease has made extensive progress, whilst the milder cases are blistered or fired; especially in the army, where firing and blistering are greatly relied on.

In a number of cases where the swelling is old, large, diffuse, and especially when it extends far forwards towards the bend of the hock, firing, even if repeated, and section of the terminal branch of the flexor metatarsi tendon fail, or only yield a very modified success; lameness persists or is remittent, being less marked after a certain amount of exercise than on leaving the stable, though some: times it is equally pronounced before and after exercise : in these rebellious cases double neurectomy of the posterior and anterior tibial nerves, the latter a branch of the external popliteal, has been recommended. This treatment of obstinate spavin by neurectomy is based on the following anatomical facts:- Opposite the point of the calcis the posterior tibial nerve divides into two parts, the internal and external plantar nerves. Behind the hock-joint the external plantar nerve gives off a fairly large branch, which passes under the tendon of the flexor perforans, and detaches several twigs, of which some ramify over the surface of the joint, whilst others penetrate into it. In front of the lower extremity of the tibia the tibial nerve gives off several branches, which enter the joint.

In neurectomy of the anterior tibial, the

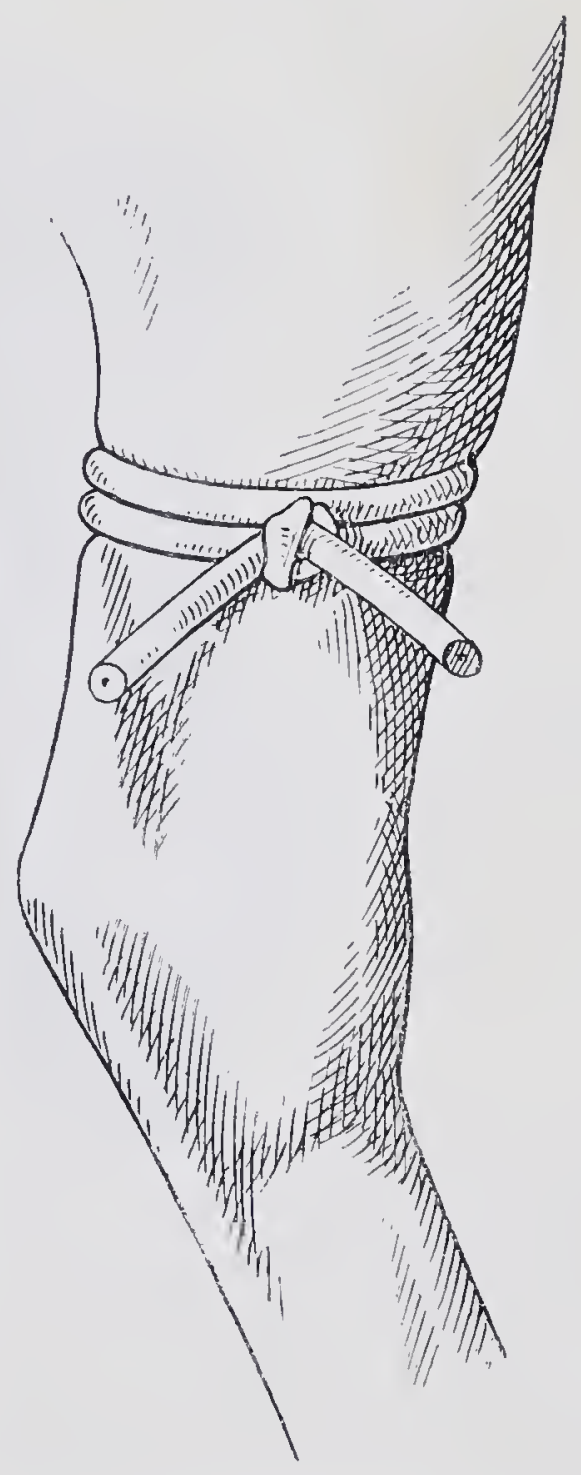

FıG. 270.-Rubber cord applied to thigh to check bleeding. In neurectomy of the posterior tibial nerve the cord would be applied rather higher. point selected is at the external surface of the lower part of the thigh, a hand's breadth above the point of the hock. The anterior tibial nerve is situated on the deep surface of the extensor pedis, between this muscle and the thin muscular portion of the flexor metatarsi which separates it from the anterior tibial artery, and from its large satellite vein-vessels which lie directly on the anterior surface of the tibia, where they are surrounded by a thick layer of connective tissue (see section, fig. 269).

The method is as follows:- The point of operation being prepared, the skin and subjacent aponeurosis are incised for a distance of $2 \frac{1}{2}$ to 3 inches, opposite the external margin of the extensor pedis V.S. 
muscle. This muscle is separated first from the tendinous, then from the muscular, portion of the flexor metatarsi, on the anterior surface of which the anterior tibial nerve is readily discovered. A fragment of this, $\frac{3}{4}$ of an inch to $1 \frac{1}{4}$ inch in length, is excised. The wound is closed by a few cutaneous sutures, with or without providing for drainage. The operation is easy. It is, however, always necessary to proceed methodically, and to talie care not to injure the tibial vein, which thrusts the muscular portion of the flexor

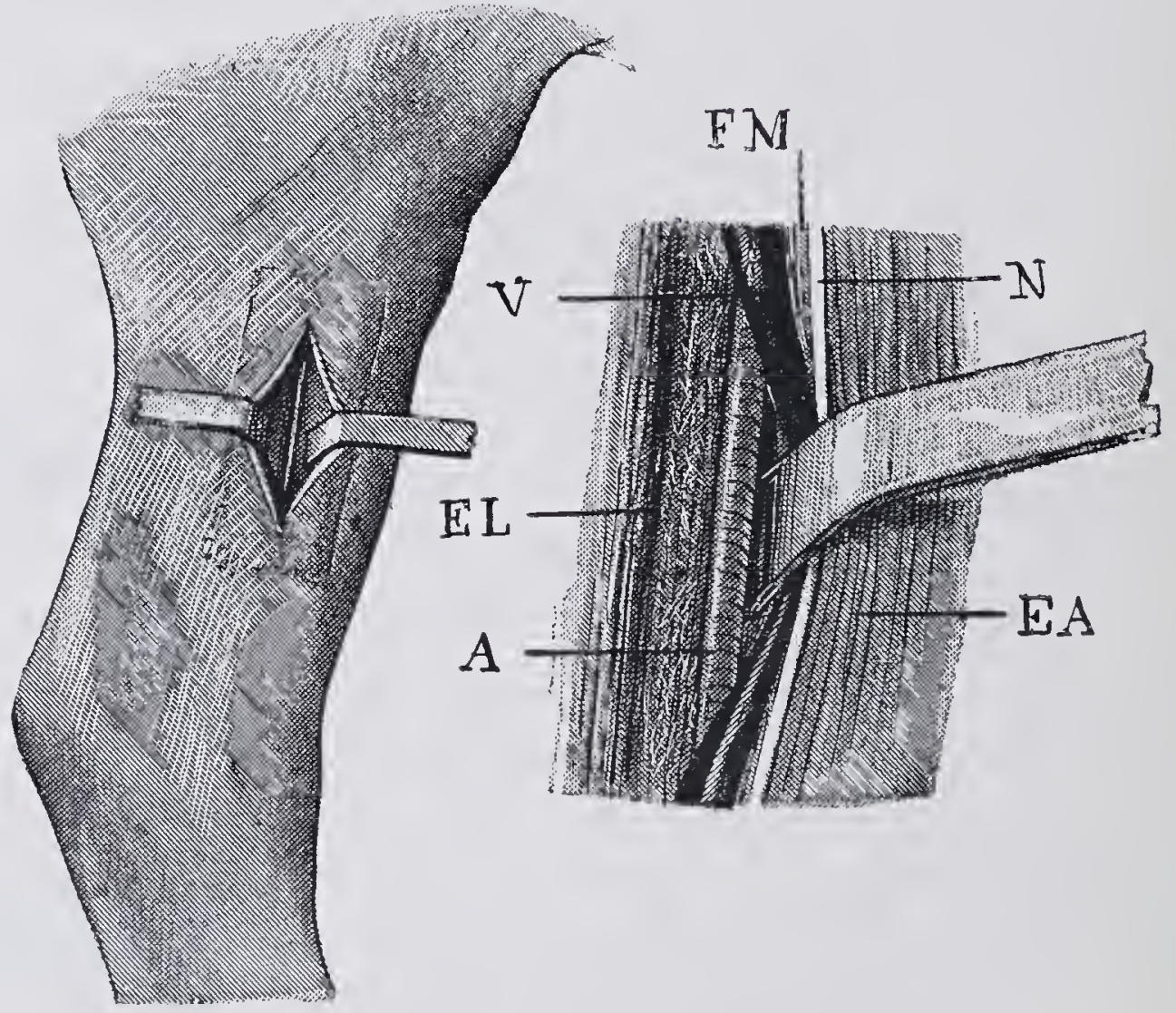

Figs. 271 AND 27.2. - Neurectomy of the anterior tibial nerve. E A, Extensor pedis muscle; E L, tendinous portion of flexor metatarsi; F M, muscular portion of Hexor metatarsi; $\mathrm{N}$, anterior tibial nerve; $V$, anterior tibial vein; $A$, anterior tibial artery. (The operation should be prrformed a little nearer the hock than indicated on fig. 271.)

metatarsi prominently forwards as soon as the extensor pedis is reflected.

Neurectomy of the posterior tibial nerve is performed as follows :-

The nerve is discovered on the inner side of the hind limb about 4 inches above the point of the hock, where it passes downward just in front of the Achilles tendon. By grasping the tendon from belind and allowing the soft tissues gradually to slip between the fingers and thumb, the nerve can be felt as a firm cord. At this point it lies about 1 to $1 \frac{1}{4} \mathrm{inch}$ in front of the Achilles tendon, and rather nearer the inner side of the limb, from which the operation is performed.

The horse is cast with the affected limb undermost, and the upper hind limb is fixed to the upper fore, so as to leave the field of operation clear. To check bleeding a rubber cord may be tightly applied about the middle of the lower thigh. A liberal space around the seat of operation is shaved, washed and disinfected, and about 4 inches above the top of 


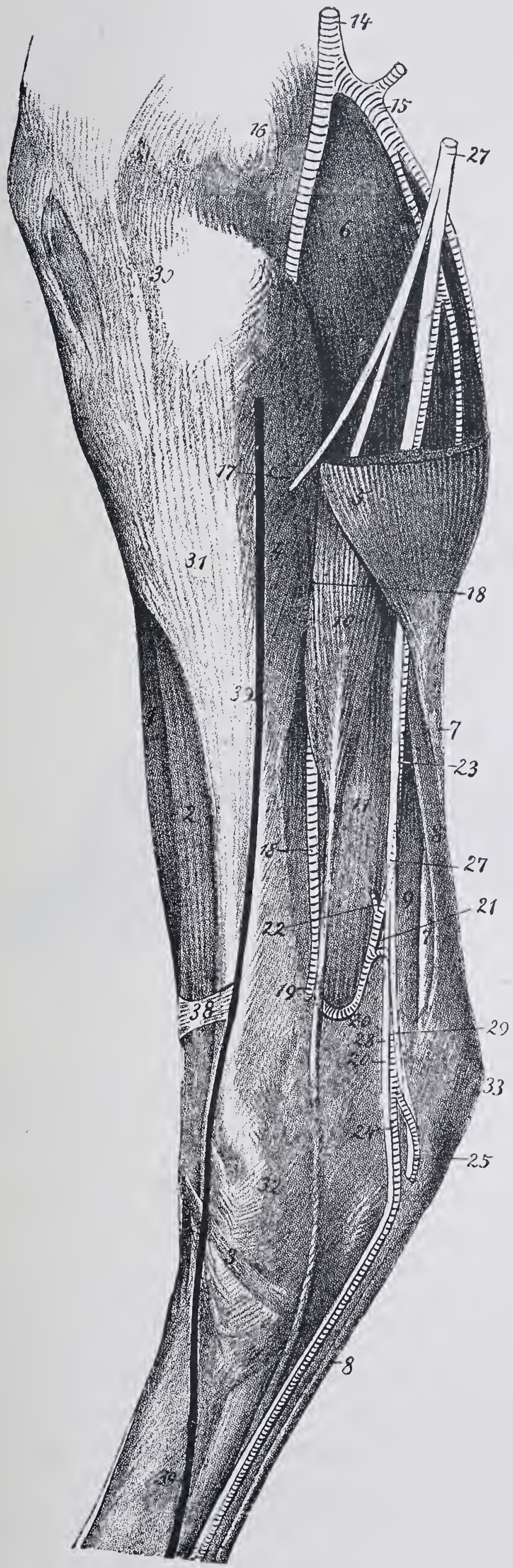

1. Extensor pedis muscle.

2. Muscular portion of the flexor neta. tarsi.

3. Internal terminal tendon of flexor metatarsi.

4. Popliteus musele.

5. Imner head of gastrocnemius.

6 . Onter head of gastrocnemius.

7. Tendo Achillis.

8. Tendon of flexor perforatus muscle.

9. Reinforcing band of flexor tendons.

10. Flexor accessorius musele.

11. Flexor perforans muscle.

14. Femoral artery.

15. Femoro-popliteal artery.

16. Popliteal artery.

17. Anterior tibial artery.

18. Post-tibial artery.

19, 20, and 21. Its S-shaped enrve.

22 and 23. Anastomosing twigs.

24 and 25. Internal and external plantar arteries.

27 Internal popliteal nerve (continued lower towards the hock as the posterior tibial nerve).

28 and 29. Internal and external plantar nerves.

30. Stifle-joint

31. Tibia.

32. Hoek.

33. Point of hoek.

38. Annular band of hoek.

39. Saphenons vein.

FIG. 273 
the os calcis and 1 full inch in front of the Achilles tendon an incision 2 to $2 \frac{1}{2}$ inches long is made parallel to the tendon. After ligaturing any bleeding vessels, two retractors are introduced into the wound and the edges drawn back to allow the subcutaneous tissue to be divided down to the underlying fascia. After again ascertaining by palpation the exact position of the nerve the fascia is divided in the same direction and to the same extent as the skin wound. A fragment of fascia about $\frac{1}{2}$ inch wide may then be removed with scissors, wheleupon the nerve, recognisable by its white colour and fibrous consistence, usually projects through the opening. A thread is passed under it with a blunt-pointed needle; the struggling which usually follows shows that the nerve has really

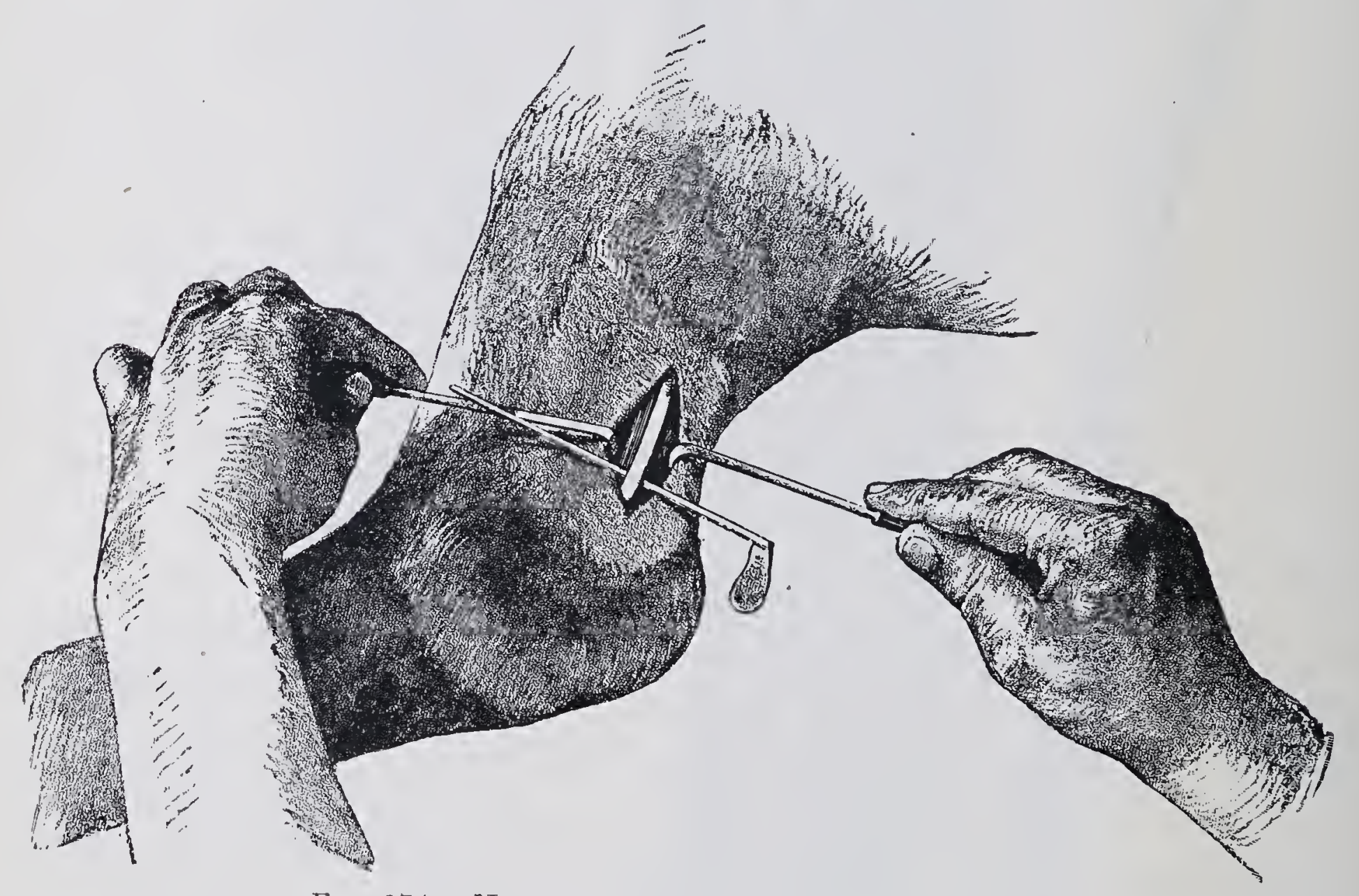

FIG. 274.-Neurectomy of the posterial tibial nerve.

been alighted on. A fragment an inch in length is then dissected free and divided as high up as possible with one stroke of the knife or with scissors.

As healing by first intention is rather difficult to attain, sutures are only necessary where rather severe bleeding occurs.

The formation of a neuroma on the central end of the divided nerve is uncommon, and when occurring is usually due to the fact that the end of the nerve has been left too long, so that it projects into the operation wound. Should it occur, however, it may necessitate a second operation for its removal, and in this event it is usually best to operate at a higher point, as the formation of fibrous tissue usually renders it difficult to find the divided end.

Double neurectomy of the anterior and posterior tibial nerves is not 
without danger, trophic changes in the extremites, local sloughing, and loss of the hoof sometimes occurring. 'I'he operation has nevertheless proved successful when all other methods have failed. Bosi, who introduced it, has had a number of very favourable results, as have Fröhner and Schimmel. Necessarily it does not modify the changes in the joint, though it may remove the lameness.

- The shoeing is of some importance. Klemm recommends raising the heels, and giving long quarters and a short toe, a suggestion supported by experience. Köster also recommended shortening the toe of the foot before treatment, and using long, wide shoes, with heels and toe-pieces.

\section{VI.-ENLARGEMENTS ON THE OUTER SURFACE OF THE HOCK.}

The above title includes all circumscribed thickenings on the outside of the hock-joint. They may be situated in the ligamentous apparatus, particularly in the outer lateral ligament, or may arise from the cuboid, outer small metatarsal, or other bone of the hock, but seldom or never from the joint. Chronic skin thickenings, and prominence of the external malleolus of the tibia, or of the small metatarsal, are often mistaken for the enlargements in question. Careful examination shows the nature of the condition, which usually results from kicks or other injuries of the outer surface of the hock. Sometimes it accompanies spavin, or forms a complication of that disease, in consequence of the chronic arthritis extending to the outer surface of the joint.

It not infrequently happens that the head of the outer small metatarsal bone is abnormally prominent, or is rendered so by the development of exostoses. It then forms a projection beyond the general vertical line of the hock, best seen when the joint is viewed from behind. This is the condition known in France as "jarde." The stock of some stallions "Saxifrage" in particular, often shows this peculiarity. Gillet, Sipierre, Goubaux and Barrier, all of whom studied the condition, state that it does not affect the hock-joint, but is invariably confined to the head of the small metatarsal and the ligament connecting the latter with the large metatarsal. Other authors, however, believe that it may extend to the hock-joint and produce on the outer surface a condition similar to spavin.

Hertwig insists that horses often go lame from this condition, and show jerking movements of the limb similar to those seen in spavin. Möller has only seen lameness where the enlargement was accompanied by spavin. As a rule, when lameness is present there is acute inflammation of the outer lateral ligament or of the periosteum. 
Diagnosis. The examination for this enlargement is similar to that for spavin. The outer surfaces of the hocks should be compared. As a rule, it is easy to discover whether the skin alone is thickened, or whether the deeper-lying structurès are involved.

The prognosis is usually favourable. Only when spavin is also present is the prognosis doubtful.

Treatment is usually unnecessary. If the ligaments and periosteum are acutely inflamed, they should be treated accordingly. In most cases rest and blistering remove the lameness, though thickening seldom disappears, and a slight blemish may always remain.

\section{VII.-CURB.}

In horsemen's parlance, this name includes all swellings on the posterior surface of the hock-joint. Seen from one side, the back of the hock-joint should appear as an absolutely straight line, stretching from the tuber calcanei to the fetlock. About 3-4 inches below the point of the calcis a swelling or convexity sometimes appears. (The French "courbe" is not identical with the English "curb." It represents a bony enlargement on the inner surface of the lower extremity of the tibia.)

In certain cases enlargements occur in and about this region, due to excessive development of the external small metatarsal, to thickening of the skin, or of the flexor pedis perforans or perforatus tendons, or to distension of the bursa tendinea of the flexor pedis perforans, which lies at this point, and is connected with the calcano-cuboid ligament. These conditions, which are usually easy of recognition, must, however, be distinguished from curb, which results from thickening of the calcanocuboid ligament (ligamentum tarsi plantare). The upper end of this ligament is attached to the posterior surface of the os calcis, whence it passes downwards, to become attached to the cuboid, to the external small metatarsal, and to the scaphoid, as well as to the large metatarsal. In consequence of sprain, it often becomes inflamed, and afterwards chronically thickened, or the inflammation may possibly induce changes in the joint itself. Thickening of the flexor pedis perforans tendon, and especially of its reinforcing ligament, is usually easy to differentiate from curb, inasmuch as it extends further downward, i.e., can be traced along the tendon itself.

Causes. The lower portion of the limb, from the point of the os calcis to the ground, may be regarded as a long lever. The pull of the gastrocnemius muscles acting on the point of the calcis tends to break this lever in two across the fulcrum, represented by the lower end of the tibia. The tissue which resists the brealing strain and holds the os calcis in line with the other bones of the limb is the calcano-cuboid ligament. It is 
easy, then, to estimate the great strains to which this ligament is subject, and to understand why it frequently becomes strained. Horses with "tied-in" and "sickle-shaped" hocks are especially predisposed to curb, and, if incautiously worked when young, generally develop it. 'The reason appear's to be that, as the calcano-cuboid ligament serves to unite the metatarsus to the os calcis, and as the calcis acts like a lever, to the end of which are attached the extensor tendons, especially the tendon of the gastrocnemius, the more nearly the bend of the hock approaches a right angle, the more powerfully does the gastrocnemius act, and, consequently, the more likely is this ligament to become strained.

In "tied-in" hocks the lower" row of bones and the upper end of the metatarsus are too slight; the distance between the calcano-cuboid ligament and the anterior margin of the hock, which represents another lever, is too short. The less this distance, the more liable is the ligament to be strained and inflamed when the limb is forcibly extended during movement.

The external causes consist in severe exertion violent attempts at extension at moments when the limb is flexed and sustaining weight, as in jumping; or in heavy or uphill draught-work. Curb sometimes results from the horse being suddenly thrown on its haunches. The more powerful the muscles of the quarter and limb, the greater the strain on the calcano-cuboid ligament, a fact which explains the frequency of curb in very powerful horses. Curb sometimes occurs as a complication of spavin, in consequence of inflammation extending to the back of the joint and to the calcano-cuboid liga-

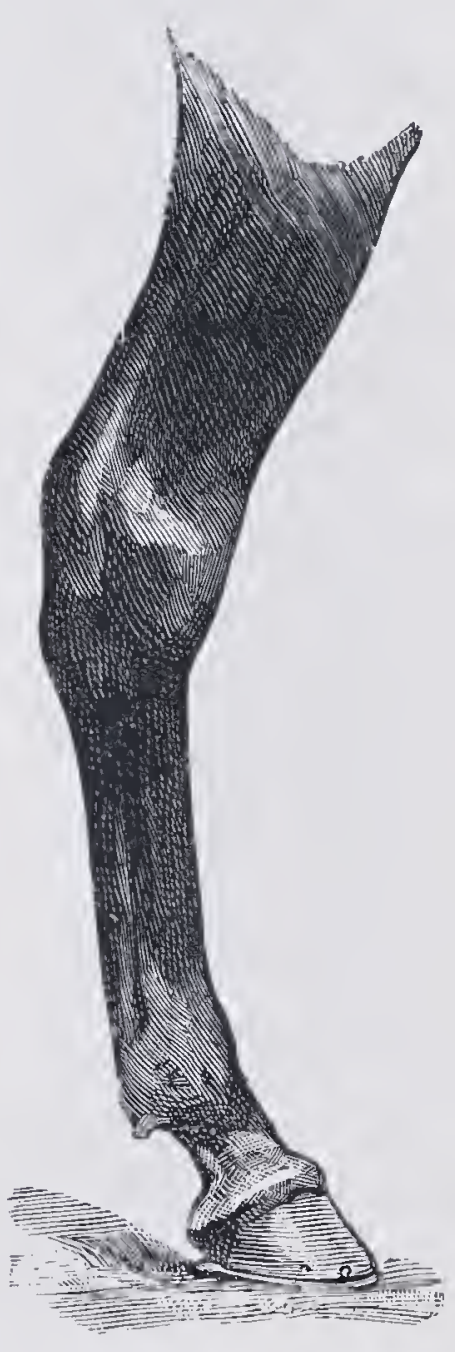

FIG. 275.-Curb. ment. This is the cause of the so-called bony curb, which, however, might better be included under the term spavin.

Symptoms. (1) Change in the form of the joint. Curb appears as a swelling in the otherwise straight line of the hock when seen from the side (fig. 275). Careful examination and palpation differentiate strain of the calcano-cuboid ligament from disease of the tendons, tendon sheaths, or skin. It is more difficult to distinguish between bony curb and strain of the ligament, though in the former additional exostoses usually exist on the imner side of the joint.

(2) Lameness is comparatively rare in curb, which in working-horses, therefore, forms little more than a blemish. Lameness, when occurring, is either due to the accompanying spavin, or is caused by inflammation in the calcano-cuboid ligament. This explains why it so often resembles 
spavin lameness, and occur's only during the development of curb. Curb, when slowly developed, may not interfere with movement, but when caused by violent injury, and accompanied by sprain or rupture of the calcanocuboid ligament, or of the reinforcing ligament of the flexor pedis perforans, is often accompanied by sudden and severe lameness. Pain is shown when weight is thrown on the $\operatorname{limb}$ and the fetlock-joint is flexed. In such cases pain and increased warmth may be detected on pressure over the affected spot. These symptoms, like the lameness, generally disappear in two to four weeks, but the swelling persists. Young horses which work hard are liable to suffer fromi relapses.

Prognosis. The gravity of curb varies greatly. In general, and particularly in old working-horses, it is simply a blemish, but animals exhibiting it should not be used for breeding, especially if the formation of the hock-joint be defective. In young animals, and in cases accompanied by chronic or intermittent lameness, prognosis depends partly on the extent to which the animal's usefulness is affected, partly on the formation of the hock-joint and the work to be done. Animals with weak "tied-in", hock-joints, if put to severe work, often suffer repeatedly, and become perfectly useless. The above is true in a still higher measure of bony curb, appearing as a complication of spavin.

The treatment varies with the nature of the condition. In acute inflammation, antiphlogistic measures, the use of cold poultices, \&c., with resolvent ointments and massage, are indicated. Cantharides blisters or the actual cautery are useful later. But it must be borne in mind that such applications are only of use in lameness consequent on acute processes. They have little effect in removing well-marked swellings, and can never completely cure old curbs. The animals must be rested as long as they show lameness. Bony curb is treated like spavin.

\section{VIII.-CAPPED HOCK.}

Like curb, the term "capped hock" is collective, and includes all swellings on the point of the os calcis, whatever their cause. Below the skin covering the tuber calcanei in the horse is usually to be found a subcutaneous bursa, lying on the upper or posterior surface of the tendon of the flexor pedis perforatus; under this, again, is a true bursa for the tendon, which glides over the cartilaginous cap of the tuber calcanei (fig. 278, e). The condition known as "capped hock" may have its origin in any of these structures. It may, therefore, consist-

(1) Of inflammation or chronic thickening in the cutis or subcutis; inflammation in the lower portion of the thigh is sometimes followed by swelling, due to gravitation of extravasated fluid (false capped hock).

(2) Of hydrops of the bursa subcutanea; this is one of the commonest causes of capped hock. 
(3) Of a swelling originating in the flexor pedis perforatus at the point where the latter covers the point of the hock, forming a cap.

(4) Of hydrops of the bursa tendinea of this tendon; or, finally,

(5) Of thickening of the point of the hock, due to disease of the tendon of the flexor pedis perforatus.

Causes. Capped hock results from external injuries produced by kiclis or striking against hard objects, from slips, violent exertion, and occasionally as a sequel to purpura hæmorrhagica. Confirmed kickers and irritable mares often have both hocks capped -an indication which it is well to note when handling such animals. The horses often strike themselves against the side of the truck or vessel when travelling by rail or water, and produce capped hock. At one time it was thought that in mares the condition was produced by the urine.

Symptoms. Though the swelling is recognised at a glance, especially if the hock be seen from the side, yet careful examination and palpation are required to determine the exact cause to which it is due. Fdema and inflammation of the skin are easily detected. Hygroma of the bursa subcutanea has an elastic character, and the swelling lies just under the skin, while hydrops of the tendon sheath is deeper seated, and is covered by the flexor pedis perforatus. Swelling of the tendon itself is characterised by greater hardness-a feature still more marked in exostoses on the tuber calcanei.

In acute inflammation, increased warmth and pain can be detected. Purulent cellulitis

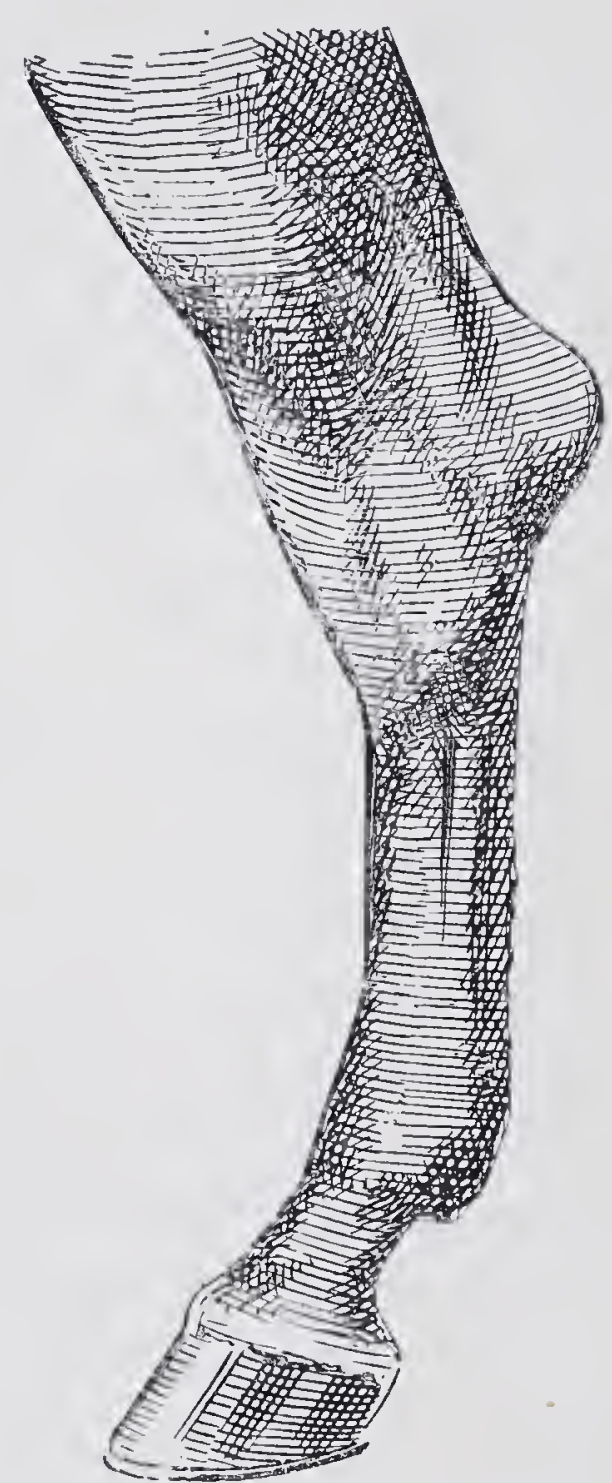

Fig. 276.--Capped hock (dropsy of the subcutaneons bursa of the hock). and disease of the bursa subcutanea may lead to well-marked and widelydistributed swelling; the tumour on the point of the hock is then rounded in shape, and may attain the size of a child's head. In aseptic inflammation, on the contrary, swelling is confined to the original spot.

Lameness is rarely marked, and never occurs in simple injury of the skin or mucous bursa. Even when purulent inflammation sets in, it seldom produces lameness. On the other hand, inflammation of the flexor pedis perforatus, of its tendon sheath, or of the tuberosity of the os calcis, may produce great difficulty in movement; infectious conditions in these structures are generally accompanied by severe lameness; but in all aseptic processes lameness is absent, or only occurs during development. 
Prognosis. True capped hock can seldom be cured, though the removal of cutaneous thickening is less difficult. Hygroma of the bursa subcutanea is usually obstinate, but can sometimes be improved, or even completely cured. Disease of the flexor pedis perforatus and of the bursa tendinea is more serious, and generally incurable, as is thickening of the os calcis, though recent cases sometimes improve under proper treatment.

As these conditions seldom produce lameness, the working powers are not much interfered with. Even in draught-horses they are far less grave than curb, \&c., and should not exclude the animal from the stud if the hocks be otherwise perfect. Infectious processes in the flexor pedis perforatus and $\mathrm{jn}$ its tendon sheath are alone dangerous.

Treatment. As a preventive the sides of the stall should be padded, and the animal, if inclined to kick at night, should be hobbled. Hock boots may be worn. Where acute inflammation exists, cold applications are indicated, as in curb. Wounds must be carefully disinfected. In disease of the skin and mucous bursa, resolvent applications and massage are most useful.

Recent cases of hydrops in the subcutaneous bursa and bursa tendinea should be treated by blistering, by the compound cantharides and euphorbium plaster, or by cantharidated collodion. The last two form a firm, unyielding covering on the surface of the skin, which exerts continuous pressure on the inflamed swelling, and assists resorption of fluid from the bursa. Cantharidated collodion is even more convenient than the plaster, though it must be repeatedly applied at short intervals, and the neighbourhood of the swelling covered so as to produce a sufficiently firm covering. Moderately active preparations are preferable to severe blisters. Exercise is very useful, except during the inflammatory
stages.

Practitioners differ as to the advisability of surgical interference, mainly because the different conditions are not always differentiated.

Puncture of hygromata with the hollow needle or slender trochar is seldom of service, the contents being soon replaced. It might perhaps be advantageously supplemented by the use of plaster or collodion, applied immediately after operation.

In capped hock the knife has been employed with very varying success. Laying open the bursa subcutanea is seldom dangerous, and sometimes removes hygromata; but the result is not certain, and depends principally on the degree of inflammation excited. Lanzillotti-Buonsanti recently operated successfully in two cases of enlargement of the subcutaneous bursa. Proceeding with strict antiseptic precautions he made a half-moon-shaped incision on the outer surface of the swelling; and through it he enucleated the bursa. Sutures were applied after the 
horse had risen, and a drainage-tube was inserted in the lower angle of the wound. A dressing was not applied. Healing occurred by primary intention. Opening of the bursa tendinea, however, is very dangerous, and in incising the bursa subcutanea care must be taken not to injure the extensor pedis perforatus. The doubtful success which has attended operation for capped hock is principally to be referred to the tendon sheath having been opened. It is better to confine treatment to external applications.

Corps-Rossartz Hell ruptures hygromata on the point of the hock by violence. For this purpose a bandage is passed round the hock whilst the animal stands on the limb, the other hind foot being lifted up. If the foot be then released, the horse makes such violent attempts to flex the bandaged limb that the bursa may be ruptured and its contents discharged. The swelling does not return. One or two cases have been reported of this method being successfully employed. It appears by no means impracticable. In men, hygroma has for a long time been treated on the same principle.

Wounds of the point of the hock may here receive consideration, as they are often of importance in the treatment of capped hock. As already stated, infectious processes in the skin and bursa almost always take a favourable course, and, though liable to be followed by some thickening, do not cause further trouble, even when the swelling is of considerable size. The same is true of wounds. It is quite otherwise in injuries of the bursa and tendon of the flexor pedis perforatus where it passes over the point of the hock, and suppuration or septic infection at this point is highly dangerous. Marked pain soon sets in, causing the leg to be continuously rested, and movement, as far as possible, avoided. The swelling extends over the joint towards the tibia, fever sets in, laminitis may attack the other foot, in consequence of the continued weight on it, and the animal prove unable to stand. It then lies continuously, and, if not destroyed, soon dies from the effects (decubitus).

The post-mortem examination shows the cartilaginous covering of the tuber calcanei to be partly or entirely destroyed, and the flexor pedis perforatus tendon more or less abraded. The severe pain and dangerous character of the disease are essentially due to movement of the tendon over the surface of the tuber calcanei, now denuded of cartilage.

Any injury of the perforatus tendon, or even of its sheath, may provolie such results, and it is therefore scarcely needful to say that in recent wounds every effort must be made to prevent infection. 


\section{IX.-LUXATION OF THE FLEXOR PEDIS PERFORATUS TENDON.}

The flexor pedis perforatus tendon, at the spot where it plays over the point of the hock, is expanded into a kind of cap. A short but powerful prolongation of the tendon is inserted into the os calcis on either side, just in front of the point of insertion of the tendo Achillis, which prevents the tendon slipping off the tuberosity of the calcis bone. Sometimes one of these ligaments is ruptured, allowing the tendon to glide off the calcis towards the opposite side, and to lie on the lateral surface of the hock. Günther describes a case of dislocation towards the inner side; others have seen it occur outwardly. The direction depends, of course, on which attachment is ruptured. As the tendon passes over the hock from within outwards, one would expect that the external attachment would most frequently suffer, and that dislocation would be towards the inner side. On the inner side the tendon either remains fixed about half-way up the astragalus, or slips down as far as the articular prominence of that bone.

Such ruptures occur during heavy work, or follow external injuries like kicks, \&c.

Symptoms. Displacement is rarely followed by severe lameness; the gait is insecure, weak, and rolling, and the animal appears to have lost full control of the limb. Standing behind the horse, the tendon is seen to glide off the tuber calcanei every time the hock is extended, and often to return to its normal position when the joint is flexed. In other cases the tendon, though displaced, can easily be returned to its position on the point of the calcis; but almost immediately becomes again luxated. When the tendon and neighbouring parts are inflamed from kicks, \&c., the swelling and pain produce a more marked lameness, otherwise movement is only mechanically interfered with. While the horse stands on the limb, the lower joints show dorsal flexion, consequent on relaxation of the flexor pedis perforatus, but the hock-joint is extended.

Prognosis. Recovery is uncertain on account of the difficulty of fixing the hock for a sufficient length of time to allow the ends of the ruptured ligament to unite. Even though, by flexing the thigh, the tendon can be replaced, it tends to slip out of position the next time the thigh is extended or weight is placed on the limb. As a rule, little improvement can be expected, and only in cases where inflammation produces local pain does the lameness even diminish.

As a rule, the animals can only do light walking-work, and are quite useless for riding or for heavy dranght.

Treatment is confined to checking the local inflammation sometimes seen in recent cases. Drouet suggests suturing the tendon, and 
Professor McQueen assures me (Jno. A. W. D.) that the tendon can be replaced and fixed by sutures, and that useful recovery follows.

\section{X.-STRINGHALT.}

The term "stringhalt" has been applied since olden times to that peculiar involuntary movement of one or both hind limbs, in which the affected member is flexed with excessive suddenness, and lifted abnormally high, whilst it still continues able to support the animal, and otherwise allows of movement in the usual way. Similar movements in the fore limbs are rare, but Frick states having seen two cases where they occurred. Sometimes the peculiar movement is seen while trotting, sometimes also while walking; as a rule, it persists in spite of work; sometimes it disappear's for a time, but generally returns with rest; often it is only noticeable when the animal is turned in small circles.

The immediate cause of this interference with movement has been sought for in different organs, but up to the present has not been clearly identified. Abildgaard considered the condition to be an "affection" of the muscles and nerves of the hind limb. Villate thought it was caused by abnormal development of the spine of the tibia. Sewell and Spooner, when making the post-mortem of a horse affected with stringhalt, found that the point of origin of the great sciatic nerve in the spinal cord was infiltrated with blood, and regarded this as the cause of the disease. Falke identified the condition with sciatica in man. Renner took it to be due to chronic inflammation of the great sciatic nerve, whilst Hochstetter attributed it to straightness of the hocks; Boccar sought the cause in contraction of the peroneus, after discovering that the condition disappeared on section of its tendon. Foelen and many others agreed with him, and reported favourable results from dividing the tendon. Goubaux, on the other hand, directed attention to the joints, especially to the hip-joint, which in his view became chronically inflamed in stringhalt.

Up to this time investigators had viewed the condition as due to a single cause, but $\mathrm{K}$. and F. Günther divided it into the following varieties:-

(1) Spavin-stringhalt, i.e., stringhalt caused by spavin.

(2) False stringhalt, produced by pain in any region of the limb; and

(3) True or involuntary stringhalt, due to irregularities in the functions of the antagonistic nerves of the lumbar and pelvic plexuses. Günther states having frequently seen hyperæmia of the great sciatic nerve without stringhalt, but says that stringhalt is sometimes accompanied by relaxation in the semi-tendinosus muscle. K. and F. Günther first drew attention to the connection between stringhalt and certain surgical diseases-as, for instance, those of the foot.

In a similar way, Dieckerhoff divided the disease into-

(1) True idiopathic stringhalt, which he considered due to contraction in the fasciæ of the thigh.

(2) Complicated stringhalt, accompanying spavin, curb, ring-bone, and other diseases.

(3) Symptomatic stringhalt, consequent on inflammation in the pedal or other regions of the limb.

Bassi differentiated two forms:-

(1) True stringhalt, caused by interference with the movement of the patella; and 
(2) False stringhalt, caused by a number of other diseases, such as spavin, \&c., but principally by contraction in the tensor fasciæ latæ, and in the extensor pedis tendon. Other forms in horses he considers due to brain disease, resulting from asymmetry of the skull, and in dogs to changes in the spinal cord following distemper.

Möller divides stringhalt into idiopathic and symptomatic forms, and includes in the first those cases due to no visible cause; and, in the second, those where a cause can be detected.

Whatever be the cause, it is quite certain that the movement is involuntary. It must, therefore, be dependent on some mechanical action, e./., contraction of the fasciæ, of the tendons, or of the ligaments, or on reflex action, originating either directly in the spinal cord, or through irritation of peripheral nerves, i.e., interference with innervation. Probably both causes may at times be effective, though, as a rule, neither can be clinically identified. Amongst mechanical causes-for we may also term the first variety mechanical-changes in the extensor pedis tendon, the fascia of the thigh, and the patella or its ligaments deserve special attention. Even though Siedamgrotzky's attempt to produce contraction by exposing the peroneus tendon, and thrusting a piece of wood under it, gave negative results; yet numerous experiments show that section of this tendon often removes stringhalt. But the fact that it sometimes fails shows that stringhalt is not due to a single cause. Amongst the others must be included contraction of the fascia of the thigh, referred to by Dieckerhoff, of the tensor fasciæ latæ, much spoken of by Hertwig, and of the lateral ligament of the patella, mentioned by Bassi. Möller has had no practical experience of the last, though he has repeatedly seen stringhalt-like movement in horses suffering from liabitual upward luxation of the patella (see "Luxation of the Patella"); and as in these cases the form of the upper surface of the trochlea of the femur possibly plays a certain part, it seems possible that division of the inner lateral ligament of the patella might prove of service. Möller several times divided the fascia of the thigh when division of the peroneus tendon had proved insufficient, but rarely saw real improvement. When section of the tendon was unsuccessful, that of the fascia always failed. Sometimes the whole of these various structures may be divided without affording relief, in which case the condition is probably reflex, and either resembles chorea minor of man, or is due to disturbance in the co-ordination of movement, i.e., ataxia (see "Paralysis of the Hind Extremities"). Symptomatic stringhalt following disease of the foot, such as pressure on the lateral cartilage, is most easily explained by regarding the reflex act as originating in these parts.

The results of peripheral irritation chiefly affect the flexor muscles, because the extensors act principally whilst weight is thrown on the 
$\lim b$, and their contraction is therefore more easily controlled. Possibly the central disturbance manifested by the affection of certain groups of muscles may sometimes be of a secondary character, the peripheral irritation in time causing changes in the central nervous matter. At any rate, temporary disease of parts removed from the centre sometimes produces chronic stringhalt. Thus, after injuries to the foot, such as pricks, treads, or laminitis, wounds of the hock, fractures of the external angle of the ilium, or even after the application of a blister, one sometimes sees stringhalt, which continues despite removal of the original irritation. Ascheberg saw stringhalt result from tetanus. In such cases one might imagine that the peripheral irritation had produced permanent interference with innervation, and that the oft-quoted dictum of Jordanus

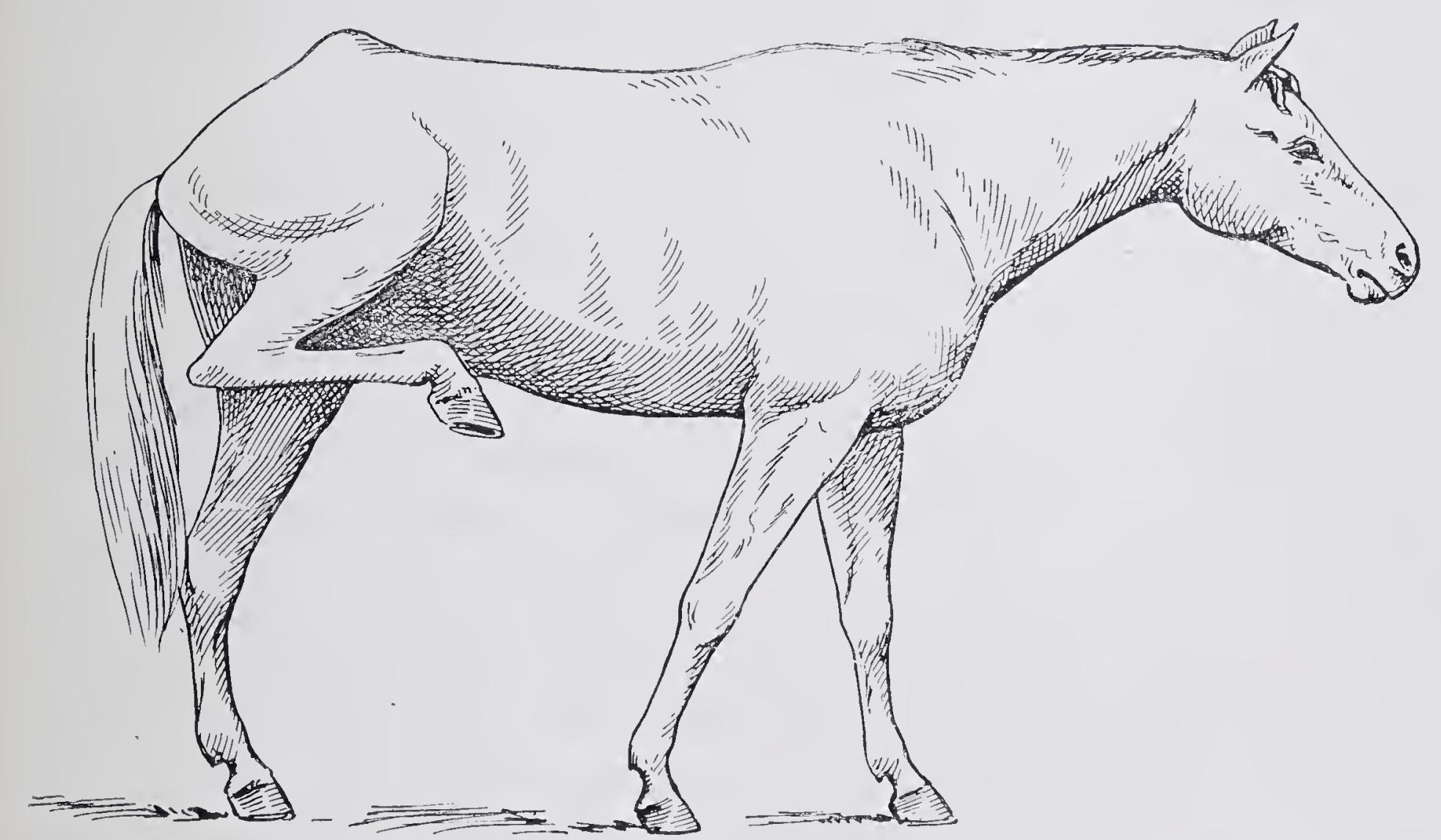

FIG. 277. - Stringhalt.

Ruffus, "Cessante causa cessat effectus," no longer applied. Dollar is of the opinion that many cases of stringhalt are due, like chorea, to localised sclerosis of the spinal cord.

Temporary stringhalt accompanies various conditions, but especially injuries to the foot; Wittlinger saw it after suppuration in the tendon sheaths of the fetlock region.

The course of the disease varies greatly. The symptomatic form accompanying sprain develops slowly, and sometimes disappears at the same time as the spavin lameness. Stringhalt often develops rapidly after external injury; some cases appear suddenly without visible cause, and are attributed (in Möller's opinion erroneously) to catching cold. Such cases are probably due to muscular rheumatism, which often produces movements resembling stringhalt. 
Prognosis is uncertain. The most favourable cases are those which have a visible cause, and which are of recent origin; such often cease as soon as the local mischief disappears. In symptomatic stringhalt, the prognosis chiefly depends on the nature of the primary disease, though it should not be forgotten that the interference with movement sometimes becomes permanent. In the absence of all apparent cause, prognosis is very uncertain, because it is never possible to say whether the methods of treatment at present known may or may not be successful, and treatment, therefore, becomes experimental.

Treatment must, therefore, in any case partake more or less of the nature of an experiment. The operation which has proved most generally successful in idiopathic stringhalt is tenotomy. It has one qualification-that, namely, of being easily performed. The operation may be performed in the standing position; the procedure is as follows:-

The hind foot is lifted as in shoeing, and the hair having been shaved from a point close below the hock, on the outer surface of the metatarsus, where the tendon in question is almost subcutaneous, the skin is washed and rinsed with carbolic solution. A twitch is then applied, and an incision made with a pointed bistoury or scalpel, immediately over the tendon and parallel to it. To penetrate the fascia lying under the skin, the point of the knife is carried right into the tendon. A slender bluntpointed tenotome is then passed under the tendon, i.e., between it and the bone, and the cutting edge being directed upwards, the tendon is divided. Excision of a portion of the tendon is of no advantage, and delays healing. Palpation of the parts will show whether section is complete. Bleeding being usually slight, the wound may be rinsed with a disinfectant and a dressing at once applied, under which healing will in four to five days be so far advanced as to allow of the wound being left open. If the instruments, the operator's hands, and the dressing be thoroughly sterile, healing happens by primary intention; and even when suppuration occurs, though the point of operation is left somewhat thickened, no further ill-effect is incurred. Complete recovery may follow either at once, or be postponed for some days, or even weeks.

The animal should be rested for eight to fourteen days after operation, and then turned loose in a box, or moved slowly on soft ground.

Möller several times divided the fascia of the thigh just in front of its union with the extensor pedis (Dieckerhoff's method) whilst the animal was standing, but restless horses should be cast for either operation. After section of the peroneus, the tenotome is passed under the fascia, which is divided from within outwards, and a dressing applied reaching down as far as the fetlock-joint, to prevent slipping off. The after. treatment is as above stated. 
Hertwig recommended cutting through the tensor vagine femoris, about 3 or 4 inches below the external angle of the ilium. This operation is more difficult, and is often followed by burrowing of pus, asepsis being almost impossible. The result is doubtful; cases are said to have been cured, though in one, which was successful, the incision was only carried through the skin on account of bleeding.

Bassi suggests dividing the internal lateral ligament of the patella. Experiments show that this operation is not easy, on account of the ligament in question being so ill-defined. The method is worth a trial in cases where the cause is thought to lie in the stifle-joint (see "Displacement of the Patella").

Starting from the hypothesis that in one case stringhalt was produced by inflammation of sensory nerves, Möller divided the anterior tibial nerve, but without success. Failure also followed subcutaneous injections of morphine and veratrine, and section of the internal insertion of the flexor metatarsi muscle. Section of the posterior tibial nerve above the hock has succeeded in cases where the cause of the disease was situated in the lower portion of the limb.

A condition in some respects resembling stringhalt has been called "straw cramp." The animals; while standing on straw, show peculiar symptoms, particularly when turning round. The affected hind limb is lifted convulsively, sometimes so violently that the animal falls. Painful conditions like inflammation in the skin of the heel or in the bend of the hock produce similar symptoms, which are then, of course, easily explained. But in some horses, particularly in coarse-bred animals, the attacks occur without any visible cause. The most careful examination fails to detect pain in the affected limb. In such cases tibial neurectomy produces no particular improvement. Similar symptoms are seen in luxation of the patella.

In a horse Möller had under observation, a similar effect was produced by irritation of the ear. If the finger were introduced into either ear, the hind limb of the same side was lifted and carried forward in an almost horizontal direction, the movement being convulsive. Though the condition only lasted a few seconds, the horse was quite useless, because the pressure of the bridle or halter on the ear immediately induced fresh attacks. Not the slightest anatomical change could be detected in the ears. 


\section{E. DISEASES OF THE METATARSUS.}

\section{I.-DISEASES OF THE TENDONS AND TENDON SHEATHS IN THE TARSAL AND METATARSAL REGIONS.}

In the horse, the tendon sheaths, both in the hock and metatarsal regions, sometimes become distended, producing conditions of great practical importance. 'Amongst the most important are--

(a) Distension of the tendon sheath of the flexor pedis perforans produces a swelling on the inner and posterior surface of the hock-joint. The sheath begins somewhat above the inner malleolus, passes downwards over the capsule of the joint, with which it sometimes communicates, then becomes somewhat flattened, and follows the course of the tendon, to appear at the posterior surface of the joint, about two inches below which it ends (fig. 279, b).

Disease of this sheath is rather frequent in the horse, and occasionally produces a hemispherical swelling as large as a child's head. Sometimes it attacks both legs simultaneously, without causing much lameness (fig. 282). It may in time decrease and even entirely disappear, especially under proper treatment. In other cases, the portion of the sheath lying at the back of the hock-joint becomes enlarged, and may be mistaken for curb, for which reason it has been called curb-gall, or soft curb.

In other cases acute inflammation sets in. Bruises and injuries may cause septic tendo-vaginitis, accompanied by severe lameness and high fever, which sometimes prove fatal. The condition is even more dangerous if inflammation extend to the joint (see "Wounds, \&c., of the Hock-joint"), or produce necrosis of the flexor pedis perforans tendon, followed by rupture. Aseptic inflammation of this sheath is often caused by drawing heavy loads, and is therefore commonest in draught-horses.

Prognosis. Infectious inflammation of this tendon sheath forms no exception to the general rule, and prognosis is therefore unfavourable, though cases have been seen to recover. The higher the fever and the greater the pain, the less probable is recovery. In aseptic disease, the degree of lameness is a fairly safe guide. Occasionally the local inflammation becomes so intense that the animal rests the leg continuously, and throws so much weight on the other foot as to induce laminitis; but this is rare, and more frequently the animals are able to work, despite considerable swelling. Complete absorption can scarcely be expected, though in exceptional cases it occurs in three to six months.

Treatment depends on the nature of the injury. Wounds near. 
the tendon sheaths, like wounds of the joint, require the strictest antiseptic precautions. In infectious inflammation, the tendon sheath may advantageously be washed out with disinfectants.

Acute aseptic inflammation usually calls for nothing more than
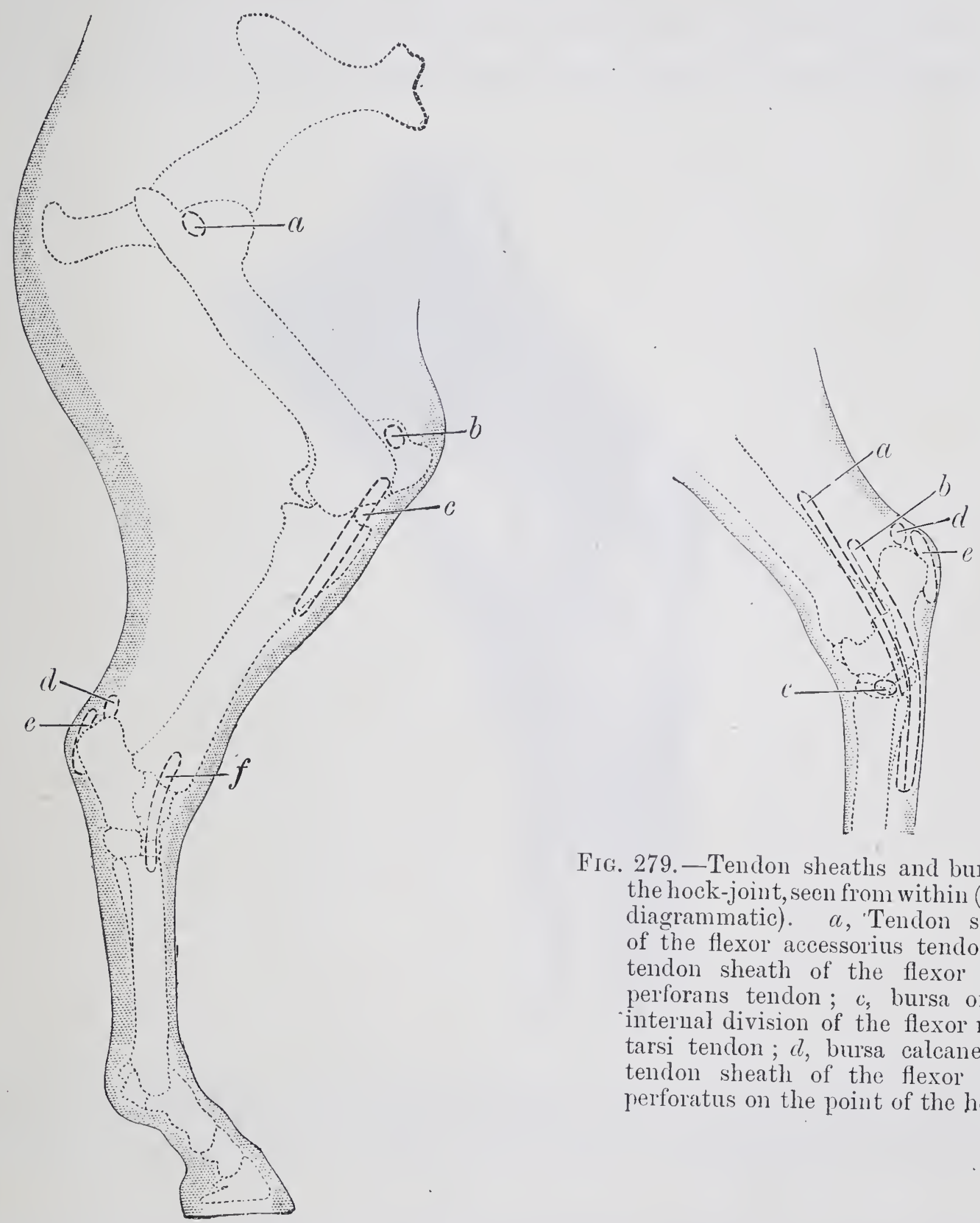

Fig. 279.-Tendon sheaths and burse of the hock-joint, seen from within (semidiagrammatic). $\quad a$, 'Tendon sheath of the flexor accessorius tendon; $b$, tendon sheath of the flexor pedis perforans tendon; $c$, bursa of the internal division of the flexor metatarsi tendon; $d$, buisa calcanea ; $e$, tendon sheath of the flexol pedis perforatus on the point of the hock.

Fig. 278.-Tendon sheaths and bursæ of the hind limb of the horse, seen from withont (semi-diagrammatic). $\quad \alpha$, Trochanteric bursa; $b$, prepatellar bursa; $c$, tendon sheath of the extensor pedis ; $d$, bursa calcanea ; $e$, tendon sheath of the flexor pedis perforatus tendon; $f$, tendon sheath of the peroneus tendon.

ordinary soothing treatment; but in cases where this proves insufficient, excellent results sometimes follow from freely firing in lines.

Chronic dropsy of the bursa is also benefited by the cautery. Peuch 
fires deeply with the pointed iron. Repeated withdrawal of contents, by means of Pravaz's syringe, is sometimes very efficacious. The operation may be repeated every four to six weeks, though careful antisepsis is required to prevent infection of the tendon sheath. For many years French operators have recommended withdrawal of the contents, followed by iodine injections. Cadiot describes the results as excellent. McCall withdraws almost the entire contents of the enlargement and then injects

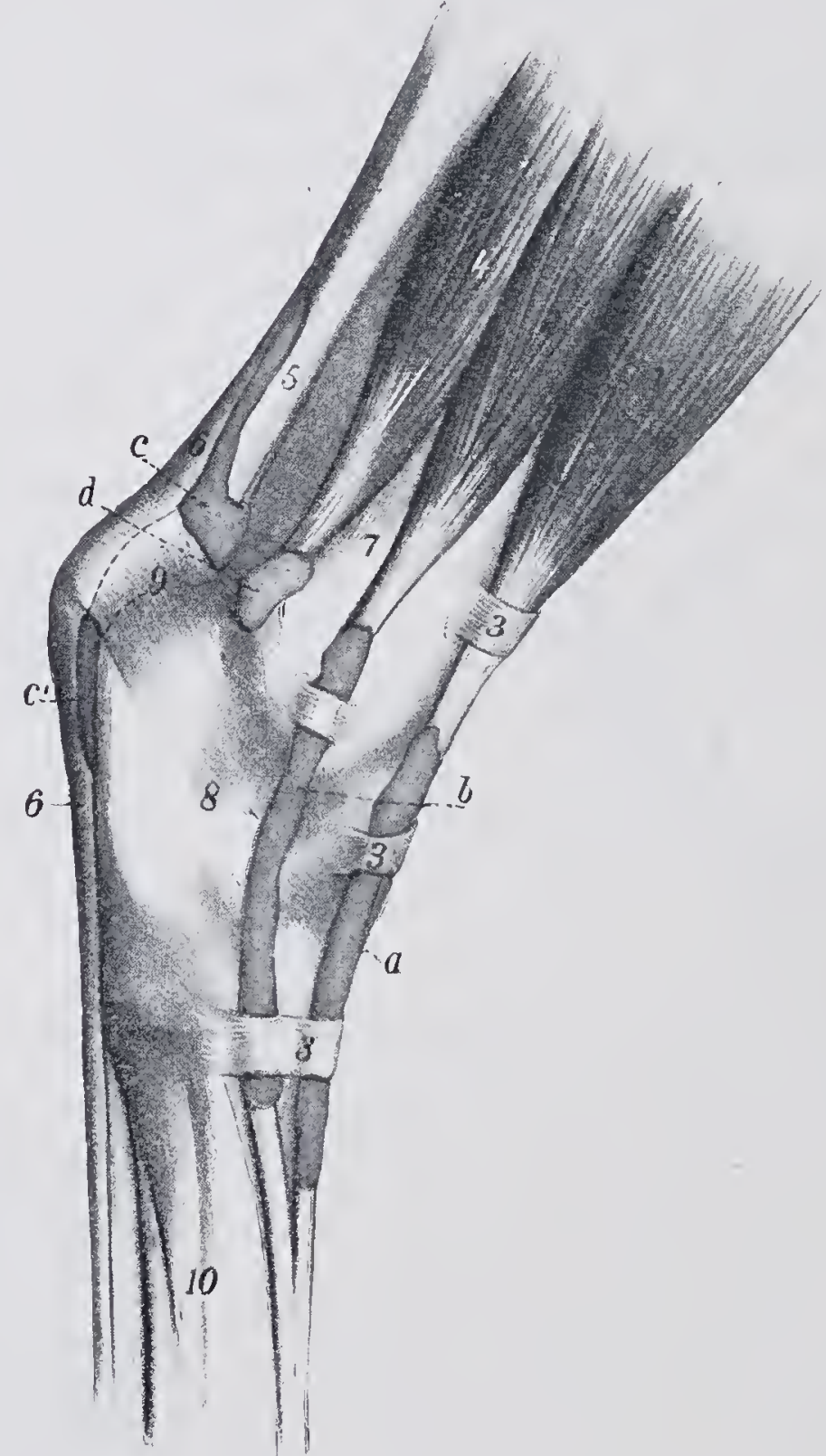

FIG. 280.-External surface of the horse's lock, to show synovial sheaths. $a$, Tendon sheath of the extensor pedis muscle; $b$, tendon sheath of peroneus musele; $c, c^{\prime}$, tendon sheath of flexor perforatus and gastroenemius museles; $d$, protrusion of the synovial eapsule of the true hoek-joint.

a $\frac{1}{2}$ per cent. alcoholic solution of sublimate, to which decolorised tincture of iodine has been added. Frick has several times withdrawn the fluid and then injected a solution containing iodine 2 parts, iodide of potassium 5 parts, and water 100 parts; thereafter he has lightly fired the parts in lines. He states that the results varied, but were never bad.

(b) Distension of the extensor tendon sheath in the hock region. The tendon of the peroneus muscle is provided, opposite the lower margin of the hock-joint and on its outer aspect, with a small sheath, which, when 
over-distended, forms an elastic swelling, varying from the size of a walnut to that of a man's fist. The condition is oftenest seen in ridinghorses, being caused when the animal is thrown on its haunches. As lameness is not a prominent symptom, the swelling is rarely treated, and only forms a blemish.

If necessary, the parts may be blistered and fired in lines. Operaion is not dangerous, though, when suppuration occurs, considerable

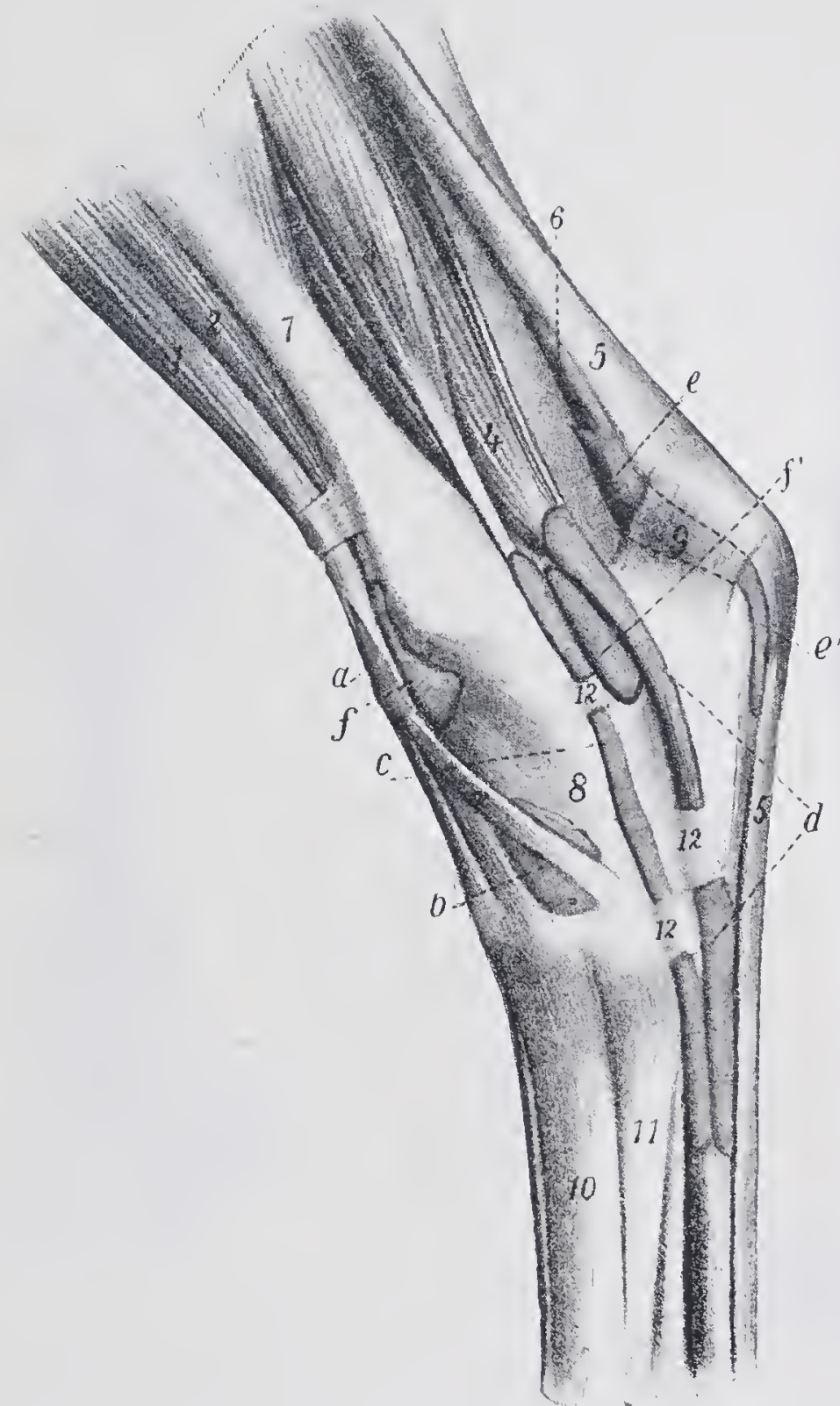

Fig. 281. - Internal surface of the horse's hock to show synovial sheaths. a, Tendon sheath of flexor metatarsi muscle; $b$, tendon sheath under inner terminal tendon of flexor metatarsi ; $c$, tendon sheath of flexor accessorius; $c$, tendon sheath of flexor perforans; $e, e^{\prime \prime}$, tendon sheath of flexor perforatus and gastrocnemius muscles; $f, f^{\prime}$, listended synovial capsule of the true hock-joint.

thickening always remains, and the remedy becomẹs worse than the disease, because everyone knows that the latter is of little importance.

(c) Distension of the sheath of the Hexor pedis perforatus. At the spot where the flexor pedis perforatus comes in contact with the posterior or upper surface of the tendo Achillis, and about eight inches above the point of the hock, a tendon sheath begins, which extends downwards, and sometimes becomes the seat of extensive swelling. In coarse-bred hor'ses, it sometimes affects both limbs, and forms above the tuber calcanei a longish swelling as large as a child's head, divided into two 
parts by the tendo Achillis and the perforatus tendon. The position and size of the swelling are best recognised by viewing the animal from behind. Sometimes the swelling is more marked on the inner, sometimes on the outer surface of the joint; the tendon can be distinctly felt in the depths. The swelling generally results from chronic bursitis, develops slowly, and seldom causes lameness.

For this reason prognosis is favourable, though the condition itself may prove extremely obstinate. Success sometimes follows repeated evacuation of the contents every three or four weeks. The operation is

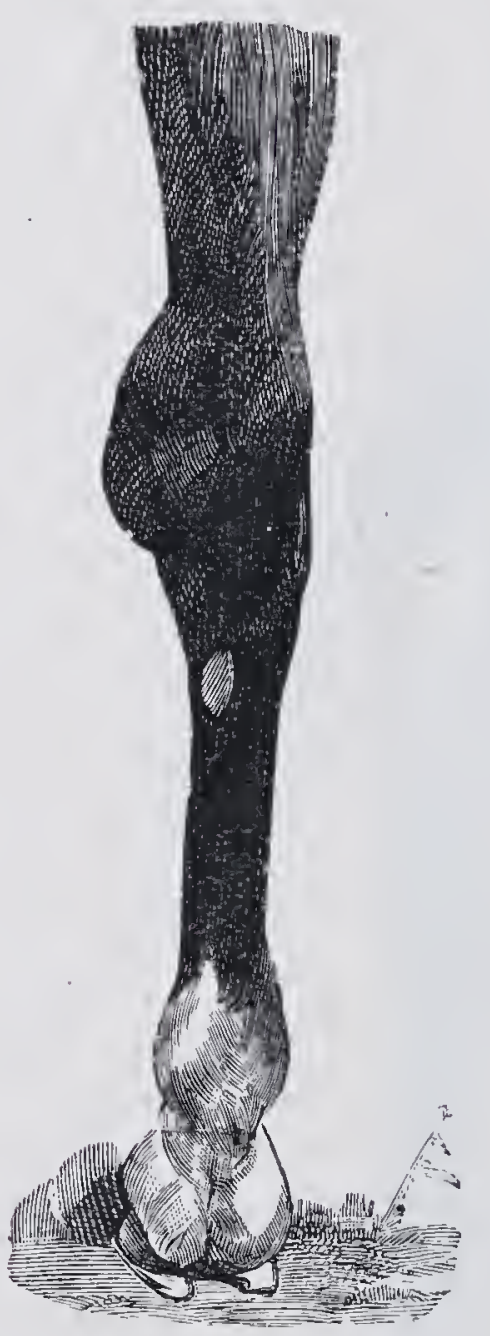

Fig. 282.-Distension of bursie of Hexor tendons in right hock (from a photograph).

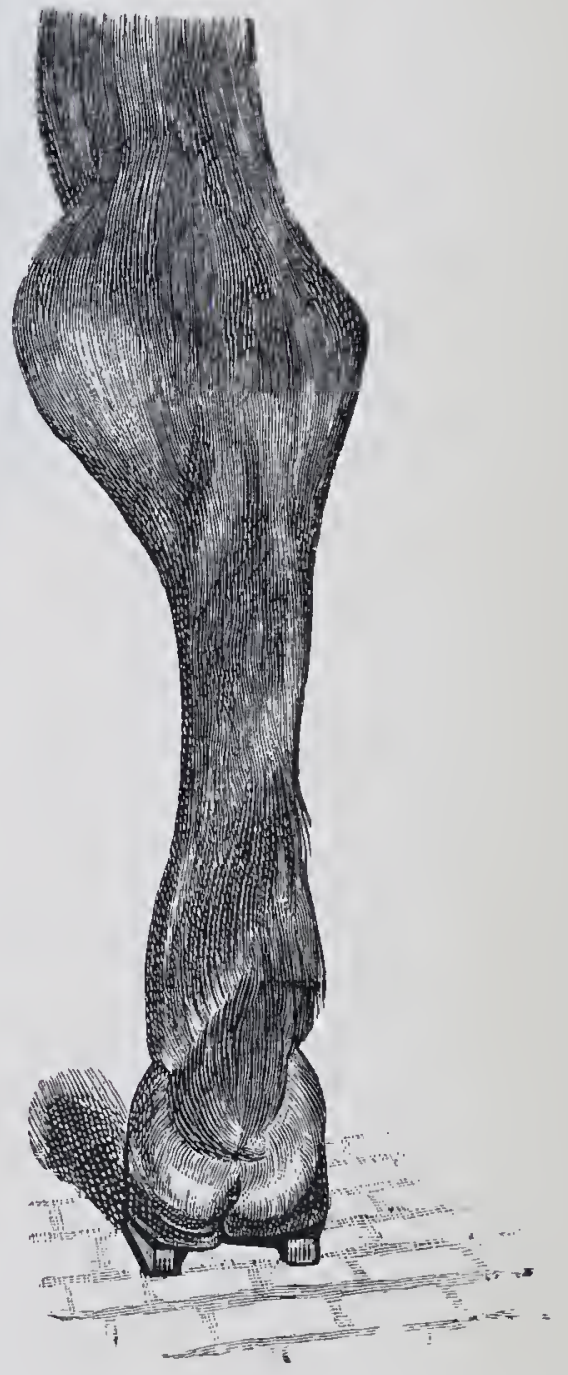

Fig. 283.-Distension of sheath of flexor pedis perforans (from a photograph).

not without danger, and Möller lost a horse from infectious inflammation of the tendon sheath. Line firing may diminish, though it seldom completely removes, the swelling.

(d) The tendon sheaths in the metatarsal and phalangeal regions become distended in a similar way to those of the fore limb, though the bursa of the extensor pedis, which lies in front of the metatarsus, at the fetlock-joint, is more often affected than in the fore limb. It gives rise to a well-marked swelling, sometimes as large as a child's head, which, though it does not cause lameness, greatly impairs the animal's appearance, and sometimes leads to inflammation of the skin, or even to abscess formation. I'hese swellings usually contain large quantities of fibrinous 
clot, which, however, cannot be detected by palpation. This explains why, in old-standing cases, neither blistering nor firing, nor even extraction of the fluid contents and injection of iodine solution, is successful, and why operation and removal of the organised contents are alone of use. Such operation is quite safe, even when suppuration occurs. Recovery follows, after strict asepsis, though considerable thickening may be left. When the swelling is extensive, a portion of the distended bursa and skin covering it may be removed, the edges of the wound brought carefully together, and a compress dressing applied (see also "Treatment of Enlarged Synovial Cavities in the Fore Limb").

Frick saw a peculiar affection of this bursa. The primary swelling and thickening of the walls of the bursa was unaccompanied by pain. Suppuration followed, and a chocolate-coloured, thin, fluid pus, containing white flocculi, was discharged. Slight lameness was then shown (supporting leg lameness), and the granulations which surrounded the wound became rather exuberant. As the wound obstinately refused to heal the horse was killed. On post-mortem examination the bursal cavity was found to communicate with the fetlock-joint. Both contained turbid, chocolate-coloured fluid. The thickened synovial membrane was covered with fungous granulations, the cartilage of the fetlock-joint showed local ulceration, and the portions of bone thus exposed were granulating. Microscopical examination of the discharges and granulations revealed the presence of tubercle bacilli.

(e) Distension of the sesamoid sheath is commoner in the hind than in the fore limb. As a rule, it is caused by irritation, and appears slowly. Juameness, which is seldom troublesome, is rarely seen unless the

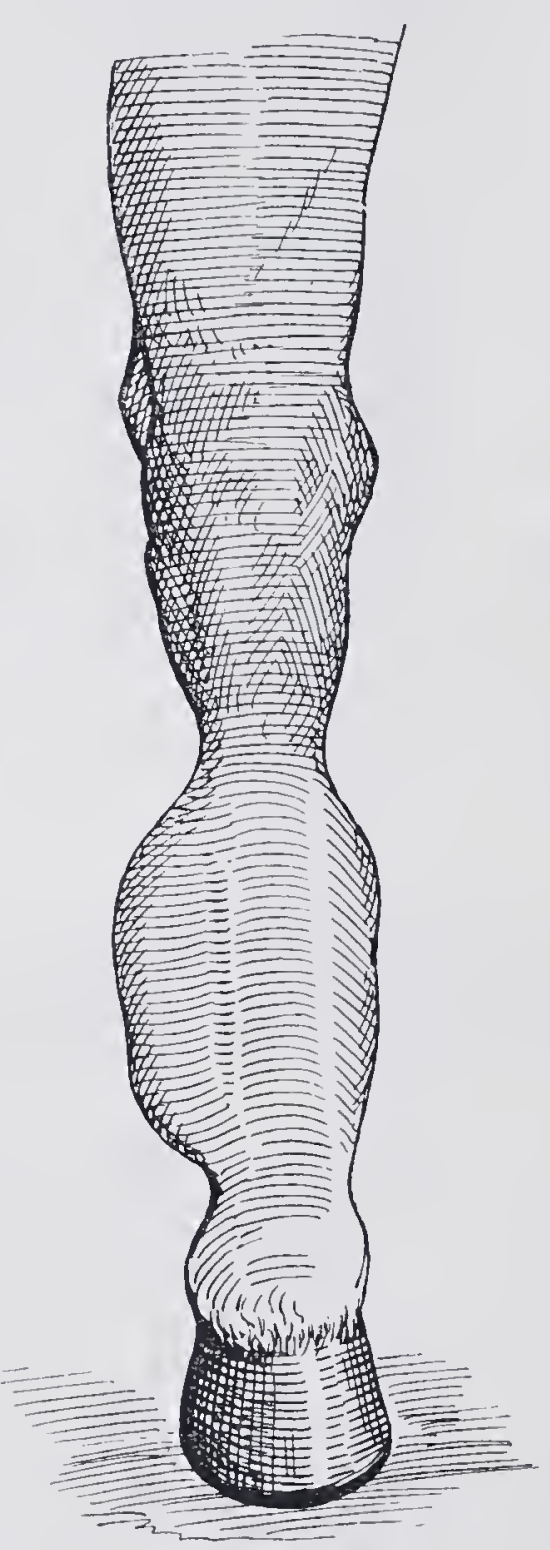

FIG. 284.- Distension of the metatarso-phalangeal (sesamoid) sheath. flexor tendons are affected; but the swelling forms a blemish difficult to remove. Careful local examination shows which condition is present, and should always be resorted to, as the lameness often disappears after long rest. Provided the swelling has not long been in existence, the parts should be tightly bandaged after work; massage often removes swelling in young horses, but firing is surer. The swelling should never be incised, for the result is often fatal. Removal of contents by Pravaz's syringe, or by trochar, is only of temporary benefit; nor does injection of iodine prevent resecretion, which may occur in a few minutes. The precautions to be observed in opening 
these swellings with the actual cautery are mentioned under "T'reatment of enlarged synovial cavities in the fore limb."

In the hind limbs such enlargements often become indurated, i.e., the tendon sheath is chronically thickened, sometimes to the extent of $\frac{1}{2} \mathrm{or}^{\circ}$ $\frac{3}{4}$ of an inch. As a rule, the flexor tendons are diseased, and there is lameness, which may become severe after hard work. It is best seen when weight is placed on the limb, and is accompanied by plantar flexion of the phalanges. The swellings are hard and immovable, the flexor tendons can scarcely be felt through them, and when the parts are firmly pressed the animal shows pain.

Such enlargements are most troublesome in riding and draught horses, though, in other animals, they interfere with usefulness and sometimes render the horse unworkable; tley are little amenable to treatment. When recent, i.e., not more than a few weeks old, they may be diminished by the use of bandages, blisters, or firing, but later the sole means of removing the lameness is neurectomy of the posterior tibial nerve. Good results often follow this operation, but occasionally the flexor tendons become elongated, producing flexion of the lock; in a case of Möller's this was so marked as to prevent the animal being used. Hendrickx has had similar results.

\section{II.-INFECTIOUS INFLAMIMATION OF THE LOWER SYNOVIAL SHEATH OF THE PERFORANS TENDON.}

Acute infectious inflammation of the above-named tendon sheath usually follows mechanical injuries like stabs with stable-forks, wounds from the plough, harrow or scythe, or contused wounds, the result of running away. It is usually attended with suppuration, and is particularly dangerous, because the tendons themselves are so frequently implicated. The symptoms consist in severe lameness, inability to stand on the limb, and discharge of a purulent synovia from the wound in the tendon sheath. There is usually cellulitis of the surrounding tissues. Fever is not infrequently present, and the animal lies continuously.

The prognosis should be reserved, because death may follow either from general infection or as a result of the animal continuously lying down, or, in the event of its standing, severe laminitis may develop in the opposite foot. When the tendon itself is injured the prognosis is always unfavourable, as necrosis almost always follows. Necrosis, however, not infrequently occurs even without the tendon being wounded. Treatment may succeed if commenced early, and consists in the most careful disinfection of the parts; in some cases it is advisable to operate and open the tendon sheath. 
Chronic purulent inflammation of this tendon sheath may also follow "cutting " or "brushing." Acute infective inflammatory processes are here particularly dangerous; very often they take the form of acute cellulitis due to local infection or local gangrene (cracked heels).

Diagnosis is sometimes difficult because of the swelling which always exists around the bursa. One symptom which always accompanies inflammation of the tendon sheath should, therefore, be particularly remembered, viz., lifting of the limb, as in gonitis; in this case, however, the foot is advanced further in the direction of the front foot of the same side (fig. 286). This peculiar position is common, and seems absolutely pathognomonic. Accurate diagnosis is very important, because treatment is scarcely advisable, except in valuable horses. The treatment, which consists in daily cleansing of the parts, washing out with disinfectants, and bandaging, is not often successful, laminitis generally occurring in the other foot, and carrying off the animal. In a few instances, however, recovery occurs, though the fetlock usually remains somewhat upright; two cases, recorded show that perfect recovery is possible, even after acute suppurative inflammation of the tendon sheaths.

Chronic septic inflammation of these sheaths is also seen in the horse. The clinical symptoms are not well marlied; swelling is moderate, because the connective tissue sur. rounding the tendon sheath is not much affected. Pain is not so great as in the acute disease, but a turbid synovia, mixed with pus, is continually discharged. Very great difficulty is found in closing the wound in the tendon sheath: healing is almost always prolonged, and often the horse is found one

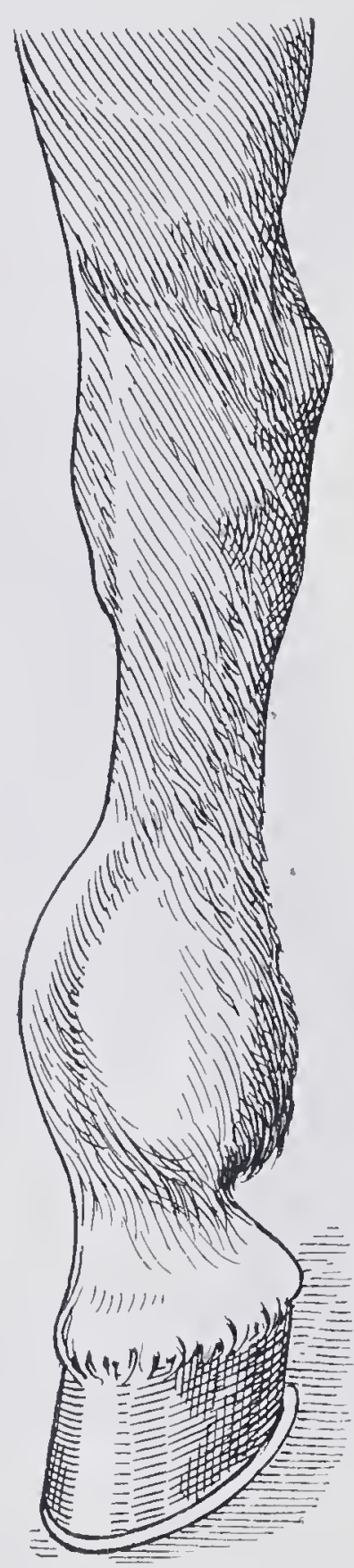

H1G. 285. - Enlargement of the bursa of the extensor pedis tendon $(i$, in schema of the tendon sheaths of the fore limb, fig. 203). morning standing almost on its fetlock, and with the toe pointed upwards, the flexor pedis perforans tendon being ruptured. The condition seems incurable. The ends of the tendon, being necrotic, are incapable of regenerative processes; on section, the necrotic part is distinguished by its yellow, opaque appearance, from the glistening, satin-like, healthy portions. Not infrequently demarcation has commenced, and can be detected by the vascular condition of the parts bordering on the necrotic 
portion. If the flexor pedis perforans is ruptured the animal should be slaughtered.

Rupture of the flexor pedis perforatus is caused in a similar way, and also follows wounds of the metatarsus. The position of the phalanges is then less altered, though the fetlock is somewhat more oblique than usual. When the horse moves, the phalangeal joints are seen to be unusually lax; they dangle from side to side each time the foot is advanced-an unmistakable symptom of this rupture.

Uncomplicated rupture of the flexor pedis perforatus is less grave; the

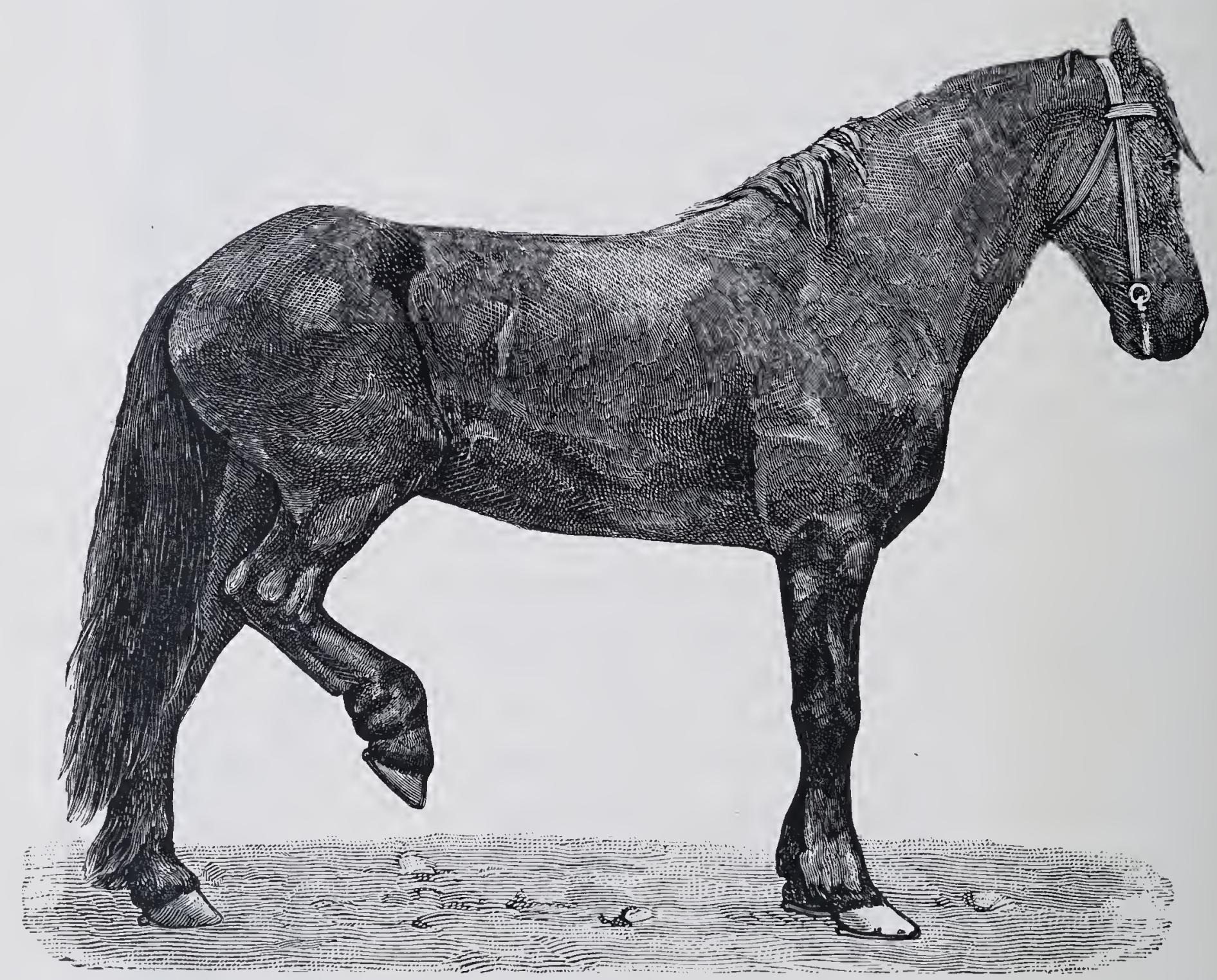

FIG. 286.-Position of the limb, in infectious inflammation of the flexor pedis perforatus sheath in the fetlock region (from a photograph).

tendon often unites, and the usefulness of the limb may be fully restored. Treatment follows general principles.

A horse was stabbed in the heel of the right hind foot with a manure-fork, and severe lameness at once set in. Being called the same day, Möller thoroughly cleansed the wound, bathed the foot in creolin solution, and in the evening applied a large antiseptic dressing. The skin wound healed by primary intention, and pain diminished, though lameness continued. Möller therefore employed for the next few days moist warm applications, but the case did not improve. The animal was then regularly exercised, which greatly diminished the lameness, until one day pain returned in an unusually severe form, and the sheath of the flexor tendon was found to be greatly distended just above the fetlock-joint, and very painful on pressure. The 
parts were again bathed, and hot poultices applied, but, as they gave no relief, blistering was resorted to. There was, however, little improvement, the swelling persisting. The sheath of the tendon was then opened under antiseptic precautions, allowing of the escape of purulent fluid, which could only be distinguished from ordinary pus microscopically, but which was then found to contain no micro-organisms. The examination was made by various persons, and every precaution was taken to avoid error.

This seemed to show that purulent tendovaginitis had existed, doubtless as a result of the wound, and yet the pus contained no micro-parasites. Whether parasites had never been present, i.e., suppuration had been brought about without infection, or whether the pus cocci had perished, is uncertain. One peculiarity of this case is, however, very remarkable, viz., that in spite of the great distension of the tendon sheath the connective tissue around it never became the seat of cellulitis. In purulent tendovaginitis the tissues round the tendon sheath are almost always attacked with active cellulitis, and in consequence are more or less markedly swollen. Dollar, called in consultation by the late Mr. Stevens, M.R.C.V.S., saw a similar case in an aged grey hunter. The horse had been stabbed in the heel with a stablefork; the wound closed, but lameness persisted. About the fourth day, swelling of the tendon sheath set in, lameness became acute, and a little offensive blood-stained pus escaped from the wound. The animal could not stand on the limb, and showed frequent lancinating pain. Dollar opened the sheath for a distance of about one inch, and prescribed continued hot

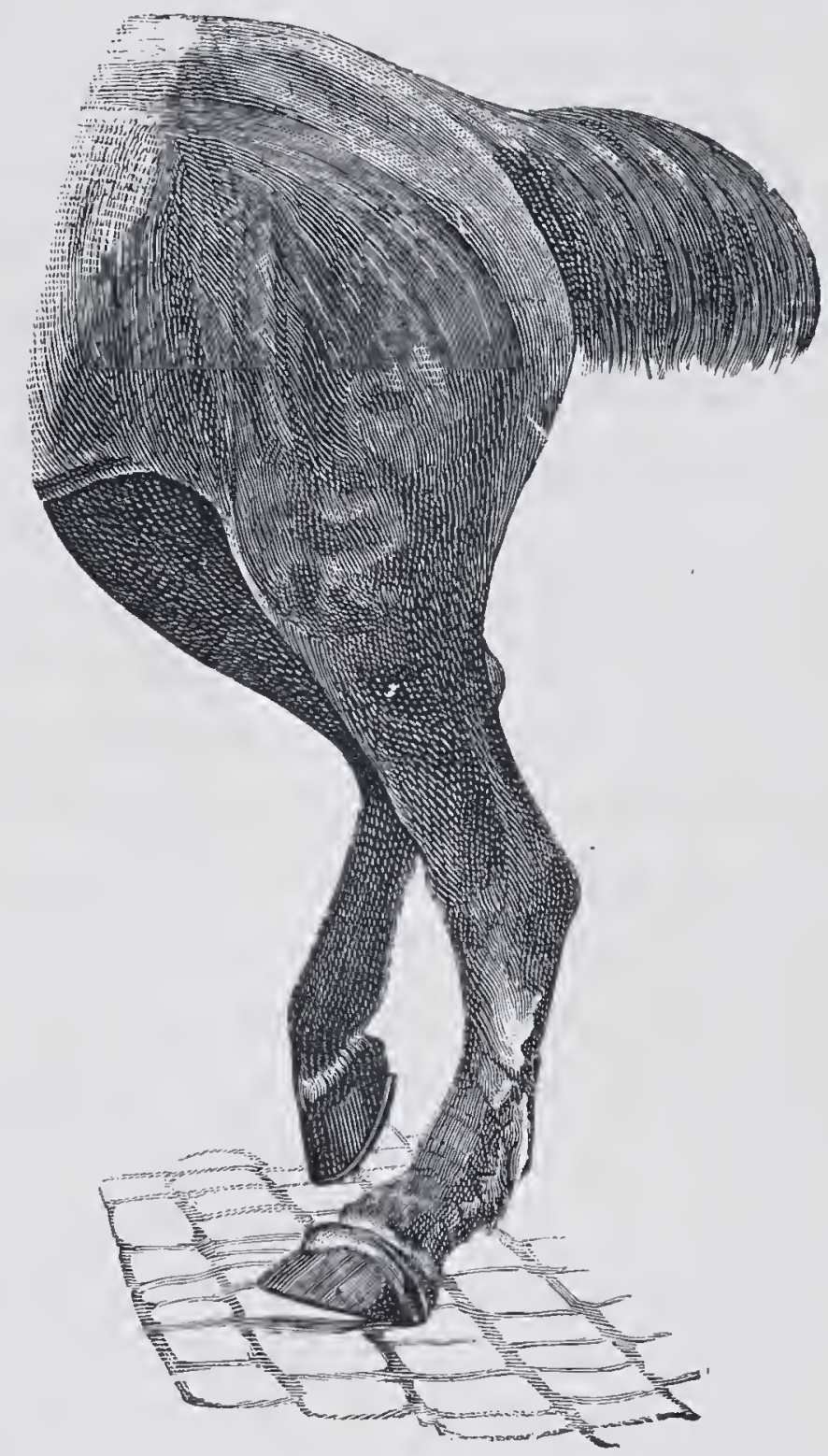

FIG. 287.- Rupture of the Hexor pedis perforans tendon (from a photograph). antiseptic baths ( 5 per cent. creolin). The limb was immersed in a long rubber bath extending from the foot to the middle of the cannon bone, and the fluid was changed every two hours. Relief was prompt. In four days the baths were discontinued, as the skin was becoming macerated. A week later the wound had closed. Lameness gradually decreased; after blistering and three months' rest, the animal again carried his owner for an entire hunting season.

\section{III.--INJURIES PRODUCED BY STRIKING (INTER- FERING) AND THEIR COMPLICATIONS.}

Striking or interfering is the term used to describe the injury inflicted by the horse's foot on the opposite leg during work. It occurs oftenest in the hind limbs, especially when the animal is trotted-the inner surface 
of the fetlock-joint is usually struck by the hoof of the opposite side; less frequently the coronet is the seat of injury. In the fore limbs, striking sometimes affects the carpus and metacarpus, particularly in badly-bred horses.

The nature of the disease produced depends partly on the tissues affected, partly on the time during which the injury continues. Thus in some cases the skin is simply bruised, in others the plantar nerves are involved; extravasations of blood or abscesses may occur on the inner surface of the fetlock-joint or splints, form on the cannon bone. Violent and repeated injuries cause excoriation and necrosis of the skin or chronic bony enlargements on the inner surface of the fetlock-joint. Occasionally, fibrous tissue enlargements, due to striking, and showing marks of injury at their most prominent point, form on the inner margin of the coronet.

Interfering is serious, because the horse can never be depended on ; riding-horses may stumble in consequence of severely bruising the plantar nerve, which lies on the inside of the fetlock-joint, and is easily injured. But such injuries are also interesting to the surgeon, because they sometimes form the origin of disease processes of a grave cliaracter. The most frequent of these is cellulitis, which either extends in the subcutis, or may seize on the subfascial connective tissue, and, taking a chronic course, continually suppurate. In other cases pus may break into the tendon sheath of the flexor pedis perforans, or into the cavity of the fetlock-joint, and produce incurable complications. Sometimes cutaneous gangrene and chronic inflammation of the cutis result. Finally, wounds from striking may be followed by grease, \&c. Wilhelm describes a case in which striking was followed by severe cellulitis, necrosis of the skin, and, in consequence of metastasis, by pneumonia, inflammation of the shoulder-joint and subacute meningitis. The animal recovered, but very slowly.

Causes. Want of "condition," faulty conformation, and large size and flatness of the hoofs are the principal causes of striking. The animal may stand with the limbs too close together, or may turn the toes outwards. Both these peculiarities are accompanied by faulty action, which predisposes to the injury mentioned. Striking is also favoured by travelling on rough ground, and by exhaustion. Young hor'ses often brush or strike while being broken in, or if, when driven, they are reined-up too short, especially before they become accustomed to work on paved streets. A very frequent cause of striking is, however, the mode of shoeing, the principal fault consisting in irregular paring of the hoof. If the inner wall be lowered and the outer left too deep, the fetlock-joint is thrust towards the middle line of the body when weight is thrown on the limb, and may be injured by the opposite foot. 
Many farriers still favour the idea that striking is prevented by lowering the inner wall, and thus perpetuate one of the most frequent causes of striling. Bad fitting, allowing the inner edge of the shoe to project beyond the wall of the foot, or leaving the clenches too long, may also produce the same result; but, in comparison with unskilful paring of the hoof, these only play a subordinate part.

Symptoms. The cause of these injuries is indicated by their position on the fetlock-joint, coronet, metacarpus, or knee. Sometimes the hair is only roughened or rubbed off, sometimes the skin is excoriated, its surface being covered with blood or discharge, but more often there is a wound penetrating the cutis. Provided injury be confined to the surface of the skin, there is little or no pain, swelling, or lameness, and animals continue at work.

But immediately inflammation extends to the subcutis, a circumscribed swelling develops, which is followed by suppuration. The pain becomes severe, the animal goes very lame, and sometimes it is unable to bear weight on the limb. In extreme cases the entire body is wet with perspiration, and fever runs high. In such cases the plantar nerve may be inflamed, though more frequently the symptoms are due to cellulitis in the subfascial tissues. The condition then becomes very grave, on account of the possibility of pus breaking into the fetlock-joint or sheath of the flexor pedis perforans. When the tendon sheath is attacked, the limb is held as in gonitis, the swelling extends upwards over the metatarsus or metacarpus, and any attempt to flex the lower joints of the limb produces great pain. There is almost always fever.

The injury may be followed by diffuse subcutaneous cellulitis, in which case the swelling extends to the hock, or to the knee, invading more particularly the inner surface of the limb. The lymph vessels are generally swollen, the lymph glands enlarged, and fever is present. This condition, which may also be caused by other injuries, is termed lymphangitis. The vena saphena may be involved, and thrombosis result, but this complication is not so frequent as was formerly supposed; it seems probable that swelling of the lymph vessels was mistaken for thrombosis. The condition has been confused with erysipelas, from which it is distinguished by the fact that the subcutaneous connective tissue, and not the cutis, is the seat of disease.

When accompanied by localised necrosis of skin, the affection becomes identical with gangrenous grease, dermatitis gangrenosa (which see). Finally, wounds caused by striking may produce a chronic dermatitis, already described as "verrucous grease." Wounds due to brushing, when not infected, generally heal rapidly, unless the injury is repeated.

The course taken by injuries due to striking varies very greatly. Provided the processes so set up remain aseptic, and the injury be not 
repeated, it is of little moment, and recovery rapidly follows. Diffuse inflammation of the subcutis in horses is also usually mild. The swelling appears suddenly, and increases for two to three days, during which pain is marked, and some fever exists; the swelling, pain, and fever then begin to diminish, and in about eight to ten days the animal is convalescent. Permanent blemishes are rare, but under some circumstances, especially if the cellulitis has been caused by infection with a particularly virulent micro-organism, abscesses form in the upper parts of the limb, severe fever sets in, septicæmia or pyæmia develops, and soon leads to death. Luckily, such complications are not frequent.

Subfascial cellulitis is graver, either when confined to the fetlock or coronet, or when appearing in a diffuse form. In the first case a circumscribed, very painful swelling develops around the fetlock-joint, and after eight to fourteen days, or sometimes longer, breaks and discharges a very offensive pus, mixed with fragments of necrotic tissue; the pain then diminishes, and recovery occurs. Sometimes the abscess re-forms, and the disease may then last for a longer time.

But if, instead of discharging outwardly, the abscess breaks into the fetlock-joint, purulent inflammation of the joint-cavity occurs, the pain and fever increase, no weight whatever can be borne on the limb, and even slight rotary movements of the foot produce severe suffering. The condition is then incurable. Injuries to the coronet may in a similar way be followed by purulent inflammation of the pedal or coronet joints, and cellulitis extend to the sheaths of the flexor pedis perforans and perforatus tendons, with equally fatal results.

The course which skin gangrene takes depends on the amount of surface implicated. Extension of necrosis inwards is especially dangerous, because the parts above mentioned may be attacked as in purulent cellulitis. (For complications resulting from the verrucous form of grease, see that section.)

Sometimes sinuses form, the granulations which follow perforation of the abscess uniting again, whilst necrotic pieces of tissue are left in the depths. The subcutaneous fascia then plays a similar part to the lateral cartilage in ordinary quittor. Such sinuses are often extremely
troublesome.

Treatment. Among the most important preventive measures is attention to the horse's general condition and to feeding. Young horses should be gradually brought into work, and not be called on for great or continued exertion until their muscular system is properly developed. To prevent striking, special attention should be paid to the shoeing, and the application of a so-called "cutting-shoe" will be found very useful, especially on the hind feet. (A very full description of shoes for "cutting" and "brushing" will be found in Dollar and Wheatley's 
"Horse-shoeing and the Horse's Foot," pp. 286 to 300.) After lowering the outer wall sufficiently to, at least, prevent it meeting the ground sooner than the inner, the edge of the latter is somewhat rounded off, and a close-fitted cutting shoe applied. Should the inner wall be too low, it must be raised by placing leather, \&c., under the shoe.

Injuries produced by striking must be treated according to their character. Complications are best prevented by carefully cleansing the wound, and smearing it with tar, creolin, or other antiseptic. Such precautions are particularly necessary in winter, and when the roads are dirty (see also "Dermatitis Gangrenosa"). Especial care is required if the skin be perforated, as the entrance of infective material into the subcutis then is greatly favoured.

When suppuration is already established, the first precaution must be to thoroughly cleanse and disinfect the wound, so as to prevent further extension. After-treatment is based on general principles. Cold applications are contra-indicated, and should be replaced by moist warmth in the form of warm baths containing a disinfectant. If cellulitis extend further upwards, the limb should be enveloped in cotton-wool or tow saturated with disinfectants, or when little assistance is available, the leg may be smeared with grey ointment. Immediately pain diminishes and swelling commences to recede, slow exercise should be given to hasten resorption.

Double precautions are required in subfascial inflammation. When located in the fetlock or near the tendon sheath, permanent lukewarm baths, containing disinfectants such as creolin, carbolic, \&c., are indicated, and immediately fluctuation can be detected, the swelling should be opened, though due care must be exercised to avoid injuring large bloodvessels and nerves, or opening the capsule of the joint or sheath of the tendons. When the joint or the tendon sheath is implicated operation is better avoided, because in such cases no good can result, and the owner is apt to conclude that the use of the knife was responsible for the fatal result.

Swelling in the soft tissues around the coronet is relieved by thinning the horn wall. Gangrene and the verrucous form of grease are treated on the lines indicated when speaking of these conditions. Rings and boots, often recommended against striking, are seldom of much value. Some horses are so apt to strike that not even the most careful shoeing is sufficient to prevent them injuring themselves, and in such cases boots may be tried. They require, however, to be so fashioned as not to be displaced if struck, nor cause injuries if tightly buckled. The best form of boot contains a zine plate exactly responding to the oval form of the fetlock.

The straw and indiarubber rings often used in draught-horses force 
the animal to move with the feet further apart, but have no permanent effect on the action, and as soon as they are removed the animal strikes as before. A "Yorkshire boot" is useful in dry weather, and can be made more effectual if before folding down the upper part a thin straw plait be wound regularly round the point struck. When the upper portion of cloth is folded down it secures the plait in position.

\section{H. DISEASES OF THE FOOT.}

Though the horse's foot and the claws of dogs and oxen are physiologically homologous, and in structure practically identical, yet the diseases to which they are subject vary in many respects in consequence of the different influences to which they are exposed. The horse's foot being more liable to injury than the ruminant's claws, becomes diseased not only more frequently, but also in different ways. The claws of carnivora again play quite a different part to the hoof and claws of herbivora.

It may, therefore, be well to first turn our attention to diseases of the foot, especially as they have been more carefully studied. As diseases of the feet are fully dealt with in Dollar and Wheatley's "Horse-shoeing and the Horse's Foot," it is here only necessary to shortly consider such of the inflammatory processes within the foot as have a direct bearing on veterinary practice.

\section{(a) Acute Inflammation of the Podophyllous Membrane.}

\section{Pododermatitis Acuta.}

Acute inflammation of the podophyllous membrane is, with few exceptions, produced by infection with micro-organisms, and in many cases is accompanied by mechanical injuries. On account of the sensitive laminx and sole being bounded on one side by the unyielding horny box, and on the other by the os pedis, inflammatory swelling, especially when of a septic nature, must be painful, and tend to necrosis. The latter, though to some extent antagonised by the great vascularity of the parts, is nevertheless not infrequent.

The surface of the podophyllous membrane possesses a well-developed stratum mucosum, formed of numerous layers of epithelium, which, however, show no horny character. Not infrequently inflammation is entirely confined to this without extending to the corium, a condition termed pododermatitis superficialis, in contra-distinction to inflammation of the deeper-lying structures corresponding to the corium and subcutaneous connective tissue, which is termed pododermatitis parenchymatoso vel profunda.

(1) Pododermatitis superficialis generally follows exposure of the 
tissue of the rete mucosum and entrance of infectious material. Let us imagine that a nail has penetrated the hoof as far as this layer, but soon after has been removed. Septic products, \&c., penetrate by the nail tract as far as the rete mucosnm, and may give rise to extended inflammation. In such cases we speak of a prick or stab. If, on the other hand, the point of entrance was an opening between the wall and sole, the condition is termed separation; while if it occur in the angle of the heel, we speak of a corn.

Aseptic inflammation of this tissue, though rare, is seen during laminitis and formation of horn tumours, \&c. In these cases the rete mucosum is sometimes thickened, as shown in laminitis, by increase in width of the white line. The horn produced during inflammation is sometimes changed in character, stained yellow, or infiltrated with bloor (corns). Infectious pododermatitis superficialis, on the other hand, is always accompanied by suppuration, if we are justified in describing the product as pus. Strictly speaking, the thick layers of rete break down under the action of the infective material, though exudation certainly occurs in the neighbouring portions of the corium. This explains the tendency of the process to remain confined to the surface, and to extend along it towards the coronet, or, when the white line is the seat of attack, to extend along the thick layers of non-horny rete cells of which it consists.

Though aseptic pododermatitis superficialis ends in resolution or chronic thickening; the infectious form is seldom followed by resolution, but the pus escapes outwardly, or into the cutis or subcutis, in which it produces inflammation. That the broken-down masses of rete do at times become absorbed is shown by the spaces occasionally found in the horn when cutting out a hoof. The same condition is often seen in the claws of herbivora. Sometimes several spaces are discovered one above the other, showing that the process has occurred repeatedly, and been interrupted by periods of normal horn formation.

As a rule, septic pododermatitis superficialis ends in perforation outwardly, though it often requires surgical assistance. The condition having been diagnosed and the horn cut away, a greasy, blackish fluid, termed horn pus, escapes, and the animal soon lecovers if the parts be properly dresserl and protected against fresh injury or infection. When, however, an artificial opening is not made the process extends, and the fluid forces its way along the laminæ as far as the coronet, which it perforates by thrusting apart the coronary band and commencement of the horny wall, appearing, therefore, at the top of the coronet "between hair and hoof." Inflammation of the lower border of the laminæ, or periphery of the sensitive sole, generally extends in the direction of the white line, and the pus, therefore, tends to escape at the heel. The discharge of fluid, V.S. 
grey or blackish material, termed "horn pus," at the coronet or heels, and the absence of marked swelling, point to the superficial nature of the attack.

(2) Pododermatitis parenchymatosa affects the corium or subcutis, and is generally associated with disease of the surface, from which it may originate, though it also results from deeper injuries, like pricks, \&c. The podophyllous membrane, or its subcutis, forms the seat of acute inflammation, accompanied by exudation; suppuration almost always occurs, the pus being precisely similar in character to that formed in other portions of the skin, and appearing thick, yellow, and creamy, while, if its formation is accompanied by necrosis, it may be offensive. The character of the discharge, in fact, is a very valuable indication for diagnostic purposes. When white, yellowish or creamy, the keratogenous membrane is evidently suppurating, or may even be undergoing partial gangrene; if reddish, or of the colour of wine lees, and stinking, it indicates diffuse gangrene of the membrane, which, again, is often accompanied by necrosis or caries of the os pedis ; when yellowish, viscous, "curdled," or containing fibrinous floceuli, and especially if escaping by a sinuous opening in the coronet or sole, it points to synovitis of the lower sesamoid sheath, or to arthritis of the pedal-joint. The significance of these peculiar characters in the pus is again controlled by the degree of pain and general disturbance.

The attack may terminate either in resolution, abscess formation, or necrosis. The first rarely happens. Much more frequently an abscess forms, similar to those in other positions, and leads to either-necrosis of the sensitive sole, purulent cellulitis, or septicæmia.

Necrosis usually depends on inoculation with particularly virulent infectious substances, though it is favoured by the position of the sensitive laminre and sole between the unyielding horn on the one side and the os pedis on the other, which causes any considerable swelling to be followed by severe compression and interference with circulation. The common occurrence of necrosis at certain times shows that infection plays an important part, though in winter the action of cold may also have a certain action. During outbreaks of gangrenous dermatitis one often sees necrosis, the dangerous consequences of which are aggravated by the difficulty with which the dead tissue separates, and by the readiness with which putrefactive products are absorbed and diffused in neighbouring structures, leading to inflammation of the laminæ or sole, and not infrequently to separation and loss of the hoof. Such a termination is preceded by excessive pain, fever, and extensive separation at the coronet. If the first point involved is one where the sensitive structure lies closely in contact with the bone (as is the case over the greater part of the sole and wall), necrosis almost always attacks the os pedis. 
Purulent cellulitis can, of course, only occur where inflammation seizes on sensitive structures possessing a subcutis, like the posterior sections

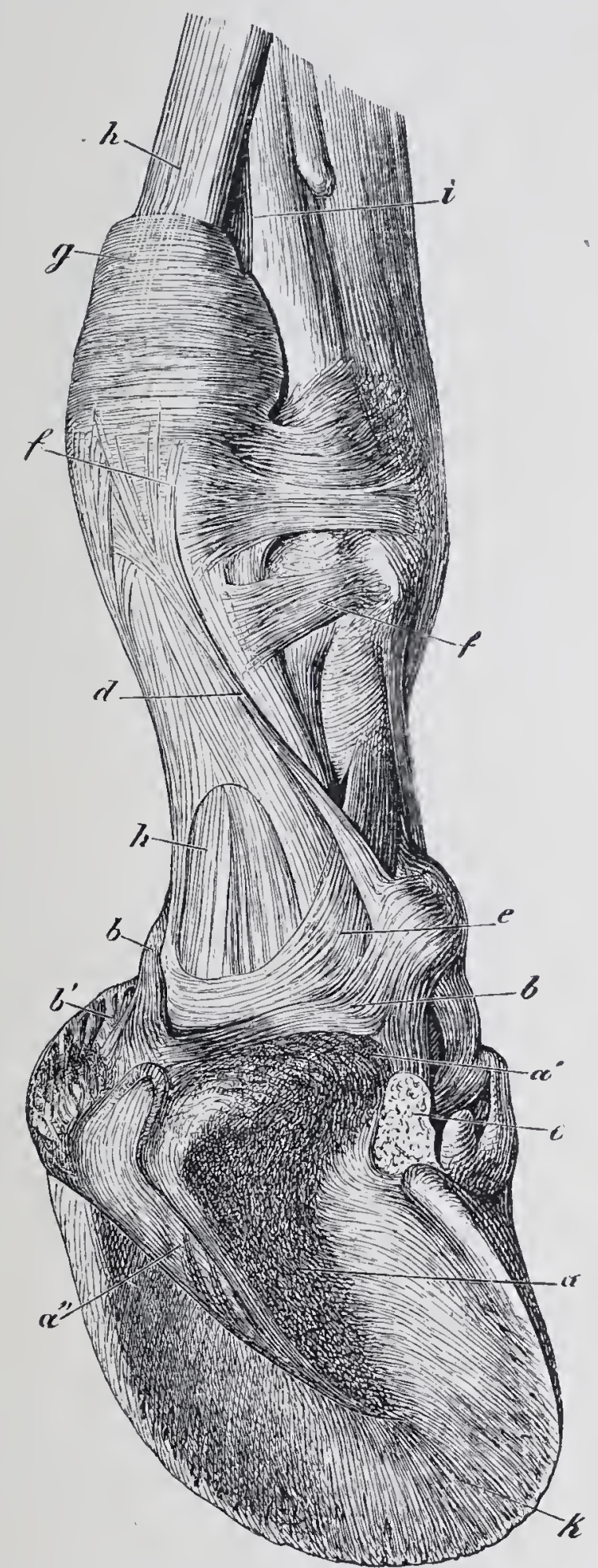

FIG. 288.-To illustrate the superficial structures involved in surgical injuries of the foot. The outer lateral cartilage and the tissues covering the lower surface of the pedal bone (sensitive frog and sensitive sole) have been removed. $a$, Plantar cushion; $a^{\prime}$, bulbar portion of plantar cushion; $a^{\prime \prime}$, cleft of the frog in which rests the "frog stay" ; $b$, origin of the so-called "suspensory ligament of the bulbs" ; $b^{\prime}$, small elastic band passing towards the lateral cartilage ; $c$, elastic band arising from lateral cartilage and becoming inserted into pastern bone - it unites with $b$; $d$, small tendon which arises from the skin and becomes attached, in common with $b$ and $c$, to the pastern bone; $e$, fibro-elastic supporting sheath of flexor pedis perforans; $f$, fibro-elastic supporting shcath of flexor pedis perforatus; $\eta$, flexor pedis perforatus tendon; $h$, flexor pedis perforans tenáon; $i$, suspensory ligament ; $k$, lower surface of pedal bone, to which the flexor pedis perforans tendon is attached.

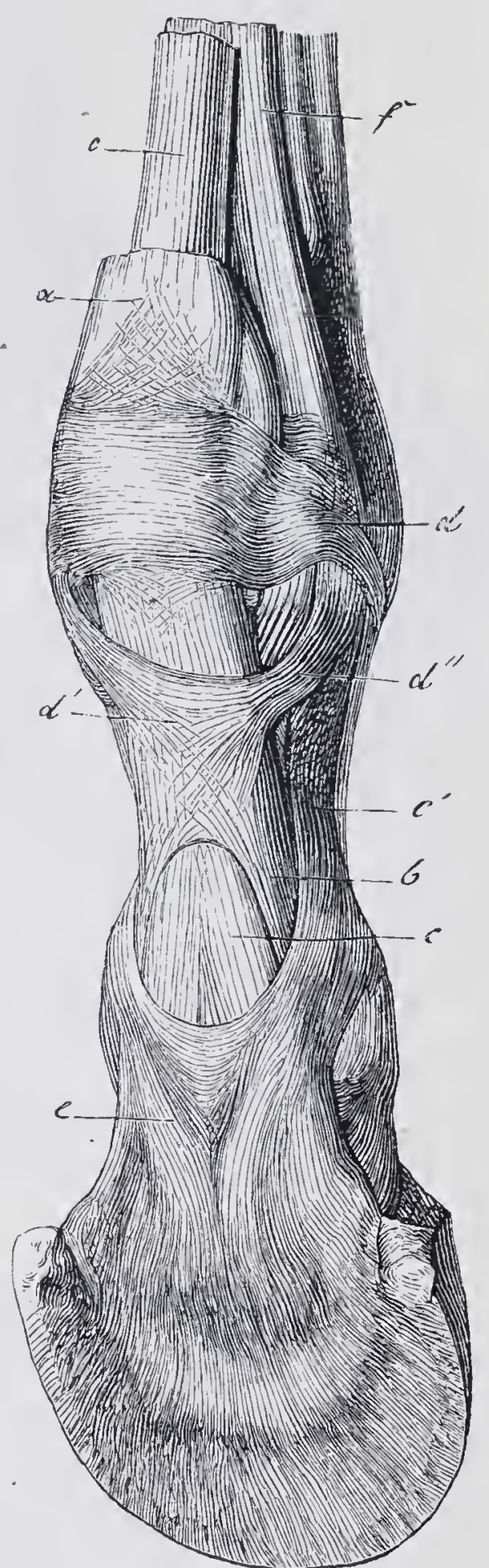

Fig. 289. - To illustrate the deeperseated structures involved in surcical injuries of the foot. Right fore foot seen from behind and slightly from one side. $a$, Flexor pedis perforatus tendon ; $b$, two limbs formed by its bifurcation ; e, flexor pedis perforans tendon; $d$, fibrous reinforcing baud of great sesamoid sheath; $d$ ', fibrous supporting sheatl inserted into suffraginis bone by four heads; $d^{\prime \prime}$, upper insertions (the lower not visible in figure); $e$, fibro-elastic plate covering the lower surface of flexor pedis perforans and inserted into suffraginis bone at $e^{\prime}$; $f$, suspensory ligament. 
of the wall and sole of the coronet and frog. Inflammation attacks the connective tissue lying below the coronary band, producing a subcoronary

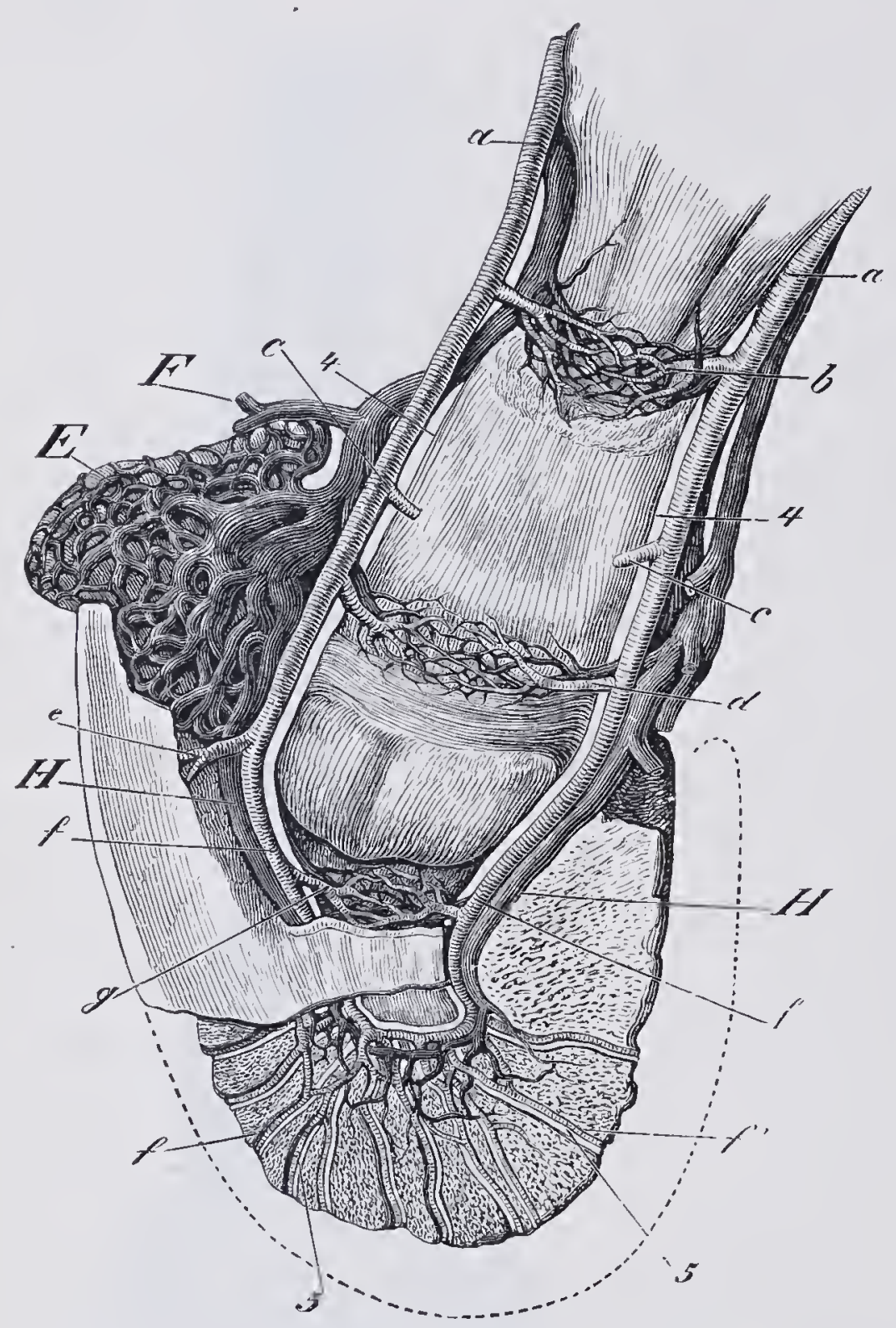

Fig. 290.-To illustrate the joints and deep-seated arteries, veins and nerres involved in surgical injuries of the foot. Right fore foot, seen from below, helind, and somewhat from one side. The outer lateral cartilage is renoved, together with sufficient of the pedal bone to render visible the vessels, \&c., in its interior. The nerves accompanying arteries $f^{\prime \prime}$, are shown too thick; thicy should be less than half as broad as figured. $a$, Digital artery ; $b$, posterior suffraginal artery ; $c$, artery of plantar cushion (cut through); $d$, posterior artery of coronary circle; $f$, plantar artery, which anastomoses with its fellow within the perlal bone, and gives off twigs $f^{\prime}$, which pass to the anterior surface of the pedal bone, just above its lower edge ; $g$, twigs of plantar artery supplying coffin joint; $E$, deep lateral layer of coronary plexus, clothing imner surface of lateral cartilage; $F$, divided ends of superficial part of coronary plexus. From these arise the digital vein (not showu); $H$, plantar vein : 4 , posterior branch of digital nerve accompanying vessels into pedal bone; 5 , twigs of posterior branch passing towards sensitive laminæ.

quite upright, or directed downwards and backwards. Swelling appears in the hollow of the heel; after some time abscesses form and break, and not infrequently the flexor pedis perforans becomes ruptured.

inflammation, which usually involves one-half of the hoof, occasionally the whole, and is recognised by the severe swelling and intense pain around the coronet. As pus may burrow into the pedal-joint, the condition is grave. When occurring about the back of the coronet, this form of inflammation generally leads to sinuses or quittors, which, however, are still more often the result of suppuration in the posterior sections of the sensitive sole or wall, where the subcutis is in direct contact with the parachondral connective tissue.

Parenchymatous pododermatitis in the sensitive frog often causes purulent cellulitis of the fibro-fatty frog, which is particularly dangerous, on account of so often extending to the flexor pedis perforans tendon and producing necrosis. Purulent cellulitis of the fibro-fatty frog is distinguished by the violent pain shown, especially on dorsal flexion of the phalanges. When weight is placed on the affected limb the parts are liept in a condition of excessive volar flexion; weight can only be borne for a moment, and the animal puts down the foot with the front of the wall 
(3) Septicæmia oftenest results from necrosis of the sensitive laminx or sole, especially when the disease attacks the os pedis. As the pedal veins are incapable of collapsing, they offer little resistance to septic material penetrating their walls, and reaching the general circulation. Spinola drew attention to the frequent connection between necrosis of the pedal bone and attacks of septicæmia or pyæmia. The constant and severe pain also causes the patients to lie continually, and, further, predisposes to fatal blood-poisoning.

Causes. With the exception of laminitis, which will later receive attention, acute inflammation of the sensitive structures of the foot is due to external injurious influences. Mechanical injuries, like bruises, wounds by driven or picked-up nails, or separations of the wall

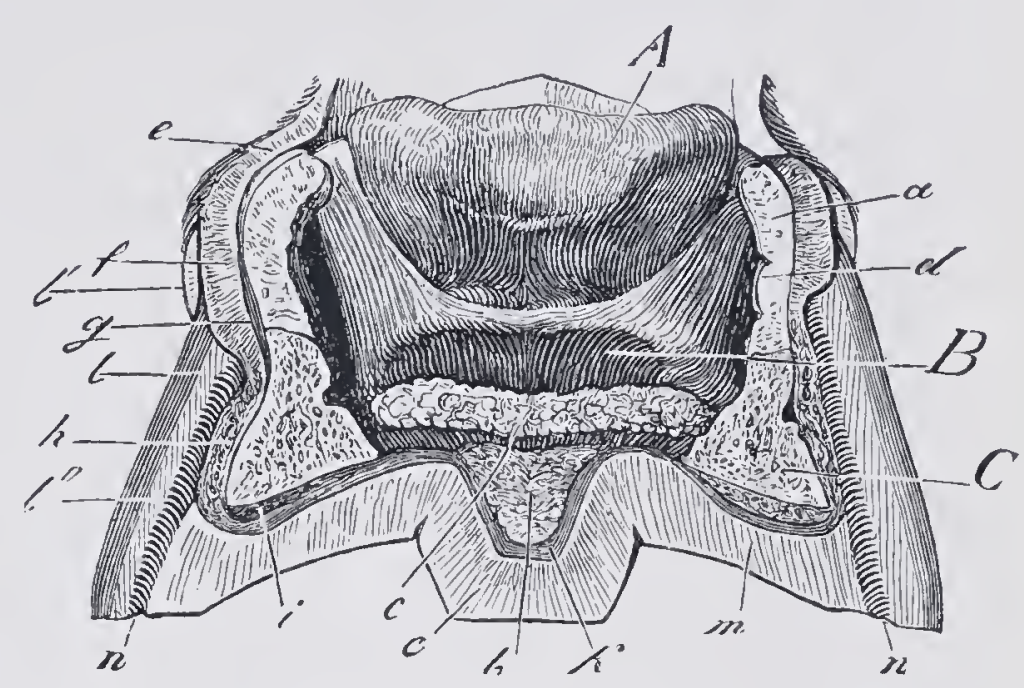

Fic. 291. - Vertical cross section of a foot seen from behind. To illustrate the structures involved in surgical injuries of the foot. $A$, Coronet bone ; $B$, navicular bone ; $C$, pedal bone; $a$, lateral cartilage ; $b$, anterior portion of plantar cushion ; c, divided part of flexor pedis perforans tendon; $\boldsymbol{l}$, postero-lateral ligaments of navicular bone; $l$, horn wall ; $m$, horn sole; $n$, white line; $o$, liorn frog. allow of irritants reaching the sensitive parts directly; in other cases the injury is thermal, but as the horny box forms so excellent a protection against ordinary changes in temperature, and burns from hot-fitting of shoes are nowadays rare, such cases are much less frequent.

Cold is more frequently injurious,

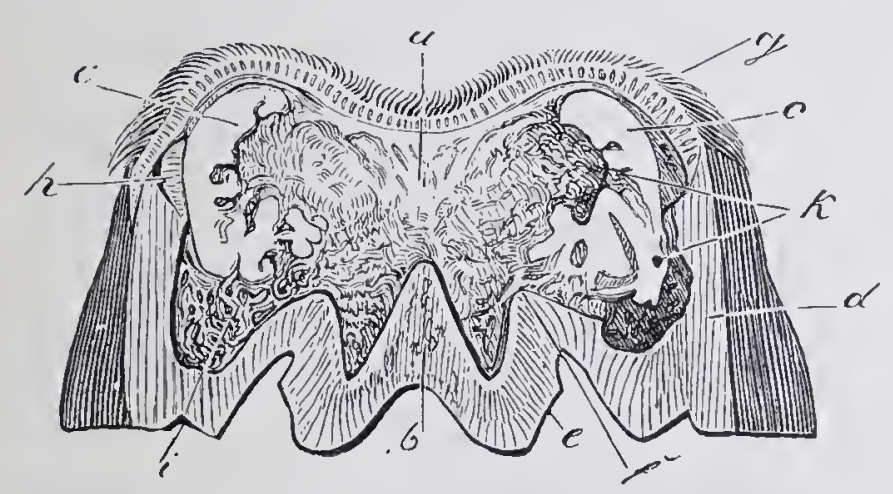

FIG. 292.-Vertical cross section of foot seen from behind (this section has been made nearer the heels than fig. 291). To illustrate the structures involved in surgical injuries of the foot. a, Posterior part of plantar cushion; $b$, median ridge of frog; $c$, lateral cartilage ; $d$, horn wall; $e$, lateral face of fros; $f$, point of mion between the bars and frog. but as soon as a fissure exists, in consequence either of pricks in shoeing, picked-up nails, the formation of sand-cracks, or separation of the wall, a way is opened for infection, which is especially liable to especially when the horn is split. Pricks in shoeing or excessive paring of corns may be followed by irritation during winter weather, especially if the roads are very dirty. Frostbite of the heels has been seen. Chemical substances, like acids, employed by farriers in treating disease of the feet, sometimes cause inflammation. But by far the most frequent and important cause of acute inflammation is infection with pus cocci and other micro-parasites. Normally, the soft parts are protected by the horny wall, 
occur, as the hoof is continually covered with dirt containing septic substances. The course and consequences of the inflammation depend chiefly on the virulence of these organisms.

Diagnosis. The presence of acute inflammation of the sensitive wall or sole may often be detected by the peculiar action of the lame leg. As pressure within the hoof is increased when weight is thrown on the limb, lameness is most marked during movement, especially over stones, though in the stall the foot is often rested.

The foot must be carefully examined to detect deformities or fissures in the horn. (The manner of conducting this examination is fully described in Dollar and Wheatley's "Horse-shoeing and the Horse's Foot," p. 318.) Palpation will detect increased warmth and pain.

Amongst indirect symptoms, increased pulsation and œdema in the lower parts of the limb are particularly noteworthy,

Prognosis. The degree of danger depends chiefly on the position and character of the inflammation. Whilst pododermatitis superficialis only proves dangerous in the region of the bars, whence it may seize on the fibro-fatty frog, and in general yields readily to treatment, parenchymatous inflammation often leads to severe complications. Any disease affecting the posterior portions of the hoof is apt to prove serious, because of the readiness with which diffuse cellulitis occurs in this region, and of the frequency with which it is succeeded by quittor formation, disease of the fibro-fatty frog, tendon sheath, or flexor pedis perforans tendon. Other things being equal, injuries to the hind are more serious than those to the front feet, because the hind limbs sustain more weight and can less easily be rested, and also because they perform a more important function than do the front-i.e., they not only support, but also propel the body. Injuries of the feet are also less important in heavy than in light horses, because the former can often be made serviceable at a walking pace, though unable to trot sound.

As in many instances it is impossible to discover the extent or character of the process, the prognosis must be based on indirect indications. The principal of these are the degree and duration of the pain. As a rule, the more stubborn and severe the lameness the graver the prognosis, especially when no direct cause is apparent, as, for example, when there is no protrusion of soft tissues. Necrosis and purulent cellulitis of the fibro-fatty frog or subcoronary connective tissue are exceedingly dangerous. The condition is also very grave when laminitis results from continuous standing on the opposite foot. Increase in pain, especially when of a lancinating character, inability to stand on the affected foot, and a rising temperature indicate grave complications, lilie suppuration, gangrene, necrosis or caries.

Treatment. Although treatment follows general principles, yet in 
acute inflammation of the sensitive structures of the foot, certain special precautions must be borne in mind. The advice to as far as possible remove the cause is eminently applicable here. Aseptic pododermatitis seldom calls for more than rest and cold applications in the form of poultices or continued irrigation. These soften the horn and permit it to yield under the pressure of the soft structures, while they directly diminish inflammation. As a precaution, however, after applying moisture for long periods, the hoof should be greased, to prevent it completely drying again. When lameness disappears, special attention must be given to shoeing.

Superficial inflammation, accompanied by suppuration, is best treated by thinning the horn, and allowing the pus to find early exit. An opening of a quarter to half an inch in circumference is sufficient to allow of the thin fluid pus escaping, after which the cavity is washed out, and injected with a disinfectant. Foot-baths are sometimes useful, though nothing but clean water should be used, unless the addition of a disinfectant be thought advisable. The opening should be covered, first, with a mass of cotton-wool saturated in a disinfecting solution, and then with a proper cotton- or wood-wool dressing to exclude sand, earth, or dirt, and plenty of clean, dry straw given as bedding. The dressing must be renewed several times a day. As a rule, with these precautions, recovery occurs in five to eight days. As already remarked, particular care is required when inflammation affects the bars.

The treatment of parenchymatous pododermatitis, accompanied by suppuration, requires greater care. To prevent the occurrence of purulent cellulitis, pus must be allowed free exit. The diseased area should, therefore, be exposed as far as possible, and neighbouring parts thinned, though without removing more horn than is absolutely necessary. Thinning the horn relieves inflammatory swelling around the suppurating centre. The next precaution is to remove all putrefying material from the inflamed cavity. For this purpose the parts are carefully washed with sublimate solution, or, if necessary, immersed in a disinfectant foot-bath. They are next rinsed with sublimate, carbolic, or creolin solution, a tampon saturated with the same material applied to the exposed soft tissues, and a pressure dressing fastened over all. It is often necessary to inject the parts with the above, or with 10 per cent. chloride of zinc solution.

A pressure dressing answers the double purpose of protecting the inflamed spot from dirt and the entrance of fresh infective material, and of preventing protrusion of soft parts, to which there is a considerable tendency, partly from removal of support, but largely in consequence of inflammatory swelling. Being irritated by contact with the margins of the opening, the soft tissues swell and cause great pain, block the 
opening, prevent escape of pus, and facilitate the production of purulent cellulitis. 'To remedy this, nothing succeeds better than steady pressure, assisted, if necessary, by astringents like alum and tannin, or even by nitrate of silver. Dusting the granulations with iodoform or other disinfectant powder also helps to combat the inflammation. Very special care is called for when the pus is offensive. Foot-baths are useful on account of their cleansing the point attacked, but as they favour prolapse of soft tissues, a pressure dressing should be applied. As soon as suppuration stops, the soft parts must be protected by means of a dressing of tar and tow, until they again become covered with horn. This dressing should not be removed until the wound has thoroughly cicatrised, when a well-fitting shoe, protecting the diseased point until the new horn becomes sufficiently strong, is applied.

In gangrene of the sensitive sole, the affected point must be completely exposed, any dead tissue removed, and the freest possible exit given to gangrenous discharge. The gangrene may be recognised by the greyish yellow colour of the soft structures, and by the pain and bleeding which follow any interference with the spot, Necrotic portions of tissue are grasped with dressing-forceps and forcibly removed, after which the parts are treated as described.

The treatment of cases complicated with purulent cellulitis of the fibro-fatty frog is described in the chapter on "picked-up" nails; that of the subcoronary connective tissue under that title.

\section{I.-PRICKS OR STABS IN SHOEING.}

'The sensitive portions of the foot are often injured during shoeing by misdirected nails, with consequences of a very varying character. The chief factor appears to be the degree of injury, though infection plays no inconsiderable rôle in determining the result. When the nail only penetrates the neighbourhood of the rete mucosum, or injures this alone, a superficial inflammation results, with the above-described consequences. But if, instead, portions of the cuticular structures containing connective tissue are affected, the usual, though not invariable, result is an attack of parenchymatous inflammation. The attack may often be avoided by immediately withdrawing the nail and closing its track by tar, wax, or by burning the horn, thus preventing infection of the wound; a proof that it is not the injury, but the infection it facilitates, which causes inflammation. A second nail should not be driven at the same spot, as it would again open the path for infection.

The os pedis is occasionally injured by driven nails, though necrosis resulting from inflammation should not be mistalien for fracture produced by a nail. 
The course of these injuries varies greatly. Superficial injuries sometimes heal without proceeding to suppuration, in six to eight days under local cooling treatment and rest. Pododermatitis superficialis produced by pricks generally causes suppuration, the pus, if not furnished with an artificial outlet, breaking through at the coronet at a point corresponding in position with the misdirected nail. Although in such cases spontaneous recovery is usual, it saves time, and the possibility of complications, if an exit for pus be provided at some point in the white line.

As pricks and stabs only occur in those portions of the sensitive structures which lie immediately in contact with the os pedis, and are umprovided with subcutis, purulent cellulitis is a comparatively rare complication, necrosis of the laminx, and even of the os pedis, being more frequently seen. As already remarked, the result depends more on the kind and degree of infection than on the extent of injury. Direct injuries of the bone lead, as a rule, to necrosis, which is apt to extend to the neighbouring sensitive structures.

The diagnosis is based partly on the fact that the horse goes lame soon after shoeing and partly on examination of the hoof. For this purpose the shoe is removed, and the ground surface of the hoof, and especially of the white line, well cut out, so that the position of the nail holes can easily be detected. (For

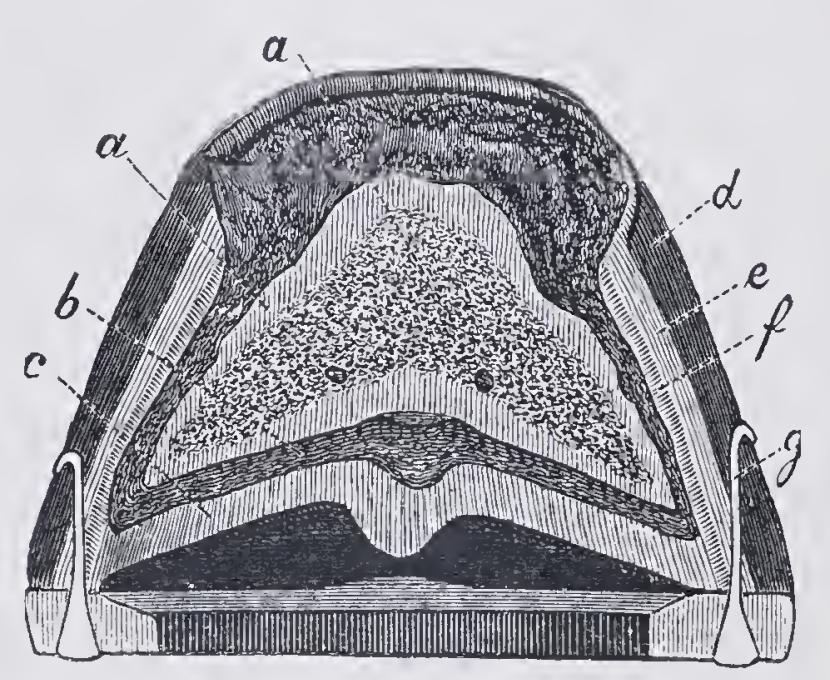

Fig. 293.-Cross section of a sound and wellshod hoof, showing the proper position of the nails. $a$, Pedal bone ; $b$, sensitive sole; $c$, horny sole; $d$, horn wall; $e$, dark-coloured outer layer of do.; $f$, laminal sheath; $g$, nails.

further directions, see Dollar and Wheatley's "Horse-shoeing and the Horse's Foot," pp. 378 et seq.) If it is necessary to expose a nail tract, it should first be followed through the horn of the sole and white line, the wall being spared, so as to preserve a sufficient bearing surface for the next shoeing.

Causes. Want of care and skill on the part of the farrier is the usual cause of stabs or pricks, though they may be due to bad feet and to the animal's restiveness, without any fault on the part of the farrier. Carelessness in shoeing is shown by the nail holes being misdirected, and by their occurring at points where there was not sufficient horn to warrant the driving of a nail. The ustual causes of stabbing are coarsely-punched nail holes and insufficient horn; stubs left in the feet rarely occasion pricks by causing the nail to deviate from its normal direction.

The first step in treatment is to remove the nail and shoe. Provided 
suppuration has not set in, cold applications, in the form of foot-baths or poultices, may be tried. The parts must be cleansed, and infection prevented by giving the animal plenty of clean bedding, and, if necessary, applying a suitable dressing. Once suppuration occurs, treatment follows the above-described principles.

\section{II.-PICKED-UP NAILS. PURULENT CELLULITIS OF THE FIBRO-FATTY FROG. RESECTION OF THE FLEXOR PEDIS PERFORANS. ${ }^{1}$}

The above title will, for convenience, be regarded as including all injuries caused by the animal treading on foreign bodies which thus penetrate the soft structures of the foot. Such bodies include nails and wire, as well as pieces of iron or glass. As the horny sole usually offers sufficient protection, such foreign bodies almost invariably enter through the frog, and may injure the fleshy and fibro-fatty frogs, or, in exceptional cases, even the flexor pedis perforans tendon, os pedis, or os naviculare. (On account of their different behaviour when injured, we distinguish(1) a horny frog; (2) a sensitive frog; and (3) a fibro-fatty frog. The two latter are usually included under the term "plantar" cushion.") Cases have been recorded of injury even to the os coronæ. The foreign body usually glides off the bars and penetrates the side or furrow of the frog.

Provided injury is confined to the surface of the fleshy frog, no bad results usually follow; but should the fibro-fatty frog be involved, diffuse purulent cellulitis may set in, suppuration may extend to the flexor pedis perforans tendon, and be followed by necrosis of the latter and by suppuration in the navicular bursa. The condition is then excessively dangerous, and animals generally die if treatment be delayed. The rapidity of the process is largely determined by the virulence of the infectious material.

Prognosis depends, therefore, firstly on the position of the injury, and afterwards on its extent and the character of the infection. The depth of the wound may be determined by the length of the foreign body removed, and sometimes by probing; but as the probe may easily become the vehicle for introducing infectious material into the depths of the wound, it should be used with considerable care.

The position of the injury is important, the most dangerous spot being the centre of the frog, immediately above which lies the navicular bone covered by the flexor pedis perforans tendon. Injuries at the point of the frog may extend to the lower surface of the os pedis, causing necrosis, which, however, is seldom so dangerous as disease of the

\footnotetext{
1 For a detailed description of several cases of resection of the tendon of the flexor pedis perforans tendon, see Cadiot and Dollar's "Clinical Veterinary Medicine and Surgery," p. 78.
} 
tendon and tendon sheath, because necrosis usually remains confined to one spot, and healing occurs after exfoliation, even though the latter

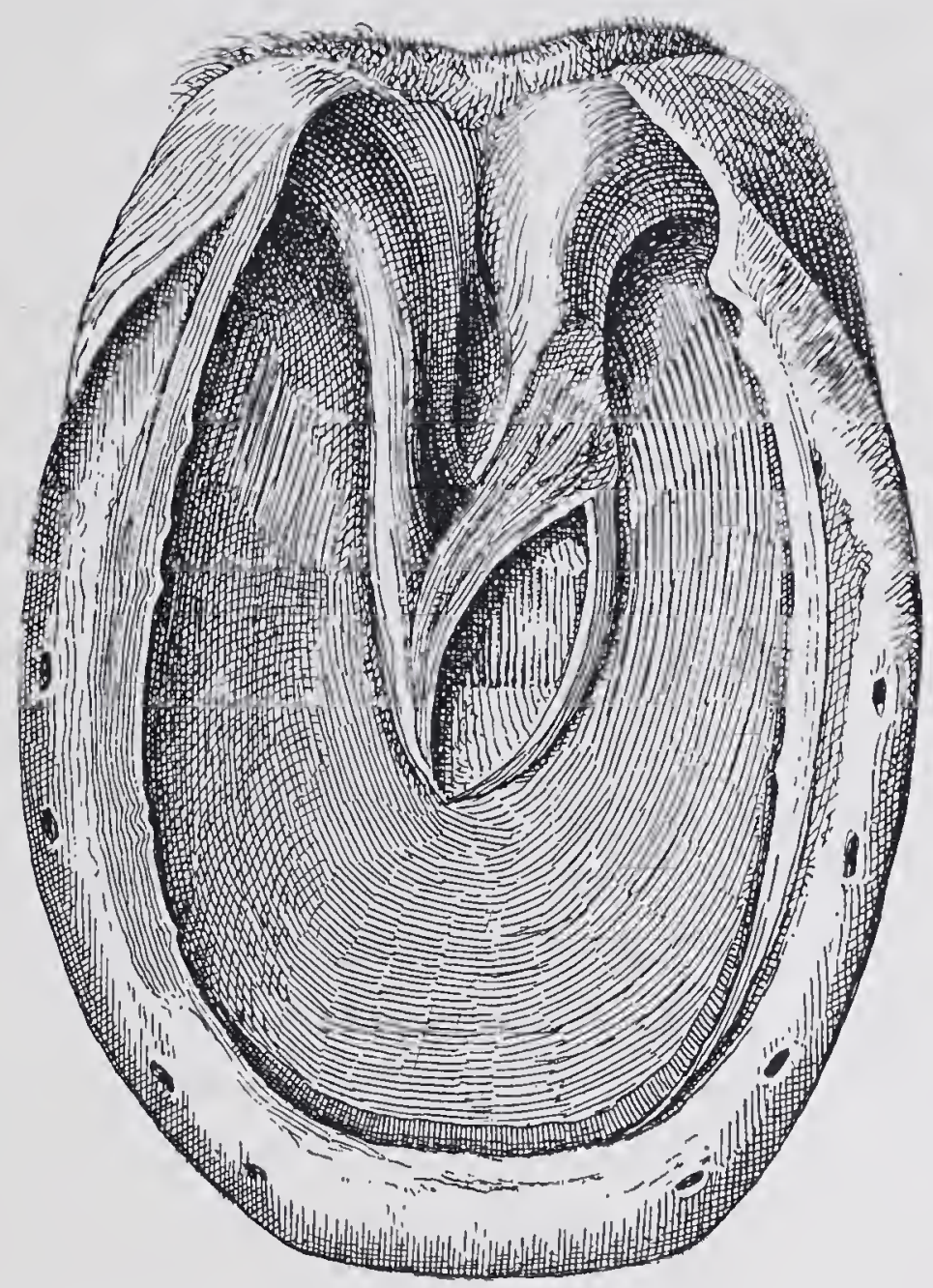

FIG. 294. - Operation for partial resection of the aponemrosis of the flexor pedis perforans tendon.

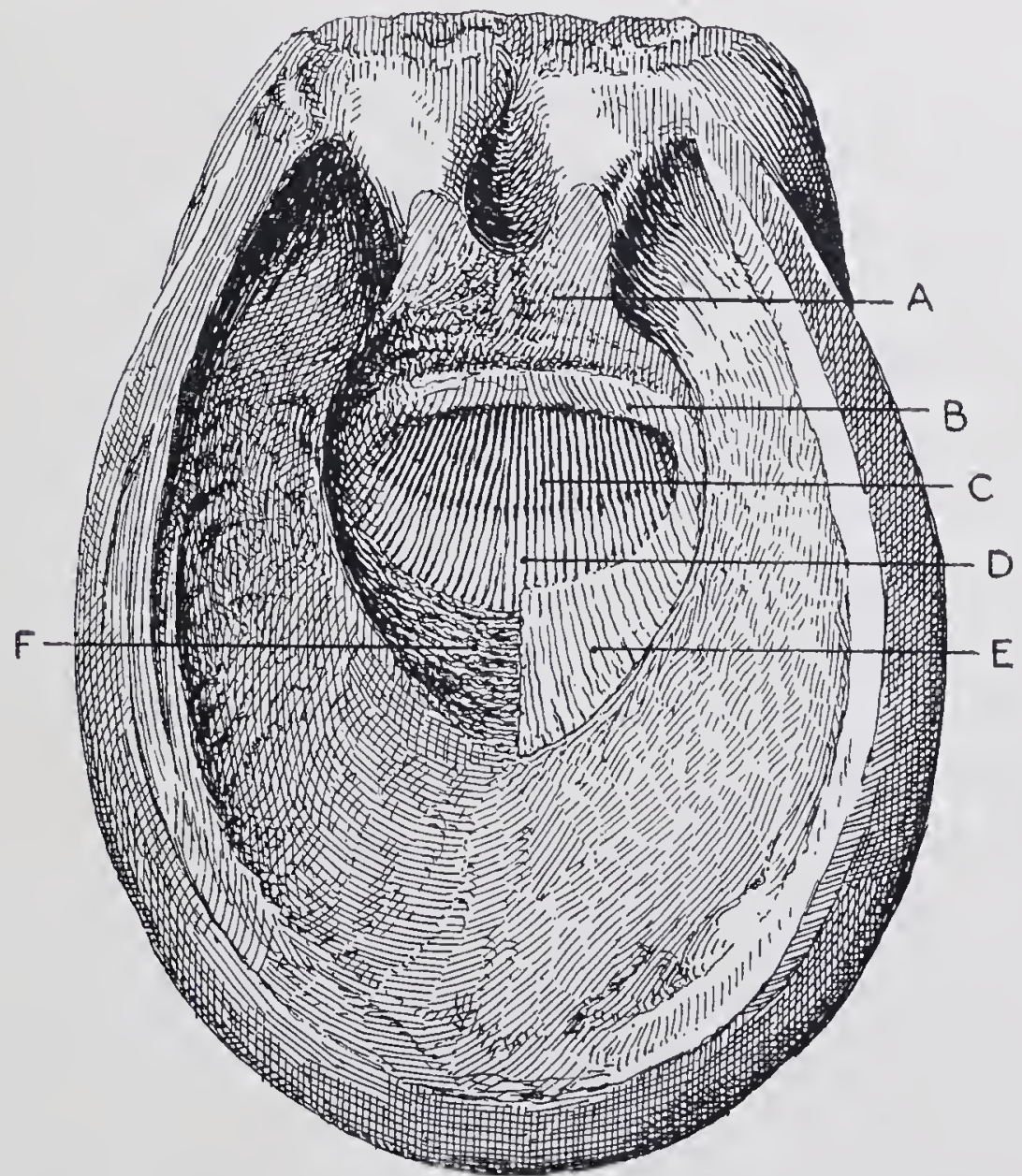

FIG. 295.-Operation for complete resection of th aponeurosis of the flexor pedis perforans tendon. A, Cut surface of frog and plantar cuslion; B, section of the aponeurosis of the flexor tendon; C, postero-inferior surface of the navicular bone; D, interosseous ligament; $\mathrm{E}$, oblique section of the aponeurosis of the flexor tendon; $\mathrm{F}$, tendinous surface of navicular bone cleaned by curetting. 
occupy a considerable time (three to four weeks). Severe lameness, and symptoms of diffuse purulent inflammation of the fibro-fatty frog, are always grave. On attempting to bear weight on the foot, the latter shows excessive volar flexion, swelling appears in the heel, and pain is exhibited on forcibly flexing the toe.

Treatment. The chief indications are to prevent infection, to disinfect the wound, and to limit inflammation. After carefully removing the foreign body and taking precautions against any particles being left behind, the entire hoof must be pared as for shoeing, and any loose fragments of horn removed from the sole or frog. The track of the nail should then be cut out until the injured soft tissues are exposed. The funnel-shaped opening, and, if possible, the wound in the soft tissues, are then washed out with clean water, or, better still, with a disinfectant; (a syringe is useful) or the parts can be swabbed with a tampon saturated with 10 per cent. chloride of zinc solution or pure "Sanitas" oil. As the chief object is to keep the wound disinfected, a dressing moistened with some disinfecting fluid is applied over all.

Should pain increase during the next few days, the wound should be cautiously probed, and any imprisoned discharge allowed to escape, after which the foot is immersed twice a day, for one to two hours at a time, in a bath containing some disinfectant. Plenty of clean straw bedding must be given, and the dressing moistened five or six times a day.

To sum up, the object of treatment is, to confine the inflammation to the point of injury, for which purpose it is necessary to keep the parts thoroughly clean, to avoid retention of pus or wound discharges, and to minimise the decomposition which is apt to occur in wounds.

When pain decreases, a dry dressing is sufficient; and as soon as inflammation or suppuration stops, a splint dressing can be applied to protect the soft parts, which are only covered with a very thin layer of horn. The sole is smeared with tar and covered with tow, which is retained by means of a pair of wooden splints placed one over the other in the form of a cross, with their ends thrust under the shoe. Provided the roads are dry, the horse can work with this protection, but in wet weather it is better to give a few days' further rest in the stable.

When suppuration is confined to a portion of the fibro-fatty frog, precautions should be taken against further infection and retention of pus, which would lead to diffuse cellulitis of the whole structure.

In cases where the flexor pedis perforans tendon and its bursa are still intact, it is sometimes useful to scrape out the wound with a curette. 'This removes the infected masses of granulation tissue which form so freely, and constitute an obstacle to the escape of wound discharges and of necrotic material. After sponging out with chloride of zinc solution, 
a tampon, moistened with the same fluid, is thrust into the wound, and a dressing applied.

Disease of the flexor pedis perforans tendon can in general only be cured by resection. The procedure is as follows:-On the day preceding operation, the sole, frog, and especially the bars, are very thoroughly thinned, the foot is cleansed, the hair clipped carefully away as high as the fetlock or knee, and the limb immersed for half an hour in a bucket of strong disinfecting solution. Whilst immersed it is subjected to a thorough scrubbing with a clean stiff brush. Immediately after removal the foot and hoof must be completely enveloper in clean cloths saturated with a disinfecting solution.

Next day the horse is cast. The affected foot is released, the sole, frog, and bars still further thinned, and the sensitive frog is exposed so as to allow any necrotic portions either of the frog or tendon to be removed. An Esmarch's bandage is then applied to the limb, commencing at the coronet and extending to above the knee, at which point a stout rubber cord under tension is passer several times round the limb and the ends tied together. This secures the operator against troublesome bleeding. The foot to be operated on may then be fastened to the other limb of the same side by means of webbing. The horse is now chloroformed.

Two methods of procedure are at the operator's disposal, in the first of which only a portion of the point of the frog is removed, in the second the whole. The first method is often sufficient to disclose the diseased tissues, and it has the advantage of producing a much smaller, and therefore more rapidly-healing, wound than the second. The sole, frog, and bars having been pared until they yield everywhere to a slight pressure with the finger, a grooved director is passed into the sinus, which is freely laid open to its extremity. Using the curved knives shown on p. 798, the edges of the wound and the plantar cushion are then removed so as to produce an elliptical opening, at the base of which lies the perforans tendon. If the superficial fibres of the tendon are necrotic the operator may confine himself to removing such diseased tissue. But much more frequently he finds it necessary to remove the entire thickness of the tendon beneath the diseased spot and to extend his incision laterally so as to operate in sound tissue. The portion of the navicular so exposed may be curetted, if it show signs of disease. The wound is then washed out with a disinfectant, dusted freely with iodoform, and filled with masses of disinfected tow, oakum, or wood-wool.

When carefully performed this partial operation is sometimes rapidly successful, but on the whole it is apt to prove uncertain, and to be followed by troublesome complications. The tendon sheath becomes the seat of suppurating synovitis; the navicular bone shows superficial 
caries; abscesses break in the flexure of the pastern, and the remaining portion of the flexor tendon becomes necrotic. In really grave cases where operation of this nature is necessary it is usually best to at once perform the operation implicating both sides of the frog.

The preliminary precautions are as before mentioned. The foot should be extended by an assistant, and the plantar cushion divided transversely close to its base, with the double-edged curved knife already referred to. The section should be at an oblique angle with the general surface of the frog and in such a direction that when prolonged inwards it will meet the posterior edge of the navicular bone. The half-detached fragment of the plantar cushion is then grasped with forceps and freed from its lateral adhesions by two cuts along the lateral lacunæ of the frog. The deep face of the plantar cushion is in contact with the aponeurosis of the flexor tendon.

Using both hands to steady the knife, the operator now divides the aponeurotic portion of the flexor tendon transversely close to the posterior border of the navicular bone with one steady cut. The portion of the aponeurosis thus freed is next divided along the middle line from before backwards as far as the upper margin of the navicular bone, the fragments are reflected and each is carefully dissected free. Again grasping the knife with both hands, the operator makes a semicircular sweeping cut along the edge of the semi-lunar crest, thus dividing the lower insertion of the aponeurosis into the os pedis. The fragments are then removed.

The fibro-cartilage covering the lower surface of the navicular bone is next removed by the vigorous use of the curette. If the primary injury extend to the point of insertion of the plantar aponeurosis the terminal fibres of the tendon must be carefully removed and the semi-lunar crest curetted, care being taken, however, not to open the joint between the navicular bone and the os pedis. If, however, the insertion of the aponeurosis is healthy the semi-lunar crest should not be touched; the fibrous layer which covers it soon becomes vascular, and granulates rapidly provided it be protected from infection. Wherever gangrene, necrosis, or caries exists it is absolutely imperative to remove the whole of the diseased tissue, and, in fact, to encroach a little on the adjoining: healthy parts. To overlook a fragment of diseased tissue is to risk the necessity for operating anew and under much more difficult conditions. The cavity is sponged with a 10 per cent. solution of chloride of zinc, rinsed with carbolic solution, powdered with iodoform, salol or calomel, firmly packed with masses of tow, moistened with carbolic or sublimate, and a moist dressing applied over all. French operators touch any point in the wound which may appear likely to undergo degenerative 
changes with tincture of iodine. The space is filled with tow, partly to check the bleeding which follows removal of the tourniquet, partly to restore the fibro-fatty frog and sensitive frog to their normal position. Next day the dressing is removed, the wound rinsed out with disinfectants, and the dressing renewed, but the cavity is only loosely filled with carbolic tow or jute. This second dressing remains in position eight to ten days, provided it is not wet through with discharge, and neither fever nor severe pain has set in. In the interval the walls of the space have everywhere become covered with granulations-in favourable cases without any trace of pus formation. The dressing is then renewed every four or five days; and as the cavity fills with granulations, less material is used. Finally, healing becomes complete; the frog may possibly appear rather shortened, but, as a rule, no other deformity remains. The French and German operators often return horses to work in four to six weelis, but it is best to keep a protective dressing splinted on the sole for some time.

A very convenient method of applying the above dressings is by means of a shoe fitted with a thin iron plate of sufficient size to fill up the entire space between the limbs of the shoe, to which it is fastened by screws. (See p. 385 of "Horse-shoeing and the Horse's Foot.")

The best results are seen in heavy draught-horses. In carriage and trotting horses slight lameness may persist for a considerable time, though it seldom proves permanent. (Full clinical details of several cases of resection of the flexor pedis tendon are given at p. 451 of "Clinical Veterinary Medicine and Surgery.")

Partial resection of the flexor tendon is not to be recommended, because the bursa podotrochlearis is generally implicated, and therefore, after removal of the necrotic portion, purulent bursitis continues, and leads to fresh necrosis of the tendon. For this reason reports of success after partial resection of the flexor pedis perforans tendon are to be received with caution. The fact that recovery has been described as occurring in eight to fourteen days, or even earlier, shows that the cases were not cases of necrosis of the flexor tendon, but only of the fibrofatty frog. In these it is often sufficient to freely scarify the parts with the curette.

\section{III.-TREADS AND INJURIES TO THE CORONET. PURU- LENT INFLAMIMATION OF THE SUBCORONARY CONNECTIVE TISSUE.}

The above injury, as the name implies, is generally produced by the animal treading with the heel of one foot on the coronet of another. Wounds of the coronet produced by carriage or omnibus wheels at first 
present somewhat similar appearances, but are oftener of a grave character, affect to a greater degree the deep-seated tissues, produce at a later stage distortion of the hoof, and, worst of all, are apt to be followed by the formation of extensive exostoses and permanent lameness. Either the coronary band, the upper portion of the wall, or the slin is divided, and the injury often extends to subcutaneous structures, to the tendon of the extensor pedis, to the lateral cartilage, or even to the os pedis, and may even lay open the pedal-joint. The danger is increased by the fact of the wound being bruised and infected from the beginning, for which reason treads never heal by primary intention, and generally show a tendency to necrosis. The structure and position of the injured part favour this ; the tendons, the os pedis, and the lateral cartilage are readily destroyed, whilst necrosis is favoured by the surroundings of the coronary band, in which acute inflammatory swellings greatly interfere with nutrition, in consequence of the unyielding character of the horny wall. Finally, as treads most frequently occur during winter, the tissues are exposed to the action of cold and dirt, which are specially injurious. At this season of the year slight injuries to the coronet are readily followed by severe gangrenous processes, which show a strong tendency to extend to the sensitive laminæ. Again, infection of the loose connective tissue lying under the coronary band often leads to diffuse cellulitis, which may extend to the pedal-joint, and give rise to incurable purulent inflammation.

Luckily, healing is usually very complete at this point, and one sometimes sees cases where large portions of the coronary band have been destroyed, yet no defect remains in the formation of horn. This is rendered possible by the papillie of the coronary band and of the neighbouring skin replacing the lost parts. (On this point compare with Dollar and Wheatley's "Horse-shoeing and the Horse's Foot," pp. 104 et seq.)

Destruction of a section of the coronary band is followed by cessation in the secretion of horn at that particular point. Acute inflammation of the band also interrupts the formation of horn, and produces a cavity in the horny wall, the width and length of which depend on the size of the region affected and on the time during which inflammation persists; the longer the time, the greater the perpendicular measurement of the cavity; the greater the extent of coronary band involved, the broader the resulting defect. When horn production is permanently checked, a depression forms in the wall, and gradually grows downwards until it extends from the coronet to the ground surface of the foot. To discover whether the horny wall will again recover its normal shape, the horn below the coronary band must be inspected.

A further obstacle to recovery consists in the papillæ of the coronary band becoming thrust out of place, and not returning to their normal 
position. During acute inflammation the papillæ of the coronary band are displaced relatively to one another, and must regain their former position before they can again produce a normal growth of horn. This is effected by the neighbouring papillæ, which have not suffered displacement. As the growth of horn starts from the periphery of the injury, the diseased and displaced papillæ are gradually brought into position by the growth of neighbouring hor'n tubules; and as repair proceeds, the normal state is restored. Sometimes the growth of horn is irregular, because it sets in before swelling has completely disappeared, and the papillæ are unable to take up their normal position; or, again, hor'n production does not proceed regularly from the periphery towards the centre of the injury. The displaced papillæ then become fixed in their abnormal position, and produce a horny prominence on the coronet, in consequence of certain of them not contributing to the formation of the wall, but producing disconnected masses of hor'n. The horny wall then shows a defect at the point in question, which detracts from its solidity. Sometimes the exterior of the wall is thickened, because horn formation goes on while the coronet is still swollen. In exceptional cases, this thickening may take place on the inner surface, producing a keratoma.

In addition to such complications, more dangerous consequences may follow treads. If, for instance, in consequence of direct injury to the joint, its perforation by pus during an attack of purulent cellulitis in the subcoronary connective tissue, or of extension of necrosis towards the depths, purulent or septic inflammation attacks the pedal-joint, the condition becomes incurable.

Necrosis of the extensor pedis tendon produces severe lameness; when the foot is extended, or attempts are made to bear weight on it, the lower part of the limb assumes a position of excessive volar flexion. The condition, however, may involve no permanent injury, the defect in the tendon being replaced by connective tissue, which firmly unites the ends. Extension of necrosis to the sensitive laminæ is dangerous, because of its tendency to involve large areas, leading to diffuse inflammation of the sensitive structures, which may be followed by loss of the hoof Necrosis of the lateral cartilage always produces quittor.

Prognosis. The consequences of treads may be exceedingly varied. The following points are of chief importance:-

(1) The position and extent of injury. The more nearly the injury approaches the band, the graver it becomes. If the slin and upper part of the horny wall are alone injured, and the wound is not deep, healing soon follows; but treads on the coronary band not only offer great obstacles to treatment, but may involve the pedal-joint, especially in the small, clean feet of well-bred horses. At the centre of the toe the

V.S. 
pedal-joint lies scarcely more than $\frac{3}{4}$ of an inch below the coronary border of the horn, but as we pass back it recedes from the coronet, and is therefore less easily injured. For this reason, and also because of the danger of injury to the extensor pedis tendon, injuries at the toe are always more dangerous than those at the side of the foot.

(2) The size of the swelling and degree of lameness. As probing gives no reliable information of the extent of the injury, and may produce perforation of the pedal-joint, or introduce septic substances into the depths of the wound, the operator, in forming his prognosis, is forced to fall back on the amount of swelling and pain. Particular reticence should be observed when the parts are exceedingly painful but swelling is limited, especially if the pain is without visible cause. The general condition, appetite, temperature, sc., must, of course, not be neglected in forming a prognosis.

Diagnosis is rarely difficult, though in horses with long hair about the feet some little care is required to discover the injury. When necrosis sets in it may be doubtful whether the original injury was a tread, or whether we have to deal with a case of spontaneous gangrene. Brushing, and similar injuries, produce wounds resembling those caused by treads both in course and consequences.

Treatment. The first and most important precaution is to clean and disinfect the wound. The hair which has been thrust into it by the heels of the other shoe, and the septic material which is always introduced in large quantities, should as far as possible be removed. The surrounding hair is clipped away, and any loose shreds of dead tissue cut off with scissors. The entire hoof, particularly the coronet, is then washed and the wound soaked with carbolic solution, or, if time allow, the foot may be immersed in a bath of antiseptic solution. The wound may also be sponged out or injected with a 10 per cent. solution of chloride of zinc. If the coronary band be much swollen, the horn should be rasped away over an area corresponding to the swelling before the final dressing is applied. Moist carbolic or sublimate dressings are preferable, and after the wound has been dusted with iodoform, a mass of tow, moistened with a disinfectant, is applied, the whole being covered with several large tampons of oakum, which are held in position by a bandage. Excessive pressure must be avoided. If the dressing be too tightly applied, it not only increases pain, but favours necrosis in the region of the coronet. Once the dressing is applied, it can be liept moist by pouring over it several times a day a disinfecting fluid. If pain diminishes and the dressing is not saturated with discharge, the latter may be left in position for two or three days, otherwise it should at once be renewed. Should suppuration occur, the moist dressing should be replaced by a dry one. 
Particular care is required during cicatrisation; horn formation at the coronet should be discouraged until all s̊velling has disappeared. Should it occur, the parts may be moistened with a 5 per cent. caustic potash or soap solution, which will check the hardening of the new horn until inflammatory swellings disappear. Irregular cicatrisation must be treated in a similar way.

Very little can be done to prevent abnormal growths of horn on the coronet. If such growths only affect a small area-if, for instance, the base is no bigger than a five-shilling piece-the newly-formed mass may be completely cut away, which will improve matters, and may possibly result in complete cure. This becomes necessary when the growth overhangs the wall, and, as is often the case, maintains chronic suppuration around its base. T'he entire base is cut away and a dressing applied to check bleeding, which is sometimes severe, and may necessitate the use of the actual cautery.

The deferred complications which follow severe accidents like those due to an omnibus wheel passing over the coronet, must be combated as they arise. Deformity of the foot, the growth of large exostoses and lameness can be treated respectively by the use of the farrier's rasp and knife, by firing and by neurectomy.

\section{INFLAMMATION OF THE SUBCORONARY CONNECTIVE TISSUE}

The abundant connective tissue underlying the coronary band is in intimate connection with the subcutaneous connective tissue of the coronary region, and is related below to the parachondrium, and through this to the fibro-fatty frog.

The subcoronary connective tissue is loose, and contains the great venous plexus of the coronet. It extends as far as the capsular ligament of the pedal-joint.

Injuries to the coronet, like treads and brushing wounds, often lead to infection of, and diffuse inflammation in, this connective tissue (cellulitis of the subcoronary connective tissue). As a rule, the disease is confined to one side of the hoof, but sometimes extends to the other, so that the swelling involves the entire coronet.

The condition is ushered in by inflammatory swelling, which appear's more or less distinctly around the coronet, and is succeeded by violent pain, especially when weight is placed on the foot. The lameness is in direct proportion to the swelling, and is usually so severe as to prevent the animal ever standing firmly on the foot. 'The lymph vessels above the hoof are sometimes swollen, though this cannot always be detected with certainty. Rotation of the phalanges causes great pain.

The disease shows a great resemblance to purulent inflammation of 
the pedal-joint, and the diagnosis may for a time appear doubtful. Lameness, appearing simultaneously with swelling, points to primary subcoronary cellulitis; in secondary cellulitis, due to purulent inflam. mation of the joint, the swelling is preceded by severe lameness. And further, while in primary cellulitis pain generally diminishes after discharge occurs, in purulent disease of the pedal-joint it persists.

Sometimes synovia is discharged, or the pedal-joint may be felt by

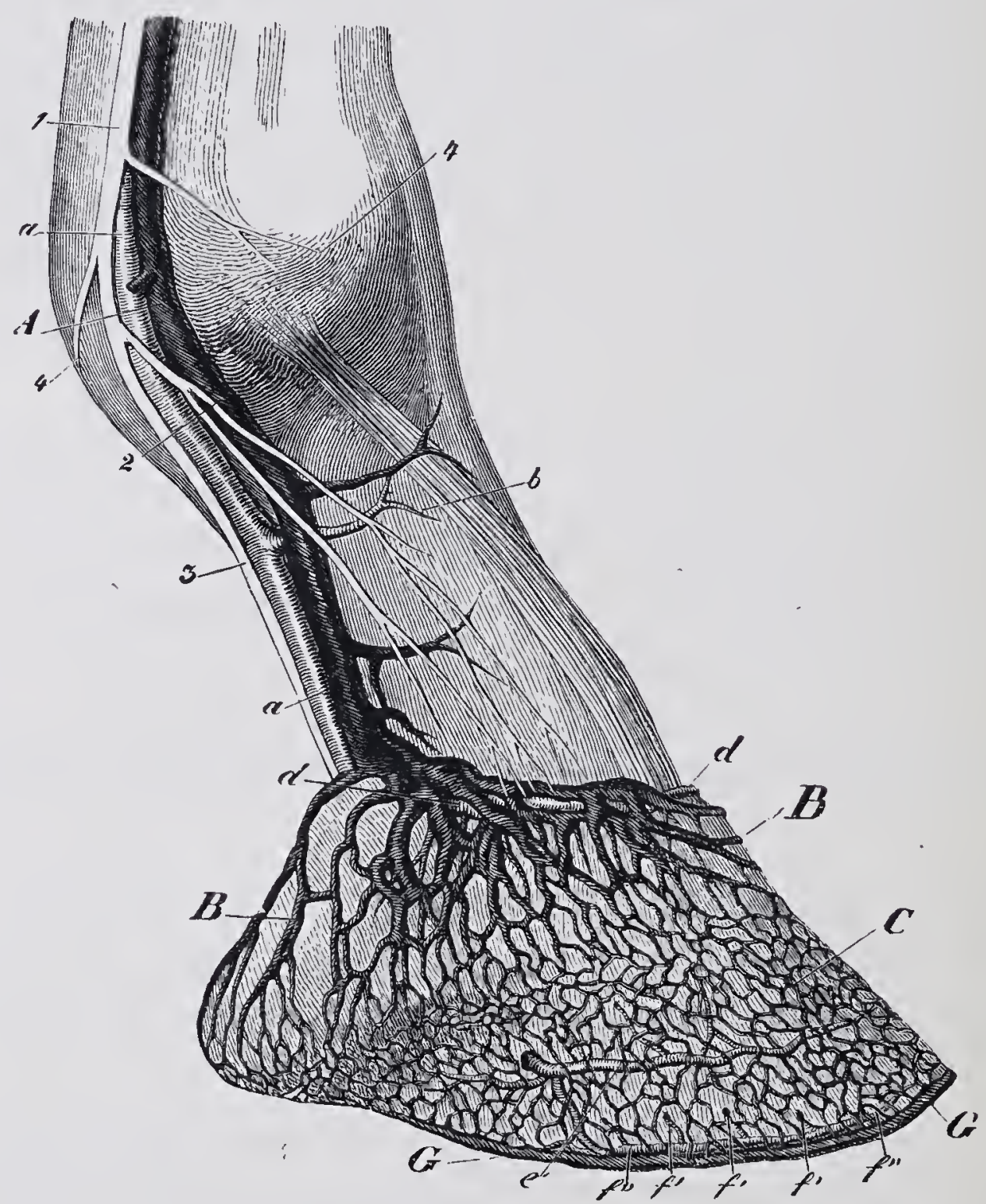

Hig. 296. - Lateral view of fore foot, with nerves and injected vessels, showing some of the structures involved in inflammation of the subcoronary connective tissue and its complications. $a$, Digital artery; $b$, perpendicular artery ; $e^{\prime}$, preplantar artery; $f^{\prime}$, twigs from the plantar artery which escape through the foramina, just above the lower margin of the os pedis, and by their anastomosis form $f^{\prime \prime}$, the circumflex artery of the toe; $A$, digital vein ; $B$, coronary venous plexus; $C$, laminal plexus; $G$; circumflex vein ; 1 , digital nerve; 2, anterior terminal branches of digital nerve; 3 , posterior terminal branches of digital nerve; 4, cutaneous branches.

introducing the finger; in such case no doubt can exist as to the nature of the condition.

The course of subcoronary cellulitis is usually unfavourable. Sometimes laminitis results from the animal continually standing on the other foot, and the patient dies from decubitus; sometimes the disease seizes on one or other of the lateral cartilages and produces quittor, but not infrequently septic inflammation occurs in the coronary band, destroys extensive tracts of tissue, and leads to fatal pyæmia or septicæmia. The 
large vessels of the coronary venous plexus (see fig. 296) particularly favour the development of pyæmia. Purulent inflammation of the pedaljoint, produced by extension of the cellulitis, is a frequent complication. Its onset is characterised by increase of pain; and if inflammation had not previously extended beyond one-half of the hoof, by the swelling involving the entire coronet; fever is a constant symptom, though in some cases it may even precede this condition.

Resolution is comparatively rare, and recovery, after perforation of the abscesses, still more so, though it has once or twice been seen.

The inflammation scarcely ever affects the fibro-fatty frog, though it may attack the connective tissue above the coronet, producing necrosis of the fascia at this point, and leading to chronic suppuration and formation of sinuses.

The prognosis is generally unfavourable, so that it is only advisable to treat animals of considerable value. On the other hand, recovery is not impossible, even when pain is severe, for sudden improvement sometimes occurs when the abscesses break.

Treatment. To prevent complications, all injuries of the coronet and its neighbourhood in which the skin is perforated, should if possible be treated antiseptically. Surface injuries are rarely dangerous. Treads on the coronet, especially when near the hoof, require particular care to avoid purulent inflammation.

If cellulitis has already appeared, dispersal will be favoured by warm baths, to which it is well to add some antiseptic. The appearance of distinct fluctuation should be the sign for immediately opening the abscess, though considerable bleeding must be expected, and almost always occurs. After discharge of the pus, which is almost always blood-stained or decomposed, the cavity should be washed out with a disinfectant, and tampons inserted to check bleeding. The tampons can be kept in position for twenty-four hours by a bandage, the pressure of which will increase their styptic action, but care must be taken not to apply it so tightly as to produce necrosis. For the next few days the abscess. cavity must be repeatedly and carefully syringed with disinfectants, and precautions taken against retention of pus; it may even be necessary to insert a drainage-tube.

As the condition is painful, a good straw bed should be given, lor there is less risk of laminitis occurring in the other foot, and hence less danger of a fatal termination from decubitus when the animal takes regular rest. 


\section{IY.-CORNS.}

The term "corn" embraces a number of diseased conditions of the posterior portions of the foot, and especially of the heels. The injury has its seat in the sensitive laminæ of the quarters, in the sensitive bars, or in the sensitive sole. The condition has, therefore, been divided into three corresponding divisions. As a rule, it consists in bleeding at the surface of the horn, resulting from pressure or rupture. As long as the hæmorrhagic spot remains uninfected, no inflammation results, but infection is followed by a pododermatitis, which may either be superficial or parenchymatous. In the latter case exudation occurs, in consequence of which this form has been termed moist corn, in contradistinction to the dry form in which inflammation is absent. In the wall or sole, the bleeding is oftenest seen at the surface of the papilla. The blood in most cases is extravasated between these and the hoof-horn, the inner surface of which it saturates, and imparts to the newly-formed horn-cells a red colour. The red spots thus formed sometimes appear as fine points if bleeding be confined to isolated papillæ, but generally as large red stains, which are only seen later when cutting out the footthat is, after the coloured layers of horn have grown down to the free surface of the hoof.

If the extravasated blood becomes infected, inflammation follows, and often proves serious-as, for instance, when pododermatitis parenchymatosa extends from the bars to the fibro-fatty frog, in which it induces diffuse purulent inflammation. A troublesome condition may also be produced by infection of the parachondrium and formation of a quittor. These two complications are unfortunately rather frequent.

Causes. In consequence of the close union between the vascular laminæ and the horn, it results that, as bleeding occurs so easily, inflammation is seldom seen without hrmorrhage. Traces of it are, however, sometimes seen in the heels. The horn of the sole then appears shining, semi-transparent, and coloured yellow, changes which point to inflammation of the loof matrix.

The fact that the posterior parts of the hoof are oftenest the seat of mechanical lesions is partly due to their greater mobility; the movement of the hoof is most apparent at this point, and rupture of soft structures, therefore, more liable to occur. Then, again, the heels carry a larger proportion of the weight, and the horn covering them is wealiest. The more frequent occurrence of corns in the inner heel and in fore feet must also be referred to the greater weight borne and the more marked impact during rapid movement.

Corns are favoured by long, weak fetlocks, and by defects in formation, especially in the front limbs. In animals which stand with the feet well 
apart, the inner heel is most generally affected; in the opposite conformation, the outer. The weaker the horn of the heels, the more readily do such injuries occur: rings on the surface of the heels suggest the existence of corns. Abnormally narrow feet are generally the seat of corns, and in contracted heels they are almost always present. But wide hoofs are also liable to them; in such case the corn being an injury of the sole, whilst in narrow hoofs the wall is more often affected.

The principal external causes are faulty shoeing, especially improper paring, the use of too short or too narrow shoes, or allowing the shoes to remain on for too long a time. As corns are very rare in unshod feet, the idea obtained currency that they were always produced by the pressure of the shoe. For this reason farriers often endeavour to prevent the

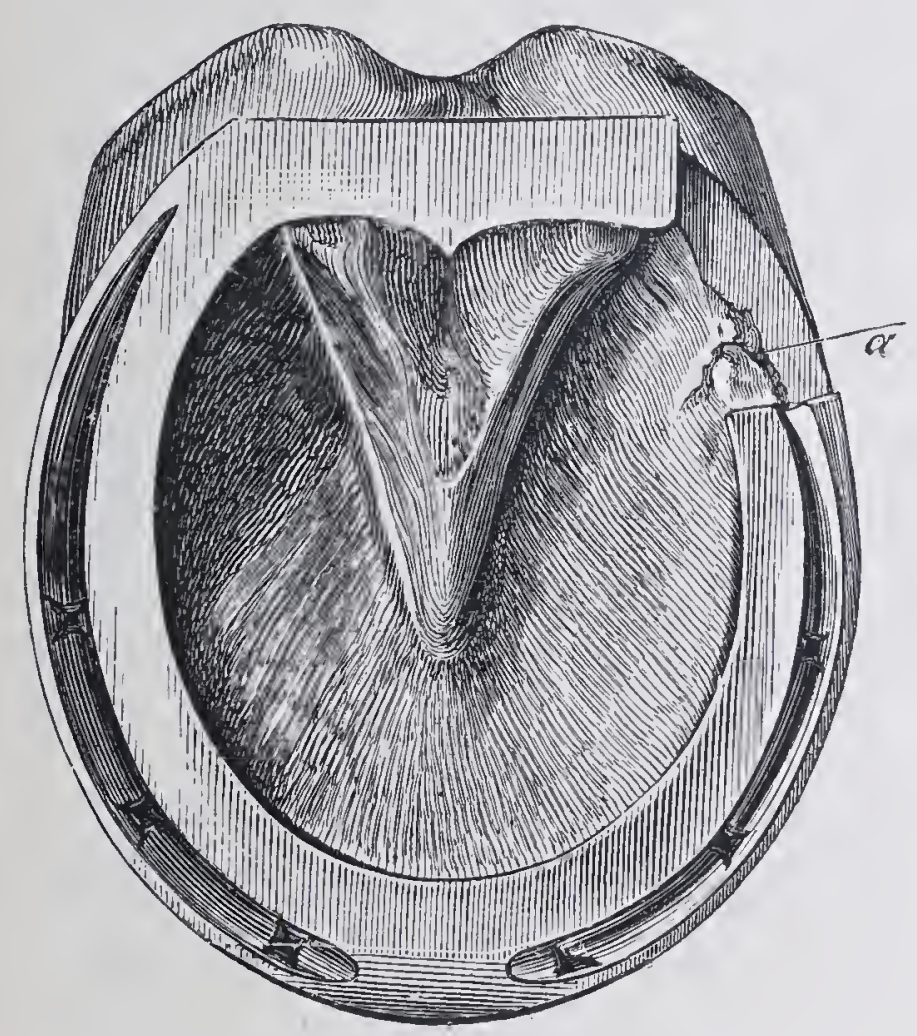

Fic. 297.-Three-quarter bar shoe, $\alpha$, Seat of the corn.

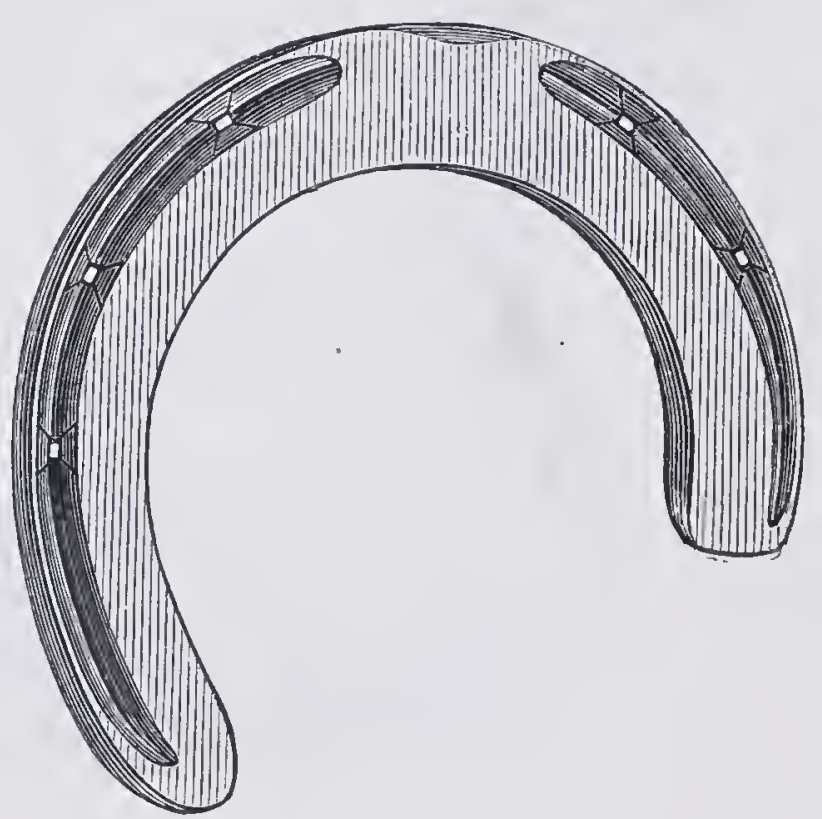

Fig. 298. -Ordinary three-quarter? shoe.

injury by rasping away the inner wall, so that it no longer touches the shoe. The result shows, however, that this idea is erroneous, for corns occur just the same, and, in addition, a sand-crack often forms at the coronet. It therefore seems clear that the heel should be supported by the shoe; if not, it descends, and leads to rupture of the sensitive laminæ. The attempt to remedy the evil thus leads to its propagation.

Prognosis. Although corns are so common that their absence in animals working in large towns is almost an exception, yet they give rise to much trouble. Severe consequences, however, only follow when the point of origin becomes infected; and as infection almost always takes place from without, it is of the greatest importance to protect the hæmorrhagic spot against the entrance of foreign material. Sometimes, however, the horn is so defective that no precautions seem sufficient to prevent it, and then the animals suffer habitually, and little hope of cure 
can be given. Suppuration, when once established, may be followed by any of the above-described complications, especially in oases of pododer: matitis parenchymatosa. Swelling of the coronet points to the later appearance of quittor, whilst severe lameness, pain on dorsal flexion of the toe, and swelling between the bulbs of the heel suggest purulent suppuration of the fibro-fatty frog, and affection of the flexor pedis perforans tendon.

Bad conformation and work on stone-paved streets naturally lessen

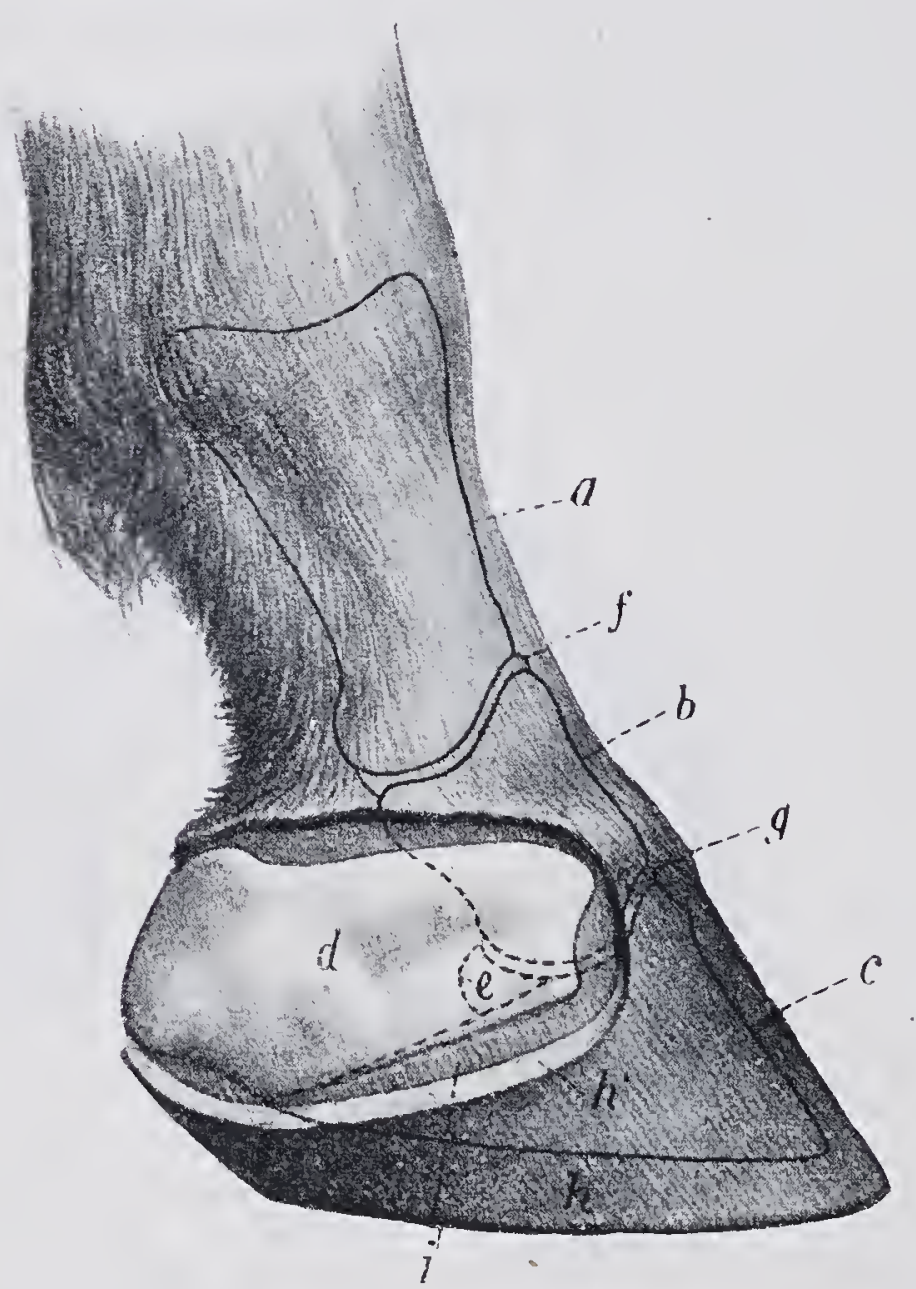

Fig. 299.- Showing the lateral cartilage and its relations. $\quad \alpha$, Os saffraginis; $b$, os coronse; $c$, os pedis; $d$, lateral cartilage ; $e$, os naviculare ; $f$, pastern-joint; $g$, coronet-joint ; $h$, horny wall; $i$, sensitive laminæ. the chances of complete recovery.

Treatment follows the principles above indicated (see treatment of "Acute Inflammation of the Podo" phyllous Membrane"). 'The immediate cause must if possible be removed, and the shoeing receive attention. For horses with broad, flat feet the shoe should be fitted long at the heels and should be broad in the "cover." After" recent suppurating corn a barshoe may advantageously be applied. On the other hand, horses with high, "blocky" feet often go better in " tips," Charlier shoes, or shoes with thin heels, provided no active inflammation exist. The regulation shoe for corn, employed by the great Compagnie des Omnibus de Paris, is a three-quarter shoe, the branch of the shoe being cut away on the side corresponding to the injury. Since adopting it the number of cases of corn has greatly diminished. From the 1st of April, 1895, to the 1st of August, 1896, only 44 cases of corn were reported amongst 15,500 horses.

In "dry" corn nothing further is required. Paring out corns is undesirable, and even injurious, because, after removal of the reddened masses of horn, the soft tissues are often exposed, and entrance of infectious material facilitated.

In parenchymatous disease, free exit must be provided for the pus; if necrosis have occurred, the dead tissue should be removed as early as possible, and the spread of infection checked, antiseptics being employed either to destroy or hinder the development of specific organisms. (For 
a detailed account of the various methods of shoeing horses with corns, see Dollar and Wheatley's "Horse-shoeing and the Horse's Foot.")

\section{V.-QUITTOR.}

The term "quittor" is applied to a chronic, purulent inflammation of the lateral cartilage and of surrounding structures, associated with necrosis and the formation of sinuses. The cartilaginous prolongations of the wings of the os pedis are surrounded by a firm connective tissue, provided with many elastic fibres, termed the "parachondrium," which is in close relation with the subcoronary connective tissue, and with the fibro-fatty frog. Suppuration in the parachondrium is always liable to be followed by necrosis of the cartilage; and, owing to the difficulty with which the dead portions separate, suppuration is obstinate, and the narrow canals or sinuses through which the discharge escapes are extremely difficult to heal. From time to time the inflammation becomes more intense, and leads to destruction of further portions of cartilage, so that, unless energetic treatment be resorted to, the condition usually continues three to five months.

A similar affection occurs in the subcoronary and subcutaneous connective tissue above the coronet, and has been termed "coronary fistula." In the same way the French discriminate between "Javart cutane" and "Javart cartilagineux."

The process is caused by infection of the connective tissue with staphylococci. Sometimes the organisms enter through the skin, as in treads, in gangrenous dermatitis, and similar diseases, but direct injury of the lateral cartilage is a rare cause of quittor formation if the wound be at once properly treated, and in by far the majority of cases the condition has its origin in corns. The inflammation thus excited extends to the connective tissue lying above the sensitive sole, or a crack forms in the horn of the wall, in consequence of which the connective tissue lying under the sensitive laminæ may become inflamed.

On examining the parts, the parachondrium is found to be thickened and infiltrated with larger or smaller abscesses, while portions of the surface of the cartilage are necrotic, some being still adherent, others completely separated; they are generally of a greenish colour.

The course of the disease is always chronic, whether it results from corns or from crackis in the horn. Recovery generally occurs, though sometimes only after complete destruction of the cartilage, a process which takes three to five months. As a rule, quittor remains confined to one side of the hoof, i.e., to one lateral cartilage, the inner cartilage being most frequently affected, because primary disease is commoner at this point. For a similar reason the destruction of cartilage commences 
at the posterior end and extends forwards. The necrotic fragment may separate partially, but, as a rule, it maintains connection with the healthy tissue in front, to which the process gradually extends. Nevertheless, spontaneous separation and recovery is possible when disease is limited to the posterior third of the cartilage. The cartilage so called is composed of two tissues: the superficial layer being truly cartilaginous, the deep fibrous. The cartilaginous layer is thickest in front. At the back it forms small plates, separated by septa of connective tissue, an arrangement which favours delimitation, inasmuch as these septa constitute boundaries at which necrosis is sometimes arrested.

Necrosis of the os pedis, suppuration in the pedal-joint, and cellulitis of the subcoronary connective tissue or fibro-fatty frog sometimes occur as complications. The first only increases the duration of the process; but inflammation of the pedal-joint generally proves fatal, whilst purulent inflammation of the fibro-fatty frog and of the subcoronary connective tissue are often the forerunners of other grave conditions (see foregoing section on "Piclied-up Nails," \&c).

Symptoms. As purulent inflammation of the parachondrium is the starting-point of quittor, the first symptom is a diffuse swelling of the coronet and of the bulb of the heel. This inflammation may in rare instances result in resolution; but generally an abscess forms, and discharges pus mixed with blood, after which the swelling partially disappears, leaving a sinus 1 to 2 inches in depth. After some time this closes up and a fresh swelling forms, which takes the same course. The process is repeated at varying intervals. Often there are several sinuses which communicate with one another. In old-standing cases the wall of the hoof is thrust outwards and the horn is ringed, while the coronet may show the scars of several old sinuses. The depth to which ringformation has extended is an approximate guide to the age of the condition, if one bear's in mind that the wear of the foot is about $\frac{3}{8}$ inch per month.

Lameness is not often very severe, and may be so slight as to allow the animals to continue at slow work. It bears no fixed relation to the seriousness of the disease. With each recurrence of inflammation, however, the pain increases, and, if the pedal-joint or fibro-fatty frog be implicated, it always becomes very marked, and then shows the peculiarities distinctive of disease in the regions named. As the capsular ligament of the pedal-joint is in close contact with the inner surface of the anterior portion of the cartilage, the pedal-joint is occasionally invaded-almost necessarily with fatal results. This termination would be more common but for the fact that the capsular ligament usually becomes inflamed and thickened by a deposit of fibrous tissue before necrosis actually extends to the cartilage in contact with it. 
Prognosis. It is very difficult to foretell the duration and probable results of quittor. As a rule, coronary sinuses heal more rapidly than those of the lateral cartilage: In consequence of the process generally extending from the posterior towards the anterior border of the cartilage, the chance of early recovery is greater the nearer the sinus lies to the posterior end and to the upper border of the cartilage; absence of swelling in front of the sinus is a specially favourable symptom. Great swelling, severe lameness, abundance of discharge, and deep sinuses suggest a long continuance of the disease and troublesome complications, though the opposite conditions do not necessarily guarantee the absence of serious changes. Quittor produced by cracks in the horn generally takes a very protracted course, being often due to necrosis of the os pedis. The probability of early recovery is in direct proportion to the recentness of the attack. In restive horses treatment is difficult, and recovery correspondingly protracted. Finally, the danger of purulent inflammation of the pedal-joint is greater in horses with small, weak feet than in heavy horses with large hoofs. In the latter, however, quittor is generally more obstinate than in the former. Speaking in general terms, quittor is less serious in hind than in front feet, probably because the lateral cartilage in the hind foot is smaller, less dense, and more vascular than in the fore, and, therefore, the exfoliation of the diseased fragment is more readily effected.

Treatment. It is of primary importance to prevent infection of the parachondrium, and, therefore, especial attention must be given to the careful treatment of corns, cracks in the horn, and other diseases likely to produce parenchymatous inflammation of the sensitive structures in the posterior portions of the foot. The freest exit must be given to inflammatory products, and the centre of disease liept as clean as possible. Injuries to the coronet are similarly treated, especially when the lateral cartilage is injured. Brïuer cured an injury of the cartilage, consequent on a tread, by carefully cleansing the wound with sublimate solution, and applying a dressing of iodoform and starch in the proportion of 1 to 5 . Even extensive injuries of the lateral cartilage may heal by primary intention.

If swelling of the parachondrium points to suppuration, the hoof must be kept soft, and a lukewarm foot-bath, containing a disinfectant, used to assist resolution. In case pus has already formed, it must be afforded free exit. Although abscess-formation always entails a quittor, suppuration may sometimes be prevented extending to the cartilage by syringing out the abscess cavity with 10 per cent. chloride of zinc or other disinfectant. When this fails other methods of treatnient can be resorted to, all of which, however, depend on immediately removing the necrotic piece of tissue, giving free exit to discharges, and checking the growth 
of pus cocci in the parachondrium. These comprise:-Injection with disinfectants. Liquor Villati has for many years enjoyed a wide reputation for this purpose. Williams recommends 10 per cent. watery solution of corrosive sublimate, to which a few drops of hydrochloric acid have been added to ensure complete solution. Carbolic acid has also been employed. In using these, the principal point is to bring them intimately into contact with the diseased membrane. For this reason it is best to first inject into the sinus, by means of a small lead syringe, some clean water or carbolic solution to, as far as possible, remove pus, a process which can be aided by gentle pressure. Then follows an injection with the active agent, which may be either liquor Villati, 5 per cent. carbolic acid, 10 per cent. chloride of zinc, or corrosive sublimate. To ensure the solution reaching the bottom of the fistula, the syringe should be provided with a long, thin nozzle.

The injections must be made daily, and continued until they cause considerable pain, when they are discontinued for a time and again resorted to until suppuration altogether ceases. Sometimes after a few days the swelling diminishes and pus ceases to be discharged, both favourable symptoms, as they point to commencing healing.

When the sinus is old, deep, or very oblique, and especially if it extends below the coronet, it is advisable to make a counter-opening. The coronary band should not be divided. After thoroughly thinning the wall a grooved director is passed to the bottom of the sinus, the wall divided from without, a small fragment of the tissue of the wall removed, and a short length of gauze passed through the sinus and tied, to secure it in position. Sometimes two sinuses exist, and require the insertion of two drains.

Winkler recently recommended spirituous sublimate solutions, followed at a later stage by acetate of lead, also dissolved in spirit. He avoids using water.

Gamgee's mixture has again come into some favour. It consists of sublimate 17 parts, acetate of lead 34 parts, spirit 136 parts, hydrochloric acid 2 parts. After clipping away the hair and enlarging the fistula, a dry dressing is applied. On the second or third day, and again on the fourth, fifth, and sixth, the parts are injected. From the seventh day onwards, the openings of the fistulæ on the coronet are only disinfected and a dressing applied. In a fortnight recovery is said to take place (Walter). To protect himself against the escaping fluid, the operator may wind a thick piece of tow around the tube of the syringe. Giesecke recommends a 5 to 20 per cent. solution of protargol.

If the canal be too narrow to allow a syringe to enter, a small fragment of sublimate, or a thread soaked in sublimate solution, may be passed to the bottom, a method of treatment which is especially useful in old sinuses 
with callous borders. After twenty-four hours the thread is removed, and exfoliation of the necrotic walls of the fistulæ assisted by warm foot= baths. Others employ the actual cautery for the same purpose, a farrier's poker being made red-hot and introduced into the sinus. This is sometimes successful, but requires care in well-bred horses with small hoofs. The instrument must not be introduced too far, as the pedal-joint might be opened, and special precautions are required in operating on the forward end of the cartilage. Neither of these methods is, in fact, safe unless confined to quittors in the posterior half of the cartilage. In enlarging the sinus, Williams employs a specially-constructed bistouri caché closely resembling the herniotome (fig. 129).

Koch recommends inserting setons. After placing the foot in a bath of 3 per cent. creolin solution, he endeavours to produce a counteropening below the coronet by passing a Gerlach's needle, and inserts a tape moistened with turpentine through the soft tissues. The parts are bathed once a day with creolin and injected with liquor Villati, and in sixteen to eighteen days the seton is removed. Möller repeatedly tried this treatment, but had no particular success, while he often found the insertion of setons a difficult matter. T'o diminish the pressure of the horny wall on the swollen parts, Giesecke recommends applying linseed poultices containing creolin, and afterwards removing the softened horn. The sinuses above the coronet are scraped out with the curette, injected with 8 per cent. solution of chloride of zinc, and finally a small mass of tow covered with sulphate of copper is introduced. This procedure may require to be repeated ; after-treatment consists of injection with antiseptic fluids.

In France, Lignières has recently recommended injections of "resinate of copper," injected daily for three or four weeks. With this treatment he cured thirty-two cases, some very serious. Considering the strong predilection towards operation shown by French veterinary surgeons, this announcement deserves some attention.

Before using foot-baths the wall should be thinned, and the hi,n removed with the rasp over a space corresponding to the swelling. H'ee curetting seems very useful in some cases, but in others only increases pain and inflammation; Möller therefore prefers sublimate threads or "pokering" to dilate the fistula.

Some cases are rapidly cured by one or other of the above-described methods, but in others no treatment seems effective. It is exceedingly difficult to exactly indicate the necessary conditions for healing, and therefore for some year's operation has been more and more practised. Although operation can only be recommended in really serious cases and after simpler methods have received careful consideration, it nevertheless offers great general advantages, especially as regards shortening the 
course of the disease. The French school, which first invented the operation, practise it with great success. If conditions are favourable to healing, operation should certainly be postponed, otherwise, and especially if careful after-treatment is possible, it should, on the contrary, at once be resorted to. When, for example, the quittor is of old standing, and has extended to the anterior portions of the cartilage, when pain is slight, and the animal able to work in spite of the discharge, it is better to try one or other of the above-described methods of treatment. An opposite condition indicates the necessity for operation. In performing this, however, a time should be selected when inflammation is not acute, or pain severe, and when suppuration is slight, for under these conditions the chance of

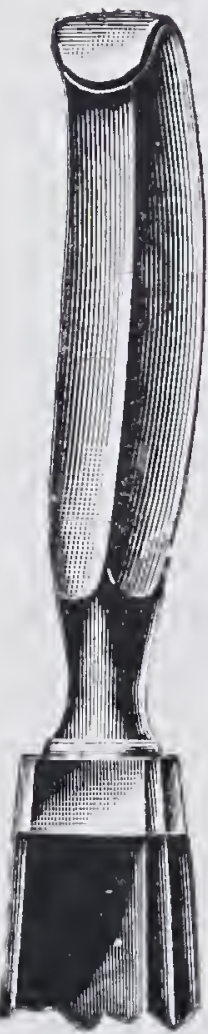

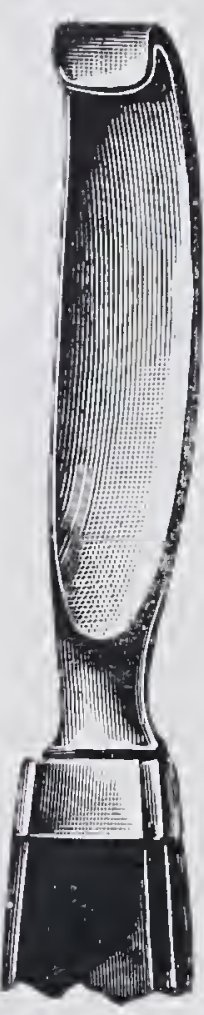

3

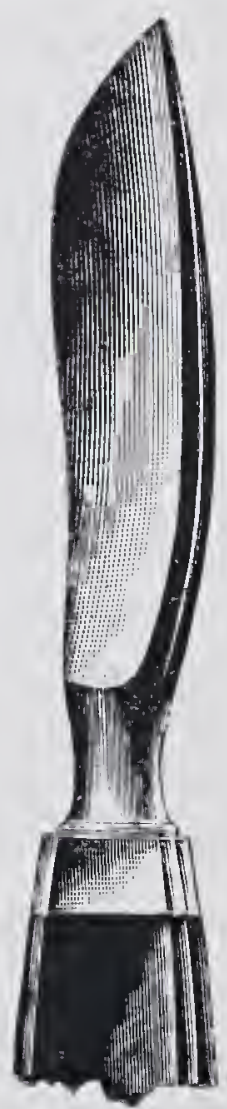

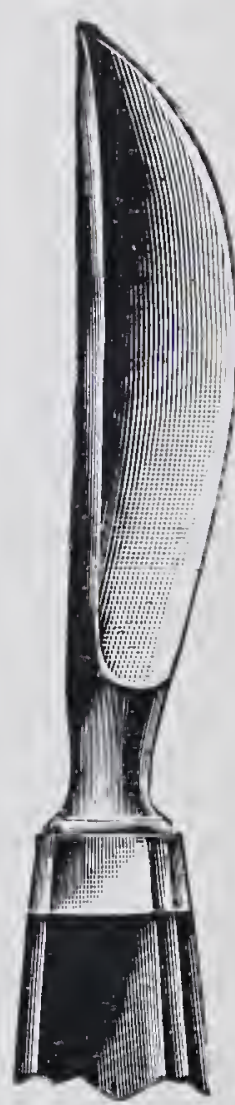

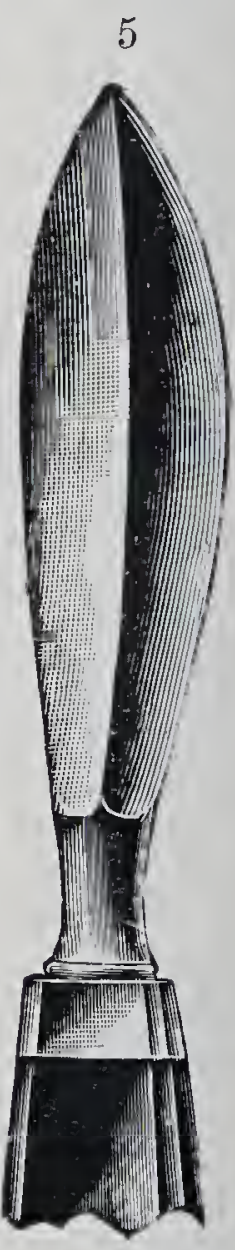

FIG. 300.-1 and 2, Double and single edged knives for thinning the horn; 3, 4, and 5, left-handed, right-handed and double-edged knives for removing the lateral cartilage in the operation for cartilaginous quittor. (French nrodels.)

aseptic wound-healing is far greater. Total extirpation of the cartilage is preferable to any partial measures, though, if the disease and swelling: on the coronet be circumscribed, the latter may be tried. But even under these circumstances it very frequently fails to stop the disease, and complete extirpation afterwards becomes necessary. The method of total extirpation is divided into three stages-(1) Preparation of the field of operation; (2) Resection of the cartilage; and (3) After-treatment. The last requires at least as much care and precaution as the operative procedure, and if after-treatment cannot be properly carried out, operation is better avoided.

After carefully cleaning and thinning the hoof, a portion of the horny wall, corresponding to the diseased cartilage, is rasped until thin enough 
to yield under the pressure of the thumb. This avoids the necessity of stripping the wall, which is very painful. The parts are then soaked in sublimate, and if operation should not immediately follow, the hoof may be surrounded with sublimate wood-wool, and liept wet with sublimate solution for twenty-four hours.

The horse is cast, and the diseased foot firmly tied to the cannon bone of the diagonal upper limb, i.e., in disease of the internal lateral cartilage of the left front foot, that foot would be tied to the right hind limb. An Esmarch's bandage is then applied from the coronet to the knee, the dressing removed, and the foot rinsed with sublimate solution.

An incision is next made with a special curved knife (see fig. 300),

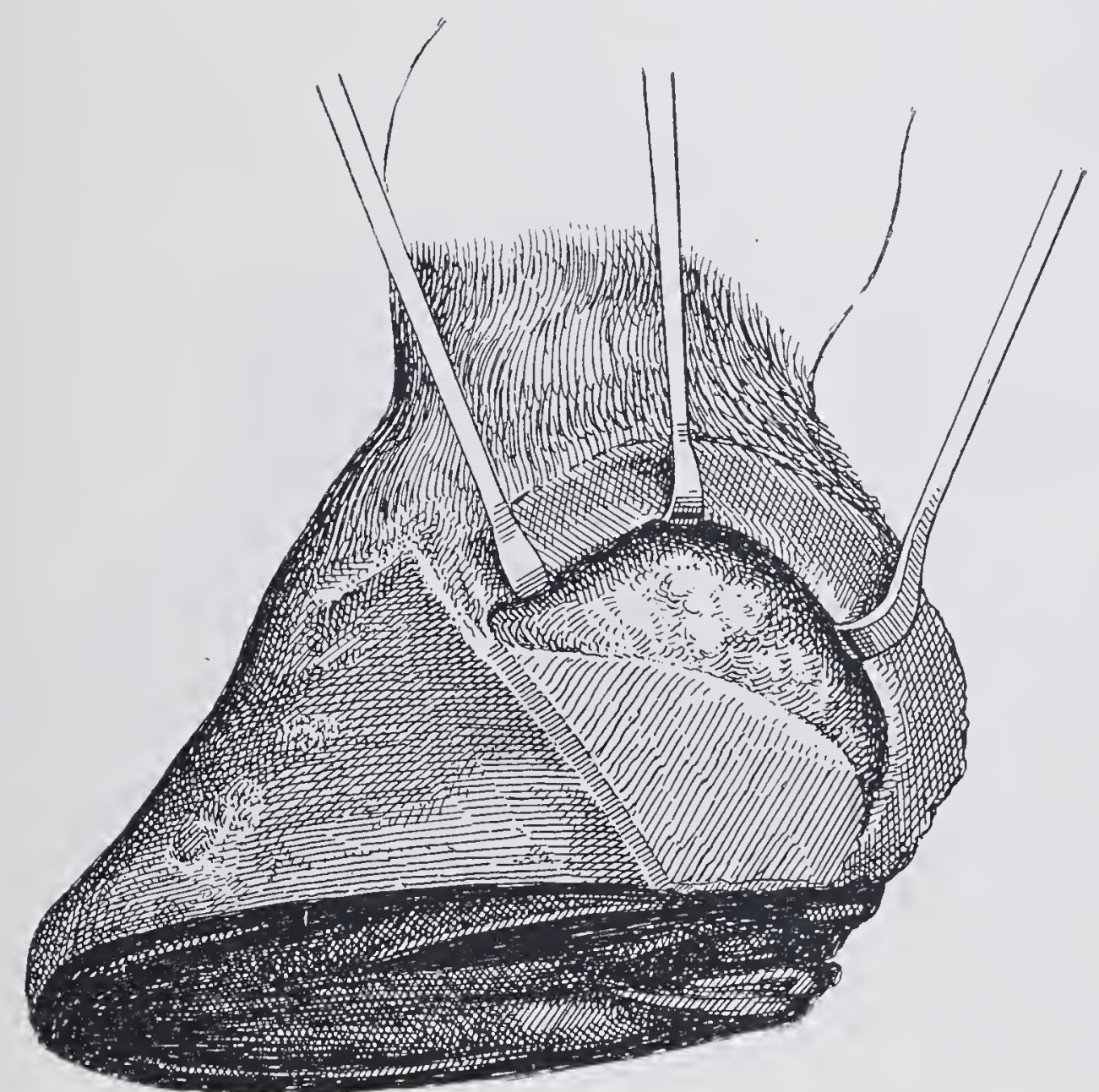

FiG. 301.-Operation for quittor. The whole of the lateral cartilage has been removed.

following the lower border of the coronary band, and corresponding to the size of the cartilage : any horn which has been left at this point is removed. The object of this incision is to separate the coronary band from the sensitive structures of the wall, with which it is continuous. The incision should not at this stage extend beyond the inner surface of the coronary band, and particular care must be taken not to divide the band, especially near the bulb of the heel.

The edge of the partially-divided coronary band is now drawn upwards with one or more retractors, and the double-edged linife passed cautiously in a perpendicular direction between the coronary band and the face of the cartilage so as to separate the two structures. The coronary band is then stripped off the face of the cartilage by gently rocking the linife 
to and fro along its long axis. The knife is used more as a lever than as a cutting instrument. Starting in the centre of the cartilage the operator first exposes the posterior, then the anterior, surface of the cartilage. Care must be taken not to cut either the coronary band or the cartilage.

The cartilage exposed, the operator seizes one of the single-edged knives, slides it into the wound with its cutting edge directed upwards and backwards, passes it behind the posterior extremity of the cartilage by executing a half turn of the instrument, and then with one stroke from within outwards, removes the posterior half of the cartilage, taking

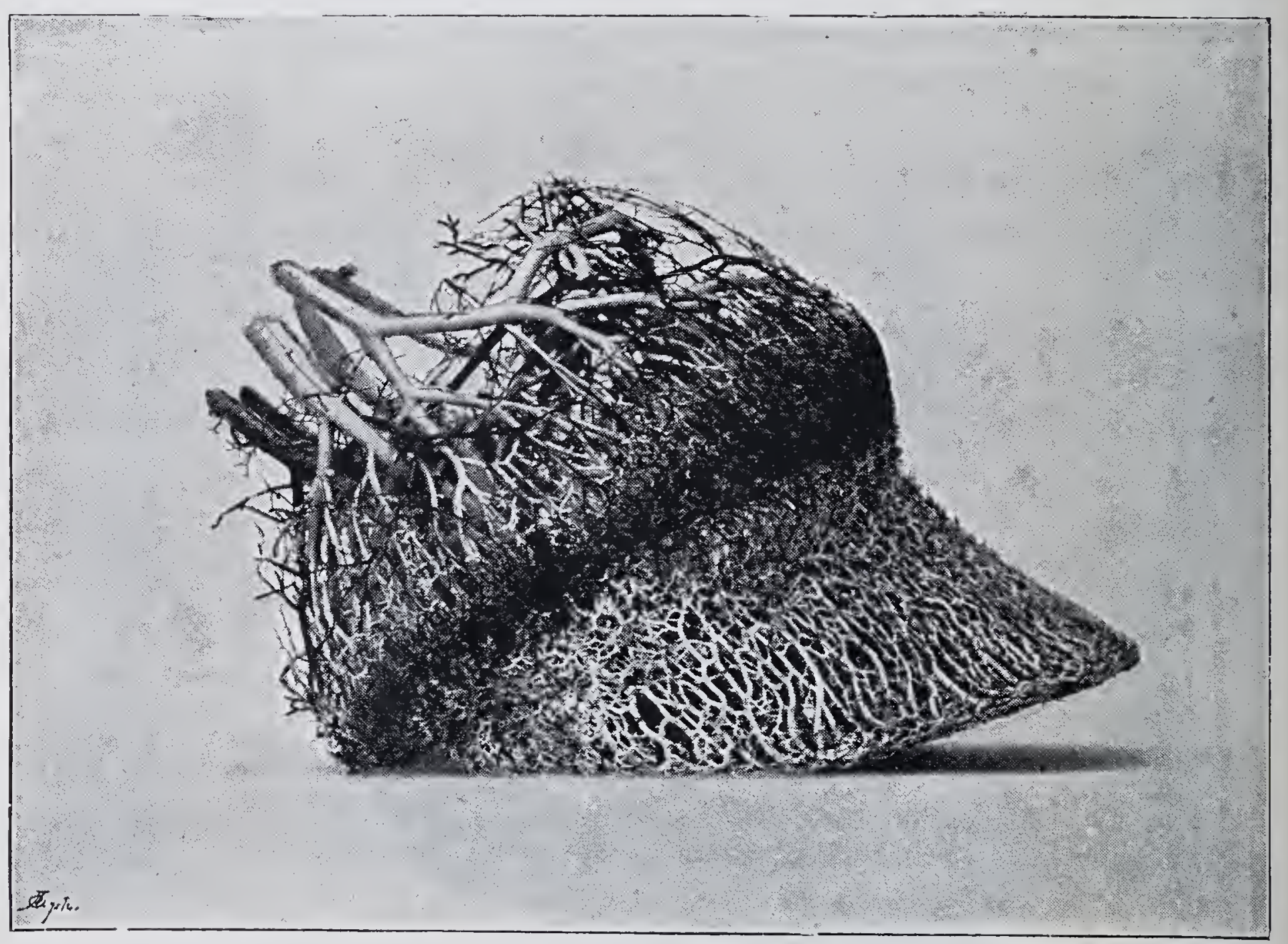

FIr. 302.-Celluloid injection preparation of the veins and arteries of the foot prepared by maceration. 'The veins are light in colour, the arteries dark.

care, however, not to injure the upper surface of the sensitive wall. In some cases there is very little cartilage at this point to remove, the greater portion having become necrotic and been replaced by fibrous tissue, which should be spared.

This completed, the toe of the foot is, by means of a cord passed round the fetlock and hoof, extended as far as possible to facilitate removal of the anterior half of the cartilage. By extending the foot, the capsular ligament of the pedal-joint is drawn away from the cartilage, and the danger of injury diminished. The knife is then held horizontally, its convex surface downwards, and the rest of the cartilage separated from underlying structures, and removed with aissecting or 
dressing forceps. By thrusting the finger under the coronary band, it is easy to discover whether portions of the cartilage have been left. Any remaining should be removed with the curette, for on their complete removal depends much of the success of operation.

The wound is washed out with sublimate solution, again examined, and any loose shreds or particles of cartilage excised with scissor's and forceps. The surface of the wound is then rubbed with 10 per cent. solution of chloride of zinc, the fistulæ scraped out with the curette, and dressed with chloride of zinc. Sublimate solution is then injected once more, and a dressing applied. French operators touch any "doubtful" spots with tincture of iodine. A couple of masses of gauze saturated with 5 per cent. carbolic solution are thrust under the coronary band to partially fill the cavity resulting from removal of the cartilage, and to check the bleeding which results after taking off the Esmarch's bandage. The hoof is then enveloper in carbolic jute or sublimate wood-wool, and a bandage firmly applied to bring the deep surfaces of the wound in contact and keep the dressing in place. A linen wrapper is placed over all, and supported by a straw rope passed round the fetlock. This ends the operation, which is, of course, carried out under antiseptic precautions, the hands being properly cleansed, and the instruments disinfected.

The first dressing remains in position twenty-four to forty-eight hours. Soon after the animal has risen, it may appear saturated with blood, but the bleeding is of little consequence, especially if the animal is not upset. It is usually best to place the horse in a stall. High-couraged horses should be operated on under chloroform.

After removing the first dressing, the hoof is washed with a disinfectant, the wound freed from blood, again rinsed out, and a smaller gauze tampon inserted. The dressing is similar to that employed after operation, and need not be renewed for eight to ten days, provided fever be not marked, or pain severe, and the covering show no signs of becoming saturated with discharge. The after-dressings are similar, though, as the granulations increase, and occupy more space, the quantity of carbolic gauze placed under the coronet should be diminished. It is of particular importance that union should start from the base of the wound. Under no circumstances should the lower portions of the coronet be allowed to come into contact with the upper part of the hoof before the deeper surfaces have completely united or the entire space above is filled up, otherwise a cavity is left at the base of the wound which, in case of suppuration occurring, would retain the discharge. For a similar reason, the newly-formed horn should be regularly trimmed away from the coronet. Excessive granulations around the coronet are removed by astringents or caustics.

$$
\text { V.S. }
$$


This treatment is continued until the defect appears completely filled up, and the coronary band is adherent to the underlying tissues throughout its entire length, i.e., until the space between the divided coronary band and its foundation is completely obliterated. A tar dressing is then applied, the wound surface being smeared with tar, and a bandage saturated with the same material wound round the hoof. If pain be slight, a bar-shoe can be put on, and the horse sent to slow work. The time occupied up to this point is from three to six weeks, so that, as a rule, operation considerably shortens the duration of the disease. Siedamgrotzky's cases, on an average, occupied thirty-one days in healing.

The popular idea that the operation renders horses useless for work on hard roads appears, after a large number of observations, to be without foundation. Many carriage-horses on which Möller operated recovered so perfectly that not the slightest trace of operation could be detected on examining the hoof; and the animals themselves worked for year's on the streets of Berlin.

Bayer has modified the above operation with the view of producing fewer and simpler incisions, facilitating the escape of discharge, and enabling the operator clearly to see each stage in the removal of the lateral cartilage.

On the day before casting the horn covering the seat of operation is thoroughly thinned, the parts are freely scrubbed with a brush and disinfecting solution, and thereafter enveloped in several thicknesses of linen saturated with a strong disinfectant.

The horse is cast and the foot secured as previously described. General anæsthesia is desirable, but if local anæsthesia is preferred cocain solution must be injected at several points around the coronet, and operation must be carried out more quickly than when chloroform is given. The hair is shaved from around the coronet and over the fetlock, and an Esmarch's bandage and tourniquet applied to the limb. (For details see Dollar's "Operative Technique," p. 163.) The horn is then completely removed from the crescent-shaped surface beneath which lies the lateral cartilage (see fig. 303). Instead of now lifting the coronary band, \&c., from the surface of the lateral cartilage, Bayer makes an incision through the sensitive structures, corresponding with, but about $\frac{1}{4}$ inch within, the incision through the horn. The ends of this incision are prolonged upwards, dividing the coronary band, \&c., as high as the upper margin of the lateral cartilage. The flap so outlined must now be carefully freed from the underlying lateral cartilage, which is next removed either wholly or in part, depending on the extent to which it is diseased. Sometimes the gouge or the chisel and mallet must be used to extirpate ossified portions of the cartilage. In the 
event of a large vessel being divided in the process it should, if possible, be ligatured. The fistulous opening on the coronet and any fistulous

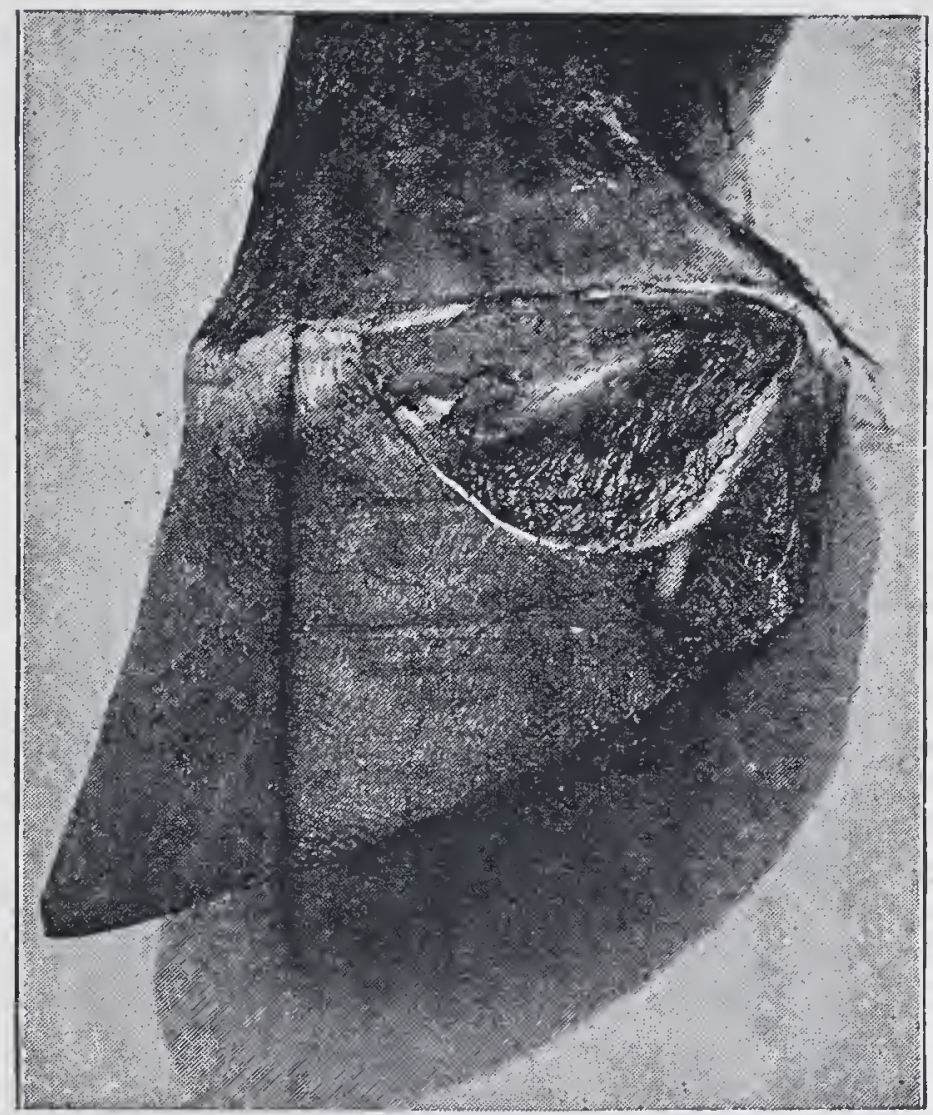

FIG. 303.-Bayer's operation (first stage).

tracts must be freely curetted. After making quite sure that no necrotic fragments have been overlooked the parts are thoroughly

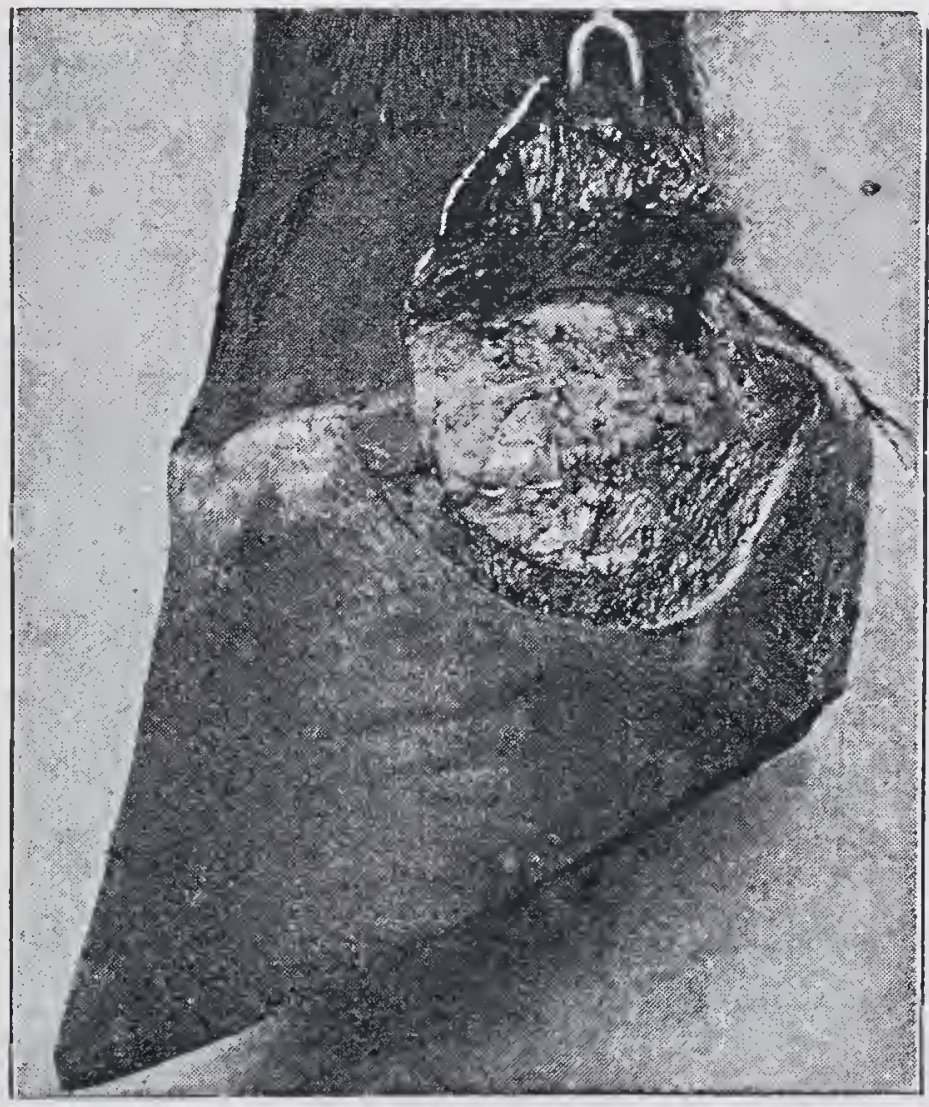

Frg. 304.-Bayer's operation (second stage).

cleansed with an active disinfectant and freely dusted with iodoform; the flap is then brought into position and secured with a series of interrupted sutures. Bayer endeavours to preserve all sound tissue, 
and therefore removes as little as possible from the coronet and, from the lateral cartilage, only diseased portions. It is quite certain, never-

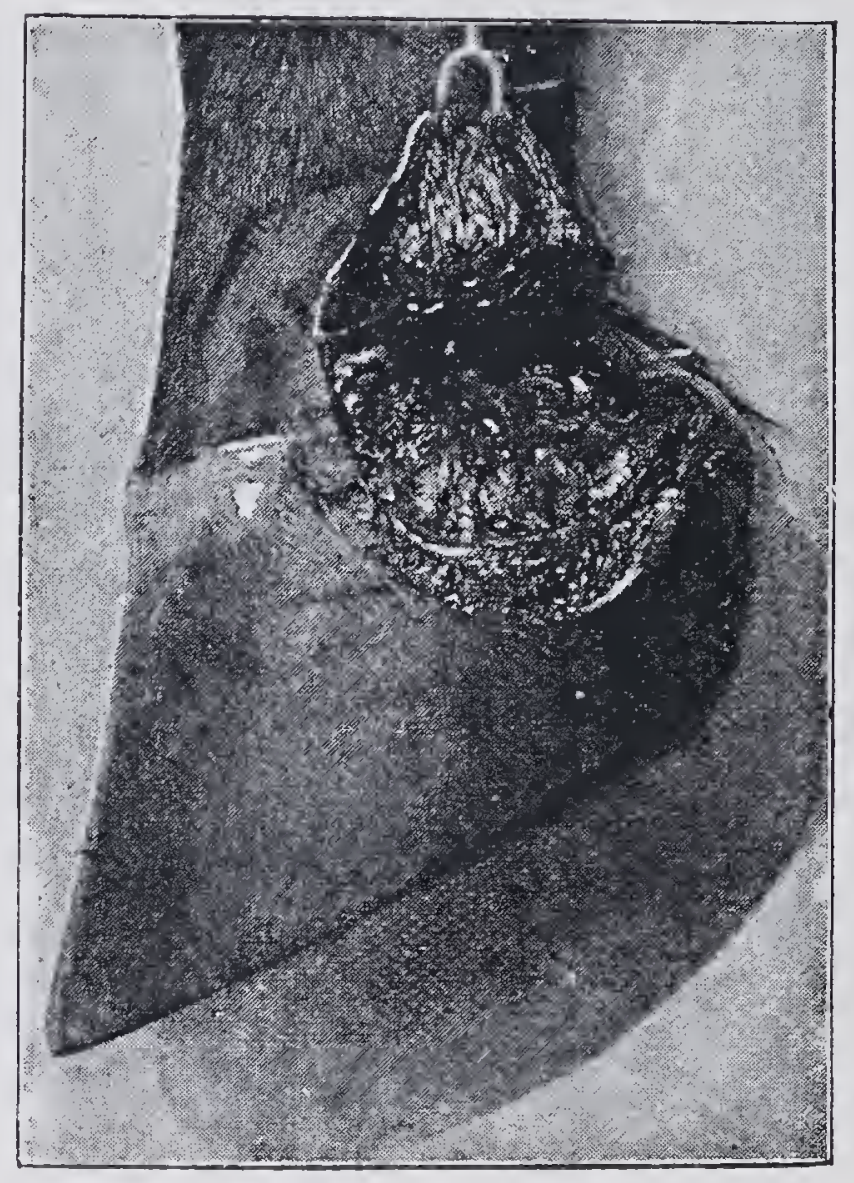

Fig. 305.-Bayer's operation (third stage).

theless, that disease processes in the cartilage extend very much farther than they appear to do, and that apparently sound portions are either

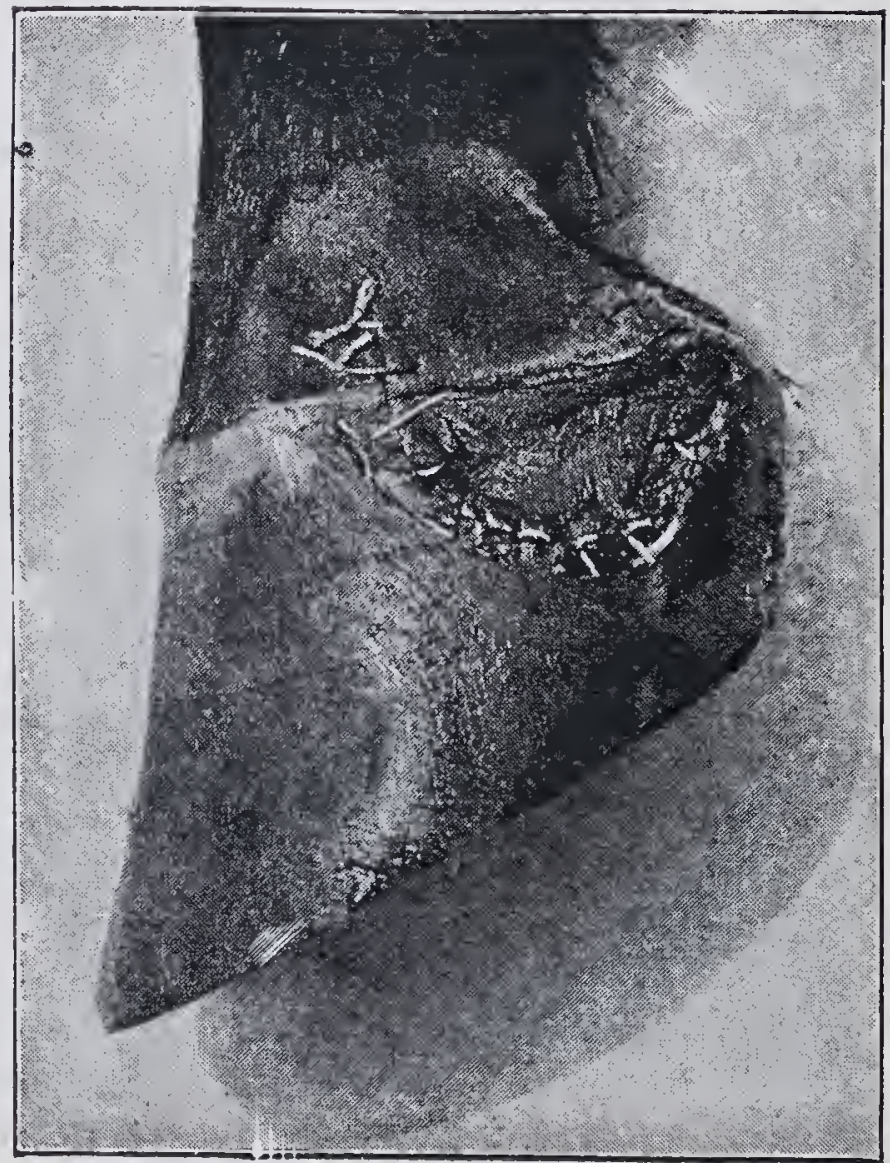

FiG. 306.-Bayer's operation (fourth stage).

in process of necrosis or very readily become infected after operation. Dollar, who has operated many times, prefers to remove the entire 
cartilage, as the operation, though more severe, is less liable to troublesome complications, and is usually final.

After the whole field of operation has been disinfected the dressing is applied. It consists of four to eight thicknesses of iodoform gauze, enveloping and stretching well beyond the wound. Over this is arranged a pad of antiseptic jute, filling up the crescent-shaped excavation in the horn, and pads of the same material are so adjusted as to bring the flap of skin and coronary substance into accurate contact with the tissues left after removal of the cartilage. A calico bandage secures the whole in place. To prevent displacing the flap the bandage should be applied from above downwards. The Esmarch bandage is only removed when the dressing has been securely fixed in position. The latter usually becomes soaked with blood in a very slort time, but this is of no importance, as the blood remains aseptic. A leather shoe or straw sandal is used to keep the parts clear of the ground.

Provided no great pain is shown and the dressing does not emit any unpleasant odour or discharge, it may be left in position as long as ten or twelve days. Increasing pain or offensive discharge necessitate a change. As, however, the second dressing can seldom be as complete or as accurately adjusted as the first, the change should only be made for good reason. This operation has given good results, and is worthy of trial in cases which prove intractable to injections and medical treatment.

\section{VI.-LAMINITIS. INFLAMIMATION OF THE SENSITIVE LAMIN EE.}

The term laminitis is used to describe a spontaneous diffuse dermatitis superficialis of the sensitive laminæ. It sometimes results from chills, sometimes from error's in diet or other causes, usually affects both fore feet, sometimes the hind feet in addition, but seldom tliese alone.

The affection starts as a severe hyperæmia of the sensitive laminæ. Exudation soon follows, and is associated with active proliferation in the well-developed layers of the rete. The immediate result is to loosen the connection between the hoof and its matrix, and as separation is favoured by the weight of the body, especially at the surface of the sensitive laminæ, the most extensive of the pathological changes occur at this point. Within twenty-four hours after onset of the disease, the parts are much reddened and swollen, and proliferation of the young epithelium is taking place on the surface of the sensitive laminæ, especially in the region of the toe. Siedamgrotzky was perhaps tle-first to point out that the pressure of the body-weight, and the pull of the flexor pedis perforans tendon on the os pedis, not only thrust the bone downwards in toto, but that the pull of the flexor tendons, which are rendered 
tense in consequence of the weight on them, tends to rotate the os pedis around its transverse axis, and so to cause the toe to sink and the wings to rise. In proportion as the toe of the os pedis recedes from the wall, the surface of the sensitive laminx becomes thickened, until finally it may measure ten times its normal thickness. This thickening consists essentially in an increase in the white line, as can afterwards be distinctly seen on examining the under surface of the hoof. As the point of the os pedis moves downwards, the horn of the sole at the toe necessarily becomes flattened, and finally takes a convex form. This condition, in connection with certain changes in the horny wall, is termed "dropped sole." It was formerly assumed that the inflammatory growth pressed the os pedis downwards. Dominik insisted, on the other hand, that the horn of the toe was dislocated upwards, but that the os pedis remained in its normal position. Neither view harmonises with our present views, and to-day no doubt can exist that Siedamgrotzliy's explanation is correct. The continuance of this abnormal form of hoof is dependent on the pressure of the ground against the toe of the hoof whilst the animal is moving. In dropped soles the wall of the toe close below the coronet appears abnormally perpendicular, but near the bearing surface takes a more horizontal direction, whilst the bearing surface itself is not only longer but also more oblique. This abnormal formation of hoof is identified with chronic laminitis.

The severe pain sometimes forces the animals to lie continuously, when death usually results. In other cases, the os pedis perforates the horny sole, purulent or septic inflammation of the sensitive structures of the foot sets in, pain increases enormously, the animal is unable to stand, and death occurs from pyæmia.

Causes. Laminitis is most common in badly-formed, flat, and pointed feet. Its onset is favoured by mechanical irritation of the sensitive structures, resulting from severe work at high speed, from continuous standing, as a result of painful lameness, from unskilful shoeing, \&c. Rapid work, after prolonged confinement to the stable, is a very common cause of the disease. Laminitis is also very common during and after long sea voyages, as animals can seldom be exercised when in transit, and their digestive system is very apt to become disordered. Chill increases the tendency to the disease, which is therefore more frequent at the time of shedding the coat, after exposure to wet, or after long rest. The external causes comprise errors of diet and chill. Heavy foods, like rye, wheat, and barley, especially when given new, are particularly dangerous; but new oats and new hay also produce laminitis. At. present it is not quite clear how such foods act, but the fact that boiling: and steaming seem to diminish the injurious effect, points to the action of a micro-organism or its toxin, and contradicts the view that the attack 
depends on the quantity of normally-digested material which enter's the circulation at any one time. Such foods are particularly dangerous to animals which are unaccustomed to them, and which work little. Chills, either from sudden cooling of the body after perspiration, or from the consumption of large quantities of cold water, also produce the disease. Finally, laminitis occurs as a complication of colic, during influenza, soon after parturition, and as a sequel of severe purgation. Imminger, who states that about 1 per cent. of mares become affected with "parturition laminitis," considers the disease due to toxic substances produced in the retained lochix. It is commonest after abortion or difficult delivery, and is then very dangerous. He says, however, that the feet seem to suffer less than the muscles.

Symptoms and course. The disease appear's suddenly, and when located in the front feet is generally recognised on first taking the animal

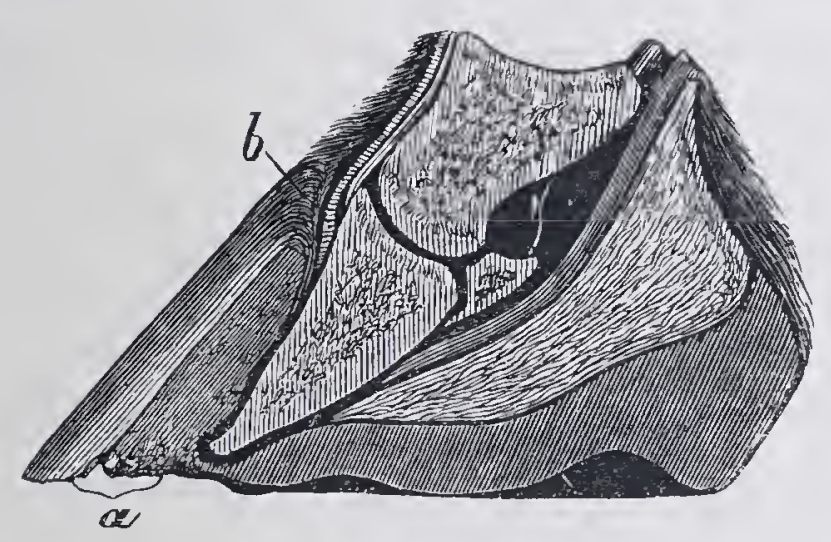

FIx. 307.-Longitudinal section of hoof three months after attack. a, Pathologically modified horn of the white line; $b$, distortion of the horn tubules in consequence of sinking of the os pedis.

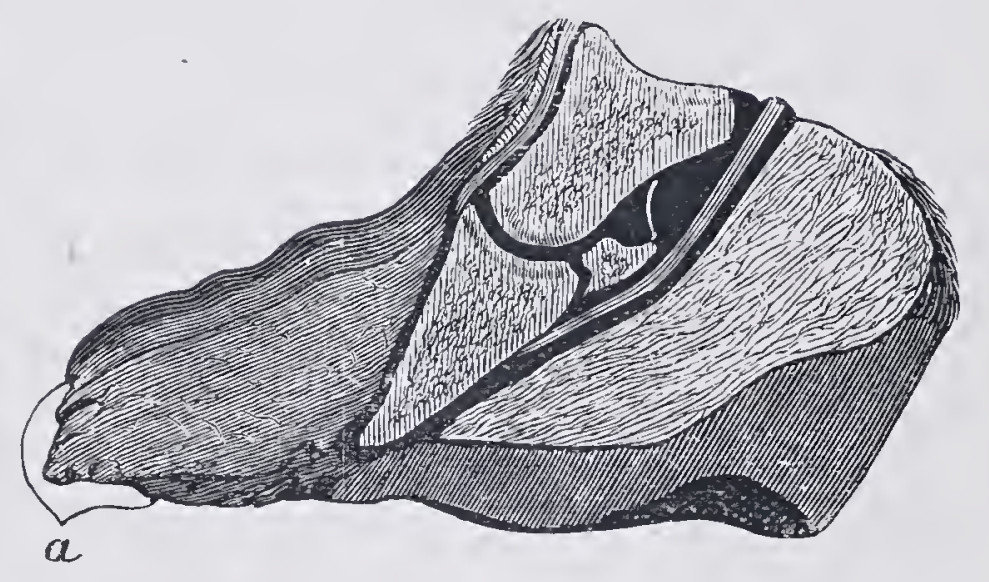

Fic. 308.-Longitudinal section of hoof one year after severe attack of laminitis.

out of the stable by its attempts to avoid placing weight on the limbs, which are thrust forward. The fore feet are moved rapidly, so as to avoid weight being thrown on them longer than is absolutely necessary, and the hind feet are thrust forward in order to take a greater share of the load, and to relieve the front feet. When turned, the horse "pivots" on the hind feet, the front feet being advanced as far as possible, and the heels first brought in contact with the ground.

In disease of the hind feet the horse thrusts the front feet as far backwards, under the body, as possible, and, in order to relieve the toes, which seem the most painful part of weight, brings the hind feet forward. Thus he stands "bunched up," and if forced to move sometimes lifts the hind legs convulsively. When in a stall or box, he generally rests the hind-quarters against the wall, and in severe cases will endeavour still further to relieve the hind feet of weight by passing his head over the side of the stall, and hanging back from it by his head. If both the hind feet and front feet are affected, movement of any kind is exceedingly 
painful. The animals can scarcely be got out of the stable, and groan when forced to move. Although the disease is so painful, horses usually stand until exhausted. Once having lain down, however, they only rise with great reluctance and a vast show of precaution, and when again on their feet usually exhibit acute pain for a time.

Pain is produced by pressing on the soles with the farrier's pincer's, especially around the toe, and the metacarpal arteries are found to be strongly pulsating: During acute attacks, fever is marked, thermometer readings sometimes rising to $103^{\circ}$ or $104^{\circ} \mathrm{F}$, whilst the surface temperature is unevenly distributed.

In favourable cases these symptoms disappear in three to five days, but generally last longer. Displacement of the os pedis can be detected by the falling-in round the coronet and depression of the sole. Once this has occurred complete recovery is no longer possible ; the soles "drop," and the animal ceases to be useful for work on stones or at a rapid pace.

Horses with dropped soles exhibit a peculiar gait: the os pedis being partly rotated around its transverse axis causes relaxation of the flexoi pedis perforans; every time the animal extends the limb, therefore, the coronet-joint shows abnormal dorsal flexion, in consequence of which the heels come first to the ground.

Laminitis is sometimes complicated with muscular rheumatism, pleurisy, pneumonia, or colic, the first two diseases being associated with rheumatism, the colic with dietetic errors. Laminitis may not become marked until the colic, pneumonia, or pleurisy has passed off.

Differential diagnosis. The following are some of the conditions which most closely simulate laminitis:-

(1) Bruising of the sole in unshod horses; this is detected by an examination of the foot.

(2) Muscular rheumatism. In this case the animal sometimes moves as in laminitis; the step is short and cautious, but the front limbs are not extended, and while symptoms of inflammation of the hoof are wanting, the muscles of the limb seem in a state of abnormal tension, and are painful on pressure.

Prognosis. The consequences depend partly on the severity of the attack, partly on the stage at which treatment is resorted to, and partly on the formation of the hoof and the character of the complications. The intensity of the disease varies greatly; it may be fairly estimated by the degree of pain.

To ensure resolution, treatment must be adopted within the first twenty-four to forty-eight hours. In this case recovery is generally complete, though it should not be forgotten that for weeks after the disappearance of symptoms the tendency to fresh attacks continues, and therefore relapses are exceedingly common. 
The prognosis is less favourable when the hoofs are already deformed, and especially when the soles are dropped. Cases following errors in diet are less favourable than those of rheumatic origin; and when all four feet are attacked, there is less hope of recovery than when the front feet alone are implicated. Finally, heavy-bodied hol'ses suffer more severely than lighter animals.

Treatment. As a preventive, all horses not in active work should be exercised daily, and should be placed on reduced diet, while attention should be given to the state of the bowels. Young horses should neither be over-worked nor over-fed especially with highly nitrogenous food. Horses just landed from a long sea voyage should be placed in loose boxes, and fed sparingly on light, easily-digested food. They should not be exercised or walked about for a week or so, as even a short journey is apt to precipitate an attack. From the earliest times laminitis has been treated by free bleeding, which, in fact, is useful if early resorted to. During the first three days bleeding from the jugular almost always produces rapid diminution of pain and inflammation. It is especially useful in cases following dietetic mischief, but is also of advantage even in rheumatic laminitis. Local bleeding, either from the coronet or sole, is less effectual; and the production of a wound in the sole is dangerous, because it may become the starting-point of septic or purulent inflammation. If, therefore, bleeding from the toe be resolved on, the parts should be treated antiseptically. Bleeding from the coronary plexus is not so dangerous, and scarification of the coronet has often been recommended. Some practitioners recommend abstracting five to six quarts of blood from the jugular and applying mustard plasters to the chest and sides of the thorax.

The second means of treatment consists in cold applications. Cold poultices may be applied to the feet, or the animal may be placed in water. When pain is severe, poultices deserve preference. Foot-baths are only of use in slight lameness, and it should not be forgotten that continued standing favours displacement of the os pedis, and, therefore, that it is preferable to allow the animals to lie, and to apply cold applications to the feet. Plenty of straw bedding should be given, the animals being liept out of draughts, and made as comfortable as possible. It is also advantageous to remove the shoes and place the horse on a soft bed, so as to oppose descent of the os pedis by distributing pressure over the sole. If in acute attacks, the horse cannot be persuaded to lie down it should be cast.

To permit of expansion of the hyperæmic tissues and diminish tension, Smith recommends dividing the horn wall at one or more points in its circumference by means of the knife or saw. 'This treatment is of doubtful value. 
In "dietetic" laminitis a dose of physic may be given, both to diminish local inflammation and remove, as quickly as possible, any injurious material from the bowels, thus preventing its absorption. Others recommend the intravenous injection of barium chloride solution. In the rheumatic form the parts should be rubbed with spirituous solutions, and the body warmly clothed.

Friis recently recommended pilocarpine in doses of 5 to 7 grains. Experiments with this drug have given varying results; some cases showed improvement, others were unaffected. It may be tried, however, especially where the attack is obstinate, and the os pedis shows no signs of displacement; but after displacement, of course, neither this nor any other drug can effect a cure. Dollar has had excellent results from hypodermic injections of bromide of arecoline (arecoline is an alkaloid obtained from areca nut). To diminish severe pain, subcutaneous injections of morphine are useful.

Regulation of diet is especially necessary, and when the attack has

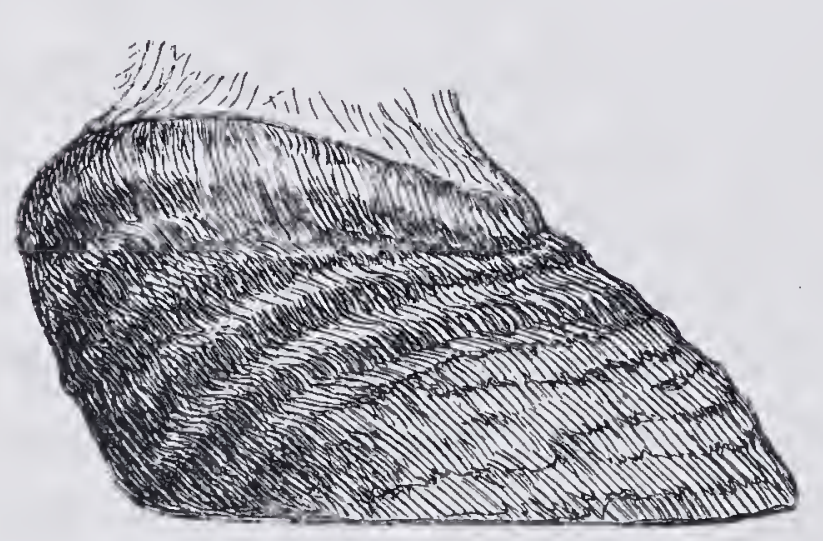

FiG. 309. - Hoof after laminitis.

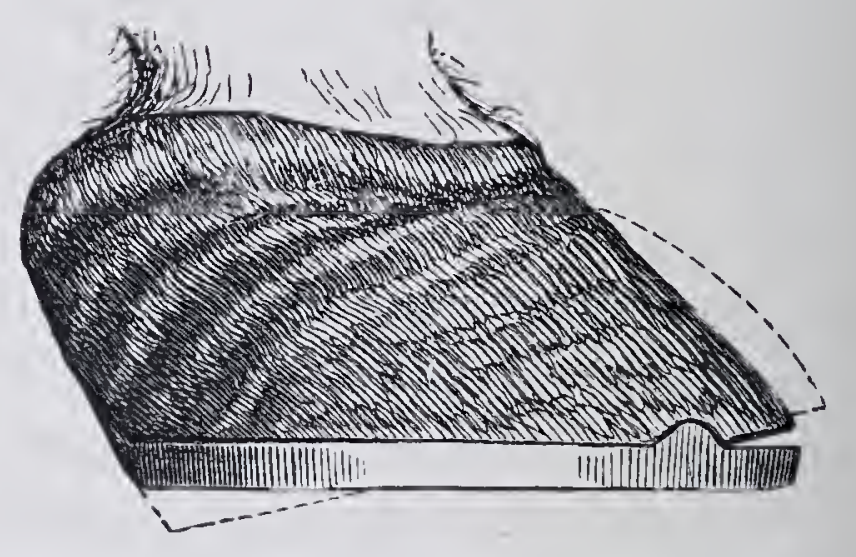

FIG. 310.-The same shod. The dotted lines indicate the previous form.

followed excessive feeding, the animals are put on short rations. For the time being, corn should be completely withdrawn; in summer grass can be given; in winter, hay and bran-mashes.

It is now well recognised that, from the outset of the attack, exercise is useful, particularly in rheumatic laminitis. Sometimes the lameness occurs whilst resting during a journey; and if the animal be started again, and pushed until it perspires freely, being afterwards carefully guarded against chill, it may completely recover. But, apart from the severe pain inseparable from this method of treatment, it may end in aggravation of the condition. The experiments made in the British army do not altogether recommend the treatment, though, under some circumstances, for example during field manœuvres, it may be useful.

The treatment of dropped sole pertains to the province of shoeing, and although the form of hoof may be improved, yet the animal's usefulness is never completely restored. As soon as acute inflammatory symptoms have disappeared, it is best to apply shoes with thick heels, and to lengthen the heels of the hoof as much as possible. (For fuller information on this 
point the reader is referred to Dollar and Wheatley's "Horse-shoeing and the Hor'se's Foot," p. 396.)

(B) Chronic Tnflamiation of the Sensitive Laminæ. Pododermatitis C'Hronica.

Chronic inflammation of the sensitative laminæ follows slight but continually-repeated mechanical irritation or other injury, the nature of which is at present imperfectly understood. The former class was studied and described by Lungwitz. Certain portions of the hoof grew with excessive rapidity and became thickened, sometimes causing hom tumours (keratomata) and chronic lameness; sometimes chronic inflammation occurs in the sensitive laminx, and interferes with horn production, producing "seedy toe."

As a rule, these processes are all very obstinate. One section has little surgical interest, and, therefore, need not be considered here. These conditions are considered at length in the above-mentioned work.

\section{I.-KERATOMA.}

The horn forming the inner surface of the wall at the point where the wall is united to the sensitive laminx may become thickened, producing a new growth, which is variously known as keratophylocele, keraphyllocele, or keratoma. The activity of secretion in the coronary band is very great; in the sensitive laminx, on the other hand, it is very small, being only well marked at the upper part of the wall just below the coronary band itself, where the horny laminæ originate. Throughout the rest of the wall secretion is almost nil. But when the tissue becomes the seat of chronic inflammation, its secretory activity is exalted, and a mass of new horn is produced, which we recognise as a keratoma. Such growths sometimes start from the coronet, in consequence of treads or other injuries producing chronic inflammation and hyperplasia in the lower papillæ of the coronary band. This pathological thickening gradually extends downwards towards the ground surface of the foot, and, pressing on sensitive structures, gives rise to chronic lameness.

In other cases the sensitive laminx form the points of origin of keratomata. In this case the irritation starts in the white line. being either set up by a misdirected nail, by separation of the wall, or by cracks in the horn. Keratomata so formed are confined to the lower portions of the wall, and though occasionally they extend upwards in the direction of the coronet, they seldom reach it.

Such thickenings, of course, press on the sensitive lamina and os 
pedis, which show a depression at the affected spot, corresponding in form to the keratoma.

Keratomata therefore result from chronic, productive inflammation. Sometimes the condition is accompanied by superficial suppuration. On the other hand, infectious inflammation of the podophyllous membrane may constitute the first phase and be succeded by a chronic productive process. For this reason the symptoms vary.

The diagnosis of keratoma during life can only be assured when the growth appears on the plantar surface. 'The white line then seems to be thrust towards the centre of the sole at the point affected, the inner border of the keratoma itself forming, in fact, the white line. Where the growth is due to a split in the horn, the wall is divided from the new growth by a space.

Keratomata, derived from the sensitive laminæ, frequently produce chronic inflammation of the latter. In such cases a secretion, which is sometimes watery, sometimes purulent, discharges from the space between the horny and sensitive laminæ. A probe may often be passed to the upper margin of the split, i.e., as far as the upper end of the keratoma.

Occasionally that portion of the

FIG. 311.-Portion of the inner surface of the wall showing changes after oldstanding corn. a, Horn tumour. external wall of the hoof corresponding to the keratoma is bulged outwards, forming a projection extend-

ing from the coronet to the bearing surface. This condition is commonest when the keratoma grows from the coronet; the surface of this protruding part is sometimes rough and inclined to split.

Keratomata usually, but not always, produce lameness, which exhibits all the symptoms of foot lameness, except acute inflammation. In exceptional cases, even these (increased warmth and pulsation) may be detected. When infectious processes are absent and the keratoma is of small size there may be no lameness, which is usually due either to pressure of the new growth on the pedal bone or to inflammation.

The prognosis depends principally on the presence or absence of lameness, and on the possibility of removing the diseased growth. It is, therefore, important to know whether the keratoma has (1) originated in the coronary region, or (2) from the lower sections of the sensitive laminæ. In the first case, its removal not only offers great difficulty, but enforces a long rest, as the hoof is divided to a considerable extent and a large amount of horn removed. In the second case, the time required for recovery is in proportion to the distance the diseased growth extends 
upwards. Keratomata, growing from the coronet, generally produce deformity of the outer surface of the wall, which is therefore an unfavourable sign. In many cases a new growth forms even after removal of the first, but this is not invariably the case.

Treatment. Unless the growth causes pain and lameness treatment is better abstained from. In shoeing, the portion of the wall below the tumour should be relieved of weight. Sometimes the lameness yields to rest and cold moist applications. Persistent lameness can only be cured by excision of the keratoma. The affected spot may be thinned with the rasp, and the keratoma removed by means of knife and forceps.

Fröhner and Gutenäcker divide the wall around the keratoma, remove the growth completely, and seek to assist healing of the operation wound by antiseptic precautions.

The great difficulty of this method is to determine the extent of the keratoma by examining the outer surface of the wall. To obviate this, the wall may be trephined and the extent of the growth ascertained by probing. The growth may also be removed by starting from the bearing surface of the wall and working upwards until its upper margin is reached. If the keratoma is small this concludes the operation. But when it is extensive and has produced degenerative processes in the laminæ and os pedis it may be necessary to excise the diseased laminæ and to freely curette the bone.

The operation wound should be treated antiseptically. Complete recovery, however, is far from being the rule. The cavity in the os pedis may possibly be filled up again, but as the tendency to new growths exists in the sensitive laminæ, and is not removed with removal of the keratoma, the disease not infrequently returns.

Similar growths occasionally affect the sole. They are commonest in flat or "dropped" feet. The tumour is usually hemispherical, the base intimately united to the velvety tissue of the sole. Treatment is usually confined to thinning the growth, applying poultices, and shoeing in leathers. Radical treatment necessitates stripping the sole, or fully exposing and wrenching away the tumour, and is rarely undertaken.

\section{CHRONIC INFLAMIMATION OF THE SENSITIVE LAMINÆ.}

Considerable spaces sometimes form between the sensitive and horny laminæ, whilst the surface of the sensitive laminæ becomes covered with a thin, turbid, fluid secretion. At this point the sensitive laminæ appear enlarged and displaced. Their surface, which in parts is completely exposed, is covered with a thin, purulent secretion, and not infrequently shows granulations. 
The process, described by Schleg as chronic ulceration of the hoof or "thrush" of the sensitive laminx, is usually confined to a spot the size of a shilling in the lower parts of the sensitive laminx. Sometimes, however, it extends upwards along the laminæ, and may even reach the coronet, but there seems no tendency to penetrate more deeply. The chronic irritation around the diseased spot sometimes causes formation of keratomata (see last chapter).

The condition is due to chronic irritation, but it is as inaccurate to describe it as ulceration as it would be to apply the same term to canker. The two processes, however, often show so great a resemblance as to be distinguished only with difficulty at the first glance. The principal difference consists in the benignant character and amenability to treatment of the disease now under consideration, as opposed to the very careful and prolonged treatment demanded by canker. The rapidly curable cases of canlier, sometimes described, are probably due to this disease.

The condition generally develops after acute inflammation caused by pricks, separation, or fissuring of the wall. Schleg saw the disease result from separation. Should the inflammatory centre become infected, healing is delayed, and the disease may become chronic. This is shown by the fact that after removal of the infected portion of wall and careful cleansing of the surface of the sensitive laminx, healing generally follows in three to four weeks.

Symptoms. Attention is first attracted by the lameness or escape of discharge through the white line during shoeing. The extent of diseased tissue can be detected by probing. Lameness is not a constant feature, but appear's immediately acute inflammation is set up by the entrance of foreign substances.

The only difficulty in diagnosis consists in distinguishing this condition from canker, a point which often can only be settled by observing the course of the disease. Healing rapidly follows removal of the portion of wall covering the diseased lamina, whereas in canker the same treatment produces active swelling and vegetations from the sensitive lamine, which extend beyond the aperture thus made, and require great care to reduce. In this respect the two conditions present a very important difference.

Treatment. The first step is to at once remove the wall covering the diseased spot by means of the rasp and guarded knife without injuring the diseased sensitive laminæ; it is, therefore, necessary to note accurately the point of division between the horny and vascular parts. After cleansing, as far as possible, the diseased laminæ, they are painted with liquor ferri sesquichlor.; a mass of tow or jute, soaked with the same fluid, is applied to the diseased spot, and fastened by means of a 
bandage. If proliferation from the surface of the sensitive structures proves troublesome nitrate of lead may be employed; Schleo used the actual cautery. The above-described dressing should then be applied and left in position for some days. When changing it, the parts are carefully examined, and if the wall has become further undermined the affected horn should at once be removed, but as a rule there is no marked tendency to extension, a feature which further distinguishes it from canker.

\section{III.-CANKER OF THE SOLE AND FROG (PODODER- IMATITIS CHRONICA VERRUCOSA VEL MIGRANS).}

Since olden times a chronic inflammatory disease of the horn-secreting structures of the foot, which shows a strong tendency to extend and seize on fresh areas, has been recognised and generally described as canker. The disease consists essentially in chronic proliferation in the papillæ and tissue of the rete, the papillæ becoming enlarged and the cellular elements of the rete undergoing active increase. True horn production is thus checked, the epithelium produced not forming horn, but breaking down and producing a greasy, stinking grey fluid. As pointed out in speaking of "grease," the disease is identical in character with so-called grease, i.e., it consists in a dermatitis verrucosa: it has also been stated that either condition may produce the other, i.e, that grease may cause canker, and canlier grease. Both conditions are very obstinate.

Symptoms and course. The commencement of the attack is generally overlooked. As a rule, a small prominence first forms on the frog and produces a grey, greasy, offensive material. On more careful examination the enlarged papillæ of the frog, which cause the swelling, can be detected with the naked eye. Lameness is rave at first, a symptom of considerable diagnostic importance, for in acute inflammatory diseases, great pain and lameness are always present. In the sensitive laminæ the process produces similar changes to the inflammation described in last chapter. Gradually, sometimes almost imperceptibly, the disease extends, attacking first the frog, then the sole and bars, passing from these to the wall, and sometimes reaching as high as the coronet; always making its way between the sensitive and horny structures, which it finally separates. The sensitive laminæ become enlarged and converted into brush - like structures, which produce exuberant vegetations immediately the wall covering them is removed.

With the extension of the process to the bars and sole the hoof expands at the quarters, whilst the frog considerably enlarges. At this stage lameness is sometimes seen during movement over rough or even over soft ground, but this is probably due to loss of the horny covering 
and accidental injury, not to the diseased process. When the condition is neglected it continuously advances, until finally it affects the entire matrix, and may lead to loss of the hoof.

Causes. The causes of canker are at present little understood, and opinions as to the nature of the disease vary greatly. The name is a very old one, and originated at a time when it was customary to describe many different disease processes, distinguished by their malignant character, by the same term. It has long been recognised that canker has no real relationship with carcinoma or cancroid, and therefore, strictly speaking, the name is unjustifiable. The disease is commonest in animals of a lymphatic temperament, and is favoured by dirty, illdrained stables, where animals stand continually on litter soiled with manure, and saturated with decomposing, ammoniacal urine. Canker is probably due to specific infection, as seems indicated both by its course and the manner in which it reacts to treatment, but the infection does not appear to be due to a single organism, but to several, which do not always agree in their method of action. Mégnin has described one which he terms Keraphyton. (The subject receives further attention in Dollar. and Wheatley's book on "Horse-shoeing and the Horse's Foot," p. 400.)

Prognosis. Although canker always takes a chronic course, yet it shows many variations which are of real importance in forming a prognosis. Under any circumstances the practitioner should be cautious in giving an opinion, though the greater number of cases recover. In forecasting the probable result the following points must be considered :-

(1) The nature of the growth. The more active the growth in the rete tissue and papillæ, and the softer the material produced, the graver the case. When the papillary growths retain their form, the case is more hopeful than when they are amorphous.

(2) The area involved. The greater the tendency to extend, and the more widespread the disease, the less the chance of recovery. Treatment is particularly difficult when the laminæ are attacked, easier when the condition is confined to the frog and sole. Rapid extension is always an unfavourable sign.

(3) The most difficult cases are those in which a predisposition to the disease exists, as shown by several feet being affected.

(4) Treatment is, of course, more difficult in restless and sensitive animals, and the prognosis correspondingly less favourable. The treatment, in fact, requires a great deal of patience, on the part not only of the veterinary surgeon, but also of the patient and owner.

Treatment. Perhaps in no other disease have so many drugs been tried as in canker. As indicated elsewhere, the selection of a proper material certainly plays a very important part in treatment, and only 
ranks second to the manner of applying it. Speaking generally, caustics, which destroy the excessive growths, together with astringents and disinfectants, appear most effective. Sublimate, chloride of zinc, carbolic acid, calomel, camphor, iodine, and many others have in turn found supporters, but, as Haubner very truly said, there is no specific against canlier. That is entirely true, and it requires long and close observation to discover the right material in any given case.

In addition to local disinfection, it is necessary, firstly, to destroy the new growths on the papillæ; and, secondly, to check proliferation and secretion in the rete tissue. Success in the first case depends on the size of the new growths. Caustics, the actual cautery, or the knife may be employed; but, whatever the means selected, care must be takien to remove all diseased tissue. 'The hoof must be thoroughly thinned, every particle of undermined hor'n removed, and the sole be reduced to a mere pellicle for a distance of $\frac{1}{2}$ an inch round the diseased area, the margins of which are easily recognised by the fact that the horn there becomes adherent to the sensitive structures beneath. By using an Esmarch's bandage (see Dollar's "Operative 'Technique," p. 163), bleeding may be entirely avoided, and the seat of operation lept continuously under observation. All fragments of diseased tissue should be removed with the knife or curette. 'L'his treatment has proved more successful than the use of caustics. Healthy tissue should naturally be spared, but not at the expense of leaving disease behind. 'The softness and yellow colour of the altered tissues enable them readily to be distinguished. 'The operation is concluded by applying a pressure dressing saturated with weak formalin solution. As considerable pain attends the above manipulation, chloroform or a large dose of morphine should be given. 'I'wo or three days later the dressing is removed. 'The field of operation will then be found covered with a cheesy adherent material, which must be removed with the knife or curette, but without wounding the sensitive structures beneath. 'The parts are then redressed with formalin or whatever dressing has been chosen.

Amongst caustics, nitrate of lead, recommended by Pütz, perhaps stands first. When strewed over the diseased surface in the form of powder, it forms a dry scab, checks extension of the growth, exerts an intense drying action, and checks proliferation and secretion in the rete tissue. Sometimes the process starts again under the dry crust, which therefore requires to be carefully examined during the next few days. Inorganic acids, particularly fuming nitric acid, can also be used, but their application is more difticult, whilst their action is certainly not more satisfactory than that of nitrate of lead. The recent experiments with sulphuric acid in the treatment of grease suggest the advisability of trying it in canker; on account of its caustic, antiseptic, and drying V.S. 
action, formalin is of great value. Fröhner recommended the cautious use of the officinal 40 per cent. solution of formic aldehyde, applied with a brush. This, however, is difficult to control, and 5 to 10 per cent. solutions are preferable; if necessary, their application can be repeated. Hell, who used formalin extensively, prefers the 5 per cent. solution, as he has seen severe results from using the 10 per cent. He applies the solution on tampons of tow. When the growth is not severe, and the secretion is alone troublesome, calomel, liquor ferri sesquichlorid., 1 per cent.; chloride of rinc, 10 per cent.; tincture of iodine, tar, \&c., may be trjed. Each has at some time been strongly recommended. Whichever be employed, a pressure dressing is always necessary. 'The actual cautery has been used to destroy the diseased tissue and excite repair, but requires supplementing with some antiseptic or chemical caustic.

Portions of undermined horn must be removed. This is a sine quâ non in treatment, and requires no slight dexterity and practice in handling the knife.

As a rule, treatment is commenced by removing the horn, and, if the hoof be very dry and hard, by immersing the foot in a warm foot-bath. It is not always possible to remove every. particle of loose horn at one operation, for as soon as bleeding occurs it is no longer safe to proceed, and is usually better to wait for a day. 'The foot is then soaked in a disinfectant, such as creolin, carbolic, chloride of zinc, or sublimate solution. After one or two hour's' immersion, and after repeatedly washing the diseased part whilst the foot is in the bath, the material selected is applied and a dressing put on. Next day this is renewed, and if the process liave extended further, the undermined horn is at once removed; otherwise, if the surface remain dry, a fresh dressing is applied.

At first the dressing is renewed daily. To checl excessive proliferation, the parts are dressed with nitrate of lead, and a pressure dressing tightly applied. In disease of the frog, bars, and sole, a shoe provided with a removable iron covering (see Dollar and Wheatley's "Horse-shoeing and the Hor'se's Foot," pp. 384 et seq.), or a plaster cast, will be found exceedingly useful. To prepare the latter, the hoof is filled with freshlyprepared plaster of Paris, mixed with chopped tow or hemp, to render it less friable, and the whole allowed to harden. When applying it, the sole should be covered with tow, so as to press as equally as possible on the growing tissues. A well-made shoe fulfils the same purpose. Even at a later stage, it is important to renew the dressing every two or three days at least, and to examine the condition of the hoof. Particular attention should be directed to the margins of the diseased spot, and to the dry scab; if only the slightest trace of grey, greasy material is present, it must at once.be ascertained whether the process is 
not spreading in deeper-seated parts. When healing is well advanced, calomel in the form of dry powder forms a very valuable application.

Mesnard ("Rec. de Méd. Vét.") claims to have had excellent results from applying a mixture of 4 parts carbide of calcium and 1 part each of neutral acetate of copper and iodide of starch. The hoof is prepared as for the treatment with nitrate of lead, and after drying is freely dusted with the above powder and a pressure dressing applied. T'he process is repeated daily.

In 1897 Imminger suggested a "radical operation," consisting in removing the velvety tissue of the sole or the sensitive laminie, where diseased. Whilst Imminger reserved this treatment for old-standing, severe cases, Eberlein practised it in every case he saw, removing the tissue with the knife "as though dealing with a tumour." 'I'he wound was then irrigated with sublimate or creolin solution, strewed with pyoktanin, iodoform or thioform, and covered with a compress dressing of gauze and jute. If no severe symptoms appeared, the tirst dressing was left in position for a fortnight and afterwards renewed every eight or ten days. After the first fortnight the surface granulated freely, and was surrounded by a margin of thin healthy horn, which gradually advanced over the wound. 'The parts, when dressed, were simply wiped and powdered with the dressing; any exuberant granulations were excised. Fröner cured 12 out of 14 horses in this way. The average duration of the cases was 27 days. Eberlein consider's this the most successful of all extant modes of treatment.

The disease cannot be regarded as cured until every point is absolutely dry, and normal horn formation has again set in. In spite of all precautions, relapses are very common, and therefore, for some months after recovery, the affected part should be kept under observation, so as immediately to check fresh onsets. Disease of the bars is exceedingly troublesome. 'The sensitive structures of the bar being in contact with those of the frog, the vegetations coalesce, and render it extremely difficult to completely expose the diseased spot. It is often necessary to excise a portion of the new growth, and insert tampons between the two, a very small tampon being first thrust into the depths, and followed by others of increasing size, so that the dressing extends into the space between the bar and frog in the form of a wedge. Many observers recommend placing the horse during the day on a thick clay bed, and, after thoroughly cleaning the hoof, to use at night tan instead of straw. Although this method appears easy, its use is attended with many inconveniences. Apart from the necessity for daily cleansing and changing the bed, the clay becomes soiled by urine and fæces, and requires frequent renewal. By working the horse on a brickfield, the same effect 
is produced more easily and thoroughly, whilst the daily exercise assists recovery.

Such treatment is useful where the ground surface only of the hoof, i.e., the frog, sole, and bars, is affected, but is valueless in disease of the wall. The regular pressure on the new growth here plays the principal part. Recovery often follows this treatment.

A somewhat similar effect is produced by using the animal on soft ground, especially if shoes can be dispensed with. 'The process makes less rapid progress while the horse is at regular work than during rest-a fact to be borne in mind. Should all other treatment fail, the animal should, if possible, be exercised. The effect is sometimes astonishingly good.

Nocard some year's ago recommended vigorous treatment with sublimate solution. After completely exposing and carefully cleansing the diseased spot, it is sprayed for two hours and a half with 1 per cent. sublimate solution. The sole is then dried, and its surface sprayed for ten minutes with iodoform ether. Nocard gives Fowler's solution inwardly to the extent of 10 drachms in eight days. Later reports by this author and others show, however, that even this treatment sometimes fails.

It need only be repeated that more is due to care in carrying out operative treatment and skill in applying the dressings than to the selection of the material, though the latter plays a by no means unimportant rôle. A change of dressing is also desirable from time to time, and the local treatment should be supplemented by internal medication with such materials as arsenic or the iodides. 'T'he use of caustics should not be pushed to excess, and when the discharge ceases it is better to replace them with milder applications and with disinfectants. Although the principles here set forth are sufficient to guide treatment, yet close personal observation is necessary, and in many cases it may appear advisable to try certain of the other materials suggested.

\section{G. DISEASES OF THE ULAHS IN CATILE ANL SHELIS.}

Although the claws of herbivora and swine are homologous with the horse's hoof, yet they differ considerably from it in their outer form, and still more in their internal arrangements. Disease processes in the claws, therefore, show a general resemblance to diseases of the hoof, though they exhibit many special features, dependent chiefly on the duplicate formation of the claws, and on their union by ligaments and soft tissues, partly on the absence of the frog and bars, and on other peculiarities of formation.

The claw consists of a horny wall surmounted by a coronary band, and of a horny sole with bulbs similar to those of the horse's heel. 'These perform the function of the frog, which, with the bars, is absent in ruminants. In medium-sized cattle the horny wall attains, on its outer part, a thickness of about $\frac{3}{16}$ of an inch, whilst the inner, which lies in contact with its fellow, is 
scarcely $\frac{1}{8}$ of an inch, and is considerably shorter than the outer on account of the sole sloping obliquely upwards to become continuous with it.

The horny sole also has a thickness of about $\frac{3}{16}$ of an inch, and reaches from the toe to the posterior third of the ground surface of the claw, where it becomes continuous with the wall. Above the wall, and between it and the tendon of the flexor pedis perforans, is a mass of firm connective tissue, to some extent corresponding to the fibro-fatty frog, and containing a large quantity of fat.

The pedal-joint (fig. 312) lies near the centre between the toe and ball of the foot, its middle point $(b)$ being somewhat nearer to the toe than to the wall, and about $\frac{3}{4}$ of an inch below the coronary border of the wall. A straight line drawn obliquely from the front of the interdigital space to the termination of the horny sole in the walls would nearly coincide with the lower border of the joint (figs. 312, 313). The posterior end of the os pedis lies between $c$ and $d$ in figs. 312,313 , behind $c$ lies the navicular bone, which completes the formation of the pedal-joint.

The coronet-joint is generally about $\frac{3}{4}$ of an inch above the coronary border of the wall of the claw; the position of the fetlock can, of course, be determined by palpation.

In each claw the tendon of the flexor pedis perforans forms with the

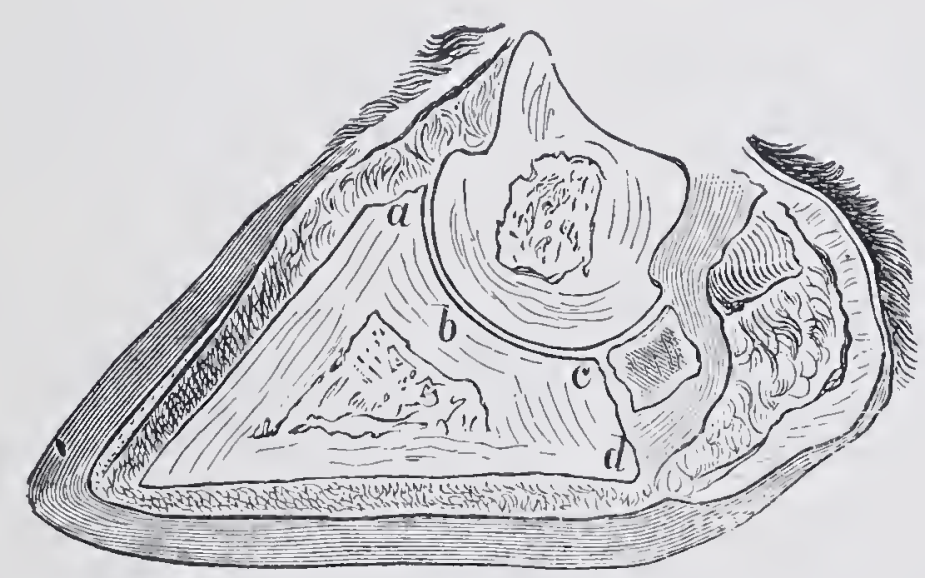

Fig. 312.-- Vertical section of an ox's claw.

navicular bone, close in front of its insertion into the os pedis, a bursa which corresponds to the bursa podotrochlearis of the horse. This lies over the posterior part of the horny sole, about the point where it becomes continuous with the bulb of the heel.

The sensitive wall and sole differ little from the corresponding portions of the horse's hoof, though the coronary band is broader', and reaches downwards almost to the middle of the horny wall. The sensitive sole presents a general resemblance to that of the horse, but the postero-internal portion possesses a modified subcutis, somewhat analogous to the sensitive frog. The surface of the sole may be divided into toe ard bulbs, a fact which accounts for the severity of all suppurating processes in the bulbar portion. The coronary band is broader than in the horse, and extends downwards for almost half the depth of the visible horn wall. Between the claws the coronary band becomes continuous with the skin, which is hairless, and covered with a thick but slightly horny epidermis. Above this, i.e., between the coronet bones, lies a large pad of fat, which is continued downwards under the inner horn wall, and assists the movement of the claws over one another.

The bone of the claw (os pedis) is united to the coronet and fetlock bones by two internal and external ligaments, and through the former to the reinforcing band of the extensor pedis (ligamentum extensorum), whilst the cruciform or interdigital ligaments unite the two claws and prevent their being 
thrust too far asunder. The interdigital ligaments lying above the interdigital space unite the coronet bones, and pass through the fatty cushion of the bulbs of the heel, to lose themselves in the sensitive sole.

This peculiar structural arrangement renders cattle less suited for moving on hard ground, and, if they be forced to do so, predisposes the soft structures of the claws to injury. The distribution of weight in the claw is different to that in the hoof. Whilst in the hoof the wall supports the principal weight and the sole is practically exempt, the sole of the claw carries by far the greater portion of weight. The same is true even when the claws are shod, and it explains the frequent occurrence of bruises of the sole.

For asimilar reason, the continued irritation of dirt and stable manure causes disease of the claw to exhibit special features. These consist in acute inflammation, which, under the action of infectious agents, lead to gangrene of soft parts and necrosis of bone, or purulent septic inflammation of the claw or coronet-joint.

The ox being less sensitive than the horse, symptoms of inflammation of the clavs are less marked than those of laminitis, and examination of the claws is more difficult than that of the hoof. Being generally macerated in manure, the claws of the hind feet are less tender in this disease than the hoof in the like condition in the horse. It is also difficult in the ox to detect slight degrees of pain. When the pain is severe the animals lie a great deal and are difficult to raise, suggesting to non-professional observers that they are injured in the back. On forcing the animal to move, it endeavours, as much as possible, to avoid placing weight on the diseased foot, and in acute attacks weight is thrown on each only for a few seconds, or not at all. At the same time the foot is placed in peculiar positions-when the pain affects the toe, most weight being placed on the bulbs of the heel, and rice rersâ: in the latter case, weight is only placed on the foot during a portion of the stride.

In these animals a local examination, although absolutely necessary for diagnosis, is very difficult to make. When the animal is standing it is sometimes possible to lift the foot, as in the horse, and thus to examine it. In restless or obstinate animals, a cord may be passed round the front fetlock, carried over the withers to the opposite side, and the front foot thus lifted. In the hind foot the cord is passed round the leg just above the hock, or round the camnon bone, and a strong stick thrust through it: Two strong men then lift the foot; the hind foot may also be diawn up to the shafts of a waggon and fastened. In any case, the animal must be firmly fixed by the head.

When the animal is lying the foot is fixed by means of cords, though it is generally best to make the animal rise. Cattle often offer great 
resistance, which may sometimes be overcome by moistening the feet with turpentine, pouring water into the ear, or by bringing a dog into the stall. If all these methods fail, Johne's method of lifting the animal may be tried: it consists in passing a girth, or strong rope, in front under the cariniform cartilage, behind under the ischium, so that it surrounds the animal's body in a circle. This rope being properly fixed, a large number of persons can assist, three or four standing at either side. Care must be takien, however, that a clear space of a yard or so is left in front of the animal, to allow it sufficient room to stretch out its head in rising.

The foot, being lifted, may be examined by pressing on and moving it. Any swelling, reddening of the neighbouring skin, wound about the claw, or discharge of pus should be noted. Both the coronet and skin of the interdigital space should be carefully examined, the foot being first cleansed.

In cattle it is extremely difficult to use foot-baths, which, therefore, are of little practical value.

Treatment is confined to carefully cleansing and keeping clean the parts by giving dry straw, and by the application of poultices and dressings.

\section{I.-BRUISES OF THE SENSITIVE SOLE AND BULBS OF THE FOOT IN OXEN.}

In the smaller herbivora the claws are exceedingly strong in comparison with the body-weight, and disease is correspondingly rarer than in oxen. In the latter, the claws of the hind feet are weaker than those of the fore, and therefore suffer most, while, of the two claws, the inner is the oftener affected.

The conditions comprised under the head of bruises vary greatly in character, some remaining aseptic and confined chiefly to the subcutaneous tissues, others being of an infectious nature and followed by suppuration or necrosis. The latter may either remain confined to the superficial layers of the sensitive sole, or may extend as far even as the bone of the claw or the flexor tendon. Bruises of the superficial layers of the sensitive sole may be followed by bleeding, varying in amount from a trifling spot up to an extravasation sufficient to undermine the entire sole. In the latter case a new sole may be formed, between which and the old sole the partially-absorbed blood forms a dark-coloured, dryish, friable cake, or, in older cases, a grey, fatty mass, corresponding in nature to the dark-coloured, liquid, horn "pus" seen in superficial injuries of the foot in horses. When the deeper layer's of the sole are injured, violent inflammation often results, leading finally to 
necrosis. In the toe, thin fluid pus develops, and the bone of the claw becomes necrotic; in the bulbs severe cellulitis is set up, the flexor tendons become necrotic, and the pedal-joint is attacked. When the injury is still deeper seated, the skin, subcutis, tendons, and tendon sheaths often become violently inflamed, the process sometimes extending to the knee or hock-joint. The animals lie continuously, and die of septicæmia.

The primary cause of the above conditions is some mechanical injury, such as unequal distribution of weight in consequence of abnormal growth of the claws, softening of the sole in stalled oxen, or in unshod oxen working on wet ground, bruises resulting from driving fat oxen over rough roads, wearing away of the sole from long journeys, careless shoeing, or the accumulation of dirt between the claws. If, as is almost unavoidable in cattle, the injured tissue becomes infected, there is scarcely anv limit to the possible complications.

Symptoms and course. The condition usually appears during a journey, the animals gradually becoming lame, the stride being shortened, and the animals showing a tendency to lie, and to move the feet uneasily when resting. When the inner claw is alone affected oxen often stand with crossed legs. Once in their stalls they at once lie down, refuse food, and are difficult to raise. An examination of the claws shows that in one or more pain is produced by pressure over the sole and bulbs of the foot. In severe cases the horn of the sole when cut appears reddened, and suppuration may have occurred, with discharge of pus at the bulb or coronet; in other instances the sensitive sole, or even the bone, may be necrotic. The coronet is then greatly swollen, painful, and, if not pigmented, shows an angry blush. In the later stages fever sets in, the animals lie continually, groan, grind the teeth, and finally die of septicremia. To ensure a proper diagnosis at an early stage the sole must be carefully thinned. When bruising is slight, and the animals are at once rested and placed on soft bedding, recovery follows in a few days, but in suppuration under the sole or bulbs there is danger of the animals dying from decubitus. The possibility of purulent inflammation and necrosis of the sensitive bulbs of the foot and underlying connective tissue constitutes a further danger. Such cases may be followed by purulent inflammation and necrosis of the perforans tendon, or be complicated with septic disease of the pedal-joint.

The prognosis must, therefore, be guided by the degree of pain and the existent changes. Necrosis of the tendon and purulent or septic inflammation of the joint is dangerous, but not absolutely hopeless, recovery sometimes following amputation of the claw; where several claws, and especially when several feet, are simultaneously attacked, however, there is little chance of the animal living.

Treatment. Unless suppuration has set in, it is often sufficient to 
give a few days' rest, and plenty of soft bedding; severe pain may be combated by cold applications; but when pus has formed, a free exit must be provided, though with as little destruction of the wall and sole as possible. The superficial form of inflammation exhibits the same peculiarities as in the horse, and a comparatively small opening is sufficient to allow the thin fluid pus to escape. After carefully cleansing the claws and injecting undermined portions of horn, a dressing is applied, which by smearing with tar can be rendered waterproof, so as to guard against the diseased spot being soiled by manure, \&c. Necrosis of the tendon or disease of the pedal-joint necessitates amputation of the claw.

\section{II.-WOUNDS OF THE SOFT TISSUES OF THE CLAW.}

On account of the comparative thinness of the horny wall, the soft tissues of the claw are often wounded in shoeing. They may also be injured by picked-up nails or other foreign bodies penetrating the sole. Such wounds generally affect the posterior parts of the sole and the bulbs of the heel, and are therefore as dangerous in cattle as in horses, the perforans tendon and pedal-joint being readily injured in either ; pricks in shoeing seldom injure more than the sensitive laminæ. The toe may be injured when the hoof is shortened, the matrix or even the bone of the claw being injured by the knife or saw. In heavy draught the point of the toe sometimes breaks off or cracks across, and is pressed inwards, severely bruising the soft tissues. These facts should be borne in mind in forming the prognosis.

Symptoms. Lameness usually sets in suddenly. Sometimes it is only slight, sometimes severe. Before examination the claws must be thoroughly cleansed. When the flexor tendons are injured pain is acute, and little or no weight can be placed on the foot, while the animals show great pain on dorsal flexion of the claw. Implication of the joint is shown by excessive pain, especially on rotation of the claw, and by swelling of the coronet, most marked above the toe. Not infrequently purulent synovia is discharged.

Treatment requires the removal of any foreign body, cleansing of the injured spot, furnishing of a proper opening for discharge of pus (the above-mentioned precautions being borne in mind), and finally the observation of precautions against infection. Clean, dry straw should be provided, and, if necessary, a dressing applied. Injuries or secondary affections of the flexor tendons are treated on the lines laid down in speaking of panaritium. 


\section{III.-LAMINITIS IN OXEN.}

Working oxen used on hard roads, and fat beasts which consume large quantities of highly-nutritious food, often suffer from a disease of the claws which closely resembles laminitis in horses. The hind feet suffer more frequently than the fore, though the fore or, indeed, all four feet are occasionally attacked.

The disease is caused either by mechanical injury or by over-feeding.

The symptoms closely resemble those in the horse. Pain is evinced whether the animal merely stands or endeavours to walk. The horn of the coronet seems to sink in, and the claws to separate from the bulbs of the heel. At a later stage suppuration occurs, the disease in this respect differing from laminitis in horses, and the pus may break through at the coronet or the horny claw may be entirely lost.

The course is usually acute, though chronic forms are seen and deformity of the wall and sole, together with "ringing" of the hoof and increased breadth of the white line may occur.

Treatment consists in absolute rest and local cold applications for working oxen. The diet should be light. When suppuration and loss of the hoof occur, they render the case so serious that treatment is seldom justifiable from an economic standpoint, and the animal should be slaughtered.

\section{IY.-WHITLOW OF THE CLAW, JOINT FELON, PANARITIUM.}

The term "panaritium" is used to distinguish an acute inflammation, rapidly leading to suppuration, and always attended with necrosis, which in man occurs under the nail, in oxen appears at different parts of the claws, and extends thence to more distant points. The use of the word is quite justifiable, for in cattle the condition attacks the part homologous with the nail, and the process develops in just the same way as in man. Panaritium is due to wound infection, followed by cellulitis, which, partly in consequence of the action of the infecting agent, partly of the anatomical formation of the diseased parts, always tends to necrosis. In oxen the claws are exceedingly exposed to such infection. Being almost always surrounded by dirt, slight injuries of the slin, which are certainly not uncommon, at once open the way to infection, and this again often extends to deeper-seated structures. The process is, therefore, not confined to the skin, but extends to the subcutis, and not infrequently to the tendon sheaths, bones, and joints, producing conditions of the gravest character. 
According to its seat we distinguish panaritium of the toe and bulbs, and interdigital panaritium, all of which have been carefully described by Harms. Being essentially due to infection, this disease sometimes extends very widely, appears more frequently in certain establishments, and even becomes enzootic, while outbreaks are favoured both by neglect of cleanliness in the stalls and by travelling on hard ground. Schleg noted inflammation of the interdigital skin, which often led to necrosis in cows at grass during the autumn. Of twenty-eight animals which stood together, eight were attacked, whilst those standing on the other side of the stable entirely escaped. The exact cause of panaritium is yet unrecognised. Flügge's bacillus of necrosis is very often present, but it cannot with certainty be regarded as the sole causative agent.

(1) Panaritium of the toe develops at the anterior end of the interdigital space. An inflammatory swelling appears, without any visible external injury, involves the coronet and skin of the interdigital space, and may extend beyond. Where pigment is absent, the skin appears reddened, swollen, and painful; and in walking, weight is only momentarily placed on the foot; the slightest pressure causes the animal to groan loudly. The attack is ushered in by fever and loss of appetite. After some days a separation occurs at the coronet, and soon afterwards a thick fluid pus is discharged. At this stage the symptoms usually moderate, granulation occurs, and is soon followed by complete recovery. Even when a piece of skin is destroyed, recovery occurs in two to three weeks.

The condition consists of cellulitis of the cutis and subcutis, accompanied by necrosis, and may therefore be described as panaritium subcutaneum. Harms views this as the first degree of panaritium.

Inflammation often attacks the connective and adipose tissue lying: above the interdigital space, and the tendons and ligaments of the pedal and coronet bones. The symptoms are then very severe; both swelling, redness, and pain are greater, scarcely any weight is placed on the foot, the swelling extends further backwards and downwards over the skin of the interdigital space, and fever, loss of appetite, stoppage of milk secretion, and general wasting ensue. The skin becomes gangrenous, or an aperture forms, from which ichorous pus is discharged in large quantities. The symptoms then moderate, and recovery follows in about three weeks under appropriate treatment. This process might be described as panaritium profundum.

But if such an attack be improperly treated, or the infectious material be particularly virulent, necrosis may extend to the coronet or pedal bone, or to one or other of the joints, and septic arthritis develop, i.e., panaritium ossium vel articulare. The animal then shows intense pain. Immediately a joint is attacked, the swelling extends to the cannon bone, 
though in disease of the pedal-joint, swelling is greatest around the interdigital space. The patient shows high fever, and the same symptoms as in panaritium profundum, but in an aggravated degree. Slight rotation of the claw produces great pain. It should, however, be remarked that in cattle the latter symptom is not so pathognomonic of arthritis as in the horse, because the swelling in and about the interdigital space may render rotation very painful. But where little or no swelling exists, the symptom becomes particularly significant. Arthritis or necrosis of the coronet or pedal bone, as a rule, necessitates amputation (see succeeding pages).

(2) Interdigital panaritium consists in infectious inflammation of the skin and deeper structures connecting the two claws, especially of the fatty tissue, which is so abundant in this region. It occurs oftenest when the claws are excessively separated, and when the oxen are worked, both of which conditions favour injury and infection of the interdigital skin. The skin between the two claws appears swollen, and protrudes somewhat in the form of a snail-hence the origin of the French term "Limace." Pain is marked, no weight can be borne on the foot, the animal lies continuously, and shows fever and loss of appetite. After a few days the skin between the claws breaks; sometimes the interdigital ligament is ruptured, and the swelling, increasing in size, causes the claws to recede from each other.

Appropriate and early treatment is generally followed by recovery, though in cases the process extends to the os coronæ or to the pedaljoint. As a rule, the latter danger is not so great as in panaritium of the toe, the pedal-joint not being so likely to become implicated from this point. Interdigital panaritium is not infrequently followed by extensive proliferation of connective tissue, which is readily injured and leads to fresh attacks of inflammation. The growth may, bowever, be readily removed by operation.

(3) Panaritium of the bulb of the foot generally attacks the inner bulb of one of the front feet; sometimes both bulbs are attacked, very seldom the external alone. The cellular inflammation rarely confines itself to the sensitive parts of the bulb, and to the connective and fatty tissue lying above them, but, as a rule, seizes on the flexor tendons, and often on the pedal-joint. Necrosis of the tendons sets in, with purulent or septic disease of their sheaths, at the point at which they pass over the navicular bone, for which reason this form might be described as panaritium tendineum.

The symptoms consist of severe pain on attempts to, place weight on the foot, and on pressure over the diseased bulbs, which are greatly swollen. Necrosis of the flexor tendons and disease of their bursæ are shown by the claws being extended; in arthritis, rotation of the claw is 
particularly painful. The excessive tenderness, and severe and more general swelling, distinguish this condition from simple bruising of the bulbs of the claws.

The course depends on the character and extent of the disease. When the bulbs and the structures underlying them are alone implicated, recovery takes place in two to four weeks. In disease of the flexor tendons or pedal-joint, amputation is the only resource.

Treatment. Preventive treatment consists in keeping injuries as clean as possible, and preventing further infection by dressings or smearing the parts with tar, and by giving plenty of dry, clean straw as bedding. When a panaritium is detected, the inflammation has usually passed the stage at which resolution is possible, and efforts must be made to provide the best possible exit for pus and necrotic tissues. The most useful agent is certainly the knife, though in many cases it cannot at once be used, and the abscess must be assisted to point by warm poultices of linseed, \&c., to which are added from 5 to 10 per cent. of some active disinfectant. In the early stages, indicated by slight reddening of the skin and shuffling with the feet, resolution sometimes follows the application of bandages saturated with sublimate or creolin solution. Fambach recommended the long-continued application of warm alum solution. As soon as an abscess is discovered, and can be reached with the knife, it should be opened, the wound being antiseptically treated and washed out with 10 per cent. chloride of zinc solution, or injected with 3 per cent. of creolin. Esser recommends using the curette, washing out the parts with 5 per cent. carbolic solution, applying an iodoform dressing which is smeared with tar, and allowing it to remain in position for eight to ten days. 'T'o keep the horn soft, moist dressings are best. Gangrenous tissue should, as far as possible, be removed with the linife and scissors.

The opening of abscesses is least dangerous in interdigital panaritium, and Harms recommends completely dividing the skin between the claws, if it has not previously been ruptured. In panaritium of the bulb the bulbs themselves should be spared as much as possible; they should never be completely divided in the longitudinal direction of the claw, as their further growth would be interfered with. In panaritium of the toe the skin is divided perpendicularly, the diseased point laid open, and free exit given to discharges.

Dressing the parts calls for particular care. In panaritium of the toe and interdigital panaritium the interdigital space is filled with tow or jute, soaked in carbolic or creolin solution, to such an extent as to prevent the claws moving. Both claws are then included in one dressing and surrounded by a bandage or cloth rendered waterproof by a free use of tar. 
As a rule, pain soon disappears after the abscess breals or is punctured. The dressing may then be left in position for several days, but the above precautions should be taken when it is renewed. If granulation appears too free, astringents like solution of alum or sulphate of copper may be applied, or the parts can be dusted with charcoal.

In oxen, the skin at the front of the interdigital space sometimes becomes inflamed, but the process remains confined to the cutis, and pain is less severe than in panaritium of the toe, though healing is difficult on account of the inevitable movement of the claws. The skin appears somewhat swollen and painful, and an inflammatory discharge flows from the surface, which, in drying, produces crusts, while the coronary edge of the horn becomes locally separated. Neglect of these symptoms may be followed by the development of panaritium. 'The animals should therefore be placed on dry straw, the diseased spot carefully cleansed, powdered with iodoform, and a triangular piece of wood corresponding to the width of the interdigital space placed between the claws and fixed in position by a strong cord or wire passed round the claws. This prevents movement of the claws, which interferes with healing. 'I'o ensure the cord or wire remaining in position, a couple of shallow grooves may be made with the knife or rasp around the lower part of the claws.

Vaeth suggests the use of chloride of zinc dissolved in water and made into a paste with an equal quantity of flour for treating inflammatory new growths and warts in the skin of the interdigital space. The material is smeared on the diseased spot and a dressing applied over it. Taeth treats warts by laying them open and rubbing in chloride of zinc, uter which they fall off in a few days.

Delmer describes in oxen a disease of the podophyllous membrane, which resembles canker in horses. The sole of the left claw of the near fore foot was entirely lost, except near the bulb, where it was as thin as paper. The sensitive sole was studded with small, greasy-looking; stinking grey masses, showing an indistinct lamination. The sensitive sole below was swollen and bluish in colour; it bled readily. The papillæ, however, showed no tendency to exuberant granulation. 'Treatment consisted in removing the diseased and broken-down horn and applying an antiseptic dressing. Recovery occurred in fourteen days. Imminger described, under the title of " canker of the claw," a disease of the claw matrix accompanied by a tendency to necrosis. It seemed due to the presence of the bacillus of necrosis (Flügge), and should probably be included under the heading of panaritium. 


\section{V.-ABNORMALITIES OF THE HORNY CLAW.}

Fissuring, \&c., of the horn, though very common in horses, is rare in oxen, and when occurring usually assumes the form of a crack extending from the bearing surface to the coronet of the outer claw in working oxen. The bearing surface of the claw usually shows contraction.

Disease of the substance of the horn is very rare, but the claw is sometimes so thin that the animal is useless for work on hard ground, on account of the sole continually being bruised and causing lameness.

Excessive growth of the toe is very common in stalled oxen. In consequence of the absence of wear the toe grows upwards, forming a kind of horn, while excessive weight is thrown on the bulbs, causing local bruises and pain. The animals avoid standing, eat less than usual, and lose condition. Shortening the toes relieves all these conditions.

Oxen with excessively turned-in or turned-out limbs necessarily have asymmetrical claws, just as similarly formed horses have irregularly shaped feet. Flat or dropped soles unfit working oxen for labour, on account of their becoining bruised.

A peculiar deformity consists in the point of one of the claws overlapping the other; the point of the inner claw often covers a considerable portion of the outer. The claws do not separate when the animals walk, and the opposed margins of the claws are bruised or the lateral ligaments of the joint are strained. The overlapping portions should be pared away.

In-and-in breeding results in some races in the claws becoming too small and the horn too thin, rendering the animals useless for draught. The cause indicates the remedy.

\section{VI.-AMPUTATION OF THE CLAWS IN OXEN.}

When purulent or septic inflammation of the pedal-joint occurs in consequence of any of the above-described forms of panaritium, or of injury from picked-up bodies, or when extensive necrosis attacks the phalanges, it is best to amputate the affected claw. Failing this, pain continues, and the animals die from decubitus, or from septicæmia. Even in the few cases which recover, the animal's general condition suffers very greatly, before anchylosis of the joint becomes complete.

The experience of Harms and others encourages the performance of this operation, very little disturbance being caused, and recovery being comparatively rapid and complete.

Esser looks on amputation as the last resort in extensive necrosis of the phalanges. Provided the animal is still able to stand and has not suffered much in condition, he prefers conservative treatment. 
Amputation may be effected either by exarticulation of the joint of the claw, or by resection of the os suffraginis. Harms prefers the first method, and lays great stress on the necessity for preserving the bulbs in order to ensure regeneration. The navicular bone is not removed.

The animal is cast, firmly fixed, and an Esmarch's bandage applied round the knee or fore arm to prevent bleeding. Harms then removes the horn in grooves at either side, and on the lower surface of the claw, by means of a farrier's knife, the grooves thus made penetrating as far as the soft tissues and converging to one point. The coronary band is

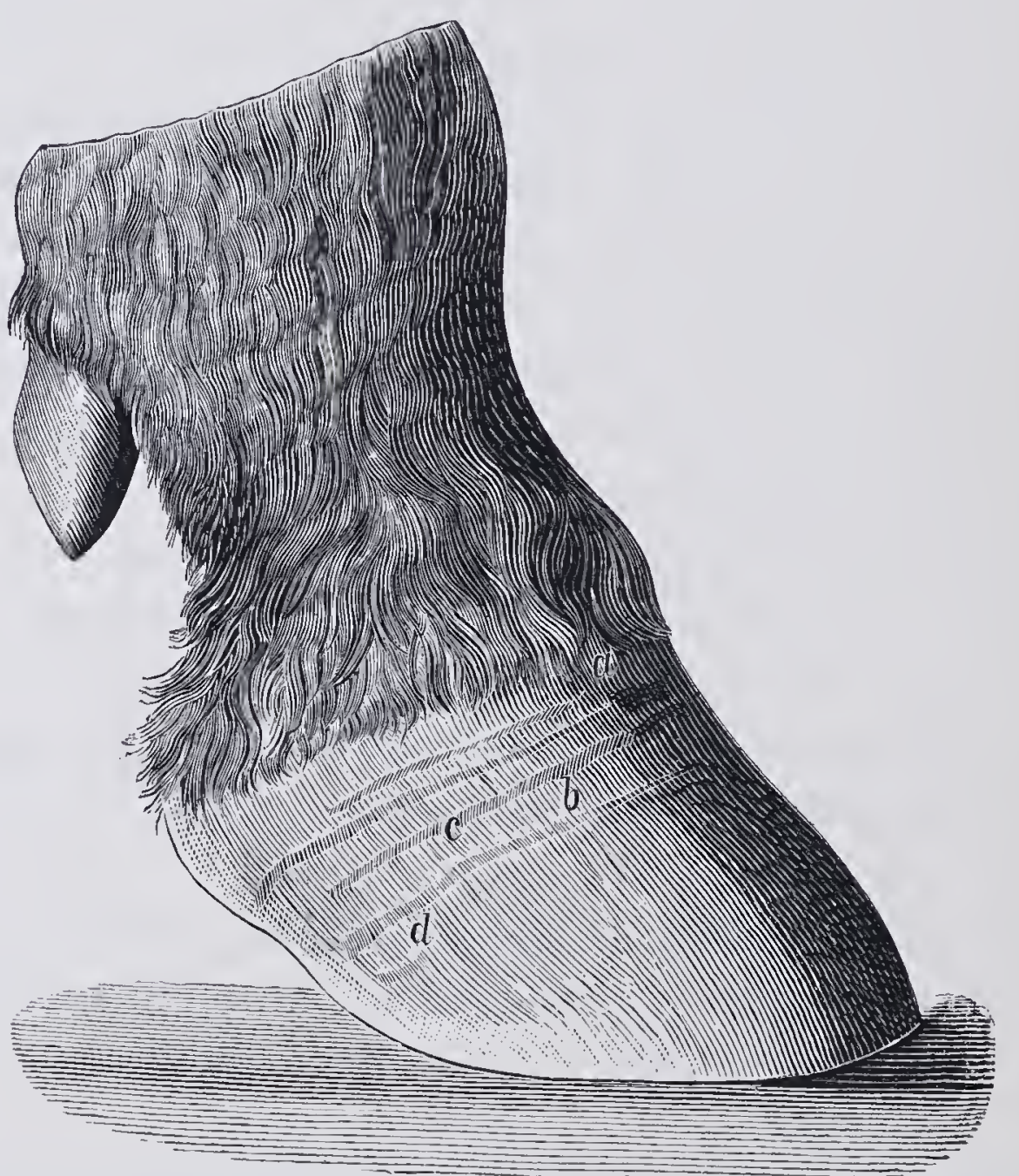

FIG. 313.- An ox's claw. The letters $a$ to $d$ correspond to those in Fig. 312, and show the position of the pedal joint.

next separated, and the pedal bone removed along with the horn of the claw.

Eggeling first thins the horn over the posterior half of the outer wall and of the bulb. With a curved linife he makes an incision, starting from the interdigital space, and lieeping below the coronet, which exposes the pedal-joint, and takes the direction $a$ to $b$ to $c$ (fig. 313). The posterior end of the pedal bone (fig. $313, c$ ) being reached, the incision is continued downwards at a right angle (fig. $313, d$ ) to prevent injuring the navicular bone. By pressing the toe downwards and backwards whilst making this incision, the pedal-joint is opened, and the inner wall of the claw and interdigital ligaments may be cut through.

Möller" repeatedly operated by this method, and recommends it. The 
greatest difficulty lies in so directing the knife as to exactly find the joint; but by bearing in mind the topographical relations given, and by using a very narrow, though not too weak a knife, the difficulty is much diminished. Cadiot proceeds as follows: Having thinned the horn over the seat of operation (fig. 314), he discovers by palpation, and by moving the claw from side to side, the exact position of the joint, which lies about 1 inch below the coronet. This he opens with a curved knife similar to that used in excising the lateral cartilage (No. 4 in fig. 300). Inserting the knife into the joint he makes the incision A, B, C (fig. 314),

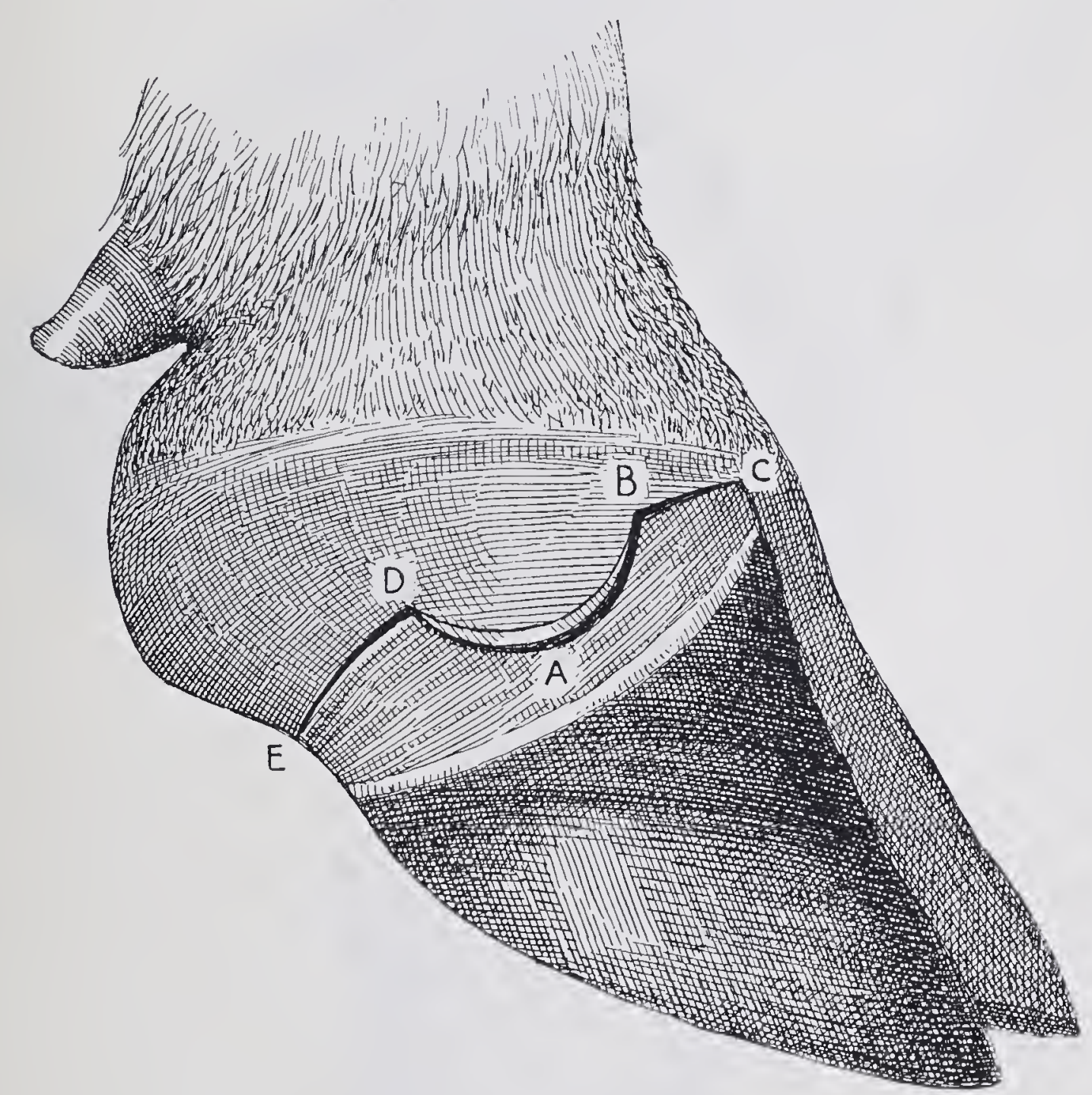

FIG. 314. --Disarticulation of the ox's claw. Primary incision.

dividing the horn, podophyllous tissue, lateral ligament of the joint, and the synovial membrane. With a knife of opposite curvature the incision $\mathrm{A}, \mathrm{D}$ is made, extending to the navicular bone. The claw is then disarticulated by plunging the knife into and through the joint and dividing the strong lateral ligament, \&c., of the opposite side. The disarticulated portion is then removed by a straight cut, D, E.

From this point the procedure is similar in all cases.

After completely dividing the claw at the joint, loose or necrotic pieces of tissue are removed with scissors or knife. Any undermined parts and granulations are thoroughly disinfected, the articular cartilage scraped away from the coronet or suffraginis bone with a curette so as to produce a better granulating surface, the operation wound covered V.S. 
with a tampon soaked in tar, and a dressing extending above the fetlockjoint applied over all. The turns of the bandage are sewn together to keep the whole firm, and plenty of tar applied. This dressing is left in position for eight to ten days. On renewing it, good granulations are generally discovered without suppuration having occurred. Recovery

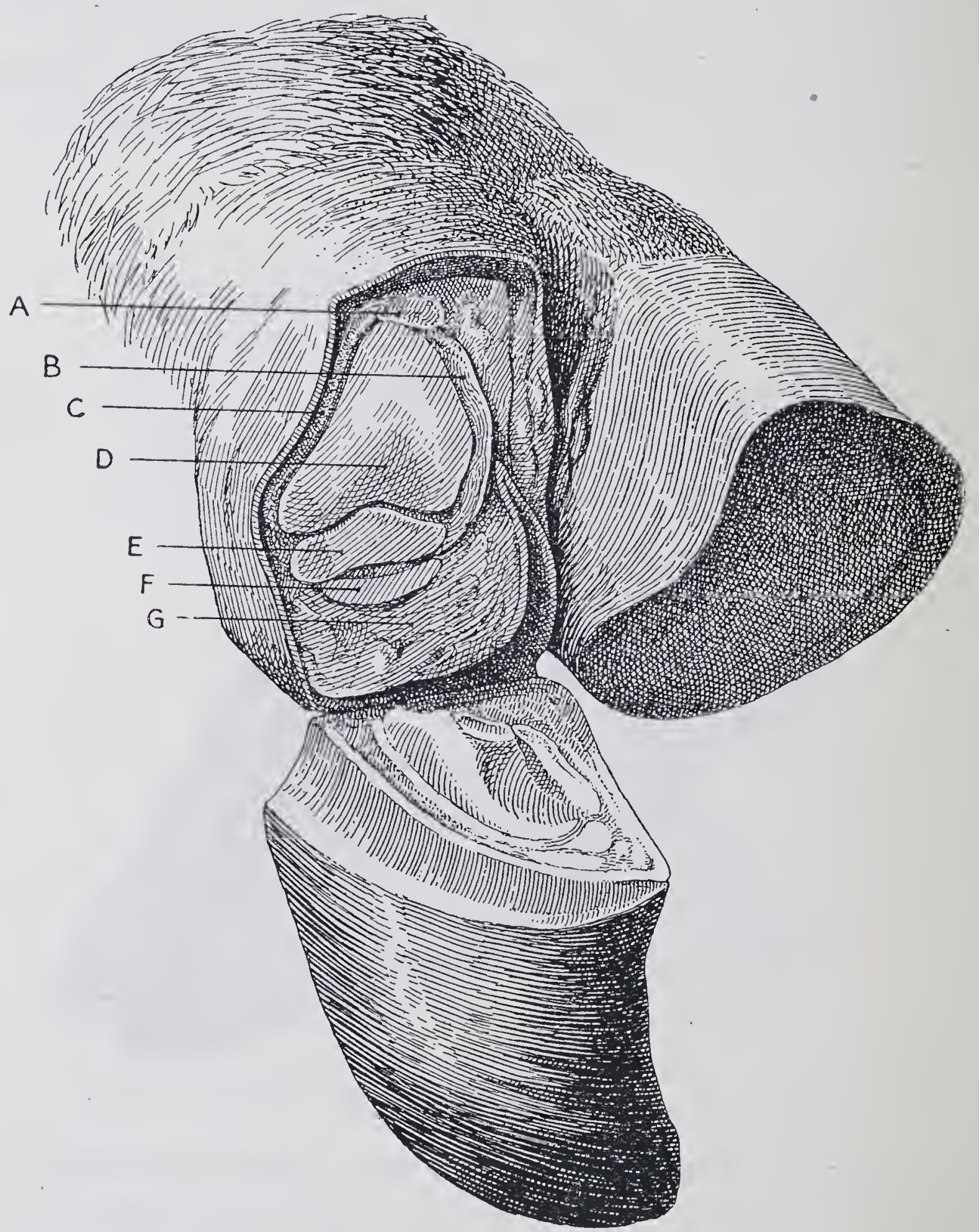

FIG. 315.-Disarticulation of the ox's claw. A, Extensor pedis tendon; B, internal lateral ligament; C, external lateral liganent; D, os coronæ; $\mathrm{E}$, navicular bone; F, perforans tendon ; G, plantar eushion.

takes four to six weeks, the stump being covered by horn, secreted partly by the remaining portions of the coronary band, but chiefly by the bulbs of the heel. 'This growth is so perfect that animals may even be again turned out to grass.

After extensive necrosis of the os coronæ, or purulent inflammation of the coronet-joint, it may be necessary to remove the coronet bone along with the lower end of the os suffraginis. The animal is fastened as 
above described, and an Esmarch's bandage applied. The skin above the bulbs is then divided, either at the outer or anterior and posterior face of the phalanges, and separated from underlying structures, the os suffraginis being then sawn through in an oblique direction, from above downwards. After checking hrmorrhage, the point of operation is cleansed, disinfected, and the flaps of skin cut to shape and sutured under the stump. The above-described dressing may be left in position for eight to ten days, and seldom requires to be renewed more than two or three times before healing becomes complete.

\section{VII.-FOOT-ROT IN SHEEP.}

Apart from foot-and-mouth disease, and from the virulent or malignant foot-rot of sheep, dealt with in works on special pathology, chronic inflammation of the claws occurs in sheep, and is accompanied by chronic suppuration in the coronet or interdigital space. The horn at the coronet is loosened, the claw undermined, the lower phalanges and their ligaments sometimes become necrotic, and the pedal and coronet joints may be attacked, though they suffer less frequently than in cattle.

Causes. As a rule, foot-rot is produced by the same causes as panaritium. Continued standing on wet pastures or manure softens the horn of the claws, produces maceration of the skin around the coronet, and while it favours inflammation of the surface, leads by infection to suppuration, which tends to extend to deeper-seated structures. Once the upper edge of the horn becomes loose, or inflammation occurs in the interdigital space, recovery is impeded by the continuous movement of the parts and the entrance of fresh infective material; at the same time, the burrowing of pus is facilitated.

Symptoms and course. Attention being drawn to the disease by the accompanying lameness, the skin of the interdigital space is found to be red and swollen. Soon afterwards the surface becomes moist, a serous, and later a purulent, fluid being discharged. As a rule, inflammation is confined to the cutis, but the coronary edge of the horn and wall of the claw become loosened, the wall and the sole often undermined, and the horny claw may even be lost, unless proper treatment be immediately undertaken. In exceptional cases panaritium sets in, producing necrosis of the ligaments, tendons, and bones.

As swelling increases and the process extends, pain gradually becomes more acute. The animals lie continuously, or are absolutely unable to bear weight on the diseased foot, and suffer severely in condition. When the superficial attack receives no attention, the infection continues to extend, the disease becomes chronic, and may last for months.

Prognosis depends on the extent of local changes and on the degree 
of pain. Particular caution is required when the animals show great pain, cease to feed, and fall off in condition. The more the claws are deformed, the slighter the chance of recovery.

Treatment. The animal should be removed to a dry place, all moistening of the parts avoided, and when the horn is loosened and undermined, provision should be made for the escape of discharges. For this purpose loose horn should as far as possible be removed, and the diseased spot carefully cleansed. When the disease is superficial, it is sufficient to smear the parts with tar, carbolic acid, or carbolic glycerin (5 to 10 per cent.), or to powder the diseased surface with iodoform or similar disinfectant. Troublesome granulations are checked by astringents like sulphate of copper, alum, sesquichloride of iron, \&c., though the cause of such growths, which often consists in the presence of dead masses of tissue or projecting pieces of horn, must be removed.

In deeper-seated disease, the treatment is similar to that in panaritium. Fixing the claws by a dressing or piece of wood placed in the interdigital space, and fastened by string, will also be found of service.

In sheep, the horn of the wall and sole sometimes grows irregularly, or becomes too long, leading to suppuration under the sole. The treatment consists in shortening the wall, thinning the sole, and giving a dry bed. Further treatment is guided by the same principles as in inflammation of the horse's hoof.

Finally, a grey fatty material sometimes accumulates in considerable quantity in the peculiar glands above the claws in sheep, and is discharged as a worm-shaped mass when the parts are pressed, or the claws forced into contact. The condition is seldom of practical importance, but is sometimes looked on by shepherds as the cause of other painful conditions. Under some circumstances, retention of this secretion may certainly lead to inflammation of the sac. A painful swelling then appears at the front of the interdigital space, and discharges more or less puriform fluid if compressed. To effect a cure, it is usually sufficient to repeatedly empty the sac by pressure.

\section{H. DISEASES OF THE CLAWS IN CARNTVORA.}

The claws surround the last phalanx, which, in carnivora, is covered by the claw matrix, just as is the os pedis in other animals. The third phalanx of the dog lies almost at a right angle to the second, with which it articulates, and just in front of the joint shows a ring-shaped depression, to receive the base of the claw. The portion of cutis which serves as matrix to the claw shows a circular thickening, the coronary band, which secretes the horn of the claw. The matrix covers the anterior portion of the third phalanx, and exhibits small furrows or laminæ. The volar surface of the third phalanx is covered with a material homologous with the sensitive sole. The claw is formed by two horny plates, lying closely together, and enclosing a space. 
They are fixed by their base into the furrow of the bone. T'wo elastic ligaments, which start from the upper end of the second phalanx, and run to the upper surface of the furrow, serve to lift the claw, and prevent it being excessively worn away. The claw does not touch the ground when weight is placed on the foot, and the weight of the body is therefore sustained by the pads of the sole and toes. These are rounded masses of connective tissue, containing elastic fibres and fatty tissue; they are provided with a strong epidermis, and are connected by ligaments with the phalanges. They are black in colour, contain neither hair follicles nor sebaceous glands, but are provided with numerous sudoriparous glands.

\section{I.-GROWING-IN OF THE CLAWS (INCARNATIO UNGUIS). DISLOCATION OF THE CLAWS. CHRONIC INFLAMI- MATION OF THE CLAW MATRIX. LOSS OF THE CLAWS.}

In cats and dogs, the dew-claw, which does not touch the ground, and therefore is not worn away, sometimes becomes abnormally long, and assumes a curved form. Its point then enters its corresponding pad, produces great pain and lameness, and occasionally suppuration in and around the pad. Treatment consists in shortening the claw by means of forceps or strong scissors, care being taken not to remove too much and thus injure the last joint of the toe. In judging of the amount to be removed, the claw of the other foot can be taken as a guide, provided it also is not overgrown. In the same way, the claws of cage-birds often grow to an excessive degree, and require to be shortened.

In dogs, the claw and its matrix are sometimes violently torn off. Sometimes only a portion of the plate of horn is separated from the last joint of the toe; sometimes the greater part of it is gone, so that the claw only remains attached to the sensitive structures by a shred; sometimes it is completely lost. The accident is commonest in large dogs, in consequence of the claw being caught in a cord, net, or similar object, is painful, and always causes lameness. Pain is especially severe if the claw remain hanging, because it then comes in contact with the ground at each step; its complete loss is less painful. Careful examination at once detects the character of the accident. If only a small part of the horny plate be lost, the parts may reunite, otherwise it is best to entirely remove the claw. It can simply be snipped away with scissors; if, however, the last joint of the toe be also injured or otherwise diseased, and if extensive change have taken place in the matrix, it is better to amputate the third phalanx by exarticulation at the last joint of the toe, which lies a little behind or above the upper edge of the nail. As a rule, strong scissors are sufficient, and by making one rapid cut the operation is rendered less painful. In the same way, a claw which is nearly separated from its matrix may be removed. No particular 
after-treatment is required, the wound merely requiring to be kept clean; some dogs will bear a dressing, others will not. A dressing is most useful when the claw alone is removed, and the third joint of the toe left. If needful, the wound or claw matrix may be smeared with tar or creolin.

Loss of the claw. Chronic inflammation of the claw matrix, gradually leading to complete loss of the claw, is not uncommon in large dogs. Kutzbach described it as panaritium. Where it originates in the coronary band, the horn of the claw is thickened and degenerated. Sometimes, however, the "sole" becomes diseased, and then nothing. abnormal is seen on the claw, but the matrix, and particularly the lower surface of the claw, discharges a turbid fluid. Horn production is in abeyance, and, as a consequence, the claw is more or less loosened. The changes in the matrix are sometimes so slight that nothing whatever unusual can be detected with the naked eye; in others, chronic inflammation of the sensitive "sole" exists, leading to suppuration or ulceration. The claw is thus loosened, and when the process extends to the other portions of the horn-secreting membrane, is completely lost. The process often affects the subcutis, or even the periostenm, causing the toe to become three to four times its ordinary thickness. The proliferating tissue contains little yellow points or yellowish-red granulations, resembling those seen in bothryomycosis.

The exact cause of this disease seems still open to discussion, though it closely resembles onychia maligna in man. In some cases herpes or eczema of the neighbouring skin had extended to the matrix of the claw, but in others no cause whatever could be discovered. Kutzner has shown that the disease is not due to want of cleanliness. It often attacks several feet, generally affecting the dew-claws, so that it might be viewed as due to disturbance of nutrition. Large dogs in the prime of life are the commonest subjects. It is most frequently seen in autumn and winter in dogs which live near the seaside and often go into the water. Frick reports the disease as quite common in Hanover; he treats it by operation. Rabe states that it is due to a microbe, the Cladothrix canis.

The animal walks with a short, painful step, lies down a great deal, and has difficulty in standing. Local examination soon shows the character of the disease. At first the "coronet" is only reddened and swollen, but exudation soon occurs, and after some time small, bluish, prominent spots develop, which later suppurate and may lead to sinus formation. The disease often persists, with remissions and exacerbations, for a long time, gradually extending to the deeper-seated tissues.

As a rule, the process cannot be checked, but leads to complete loss of the claw, though Frick has had occasional success by early injection of 
iodine tincture into the coronary band, and therefore the prognosis principally depends on the number of diseased claws. Where confined to single claws, amputation at the last joint offers the most satisfactory results, otherwise the loose claw should be removed, the matrix cleansed and disinfected, smeared with tar or other disinfectant, and a bandage applied. Kutzbach recommends liquor arsenicalis internally, and locally pencilling with nitrate of silver. Attempts to save the diseased claw generally fail. In this respect the condition shows a great resemblance to canker and certain forms of chronic laminitis in horses. Unlike canker, however, it shows no tendency to produce vegetations from the matrix of the claw.

\section{II.-BRUISES AND WOUNDS OF THE PADS OF THE SOLE AND TOE.}

The carpal-pads of carnivora do not touch the ground during movement, and the body-weight is sustained by those of the sole and toes. Continued exercise on rough, hard ground may wear these away, and cause the animal to show pain when walking, a condition most commonly seen in sporting-dogs during the winter. In addition, the pads may be injured by treading on sharp objects, like pieces of glass, which produce more or less serious wounds, or by the animal stepping in caustic chemical substances like quicklime, acids, \&c.

The naturally black colour of the pad is lost when its epidermis is excessively worn, and the surface then appears more or less red, painful on pressure, and abnormally warm. The animal rests a great deal, and when on rough ground, takes short steps, exhibits pain, and has a desire to lick the pads. Should the epidermis be completely worn away at any point, suppuration, or even necrosis, of the pad may occur. Severe lameness results, appetite is lost, and fever sets in. Wounds of the pad generally suppurate, and may even become gangrenous.

The prognosis is generally favourable when rest can be given and extensive necrosis has not occurred. In the latter case the phalangeal joints may become the seat of purulent or septic inflammation, though such a result is rare.

Treatment. When the pads are sore, the animal should be rested and placed on a dry, clean bed. In eight to fourteen days the epidermis will have grown again. If the parts be wounded or suppurating, the undermined epidermis is carefully removed with scissors, the wound cleansed, and any foreign body removed. Abscesses, when present, are freely laid open. Further treatment is scarcely desirable, both because the animal resists, and because it keeps the wound thoroughly clean by 
licking the surface. A surgical dressing is seldom needed unless granulation appears too active or cicatrisation is checked by the animal's interference. A dressing may then be applied, or the diseased spot may be smeared with some bitter-tasting substance, like aloes, which repels the animal, and at the same time assists healing. Severe itching is sometimes removed by pencilling with cocain solution, but precautions are required against absorption of the drug. 


\section{N D E X.}

AвDOMEn, survical diseases of PAGE

, . . . . 261 261

Abdominal hemia. . . . . 263

,, parietes, bruises of . . 251

, wolmds 251

,, ,, with injuries to contained organs . 257

, $\quad$, with prolapse of internal organs . 255

Abnormalities of the horny claw . $\quad 831$

Abscess of the shoulder . . . 222

,, in sub-parotid lymph glands : 80

Abscess, slioulder . . . . 228

Actinomyces fungus . . . $\quad . \quad 87$

Actinomycosis in the parotideal region .

$\begin{array}{cc},, & \text { of the lips and cheeks } \\ ,, & ,, \quad \text { maxillæ } \\ ,, & ,, \quad \text { tongue }\end{array}$

Acute inflammation of the udder . $\quad 44$

tympanites, treatment of . . 322

Alæ of the nostrils, wounds of $\quad 59$

Alveolar periosteum, inflammation of, in carnivora

Alveolar periostitis

Alveoli, diseases of

neoplasms of .

Amputation of the bladder

Anal fistula

$$
\text { , , claws, in oxen }
$$

, region, inj

tumours

Anterior surface of the knee, injuries to

Antiseptics in chronic catarrh of the gnttural pouches

Anus, absence of

$\cdot \quad \cdot 335$

"diseases of . . 335

", inflammation of the mucous membrane of . . . . 340 preternaturalis . . . . $\quad .259$ prolapse of . . . . 343

stenosis of .

, surgical diseascs of the $\quad . \quad 335$

, tumours in . . . 354

Aorta, posterior, thrombosis of . $\quad 677$

Apparatus for prevention of vertebral fracture when casting

Articular and tendinous synovial cavi-

ties in the fore limb, enlargement of

Articulation of the jaw, inflammation of .

Articulation, sacro-iliac, diastasis of

Aspirator, Dieulafoy's .
Atheroma of the false nostril . . 5

Atlas, fracture of . . . . . 140

Atresia ani et recti . . . . 335

Anditory meatus, external, inflamma-

tion of . . . . 110

B.

Balanitis . . . . . . 389

, in carnivoria . $\quad .393$

,, ,. horses . . . 389

,, , oxen . . . . 391

," , swine . . . . 392

Basedow's disease. . . . 153

Biceps femoris nuscle, displacenent of . 680

, muscle, rupture of . . . 509

Birds, impaction of the crop in . . 170

Bladder, amputation of . . . 385

,. puncture of . . . . 373

,2 tumour's in . . $\quad 386$

", urinary, paralysis of . . 377

,2 inflammation of ${ }^{\circ} .380$

Bleeding from the nose . . . 75

Bog-spavin . . . . 717

Bones, fracture of the cranial . . 123

, of the face, fractures of $\quad . \quad 90$

," hock, fractures of . . 711

", ", knee, fractures of . . 539

turbinated, necrosis of . 72

Bothryomycosis of the lower jaw . 103

, , , udder . . 456

Bouley's forceps . . . . . 364

Bowcl fistula . . . . . 259

, injury of . . . . . . 260

, intussusception of . . . 328

, invagination of . . . . . 328

,, puncture of, in liorses . . . 315

,, surgical diseases of . . . 310

, sutures, Gély's . . . . . 313

," Lembert's . . . 313

", ", Wölfler's • • • . 313

," treatment of injured . $\quad . \quad 258$

Brachial plexus, paralysis of $\quad . \quad 519$

Braiu, concussion of . . . . 126

Bräuer's trochar in distension of rumen • 327

Breast, tumours of . . . . 243

Bruises of the abdominal parietes . . 251

719 , , , bulbs of the foot, in oxen 823

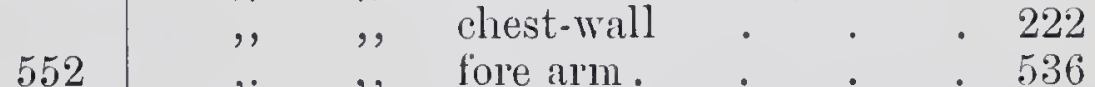

$98 \quad, ", \quad$ ", liead . . . . 1

$480 \quad$, ," mammary gland . . 440

246 ,,, metacarpus . . . 562 
PAGE Bruises of the pads of the sole and toe . 839 ,, 2 sensitive sole, in oxen . 823 , ,, tissues of the neck . 137 ,, udder . . . . 440 ,, , withers . . . . 241 , produced by the collar or other portions of the liarness.

Bulbs of the foot, bruises of, in oxen

Bursa of biceps muscle, inflammation of Bursa of the flcxor pedis, inflammation of

Pursitis glutæi medii

$$
\text { ,, intertubercularis }
$$$$
, \text { præpatellaris }
$$

\section{C.}

Caiculi, lacteal .

$$
\begin{array}{ll}
,, & \text { salivary } \\
, . & \text { urethral }
\end{array}
$$$$
,
$$

Calculus forceps.

, in ruminants
, in the horse
arinary. . .

, spoon

Campylorhinus

Cancer of the tail

Canker of the ear, external

$$
\text { , , , ,, internal. }
$$$$
\text { ,, ,, frog }
$$$$
\text { ,, }, \text { sole of the foot }
$$

Canula and trochar for puncture of the rumen

Capped elbow in dogs . $\quad . \quad$ • $\quad . \quad 535$$$
, \text { in horses }, 531
$$

,, , treatment of, by elastie ligature

\section{,, hock forms of. \\ Caput obstipnm .}

Caries, dental

Carnivora, diseases of the claws in

Carotid artery, injury of

Castration of cryptorchids

Catarrh of the guttural pouches ", $\because, \quad$, use of antiseptics in . . . . . 120

Catarrh of the udder . . • . 447

Catarrhal mastitis, infectious . . 455

Catheter, Giinther's . . . . 117

Catheterisation in dogs . . . . . 379

, horses . . . 379

Catheterism oxen • .

Cattle, diseases of the elaws in . $\quad 820$

, hysroma of the subcutaneous bursa of the tuber ischii in . 483 trimours of the knee in . . 546

Cervical muscles, cramp of . . . 142

$$
,, \quad \text { inflammation of } \quad 143
$$

, vertcbrie, dislocations of . . 141

,", fractures of . . 140

" • • 419

Chceks, diseascs of . . . . . . 1

$\begin{array}{llll},, & \text { inflammation, acute, of . . . } & 3 \\ , & \text { tumours of }\end{array}$
Chest, tapping the

Chest-wall, wounds and bruises of the soft parts of

Clironie catarih of the suttural pouches. 11 , inflammation of the hock . 719

Circus movements after cervical injury . 147

Cirsocele . . . . . . 417

Clam, Bordonnat's . . . . . $\quad$. 278 Combe's . . . . . 278

Claw, anatomy of . . . . $\quad$. 820

, ehronic inflammation of matrix of 837

,, horny, abnormalities of . . 831

, matrix, chronie inflammation of . 837

, whitlow of : . . . . 826

, wounds of soft tissue of . . 825

, amputation of, in oxen . . 831

,, diseases of, in carnivora . . $\$ 36$

,$\quad$,,$\quad$ in eattle and sheep . 820

., dislocation of . . . 837

,, growing in of . . . . $\quad$. 837

, 1 loss of . . . . . 837

Cloaca formation . . . . . . 335

Closure of the mammary dnct • $\quad$ • 457

Conurus cerebralis, diagnosis of position of $\quad$ : 131

,, , difficulties in removing . . . 134

, " in shecp . • . 131

- 132

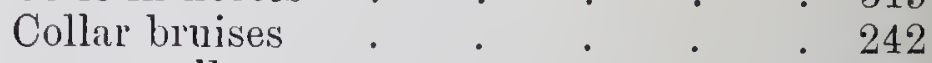

, galls . . . . . . . 233

Colon, twist or rotation of . . . $\quad$. 330

Commotio cerebri. . . . . 126

, spinalis . . . . . 463

Compression of the csophagus . . 176

Concretions, salivary . • . . 81

Concussion of the brain . . . 126

Congenital defects of the testicle . . 407 malformations . $\quad 335,356$

Contracted tendons in foals . . . 582

Contusions and distortions of the shoulder-joint . . . . . 505

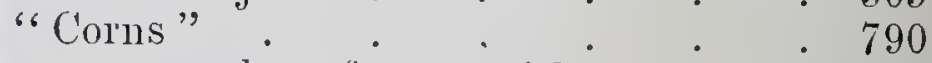

, shoes for use with . . 792

Coronary joint, sprain of . . . . 596

Coronct, injuries to the . • . 783

, joint, inflammation of the posterior ligaments of the . 622

, treads of . . . . . 783

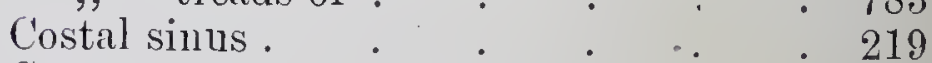

Coxitis. . . . . . . 665

Cracked heels . . . . $\quad . \quad$ • $\quad$ • 644

Cramp of the muscles of the neek . • 142

Cranial bone, injurics of . . . 123

, fractures . . . . . 123

Cranial cavity in sheep and cattle,

trephining the . . . . . 131

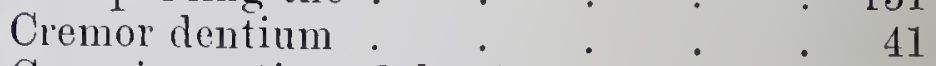

Crop, impaction of the, in birds . $\quad$ • 170

Crural hernia . • • • • 298

Cryntorchids, castration of . . . 407

Curb . . 742

" conformation predisposing to $\quad$ - 742

Curvature of the spine . . . . 470

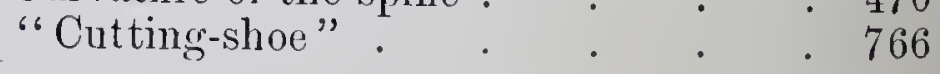


Cystitis

Cystoplegia

Cystotony

Cysts of the udder

,, dentigerous.

$\because$ dermoid

D.

DEFormitres of the tracliea .

Dental caries

\begin{tabular}{|c|c|}
\hline cyst . & . \\
\hline fissures & . \\
\hline fistula & ? \\
\hline for dogs & - \\
\hline $\begin{array}{l}\text { forceps } \\
\text { instrumen }\end{array}$ & ts, Giinther's \\
\hline tartar & .. \\
\hline tumours & . \\
\hline
\end{tabular}

Dentigerous cysts.

Dermatitis chronica verrucosa

$\begin{array}{lll}, & \text { eczematosa } \\ , & \text { erythematosa } \\ ,, & \text { gangrenosa. }\end{array}$

Dermoid cysts

Diaphragmatic heruia • • 114

Diastasis of the sacro-iliac articulation . 480

Digestive tract, foreign bodies in . . 310

Dilatation of the anus . $\quad$. $\quad . \quad 349$

" " rectum . . . 349

Diplegia, laryngis . • . . . 187

Discomyces equi, abscess due to . . 229

Diseases of the abdomen . . . 251

alveoli • . . 42

anus . $\quad . \quad 335$

cheeks . . . . 1

claws in carnivora. . 836

, in cattle and

ear $\begin{array}{ccc}\text { sheep } & \cdot & 820 \\ & \cdot & \end{array}$

elbow . . . $\quad 526$

face and lower jaw . 90

female organs of genera-

tion .

fore arm

fore limb

foot

cuttural pourel

head

hind limb

hock

knee

larynx .

lips

lower thigh or les.

male organs of genera-

tion .

mammary gland

metacarpus

metatarsus ... . 754

moutl . . . . 10

neck . . . 137

nose and nostrils . . $\quad 59$

œesophagus . . . 160

pelvis . . . . 463
592

160

403

655

335

76

405

503

498

123

463

687

310

stomach and bowels

submaxillary lymph glands . . . 105

tail . . . . 491

teeth. . . 25

frontal and superior maxillary sinuses . 64

tendons in the metatarsal regions

tendons in the talsal regions . . . 754

testicle . . . 407

thigh : $: 699$

thorax. . . . 217

tongue . . . . 13

tracliea..$\quad 182$

minary organs . . 356

Dislocation of the cervical vertebræ . 141

,, clitws. • . . 837

," $\quad, \quad$ lower jaw. $\quad . \quad 97$

Displacement of the biceps femoris muscle . . 680

$\begin{array}{llll}, & \text { patella } & \text {. } & 688 \\ ,, & \text { teeth } & 28\end{array}$

Distortion of the neck . . . 142

, , , , $\quad \begin{gathered}\text { Knudsen's ironl } \\ \text { splint for . }\end{gathered}$

$, \quad, \quad, \quad$ results of. $\quad 148$

,",, varieties of . 142

Diverticula of the oesophagus _. . 173

Dorsal vertebræ, fractures, luxations and sub-luxations of .

463

E.

EAR, injury of the external . . . 107

" fistula of . . . . 113

", cedema of the free border of . $\quad$. 109

Ectasiæ of the wesophagus . . . 173

Elbow, capped, in the dog . . . $\quad 535$

, . in the hor'se . . 531

, diseases of . . . . . . 526

, joint, inflammation of . . 530

,$\quad$, luxation of . . . 529

Electricity in facial paralysis. . $\quad 9$

Embolisn, pulmonary • • • . 158

Empyema of the facial sinuses . . 64

Enlargement of articular cavities in fore $\operatorname{limb}$. . . 552

,, outer surface of the hock. 741

, synovial cavities in fore limb.

, tendinous syinovial cavities in fore limb. 
Epididymitis

Epididymis, inflammation of.

Episparia

Epulis

Exonmbalos . $\cdot 57$

Exophthalmic goitre . . . . 158

Exostosis on the posterior border of the lower jaw.

Extermal auditory meatus, inflammation

, canker of the

, ear, injury of

,, , inflanmation of

Extraction of teeth

$$
\text { , " precautions in }
$$

\section{F.}

FACE, diseases of .

", empliysema of

,, fracture of the bones of

Facial nerve, distribution of .

$$
\text { , } \text { œdema. }
$$

,, paralysis

, sinuses, empyema of .

, , trephining of .

False inguinal hernia

Female organs of generation, diseases of

Femul, fracture of

Fe'tlock galls

$$
\text { luxation of }
$$

, region, dermatitis in

Fibro-fatty frog, purulent cellulitis of Firing and operating for spavin, methods of

Fissure, dental

of the male meatus urinarius $\quad 356$

Fistula, anal

$$
\begin{array}{ll}
, & \text { bowel } \\
, & \text { dental } \\
, & \text { Desopliageal } \\
, & \text { of the cheek } \\
, & \text { pharyngeal } \\
, & \text { phecto-vaginal } \\
, & \text { recto-vaginalis } \\
, & \text { salivary }
\end{array}
$$

Flexor metatarsi muscle, rupture of $\quad 704$ , pedis, inflammation of the bursa of 550

, pedis perforans tendon, resection of .

, pedis perforatus tendon, luxation of . . . . .

"tendons, inflammation of

'Furaut's ", rupturc of.

Foot diseases of tendons in

Foot-rot in sheep. . . . . 835

Forceps, adjustment of . . . . 50

$$
\text { choice of }
$$$$
48,49,52
$$

Forcible dilatation in stricture of anus . 352

Fore arm, bruises of . . . 537
Fore arm, diseases of PACE

. 526

, , wounds of . . . . 537

Fore limb, diseases of . . . . 498

enlargement of articular cavities • . 552

enlargement of synovial cavities . . . 552

enlargement of tendinous syovial cavities. . . 552

Foreign bodies in the digrestive tract . 310

," , , mouth . . 10

," ,, , nostrils . . 61

,, , , , wopopagus of carnivora. . 170 osophagus of cattle . . 163 nesophagus of horse . . 162 oesophagus of swine $\quad \cdot 169$

", trachea $\quad: \quad 192$

$,, \quad, \quad$ bones of the face : $\quad$. 140

," , , , , lock. . 711

:, , ", ,, knee . . 539

, ,, caudal vertebræ . . 468

". , cervical ,, . . 140

, , , cranial bones. . . 123

., $\quad$, dorsal vertebre $\quad$. 463

" . femur. . . . 657

," , frontal bone . . . 126

,, horn corc . . . . 127

." , liumerus . . . 501

", ," liyoid bone . . . 21

, , , jaw, splints for . . $\quad 95$

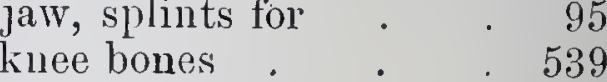

lower jaw . . 90

lumbar vertebræ . . 463

malleoli of the tibia . 702

metacarpus . . . 561

nasal bones . . . 59

navicular bone . $\quad 632$

os astragalus. . . 712

os calcis. . . . 711

os coronæ . . . 603

os pedis . . . 603

patella. . $\quad .694$

pelvis . . . . 472

phalanges . $\quad . \quad 599$

premaxillary bone. . 22

radius. . . 528

ribs . . . . 217

sacral vertebre . . 468

scapula. • . . 498

sesamoid bones . . 624

sternum . . . 220

tibia . $\quad .700$

trachea. . . $\quad 189$

, " ulna . . . . 526

Fracture costarum . . . . $\quad$. 217

Friebel's extension apparatus for contracted tendons.

Fros, canker of the

Frog setons

Frontal bone, fractures of the . . 126

Fulcra . . . . . 50

Funiculitis chronica $\quad . \quad$. $\quad . \quad 417$ 
INDEX.

G.

GaLLs, collar

fetlock

, knee

,

, wind

Gangrenous dermatitis .

.

,, inflammation of the inder.

Gastrocnemins muscle, rupture of .

Generation, diseases of the female organs

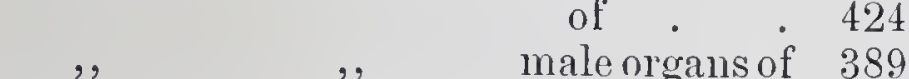

Gid or sturdy in sheep. . . . 131

Gland, salivary, wounds and injuries of , $:$ inflammation of . . 76 $:$ tumours of .

,, sub-maxillary lymph, inflammation of

, sub-parotid lymph, abscess in . 80

, thyroid, results of extir)ation of 152

Glossitis aclinomycotica chronica interstitialis fibrosa.

Glossoplegia .

Gluteus medius muscle, inflanmmation of the tendons of

Goitre.

, cystic
,,$\quad$ exophthahic
,$\quad$ fibrous .
,, real .
varicose .

Gonitis

,, chronica sicca

rrease

, due to exposure to sun's rays inoculability of

Growing in of the claws

Growtlıs, new, in the tongue $\quad \cdot 16,28$

, , , in the subniaxillary $\begin{array}{rr}\text { lymph glands • } & 105 \\ \text { Gums, neoplasms of } & 57\end{array}$

Giinther's catheter for guttural pouch . 117

, dental instruments . . 47

"Gut tie" . . . . . . 305

Guttural pouches, air in. . . . 121

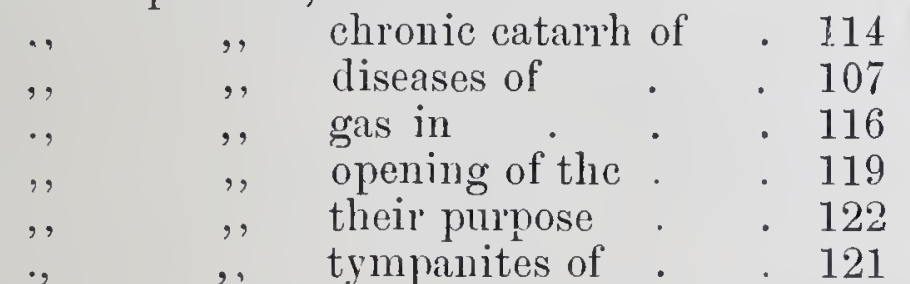

H.

HÆMATOCELE

\section{Hxmothorax}

Head, diseases of the ,, cedema of .

Hemiplegia laryngis

Hernia, abdominal

cruralis

$$
\text { cure of }
$$

diagnosis of

diaphragmatica

essential constituents of

false inguinal.
Hernia, incarcerated inguinal

PAGF

289

280

297

281

in ruminants $\cdot \quad \cdot \quad 294$

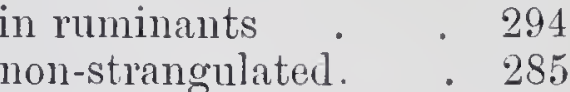

inguinalis

interstitialis * 290

interna abdominalis . . 305

methods of cure . . . 269

non-strangulated . . . 268

pelvic . . 305

perineal . . . . . 299

, in swine . . . 295

peritoneal . . . . 305

scrotalis . . . . . 280

strangulated . . . . . 265

umbilical . . . . . 273

ventralis. . . . . 301

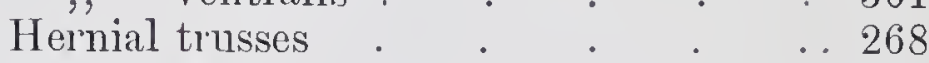

Herniotome . . . . . . 290

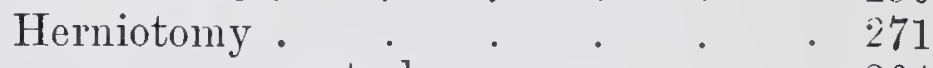

, ventral . . . . 304

Hind extremities, paralysis of . . 483

, $\quad \operatorname{limb}$, diseases of . . . . 655

," ," paralysis of . • . . 667

Hip-joint, inflammation of . . . 665

," lameness . • . . 683

Hock, bones of, fracture of . . . 711

, capped. . . . . . 744

, chronic inflammation of the $\quad 719$

, diseases of . . . . . 711

,, enlargement of outer surface of. 741

, joint, acute inflammation of . 713

, ,, luxation of . • $\quad 713$

, , sprain of . . . . 713

, , wounds of . . . . 714

Horn core, fracture of . • . $\quad$ • 127

", ", splint for . . 127

Horns, looscning of • • • • 129

Horny claw, abnormalities of . . 831

Humerus, fracture of . . . . 501

Hydrocele : . . . . . 415

Hygroma of the knee • • • • 541

, subcutaneous bursa of the tuber ischii in cattle . . . 183

Hyoid bone, fracture of . . . $\quad 21$

Hyperdentition . • . . . . 26

Hyovertobrotomy . . . . . 117

Hypospadia . . . . . . 356

I.

IMPACTION of the clop in birks . . 170

Incarnatio unguis. . . . . . 837

Infectious catarrhal mastitis . • . 455

inflammation of the lower synovial sheath of the perforans tendon . . 760

Inflammation of the alveolar poriosteum in carnivora . 53

,, alveolar periostem in herbivora . 42 articulation of the jaw. 
Inflammation of the bursa of biceps muscle bursa of the flexor pedis

buisa of the ligamentum nuchx. claw matrix . connective tissue surrounding rectum . external auditory meatus or Otorrhoea

elbow-joint .

epididymis .

flexor tendons

hip-joint

hock, chronic

liock-joint

jugular vein .

knee-joint

larynx .

ligamentum uuchæ. 154 mucous membrane of the anus

mucous membrane of the rectum podophyllous mem-

brane.

postea spinatus muscle, tendou of

posterior ligaments of coronet-joint.$$
\text { , }
$$$$
\text { , }
$$$$
,
$$$$
\text { , }
$$$$
\text { , }
$$

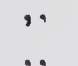$$
,
$$$$
,
$$$$
\text { , }
$$$$
\text { , }
$$$$
\text { , }
$$$$
,
$$$$
,
$$$$
,
$$$$
\text { , }
$$$$
\text { , }
$$$$
\text { , }
$$$$
\begin{aligned}
& ,, \\
& ,,
\end{aligned}
$$$$
\text { , }
$$$$
\text { , }
$$
prepuce in carni-

vola.

$$
\text { , horse. }
$$$$
\text { , oxen. }
$$$$
\text { , }
$$
swine .

salivary glands sensitive laminx. shoulder-joint spermatic cord stifle-joint sub-maxillary lymiph glands . 105 sub-coronary connective-tissue suspensory and check ligaments synovial sheath of the perforans tendon tendons of gluteus medius muscle . 666 testicle. . . 413 , tongue . . 15, 16 udder • • 441,449 uretlira . . 375 urinary bladder . 380 uterus • • 424 vagina . . . 424

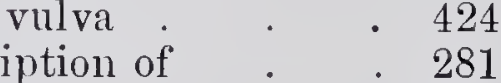

, hernia

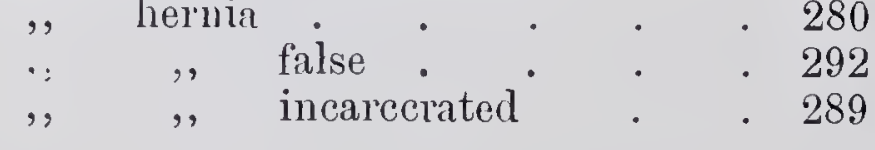

AGE

Ir

nguinal hernia, in dogs

,, ,, in ruminants.$\quad$. 294

,, , non-strangulated. .285

Injuries of the carotid artery . . 137

,, ,, external ear . . . 107

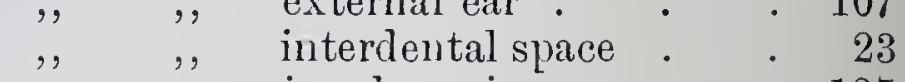

,, , , jugular vein . . . 137

,. $\quad$, knee in the horse . $\quad .540$

$,, \quad,, \quad$ larynx . . . . 182

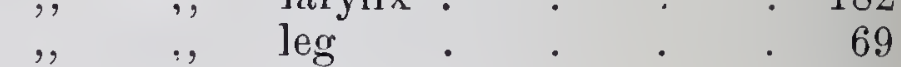

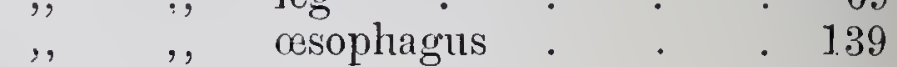

", ., penis . . . . $\quad .394$

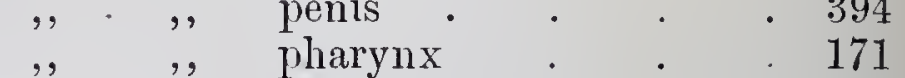

,, $\quad$,, lecurrent nerve $\quad$. $\quad$. 138

, ., stifle-joint . . . 695

., $\quad$, sympathetic nerve. 138

,. :, thigh . . . . 699

," $"$ tongue. . . . 13

,. ., urethra. . . . 375

,. ., uterus . . . . 425

,, , vagina . . . . 425

, ., vilva. 424

,$\quad$ produced by saddle. . . 233

,, produced by striking (inter-

ference) . . . . 763

to the anal region : $\quad \cdot \quad \cdot 338$

., ,, blood-vessels of the neck 137

.,, coronet. . . . $\quad 783$

, . . cranial bones. . . 123

,, muscles and fascire of the quarter • . 655

$\begin{array}{llll}, & \text { pharynx and } \text { osophagus } & 171 \\ ,, & , & \text { rectum }\end{array}$

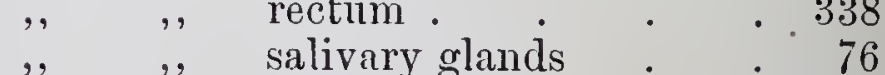

,", skull . . . 129

,, , vertebræ . . . 466

Instruments for performing laryngotomy 208 ,

, , trephining . . 209, 215

Interdental space, injuries of $\quad \cdot \quad \cdot \quad 23$

"Interfering," injuries from striking or" 764

Internal abdominal hernia . . . 305

,, herniotorny . . . . 271

, organs, prolapse of . $\quad . \quad \cdot 255$

, popliteal nerve, paralysis of $\quad 670$

Intestinal trochar, Friedberger's . . 317

Intussusception . . • . . 328

Invagination of the bowel * . . 328

Inversion of the urinary bladder : . 382

, , , uterus. . . . 431

,, vagina. . . . 428

Iodide of potassium in actinomycosis : 18

Iodine in actinomycosis . . 18

Iron splint for distortion of the neck : 148

rregularities in development of teeth . 26

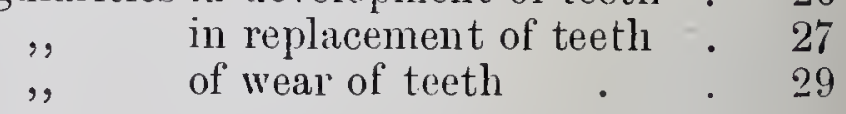

Javart cartilaginenx . . . . 793

". cutané . . : : . 793

Jaw, inflammation of the articulation of 98 , lower, diseases of . • . $\quad .90$

,,$\quad$, dislocation of $\quad \cdot \quad \cdot \quad \cdot \quad 97$ 
Jaw, lower, fracture of

, ,, splints for.

, overshot

, paralysis of .

, periostitis and exostosis on the pos-

terior border of the lower.

,, prognathous.

, tumours on.

,, undershot

Joint felon

Jugular vein, fistula of .

,$\quad$, inflammation of

, , , injury of .

,, , thrombosis

\section{K.}

Keratona

Keratomata, excision of

Knee, anatony of.

, diseases of .

, fracture of the bones of $\quad$. $\quad .539$

, cralls

, injuries to, in the hor'se . . 540

, joint, chronic inflammation of . 549

$\therefore$ tumours of, in cattle . . . 546

"Knuckling over" in foals . • • 582

Kyphosis

Kypho-skoliosis • • •

L.

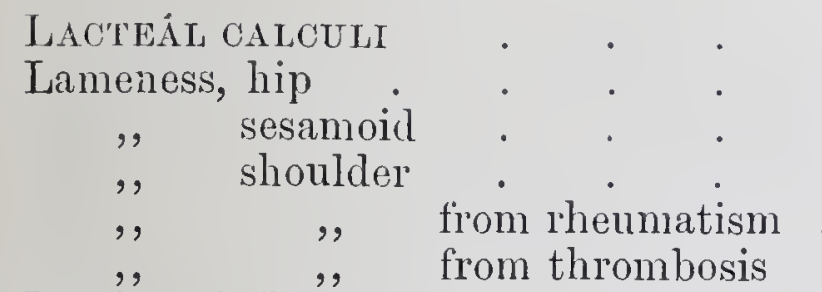

Laminæ, inflammation of sensitive 805

Laminitis ,$\quad$ exercise in

Laparotomy

Laryngismus stridulus

Laryngitis

, chronic

,, -spasmus .

Laryngotomy

instruments

$\begin{array}{ll}, & \text { inflamination of } \\ ,, & \text { injury to } \\ ,, & \text { method of opening } \\ , & \text { neuroses of } \\ ,, & \text { tumours in }\end{array}$

Lateral cartilage, ossification of the

Leg, wounds and injuries of .

Ligration, method of in prolapsus recti

Lips, bluises of

, diseases of .

,

inflammation, active, of . . 3

, paralysis of.

, tumours of
Liquor Villati

Loosening of holns . . . . $\quad$ • 129

Lordosis . . . . . 470

Loss of the claws . . . . . $\quad$. 837

Lower jaw, dislocation of . . . . . . 97

,, , fracture of . . . . 90

,, splints for fractule of . . 95

Lumbar vertebræ, fractures, luxations,

and sub-luxations of . . . . 463

Lumbo-dorsal fascia, necrosis of . . 497

Luxatio patellæ . . . : . 688

Luxation of the dorsal vertebræ . . 463

,,, elbow-joint. $\quad . \quad 529$

,, , femur. . . 661

,, , , varieties of. • 663

,, , flexor pedis perforatus

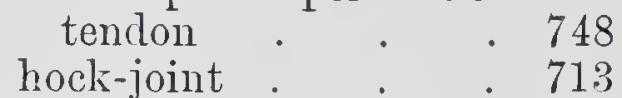

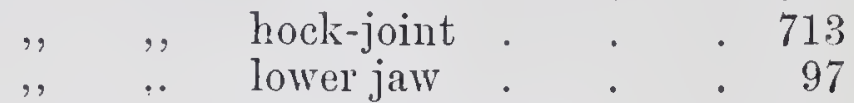

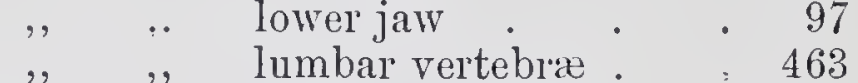

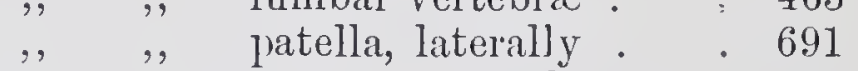

,, ., , upwards . . 688

,, ., phalanges . . . . 594

: , sacro-iliac articulation . 480

,,$\quad$ shoulder-joint . . 504

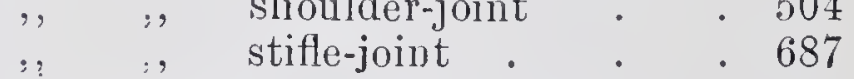

,, $\quad$, symphysis ossimm pubis 482

Lymphadenitis chronica suppurativa . 230

\section{M.}

Makroglossia . . . . . 16

Nakrostomy . . 2

"Maladie du cöit" . . . . . 485

Male meatus urinarius, fissuring of . 356

Male organs of generation, diseases of . 389

Malformations, congenital . . 335, 356

Malleoli of tibia, fracture of . . $\quad 702$

Mammary dnct, closure of . . . 457

,$\quad$, stenosis of . . . 457

, gland, bruises of . . . 440

", $\quad$, diseases of . . . . 439

," $\quad$, structure of. $\quad . \quad 439$

,, wounds of ., . . 440

Manège movements in disease of middle

ear . . . . . 112

Mastication, paralysis of muscles of $\quad \cdot \quad 99$

Mastitis acuta. . . . . . 441

,, apostematosa . . . $\quad .449$

,, catarrhalis . . . . 447

, gangrenosa . . . . . 451

,, phlegmonosa. . . . 445

, tuberculous . . . . 455

Matrix, claw, chronic inflammation of

the . . . . . . 837

Maxillæ, actinomycosis of the . . . 103

Maxillary sinus, trephinings. . . $\quad 67$

Mechanical injuries . . . . 13

, pneumonia . . . . 162

Median neurectomy . . . . 617

Metacarpus, bruises of . . . . 562

, diseases of $\quad \cdot \quad .560$

," fractures of $\quad . \quad 561$

," wounds of . $\quad . \quad . \quad 562$

Metatarsus, diseases of . . . . 754 
Month, angular or shar]?

, diseases of .

, foreign bodies in

, gag for eattle.

"Nouth-rot", oxen

Mouth, shear-like

$$
\begin{array}{ll}
, & \text { step-formed } \\
, & \text { smooth . } \\
, & \text { wave-formed }
\end{array}
$$

Mucoid degeneration of the turbinated bones .

Mucous membrane of the rectum, inflammation of

Mud-fever

Muscles of mastication, paralysis of

Myopathic paralysis of the larynx.

Myotomy of biceps femoris muscle

N.

NAILS, picked-up.

Nasal bones, fracture of

Navicular bone, fracture of . , disease

Neck, 'diseases of theories of$$
, \text { distortion of }
$$

,2, varieties of

, paralysis of muscles of

, wounds and bruises of the tissues of the

Necrosis of the lumbo-dorsal faseia

Neoplasms of the gums and alveoli

Neurectomy, median

$\begin{array}{ll},, & \text { plantar } \\ ,, & \text { spavin } \\ \text { ulnar } & \text { ulnar }\end{array}$

Neuroses of the larynx .

New growths in the tongue.

Nose, bleeding from

,, diseases of .

Nostril, false atheroma of

Nostrils, diseases of

, foreign bodies in

, tumours in

,, wounds of the ale of

\section{O.}

CEDEMA, facial

Erlema of glans penis, elastic bandages in sophageal fistula

,, forceps, Delvos' and Hert-

wig's.

, screw . . . . 165

Wo snare, Meier's' . . . 165

Gophatomy

Esophagus, compression of. . . . 176

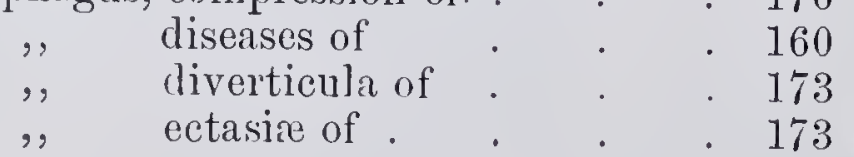

PAEE
31
10
10
164
322
53
33
35
38
34

73

340
644
99
188
681

Cisophagus, foreign bodies in

$\begin{array}{ll},, & ,, \\ ,, & ,\end{array}$

PAGE

in horse . 162

in swine . 169 of carni-

vora . 170

incision into . . . 166

injury of . . . 139,171

paralysis of. . . . 176

psorospermia in . . . 181

rupture of . . . . 173

spiroptera sanguinolenta in 181

stenosis of . . . . 176

tumours of . . . . 178

ventriculosus . . . 173

Onarthritis . . . . . 506

Opening the guttural portches . 119

Orchitis . . . . 413

Os astragalus, fracture of . . . 712

,, calcis, fracture of . . . . 711

,, coronæ, fracture of . . . . 603

,, pedis, fracture of . . . . 603

Ossification of the lateral cartilage $\quad 628$

Osteomyelitis . . . . 24, 45

Osteoporosis . . . . . 104

Othæmatoma . . . . . . 109

Otitis externa . . . . . 110

Otorrhœa : . . . . . 110

Ovary, treatment of cysts in. . . 461

()xen, laminitis in . . . 826

P.

PAD of the sole and toe, hruises and wounds of .

Panaritium

Paracentesis abdominis. $\quad \cdot \quad \cdot \quad \cdot 261$

,. thoracis . . . . 244

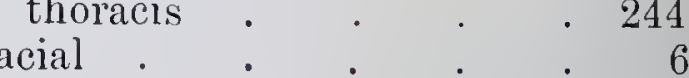

,, masticatory. . . 99

nervi radialis . . . 513

of the ants . . . . 349

,, brachial plexus . . 519

,$\quad$ crural nerve . . 673

,, great sciatic nerve . 571

, lind extremities . 483

,$\quad$ lind limb . . . 667

,, internal popliteal nerve 670

, jaw (so-called) . . 99

,, lips. . . . 4

,, muscles of mastication 99

, ,, neck. . 142

,", , tail . . 493

,, obturator nerve . $\quad 676$

,, assophagus . . 20, 176

. palate . . . 20

, penis . . . 394

, pharynx . . 20,176

,, quadriceps femoris

muscle . . 673

radial nerve . 513

rectum . $\quad . \quad 349$

supra-scapular nerve . 510

tongue . . . 19

mrinary bladder . . $\quad 377$ 
Paraphimosis

PAGE

Paraplegia

Paraproctitis apostematosa.

Parasites in the trachea

Parotideal region, actinomycosis in the.

Parotitis

Patella, displacement of:

, fractule of.

, luxation of

, linture of the straight liva-

Pelvic hernia

$$
\text { ments of }
$$

Pelyis, Anker's knife for

fiseases of

Pcnetrating wounds of the abdomen

Penis, amputation of

", injuries of .

, paralysis of

, tumonr's of.

Pentastomum taenioiles

Perforans tcndon, infectious inflamma-

tion of the lower synovial sheath of .

Pericarditis, septic, in cattle.

Pericardium, tapping the in the dos '248

Perilaryngitis chronica fibrosa . . 182

Perineal hernja . . . . . 299

Periorchitis . . . . 413

Periosteotomy in splint lanemess . . 591 kuives. . . 735

Periostitis, alveolar 42,53 ,, on the posterior border of the lower jaw 、 . 101

Periproctitis .

Peritoneal hernia.

Pervious urachus.

Phalanges, anatomy of . . . $\quad .592$ ,$\quad$ diseases of . . $\quad 592$

, fractures of . . . . $\quad 599$

"luxations of.$\quad 594$

Pharynx, diseases of . . . 160

, foreign bodies in . . 160

", , , of horse 162

, , , , of carrivora 170

", injuries to the : . . 171

, paralysis of . . . 176

Phimosis

tumours in . . . 179

Phlebitis

venæ jugularis

Phlegmonous mastitis .

Picked-up nails .

Plantar neurectomy

Plcura, wounds of

Pleurisy

Pneumonia, inechanical

Preumothorax

Pododermatitis acuta

$$
\text { " chronica } \cdot \text { verucosa vel }
$$$$
\text { migrans . } 815
$$$$
\begin{array}{lll}
\text { parenchymatosa } & \text {. } & 770 \\
\text { superficialis } & . & 768
\end{array}
$$

Podophyllous membrane, acute inflammation of .

Poll-evil

Polyodontia

$$
\text { V.S. }
$$

Postea spinatus muscle, inflammation of the tendon of . $\quad .510$

Posterior aorta, thrombosis of . . 677 , ligament of the coronet-joint, inflammation of .

Pre-maxillary bone, fracture of $\quad 022$

Prepuce, anatomy of, in horse . . 389

391

393

389

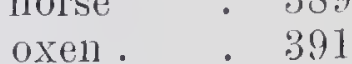

swine $\quad 392$

Pressure in treatment of tendinitis . 576

"Pricks" in shoeing. . . . 776

Proballg for" cattle. . . . 164

Proctitis . . . . . . 340

Proguathous jaw . . . . . 28,31

Prolapse of internal organs . . . . 255

the allus $\cdot 343$

"rectun. . . . 343

" virinary bladder . . $\quad 382$

". uterus. . . 431

vacina $4 \cdot 28$

Prolapsus recti cum invaginatione . 343

, et ani. . . 343

uterí. . . . 431

vaginæ. . . . 428

et inversus vesicæe $\quad 382$

Prostate, castration for enlarged . . 405

diseases of $\cdot 403$

Pulmonary embolism . . . . 158

Pulpitis . .44

Punctio intestini . . . . . 315

vesicæ. . 373

Puncture of the abdomen . . . 261

blatder . . . 373

in horses . . 315

through the abdominal walls 318

through the rectum . . 320

rumen . . . $\quad 321$

thorax . . . 245

Purulent cellulitis of the fibro-fatty frog. 778

,, fluid, puncture of the thorax

for removal of . . . . 248

, inflammation of the nidder . 449

, , , , subcoro-

nary connective tissue . 783

Q.

Quarteli, diseases of . . . . 655

injuries to the minscles and

fascie of the . 655

"Quidding" the food . . . . 25

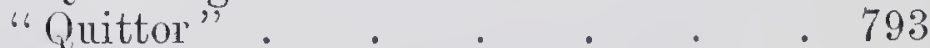

, setons in . $\quad 797$

,, excision of latcral cartilage in 798

\section{R.}

RADJAt nerve, paralysis of . . . 513

Radius, fracture of . . . . 528

Ranula. . . . 20,78 
Rectal tumours

Recto-vaginal fistula

Rectum, diseases of

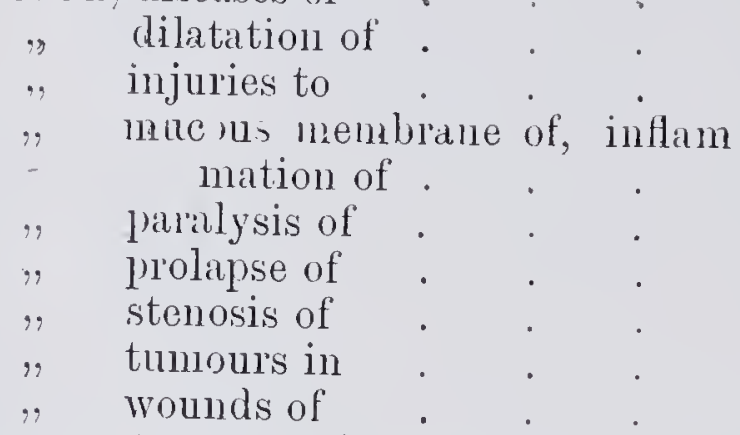

Recurrent nerve, injury of . . . 138

Removal of conurus cercebralis . . 132

Resection of the flexor pedis perforans . 778

Retention of the testicles . . . 407

Rhaclitic ring-bone . . . . . 606

Rlinoscleroma

Ribs, fracture of .

Riding, errors in .

Rima glottidis, spasm of . $\quad 187$

"Ring-bone" . 605

$$
\text { , rhachitic }
$$

Ring-bones, importance of shoeing in $\quad \cdot 610$

"Roaring"

operation for

Rotation of the colon in horses

Rumen, puncture of

liumellotomy

"Ruminant's moutlı",

Rupture of the licepss muscle.

\section{flexor tendous}

" metatarsi muscle gastroenemii muscle. oesophagus

rectus femoris and vasti muscles

stritight ligaments of the patella

suspensory ligament.

tendo-Achillis muscle

tibio-fibular interosseous ligament

\section{S.}

SACro-ILIAC articulation, huxation of . 480

Sacrum, fracture of

Saddle-bed, injuries in .

$$
\text { , -galls . }
$$$$
\text { ,, }, \text { causes of }
$$

Sadding, faults in

Salivary calculi and concretions

$$
\begin{aligned}
& \text {, fistulie } \\
& \text { slands, diseases of } \\
& \text {, inflammation of. } \\
& , \text { injuries to } \\
& \text {; } " \text { tumours of . } \\
& \text {,, } \quad \text {, wounds of }
\end{aligned}
$$

Sarcocele

Sauberg's vaginal ring .

Scapula, fractures of

Scirrhous cord
Scirrhous cord, bothryomyces in PAGE caused by discomyces equi . . . 418 staplyylococei in . $\quad 418$

Scrotal hernia

Scrotum, diseases of . . 405

Sensitive laminæ, inflannmation of $\quad .805$ sole in oxen, bruises of the

Sesamoid bones, fractures of . lameness . . . 625

Seton needles . . . . . 643

Setons in quittor . . . 797

Sewell's frog seton needle . . . . 643

"Sliar'l mouth" . 31

Shears, Professor Möller's tooth . . $\quad 35$

Shear-like moutlı. . . 33

Sheath, tumours on $\quad \cdot \quad \cdot \quad \cdot \quad \cdot \quad \cdot \quad \cdot \quad 398$

Sheep, cnenurus cerebralis in. - . . 131

," diseases of the claws in . . 820

, foot-rot in.

Slines for use with "coins". . . 792

Sloeing after tendinitis . . . 577

, pricks or stabs in . . . 776

Shoulder abscess . . . . . . 229

" joint, contusions and distortions of . $\quad 505$

. diseases of $. \quad . \quad .503$

.. luxation of . . . 504

lameness . . . . 521

., , from rheumatism . 522

. ", thrombosis . 524

," region, diseases of . . . 498

Side-bone .

Sinuses, frontal and superior, diseases

of . . 64

Skoliosis . . . . . .

Skull, diseases of . . . . . . . 123

,' wounds of . . . . . $\quad . \quad 129$

"Smootl mouth" . . . . 38

Sole, bruises of the pads of . . . $\quad$. 839

,, canker of the . . . . 815

"Sore-shins" . . . . . 591

Spasm of the rima glottidis: . $\quad 187$

Spasmus glottidis. . . . 187

Spavin. . . . . 719

bog. $\quad . \quad .717$

,. conlormation predisposing to . 722

, methods of firing and operating for

leurectony $\cdot \quad 733$

1remectomy in . . . 737

patlological anatomy of . $\quad 720$

test for. . . 726

theory of production . $\quad 719$

treated by tibial neurectomy . 737 opening bursa of flexor metatarsi . 736 periosteotomy . . 734 section of flexor metatarsi tendon . 736

Spermatic cord, hydrocele of. . . . 416

416
417

Spinal column, diseases of . $\quad . \quad$. 417

, curvature, factors in producing : 471

Spinaventosa . . $: 103$

Spine, curvature of $\quad . \quad . \quad . \quad 570$

Splints. $\quad . \quad 586$ 
Splints, cause of

for fracture of lower jaw

". in the horse

theory of production of

Sprain of the eoronary joint .

.. . . hock-joint

Stabs in shoeing

$$
\begin{aligned}
& \text { hock-joint } \\
& \text { stifle-joint }
\end{aligned}
$$

Stenosis of the mammary duct

$$
\begin{aligned}
& \text { nesophagus } \\
& \text { reetum. } \\
& \text { traehea. }
\end{aligned}
$$

Stenson's duet, division of

Step-formed mouth

Ster"ual sinus

Sternum, fraeture of

Stifle-joint, diseases of

, inflammation of

," , injuries to

,, , luxation of

," , sprain of

Stockfleth's wooden ring in prolapsus

ani

Stomael, surgical diseases of

Strangulation of the tongue .

Strictures of the urethra

Striking or interfering injuries produced

by . • 76

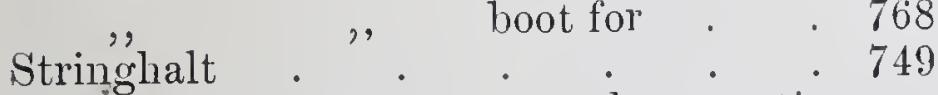

,, attempts to produce artificially

forms of

treated by section of thigh faseia tenotomy in

Struma

Sturdy ill cattle . . . . . 134

Sub-eoronary conneetive tissue, inflanmation of .

Subluxations and fractures of the cervical vertebrx

Sub-maxillary lymph slands, inflannmation of .

Sub-parotid lympli glands, abscess in

Superior maxillary sinus, trephining the

Supernumerary teeth . . . . 26

Suprascapular nerve, paralysis of . . 510

Sursical diseases of the abdomen . . 251

Suspensory ligament, inflammation of . 570

,, , lupture of . . 565

Suture, Gély's bowel • • • • . 313 ,L Lembert's bowel . . . 313 , Wölfler's bowel . . . . 313

Swine, foreign bodies in cesophagus of . 169 , perineal hernia in . . . 295

Sympathetie nerve, injury of . . . 138

Symphysis ossium pubis, luxation of . 482

Syringe, Zehden's .... 133

$\mathrm{T}$.

TANIA canurus in sheep

Tail, diseases of
Tampon-canula . . . . . 186

Tapping the eliest . . . . 244

," ", perieardium in the $\log .248$

Tartar, dental •. • . 41

Teeth, cavities in . . . . . 40

, diseases of . . . . 25

,. displacement of . . . . 28

extra or adventitious . . . 26

" extraction of . . . 51

" extraction of by punehing. • 46

" $"$ precautions in .51

,,$\quad$ fissures in . . 40

," irregular development . . 26

,, replacement . . 27

$" \quad$ wear 29

, premature wear of . . . 37

, superumerary . . . . 26

Te'mporal and superior maxillary sinuses, diseases of . . . . . 64

Tender-mouthed horses, treatment in . 24

Tendinitis . . . 570

- , after influenza . . . 572

Tendo Aehillis, rupture of . . . . 708

T'endons, contraeted in foals . . . 582 rupture of . . . 565

'Tendon sheaths, disease of, in tarsal and metatarsal regions . . .754

enlarged, opening by cautery . . of fore limb, sehema of . . 554

Tenotomy . . . . . 578

Testicle, eareinoma of . . . . 414

, eongenital defeets of $\quad \cdot \quad 407$

", diseases of $\quad \cdot \quad \cdot \quad \cdot \quad \cdot 413$

" retention of . . . 407

". tumours of . . . . 414

Testicles, supernumerary . . . 408

Thigh, diseases of . . . . . 699

, injuries of . . . . . 699

" lower, wounds of . 699

Thoraeic cavity, wounds of . . . 224

injuries, diagnosis of . . 224

Thoracocentesis . . . . . 244

Thorax, diseases of . . . . 217

,, puncture of . . . . 245

Thrombosis of the jugular vein . 157

$" \quad \begin{gathered}\text { posterior aorta and } \\ \text { branches }\end{gathered} .677$

Thyroid gland, results of extirpating . 152

Tibia, fracture of . . . . 700

Tibio-tibular interosseous ligament, rupture of . . . . . 704

Toe, bruises of the pads of $\quad . \quad$. 839

, wounds of the pals of . . 839

Tollgue, actinomyeosis of . . . 17

, acute inflammation of . 15

", chronic inflammation of . . 16

, diseases of . . . . 13

," meehanical injuries to . . 13

" new growth in the . $\quad 16$

," paralysis of . . . . . 19

,, strangulation of $\quad . \quad$. 12

," tumours in . $\quad . \quad$. $\quad . \quad 22$ 
Tooth clisels and rasps

, cutter

, forceps, Frick and Hauptrer's

", pickers Giunther's

, scalers

screw

"shears, Metznik's

", substance, diseases of .

T'orticollis

Trachea, deformities of .

diseases of

,. foreign bodies in

fractures of

parasites in

stenosis of

Tracheal canula, Barthèlemy's

$\begin{array}{lll}\text {," } & , & \text { double tube } \\ , & , & \text { Leblanc's } \\ , & \text { Peuch's } & \text { Pechar, Hayne's . }\end{array}$

,, tumoure

Tracheotome, Tl

Trachétomie sors cicon's

Tracheotomy

$\begin{array}{ll}, & \text { dangers of } \\ , & \text { ill effects of } \\ , & \text { tube, Coleman's } \\ & \text { tubes or canulæ }\end{array}$

'Tread"s of the coronet

'I'rendelenberg-canula

Trephines

Trephining instruments

,, position for cranial cavity in sheep or cattle.

facial sinuses

, superior maxillary and frontal simuses

Trochar and canula, special .

$$
\begin{aligned}
& \text { Bräuer's . } \\
& , \quad \text { Zpening by the } \\
& \text { Zehden's. }
\end{aligned}
$$

Tuberculosis of the udder

Tumours, actinomyces.

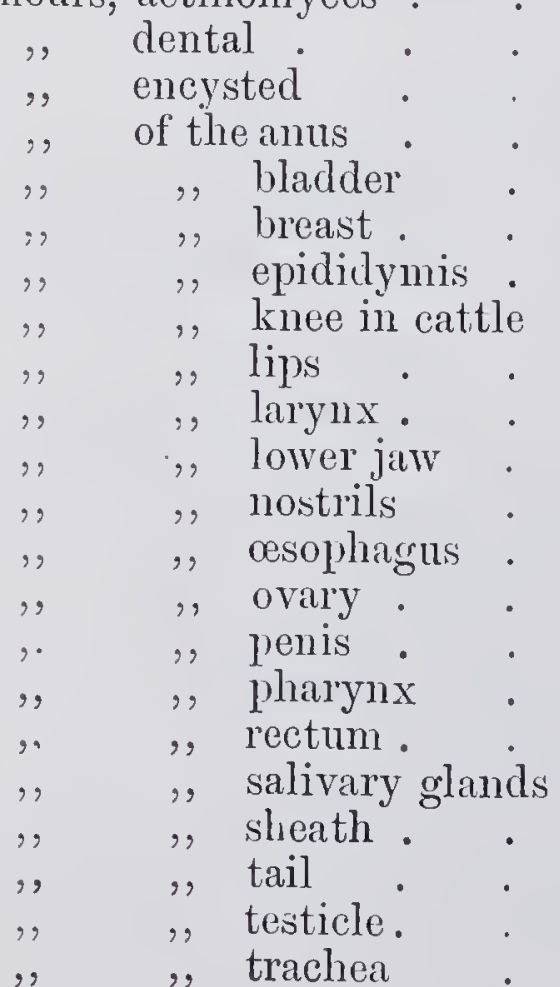

PAGE

32

49

48

50

41

37

36

35
Thmol PAGE

$\cdot . \quad \cdot 460$

",$\quad$ urethra . . . . 386

", uterus . . . . 436

Turbinated bones, excision of $\quad . \quad 436$

,, $\quad$, mucous degeneration

of . म . $\quad 73$

Twist of the colon . $\quad . \quad . \quad .330$

,", ,, method of detection : 331

"Tympanites "' operation for • • 332

Tympanitic colic. $. \quad . \quad .315$

\section{$\mathrm{U}$.}

UDDEk, actinomycosis of • . . 456

, , acute inflammation of $\quad . \quad$. 441

" bothryomycosis of . . . . 456

", bruises of . . . . 440

, catarrh of . . . . 447

", chronic inflammation of . $\cdot 455$

, cysts of . . . . 460

, gangrenous inflammation of . 451

, purulent inflammation of . . 449

,, tuberculosis of . . . . . 455

", tumours of . . . . 460

, wounds of . . . . $\quad .440$

Ulna, fracture of . . . . 526

Ulnar neurectomy $\quad . \quad \cdot \quad \cdot \quad \cdot 621$

Umbilical hernia . . . . . 273

", Bordonnat's clain for 278

,, $\quad$, Combe's clam for . 278

Urachus, pervious Degive's treatment • 275

• • , . 356

Urinary bladder, inflammation of . 380

$,, \quad, \quad$ inversion of . . 382

$, \quad,, \quad$ in female

. paralysis of animals 382

", paralysis of . . 377

$, \quad, \quad$ prolapse of . . 382

, , , in female

animals 382

, calculi • . • . . 357

, , composition of . . 357

,, in the $\operatorname{dog} . \quad . \quad .371$

, ",, horse $\quad . \quad 358$

,", swine . $\quad 370$

", organs, diseases of . . . 356

Uretlira, anatomy of, in ox . $\quad . \quad$. $\quad 366$

, inflammation of . . . 375

,$\quad$ injuries of . . . . $\quad .375$

, strictures of . . . . 375

, tumours of . . . . 386

Urethral calculi in the horse $\quad . \quad 359$

", , in ruminants . . 365

Urethrotomy, complications of, in herbivora. . . 370

in horses $\quad . \quad \therefore \quad 360$

in ruminants. $\quad . \quad 367$

in the sheep . . . 369

Uterine crutch, Günther's . . . . $\quad .433$

Uterus, inflammation of $\quad . \quad . \quad .424$

, injuries of . . . . . 424

, myxoma of . . . . $\quad . \quad 438$ 
INDEX:

terus, prolapse of

"Whistling" : . : : 188

", reduced by injection of water . . 435

Whitlow of the claw . . . : 826

Wind galls . . . . . 558

Withers, bruises of . . . . 241

Wooden ring, Stockfleth's • • 348

Working oxen, injuries in . . . 130

V.

VAGINA, cysts of . .436

inflanmation of . $\quad . \quad 424$

injuries of

" occlusion of . . . . $\quad .425$

prolanse of 428

,2 tumours of . . . . 436

wounds of . . . . 425

Vaginal ring, Sauberg's . . . . 430

, speculum . . . . 361

" trusses . . . . . 430

Vaginitis . . . . . 427

. . infectious, in cows . . 428

Varicocele . . . . 415

Venous fistulæ . . . . . 15 T

Ventral hernia . . . . . 501

Veratrin in impaction of the asophagus . . . . 169

subcutaneous injection of, in paralysis of suprascapnlar nerve

Wounds and bruises of the head

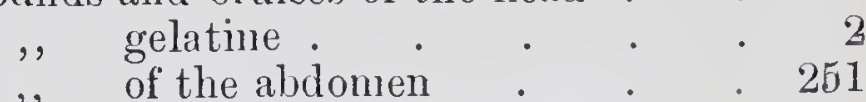

$, ", \quad$ ale of the nostrils . $\quad 59$

$"$ " chest-wall 222

.$\quad$, forearm . . . 537

," head . . . 1

$\because \quad$ ", lower thigh or leg. $\quad 699$

, niammary gland . . 440

". ". metacarpus . . . 562

, neck . . 138

" " $"$ pads of the sole and toe. 839

,,, pleura. . . . 224

", ". . . . 339

,, , salivary glands . . $\quad 76$

$\therefore$, shoulder joint 506

, $"$ skull . . . 129

" " soft tissue of the claw 825

" " thigh, lower. . . 699

", thoracic cavity 224

", ., tissues of the neck : 137

" udder. . . 440

" $"$ uterus . . . . 426

Vertebræ, dislocation of $: \quad \cdot \quad \cdot 141$

,e fractures of . $140,463,468$

, injuries to . . . . 466

Viborg's triangle . . . . . . 119

Villat's solution . . . . . . 220

Vulva, inflammation of. $\quad . \quad$. 424

, injuries to . . . . 424

", occlusion of . . . . . 424

W.

Walits

Wave-formed mouth

ZEHDEN's syringe and trochar for removal of coenurus cerebalis

THE END. 





\section{WILLIAM R. JENKINS'}

\section{BHTALOUUE OF VETERINARY BOOKS}

\section{4}

(*) Single asterisk designates New Books.

(**) Double asterisk designates Recent Publications.

ANDERSON. "Vice in the Horse" and other papers on Horses and Riding. By F. L. Anderson. Demy, 8 vo, cloth.............................2 00

- "How to Ride and School a Horse." With a System of Horse Gymnastics. By Edward L. Anderson. Cr. 8 vo ............................1 00

ARMSTEAD. "The Artistic Anatomy of the Horse." A brief description of the various Anatomical Structures which may be distinguished during Life through the Skin. By Hugh W. Armstead, M.D., F.R.C.S. With illustrations from drawings by the author. Cloth oblong, $12 \frac{1}{2} \times 10 \ldots \ldots \ldots \ldots \ldots \ldots . \ldots 35$

BACH. "How to Judge a Horse." A concise treatise as to its Qualities and Soundness; Including Bits and Bitting, Saddles and Saddling, Stable Drainage, Driving One Horse, a Pair, Four-in-hand, or Tandem, etc. By Captain F.W. Bach.

$12 \mathrm{mo}$, cloth, fully illustrated. 
$\left.{ }^{* *}\right)$ BANHAM. "Anatomical and Physiological Model of the Horse." Half life size. Composed of super. posed plates, colored to nature, showing internal organs, muscles, skeleton, etc., mounted on strong boards, with explanatory text. By George A. Banham, F.R.C.V.S. Size of Model $38 \times 41$ in...7 50

$\left.{ }^{(* *}\right)$ - "Tables of Veterinary Posology and Therapeutics," with weights, measures, etc. By Geo. A. Banham, F.R.C.V.S. New edition. 12mo, eloth........100

BAUCHER. "Method of Horsemanship." Including the Breaking and Training of Horses..........100

$\left.{ }^{*}\right)$ BELL. "'The Veterinarian's Call Book (Perpetual)," By Roscoe R. Bell, D.V.S., editor of the American Veterinary Review. Revised for 1903.

A visiting list, that can be commenced at any time and used until full, containing much useful information for the student and the busy practitioner. Among contents are items concerning: Veterinary Drugs; Poisons; Solubility of Drugs; Composition of Milk,Bile, Blood, Gastric Juice, Urine, Saliva; Respiration; Dentition; Temperature, etc., etc. Bound in flexible leather, with flap and pocket ........ 125

BRADLEY. "Outlines of Yeterinary Anatomy." By O. Charnock Bradley, Member of the Royal College of Veterinary Surgeons; Professor of Anatomy in the New Veterinary College, Edinburgh.

The author presents the most important facts of veterinary anatomy in as condensed a form as possible, consistent with lucidity. $12 \mathrm{mo}$.

Complete in three parts.

PART I.: The Limbs (cloth).................125

PART II.: The Trunk (paper) .............. 25

PART III.: The Head and Neck (paper).......... 25

THE SET COMPLETE .................... 50 
CADIOT. "Roaring in Horses." It,s Pathology and Treatment. This work represents the latest development in operative methods for the alleviation of roaring. Each step is most clearly defined by excellent full-page illustrations.' By P. J. Cadiot, Professor at the Veterinary School, Alfort. Trans. Thos J. Watt Dollar, M.R.C.V.S., etc. Cloth....75

- "Exercises in Equine Surgery." By P. J. Cadiot. Translated by Prof. A. W. Bitting, M.D., V.S. ; edited by Prof. A. Liautard, M.D.V.S. 8vo, eloth, illus-

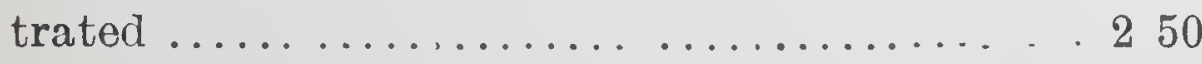

(")- "6 A Treatise on Surgical Therapentics of the Domestic Animals." By P.J. Cadiot and J. Almy. Translated by Prof. A. Liautard, M,D., V.S. 2 Parts ready.

Part I, Vol. I, 8vo, 93 pages, 45 illustrations......1 00 Part II, Vol., I, 8vo, 96 pages................1 00 Part III, Vol., I, 8vo, 134 pages, 33 illustrations..100 Part $I V$, in preparation, to be ready in 1903.

$\left({ }^{*}\right)$ - Clinical Veterinary Medicine and Surgery." By P.J. Cadiot. Translated, edited, and supplemented with 49 new articles and 34 illustrations by Jno. A. W. Dollar, M.R.C.V.S. Royal 8vo, 619 pages, 94 black and white illustrations..................5 25 See also "Dollar."

${ }^{(* *)}$ CHAPMAN. "Wanual of the Pathological Treatment of Lameness in the Horse," treated solely by mechanical means. By George T. Chapman. 8vo, cloth, 124 pages......................2 00

CHAUVEAU. "The Comparative Anatomy of the Domesticated Animals." By A. Chauveau. New edition, translated, enlarged and entirely revised by Geo. Fleming, F.R.C.V.S. 8vo, cloth, 585 illus.. 625 
CLARKE. "Chart of the Feet and Teeth of Fossil Horses." By W. H. Clarke...................25

CLEAVELAND. "Pronouncing Medical Lexicon." Pocket edition. Cloth....................75

CLEMENT. "Veterinary Post Mortem Examina. tions." By A. W. Clement, V.S. Records of autopsies, to be of any value, should accurately represent the appearances of the tissues and organs so that a diagnosis might be made by the reader were not the examiners' conclusions stated. To make the pathólogical conditions clear to the reader, some definite system of dissection is necessary. The absence in the English language, of any guide in making autopsies upon the lower animals, induced Dr. Clement to write this book, trusting that it would prove of practical value to the profession. $12 \mathrm{mo}$, cloth, illustrated. .75

(") COURTENAY. "Manual of the Practice of Veterinary Medicine." By Edward Courtenay, V.S. Revised by Frederick T. G. Hobday, F.R.C.V.S. Second edition. Crown, 8 vo, cloth.....................2 75

COX. "Horses : In Accident and Disease." The sketches introduced embrace various attitudes which have been observed, such as in choking; the disorders and accidents occurring to the stomach and intestines; affection of the brain; and some special forms of lameness, etc. By J. Roalfe Cox, F.R.C.V.S. 8vo, cloth, fully illustrated $. . . \ldots \ldots \ldots \ldots \ldots \ldots \ldots \ldots . \ldots 150$

CURTIS. "Horses, Cattle, Sheep and Swine." The origin, history, improvement, description, characteristics, merits, objections, etc. By Geo. W. Curtis, M.S.A. Superbly illustrated. Cloth, $\$ 200$; half sheep, $\$ 2.75$; half morocco................3 50 
$\left.{ }^{(*)}\right)$ DALRYMPLE. "Veterinary 0bstetrics." A compendium for the use of advanced students and Practitioners. By W. H. Dalrymple, M. R. C. V.S., principal of the Department of Veterinary Science in the Louisiana State University and A. \& M. College; Veterinarian to the Louisiana State Bureau of Agriculture, and Agricultural Experiment Stations; Member of the United States Veterinary Medical Associations, etc. $8 \mathrm{vo}$, cloth, illus..........2 50

DALZIEL. "The Fox Terrier." Illustrated. (Monographs on British Dogs). By Hugh Dalziel............1 00

— "The St. Bernard." Illustrated..................100

— "The Diseases of Dogs." Their Pathology, Diagnosis and Treatment, with a dictionary of Canine Materia Medica. By Hugh Dalziel. 12mo, cloth..........80

— "Diseases of Horses." 12mo, cloth.................100

- "Brenking and Training Dogs." Being concise directions for the proper education of dogs, both for the field and for companions. Second edition, revised and enlarged. Part I, by Pathfinder: Part II, by Hugh Dalziel. 12mo, cloth, illus ....2 60

- "The Collie." Its History, Points, and Breeding. By Hugh Dalziel. Illustrated, 8vo, cloth..........100

— "The Greyhound." 8vo, cloth, illus................1 0.0

$D A N A$. "Tables in Comparative Physiology." By Prof. C. L. Dana, M.D......................25

DANCE. "Veterinary Tablet." Folded in cloth case. The tablet of A. A. Dance is a synopsis of the diseases of horses, cattle and dogs, showing at a glance the causes, symptoms and cures ................75 
(*)DE BRUIN. "Bovine Obstetries." By M. G. De Bruin, Instructor of Obstetries at the State Veterinary School in Utrecht. Translated by W. E. A. Wyman, formerly Professor of Veterinary Science at Clemson A. \& M. College, and Veterinarian to the South Carolina Experiment Station.

$8 \mathrm{vo}$, cloth, 382 pages, 77 illustrations.........5 00

Synopsis of the Essential Features of the Work

1. Authorized translation.

2. The only obstetrical work which is up to date.

3. Written by Europe's leading authority on the subject.

4. Written by a man who has practiced the art a lifetime.

5. Written by a man who, on account of his eminence as 5. Written by a man who, on account of his eminence as
bovine practitioner and teacher of obstetrics, was selected by Prof. Dr. Fröhner and Prof. Dr. Bayer (Berlin and Vienna), to discuss bovine obstetrics, both practically and scicntifically.

6. The only work containing a thorough differential diagnosis of ante and post partum diseases.

7. The only work doing justice to modern obstetrical surgery and therapeutics.

8. Written by a man whose practical suggestions revolutionized the teaching of veterinary obstetrics even in the great schools of Europe.

9. 'The only work dealing fully with the now no longer obscure contagious and infectious diseases of calves.

10. Absolutely original and no compilation.

11. The only work dealing fully with the difficult problem of teaching obstetrics in the colleges.

12. The only work where the practical part is not overshadowed by theors.

- A veterinarian, particularly if his location brings him in contact with obstetrical practice, who makes any pretence towar being scientific and in possession of modern knowledge upon this subject, will not be without this excellent work, as it is really a very valuable treatise. It contains nearly 400 pages, numerous illustrations, and is put together in Jenkins' best style. - Prof. Roscoe $R$. Bell, in the American Veterinary Review.

In translating into English Professor De Bruin's excellent textbook on Bovine Obstetrics, Dr. Wyman has laid British and American veterinary surgeons and students under a debt of oratitude. The work represents the happy medium between the booklets which are adapted for cramming purposes by the student, and the ponderous adapted for cramming purposes by the student, and the ponderous to the requirements of the everyday practitioner. . It contains seventy-seven excellent illustrations . . Both translator and publisher have done their work in a way that deserves praise and we can strongty recommend the work to veterinary students and practi

This grand volume, written by Europe's leading authority on the subject, who has practiced the art for a lifetime, is the most recent and up-to-date obstetrical work. It discusses bovine obstetrics, both practically and scientifically, and contains thorough differential diagnoses of ante and post mortem diseases. It deals fully with the now no longel obscule contagious and infectious diseases of calves, and is the only work of the kind in which the practical part is not overshadowed by theary.-American Agriculturist, Aug., 190\%.

See also "Wyman." 
(*)DOLLAR. "Clinical Veterinary Medicine and Surgery." By l'. J. Cadiot. Translated, edited, and supplemented with 49 new articles and 34 illustrations by Jno. A. W. Dollar, M.R.C.V.S. Royal 8vo, 619 pages, 94 black and white illustrations......5 25 . This work, containing as it does the ripe experience of the author, who may be considered one of the foremost surgeons and clinicians of the day, contains a vast amount of exact scientific information of the utmost value to the busy workaday practitioner, while for the student of either human or compulative medicine, no better book could be placed in their hands, that will give them a clear insight into the many intricate problems with which they are daily confronted. . . - American Veterinary Review.

See also "Cadiot."

(*)_ "Operative Technique." For veterinary surgeons. Being the first volum $\theta$ of a new work on the prastice of veterinary surgery. By Jno. A. W. Dollar,

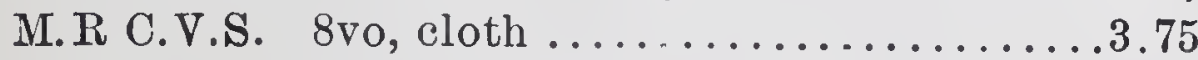

$\left({ }^{* *}\right)$ - "A Hand-book of Horse-Shoeing," with introductory chapters on the anatomy and physioiogy of the horse's foot. By Jno. A. W. Dollar, M.R.C.V.S., translator and editor of Möller's "Veterinary Surgery," "An Atlas of Veterinary Surgical Operations," etc.; with the collaboration of Albert Wheatley, F.R.C.V.S. 8vo, cloth, 433 pp., 406 illustrations ..4.75

_ "Roaring in Horses." By Prof. P. J. Cadiot. Translated by Dr. Dollar. Cloth, illustrated.............75 See also "Cadiot."

- "Operative Veterinary Surgery." By Prof. Dr. H. Möller. Translated by Dr. Dollar. 8 vo, cloth, illustrated. 
DUN. "Veterinary Medicines, their Actions and Uses." By Finlay Dun, V.S., late lecturer on Materia Medica and Dietetics at the Edinburgh Veterinary College, and Examiner in Chemistry to the Royal Coliege of Veterinary Surgeons. New (tenth) revised and enlarged English edition. 8vo, cloth.......3 75

The new volume has been carefully revised, adapted to the official British Pharmacopoeia, 1898, and brought up to date by Prof. James Macqueen, F.R.C.V.S., Royal Veterinary College, London. Clinical experiments and therapeutic observations-which form the basis of the rational treatment of disease-dilections for using mallein, tuberculin and Black-quarter vaccine, and all the more important recently introduced medicines have been added of diseases and remedies, suppla of diseases and remedies, supplemented by a copious index of medicines, will enhance the usefulness of the book to students and practitioners.

DWYER. "Seats and Saddles." Bits and Bitting, Draught and Harness and the Prevention and Cure of Restiveness in Horses. By Francis Dwyer. Illustrated. 1 vol., 12mo, cloth, gilt.............1 50

FLEMING. " Veterinary 0bstetrics." Including the Accidents and Diseases incident to Pregnancy, Parturition, and the Early Age in Domesticated Animals. By Geo. Fleming, F.R.C.V.S. Illustrated .....6 25

- "Operative Veterinary Surgery." Part I, by Dr. Geo. Fleming, M.R.C.V.S. This valuable work, one of the most practical treatises yet issued on the subject in the English language, is devoted to the common operations of Veterinary Surgery ; and the concise descriptions and directions of the text are illustrated with numerous wood engravings.

$8 \mathrm{vo}$, cloth...........................2 75

(*) Part II, containing nearly 500 pages and 400 illustrations, edited and passed through the press by Principal W. Owen Williams, F.R.C.V.S.

$8 \mathrm{vo}$, cloth.............................. 25 
FLEMING. "Tuberculosis." From a Sanitary and Pathological Point of View. By Geo. Fleming, F.R.C. V.S..................................25

- "The Contagious Diseases of Animals." Their in fluence on the wealth and health of nations. 12mo, paper...25

- "The Comparative Anatomy of the Domesticated Animals." By A. Chaveau. Translated by Dr. Fleming. 8vo, cloth, illustrated ...................6 25 See also "Chaveau."

- "Human and Animal Variolæ." A Study in Comparative Pathology. Paper.......................25

- Animal Plagues." Their History, Nature, and Prevention. By George Fleming, F. R. C. V. S., etc. First Series. 8 vo, cloth .................6 00 Second Series. 8 vo, cloth ................3 00

- "Roaring in Horses." By Dr. George Fleming, F.R.C.V.S. A treatise on this peculiar disorder of the Horse, indicating its method of treatment and curability. 8vo, cloth, with col. plates .....1 50

FLEMING-NEUMANN. "Parasites and Parasitic Diseases of the Domesticated Animals." A work to which the students of human or veterinary medicine, the sanitarian, agriculturist or breeder or rearer of animals, may refer for full information regarding the external and internal Parasites-vegetable and animal-which attack various species of Domestic Animals. A Treatise by L. G. Neumann, Professor at the National Veterinary School of Toulouse. Translated and edited by George Fleming, C. B., L.L. D.,F.R.C.V.S. 873 pages, 365 illustrations, cloth.7 50 
GRESSWELL. "Diseases and Disorders of the Horse." By Albert, James B. and George Gresswell.

Crown, 8 vo, illustrated, cloth............... 75

- Manual of "The Theory and Practice of Equine Medicine."

By James B. Gresswell, F.R.C.V.S., and Albert Gresswell, M.R.C.V.S., second edition, enlarged, 8 vo, cloth...........................2 75

- "Veterinary Pharmacology and Therapentics." By James B. Gresswell, F.R.C.V.S. 16mo, cloth ...1 50

- "The Bovine Prescriber." For the use of Veterinarians and Veterinary Students. By James B. and Albert Gresswell, M.R.C.V.S Cloth.............75

- "The Equine Hospital Prescriber." For the use of Veterinary Practitioners and Students. By Drs. James B. and Albert Gresswell, M.R.C.V.S. Cloth.....75

- "Veterinary Pharmacopæia, Materia Medica and Therapeutics." By George and Charles Gresswell, with descriptions and physiological actions of medicines. By Albert Gresswell.

Crown, 8vo, cloth......................2 75

GOTTHEIL. "A Manual of General Histology." By Wm. S. Gottheil, M.D., Professor of Pathology in the American Veterinary College, New York; etc., etc.

Histology is the basis of the physician's art, as Anatomy is the foundation of the surgeon's science. Only by knowing the processes of life can we understand the changes of disease and the action of remedies; as the architect must know his building materials, so must the practitioner of medicine know the intimate structure of the body. To present this knowledge in an accessible and simple form has been the author's task.

8vo., cloth, 148 pages, fully illustrated 
HASSLOCH. "A Compend of Veterinary Materia Medica and Therapenties." By Dr. A. C. Hassloch, V.S., Lecturer on Materia Medica and Therapeutics, and Professor of Veterinary Dentistry at the New York College of Veterinary Surgeons and School of Comparative Medicine, $\mathrm{N}$. Y. $12 \mathrm{mo}$, cloth, 225 pages ..1 50

HEATLEY. "The Stock Owner's Guide." A handy Medical Treatise for every man who owns an ox or cow. By George S. Heatley, M.R.C.V. 12mo, cloth...125

- “The Horse Owner"s Safeguard." A handy Medical Guide for every Horse Owner. 12mo, cloth.....150

- "Practical Veterinary Remedies." 12mo, cloth.......100

HILL. "The Management and Diseases of the Dog." Containing full instructions for Breeding, Rearing and Kenneling Dogs. Their Different Diseases. How to detect and how to cure them. Their Medicines, and the doses in which they can be safely administered. By J. Woodroffe Hill, F.R.C.V.S. 12mo, cloth, extra fully illustrated.......................200

(*)HILL. "The Diseases of the Cat." By J. Woodroffe Hill, F.R.C.V.S. 12mo, cloth, illustrated.......125

Written from the experience of many years' practice and close pathological research into the maladies to which our domesticated feline friends are liable-a subject which it must be admitted has not found not prominence in veterinary literature to which it is undoubtedly entitled.

HINEBAUCH. "Veterinary Dental Surgery." For the use of Students, Practitioners and Stockmen.

$12 \mathrm{mo}$, cloth, illustrated..................2 00

Sheep................................. 275 
HOARE. "A Manual of Veterinary Therapeutics and Pharmacology." By E. Wallis Hoare, F.R.C.V.S. 12mo, cloth, 560 pages......................2 75

"Deserves a good place in the libraries of all veterinarians. * * * Cannothelp but be of the greatest assistance to the young veterinarian and the every day busy practitioner."-American Veterinary Review.

(")HOBDAY. "Canine and Feline Surgery." By Frederick T. G. Hobday, F.R.C.V.S., Professor in Charge of the Free Out-Patients' Clinique at the Royal Veterinary College, London, The work contains 76 illustrations in the text. Demy 8vo, 152 pp., full-bound cloth.2 00

(**)HUNTING. The Art of Horse-shoeing. A manual for Farriers. By William Hunting, F.R.C.V.S., expresident of the Royal College of Veterinary Surgeons. One of the most up-to-date, concise books of its kind in the English language. 8vo, cloth, with nearly 100 illustrations...................100

(**)TENKINS. "Model of the Horse."..........750 See also "Banham."

(**)KOBERT. "Practical Toxicology for Physicians and Students." By Professor Dr. Rudolph Kobert, Medical Director of Dr. Brehmer's Sanitarium for Pulmonary Diseases at Goerbersdorf in Silesia (Prussia), late Director of the Pharmacological Institute, Dorpat, Russia. Translated and edited by L. H. Friedburg, Ph.D. Authorized Edition. 8vo, cloth.2 50

KOCH. “ "Etiology of Tuberculosis." By Dr. R. Koch. Translated by T. Saure. 8vo, cloth...........100 
KEATING. "A New Unabridged Pronouneing Dictionary of Medicine." By John M. Keating, M.D., LL.D., Henry Hamilton and others. A voluminous and exhaustive hand-book of Medical and scientific terminology with Phonetic Pronunciation, Accentuation, Ftymology, etc. With an appendix containing important tables of Bacilli, Microcci Leucomaines, Ptomaines; Drugs and Materials used in Antiseptic Surgery; Poisons and their antidotes; Weights and Measures; Themometer Scales; New Officinal and Unofficinal Drugs, etc., etc. $8 \mathrm{vo}, 818$ pages .....5 00

LAMBERT. "The Germ Theory of Disease." Bearing upon the health and welfare of man and the domesticated animals. By James Lambert, F.R.C.V.S. 8 vo. paper............................25

$\boldsymbol{L} \boldsymbol{A} \boldsymbol{W}$. "Farmers' Veterinary Adviser." A Guide to the Prevention and Treatment of Disease in Domestic Animals. By Prof. James Law. Illus., 8vo, cloth.3 00

$\left.{ }^{* *}\right) \boldsymbol{L E G G E . ~ " C a t t l e ~ T u b e r c u l o s i s . " ~ A ~ P r a c t i c a l ~ G u i d e ~}$ to the Farmer, Butcher and Meat Inspector. By T.M.' Legge, M.A., M.D., D.P.H., Secretary of the Royal Commission on Tuberculosis, 1896-98; author of "Public Health in European Capitals," and " Harold Sessions, F.R.C.V.S." Cloth................100

(*)LIAUTARD. " $A$ Treatise on Surgical Therapentics of the Domestic Animals." By Prof. Dr. P.J. Cadiot and J. Almy. Translated by Prố. Liautard.

Part I, Volume I.................. 100

Part II, " $\quad$....................1 00

Part III, “ $\quad$ "...................1 00

(Part IV in preparation.)

See also "Cadiot." 
LIAUTARD. "Median Neurotomy in the T'reatment of Chronic Tendinitis and Periostosis of the Fetlock." By C. Pellerin, late Repetitor of Clinic and Surgery to the Alfort Veterinary School. Translated with additional facts relating to it, by Prof. A. Liautard, M.D., V.M.

Having rendered good results when performed by himself, the author believes the operation, which consists in dividing the cubito-plantar nerve and in excising a portion of the peripherical end, the means of improving the conditions, and consequently the values of many apparently doomed animals. Agriculture in particular will be benefited.

The work is divided into two parts. The first covers the study of Median Neurotomy itself; the second, the exact relations of the facts as observed by the author. 8vo., boards.....................100

- "Manual of Operative Veterinary Surgery." By A. Liautard, M.D., V.M., Principal and Professor of Anatomy, Surgery, Sanitary Medicine and Jurisprudence in the American Veterinary College; Chevalier du Merite Agricole de France, Honorary Fellow of the Royal College of Veterinary Surgeons (London), etc., etc. $8 \mathrm{vo}$, cloth, 786 pages and nearly 600 illustrations.........................6 00

${ }^{*}$ )-- "Animal Castration." A concise and practical Treatise on the Castration of the Domestic Animals. The only work on the subject in the English language. Illustrated with 52 cuts, having a fine portrait of the author. A new revised and enlarged edition, 1902. $12 \mathrm{mo}$, cloth..........................200

The most complete and comprehensive work on the subject in English veterinary literature.-Ameriean Agri-

- "Exercises in Equine Surgery." By Prof. Dr. P. J. Cadiot. Translated by Prof. Bitting and edited by Dr. Liautard. 8vo, eloth, illustrated ..............250 See also "Cadiot." 
LIAUTARD. "On the Lameness of Horses." By A. Liautard, M.D.,V.S. ................... 50

- "How to Tell the Age of the Domestic Animal." By Dr. A. Liautard, M.D., V.S. Profusely illustrated. $12 \mathrm{mo}$, cloth .........................50

- "Vade Mecum of Equine Anatomy." By A. Liautard, M.D.V.S. Dean of the American Veterinary College. 12mo. cloth. New edition, with illustrations...200

— "Translation of Zundel on the Horse's Foot." Cloth ..2 00

LONG. "Book of the Pig." Its selection, Breeding, Feeding and Management. 8vo, cloth ........4 25

$\left.{ }^{(* *}\right)$ LOWE. $\quad{ }^{66}$ Breeding Racehorses by the Figure System." Compiled by the late C. Bruce Lowe. Editerl by William Allison, “The Special Commissioner," London Sportsman, Hon. Secretary Sporting League, and Manager of the International Horse Agency and Exchange. With numerous fine illustrations of celebrated horses. Quarto, cloth .....7 50

$\left.{ }^{*}\right) L U D L O W . \quad$ "Science in the Stable"; or How a Horse can be Kept in Perfect Health and be Used Without Shoes, in Harness or under the Saddle. With the Reason Why, Second American Edition. Enlarged and Exemplified. By Jacob R. Ludlow, M.D. Late. Staff Surgeon, U. S. Army. Paper, 166 pages..... 50

LUPTON. "Horses: Sound and Unsound," with Law relating to Sales and Warranty. By J. Irvine Lupton, F.R.C.V.S. 8vo, cloth, illustrated......1 25

- "The Horse." As he Was, as he Is, and as he Ought to Be. By J. I. Lupton, F.R.C.V.S. Illus., Crown, \&vo. .1 40 
MAGNER. "Facts for Horse Owners." By D. Magner. Upwards of 1,000 pages, illustrated with 900 engravings. $8 \mathrm{vo}$, leather binding. ..............750

MAYHEW. "The Illustrated Horse Doctor." An accurate and detailed account of the Various Diseases to which the Equine Race is subject; together with the latest mode of Treatment, and all the Requisite Prescriptions written in plain English. By E. Edward Mayhew, M.R.C.V.S. Illustrated.

8 vo, cloth.............................2 75

McBRIDE. "Anatomical Outlines of the Horse." $12 \mathrm{mo}$, cloth. Reduced to...................150

Mc COMBIE. "Cattle and Cattle Breeders." Cloth.....1 00

(")M'FADYEAN. "Anatomy of the Horse." Second edition completely revised. A Dissection Guide. By John M'Fadyean, M.B., B.Sc., F.R.S.E., Principal of the Royal Veterinary College, London.

$8 \mathrm{vo}$, cloth

This book is intended for Veterinary students, and offers to them in its 48 full-page colored plates 54 illustrations and excellent text, the most valuable and practical aid in the study of Veterinary Anatomy, especially in the dissecting room.

- "Comparative Anatomy of the Domesticated Animals." By J. M'Fadyean. Profusely illustrated, and to be issued in two parts.

Part I-Osteology; ready. Paper, 2.50; cloth...2.75 (Part II in preparation.) 
MILLS. "How to Keep a Dog in the City." By Wesley Mills, M.D., V.S. It tells how to choose, manage, house. feed, educate the pup, how to keep him clean and teach him cleanliness. Paper........25

MOLLER. "Operative Veterinary Surgery." By Professor Dr. H. Moller, Berlin. Translated and edited from the 2nd edition, enlarged and improved, by John A. W. Dollar, M.R.C.S.

Prof. Moller's work presents the most recent and complete exposition of the Principles and Practice of Veterinary Surgery, and is the standard text-book on the subject throughout Germany.

Many subjects ignored in previous treatises on Veterinary Surgery here receive full consideration, while the better known are presented under new and suggestive aspects.

As Prof. Moller's work represents not only his own opinions and practice, but those of the best Veterinary Surgeons of various countries, the translation cannot fail to be of signal service to American and British Veterinarians and to Students of Veterinary and Comparative Surgery.

1 vol., 8vo. 722 pages, 142 illustrations ......5 25

MORETON. "On Horse-breaking." $12 \mathrm{mo}$, cloth.... 50

MOSSELMAN-LIENAUX. "Veterinary Microbiology." By Professors Mosselman and Liénaux, Nat. Veterinary College, Cureghem, Belgium. Trans lated and edited by R. R. Dinwiddie, Professor of Veterinary Science, College of Agriculture, Arkansas State University. $12 \mathrm{mo}$, cloth, 342 pages......2 200 
NOCARD. "The Animal Tuberculoses, and their Relation to Human T'uberculosis." By Ed. Nocard, Prof. of the Alfort Veterinary College. Translated by H. Scurfield, M.D. Ed., Ph. Camb. 12mo, cloth, 143 pages....1 00

Perhaps the chief interest to doctors of human medicine in Professor Nocard's book lies in the demonstration of the small part played by heredity, and the great part played by contagion in the propagation of bovine tuberculosis. It seems not unreasonable to suppose that the same is the case for human tuberculosis, and that, if the children of tuberculosis parents were protected from infection by cohabitation or ingestion, the importance of heredity as a cause of the disease, or even of the predisposition to it, would $\mathrm{d}$ windle away into insignificance.

PEGLER. "The Book of the Goat." 12mo, cloth......175

PELLERIN. "Median Neurotomy in the Treatment of Chronic Tendinitis and Periostosis:of the Fetlock." By C. Pellerin, late repetitor of Clinic and Surgery to the Alfort Veterinary School. Translated, with Additional Facts Relating to It, by Prof. A. Liautard, M.D., V.M. $8 \mathrm{vo}$, boards, illustrated.............100

See also "Liautard."

PETERS. "A Tuberculous Herd-Test with Tuberculin." By Austin Peters, M. R. C. V.S., Chief Inspector of Cattle for the New York State Board of Health during the winter of 1892-93. Pamphlet...25

REYNOLD. "Breeding and Management of Draught Horses." 8 vo, cloth................... 40 
FOBERGE. "The Foot of the Horse," or Lameness and all Diseases of the Feet traced to an Unbalanced Foot Bone, prevented or cured by balancing the foot. By David Roberge. 8vo, cloth...........5 00

SEWELI. "The Examination of Horses as to Soundness and selection as to Purchase." By Edward Sewell, M.R.C.V.S. 8vo, paper............1 50

.... It is a great advantage to the business man to know something of the elements of law, and nobods ought either to buy or own a horse who does not know something about the animal. That something this book gives, and gives in a thoroughly excellent way....

-Our Animal Friends.

SMITH. "A Manual of Veterinary Physiology." By Veterinary Captain F. Smith, M.R.C.V.S. Author of "A Manual of Veterinary Hygiene."

Throughout this manual the object has been to condense the information as much as possible. The broad facts of the sciences are stated so as to render them of use to the student and practitioner. In this seeond edition-rewritten-the whole of the Nervous System has been revised, a new chapter dealing with the Development of the Ovum has been added together with many additional facts and illustrations. About one hundred additional pages are given. Second edition, revised and enlarged, with additional illus-

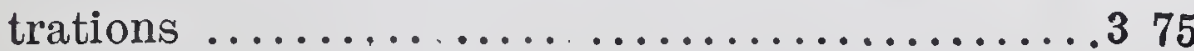

- "Manual of Veterinary Hygiene." 2nd edition, revised. Crown, 8 vo, cloth .......................3 25

(**)STRANGEWAT. "6 Veterinary Anatomy॰" Edited by I. Vaughan, F.L.S., M.R.C.V.S. New edition revised, with several hundred illustrations, $8 \mathrm{vo}$, cloth...50 00 
SUSSDORF. "Large Colored Wall Diagrams." By Prof. Sussdorf, M.D. (of Göttingen). Text translated by Prof. W. Owen Williams, of the New Veterinary College, Edinburgh. Size, 44 inches by 30 inches.

$$
\begin{array}{ll}
\text { 1.-Horse. } & \text { 4.-Ox. } \\
\text { 2.-Mare. } & 5 .- \text { Boar and Sow. } \\
\text { 3.-Cow. } & 6 .- \text { Dog and Bitch. }
\end{array}
$$

Showing the position of the viscera in the large cavities of the body.

Price, unmounted 175 each

" mounted on linen, with roller... ...3 50 “

$\left(^{*}\right)$ THOMPSON. "Elementary Lectures on Veterinary Science." For agricultural students, farmers and stock keepers. By Henry Thompson, M.R.C.V.S., lecturer on Veterinary Science at the Aspatria Agricultural College, England.

$8 v o$, cloth, 397 pages, 51 illustrations .......... 375

It is complete yet concise and just the up-to-date book for a beginner:

\section{$V A N$ MATER. "A Text Book of Veterinary 0ph-} thalmology." By George G. Van Mater, M.D., D.V.S., Professor of Ophthalmology in the American Veterinary College; Oculist and Aurist to St. Martha's Sanitarium and Dispensary; Consulting Eye and Ear Surgeon to the Twenty-sixth Ward Dispensary; Eye and Ear Surgeon, Brooklyn Eastern District Dispensary, etc. Illustrated by one chromo lithograph plate and seventy-one engravings.

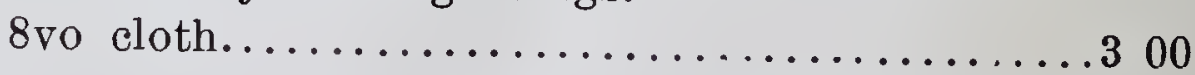

. . We intend to adopt this valuable work as a text book.-E. J. Creely, D.V.S., Dean of the San Francisco Veterinary College. May $16 \mathrm{th}, 1902$. 
VETERINARY DIAGRAMS in Tabular Form. Size, $28 \frac{1}{2}$ in. $x 22$ inches. Price per set of five...4 75

No. 1. "The External Form and Elementary Anatomy of the Horse." Eight colored illustrations1. External regions; 2. Skeleton; 3. Muscles (Superior Layer); 4. Muscles (Deep Layer); 5. Respiratory Apparatus; 6. Digestive Apparatus ; 7. Circulatory Apparatus ; 8. Nerve Apparatus ; with letter-press descrip-

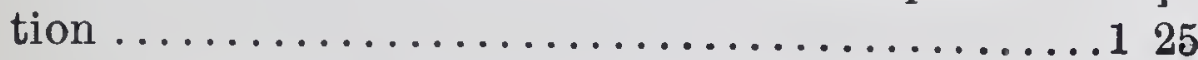

No. 2. "The Age of Domestic Animals." Forty-two figures illustrating the structure of the teeth, indicating the Age of the, Horse, Ox, Sheep, and Dog, with full description ..........................75

No. 3. "The Unsoundness and Defects of the Horse." Fifty figures illustrating-1. The Defects of Conformation; 2. Defects of Position; 3. Infirmities or Signs of Disease; 4. Unsoundnesses; 5 . Defects of the Foot; with full description...................... 75

No.4. "The Shoeing of the Horse, Mule and 0x." Fifty figures descriptive of the Anatomy and Physiology of the Foot and of Horse-shoeing............75

No. 5. "The Elementary Anatomy, Points, and Butcher's Joints of the Ox." Ten colored illustrations -1. Skeleton; 2. Nervous System; 3. Digestive System (Right Side); 4. Respiratory System ; 5 . Points of a Fat Ox ; 6. Muscular System; 7. Vascular System; 8. Digestive System (Left Side); 9. Butcher's Sections of a Calf; 10. Butcher's Sections of an Ox; with full description ......................... 25

WALLEY. "Four Bovine Scourges." (Pleuro-Pneumonia, Foot and Mouth . Disease, Cattle Plague and Tubercle.) With an Appendix on the Inspection of Live Animals and Meat.

Illustrated, 4to, cloth. 
(') W ALLEX. “A Practical Guide to Meat Inspection." By Thomas Walley, M.R.C.V.S., formerly principal of the Edinburgh Royial (Dick) Veterinary College: Professor of Veterinary Medicine and Surgery, etc. Fourth Edition, thoroughly revised and enlarged by Stewart Stockman, M.R.C.V.S., Professor of Pathology, Lecturer on Hygiene and Meat Inspection at Dick Veterinary College, Edinburgh.

12mo, cloth, with 45 colored illus., 295 pages....3 00

An experience of over 30 years in his profession and a long official connection (some sixteen years) with Edinburgh Abattoirs have enabled the author to gather a large store of information on the subject, which he has embodied in his book. Dr. Walley's opinions are regarded as the highest authority on Meat Inspection.

This fourth is an entirely re-written and enlarged edition, and while Dr. Stockman is indeed indebted to the old for much useful information, this up-todate work will hardly be recognized as the old "Walley's Meat Inspection." It will be found the most valuable work on the subject in the English language, has many illustrations, many of which are colored, and a complete index.

WILLIAMs. "Principles and Practice of Veterinary Medicine." Author's edition, entirely revised and illustrated with numerous plain and colored plates. By W. Williams, M.R.C.V.S.

8 vo., eloth........................6 00

- "Principles and Practice of Veterinary Surgery." Author's edition, entirely revised and illustrated with numerous plain and colored plates. By W. Williams, M.R.C.V.S.

$8 \mathrm{vo}$, eloth ......................6 00 
THE MOST COMPLETE, PROGRESSIVE AND SCIENTIFIC BOOK ON THE SUBJECT IN THE ENGLISH LANGUAGE

$\left.{ }^{*}\right)$ WINSLOW. "Veterinary Materia Medica and Therapeutics." By Kenelm Winslow, B.A.S., M.D.V., M.D., (Harv.); formerly Assistant Professor of Therapeutics in the Veterinary School of Harvard University; Fellow of tine Massachusetts Medical Society; Surgeon to the Newton Hospital, etc.

\section{Second Exition Revised.}

8 vo, cloth, 750 pages.................6 00

Your letter received and I am pleased to know that we are to have an American Materia Medica.-J.H. Wattles, Sr., M.D., D.V.S., The Western Veterinary College, Kansas City, Mo.

. Am delighted with it. It is remarkably correct, complete and up to date and is bound to supersede any other work on the same subject heretofore before the profession.

No practitioner's library is complete without it and it will be indispensable for students, as it does away with the necessity of their having a number of collateral books on the subject.

It will be adopted as the text book in the Chicago Veterinary College.-Dr. E. L. Quitman, Chicago Veterinary College.

. . The book is of admirable merit and full of valuable information from beginning to end, very explicit, rich and interesting, and should be in the hands of every student as well as practitioner of the art of Veterinary Medicine.-Thurston Miller, M.D., Professor of Materia Medica, Therapeutics and Chemistry, San Francisco Veterinary College.

. I I have found the book very satisfactory as a reference book to be used in connection with lectures. . - -Dr.W.A. Landacre, College of Pharmacy, Ohio State University.

I consider it the only work on materia medica and therapeutics suitable to the American veterinary practitioner. It deserves a wide distribution among veterinarians. I have recommended it to $\mathrm{my}$ students,-John J. Repp, V.M.D., Iowa State College, Ames, Iowa. 
(**)WYMAN. "The Clinical Diagnosis of Lameness in the Horse." By W. E. A. Wyman, V.S., formerly Professor of Veterinary Science, Clemson A. \& M. College, and Veterinarian to the South Carolina Experiment Station. 8vo, cloth, illustrated....2 50

(*) " "Bovine Obstetries." By M. G. De Bruin, Instructor of Obstetrics at the State Veterinary School in Utrecht. Translated by W. E. A. Wyman, M.D.V., V.S., formerly Professor of Veterinary Science, Clemson A. \& M. College, and Veterinarian to the South Carolina Experiment Station.

8 vo, cloth, 382 pages, 77 illustrations.........5 00

See also "De Bruin."

$\left({ }^{*}\right)$ - "Tibio-peroneal Neurectomy for the Relief of Spavin Lameness." By W. E. A. Wyman, M.D.V., V.S. $8 \mathrm{vo}$, boards, 30 pages.

Anyone wanting to perform this operation should procure this little treatise; he will find it of considerable help.-The
Veterinary Journal, Sept., 1902.

ZUNDEL. "The Horse's Foot and Its Diseases." By A. Zundel, Principal Veterinarian of Alsace Lorraine. Translated by Dr. A. Liautard, V.S. 12mo, cloth illustrated......................... 00

ZUTLL. "Typhoid Fever; or Contagious Infiuenza in the Horse." By Prof. W. L. Zuill, M.D.,D.V.S.

Pamphlet .............................25

Our Boolss are for sale by all booksellers, or will be sent prepaid for the prices here quoted. .8000

WILLIAIT R. JENKINS, 851 and 853 Sixth Avenue, NEW YORK 
Pexaluhting 


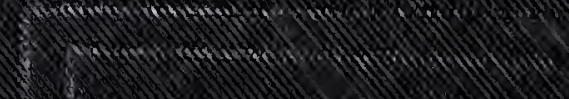

